
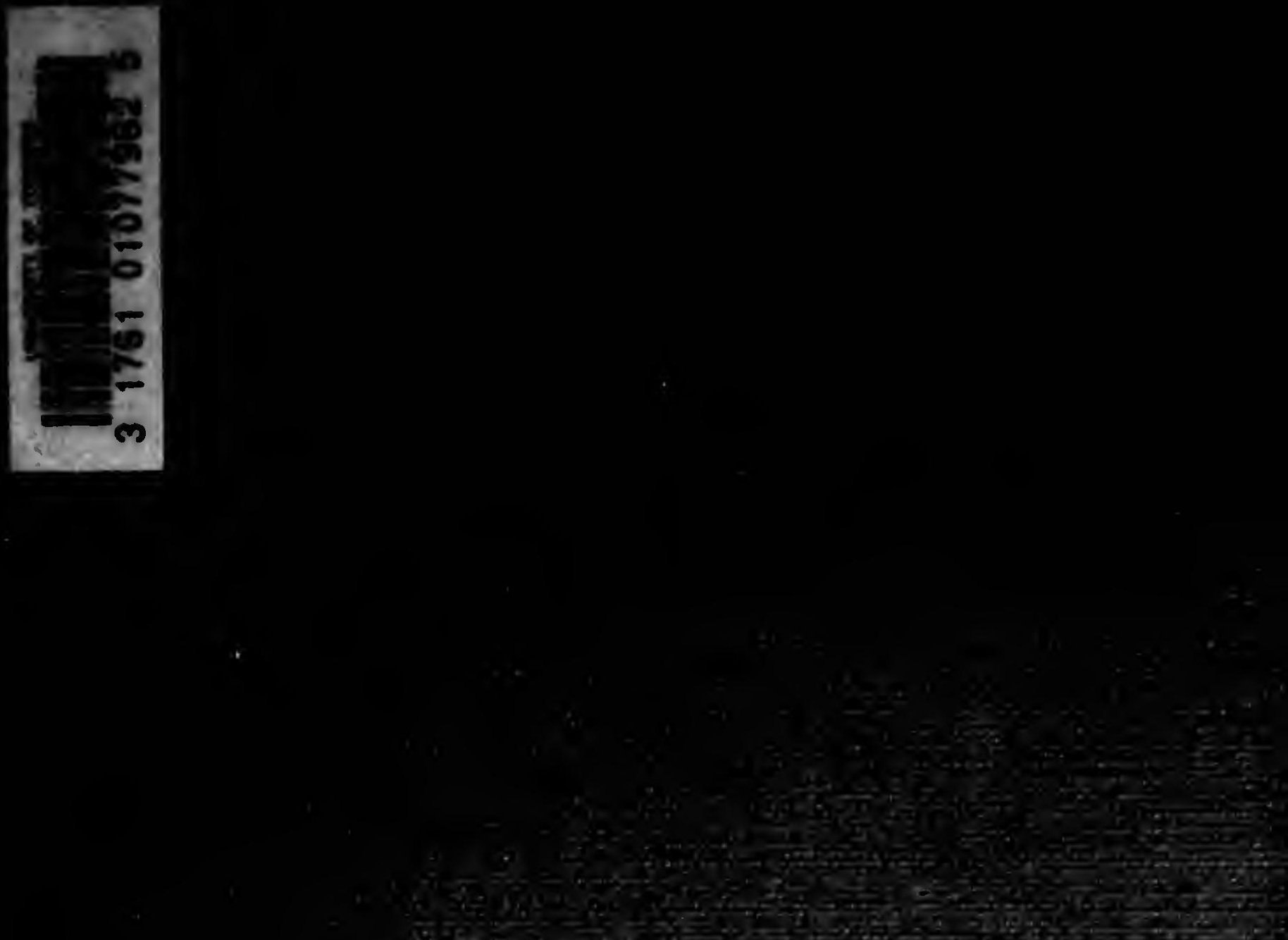

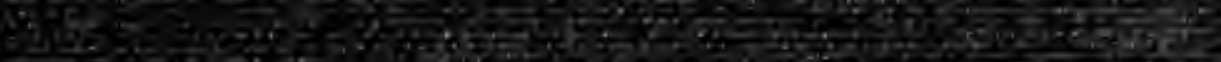

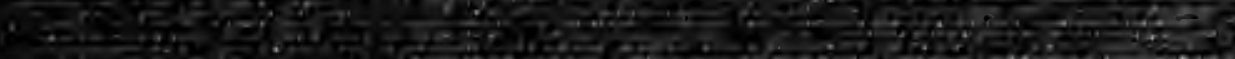
.

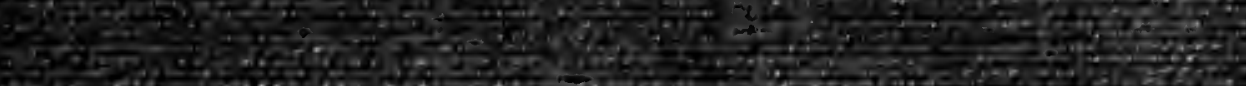
It

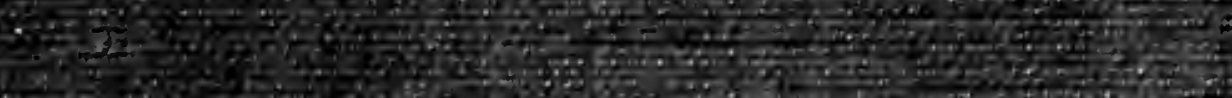
w. 2.

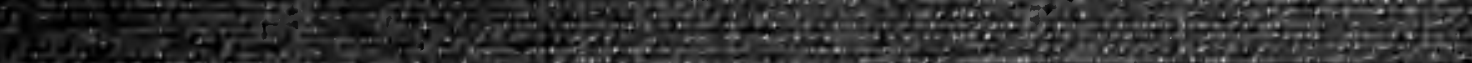
St

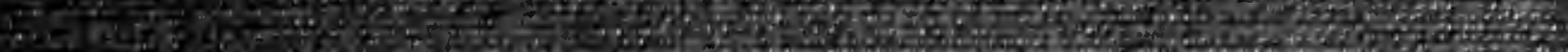

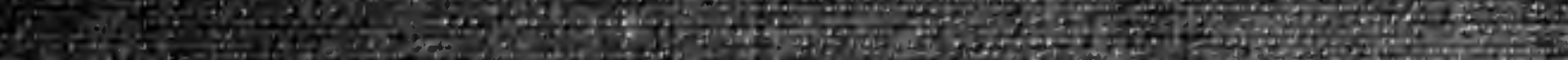

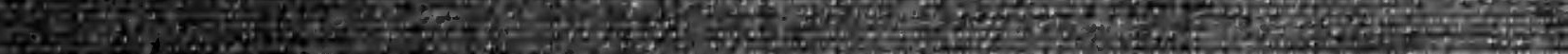

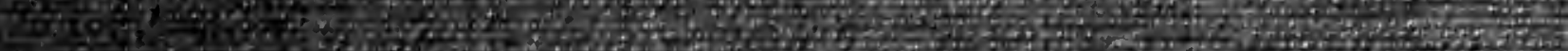

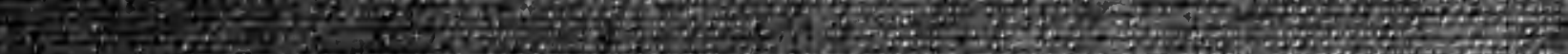
15.

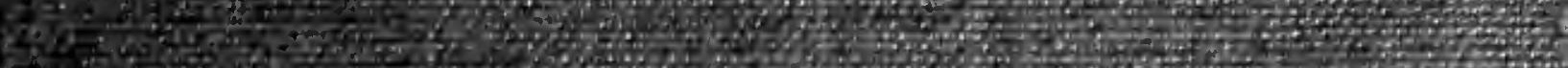
b t

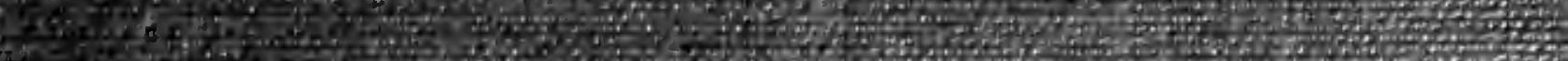
(2) r.

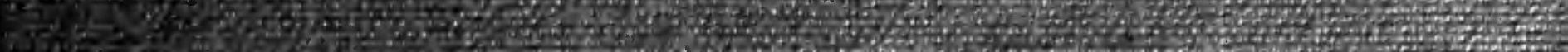

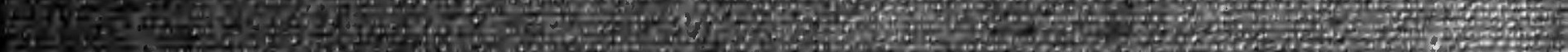

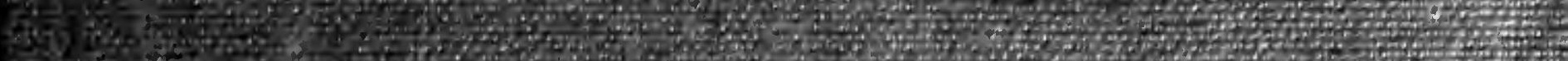
2. wer 2.1, S.

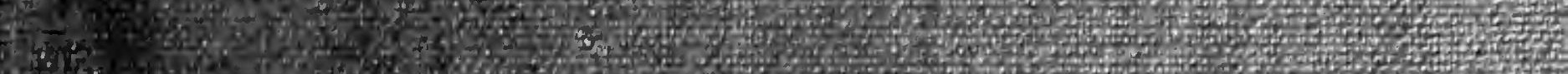
6.

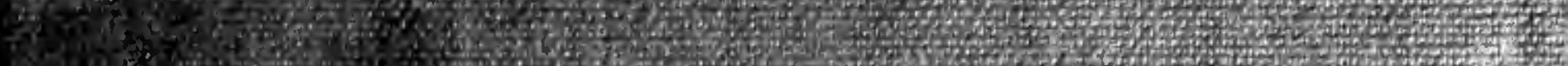
int

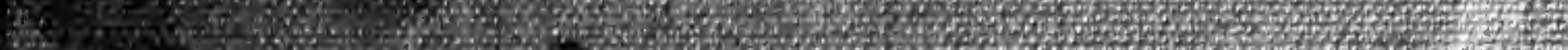
W.

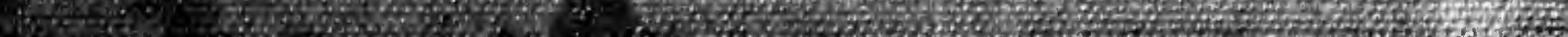






\section{PHOTOMETRIE DER GESTIRNE}

rON

\section{Prof. Dr. G. MÜLLER}

OBSERVATOR AM KÖNIGLICHEN ASTROPHYSIKALISCHEN OBSERVATORIUM ZU POTSDAM.

MIT 81 FIGUREN IM TEXT.

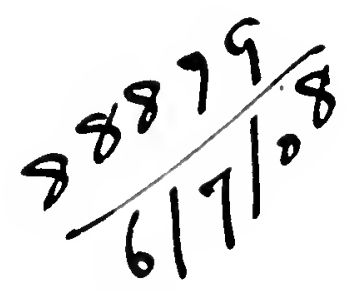

LEIPTIG

VERLAG VON WILHELM ENGELMANN

1897. 
Nlle Rechte, besonders das der Übersetzung, vorbehalten.

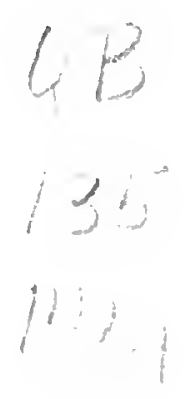




\section{VORWORT.}

$V_{\text {on }}$ den drei Hauptzweigen der hent allgemein mit dem Namen - Astrophysik a bezciehneten Diseiplin, welche nach Zöllners Definition als eine Vereinigung der Physik und Cliemie mit der Astronomie betrachtet werden kann, ist die $A$ stroph oto metrie bei Weitem der :ilteste. Während die Astrospectroskopie und die Astrophotographie erst in den letzten Jahrzehnten entstanden sind, fiihrt die Helligkeitsbestimmung der Gestirne ihren Ursprung bis zu den Zeiten des Ptolemä us zurilek. Merkwiirdiger Weise hat aber die Photometrie stets nur einen sehr beseheidenen Platz in der Astronomie eingenommen, und die Resultate; welehe auf diesem Gebiete bis in das gegenwärtige Jalhrhundert linein errungen worden sind, bleiben weit hinter den auf auderen Gebieten der Astronomie erreichten Erfolgen zuriick. Es scheint, als ob die allmähliche Loslösung von der alten Astronomie und die immer engere Vereinigung mit den beiden oben genannten jungen Seliwestern der Astrophotometrie zum Heil gereicht hätte, und als ob dieselbe erst dureh die Zufuhrung dieser nenen Krüfte aus einem gewissen Zustande der Erstarrung zu neuem Leben erweckt worden sei.

Trotz des hohen Alters der Himmelsphotometrie existirt bis jetzt ein eigentliches Lehr- oder Handbueh derselben noch nicht. Wir besitzen awar vortreffliehe Sehriften über die Photometrie in Allgemeinen, deren Studium nicht warm genug empfohlen werden kann, in dencu aber speciell den Liehterseheinungen am Himmel mur ein verhältnissmässigr geringer Raum gewidmet ist. Dahin gehören in erster Linie die classsisehen Werke von Bouguer und Lambert. Die "Photometriak des Letzteren ist neuerdings dureh die vortreffliehe Übersetzung Andings wieder in den Vordergrund des Interesses geriiekt worden, und der Werth des Buches ist noeh dadureh wesentlich erhöht, dass Anding in einer Reihe von kurzgefassten Anmerkungen einen Überllick iiber dic W'eiterentwieklung der P'hotometric seit den Zciten Lamberts hinzngefiigt und 

IV
Hahei unch die wichtigsten Fortschritte anf dem engeren Gebiete der
Himmelsphotometric herheksichtigt hat.

Iin lie Mitte des gerenwiirtigen Jihhrhunderts ist dureh die Arbeiten soidels und hesonders durch die geistrollen Schriften Zöllners ein lebhafteres lnteresse fir die lichterscheinmugen der Himmelskörper geweckt worden. Sicitdem hat dic heobachtende Thiitigkeit die erfrenlichsten Forfuchritte gemacht, nyd dass im Kusammenhange damit anch die theoretische Forschum nicht \%urtickgeblieben ist, beweisen nenerdings die zahlruindu Irlueiten Sceligers, die nach den verschiedensten Richtungen hin interiss:mte und vielversprechende Anshlicke eroffinet haben.

sulum limest durfte der Zeitpmukt gekommen sein; wo ein ausfiihrlindures Work filor len bisherigen lintwieklungsgang und den angenhirklichen Stamlpmukt der Astrophotometrie einen dringenden Bediurfnisse cutspricht. Der Wimseli, diese Liicke in der astronomischen Litteratur

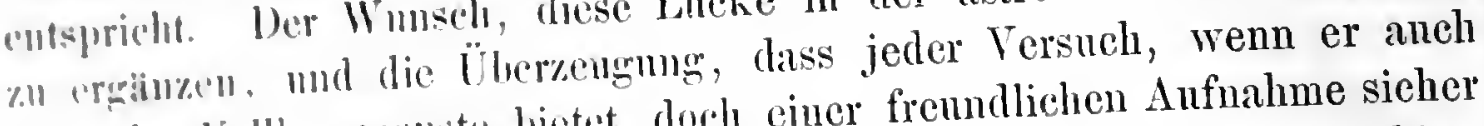

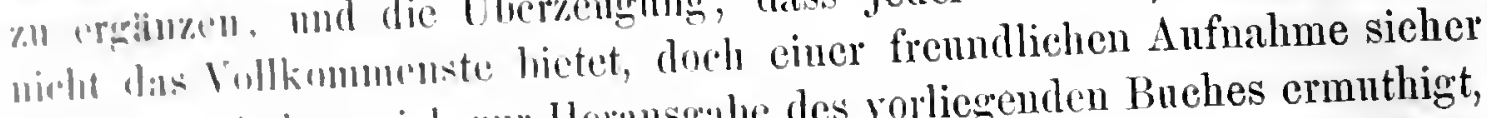
suin kimn. hilkn mich zur llerausgahe des vorliegenden Buches ermuthigt, whlar allen denjenizen, die sich mit der Lichtmessung der Gestirne heshifrigen whllw, als Wegweiser, und denjenigen, die mit diesem Ge-

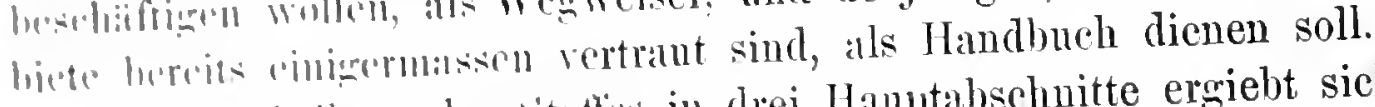

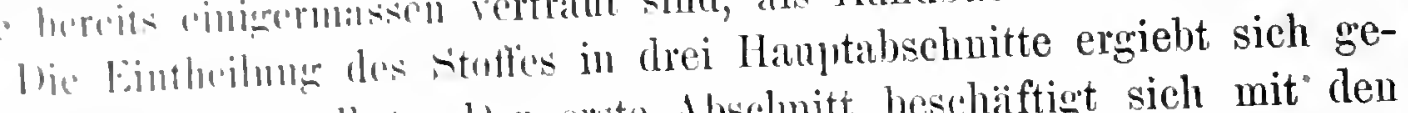

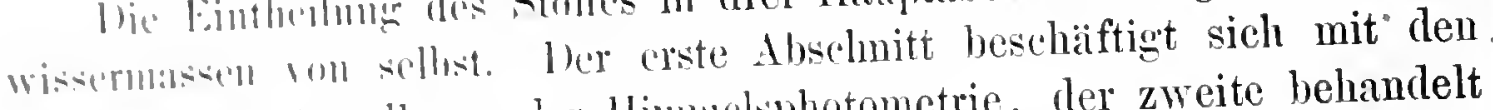

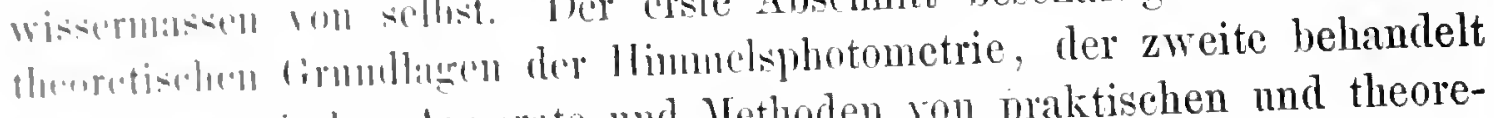

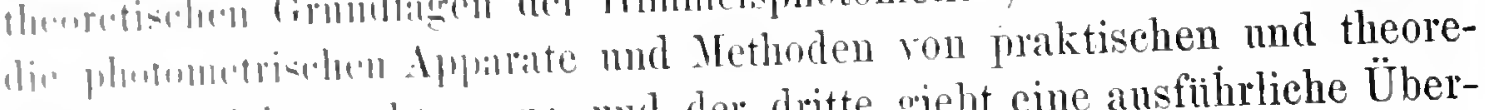

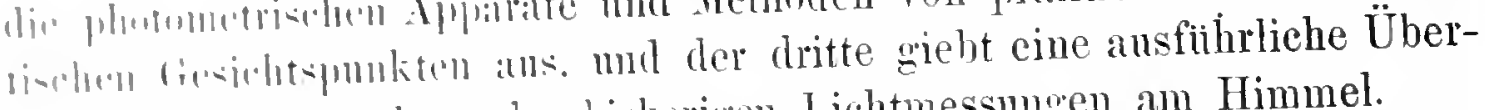
sinh Hur die lisultan du hisherigen Lichtmessmgen am Himmel.

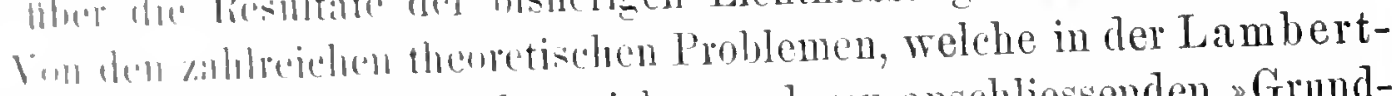

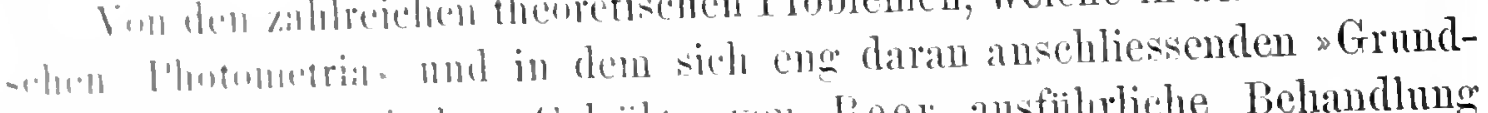

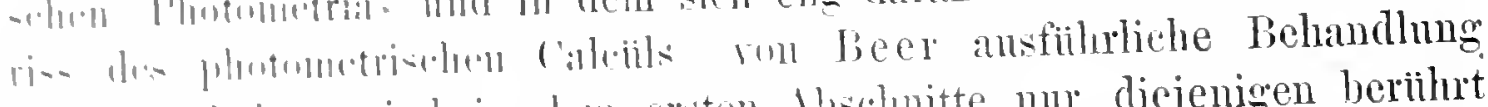

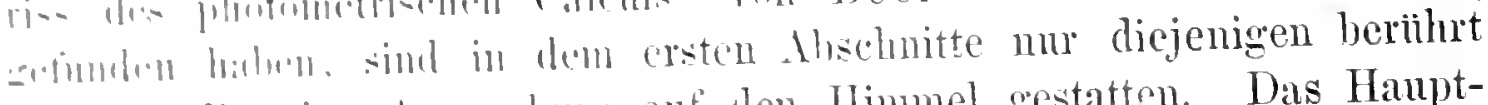

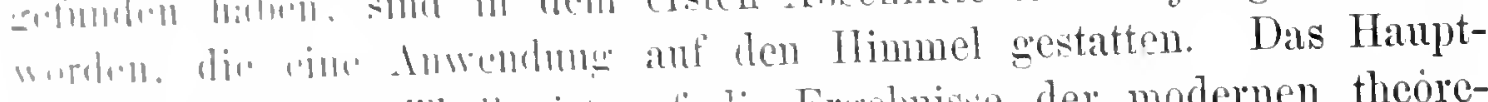

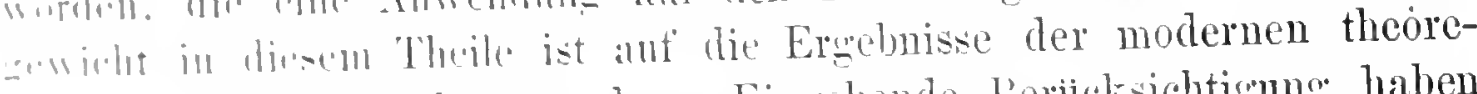

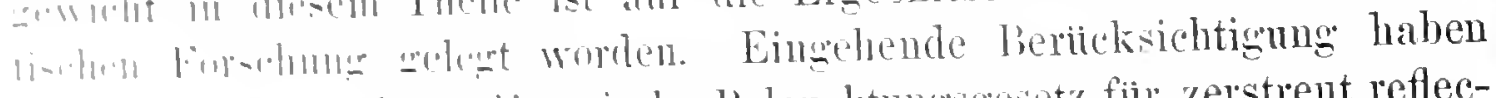

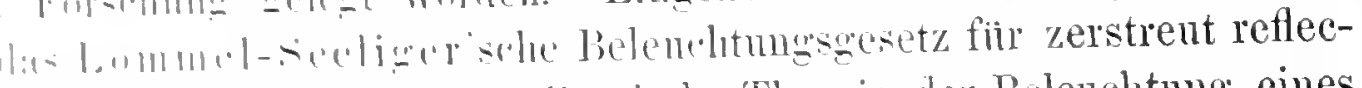

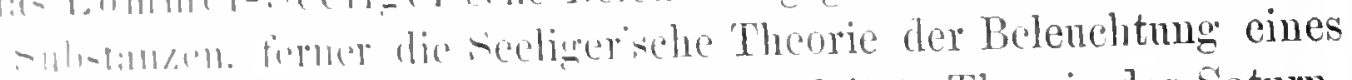

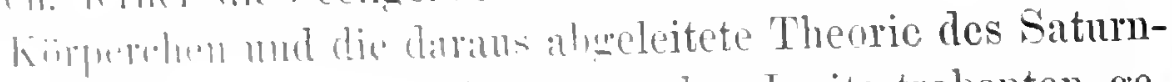

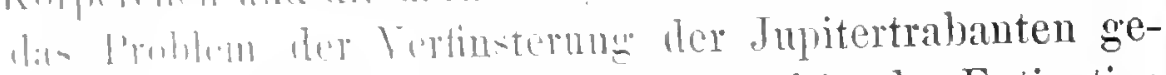

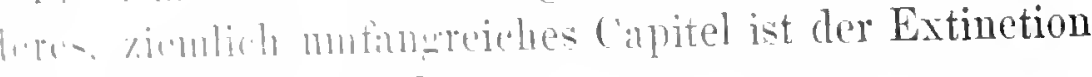

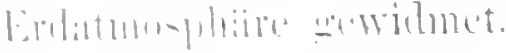

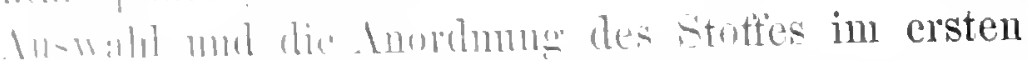

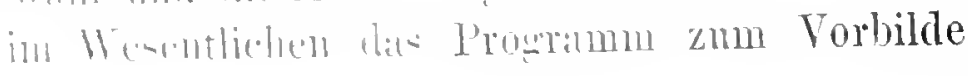


geuommen, welehes Herr Secliger in seinen Vorlesungen ther theoretische Astroplotometrie an der Munchener Universitait innezuhalten pflegt, und ich bin demselben fir die liobenswurdige Bereitwilligkeit, mit der er mich durch Rath ind 'That unterstutut hat, zu aufrichtigstem Danke verptliehtet.

Bei der Eintheilung des zweiten Abschnittes sind zmuichst die beiden grossen Gruppen der Auslöschungs- und der Gleichheitsphotometer vonoinander getrennt worden, und innerhalb jeder dieser Gruppen ist eine Reilie von Unterabtheilungen gewïhit, entsprechend den verschiedenen Methoden, welehe dic messbare Verïnderung der von einer Lichtquelle ausgehenden lebendigen Kraft ermögliehen. Natturlich .sind in erster Linie diejenigen Apparate und Messungsmethoden beriteksichtigt worden, die bereits .mit Erfolg zu Untersuchungen am Himinel angewandt wurden; doch ist es keineswegs vermieden, anch solche Photometer wenigstens kurz zu erwialmen, die bisher zwar nur im Laboratorium Verwendung gefunden haben, die sich aber vielleicht mit geringen Modificationen in /atkunft auch \%u Messungen am Himmel eignen diirften: Absolute Vollstiindigkeit wirl dabei wolıl schwerlich erreicht worden sein; doch hoffe ich, dass mir kein irgendwic wichtigeres Instrument entgangen ist. Die ausfiihrlichste Behandlung ist den drei hervorragendsten Messappariaten der modernen Himmelsphotometrie, den Keilphotometer, dem Züllnerschen Astrophotometer und dem Pickering'schen Meridianphotometer, zu Theil geworden. Es kam mir dabei nicht nur auf eine genaue Beschreibung der gebrïuchlichsten Formen dieser Apparate an, sondern es selien mir auch geboten, an den Vol\%iigen und Mängeln derselben sorgfiiltige Kritik zu iiben und anf Grund eigener Erfahrmugen Rathschlige zur zweckmässigsten Handhabung derselhen hinzuzufigen.

Die Spectralphotometer, die bisher zwar nur in beschriinktem Masse anf den Himmel angewendet worden sind, denen aber in Zukunft zweifellos eine wichtige Rolle zukommen wird, sind in einem Cappitel fiur sich behandelt, und in dem Schlusseapitel des zweiten Abschnittes ist noch anhangsweise eine knappe Ühersicht iiber diejenigen Apparate und Methorlen gegeben, welehe nieht zur Messung der physiologischen Intensitiit des Lichtes, sondern zur Feststellung der thermischen und chemischen Energie dienen, wobei namentlieh anf die photographische Photometrie Riicksicht genommen ist. Über die Berechtigung dieses letzteren Cilpitels liisst sich vielleicht streiten; fuir mich ist hei der Abfassmng desselben der Gedanke bestimmend gewesen, dass bei dem ersten Versuche einer Zusammenstellung aller zu Intensitiitsmessuugen am Himmel geeigneten Verfahren auch die eng damit in Beriihrung stehenden Gebiete nicht ganz unerwïhnt bleiben sollten, und dass hier vielleicht ein Überselıreiten der keineswegs sicheren Grenzen willkommener sein diirtte, als ein zu strenges Aussondern. 
In dem dritten Alsschnitte ist das Capitel iber die Fixsterne bei Weitem das umfangreichste geworden. Es ergab sich dies ganz naturgemaiss daraus, dass auf diesem Gebiete, namentlich in Betreff der verinderlichen Sterne, die Astrophotometric bisher relativ die meisten Erfolge :ufauwcisen hat. Die verschiedensten Helligkeitscataloge, sowohl die anf Schịt\%nngen, als die auf Messungen beruhenden, sind in historischer Reihenfolge kritisch besprochen und die Beziehungen zwischen ihnen erürtert worden.

Bei den verinderlichen Sternen konnten natiirlich nur die Hauptverteter der cimzehen Gruppen etwas näher besprochen werden. Die urspringlich geplinte Mittheilunğ. cines Verzeichnisses der sämmtlichen gergenwiirtig als sicher erkannten Veränderlichen mit den Elementen ihres lichtwechsels ist schliesslich unterblieben, weil der ganz kürzlich ershichene neneste Citalog ron Chandler in dieser Beziehung allen Anfordermigen entisprechen diirfte.

Bri den iibrigen Capiteln des dritten Abschnittes, mit Ausnahme höehstons lesjonimen iher die Planeten mul Satelliten, war von vornherein durch don Mancel an anseichendem Beobachtungsmaterial ein geringer Umfang Feboten. Dor Inhalt dieser Capitel zeigt recht dentlich, wie wenig auf manchen Cielieten der Astrophotometrie bisher geleistet worden ist, und

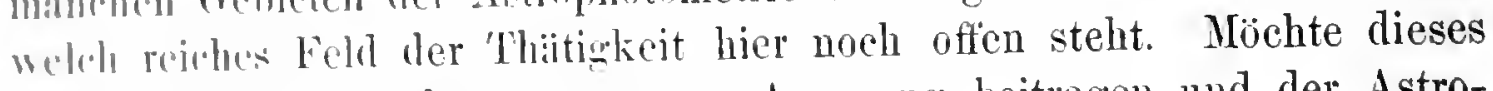
Bun in dicser Bezichung etwats zur Anregmug beitragen und der Astroflummutrie nene, Fremole zufialiren!

Im Luhanle ist eine ansfiilnliche Tafel der nach den verschiedenen beholuturathenrien herechneten Phasencorrectionen mitgetheilt, welche hei l'utersulnungen ibler die Ilelligkeit von Planeten und Trabanten von Mirth s sin kam: ferner sind die mittleren Extinctionstabellen für Potsdam

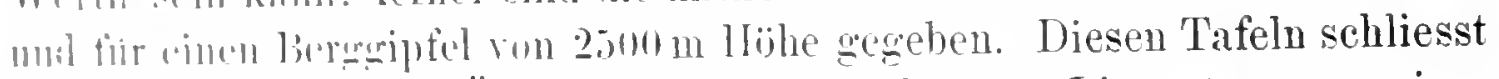

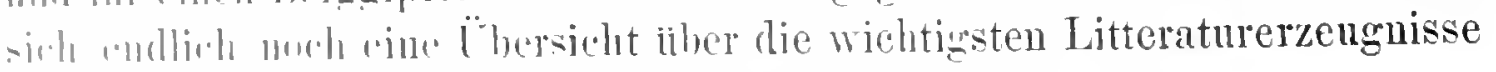
: Inf Alun

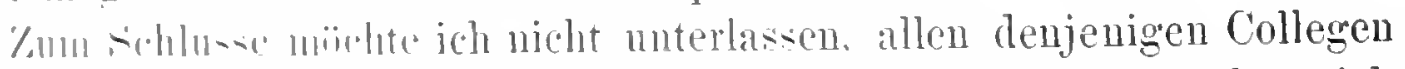
mnd livenum meinen verhindlinsten Damk auszusprechen, welche mich

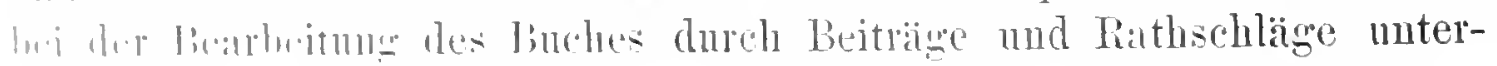

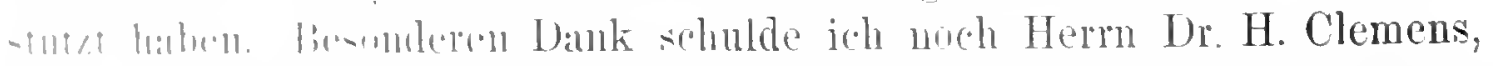

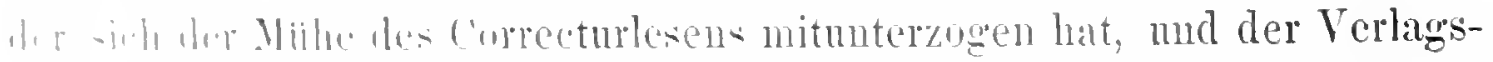

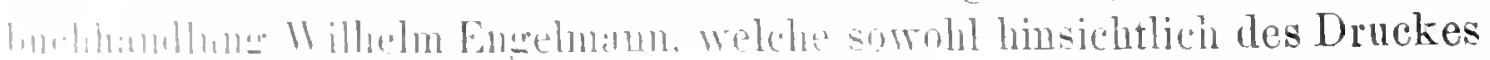

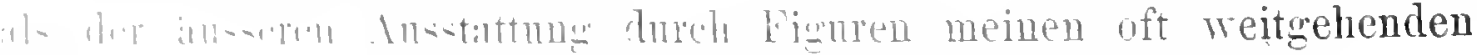

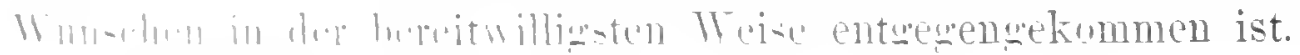
YI: $11 \% ! 17$.

(i. Hiiller. 


\section{INHALTSVERZRICHNISS.}

\section{Abschnitt.}

\section{Grundzïge der theoretischen Astrophotometrie.}

Capitel I. Die photometrischen Inaptgesetze

1. Allgemeine Definitionen . . . . . . . . . . . . . . . 3

2. Das Gesetz vom Quadrate der Entfernung. . . . . . . . . . . . 6

3. Zusammensetznng der von mehreren letichtenden Pankten ausgehenden Lichtbewegungen

4. Die physiologische Intensitït und das Fechner'sche psychophysische Gesetz.

5. Beleuchtung von Flïchen durch leuchtende Punkte. Das Gesetz vom Cosinus des Incidenzwinkels . . . . . . . . . . . . . . . .

6. Belenchtung von Flächen durch leuchtende Flïchen. Das Lambert'sche Gesetz vom Cosinus des Emanationswinkels.

7. Zerstreut reflectirende Substanzen. Die Bouguer'sche Reflexionstheorie. Das Lommel-Seeliger'sche Beleuchtungsgesetz. . . . . . . . . . . 35

8. Begriff der Albedo. . . . . . . . . . . . . . . . . 52

Capitel II. Anwendung der photometrisclien Grundprineipien auf die wichtigsten Anfgaben der Himmelsplotometrie. . . . . . . . . . . . . . . . 56

1. Beleuchtung der Planeten und Monde. . . . . . . ...... 56

a. Berechnung der von den Phasen eines beleuchteten Himmelskörpers nach der Erde gesandten Lichtmenge. Bestimmung der Albedo . 58

b. Die Lichtvertheilung auf einer Planetenscheibc . . . . . . . . 67

c. Mittlere scheinbare Helligkeit eines Planeten . . . . . . . . 78

d. Beleuchtnng der Planetentrabanten . . . . . . . . . . . . 79

e. Berechnung des aschfarbenen Mondlichtes . . . . . . . . . 82

2. Beleuchtnng eines Systems kleiner Körper. Die Secliger'sche 'l'heorie des Saturnringes. . . . . . . . . . . . . . . 86

3. Die Verfinsterangen der Jupitersatelliten . . . . . . . . . . 101

Capitel III. Die Extinction des Lichtes in ler Erdatmosphïre . . . . . . . . 110

1. Die Lambert'sche Extinctionstheorie . . . . . . . . . . . . . . 112

2. Die Bonguer'sche Extinctionstheoric . . . . . . . . . . . . . 116

3. Die Laplace'sche Extinctionstheorie. . . . . . . . . . . . . . . . . 122

4. Die Maurer'sche Extinctionstheorie . . . . . . . . . . . . . 128

5. Vergleichung der Theorien mit den Beobachtungsergebnissen. Die Durcllïssigkeitscoefficienten der Erdatmosphïre . . . . . . . . . . . 131

6. Die selective Absorption der Atmosphüre. Die Langley'schen Untersuchungen. . . . . . . . . . . . . . . . . 139 


\section{Alschnitt. Die photometrischen Apparate.}

Finloitun:

lapifel I. Photometer, hei llenen das Versehwinden von Lichteindriicken beobachent wirl

1. Ausliselıung des Lichtes dureh Jitendvorrichtungen . . . . . . . 157

^. Blenden vor dem Ohjectiv. Die Photometer von Köhler, Reissig, Dawes, linmbel, 'llhry, Lamont. • D Photometer von Hirseh,

1. Blenden \% wischen Ohjectiv und Oentar. Dio Photoneter von Hirseh, 175 Уашен, Іаешу. . . . . . . . . . 177

c. 1) Parkhnrst'sche Deflectionsphotometer Medien. . . . . . . . . 180

2. Auslizchung des lichtes Hureh absortirente Mor, Qmetelet, Albert. . . . 180

Capilal H. Photometr. hei lenen lin (ileichloit zweier Lichteindricke be-

"rehoilt wipl bes photometriselien Inuptgesetzes vom Quadrate der Ent-

forsung. . . . . . . . . . 195

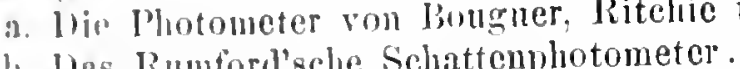

1. Mas Rumforl'sche Schatteuphotometer. . . . . . . . 199

c. Has limscrisehe Fleckplotometer . . . . . . . . . . 200

(1) 1:14 1lersehel'sche Astrometer........... 204

c. Mas Stcinheil'sche l'rismenphotometer . . . . . . . . . . 210

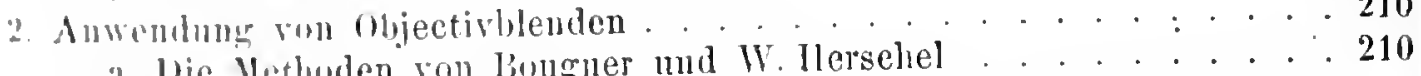

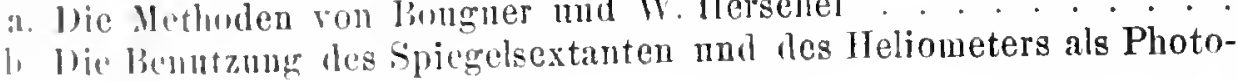
meter

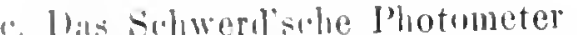

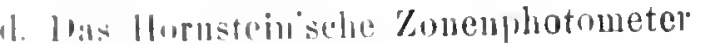

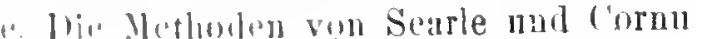

: Anwomlung ron rotiremden scheiben. Die photometrischen Methoden run 'Talhor, secehi. Almey

4. Anwmilnug von spiegehiden Kingeln. Die photumetrisehen Methoden

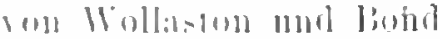

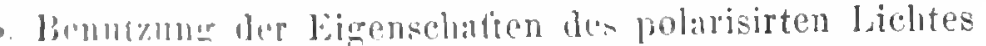

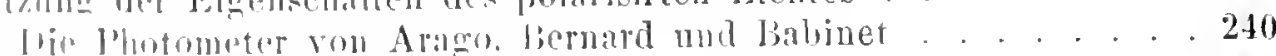

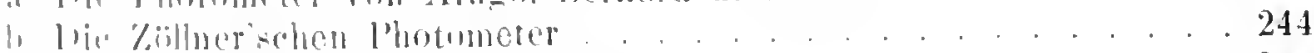

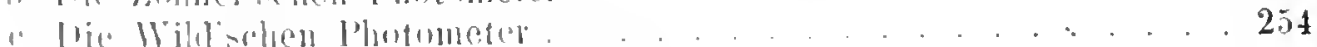

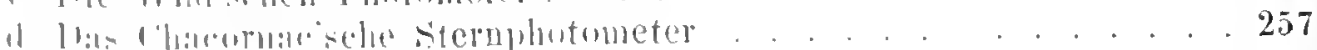

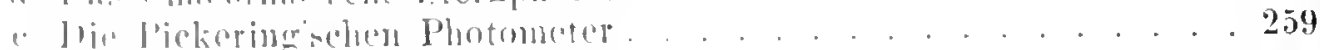

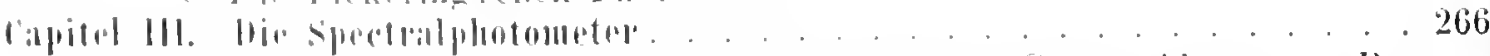

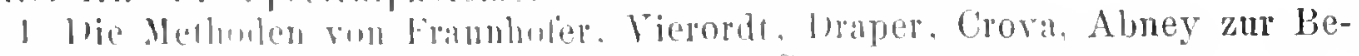

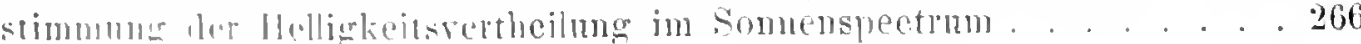

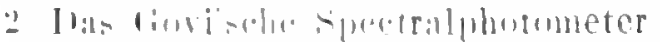

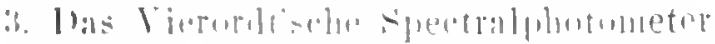

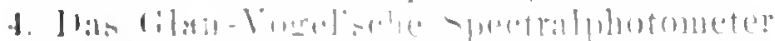

5. Dat (

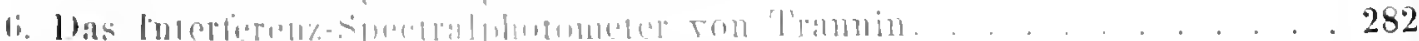

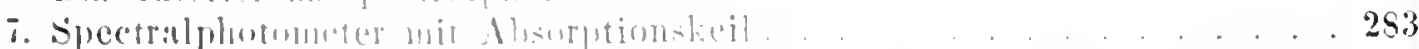

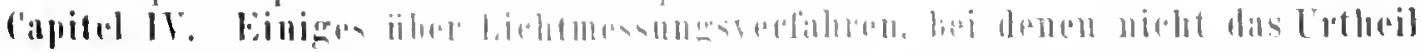

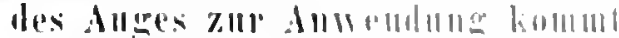

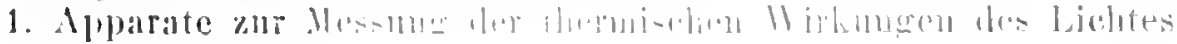


Dio wichtigaten Actinometor

D. Das

b. Das Langloy'scho Bolouneter . . . . . . . . . . . . 290

c. Das Boys'sche Radiomikromoter. . . . . . . . . . 200

d. Das Crookes'Bcho Radiometer und das Zibllner'sehe Sealenphotometer 291

2. Apparate zur Messang ler chemischen Wirkungen des Lichtes . . . . . 292

a. Dus chemische Photometer ron Bunsen und Roscoe . . . . . . . 292

b. Das Selenphotometer. . . . . . . . . . . . . . . . 293

c. Die Plotographio als photometrisehes Hulfsmittel . . . . . . . . 294

\section{Abschnitt.}

\section{Resultate der photometrischen Beobachtungen am Himmel.}

Capitel I. Die Sonne. . . . . . . . . . . . . . . . . . 30i

1. Das Licht der Sonne verglichen mit anderen Lichtquellen . . . . . 308

a. Sonne nnd kilnstliches Licht . . . . . . . . . . . . . 30s

b. Sonne und Vollmond. . . . . . . . . . . . . . . . . .312

c. Sonne und Fixsterne . . . . . . . . . . . . . . 316

2. Die Vertheilung der Helligkeit anf der Sonnenscheibe. . . . . . . . 318

3. Die Helligkeit der Sonnencorona. . . . . . . . . . . . . . . . . . 32!

Capitel II. Der Mond. . . . . . . . . . . . . . . . . . . . . . . . . 335

1. Das Licht des Mondes verglichen mit anderen Lichtquellen . . . . . 336

a. Mond und künstliches Licht . . . . . . . . . . . . 336

b. Mond verglichen mit Planeten und Fixsternen . . . . . . . . 339

2. Die Lichtstärke der Ioudphasen . . . . . . . . . . . . 340

3. Die Albedo des Mondes und die Vertheilnng der Helligkeit auf der Mondseheibe. . . . . . . . . . . . . . . . 343

Capitel III. Die Planeten und ilıre Trabanten . . . . . . . . . . . . . . 347

1. Mercur . . . . . . . . . . . . . . . 350

2. Venus. . . . . . . . . . . . . . . . . . . . . . . . . .

3. Mars . . . . . . . . . . . . . 369

4. Die Marstrabanten. . . . . . . . . . . . . . . . . . . 373

5. Die kleinen Planeten . . . . . . . . . . . . . . . . . . . 375

6. Jupiter . . . . . . . . . . . . . . . . . . . . . . . . . 381

7. Die Jupitersatelliten . . . . . . . . . . . . . . . . . 355

8. Saturn . . . . . . . . . . . . . . . . . . . . . . 393

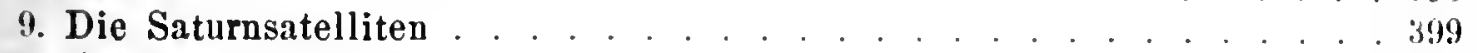

10. Uranus . . . . . . . . . . . . . . . . . . . . 401

11. Die Uranussatelliten . . . . . . . . . . . . . 403

12. Neptun . . . . . . . . . . . . . . . . . . . 4115

13. Der Neptunsatellit. . . . . . . . . . . . . 406

Capitel IV. Die Cometen nnd Nebelflecke . . . . . . . . . . . . . 407

Capitel V. Die Fixsterne . . . . . . . . . . . . . . . . . . . . . . . . . .425

1. Die Helligkeitsverzeichnisse der Fixsterne . . . . . . . . . 425

a. Helligkeitsverzeichnisse, welche auf Grössenschïtzungen beruhen 429

b. Irelligkeitsverzeichnisse, welche ans photometrischen Messungen hergeleitet sind . . . . . . . . . . . . . 443

c. Beziehungen zwischen den Grössenschätzungen und den photometrischen Messungen . . . . . . . . . . . . . . 454

2. Die verïnderlichen Sterne . . . . . . . . . . . . 459

a. Die temporären oder nenen Sterne . . . . . . . . . 472

b. Die Veriinderlichen ron langer Periode . . . . . . . . . 4s1

c. Die nnregelmässig Veriinderlichen . . . . . . . . . . . . 45.) 
d. Die regelmiissig Verïnderlichen von knrzer Periode. Der Lyra'Typns

c. Die Verinderlichen vom Algol-Typus

3. Dio spectralphotometrischon Beobachtungen der Fixsterne . . . . . 500

1. Die photogruphischen Ilelligkeiten der Fixsterne . . . . . . . 505

\section{Auliang.}

I. Tafel ther nach den Theorien von Lambert, Lommel-Seeliger und Euler herechueten, vom l'hasenwinkel abhiingigen Reduetionen auf volle $\mathrm{Be}$ lenchtung eines Plancten filr Potsdam (Meereshöhe $100 \mathrm{~m}$ ) und für den

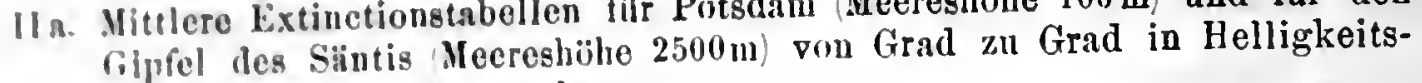
logarithasen und Grösenclassen

IIb. Nittlere lixtinctionstabelle fïr Potsdaun zwischen $50^{\circ}$ und $88^{\circ}$ Zenithdistanz ion Zelintel zu Zehntel Grad in Helligkeitslogarithmen . . . . . . . 516

III. litteraturverzeichniss . . . . . . . . . . . . . . . . 517

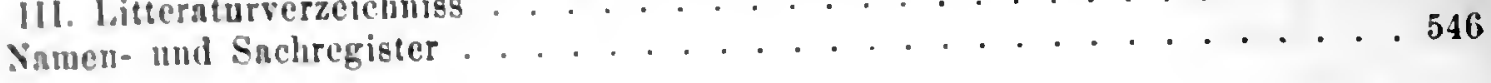




\title{
I. ABSCHNITT.
}

\author{
GRUNDZÜGE
}

DER

\section{THEORETISCHEN ASTROPHOTOMETRIE.}





\section{Capitel I. \\ Die photometrischen Hauptgesetze.}

\section{Allgemeine Definitionen.}

Die Undulationstheorie des Lichtes geht bekanntlieh von der Anschauung aus, dass ein sehr elastisches Medinm von ausserordentlich geringer Dichtigkeit, der sogenannte Lichtäther, den ganzen Weltraum sowohl als auch die Zwischenräume zwischen den Molekülen der ponderabelen Körper erfillt. Dureh die von einem leuchtenden Gegenstande ausgehenden Impulse werden die nnmittelbar benachbarten 'Theilehen dieses Äthers in Sehwingungen versetzt, die Erregung pflanzt sich in dem elastisehen Medium nach allen Richtungen mit eonstanter Geschwindigkeit fort, und die Empfindung, welche dureh die das Auge erreiehenden Schwingungen in dem Nervensystem hervorgebracht wird, nemnen wir Licht. Diese Empfindung wird je nach der Anzahl der Sehwingmugen, welche in einer bestimmten Zeiteinheit das Auge treffen, versehieden sein. Wir sprechen in diesem Simne ron der Farbe des Lichtes und unterscheiden alle möglichen Niianeen zwisehen dem iussersten Roth mit etwa 458 Billionen und dem äussersten Violett mit etwa 727 Billionen Schwingungen in der Seeunde. Aber auch bei gleieher Farbe kann der Lichteindruck anf das Ange sehr verschieden sein je wach der Stiirke der einwirkenden Impulse. Wir spreehen in diesem Sinne ron der Intensitait des Lichtes, und diese kann ebenfalls innerhalb sehr weiter Grenzen variiren, von einer kaum wahrnehmbaren Empfindung an bis zu einem Eindrucke, den das menschliche Sehorgan nicht ohne Gefahr zu ertragen vermag. Mit der Bestimmung ind Vergleichung der verschiedenen Intensitiitsgrade beschäftigt sich die Photometrie.

Die Schwingungen der einzelnen Äthertheilchen werden als geradlinig und senkrecht zur Fortpflanzungsrichtung der Bewegung vorausgesetzt. 
Nach den Principien der Mechanik wird dann die Bewegungsggleichung rines cinzelnen Theilchens, dessen Entfernung von der Gleichgewiehtslage \%ur Zeit $/$ mit $x$ bezeichnet sein möge, lauten:

$$
m \frac{d^{2} x}{d t^{2}}=-C x
$$

wo $m$ dic Masse des Theilehens und $C$ irgend eine Constante ist. Setzt man 110 ch $\frac{C}{m}=c^{2}$, so wird:

$$
\frac{d^{2} x}{d t^{2}}=-c^{2} x
$$

Dic Anflisung dieser Differentialgleichung giebt:

$$
x=a \cos (c t+b),
$$

und fir die reschwindigkeit $r$ des 'l'heilchens folgt daraus:

$$
r=\frac{d \cdot r}{d t}=-a c \sin (c t+b) \text {. }
$$

II: As dic Interrationsonnstanten a und $b$ anbetrifft, so folgt zunächst, wemn m:11 die \% it ion dem Inomente an rechnet, wo das Theilchen die

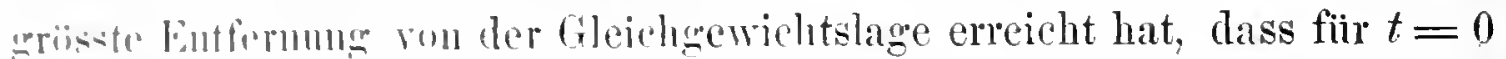
andh dir (irshwindigkeit $r=0$ sein muss und daher anch $b=0$ zu soptrill ist. Mall halt allso:

$$
\begin{aligned}
& r=a \cos c t \\
& r=-a c \sin c t .
\end{aligned}
$$

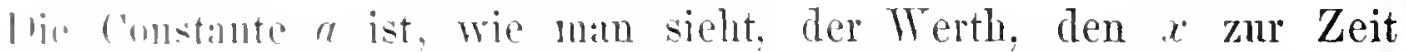

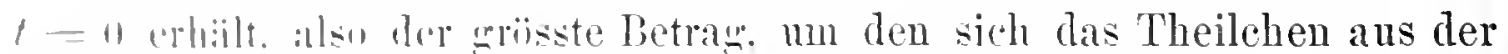

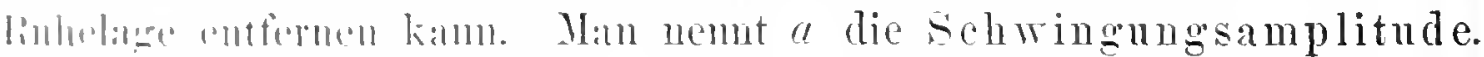

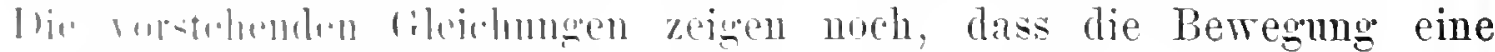

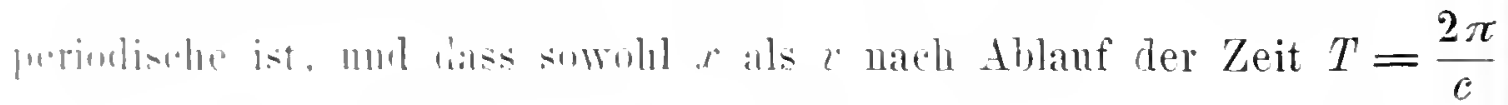
immu "jullu dirsolhen Werthe ahalten; die Grösse $T$ heisst die

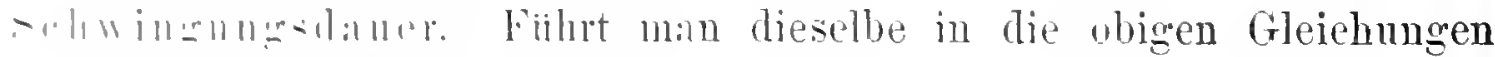
(in. - .."

$$
\begin{aligned}
& r=\| \cdots \frac{2 \pi t}{T}, \\
& r=-a \frac{2 \pi}{T} \sin \frac{2 \pi t}{T} .
\end{aligned}
$$

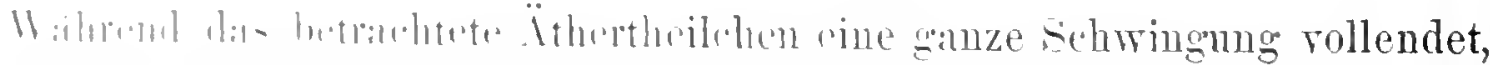

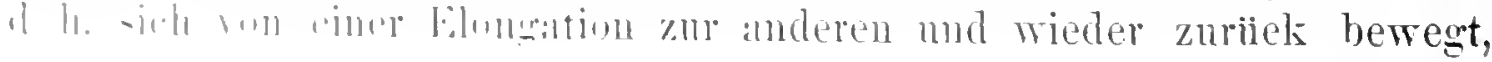


hat sich die Erregung weiter ausgebreitet, und es wird ein Theilehen geben, welches sich genau in demselben Selıwingungszustande befindet, wie das erste. Bezeichnet man die Entfernung der beiden Theilchen mit 2., und ist $V$ die Fortpflanzungsgeschwindigkeit der Ätherbewegung, so hat man:

$$
\lambda=V T \text {. }
$$

Die Entfernung 2. wird die Wellenlänge des Lichtes genannt.

Unter Intensitiit des Lichtes im weitesten Sinne versteht man eine

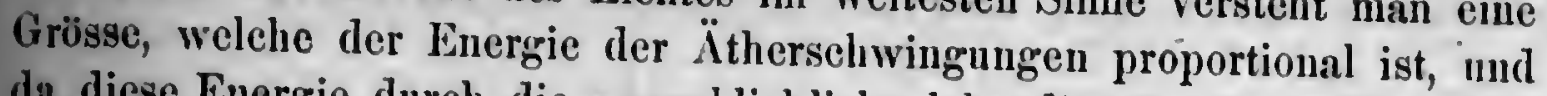
da diese Energie durch die augenblickliche lebendige Kraft der Theilchen gemessen wird, so kann man die Intensitait ausdricken dureh $y m v^{2}$, wo $\gamma$ eine Constante ist. Je weiter die Theilehen von der Rulielage entfernt sind, desto mehr nöhert sich die lebendige Kraft dem Werthe 0 und wird andererseits am grössten, wenn die Theilchen die Ruhelage passiren. Da aber das Auge nicht im Stande ist, den schnellen Schwingungen zn folgen, so wird nicht die augenblickliche lebendige Kraft in jedem Stadium der Schwingung zur gesonderten Wirkung gelangen, sondern es wird nur der Mittelwerth aus allen Werthen, welehe die lebendige Kraft wïlrend der Schwingungsdauer $T$ erbalten kann, in Betracht zu ziehen sein. Wir nehmen daher für die Intensität $J$ den Ausdruek an:

$$
J=\frac{\gamma m}{T} \int_{0}^{T} v^{2} d t
$$

Durch Substitution des Werthes ron $v^{2}$ erhïlt man:

$$
J=\frac{\gamma m}{T^{-}} a^{2}\left(\begin{array}{c}
2 \pi \\
T
\end{array}\right)_{0}^{2} \int_{0}^{T} \sin ^{2} \frac{2 \pi t}{T} d t
$$

Der Werth des Integrales ist $\frac{1}{2} T$; folglich:

$$
J=\gamma m \frac{a^{2}}{2}\left(\frac{2 \pi}{T}\right)^{2}
$$

und wenn fuir $T$ der Werth $\frac{\lambda}{V}$ substituirt und eine einzige Constante $I$ eingeführt wird:

$$
J=I V^{2}\left(\frac{a}{i}\right)^{2}
$$

Die Fortpflanzungsgeschwindigkeit $V$ liingt von der Wellenlïnge ab; doch sind die Unterschiede zwischen den fül die einzelnen Wellenlïngen gilltigen Geschwindigkeiten so gering, dass man olıne grossen Fehler $V$ als Constante betrachten darf. Man kann dann schreiben:

$$
J=K^{a^{2}}
$$


Die Intensitit eines Lichtes ron der Wellenlinge $\lambda$ wird also gemessen durch das Quadrat der Amplitude, welehe dic Lichtsehwingungen besitzen.

Hut man es nicht mit homogenem Lichte von der Wellenlänge $\lambda$ zu thun, souderi mit zusammengesetztem Lichte von allen möglichen Wellenlingen, so wird jeder Farbe eine besondere lebendige Kraft, also auch sin besonderer Werth von a angehören. Man muisste also allgemein die Summe der Werthe $\left(\frac{a}{i}\right)^{2}$ ther alle mögliehen Werthe von $\lambda$ bilden und hiltte streng genummen statt des obigen Ausdruckes zn setzen:

$$
I=K \geq\left(\frac{a}{i}\right)^{2}
$$

1) is der /stimmenhang \%wischen a und $\lambda$ nicht genigend bekannt ist nnd :nll die Gren\%,u, wo die Liehtwirkung beginnt, schwer zu bestimmen sind, so sieht mall, dilss eine ganuz strenge Definition der Lichtintensität

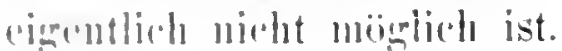

\section{Dis fiesetz vom Quadrate der Entfernung.}

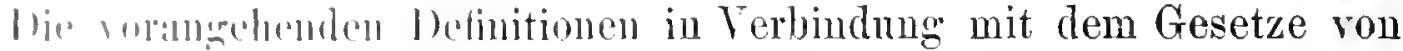

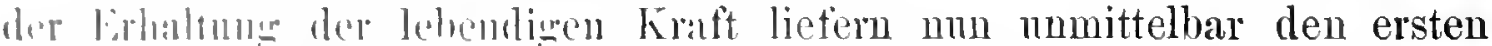

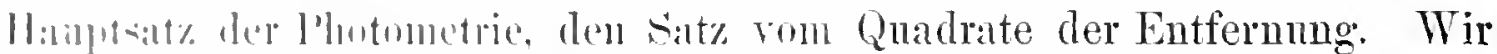

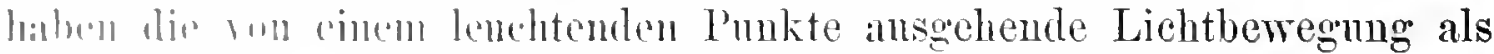
cimn lirilur lun :mf cinander folgenden Impulsen anfoefasst. Diese Be-

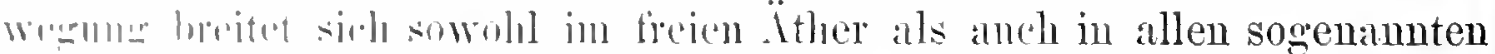

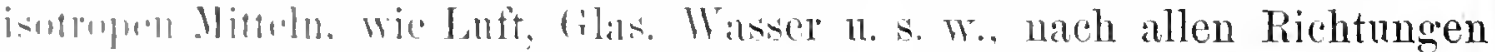

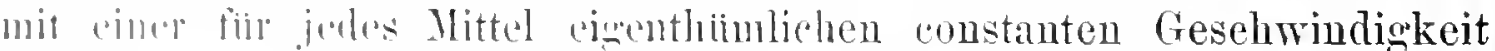
atis: nun in den krystallinischen oder anisotropen Medien ist die Fort-

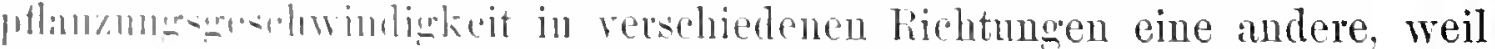

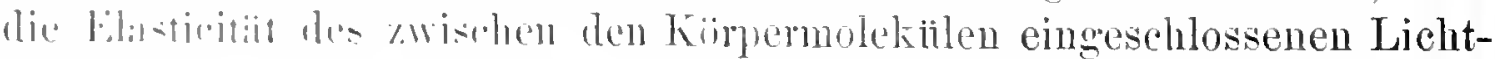

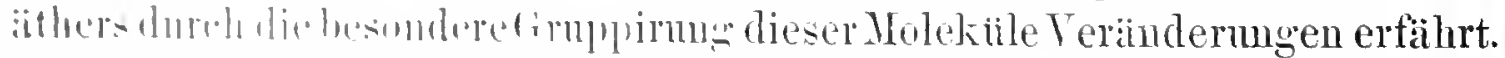

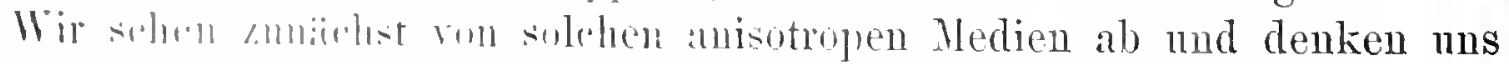

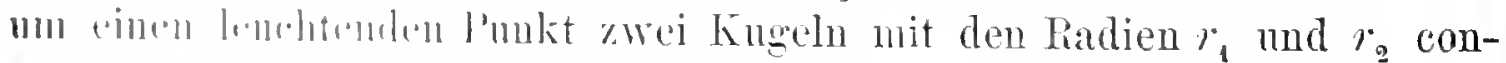
strnirt: dinn hetinden sich simmtliche Theilchen der einen Kugelobertliche

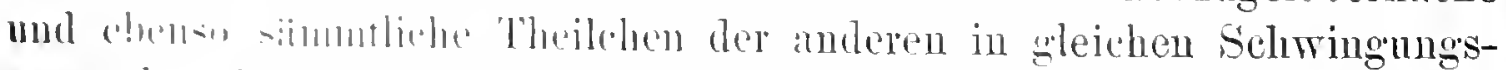

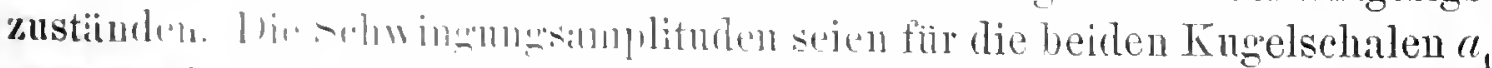

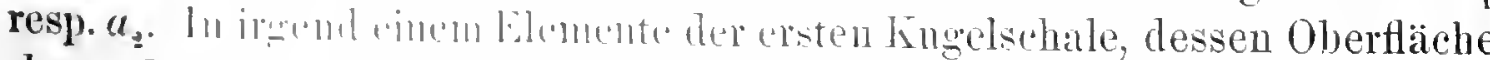

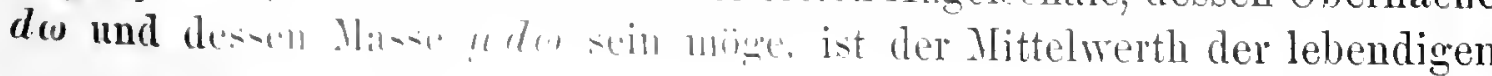
Kraft während viner 
oder nach Einsetzen des Werthes von $t^{2}$ und Auflösen des Integrales wie frther dureh $\mu d \omega\left(\frac{\pi}{T}\right)^{2} \omega_{i}^{2}$. Will man die lebendige Kraft nicht flir ein einzolnes Theilehen, sondern fur die gan\%e Kugel angeben, so hat man, da die Amplitude $a$, fur alle Theilchen dieselbe ist, in dem vorstehenden Ausdrucke nur do durch die Kugeloberfliche $4 r_{i}^{2} \pi$ zu ersetzen und findet demuach fur den Mittelwerth der lebendigen Kraft auf der ganzen Kugel wahrend einer Schwingung den Werth $4 u a_{i}^{2} r_{1}^{2} \frac{x r^{3}}{T^{2}}$. Entsprechend findet man flir die zweite Kugeloberfliiehe den Werth $4 ! 1 a_{2}^{2} r_{3}^{2} \frac{\pi^{3}}{T^{3}}$, und da mach dem Satze von der Erhaltung der lebendigen Kraft die beiden Ausdrilcke einander gleich sein sollen, so ergiebt sich ohne Weiteres:

$$
a_{1} r_{1}=a_{2} r_{2} \text {, }
$$

oder in Worten: Die Sehwingungsamplituden einer Lichthewegung an awei Stellen, deren Entfernungen vom liehterregenden Centrum verschieden sind, verhalten sich umgekehrt wic diese Entfernungen. Nun wird aber nach unserer friberen Definition die Lichtintensitit gemessen dureh das Quadrat der Sehwingungsamplitude; es folgt daher, wenn man die Intensität in einem Punkte der ersten Kugel mit $J_{1}$ und in einem Punkte der zweiten Kugel mit $J_{\mathrm{a}}$ bezeiehnet, ummittelbar die Proportion:

$$
J_{1}: J_{2}=r_{2}^{2}: r_{1}^{2}
$$

d. h. die Intensitaten verhalten sieh $1 \mathrm{mgekehrt}$ wie die Quadrate der Entfernungen. Dieser Satz, weleher das Fundament der ganzen Photometrie bildet, ist dureh zahlreiche Beobaehtungen experimentell bewiesen, und es ist ron besonderem Interesse, dass durch die Beobachtungen an den Himmelskörpern seine Gültigkeit anch für die enorm grossen Distanzen, die in der Astronomie ins Spiel kommen, festgestellt worden ist.

\section{Zusammensetzung der von mehreren leuchtenden Pnnkten ausgehenden Lichtbewegungen.}

Sind statt eines einzigen lichterregenden Centrums deren zwei vorhanden, so fragt es sich, welche Bewegung einem bestimmten Äthertheilchen unter dem gemeinschaftlichen Einflusse der yon beiden Punkten ausgehenden Vibrationen ertheilt wird. Wir wollen der Einfachheit wegen dabei annehmen, dass die beiden Lichtbewegungen gleiehe Wellenlingen haben, und wollen ferner noch für das betrachtete ithertheilchen gleiehe 
Schwingungsphase vornussetzen, d. h. uns denken, dass dieses Theilchen unter der alleingen Wirkung der einen Lichtbewegung seine einzelnen Schwingungen gensu \%u denselben Momenten beginnen und vollenden wilrde, wie unter der alleinigen Wirkung der anderen. Die von der crsten Lichtquelle :ursgehenden Impulse mögen nun, für sich allein betranlitet, in eincm geyissen Momente dem Theilchen die Geschwindigkeit $v_{1}$ in cincr bestimmten Schwingungsebene ertheilen, und entsprechend möge das 'Theilchen, falls es nur der zweiten Lichtbewegung ausgesetzt wäre, in demselben Momente die Geschwindigkeit $v_{z}$ annehmen und zwar im Allemenenen in irgend einer anderen Schwingungsebene, die mit der crsteren den Winkel it einsehliesst. Dann setzen sich diese beiden Geschwindigkeiten nill den Lehren der Mechanik zu einer resultirenden rosolwindigkeit $l^{*} \%$ summen, die gremäiss dem Satze rom Kräfteparallelogr:mum durde die Gleichung ansgedriickt ist:

$$
r^{2}=r_{1}^{2}+r_{2}^{2}+2 r_{1} r_{2} \cos \vartheta
$$

Ist dor Winkel it = "), erfolgen also dic ron beiden lenchtenden Punkten lnorveredranditen schwingungen in derselben Ebene, so wird $V^{2}=\left(v_{1}+v_{2}\right)^{2}$. Ist dispogen it = $=90^{\prime \prime}$, so ergielst sich $V^{z}=v_{1}^{2}+v_{2}^{2}$, und wenn endlich it $=141^{\prime \prime}$ ist, so folgt $l^{\circ}=\left(r_{1}-r_{2}\right)^{2}$, und in dem speciellen Falle, "r $r_{1}=r_{\text {a }}$ is. $Y^{*}=0$. Man sieht also, dass die ron zwei Centren ausEehoulen livhtwirkungen sich sowohl rerstärken als verminderu, in einem hestimutrol falle sich sugrar wimzlich rernichten kümen. Indessen findet

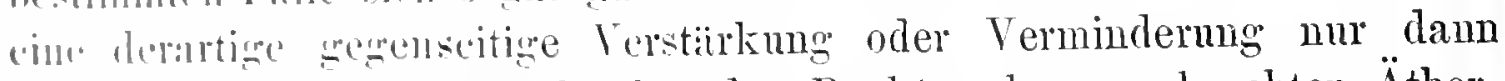
st:ıtr. Wenln die ron den lenchtenden Punkten hervorgebrachten Äthersohwingm!nen constant in denselben Ebenen ror sich gehen, d. h. wenn manl os mit sugenanntem linear polarisirten Lichte zu thun hat. Hamdelt "sich aber. wie hier voransgesetzt werden soll, um natior-

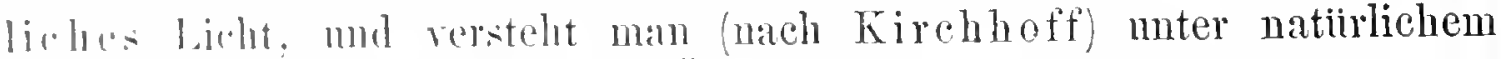
hichte shlhes. hei welkhem die Äthertheilchen fortwïhrend ihre Schwin-

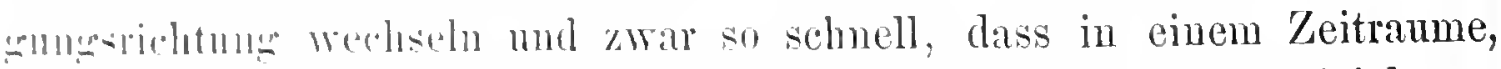

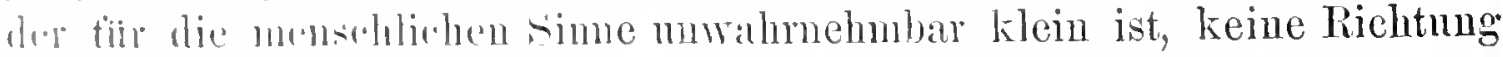
di. :mkten iibrwient, so wird man die gemeinschaftliche Wirkung der lnihln l.m.htemlon l'unkte anf ein gewisses äthertheilchen fiir eine be-

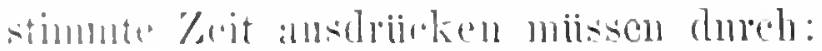

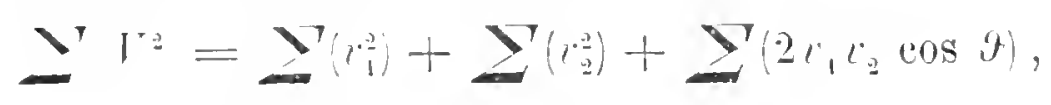

wn die summen iiher alle mightichen Combinationen fom $r_{4}$ und $r_{2}$ inner-

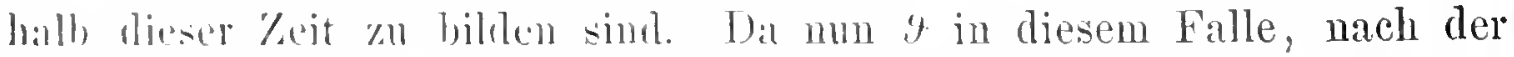
whigen betinition sun matiolichem Lithte, alle migghehen Werthe zwischen 
+1 und -1 durchlanfen kann, so wird der Mittelwerth fur Zeiten, die ausere sinnliche Wahruehmung verlangt, Null sein, und man hat daher:

$$
\Sigma\left(V^{z}\right)=\Sigma\left(v_{i}^{*}\right)+\Sigma\left(v_{i}^{2}\right)
$$

Nun sind aber diese Summen, wenu man sic sich thber die Dauer einer gauzen Selwwingung ausgedelunt denkt, unmittelbar proportional den im Vorangehenden als Intensitiit der Lichtbewegung definirten Grössen. Bezeiehnet man daher diese Intensititen flir die beiden einzelnen Bewegungen mit $J_{1}$ und $J_{2}$ und fur die resultirende mit $J$, so ergiebt sieh:

$$
J=J_{1}+J_{2} \text {. }
$$

Dieser Satz liisst sich ohne Sehwierigkeit anch anf beliebig vicle leuchteude Punkte ausdehnen, und da man sich jede leuchtende Flïche ans lauter leuchtenden Punkten zusammengesetzt denken kann, von denen jeder unabläingig von den anderen eine Wellenbewegung erregt, so folgt oline Weiteres der wiehtige Grundsatz, dass die von einer leuchtenden Fliche hervorgebrachte Liehtintensitiit der Ausdehnung dieser Fliehe proportional ist.

\section{Die physiologische Intensität und das Fechuer'sche psychophysische Gesetz.}

Nach der bisherigen Definition wird die Intensitiit eines leuchtenden Punktes dureh den Mittelwerth der lebendigen Kraft des Äthers wiilnend einer Schwingungsdauer gemessen. Denken wir uns an der durch das Licht erregten Stelle unser Auge, so fragt es sich, ob die ganze lebendige Kraft in den Sehnerven wirksam ist, ob wir die ganze lebendige Kraft messen können. Diese Frage ist entschieden zn verneinen; denn ein Theil der Kraft äussert sich als Wärme, ein Theil als chemische Reaction, und nur ein gewisser Theil, der sich zunäichst nicht sicher bestimmen lässt, afficirt die Nerven unserer Angen so, dass dadurch der Eindruek des Lichtes herrorgebracht wird. Nenuen wir die Intensitits-Empfindung $E$, so ist zunaichst klar, dass diese Empfindungsgrösse eine Function der objectiven Intensitiit $J$ ist, also:

$$
E=f(\cdot J) .
$$

Wir wissen von der Bezielnung zwischen $E$ und $J$ zunaichst nur so viel, dass $E$ mit wachsendem $J$ ebenfalls waichst, ferner, dass $E$ bei einem bestimmten Werthe ron $J$ verschwindet. Macht man die Annahme, dass unsere Netzhaut iiberall dieselbe Reizbarkeit besit\%t, ferner, dass die Beschaffenheit der Funetion $f$ ron der Lage der gereizten Stelle der Netzhant nuabuingig ist, und denken wir uns zwei leuchtende Punkte, 
deren objective Intensitiiten oder lebendigen Kräfte durch $J_{1}$ und $J_{2}$ ansgedrllekt sein mögen und welehe die Empfindungsgrö̈ssen $E_{1}$ und $E_{\mathrm{q}}$ hervorhringen mögen, so ist ans der Gleichheit von $E_{1}$ und $E_{2}$ nothwendig auch :uf die Gleichheit von $J_{1}$ und $J_{2} z u$ schliessen, ganz gleichguiltig, welehes die Beschatfenheit der Function $f$ sein mag. Sind die Empfindungsgrössen zweier Liehtquellen verschieden, so können wir nur so viel mit Sicherheit schliessen, dass die eine heller oder schwiicher als die andere ist, aher es ist nnmöglich anzugeben, um wieviel. Sind wir aber im Stande, durch irgend ein Mittel dic lebendige Kraft der cinen (der stirkeren) lichtinelle in messbarer Weise soweit zu ändern, bis die physiologischen Eindriicke für unser Urtheil gleich sind, so können wir ans der Grösse der Veriunlermug anf das urspriungliche Verhältniss der lohendigen Krifte ler beiden Lichtquellen schliessen. Hiermit ist die Grundhedingung fitr die Construction eines brauchbaren photometrischen Aplitrites anspesprochen. Wir werden im aweiten Abschmitte ansführlich dire littel \% hesprechen haben, welche uns zu Gebote stehen, um die lebendigo Kraft einer Livlityuelle messhar zu reriudern. Im Vorigen ist still-

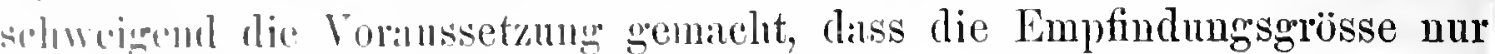
ron der lohndigen Kraft der Liehtbewegung abhängt; dies ist aber nicht ller Fill. sondern $E$ hïngt anch noch ron der Farbe des Lichtes ab, "ie atul shon of an mul für sich cine Function der Wellenlänge war. llan halt alsin richtimere:

$$
E=p(J, \lambda) \text {. }
$$

ln Welcher Weise $E$ von der Farbe abbingt, lïsst sich nicht mit

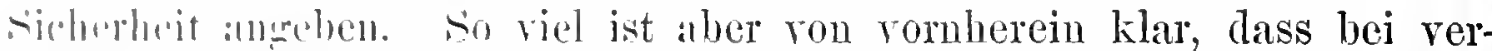

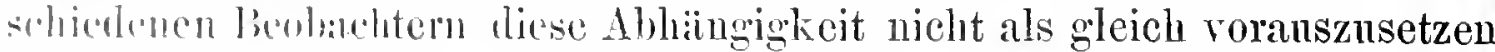

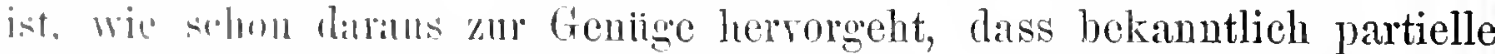
Fumbuhlindheit rorkmunt. Handelt es sich $\mathrm{mm}$ Lichtbewegungen ron versolichlener Vellenliinge, so wird man durch blosse Veränderung der W.bulizen Kralt der comen zwar die Amplitule der Bewegung, aber

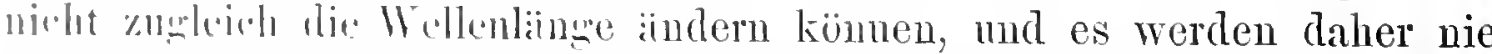

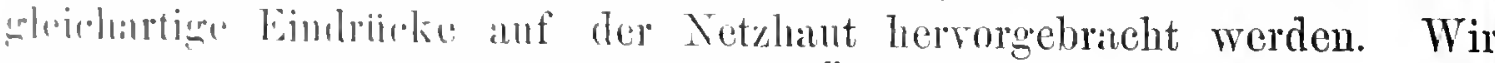

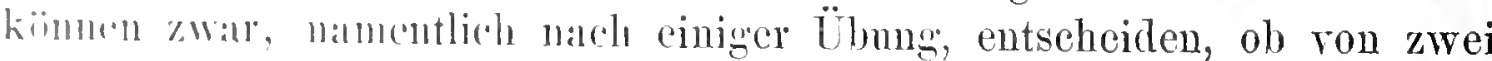

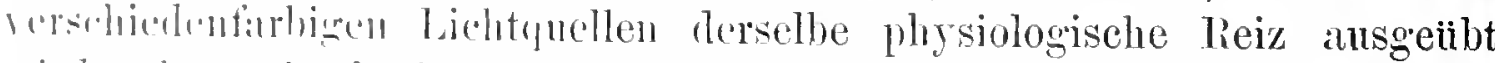
wirl. alke wir dirfen muter keinen Unstainden, ebenso wie bei gleich-

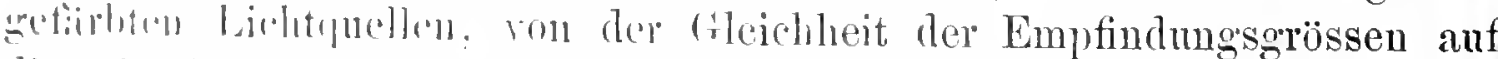

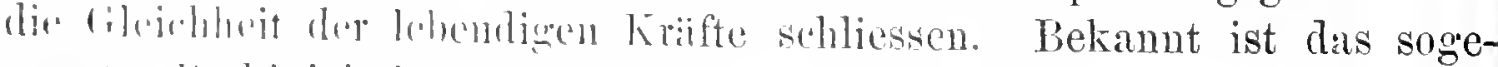
nanute I'urkinjesche I'himomen' '), welches zeigt, dass, wenn zwei

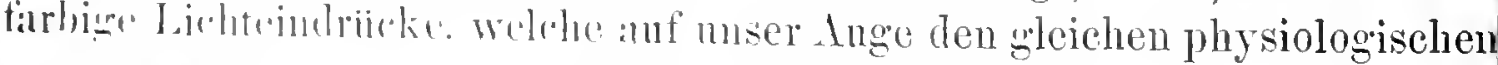

1 l'urkinje. Zur Physiologie der Sinne. 1Bd. II, p. 109 
Eindruck machen, im gleiehen Verbiiltnisse geselıwälıt werden, dann bei der geringeren Lichtstilike die blanen Farben deutlich heller erscheinen als die weniger brechbaren. Dovel) hat interessante Versuche llber den Kinfiuss einer weissen Belenchtung anf die relative Intensitit verschiedener Farben angestellt und gefunden, dass bei grosser Beleuchtungsstïrke die rothen, bei geringerer die blawen Eindrheke thberwiegen. Hierher gehört anch die alltïgliche Wahrnehmung, dass man in einem dunklen Zimmer zuerst dio blauen Gegenstinde bemerkt und daun erst die rothen, sowie ferner, dass umgekehrt das Auge den sehr hellen Gegeustïnden unwillkurlich eine bläuliche Fïrbung beilegt. Ausfthrliches uber diesen Gegenstand findet man in Helmholt $z^{\prime}$ 's physiologischer Optik, wo auch neuere Versuehe von A. Königº tiber den Helligkeitswerth der Spectralfarben hei rerschiedener absoluter Intensitiit besprochen sind.

Fur die messende Photometrie sind diese Thatsachen von der grössten Bedentung, und man sieht, dass, so lange es sich um die Messung verschiedenfarbiger Lichtquellen landelt und so lange das menschliche Auge in letzter Instanz zu entscheiden hat, von vornherein der zu erreichenden Genauigkeit gewisse Schranken gesetzt sind, dic unter Umstiinden bei anormalen Angen ziemlich weit sein können. Für die Technik, wo es sich beispielsweise um die Vergleichung von blïulichem elektrisehen Lichte und röthlichem Gaslichte handelt, sind diese Fragen von der allerhöehsten Bedeutung, und es wird Aufgabe der Praxis sein, geeignete Methoden zu ersinnen, die cine möglichst sichere Beurtheilung der physiologischen Intensitiit gestatten. Einen Weg dazn eröffinen die spectralphotometrischen Beobachtungen, bei denen das Lieht in seine einzelnen Bestandtheile zerlegt, die Vergleichung in den versehiedenen Farbenbezirken ausgefiilırt und aus der Summirung der Einzelwirkungen auf das urspriingliche Verhïltniss der lebendigen Krïfte geschlossen wird. Dieser verhiiltnissmässig neue Zweig der Photometrie hat jedenfalls eine grosse Zukunft und rerdient eine immer grössere Beachtung. In der Astrophotometrie ist die Verschiedenheit der Farben ebenfalls eine Quelle der Unsicherheit. Zwar kommen am Himmel keine so erheblichen Farbenunterschiede vor wie in gewöhnlichen Leben; wirklich grime und blane Sterne giebt es nicht, und die meisten Sterne besitzen eine gelblich weisse oder weisslich gelbe Färbung. Inmerhin sind aber die Unterschiede zwischen einem weissen nnd einem röthlichen Sterne so erheblich, dass die directe Vergleichungr ungemein schwierig ist und insbesondere die Vereinigung

1) Berl. Monatsber. 1852, p. 69.

2) A. König, Über den Helligkeitswerth der Spectralfarben bei verschiedener absoluter Intensitiit. (Beiträge zur Psychologie und Physiologie der Sinnesorgane. Festschrift für H. v. Helmholtz, Hamburg 15!11, p. 309. 
der vou verschiedenen Beobachtern erhaltenen Resultate mit grosser Vorsicht auszufuhren ist. Die Spectralphotometrie wirde anch hier der einzig richtige Werg sein; doch stellt sich der allgemeinen Anwendung derselben suf den llimmel ein hedenkliches Hinderniss entgegen in der geringen lichtstirke der Steme, welche muäichst nur bei den helleren eine erfolgreiche V'ergleichung in den verschiedencu Spectralbezirken gestattet. Wir werlen auf diesen l'unkt in späteren Capiteln eingehender zuriickkommen.

\%/n weiteren Betrachtmigen tiber die physiologische Empfindungsurriosse, welche, abgeschen von der Wämewirkung und der ehemischen lieaction, das cinzige Mass fiu die urspringliehe lebendige Kraft der louphtenden l'mnkte grieht, mögre zuniachst wieder homogenes oder wenigstens Wrisses Licht vorinsgesetyt werden. Es fragt sich nun, wie die Emptindungrunrïsse sich indert, wemn die Intensitiit des objectiven Lichtes vriutert wirl. Wine einfuche Vorstellung davon giebt die Erscheinung, dass: man die Sterne :m Tage mit blossem Auge nicht sehen kann, ob-

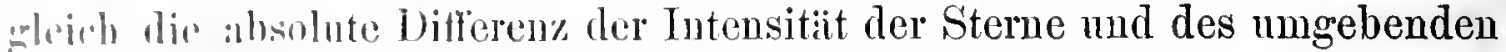

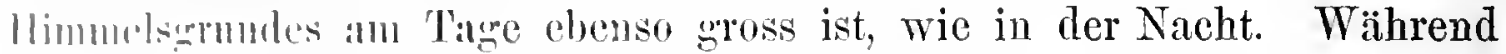

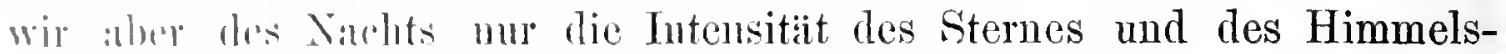

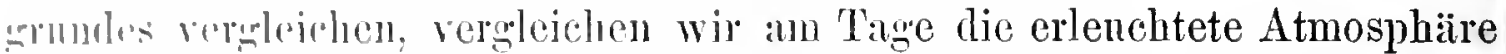
mit ler simme ron stern und erleuchteter Atmosphäre, also zwei stärkere Jichunduldicke. Man sicht also, dass das menschliche Ange die Differenz

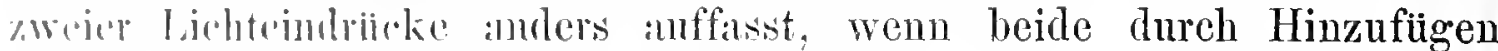

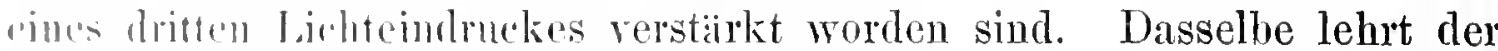

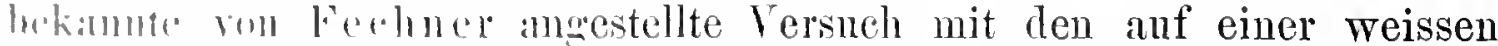

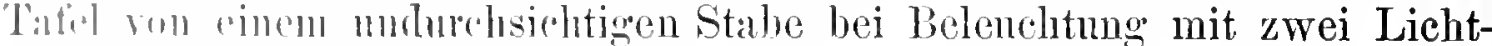

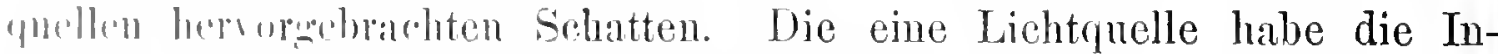
tensitiit h, llie andere die Intensitiit $I I$; dimn erhält der durch $h$ hervor-

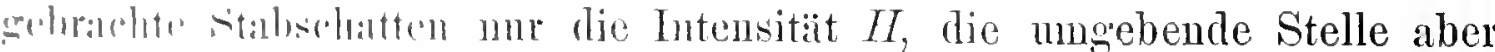
dir. lutrusitiit $I I+h$. Tst mun $I I \mathrm{im}$ Vergleich zu $/$ sehr klein, so wird

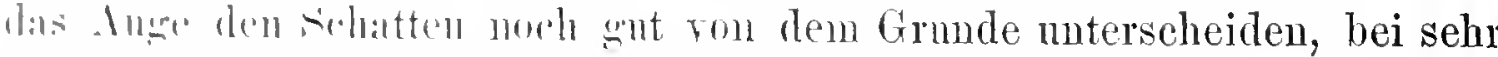

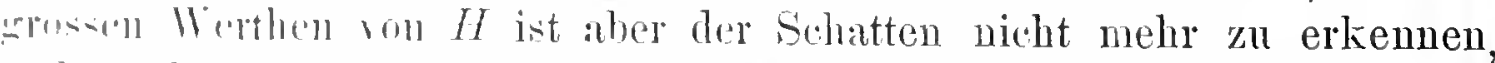
mul us font also hicrams, dass das Ange dieselbe Intensitiitsdifferenz je man lmatimulan anders mutfast, also keinen richtigen Massstab fuir

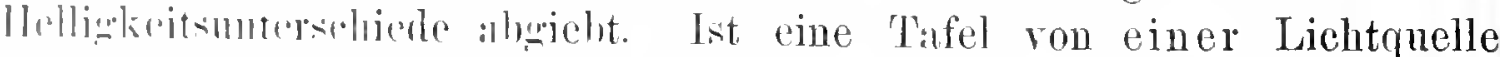

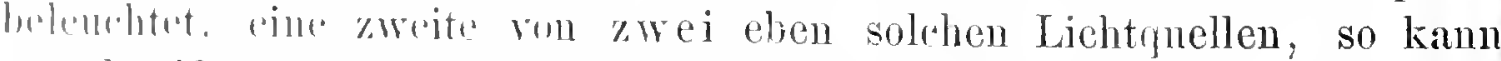
man den l ntershied in der lutensitit erkennen; wird aber die eine Tafel von

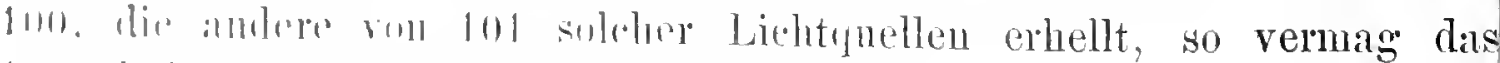

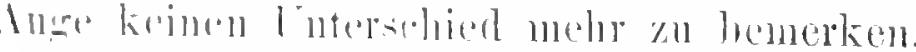

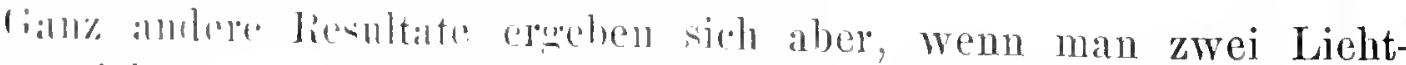

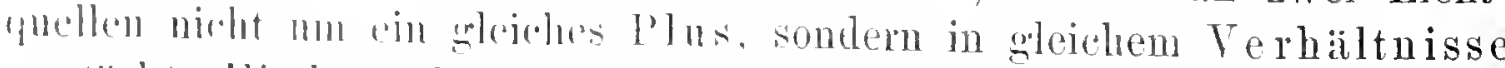

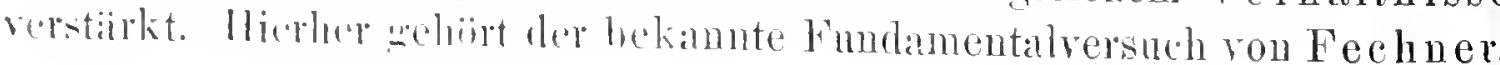


welcher zwei benachbarto Wolkenflichen, deren Helligkeitsdifferenz eben noch merklich war, einnal mit blossem Auge, das andere Mal durch absorbirende Gliser, die also einen bestimmten Procentsatz des ursprlluglichen Lichtes beider Wolkenflichen absorbirten, beobachtete. In zweiten Falle wurde der urspringlieh eben noch merkliche Unterschied der Helligkeit keineswegs geringer, sondern nach dem Urtheile vieler Beobachter mindestens ebenso anffalleud, selbst wenn die Absehwächung innerhalb weiter Grenzen modificirt wurde. Allgemein ergiebt sich aus diesen und zahlreichen :ihnlichen Versuchen, dass bei den versehiedensten Helligkeitsgraden die Differenz der Intensitäten, welche rom Auge gerade noch unterschieden werlen kömnen, nahezu denselben Bruchtheil der ganzen Helligkeit bildet. Fechner') hat dies in dem nach ihm benannten psychophysisehen Gesetze zuerst mit voller Klarheit ausgesprochen, obgleich vor ihm schon Andere, namentlich Bouguer, Arago, Masson und Steinheil auf die Bedeutung der Erscheinungen hingewiesen und entsprechende Versuche zur Bestimmung der Empfindungsgrenze angestellt hatten. Nach Bonguer ${ }^{2}$ ) kann man noeh ${ }^{1}$ r der Liehtstïrke unterseheiden; Arago ${ }^{3}$ ) beobachtete, dass bei Bewegung der lenehtenden Objecte noeh feinere Untersehiede bemerkt werden konnten und bestimmte den Lichtquotienten nnter den giinstigsten Bediugungen zu T3T. Masson l) fand im Mittel den Factor $\mathrm{zu} \tau_{\frac{1}{00}}$, und nahe denselben Werth lieferten die Versuche von Fechner, wiihrend Steinheils) aus photometrischen Messungen den etwas abweichenden Werth $\frac{1}{38}$ erhielt.

Ohne nïher auf diese Versuche und die damit zusammenhïngenden Fragen einzugehen, iiber welche die Lehrbiicher der physiologischen Optik noch manche interessante Einzelheiten enthalten, geniigt es für unsere Zwecke herrorzuheben, dass etwa 1 Procent als diejenige Grösse anzusehen ist, welche unter giinstigen Umständen noch als Helligkeitsunterschied wahrgenommen werden kamm.

Es verdient hier nieht merwähnt zu bleiben, dass das psyehophysische Gesetz anch auf anderen Gebieten der Sinnesempfindungen Guiltigkeit hat. So ist nachgewiesen, dass der Unterschied zweier Tonhöhen sich gleich bleibt, wenn das Verhailtniss der Differenz der Schwingungsdauern zur ganzen Schwingungsdamer constant ist; und auch bei der Beurtheilung der

1) Fechner, Über ein psychophysisehes Grundgesetz uud dessen Beziehnng zur Schätzung der Sterngrüssen. (Abhandl. der K. Saichs. Ges. der Wiss. Bd. 4, p. 455.)

2) Bonguer, Traité d'optique sur la gradation de la lumière. Onvrage posthume. Paris, 1760 , p. 25.

3) Arago, Sïmmtl. Werke. Deutsehe Ausg. von IIankel, Bd. 10, p. 210.

4) Annales de chimie et de plysique. Série 3 , tome 14, p. 157. 5) Steinheil, Elemente der Helligkeitsmessungen am Sternenhimmel. (Denk-
schriften der K. Bayer. Akad. d. Wiss. Math.-Phys. Classe, Bd. II.) München, 1836, p.80. 
Differen\%en vou Gewichten und Liniengrössen findet Ähnliches statt. Die Allgemeinglittigkeit les Gesetzes ist namentich durch Untersuchungen von li. H. Weber nachgewiesen worden, und es wird daher dieses Gesetz auch hiufig als das Weber'sche bezeichnet.

In seiner Anwendung anf die Photometric liasst sich das Gesetz in der folgenden Form ansirticken. Ist $E$ dic Empfindungsgrösse, welche der objectiven Helligkeit $h$ entsprieht, und ist $d E$ die Zunahme der Empfindungsstiirke, welehe durch einen Zuwachs $d h$ der objectiven Helligkeit hervorgernfen wirl, so hat man:

$$
d E=c \frac{d h}{h},
$$

wo e cine Constunte ist. Durch Integration folgt:

$$
E=c \log h+C \text {. }
$$

Fiftr zwei andere Werthe $E_{0}$ und $h_{0}$ hat man ebenso:

$$
E_{0}=c \log h_{0}+C \text {, }
$$

mull dirans fillat:

$$
E-E_{0}=c \log \frac{h}{h_{0}}, \quad \text { oder: } \quad h=h_{0} e^{\frac{E-E_{0}}{c}} .
$$

Lis dart nirlit rerschwiegen werden, dass das Fechner'sehe Gesetz nimit mmmschrankte Ciiltigkeit besitat, vielmelır eine untere und obere lirmo hat und hei sehr kleinen sorohl als bei sehr grossen Helligkeiten murnall wird. Fechuer hat bereits selbst auf diese Ausnahmen hinmenirsoll mul die mutere Grenze dureh den Einfluss des subjectiven lijpenlinhts des Anges zu exklairen rersucht. Die Selnerven werden nimblich nirht mur durch dis ron aussen kommende Licht gereizt, sondern

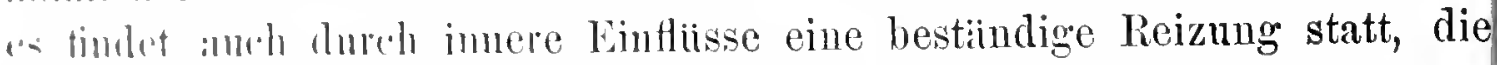
bu m.'ll

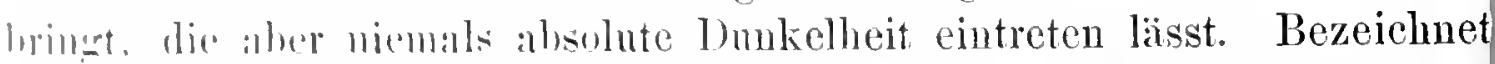
man diesp lizenlinh des Anges, welches zu dem von anssen kommenden I.johe sich hinzunddirt, nit $H_{0}$, so mitisste das Fechner'sche Gesetz

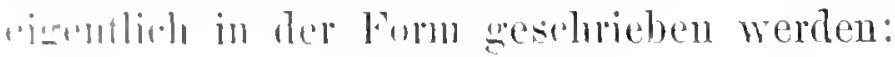

$$
d E=c \frac{d h}{h+I_{0}}
$$

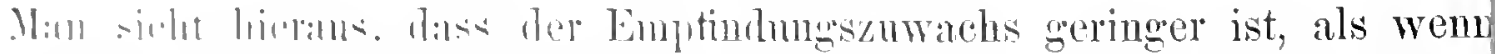

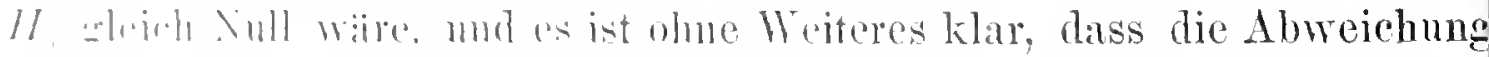
mo J

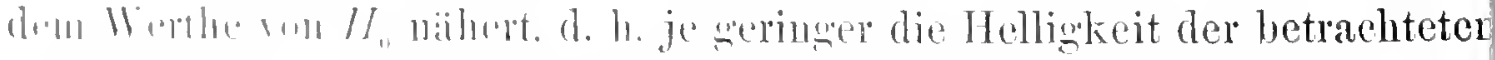

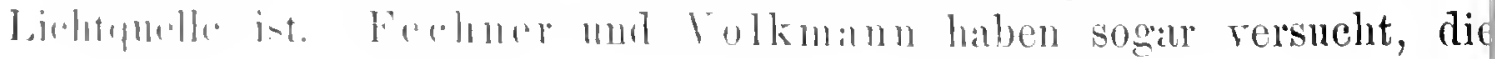


Intensitilt des Eigenlichtes zu bestimmen; indessen sind diese Bestimunngen nicht ganz einwurfsfrei und ergeben ofienbar zu kleine Werthe. Wenn dins Eigenlicht des Auges wirklieh vorhanden ist, so muss die objective Intensitït eine gewisse Stlirke hahen, um uberhaupt wahrgenommen zu werden. Kleinere Grade der Intensität uben keine Wirkung mehr auf das Auge aus. Feehner hat die kleinste noch erkennbare Belenchtung die Reizschwelle genannt.

Was die obere Grenze des Fechner'schen Gesetzes anbelangt, so liisst sich dieselbe ebenso wenig wie die untere mit Sicherheit angeben, aber es ist ohne Weiteres klar, dass bei einer gewissen Stärke des Reizes das Seliorgan geschädigt wird. Wir können olne Schutzmittel nicht das directe Sonnenlieht vertragen, und auch schon bei weniger intensiven Lichtquellen findet eine Überreizung der Nerven statt, bei weleher es nicht mehr möglich ist, Empfindungsunterschiede wahrzunchmen.

Fur die Astrophotometric ist das Fechner'sche Gesetz ron der fundamentalsten Bedeutung, und es rechtfertigt sich ganz von selbst eine ausfuhrliche Besprechung desselben an erster Stelle in einem Lehrbuche uber Astrophotometrie, weil sich die wichtigsten Folgerungen hinsichtlich des Masses, in welchem photometrische Beobachtungen anzugeben sind, sowie hinsichtlich des Ausgleichungsverfahrens an dasselbe knijpfen. Di es bei allen photometrischen Messungen in letzter Instanz auf die Empfindungsgrösse $E$ ankommt, so sieht man sofort aus der Formel:

$$
E=c \log h+C,
$$

dass es nicht die objectiven Helligkeiten (lebendigen Kräfte) selbst, sondern die Logarithmen derselben sind, welehe psychiseh zur Empfindung kommen, und es ergiebt sich daraus die Nothwendigkeit, die Helligkeitslogarithmen als Mass in die messende Astrophotometrie einzuführen. Schon Fechner hat selbst anf den Zusammenhang seines Gesetzes mit der messenden Astronomic hingewiesen. Bekanntliel sind schon von Alters her die Sterne nach dem Eindrucke, den ihr Licht anf das Auge macht, in gewisse Helligkeitsclassen, sogenamnte Sterngrössenclassen, eingetheilt worden, und zwar wurden fiir die mit blossem Auge sichtbaren Sterne sechs Abtheilungen gewiillt mit der Bedingmug, dass der Helligkeitsuntersehied zwisehen je zwei anfeinander folgenden Abtheilungen derselbe sein sollte. Später hat man diese Helligkeitsscala anch auf die teleskopischen Steme ausgedehnt, und es ist so cine znnïchst willkiirliche Scala zur Beurtheilung ron Helligkeiten der Sterne entstanden. Es fragt sich, ob diese Seala in dem Fechner schen Gesetze begriindet ist? Man denke sich Sterne, welehe in dem eben angedenteten Simne Repr:isentanten der anfeinander folgenden Grössenclassen 1, 2, $3 \ldots m$ sind; 
ihre olijectiven Lichtstärken seien $h_{1}, h_{2}, h_{3} \ldots h_{m}$ und die Empfindungsgrössen $E_{1}, E_{z} \ldots E_{m}$. Nach dem Fechner'sehen Gesetze ist:

$$
\begin{aligned}
& E_{m}=c \log h_{m}+C \\
& E_{m-1}=c \log h_{m-1}+C ;
\end{aligned}
$$

also:

$$
E_{m}-E_{m-1}=c \log \frac{h_{m}}{h_{m-1}} \text {. }
$$

Wäre nun das Fechner'sche Gesetz auf die Grössenclassen anwendbar, so milsste $E_{m}-E_{m-1}$ fiir je zwei beliebige anf einander folgende Grössenclassen constant sein; man miisste also laben:

$$
c \log \frac{h_{m}}{h_{m-1}}=k
$$

oder, wenn man $l_{c}^{l}$ dureh eine einzige Constante $\log \frac{1}{\varrho}$ ersetzt:

$$
\frac{h_{m-1}}{h_{m}}=\varrho .
$$

In der That haben num alle bisherigen Untersuchungen ergeben, dass innerhall, gewisser Crenzen das Helligkeitsverhailtniss zweier nm eine Ciriissonlalsse ron einumder verschiedenen Sterne als constant anzusehen ist mul dass also die Grössenschätzmngen als eine Bestätignng des Fechner"schen Ciesetzes betrachtet werden kömnen. Im letzten Absehnitte wird ansfiilulich iiher diese Untersuchungen berichtet werden; hier genügt as herrormhleben, dass mit Ansnahme der lelleren Grössenclassen, wo "twats stiolkere Abweichungen zu bemerken sind, für das Helligkeitsverhailtniss zweier auf cinander folgenden Classen mit geniigender Sieherheit lic Zahl 2.5 angenommen werden kann. Wäre das Fechner'sche Gesutz in aller strenge anf Sterngrössen von den hellsten bis zu den shwiidsten Sternen anwendhar, so hätte man streng:

mler:

$$
h_{1}=\frac{h_{2}}{h_{3}}=\frac{h_{3}}{h_{4}} \cdots=\frac{h_{m-1}}{h_{m}}=\rho,
$$

$\ln h_{1}-\operatorname{lng} h_{2}=\log h_{2}-\log h_{3}=\cdots=\log h_{m-1}-\log h_{m}=\log \varrho$, wher wem man alle Gleichungen addirt:

$$
\log h_{1}-\log h_{m}=(m-1) \log \varrho .
$$

Setzt maln. da die Einheit heliebign angenommen werden kann, die Hel-

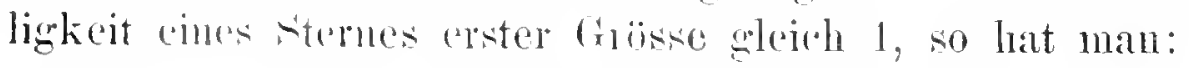

oder:

$$
\log m=m-m-1) \log 0,
$$

$$
m=1-\frac{\log h m,}{\log h^{\prime}},
$$


d. h. die ideale Grössenclasse $m$ eines Sternes, dessen Helligkeitsverhailtniss zu einem Sterne 1. Grösse durch $h_{m}$ ausgedruckt ist, wird gefunden, weun man $\log h_{m}$ durch eine Constante $\log \varrho$ dividirt und den Quotienten ron 1 subtrahirt. Es hat sich in der Photometrie die Bezeichnung mit Sterngrössenclassen so eingebtirgert, dass es kaum noch rathsam sein durfte, dieselbe wieder durch eino andere Schreibweise verdrängen und z. B. alle Angaben in Helligkeitslogarithmen unachen zu wollen. Es wird sich daher empfehlen, fur die Coustante $\varrho$ einen ganz bestimmten Werth einzufthren und als photometrische Sterngrösse denjenigen Werth zu definiren, weleher sich bei der Division der Helligkeitslogarithmen durch den Logarithmus dieser Constanten ergiebt. Man bedient sich jetzt allgemein des Werthes $\log \varrho=-0.4$ oder $\varrho=0.359$ oder $\frac{1}{\varrho}=2.512$.

Das Fechner'sche Geset\% ist auch, wie hier noch kurz erwithnt werden soll, fur die Ansgleichung der photometrischen Beobachtumgen von hoher Bedeutung. Es mögen von einem Sterne eine Anzahl Helligkeitsbestimmungen $h_{1}, h_{2} \ldots h_{n}$ vorliegen, denen die Empfindungsstiirken $E_{1}, E_{2}$ $\ldots E_{n}$ entsprechen sollen. Der wahrscheinlieliste Werth für die Helligkeit des Sternes sei $x$ und die zugehörige Empfindungsstiirke sei $E_{0}$. Nach dem Fechner'schen Satz hat man dann:

Mithin ist:

$$
\begin{aligned}
& E_{1}=c \log h_{1}+C, \\
& E_{0}=c \log x+C .
\end{aligned}
$$

Ebenso wird:

$$
E_{1}-E_{0}=c \log \frac{h_{1}}{x} \text {. }
$$

$$
\begin{aligned}
& E_{\mathrm{z}}-E_{0}=c \log \frac{h_{2}}{x}, \\
& \cdot \cdot \cdot \cdot \cdot \cdot \cdot \\
& E_{n}-E_{0}=c \log \frac{h_{n}}{x} .
\end{aligned}
$$

Die Verschiedenheit der Grössen $E_{1}, E_{2} \ldots E_{n}$ wird einerseits durch die rein zufalligen, auf der unvollkommenen Urtheilsfahigkeit des Aurges beruhenden Messungsfehler bedingt, andererseits durch inssere Einfliisse, wie wechselnde Durchsichtigkeit der Atmospliaire n. s. w., hervorgebracht. Betrachtet man die Grössen $E_{1}-E_{0}, E_{2}-E_{0}, \ldots E_{n}-E_{0}$ als Beobachtungsfehler und legt der Ausgleichung das Gauss'sche Fehlergesetz zu Grunde, nach welchem die Summe der Fehlerquadrate ein Minimum werden muss, so ergiebt sich die Gleichung:

$$
c^{2}\left(\log \frac{h_{1}}{x}\right)^{2}+c^{2}\left(\log \frac{h_{2}}{x}\right)^{2}+\cdots+c^{2}\left(\log \frac{h_{n}}{x}\right)^{2}=\text { Minimum }
$$


und hieraus folgt \%ur Bestimmung des wahrscheinlichsten Helligkeitswerthes $x$ die Gleichung:

$$
\log \frac{h_{1}}{x}+\log \frac{h_{3}}{x}+\cdots+\log \frac{h_{n}}{x}=0
$$

oder:

$$
\log x=\frac{\log h_{1}+\log h_{2}+\cdots+\log h_{n}}{n} .
$$

lis geht hierans unmittelbar hervor, dass man bei Ableitung des plausibelsten Helligkeitswerthes aus eincr Reilie von Einzelbestimmungen am Rationellsten verfilurt, wemn man mit den Helligkeitslogarithmen (oder was (anselhe, mit Stemgrössen) anstatt mit den Helligkeiten selbst operirt.

Auf die Bedentumg dieses Reehnungsverfahrens bei photometrisehen Messungen ist sehon wiederholt, am Eingehendsten wohl ron Seeliger ${ }^{1}$, hingewiesen worden, weleher aneh noch eine andere Ansgleichungsformel anfigestellt hat, deren Anwendung sich namentlich dann empfiehlt, wenn die durch inssere Unstinde bedingten Messungsfehler die reinen Beobarhtunrsfeller wesentlich iiberwiegen. In der Praxis ist das Rechnungsverfahren mit den llelligkeitslogarithmen bereits seit geraumer Zeit und zwall durch seidel"j cingefiblut worden, und man kaun sagen, dass die Astrophotometrie damit in eine nene Phase der Entwicklung eingetreten ist.

Lis ist noch ron Interesse zu sehen, welche Genauigkeitsgrenze allen photonctrischen Angaben, die in Helligkeitslogarithmen oder Sterngrössen renacht werlen, von vornherein gesetzt ist. Wenu sich $h$ um die Grösse dh indert, so :indert sich $\log h$ um die Grösse $\frac{d h}{h}$ Mod., nnd da nach den when hesprochenen Litersuchmgen als äusserste Grenze für einen gerade nowl erkenubaren Lichtnuterschied etwa 1 Procent angenommen werden killm, also $\frac{d h}{h}=\frac{1}{1011}$ zu setzen ist, so folgt, dass unter keinen Umständen cine rrössere Helligkeitsdifferenz als 0.0043 im Helligkeitslogarithmus oler etwal 0.01 sterngrissen bestimmt werden kann. In Wirklichkeit ist allerdins eine solehe Genanigkeit bei Hessungen am Himmel anch nicht

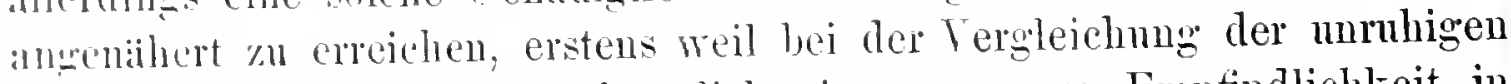
pmktfirmigen stermbilder sehwertich eine so grosse Empfindlichkeit in der brurtloihung ron llelligkeitsmutersehieden roransgesetzt werden darf, wic oben angenommen wurde, und zweitens, weil bei allen photometriselen sturnmessmoen die änseren Lmstiunde, insbesondere die sehwankende Durchsichtimkeit der Lutt. Aie Extinction in der Atmosphäre u. s. w., einen selur stïreuden. schwer controlipbaren Einfluss ansiben.

1 Astron. Nachr. 13d. 132, Nr. 315 s.

2 Abhandl. der $\bar{h}$. Biyer. Akad. der Tiss. II. Classe, Bd. 9, Abth. 3. 


\section{Beleuchtnug rou Flächen dureh leuchtende Punkte. Das Gesetz vom Cosinus des Incidenzwinkels.}

Wir haben in Vorangehenden von der objectiven Intensitiit oder Lenchtkraft einer Liehtquelle gesprocheu und die beiden wichtigsten Gesetze anfgestellt, welehe fur diese Intensität gelten. Indem wir weiter zunäichst nur die Nervenfasern des menschliehen Auges als die Licht empfangende Stelle betracliteten, haben wir den Begriff der pliysiologischen Intensitait eingefuhrt und die daftir geltenden Gesetze besprochen. Wir kinnen aber nicht immer das von einem Punkte ausgehende Licht direct unit deñ Auge betraehten, in vielen Fïllen wird uns eine Lichtquelle erst indireet, d. l. dadureh, dass sie anf Gegenstiinde in unserer Umgebung einwirkt, bemerkbar. Wir sagen von einem Körper, der in den Bereich einer Lichtbewegung kommt, er wird von der Lichtquelle beleuchtet oder ses fällt Licht von der Lichtquelle auf denselbeus, und wir fullıren zur nïheren Festlegung des Begriffes den Ausdruck Lichtmenge ein. Von dem Standpunkte der Newton'schen Emanatioustheorie ans, nach weleher die Empfindung des Liehtes dadureh hervorgebracht wird, dass von einem lenchtenden Körper ans kleine Theilchen mit grosser constanter Geschwindigkeit geradlinig nach allen Richtungen fortgeschleudert werden, hat dieser Ausdruck niehts Befremdendes, da das Licht danach gewissermassen als etwas Greifbares und Materielles aufzufassen ist und das Wort Menge ganz von selbst verständlich ist. Für die Undulationstheorie ist der Begriff allerdings fremdartig, aber er ist als ein sehr bequemer immer beibehalten worden und hat sich allgemein eingebuirgert. Wir haben danach die Lichtmenge, welche ron einem leuchtenden Punkte auf irgend einen Körper iibergeht, als die Summe aller lebendigen Krïfte der Lichtbewegung in den einzelnen Punkten dieses Körpers zu definiren. Es sei $P$ (Fig. 1) ein lenchtender Punkt, in der Entfernung $r$ befinde sich das irgendwie gestaltete zunächst als eben zu betrachtende Element $d f$; es soll die Liehtquantitiit $d q$ bestimmt werden, welche von $P$ anf $d f$ iibergeht. Denkt man sich die Pyramide oder den Kegel eonstmirt, welcher df als Grundfliche und $P$ als Spitze hat, und denkt man sich un $P$ zwei Kugeln mit den Radien 1 und $r$ gelegt, so werden diese ans der Pyramide

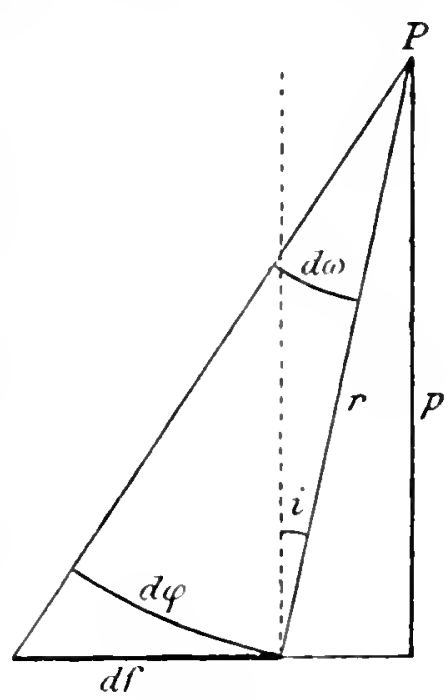

Fig. 1. oder dem Kegel die Elemente $d \omega$ und dip heranssehneiden. Nach unserer Definition ist die Summe der lebendigen Kräfte in $d \omega$ dieselbe, wie in $d \rho$ 
und in $d f$, oder anch die auf $d \omega, d \varphi$ und $d f$ auffallenden Lichtquantitiiten sind dieselben. Bezeichnen wir num, um einen bestimmten Begriff zn fixiren, die Lichtmenge, welche auf die Eiuheit der Fläche in der Entfermung 1 senkreeht :uffallt, mit $J$, so ist klar, dass auf die ganze Fliche du die Quantitiit $J d \omega$ gelangen muss, da die Einzelwirkungen sich ja summiren missen. Dieselbe Quantitit fitlt aber auch anf $d \varphi$ und if, mud man hat daher das gesuchte $d q=J d c$. Nun ist aber:

mithin:

$$
d \omega: d \varphi=1: r^{\circ}, \quad \text { und: } \quad d \varphi=d f \cos i
$$

$$
d q=J \frac{d \cdot f \cos i}{r^{2}}
$$

Lis ist aber anch:

$$
\operatorname{eos} i=\frac{p}{r}
$$

wenn $l$ das Perpendikel von $P$ anf die Verlangerung von $d f$ ist; daher alliclt:

$$
d q=J d f p \frac{1}{r^{3}}
$$

Der Winkel $i$, den die Normale anf dem Elemente df mit der Richtung miclı dem lenchtenden Punkte zu bildet, wird der Incidenzwinkel wellannt, nud die voranstehende Formel bildet eins der wichtigsten Fundamentalgesetze der Photometrie, welehes aussprieht, dass die von einem lenchtenden Punkte anf ein ebenes Element ausgesandte Lichtmenge dem Cosinns des Tneidenzwinkels proportional ist.

Die (riosse $J$, also die Lichtmenge, welche von einem lenchtenden P'mukte anf die Einheit der Fliidhe in der Einheit der Entfermung senkrecht selangt, indert sich von Lichifgnelle zn Lichtquelle und ist ein Mass fiir die Energie dos Lenchtens oder der Ätherbewegung, welche von der betreffenden Lichitquelle ansugeht. Man hat diese Grösse anch die Dichtigkeit des Liclites genannt, ganz im Simne der Vorstellung, dass das Licht eine Materie ist, die, wenn sie sich ron einem Punkte ans strallenfibming anf verschiedene um diesen Punkt concentrisch gelegte Kunelschalen ansheitet, naturgemaiss anf der inneren dieser Schalen diehter vertheilt ist als anf der insweren. Seln glioklich ist diese Bezeiehnung in Sinne der ludulationstheorie nicht gerade gewihlt, aber sie ist all-

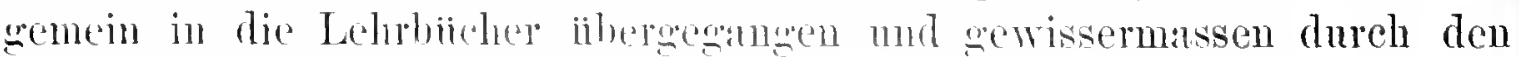
Sprachgehanch sanctimirt: sie grebt iilnigens cine reeht gute Vorstellung ron den Vorgangen und kimn diher mberlenklich beibehalten werden.

Die Lichtmenge oder, wie man sie auch nennt, die wahre oder objeetive Helligkeit dy des Flachenelementes df ist, wie man leicht

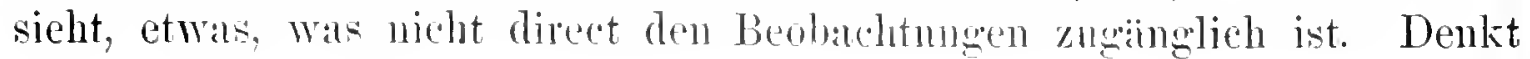


Inan sich an Stelle des Elementes df eine photographische Platte oder ein empfindliches Thermoelement, so wllrde man allerdings in diesen Faillen eine Art Mass fur die anftallendo Liehtmenge haben, insofern dieselbe andere messbare Wirkungen veranlasste, im ersten Falle die Zerlegung der Silbersalze, in anderen die Ablenkung der Galvanometernadel; aber die Wirkung äussert sich in beiden Fïllen nur in Betreff eines kleinen Theiles der von dem leuchtenden Punkte ansgehenden Lichtbewegung, da entweder nur die sogenamnten ehemischen Strahlen oder die sogenaunten Wainnestrahlen in Thaitigkeit treten. Mit dem Auge, das in letzter Instan\% wieder unser hauptsïchlielistes Hulfsmittel ist, nelımen wir die auf ein Element auffallende Lichtquantitait erst indirect durch Vermittlung dieses Elementes wahr, und dabei ist durch allerlei Vorgiinge, wie Brechung, Reflexion, Absorption u. s. w. die ursprünglich empfangene Lichtmenge so modifieirt, dass schliesslich etwas ganz Anderes in unserem Sehorgan zur Empfindung gelangt.

Geht man von dem oben aufgestellten Belenchtungsgesetze aus und dehnt die Betrachtung von dem ebenen Flaichenelemente auf eine beliebig grosse, beliebig gekrilmmte Fliche aus, so ergeben sich eine Menge von interessanten Anfgaben, deren Behandlung in ein eigentliches Lehrbuch des photometrischen Calclils gehört, und von denen im Hinblick anf die Himmelsphotometrie hier nur die wichtigsten angedentet werden können. Es sei ein Kreis mit dem Radius $a$ (Fig. 2) ron cinem Punkte $P$ ans beleuchtet, welcher in der Entfermung $P C=c$ senkreeht iber dem Mittelpunkte $C$ des Kreises liegt. Man beschreibe um dis Centrum zwei concentrische Kreise mit den Radien $r$ und $r+d r$; ausserdem ziche man ron $C$ aus zwei unendlich nahe, den Winkel d'p einsehliessende Radien, dann wird ein kleines Flïchenelement ausgeschnitten, dessen Grösse gegeben ist durch $r d i p d r$.

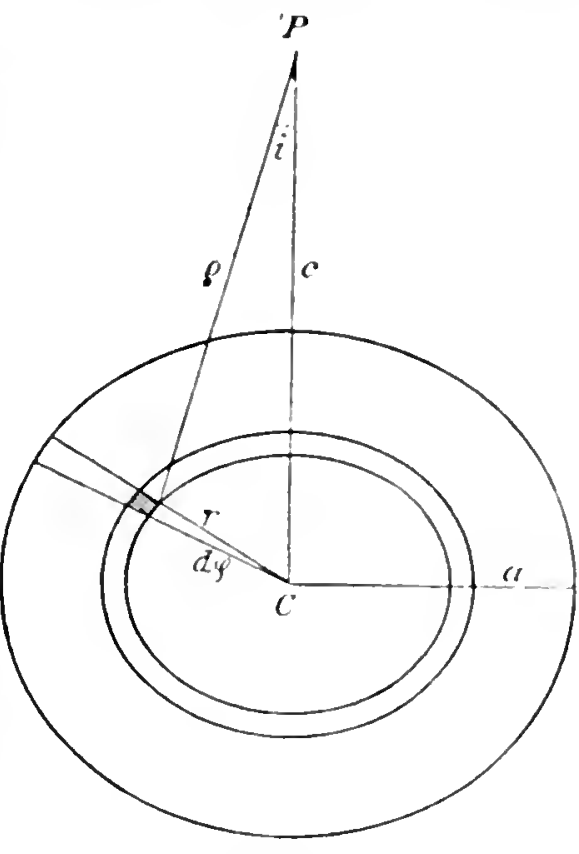

Fig. 2. Die auf dieses Element fallende Lichtmenge wird, da die Entfernung $\varrho$ vom leuchtenden Punkte $=\sqrt{r^{2}+c^{2}}$ und ausserdem $\cos i=\frac{c}{\sqrt{r^{2}+c^{2}}}$ ist, nach der obigen Formel ausgedriickt durch:

$$
d q=\frac{J c r d r d p}{\left(r^{2}+c^{2}\right)^{\frac{3}{2}}}
$$


Will man die gan\%e anf die Kreisfliche fallende Lichtquantität $Q$ kennen, so hat mill!:

$$
Q=J c \int_{0}^{2 \pi} d r \int_{0}^{\|} \frac{r d r}{\left(r^{2}+c^{2}\right)^{\frac{3}{2}}}, \quad \text { oder: } Q=2 \pi J\left\{1-\frac{c}{\sqrt{c^{2}+a^{2}}}\right\} .
$$

Denkt man sich den Kreis unendlich gross $(a=\infty)$, so wird die ron dem Punkte $I$ anf die ganze Hemisphäre ansgehende Lichtmenge $Q=2 \pi J$.

Wir Wollen nun grunz allgemein eine beliebige geschlossene Fläche betrachten, die als eonvex angenommen werden soll. Die Flïche wird von dem Punkte $P$ ans belenehtet (Fig. 3). Denkt man sich eimen Kegel

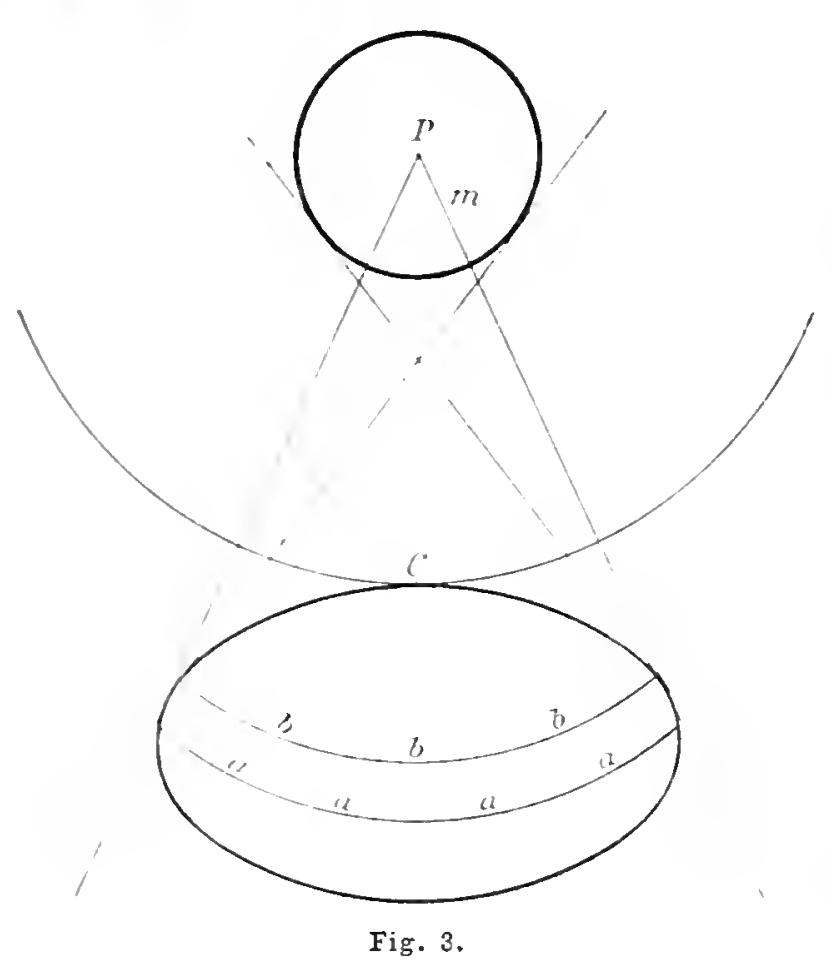
mit der Spitze in $P$, welcher die Fläche umhiillt und dieselbe längs der Curve $a, a, a \ldots$ beriihrt, so folgt ohne Weiteres, dass alle jenseits dieser Curve gelegenen Punkte der Fläche uberhaupt kein Licht ron $P$ erhalten kömnen; sie befinden sich im Schatten. Um $P$ sei eine Kingel mit dem Radius $m$ besehrieben, und es seien an diese Kugel und die Fläehe alle gemeinschaftlichen Beriilhrungsebenen construirt. Die dieser umhiillenden conoidisehen Fläehe und der urspriinglichen Fläche gemeinsamen Punkte $b, b, b \ldots$ bilden eine $\mathrm{Be}-$ riihrungsenre, und da fiir sämmtliche Elemente dieser Curre $p$ denselben Werth hat, so folgrt. dass die Belenchtung lings dieser Curve umgekehrt proportional dem C'ubus der Entfermung der Elemente rom lenehtenden Pnnkte $P$ ist. Legt man $n$ m $P$ eine ganze Schaar ron Kugeln, deren Rialien immer srösser werden. so findet man nene Beriilurungsemren und zwall inmer engere, bis endlich die beriblumgseurve für eine bestimmte Kngel in einen einzigen Punkt iibergeht, den sogenamnten wlainzenden P'unkt C', welcher miter allen Punkten der Fläehe die grösste Beleuchitung erhält. Er liegt nicht nur dem lenchtenden Punkte am nächsten sonden der Winkel $i$ hat fuir ein dort befindliches Element den Maximal werth $\frac{\pi}{2}$. Lext man durch $P$ und $C^{\prime}$ Ebenen. su schmeiden diese di 
Fliehe lïngs Curven, auf welehen dic Belenchitung von $C$ aus bestindig ahnimmt bis zum Schnittpunkte mit der Berllırungseurve $a, a, a \ldots$, wo die Beleuchtung Null wirl. Man nennt diese Curven Belenchtungrmeridiane, während die Berilhrungseurven Beleuchtungsparallele heissen. Denkt man sich diejenigen Punkte der Fliiche mit einmder verhunden, in denen die Belenchtung gleich intensiv ist, so erhïlt man_Curven, welehe im Allgemeinen die oben eharakterisirten Beruhrungseurven selneiden werden; man nenut solehe Curven gleicher Helligkeit Isophoten. Ist die betrachtete Fläche eine Kugel, so fallen Isophoten und Beriihrungscurven uusammen; beim Ellipsoide ist dies sehon nicht melı der Fall, dem dort werlen auf jeder Berlihrungseurve immer nur zwei Punkte sein, die von dem leuchtenden Punkte gleich weit entfernt sind, die also gleiche Beleuchtung erhalten.

Man denke sich wieder eine beliebig gestaltete Flaiche $F$, die nach allen Richtungen eonvex sein möge, von einem leuchtenden Punkte $P$ aus belenchtet. Es sei von $P$ als Spitze der die Fläche umhiillende Kegel eonstruirt, und es sei mit dem Radius 1 um $P$ eine Kugel gelegt; aus dieser Kugel wird dureh den Kegel ein Flichenstick $\varphi$ herausgesehnitten, und man nennt ip die scheinbare Grösse der Fläche $F$, vou $P$ aus geselren. Es ist klar, dass auf $\rho$ dieselbe Lichtmenge libergeht, wie auf die gamre Fliche $F$, nur mit dem Unterschiede, dass dic Beleuchtung auf der Kugel\%one in allen Punkten gan\% gleichmïssig vertheilt ist, anf der Flïche jedoeh nicht. Da nun die auf $p$ fallende Lichtmenge nach dem Obigen gleich $J_{\text {।p }}$ ist, so ergiebt sich der wiehtige Satz, dass die Lichtquantitiit, welche eine Fliache $F$ von einem leuchtenden Punkte $P$ aus erhält, proportional ist der scheinbaren Grösse derselben, gesehen von $P$ aus.

Mit Hülfe dieses Satzes liisst sich \%. B. ganz einfuch die Lichtmenge berechnen, welche auf eine Kugel rom Radius $a$ von einem leuchtenden Punkte aus gelangt, dessen Entfermung rom Mittelpunkte der Kingel gleich $c$ ist. Der unhiillende Kegel wird in diesem Falle ein gerader Kegel und das Fliichenstiickchen ip ist eine Kugelealotte, deren Fliiche ausgedrickt ist durch $2 \pi\left\{1-\frac{\sqrt{c^{2}-a^{2}}}{c}\right\}$. Man hat also fuir die Lichtmenge $Q$, welche anf die Kugel iibergeht, den Ausdruck:

$$
\left.Q=2 \pi J \mid 1-\frac{\sqrt{c^{2}-a^{2}}}{e}\right\} \text {. }
$$

Der Satz von dem Zusammenhange zwisehen Lichtquantität und scheinbarer Grösse lässt cine vielfache Anwendung zu. So kann man die Aufgabe stellen, alle möglichen Lagen cines leuchtenden Punktes zu crmitteln, bei denen eine Fläche $F$ stets dieselbe Lichtmenge erhält. Wrem $Q$ 
constaut sein soll, so muss es auch $\varphi$ sein, und es reducirt sich die Aufgabe daher darauf, alle Lagen von $P$ zu finden, von denen aus gesehen $F$ dieselbe scheinbare Grösse hat. Für eine Kugel ist natürlich der gesuchte Ort wieder eine Kugelfläche, die mit der ersteren concentrisch ist. Man nent Fliichen, von deren sämmtlichen Punkten aus gesehen eine bestimmte Flïche dieselbe schembare Grösse bat, Flächen constanter Kírgeloftinumıg.

Ls ist hier nicht der Platz, nüher auf diese vom rein mathematischen Standpunkte ans höchst interessanten Probleme einzugehen, es soll nur noch kur\% der liall beriihrt werden, der bei astronomischen Aufgaben am Hiinfigsten cintreten wird, dass der lenchtende Punkt schr weit von der erlenchteten Flaiche entfernt ist, dass also fiir alle Elemente der Fläche die Distanz rom lenchtenden Punkte als eonstant angesehen werden kann. Be\%cichnet mam hicr mit $J$ die Lichtquantitiit, welehe auf die Flächencinheit senkreclit anffiillt, so ist die Quantitiit, welehe auf ein Element $d f$ gnelinght, alusgedriickt durch:

$$
d \eta=d f J \cos i
$$

1)er umhiillende Kegel gelit in diesem Falle in einen Cylinder iber, dessen Axr der Richtmig des einfillenden Lichtes parallel ist; die Belenchtmmarente ist die Cnre, in welcher die Fläche von diesem Cylinder beriilut wird. Fragt man mach den Curven gleicher Beleuchtung, so ist offenbil die Bedingmg daftur: $\cos i=$ const. Lantet die Gleichung. der hetralditeten Fliache: $F(x, y,:)=$.0 , und bildet die Richtung des einfallenden I idites mit den Coordinatenaxen die Winkel $a, \beta, \gamma$, so wird der C'osinns des Winkels zwischen der Normalen an irgend einem Punkte der libirle und der libchtung des einfallenden Liehtes bekauntlich durch die Gleirhung answedriickt:

$$
\cos i=\frac{\frac{\partial F}{\cos c+\frac{\partial F}{\partial y} \cos \beta+\frac{\partial F}{\partial y} \cos \gamma}}{\sqrt{\left(\frac{\partial F}{\partial r}\right)^{2}+\left(\begin{array}{l}
\partial F \\
\partial y
\end{array}\right)^{2}+\left(\begin{array}{l}
\partial F \\
\partial z
\end{array}\right)^{2}}} .
$$

Fiir ein Ellipsuid, dessen Mittelpunktsgleiehmng

$$
\frac{r^{2}}{a^{2}}+\frac{y^{2}}{b^{2}}+\frac{a^{2}}{c^{2}}-1=0
$$

lautet, laat main dinilch \%. B.

$$
\cos i=\frac{a^{2} \cos \alpha+\frac{b^{2}}{b^{2}} \cos \beta+\frac{i}{c^{2}} \cos \gamma}{\sqrt{\left(\frac{r^{2}}{a^{2}}\right)^{2}+\left(\frac{n}{b^{2}}\right)^{2}+\left(\frac{\vdots}{r^{2}}\right)^{2}}} \text {. }
$$


Da unn fur alle Punkte, die gleieh stark beleuchtet werden, eos $i$ constant sein soll, so folgt, dass der Durehschnitt des Ellipsoides mit derjenigen Fläehe, welelie dureh die Gleichung

$$
\left[\frac{x}{a^{2}} \cos a+\frac{y}{b^{z}} \cos \beta+\frac{z}{c^{z}} \cos ;\right]^{z}=\text { eonst. }\left[\left(\frac{x}{a^{2}}\right)^{2}+\left(\frac{y}{b^{2}}\right)^{2}+\left(\frac{z}{c^{2}}\right)^{2}\right]
$$

reprilsentirt wird, eine sogenaninte Isophote darstellt. Die vorstehende Gleichung gehört aber einem Kegel zweiten Grades an, dessen Spitze im Mittelpunkte des Ellipsoides liegt. Fur den speeiellen Fall, wo $i=90^{\circ}$ ist, also an der Belenehtungsgrenze, hat man fur deu Kegel die Gleichung:

$$
\left(\frac{x}{a^{2}} \cos \alpha+\frac{y}{b^{2}} \cos \beta+\frac{\ddot{x}}{c^{2}} \cos \gamma\right)^{2}=0,
$$

d. h. der Kegel geht daun in zwei zusammenfallende Ebenen tiber, und die Beleuehtungsgrenze ist eine ebene Ellipse.

Für die Kugel ist es klar, dass bei sehr weit entferntem leuchtenden Punkte, wenn man die Strahlungsriehtung als Axe der Kngel ansicht, die Beleuclitungsgrenze in den $\ddot{A} q u a t o r$ fällt und die Isophoten Parallelkreise siud, ferner dass die Beleuchtung an irgend einem Punkte dem Sinus der Breite proportional ist.

6. Beleuchtung von F]äehen dureh lenchtende Fläichen. Das Lambert'sehe Gesetz vou Cosiuns des Emanationswinkels.

Anstatt leuchtender Punkte sollen im Folgenden selbstlenclitende Fläehen betraelitet werden, und zwar soll ganz allgemein die Lichtquantitiit ermittelt werden, welche vou einer beliebig gestalteten leuchtenden Flïclıe auf eine andere ebenfalls gan\% beliebige Fliche gelangt. Um von dem einfachsteu Falle auszugehen und einige nene wichtige Définitionen einzufiilıren, sei df (Fig. 4 ) ein kleines ebenes selbstleuchtendes Fläehenelement, welehes nach allen Richtungen auf die ganze Hemisphäre Licht aussendet. In der Entfermung $r$ von demselben befinde sich ein zweites elyenes Flächenelement $d o$, welches von dem ersteren Licht zugesandt erhïlt. Die

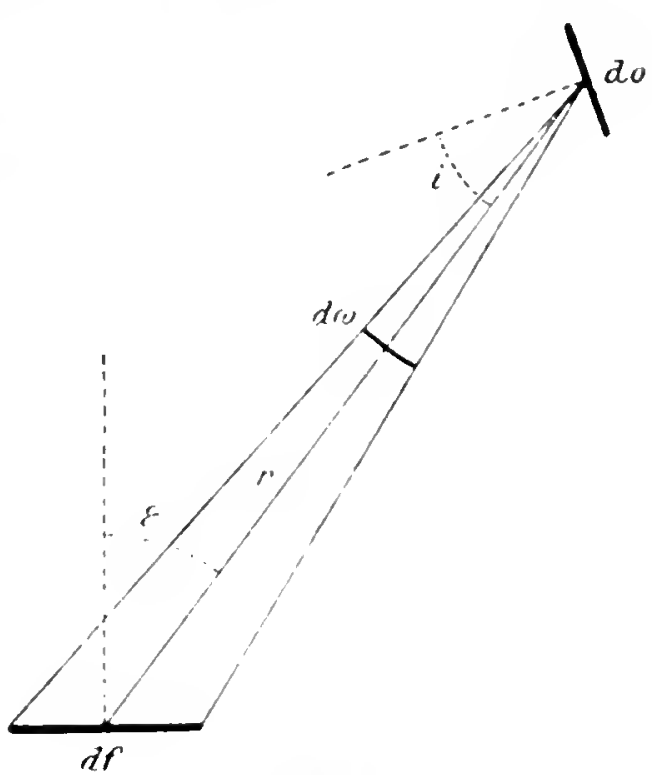

Fig. 4. Normalen zu den beideu Elementen mögen mit der Verbindungslinic 
derselben die Winkel $\varepsilon$ und $i$ einsehliessen, von denen der erstere der Limanationswinkel oder Ausflusswinkel genannt wird. Denkt man sich dis lenehtende lilement aus lauter leuehtenden Punkten zusammengeset\%t, rou denen jeder einzelne der A usgangspunkt einer Lichtbewegung. scin möge, so wird sich die Wirkung derselben anf do summiren, und mill wird daher in erster Linie sagen können, dass die Liehtmenge, welehe ron af anf do ïbergeht, proportional sein muss der Grösse des lenchtenden Elementes. Nach dem Friheren muss diese Liehtmenge aber anch noeh proportionil sein der Grösse des beleuchteten Elementes, dem Cosinns des Incidenwwinkels i mnd dem nmgekehrten Quadrate der Entfermun; ferner muss die knergie der Lichtentwicklung in den einzelnen l'unkten ron df als constanter Factor $J$ anftreten, und endlich wird die lichtwirknug anch now in irgend einer Weise rou dem Emanationswinkel $\varepsilon$ आhaingen. Wir wollen diese Abhängigkeit zunäehst ganz allgemein dureh die Funtion $f(s)$ bezeichnen.

Dic Lichtnuenge $d L$, welche von $d f$ auf do iibergeht, ist demnach :msigedriickt dureh die Formel:

$$
d L=J d f d o \cos i \frac{1}{r^{2}} f\left(\dot{c}_{l} .\right.
$$

Den Finctor $J$, welcher die Stairke der von dem Elemente df ausgehenhon Livhthewewn chankterisirt, nemt man die Liehtintensität oder beulkhaft des Elementes. Betraehtet man mur die Flicheneinheit des belunchteten Elementes do, so ergiebt sich die Lichtmenge $d L^{\prime}$, welche ron dem lenchtenden Elemente df anf diese Flächeneinheit iibergeht, aus der riloidhung:

$$
d L^{\prime}==\int d f \cos i \frac{1}{\gamma^{2}} f(\varepsilon) \text {. }
$$

Man nemut diese Grisse allgemein die Belenchtung im Elemente do.

Wird forner $i=0$, sn fiillt das Lieht senkrecht auf; man nemnt die von dem lenchtenden Elemente df ant' die Flicheneiuheit senkrecht gelinguende Lirlitnenge $D=J d f \frac{1}{r^{-2}} f(c)$ die Dichtiggkeit der Belenchtmug. Wird eudlich noch statt $r$ die Einheit der Entfermung angenommen, \&n erhailt man die ron df auf die Flächeneinheit in der Entfermung 1 sonkrecht anffillende Lichtmenge $d_{y}=J d f f(\varepsilon)$; man nemnt diese Grijste. Welche rom der Lage, Grösse und Entfermung des bestrahlten klencutes nubbhängig ist, ganz allgemein die unter dem Winkel $\varepsilon$ anszestrahlte Lichtmenge.

Ls ist bisher voranswesetzt worden, dass die Flächenelemente im Verhiiltniss zu den Entfernungen als schr klein zu betrachten sind. Nimmt 
man sie so klein an, dass alle von irgend cinem Punkte vou $d f$ nach irgend einem lunkte von $d o$ gezogenen Linien unter einander parallel sind, so hat man es mit einem mendlich dunnen, unter dem Winkel $\varepsilon$ ausgehenden Liehteylinder zu thun. Eine Ausbreitung des Lichtes in Raume findet nicht statt, und die Fliicheneinheit des zur Cylinderaxe senkrechten Querschnittes erhailt daher von $d f$ in allen Entfernungen die Lichtmenge o df $f(\varepsilon)$. Diese Grösse wird häufig als Dichtigkeit des unter dem Emanationswinkel $\varepsilon$ vou df ausgehenden Lichtcylinders bezeichnet, und man sieht, dass diese Bezeichnung mit dem oben gewiilhlten Ausdruck susgestrahlte Liehtmenge gleiehbedentend ist.

Wir wollen uns nun an der Stelle des belenchteten Elementes do das menschliche Auge denken. Betrachtet man die Wirkung des lenchtenden Elementes off auf dasselbe, so spricht man von der Helligkeit des lenchtenden Elementes $d f$, und zwar unterscheidet man die wirkliche und die seheinbare Helligkeit. Unter der wirkliehen Helligkeit versteht man die Liehtquantitait, welche die Flächeneinheit des Elementes $d f$ senkrecht anf die Fläeheneinheit des Auges gelangen lässt, also nach dem Obigen die Grösse: "Dichtigkeit der Beleuchtụng dividirt durch die Grösse des Elementes dfa. Wenn man daher die wirkliche Helligkeit mit $H$ bezeichnet, so ist

$$
H=\frac{D}{d f}=J \frac{1}{r^{2}} f(\varepsilon) .
$$

Hat man an Stelle des einen leuchtenden Elementes eine selbstleuehtende Fläiche, deren einzelne Elemente $d f_{1}, d f_{2}, d f_{3} \ldots$ mit den Lenchtkräften $J_{1}, J_{2}, J_{3} \ldots$ begabt sind, ausserdem die Emanationswinkel $\varepsilon_{1}, \varepsilon_{2}, \varepsilon_{3} \ldots$ und die Entfernungen $r_{1}, r_{2}, r_{3} \ldots$ besitzen, so gelangt von der ganzen Fläche die Lichtquantitit

$$
J_{1} \frac{1}{r_{1}^{2}} f^{\prime}\left(\varepsilon_{1}\right) d f_{1}+J_{2} \frac{1}{r_{2}^{2}} f\left(\varepsilon_{2}\right) d f_{2}+\cdots
$$

senkrecht anf die Fliichencinheit des Anges; man spricht dann ron einer mittleren wirklichen Helligkeit der leuchtenden Flaiche und rersteht darunter den Qnotienten

oder

$$
\frac{J_{1} \frac{1}{r_{1}^{2}} f\left(\varepsilon_{1}\right) d f_{1}+J_{2} \frac{1}{r_{2}^{2}} f\left(\varepsilon_{2}\right) d f_{2}+\cdots}{d f_{1}+d f_{2}+d f_{3}+\cdots}
$$

$$
\frac{\sum\left(J \frac{1}{r^{2}} f(\varepsilon) d f\right)}{\sum(d f)}
$$


Um den Begriff der scheinbaren Helligkeit zu fixiren, denke man sich in der obigen Figur un do eine Kugel mit dem Radius 1 construirt, welche ans der Pyramide, die $d f$ zur Grundfläche und in do die Spit\%e hat, das Element de heransselmeidet. Man nennt $d \omega$ die seheinbare Grösse des Elementes $d f$ und rersteht unter der seheinbaren Helligkeit ron af die von diesem Elemente ausgehende Dichtigkeit der Belenehtung, dividirt dureh die scheiubare Grösse des Elementes $d f$. Bereiehnet m:m diese scheinbare Helligkeit mit $h$, so wird also:

$$
h=\frac{D}{d \omega}=\frac{J d f \frac{1}{r^{2}} f(\varepsilon)}{d \omega},
$$

und da $d \omega=\frac{d f \cos \varepsilon}{r^{2}}$ ist, so wird:

$$
h=\frac{J f(\varepsilon)}{\cos \varepsilon} .
$$

Inn sieht also hieraus, dass die scheinbare Helligkeit eines Fläehenclementes von der Entfermug rom Auge ganz mabhängig ist und nur durch die Leunhtkraft und den Emanationswinkel bestimmt wird. Hat man wieder eine lenchtende Fliiehe statt eines einzelnen Elementes, so besichnet man entsprechend wie oben mit mittlerer seheinbarer Flä chen helligkeit den Quotienten ans der gesammten Lichtmenge, welche ron der sanzen Fliche senkrecht anf die Flacheneinheit des Auges gelanıgt. und der scheinbaren Grösse der gamzen Fläche, also die Grösse

$$
\frac{\sum\left(J \frac{1}{r^{2}} f(c) d f\right)}{\sum(d(v)}
$$

Wir haben bisher die Abhängigkeit der Lichtwirkung von dem Emanationswinkel ganz allgenein dureh die Function $f(\varepsilon)$ bezeichnet. Wenn jeder Pmkt des lenchtenden Elementes als Ansangspunkt einer nach allen livchtungen gleich enerwischen Lichtbewegme aufzufassen ist, so sollte es anf den ersten Blick scheinen, als misste das Element nach allen Richtnngen dieselbe Liehtmenge ansstrahlen. Diese Ansicht ist in der That ron Enler vertreten und ron $L$ aplace spaiter acceptirt worden, und sie wirde anch hurdhans einwurfsei sein, wemn das le uchtende Element als eine rein mathematische Fliche angesehen werden dirite. Dies int alher keineswegs statthaft. und wir werden sogleich selın. zu welkh wimzlich anderem Belenchtungsesetze man gelangt, wenn mall die allein richtige tmiahme macht. das bei jedem selbstlenchtenden 
Körper das Licht nicht nur von den Oberflächentheilchen ausgesandt wird, sondern auch aus einer gewissen Tiefe unterhalb der eigentlichen Oberlliche herkomnt. Nach der Euler'schen und Laplace'sehen Anschaunngsweise wiire die Funetion $f(\varepsilon)$ gan\% unberticksichtigt zu lassen, und nach den obigen Formeln mltsste daher die seheinbare Helligkeit eines leuehtenden Elementes ausgredrllekt sein dureh $h=\frac{J}{\cos \varepsilon}$, d. h. die seheinbare Helligkeit des Elementes $d f$ mitsste immer grösser werden, je grösser der Emanationswinkel wird. Danach musste also eine gllihende Metallplatte, von der Seite her betrachtet, viel heller beurtheilt werden, als senkreeht von vorn geschen, und eine gluhende Metallkugel musste am Rande beträchtlieh intensiver erseheinen, als in der Mitte.

Die Euler'sche Vorstellungsweise ist von Lambert in seinem Hauptwerke1) uber die Plotometrie nicht acceptirt worden. Er nalum die von einem Fliachenelemente of ansgehende Lichtquantitait nicht unabhïngig von dem Ausflusswinkel an, sondern stellte das nach ihm benannte Emanationsgesetz auf, wonach die Lichtquantitiit proportional dem Cosinus des Emanationswinkels sein soll. Danach wäre also $f(\varepsilon)=\cos \varepsilon$ zu' setzen; die wirkliche Helligkeit eines leuehtenden Elementes wiire $H=J \frac{1}{\gamma^{2}}$ und dic scheinbare Helligkeit $h=J$. Es wiirde also das bemerkenswerthe Resultat folgen, dass die scheinbare Helligkeit iuberall die gleiehe wïre. Eine gliihende Metallplatte miisste von allen Richtungen ans betrachtet gleieh hell erseheinen, mnd eine lenehtende Kngel wiirde :m Rande eben so hell ausschen wic in der Mitte. Lambert glaubte eine der wichtigsten Stlitzen für seinen Satz darin zu erblieken, dass die Sonnenseheibe an allen Punkten gleich hell erschienc. Es war ihm unbekannt, dass bereits Bonguer dureh geniigend zuverlïssige Messungen den Beweis erbracht hatte, dass die Randpartien der Sonne betrïichtlich lichtschwïcher sind, als die Centralpartien; er hatte das Vorhandensein einer Sonnenatmosphïre gar nicht in Betracht gezogen und komnte daher aneh nicht zn dem cinzig richtigen Sehlusse gelangen, dass die Erscheinungen an der Sonne wegen des uncontrolirbaren Einflusses einer unbekannten Sonnenatmosphäre iiberhaupt nicht zn Gunsten odes Ungunsten irgend welcher Emanationstheoric entscheiden können. Wodureh Lambert speeiell zu scinem Emanationsgesetze gefiihrt worden ist, lïsst sich nicht mit Sicherheit angeben, soviel aber steht fest, dass er von der Tragweite desselben flir die ganze Photometrie iiberzengrt gewesen ist und dass er Alles ver-

1) Lambert, Photometria sive de mensura et gradibus luminis, colorum et ambrae (1760). Deutsche Ausgabe von Anding, Leipzig, 1892. 
sucht hat, $\mathbf{m}$ anf experimentellem und theoretischem Wege die Richtigkeit seines Satzes zu beweisen. Leider verfügte Lambert uber durchaus migenllgende Huilfsmittel, und die Versuche, durch Beobachtungen sein Geset\% plausibel \% machen, miissen als durehaus unzureichend bezeichnet werlen. An selbstleuchtenden Substanzen, also etwa glïhenden Platten oder Kugehn, sind ron ilm iberhanpt keine Experimente angestellt worden. Auch der Limbert'sche Versuch eines theoretisehen Beweises ist wegen der willkiirlichen Ammahmen, die dabei zn Grunde gelegt sind, als verfullt \%u betrachten, und dasselbe gilt in noch viel stïrkerem Grade ron den Beweisen, die später Beer ${ }^{1}$ ) und liheinaner'2) zur Stütze des Limmbertschen Gesetzes hinmgefïgt haben, und die, wie sehon Zöllner ${ }^{3}$ ) dirrugethan lıat, nur auf einem Kreisschlusse bermhen. Zöllner gebiilurt olne /wweifel das Verdienst, znerst anf die Unhaltbarkeit der hisherigen Versuche, das Lambert'sche Gesetz zu beweisen, aufmerksam semacht und gleichzeitig den Weg gezeigt zu haben, anf dem man :lloin \% klatren Anschamungen gelangen komte. An der Richtigkeit des

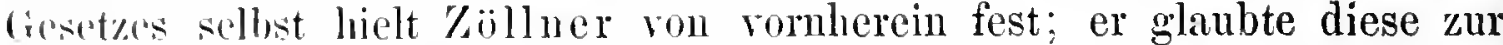
(ieniinge durch alle experincutellen Versuche ansser Zweifel gestellt und er versuchte nur, anf plansiblere Weise als bisher anch theoretisch den Sitt\% \% stiitz'n. Er verwies anf die mit dem Lichte nahe verwandten Ersheinumen der strihlenden Wäime, machte daranf aufmerksam, dass anch bei der Wärmeansstralıhng die Intensitit ron dem Cosinus des Emanatimswinkels abliangt nud dass bereits Fourier eine rollkommen aus-

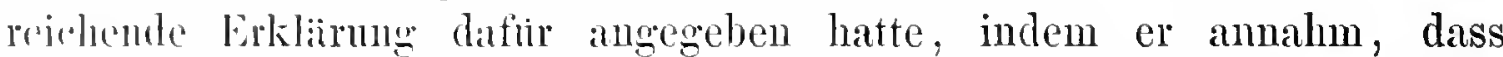
nirlit nur die an der Oberfliche eines Körpers gelegenen Molekiile, smolern anch die unter der Oberfliche befindlichen als Sitz der Wärmeinstrallung zu hetrachten seien. Indem Zällner diese Fourier'sche Hypo-

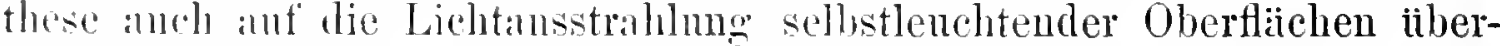
trug, solumte er zu cinem einwurfsfreien Beweise des Lambert'schen Binanatimsurestzes. Damit hat Züllner einen wichtigen Schritt gethan, desen bedentume fïr die Entwicklung der theoretischen Photometrie noch

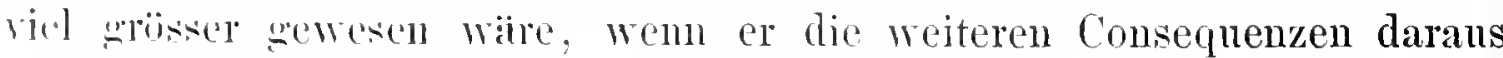

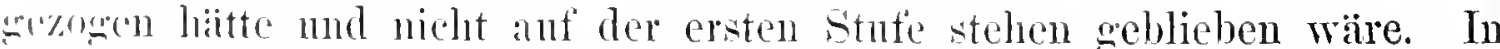
dou Irothme befangen, dass das Lambert'sche Gesetz durch alle Beobachtmowen manferhtlar nacherewesen sei, so anch heispielsweise für die

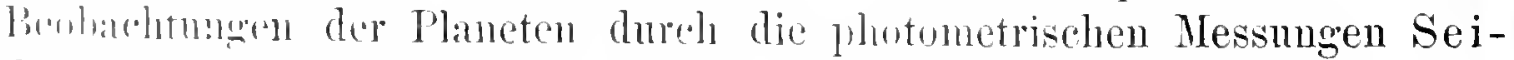

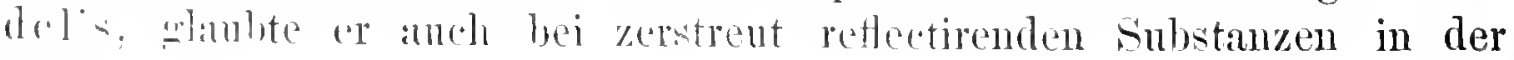

1. Heer. Grundriss des photometrischen (alciiles. Braunschweig, 1854. p. 6.

-) Rheinauer, Grundziige der Photometrie. Halle, 1462. p. 2.

3 Zijllntr. Piotometrische Untersuchmeen mit besonderer Rücksicht auf die physische beschaffenheit der Himmelsküper. Leịzig, 166j. p. 12. 
weiteren Verfolgung der Fourier'sehen Vorstellungen eine Ableitungr des Lambert'schen Gesetzes zu finden. Er hat aber dabei einen sehr wichtigen Umstand ubersehen, dass nimlieh bei den zerstrent reflectirenden Substanzen sowohl beim Eindringen des Lichtes bis zu einer gewissen Tiefe als beim Ausstrahlen eine Absorption stattfindet, welche die Lichtorseheinungen wesentlich modifieirt. Wir werlen im näehsten P'aragraphen zeigen, dass die Berlieksiehtigung dieser Umstiinde \% einem wesentlielı anderen Gesetze als dem Lambert'schen fuhrt. Soviel lier nur in Kulire uber Zöllner's Stellung zum Lambert'selıen Emanationsgesetze.

Wir kehren nach dieser Abschweifung zu den selbstlenchtenden Oberflïchen zurlick und wollen zmuiichst den strengen Beweis angeben, den zuerst Lommel fur das Lambert'sche Enamationsgesetz auf Grund der Fourier'schen Anschauungsweise aufgestellt hat. Dieser Bewcis findet sieh ganz versteckt in ejner Abhandlung Lommel's1) iber das Fluoreseenzlicht, in weleher ausserordentlieh wichtige Bemerkungen über die Grundsiitze der Photometrie enthalten sind. Es sei (Fig. 5) $M N$ die Oberfliche eines leuchtenden Körpers, $A B=d \varphi$ ein Oberflichenelement. In der Riehtung $A P$, welche mit der Normale $A C$ den Winkel $\varepsilon$ bildet, mögen die Strahlen (unter einander parallel) aus dem Körper austreten; damn werden nach der Fouriersehen Ansehaung alle diejenigen Volumelemente an der Liehtansstrahlung sich betheiligen, welehe in dem schiefen Cylinder enthalten sind, der $A B$ zur Basis hat und dessen Axe der Riehtung $A P$

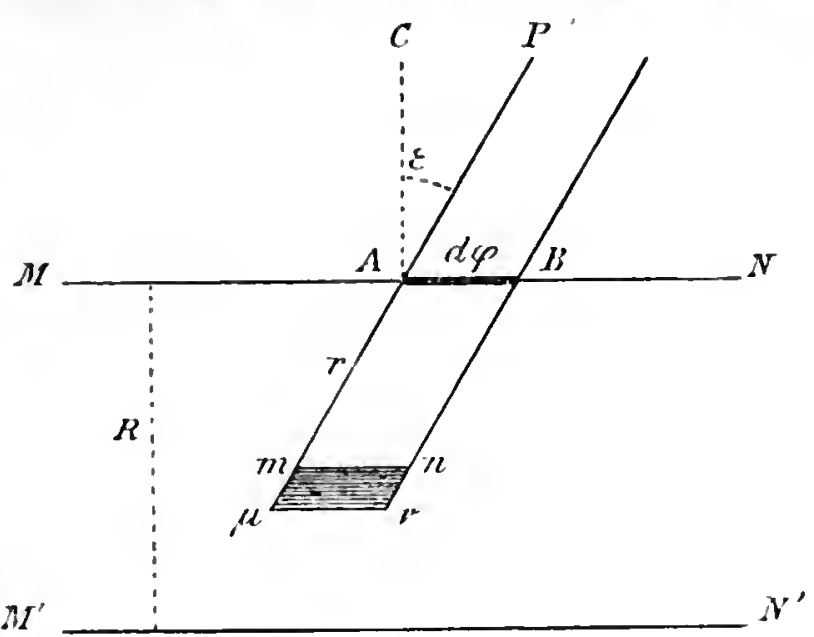

Fig. 5. parallel ist. Die Tiefe, ans weleher noch Licht hervordringen kann, hängt von dem Grade der Durehsichtigkeit des lenchtenden Körpers al). Bei den sogenannten undurchsielitigen Körpern dringt das Licht nur aus ganz geringer Tiefe unter der Oberfliiche hervor, sie werden erst in unendlich dimnem Zustande durchsichtig; während z. B. gliilıende Gasmassen das Lieht aus riemlich tiefen Schichten hervorkonmen lassen. Wir wollen uns hier zunächst nur mit Körpern der crsten Gattung beschïftigen und ein kleines Volumelement m.nuv in Betracht ziehen, welehes von dem Oberflïchenelemente $A B$ um die Strecke $A m=r$ entfernt ist. Die Leneht-

1) Wiedem. Annal. Bd. 10, p. 449. 
kraft des Körpers, den wir als gleichmässig leuchtend annehmen wollen, sei $f$, d. h. die Volumeinheit möge in der Entfernung 1 auf die Flächeneinheit senkrecht die Lichtmenge $J$ ausstrahlen, vorausgesetzt, dass keine Absorption stattfindet. Das Volumelement $m n \mu \nu$, dessen Iuhalt $=d$ ip $d r \cos \varepsilon$ ist, wlirde also ohne Absorption die Lichtquantität

$$
q=J d \uparrow d r \cos \varepsilon
$$

anssenden. Bezeichnen wir nun dic Änderung, welche $q$ auf einem sehr kleinen Wege $d r$ erleilet, mit $d q$, so ist klar, dass $d q$ negativ sein muss, weil eine lichtabnahme stattfindet, ferner dass es proportional dem zurilckgelegten Wege $d r$; ebenso proportional der ursprïnglichen Energie $q$ scin muss, und endlich, dass es infolge der dem Körper eigenthümlichen Alsorption mit einem gewissen Absorptionseoefficienten zu multipliciren ist. Man hat also:

$$
d q=-l i q d r, \quad \text { oder }: \frac{d q}{q}=-k d r
$$

Durch lntegration iiluer die ganze Strecke $r$ findet man:

$$
\log \frac{q_{0}}{q}=-k r, \quad \text { oder }: \quad \frac{q_{0}}{q}=e^{-k r}
$$

wo $\eta_{0}$ die Lichltguantitiit ist, wclche anstatt der urspriinglichen Quantität

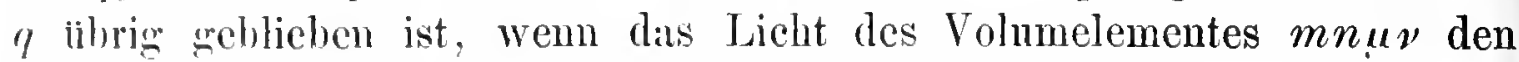
Kïrper verlïsst. Dureh Substitution von y hat man:

$$
q_{\mathrm{n}}=J d \boldsymbol{d} d r \cos \varepsilon e^{-k r} .
$$

Simmtliche in dem schiefen Cylinder enthaltenen Vohmelemente senden also dic lichtnenge aus:

$$
c_{\imath}=\operatorname{Idr} \cos \varepsilon \int_{i j}^{\prime \prime} d r e^{-k r},
$$

wo die Integration $v 01 v=0$ an bis zu cinem Werthe $r=0$ anszuführen ist. für welchen ibluerhaupt kein Licht mehr ans dem Körper hervordrin-

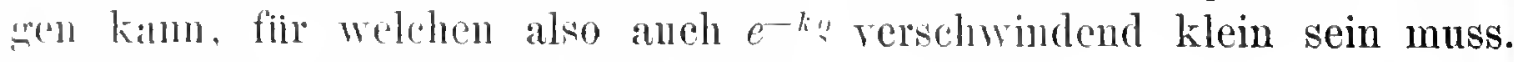
Die Insfiihrung der Integration liefert:

$$
()=I d_{i j} \cos \varepsilon \frac{1}{l_{i}}\left\{1-e^{-k g}\right\},
$$

und da nath dom eben Gesagten $e^{-7 " n g l e i c h ~ N u l l ~ s e i n ~ s o l l, ~ s o ~ w i r d: ~}$

$$
Q=\frac{1}{l_{i}} \cdot J d y \cos \varepsilon \text {. }
$$


Bei senkrechter Ausstrahlung $(\varepsilon=0)$ wilrde man haben:

$$
Q_{0}=\frac{1}{l} J d r
$$

mithin :

$$
\frac{Q}{Q_{0}}=\cos \varepsilon \text {. }
$$

Es ist hiermit der Lambert'sche Satz vom Cosinus des Emanationswinkels ganz streng bewiesen, allerdings un für selbstlenchtende Körjer und auch bei diesen nur fur sogenamite undurehsichtige Substanzen. Wilrde der durch die Flichen $M N$ und $M^{\prime} N^{\prime}$ begrenzte Körper ein durchsichtig glihender sein, so milsste man, um die gesammte von allen Volumelementen des schiefen Cylinders durch dip ansgesandte Liclitmenge zu erhalten, die Integration von $r=0$ bis $r=\frac{R}{\cos \varepsilon}$ ausfuhren, wenı $R$ die Dicke des betrachteten Körpers ist. Man erhielte damn:

ebenso:

$$
Q=\frac{1}{k} J d \varphi \cos \varepsilon\left\{1-e^{-k \frac{k}{\cos \varepsilon}}\right\}
$$

und mithin:

$$
Q_{0}=\frac{1}{l} \cdot \operatorname{lip}\left\{1-e^{-k R}\right\}
$$

$$
\frac{Q}{Q_{0}}=\cos \varepsilon \frac{1-e^{-k \frac{k}{\cos \varepsilon}}}{1-e^{-k R}} .
$$

Fïr $\varepsilon=0$ und $\varepsilon=90^{\circ}$ wird dieser Ausdruck, ebenso wie bei dem cinfachen Lambert'schen Emainationsgesetze, gleich 1 resp. wleich 0, nud im Allgemeinen nimmt der Werth mit wachsendem $\varepsilon$ bestiindig alb, bleiht jedoch stets grösser als eos $\varepsilon$. Fist für eine mendlich dieke Sehicht geht der Ausdruck in das reine Emanationsgreset\% iiber.

Wir wollen diesen Gegenstand, so interessant er anch namentlich im Hinbliek anf das Verhalten aller lenchtenden Flammen und aller grliihenden Gasmassen ist, hier nicht weiter verfolgen, sondem kehren zo den sogenannten undurchsichtigen selbstlenchtenden Fliichen zuriick, fiir welche das einfache Lambert'sche Emanationsgeset\% als griltig nachgewiesen worden ist, um noeh einige allgemeine Betrachtmugen iiber die gregenseitige Belenchtung von Flaichen anzukniipfen und einige speeielle Aufgalben zu behandeln, die fiir die Astrophotonetrie ron Bedeutung sein kömen. lieiläufig verdient noch erwilhnt zu werden, dass anch experimentell die Inwendbarkeit des Emanationsgesetzes anf die Lichtausstrahlung gliihender Metallstreifen, also undurchsichtiger selbstleuchtender Körper, in neuerer Zeit dureh Versuche von M̈̈lle ${ }^{1}$ ) in Strassburg ansser Zweifel gestellt worden ist.

1) Elektrotechnische Zeitschrift. Bd. 5, p. 370 und 405.

Măller, Photometrie. 
Is sei $f$ (Fig. 6) eine selbstlenchtende Fläche und $F$ eine andere daron helenchtete belicbige Fliiche; $d f$ sei eil Oberflächenelement der

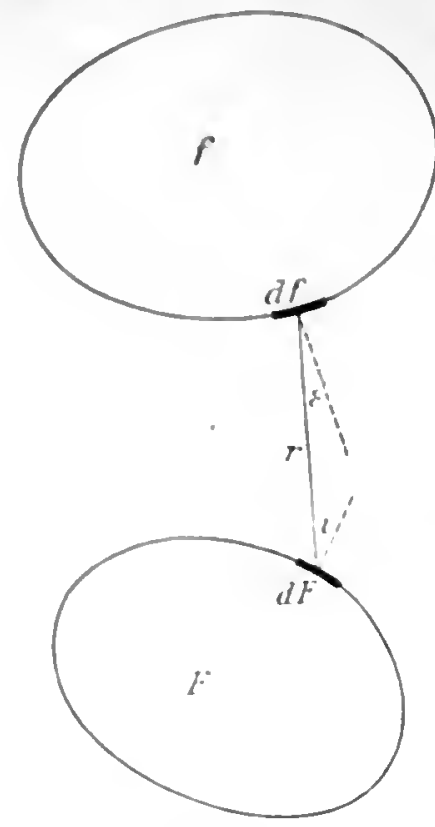

Fig. 6. ersten, $d F$ ein Element der zweiten Fläehe. Ist die Fliche $f$ gleichmässig leuchtend, ist also die Leuchtkraft $J$ in allen Punkten die gleiche, so ist nach den bisherigen Erörterungen die Lichtquantitiit $d L$, welche ron dem Element $d f$ auf das Element $d F$ übergeht, ansgedriickt durch:

$$
d L=J d f d F \cos i \cos \varepsilon \frac{1}{r^{2}} .
$$

Um die Lichtmenge zn haben, die ron dem granzen Körper $f$ auf das Element $d F$ gelangt, hat mam zul summiren iiber sämmtliche Theilchen von $f$, die ron $d F$ aus frei sichtbar sind, und un endlich die ganze Iichtquantitait zu haben, die von $f$ anf $F$ gelangt, hat man eine zweite summation iiber alle Theilchen $d F$ zu bilden, auf die von $f$ ans iiberhaupt Licht gelangen kaun. Ilan hilt also fïr die lichtmenge $L$, welche ron $f$ auf $F$ gelangt, den Insinurk:

$$
L=J \sum \sum\left(d f d F \cos i \cos \varepsilon \frac{1}{r^{2}}\right) \text {. }
$$

Denkt miln sich mun umgekehrt $F$ als gleichmissig lenehtende Fläche mit de' lachlitkrift $f^{\prime}$. so wirde entsprechend die Lichtmenge $L^{\prime}$, welche

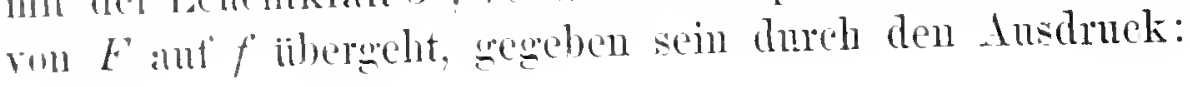

$$
L^{\prime}=J^{\prime} \Sigma \Sigma\left(d F d f^{\prime} \cos i \cos \varepsilon \frac{1}{r^{2}}\right) \text {. }
$$

und dat die bnprelsummen einander greich sind, so erhält man unmittelbar:

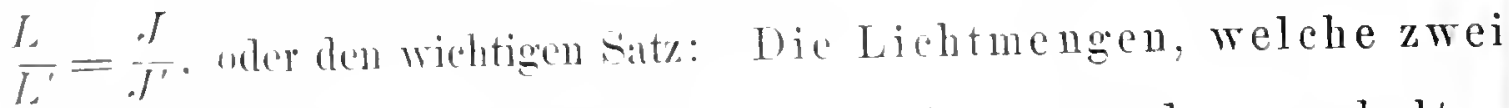

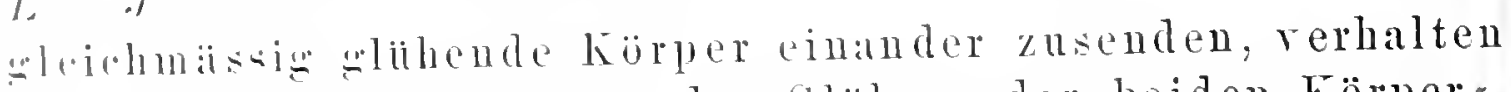
sich wie die Tutensititen des Glibens der beiden Körper", mber: Dic Lichtynantitäten, welche zwei leuchtende Flächen

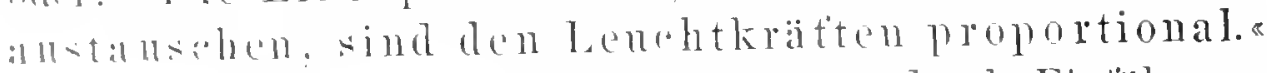

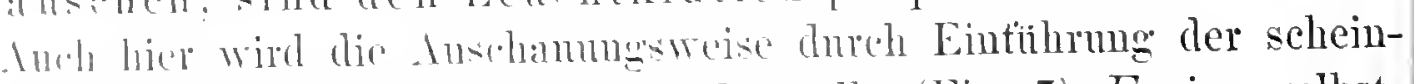

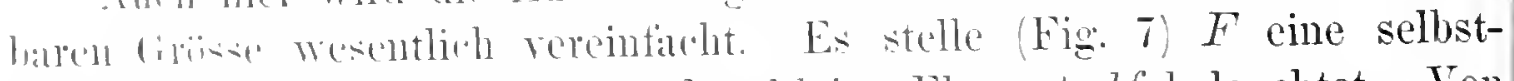

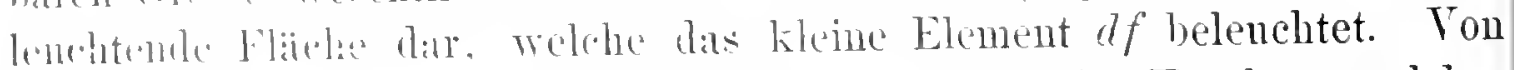

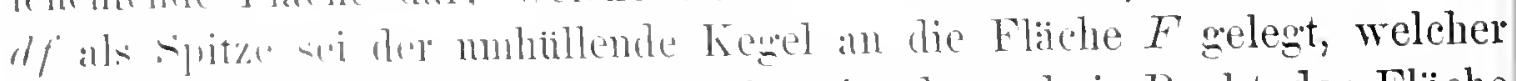

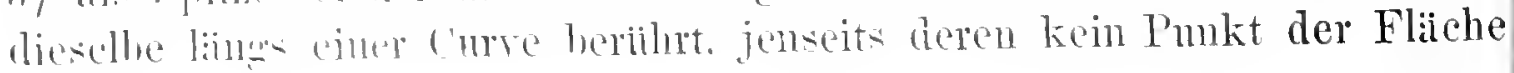


Licht nach $d f$ schicken kann. Coustruirt man um $d f$ als Centrum eine Kugel mit dem Radius 1, so wird aus dieser dureh deu Kegel ein Stilck lerausgeschnitten, welelies man die seheinbare Grösse der leuchtenden Fläche $F$ nennt. Auf irgend einem Radius liegen die Elemente do und dF. Nach den bisherigen Sitzen ist die Ijichtmenge $d L$, welche von $d F$ auf $d f$ d libergelit, gegeben durch den Ausdruck:

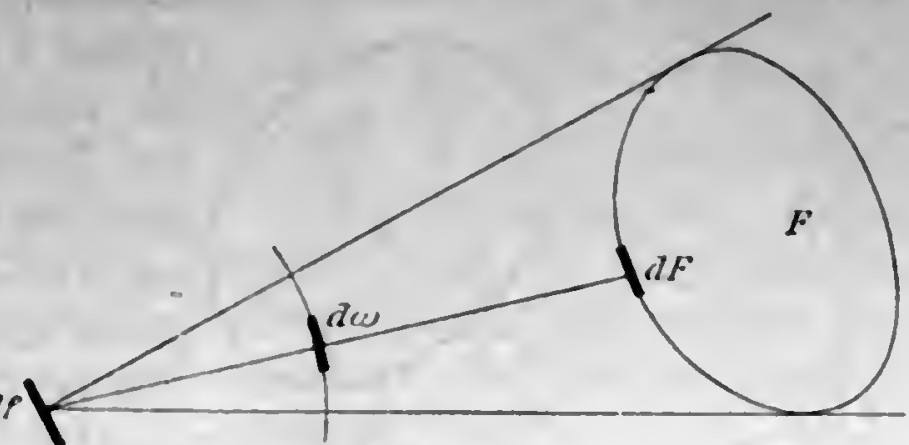

Fig. 7.

$$
d L=J d F d f \cos i \cos \varepsilon \frac{1}{r^{2}} .
$$

Waire das Element $d \omega$ mit derselben Lenehtkraft $J$ wie die Fläche $F$ selbstlenchtend, so wirde es nach $d f$ die Lichtmenge $d L^{\prime}=I d \omega$ df $\cos i$ senden, da $\varepsilon$ in diesem Falle gleich Null wäre. Nnn sei aber $d \omega=$ $\frac{d F \cos \varepsilon}{r^{z}}$; folglich wird $d L=d L$, und es gelangt also von $d \omega$ dieselbe Lichtmenge auf $d f$ wie ron $d F$. Dasselbe gilt von den entsprechenden Elementen der Fläehe $F$ und der Hülfskugel, und man kamn daher die gesammte Lichtmenge, welehe von einer beliebigen Fliclie $F$ mit der gleichmässigen Lenchtkraft $J$ auf ein Element df iibergeht, ersetzen durch die von der seheinbaren Grösse der Fläche ausgehende Lichtquantitiit, vorausgesetzt, dass anch die seheinbare Figur iiberall die Leuchtkraft $J$ besityt; ja man kamn diese Substitution aneh dann noch einführen, wenn die lenchtende Fläche nicht gleichmässig lenchtend ist, rorausgesetzt nur, dass man in jedem Punkte der Hiilfskugel dieselbe Intensitiit annimmt, welehe in dem entsprechenden Punkte der Fliiehe herrscht.

Dieser Hiilfssatz kann num dazu benutzt werden, um eine ganze Reihe ron Aufgaben zu lösen, welche die Belenehtung eines horizontalen Elementes dureh einen irgendwie gelegenen selbstlenehtenden Kreis oder eine Ellipse, durch ein sphärisehes Dreieck, dureh eine selbstlenchtende Kngel oder ein Ellipsoid n. s. w. behandeln. Anding hat in seiner deutschen Ausgabe von Lamberts *Photometria * in der Anmerkmog zu $\$ 140$ daranf aufmerksam gemacht, dass bei allen lenehtenden Flïehen, die einen Mittelpunkt haben, die Beleuehtungsanfarabe sich daranf reducirt, die Lichtcquantitait zu ermitteln, welche die betreffende Fliiche anf das horizontale Element sendet, wenn der Mittelpumkt senkrecht iiber demselben, also im Zenith, liegt. 
In der 'That sei (Fig. S) $F$ irgend eine Fläehe mit Mittelpunkt, $d F$ ein Oberllichenelement, $C$ der Mittelpunkt, if das belenchtete horizontale

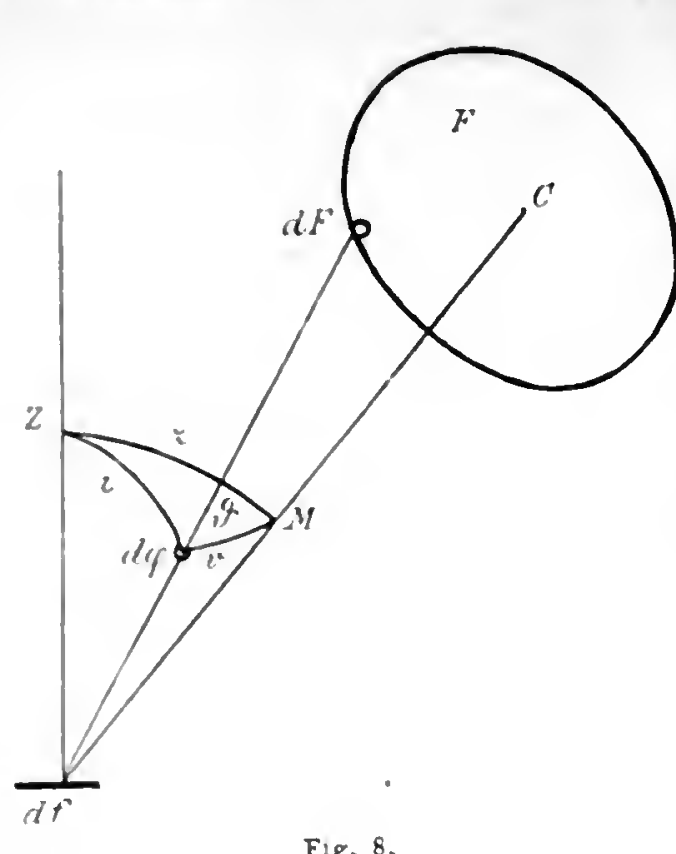

Fig. 8 . Element. Auf der Huilfskugel mit dem Radius $1 \mathrm{um}$ df als Centrum sei $d \varphi$ die scheinbare Grösse von $d F, Z$ sei das Zenith und $M$ die Projection des Mittelpunktes; daun ist in dem sphärischen Dreiecke $Z M d p$ die Seite $Z d r p$ gleich dem Incidenzwinkel $i$, die Seite $Z M$ ist die Zenithdistanz $\approx$ des Mittelpunktes $C$; die dritte Seite sei mit $v$ bezeichnet, ferner heisse $\vartheta$ der Winkel zwischen $v$ und $x$. Dann hat man:

$$
\cos i=\cos x \cos x+\sin x \sin v \cos \vartheta \text {. }
$$

Die Lichtquantität, welche von $d F$ nach af gelangt, ist nach dem obigen Hiilfssatze insgedriickt durch:

$$
d L^{\prime}=J d f d i p \cos i,
$$

mler, namb sulsstitution ron cos $i$, durch:

$$
\| L^{\prime}=J d f^{\circ} \cos : \cos r d p+J d f \sin : \sin r \cos \vartheta d p .
$$

Xim riveht as hei riner Mittelpunktsfiche zu jedem Elemente $d F$ ein zwrite's. fiir wolches, denselben Werth hat, fiir welches aber $\vartheta$ in den Wroth,+ , iiberoht. Bei der Integration iiher alle Elemente $d . F$ fällt

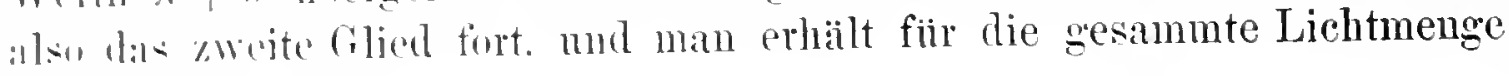
de'll Wirth:

$$
L^{\prime}=\operatorname{s} d f \int \cos \therefore \cos r d y,
$$

mul dil ans: annstant jist. so wird:

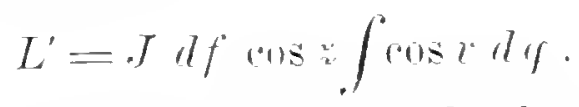

Fo fillut alsu der wichtige Satz. dass die Lichtmenge, welche eine littelpunktstäche anf eju horizontales Element wirft, stets propurtimal dom Cusinus der Zenithdistanz des Mittelpunktes

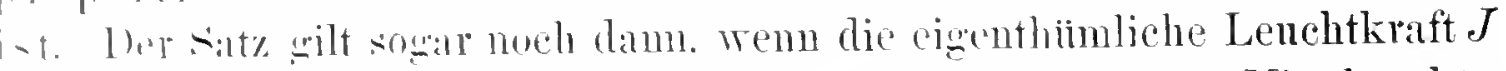

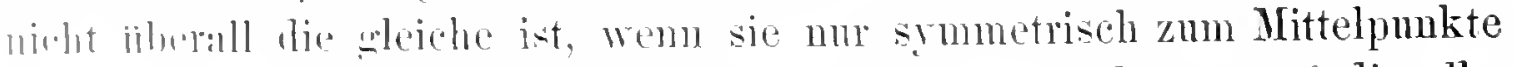

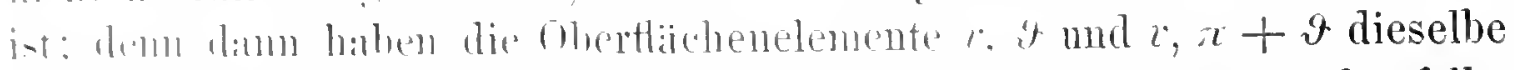

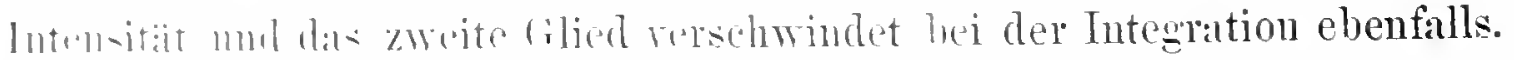

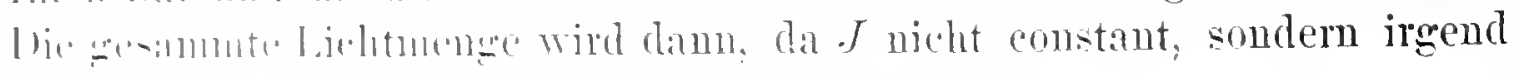

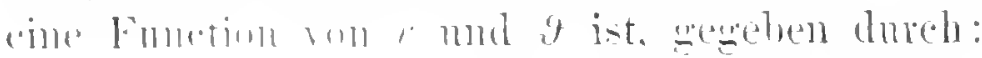

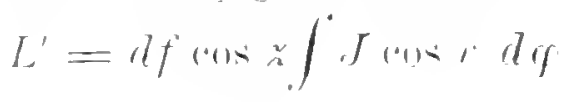


Aus dem Anding'sehen Satze folgt ohne Weiteres, dass alle Beleuchtungsaufgaben bei Mittelpunktsflächen sich auf den Fall $z=0$ reduciren, und dass es also bei jeder speciellen Anfgabe auf die Lösungr des Integrales: $\int J \cos i d$, ankomnt. Denkt man sich nun an die scheinbare Fläche im 'Zenith eine tamgirende Ebene gelegrt, so ist $\cos i$ drp nichts Auderes als dic Projection ron dif auf diese Ebene. Ist die lenchtende Fliche \%. B. eine Kugel, deren scheinbarer Radius $=s$ ist, so wird die Projection der scheinbaren Fliiche auf die erwälnte Tangentialebene ein Kreis mit dem Radius $\sin s$, und die Lichtmenge, welehe von der leuchtenden Kugel in 'Zenith anf das horizontale Element af abergeht, wird nach dem Vorangehenden:

$$
L^{\prime}=J \text { df } \pi \sin ^{2} s .
$$

Befiudet sich die Kugel nicht in Zenith, so muss man noch mit dem Cosimus der Zenithdistan\% des Mittelpunktes multipliciren, um die Lichtmenge zu finden.

Man sieht, dass durch den Anding'sehen Hulfssatz die Lösung ron Aufgaben wesentlich erleichtert wird, die bei Lambert und in dem erwilhnten Beer'schen Lehrbuche des photometrischen Caletils einen nicht unerheblichen Aufwand von Reehnumgen und Entwicklungen erfordern. So liisst sich sehr einfach die Belenchtung durch eine sphärische Ellipse bestimmen. Es geniigt auch hier wieder der Fall, dass der Mittelpunkt der Ellipse im Zenith liegt. Sind damn $s_{1}$ und $s_{z}$ die Winkel, nnter denen die beiden Halbaxen der Ellipse, von $d f$ ans gesehen, erscheinen, so wird die Projection der scheinbaren Fliche auf die Tangentialebene elsenfalls eine Ellipse mit den Halbaxen $\sin s_{1}$ und $\sin s_{2}$, und der Inlaalt der Projection wird daher:

$$
\pi \sin s_{1} \sin s_{\beth} .
$$

Die von der sellsstlenehtenden sphïrischen Ellipse auf of gesindte Lichtmenge ist daher:

$$
L^{\prime}=J \pi d f \sin s_{1} \sin s_{z},
$$

oder wem der Mittelpunkt der Ellipse die Zenithdistany : hat:

$$
L^{\prime}=J \pi d f \sin s_{1} \sin s_{2} \cos \approx .
$$

Ebenso leicht lassen sich die Fälle behandeln, wo die lenchtende Fläche ein sphiirisches Dreieck oder ein sphärisches Polygon, eine sichelfömige Figur wie bei den Phasen einer Somnentinsterniss u. s. w. ist, ebenso die Fälle, wo die belenchtete Flaiche nicht ein kleines horizontales Element ist, sondern ebenfalls eine beliebig gestaltete Fliiche reprïsentirt. Eine ausfuihliehere Bchandlung dieser Auforaben wiirde den Rahmen dieses 
Buches wesentlich tibersehreiten, und es muss daher auf die Lehrbüeher ron Lambert und Beer verwiesen werden.

\section{Zerstrent reflectirende Substanzen. Die Bongner'sche Reflexions- theorie. Das Lommel-Seeliger'sche Belenchtungsgesetz.}

Dic Berechumg der Lichtquantitit, welehe von einem leuehtenden l'unkte oder einer lenchtenden Fliiehe anf eine andere Flïehe objectiv gesandt wird, hat, wie schon melirfach im Friiheren betont wurde, im Grossen und Ganzen nur ein theoretisches Interesse, da diese Lichtmenge nicht ron unserem luge direct wahrgenommen wird und auch nur unter bestimmten Voranssetzmngen und nur in gewissem Betrage anf andere Weise (durch Photographie, durch elektrische Wirkungen ete.) gemessen werden kann. Fiir muser Auge wird diese Erlenehtung erst dadureh wahlonehmbar, dass die anffallende Lichtmenge ron der beleuchteten Fliiche wieder allswestrahlt wird, und was in moerem Sehorgan zum Bewustsein gelanut, ist erst dic durch Zuriickstrahlung mehr oder weniger monliticirte ursuriugliche objective Lichtmenge. Wieriel ron dem auffillenden Lichte zuriickgeworfen wird und mach relchen Gesetzen, hängt cinrig und allein ron der phrsischen Beschaffenheit der belenchteten substanz alb, und es wird wohl schwerlich möglich sein, ein allgemein

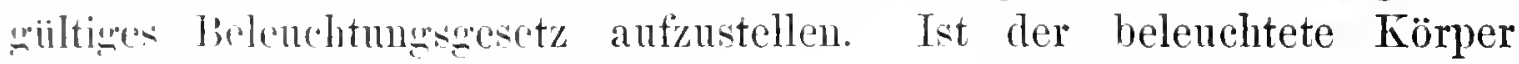
"putisch rollknumen durchsichtig, so ist klar. dass alles auffallende Licht hindureligelassen wird und dass iiberhanpt nichts mehr reflectirt werden kiln1! einen solchen absolut durchsichtigen Körper wiirde man s'iner :insteren Begrenznng nach iiberhanpt gar nicht wahmehmen kömnen. In der Nitur wicht es solche alsolut durchsichtigen Körper nicht. Je weniger durrhsichtig ein Körper ist, desto mehr Licht muss er reflectiren, wemn der satz von der Erhaltung der Enerwie Guiltigkeit laben soll. Sinh den Fresnchechen Lutersuchungen ist die Lichtquantität $L$, welche

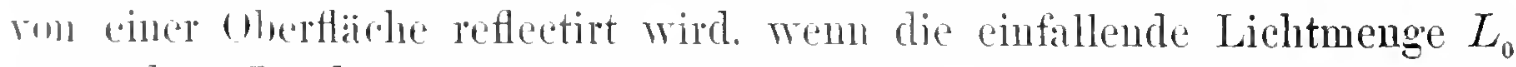
muter dem Incidenzwinkel $i$ anfiallt und nnter dem Brechungswinkel $r$

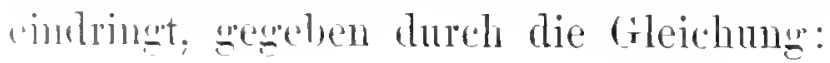

$$
L=L_{2} L_{0}\left\{\frac{\operatorname{tang}^{2}(i-r)}{\left.\mid \operatorname{tang}^{2} i+r\right)}+\frac{\sin ^{2}(i-r)}{\sin ^{2} i+r \mid}\right\},
$$

wihlermt die in den Körner eindringende Lichtmenge $D=L_{0}-L$ sein muss.

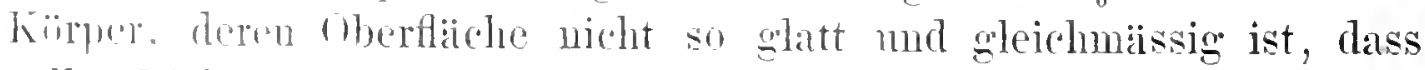
nach allen Ridhtnnwen cine regelmässige Reflexion nach den Fresnel'schen cresctzen stittlinden kimn, nement nilu zerstrent reflectirende und nimmt 
an, dass bei ihnen das auffallende Licht unregehnissig naeh allen Richtungen zurllckgestrahlt wirl. Aber ebenso wenig, wie es rollkommen spiegelnde Substanzen giebt, durfte es auch vollkommen diffus reflectirende geben, und selbst sehr rauhe Oberflichen zeigen bei grossen Einfalls- und Reflexionswinkeln bekauntlich vollkommene Spiegelbilder. Die zerstreute Reflexion wird wesentlich ron der Beschaffenheit der Substanz abhängen, und zwar voraussichtlich in erster Linie von der Absorption, welche das Licht in der Substan\% selbst erleidet. Denn dass eine solehe Absorption in der That stattfinden muss, folgt sehon allein aus dem Vorhandensein einer speeifiselıen Körperfarbe. Seeliger1) hat darauf aufmerksam gemacht, wie instructiv in dieser Beziehung die Betrachtung pulverisirter Farbstoffe ist. Je feiner das Pulver ist, desto weniger intensir tritt seine lüirbung hervor, desto weisslicher erseheint die Farbe, weil das Licht in diesem Falle nur von den allerobersten Sehichten zurlickgeworfen wird, wiihrend die Färbung bei gröberem Pulver, wo das Lielit infolge der grossen Zwischenriume tiefer in den Körper eindringen kamn, entschieden deutlicher $z u$ bemerken ist.

Das Studium der Liehtansstrahlung zerstreut refleetirender Substan\%en gehört zu den sehwierigsten Capiteln der Photometrie und ist aneh fiur die Astronomie ron der grüssten Bedentung, weil die Planeten und Monde zweifellos das Sonnenlieht zum grössten Theile diffus reflectiren. Lambert liat sieh die Lösung des Problems allerdings sehr leicht gemaeht, indem er einfach aunahm, dass die beleuchteten Elemente des Körpers ilırerseits wieder als selbstlenchtend betrachtet werden diirften und dass daher dasselbe Emanationsgesetz gelten miisse, wie für selbstlenchtende Körper. Min denke sich ein zerstrent reflectirendes Nliichenelement $d s$ von einer Lichtquelle (einem lenehtenden Punkte oder einer lenehtenden Fliiche) unter dem Incidenzwinkel $i$ belenchtet. Ist damn $L$ die Lichtmenge, welche anf die Flächeneinheit senkrecht auffällt, so erhïlt $d s$ nach dem Friiheren die Lichtquantitit $L d s \cos i$. Von dieser Liehtmenge wird mun das Elenent naeh jeder Richtung einen gewissen Bruehtheil reflectiren, und es sei die in senkreehter Richtung ansgestrahlte Lichtmenge durch $c L d s \cos i$ ansgedrickt. Wenn sich nun das diffus reflectirende Element, wie Lalmbert aunimmt, wie ein selbstleuehtendes verhalten soll, so miissen sich nach dem Emanationsgesetze die in rersehiedenen Richtmugen ansgestrahlten Lichtmengen wie die Cosinus der Emanationswinkel verhalten, und es ist daher die unter dem Winkel $\varepsilon$ ansgestrahlte Lielitmenge

$$
d_{I}=c L \cos i d s \cos \varepsilon \text {. }
$$

1) Sitzungsb. der math.-phys. Classe der K. Bayer. Akad. der Wiss. Bd. 18 (1888), p. 228. 
Denkt man sich nun um $d s$ eine Halbkugel mit dem Radins 1 construirt, so wird einem Elemente do derselben die Lichtquantität

$$
\| Q=d q d \omega=c L d s \cos i \cos \varepsilon d \omega
$$

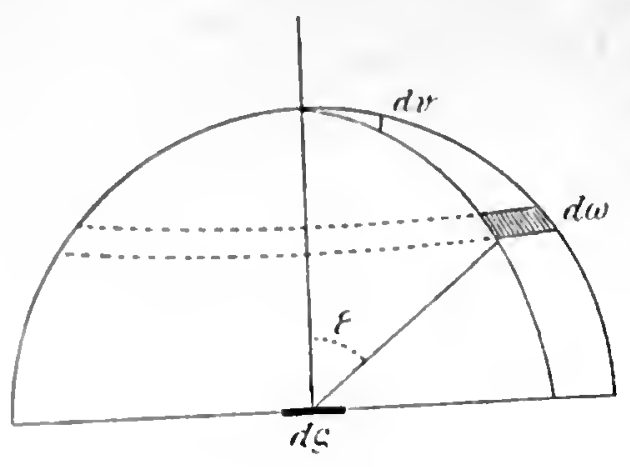

Fig. 9. zugesandt, und die ganze Halbkugel erhailt daher, da sich das Element $d(1)$, wie nebenstehende Figur 9 zeigt, dureh $d \varepsilon \sin \varepsilon d r$ ausdriicken liisst, die Lichtmenge:

$$
Q=c L d s \cos i \int_{0}^{\frac{\pi}{2}} \cos \varepsilon \sin \varepsilon d \varepsilon \int_{0}^{2 \pi} d t
$$

oder:

$$
Q=c L d s \cos i \pi .
$$

Fis wird aher diese wanze ansgestrallite Liehtmenge anch irgend ein limmitheil der von dem Elenent empfangenen Liehtmenge $L$ as $\cos i$ sein, (i. h. Milln wird haben:

$$
(j=I \operatorname{ds} \cos i A \text {, }
$$

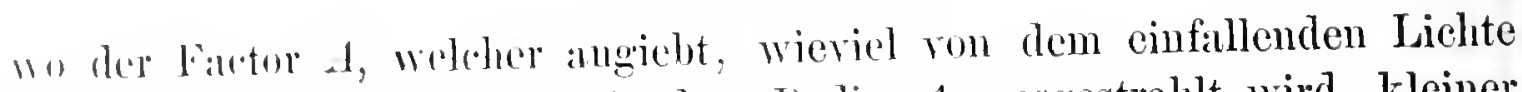

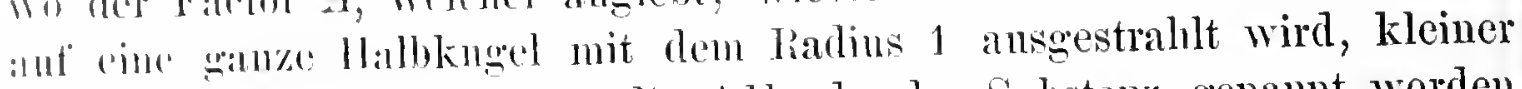
als I ist und rom Lambert die Albedo der Substamz genamnt worden ist. Ins den heiden Gleielnumgen fiir $Q$ folgt: $c=\frac{A}{\pi}$, und man hat daher

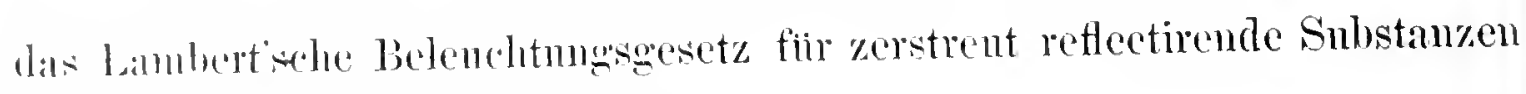
in der bekalnuten Form:

$$
d_{1}=\frac{A}{\pi} L d s \cos i \cos \varepsilon,
$$

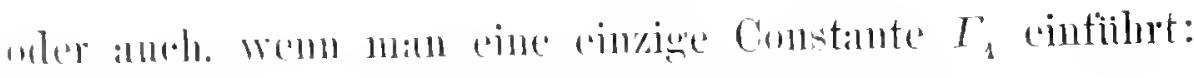

$$
d_{l}=l_{1} d_{\text {s. } \cos i \cos \varepsilon} \text {. }
$$

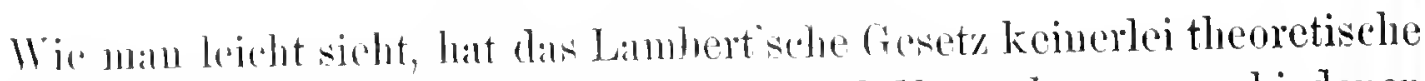

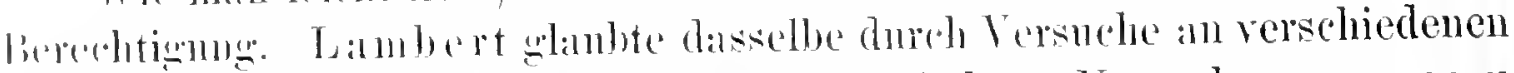
substanzen bewiesen zu haben, indessen sind diese Tersuche so mongentu

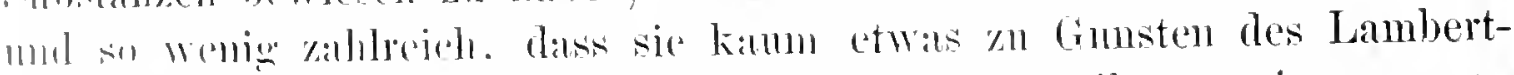

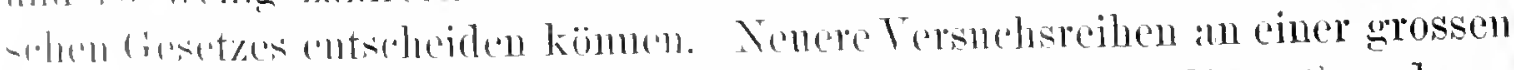

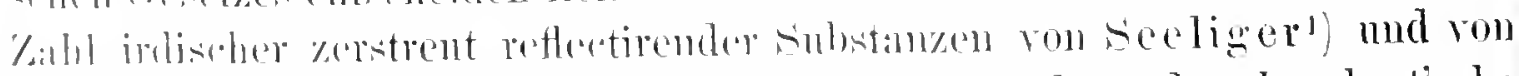

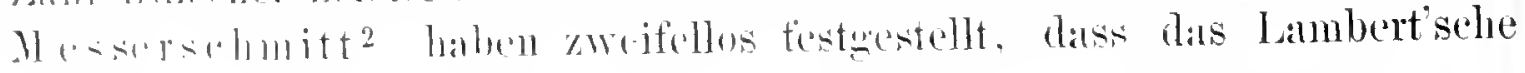

1. Sitzun der math.-phrs. Classe der K. Bayer. Akad. der Wiss. Bd. 1s 201.

2 Unserschmitt. Über diftuse Reflexion. Diss inaug. Leipzig, 1558. 
Gesetz mur gan\% ansnalunsweise als Annilierung an die. Walırheit betrachtet werden kann, dass die Lichtmenge vom Azimuth abhüugig ist ete., und dass es daher fur diffus reflectirende Kürper aus der lieihe der photometrischen Grundgeset\%e gestrichen werden nuss. Der Umstand, dass dieses Geset\% so lange eine unumschriinkte Herrschaft ausgetibt hat, erklïrt sich wohl an besten durch die theraus einfache und elegante lorm, in welcher es erscheint, und aus dem Mangel an zurerlissigen Beobachtungen, welche gegen dasselbe stimmen komnten. Fur die Astrophotometrie schien es \%war durch die Zölluer'schen Messungen an Monde und an Planeten Mars nicht bewiesen, dafuir ergaben aber die Seidel'schen Beobachtungen an der Venus eine vollkommene Bestätigung, und unter geeigneten Annahmen tiber die Oherflichenbeschaffienheiten dieser Himmelskürper liessen sich diese seheinbaren Widersprtiehe sehr gut anch mit dem Lambert'schen Gesetze vereinigen. Niiheres dariber später. Züllner hat zwar, wie wir gesehen haben, bei den selbstlenchtenden Substanzen den Weg \%u einem strengen Beweise des Lambert'sehen Emanationggesetzes gewiesen, indem er die Fourier'sche Anschanungsweise von dem Hervordringen der Wirmestrahlen aus dem Inneren der Körper anch auf die Lichtstralılen in Anwendung brachte, er hat aber, indem er versuchte, diese Überlegungen bei der liehandlung der nicht selbstlenchtenden Substanzen einzufiihren, den Fehler gemacht, nur eine Lichtabsorption bei der Ansstrahlung der Rammelemente aus dem Imneren anzunehmen, nicht aber anch entsprechend eine Lichtschwiichung sehon bei dem Eindringen des Lichtes in das Innere. Dieser Umstand hat ihn wieder anf das Lambertsehe Geset\% zuriekgreführt nud ihn verhindert, schon vor 30 . Iahren diejenigen Fortschritte in der theoretisehen Photometrie :mzubahnen, welche wir den neneren Forschungen ron Lommel und Seeliger verdanken.

Nicht ohme Interesse ist die Vorstellung, die sich Bouguer von der physikalischen Besehaffenheit der diffus reflectirenden sulstanzen semacht hat. Er nahm an, dass die Oberflaiche eines solehen Körper's wegen seiner Rauhheit keine vollkommen geonetrische Flïche sein kïnne, sondern dass sie aus einer \%ahllosen Menge von kleinen spiegchnden Elementen bestiinde. die unter allen möglichen Winkeln gegen die Oberfliche des Kürpers geneigt wären und das Lieht nach den Gesetzen der Spiegelun" zmrickwtirfen. Bouguer hat diese Auschanmosweise in seinem "Traité d'optique mit grosser Consequenz durehzufiihren versucht, es ist ilnu aher nicht gelungen, ein allgemein giiltiges Belenchtmunsoget\% fuir alle zerstrent reflectirenden Substanzen anf\%nstellen. Seine Theorie wilt hente als veraltet, es verdient aber vielleieht hervorehoben zu werden, dass Seelig̈er in nenerer Zeit die Bouguer sehe Vorstellung unter gewissen Voranssetzungen etwas weiter verfolut lat. Wir denken uns anf ane zerstrent reffectirende 
Oberfläche, die im Grossen und Ganzen eine Ebene sein, aber im Speciellen „uns lauter kleinen spiegelnden Elementen bestchen möge, aus grosser Entferumng Licht unter dem Incidenzwinkel $i$ auffallend. Dasselbe möge unter dem Emanationswinkel $\varepsilon$ wichler ausgestrahlt und ron grosser Entfernung: aus betrachtet werdeu. Damn ist klar, dass, wenn die Lichtwirkung einzig. und allein in einer Spiegrelıng bestehen soll, nur solehe Elemente dem Beobachter Licht zuschicken künnen, deren Normale mit der Richtung der cin- und austretenden Strahlen in einer Ebene liegt und den Winkel \%ischen diesen heiden Richtungen halbirt. Dieser Winkel sei $x$, und dic 'sahl der kleinen spiegeluden Elemente, die dieser Bedingung genügen und deren Ebenen unter einander natiurlich parallel sein miissen, sei $n$. Dann wird die Lichtmenge, welche das betrachtete Flächenstück den Beobachter zusendet, proportional der Anzahl der Elemente und ausserdem cine l'unction des Winkels $\frac{r}{2}$ sein, welche die Abhängigkeit der Intensitait des erespiegelten Lichtstrahles ron dem Einfallswinkel ansdriickt. Man wird ilso haben:

$$
\text { l } q=l i n f\left(\frac{x}{2}\right) \text {, }
$$

Wo li cine Constante ist.

M:tn denke sirl mun un einen Punkt der Fläche cine Kugel beNhriehen. dimun wire in nebenstehender Figm 10 die Richtung des

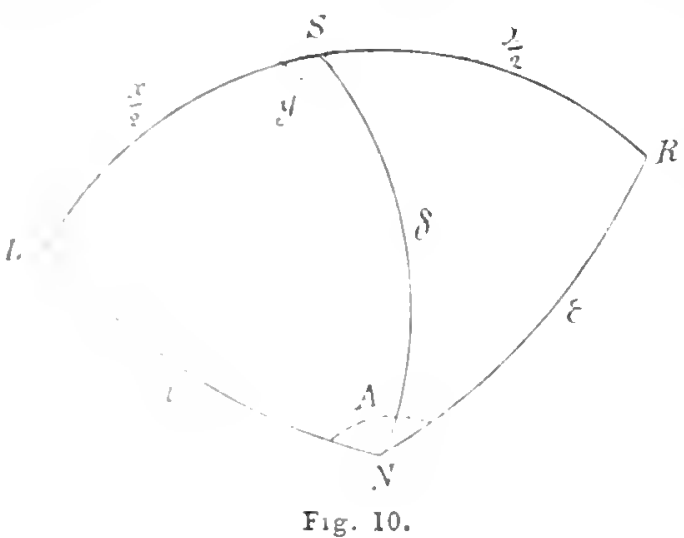
einfallenden Lichtes an der Sphäre durch deu Punkt $L$, die Richtung des alusstrahlenden Lichtes durch $R$, die Normale zur Flaiche durch $N$ und die Tormale zn den wirksamen Spiegelelementen durch $S$ markirt. Die Eeiten in den sphïrischen Dreiecken sind $L S=S R=\frac{r}{2}, L N=i$ und $M R=:$ : ansserdem sei NS, d. h. der

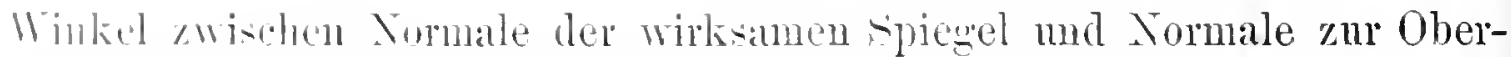

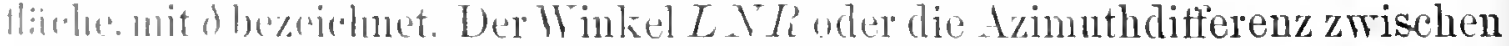

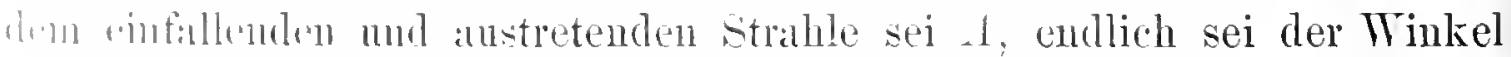

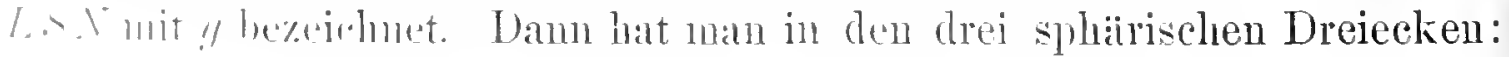

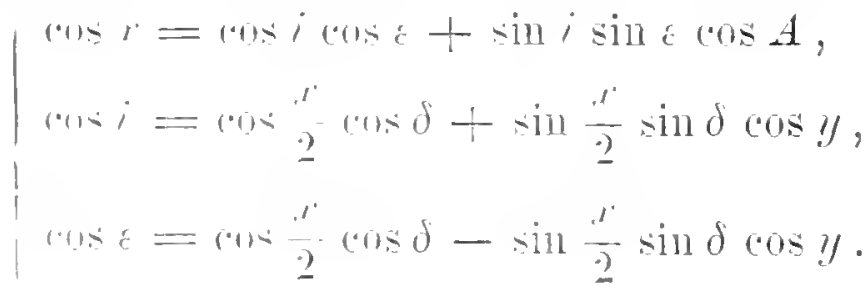


Aus den beiden letzten dieser Gleielungen folgt:

$(3)$

Nun ist aljer:

$$
\cos i+\cos \varepsilon=2 \cos \frac{x}{2} \cos \delta .
$$

$$
2 \cos \frac{x}{2}=\sqrt{2} \sqrt{1+\cos x},
$$

oder, wenn man den Werth von $\cos x$ ans der ersten der Gleichungen $(2)$
cinträgt:

$$
2 \cos \frac{x}{2}=\sqrt{2} \sqrt{1+\cos i \cos \varepsilon+\sin i \sin \varepsilon \cos A} .
$$

Man erhält daher aus Gleichnng (3):

$$
\cos \delta=\frac{\cos i+\cos \varepsilon}{\sqrt{2} \sqrt{1+\cos i \cos \varepsilon+\sin i \sin \varepsilon \cos A}} .
$$

In den Gleichnngen (2) und (4) sind die Grössen $x$ und $\delta$ durch die Einfalls- und Emanationswinkel und die Azimuthdifferenz $A$ ansgedriiekt. Kennt man also die Form der Funetion $f$ und kennt auch $n_{3}$ so ist die Liehtquantitiat durch die Grössen $i, \varepsilon, A$ in jedem Falle gegeben. Was die Grösse $n$ anbetrifft, also die Anzalıl der in einer bestimmten lichtung spiegelnden Elemente, so hängt diese natiirlieh vollkommen vou der Bcschaffenheit der zerstreut reflectirenden Oberflizehe $a b$; man wird aber in jedem Falle eine willkirliche Function of annehmen kïmen, die $n$ albhïngig erseheinen liisst von dem Winkel $\delta$, eigentlich auch noch von derr Azimuth der Normale der kleinen Spiegel. Vernachliissigen wir letztere Abhängigkeit und setzen also willkiirlich $n=\varphi(\delta)$, so wird dic ansgestrahlte Lichtmenge:

$$
q=\operatorname{li} \mathscr{f}(\delta) f\left(\frac{x}{2}\right)
$$

In den beiden Fällen, wo die Azimuthdifterenz $A$ zwischen einfallenden nnd anstretendem Strahle 0 resp. $150^{\circ}$ ist, werden die Beziehungen sehr einfach. Man hat nach den Gleichungen $(2)$ und $(t)$

$$
\begin{aligned}
& \text { im ersten Falle: } x=i-\varepsilon \text { und } \delta=\frac{1}{2}(i+\varepsilon), \\
& \text { im zweiten Falle: } x=i+\varepsilon \text { und } \delta=\frac{1}{2}(i-\varepsilon),
\end{aligned}
$$

und demnach ergeben sich damn die Belenclitungsgesetze:

$$
\begin{aligned}
& q=\operatorname{li} \varphi\left(\frac{i+\varepsilon}{2}\right) f\left(\frac{i-\varepsilon}{2}\right), \\
& q=\operatorname{li} \varphi\left(\frac{i-\varepsilon}{2}\right) f\left(\frac{i+\varepsilon}{2}\right) .
\end{aligned}
$$


Weiter hat Seeliger den Gegenstand nicht rerfolgt, und weiter lässt er sich anch nicht verfolgen, wenn man nicht noch speciellere Annahmen ther die Anordumng der spiegelnden Einzelelemente machen will. Dazu liegt aber keinerlei Auregung vor, da die gauze Bonguer'sche Vorstellungsweise etwas gekilnstelt ist und inzwischen auch durch die ron Lommel und Seeliger ansgrebante Absorptionstheorie vollständig verdrängt worden ist.

Wir haben hereits oben gesehen, zu wchchen Resultaten bei den selbstlenchtenden Köpern die Annahme, dass das Licht aus einer rewissen 'liefe muter der Oberfliche herrordringt und auf seinem Wege eine grewisse Absorption erleidet, grefiihrt hat, und werden diese Hypothese Inu :uch anf die zerstrent reflectirenden Körper ausdehnen, indem wir romussetzen, dass das anffallende licht bis zu einer gewissen Tiefe in den Körper eindringt, eine Absorption auf seinem Wege erleidet, von den untroftenen Rimumelementen des Körpers wieder zur Umkehr gezwungen wird und endlich anf seinem Riickwege eine neue Schwächnng dureh Ahsolption erleidet. Dabei ist noch die Annalume zu Grunde zu legen, dass dic eimzolnen Volumelemente des Körpers die Fähigkeit haben, dis: Licht March allen Seiten mit der nämlichen Intensitä zur Umkehr zu bringen, und diss anch die Alsorption in allen Richtungen gleich stark ist. Minn denke sich den helenchteten zerstreut reflectirenden Körper als plamparallele l'latte, mod es sei (Fig. 11) $d r$ ein kleines Volumelement

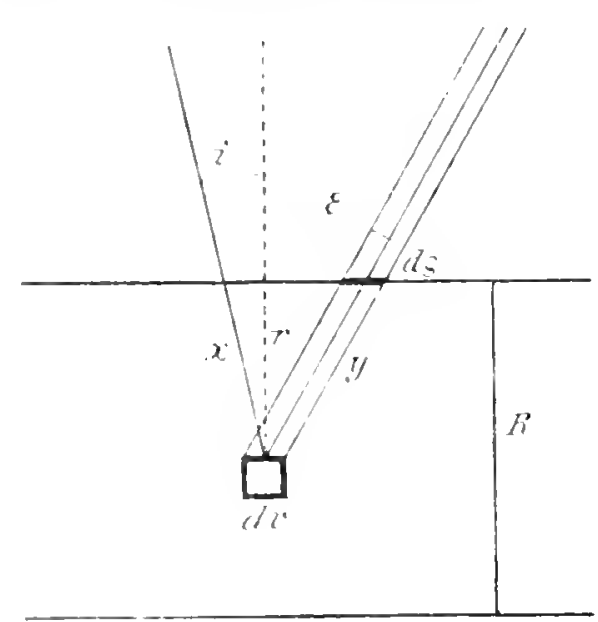

Fig. 11. in Inneren derselben. Das Licht falle in einer Iiichitung, die mit der Normale zum Kïrper den Winkel i einschliesst, anf. Es soll die in der Richtung $\varepsilon$ dureh das Oberflichenelement $d s$ ausgestrallte Lichtmenge, oder was nach unseren friiheren Detinitionen dasselbe ist, die Diehtigkeit des Lieltes in dew unter dem Emanationswinkel c ans dem Körper anstretenden Licht"ylinder gefunden werden. Ist $L$ die Lichtruluntitiit, welche auf die Tolumeinheit an der Oberflïche des Körpers rou der lichtepnelle gesandt wird, und ist $l$ der Whomptionsonfticient der substanz fiir die eindringenden Strahlen, so

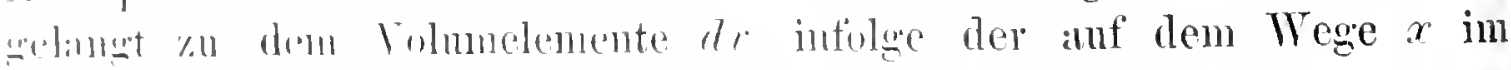

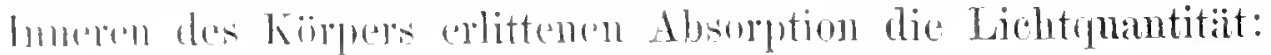

$$
d q^{\prime}=L d r e^{-k x} .
$$

11 irrl mu

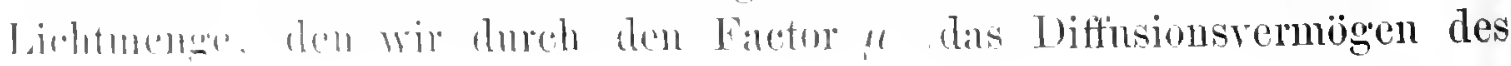


Körpers) bezeichnen wollen, naeh allen Richtungen ringsum zurllekgeworfen, so wurde die von $d v$ in irgend einer Richtumg, also anch tunter dem Emanationswinkel $\varepsilon$ ansgestrahlte Lichtmenge ansgedrllekt sein dureh:

$$
\frac{11}{4 \pi} d q^{\prime} \text {. }
$$

Da aber auf dem Wege $y$ wieder eine Absorption stattfindet, so wirl, wenn der Absorptionscoefficient filr die anstretenden Strahlen mit $k^{\prime}$ bezeichnet ist, die von $d v$ unter dem Winkel $\varepsilon$ wirklich ansgestrahlte Lichtmenge $d q$ gegeben sein durch die Gleichung:

$$
d q=\frac{\mu}{4 \pi} d q^{\prime} e^{-k^{\prime} y}=\frac{\mu}{4 \pi} L d v e^{-\left(k x+k^{\prime} y\right)} .
$$

Dass im Allgemeinen der Absorptionseoefficient flir die anstretenden Strahlen etwas anders sein wird als für die einfallenden, erklït sich dadureh, dass die Farbe des Liehtes beim Eindringen in die Substanz geïndert wird. Ist das auffallende Lieht $z$. B. weisses und der Körper ein blaner, so werden beim Eindringen die rothen, gelben und griinen Strahlen besonders stark absorbirt werden, wïhrend nach der Umkehr, wo in der Hauptsache fast unr noch blaue Strahlen ubrig sind, die Lichtschwiichnng geringer sein wird, also $k^{\prime}<l$.

In der obigen Figur ist $x=\frac{r}{\cos i}$, ferner $y=\frac{r}{\cos \varepsilon}$ und das Volumelement $d r=d s d r$; daher hat man:

$$
d q=\frac{\prime \prime}{4 x} L d s e^{-\left(\frac{k}{\cos i}+\frac{k^{\prime}}{\cos s} r\right.} d r .
$$

Um die gesammte Liehtquantitït zu haben, welche durch das Oberflichenelement $d s$ in das Ange gelangt, hat man voll $r=0$ bis $r=R$ zu integriren, wemn $R$ die Dicke der Selicht vorstellt, bis zu der iberhaupt noeh Lieht ans der Tiefe hervorkommen kann, fiir welche also die Grösse $-\left(\frac{k}{\cos i}+\frac{k^{\prime}}{\cos \varepsilon}\right) R$

Man hat also:

$$
\text { verschwindend klein sein muss. }
$$

$$
\begin{aligned}
& I=\frac{l l}{1 \pi x} L d s \int_{0}^{l i} e^{-\left(\frac{k}{\cos i} \div \frac{k^{\prime}}{\cos t}\right)} d r . \\
& r\left\{\frac{l i}{\cos i}+\frac{l^{\prime}}{\cos \varepsilon}\right\}=x, \\
& d i=\frac{k \cos \varepsilon+k^{\prime} \cos i}{\cos i \cos i} d r,
\end{aligned}
$$

Setzt man

so wird: 
und mithin:

$$
\eta=\frac{\prime l}{4 \pi} L d s \frac{\cos i \cos \varepsilon}{k \cos \varepsilon+l^{\prime} \cos i} \int_{0}^{R \frac{k \cos \varepsilon+k^{\prime} \cos }{\cos i \cos \varepsilon}} e^{-z d z}
$$

oder:

$$
\eta=\frac{\prime \prime}{1 \pi} L d s \frac{\operatorname{eos} i \cos \varepsilon}{l_{i} \cos \varepsilon+k^{\prime} \cos i}\left\{1-e^{-l i \frac{k \cos \varepsilon+k^{\prime} \cos i}{\cos i \cos \varepsilon}}\right\} \text {. }
$$

Nich unserer obigen Anmahme ibber $R$ ist das zweite Glied versehwindend klein, mul man hat daher:

$$
q=i_{4 \pi}^{\prime \prime} L d s \frac{\cos i \cos \varepsilon}{l_{i} \cos \varepsilon+k^{\prime} \cos i} .
$$

Setzt man noeh $\frac{l_{i}}{l_{i}^{\prime}}=i$. und bezeichnet $L \frac{1}{4 \pi} \frac{\mu}{l^{\prime}}$ mit $\Gamma_{2}$, so hat man:

$$
\eta=\Gamma_{2} d s \frac{\cos i \cos \varepsilon}{\cos i+\lambda \cos \varepsilon} .
$$

In Allgeneinen wird man $l_{i}=l^{\prime}$ setzen können, ohne einen erheblirhen Felıler zu begehen; man hat dann $\lambda=1$, und erhält das sogenannte Lommel-neeligersehe Belenehtungsgesetz in der einfaehsten Form:

$$
\eta=\Gamma_{2} d s \frac{\cos i \operatorname{eos} \varepsilon}{\cos i+\cos \varepsilon} .
$$

Die soleinhare Helligkeit ergiebt sich mach den Definitionen anf Seite 28 ans der cileichung: $h=I_{\mathrm{a}} \frac{\cos i}{\cos i+\cos \varepsilon}$, und fiir den speeiellen Fall $i=0$ finlut die selueinlalre Helligkeit:

$$
h_{0}=\Gamma_{2} \frac{1}{1+\cos \varepsilon} .
$$

Fiir die beiden extremen Annalmen $\varepsilon=0$ und $\varepsilon=90^{\circ}$ wird:

$$
h_{11}=\stackrel{1}{2}_{2} I_{\underline{2}} \text { und } h_{91}=\Gamma_{2} \text {. }
$$

lis sulte allon nilch dem obigen Gesetze die scheinbare Helligkeit der

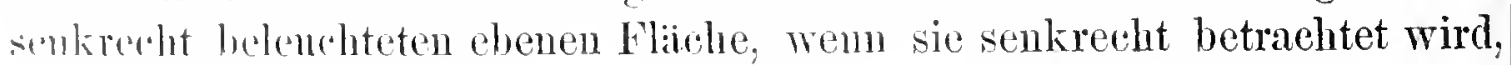
hallh sn wruss sein, als wenn sie ganz ron der Seite angesehen wird, wihnend nate dem Lambert'schen Emanationsgesetze die seheinbare Hellig-

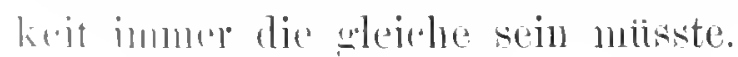

Ilic oligen Endformetn gelten nur dann, wemn die einzelnen Volum-

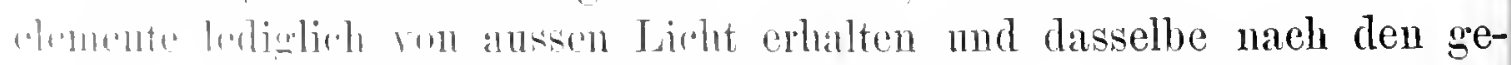

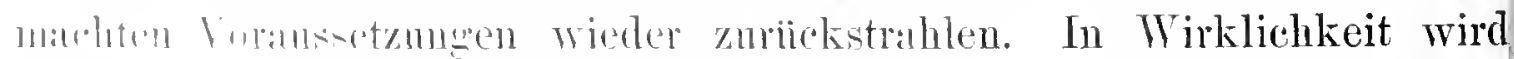

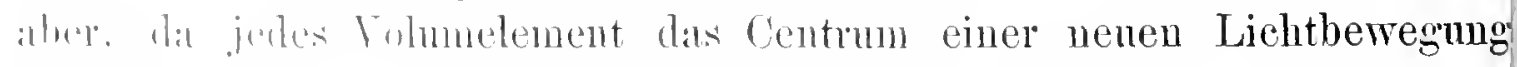

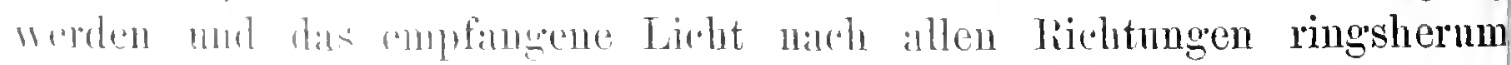


wieder ansstrahlen soll, aueh jedes Element von allen ubrigen Nachbarclementen Liclit empfangen, und infolge dessen wird der Ausdruck filr die Gesammtquantität riemlich eomplieirt werden. Lommel') hat diese Anschaumngsweise ganz, eonsequent durehgefthrt in einer Abhandlung uber die difluse Zurlickwerfung und ist zu ansserordentlich verwickelten Ausdrltcken gelangt. Besehrïnkt man sich jedoch nur auf die inneren Reflexe erster Ordnung, so kommt man zu einer verhiltnissmiissig einfachen Endformel, und es durfte bei der Wichtigkeit, welehe die neue Inschaunngsweise fur die Entwicklung der theoretischen Photometrie und im Speeiellen auch für die Astrophotometrie zweifellos besitzt, wilnschenswerth erșcheinen, die Ableitung dieser Endformel hier ausfulhrlich mitzntheilen.

Man denke sieh wieder den diffus reflectirenden Körper als planparallele Platte von der Dicke $R$, und es seien (Fig. 12) $d v$ und $d v^{\prime}$ zwei Volumelemente im Inueren derselben, deren senkrechte Abstinde von der Oberfliiche $r$ und $r^{\prime}$ und deren gegenseitige Entfernung $\boldsymbol{a}$ seiu mögen. Die Lichtstrahleu sollen parallel unter dem Incidenzwinkel $i$ anffallen und nach dem Austritte aus dem Körper unter dem Emauationswinkel $\varepsilon$ ron grosser Entfernung ans betrachtet werden. Nach dem Vorausgehenden ist die Lichtquantititit, welche ron aussen

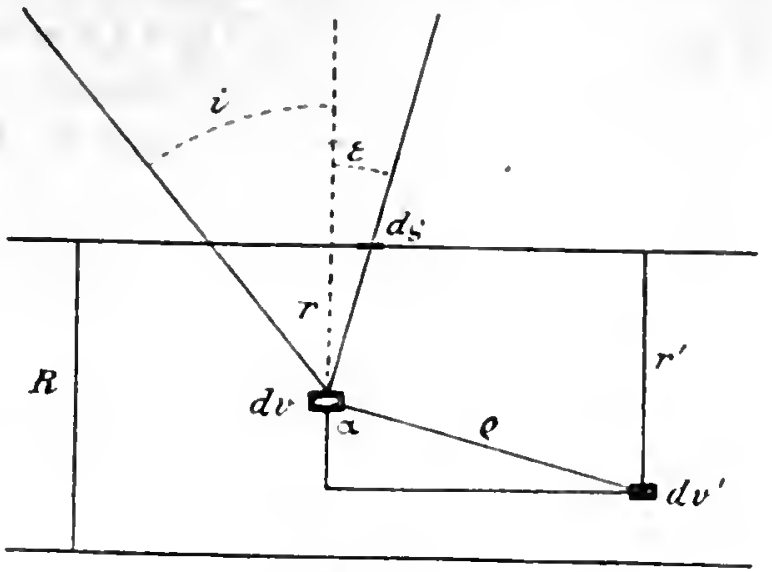

Fig. 12.

bis zu dem Elemente $d v$ gelangt, grleich $L e^{-\frac{k r}{\cos i}} d r$, wenu $l_{i}$ der $A$ b)sorptionscoefficient der Substanz ist. Ebenso erhïlt das Element $l \ell^{\prime}$ von aussen die Lichtmenge $L e^{-\frac{k r^{\prime}}{\cos i}} d r^{\prime}$. Nehmen wir mun wie friher all, dass das Element $d \ell^{\prime}$ von dieser Lichtmenge einen gewissen Bruchtheil, der dureh den Factor " bezcichnet werden soll, narh allen Seiten ringsum zuriickwirft, so wird aluch $d r$ einen Theil daron erhalten, der mit Riicksicht auf die Entfernung o und die anf dieser Strccke stattfindende Absorption anssgedriiekt wird durch:

$$
\frac{\mu}{4 \pi} \frac{L d r d r^{\prime} e^{-\frac{k r^{\prime}}{\cos i}} e^{-k \eta}}{Q^{z}} .
$$
(1887), p. 95.

1) Sitzungsb. der math.-phys. Classe der K. Bayer. Akad. der Wiss. Bd. 17
p. 95 . 
line solche Lichtmenge erhiilt nun $d v$ anch von allen iibrigen Volumclementen des Körpers, und es ist daher die gesammte Lichtmenge l, welche anf das Element $d v$ gelangt, gegeben durch die Gleichung:

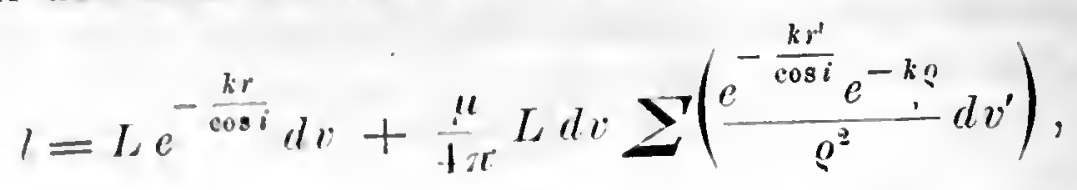

wo die summe Hiber simmutliche Elemente des Körpers zu bilden ist.

Streng genommen misste nnn anch noch das Licht beriicksichtigt werden, welehes $d r$ seinerseits wieder von den andern Elementen erhält mol rou welehem es ebentalls cinen Procentsatz nach $d v$ wirft; wir wollen alher ron diesen lieflexen höherer Ordnung; wie schon oben gesagt, Abstamd nehmen.

Von der Lichtmenge l, welehe $d r$ erhiilt, wird num nach allen Rich-. tmungen ein Theil wieder ansestrahlt, weleher durch den Reflexionseoeffi"inten! bezeidnet ist. Die Qnantitiit. 4, welche nnter dem Richtungswinkel \& hurch das Obertiachenelement $d s$ parallel anstritt, ist unter leriicksinhtignug der Absorption in Inneren des Körpers gegeben dureh:

$$
y=\frac{\mu}{4 x} l e^{-\frac{k i}{\cos \varepsilon}}
$$

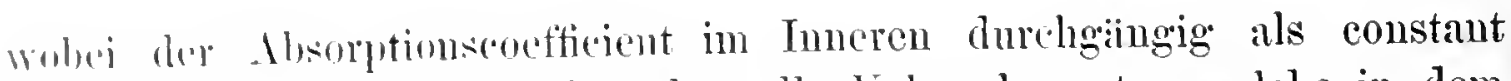
angrommmen ist. Nun werfen aber alle Volumelemente, welche in dem solicfin, durch ds und dr gelegten Cylinder sich befinden, Licht durch Is in las Ange. Din (resimmtenautitit (), welehe ins Ange gelangt, int dilluel:

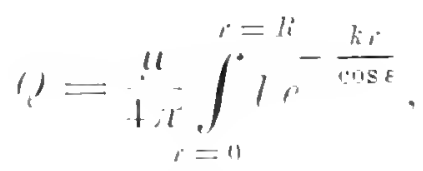

meler wem man den Werth rom / einsetat mud fiir $d r$ den Werth $d s$ dr sintiilut:

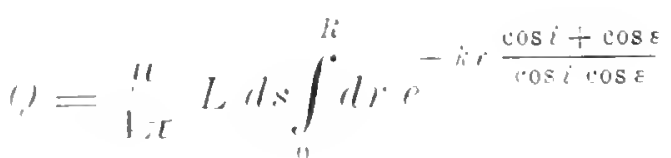

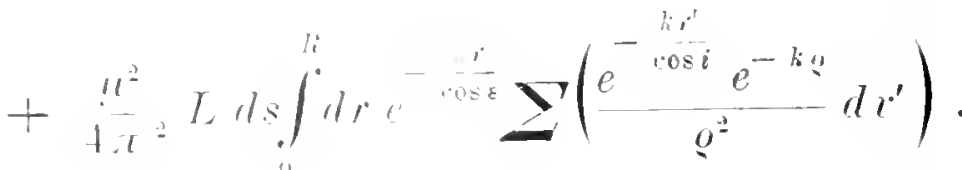

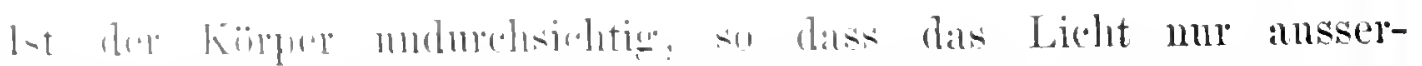

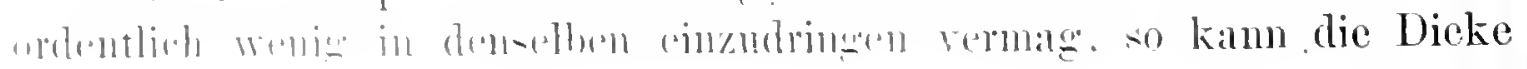


$R$ schon als sehr gross angesehen werden, und man kann direct $R=\infty$ sctzen. Man hat damn, da das erste Glied sich leieht bereehnen lisst:

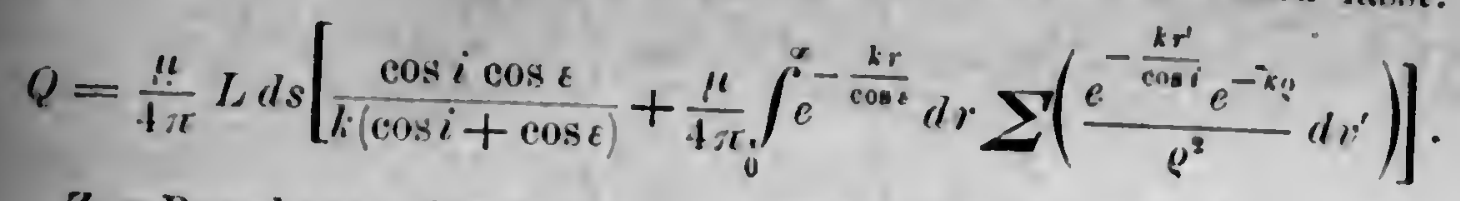

Zur Berechnung der Summe in zweiten Gliede denke man sich von do als Spitze aus einen geraden Kreiskegel mit dem halben Oeflinungswinkel a und der Seitenlïnge $\varrho$ construirt und ebenso einen zweiten Kegel unit dem halben Oeffuungswinkel $a+d \alpha$ und der Seitenlänge $\varrho+d \varrho$, so werden die Grundflachen dieser beiden Kegel einen ebenen Kreisring bilden, dessen Flaicheninhalt gegeben ist durch:

$$
2 \pi\left(r^{\prime}-r\right)^{2} \text { tang } \alpha \sec ^{2} \alpha d \alpha \text {. }
$$

Das Volnmen eines körperlichen Ringes mit dieser Fliiche als Basis und mit der Höhe $d r^{\prime}$ ist dann ausgedriekt durch:

$$
2 \pi\left(r^{\prime}-r\right)^{2} \operatorname{tang} a \sec ^{2} \alpha d u d r^{\prime} \text {. }
$$

Alle Elemente dieses Ringes, zu denen auch $d v^{\prime}$ gehört, erhalten von anssen die glejehe Lichtmenge, und man kann daher die obige Summe anstatt iber alle einzelnen Elemente $d r^{\prime}$ soglejeh tiber alle solchen Ringe bilden und $d r^{\prime}$ direet durch den voranstehenden Werth ersetzen. Da ferner noch $\varrho=\left(r^{\prime}-r\right)$ sece ist, so hat man:

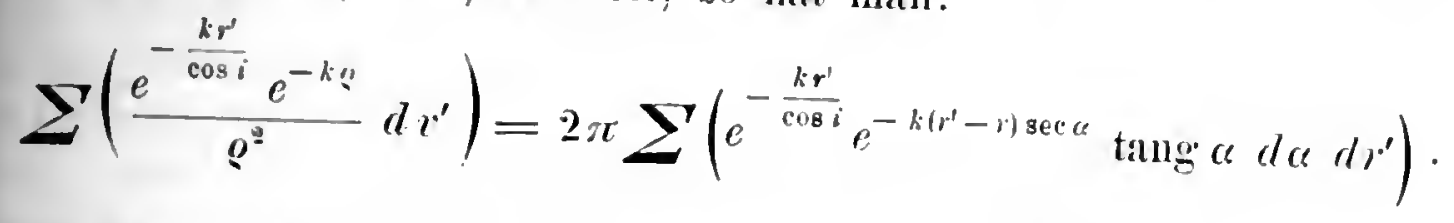

Setzt man noch sec $a=y$, mithin ting $a d u=\frac{d y}{y}$, so wird:

$$
=2 \pi \int_{i}^{\infty} e^{-\frac{k v^{\prime}}{\cos i}} d r^{\prime} \int_{i}^{e^{-k\left(r^{\prime}-r\right) y}} \frac{l !}{!} d !
$$

In Bezug anf $r^{\prime}$ sind die Integrationsgrenzen eigentlich " und $R$, da aber der Körper mulurehsichtig ist, so werden sie, wie oben, 1 und $\infty$. In Bezug anf a sind die Grenzen, da man sich die schicht, in welcher $d r^{\prime}$ liegt, ron umbegrenzter Ausdehnung denken kamu, " und $\frac{\pi}{2}$, in Bezug auf " werden sie also 1 und $\infty$. Setrt man endlich noch $r^{\prime}-r=x$, mithin $d r^{\prime}=d x$, so erhalten die Integrationsgrenzen in Bezug anf $x$ die Werthe $-r$ und $\infty$. und man hat daher, wemn man 
noch das Integral in Bezug auf $r^{\prime}$ in zwei Theile, von $-r$ bis 0 nnd von 0 bis $\infty$, theilt:

$$
\begin{aligned}
\sum\left(\frac{e^{-\frac{k r^{\prime}}{\operatorname{cosi}}} e^{-k y}}{\varrho^{2}} d r^{\prime}\right) & =2 \pi \int_{-r}^{0} e^{-\frac{k(x+y)}{\cos i}} d x \int_{1}^{\infty} \frac{e^{-k x y}}{y} d y \\
& +2 \pi \int_{0}^{\infty} e^{-\frac{k(x+r)}{\cos i}} d x \int_{1}^{\infty} \frac{e^{-k x y}}{y} d y \\
& =2 \pi e^{-\frac{k r}{\cos i}} \int_{0}^{r} e^{\frac{k x}{\cos i}} d x J(x)+2 \pi e^{-\frac{k r}{\cos i}} \int_{0}^{\infty} e^{-\frac{k x}{\cos i}} d x \dot{J(x)}
\end{aligned}
$$

wemn fìr den sogenamnten Integrallogarithmus $\int_{1}^{\infty} \frac{e^{-k x y}}{y} d y$ die übliche Benemmun. $J(x)$ eingefiihrt wird.

Bezcichuet nam dic beiden Glieder der rechten Seite der kürzeren schureibweise wegen mit $2 \pi \mathrm{X}$ und $2 \pi Y$, so ergiebt sich nun durch Substitution in die frihlhere Gleichnng fuir $Q$ :

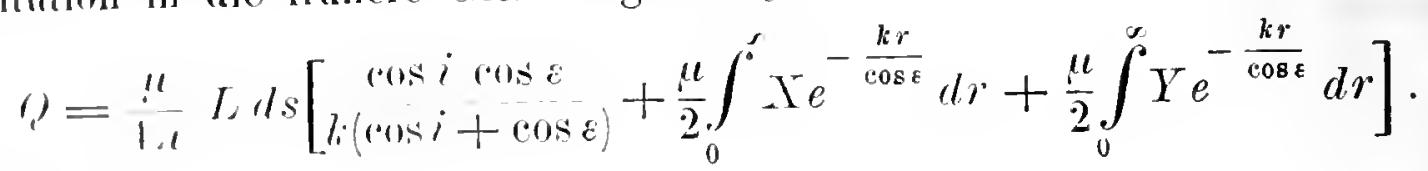

Sill ist:

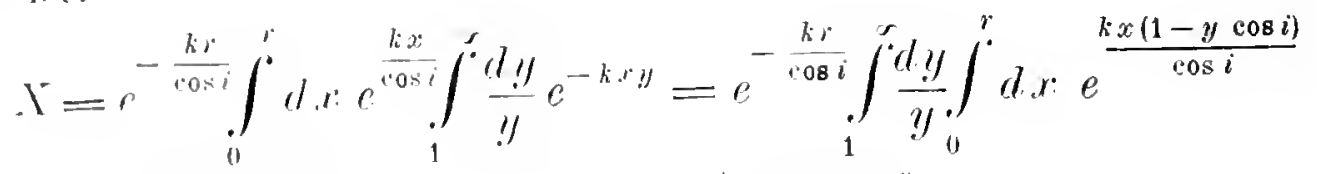

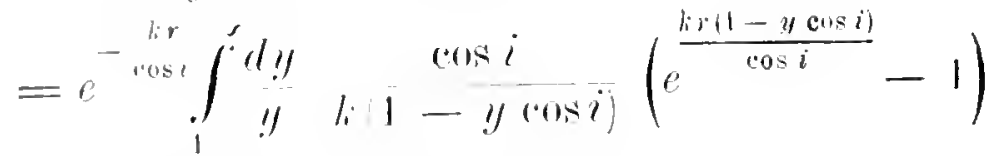

$$
\begin{aligned}
& =\operatorname{li}_{i} \int_{i}^{n} \frac{1 ! !}{! !} \frac{1}{1-! \cos i}\left(e^{-k i y}-e^{-\frac{k r}{\cos i}}\right) \text {. }
\end{aligned}
$$

Yitlin wird:

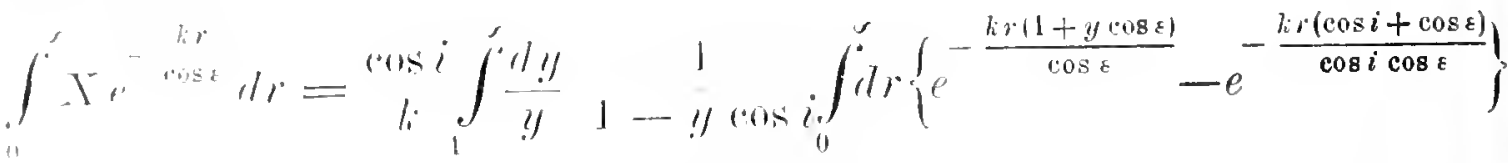

$$
\begin{aligned}
& =\frac{\cos i}{l i} \int_{1}^{2} \frac{d ! !}{! !} i-\frac{1}{1}\left(\frac{\cos \varepsilon}{1+! n \cos \varepsilon}-\frac{\cos i \cos \varepsilon}{\cos i+\cos \varepsilon}\right)
\end{aligned}
$$

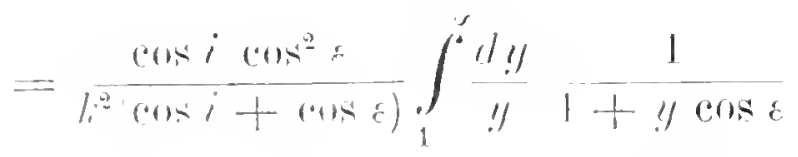

$$
\begin{aligned}
& \frac{\operatorname{loc} i \cos ^{2} i}{\left.\operatorname{los}^{2} i+\cos c\right)} \operatorname{lng}\left(\frac{1+\cos \varepsilon}{\cos \varepsilon}\right) \text {. }
\end{aligned}
$$


(ianz in ähnlicher Weise lisst fich das letyte Glied in der obigen Gleichung filr $Q$ bereehnen. Man findet:

$$
\int_{0}^{r} Y e^{-\frac{k r}{\cos i}} d r=\frac{\cos ^{2} i \cos \varepsilon}{l^{2}(\cos i+\cos \varepsilon)} \log \left(\frac{1+\cos i}{\cos i}\right) \text {. }
$$

Durch Substitution erhiilt man nun endlich:

$$
Q=I_{a} d s \frac{\cos i \cos \varepsilon}{\cos i+\cos \varepsilon}\left[1+u^{\prime} \cos \varepsilon \log \left(\frac{1+\cos \varepsilon}{\cos \varepsilon}\right)+u^{\prime} \cos i \log \left(\frac{1+\cos i}{\cos i}\right)\right] \text {, }
$$

wo noch gesetzt ist:

$$
I_{2}=\frac{1}{4 \pi l_{i}} \mu L \quad \text { und } \quad u^{\prime}=\frac{\mu}{2 l_{i}} .
$$

Diese Gleiehung müsste nun an Stelle des auf Seite 46 entwickelten einfachen Beleuchtungsgesetzes eingeführt werden und wirde bei undurehsichtigen, diffus reflectirenden Substanzen das Lambert'sche Emanationsgesetz zu ersetzen haben. Mam sieht tibrigens ans dieser Form, ebenso wie aus der vereinfachten, dass bei der hier durehgefthrten Ánschanungsweise auch das Geset\% rom Cosinus des Incidenzwinkels verschwindet, dass rielmehr die Formel in Bezug anf Emanations- und Incidenzwinkel gaumz symmetriseh ist, so dass dieselben beliebig mit einander vertauscht werden können. Es wiirde daraus folgen, diss die Helligkeit gan\% nuabhängig rom Azimnthe sein misste, mid dass es dalier anch gleichgnlltig wäre, ob Beobachter und Lichtrquelle sich auf derselben oder anf entgegengeset\%ter Seite der Normale zur Fläehe befinden. Maln weiss aber, insbesondere durch Beobachtungen ron Seeliger und Messerschmitt an einer grossen Reihe ron diftits reflertirenden Sul,stumzen. dass dies nicht der Fall ist, mud dass in Allgemeinen die mrickgeworfene Lichtmenge :mo grössten ist, wemn lichtquelle und Auge im Azimuth mm $190^{\circ}$ von einander entfernt siud. Lis greht daraus hervor, diss ancli das nene Lommel-Seeliger'sche Belenchtmugsgeset\% keinesweg's vollkomnten den thatsächlichen Verhiiltnissen entspricht und nur in gewissen Fïllen als eine Nihermugsformel zn betrachten ist. Dies liisst sich anchl ron rornherein sehon deswegen erwarten, weil die Amnalunen, welche der Theorie zu Grunde gelegt wurden, sicher nicht der Wirklicheit contsprechen und weil es schwerlich Substanzen geben wird, deren winzelne Theilchen das empfangene Licht mit gleicher Stairke nach allen lichtmugen zerstrenen und in denen die Absorption nach allen Seiten gleich gross ist; anch wird man kaum Substanzen finden, bei denen die Ausstrahlung ans den Innern ganz allein zur Geltung kommt und bei denen keinerlei directe Spiegelung zur Wirkmng gelangt. Im Allgemeincn werden beide Liclit- 
wirkungen жu bericksichtigen sein, und da jede einzelne Substanz je naeh der Oherflächenbeschatỉenheit und der inneren Anordnung der Theilchen ein verschiedenes Verhalten zeigen wird, so scheint eigentlich jede Hoffmung ansgreschlossen, die Lichterscheinungen bei diffus reflectirenden Kiirpen dureh ein einziges Belenchtmosgesetz zu umfassen und im gegrehenen lialle in Vorans zu berechen. Immerhin verdient die LommelSecliger'sche Theorie, wem sie anch das Problem nicht vollständig zu lösen vermag, durch die Exactlieit der Amahmen, anf die sie sich stützt, cutselichencu Voryg ror der Lambert'schen Theorie, welehe jeglieher fosten stibte enthehrt.

\section{Begrifl der Albedo.}

Bercits in vorangelenden Paragraphen ist kurz ron der Albedo rines Körpers die Rede gewesen. Sach der von Lambert eingeführten Dhinition ist dirmuter eine Zalul zu verstehen, welche angiebt, wie sich dir wn cinem belenrliteten Element nich allen Richtungen diffus ans-

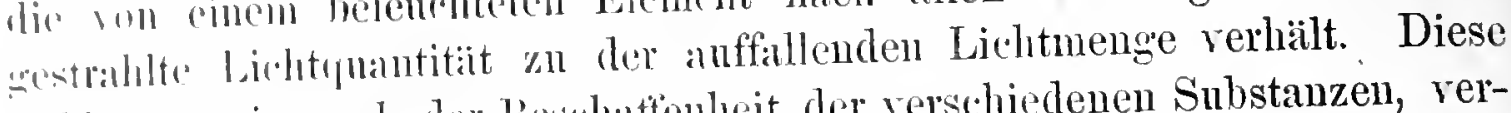
ball muss, je mallh der beschatfenheit der rerschiedenen Substanzen, verstheden sin. boi eincm ahsolut weissen Körper, d. h. bei einem sol-

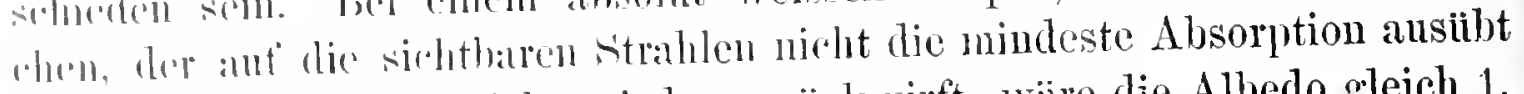

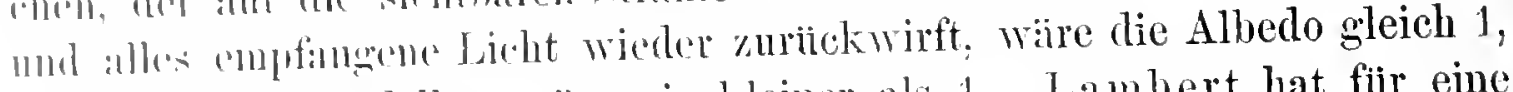
in allen iihrigen fillen wiire sie kleiner als 1. Lambert hat für eine Trzall wn substmzen dic Mbedo bestimmt und findet dieselbe in den

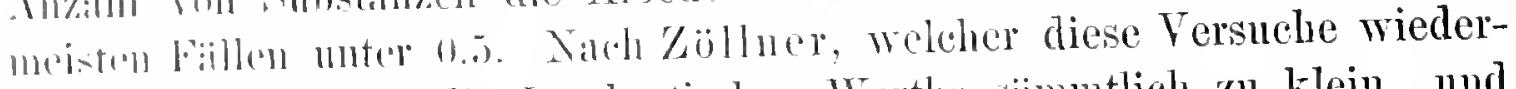
lmlt hat. sind alder die Lambertschen Werthe simmtlich zu klein, und

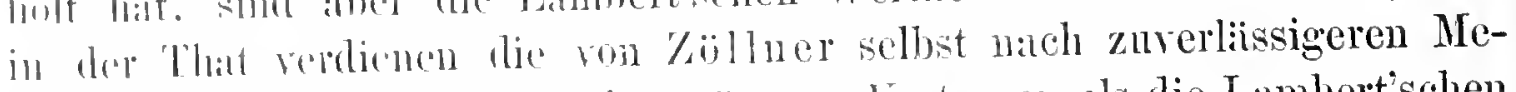

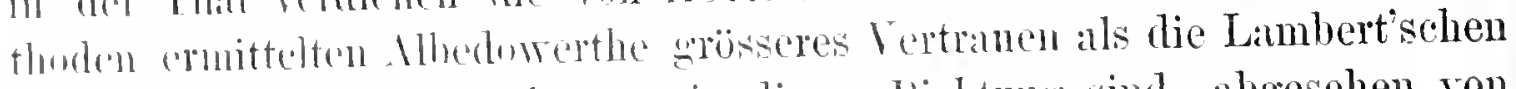

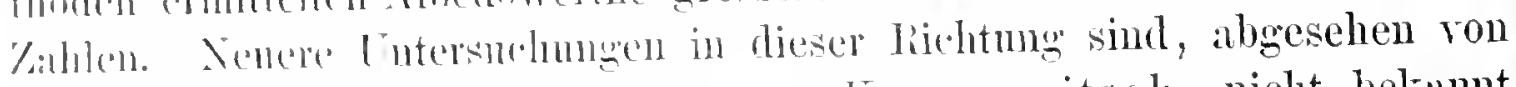
rinigen veremzelten Bestimnungen ron Kononowitsch, nieht bekannt

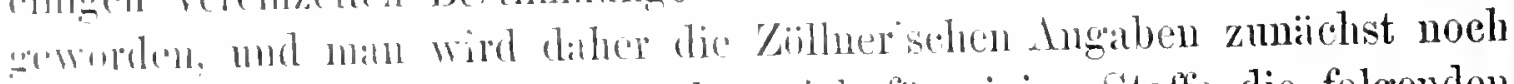

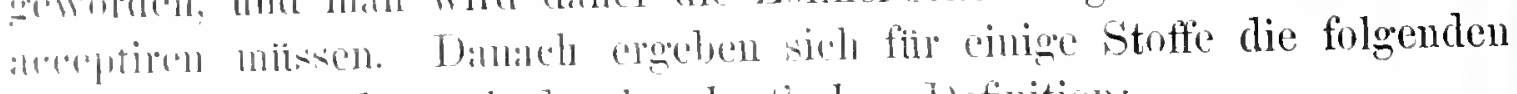

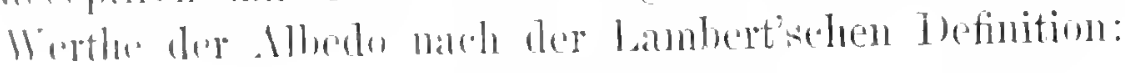

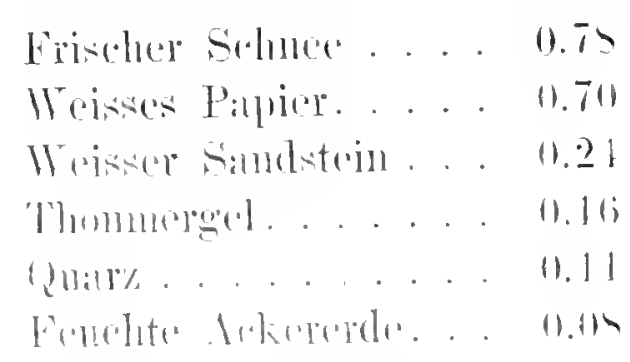

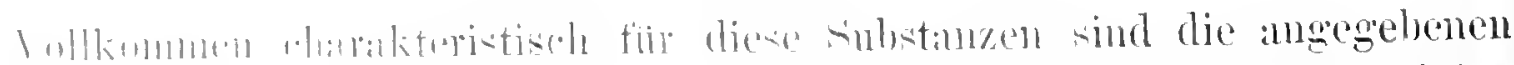

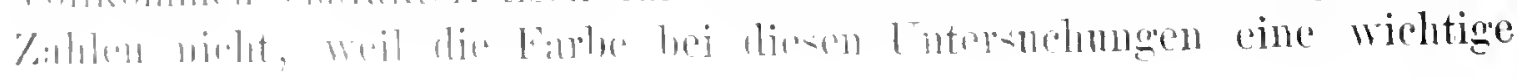


liolle spielt. Der Begrifl der Albedo ist streng genommen mur gilltig fiir homogenes Licht oder wenn einfallendes nnd austretendes Licht gleiche Farbe haben, was fast niemals der Fall sein wird. Rationelle Albedobestimmungen mltssten bei jeder Substan\% in allen möglichen Fitrhen, etwa mit Hulfe des Spectrilphotometers, vorgenommen werden, und wenn es sich, wie in der Astrophotometrie, beispielsweise um die Untersuchungr einer Planetcnoberliiche hambelte, so kümte man, al)geschen von einer Menge anderer Unstinde, unr dam anf' die stof'liche Verwandtschaft mit irgend einer irdisehen Subst:m\% schliessen, wem die Albedowerthe hei beiden untersuchten Körpern fur alle Farben ibloreinstimmten.

Die Lambert'sehe Definition der Albedo ist vollkommen correct, so laugre man auch das Lambert'sehe Belenchtungssgeset\% gelten liisst. Dem da in diesem Falle das austretende licht lediglich rom Emamationswinkel ahhïngt, so liat die Albedo fir alle Werthe des Incidenzwinkels denselben Betrag. Wird aber ein anderes Beleuchtungsgeset\%, \%. B. das LommelSceliger'sche, \%n Grunde gelegt, bei welchem dis anstretende Licht eine Function von Inciden\%- und Emanationswinkel zugleich ist, so ergiebt sich fir jeden Werth des Incidenzwinkels eine andere Albedo, und die Lambertsche Definition verliert dam ihre Bedentung. Seeliger-1) hat anf diesen Unstand anfmerksam gemacht und cine andere Definition der Albedo in Vorschlag gehracht, welche gan\% allgemein fiir jedes Belenchtungsgeset\% Giiltigkeit hat.

Man denke sich ein Fliichenelement $d_{\sigma}$ unter dem Incidenzwinkel; ron einer Liehtquelle belenehtet, welehe anf die Flidehenemheit senkrerlit die Liehtmenge $L$ werfen möge: dimn erhialt do die Lichtnenge $L$ cos $i d \sigma$. Wird das zunäichst als unbekimnt voramsgesetzte Belenclitnngsugeset\% nit $f(i, \varepsilon)$ bezeichnet, so ist die Lichtmenge $d q$, welehe dis likement $d_{\sigma}$ in der Richtung des Emanationswinkels $\varepsilon$ anf ein in der Entfermm!g 1 senk-

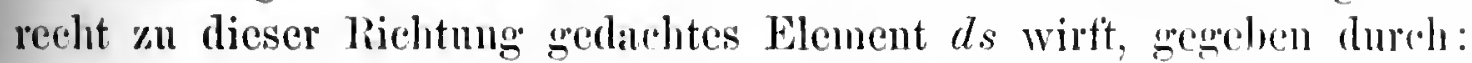

$$
d q=C L d i d s f(i, i) \text {, }
$$

wo $C$ für jedes Belenchtungsgesetz $f(i, \dot{s})$ eine andere Constante ist. Wird num um $d \sigma$ eine kugel mit dem Radius 1 construirt. so orhiilt die sanze Halbkugel die Lichtmenge:

$$
\eta=C L d \sigma \geq[f(i, c) d \cdot s] .
$$

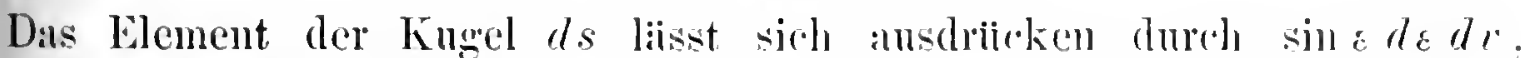
mil man hat daher:

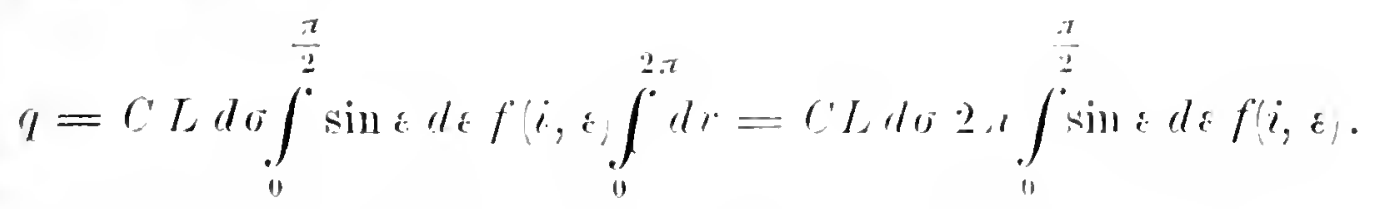

1) Abhandl. der K. Iayer. Akad der Wiss. Il. Classe. Ba. 16, p. 430. 
Das Verhibltuiss $A$ dieser gauzen anf die Halbkugel ausgestrahlten Liehtmenge zu der :uf $d o$ anffallenden ist mithin:

$$
A=\frac{q}{L \cos i d \omega}=2 \pi C \int_{0}^{\frac{\pi}{2}} \frac{f(i, \varepsilon)}{\cos i} \sin \varepsilon d \varepsilon .
$$

Ls gelit hierans hervor, dass im Allgemeinen $A$ noch rom Incidenzwinkel $;$ abhaingt. Nur wem $f(i, \varepsilon)=\cos i \varphi(\varepsilon)$ wäre, wie es z. B. trei dem Lambert'sehen Gesetze der Fall ist, würde die obige Definition der Albedo branchbar sein, in allen anderen Fällen müsste jedesmal der Incilenzwinkel angegeben werden, für den der betreffende Mlledwwerth giiltig sein soll. Man könnte diese Unklarheit vermeiden, wrmi mall unter Albedo den Werth verstehen wirrde, den $A$ für senkrecht anfiallemdes Licht, also fuir $i=0$, ammimut. Es ist aber vielleicht noch richtiger, den Seeliger'schen Vorschlag zn aeceptiren und unter Albedo den Mittelwerth aller $A$, die sich fuir simmtliche Werthe des Ineidenzwinkls $i$ arehen, zu verstehen. Denkt man sich wieder um das Element to mit clem liatlins I cine Kugel construirt, so wäre zur Bildung des Fesurliten Nittelwertles, der mit $A^{\prime}$ bezeichnet werden soll, die Summe

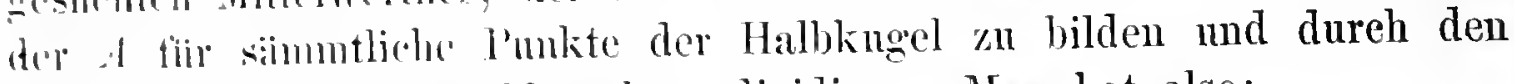

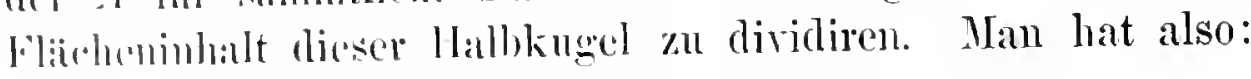

$$
A^{\prime}=\underbrace{1}_{\pi} \sum A \text {. }
$$

Zhoht man zunähst eine schmale Kugelzone in Betracht, so ist der Inhalt dereselben $2, r$ sin $i d i$, die Summe aller für die Zone giiltigen $A$. ist diller 2 a 1 sin $i d i$. mul die summe aller fuir die ganze Halbkugel

wiiltigen $A$ ist mithin $2 . \int_{11}$ l sini di. Es wird also:

$$
A^{\prime}=\int_{i}^{\frac{\pi}{2}} 1 \sin i d i \text {. }
$$

mine nall substitution des obigen Werthes rom at:

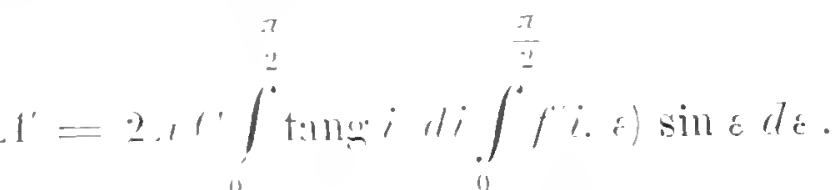

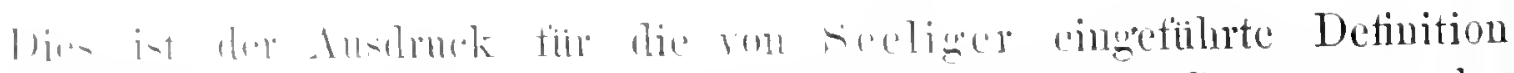

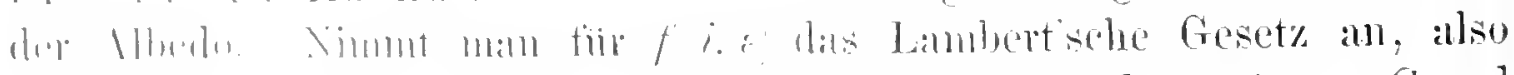

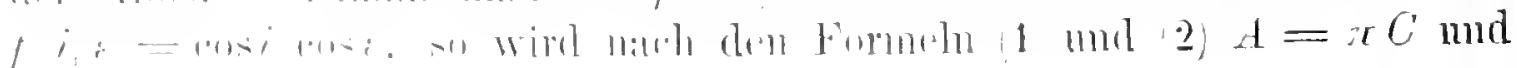


$A^{\prime}=\varkappa C$; es stimmen also in diesem Falle, wie zu erwarten war, die Lambert'sche und Seeliger'sche Albedo uberein.

Substituirt man das Lommel-Secliger'sche Belenchtungsgeset\% in der vereinfachten anf Seite 46 angegebenen Form:

$$
f(i, \varepsilon)=\frac{\cos i \cos \varepsilon}{\cos i+i \cdot \cos \varepsilon}
$$

so erhiailt man alls Formel (2) den Werth:

$$
A^{\prime}=2 \pi C \int_{0}^{\frac{\pi}{2}} \sin i d i \int_{0}^{\frac{\pi}{2}} \frac{\sin \varepsilon \cos \varepsilon}{\cos i+\lambda \cos \varepsilon} d t \text {. }
$$

Zur Auflösumg des zweiten Integrales setze unan $\cos i+\lambda \cos \varepsilon=x$, also $\sin \varepsilon d \varepsilon=-\frac{d x}{i}$; dann erhült man:

$$
A^{\prime}=\frac{2 \pi C}{i_{0}^{2}} \int_{0}^{\frac{\pi}{2}} \sin i d i\left\{\lambda_{0}+\cos i \log \cos i-\cos i \log (\lambda+\cos i\}_{j},\right.
$$

oder wenn man noch $\cos i=$ ?/ setzt:

$$
A^{\prime}=\frac{2 \pi C}{\ell^{2}}\left\{j-\int_{i}^{0} y d y \log y+\int_{1}^{0} y d y \log (i+y)^{\prime}\right\} .
$$

Nach Auflösung der beiden einfachen Integrale wird endlich:

$$
A^{\prime}=\frac{\pi C}{i}\left\{1-i \log i+i^{2}-1 \log (1+i)\right\},
$$

wo die sämmtlichen Loganithmen natiirliche sind.

Die Seeliger'sche Albedo ist hiernach bei Zugrundelegrmg des LommelSeeliger'schen Beleuchtungsgesetzes in der That vom Einfallswinkel gainzlich nnabhängig; sie wird nur durch die Grössen $C$ und i. bestimmt, welche Constanten repriisentiren, die jeder Substanz eigenthiimlicl sind mud ron der Reflexions- und Absorptionsfähigkeit derselben ahbängen. Sieht man, wie im Friheren, von der Farbeniandermg in Innern des Körpers al) und nimmt das Absorptionsvermögen für ein- und anstretende strahlen als gleich an, setzt also $i=1$, so wird die Albedo cinfach gleich $x C^{\prime}$, also nur durch den Proportionalititsfictor des Lommel-Secligerschen Gesetzes bestimmt. 


\section{Capitel II.}

\section{Anwendung der photometrischen Grundprincipien auf die wichtigsten Aufgaben der Himmelsphotometrie.}

\section{Brleuchtung der Plancten nud Monde.}

Durch die Eutwicklungen des vorigen Capitels sind wir in den stand gesetzt, die Lichterseheinmgen derjenigen Himmelskörper theoretiseh \% stmdiren, welehe nieht wie die Fixsteme unendlich weit von uns entfornt sind und rigenes Lirht besitzen, soudern uns nur dureh reflectirtes simmonlicht sichthar werden. Da infolge der Bewegungen im Sonnen-rstem diese Ilimmelskibler in sehr versehiedene Entfermungen ron der Frole kommen, und ankserdem die Grösse des fiur uns sichtbaren Theiles

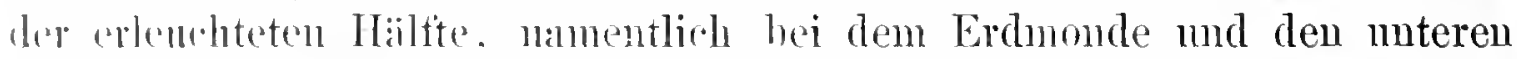
[']:meton. selu starken Veriudermgen unterworfen ist, so schwankt die

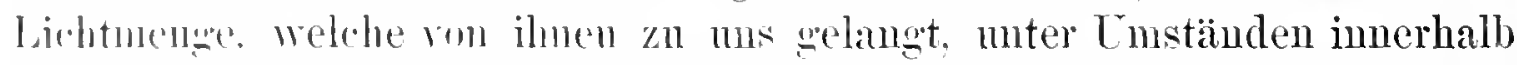
writer Gienten. men da wir diese Schwamkungen durch photometrische

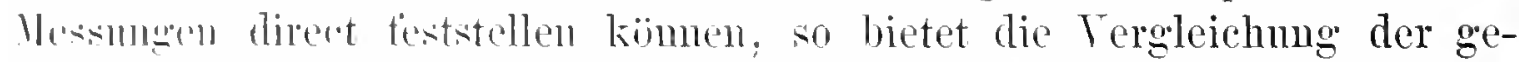
tundemen liesultate mit den anf theoretischem Wege berechueten Werthen

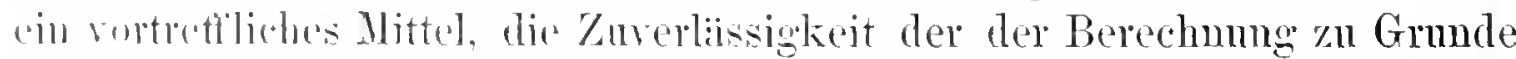

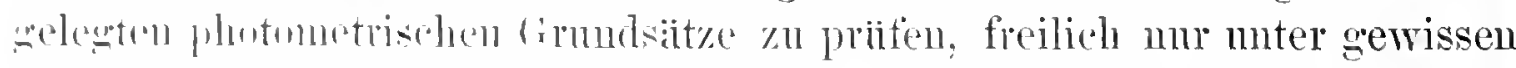

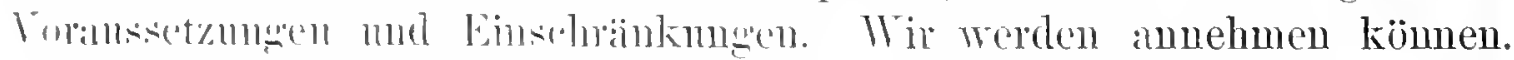

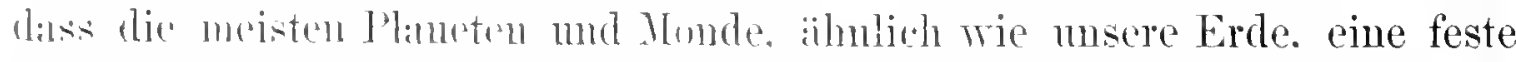

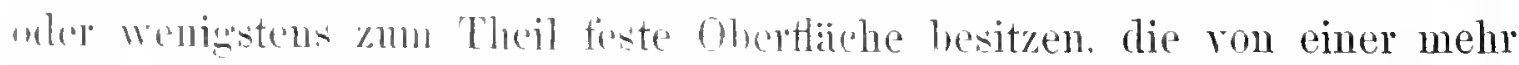

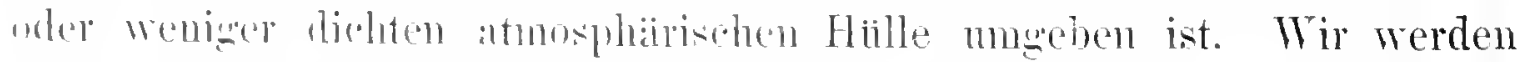

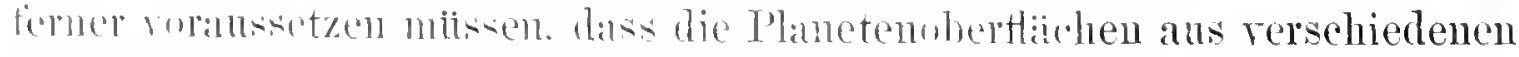

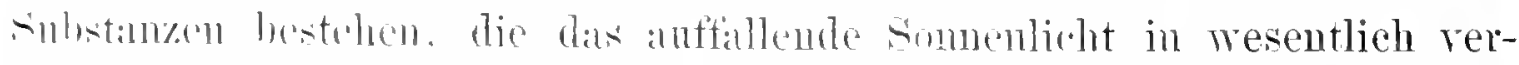

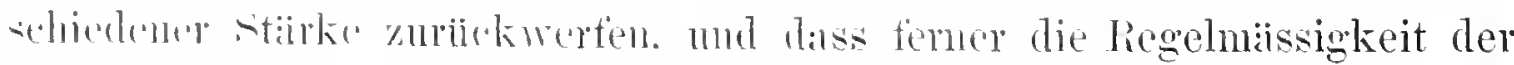

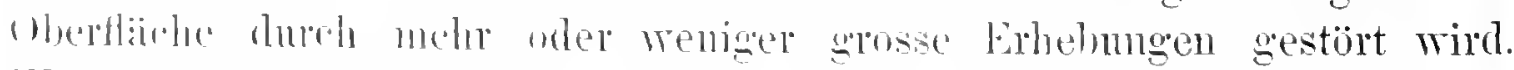

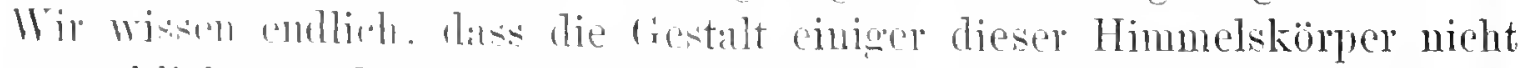

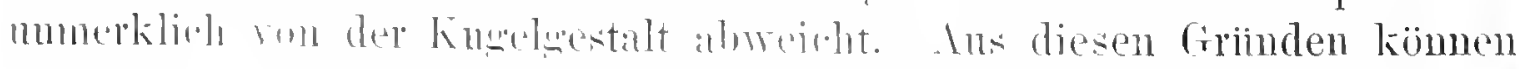

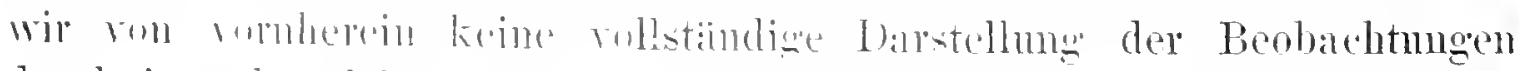

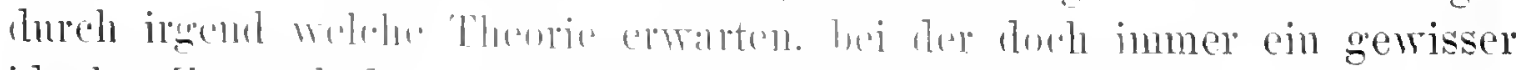

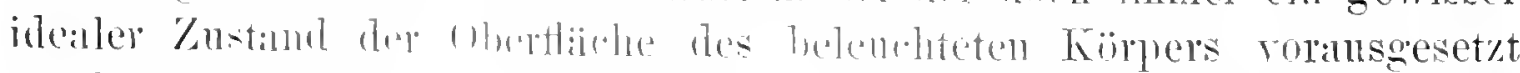
werden mus. Latu kimmut noh. wis wir in rorigen Capitel bereits 
unseinandergesetzt haben, dass bei den irdischen zerstrent reflectirenden Substanzen keines der bisher anfgestellten Belenelitungsgesetze sich als vollkommen unanfechtbar erwiesen hat, und dass daher etwas $̈$ hunliches anch bei den anderen Himmelskörpern zu erwarten ist.

Wir wollen bei den folgenden Betrachtungen die Planeten und Monde zunächst als vollkommen kugelförmig ansehen, ferner annehmen, dass jede Oberfläche uberall dieselloc ihr eigenthlimliche mittlere Reflexionstihigkeit hesitzt, endlieh wollen wir der Berechnung der. von ilnen »urlickgeworfenen Lichtquiutititen drei versehiedene Beleuchtungsgesetze 211 Grunde legen, indem wir zn den beiden in vorigen Capitel ansfilhrlich behandelten Gesetzen von Lambert und Lommelseeliger noch ein drittes hin\%ufitgen, welehes im Voransgehenden hereits ebenfalls kur\% erwiilunt worden ist, und welehes anf der einfachen Vorstellung berulit, dass das von einer selbstlenchtenden oder zerstrent reflectirenden Oberfliiche ausgesandte Licht gainzlich vom Emanationswinkel unabhïngig und lediglich den Cosinus des Ineidenzwinkels proportional ist. Dieses Belenchtmngsgeset\%, welches das Euler'sehe renamnt werden kiun, weil es ron diesem Mathematiker an Eingehendsten behandelt worden ist, verdient hier deswegen noch eine besoudere Berieksiehtignung, weil es bis in die neneste Zeit ron vielen Astronomen zur Berechmung der Planetenhelligkeiten benutzt worden ist. lis wird sich später bei der Besprechung der nenesten Ergebnisse der Planetenforschung zeigen, dass dieses Geset\%, wie nach den bisherigen Erörterungen auch von vornherein zu erwarten ist, am Wenigsten vou allen den thatsaiehlichen Verhiiltnissen entspricht. Die drei in Frage kommenden Belenchtnngsgesetze sind durch die folgenden Formoln reprissentirt, wenn $d_{\eta}$ die Lichtmenge ist, welche ein unter dem Inciden\%winkel $i$ gretroffenes Oberflichenelenent $d s$ unter dem Emanationswinkel $\varepsilon$ wieder ausstralult:

$$
\begin{array}{ll}
d \tau_{1}=I_{1} d s \cos i \cos \varepsilon & \text { (Limbert'sches Gesetz), } \\
d q_{2}=I_{2} d s \frac{\cos i \cos \varepsilon}{\cos i+i \cos \varepsilon} & \text { (Lommel-seeliger sches (iesetz), } \\
d q_{3}=I_{3} d s \cos i & \text { (Euler sches Gesetz). }
\end{array}
$$

Die Constanten hïngen ron der Intensitiit des anffallenden Lirlites und ansserdem ron der Retlexionstihigkeit resp. von der Diffusions- und Absorptionsfühigkeit im Innern der betreffenden Subst:lnz a h. 
a. Bercehnung der ron den Phasen eines beleuchteten Himmelskörpers nach der Erde gesandten Lichtmenge.

Bestimmung der Albedo.

Lis soll nun zuerst die Aufgabe behandelt werden, diejenige Liehtmenre жn berechnen, welehe eine Plametenkugel der Erde zusendet bei einer belichigen Stellung von Sonne, Planet und Erde zu einander. Man denke

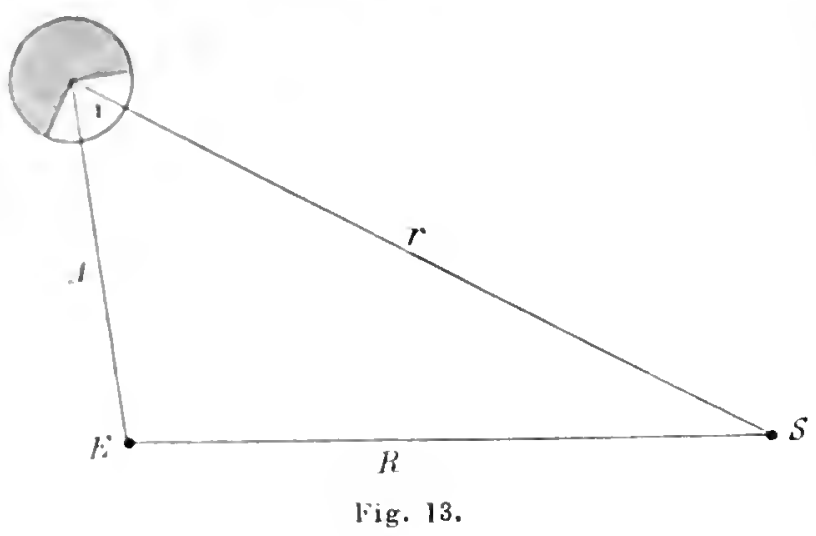
sich dieMittelpunkte der drei Himmelskörper mit einander verbunden und bezeichne in dem nebenstehenden Dreieck (Fig. 13) die Entfermung Erde-Sonne mit $R$, die Entfermung Erde-Planet mit $A$ und die Entfermung SonnePlanet mit $r$, ferner den Winkel an Planeten mit a, dann reprïisentirt a die Grösse des verfinsterten Theiles der Planeten-

kind und wirl daher jetzt allgemein der Phasenwinkel genamnt, während lambert diese Bezeichmmg fiur das Supplement ron a gewählt hatte. Wo" linkel "liisst sirh durch die drei Entfermungen $r, \Delta, R$, deren muncrishe Werthe fir jeden Zeitpunkt ans den astronomischen Ephemepirlen dntmonnmen werden können, berechnen. Man hat:

Int mithin:

$$
l^{2}=r^{2}+l^{2}-2 l^{2} l \cos a,
$$

$$
\operatorname{ros} r=\frac{f^{2}+r^{2}-r^{2}}{2 r l} \text {, }
$$

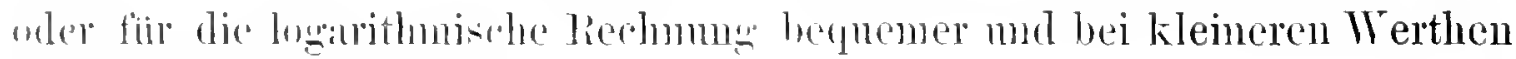
vols a comptehlemswerther:

$$
\therefore \sin \frac{1}{2}=i \sqrt{\frac{1 i+1-i+1-1}{i}} .
$$

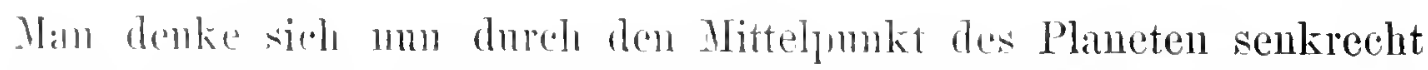

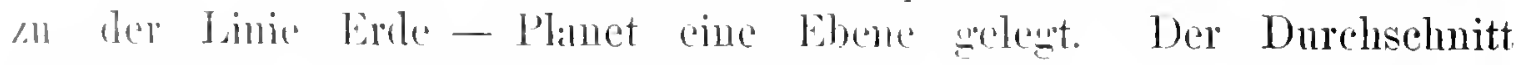

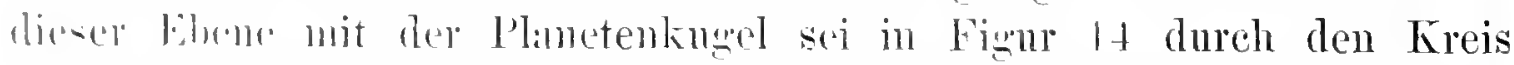

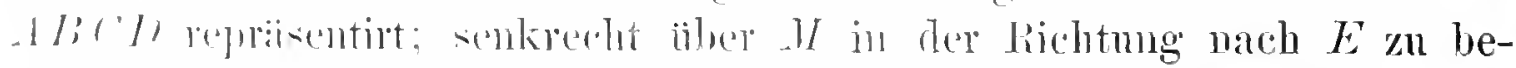

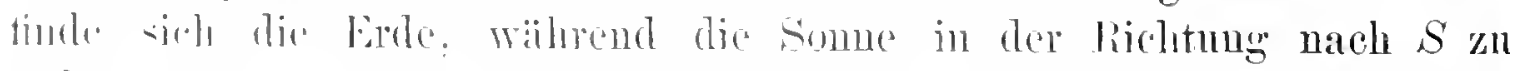

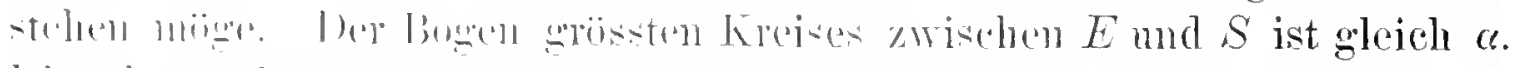

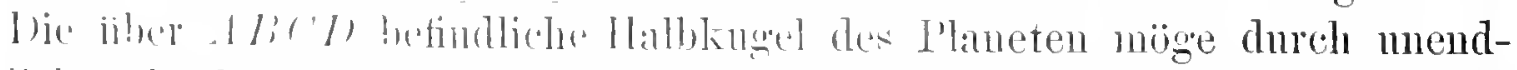

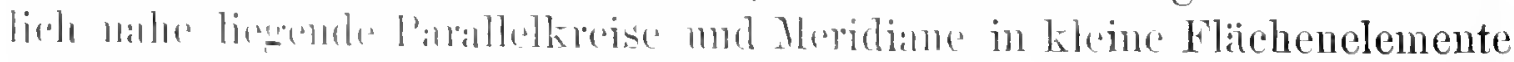

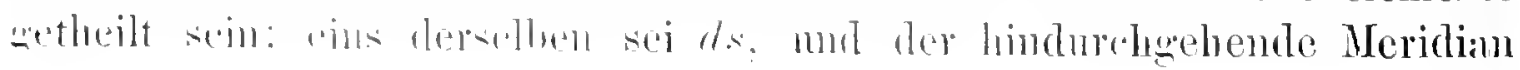


schneide den dureh $E$ und $S$ gelegten grössten Kreis im Punkte $F$. Verbindet man $d s$ mit $S$ und $E$ durch Bogen grössten Kreises, so ist leicht ersichtlich, da bei der grossen Entferunng der Himmelskörper alle auffallenden und ebenso alle zurltckgeworfenen Lichtstrahlen als parallel unter einander angesehen rerden durfen, dass der Bogen zwischen $d s$ und $S$ nichts Anderes als der Incidenzwinkel $i$ und ebenso der Bogen zwisehen $d s$ und $E$ inichts Anderes als der Emanationswinkel $\varepsilon$ ist. Flihrt man noch andere Coordinaten ein, indem man die Breite des Elementes ds mit $\psi$ und die Liinge in dem durch $E$ und $S$ gelegten grössten Kreise, von $E$ ans gezililt, mit $\omega$ bezeichnet, so hat min in den beiden bei $F$ rechtwinkligen sphïi-

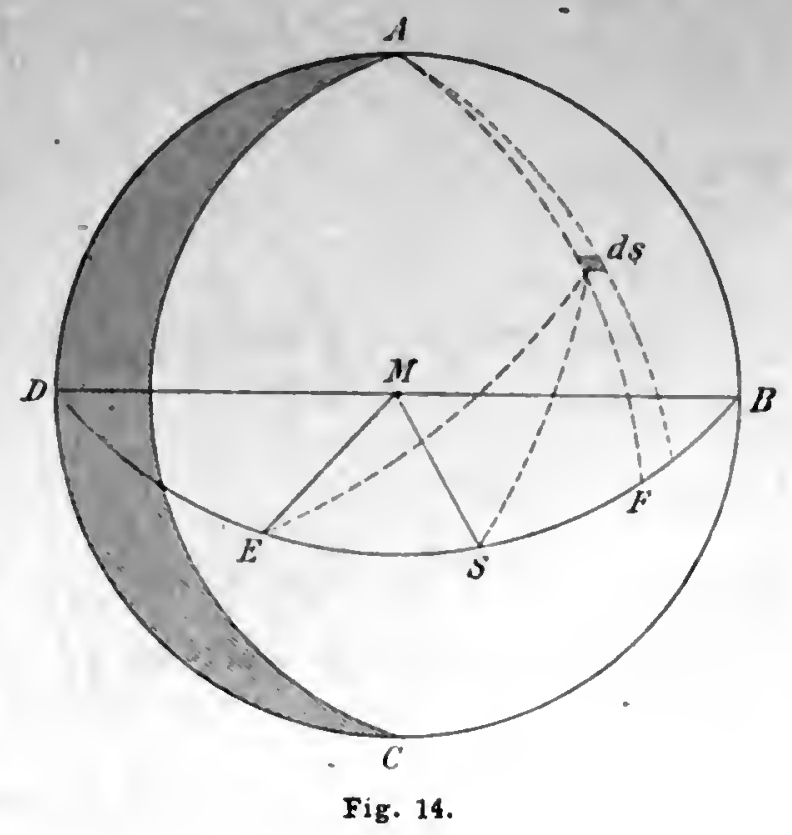
rischen Dreiceken FSds und FEds die Beziehungen:

$$
\begin{aligned}
& \cos i=\cos \psi \cos (\omega-\omega), \\
& \cos \varepsilon=\cos \psi \cos \omega .
\end{aligned}
$$

Ist endlich noch der Halbmesser der Planetenkugel gleich $\varrho$, so hat das Oberflächenelement $d s$ in der Meridiamrichtung die Grösse $\varrho d \psi$ und in dem Parallelkreise die Grösse $\varrho d \omega \cos \psi$; mithin ist der Fliicheuinhaılt ron $d s=Q^{2} \cos \psi d \omega d \psi$.

Setzt man diese Werthe in die obigen drei Belenchtungsforneln ein, so crhält man für die ron einem Planetenoberflichenelemente bei dem Phasenwinkel " nach der Erde ansgestrahlte Lichtmenge die drei Werthe:

$$
\left\{\begin{array}{l}
d q_{1}=r_{1} \rho^{2} \cos ^{3} \psi d \psi \cos (\omega-a) \cos \omega d \omega, \\
d q_{2}=\Gamma_{2} \varrho^{2} \cos ^{2} \psi d \psi \frac{\cos \omega-\cos (\omega-\omega)}{\cos (\omega-\omega)+i \cos \omega} d \omega, \\
d q_{3}=r_{3} \varrho^{2} \cos ^{2} \psi d \psi \cos (\omega-(\omega) d \omega .
\end{array}\right.
$$

Um die von der ganzen Planetenkngel zur Erde gresindte Lichtquantität zu haben, miissen diese Formeln iiber den ron der Erde ans sichtbaren, von der Some belenchteten Theil der Oberflaiche integrirt werden. Wie man aus der obigen Figur ersieht, sind die Integrationsogrenzen in Bezuy" aut

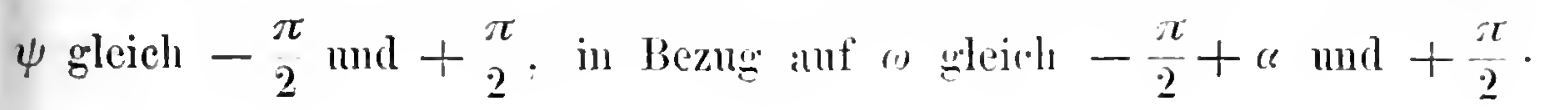


Behandelt man die verschiedenen Gesetze fiir sich, so hat man zunichst fur das Lambert'sehe Geset\%:

$$
\mu_{1}=r_{1} \rho^{2} \int_{-\frac{\pi}{2}}^{\frac{\pi}{2}} \cos ^{3} \psi d \psi \int_{\omega-\frac{\pi}{2}}^{\frac{\pi}{2}} \cos (\omega-\omega) \cos \omega d \omega .
$$

lis ist ilber:

$$
\int_{-\frac{\pi}{2}}^{\pi} \cos ^{3} \psi d \psi=\int_{-\frac{\pi}{2}}^{\frac{\pi}{2}} \cos ^{2} \psi d(\sin \psi)=\int_{-\frac{\pi}{2}}^{\frac{\pi}{2}}\left[1-\sin ^{2} \psi\right] d(\sin \psi)=\frac{4}{3} .
$$

Fermer ist einfiach:

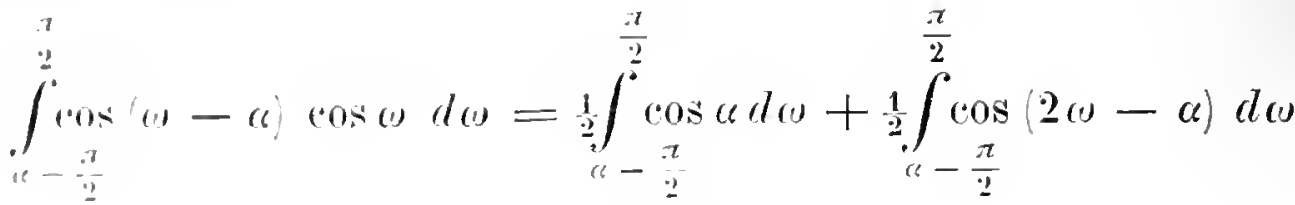

$$
\begin{aligned}
& =\frac{1}{2}[(t-c) \cos \theta+\sin c] \text {. }
\end{aligned}
$$

liller:

$$
\eta_{1}=I_{1} \theta^{2} \frac{2}{3}\{\sin \theta+(\pi-c) \cos \theta\} \text {. }
$$

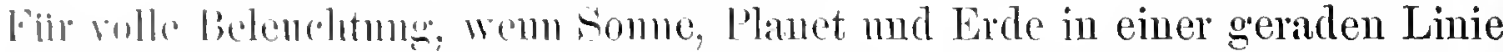

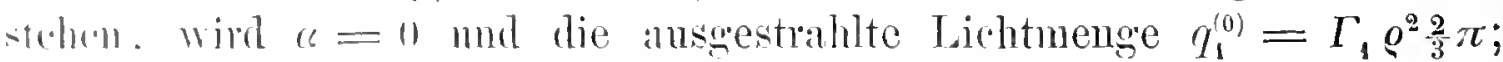
llall hat dallere alleh:

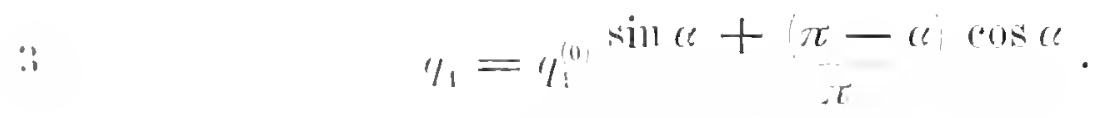

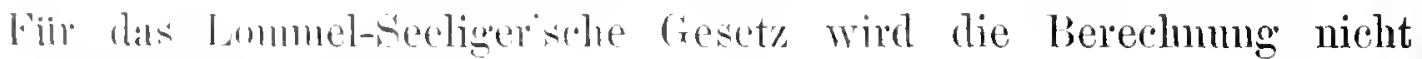

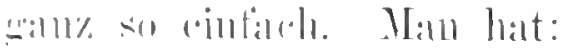

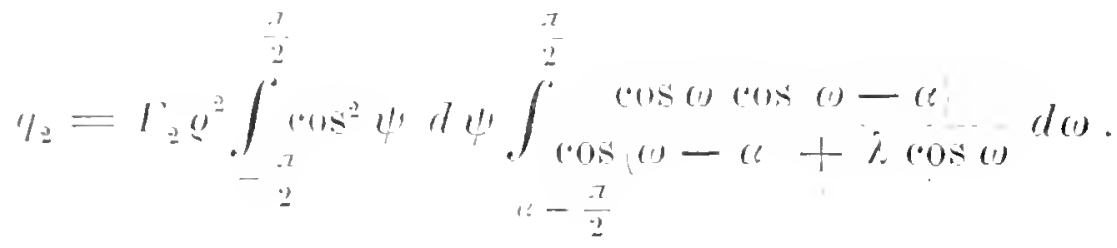

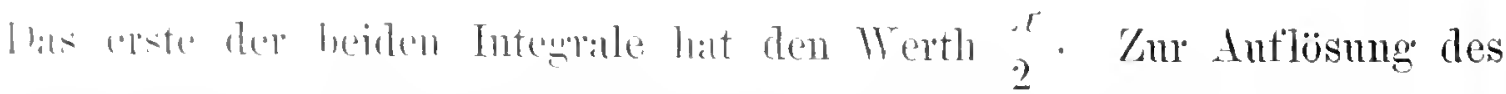

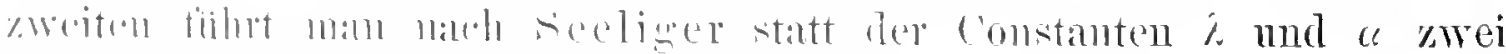

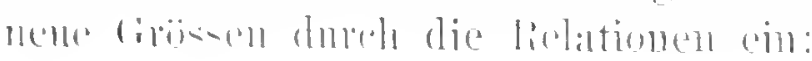

$$
\begin{aligned}
& \text { sinu } u=m \text { sin } u \text {. } \\
& \text { i. }+1016=11101011
\end{aligned}
$$


Daun wird:

$$
q_{2}=\frac{r_{2} e^{2} x}{4 m} \int_{n-\frac{\pi}{2}}^{\frac{\pi}{2}} \frac{\cos \alpha+\cos (2 \omega-(\omega)}{\cos (\omega-\mu)} d(\omega)
$$

oder wenu man $u-" 1=$ y set»t:

$$
\begin{aligned}
& \frac{\pi}{2}-\mu
\end{aligned}
$$

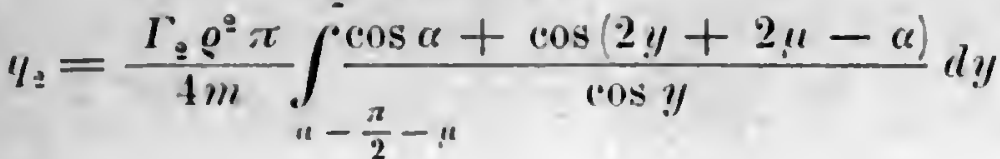

$$
\begin{aligned}
& \frac{\pi}{2}-11 \\
& =\frac{l_{2} \varrho^{2} \pi}{4 m} \int \frac{d y}{\cos y}[\cos (\iota-\cos (2 u-\alpha)+2 \cos y\{\cos (2 u-\alpha) \cos y-\sin (2 u-\alpha) \sin y\}] \\
& "-\frac{\pi}{2}-" \\
& \frac{\pi}{2}-u^{\circ} \quad \frac{\pi}{2}-\mu \\
& =\frac{\Gamma_{2} \varrho^{2} \pi}{2 m} \int_{\alpha-\frac{\pi}{2}-\mu}^{\sin \mu} \frac{\sin (\mu-\alpha)}{\cos y} d y+\frac{\Gamma_{2} \varrho^{2} x}{2 m} \int_{\alpha-\frac{\pi}{2}-\mu}^{2} \cos (\eta+2 \mu-\alpha) d ! \text {. }
\end{aligned}
$$

Diese Integrationen lassen sich leicht ansfuhren, mod man hat daher endlich:

(4) $\eta_{z}=\frac{\Gamma_{z} \varrho^{2} \pi}{2}\left\{\frac{2 \cos \frac{a}{2} \cos \left(u-\frac{c}{2}\right)}{m}+\frac{\sin ! \sin (u-(i)}{m} \log \left[\cot \frac{a-! \prime}{2} \cot \frac{\prime \prime}{2}\right]\right\}$.

Fiir volle Beleuchtung wird $a=n$, folglich $"=0$ und $n=1+i$. und man hat daher fuir die von dem voll belenchteten Planeten nach der Erde ausgestrahlte Lichtmenge $q_{2}^{(0)}$ den Werth:

$$
q_{2}^{(0)}=\frac{\Gamma_{2} o^{2} x}{1+i}
$$

Von Wichtigkeit zur Bestimmung ron $\eta_{2}$ ist die Kenntuiss der Cirösse $\lambda_{\text {. }}$ d. h. des Verhältnisses der Absor|ptionscoefficienten für die ein- mul anstretenden Strahlen. Die Coefficienten werden in den neisten Fiillen nicht wesentlich ron einander verschieden sein, und man wird daher kaum einen grossen Fehler begehen, wemn man $i=1$ setzt. Fuir $i=1$ wird aber $m \cos u=1+\cos \alpha$; ferner ist tan $" 1=\frac{\sin u}{1+\cos u}=\tan \frac{a}{2}$ oder $"=\frac{a}{2}$ und $m=2 \cos \frac{a}{2}$. Durch substitution in (4) erhialt man daher: 
(6)

$$
q_{2}=\frac{I_{2} \varrho^{2} \pi}{2}\left\{1-\sin \frac{\alpha}{2} \operatorname{tang} \frac{\alpha}{2} \log \cot \frac{\alpha}{4}\right\} \text {. }
$$

Fir volle Beleuchtung oder $a=0$ wird die ausgestrahlte Lichtmenge:

$$
q_{2}^{(0)}=\frac{I_{2} \varrho^{2} \pi}{2}
$$

mithin ergiebt sich:

$$
\eta_{2}=q_{9}^{(0)}\left\{1-\sin \frac{a}{2} \operatorname{tang} \frac{a}{2} \log \cot \frac{c}{4}\right\} \text {. }
$$

Fiir das dritte der obigen Gesetze, das Euler'sche, hat man:

$$
I_{3}=I_{3} \varrho^{2} \int_{-\frac{\pi}{2}}^{\frac{\pi}{2}} \cos ^{2} \psi d \cdot \psi \int_{\omega-\frac{\pi}{2}}^{\frac{\pi}{2}} \cos (\omega-\omega) d \omega,
$$

worans sich mmittelbar ergiebt:

$$
t_{3}=I_{3} q^{2} \pi \cos ^{2} \frac{a}{2} .
$$

Bei voller Belenchtung wird die answestrahlte Liehtmenge $q_{3}^{(0)}=\Gamma_{3} \varrho^{2} \pi$, Innd mant hat daher:

$$
q_{3}=q_{3}^{(j)} \cos ^{2} \frac{c}{2}
$$

Die in Vor:mgehenden fiir die drei betrachteten Beleuchtungsgesetze aufgestellten Formeln geben ganz allgemein die ron der ganzen belenchteten l'ametenphase in der Richtmo nach der Erde ansgestrahlte Liehtmente oder natch mseren triiheren Detinitionen die Lichtquantität, welche anf die Flächeneinheit in der Entferunng I senkrecht anuffällt. Diese Grösse ist aber der Berbachtung nicht direct zugäughtich. Was wir mit dem Photometer nder mit dem Ange messen, ist eine Grösse, die der auf das Fernruhrohjertir uder die Pupille des Anges senkrecht anffallenden Lichtmenge proportional ist. Will man also die theoretisch berechneten Helligkeitswerthe mit den Beobachtnugen rergleichen, sn miissen zunächst die oben

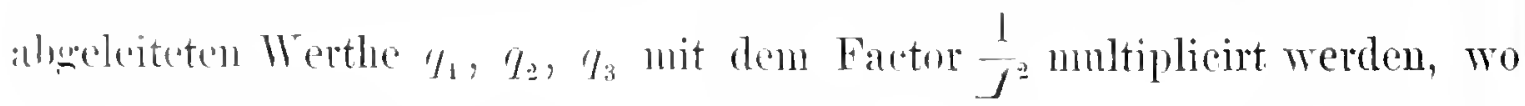
I die jertesmalige Entfermmg des l'kaneten rom der Erde ansdriiekt. Fermere ist zn hearhten. dass die in den Formeln anftretenden Grössen $r_{1}, r_{2}, r_{3}$ m diun als Constanten angesehen werden diufen, wenn die

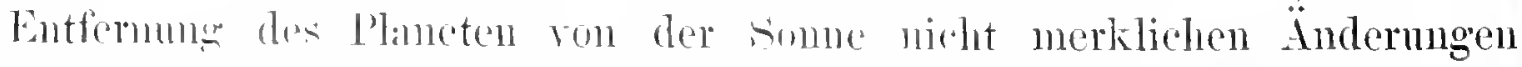
unterwerfen ist. Fo wird nöthign sein, diese Grössen etwas näher zu 
betrachten. Nach den Erörtermugen in vorigen Capitel ist $\Gamma_{1}=\frac{A_{1}}{\pi} L$ (S. 40), wo $A_{1}$ die Lambert'sehe Albedo ist. Ferner ist $I_{2}=\frac{1}{4 \pi} \frac{\mu}{k} L$ (S. 51), wo $\mu$ und $l$ das Diffusions- und Absorptionsvermö̈gen des beleuchteten Körpers bezeichnen. Nun ist aber fir das Lommel-Seeliger'sehe Gesetz in seiner einfachsten Form die Seeliger'sche Albedo, die wir $A_{2}$ nenuen wollen, ausgedrickt dureh $\frac{1}{4} \frac{\mu}{l_{i}}$, es wird also $I_{\mathrm{a}}=\frac{A_{9}}{x} L$. Endlich lisst sich durch eine :ihuliehe Betrachtung, wie durch die auf Seite 40 angestellte, leicht zeigen, dass $I_{3}=\frac{A_{3}}{2 \pi} L$ ist, wo $A_{3}$ dieselbe Bedeutung lat, wie die Lambert'sehe Albedo. In allen drei Ausdrieken bedentet $L$ die Liehtmenge, welehe anf die Flaicheneinheit des Planeten von der Sonne senkreeht ansgestrahlt wird. Betrachtet man die Somne als eine selbstlenehtende Kugel mit der Lenehtkraft $J$, so ist nach den Entwicklungen auf Seite $37 L=J \tau \sin ^{2} s$, wo $s$ der seheinbare liadins der Sonne ist, vom Planeten aus gesehen. Wir wollen mu statt der Grössen $q_{1}, q_{2}, q_{3}$ die der Beobachtung zug:inglichen Liehtmengen bestimmen, welche von der gesammten belenchteten Planetenphase senkrecht anf die Fliieheneinheit des Fernrohrobjectivs oder der Angenpupille gesandt werden. Bezeichnen wir diese durch $Q_{1}, Q_{3}, Q_{3}$ und setzen die oljigen Werthe für $\Gamma_{1}, \Gamma_{2}, \Gamma_{3}$ ein, so ergiebt siel, wem man noch den seheinbaren Halbmesser $" ;$ des Planeten, ron der Erde aus gesehen, durch die Relation $\sin \sigma=\frac{\varphi}{d}$ einführt:

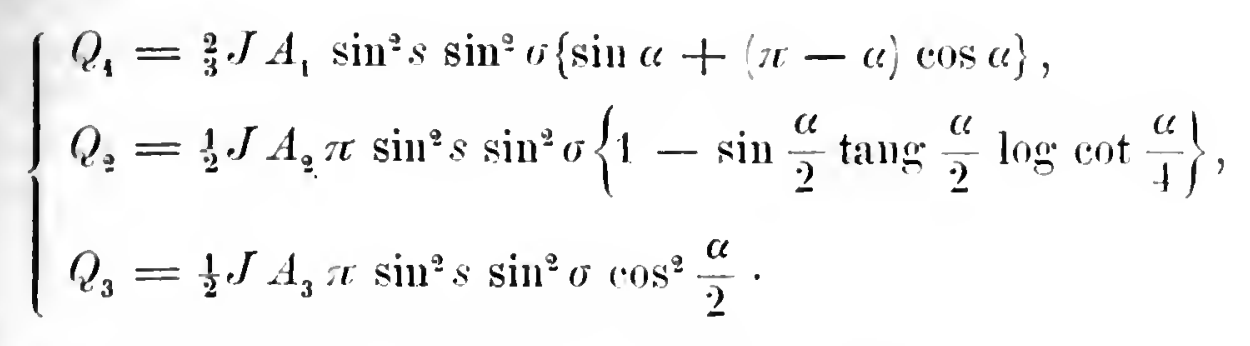

Für volle Belenchtung gehen diese Werthe iiber in:

$$
\left\{\begin{array}{l}
Q_{1}^{0)}=\frac{2}{3} J A_{1} \cdot \sin ^{2} s_{0} \sin ^{2} \sigma_{0}, \\
Q_{0}^{(0)}=\frac{1}{2} \cdot A_{2} \pi \sin ^{2} s_{0} \sin ^{2} \sigma_{0}, \\
Q_{3}^{0}=\frac{1}{2} \cdot A_{3} \pi \sin ^{2} s_{0} \sin ^{2} \sigma_{0},
\end{array}\right.
$$

wo $s_{0}$ und $\sigma_{0}$ die scheinbaren Halbunesser ron Somme nurd Planet zur Zeit der Opposition sind. Aus den Gleichungen (10) und (11) erhält man noch, wenn man statt der scheinbaren Halbmesser wieder die Distanzen einfiilnt: 


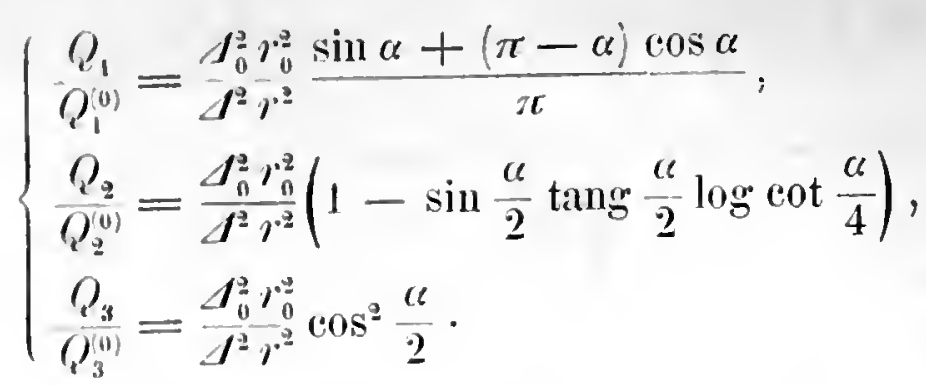

Diese Werthe sind direct mit den Resultaten vergleichbar, welche wir dureh photometrische Messungen oder Schätzungen erhalten könmen. Zu ihrer bequemen Berechnumg soll die im Anhange mitgetheilte Tafel dienen, welche die Log:urithmen der ron dem Phascuwinkel abhängigen Faetoren und die ans diesen Logarithmen durch Division mit 0.4 hervorgehenden Differenzen in Sterngrössenclissen enthält. Ein Überblick iiber diese Tafel \%eigt, wie stark dic mach den rerschiedenen Theorien berechmeten Helligkeitswerthe ron einander alweichen.

Die Formehn (10) oder 11 , kümneu noch dazn benutzt werden, die Illndo eines I'laneten zu berechnen. Zn diesem Zweek ist es aber \%uniahst erforderlich, die Grösse $J$, welche nicht dureh Beobaehtungen (runitfelt werlen kam, ans denselben zn eliminiren. Nemnen wir $L^{\prime}$ die linhtnenge oder Belenchtmng, welche von der somne direct anf die Flächenconhoit dus Fermmbroljecotivs oder der Angempupille gesandt wird, so ist (M)ruso wie when:

$$
L^{\prime}=J x \sin ^{2}, S^{\prime}
$$

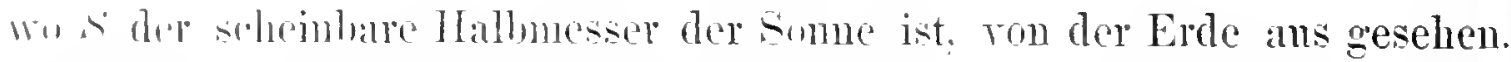

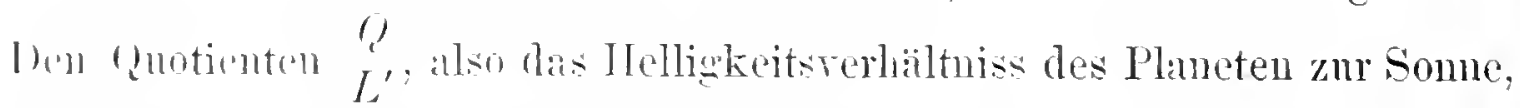

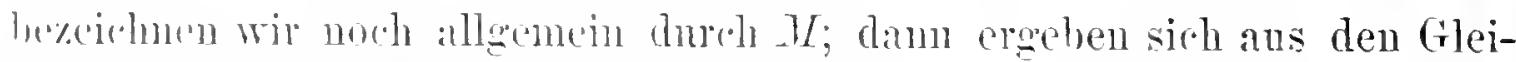
chungen in dio folgenden stbedowerthe:

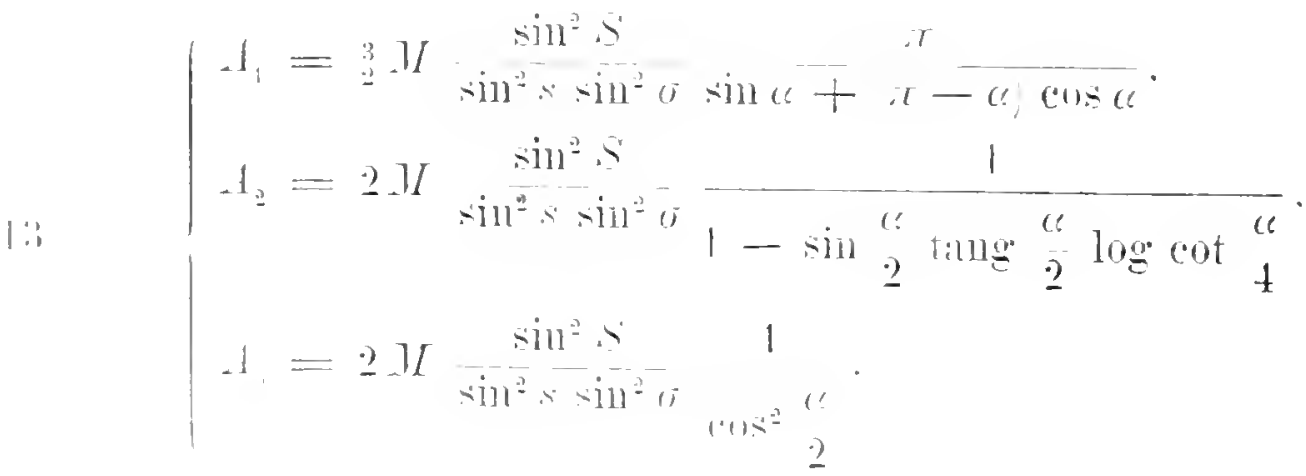

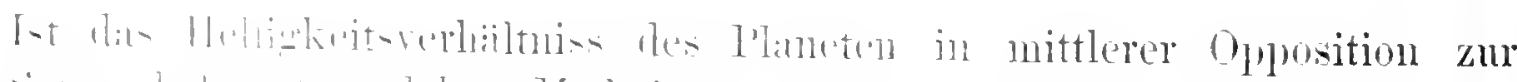

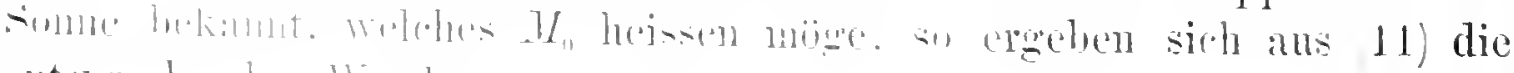

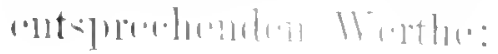




$$
\left\{\begin{array}{l}
A_{1}=\frac{3}{2} M_{0} \frac{\sin ^{2} S}{\sin ^{2} s_{0} \sin ^{2} \sigma_{0}}, \\
A_{2}=2 M_{0} \frac{\sin ^{2} S}{\sin ^{2} s_{0} \sin ^{2} \sigma_{0}}, \\
A_{3}=2 M_{0} \frac{\sin ^{2} S}{\sin ^{2} s_{0} \sin ^{2} \sigma_{0}} .
\end{array}\right.
$$

Die Albedowerthe fur das Lommel-Seeliger'selie und das Euler'sehe Gesetz stimmen nach diesen Formehn mit einander tuberein, und ans den leiden ersten Gleiehungen folgt: $A_{2}=\frac{1}{3} A_{1}$. Es ist aber dabei zu beachten, dass diese Beziehungen nur gelten, wemn in dem Lommel-Seeligerschen Gesetze die Grösse $\lambda=1$ gesetzt werden darf. Flir einen beliebigen Werth ron $\lambda$ ergiebt sich statt der zweiten Formel in (11) die folgende:

$$
Q_{q}^{(0)}=f L^{\prime} \frac{\mu}{k} \frac{1}{1+\lambda} \frac{\sin ^{2} s_{0} \sin ^{2} \sigma_{0}}{\sin ^{2} S} .
$$

Ferner ist nach Formel (3) auf Seite 55 die Seeliger'sehe Albedo bei einem heliebigen Werthe von $\lambda$. ansgedriickt dureh:

$$
A_{2}=\frac{1}{4 \lambda} \frac{\mu}{k}\left\{1-\lambda \log \lambda+\frac{\lambda^{2}-1}{\lambda} \log (1+\lambda)\right\} \text {. }
$$

Ans den beiden letzten Formeln erlült man daher den Ausdruck:

(15) $A_{2}=M_{0} \frac{1+\lambda}{\lambda}\left\{1-\lambda \log \lambda+\frac{\lambda^{2}-1}{\lambda} \log (1+\lambda)\right\} \frac{\sin ^{2} S}{\sin ^{2} s_{0} \sin ^{2} \sigma_{0}}$,

welcher für $\lambda_{0}=1$ unmittelbar in der obigen Ausdruck in (14) iibergeht. Setzt man in $\langle 15\rangle \lambda=\frac{1}{2}$, so wird:

fur $\lambda=\frac{3}{4}$ wird:

$$
A_{2}=2.22 M_{0} \frac{\sin ^{2} S}{\sin ^{2} s_{0} \sin ^{2} \sigma_{0}} ;
$$

und fuir $\lambda=2$ wird:

$$
A_{2}=2.08 M_{0} \frac{\sin ^{2} S}{\sin ^{2} s_{0} \sin ^{2} \sigma_{0}}
$$

$$
\text { - } A_{2}=1.89 M_{0} \frac{\sin ^{2} S}{\sin ^{2} s_{0} \sin ^{2} \bar{\sigma}_{0}} .
$$

Man sieht also, dass die Werthe der Seeliger'schen Albedo zwar nicht sehr erheblich, aber doch immerhin merklich geändert werden, wenn man die Grösse $\lambda$ von $\frac{1}{2}$ bis 2 variiren lässt.

Es verdient noch erwähnt zu werden, dass die obigen Formehn (13) oder (14) anch benutzt werden können, um umgekehrt, wenn die Albedo eines Himmelskörpers und seine Helligkeit bekamut sind, den Durchmesser 
desselben zu berechnen. In der Praxis ist dies von Bedeutung bei der grossen Zahl der Asteroiden und bei den kleinen Planetenmonden, deren Durehmesser mit den gebräuehlichen Messungsmitteln der Astronomie nicht mit Sicherheit bestimmt werden können." Wir wollen annehmen, dass das Helligkeitsverhailtniss cines kleinen Planeten zu einem der Hauptplancten (beide zunächst in mittlerer Opposition gedacht) durch photometrisehe Bcobachtungen sicher bestimnt wäre; es möge mit $H_{0}$ bezcichnet sein. Wir wollen ferner das Verhältniss der Albedowerthe der beiden Gestirue $a$ nennen, die scheinbaren Halbmesser der Sonne, ron den beiden Planeten aus gesehen, mit $s_{1,0}$ resp. mit $s_{2,0}$ bezeichnen, ebenso die scheinbaren Radien der beiden Planeten, von der Erde aus gesehen, mit $\sigma_{1,0}$ resp. mit $\sigma_{2,0}$, so ergiebt sich ans den Formeln (14), wenn für beide Himmelskörper dasselbe Belenchtungsgesetz als gültig angenommen werden darf, zur Bestimmung der unbekannten Grösse $\sigma_{i, 0}$ dic Gleichnng:

$$
\sin ^{2} \sigma_{1,0}=\frac{H_{0}}{a} \frac{\sin ^{2} s_{2,0} \sin ^{2} \vec{\sigma}_{2,0}}{\sin ^{2} s_{1,0}}
$$

Ist das Helligkeitsverhältniss $H$ der beiden Planeten nicht für die mittleren Dppositionen, sondern fiir beliebige Stellungen derselben bekannt, wo ihre Phasenwinkel $a_{1}$ resp. c $\iota_{3}$ sein mögen, so erhält man statt der einen (rleichun! (16) die drei Gleichnngen:

17

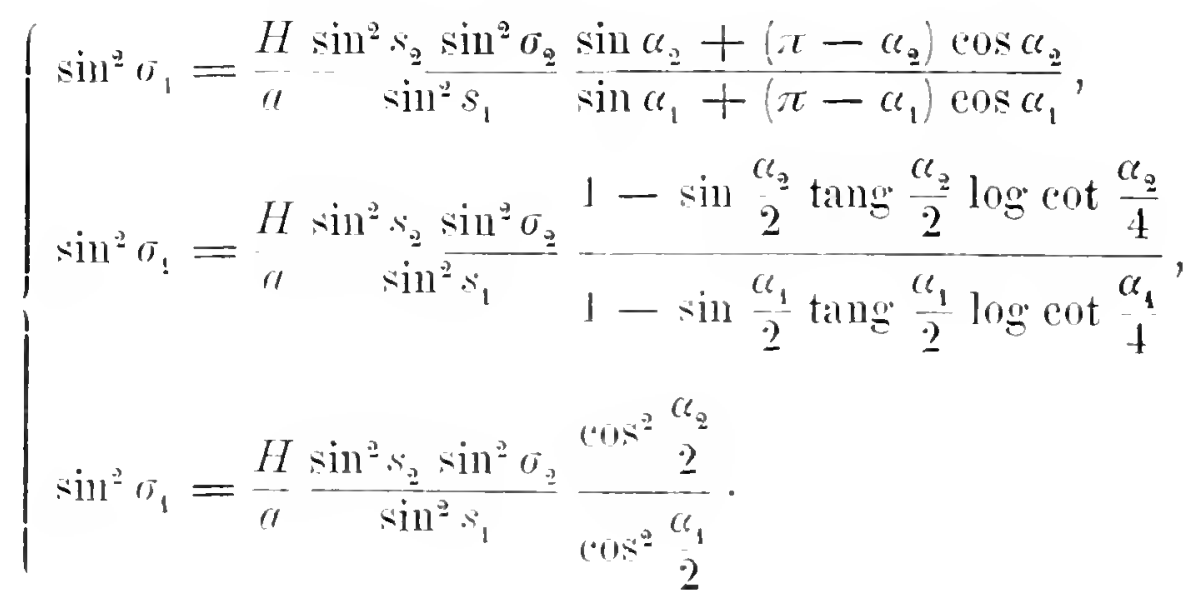

Yan findet also in diesem Falle verschiedene Dnrchmesserwerthe, je nach ilem lieleuchtungsgesetze, welches min der lierechmmug zu Grunde legt.

bei allen vorangehenden Betrathtnngen sind die Gestalten der Planeten als wilkonmen kngelfurmig voransesetzt worden, nud es tritt daher die Frage ant, in weleher Weise die entwickelten belenchtmosformeln modi-

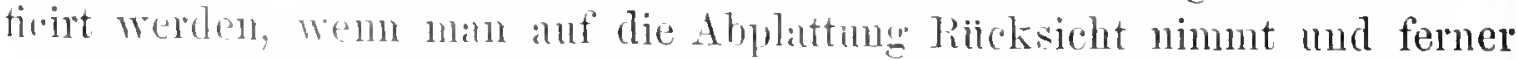

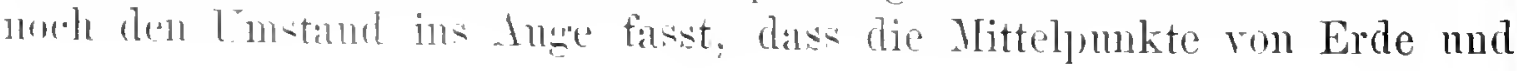


Sonne nicht genau in der Aequatorebene des Planeten liegen. Seeliger'y hat diese Anfgabe gelöst und die Belenchtungsformeln ganz allgemein anstatt fur die Kugel fur das Rotationsellipsoid entwickelt, wobei er sowohl das Lommel-Seeliger'sehe als das Lambert'sehe Beleuchtungsgesetz zu Grunde gelegt hat unter der vereinfachenden Annahme, dass die dritten und höheren Potenzen des Phasenwinkels ce vernachlässigt werden durfen. Diese Vereinfaehung ist bei den Verhältnissen in unserem Planetensystem im Allgemeinen durchaus statthaft, weil gerade bei denjenigen Planeten, die eine merkliehe Abweiehung von der Kugelgestalt zeigen, den Jupiter höchstens ausgenommen, die Phasenwinkel nur verhültnissmïssig kleine Werthe erreichen können.

Es ist nieht möglieh, den Gang der Seeliger'schen Untersuchung hier in voller Ausfihrliehkeit wiederzugeben; es muss daher auf die Originalabhandlung selbst verwiesen werden. Im Folgenden mögen nur die beiden Endformeln mitgetheilt werden, welche sich bei Berticksichtigung der ellipsoidisehen Gestalt austatt der beiden ersten Formeln (10) ergeben:

$$
\left\{\begin{array}{l}
Q_{1}=2 \pi J A_{1} \sin ^{2} s \sin ^{2} \sigma \cos \alpha\left\{P \cos ^{2} E+R \sin ^{2} E\right\} \\
Q_{9}=\frac{1}{2} \pi J A_{2} \sin ^{2} s \sin ^{2} \sigma\left\{1-\sin \frac{\alpha}{2} \operatorname{tang} \frac{\alpha}{2} \log \cot \frac{\alpha}{4}\right\} \sqrt{1+\frac{a^{2}-b^{2}}{b^{2}} \sin ^{2} E}
\end{array}\right.
$$

In diesen Formeln sind $a$ und $b$ die beiden Halbaxen des Planeten, unter $\sigma$ ist hier der scheinbare grosse Halbmesser $z$ verstehen, $P$ und $R$ sind zwei Grössen, welche uur von der Abplattung ablï̈ngen und deren Zahlenwerthe ans einer am Schlusse der Seeliger'schen Abhandlung mitgetheilten Tafel entnommen werden können, und $E$ ist der Erhebungswinkel der Erde ïber der Äquatorebene des Planeten.

\section{b. Die Lichtvertheilung anf einer Planetenscheibe.}

Wir kehren im Folgenden wieder zu der Ammahme zuriick, dass dic Gestalten der Planeten kugelförmig sind, und wollen nun noch einige Betrachtungen über die Lichtvertheilung auf einer erlenchteten Planetenoberfläche austellen. Es soll also nicht mehr, wie in Vorangehenden, die gesammte Lichtmenge, welehe die Planetenphase auf das Fermrohrobjectiv wirft, untersucht werden, sondern die Flächenhelligkeit an irgend einem Punkte der belenchteten Scheibe. Wemu es gelänge, dureh photometrische Messungen die scheinbare Helligkeit an jeder beliebigen Stelle der Oberfliche zu bestimmen, so wïrde man einerscits ein vortreffliches

1) Abhandl. der K. Bayer. Akad. der Wiss. II. Classe, Bd. 16, p. 405. 
Mittel hahen, die versehiedenen Beleuchtungsgesetze einer strengeren Prifing als bisher „u mnterwerfen, nnd andererseits würde die Möglichkeit gegelıen sein, iber dic Reflexionsfähigkeit an verschiedenen Punkten der Planetenoberfliichen und damit anch bis zu einem gewissen Grade liher die physische Beschaffenheit dieser Himmelskörper Aufsehluss zu erlangen. Leider ist es infolge der Schwierigkeiten, welche sich hauptsiichlich wegen der Kleinheit der Planetenscheiben und znm Theil auch wegen der Unvollkommenlieit der photometrischen Messungsmethodeu entgegenstellen, bisher nicht gelungen, branchbare Beobachtungen iiber die Lichtvertheilung anf einer Planctenoberflïche zu erhalten. Es ist hei den meisten Plimeten nicht einmal mit Sicherheit entschieden, an welchen Stellen der Oberflïche die grösste oder geringste Helligkeit stattfindet, geschweige denu, dass die Helligkeitsverhältnisse in Zahlenwertheu angegeben werden könuten, und selbst bei dem Monde, der doch in dieser Beziehmng weniger Schwierigkeiten bieten sollte, weichen die hisher ermittelten Angaben ïber die Helligkeitsverhältnisse von hellen und dunklen Stellen, ron Rand und Mittelpartien, ganz erheblich von cinander all.

Nit der theoretischen Seite der Frage hat sich vor einiger Zeit Anding heschäftigt und ist dabei zu einigen Resultaten gelangt, die der Hervorhehnng werth sind. Es handelt sich ganz allgemein um die Bestimmung der scheinbaren Helligkeit eines beliebig gelegenen Planetenoherflichenelementes. Nach unseren friiheren Definitionen wird die scheinbare llelligkeit $h$ eines Flïchenelementes $d s$ erhalten, wemn man die in der Richtmug \& von demselben ausgestrahlte Lichtquantitit $d q$ durch die scheinbare Grösse des Elementes, also durch $d s \cos \varepsilon$, dividirt. Diese lefinition gilt natiirlich sowohl fiir selbstlenclitende als zerstrent reflectirende Flichen. Zieht man wieder die drei rerschiedenen Belenchtung:sgesetze in Betralcht, wie sie durch die Formeln anf Seite 57 repräsentirt sind, so craicht sich die scheinbare Helligkeit eines Planetenoberflichenelementes ans den rileichungen:

$1 ! 1$

$$
\begin{cases}h_{1}=I_{1} \cos i & \text { (Limbertisches Gesetz) } \\ h_{2}=I_{2} \frac{\cos i}{\cos i+\cos c} & \text { (Lommel-Seeliger'sches Gesetz) }, \\ h_{3}=I_{3} \cos i & \text { (Enlerisches Gesetz) }\end{cases}
$$

whei ler binfachlecit wegen bei dem zweiten Gesetze wieder $\lambda=1$ mmenommon wroden ist. Fihnt man statt der Winkel $i$ und $\varepsilon$ die 
anf Seite 59 erkliirten Winkel 0 und $\psi$ vermittelst der Relationen $\cos i=\cos \psi \cos (\omega-(c)$ und $\cos \varepsilon=\cos \psi \cos \omega$ ein und substituirt fur $\Gamma_{1}, \Gamma_{2}, I_{3}$ die fruhoren Werthe, so gehen die obigen Gleichungen llber in:

$$
\left\{\begin{array}{l}
h_{1}=J A_{1} \sin ^{2} s \cos \psi \cos (\omega-\alpha), \\
h_{2}=J A_{2} \sin ^{2} s\left[\frac{1}{2}+\frac{1}{2} \operatorname{tang} \frac{\alpha}{2} \operatorname{tang}\left(\omega-\frac{\alpha}{2}\right)\right], \\
h_{3}=\frac{1}{2} J A_{3} \sin ^{2} s \cos \alpha\{1+\operatorname{tang}(\omega \operatorname{tang} \omega\} .
\end{array}\right.
$$

Ans diesen Formeln liisst sich sofort die theoretisch verlangte Lichtvertheiling bei voller Beleuchtung, also bei $\alpha=0$, thbersehen. Nach der zweiten und dritten Gleichung wird die scheiubare Helligkeit, abgesehen natlirlich ron Versehiedenheiten der Albedo, an allen Stellen der Planetenscheibe constant. Nach dem Lambert'schen Geset\%e wird dagegen die seheinbare Helligkeit proportional dem Werthe $\cos \psi \cos \omega$, sie nimmt also von der Mitte der Scheibe, wo $\psi$ und $\omega$ gleich Null sind, bestiindig nach dem Rande zu ab und wird in unmittelbarer Niihe des Randes, wo w nahe gleich $90^{\circ}$ ist, verschwindend klein. Nach dem blossen Aublicke einer voll beleuchteten Planetenseheilse zu urtheilen möchte man von vornherein geneigt sein, dem Lommel-Seeliger'schen und dem Euler'schen Gesetze vor dem Lambert'schen den Vor\%ug zu geben. Die scheinbare Helligkeit in der Mitte einer voll belenchteten Planetenseheibe, welche mit $h_{1}^{(0)}, h_{2}^{(0)}, h_{3}^{(0)}$ bezeichnet werden möge, wird, da $\psi$, ") und $\alpha$ in diesem Falle gleich Null sind, gegeben durch die Gleichungen:

$$
\left\{\begin{array}{l}
h_{1}^{(0)}=J A_{1} \sin ^{2} s, \\
h_{2}^{(0)}=\frac{1}{2} J A_{2} \sin ^{2} s, \\
h_{3}^{(0)}=\frac{1}{2} J_{3} \sin ^{2} s,
\end{array}\right.
$$

und wenn man diese Werthe in (20) substituirt, so erhiilt mam ganz allgemein die scheinbare Helligkeit in irgend einem Punkte der Planetenscheibe bei beliebiger Stellung von Sonne, Planet und Erde alusgedriickt im Verhältniss zur centralen scheimbaren Helligkeit in der Opposition. Man hat:

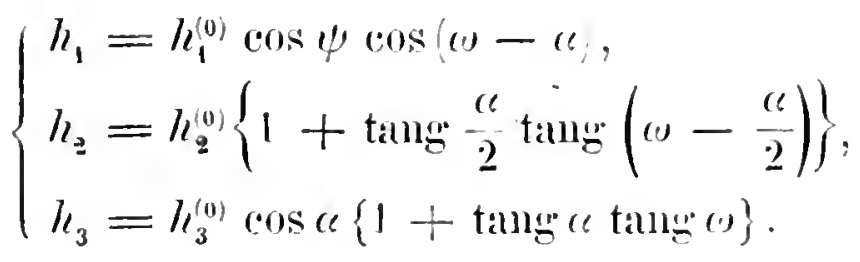

Aus diesen Gleichungen laisst sich unn in Bezmg anf die Helligkeitsrertheilung anf einer Planetenscheibe Folgendes ermitteh. Es stelle 
Figur 15 die theilweise beleuchtete seheinbare Planetenoberfläche dar, und der horizontale Durchmesser repräisentire den Durchsehnitt mit einer

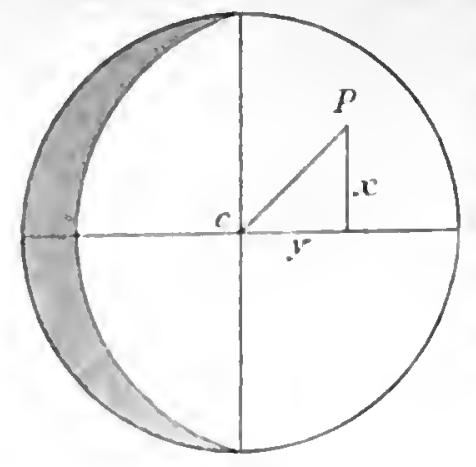

Fig. 15. senkrecht zur Papierebene gedachten Ebene, welchè die Mittelpunkte von Erde und Sonne (erstere senkreeht iiber dem Centrum c) enthalte. Sind dann $x$ und $y$ dic rechtwinkligen Coordinaten irgend eines Punktes $P$ anf der Planetenseheibe, so hat man (den Radius des Planeten gleich 1 geset $\%$ ):

$$
x=\sin \psi \quad \text { und } \quad y=\cos \psi \sin \omega .
$$

Substituirt man diese Werthe in die erste der obigen Gleichungen (22) und bezeichnet den Quotienten $\frac{h_{1}}{h_{1}^{(0)}}$ mit $a$, so ergiebt sich:

$$
r^{2} \cos ^{2} u+\eta^{2}-2 y a \sin u+\left(a^{2}-\cos ^{2} \alpha\right)=0 .
$$

Diese (ileichnng reprïscntirt den greometrisehen Ort aller Punkte der l'linetenscheibe, welche dieselbe Helligkeit $a$ besitzen. Es ist, wie man loicht sehen kilnn, die Gleichung einer Ellipse, deren kleine Axe in der obigen !-Axe liegt. deren ('entrum ron dem Mittelpunkte der Scheibe um lias Stick a sina entfernt ist nud deren Halbaxen die Werthe $\sqrt{1-a^{2}}$ und $\cos a \sqrt{1-a^{2}}$ haben. Die Curven gleicher Helligkeit auf einer l'lanetenscheibe sind also, falls dąs Lambert'sche Gesetz gilt, im Allيnemeinen Ellipsen mit rerschiedenen Mittelpunkten; auch die Axen haben verschiedene Werthe; $11 n$ ist das Axenrerhialtuiss bei allen Ellipseu ronstant gleich 1 : $\cos u$.

Fiir die roll belenchtete Scheibe, also für $a=0$, gehen die Ellipsen weicher Helligkeit in Kreise mit dem Radius $\sqrt{1-a^{2}}$ iiber, deren Mittelpunkte mit dem C'entrum der Scheibe zusammenfallen. Das Maximum der Helligkeit findet in Centrum statt. und die Helligkeit nimmt, wie shon when erwähnt wurde, nach allen Seiten sleichmässig ron der Mitte nath dem hiande $z$ alb. Fiir Werthe ron " zwischen 0 und $90^{\circ}$, also hoi molu als hall, belenditeter scheibe, findet das Maximum der Helligkeit nirlit in Centrum der scheibe statt, sondern, wie unmittelbar aus

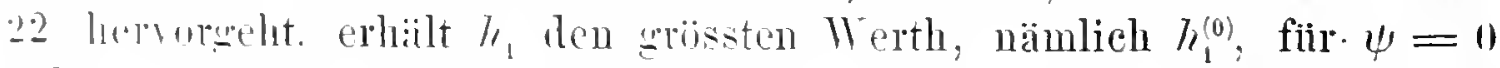
mul $"=$ ". A. h. also in demjenigen I'nukte der Scheibe, welcher dem sonkrent rom dor smne helenchteten Elemente der Oberflache entspricht.

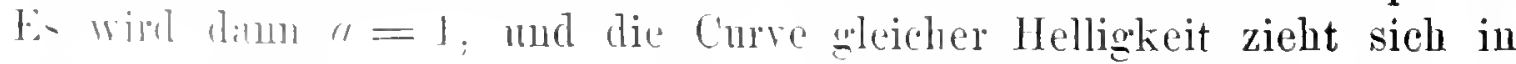

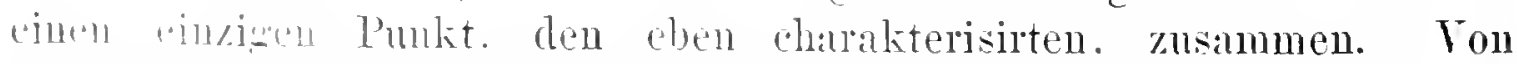

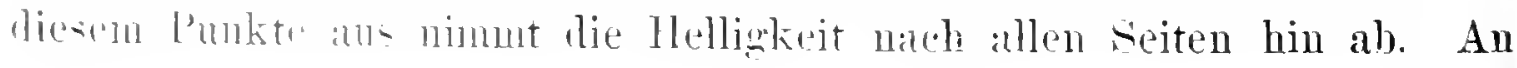


dem sogenaunten positiven, der Sonne zugewandten Rande (in unserer Figur dem reehten Rande) wird $\omega=90^{\circ}$, und es folgt daher fur einen l'unkt in der $y$-Axe aus (22) fitr $a$ der Werth since, die Helligkeit wird an diesem Rande nicht, wie bei voller Selieibe, gleich 0, und die Ellipse gleicher Helligkeit hat die Axen $\cos \alpha$ und $\cos ^{2} \alpha$; ihr Miftelpunkt ist von dem Centrum der Scheibe um die Streeke $\sin ^{2} \alpha$ entfernt. An dem uegativen Rande wird $\omega=-\left(\frac{\pi}{2}-\alpha\right)$, und daher wird dort nach (22) die Helligkeit gleich Null. Die Ellipse gleicher Helligkeit reducirt sich hier auf eine Halbellipse mit dem Mittelpunkt im Centrum der Scheibe und den Halbaxen 1 und $\cos c$, failt also mit dem negativen Rande selbst zusammen.

Ist \& gerade gleich $90^{\circ}$, ist also die beleuchtete Planetenscheibe ein Halbkreis, so wird $\cos \alpha=0$, die Ellipsen gleicher Helligkeit gehen, di die halben kleinen Axen derselben gleich Null werden, in gerade Linien ulber, die zur Beleuchtungsgrenze parallel sind. In der Beleuchtungsgrenze, dem negativen Rande selbst, ist die Helligkeit gleich Null, sie nimmt nach dem positiven Rande hin bestiindig zu und erreicht das Maxinum in diesem Rande, in dem Endpunkte der $y$-Axe. Wird endlich " noch grösser als $90^{\circ}$, so dass die beleuchtete Planetenscheibe dic Gestalt einer Sichel hat, so kommen nur Werthe von w zwischen $a-\frac{\pi}{2}$ und $\frac{\pi}{2}$ in Betracht; fiir den ersteren wird die Helligkeit gleich Null, und die Curve gleicher Helligkeit faillt also wieder mit dem von der Sonne abgewandten negativen Rande zusammen, für den letzteren wird die Grösse $a$ in Äquator gleich sin c, die Curve gleicher Helligkeit faillt aber nicht mit dem positiven Rande zusammen, sondern ist ein Stiick einer Halbellipse, deren Halbaxen $\cos \alpha$ und $\cos ^{2} \alpha$ sind, und deren Mittelpunkt in der Entfernung $\sin ^{2} a$ vom Centrum der vollständig gedachten Scheibe liegt. Die grösste Helligkeit auf der sichelförmigen Planetenscheibe erreicht, da $a$ nie grösser als $\sin \alpha$ werden kann, niemals den Werth 1; es ist also kein Punkt so hell, wie das Ceutrum der voll beleuchteten Scheibe. Im Allgemcinen folgt aus den Betrachtungen iiber die nach dem Lambert'schen Gesetze bei irgend einer Plalse stattfindende Helligkeitsvertheilung, dass nach dem negativen Rande hin die Helligkeit stets bis Null abnimmt, wïhrend nach dem positiven Rande $\%$, je nach der Gestalt der Phase, entweder Abnabme (aber nicht bis Null) oder Zunahme stattfindet; es wird infolge dessen der positive Rand stets schärfer begrenzt erscheinen müssen als der negative, eine Erscheinung, die allerdings, wie Anding in der erwähnten Schrift hervorgehoben hat, dureh die Bengung 
am Objectivrande des Fermrohrs erheblich modificirt und zwar zum Theil verwischt werden kann.

Wesentlich anders als nach dem Lambert'schen Gesetze ergiebt sich die Lichtvertheilung auf einer Planetenscheibe, wenn man das LommelSeeliger'sche Gesetz in Betracht zieht. Fiihrt man wieder die rechtwinkligen Coordinaten eines Punktes der Planetenscheibe durch die Relationen $x=\sin \psi$ und $y=\cos \psi \sin \omega$ ein und bezeichnet $\frac{h_{2}}{h_{2}^{(0)}}$ wieder mit $a$, so erhält mam aus der zweiten der Gleichungen (22) für den geometrischen Ort der P'unkte gleicher Helligkeit die Formel:

$$
x^{2}+y^{2}\left\{\frac{1+2 b \cos \alpha+b^{2}}{(1-b \cos \alpha)^{2}}\right\}=1,
$$

wo noch der Abkiirzung wegen $b$ eingefuihrt ist fiur die Grösse: $\frac{2-a}{a}$. Dies ist die Gleichmng einer Ellipse, deren Mittelpunkt im Centrum der scheibe liegt, deren grosse Halbaxe 1 mit der Verbindungslinie der Pole zusimmenfillt, und deren kleine Halbaxe gleich $\frac{1-b \cos a}{\sqrt{1-2 b \cos a+b^{2}}}$ ist. Dic Curen weicher Helligkeit sind also nach dem Lommel-Seeliger'schen Gesetze stets Halhellipsen, welche durch die Pole gehen. In Betreff der Liehtrertheilung anf der Scheibe ergieht sich aus der zweiten Gleichung 22 ummittellsar, diass bei $a=0$ für alle Werthe ron $\omega$ die scheinbare

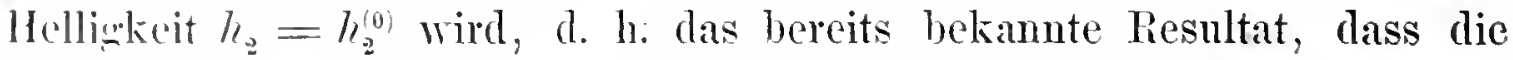
voll belenthtete Scheibe in allen Punkten dieselbe Helligkeit besitzt. Bei mehr als halb belenchteter Scheibe, also bei Werthen ron a zwischen 0 und $90^{\circ}$, kommen für $"$ alle Werthe zwischen $-\left(\frac{\pi}{2}-a\right)$ und $+\frac{\pi}{2}$ in Betracht, mud die Helligkeit nimmt von 0 am negatiren Rande bis zu dem Werthe $h_{z}=2 h_{z}^{0}$ am positiven Rande contimuirlich zu; die Helligkrit in centrmm der Scheibe ist gleich $h_{a}^{(0)}\left(1-\operatorname{tang}^{2} \frac{c}{2}\right)$. Anch bei halh belenthteter Scheibe und bei sichelformiger Gestalt ist die Helligkeit am nerativen liande stets gleich Null und am positiven Rande $=2 h_{a}^{(0)}$. Die Lichtrertheilmm, mach dem Lommel-Seeliger schen Gesetze unterscheidet

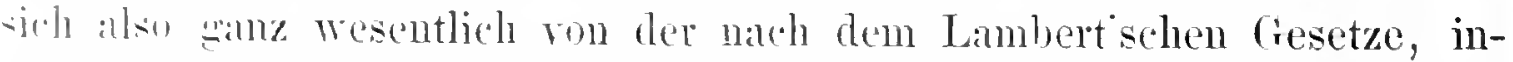
when dis Maximum der Helligkeit stets am pusitiven Ratinde liegt und lxidlun I'hliken denselben Werth, nimlich den doppelten Betrag der

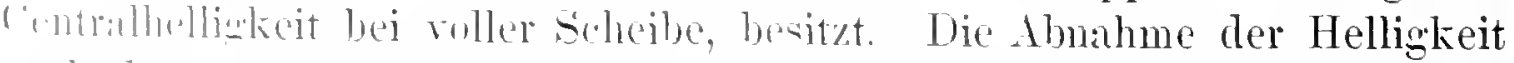

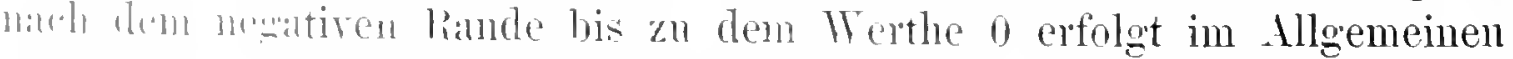

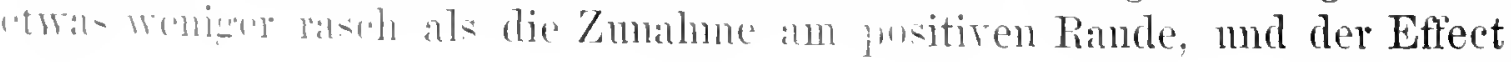


davon ist, dass der erstere verwaschen, ler letatere dagegren scharf begrenzt enseheint. Der Untersehied in dem Aussehen der heiden Ränder muss noch deutlieher in's Auge fallen, als bei der Helligkeitsvertheilung nach dein Lambert'sclien Gesetze.

Was endlich das dritte Beleuehtungggeset\% anbelangt, $80^{\circ}$ ergiebt die Substitution der Werthe von $x$ und y in die letzte der Formeln (22) fur den geometrischen Ort der Punkte gleicher Helligkeit die Gleiehung:

$$
x^{2}+y^{2} \frac{1-2 a \cos \alpha+a^{2}}{(u-\cos \alpha)^{2}}=1 \text {, }
$$

welche wieder einer Ellipse nit den Halbaxen 1 und $\frac{a-\cos \alpha}{\sqrt{1-2 a \cos a+a^{9}}}$ angehört, deren Mittelpunkt im Coordinatenanfange liegrt. Die Lichtvertheilung auf der Seheibe selbst ist bei voller Beleuchtung dieselbe wie nach dem Lommel-Seeliger'sehen Gesetze, d. h. es haben alle Punkte die constante Helligkeit $h_{3}=h_{3}^{(0)}$. Bei allen auderen Phasen nimmt auch hier, wie aus der Betrachtung der Gleichung (22) hervorgeht, dic Helligkeit nach dem negativen Rande zu beständig al und ist längrs eines unendlich schmalen Streifens verschwindend klein, dagegen wiichst dic Helligkeit nach dem positiven Rande hin beträchtlich stairker an als nach dem Lommel-Seeliger'sehen Gesetze, sie wird sogar an Rande selbst lïngs eines unendlich sehmalen Streifens bei allen Phasen muendlich gross. Die beiden Ränder missten also nach dem Euler'schen Gesetze am meisten von einander versehieden erscheinen, und es ist wohl schon bei einer gan\% flichtigen Betrachtung der Planetenoberflaichen einleuchtend, dass dic durch das Euler'sehe Gesetz in Bezng auf die Lichtvertheihmg verlangten Verhältnisse in Wirklichkeit nicht vorhanden sind.

Selbstverstiandlich dauf nicht ausser Acht grelassen werden, dass die Anordnung der Helligkeit auf einer Planetenscheibe, wie sie sich nach dem Vorangehenden mit Zugrumdelegrnng der versehiedenen Beleuchtumgsresetze ergiebt, mur für den idealen Fall grilt, dass die Oberflaiche eine gleichmässig rauhe ist und an allen Punkten dieselbe Reflexionsfilhigkeit besitzt. In Wirklichkeit werden die Verhältnisse gan\% wesentlich modificirt, und zwar einmal dureh das Vorhandensein einer mehr oder weniger dichten Atmosphäre, damn durch die verschiedenen Albedowerthe, welche zwcifellos den einzelnen Partien einer Planetenoberflaiche zukommen, und cndlich wicht zum Wenigsten durch Erhebungen, welche infolgre des Schattenwurfes ganz besondere Erscheinungen hervornfen.

Von der Wirkung der Atmosphäre auf das Aussehen versehiedener Stellen der Planetenseheilse kamn man sich nur eine mgefihre Vorstellung. machen, wemn man nicht im stande ist, den Grad ilner Diehtugkeit in 
Rechmung zn bringen. Im Allgemeinen wird bei roller Beleuehtung ein Abnehmen der Mlelligkeit von der Mitte nach dem Rande hin zu erwarten sein; es kinn also durch die Atmosphiire derselbe Effect hervorgebracht werden, der sich nach dem Lambert'sehen Gesetze anch ohne Vorhandensein eimer Atmosphiare erklïren liisst. Bei nieht voll belenchteter Seheibe wiirde sich dir Mirkung der Atmosphüre in der Weise äussem muissen, dass der von der Somme alhgewandte Rand verwasehen und undeutlich erseheint, während der positim liand seharf hegrenzt ist. Die ron den sammtlichen Belench-

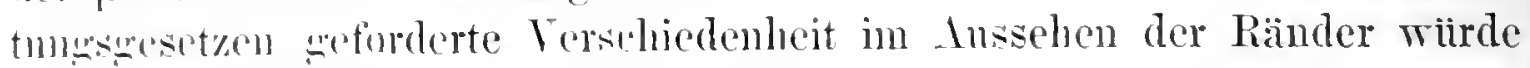

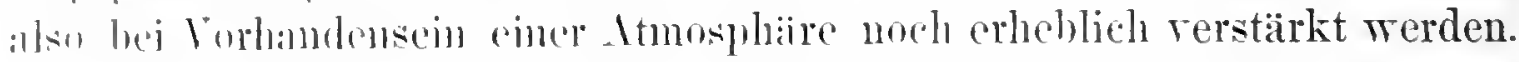

(i:m\% nuncontmolirbar ist natiolich der Intheil, welehen die rersehiedene

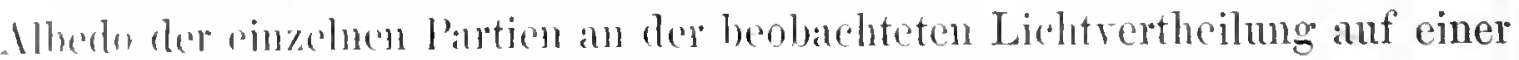

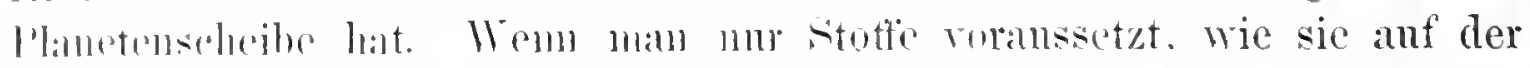

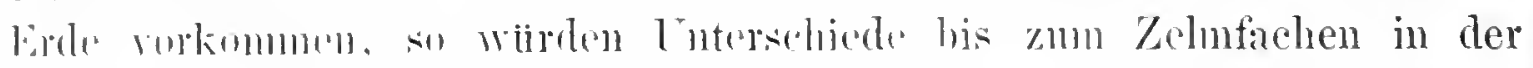

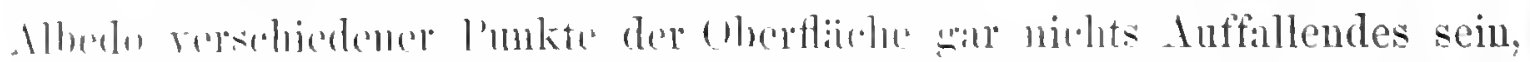

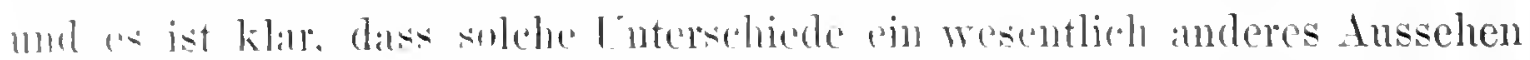

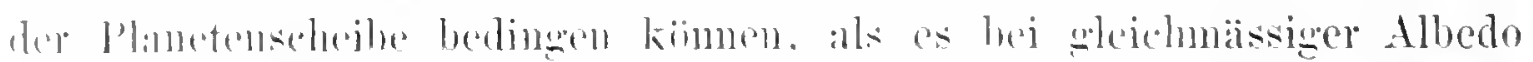

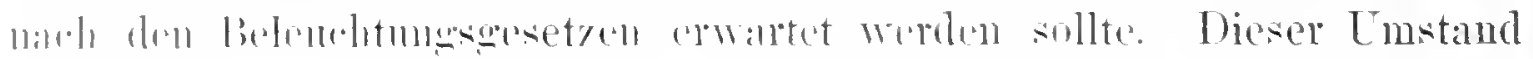

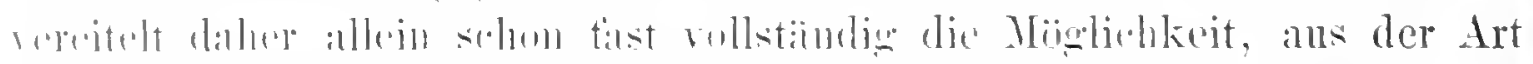

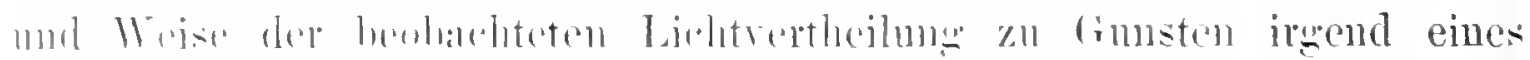

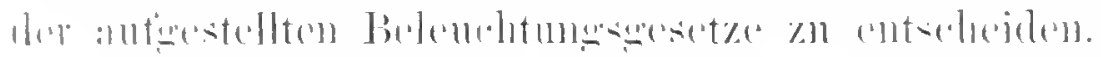

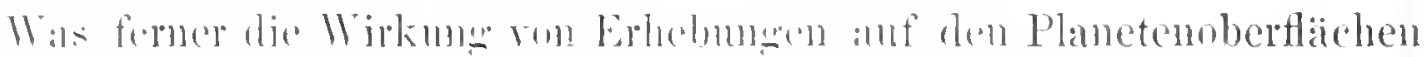

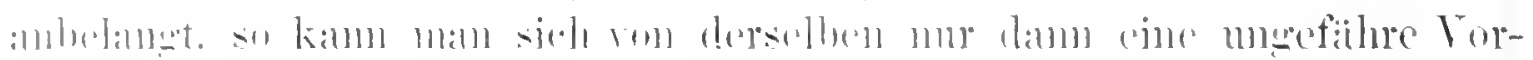

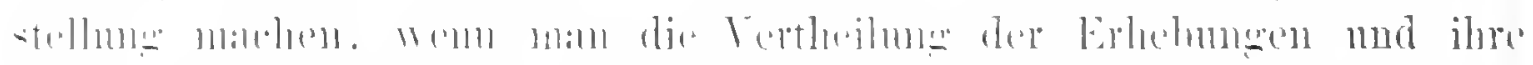

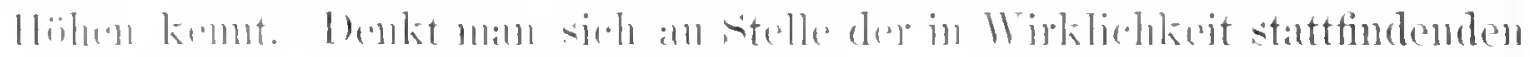

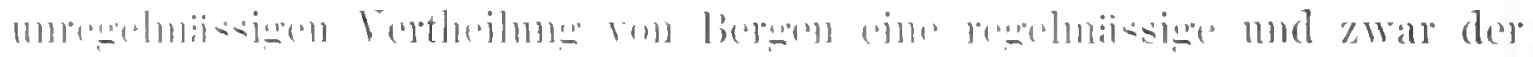

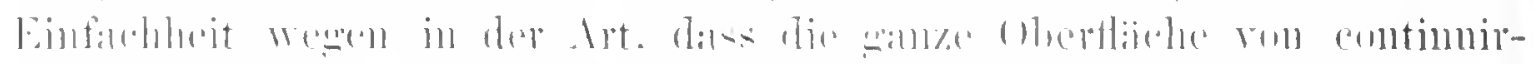

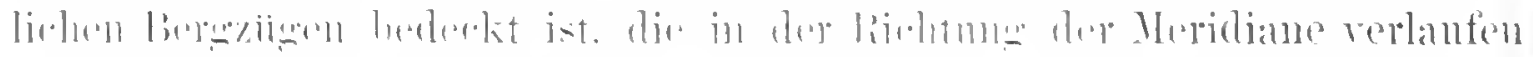

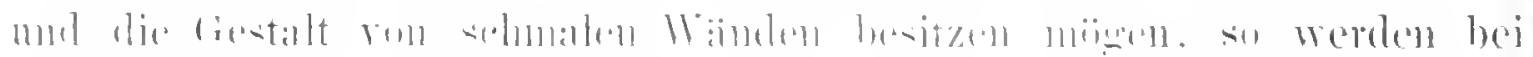

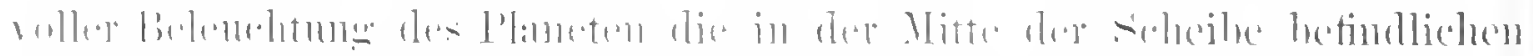

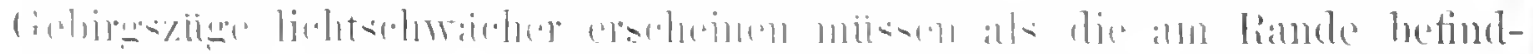

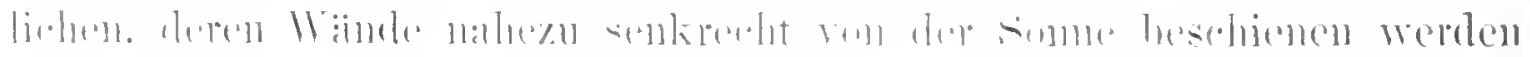

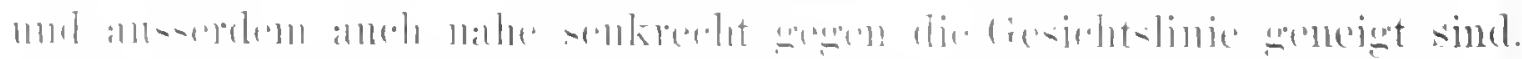

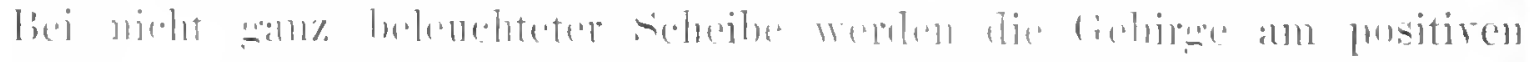

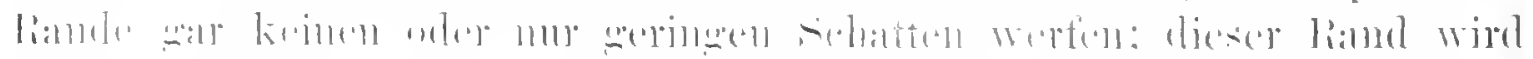

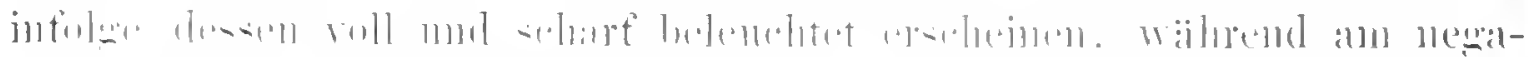

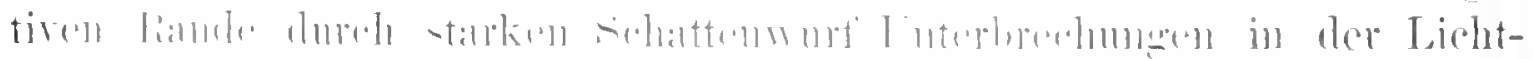

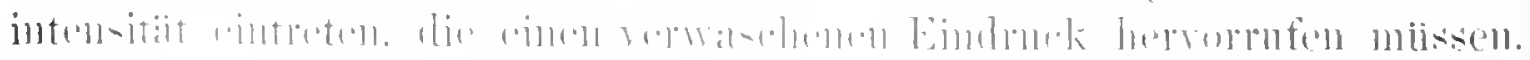

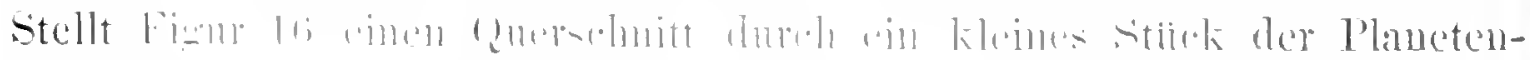

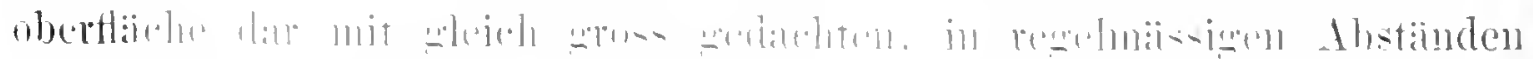

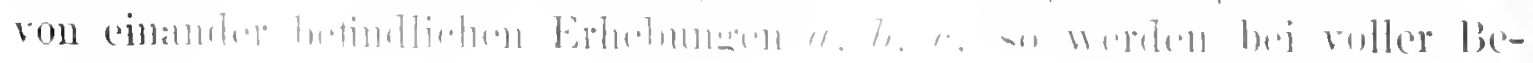


leuchtung des Planeten sowohl die beiden Seitenwiinde jeder Erhebung als auch die zwischen je zweien derselben befindlichen Vertiefungen Licht nach der in der Riehtung $E$ stehenden Erde senden. Ist die Sonne dagegen in $S$, so erhalten die linken Seitenwände tiberhaupt_kein Lieht von derselben, und aueh die Vertiefungen erseheinen finster, weil sie «um Theil oder gan\% im Sehatten der benaehbarten Erhebungen liegen. Je hoher die Berge sind und je geringer die $\mathrm{Ab}$ stilnde zwisehen ihnen, desto merklicher wird schou beieiner geringen Entfernung von der Opposition die beobachtete Liehtrerminderung sein. Dieser Umstand ist nicht unwielitig, weil bei einigen kleinen Planeten ein merkliehes Anwaehsen der Gesammthelligkeit unmittelbar vor der Opposi-

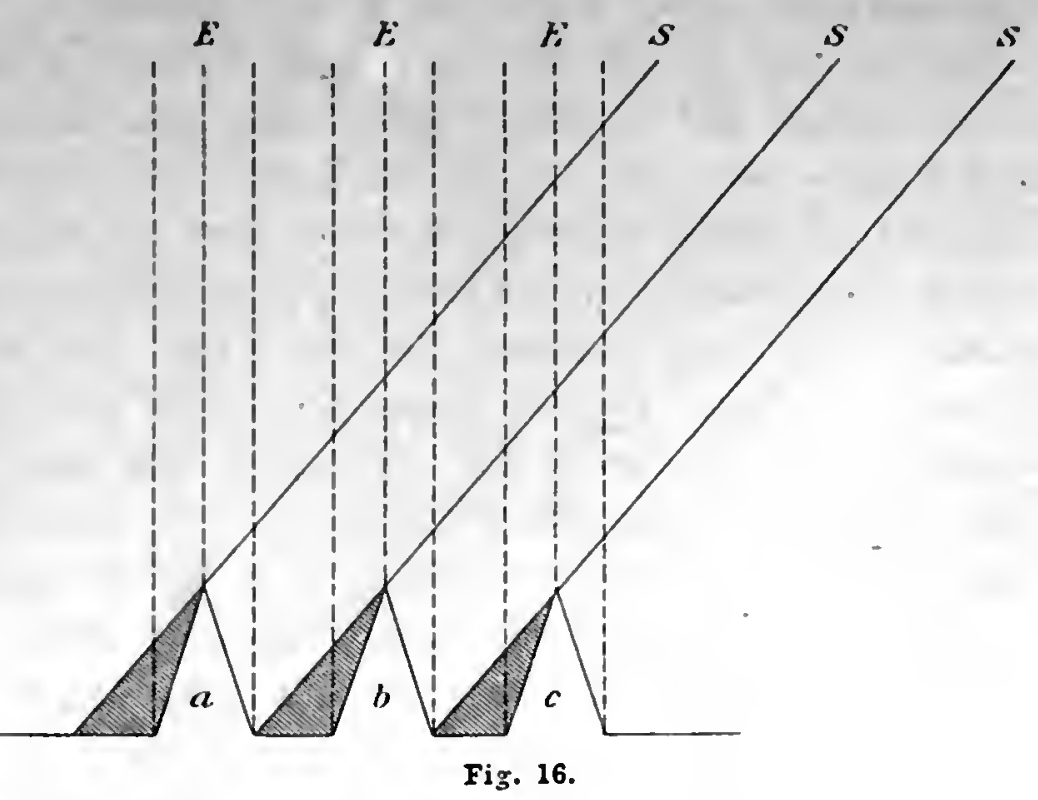
tion und ebenso eine Abnahme nach derselben in den beobachteten Helligkeiten angedeutet zu sein scheint.

Es ist wiederholt die Frage anfgeworfen worden, ob es nicht möglich wäre, unter gewissen plansibelen Amahmen iber die Anordnung und die Grössenverhältnisse der Erhebungen ạf einer Planetenoberfliche mit Benutzung der bekannten Beleuehtungsgesetze einen theoretisehen Ausdruck fuir die von den Phasen einer Planetenkugel ausgesandte Gesammtliehtmenge abzuleiten. Mit Riicksicht auf die complicirten Terhiiltnisse, welche sich darbieten, könnte man diese Frage von vornherein remeinen; es ist aber bemerkenswerth, dass ein Versueh zur Lösumg der Anfgabe hereits geemacht worden ist und zwar ron Zöllner in dem zweiten Absehnitt seiner „Photometrischen Untersuchungen \&, weleher die Überschrift trägt »Theorie der relativen Liehtstairke der Mondphasen «. Wem der Zöllner'sche Versuch auch als verfehit zu bezeichnen ist, weil die vou ihm gemachiten Voranssetzungen schwerlich aceeptirt werden können, und ansserden, wie ron mehreren Seiten nachgewiesen worden ist, in scinen mathematisehen Entwicklungen Fehler enthalten sind, so rerdient derselbe doch noch an dieser Stelle eine kurze Besprechung.

Zölluer wendet durchweg das Lamhert sche Beleuchtungsgesetz an und gelit bei seinen Untersuchumgen ron einem streng beweisbaren Satze 
aus, den er in der folgenden Form ausspricht: „Die Erleuchtung eines auf der Erde gelegenen Fläichenelementes durch die Phasen der als homogen und kugelförmig angenommenen Mondoberfläche bleibt dieselbe, wenn die Mondkugel dureh einen homogenen Kreiscylinder ersetzt wird, dessen Axe senkrecht zu der durch Sonne, Erde und ihn selber gelegten Ebcne steht und dessen Höhe sich zu dem, dem Monddurchmesser gleichen, Durehmesser seiner Basis wie $2 \mathrm{zu} 3$ verhält." Diesen an und für sich riehtigen Satz glaubt Zöllner auch in dem Falle anwenden zu dürfeu, wenu Kugel und Cylinder nicht eine gleichmässig rauhe, sondern mit Erhebungen bedeckte Oberfläche haben. Er sagt: "Indem man nun den Einfluss zu crmitteh sucht, welchen eine regelmässige Vertheilung von schattenwerfenden Körpern auf das Phasencrlenchtungsgesetz eines Cylinders ausiilot, kann man jederzeit anf der Kugcl eine solche unregelmässige Vertheilung jener Körper amnehmen, dass sowohl für den Cylinder mit regelmïissiger als anch fuir die Kugel mit umregelmässiger Vertheilung. vou Erhebungen dasselbe Phasenerlenchtnngsgesetz stattfindet." Er glaubt daher fiir die irgendwie mit Bergen bedeckte Mondoberfläehe einen regelmässig cannclirten Cylinder substitniren zu diurfen, dessen Furchen durch je zwei Ebenen gebildet werlen, die unter einem gewissen Winkel gegen einimder geneigt sind und sich in einer zur Cylinderaxe parallelen Kante schneiden. Es liegt auf der Hand, dass die Bereehtigung zu dieser Substitution strenger dargethan werden miisste, md man wird sehwerlich dem Siltze zustimmen kömen, mit dem Zöllner die einleitenden Betrachtungen zu seiner Mondtheorie schliesst: » Die befriedigende Übereinstimmung der anf diese Weise entwickelten Theorie mit den Beobachtungen wird zeigen, dass man zu den bei ihr gemachten Voraussetzungen berechtigt war. *

Inter der weiteren Annilume, dass anf dem ramehirten Cylinder die Anzahll der Erhebmgen nuendlich gross ist und die Höhe derselben im Verhältnisse zn deu Dimensionen des Crlinders sehr klein, ist nun Zöllner zu einer sehr einfachen Formel für die ron den Phasen eines solehen Cylinders reflectirte Lichtmenge $L$ gelangt. Bezeichnet nämlich $\beta$ den Winkel, wekchen die seitenfliichen der einzehen Erhebungen mit ihrer Basis bilden, und wirl nach der Lambert schen Schreibweise statt des Phisenwinkels a das supplement desselben $1=150^{\circ}-a$ eingefiihrt, so lautet die Zölhnersche Formel:

$$
L=\gamma\{\sin \langle\gamma-\beta-(\gamma-\beta) \cos r-\beta\rangle\},
$$

wo $\gamma$ eine Constante ist, die ron der Lendelitkraft der somne, ron den Dimensionen des Cylinders, ron seiner Allsedo. endlich noch ron den Entfermugen desselhen ron some und Erde abhängt. Fiir $\beta=0$ geht die Gleichung umnittelbal in die hekannte Lambort solhe Belenchtunges- 
formel tuber. Zöllner hat nun gezeigt, dass die von ihm zwischen Vollmond und Quadratur angestellten photometrischen Mondbeobachtungen dnreh die obige Formel genttgend dargestellt werden, wenn man für $\beta$ den Werth $52^{\circ}$ annimmt, und glaubt in der Übereinstimmung seiner Theorie mit den. Beobachtungen den Beweis zu erblicken, dass dic bei den thenretischen Entwicklungen vorausgesetzten Einflusse in der That anf dem Monde vorhanden sind, wenn er aueh vorsiehtiger Weise bemerkt, dass man sich hiiten mlisse, der Constanten p̈ hinsichtlich ihrer physisehen Bedeutung einen allzn grossen Werth beizulegen.

Nenerdings ist von Searle 1) und Seeliger²) ubereinstimmend nachgewiesen worden, dass die mathematischen Entwicklungen Zöllners einen Irrthum enthalten, insofern bei den vorkommenden Integrationen unrichtige Grenzen zur Anwendung gekommen sind. Infolge dessen gilt die obige Zöllner'sche Formel nicht unumschränkt, sondern nur für eil gan\% bestimmtes Phasenintervall. Es sind nämlich bei der Behandlung des Problems die beiden Fïlle zu unterscheiden, wo $v<2 \beta$ und wo $v>2 \beta$ ist. Nach den Entwicklnngen Searles lautet die Formel:

$$
L=\gamma 2 \cos \beta[\sin (v-\beta)-(v-\beta) \cos (v-\beta)],
$$

wenn $v<2 \beta$ ist; dagegen :

$$
L=\gamma[\sin v-v \cos v-2 \beta \sin \beta \sin (\gamma-\beta)],
$$

wenn $v>2 \beta$ ist. Da die Züllner'sehen Beobachtungen nur bei Werthen von $v$ zwischen $110^{\circ}$ und $180^{\circ}$ angestellt sind, so hiitte bei der Vergleichung mit der Theorie nur die zweite der obigen Formeln zur Anwendung kommen diirfen. Wenn also Zöllner trotzdem mit Benutzung: der in seinem Falle unrichtigen ersten Formel die Beobachtungen befriedigend dargestellt hat, so beweist dies nur, dass die Formel weiter nichts als eine branchbare Interpolationsformel ist, dass ihr aber eine physikalische Bedeutung nuter keinen Umständen zuerkannt werden darf.

Seeliger hat noch darauf hingewiesen, dass die Zijllner'sche Annahme einer unendlich grossen Zahl ron sehr wenig tiefen Caniilen eigentlich nur einer Hypothese über die Oberfläehenbeschaffenheit des Cylinders in seinen kleinsten Theilen gleichkomme, und diss daher das Zöllner'sche Resultat auf dasselbe hinauslaufe, als wenn man irgend ein beliebiges nicht näher zu definirendes photometrisches Hauptgeset\% zu Grunde gelegt hätte; die Züllner'sehe Formel hätte schon deshalb

1) Proc. of the Amer. Acad. of arts and sciences. Vol. 19, 1884 , p. 310.

2) Vierteljahrsschrift der Astr. Gesellsch. Jahrg. 21, 1886. p. 216. 
keinen anderen Werth, als den einer einfachen Interpolationsformel. So interessant und anregend in gewisser Beziehung die Zöllner'schen Untersuchungen zweifellos sind, so wird man nach dem Gesagten doch zu dem Schlnsse kommen, dass Zöllner sich umsonst an ein Problem gewagt hat, dessen strenge Lösung aus den verschiedensten Gründen ïberhaupt nicht möglieh ist.

\section{c. Mittlere sclieinbare Helligkeit eines Planeten.}

In den meisten Lehrbüchern der Photometrie, besonders in dem Lalmbert'schen Werke und den sich eng an dasselbe anschliessenden Schriften ron Beer und Rheinauer, ist der Bereehnung der seheinbaren mittleren Helligkeit einer Planeteuphase ein grösserer Platz eingeräumt worden, als diesclbe verdient, weil diese Grösse eine nur in der Vorstellung beruliende ist, die mit directen Beobachtungen niemals verglichen werden kaum. Uns interessirt an den Planeten eigentlich nur die in muscre Instrunente oder in das Ange gelangende, von der Planetenphase herkommende gesammte Lichtmenge und ferner die Vertheilung der Helligkeit in den einzelnen Punkten der siclitbaren hellen Scheibe, dagegen hat die Angabe ciner mittleren scheinbaren Helligkeit so gut wie gar keinen \%weck, zmmal wir dieselbe nur unter der zweifellos umrichtigen Amalme berechnen kïnnen, dass die Reflexionsfïhigkeit an allen Punkten der Planetenoberffiache denselben Werth hat. Es soll im Folgenden nur kury und mehr der Vollständigkeit wegen anf diesen Punkt eingegangen werden. Nach den Definitionen anf Seite $2 S$ versteht man unter der mittleren scheinharen Helligkeit einer lenehtenden (oder beleuchteten) Fliache das Verhïltniss der von der ganzen Flïche anf die Flächeneinheit (des Objectivs oder des Anges) scnkrecht gresandten Liehtquantität zu der scheinbaren Grösse dieser Flaiche. Betrachtet man in Figur 15, welche die seheinhare Flaiche eines l'laneten darstellt, den erlenchteten Theil, so besteht derselbe ans ainem Hablbreise mit dem Radius $\frac{O}{d}$ (wenu $g$ der wahre Halhmesser des Planeten und I seine Entferumng rou der Erde ist und ans ciner Halbellipse mit den Halbaxen $\frac{\varrho}{f}$ mod $\frac{\varrho}{d} \cos a$. Die rheinbare Grösse des erlenchteten Theiles ist daher ansuedriickt durch

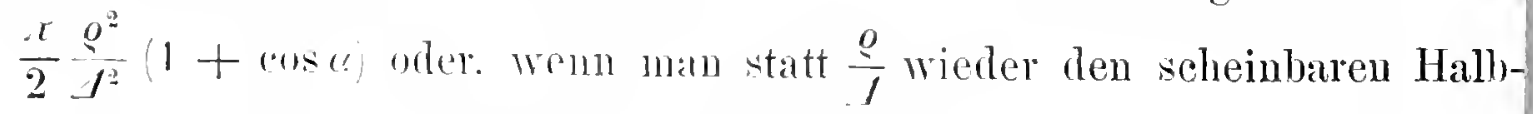
messer o einfiuhrt, durch $\frac{1}{2} \sin ^{2} \sigma 11+$ cos a). Dividirt man mit diesem Werthe in die friilieren Formeh (10), wetche fiu die rerschiedenen Be- 
leuchtnngsgesetze die zur Erde gesandten Lichtquautitaten ansdrucken, so erhält man unmittelbar die gesuchten mittleren scheinbaron Helligkeiten:

(23)

$$
\left\{\begin{array}{l}
H_{\mathrm{i}}=\frac{1}{3} A_{1} J \sin ^{2} s \frac{\sin \alpha+(\pi-\alpha) \cos \alpha}{\pi(1+\cos \alpha)}, \\
H_{2}=A_{2} J \sin ^{2} s \frac{1-\sin \frac{\alpha}{2} \operatorname{tang} \frac{\alpha}{2} \log \cot \frac{\alpha}{4}}{1+\cos \alpha} \\
H_{3}=\frac{1}{2} A_{3} J \sin ^{2} s .
\end{array}\right.
$$

Bemerkenswerth ist, dass wach dem Enler'sehen Gesetze die mittlere scheinbare Helligkeit rom Phasenwinkel ganz unabhängig wird, mithin bei allen Beleuchtungsphasen constant bleibt. Fur voll belenchtete Planetenscheiben erhält man die mittleren scheinbaren Helligkeiten aus den Gleichungen:

$$
\left\{\begin{array}{l}
H_{1}^{(0)}=\frac{2}{3} A_{1} J \sin ^{2} s \\
H_{0}^{(0)}=\frac{1}{2} A_{2} J \sin ^{2} s \\
H_{3}^{(0)}=\frac{1}{2} A_{3} J \sin ^{2} s .
\end{array}\right.
$$

Man sieht, dass die Werthe von $H_{2}^{(0)}$ und $H_{3}^{(0)}$ unit den entsprechenden Insdricken in den Gleichungen (21) ibereinstimmen, was anch ohne Weiteres zn erwarten ist, weil, wie wir gesehen haben, nach dem zweiten und dritten Beleuchtungsgesetze die scheinbare Helligkeit in allen Punkten der voll belenchteten Seheibe die gleiche sein muss.

\section{d. Beleuchtung der Planetentrabanten.}

Wenn man die Lichtguantitiit berechnen will, welche von einem Planetentrabanten bei beliebiger Stellung von Somne, Erde, Planet und Satellit nach der Erde gesandt wird, so ist zn beachten, dass diese Lichtmenge sich ans zwei Theilen zusammensetzt, erstens aus dem direct von dem Trabanten zuriiekgeworfenen Sonnenlichte und zweitens ans denjenigen Lichte, welehes vom Planeten selbst nach seinem Satelliten und von diesem wieder nach der Erde refleetirt wird. Der \%weite Theil ist im Verhältnisse zum ersten ausserordentlich geringfiigig und wird in der Praxis bei photometrisehen Messungen kanum merklich sein; indessen bietet die theoretisehe Behandlung des Falles doch ein gewisses Interesse.

Der Einfachheit wegen soll rorausgesetzt werden, dass die Mittelpunkte der vier in Betraeht kommenden Himmelskörper alle in einer und derselben Ehene liegen; ferner sollen die Dimensionen derselben im Verhültnisse zu den Entfernungen als sehr klein angenommen werden. Wir wollen der Berechnung zun:ichst das Lambert sche Belenehtungsgesetz zu Grunde legen. Ist $q$ ' das direct ron der 'Trabantenphase reflectirte 
Somnenlicht, $q^{\prime \prime}$ das rom Planeten auf den Trabanten iibergehende und von diesem wieder nach der Erde gesandte Licht, so wird die Gesammtmenge $Q_{1}$, welche die Flächeneinheit des Fermrohrobjectivs senkrecht von der Trabantenpliase erhïlt, ansgedriickt durch $Q_{1}=q^{\prime}+q^{\prime \prime}$. Es sei:

a der Phasenwinkel des Trabanten,

$A_{1}$ die Albedo des Trabanten,

$\sigma$ der schcinbare Halbmesser des Trabanten, von der Erde aus gresehen,

s der scheinbare Halbmesser der Sonne, rom Trabanteu aus gesehen, damn erhailt man $q$ ' unmittelbar aus der ersten der Gleichungen (10). Ls ist:

$$
q^{\prime}=\frac{2}{3} J A_{1} \sin ^{2} s \sin ^{2} \sigma[\sin \alpha+(\pi-\alpha) \cos \alpha] .
$$

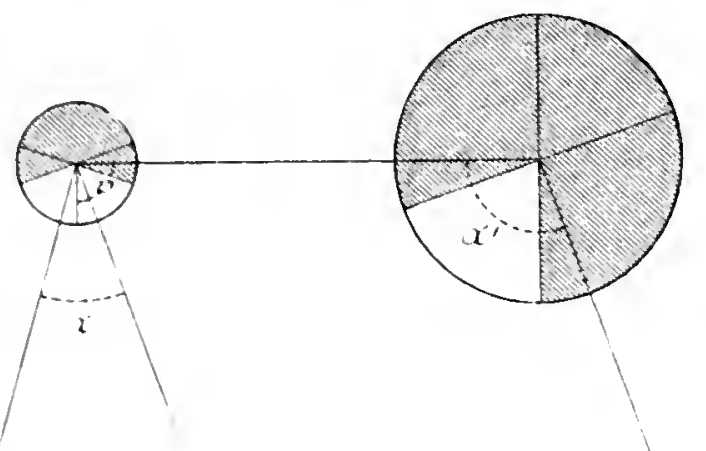

$E$

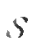

Fig. 17 .

$$
d q=\frac{2}{3} J A_{1}^{\prime} \sin ^{2} s^{\prime} \sin ^{2} \sigma^{\prime}\left[\sin \alpha^{\prime}+\left(x-\alpha^{\prime}\right) \cos \alpha^{\prime}\right] d s .
$$

Dabei ist:

a' der I'hasenwinkel des Planeten in Bezng auf den Trabanten,

$A_{i}^{\prime}$ die Albedo des Planeten.

$\sigma^{\prime}$ der scheinbare Halbmesser des Planeten, rom Trabanten ans gesehen,

ter scheinbare Hallmesser der Somne, rom Planeten aus gesehen.

Xach den hai der Mbleitmng des Lambertschen Belenchtungsgesetzes angestellten Betrachtungen 'Seite 40 ist num die Lichtquantität $d q^{\prime \prime}$, welche ein beliebing welegenes Trabuntenelement ds ron dem empfangenen Lichte 
wieder nach der Flaieheneinheit auf der Erde senkreeht sendet, gegeben durch die Gleichung:

$$
d q^{\prime \prime}=\frac{A_{1}}{d^{z} \pi} d q \cos i^{\prime} \cos \varepsilon,
$$

wo $\Delta$ die Entfernung des Trabanten ron der Erde, $i^{\prime}$ der Incidenzwinkel der als parallel vorausgesety.ten, rom Planeten auf das Trabantenelement gelangenden Liehtstrahlen und $\varepsilon$ der Emanationswinkel am Elemente $d s$ ist. Un die gesammte Liehtmenge $\bar{q}^{\prime \prime}$ zu haben, ist zu integriren den rom Planeten beleuchteten, von der Erde aus sichtbaren Theil der Trabantenkugel, der in der obigen Figur dureh den Winkel $v$ bezeichnet ist. Ersetzt man $i^{\prime}$ und $\varepsilon$, wie frilher, dureh die Winkel $\omega$ und $\psi$, und rechnet die Liingen $\omega$ von demjenigen Punkte der Trabantenscheibe, liber welehem die Erde senkreeht steht, und die Breiten $\psi$ ron der durch die Vittelpunkte der vier Himmelskörper gehenden Ebene, so hat man die Relationen:

$$
\begin{aligned}
\cos i^{\prime} & =\cos \psi \cos \left[150^{\circ}-\left(\alpha^{\prime}-\alpha\right)-\omega\right], \\
\cos \varepsilon & =\cos \psi \cos \omega, \\
d s & =\varrho^{2} \cos \psi d \omega d \psi,
\end{aligned}
$$

wo noch $\varrho$ der walıre Halbmesser des Trabanten ist.

Die Integrationsgrenzen in Bezug auf $\psi$ sind $-\frac{\pi}{2}$ und $+\frac{\pi}{2}$, in Bezug anf $\omega$ sind dieselben $\frac{\pi}{2}-v$ und $\frac{\pi}{2}$ oder, da $\iota=\alpha^{\prime}-\alpha$ ist, $\frac{\pi}{2}-\left(a^{\prime}-a\right)$ und $\frac{\pi}{2}$.

Substituirt man die Werthe ron $\cos i^{\prime}, \cos \varepsilon, d q$ und $d s$, so erhiilt man endlich:

$$
\begin{gathered}
q^{\prime \prime}=\frac{2}{3 \pi} J A_{1} A_{1}^{\prime} \frac{g^{2}}{d^{2}} \sin ^{2} s^{\prime} \sin ^{2} \sigma^{\prime}\left[\sin \alpha^{\prime}+\left(\pi-\alpha^{\prime}\right) \cos \alpha^{\prime}\right] \int_{-\frac{\pi}{2}}^{\frac{\pi}{2}} \cos ^{3} \psi d \psi \times \\
\int_{\frac{\pi}{2}-\left(c^{\prime}-\alpha\right)}^{\frac{\pi}{2}} \cos \omega \cos \left[180^{\circ}-\left(\alpha^{\prime}-(\omega)-\omega\right] d(\omega) .\right.
\end{gathered}
$$

Beachtet man noch, dass $\frac{\varrho}{d}=\sin \sigma$ ist, so hat man nach Ausfiuhrung der Integrationen:

$$
\begin{array}{r}
q^{\prime \prime}=\frac{4}{9 \pi} J A_{1} A_{1}^{\prime} \sin ^{2} s^{\prime} \sin ^{2} \sigma \sin ^{2} \sigma^{\prime}\left[\sin \alpha^{\prime}+\left(\pi-\alpha^{\prime}\right) \cos \alpha^{\prime}\right]\left[\sin \left(\alpha^{\prime}-\alpha\right)\right. \\
\left.-\left(\alpha^{\prime}-\alpha\right) \cos \left(\alpha^{\prime}-\alpha\right)\right] .
\end{array}
$$

If üller, Photometrie der Gestirne. 
Addirt man die Werthe von $q^{\prime}$ und $q^{\prime \prime}$ und setzt noch, was ohne erheblichen Fehler gestattet ist, $s$ und $s^{\prime}$ einander gleich, so hat man endlich die gesuchte Lichtmenge:

$$
\begin{array}{r}
Q_{1}=\frac{2}{3} J_{A_{1}} \sin ^{2} s \sin ^{2} \sigma\left[\sin \alpha+(\pi-\alpha) \cos \alpha+\frac{2 A_{1}^{\prime} \sin ^{2} \sigma^{\prime}}{3 \pi} \times\right. \\
\left.\left\{\sin \alpha^{\prime}+\left(\pi-\alpha^{\prime}\right) \cos \alpha^{\prime}\right\}\left\{\sin \left(\alpha^{\prime}-\alpha\right)-\left(\alpha^{\prime}-\alpha\right) \cos \left(\alpha^{\prime}-\alpha\right)\right\}\right] .
\end{array}
$$

Mit Zngrundeleg'ung des Lommel-Seeliger'schen nnd des Euler'sehen Belenchtungsgesetzes ergeben sich ohne besondere Sehwierigkeiten-die entsprechenden Formeln:

$$
\begin{gathered}
Q_{2}=\frac{1}{2} \pi J A_{2} \sin ^{2} s \sin ^{2} \sigma\left[1-\sin \frac{\alpha}{2} \operatorname{tang} \frac{\alpha}{2} \log \cot \frac{\alpha}{4}+\frac{1}{2} A_{2}^{\prime} \sin ^{2} \sigma^{\prime} \times\right. \\
\left(1-\sin \frac{a^{\prime}}{2} \operatorname{tang} \frac{a^{\prime}}{2} \log \cot \frac{\alpha^{\prime}}{4}\right)\left(1-\frac{1}{2} \cos \frac{\alpha^{\prime}-\alpha}{2} \cot \frac{\alpha^{\prime}-\alpha}{2} \log \frac{\left.1+\sin \frac{\alpha^{\prime}-\alpha}{2}\right)}{\left.\left.1-\sin \frac{\alpha^{\prime}-\alpha}{2}\right)\right],}\right. \\
U_{3}=\frac{1}{2} \pi J A_{3} \sin ^{2} s \sin ^{2} \sigma\left(\cos ^{2} \frac{\alpha}{2}+\frac{1}{2} A_{3}^{\prime} \sin ^{2} \sigma^{\prime} \cos ^{2} \frac{\alpha^{\prime}}{2} \sin ^{2} \frac{\alpha^{\prime}-\alpha}{2}\right) .
\end{gathered}
$$

Die Werthe der Phasenwinkel a und a' lassen sieh sehr beqnem durch die heliocentrischen, geocentrischen and planetocentrischen Längen der einzelnen Himmelskorper, wie sie in den astronomischen Ephemeriden angegeben sind, ansdriicken.

\section{e. Berechung des aschfarbenen Mondlichtes.}

Eine Aufgabe, welche nit der soeben behandelten grosse Älınlichkeit hat. bezieht sich anf die Bestimmnng des sogenamnten aschfarbenen Lichtes des Dondes. Bekamntlich erseheint der ron der somne nicht direct belenchitete Theil der Mondscheibe nicht vollkommen dunkel, sondern lenchtet nit rinem schwachen Lichte, welches namentlich einige Tage nach dem Nennoud mit blossem Ange deutlich wahrzmehmen ist. Dieses Licht riihrt ron den Somnenstrahlen her, welche ron unserer Erde nach dem Ifonde hin reftertirt und ron diesem wieder niel der Erde zurickgeworfen werden. Seine Berechmmg hat deshalb ein besonderes Interesse, weil es muter sewissen vereinfichenden tmolhnen möglich ist, daraus einen Werth fïr die mittlere Reflexionsfäligkeit der Erde abzuleiten. Man denke sich in Figur is die Nittelpunkte rom somme, Mond and Erde in einer Ehene liegend nud die Sommenstrahlen muter sich parallel auf

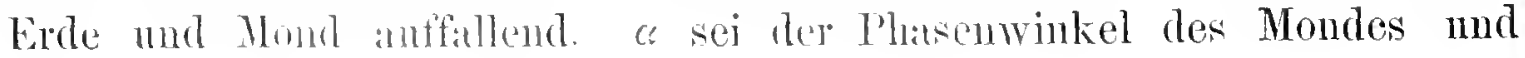


folglich $x$ - " der Phasenwinkel der Erde in Bezug anf den Mond. Der zwischen den Punkten $a$ und $b$ liegende Kreisbogen bezeiehnet denjenigen 'Theil der Erdoberfliche, von welehem Ilberhaupt nur Litht nach dem Monde gelangen kann; ferner bezeichnet der Bogen zwisehen $c$ und d. denjenigen Theil des Moudes, welcher fitr einen Beobachter auf der Erde von der Sonne beleuchtet erseheint, dagegen der Bogen zwischen $d$ und $e$ den im aschfarbenen Lichte lenchtenden Theil der Mondscheibe. Wir wollen zuniichst wieder das Lambert'sche Betenchtungsggeset\% ж. Grunde legen und die folgenden Bezeichnungen einflihren:

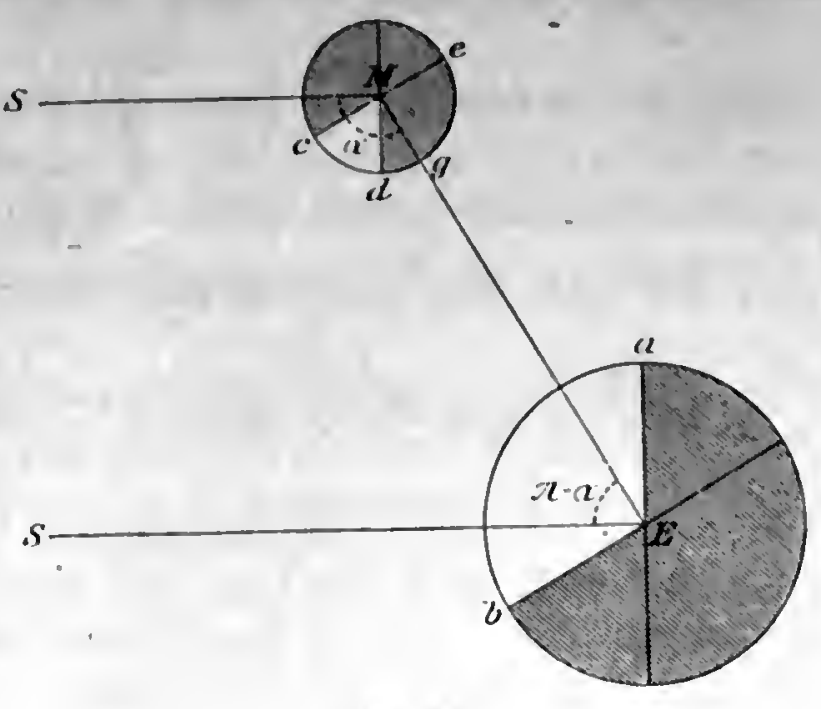

Fig. 18 ,

$A_{1}^{\prime}=$ Albedo der Erde,

$S=$ scheinbarer Halbmesser der Sonne, von der Erde aus gesehen, $\sigma^{\prime}=$ scheinbarer Halbmesser der Erde, rom Monde ans gesehen.

Ist dann $d q$ die Lichtmenge, welche von der gesammten Erdphase senkrecht auf ein Oberfliichenelement $d s$ des Mondes geworfen wird, so hat man nach der ersten der Gleichungen (10), da anstatt c hier der Werth $\pi$ - $a$ zul setzen ist:

$$
d q=\frac{2}{3} J A_{1}^{\prime} \sin ^{2} S \sin ^{2} \sigma^{\prime}(\sin \omega-a \cos c) d s .
$$

Die Lichtquantitït $d q^{\prime}$, welche von diesem Elemente $d s$ nun wieder nach der Erde (senkreeht anf die Flïcheneinheit) zuriickgeworfen wird, ist nach dem Friberen ausgedriickt durch:

$$
d q^{\prime}=\frac{A_{1}}{\Delta^{2} \pi} d q \cos i^{\prime} \cos \varepsilon,
$$

wo $A_{1}$ die Albedo des Mondes, $\boldsymbol{A}$ die Entfermung des Mondes von der Erde, $i^{\prime}$ der Ineidenzwinkel der ron der Erde anf ein Mondelement reflectirten Strahlen und \& der Emanationswinkel der vou dem Elemente wieder nach der Erde zuriickgeworfenen Strahleu ist. In dem vorliegenden Falle miissen die Werthe ron $i^{\prime}$ und $\varepsilon$ stets eininder soleich sein, und man erhält daher; wenn $\varepsilon$ und $d s$ wieder durch die ron Punkt $g$ aus auf der Mondoberfliehe geziihlten Längen (") und durch dic anf die Zeichnungsebene bezogenen Breiten $\psi$ ausgedrickt werden, durch Substitution dic Gleichung:

$$
d q^{\prime}=\frac{2}{3 \pi} J A_{1} A_{1}^{\prime} \frac{\varrho^{2}}{d^{2}} \sin ^{2} S \sin ^{2} \sigma^{\prime} \sin \left(-\omega \cos (\zeta) \cos ^{3} \psi \cos ^{2} \omega d \omega d \psi\right. \text {. }
$$


Um die Lichtmenge zu haben, welche von dem ganzen im aschfarbenen Lichte leuchtenden Theile der Mondoberfläche herrïhrt, hat man die vorstehende Gileichung in Bezug anf $\psi$ zwischen $-\frac{\pi}{2}$ und $+\frac{\pi}{2}$ und in Bezug auf $\omega$ zwischen $-\left(\alpha-\frac{\pi}{2}\right)$ und $+\frac{\pi}{2}$ zu integriren. Man findet leicht, wen! man $\frac{\varrho}{d}$ noch durch den scheinbaren Halbmesser $\sigma$ des Mondes (von der Erde aus gesehen) ansdriickt:

$$
q^{\prime}=\frac{4}{9 \pi} J A_{1} A_{1}^{\prime} \sin ^{2} S \sin ^{2} \sigma \sin ^{2} \sigma^{\prime}(\sin \alpha-\alpha \cos \alpha)(\alpha-\sin \alpha \cos \alpha) .
$$

Durch photometrische Beobachtungen kanu man diese Grösse nicht bestimmen, weil sich das von dem aschfarbenen Theile des Mondes herriihrende Licht nicht ron dem durch den beleuchteten Theil ausgesandten trennen liisst. Dilgegen ist es nicht unmöglich, durch geeignete Methoden die Wlichenhelligkeiten gleich grosser Stiicke auf dem hellen und dunklen Theile der Mondscheibe mit einander zu vergleichen, und in der That sind derartige Messungen bereits von Zöllner und in neuester Zeit von mir selhst versucht worden. Theoretisch liisst sich die Fliichenhelligkeit an jeder heliebigen Stelle auf dem aschfarbenen Theile des Mondes leieht hestimmen. Die scheinbare Helligkeit des Oberflichenelementes $d s$ erhailt man nach dem Friheren, wenu man die ron demselben nach der Erde s.elangende Lichtquantitiit $d \eta^{\prime}$ durch die scheinbare Grösse des Elementes, ron der Erde ans gesehen, dividirt. Diese scheinbare Grösse ron $d s$ ist aber gleich $\frac{d s \cos \varepsilon}{d^{2}}$; mithin erhält man die scheinbare Helligkeit $h_{1}^{\prime}$ an irgend einem Punkte, der dnreh die Coordinaten $\omega$ und $\psi$ bestimmt ist, ans der Gleichung:

$$
l_{1}^{\prime}=\frac{d \eta^{\prime} A^{2}}{d s \cos \varepsilon}=\frac{2}{3 \pi} J_{A_{1}} A_{1}^{\prime} \sin ^{2} S \sin ^{2} \sigma^{\prime}(\sin \alpha-\alpha \cos \alpha) \cos \psi \cos \omega .
$$

Dass diese formel und infolge dessen auch das dabei voransgesetzte Lambert'sche Gesetz der Wirklichkeit nieht entspricht, goeht daraus hervor; diks niach derselhen furr $\omega=90^{\circ} h_{i}^{\prime}$ rersehwinden miisste, die Helligkeit :m Riande also gleich Null sein sollte, wihrend thatsächlich der Rand scharf herentent nud sogall eher heller als die iibrigen Partien der nicht belenchtoten solloibe erwoheint.

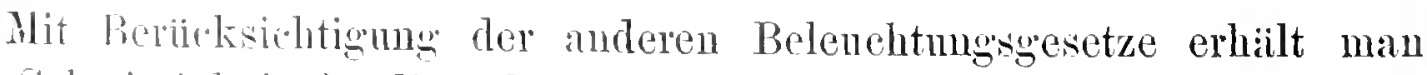
ohne Schwirigkit fü die scheinbare Helligkeit des aschfarbenen Theiles an irgend einem l'unkte dere seheibe die Formeln: 


$$
\begin{aligned}
& h_{3}^{\prime}=f J A_{2} A_{2}^{\prime} \sin ^{2} S \sin ^{2} \sigma^{\prime}\left\{1-\cos \frac{a}{2} \cot \frac{a}{2} \log \cot \left(45^{\circ}-\frac{a}{4}\right)\right\}, \\
& h_{3}^{\prime}=\frac{1}{6} J A_{3} A_{3}^{\prime} \sin ^{2} S \sin ^{2} \sigma^{\prime} \sin ^{2} \frac{a}{2} .
\end{aligned}
$$

Man sieht, dass naeh diesen beiden Formeln die scheinbare Helligkeit von $\omega$ und $\psi$ unabhängig ist und daher in allen Punkten des aschfarbenen 'Theiles gleieh sein sollte, ein Resultat, welehes mit der direeten Beobachtung jedenfalls besser harmonirt, als das Ergebniss nach der Lambert'sehen Theorie.

Hat man dureh irgend ein Verfahren das Helligkeitsverhiiltniss des aschfurbenen Lichtes zu dem beleuchteten Theile des Mondes bestimmt, so geben die soeben abgeleiteten Formeln in Verbindung mit den früheren Gleichungen (22) ein Mittel an die Hand, um einen angen:iherten Werth fir die mittlere Albedo der Erde abzuleiten. Wir wollen annehmen, dass die beiden verglichenen Stellen der Mondseheibe in der Nähe des Äquators gelegen sind, so dass also $\psi=0$ zu setzen ist; ferner soll die Liinge der anf dem hellen Theile gemessenen Stelle co, die Länge der auf dem dunklen Theile betrachteten $\omega^{\prime}$ heissen; endlich wollen wir noch die scheinbaren Halbmesser der Sonne $S$ und $s$, von der Erde und dem Monde ans gesehen, als gleich betrachten, dann erhält man durch Division der obigen Gleichungen in die Gleichungen (22) die Helligkeitsverhiiltnisse:

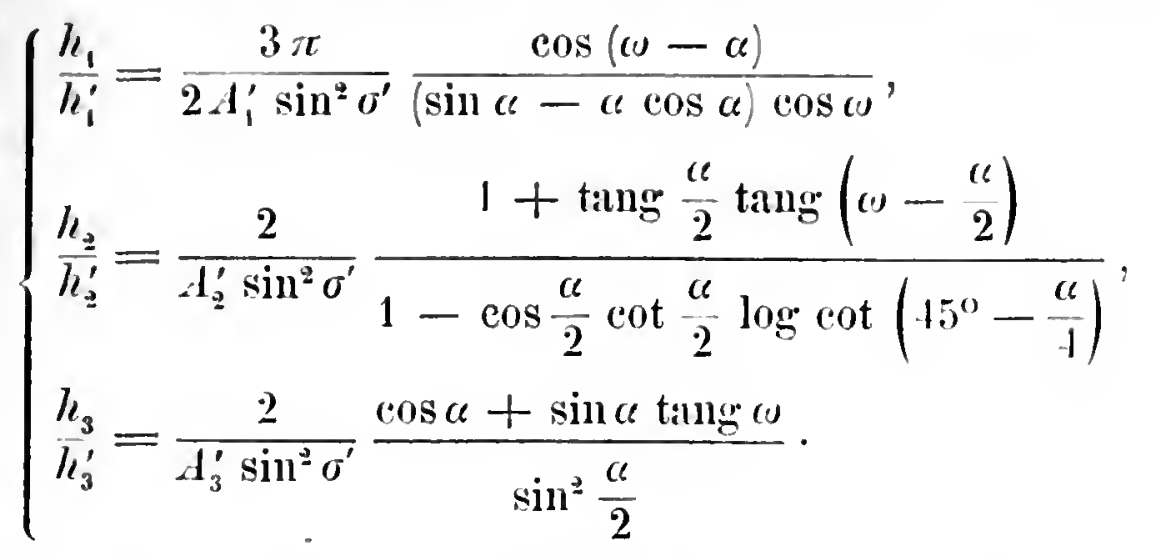

Mit Hülfe dieser Gleichungen kamn man die mittlere Nlbedo der Erde berechnen. Es darf aber dabei nicht vergessen werden, dass die Formehn nur gelten, wenn die beiden verglichenen Stellen der Uondoberfläche dieselbe Reflexionsfahigkeit besitzen, eine Voranssetzung, die nicht olne Weiteres acceptirt werden kann. In der Praxis wird man daher gut thun, die Beobachtnngen bei verschiedenen Iondphasen und an möglichst vielen Punkten der Mondscheibe anzustellen und aus allen so erhaltenen Werthen der Albedo einen Mittelwerth zu bilden. 


\section{Beleuchtung eines Systems kleiner Körper. Die Seeliger'sche Theorie des Saturnringes.}

Bei ciner Reihe von optischen Erscheinungen der Erdatmosphäre tritt die Aufgabe auf, die Lichtmenge zu bestimmen, welche ein Aggregat ron unendlich vielen ganz znfällig vertheilten kleinen Körperchen, deren Dimensionen im Verhältniss zu ihren gegenseitigen Entfernungen als klein anzusehen sind, nach einer beliebigen Richtnng anssendet, wenn dasselbe in irgend einer anderen Richtung ron der Sonne belenchtet wird. Hicrher gehören die Untersuchungen iiber die Reflexion des Lichtes an den in der Atmosphïre vertheilten Wasserbliischen und die damit im Zusammenhange stehenden Erscheinungen der Morgen- und Abendröthe, ferner die Versuche zur Erklärung der blanen Farbe des Himmels und endlich die Untersuchungen iiber die Intensität des diffusen Tageslichtes. Alle diese Probleme, deren theoretische Behandhung zum Theil mit grossen Schwierigkeiten rerknipft ist, liegen schon ansserhall, der Grenze des cigentlichen Gebietes der Astrophotometrie und kömnen daher hier mit Fug und Reeht unberiicksichtigt bleiben. Was die Astrophotometrie im engeren Simne anbetrifft, so kommt die bezeichnete Aufgabe zur Verwendung bei dem Zodiakallicht, sofern dasselbe als Licht betrachtet werden diuf, weldhes von einer nugehener grossen Menge ron Meteoroiden zwischen Somme und Erde reflectirt wird, und vor Allem bei dem Saturminge, welcher nach der jetzt allgemein aceptirten Maxwell-Hirn'sehen Ansicht ans getremten Theilchen besteht, die sich wie ein dichter Schwarm von Satelliten nm den Satum bewegen. Soweit die Anfgabe unter gewissen vereinfachenden Ammahmen iiberhant eine Lösmng zulaisst, ist sie bisher nur von Seeligeri) ansfiilnlich und erschöpfend behandelt worden. Im Folgenden sollen die wichtigsten Lromisse dieser theoretischen Untersuchmoen wiederwegehen und namentlich etwas ansfilmblicher anf die Beleuchtung des Saturminges eingegangen werdent.

Man denke sich znnïchst ein irgendwie gestaltetes Sustem ron einzehnen getremten Körperhen und führe die Beschränkung ein, dass diese Theilchen saimmtlich wleich gross sind und eine kugelfümige Gestait besitzen, ferner dass ilne gregenseitigen Abstände gross sind im Terhältniss zn ihren Dimensionen. I)iese Beschrinkmugen erleichtern wesentlich die Lösung der Anfgabe, sie sind aber nicht mbedingt erforderlich; dem,

1) Abhandl. der K. Iiager. Akat. der Wiss. II. Classe. Bd. 16, p. 405 und Bd. 15, p. 1 . 
wie Seeliger gezeigt hat, lässt sich anch der allgemeinere Fall behandelu, wo das System aus Kugeln von beliebiger Grösse in beliebigem Mischungsverhältuisse besteht. Es werde endlich noch die Lichtquelle, die Sonne, als ein lenclitender Punkt angesehen.

Wenn ein soleher Schwarm von Körperehen in einer gewissen Richtuug belenchtet wird, so ist klar, dass ein einzelnes bestimmtes Partikelchen im Inneren der Masse einerseits von anderen Theilehen beschattet, andererseits, wenn es ron aussen her in einer gewissen Richtung betrachtet wird, durch andere davor liegende Partikelchen theilweise verdeckt werden kann. Die beschatteten und rerdeckten Theile sind im Allgemeinen von einander versehieden, nur im Moment der genauen Opposition fallen sie zusammen. Sobald dic Opposition voriuber ist, treten zu den verdeckten Partien noch die beschatteten hinzu, und es lisst sich daraus sofort ersehen, dass die Helligkeit einer solehen wolkenartigen Nasse in der Nihe der Opposition merklich variiren kann, besonders dann, wenu die Masse wenig durchsichtig ist, die Theilchen also verhältnissnäissig nahe bei einander liegen. Diese Lichtänderung in der Nähe der Opposition ist von dem Beleuchtungsgesetze, welches auf die einzelnen Theilchen anzuwenden ist, so gut wie gänzlich unabhängig. Erst bei grössseren Phasen kommt die Form dieses Gesetzes in Frage, und in diesem Falle ist daher die theoretische Behandlung des Problems am schwierigsten und unsichersten.

Ein unendlich kleines Element einer im Inneren der Masse gelegenen Kugel sende, wenn es frei waire, dem Auge des Beobachters die Lichtmenge $d q^{\prime}$ zu. Der Radins der saimmtlichen Kugeln sei $\varrho$. Nun kamı dieses Element durch andere Kugeln beschattet oder verdeckt sein, und es wird daher die wirkliehe Lichtmenge desselben, die mit $d q$ bezeichnet werde, im Allgemeinen kleiner scin als $d q^{\prime}$. Es handelt sich darum, einen Durehschnittswerth für $d q$ zu bestimmen, wenn sehr viele soleher Elemente in Frage kommen. Ist nun $p$ die Anzahl der Fälle, in denen ein Element ganz frei liegt, $p^{\prime}$ dic Anzahl der Fiille, in denen es beschattet oder verdeckt ist, so gelangt ron den $p$ Elementen im Ganzen die Lichtmenge $p d q^{\prime}$, von den $p^{\prime}$ Elementen dagegen die Liehtmenge Null in das Auge. Der Mittelwerth aller Lichtmengen ist daher:

$$
d_{l}=d q \frac{p}{p+p^{\prime}}
$$

oder, wenn $\frac{p}{p+p^{\prime}}$ mit $w$ bezeiclnet wird:

$$
d q=\| d q^{\prime}
$$


Sind die Kugeln ganz zufällig innerhalb der Masse vertheilt, so ist $w$ die Wahrscheinlichkeit dafür, dass ein unendlich kleines Element im Imneren weder beschattet noch verdeckt ist. In Figur 19 bedeute $R$ eiuen irgendwie gestalteten Raum, der mit zorstreut refleetirenden Theilehen angefullt ist; $d f$ sei ein unendlich kleines Element. Von $d f$ werden awei Gerade nach der Sonne und nach der Erde gezogen, und um diese als Axe \%wei Kreiscylinder construirt gedacht mit den Durchmessern 2 $\varrho$, welche sich an dem unteren Ende durchschneiden. Der von den Cylindern innerhalb der Masse eingesehlossene Raum heisse $V$. Wenn s

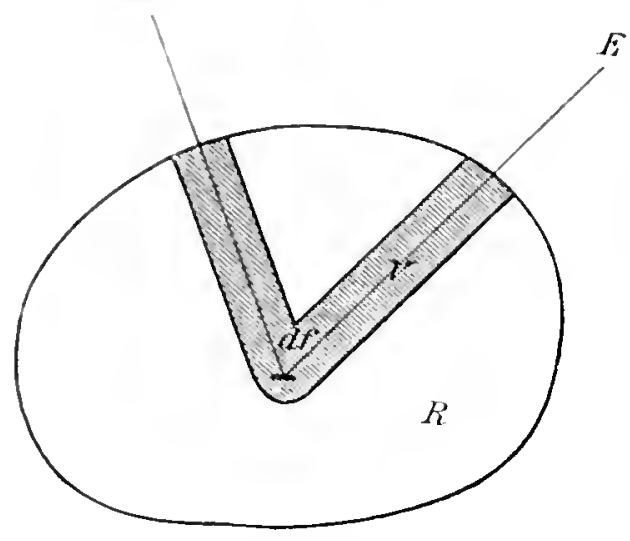

Fig. 19. nun von den sämmtlichen Kugeln, welehe in

s $R$ vertheilt sind, keine einzige so liegt, dass ihr Mittelpunkt in den Raum $V$ fällt, so ist das Element $d f$ weder beschattet noch verdeekt. Man kann also $w$ anch definiren als die Wahrscheinlichkeit dafür, dass sämmtliehe Kugelmittelpunkte ausserhalb des Ranmes $V$ liegen. Die Dimensionen der eimzelnen Kugeln mögen im Verhältniss zur Ausdehuung der ganzen Masse $R$ als sehr klein voransgesetzt sein; dann wird man $w$ für alle Elemente einer und derselben Kngel als nahe goleich ammehmen kömnen und hat damn für die rou einer ganzen Kingel ansgesandte Lichtmenge die Gleichung:

$$
q=u \cdot q^{\prime} \text {. }
$$

$q^{\prime}$ ist die Liehtquantität, welehe eine einzelne Kugel nach der Erde ansstrahlen wiirde, wenn sie isolirt läge. Nach den Entwicklungen des vorigen Paragraphen wird aber $q^{\prime}$ ausgedriickt durch die Formel:

$$
q^{\prime}=\Gamma f^{\prime}(a),
$$

wo $f(a)$ die Abhängigkeit von dem Phisenwinkel a angiebt, die je nach rlem zn Grunde gelegten Belenehtungsgesetze versehieden ist, und $\Gamma$ eine ('onstante bedentet, die von der Grösse der Kugel, ron der Reflexionsfïhigkeit n.s. w. abhängt. Die Anzahl der siimmtlichen in $R$ enthaltenen Kungehn sei $Y$. Ist nun diese Anzahl gross, so wird man ohne erheblichen Fehler annehmen diurfen, dass bei zuffilliger Anordmung der Theilchen der ganze lamm nahezn gleirhmïssig mit Kugeln angefuillt ist. Unter clieser Foransetzung sind in der Rammeinheit $\frac{N}{R}$ Kugeln enthalten, und in eincm Volnnelemente $d r$ beträgt die Inzahl der Kugeln $\frac{N}{R} d v$. Dal narch Ohigem die durrhohnittliche Lidhtmenge, welche eine einzelne Kugel 
anssendet, gleich $w q^{\prime}$ ist, so wird die von dem Volumclemente $d v$ ansgehende Lichtmenge gegreben dureh:

$$
d Q=i \frac{N}{R} d v u q^{\prime}
$$

oder nach Snbstitution des Werthes von $q^{\prime}$ durch:

$$
d Q=I f(\alpha) \cdot u \frac{N}{R} d v
$$

Das Volumelement $d v$ kann man sich ersetzt denken durch $d x d \sigma$, wo $d x$ das Element der Gcraden ist, die von $d v$ nach dem Bcobachter hin gezogen ist, und $d \sigma$ die scheinbare Grösse von $d v$ reprïsentirt. Man hat also:

$$
d Q=r f(\alpha) w \frac{N}{R} d x d \sigma
$$

und daher folgt für die Lichtquantität aller derjenigen Kugcln; welche llberlaupt einen Beitrag zu der Helligkeit vou do liefern, der Werth:

$$
Q=\Gamma f(\alpha) \frac{N}{R} d \cdot \int_{0}^{\mathrm{x}} u d x
$$

wobei also $f(\alpha)$ als constant angesehen wird, und $X$ die Lïnge der Strecke innerhalb der Masse von dem Elemente $d v$ an bis zu der äusseren Begrenzung in der Richtung nach dem Beobachter zu bedentet.

Die mittlere scheinbare Helligkeit von $d \sigma$, oder, wic man sie auch nennen kann, die Flächenhelligkeit von $d \sigma$, d. h. nach dem Friiheren die ausgesandte Lichtquantitä, dividirt durch die seheinbare Grösse, wird nmn:

$$
J=r f(\alpha) \frac{N}{R_{0}} \int_{0}^{x} u^{*} d r .
$$

Die Wahrscheinlichkeit $u$ ist eine Function der Lage der betrachteten kleinen Kugel innerhalb des Raumes $R$ und hängt ausserdem noch ron den Richtungen nach Sonne und Erde ab; cine Bestimmung dieser Grösse ist nur unter gewissen Voraussetzungen möglich.

Befindet sich nur eine einzige Kugel in dem liaume $R$, so bedeute w, die Wahrscheinlichkeit dafiir, dass der Mittelpunkt derselben ausserhalb des Raumes $V$ oder innerhalb des liammes $R-V$ liegt. Ist daun ferner $w_{a}$ die Wahrscheinlichkeit dafüir, dass eine zweite Kugel clieselbe 
Bedingung erfillt, während der Mittelpunkt der ersten bereits im Raume $R-V$ liegt, und sind $w_{3}, w_{4} \ldots w_{N}$ die entsprechenden Werthe fiir $3,4 \ldots . . N$ Kugeln, so ergiebt sich nach den Gesetzen der Wahrscheinlichkeitsrechmung:

$$
w=w_{1} w_{2} w_{3} \ldots w_{N} .
$$

Num ist aber die Wahrscheinlichkeit dafür, dass eine erste Kugel innerhalb von $V$ liegt, ansgedrickt dureh $\frac{V}{R}$; folglich ist die Wahrscheinlichkeit $w_{1}$ dafiur, dass sie ausserhalb ron $V$ liegt, gegeben durch:

$$
w_{1}=1-\frac{V}{R} \text {. }
$$

Lient aber eine Kugel bereits in $R$, so bleibt für den Mittelpunkt einer wweiten Kugel nur der Ramm $\dot{R}-l$ iibrig, wo $k=\frac{32}{3} \varrho^{3} \pi$ ist (siehe nebenstehende kJeine Figur 20\%. Da num die erste Kugel

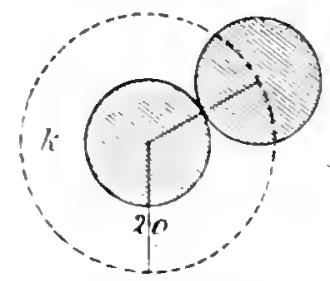

Fig. 20. theilweise im Raume $V$ liegen kamm, so lässt sich die Wahrscheinlichkeit $\varkappa_{2}$. falls $\varepsilon_{1}^{\prime}$ einen ausserordentlich kleinen positiven echten Bruch bedentet, ansdricken durch:

$$
u_{2}=1-\frac{V-\varepsilon_{1}^{\prime} l_{i}}{R-l_{i}} .
$$

Kommnt noch eine dritte Kngel hinzu. so bleibt für den Mittelpunkt derselben ein límm uibrig, der grösser ist als $l i-2 l$, weil das $k$ der zweiten und dritten Kngel sich mit dem der ersten nud zweiten zum Theil deckt. Bezeichnet also $\varepsilon_{2}$ einen echten Bruch, so ist der für das Centrum der dritten Kngel iiberhanpt rerfighbare Ram $R-2 \varepsilon_{a} l_{i}$. und da die Kugeln zum Theil wieder in den Raum $T$ hineinreichen kïnnen, so wird:

$$
\|_{3}=1-\frac{\Gamma-2 c_{2}^{\prime} l_{i}}{R-2 \varepsilon_{2} l_{i}} .
$$

Gallz allgemein wird fermer:

und mithin:

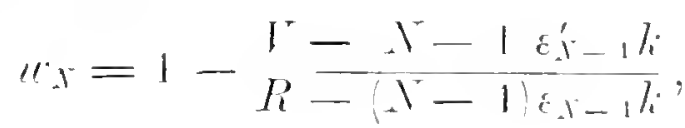

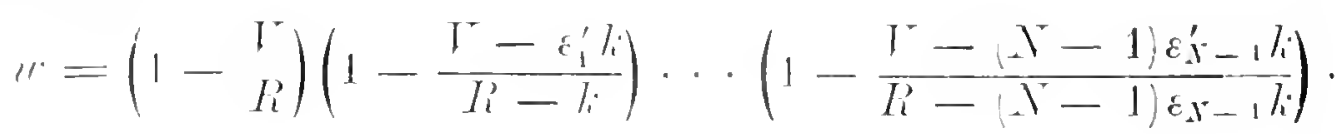

Eine strenge Berechnnng der Cirisse " ist im Allgemeinen nicht möglich. Wenn die Anzahl der Kngehn selnr gross, das betraehtete System also selur dicht angenommen werden muss. Tur damn lässt sich ein 
Näherungsasusdruck einfuhren, wemn die Materie dtunn vertheilt ist, wie man es $\%$. B. bei dem das Zodiakallicht veranlassenden Meteoroidenringe und walrseheinlich auch bei dem Saturnringe voranssetzen darf. Der Gesammtinhalt aller Kugeln kann in diesem vereinfacliten Falle, der hier allein weiter verfolgt werden soll, im Vergleich zu dem ganzei Raume $R$ als klein angesehen werden, und man wird keinen sehr grossen Fehler begelien, wenn man bei der Entwicklung des obigen Ausdruckes von $w$ alle Glieder fortlisst, in denen der ausserordentlich kleine Factor $\frac{k}{h}$ oder eine Potenz desselben anftritt. Man erhält dann einfach:

$$
u=\left(1-\frac{V}{R}\right)^{x}
$$

Nun kann man die weitere vereinfachende Annahme machen, die jedenfalls in den oben erwähnten Fällen gestattet sein wird, dass $\frac{V}{R}$ klein ist und dass infolge dessen dic höheren Potenzen von $\frac{V}{R}$ gegen die erste vernachlïssigt werden dürfen; dann wird:

oder:

$$
\log u=N^{r} \log \left(1-\frac{V}{R}\right)=-N \frac{r}{R},
$$

$$
w=e^{-x \frac{r}{R}} \text {. }
$$

Dureh Sulbstitution dieses Werthes in Gleichung (2) ergiebt sich mun:

$$
J=I f(c) \frac{N}{R} \int_{0}^{x} e^{-x \frac{r}{R}} d x .
$$

Diese Gleichung sehliesst alle Fülle der Belenchtung eines Systems kleiner Körper, die nieht allzu dicht vertheilt sind, in sieh. Thre Auflösung ist im Allgemeinen, da $V$ rom Phasenwinkel itbhïngt, :insserst schwierig, inshesondere bei einer beliebigen unregehmiissigen Gestaltung der ganzen Masse, wie sie z. B. beim Zodiakillieht itmzunelmmen ist. Seeliger hat den Fall einer kugelfürmigen homogenen Staubwolke unter der Voraussetzung, dass der Phasenwinkel nicht zu klein ist, ansfiulnlich behandelt und gelangt dabei zu einem verhältnissmässig einfachen Ausdrucke; unter der speeiellen Annahme, dass die Masse so dieht ist, dass sie als undurchsiehtig betraeltet werden darf, findet er die Gesammtlichtmenge identiseh mit der ron einer festen Kugel ansgesandten, welche denselben Durchmesser wie die Stanbwolke besitzt.

Von besonderem Interesse ist der Fall. wo der Raum $R$ von zwei parallelen Ebenen begrenzt wird, wic mim es z. B. beim Satturnringe 
annimmt. Ist in Figur $21 H$ die Gesammtdicke der Schicht, $h$ der senkrechte Abstand eines Volumelementes von der oberen begrenzenden libene, sind ferner $i$ und $\varepsilon$ die Winkel, welche die Normale zu dieser Ebene mit den Riehtungen nach Soune und Erde hin bildet, so hat man $h=x \cos \varepsilon$ und mithin dureh Substitution in (3):

$$
J=I f(\alpha) \frac{N}{R \cos \varepsilon} \int_{0}^{H} e^{-N \frac{r}{R}} d h .
$$

Das Volumen $V$ besteht aus vier Theilen, aus den beiden cylindrischen Riiumen $V_{0}$ und $V_{1}$, aus dem beiden Cylindern gemeinsamen Stuick

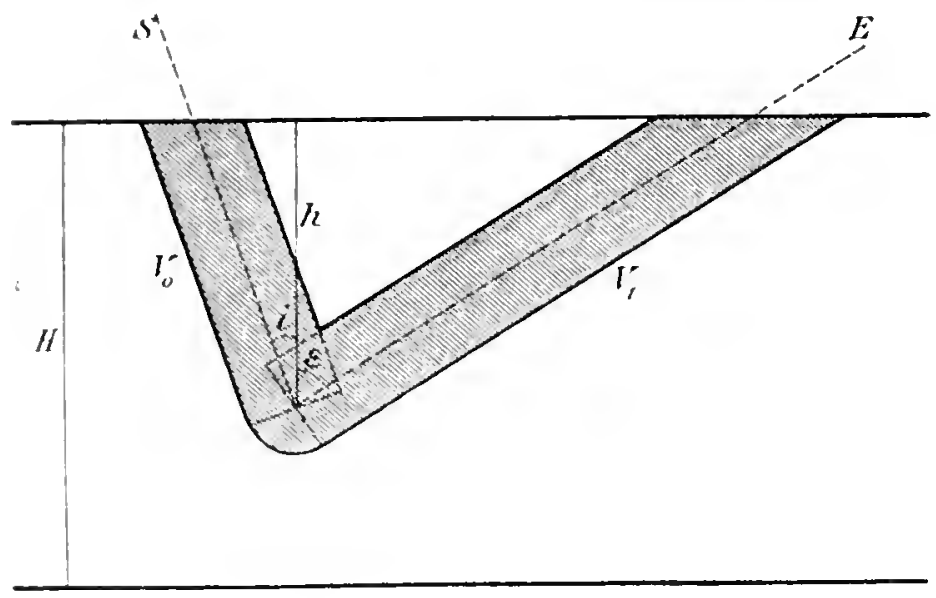

Fig. 21.

mithin ist:

und :

$$
\begin{aligned}
& r_{0}=Q^{2} \pi \frac{h}{\cos i}, \\
& r_{1}=Q^{2} r \frac{h}{\cos \varepsilon} ;
\end{aligned}
$$

$G$ und einem kleinen von der Kugel begrenzten Stiiek, das ohne Bedenken vernachlaissigt werden kann. Für den Fall, dass der Phasenwinkel nicht sehr klein ist, kann man auch das Stiick $G$ gegeniiber dem Inhalte der beiden Cylinder vernachlïssigen und hat dann $V=V_{0}+V_{1}$. Nun kann man angenähert setzen:$$
V=e^{2} x h_{1} \frac{\cos i+\cos \varepsilon}{\cos i \cos \varepsilon},
$$

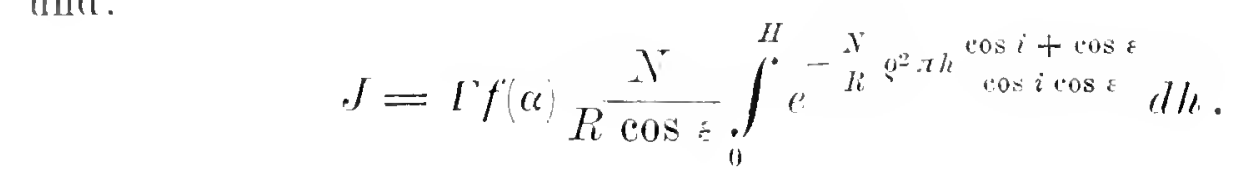

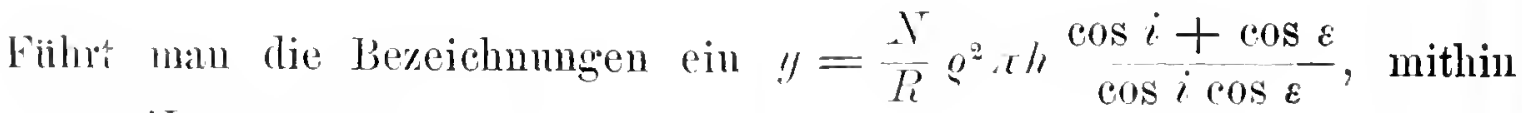
$d !=\frac{T}{l} e^{2}, \frac{\cos i+\cos \varepsilon}{\cos i \cos \varepsilon} d h$, so folut:

$$
I=\begin{aligned}
& l f(c) \quad \cos i \\
& e^{2} x \cos i+\cos \varepsilon \\
& \int_{0}^{l} e^{-y} d ! y
\end{aligned}
$$


wo noch die obere Grenze $Y$ gegeben ist durch die Gleichung:

$$
Y=\frac{N}{R} \varrho^{2} x H \frac{\cos i+\cos \varepsilon}{\cos i \cos \varepsilon} .
$$

Daraus erhiilt man sofort, wenn man noch statt $\frac{\Gamma}{\varrho^{2} \pi}$ die nene Constante $\gamma$ cinfuhrt:

$$
J=\gamma f(\alpha) \frac{\cos i}{\cos i+\cos \varepsilon}\left(1-e^{-r}\right),
$$

und da das zweite Glied fur den Fall, dass der Raum $R$ als nahęu undurchsichtig oder, was dasselbe ist, $H$ als sehr gross angesehen werden darf, zu vernachlïssigen ist, so ergielot sich:

$$
J=\gamma f(\alpha) \frac{\cos i}{\cos i+\cos \varepsilon} .
$$

Dieser Ausdruck stimmt unter der Voraussetzung, dass $f(\alpha)$ als constant $z u$ betrachten ist, mit dem Werthe uberein, der für die Flächenhelligkeit eines festen Körpers nach dem Lommel-Seeliger'schen Gesetze gefunden wird.

Die Formeln (5) und (6) werden ungenau bei kleinen Werthen von $\alpha$, weil dann das beiden Cylindern gemeinsame Stick nicht unberiicksichtigt bleiben darf. Von besonderem Interesse ist der Fall $a=0$. Bei dieser Stellung fallen die beiden Cylinder $V_{0}$ und $V_{1}$ in einen einzigen zusammen, und das Volumen $V$ lässt sich (wieder mit Vernachlässigung der sehr kleinen Halbkngel am unteren Ende) ansdriicken durch:

Mithin wird aus (4):

$$
V=e^{2} \pi{ }^{h} \cos ^{\prime}
$$

$$
J_{0}=I f(0) \frac{N}{R \cos i} \int_{0}^{H} e^{-\frac{N}{h} l^{2} \pi \frac{h}{\cos i}} d l,
$$

oder nach Ausfithrung der Integration:

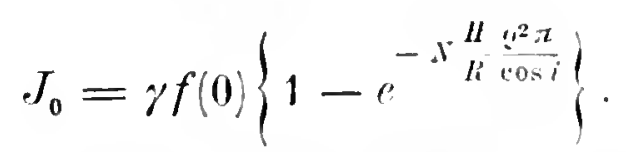

Einen wesentlich hiervon verschiedenen Werth erhält man, wenn man die obige Formel (5) anf den Fall $a=0$ anwendet. Da hierbei $i=\varepsilon$ sein muss, so wird dic Flächenhelligkeit, die wir jetzt $J_{n}^{\prime}$ nennen wollen, ansgedriickt durch:

$$
J_{0}^{\prime}=\frac{1}{2} \gamma f(0)\left\{1-e^{-2 x x^{\frac{H}{h}} \frac{g^{2}-2}{\cos i} i}\right\} .
$$


Aus der Vergleichung mit Formel (7) folgt dann:

$$
\frac{J_{0}}{J_{0}^{\prime}}=2 \frac{1-e^{-\lambda}}{1-e^{-2 \lambda}},
$$

wobei zur Abkiurzung gesetzt ist $\lambda=N \frac{H}{R} \frac{\varrho^{2} \pi}{\cos i}$.

Ist das System fast undurchsichtig, also $H$ und demnach auch $\lambda$ als selu grross anzusehen, so wird $\frac{J_{0}}{J_{0}^{\prime}}=2$. Daraus ersieht man, dass bei einem sehr dichten System von kleinen Körpern die Helligkeit in unmittelbarer Nihe der Opposition doppelt so stark anwachsen kann, wie bei einem festen Körper, für welchen das Lommel-Seeliger'sche Belenchtungsgesetz Gültigkeit hat.

Ist das System nicht als fast undurchsichtig zu betrachten, so wird der Quotient $\frac{J_{0}}{J_{0}^{\prime}}$ stets kleiner als 2, und weun endlich die Masse äusserst durchsichtig, also $\lambda$ sehr klein ist, so nähert sich der Bruch $\frac{1-e^{-\lambda}}{1-e^{-2 \lambda}}$ dem Grenzwerthe $\frac{1}{2}$, und mithin $\frac{J_{0}}{J_{0}^{\prime}}$ dem Grenzwerthe 1 . Bei sehr durchsichtigen Massen, wie sie z. B. beim Zodiakallicht in Betracht kommen mögen, wird die Helligkeitszmahme in der Nähe der Opposition nicht so sehr ins Ange fallen.

Es soll num noch etwas specieller auf die Seeliger'sche Beleuchtungstheorie des Saturnsystems eingegangen werden, welche deshalb von besonderem Interesse ist, weil ihre Ergebnisse durch die nenesten Helligkeitsmessungen des Planeten Saturn in vollem Umfange bestätigt werden. Dass der Saturmring als ein Aggregat von getrennten Massentheilchen zu betrachten ist, diirfte gegenwiirtig bei den Astronomen kaum noch anf Widerspruch stossen, nachdem insbesondere durch die Naxwell'schen Lntersnchungen festgestellt ist, dass die Amnahme eines festen Zustandes wenig Wahrscheinlichkeit für sich hat. Wie schon der blosse Angenschein lehrt, kann der Saturnring nicht als ein rollkommen lomogenes rehilde angesehen verden. Er hesteht aus dem der Planetenkugel am n:̈ichsten liegenden sogrenannten dunklen Ringe, dem sehr hellen inneren linge nud dem durch die Cassini'sche Tremnngslinie daron geschiedenen, etwas schwicheren insseren Ringe. Die beiden letzten Theile, die hier allein in Betracht zu ziehen sind, wird naln als ziemlich dicht und nahezu undurchsichtig roranssetzen diirfen. In roller Strenge ist die Theorie natiirlich nicht anwendbar, weil sie cine vollkommen gleichmässige Vertheilung der einzelnen Partikelchen rerlangt, wïhrend in Wirklichkeit 
die Theilehen an einigen Stellen des Ringes dichter, an anderen dumer stehen werden. Auch ist es sehwerlieh statthaft, den Saturnring als einen vollkommen regelmassigen, ron zwei parallelen Ebenen begrenzten eylindrisehen Raum zu betriehten. Das Resultat der Untersuehung, wird daher unr ein genihlhertes sein köuneu.

Bei den voraugehenden allgemeinen Betrachtungen war der Raum $V^{\text {r }}$ berechnet worden mit Vernachlaissigung des kugelförmig begrenzten Stllekes an Durchsehnitt der beiden Cylinder und des den beiden Cylindern gemeinsamen Stltekes. Die erstere Vereinfachung wird statthaft sein, da die einzelnen Kugeln gegenuber deu Cylindern $V_{0}$ und $V_{1}$ stets klein sind; dagegen wird es rathsam sein, beim Saturnringe das gemeinsame Stuck $G$ mit zu hericksichtigen. Man hat dann:

$$
V=V_{0}+V_{1}-G \text {. }
$$

Diese Gleichung gilt jedoch nur fitr alle diejenigen Volumelemente des Ringes, fïr welehe der zugehörige Raum $G$ günzlich imnerhalb des linges liegt und nicht ron der oberen Ringebene gesehnitten wird. Ist dies letztere der Fall, so bleibt ein Theil von $G$, der mit $\Sigma$ bezeiehnet werden soll, ansserhall, des Ringes, und es wird dann:

$$
V=V_{0}+V_{1}-G+s \text {. }
$$

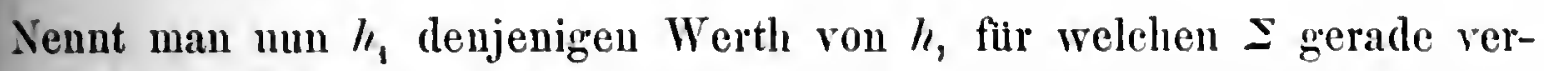
schwindet, so wird die Flïchenhelligkeit des Saturnringes nach Gleichung $(4)$ ausgedrickt durch:

(8) $J=I \cdot f(a) \frac{X}{R \cos \varepsilon}\left\{\int_{0}^{h_{1}} e^{-\frac{N}{R}\left(r_{0}+b_{1}-G+\Sigma\right)} d l l+\int_{h_{1}}^{H} e^{-\frac{N}{h}\left(r_{0}+r_{i}-G\right)} d l\right\}$.

F'thrt man noch die Elevationswinkel $A$ und $A^{\prime}$ von Erde und Somne iiber der Ringebene ein durch die Relationen $A=90^{\circ}-\varepsilon$ und $A^{\prime}=90^{\circ}-i$, so ist mit ausreichender Genanigkeit:

$$
\begin{aligned}
& V_{0}=e^{2} x \frac{l 1}{\sin A}, \\
& V_{1}=g^{2} x \frac{l 1}{\sin A^{\prime}} .
\end{aligned}
$$

Die Berechnung der Rïume $G$ und $\searrow$ ist etwas umstiindlich und soll hier ibergangen werden. Nach den Seeliger'schen') Entwieklungen ist: und $495 \mathrm{ff}$.

1) Abhandl. der K. Bayer. Akad. der Wiss. II. Classe, band 16, Seite $477 \mathrm{ft}$. 


$$
G=\frac{4}{3} \varrho^{3} \frac{1+\cos \alpha}{\sin \alpha},
$$

und:

$$
\mathbf{S}=\frac{\left(\sin A+\sin A^{\prime}\right)^{2}}{\sin \omega \sin A \sin A^{\prime}} \frac{\varrho^{3}}{\cos !}\left\{\cos \varphi-\frac{1}{3} \cos ^{3} \varphi-\left(\frac{\pi}{2}-\varphi\right) \sin \varphi\right\},
$$

wobei die Grössen "״ und $\varphi$ bestimmt sind durch die Gleichungen:

$$
\begin{aligned}
& \operatorname{tang} u=\frac{\cos A \sin \beta}{\sin A+\sin A^{\prime}} \sin \alpha, \\
& \varrho \sin \varphi=\frac{h \cos \varphi \sin \alpha}{\sin A+\sin A^{\prime}} .
\end{aligned}
$$

$\beta$ ist dabei der Winkel zwischen der durch Saturn, Sonne und Erde gelegten Ebene und der durch Saturn und Erde senkrecht zur Ringebene gelegten Ebene.

Der Grenzwerth $l_{1}$ ist endlich wach Seeliger bestimmt durch:

$$
l_{1}=\frac{g\left(\sin A+\sin A^{\prime}\right)}{\sin \iota} \text {. }
$$

Man hait nun:

$$
\begin{aligned}
& V_{0}+V_{1}-G=Q^{2} \pi l \frac{\sin A+\sin A^{\prime}}{\sin A \sin A^{\prime}}-\frac{4}{3} \varrho^{3} \frac{1+\cos \alpha}{\sin \alpha}, \\
& V_{0}+V_{1}-G+\Sigma=
\end{aligned}
$$

$\frac{\left(\sin A+\sin A^{\prime}\right)^{2} \varrho^{3}}{\sin \left(\sin A \sin A^{\prime} \cos \varphi\right.}\left\{\cos q-\frac{1}{3} \cos ^{3} \varphi+\left(\frac{\pi}{2}+\varphi\right) \sin \varphi\right\}-\frac{4}{3} \varrho^{3} \frac{1+\cos \alpha}{\sin \alpha}$.

Da der Phasenrinkel a beim Saturn stets klein ist (im Maximum 6.5), so kann man ohne erheblichen Fehler setzen:

$$
1+\cos a=2 \text {. }
$$

Ferner sind die Elerationswinkel $A$ und $A^{\prime}$ stets nur nm sehr kleine Betrïge ron einander versehieden. und man darf daher anch setzen:

$$
\frac{\sin A+\sin A^{\prime} z}{\sin A \sin A^{\prime}}=4 \text {. }
$$

Endlich ist der Winkel " ron derselben Ordnung wie $a$, und man darf daher cos $\mu$ gleich 1 setzen.

Mit diesen Vereinfalchungen eroiebt sich:

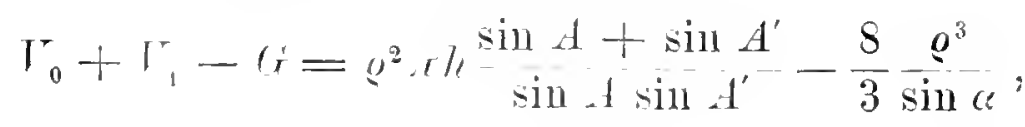

$$
\begin{aligned}
& r_{0}+r_{1}-G+\Sigma=\frac{4 Q^{3}}{\sin q}\left\{\cos q-\frac{1}{3} \cos ^{3} \psi+\left(\frac{\pi}{2}+q\right) \sin \psi-\frac{2}{3}\right\} .
\end{aligned}
$$


Das aweite Integral in Glciclung (8) lässt sich mun durch Einfuhrung des ersten dieser Werthe leicht berechneu. Man hat:

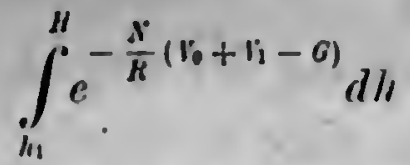

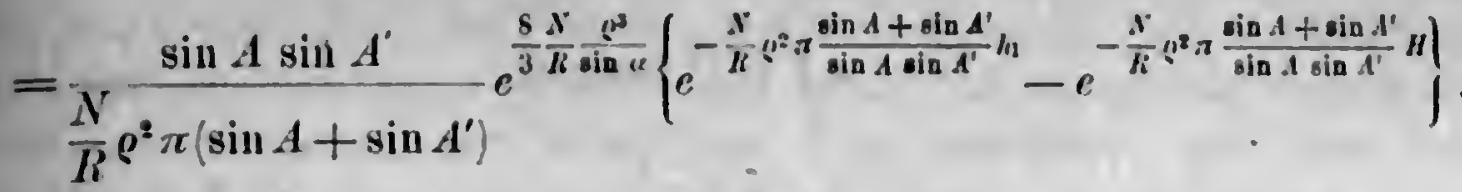

Das zweite Glied in der Klammer ist zu vernachlässigen, weil der Ring als undurehsichtig und mithin $H$ als sehr gross angenommen werden kann. Fillurt man noch die Bezeichnungen ein:

$$
\begin{aligned}
& \delta=\frac{32}{3} \frac{\varrho^{3} \pi}{R}, \\
& \xi=\frac{N \delta}{\sin \alpha},
\end{aligned}
$$

so wird, wenn man den Werth von $h_{1}$ substituirt:

$$
\int_{h_{l}}^{H} e^{-\frac{N}{h}\left(r_{0}+r_{1}-G\right)} d h=\frac{32}{3} \frac{\varrho}{\xi \sin \alpha} \frac{\sin A \sin A^{\prime}}{\sin A+\sin A^{\prime}} e^{-\frac{3 \pi-2}{8 \pi}} .
$$

Das erste Integral in Gleichung (8) lïsst sieh, wenn man die Variable $/$, durch of ersetzt, in der Form schreiben:

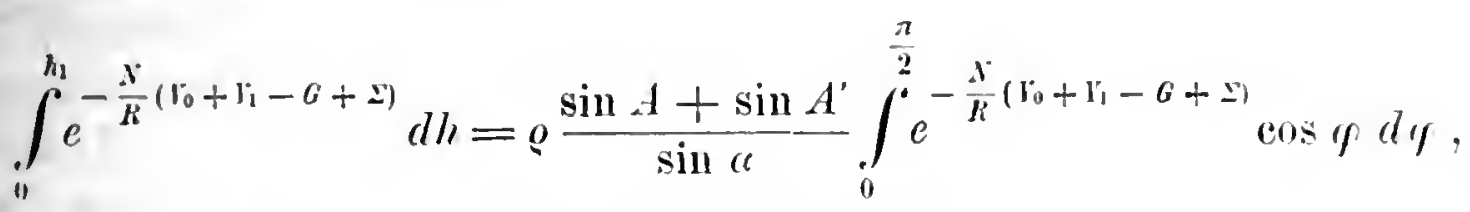

und wenn man die Grösse (D) einfihint durch die Substitution:

$$
\Phi=\frac{3}{8 \pi}\left\{\cos r-\frac{1}{3} \cos ^{3} \varphi+\left(\frac{\pi}{2}+\tau\right) \sin \varphi-\frac{2}{3}\right\} .
$$

so wird:

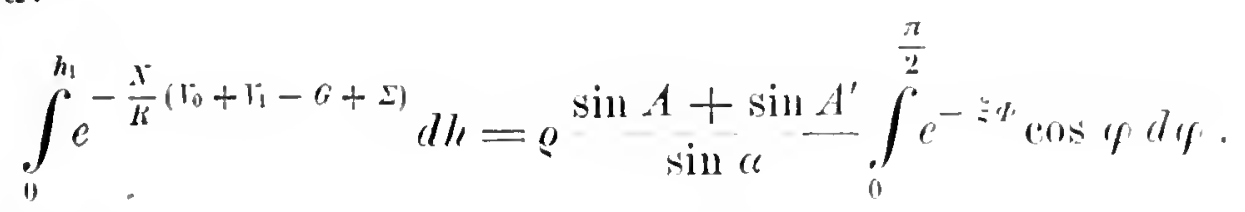

Dureh Substitution von (10) und (11) in (5) ergiebt sich nun:

$$
J=I f a \frac{\left.g^{\prime} \sin A+\sin A^{\prime}\right)}{R \delta \sin A}\left\{\int_{0}^{\frac{\pi}{2}} e^{-5 t} \cos y d y+\frac{5}{3} c^{-\frac{3 \pi-2}{3 \pi}}\right\} \text {. }
$$


Da $f(\alpha)$ durchweg als nahezu constant angesehen worden ist, so kann man noch den Werth $\frac{\Gamma f(a) \varrho}{R \delta}$ durch eine einzige Constante $\Gamma^{\prime}$ ersetzen und erhält dann, mit Einftihrung der Bezeichnungen:

$$
\begin{aligned}
& \mathfrak{A}=\xi \int_{0}^{\frac{\pi}{2}} e^{-\xi \omega} \cos \varphi d \varphi, \\
& \mathfrak{B}=\frac{8}{3} e^{-\frac{\xi \pi-2}{5 \pi}} \\
& \mathfrak{C}=\mathfrak{A}+\mathfrak{B}
\end{aligned}
$$

die Endgleichung:

$$
J=\Gamma^{\prime} C \frac{\sin A+\sin A^{\prime}}{\sin A} .
$$

Der Bruch $\frac{\sin A+\sin A^{\prime}}{\sin A}$ unterscheidet sich stets uur wenig ron dem Werthe 2, und da 5 von $\xi$ allein abhängt, so folgt ohne Weiteres, dass die Flächenhelligkeit des Saturnringes stets nahezu dieselbe sein muss, mag der Ring ganz schmal erscheinen oder weit geöffnet sein, ein Resnltat, welches durch die directen Beobachtungen bestätigt zu werden scheint.

Der Werth ron $\mathfrak{A}$ kann nur durch mechanische Quadratur oder durch Reihenentwickhng ermittelt werden. Die Seeliger'sehen Abhandhungen enthalten Tafeln, ans denen die numerischen Werthe dieser Grösse, ebenso der Grössen $\mathfrak{B}$ und $\mathfrak{C}$, für rerschiedene Werthe ron $\xi$ entnommen werden können. Da $\xi$ rom Phasenwinkel $a$ abhängt, so folgt das wielıtige Resultat, dass die Ringhelligkeit mit dem Phasenwinkel variirt. Es ist aber $\xi$ auch ron $T \delta$ abhängig, und diese Grösse ist ein Mass fuir die Dichtigkeit, wit welcher die einzelnen Partikelehen in dem linge rertheilt sind. Bezeichnet man nämlich das ganze von simmtlichen $T^{\top}$ Kugehn eingenommene Volumen mit $K$, so ist die Dichtigkeit $I$ der Materie ansgedriickt dureh: $L=\frac{\pi}{R}$. Nun ist aber $K=N^{4} \frac{4}{3} Q^{3} \pi$, und da nach Obigem $\delta=\frac{32}{3} \frac{g^{3} \pi}{R}$ gesetzt war. so ist $L=\frac{1}{8} N \delta$. Fiir $N \delta=0.4$ wird z. B. $\left.I\right)=0.05$, d. h. etwa $\frac{1}{20}$ des gesammten Rammes des Satururinges wiirde in diesem Falle mit Materie erfiillt sein.

Sceliger giebt eine Zusammenstellung der Werthe ron log c fuir verschiedene Annihmen ron $T \delta$, nnd es erriebt sich ans dieser Tabelle, dass die geammte Lichtrariation innerhalh des in Betracht gezogenen 
Phaseniutervalles ron 0 bis $5^{\circ}$ sehr betrichtlich ist, in der Hauptsache aber sich sehon in unmittelbarer Nihe von $\iota=0$ alsspielt, nnd \%war um so schneller, je kleiner $N \delta$, d. l. je geringer die Dichtigkeit der Ringmaterie angenominen wird.

In der Praxis ist es bisher noch nicht mit Erfolg versicht worden, die Fliichenhelligkeit des Saturnriuges \%u bestimmen. Die vorhandenen znverlissigen Messungen bezichen sich auf die Lichtquantitiit, welche das ganze Saturnsystem, also Kugel und Ring zusammen, nach der Erdé sendet, und un diese Ergelnnisse mit der Theorie 7 vergleichen, ist es daher noch erforderlich, das rom Planeten selbst ansgestrahlte Licht \%n berechnen. Dabei muss Ritcksicht genommen werden anf die theilweise Bedeckung von Ring und Kugel. Ist $Q_{s}$ die Lichtmenge, welehe die frei gedachte Saturnkugel uns musenden wirde, ferner $Q_{F}$. die Jichtmenge des vom Ringe verdeckten Theiles der Kugel, $R$ die scheinbare Fliiche des frei gedachten Ringes, $F$ die scheinbare Fliiche des vom Saturn verdeckten Theiles des Ringes, so ist die Gesammthelligkeit des ganzen Systems:

$$
Q_{B}=(R-F)_{e} J+Q_{S}-Q_{k^{\prime}},
$$

wobei \%uniichst auf die gegenseitige Beschattung vou Ring und Kugel keine Riicksieht genommen ist. Der Einfachheit wegen soll der Saturnkörper als Kingel mit dem scheinbaren Radius $a$ betrachtet werden, ferner soll die Voraussetznng gemacht werden, die in aller Strenge allerdings nur für das Lommel-Seeliger'sche und das Euler'sehe Beleuchtungsgesetz und auch für diese uur beim Phasenwinkel 0 gilt, dass nämlich die Planetenscheibe in allen Punkten gleichmässig hell ist; dann kann man setzen:

$$
Q_{F}: Q_{S}=F^{2}: a^{2} x \text {. }
$$

Bei Anwendung des Lommel-Seeliger'sehen Gesetzes ist aber naeh Formel (6) S. 62:

$$
Q_{S}=\frac{\Gamma_{2} a^{2} \pi}{2}\left\{1-\sin \frac{\alpha}{2} \operatorname{tang} \frac{\alpha}{2} \log \cot \frac{\alpha}{4}\right\},
$$

und wenn man den Ausdruck in der Klammer mit $D$ bezeichnet, so wird:

$$
Q_{s}-Q_{F^{\prime}}=\frac{\Gamma_{2} D}{2}\left\{a^{2} x-F\right\} \text {. }
$$

Führt man nun noch die Bezeichnungen ein:

$$
\begin{aligned}
& X=\frac{R-F}{a^{2} \pi}, \\
& Y=\frac{a^{2} \pi-F}{a^{2} \cdot T}, \\
& B=\frac{2 J}{\Gamma_{2}},
\end{aligned}
$$


so wird durch Sulostitution in Gleichung (13):

$$
Q_{B}=\frac{a^{2} \pi \Gamma_{2}}{2}\{B X+D Y\}
$$

Nun ist aber nach dem Friheren $\frac{a^{2} \pi}{2} \Gamma_{2}$ niehts Anderes als die Lichtquantitiit, welehe die Saturnkugel allein ohne Ring bei voller Belenchtung anssendet, und wenn diese $Q_{(0)}$ genannt wird, so ergiebt sich:

$$
Q_{B}=Q_{(0)}\{B X+D Y\} \text {. }
$$

Eine gam\% analoge Formel mit anderen Werthen für die Grössen $X$ und $Y$ hat Seeliger auch mit Zugrundelegung des Lambert'schen anstatt des Lommel-Seeliger'schen Gesetzes abgeleitet. Für die rersehiedenen Grössen $\mathrm{X}, 1, D$ u. s. w. sind ron ihm Tafeln berechnet, und es ist noch zu erwïlınen, dass bei Berechnung dieser Grössen auch auf die Abplattung des Saturnkörpers Riicksicht genommen ist. Der Schattenwurf ron dem Ringe anf den Satum und umgekehrt ist ron Seeliger ebenfalls in Rechunng gezogen. und es sind fuir die Grössen $\mathrm{I}$ und $Y$ kleine Correctionen al)greleitet worden, die jerloch in der Praxis olme Bedenken vernachlässigt werden dürfen, weil sie im Vergleich zu der bei photometrischen Messumgen erreichbaren Genanigkeit rersehwindend klein sind. Setzt man noch

$$
\sin A+\sin A^{\prime}(5 X=m \text {. }
$$

ferner

$$
D Y=n
$$

und fiihrt statt der Giösse $\frac{2 I^{\prime}}{\Gamma_{a}}$ eine nene Constante $\gamma$ ein, so wird:

$$
Q_{B}=Q_{10}\left\{y^{\prime} m+n\right\} \text {. }
$$

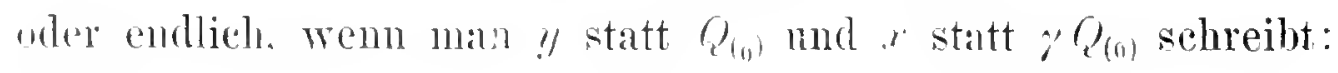

$$
\eta_{B}=m \cdot r+m !
$$

Wit Hiilfe dieser Gleichnng laisst sieh jede beolaldhtete Helligkeit des ganzen satumststems anf die Helligkeit bei verschwundenem Ringe reduriven.

Dir photometrischen Ersheinungen, welehe der dunkle Saturnring zeigt, sind ron Seeliner henfalls theoretisch rerfolgt worden unter der Annahme, dasis dieser Theil des linges ans Partikelchen besteht, welehe weniger dirht angerindnet sind, als in dem hellen Ringe. so dass das Licht theilweise durrhacheinen kinn. 
Bine direete Stlitze erhält diese Annahme dureh Beobachitungen des Trabanten Japetus, wenn derselbe dureh den Selıtten des Saturnsystems hindurchgeht. Eine derartige Beobaehtungsreihe ist nenerdings von Barnard ansgefuhrt worden, und es folgt aus den Beobaehtungen, dass der dunkle Ring in den dem Planeten am niiebsten liegenden Theilen fast ganz durehsiehtig ist, und dass die Undurehsichtigkeit erst mit der Annäherung an den hellen Ring allmählieh zunimmt. Eine ausfuhrliehere Behandlung dieses Problems ist vor-Kurzem ron Buchhol\%y rersueht worden.

Die Seeliger'schen Betrachtungen fuhren noeh zu dem Sehlusse, dass die 'Theilchen des dunklen Ringes eine etwas andere Reflexionsfahigkeit habeu mlissten, wie die des hellen Ringes, und Seeliger maelit daranf aufmerksam, dass nach den bisherigen Beobachtungen im Laufe der Zeit Verinderungen innerhalb des Saturnringes vor sich gegangeu zn sein seheinen, und zwar dass möglieher Weise die Albedo der den dunklen Rinır bildenden 'Theilehen sieh vergrössert hat.

\section{Die Verfinsterungen der Jupitersatelliten.}

Die Anwendung der photometrisehen Hauptgesetze bietet noch ein besonderes Interesse in dem Falle der Verfinsterung eines Himmelskürpers durch einen anderen. Hierher gehören die Lichterseheinungen des Mondes wiihrend einer totalen Mondfinsterniss und die Bedeekungen der Satelliten ron ihren Hauptplaneten. Die erstere Aufgabe scheint auf den ersten Blick die einfachere zu sein, weil es sich dabei nur um drei Himmelskörper (Sonne, Mond und Erde) handelt, und die ganze Erscheimung sieh zin der Zeit abspielt, wo die Mittelpunkte derselben sich in einer geraden Linie befinden. In Wirklichkeit aber ist die theoretische Behandlung dieses Problems deshalb ersehwert, weil bei der verhältnissmässig geringen Entfernung des Erdsystems ron der Sonne diese letztere nielit als lenchtender Punkt, sondern als eine Scheibe von ungleichmaissiger Helligkeit angenommen werden muss, und weil ferner die complicirte Form der Sehattengrenze anf dem Monde, ansserdem die Wirkmng des Halbschattens streng in Rechnung zu ziehen sind.

Eine vollstiindige Lösung dieser interessanten Aufgabe ist erst in allernenester Zeit von v. Hepperger ${ }^{2}$ ) und noch cingehender und erfolg-

1) Astron. Nachr. Bd. 137, Nr. 3280.

2) Sitznngsb. der Wiener Akad. der Wiss. Math.-naturw. Classe. Bd. 104. Abth. II a, p. 189. 
reicher von Seeliger $\left.{ }^{1}\right)$ versucht worden, nachdem Letzterer bereits früher gelegentlich einer Besprechung ${ }^{2}$ ) der Arbeiten von Brosinsky und Hartmann iber die Vergrösserung des Erdschattens bei Mondfinsternissen die Frage kur\% berihrt hatte. Da eine ansführliehe Darlegung der theoretischen Entwicklungen den Rahmen dieses Buches erheblich übersehreiten wiirde, so muss hier der blosse Hinweis auf die genannten Arbeiten genuigen. Übrigens hat sich die praktische Photometrie mit dem Problem der Mondverfinsterung bisher so gut wie gar nicht beschäftigt, und erst ror Kurzem ist ron Ver $\mathrm{y}^{3}$ ) ein erster Versuch gemacht worden, die Helligkeitsvertheilung auf der verdunkelten Mondscheibe durch wirkliche photometrische Messungen anstatt durch blosse Schätzungen zu bestimmen.

Was num die aweite der oben erwähnten Aufgaben, das Studium der Verfinsterungen der iibrigen Planetentrabanten, anbelangt, so ist die photometrische Beobachtung dieser Phänomene, wic zuerst Cornu ${ }^{4}$ nachwewiesen hat, speciell im .Jupitersystem, deshalb ron ansserordentlicher Bedeutung, weil daraus mit viel grösserer Sirherheit als bisher der Zeitpunkt bestimmt werden kann, zu welchem sich der Trabant in einem newissen Stadinm der Verfinstermg betiudet, und weil daher auch die praktische Verwendung der Tupitertrabintenbedeckungen zu Längenbestimmungen eine ganz nene erhölhte Wichtigkeit erlangt hat. Die theoretische Seite dieses Problems ist sehr ansfihrlich ron Obrecht5), IVollmann and Anding behandelt worden mit roller Beriicksichtisun aller dabei ins spiel kommenden Factoren. Ersterer hat dabei eine pleichmässige Vertheilung der Holligkeit anf der Trabantenscheibe rorausuresetzt. die sowohl durch das Lommel-Seeliger seche als auch durch das Eulersche Belenchtmostesetz. jedoch mur bei roller Beleuchtung, gefordert wird. die beiden anderen haben ihren Betrachtungen das Lambert'sche Gesetz zu Grunde gelest. In einer Besprechung der Obrecht schen Arbeit liat seeliger" noch einige wichtige Bemerkmoen iber die Bedeutung

1. Abhandl. der K. Bayer. Akad. der Wiss. II. Classe, Bd. 19, Abth. II, 1). 355.

2 Vierteliahrsschrift der Astron. Gesellschaft. Jahrg. 27 (1892), p. 1 S6.

: Astrophrsical Journal. Vol. II, p. 293.

4, Comptes Rendus. Tome 96, p. 1609

5) Innales de lobserr. de Paris. Mémoires, tome 18. (Siehe auch Referat dariiber: Viertelj-Sehrift ler Astr. Ges. Jahrg. 20 1555. p. 176.

6. Wellmann. Zur Photometrie der Jupiters-Trabanten. Berlin 1ss7.

i Anding. Photometrische Cntersuchungen über die Verfinsterungen der Jupiterstrabanten. Preisschritt der Unir. Miinchen. Minchen 1559.

S) Vierteljahrschuit der Astr. Gesellschaft. Jahrg. 20 (1585, p. 176. 
versehiedener Beleuchtungsgesetze für das vorliegende Problem hinzugefugt.

Die strenge Lðsung der Aufgabe fillırt zu ziemlich complicirten Entwicklungen. Unter gewissen Voraussetzungen gelangt mä aber \%u verhältnissmäissig einfachen Ansdrlicken, dic im Folgenden etwas naher betraclitet werden sollen.

Die Aufgabe selbst ist \%unihelist folgendermassen "u prïcisiren. Eiu Jupitertrabant tritt in den Sehatten seines Planeten; dabei wirl allmällieh ein inmer grösseres Stlick seines Seheibchens verfinstert, bis er zuletzt gan\% unsielitbar wird. Bei den ersten 'Trabanten betright die ganze Dauer des Phiinomens $4^{\mathrm{m}} 19^{\mathrm{s}}$, bei dem vierten $16^{\mathrm{m}} 27^{\mathrm{z}}$. Es soll num die Helligkeitsabnahme des Trabanten als Funetion der Zeit ermittelt werden.

Streng genommen misste man zunïchśt auf die Bewegungsverhältnisse im Jupitersystem Rícksicht nehmen. Bei der verhiiltnissmaissig kurzen Daner der Erscheinnug wird aber kein grosser Fehler entstehen, wemn man die Verschiebung der Schattengrenze auf dem Trabanten der Zeit proportional setzt. Zur weiteren Vereinfachung werde die Gestalt der Trabanten als kugelformig angeselien, ferner werde die Wirkung der Jupiteratmosphiire ansser Aclit gelassen. Zweifellos wird durch dieselbe eine Brechung und Schwächung der Somnenstrahlen hervorgebracht, und die Sclattengrenze anf dem Trabanten wird infolge dessen nicht scharf erscheinen; aber bei der g:inzlichen Unkenntniss von der Höhe und Dichtigkeit dieser Atmospluaire fehlt jeder Anhalt fiir eine rechnerische Berücksichtigung ihres Einflusses. Bei der grossen Entfermung des Jupitersystems von der Sonne wird es ohne merklichen Fehler erlaubt sein, alle von einem beliebigen Punkte der Sonne nach einem heliebigen Punkte des Satelliten gelangenden Strahlen als parallel muter cinauder zu betrachten; man kamn also die Somne als eine punktförmige Liehtyuelle ansehen und brancht anf die Wirkung des Halbschattens keine Riurksicht zn nehmen. Die Schattengrenze, welche dureh den Durchschnitt des Kernschattenkegels des Jupiter mit der Trabintenkugel entsteht, projicirt sich auf der Trabantenscheibe als eine Linie ron gewisser Krimmmnng. Da der Trabant im Verhiailtniss zur Jupiterkugel selur klein ist, so kommt nur ein kleines Stiuck dieser Curve in Frage, und dieses stiick darf ohne allzu grossen Fehler als geradlinig angesehen werden. Sieht man ferner von dem Phasenwinkel, der beim Jupitersystem bis zu $12^{\circ}$ steigen kann, ganz ab und beriieksichtigt nur den Fall der vollen Beleuchtung, so redncirt sich das ganze Problem auf die folgende Iufgabe: Es soll die Helligkeitsabnahme einer beleuchteten Kreisscheibe ermittelt werden, wenn dieselbe ron einem mit gleichfürmiger Gesehwindigkeit über sie hinweg gehenden geradlinig begrenzten dunklen Schirme bedeckt wird. 
Die Figur 22 stelle die scheinbare Trabantenseheibe dar; der Radius derselben sei $r$, und der kürzeste Abstand der Schattengrenze rom

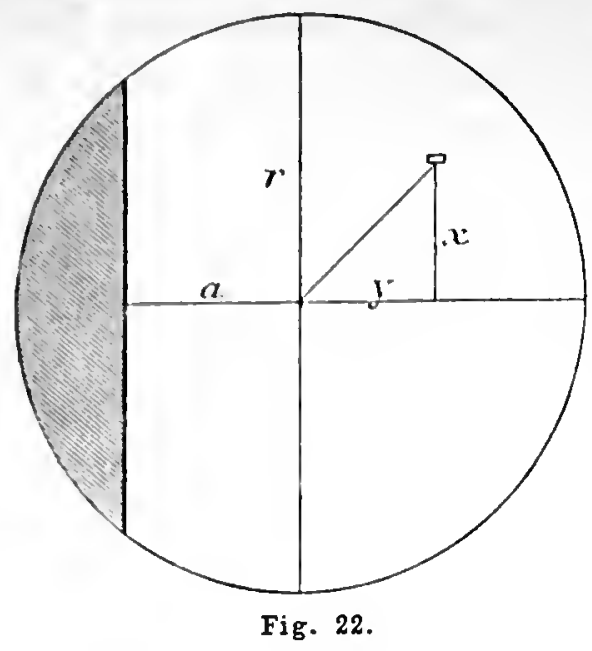
Mittelpunkte heisse $a$. Die Coordinaten irgend eines kleinen Elementes der Scheibe, bezogen auf ein rechtwinkliges Coordinatensystem, dessen $y$-Axe mit der Geraden $a$ zusammenfällt, mögen $x$ und $y$ sein. Die scheinbare Helligkeit irgend eines Elementes der Scheibe wird im Allgemeinen bei voller Beleuchtung eine Function des Abstandes rom Centrum sein. Die Lichtquantität, welche dureh ein solches Element zu dem Beobachter gelangt, wird also ausgedriickt werden können dureh:

$$
d \eta=i f\left(\sqrt{x^{2}+y^{2}}\right) d x d y
$$

wo $\gamma$ eine Constante ist, und wo das Beleuchtungsgesetz, ron welchem die scheinbare Helligkeit abhängt, zunächst noch unbestimmt gelassen werden soll. Die gesammte Lichtmenge, welche der Trabant in dem Moment anssendet, wo die Schattengrenze anf der Scheibe den kürzesten Abstand a vom Centrum hat, ist daher, falls mehr als die Hälfte der Scheibe belenchtet ist:

$$
Q=2 \gamma \int_{-a}^{r} d y \int_{0}^{\sqrt{r^{2}-y^{2}}} f \sqrt{x^{2}+y^{2}} d x
$$

Es sollen nun die bekinnten Beleuchtungsgesetze auf diese Gleichung angewendet werden. Nach den Formeln (22) (S. 69) ist beim Lambertschen Gesetze die scheinbare Helligkeit eines Elementes einer Planetenscheibe'), für den Fall, dass der Planet voll belenchtet ist, ansgedrückt dureh:

$$
h_{1}=C \cos \psi \cos \omega,
$$

wo $C$ eine Constante bedeutet. Es ist also in der obigen Gleichung $\cos \psi \cos \omega$ statt $f \sqrt{x^{2}+y^{2}}$, zu setzen. Die Winkel $\psi$ und $\omega$ hängen mit den Coordinaten ir und y nach deu Erliantermgen auf Seite 70 , wenn alle Distanzen in demselben Masse wie der seheinbare Radius $r$ ansgedrickt werden, durh die lielationen zusanmen:

$$
\begin{aligned}
& r=r \sin \psi \\
& y=r(0), \sin (\omega)
\end{aligned}
$$

1. Das Licht, welches vom Planeten auf den Trabanten reflectirt und ron diesem wieder nach der Erde geworfen wird, ist hier gïnzlich zn rernachlïssigen. 
Daraus ergiebt sich:

$$
f\left(\sqrt{x^{2}+y^{2}}\right)=\cos \psi \cos \omega=\sqrt{1-\frac{x^{2}+y^{2}}{r^{2}}},
$$

und dureh Substitution in (1) wird:

$$
Q=2 \gamma \int_{-a}^{r} d y \int_{0}^{\sqrt{r^{2}-y^{2}}} d x \sqrt{1-\frac{x^{z}+y^{2}}{\gamma^{2}}} .
$$

Um das zweite Integral anfaulösen, ist zuniiehst $y$ als eonstant anzunehmen. Setzt man $1-\frac{y^{2}}{r^{2}}=b$ und $\frac{1}{r^{2}}=c$, so wird:

$$
\begin{aligned}
& \int_{0}^{\sqrt{r^{2}-y^{2}}} d x \sqrt{1-\frac{x^{2}+y^{2}}{r^{2}}}=\int_{0}^{\sqrt{r^{2}-y^{2}}} d x \sqrt{b-c x^{2}} \\
= & {\left[\frac{1}{2} x \sqrt{b-c x^{2}}+\frac{b}{2 \sqrt{c}} \arcsin x \sqrt{\frac{c}{b}}\right]_{x=0}^{x=\sqrt{r^{2}-y^{2}}} } \\
= & \frac{r}{2}\left(1-\frac{y^{2}}{r^{2}}\right) \arcsin 1 \\
= & \frac{r \pi}{4}\left(1-\frac{y^{2}}{r^{2}}\right) .
\end{aligned}
$$

Damit erhält man aus Gleichung (2):

$$
Q=\frac{\gamma r \pi}{2} \int_{-a}^{r}\left(1-\frac{y^{2}}{r^{2}}\right) d y
$$

oder endlich:

$$
Q=\frac{\gamma \pi}{2 r}\left\{r^{\cdot 2}(r+a)-\frac{1}{3}\left(r^{3}+a^{3}\right)\right\} .
$$

Wäre die Scheibe ganz unbedeckt, so würde man zn set\%en haben $a=1$. und erhielte dann die Lichtmenge:

$$
Q_{0}=\frac{\gamma \pi}{2 r} \frac{4}{3} r^{3}
$$

Dureh Division von $(3)$ und $(4)$ wird dam endlich:

$$
\frac{Q}{Q_{0}}=\frac{1}{2}+\frac{3}{4} \frac{a}{r}-\frac{1}{4} \frac{a^{3}}{r^{3}}
$$

oder, wemn man die nene Bezeichnung $\frac{a}{r}=\cos \varphi$ einfuhrt:

$$
\stackrel{Q}{Q_{0}}=\frac{1}{2}+\frac{3}{4} \cos r-\frac{1}{4} \cos ^{3} r .
$$


Wird also die Helligkeit des Trabanten während der Dauer einer Verfinsterung in Einheiten der Helligkeit ansgedrückt, welehe derselbe vor dem Beginn der Verfinsterung lat, so giebt die Gleichung (5) die Abhängigkeit der angenblicklichen Lichtstärke von dem Werthe von $a$ an, oder, da a der Zeit proportional ist, die Abhängigkeit von der Zeit. Für die Mitte der Verfinsterung ist $a=0$ zu setzen, und man erhailt daun aus (5) $\frac{Q}{Q_{0}}=\frac{1}{2}$, die Lichtstärke ist also anf die Hailfte herabgesunken. Dies ist anch von vornherein zu erwarten, da, wie wir friiher gesehen haben, nach dem Lambert'sehen Beleuchtungssesetze die scheinbare Helligkeit auf einer Planetenscheibe ron der Mitte nach allen Seiten hin gleichmässig abuimmt.

Ist die Schattengrenze über die Mitte der Scheibe binausgeriiekt, also weniger als die Hälfte des Trabanten erleuchtet, so erhält man die zugehörigen Werthe ron $Q$ aus den Formeln (3) und (5), wenn man $a$ negativ rechnet.

Bei Anwendung des Lommel-Seeliger'schen und des Euler'schen Belenchtungsgesetzes wird die Bestimmung der Lichteurve noch einfacher. Demn in beiden Fïllen ist bekanntlich bei roller Beleuchtung des Trabanten die scheinbare Helligkeit an allen Punkten der Scheibe gleich, und die Function $f \overline{x^{2}+y^{2}}$, kaun daher gleich 1 gesetzt werden. Die Gleichung (1) geht damn sofort in die folgende iiber:

$$
Q=2 \gamma \int_{-a}^{r} d \eta \int_{0}^{\gamma^{2}-y^{2}} d x=2 \gamma \int_{-l}^{r} \sqrt{r}-\eta^{2} d \eta
$$

Sach Austiihrung der einfachen Integration hat man:

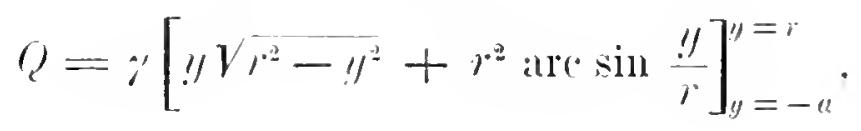

und, wemm man wieder wie when $\frac{a}{r}=$ ass if setat:

$$
\eta=\gamma r^{2}\{x+\sin q \cos q-q\} \text {. }
$$

Für die Helligkeit bei mbederkter Scheibe eroiebt sich. dal $a=r$ ist:

und mithin wird endich:

$$
r_{11}=i^{2} x \text {, }
$$

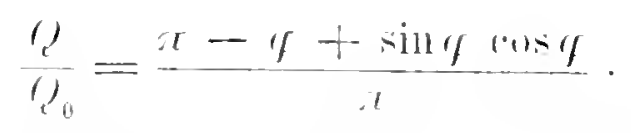

Selbstrerständlich redncirt sich anch hier fiir die Mitte der Verfinsterung, 
d. L. fur $a=0$, die Helligkeit anf die Hulfte des ursprungliehen Betrages. Zur Vergleichung der beiden dureh die Formeln (5) und (8) repräsentirten Liehteurven kann man die Werthe von $\frac{Q}{Q_{0}}$ fur versehiedene Werthe von $a$, d. h. fur versehiedene Stadien der Bedeckung, bereehnen. Man erhält so \%. B. die folgenden Werthe:

\begin{tabular}{|c|c|c|c|c|c|}
\hline$\frac{11}{r}$ & $\begin{array}{c}\text { Lambert'sches } \\
\text { Gesetz }\end{array}$ & \begin{tabular}{|} 
Lommol-Secliger- \\
sches und Énler'sches \\
(ieset:
\end{tabular} & $\frac{a}{r}$ & $\begin{array}{c}\text { Larnbert'sches } \\
\text { Gesetz }\end{array}$ & $\begin{array}{c}\frac{8}{20} \\
\text { Lommel-Secliger- } \\
\text { sches und Euler'sches } \\
\text { Geselz }\end{array}$ \\
\hline $\begin{array}{l}0.6 \\
0.5 \\
0.4 \\
0.3 \\
0.2 \\
0.1 \\
0.0\end{array}$ & 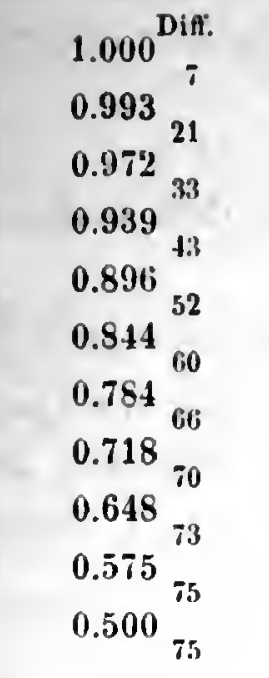 & 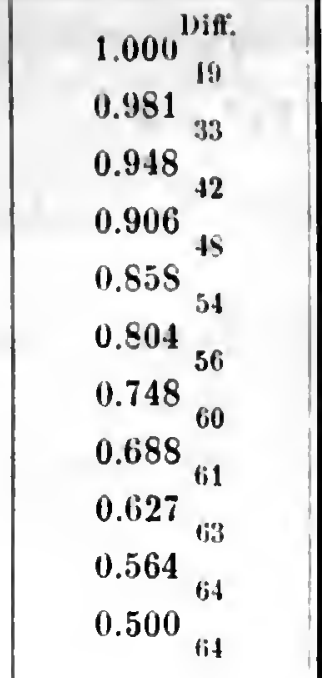 & $\begin{array}{r}0.0 \\
-0.1 \\
-0.2 \\
-0.3 \\
-0.4 \\
-0.5 \\
-0.6 \\
-0.7 \\
-0.8 \\
-0.9 \\
-1.0\end{array}$ & $\begin{array}{l}0.300^{\text {Diff. }} \\
0.425 \\
0.352 \\
0.282^{73} \\
0.216 \\
0.156 \\
0.104 \\
0.06 \\
061 \\
0.025^{33} \\
0.007 \\
0.000\end{array}{ }^{31}$ & $\begin{array}{l}0.500^{\text {Difi. }} \\
0.436 \\
0.373^{63} \\
0.312^{61} \\
0.252^{60} \\
0.196 \\
0.142^{54} \\
0.094 \\
0.052 \\
0.019 \\
0.000\end{array}$ \\
\hline
\end{tabular}

Betraehtet man die Werthe von a als Abseissen, die zugehörigen Helligkeitswerthe als Ordinaten, so sieht man, dass die beiden den oljigen Zahlemreihen entspreehenden Curven sich bei $a=0$ schneiden, und dass die dem Lambert'sehen Gesetze zugehörige in der ersten Hälfte der Verfinsterung oberhalb, in der zweiten unterhalb der anderen Curve liegt. Bei $a=-0.5 r$ ist nach dem Lambert'sehen resetze die Lichtstiirke des Trabanten, in Grössenclassen ausgedriickt, um 2.02 geringer als vor Beginn der Verfinsterung, naeh den anderen Gesetzen nu um 1.77. Bei $a=-0.8 r$ werden die entsprechenden Zahhen 3.55 und 3.21 Grössenclassen u. s. w. Wemu es möglich wïre, die IJelligkeiten der Trabanten noch in diesem vorgeriickten Stadium der Verfinsterung mit einiger Sicherheit zu messen, so liesse sich ans solchen Beobachtnngen ein Urtheil dariiber gewimnen, welches von den zu Grunde gelegten Belenehtungsgesetzen den Vorzug rerdient. Beide Curven zeigen das Charakteristische, dass die Ordinaten sich am schmellsten um die Mitte der Erseheinung indern, und dass an dieser Stelle ein Wendepunkt 
punkt vorhanden ist. Dies liisst sich auch numittelbar ans den Gleichungen $(3)$ und $(7)$ ableiten, wenn man die zweiten Differentialquotienten nach " bildet. Man hat aus (3) sofort:

$$
\frac{d^{2} Q}{d a^{2}}=-\frac{a \gamma \pi}{r}
$$

und da der \%weite Differentialcuotient einer Function verschwinden muss, wem die betreffende Curve einen Wendepunkt haben soll, so sieht man, dass die dem Lambert'schen Gesetze cntsprechende Lichteurve für $a=0$, d. h. also in der Mitte der Verfinsterung, einen solehen besitzt.

Ans Gleichmng ( 7 ) wird entsprechend nach kmrzer Rechnung:

$$
\frac{d^{2} Q}{d a^{2}}=-\frac{2 a \gamma}{\sqrt{r^{2}-a^{2}}}
$$

und es folgt daher anch fiir die durch (7) reprissentirte Lichteurve ein Wendepunkt bei $a=0$.

Se eliger hat noch ganz allgemein gezeigt, dass, wie anch die Form der Function $f \sqrt{x^{2}+y^{2}}$, beschaffen sein möge, die Liehtcurve des Trabanten waihrend der Verfinsterung stets einen Wendepunkt für $a=0$ besitzen muss. In der That ergiebt sieh ans Gleiehung (1) sofort:

$$
d Q=2 \gamma \int_{0}^{\sqrt{r^{2}-a^{2}}} f\left(\sqrt{a^{2}+r^{2}}, d x\right.
$$

Daraus folgt dam weiter:

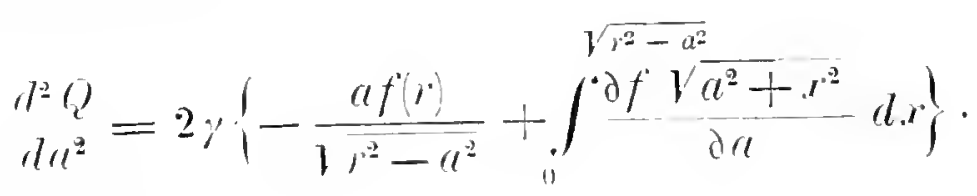

Nim kilnn man s.hreiben:

$$
\partial f^{\prime} \sqrt{a^{2}+r^{2}} \frac{2 a}{\partial a}=\frac{\partial a^{2}+r^{2}}{\partial\left(a^{2}\right)},
$$

und dat allowemein wilt:

$$
\frac{\partial f \prime \prime+r}{\partial \|}=\frac{\partial f u+r}{\partial n+r} .
$$

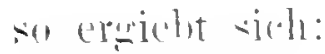

$$
\partial f \frac{u^{2}+\overline{r^{2}}}{\partial u^{2}}=2 u^{\prime \prime} \frac{\sqrt{u^{2}+u^{2}}}{\left.\partial u^{2}+u^{2}\right)} \text {. }
$$


Fulırt man noch die neue Variable : ein durch die Substitution $a^{2}+x^{2}=x^{2}$, woraus folgt $d x=\frac{z d z}{\sqrt{a^{2}-a^{2}}}$, so wird:

$$
\begin{aligned}
\frac{\partial f\left(\sqrt{a^{2}+x^{2}}\right)}{\partial a} d x & =2 a \frac{\partial f(z)}{\partial\left(z^{2}\right)} \frac{z}{\sqrt{i^{z}-a^{2}}} d z \\
& =a \frac{\partial f(z)}{\partial z} \frac{d z}{\sqrt{i^{2}-a^{2}}} \\
& =a \frac{f^{\prime}(z)}{\sqrt{i^{2}-a^{2}}} d z
\end{aligned}
$$

Setat man diesen Werth in Gleichung (9) eiu und beachtet, dass für * die Integrationsgrenzen $r$ und $a$ sind, so erhält man:

$$
\frac{d^{2} Q}{d a^{2}}=-2 a y\left\{\frac{f(v)}{\sqrt{r^{2}-a^{2}}}-\int_{a}^{r} \frac{f^{\prime}(z)}{\sqrt{a^{2}-a^{a}}} d x\right\}
$$

Dieser Ausdruck rerschwindet jedenfalls fitr $a=0$, und es findet sich also an dieser Stelle unter allen Umständen ein Wendepunkt; es ist aber nicht nothwendig, dass dies der einzige Wendepunkt ist, deu die Lichteurve haben kann. Seeliger hat naehgewiesen, dass, weun das Belenchtungsgesetz z. B. die willkürliche Form hätte $f\left(\sqrt{x^{z}+y^{2}}\right)=x^{2}+y^{2}$, die allerdings durchaus unwahrscheinlich ist, weil nach ihr die scheinhare Helligkeit in der Mitte der Scheibe gleich Null sein miisste, dann drei Wendepunkte anstatt des einen auftreten.

Der Umstand, dass jedes beliebige Belenchtungsgesetz auf eine Lichtcurve fubrt, die in der Mitte der Verfinsterung einen Wendepunkt besitzt. an dieser Stelle also gradlinig verliuft, lïsst es fiur die praktische Verwerthung vou photometrischen Beobachtungen eines solchen Phïnomens empfehlenswerth erscheinen, wie schon von Corn n hervorgehoben worden ist, die Messungen sämmtlich anf denjenigen Moment zn reduciren, wo die Lichtstärke des Trabanten halb so gross ist, wie vor dem Beginn der Verfinsterung, d. l. also anf den Zeitpunkt, wo der Mittelpunkt der Trabantenscheibe durch den Mantel des Tangentenkegels hindurehigeht, welcher rom Mittelpunkte der Sonne aus an den Jupiter gelegt werden kann. 


\section{Capitel III. \\ Die Extinction des Lichtes in der Erdatmosphäre.}

Die unsere Erde umgebende Lufthiille iibt auf das von den Gestirnen zu uns gelangende Licht eine absorbirende Wirkung aus. Die Sterne erscheinen uns in der Ebene schwächer als auf hohen Bergen, und auf diesen wieder schwächer, als es ohne das Vorhandensein einer Atmosphäre der Fall sein wiurde, und auch an ein und demselben Orte rariirt die Helligkeit eines Sternes mit seiner Erhebung iiber den Horizont. Je weiter er rom Zenith entfernt ist und je grösser der Weg ist, den die Lichtstrahlen in der Erdatmosphäre zu durchlaufen haben, desto stärker ist auch die Absorption, welche dieselben erfahren. Aus den photometrischen Messungen geht herror, dass in nnmittelbarer Nähe des Horizontes ein Stern bereits mehr als 95 Procent von seinem urspriinglichen Lichte eingebüsst hat und $\mathrm{nm}$ mehrere Grössenclassen schwiicher erscheint als im Zenith.

Es ist klar, welch wichtige Rolle die Extinction in der Astrophotometrie spielt, und dass eine möglichst genaue Bestimmung dieses Reductionselementes eine der ersten Grundbedingungen für die Ausfiihrung ron branchbaren photometrischen Messungen am Himmel ist. Der Gegenstand ist dither auch stets mit dem grössten Eifer sowohl anf theoretischem als auch auf praktischem Wege rerfolgt worden, und wir besitzen bereits eine riemlich nmfangreiche Litteratur iiber denselben. Leider stellen sich einer vollkommenen Lösung der Anfgabe Hindernisse verschiedener Art entgegen. die zum Theil ganz nniiherwindlich sind. Sie beruhen einmal auf der nicht geniigenden Kenntniss der Ausbreitung unserer Atmosphäre und des (iesetzes, nach welehem die Dichtigkeit derselben mit der Höhe abnimmt, dann aber vor Allem auf den Verändertugen, denen die Zusammensetzung unserer Lufthïlle infolge der neteorologischen Vorgänge bestiindig unterworfen ist. Temperatur. Luftdruck und Fenchtigkeitsgrehalt der Luft wechseln ohne Aufhören und modificiren die Absorptionsfäligheit der Atmosphäre. Man wird daher nicht mur an rerschiedenen Orten der Erdoherfliche. sondern anch an demselben Orte zu rerschiedenen Jahresziten, unter I mständen sugrn zu rerschiedenen Stunden des Tages, eine andere Wirkung der Extinction erwarten können. Dazu kommt der sainzlich uncontrolirbare Einfluss. den namentlich in den tieferen Schichten der Atmosphäre lorale Terhailtnisse, unter anderen die Anwesenheit von stanb- und Rambly rartikekhen, ansiben. Erschwerend 
wirkt auch der Umstand, dass beim Durchgange des Lichtes dureh die Atmosphäre neben der Quantität desselben auch die Qualitït Änderungen erleidet, indem die versehiedenen Strahlengattungen, aus denen es znsammengesetzt ist, ungleich dureh die Luftsehichten beeinflusst werden. Dic Wahrnehmung, dass alle Gestirne, wenn sie in die Nähe des Horizontes kommen, röthlich gefürbt erscheinen, dentet darauf hin, dass die brechbareren Liehtstrahlen viel mehr dureh die Atmosphäre absorbirt werden als die rothen; infolge dessen werden auch die Extinctionserselieinungen verwickelter, wenn man es nieht mit homogenem Liehte zu thun hat, sondern, wie es bei den Sternbeobachtungen der Fall ist, mit zusammengesetztem.

Eine einheitliche Theorie kaun allen diesen störendeu Einflussen unmöglich gerecht werden, und man wird sieh daher begntigen müssen, den theoretischen Betrachtungen einen idealen mittleren Zustand der Erdatmosphäre zu Grunde zu legen. Eine gewisse Verwandtsehaft des Problems mit dem Refractionsproblem springt sofort in die Augen; es liegt daher nahe, diejenigen Annahmen uiber die Constitution der Atmosphäre, welche allgemein bei der Behandlung der Refraction acceptirt worden sind, aueh auf die Extinetion anzuwenden, d. l. also in erster Linie vorauszusetzen, dass die Atmosphïre aus eoncentrischen Schichten besteht, deren Dichte und Absorptionsvermögen nach einem regelmässigen Gesetze von der Erdoberfläche nach anssen zu abnimmt.

Zwei Fragen sind es vornehmlieh, die uns bei dem vorliegenden Probleme interessiren: 1) Nach welehem Gesetze nimmt die Helligkeit eines Sternes rom Zenith bis zum Horizonte ab? und: 2) Welches wiirde die Lichtintensität eines Sternes sein, wenn die Atmosphïre gar nicht vorhanden wäre? Die Beantwortung der ersten Frage ist fiir die praktische Astrophotometrie von der höehsten Bedentung, weil daron die Möglichkeit abhängt, Messungen von Sternen, die rersehiedene Zenithdistanzen haben, mit einander zu vergleichen. Die zweite Frage hat mehr theoretisches als praktisches Interesse; ihre Beantwortung verspricht Aufsehluss dariiber, wie die raumdurchdringende Kraft unserer Fermröhre olme Vorhandensein der Atmosphäre zunehmen und welcher Gewinn der Astronomie eventuell schon dureh Errichtung ron festen Beobachtungsstationen auf hohen Bergen erwachsen wiirde.

Lambert1) und Bouguer ${ }^{2}$ ), die beiden Begrinder der wissenschaftliehen Photometrie, sind die ersteu gewesen, welche das Extinctionsproblem theoretisch und praktisch zu lösen rersucht haben. Die von ihnen

1) Lambert, Photometria sive de mensura et qradibus luminis, colorum et nmbrae. Deutsche Ausgabe von E. A nding, Heft 2, p. 61. (Ostwald's Klassiker Nr.32.,

2) Bonguer, Traité d'optique. Livre III, section 4, p. 315. 
alggeleiteten Formeln haben bis heutigen Tages Bedeutung behalten, und der aus deu Bouguer'schen Messungen hervorgehende Werth des Durehlisssigkeitscoefficienten der Erdatmosphïire gilt als einer der besten für diese wiehtige Constante.

line noch eingehendere und rationellere Behandlung hat das Problem durch Laplacel) erfahren, welcher sieh dabei stieng an die der Refractionstheorie zu Grunde liegenden Voranssetzungeu angesehlosseu hat. Seine Theorie ist in neuerer Zeit in einigen Punkten dureh Maurer ${ }^{2}$ ) Ind Hansdorff ${ }^{3}$ ) modificirt worden, ohne dass damit jedoch ein wesentlicher Fortschritt eryielt worden wäre.

Im Folgendeu sollen die cinzelnen Extinctionstheorien etwas näher behindelt werden, und es wird sich empfehlen, unmittelbar daran eine kurze Besprechung der wichtigsten Ergebnisse der praktischen Astronomie :uf diesem Gebicte anzuschliessen, insbesondere die ron Seidel in München, sowie die von mir in Potsdam und auf dem Säntis ausgefiihrten Arbeiten mit den Resultaten der Theorie zu vergleichen. Auch wird es wünsehenswerth sein, wenigstens in aller Kiirze auf die wichtigen Untersuchungen Langley's einzugehen, in welchen die Frage von einem ganz nenen interessiunten Gesichtspunkte ans betrachtet wird.

\section{Die Lambert'sche Extinctionstheorie.}

Fis stelle in Figur $23 A A^{\prime} B b^{\prime}$ die vou parallelen Ebenen begrenzte Schieht eines vollkommen homogenen Mittels dar, und $a b$ bezeichne den IV eg eines Lichtstrahles durch diese Schicht. Man kann sich die absorbirende Wirkung des Mittels so denken, dass der Lichtstrahl beim Durchlaufen einer unendlich kleinen Strerke $d s$ stets einen gleich grossen Theil ron derjenigen Intensität, die er an Anfange der Strecke besass, verliert. Driekt man diesen Lichtverlust durch den constiuten Faetor $\lambda$.

ans, nenut die Intensitiit des Lichtstraliles, venn er im Punkte $P$ angelangt ist. i und wem er im l'unkte $I^{\prime r}$ math Inrchlitufen des Weges ds angelamgi

1) Laplace, Mécanique cúleste. Tul. IV. 1. 282.

2) Manrer, Die Extinction des Fixstemlichtes in der Atmosphäre in ihres leziehung zur astron. Refaction. Diss. iname. Zürich, 1482.

3 . Ferichte der K. Siichs. Gen. der Wiss. Jahrg. 1S95, Heft 4. p. 401. 
ist, $i-d i$, so hat man olune Weiteres: $d i=-\lambda i$. Die Grösse $\%$ ist unendlich klein, und man kann sie durch die ebenfalls unendlich kleine Grösse $d s$ ersetzen, wenu man diese letatere mit einor gewissen positiven Constante $y$ nultiplicirt. Daun erhailt man:

$$
d i=-v d s i
$$

orler durch Integration:

mithin:

$$
\log i=-\nu s+\text { Const. }
$$

$$
i=C e^{-r s} \text {. }
$$

Bedeutet noch $J$ die Intensitiit des Lichtstrahles beim Eintritte in die Schicht im Punkte $a$, wo $s$ gleich Null ist, so hat man $J=C$ und mithin $i=J e^{-r s}$. Die Grösse $r$, welehe für das betrachtete homogene Mittel charakteristiseh ist, nenut man den Extinctions- oder Absorptionscoefficienten der Substan\%. Ersetzt man noch $e^{-r}$ durch eine andere Constante $c$, so geht die Gleichung in die allgemein gebräuchliche tiber:

$$
i=J c^{i} \text {. }
$$

Darin heisst $c$ der Trumsmissions- oder Durchliissigkeitscoefficient der Substanz; er bezeichnet das Verhältniss der nach Durchlaufen der Wegeinheit austretenden Lichtmenge zu der in dieselbe eindringenden. Ist das Medium, in welches der Lichtstrahl eintritt, nicht homogen, so bleibt " keine Constante, sondern ändert sich ron Punkt zu Punkt auf dem Wege $s$; man wird im Allgemeinen haben $\nu_{s}=f(s)$. Aus der Gleichmog (1) folgt dann :

$$
\log i=-\int \nu_{s} d s+\text { Const. }
$$

und wenn man das Integral zwischen den Grenzen 0 und $s$ bildet, wobei an beachten, dass $\log i$ für $s=0$ in $\log J$ ibergeht, so hat man:

$$
\log \frac{i}{J}=-\int_{0}^{s} v_{s} d s .
$$

Diese Gleichung ist numittelbar anf das Extinctionsproblem anwendbatr; wenn man die Erdatmosphäre aus lanter unendlich schmalen, concentrischen Luftschichten zusammengesetzt denkt, deren Absorptionsfihigkeit von der Erdoberflaiche an mit der Höhe bestaindig abnimmt. Ist das Geset\% dieser Abnahme bekannt, so liisst sich die Aufgabe streng lisen. Lambert hat nun die rereinfachende Voraussetzung gemacht, dass der Weg des Lichtstrahles durch die Atmosphïire als greradlinig zu betrachten sei. In aller Strenge ist diese Ammahme nur für solche Strahlen zuliassigr, die senkrecht in die Atmosphäre eindringen. also für Sterne im Zenith des 
Beobachtungsortes. Je weiter die Sterne vom Zenith entfernt sind, desto stärker weicht der Weg der Lichtstrahlen infolge der Brechung in den einzelnen Luftsehichten von der geraden Linie ab, und in unmittelbarer Nilie des Horizontes ist die Refractionskriimmung bereits sehr merklich. Dadureh, dass Lambert die Brechung in der Atmosphäre vernachlässigt, findet er die Wegliingen dureligängig zu klein, und daher müssen auch die nach seiner Formel bereehneten Extinetionen im Allgemeinen zu klein sein; indessen ist der Fehler vom Zenith bis zu wenigen Graden über dem Horizont kamm merklich.

In der nebenstehenden Figur 24 bedeute $A A$ die Oberfliiche der Erde,

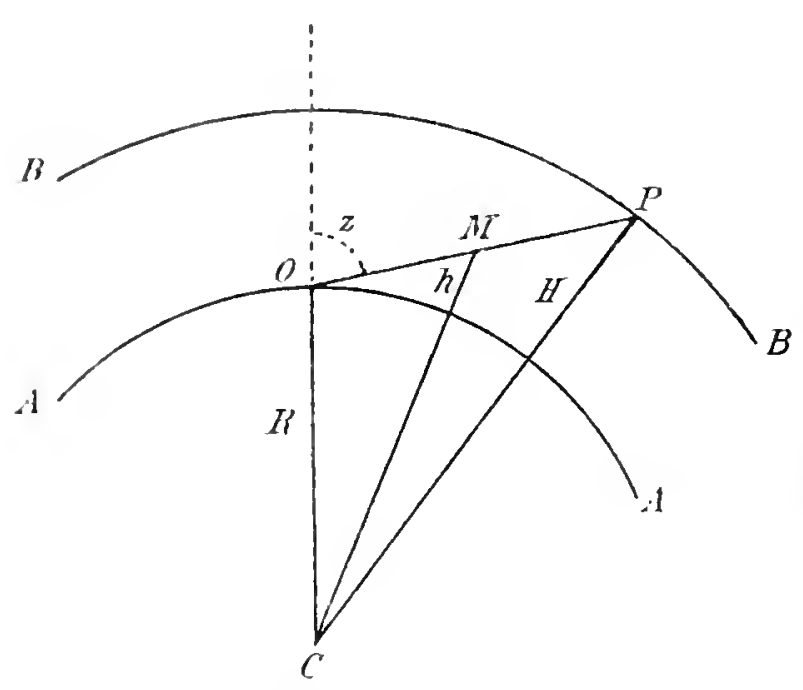

Fig. 24. $B B$ die Grenze der Atmosphäre. Die Höhe der letzteren sei mit $H$ bezeichnet und der Radius der Erde mit $R . \quad P O$ sei der Weg eines Lichtstrahles, weleher bei $P$ in die Atmosphäre eindringt und in $O$ die Erdoberflaiche erreieht; derselbe bilde mit der Zenithrichtung $C O$ den Winkel $\therefore$ Endlich sei II ein Punkt auf dem Wege des Lichtstrahles, dessen Abstand vom Erdmittelpunkte $R+h$ heissen möge. Bezeichnet man noch die Strecke $M O$ mit $s$, so hat man in dem Dreicek $M O C$ :

$$
(R+h)^{2}=R^{2}+s^{2}+2 R s \cos i
$$

woraus sich ergiebt:

$$
s=-R \cos z=\sqrt{R^{2} \cos ^{2}{ }^{2}+h^{2}+2 R h} .
$$

Da s eine positive Linge sein muss, so kann nur das positive Vorzeichen der Wurzelgrosse in Frage kommen. Setzt man noch $h^{2}+2 R h=y^{2}$ : so wird:

$$
s=-R \cos s+\sqrt{y^{2}+R^{2} \cdot \cos ^{2} x},
$$

und durch Differentiation:

$$
d s=\frac{y d !}{\sqrt{y^{2}+R^{2}} \cos ^{2}} .
$$

Wir wollen nmn rie llellinkeit eines Lidhtstrables beim Eintritt in die Atmosphïre $J$ und hei der Ankunft an der Erdoberfliche $J_{z}$ nennen, fermer 
noch die ganze Weylïnge in der Atmosphäre unit $S$ bezeiehnen; danı erhält man ans Gleiehumg $\langle 3\rangle$ ummittelbar:

$$
\log \frac{J_{s}}{J}=-\int_{0}^{s} v_{s} d s=+\int_{s}^{0} v_{s} d s,
$$

oder wenn man den obigen Werth von $d s$ substituirt:

$$
\log \frac{J_{z}}{J}=\int_{s=s}^{s=0} v_{s} \frac{y d y}{\sqrt{y^{2}+R^{z} \cos ^{2} z}} .
$$

An der Erdoberfliche (fur $s=S$ ) wird $h$ und mithin auch $y=0$; an der Grenzeder Atmosphäre dagegen (fur $s=0$ ) geht $h$ in $H$ iber, und der entsprechende Werth von $y$, der mit $Y$ bezeiehnet werden möge, wird $=\sqrt{H^{2}+2 R H}$. Mithin kommt:

$$
\log \frac{J_{x}}{J}=\int_{0}^{r} v_{s} \frac{y d y}{\sqrt{y^{2}+R^{2} \cos ^{2} r}} .
$$

Nun ist $\frac{1}{\sqrt{y^{2}+R^{2} \cos ^{2} z}}=\frac{\sec z}{\sqrt{R^{2}+y^{2}+y^{2} \operatorname{tang}^{2} \approx}}$, oder durch Reihenentwicklıng

$=\sec \approx\left[\left(R^{2}+y^{2}\right)^{-\frac{1}{2}}-\frac{1}{2}\left(R^{z}+y^{2}\right)^{-\frac{3}{2}} y^{2} \tan g^{2} z+\frac{1 \cdot 3}{2 \cdot 4}\left(R^{z}+y^{2}\right)^{-\frac{3}{2}} y^{4} \operatorname{tang}^{4} z-\cdots \cdot\right]$; folglich ergiebt sich aus (4) die Gleichung, welche unter dem Namen der Lambert'schen Extinctionsgleichung bekannt ist:

(5) $\log \frac{J_{s}}{J}=A \sec z-\frac{1}{2} B \sec z \operatorname{tang}^{2} z+\frac{1 \cdot 3}{2 \cdot 4} C \sec z \operatorname{tang}^{4} z-\cdots$, wo die Coefficienten $A, B, C \ldots$ die Werthe haben:

$$
\begin{aligned}
& A=\int_{i}^{Y} \frac{x_{s} y d y}{\left(R^{2}+y^{2}\right)^{\frac{1}{2}}}, \\
& B=\int_{i}^{Y} \frac{v_{s} y^{3} d y}{\left(R^{2}+y^{2}\right)^{\frac{3}{2}}}, \\
& C=\int_{i}^{F} \frac{x_{s}^{\prime} y^{5} d y}{\left(R^{2}+y^{2}\right)^{\frac{5}{2}}} \text { u. s. w. }
\end{aligned}
$$

Für das Zenith eines Beobachtungsortes geht die Lambert'sche Extinctionsformel(5), wenn man die Helligkeit des Sternes im Zenith mit $J_{0}$ bezeichnet, in den einfachen Ausdruck iiber:

$$
\log \frac{J_{0}}{J}=A
$$


Der Coefficient $A$ ist also der Logarithmus der Zahl, welche angiebt, wievielmal sehwächer ein Stern im Zenith eines Beobachtungsortes erscheint, als wenn gar keine Atmosphäre vorhanden wäre. Man nennt diese Zahl gewöhnlich den Transmissionscoefficienten der gesammten Erdatmosphäre.

Aus den Gleichungen (5) und (6) erhält man noch:

(7) $\log J_{0}-\log J_{z}=A(1-\sec z)+\frac{1}{2} B \sec \approx \operatorname{tang}^{2} \approx-\frac{1 \cdot 3}{2 \cdot 4} C \sec \approx \operatorname{tang}^{4} \approx+\cdots$

Diese Form ist fiur die praktisehe Verwendung in der Astrophotometrie dic bequemste. Sie licfert unmittelbar die sogenaunte Zenithreduction, die man meistens dureh of $(i)$ bezeichnet, d. h. die Grösse, die zu einem bei beliebiger Zenithdistanz beobachteten Helligkeitslogarithmus hinzugefügt werden muss, um den fiir das Zenith giiltigen Helligkeitslogarithmus zu erhalten. Die Coefficienten $A, B, C \ldots$ in der Lambert'schen Formel lassen sich nur damn berechnen, wenn man eine Annahme über die Höhe der Erdatmosphäre nnd iiber die Änderung des Absorptionscoefficienten $\nu_{s}$ mit der Länge des durehlanfencu Weges macht. Lambert hat dieses Verfahren nicht eingeschlagen. sondern mur daranf hingewiesen, dass man anf rein empirischem Wege, ans photometrischen Beobachtungen eines und desselben Sternes in rerschiedenen Höhen über dem Horizonte, numerische Werthe fiir die Coefticienten $A, B, C$. . ableiten kann. Anf diese Weise erhiilt man allerdings eine Formel, die den Gang der Extinction ziemlich gut bis nahe an den Horizont darstellt; ans mehrjährigen photometrischen Messungen in Potsdam sind fiir die beiden ersten Coefficienten der Lambertschen Formel die Werthe gefunden worden:

$$
\begin{aligned}
& A=-0.050+11, \\
& B=-0.0000911 .
\end{aligned}
$$

Es ist aber klar, dass die so bestimmte Lambert'sche Extinctionsformel nur den Werth einer blosken Interpolationsfurmel haben kann, und dass ihr keinerlei physikalikche Bedentung zukommt.

Beiliunfig bemerkt, ereiebt sich nit dem angefiilnrten Zahlenwerthe ron $A$ ans $\langle 6\rangle$ der Transmiscionscoefticient der gesammten Erdatmosphäre zn 0.5309 , d. h. die Atmosphizre absorlint 17 Procent ron dem senkrecht in sie eindringenden Lichte.

2. Die Bouguer'sche Extinctionstheorie.

Weit mindlicher als ron Lambert ist das Extinctionsproblem rou Bouguer behandelt worden. Obgleich anch er den Weg des Liehtstrahles 
in der Atmosphäre als geradlinig annimmt, so hat doch seine Endformel einen höheren Werth als den einer blossen Interpolationsformel, weil er seine Theorie anf eine bestimnte Voraussetzung llber die Abnalume der Dielıtigkeit mit der Höhe der Atmosphïre grllndet. Die ans seinen Untersuchungen hervorgehende Extinctionstabelle ('Traité d'optique, p. 332) weicht bis zu Zenithdistanzen ron mehr als $80^{\circ}$ noch nicht merklich von den besten neueren Extinctionstabellen ab. Sein Verfahren ist das folgende. Es stelle (Fig. 25) $A A$ die Erdoberfliache dar, $G G$ die Grenze der Atmosphïre, $B B$ eine beliebige Schicht derselben in dem Abstande $x$ von der Erdoberfläche. $O$ ist ein Punkt der ErdoberHïche, $C$ der Mittelpunkt derselben, derErdradiusheisse $a$. Bonguer uirnmt nun fur die ganze Atmosph hïre das Mariottesclie Gesetz als gilltig an, er vernachlissigt die Temperaturabnahme mit der Höhe und setzt also ganz allgemein: $\frac{\varrho}{\varrho_{0}}=\frac{p}{p_{0}}$, wenn $\varrho$ und $p$ Dichtigkeit und Druck an irgend einem Punkt der Atnosphäre, $\varrho_{0}$ und $p_{0}$ die entsprechenden Grössen an der Erdoberfliache vor-

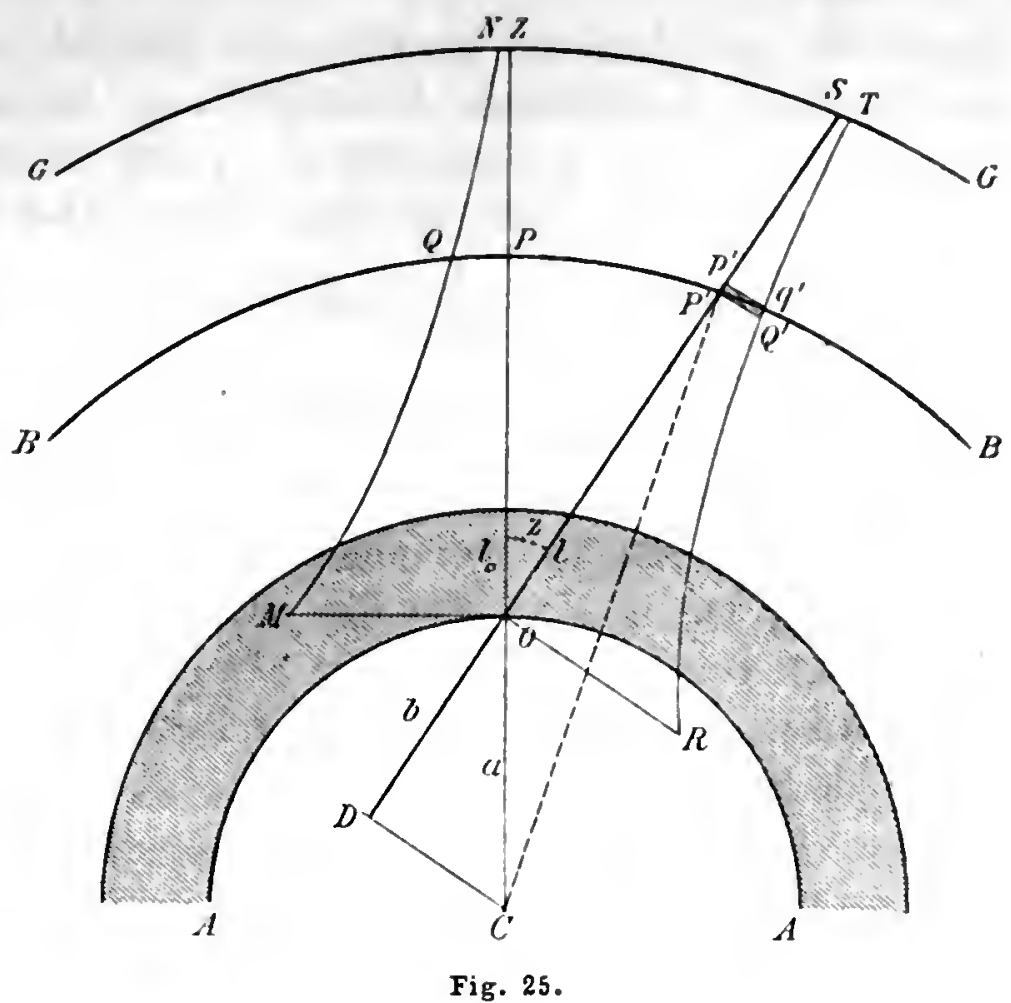
stellen. Auf der nach dem Zenith $Z$ gerichteten Linie $O Z$, die als $A$ bscissenaxe betrachtet werden soll, mögen num in den einzelıen Punkten die zugehörigen Dichtigkeiten $Q$ als Ordinaten aufgetragen werden; dureh die Endpunkte derselben sei eine Curve $M Q N$ gelegt, die an der Grenze der Atmosphäre, wo die Dichtigkeit unendlich klein ist, die Abscissenaxe fast beriihrt. Die von dieser Curve und der Axe $O Z$ begrenzte Fläche $O M N Z$ ist offenbar der gesammten iiber dem Beobachtungsorte $O$ ruhenden Luftmasse proportional, und ebenso ist das Flaichenstiick $P Q N Z$ dem in $P$ geltenden Luftdrucke oder, was dasselbe ist, der iiber $P$ befindlichen Luftmasse proportional. Da nach dem Mariotte schen Gesetz diese beiden Flächenstiicke sich wie die zugehörigen Urdinaten $I L()$ und $Q P$ verhalten sollen, so ergiebt sich leicht, dass die Curve $M Q N$ eine logarithmische Linie scin muss, deren Gleichung die Form liat: $y=g_{0} q^{x}$, wo $q$ eine 
Constante bedentet. Diese Curve hat die besondere Eigenschaft, dass in allen Punkten derselben die Subtangente $y \frac{d x}{d y}$ einen constanten Werth lıat. Die Fliiche $O M N Z$ wird ausgedriickt durch das bestimmte Integral $\int_{y=0}^{y=} y d x$, und da aus der Gleichung der Curve ohne Weiteres folgt: $\frac{d y}{y}$ $=d x \log q$, so wird die gesammte über $O$ ruhende Luftmasse gegeben lureh $\frac{Q_{n}}{\log q}$. Bouguer denkt sich nun die gesammte Atmosphäre ersetzt durch eine gleichmaissig absorbirende Schicht, welche iiberall die an der Erdoberfliche herrschende Dichtigkeit $\varrho_{0}$ besitzt. Bezeichnet man die Höhe derselben mit $l_{0}$, so wird die ganze Luftmasse iiber $O$ auch ausgedriiekt durh $\varrho_{0} l_{0}$, und es folgt daher, dass die Höhe der homogenen Atmosphiare gleich ist $\frac{1}{\log q}$, a. h. gleich dem constanten Werthe der Subtangente $y \frac{d x}{d y}$. Der numerische Werth dieser Subtangente lässt sich angen:ihert ans Beobachtungen des Luftdruckes in versehiedenen Höhen iiber der Erdoberfliche bestimmen, und Bonguer hat selbst aus Beobachtungeu vou De la Hire für die Höhe der homogenen Atmosphäre die Zahl 3911 Toisen $=7623$ Meter abgeleitet, ein Werth, der, wie auch schon Boun w nelbst ans eigenen Beobachtnngen gesehlossen hat, ein wenig zn klein sein diirfte.

Es sei num weiter $\$ O$ der als geradlinig angenommene Weg eines Lichtstrahles, der mit der Zenithrichtung $Z O$ den Winkel z bildet. Trägt man wieder in jedem Punkte der Linie $S O$ senkreeht zu ihr die der betreffenden Lufterhicht entsprechende Dichtigkeit anf und legt durch die Endpunkte dieser Senkrechten eine Curve, so reprïisentirt die Fläche ORTS, die mit $F$ bezeichnet werden möge, die gesammte Luftmasse, welche das Licht bei der Zenithdistanz $\approx$ zu passiren hat. In der ron Bouguer substituirten homogenen Atmosphüre durchläuft das Lieht die Weglänge l, und es kamn daher die gesammte Luftmasse auch dureh $g_{0} l$ ansgedriekt werden. so dass man hat $F=Q_{0} l$.

Es handelt sich mu darum, den Fliicheninhalt $F$ zn bereehnen. Die Linie $S O$ werde iiber O hinams rerliingert und rom Erdmittelpunkte $C$ eine Senkrerhte C'D anf diese Linie gefällt; das Stiiek $O D$ möge mit $b$ bezeichnet sein. Wir betrachten mu das Flächenelement $P^{\prime} Q^{\prime} p^{\prime} q^{\prime}$. Man hat:

oder:

$$
P^{\prime} D^{2}=\left(P^{\prime} Q^{2}-(C D)^{2},\right.
$$$$
P^{\prime} D^{2}=\|+r^{2}-\left(a^{2}-b^{2}=b^{2}+2 a x+x^{2}\right. \text {. }
$$ 
Mithin wird:

$$
d\left(P^{\prime} D\right)=P^{\prime} p^{\prime}=\frac{(a+x) d x}{\sqrt{b^{2}+2 a x+x^{2}}} .
$$

Die Strecke $P^{\prime} Q^{\prime}$ entspricht der Luftdichtigkeit im Punkte $P^{\prime}$; wir wollen die Dichtigkeit in irgend einem Punkte nach Bouguer mit $\mathfrak{g}_{0}(1-u)$ bereiehnen, wo $u$ an der Erdoberfliche den Werth 0 und an der Grenze der Atmosphiäre den Werth 1 hat; damn ist das Flächenelement $P^{\prime} Q^{\prime} p^{\prime} q^{\prime}$ gegeben dureh:

$$
\frac{\varrho_{0}(1-u)(a+x) d x}{\sqrt{b^{2}+2 a x+x^{2}}}
$$

und die gesammte Flïehe $F$ wird bestimmt dureh:

$$
F=\varrho_{0} \int_{u=0}^{u=1} \frac{(1-u)(a+x) d x}{\sqrt{b^{2}+2 a x+x^{2}}} .
$$

Um $x$ als Function von $u$ zu haben, beachte man, dass nach dem Obigen $l_{0}=y \frac{d x}{d y}$ und $y=\varrho_{0}(1-u)$ zu setzen ist. Daraus folgt: $d y=-\varrho_{0} d u$, wobei das negative Zeichen zu vernachlässigen ist, weil es nur aussagt, dass $y$ wäehst, wenn $u$ abnimmt, und weil es hier nur anf die absolute Weglänge ankommt. Man hat daher:

$$
d x=\frac{l_{0} d u}{1-u},
$$

und daraus dureh Reihenentwieklung und Integration:

$$
x=\operatorname{lo}_{0}\left\{u+\frac{u^{2}}{2}+\frac{u^{3}}{3}+\cdots\right\} \text {. }
$$

Substituirt man die Wertlıe ron $d x$ und $x$ in die obige Gleichung fuir $F$ und entwickelt die Quadratwurzel im Nenner in eine Reihe, so findet man nach Ausfiuhrung der Integration:

$$
F=o_{0} l_{0}\left[\frac{a}{b}-\frac{a^{2}-b^{2}}{2 b^{3}} l_{0}+\left(a^{3} l_{0}-a b^{2} l_{0}-\frac{1}{3} a^{2} b^{2}+\frac{1}{3} b^{4}\right) \frac{l_{0}}{2 b^{5}} \cdots\right] .
$$

Ans der Figur ergielot sich, dass $b=a \cos \therefore$ ist. Fiihrt man diesen Werth ein und ersetzt noch nach Obigem $F$ durch das Product $\varrho_{0} l$, so erhält man endlich:

$$
l=l_{0}\left[\sec z-\frac{l_{11}}{2 a} \tan ^{2} z \sec z+\left(l_{0}-\frac{1}{3}\left(\cos ^{2} z\right) \frac{l_{0} \tan ^{2} z}{2 a^{2} \cos ^{3} x} \cdots\right] .\right.
$$

Diese Gleichung ermöglicht es, für jede Zenithdistanz die Weglänge in der supponirten homogenen Atmosphlïre zu berechnen, falls die Höhe derselben $l_{0}$ als bekannt vorauszusetzen ist. Bis zu Zenithdistanzen ron etwa 
$\$ 2^{\circ}$ erhält man daraus $l$ genau genug, wenn man sich auf die drei ersten Glieder der Reihe beschriinkt. Dariber hinaus wird die Berechnung unsicher, und für $:=90^{\circ}$, also im Horizonte, versagt die Formel giinzlich. Um die Weglänge im Horizonte zu berechnen, hat Bouguer die Gleichung (S) unter der Berlicksiclitigung, dass $b=0$ wird, entwickelt, und findet so fur die gesuchte Grösse die unendliche Reihe:

$$
\left(2 a l_{0}\right)^{\frac{1}{2}}-\frac{a l_{0}-3 l_{0}^{2}}{6\left(2 a l_{0}\right)^{\frac{1}{2}}}-\frac{7 a^{2} l_{0}^{2}-18 a l_{0}^{3}+15 l_{0}^{1}}{120\left(2 a l_{0}\right)^{\frac{3}{2}}}-\cdots
$$

Mit Zingrundelegung des oben erwähnten Werthes ron $l_{0}=3911$ Toisen hat Bouguer eine Tafel berechnet, aus welcher für jede Höhe eines Gestirnes die Weglainge in der lomogenen Atmosphäre, ausgedriickt in Toisen, entnommen werden kimn.

Die Einfuihrung der homogenen Atmosphäre und die Berechnung der Weglängen in derselben ermöglicht num sofort die Lösung des Extinctionsproblems. Fiir ein homogenes Medium gilt dic einfache Gleichung $i=J c^{s}$, worin $s$ die durchlaufene Wegstrecke, $c$ den sogenannten Transmissionscoefficienten für die Läingeneinheit, $J$ die Helligkeit beim Eintritte und $i$ die llelligkeit beim Terlassen der Strecke $s$ bedentet. Nennt man nun $J_{z}$ die an der Erdoberfliche beobachtete Lichtstiirke eines Sternes bei der Zenithdistanz ¿, $J$ seine Helligkeit ansserhalb der Atmosphäre, so hat man fiir die homogen gedachte Atmosphäre:

$$
J_{z}=J c^{l} \text {. }
$$

Fiir das Kenith wird:

$$
J_{0}=J_{C^{l_{1}}}
$$

und darams folgt:

$$
l_{0} \log c=\log \frac{J_{i 1}}{J}
$$

Dils Verhiiltniss $\frac{J_{0}}{J}$ der Helligkeit eines Sternes im Zenith zu seiner Helligkeit ansserhalb der Atmosphäre nennt man, wie schon im vorigen Paragraphen erwähut ist, den Durchlissigkeitseofficienten der gesammten Atmosphïire. Bezeichet man denselben mit $p$. so lat man $\log p=l_{0} \log c$. lits 10$)$ ind (11) folgt:

mithin :

$$
\log \frac{J_{11}}{J_{z}}=\left(I_{0}-1\right) \log c
$$

$$
\log \frac{J_{0}}{J_{z}}=-\log p\left(\frac{l}{I_{0}}-1\right)
$$


Dies ist die Bonguer'sche Fxtinctionsgleichung in derjenigen Form, welche fur die Berechnung am bequemsten ist. Setzt man fur $\frac{l}{l_{0}}$ den Wertlı aus (9) ein, so sielit man, dass die Bouguer'sche Formel mit der Lambert'schen in der Fonn nahe libereinstimmt. Wiihrend aber die Lambert'seho Theorie an dem wichtigsten Punkte stehen bleibt und die Bestimmung der einzelnen Coefficienten lediglich den Beobachtungen uberlisst, hat die Bouguer'sche Formel physikalische Bedeutung, und das einzige hypothetische Element bleibt die Ermittelung der Höhe $l_{0}$ der homogenen Atuosphläre. Der Transmissionscoefficient $p$ kann nach der Gleichung (12) aus zwei Helligkeitsmessungen desselben Gestirnes bei versehiedenen Zenithdistanzen bestimmt werden, wenn die zugehörigen Weglängen nach (9) berechnet sind. Bouguer liat selbst aus photometriselien Beobachtungen des Vollmondes bei Zenithdistanzen ron $70^{\circ} .7$ und $23^{\circ} .8$ den Werth $p=0.8123$ abgeleitet und mit Hilfe dieses Werthes eine Tabelle berechnet, ans welcher die Helligkeiten eines Sternes bei beliebigen Zenithdistanzen entnommen werden können, wobei die Helligkeit ansserhalb der Atmosphäre mit 10000 bezeichnet ist. (Traité d'optique, p. 332.)

Die Bouguer'schen Tabellen gelten für einen Beobachtungsort im Niveau des Meeres, und die Wegliinge $l_{0}$ entsprielit der ganzen Masse der Atmosphäre. Für einen höher gelegenen Beobachtungsort ist die darüber befindliche Luftmasse geringer und die Helligkeit der Sterne wird grö̈sser. Anch die Abnahme der Lichtstïrke vom Zenith nael dem Horizonte zu ist an einem solchen Beobachtungsorte geringer. Betrachtet man den Punkt $P$, so ist die dariber ruhende Luftmasse repräsentirt dureh die Flaiche $P Q Z N$; man kann sich dieselbe wieder ersetzt denken dureh eine homogene Luftmasse von der Diehtigkeit $\varrho_{n}$, deren Höhe $l_{0}^{\prime}$ sein mïge. Es verhalten sich aber die iber $P$ und $O$ befindlichen Luftmassen wie die Dichtigkeiten an diesen beiden Orten oder anch wie die entsprechenden Barometerstände, welche $b$ und $b_{0}$ heissen mögen. Man hat also:

und daher:

$$
\varrho_{0} l_{0}^{\prime}: \varrho_{0} l_{0}=b: b_{0}
$$

$$
l_{0}^{\prime}=\frac{l^{\prime}}{b_{0}} l_{0} \text {. }
$$

Man kamn demmach für jeden Ort, dessen Barometerhöhe l gregeben ist, die Höhe der entsprechenden homogenen Atmosphïre berechnen, wenn die fuir das Mecresniveau geltende Höhe $l_{0}$ bekannt ist. Die Wegliangen $l^{\prime}$ am Beobachtungsorte $P$ fiir beliebige Zenithdistanzen ergeben sich aus Gleichung (9), weun man $l_{0}$ dureh $\frac{b}{b_{0}} l_{0}$ ersetzt, und man kann daher für jeden Ort mit Leichtigkeit die Zenithreduction $p(x)$ vorausberechnen. 
Ist noch $J_{0}^{\prime}$ die Zenithhelligkeit eines Stcrnes am Beobaehtungsorte $P$, so ist nach Gleichung (11) $J_{0}^{\prime}=J c^{l_{s}}$ oder $\log \frac{J_{0}^{\prime}}{J}=l_{0}^{\prime} \log c$. Da aber anch $\log \frac{J_{0}}{J}=I_{0} \log c$ ist, so hat man:

$$
\log \frac{J_{0}^{\prime}}{J}: \log \frac{J_{0}}{J}=l_{0}^{\prime}: l_{0}=b: b_{0} .
$$

Bezeichnet man dic Grösse $\frac{J_{0}^{\prime}}{J}$, d. h. den Transmissionscoefficienten der iiber $P$ befindlichen Luftmasse, mit $p^{\prime}$, so wird:

$$
\log p^{\prime}: \log p=b: b_{0},
$$

d. h. die Logarithmen der Transmissionseoefficienten für zwei Beobachtungsorte verhalten sich wie die entspreehenden Barometerstände.

\section{Die Laplace'sche Extinctionstheorie.}

Wie schon oben bemerkt wurde, schliesst sich die ron Laplace anfgestellte Extinctionstheorie eng an die Refractionstheorie an und unterscheidet sich ron der Lambert'sehen und Bouguer'schen Behandlung des

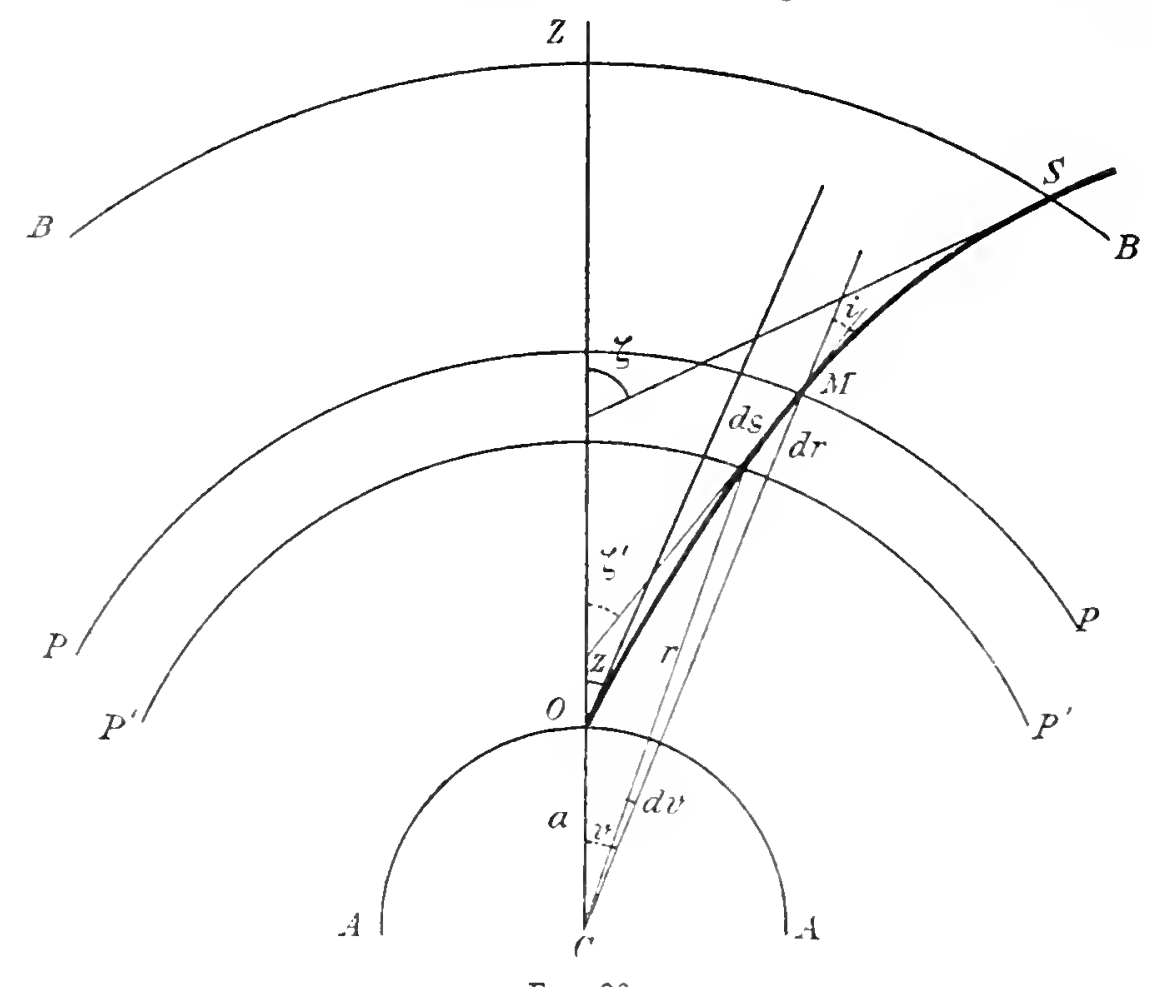

Problems wesentlich dadurch, dass sie ant die Krimmmng des Weges, welchen die Lichtstrahlen in der Atmosphïre durchla ufen, Riicksicht nimmt. Es bedente in Figur 26 A die Erdubertiiche. $B B$ die Grenze der Atmosphäire, P P $P^{\prime} P^{\prime}$ ene unendlich diinne Schicht derselben, deren Abstand 
von Centrum der Erde $r$ sein möge. In $S$ trete ein Lichtstrahl in die Atmosphäre und erreiche in $O$ die Erdoberfliche. Der Winkel, den die Tangente an die Refractionseurve im Punkte $S$ mit der Zenithrichtung $O Z$ bildet, ist die wahre Zenithdistanz $\zeta$, wïhrend der Winkel der Tangente im Punkte $O$ mit der Richtung $O Z$ die scheinbare Zenithdistany $\approx$ darstellt. Streng genommen mlisste die wahre Zenithdistan\% anch von dem Punkte $O$ ans gereehnet werden, indessen ist der Untersebied bei der verhïltnissmässig geringen Höhe der Atmosphäre und der mässigen Krümmung der Refractionseurve, sowie bei der bedeutenden Entfernung der Himmelskörper nur geringfügig und selbst beim Monde fast ganz »u vernachlässigen. Der Lichtstrahl trifft die in Betracht zu ziehende Schicht in $M$, der Einfallswinkel an der Grenze der Sehicht sei $i$, und der innerhalb derselben \%uriickgelegte Weg, der als geradlinig aufzufassen ist, sei $d s$. Nennt man noch " den Brechungsexponenten aus dem luftleeren Raume in die betrachtete Sehicht, so gilt die bekannte der Refractionstheorie zu Grunde liegende Gleichung:

$$
r \mu \sin i=\text { Const. }
$$

Ftir einen Punkt der Erdoberfliiche geht $i$ in die scheinbare Zenithdistanz : uber, $r$ in den Erdhalbmesser $a$, und der Brechungsexponent erhält den Werth $\mu_{0}$; man hat also auch:

$$
a \iota_{0} \sin x=\text { Const. }
$$

Nennt man die Helligkeit des Liehtstrahles, wenn er in $M$ angelangt ist $J_{z}$, so hat man nach Gleichung (1):

$$
\frac{d J_{\bar{z}}}{J_{\bar{z}}}=-r d s,
$$

wo $\nu$ der Absorptionscoefficient der unendlich sehmalen Luftschicht $P P P^{\prime} P^{\prime}$ ist. Man kann sich nun vorstellen, dass die Absorption dureh die saimmtlichen Massentheilehen hervorgebracht wird, anf welche der Lichtstrahl beim Durchlaufen der Strecke $d s$ triff't, und es ist daher klar, dass die Absorption um so grösser sein wird, je grösser die Anzahl der im Wege stehenden Partikelchen ist, d. h. je dichter das Medium ist. Man wird daher den Absorptionscoefficienten der Dichtigkeit $Q$ proportional annchmen diurfen und erhäilt dann dureh die Substitution $"=$ li $\underline{Q}$ die Gleichung:

$$
\frac{d J_{\bar{z}}}{J_{\tilde{z}}}=-l_{i} Q d s \text {. }
$$

Die Weglänge $d s$ lïsst sich nach der Figur ansdriicken durch:

$$
d s=\frac{d r}{\cos i}
$$


mithin wind:

$$
\frac{d J_{z}}{J_{z}}=-\operatorname{sic} \frac{d r}{\cos i^{\circ}}
$$

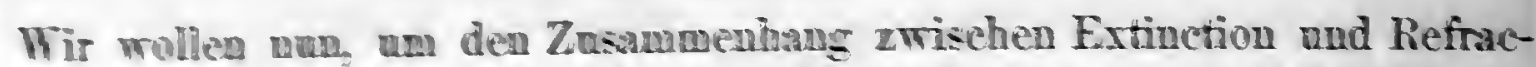
bion näber darealegen, die Refractionsenerte betrachten aud zunächst in $d s$ eine Twpreste su dioselbe legen, welche mit der Normalen $O Z$ den Winkel sै bilden wugre. Dam ist $==r+i$, within $d \xi=d r+d i$. Man nennt dit das Elemeat der Refraction. Ans Gleichung (14) ergiebt sich durch logariohmische Differentiativa:

$$
\begin{aligned}
& \frac{d r}{\pi}+\frac{d u}{d}+\cos i d i=0 \text {. }
\end{aligned}
$$

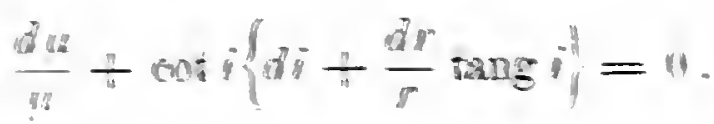

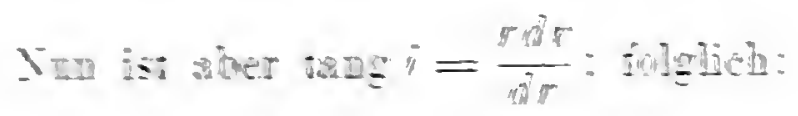

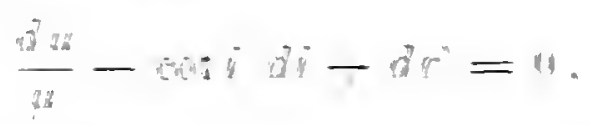

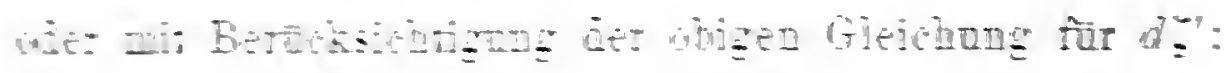

$$
y=-\frac{1}{2 x} \sin 2=
$$

Z ज

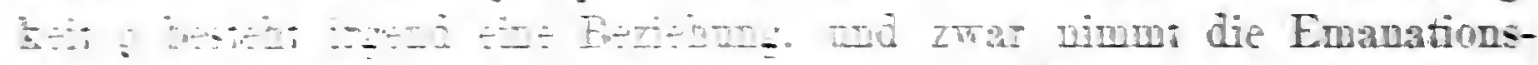

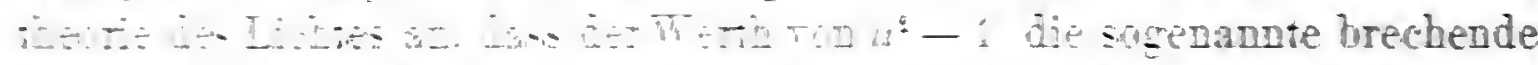
E.t.

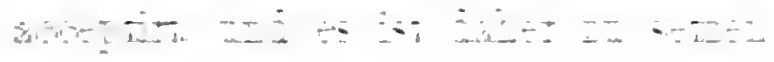

I tos

$$
\frac{i}{i}=\frac{i}{i}
$$

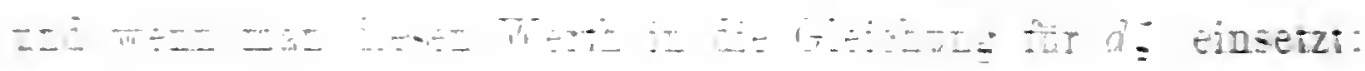

$$
\because=-\frac{2}{2} \cdot 3
$$

$\therefore=0$

$$
\because=\tau
$$


Um de in dieser Gleichung durch andere Grüssen auszudrucken, muss man das Gesetz keunen, nach welchem die Luftliehtigkeit mit der Höhe uber der Erdoberfluche abnimmt. Man hat dabei Folgendes zu beachten. Es reien $p$ und $\varrho$ Luftdruck und Dichtigkeit an einem Punkte der Atmosphäre, der vom Erdmittelpankte die Entfernung $r$ besitzt, $g$ sei die entiprechende Scliwere; dann ist die Änderung des Luftdruckes $d p$ fur eine Änderung des Abstandes $d r$ bekanntlich gegeben durch die Gleichung:

$$
d p=-g \varrho d r,
$$

und da nach dem Gravitationsgesetze $g=g_{0}\left(\frac{a}{r}\right)^{2}$ ist, wo $g_{0}$ die Sebwere an der Ërdoberfläclıe bezeichnet, so wird:

$$
d p=-g_{0}\left(\frac{a}{r}\right)^{2} e d r .
$$

Nennt man noch $l_{0}$ die Höhe einer Luftsåule von der Dichtigkeit $\varrho_{0}$, welche dem an der Erdoberfläcbe stattfindenden Drucke $p_{0}$ das Glcichgewicht hält, so ist:

$$
p_{0}=\varrho_{0} g_{0} l_{0}
$$

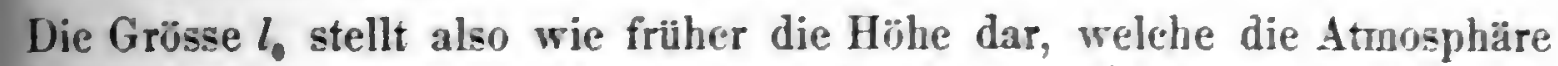
haben wỉrde, wenn sie durchweg die Dichtigkeit der untersten Schichten besåsse. Es folgt nun:

$$
\frac{d p}{p_{0}}=-\left(\frac{a}{r}\right)^{2} \frac{Q}{Q_{0} l_{0}} d r
$$

Tach dem Mariotte'schen Gesetz, welches Laplace ebenso wie Pouguer für die ganze Atmospluäre als guiltig annimmt, ohne die Temperatur abnahme mit der Höhe za berücksichticen. ist aber:

nnd folglich anch:

$$
\frac{p}{p_{0}}=\frac{Q}{\varphi_{0}}
$$

$$
\frac{d p}{p_{0}}=\frac{d \varrho}{\varrho_{0}} .
$$

Durch Substitution in 19 wird daber:

$$
d Q=-\left(\frac{a}{r}\right)^{2} \frac{Q}{l_{g}} d r .
$$

nnd Tenn man diesen Werth in 15, einsetzi, so ergieft sich:

$$
d=\frac{c \mu_{0}}{2 l_{0} \mu^{3}}\left(\frac{a}{r}\right)^{3} \sin : \frac{0 d r}{\cos i}
$$


Die beiden Gleichungen (17) und (20) geben nun eine Beziehung zwisehen Extinetion und Refraction. Man erhält durch Elimination von $\frac{\varrho d r}{\cos i}$ sofort:

$$
\frac{d J_{g}}{J_{g}}=-\frac{2 k l_{0}}{c \mu_{0}} \frac{\mu^{3}}{\left(\frac{a}{r}\right)^{3}} \frac{d \zeta^{\prime}}{\sin \approx}
$$

Die Grösse $\frac{\mu^{3}}{\left(\frac{a}{r}\right)^{3}}$ wird ohne erheblichen Fehler gleich 1 gesetzt werden diirfen; denn bei der verhältnissmäissig geringen Ausbreitung der Erdatmosphäre unterscheidet sich $r$ nur wenig von $a$, und der Brechungsexponent " weicht ebenfalls nur wenig von der Einheit ab (an der Grenze der Atmosphäre ist $u=1$, an der Erdoberfläche ist $\mu_{0}=1.000294$ ). Daher wird:

$$
\frac{d \cdot J_{\tilde{z}}}{J_{z}}=-\frac{2 l_{i} l_{0}}{c_{i} \mu_{0}} \frac{d \zeta^{\prime}}{\sin \nu}
$$

oder wenu man $\frac{2 k l_{0}}{c \mu_{0}}$ durch eine nene Constante $K$ ersetzt:

$$
\frac{d J_{z}}{J_{z}}=-\frac{K}{\sin z} d z
$$

Lies ist die sogenamute Laplace'sche Extinctionsformel. Integrirt man dieselbe uiber die sämmtlichen Schichten der Atmosphäre, nennt die Helligkeit des Lichtstrahles ausserhalb der Itmospläre $J$ und bezeichnet mit $»$ Refraction $*$ den Gesammtbetrag der Refraction bei der Zenithdistanz $\approx$, so erhïilt man:

$$
\log \frac{J_{z}}{J}=-\mathrm{sin}^{-} \times \text {Refraction. }
$$

Nun wird allgemein der Werth der Refiaction gegeben dureh den Ausdruck $\varepsilon_{z} \operatorname{tang}{ }^{2}$, wo der Zahlenwerth von $a_{z}$ aus den bekamnten Refractionstafeln zu entuehmen ist. Man hat daher auch:

$$
\log \frac{J_{z}}{J}=-h^{2} \cos _{z} \text { se. }
$$

Fiir $x=0$ geht diese Gleichnng iiber in:

$$
\log _{J} J_{0}=-K \iota_{0} .
$$

Aus (22) und (23) erhält man eudlich, weun man den Transmissions- 
coefficienten der gesammten $\Lambda$ tmosphïre $\frac{J_{0}}{J}$ wie fruher mit $p$ bezeichnet, die Laplace'sche Zenithreduction $p(*)$ in der Form:

$$
p(z)=\log \frac{J_{0}}{J_{z}}=-\log p\left\{\frac{\alpha_{z}}{c_{0}} \sec z-1\right\} .
$$

Aus der Refractionstheorie ergiebt sich noch, dass die Grösse $\alpha_{z}$ durch eine Reihe ausgedrlickt werden kamn, die nach Potenzen ron tang ${ }^{2}:$ fortschreitet. Man hat nitmlich:

$$
\alpha_{z}=\alpha_{0}\left\{1+a \text { tang }^{2} z+b \text { tang }^{4} z+c \text { tang }^{6} z+\cdots\right\},
$$

wo die Cöefficienten $a, b, c \ldots$ Constanten sind, deren numerische Werthe je nach den Hypothesen, die man über die Abnahme der Temperatur in der Atmosphäre machen will, versehieden sind. Setzt man den Werth furr $a_{x}$ in die obige Gleichung (22) ein, so geht dieselbe iiber in:

$\log \frac{J_{z}}{J}=-K \alpha_{0} \sec z-K \alpha_{0} a \sec z \operatorname{tang} z-K \alpha_{0} b \sec \approx \operatorname{tang}{ }^{4} z-\cdots$,

welehe der Form n:ch ganz mit der Launhert'schen Extinctionsformel (5) thereinstimmt.

Stellt man den Laplace sehen Ausdruck der Extinetion (24) dem Bougnerschen (12) gegeniiber, so sieht man, dass die beiden vollkommen identiseh werden, wenn man das Verhältuiss der Weglïngen $\frac{l}{l_{0}}$ dureh $\frac{\alpha_{\varepsilon}}{u_{0}} \sec \approx$ ersetzt. Die Laplace'sche Grösse $a_{z}$ see $\approx$ entspricht also der jedesmaligen Weglïnge in der homogen gedachten Atmosphäre, wenn die Weglïnge im Zenith als Einheit gew:ïllt ist. Da aber die Laplace'sche Theorie auf die Krimmung des Weges Rüeksicht nimmt, so ist einleuchtend, dass die so ausgedrückten Weglängen den Vorzug vor den Bonguer'schen rerdienen, und dass hierin der Fortschritt der Laplace'sehen Extinctionstheorie zu erblicken ist. Bis zu Zenitlddistanzen rou etwa $85^{\circ}$ weichen ubrigens die Bonguer'schen Werthe nur so nubedentend von den Laplacesehen ab, dass es fuir die Praxis vollkommen gleichgiiltig ist, welehe man benutzt. In jedem Falle setzt die Bereehnung der Zenithreduction of $(x)$ die Kenntniss des Transmissionseoefficienten $p$ vorans, der durch Beobachtungen desselben Gestirnes in rerschiedenen Zenifldistanzen crmittelt werden kann.

Die gebräuchlichen Refractionstafeln geben die Werthe ron $\omega_{z}$ firr einen gewissen mittleren Zustand der Atmosphï̈re, also für einen bestimmten Barometerstand und eine bestimmte Temperatur. So gelten die Bessel'schen Tafeln fuir einen Luftdruck ron 751.5 Millimeter und fiur 903 Celsius. Un die Werthe $\alpha_{z}$ für einen beliebigen anderen Zustand der Atmosphüre 
zu erhalten, hat man nach Bessel die mittleren Werthe mit dem Ausdrucke $(B \times T)^{d} \gamma^{\lambda}$ zu multipliciren, wo $A$ und $\lambda$ Grössen sind, die von der scheinbaren Zenithdistanz abhängen, wälurend $B$ dem Barometerstande proportional ist, $\gamma$ von der 'Temperatur der Luft (der insseren Temperatur) und $T$ ron der Temperatur am Barometer (der imneren Temperatur) abhängt. Nlle diese Grössen sind ron Bessel in Tafeln gebracht, und es ist daher leicht, die Werthe $\alpha_{z}$ und daher anch die Zenithreduction $\varphi(:)$ fiir jeden Beobachtungsort zu berechnen, wenn man ansserdem den Transmissionscoeffieienten für denselben kemnt. Dal aber $\log p=-\hbar \alpha_{0}$ ist und $K=$ $\frac{2 l_{l} l_{0}}{c \|_{0}}$, endlich noch $l_{0}$ dem Luftarucke $p_{0}$ proportional ist, so folgt, ehenso wie bei der Bouguer'sehen Extinctionstheorie, dass der Logarithmus des Transmissionscoefficienten dem jedesmaligen Barometerstande proportional ist und daher für jeden Ort im Voraus berechnet werden kamn, wenn er fiur irgend einen Ort, z. B. fuir das Meeresnireau, aus Beobachtungen bestimmt worden ist.

\section{t. Die Manrer'sche Extinctionstheorie.}

In newerer Zeit ist ron J. Manrer in Zürich eine Bearbeitung des Fatinctinsproblems rersucht worden, welehe ebenfalls die Refractionstheorie zu Huilfe nimmt, sich aber von der Laplace'schen Behandlung im Nosentlichen darin muterscheidet. dass sie fïr die Beziehung zrischen dem Brechnugsexponenten ", einer Luftsehicht und der zugehörigen Dichte $\boldsymbol{g}$ den Ausdruek aceptirt:

$$
" 1-1=c Q .
$$

wibluend laphace nach den Auschammon der Emanationstheorie des Lichtes die Dichtigkeit proportinul der sogenamten brechenden Kraft $11^{2}-1$ setzt. Ohyleich die Frage noh keineswegs endgiiltig entschieden ist. so surechen doch die meisten Tutersudhungen, besonders die ron Dale und Gladstome. Lambolt. Mascat angestellten, mehr zu Gunsten der ersteren Beziehmos; und der von Manrer cingeschlagene Wese hat daler seine volle Berechtizune.

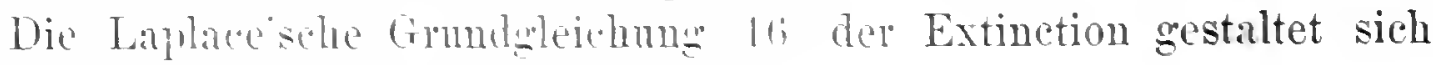
mit der Manrersoben Amalne nm in:

$$
2-1
$$

wem noch - i durch eime nene cinstante $C$ ersetzt ist. In Figur 27 ist II ein Punkt anf dem Wege des Lichtstrahles durch die Atmosphäre 
an der Grenze zweier unendlich dunnen Schichten derselben; der in der unteren Schicht durchlanfene Weg sei ds. Verlingert man die 'Tangenten an die Wegcurve und fillt ron $C$ :us die Senkrecliten $C D$ und $C E$ anf dieselben, so ist, weun $C D$ mit $t$ bezeicluet wird, $C E=t+d t$. Der Winkel qwischen den 'T:ungenten ist gleich dem Element der Refraction, welehes mit $d$ (Refr.) be\%ciclsnet werden soll. Man laat nun in dem unendlich sehmalen Dreicek $M E F$ mit geniigender Genanigkeit:

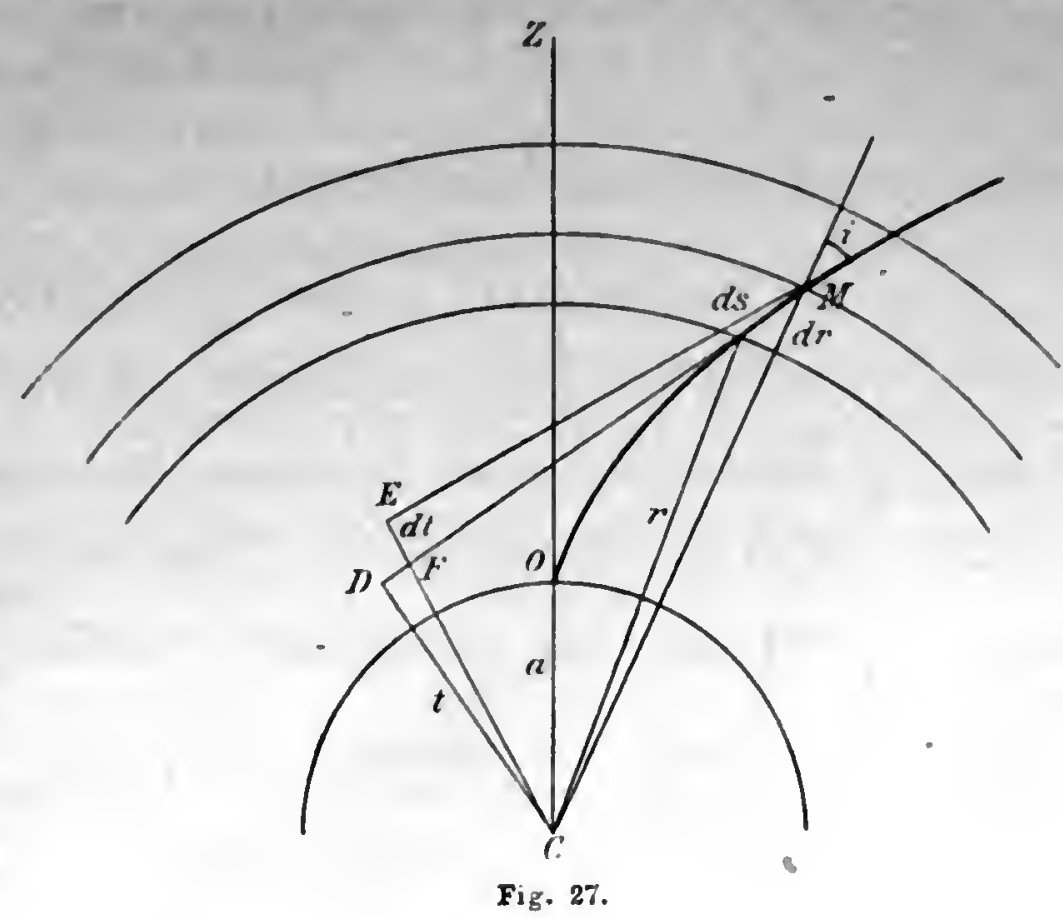

Ferner ist:

$$
d(\text { Refi })=\frac{d t}{\sqrt{r^{2}-t^{2}}} .
$$

oder auch:

$$
d s=\frac{d r}{\cos i}=\frac{r d r}{\sqrt{r^{2}-t^{2}}},
$$

mithin auch:

$$
d s=\frac{t d t}{\sqrt{r^{2}-t^{2}}}+\frac{r d r-t d t}{\sqrt{r^{2}-t^{2}}},
$$

$$
d s=t d(\text { Refr. })+\frac{r d r-t d t}{\sqrt{r^{2}-t^{2}}} .
$$

Setzt man diesen Werth in die obige Gleichung $(25)$ ein und erlanbt sich die Vereinfachung, in dem Factor " 1 statt des ron Schicht zu Schicht verïnderlichen Werthes von " iiberall einen Mittelwerth "' einzufiilnen, so erhält man durch Integration iiber die ganze Atmosphicire:

(26) $\log \frac{J_{\bar{z}}}{J}=-C\left(\iota^{\prime}-1\right)\left[\int t d(\operatorname{Refr} \cdot)+\int \frac{r d r-t d t}{V r^{2}-t^{2}}\right]_{\text {Erdoberfäule }}^{\text {Grenze der Atmosphäre }}$,

wo $J$ wieder dic Helligkeit ausserhalb der Atmosphïire bedentet, und wo die Integrale zwischen denjenigen Grenzen in Bezng anf $r$, Ind die Refraction zu nehmen sind, die der Erdoberfläche und der Ilöhe der gesammten Atmosphiare entsprechen. Ua $t=r \sin i$ oder mit Beriicksich- 
tigung der Gleichungen (14) und (15) $t=\frac{a \mu_{0} \sin \approx}{\mu}$ ist, so sind die Grenzen in Bezın anf $t$ an der Erdoberfläche $a \sin \%$ und an der Grenze der Atmosphäre $a \mu_{0} \sin \%$ In Bezıng auf $r$ sind die Grenzen, wenn die Höhe der Erdatmosphäre mit $H$ bezeichnet wird, resp. $a$ und $a+H$. Der VTerth des zweiten Integrales wird damn

$$
=\sqrt{(a+H)^{2}-a^{2} \mu_{0}^{2} \sin ^{2} z}-a \cos \%
$$

Das erste Integral hat nach Substitution des Werthes von $t$ die Form " $\sin \approx \int \frac{\underline{\prime}_{0}}{\|} d$ (Refr.). Da $\mu$ an der Grenze der Atmosphäre $=1$, an der Erdoberfliche $=1.000294$ ist, so wird man keinen sehr grossen Fehler begehen, wemı man den veränderlichen Quotienten $\frac{\mu_{0}}{\|}$ innerhalb des Integralzeichens durch den constanten Werth $\frac{\mu_{0}+1}{2}$ ersetzt; damit wird aber der Werth des ersten Integrales sofort

$$
=a \frac{\mu_{0}+1}{2} \sin : \times \text { Refr. }
$$

wemn man unter »Refr. " die für die gesammte Atmosphäre bei der scheinharen Zenithdistanz : griltige Refraction versteht. Man hat nun:

(27) $\log _{J} J_{\tilde{z}}=-C\left(\prime^{\prime}-1\right)\left[a \sin : \frac{\prime_{0}+1}{2} \times\right.$ Refr. $\left.+\sqrt{(a+H)^{2}-a^{2} u_{0}^{2} \sin ^{2} x_{n}}-a \cos r\right]$.

Fiir : $=0$ geht dieselbe iiber in:

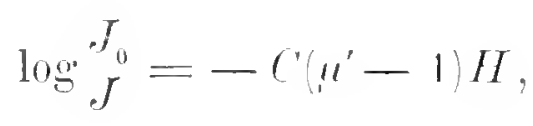

und es folgt daher durch subtraction, wenn man wieder den Transmissimsonefticienten $y=\frac{J_{0}}{J}$ einfiilnt:

$$
y(:)=\operatorname{lng} \frac{J_{11}}{J_{3}}
$$

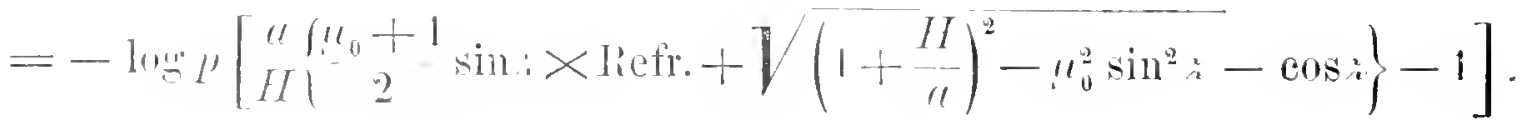

Dies ist die Mantrersche Endformel der Extinction. Sie hat vor der Laplace-

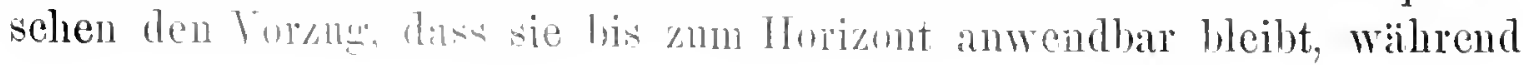


jene fur $z=90^{\circ}$ unendlich grosse Werthe fur $\varphi(z)$ ergiebt. Der schwache Punkt der Maurer'schen Theoric liegt darin, dass sie dureh Einfuhrung eines mittleren Werthes fur " jeder Hypothese Uber dic Änderung des Brechungsexponenten von Luftschicht zu Luftschicht ans dem Wege geht. Die Maurer'sche Formel wird daher in diesem Sinne anch nur als eine Interpolationsformel zu betracliten sein. Die Anwendung derselben verlangt Ubrigens noch eine bestimmte Annalıme Hber das Verhuiltniss der Atmosphärenhöhe zum Erdradius. Maurer sehlägt dafur die Zahlıl ifo vor und vertritt die Ansieht, dass wenn anch wirklich die Atmosphäre sich weiter als etwa 64 Kilonneter ansbreiten sollte, die jenseits dieser Grenze befindlichen Luftschichten bereits so unendlich dinn sein inlissten, dass ihr Einfluss anf die Refraction und Extinction unter allen Umstiinden zu vernachliassigen wäle.

\section{Vergleichung der Theorien mit den Beobachtungsergebnissen. Die Durchlïssigkeitscoefficienten der Erdatmosphäire.}

Die Bestimmung der fur die praktische Astrophotometrie uberaus wichtigen Zenitlureductionen ist auch auf rein empirischem Wege versucht worden und zwar zuerst ron Seidel in Minchen. Derselbe benutzte dazu die Helligkeitsvergleiehungen, welche er in den Jahren 1S\$4-18.18 zwischen den Fixsternen erster Grösse mit Hülfe des Steinheil'schen Prismenphotometers angestellt hatte. Indem er das am liäfigsten beobachtete Sternpaar (Wega und Capella) answiilhlte, ermittelte er \%unïchst aus denjenigen Vergleichungen, wo die Zenithdistanzen beider Sterne nahe gleich waren, einen vorliiufigen Wertlı fuir das wahre IIelligkeitsverhïltniss derselben und erhielt damm mit Zugrundelegung dieses Werthes aus den Hbrigen Beobachtungen eine Reihe von Bestimmungen für verschiedene Differenzen $p\left(x_{1}\right)-\tau\left(x_{2}\right)$, ans denen sich durch ein Nihermngsverfalnen eine vorlaufige Extinctionstabelle alleiten liess. Mit Anwendung dieser vorlänfigen Tabelle anf die anderen beobachteten Sternpare ergab sich dann durch wiederholte Ausgleiehmngen und Interpolationen die definitive Extinctionstabelle ${ }^{1}$, welehe die siimmtlichen Messmugen relativ an besten darstellte. Seidel hat diese Tafel später noch an einem grösseren Beobachtungsmaterial, welehes sich auf 208 Fixsterne ron der ersten bis zur fïnften Grössenclasse erstreckte, gepriift, wobei sich aber keine Veranlassung zu irgend welehen Ändermogen heransgestellt hat ${ }^{2}$.

1) Abhandl. d. K. Bayer. Akad. d. Wiss. II. Classe. Bd. 6, p. 581.

2) Abhandl. d. K. Bayer. Akad. d. Wiss. II. Classe, Bd. 9, p. 503. 
Wesentlich abweichend von dem Seidel'sehen Verfahren ist dasjenige, welches von mir bei der empirischen Ableitung der Extinctionstabelle für Potsd:am in den Jahren 1879-1881 angewandt worden ist. Mit Hülfe des 'Zölluer'schen Photometers wurden fünf hellere Sterne ( $\alpha$ Cygni, $\eta$ Ursae majoris, $\delta$ Persei, a Aurigae und a Tauri) bei möglichst vielen Zenithdistanzen mit dem Polarstern verglichen, für welchen wegen der geringen Änderungen seiner Höhe uber dem Horizonte $\varphi(z)$ nahezu constant angenommen werden kann. Aus dem sehr umfangreichen Beobachtungsmaterial wurde nun zunïchst für jeden einzelnen Stern dureh ein graphisches Verfalren einc Extinctionseurve bestimmt, und aus der Vereinigung dieser fünf Limzelenrven wurde dann nach Ansgleichnng der Differenzen die inittlere Extinctionstabelle fiir Potsdam bis zur Zenithdistanz $80^{\circ}$ hergeleitet. Bei grösseren Zenithdistanzen als $80^{\circ}$ war das Verfahren etwas anders. Es wurden helle Gestirne, meistens Planeten, beim Anf- oder Unterg:mace beobachtet. Ans der Vergleichung je zweier Messungen dessclhen Objectes ergaben sich dann Werthe von $r\left(x_{1}\right)-r\left(x_{2}\right)$ für :lle möglichen Werthe der Zeuithdistanzen $\approx_{1}$ und $\approx_{2}$ zwischen $80^{\circ}$ und ss ${ }^{\circ}$, aus denen sich wach der Methode der kleinsten Quadrate der wahrscheinlichste Verlanf der Extinctionseurve zwischen $80^{\circ}$ and $88^{\circ}$ \%enithdistinz ermitteln liess. Beide Curventheile wurden endlich an cinauder gefiigt, und so entstand die Extinctionstabelle für Potsdam '). lis ist noch zn erwïlnnen, dass die Messnugen für den zweiten Theil ohne Ausuahme alu aussergewöhnlich klaren Tagen angestellt worden sind, wïhreud die Vergleichungen der fünf Sterne mit dem Polarstern anch an mittelmäissig' g'uten Tagen ausgeführt wurden. Infolge dessen entsprechen die beiden Theile der Tabelle streng genommen nieht ein und demselben mittleren Luftzustande. Fiir die praktische Verwendung der Tabelle wird diese kleine Ungleichmässigkeit eher zum Vortheil als zum Schaden sein, weil man es im Allgemeinen streng vermeiden wird, photometrische Beobachtungen in der Nïhe des llorizontes bei anderem als dem allerbesten Luftunstande auszuführen, wïhrend man sich nicht schenen wird, in Höhen iiber $10^{\circ}$ auch bei weniger ausgezeichneter Luft zu heobachten. Dagegen darf bei allen theoretischen Untersuchungen, die sich anf die Potsdamer Tabelle stitzen, diese Ungleichmässigkeit, wie us bereits mehrfach geschehen ist, durchaus nicht ausser Acht geclassen werden.

Bei der Ableitung der cinzehneu Extinctionscurren fur die fünf Sterne ergaben sich Unterschiede zwischen denselben, die im Zusammenhange

I I'ubl. 1. Astrophys. (1bs zu Potsdam. Bd. 3. p. 255. 
Init der Farbe dieser Sterne zu stchen schienen. Die gesammte Lichtabnalıme vom Zenith bis zu $80^{\circ}$ Zenithdistan\% war an grössten bei de॥ gelben und röthlichen Sternen, am kleinsten bei den weissen, wilhrend mun von vornherein wegen dor stärkeren Absorption der banen Strahlen in der Atmosphüre elier das Gegentheil häitte erwarten sollen." Vielleicht ist diese Erscheinung, deren Realitiit noch weiterer Bestätigung bedarf, dureh physiologiselse Finflisse zu erklïren.

Die Vergleichung der mittleren Potsdamer Extinctionstabelle mit der Minchener zeigt im Grossen und Ganzen eine selır befriedigende Übereinstimmmug. Von 0 bis 50$)^{\mathbf{0}}$ Zenithdistan\% sind die Seidel'sehen Werthe "war durehgängig etwas kleiner, von 55" bis $79^{\prime \prime}$ etwas grösser als die Potsdamer, so dass man anf systematische Unterschiede schliessen könnte; indessen ist der numerisehe Betrag der Diflerenzen so geringfügig, dass er fiir die praktische Anwendung gar nicht in Betracht kommt, und dass his $\%$ Zenithdistanzen von etwa $50^{\circ}$ beide Tabellen als durehaus grleichwerthig anzusehen sind. Für grö̈ssere Zenithdistanzen als $80^{\circ}$ verdient die Potsdamer Tafel zweifellos den Vor\%ng, weil sie auf einer grösseren Anzahl von Beobachtungen in der Nähe des Horizontes berulit.

Bei Benutzung der empirischen Extinctionstabellen (der Miinchener oder Potsdamer), deren Brauchbarkeit anch an anderen Beobachtungsorten in der Nähe des Meeresniveans zur Geniige nachgewiesen ist, wird selbstrerstïndlich ein, so weit das Ange zu beurtheilen vermag, klarer und dunstfreier Himmel vorausgesetzt. Jede Staub- oder Dunstschieht muss eine betriiehtlich stärkere Lielitabnahme bedingen, und der Standpunkt des Beobachters (ob) auf freien Felde oder anf einem erhöhten Punkte oder inmitten einer grossen Stadt, wo Raueh- und Stanbtheilchen fast nie fehlen) ist von der grössten Wichtigkeit. Zweifellos werden anch bei scheinbar ganz reinem Himmel Schwankungen in der Absorptionswirkung der Atmosphü̈re vorkommen, die durch Änderungen des Lnftdrnekes, der Temperatur und namentlich des Fenchtigkeitsgehaltes hervorgerufen werden; man sollte infolge dessen zn verschiedenen dalıreszeiten an ein und demselben Orte Unterschiede in den Extinctionswerthen erwarten. Indessen sind diese Schwankungen verhailtnissmïissig so gering, dass sie nicht erheblich die muermeidliche Unsicherheit der photometrischen Messungen iibersteigen diirften. Das Richtigste wiire es, fuir jede Beobachtungsreihe durch besondere Messungen den Verlanf der Extinctionseurve zu bestimmen. Da aber ein derartiges Verfahren einen verhältnissmässig grossen Zeitanfwand erfordert, so wird man nur ansnalimsweise davon Gebrauch machen können. In den meisten Fällen wird man sich doch mit der mittleren Extinctionstabelle begniigen miissen, wobei nach Möglichkeit die Vorschrift innezuhalten ist, die Beobachtungen so 
zu arrangiren, dass grosse Unterschicde in den Zenithdistanzen iiberhaupt nicht vorkommen. Auch Vergleichungen von Gestirnen in sehr verschiedenen Azimuthen sind, wenn irgend ang:ingig, zu vermeiden, weil erfahrungsmïssig locale Verhältnisse, z. B. die Anwesenheit von grossen Wassermengen oder ansgedehnten Wäldern, den regelmässigen Verlauf der Extinction stören kïmen. Im Allgemeinen wird man behaupten diurfen, dass bei Anwendung der mittleren Extinctionstabelle innerhalb des Intervalles von 0) bis $60^{\circ}$ Zenithdistanz selten ein Fehler hervorgebracht werden kann, der gegeniblser der Ungenanigkeit der photometrischen Messungen selbst irgendwio ins Gewieht fiele.

Zur Vervollstïndigung sei noch erwähnt, dass ausser an den beiden in der Ebcne gelegenen Orten Mïnchen und Potsdam, anch noch anf einen 2500 Meter hohen Berggipfel (dem Säntis in der Schweiz) die Extinctionscurve durch ein umfangreiches Beobachtumgsmaterial von mir empirisch bestimunt worden ist $\left.{ }^{1}\right)$. Es wurde dabei das gleiche Beobachtungsverfahren wie in Potsdam eingeschlagen, nur mit dem Unterschiede, dass mehr Sterne (13 statt 5) benutzt und alle so weit wie möglich bis zum Ilorizonte verfolgt wurden. Das Sintismaterial ist daher weit homogener als dis Potsdamer, und da ausserdem auf dem hohen Berge gleichmilissigere Durchsichtigkeitsverhältnisse vorausgesetzt werden dürfen als in der Ebene, so eignet sich dieses Material am besten zu theoretischen Untersuchungen iiber die Extinction.

Im Anhange sind die mittleren Extinctionstabellen fuir Potsdam und fiir den Saintis ansfiilnlich mitgetheilt, und zwar sind die Zenithreductionen o $p\left(\begin{array}{l}n \\ 2\end{array}\right)$ sowohl in Helligkcitslogarithmen als in Grössenclassen angegeben.

Es fragt sich num, wie sich die empirisch bestimmten Extinctionstabellen zu den Ergebnissen der theoretischen Forsehung verhalten. Wir haben geschen, dass die Endgleiehnngen der verschiedenen Theorien sïmmtlich anf die Form gebracht werden können:

$$
\log \frac{J_{0}}{J_{\tilde{z}}}=-\log p[F(i)-1] \text {. }
$$

Darin bedentet fl durehweg den Transmissionscoeffieienten der ganzen Atmosphire, und $F(:)$ ist cine Function der Zenithdistmz, die je nach den Anmahmen iitrer die C'onstitntion der Atmosphäire nach den einzelnen Theorien verschiedene Zalnlenwerthe haben kimm.

Sieht man ron der Lambertsehen Interpolationsformel ab, so giebt die folgende kleine Tabelle eine Ühersicht der verschiedenen Werthe

1, Publ. d. Astropliys Obs. zu Potsilam. Bd. s, p. 1. 
von $F^{\prime}(*)$ oder, was dasselbe ist, der Wegliingen in der homogen gedachten Atmosphäre, sowie gleichzeitig der daraus bereehneten Werthe der Zenithreduetionen $p(z)$, wobei fur den Transmissionscoeffieionten $p$ der Werth 0.835 zu Grunde gelegt ist. Argument dieser Tabelle, welche fur Orte in der Nihe des Mecresnireans gilt, ist die scheinbare Zenithdistan\%. In der letzten Columne stehen zurVergleichung die der Potsdamer mittleren Extinetionstabelle entnommenen Werthe ron $r(x)$.

\begin{tabular}{|c|c|c|c|c|c|c|c|}
\hline \multirow{2}{*}{$\begin{array}{l}\text { Scheíbare } \\
\text { Zenitl1- } \\
\text { distanz- }\end{array}$} & \multicolumn{2}{|c|}{ Bonguer } & \multicolumn{2}{|c|}{ Laplace } & \multicolumn{2}{|c|}{ Maror } & \multirow{2}{*}{$\begin{array}{c}\text { Potsdamer } \\
\text { empirische } \\
\text { Tabelle } \\
y(l)\end{array}$} \\
\hline & $F(n)$ & $p(2)$ & $F(z)$ & $p(z)$ & $F(2)$ & $p(z)$ & \\
\hline $0^{\circ}$ & 1.000 & 0.000 & 1.000 & 0.000 & 1.000 & 0.000 & 0.000 \\
\hline 10 & 1.015 & 0.001 & 1.015 & 0.001 & 1.014 & 0.001 & 0.000 \\
\hline 20 & 1.064 & 0.005 & $1.06 \mathrm{i}$ & 0.005 & 1.064 & 0.005 & 0.004 \\
\hline 30 & 1.155 & 0.012 & 1.154 & 0.012 & 1.154 & 0.012 & 0.011 \\
\hline 40 & 1.305 & 0.024 & 1.304 & 0.024 & 1.300 & 0.023 & 0.024 \\
\hline 45 & 1.414 & 0.032 & 1.413 & 0.032 & 1.406 & 0.032 & 0.035 \\
\hline 50 & 1.556 & 0.044 & 1.553 & 0.043 & 1.546 & 0.1143 & 0.048 \\
\hline 55 & 1.742 & $0.05 \mathrm{~s}$ & 1.739 & 0.059 & 1.728 & 0.057 & 0.067 \\
\hline 60 & 1.990 & 0.078 & 1.993 & $0.07 \mathrm{~s}$ & 1.972 & 0.076 & 0.092 \\
\hline 65 & 2.350 & 0.106 & 2.354 & 0.106 & 2.315 & 0.103 & 0.128 \\
\hline 70 & 2.900 & 0.149 & 2.899 & 0.149 & 2.524 & 0.143 & 0.180 \\
\hline 72 & 3.200 & 0.172 & 3.201 & 0.172 & 3.108 & 0.165 & $0.20 \mathrm{~S}$ \\
\hline 74 & 3.550 & 0.202 & 3.579 & 0.202 & 3.442 & 0.191 & 0.241 \\
\hline 76 & 4.060 & 0.240 & 4.060 & 0.240 & 3.964 & 0.224 & $0.2 S 2$ \\
\hline 78 & 4.690 & $0.2 \$ 9$ & 4.694 & 0.289 & 4.397 & 0.266 & 0.3332 \\
\hline 80 & 5.560 & 0.357 & 5.563 & 0.357 & 5.084 & 0.320 & 0.394 \\
\hline 81 & 6.130 & 0.402 & 6.129 & 0.402 & 5.506 & 0.353 & 0.432 \\
\hline 82 & 6.520 & 0.456 & 6.S18 & 0.456 & 6.001 & 0.392 & 0.477 \\
\hline 83 & 7.670 & 0.522 & 7.676 & 0.523 & 6. .573 & 0.436 & 0.533 \\
\hline 84 & 5.770 & $0.60 \mathrm{~S}$ & 8.768 & 0.608 & 7.252 & 0.490 & 0.607 \\
\hline$\$ 5$ & 10.200 & 0.721 & 10.196 & 0.720 & 8.049 & 0.552 & 0.707 \\
\hline 86 & 12.140 & 0.572 & 12.125 & 0.871 & 8.957 & 0.625 & 0.516 \\
\hline 87 & 14.877 & 1.057 & 14.835 & 1.083 & 10.114 & 0.714 & 1.045 \\
\hline 85 & 19.030 & 1.412 & 18.835 & 1.397 & 11.435 & 0.817 & 1.3333 \\
\hline
\end{tabular}

Aus dieser Zusammenstellung geht zunaichst hervor, dass die Bouguersehen und Laplace'schen Werthe bis zu Zenithdistanzen ron etwa 8." rollkommen miteinander iibereinstimmen und erst ron da an grössere $\mathrm{Al}_{\text {)- }}$ weichungen ergeben, wie anch von vornherein zu erwarten ist, weil Bonguer für $:=90^{\circ}$ einen endliehen Werth ron of $(:)$ berechnet, während die Laplace'sche Theorie dafiir einen unendlich grossen W'erth ergiebt. Die Maurer'sche Theorie liefert durchwegr kleinere Wertle als die beiden 
anderen. Die Vergleichung der theoretischen Werthe von $p(x)$ mit der Potsdamer empirischen Extinctionstabelle zeigt fuir Boug u er und Laplace in Allgemeinen eine sehr befriedigende Übereinstimmung, da die Differenzen zwischen Rechnung und Beobachtung bis zu $87^{\circ}$ Zenithdistanz niemals den Betrag von 0.04 im Helligkeitslogarithmus oder von 0.1 Grössenclassen merklich iibersteigen. Zwar spricht sich in diesen Differenzen ein systematiseher Charakter ans, indem die beobachteten Werthe bis zur 'Zenithdistanz $84^{\prime \prime}$ durehweg" grösser, dariiber hinaus durehweg. kleiner sind als die nach den Theorien berechneten; aber dieser systematische Charakter ist wohl lediglich anf die oben erwähnte Ungleichmäissigkeit der Potsdamer Extinctionstabelle zuriiekzuführen; er versehwindet grinzlich, wie vor Kurzem von Kempf') nachgewiesen ist, wenn mail die Lalplace'sche Theorie anf dic beiden Theile der Potsdamer Tabelle getrennt anwendet. Was die Manrer'sche Theoric betrifft, so geniigt dieselbe nach der obigen Tabelle den Beobachtungen in keiner Weise. Wollte man nach dieser Theorie eine leidliche Übereinstimmung zwisehen den berechucten und beobachteten Extinctionswerthen $p(x)$ erzielen, so niisste man fiir den Transmissionscoefficienten $p$ statt der benutzten Zahl 0.S35 einen viel kleineren Werth (etwa 0.765) zu Grunde legen. Nach allen bisherigen Untersuchungen ist aber ein so kleiner Werth des Transnissionseoefficienten so gut wie ansgeschlossen.

Dic Laplace'sche Theoric verdicut jedenfalls ror allen anderen den Vor\%ug, und da die Berechnung der Extinctionswerthe mit Benutzung der bekannten Refractionstafeln ansserordentlich einfach ist, so steht ihrer :llgencinen Anwendung aif photometrische Vessungen Nichts im Wege. Lhre Branchlarkeit ist iilnrigens nicht nur fiur Beobachtungsorte in den mutersten Schichten der Itmosphïre, sondern, wie meine Untersuchungen :nuf dem Sintis gezeigt haben, anch fiir eine Meereshöhe von 2500 Meter dargethan. Dic Übereizstimmung zwischen Theorie und Beobachtung: ist in den höheren Luftschichten sogan noch besser als in der Ebene, Withrcheinlich weil dort ein idealerer Znstand der Atmosphaire stattfindet, namentlich alle verumreingenden Bestandtheile, wie Staub und Dunst, griuzlich felilen.

Ein Überblick iiber die im Anhange mitgetheilten Extinctionstabellen fiir Potsdam nnd den Süntis zeigt noch ummittellar den Untersehied awischen einer nicderen und höheren Beobuchtungsstation. Bei einer Zenithdistanz ron $71^{\circ}$ ist die Helligkeit eines Sternes am Neeresnivean um 11.t5 Cröisencliksen, dagegen auf einem 2500 Meter hohen Berge

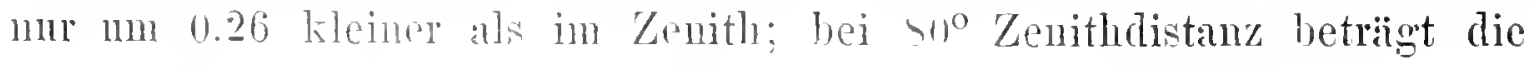


Lichtabschwiichung gegenib)e dem Zenith in der libene ungefilhr eine Grössenclasse, anf dem Berge nur 0.64; bei 85" Zenithlistan\% endlieh luat cin Stern von seiner '/enithhelligkeit an der unteren Station eine volle Grössenclasse melır eingehilsst als anf der oberen. Der Unstand, dass man anf einem hohen Berge in der Nïhe des. Horizontes mit blossem Ange mehr Sterne sieht als in der Ebene, liisst den Anblick des restirnten llimmels daselbst etwas fremdartig erseheinen und rerleitet leicht zur Überschützung des Durchsichtigkeitszıwaehses. In Zenith selbst ist der Helligkeitsgewinn, wenn man ans den unteren Schichiten der Atmosphäire in die höheren anfsteigrt, verhïltnissmäissig unbedentend; mach der 'Theorie dlirfte die Zenithhelligkeit eines Sternes an einem 2:00 Meter hohen Beobachitungsorte noch nieht um 0.1 grösser sein als in der Ebene. Eine direete empirisehe Bestimmung dieses Betrages wiire im hohen Grade erwiinseht. Bisher ist eine solehe erst einmal und zwar in Jahre IS9.1 von Kempf und mil durch gleichzeitige Beobachitungren in Catania und anf dem Gipfel des $̈$ tna versucht worden; doch ist dieser Versuch, dessen Ergebnisse noeh nicht veröflentlicht sind, keineswers als entscheidend zu betrachten. Soviel steht fest, dass die mehrfach anfgestellte Frage, ob die Errichtung von festen Observatorien auf hohen Bergen \% empfehlen sei, verneint werden miisste, falls es sich ledighich un die Zunahme der Sternhelligkeit handelt, weil der Gewinn von weniggen Zehntel Grössenelissen, noch dazn erst bei niedrigem Stande der Sterne, in keinem Verhiiltnisse 'מu den beträichtlichen Kosten nud der schwierigen Unterhaltung solcher Stationen stehen wiirde.

Was nun noch die Frage nach der Helligkeit der Gestime ansserhalb der Erdatmosphiare betrifit, so liisst sich dieselbe natiirlich mur anf Grund der Theorien ans Beobachtungen in versehiedenen Zenithdistanzen heantworten, und es existirt hereits eine zicmlich grosse Anzahl von Bestimmungen des Transmissionscoefficienten $p$ fiir verschiedene Beobalchtungsorte. Die wiehtigsten derselben sind in der folgenden Tabelle zusammengestellt mit Angabe des Beobachters, der Station, der Hijhe derselhen ïber dem Meere und des zugehürigen mittlereu Baroneterstandes. Da die absorbirende Luftmalsse an den einzehen Stationen sehr verschieden ist, so sind die in Columne 5 mitgetheilten direct crmittelten Coefficienten noch auf den Barometerstand 760 Nillimeter, also auf die gamze $\Lambda$ tmosphïre, redneirt worden; die reducirten Werthe finden sich in der vorletzten Columne, und in der letzten Columne ist der ILelligkeitsbetrag in Grössenclassen angegeben, $11 n$ welchen das senkrecht in die Atmosphäre eindringende Licht eines Sternes am Meeresnivean geschwächt erseheint. 


\begin{tabular}{|c|c|c|c|c|c|c|}
\hline Boobachter & Beobachtnngs-Station & $\begin{array}{l}\text { IIöhe } \\
\text { ūber } \\
\text { dem } \\
\text { Meere } \\
\text { in } \\
\text { Metern }\end{array}$ & Bar. & $\begin{array}{l}\text { Beobacht. } \\
\text { Transmiss.- } \\
\text { Coefficient. }\end{array}$ & $\begin{array}{c}\text { Trausm. } \\
\text { fūr eine } \\
\text { Atmosphăre }\end{array}$ & $\begin{array}{c}\text { Ab- } \\
\text { sorbirte } \\
\text { Lichtmenge } \\
\text { in Stern- } \\
\text { grössen }\end{array}$ \\
\hline Bouguer 1) & Croisie (Bretagne) & - & $\begin{array}{l}\operatorname{mm} \\
760\end{array}$ & 0.812 & 0.812 & 0.23 \\
\hline Pritchard $\left.{ }^{2}\right)$ & Cairo & 33 & 759 & 0.843 & 0.843 & 0.19 \\
\hline 'Trépied $\left.{ }^{3}\right)$ & Paris & 59 & 758 & 0.810 & 0.509 & 0.23 \\
\hline Wolft 4 & Bonn & 62 & 756 & 0.806 & 0.805 & 0.24 \\
\hline Pritchard') & Oxford & 64 & 756 & 0.791 & 0.790 & 0.26 \\
\hline Abney 5 & Derby & - & 754 & 0.850 & 0.849 & 0.18 \\
\hline Miiller $\left.{ }^{6}\right)$ & Potsdam & 100 & 752 & 0.835 & 0.833 & 0.20 \\
\hline Stampfer ${ }^{i}$ & Wien & 202 & 744 & 0.824 & 0.821 & 0.21 \\
\hline Seidel's) & München & 529 & 716 & 0.804 & 0.793 & 0.25 \\
\hline Abney ${ }^{5}$ & Grindelwald & 1057 & 676 & 0.838 & 0.820 & 0.22 \\
\hline Langley & Casa dẹl Bosco am & 1440 & 660 & 0.90 & $0.8 S 6$ & 0.13 \\
\hline Miiller $\left.{ }^{10}\right)$ & Säntis & 2504 & 569 & 0.579 & 0.842 & 0.19 \\
\hline Abney 5 ) & Faulliorn & $26 \$ 3$ & $5+6$ & 0.921 & 0.892 & 0.12 \\
\hline Miiller und Kempf $"$ & Ätnaobservatorinm & 2942 & 540 & $0 . \$ \$ 0$ & 0.835 & 0.20 \\
\hline Limgley 12 & Mount Whitney & 3543 & 500 & 0.92 & 0.881 & 0.14 \\
\hline
\end{tabular}

Die Zahlenangaben für den Transmissionscoefficienten der ganzen Atmosphiare in der obigen Tabelle sind nicht als gleiehwerthig anzusehen. Wihrend einige derselben, so namentlich die Miunchener und Potsdamer, aus einem sehr grossen Beobachtmngsmaterial hergeleitet sind, beruhen andere mur anf rereinzelten Messungen, und die Langley'schen Werthe griinden sich sogar nur anf renige nieht seln zurerlässige Helligkeitssehaitzungen. Zur Ableitung cines Mittelwerthes miisste man den einzelnen Angaben verschiedene Gerichte beilegen, wobei eine gewisse Willkiur nicht zn vermeiden wäre. Als ein branchbarer Durchschnittswerth für den Transmissionscoefficienten th diirfte sich die Zahl 0.835 empfehlen;

1) Bouguer, Essai d'optique sur la gradation de la lnmière. Paris 1729, p. 163.

2 Memoirs of the R. Astr. Soe. Yol. 47, p. 116.

3 Comptes Rendus. Tom. S2, p. 559.

4 Wolff', Phot. Beob. an Fixsternen ans den Jahren 1S76-1s83. Berlin 1ㄴ. p. 34.

5 Phil. Trans of the R. Soe of London. 1893. p. 24-42. Die obigen Werthe sind ans den a. a. O. mitgetieilten Beobachtnugen ron mir berechnet worden.

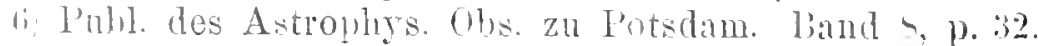

7 Entmommen ans der muter 4 citirten Abhandl. ron Wolfi, p. 31 u. 32.

- Abh. d. K. Bayer. Akad. d. Wiss. 11. Classe, Bd. 6, p. 619.

9 Auerican Jommal of science. 3 Ser. Vol. 20, p. 35.

10) P'nbl. des Astrophys Obs. zu Potsdam. Bd. \&, p. 39.

11, Nuch nicht unhlicint.

12. Profenoional jatpers of the Signal Service. No. 15, p. 155. 
darans wurde folgen, dass die Storue ausserhalb der Erdatmosphïre um rund 0.2 Grössonelassen heller erscheinen als im Zenith eines Beobachtungsortes im Nivean des Meeres bei besonders gunstigem Inftznstande.

\section{Die selective Absorption der Atmosphäre. Die Langley'schen Untersuchungen.}

Die Extinction des Lichtes in der Erdatmosphaire ist nicht fur alle Strahlengattmugen dieselbe, vielnehr ibt die Lufthulle eine selective $\mathrm{Ab}$ sorption aus, welche sich in zweifacher Weise änssert. Znnäichst werden Strahlen von gewisser Wellenlänge fast vollständig von dem in der Atmosphäre enthaltenen Wasserdampf aufgehalten. Es treten daher an bestimmten Stellen des Speetrums Absorptionslinien, ähnlich den bekannten Frannhofer'seheu Linien, anf, welche je nach der Quantitït des vorhandenen Wasserdampfes und der Länge des in der Atmosphäre von den Lichtstrahlen durehlaufenen Weges in Bezng anf Intensitiit und Breite variiren. Diese Wirkung ist eine discontinuirliche nnd erstreckt sich uber ein verhältnissmaissig kleines Gebiet im gelben und rothen Theile des Spectrums. Eine bemerkenswerthe Schwächung im Gesammtlichte eines Sternes wird durch diese Absorptionsstreifen nicht hervorgebracht.

Wesentlieh anders ist die zweite $A$ rt der selectiven $\mathrm{Absor}$ ption, welehe sich continuirlich iiber das ganze Spectrum ansdehnt und in der Weise zn Tage tritt, dass die blauen und violetten Strahlen stärker ansgelöscht werden als die griuen, und diese wieder stärker als die gelben und rothen. Wenn man diese Absorption als eine rein mechanisehe ansieht, hervorgebracht dureh die in der Luft befindlichen Partikelchen der verschiedensten Art, welehe cine allgemeine Diffiaction verursachen, so erklïrt sich der continuirlich wechselnde Grad der Lichtschwächung im Spectrum durch die Beziehung, welche zwischen den Dimensionen dieser Partikelchen und der Wellenlainge existirt. In welchem Betrage sich die Absorptionsfähigkeit der Atmosphäre für die einzelnen Strahlengattungen iudert, geht ans der folgenden Tabelle hervor, in welcher die zuverliissigsten Werthe der Transmissionscoefficienten zilsammengestellt sind. Die von nir fü Potsdam gefundenen Resultate ${ }^{1}$ ) beruhen anf Vergleichmgen des Somnenspectrums und des Spectrums einer Petroleumflamme init Iiilfe des Spectralphotometers, die Abney'schen ${ }^{2}$ Z Zahlen sind ans Ilelligkeitsvergleichnngen

1) Astr. Nachr. Bd. 103, No. 2464 mnd Publ. des Astrophys. Obs. zu Potsrlam. Bd. 8, p. 7, Anmerkung.

2 Phil. Trans. of the R. Snc. of London. 1557. 1. 251-2s3. NB. Die Werthe sind aus Mascart's Traité d'optique, 'Tome III, p. 3\%”, entuommen. 
verschiedener 'Theile des Somnenspectrums mit dem Gesammtlichte der Sonne abgeleitet, und die Langley'schen ${ }^{1}$ ) Angaben griinden sich auf Messungen mit dem Bolometer, sind also eigentlich streng genommen nieht direct mit den anderen Werthen vergleichbar, weil sie sich auf die Wairmewirkung der Sonne, nicht anf die Lichtwirkung beziehen. Die in der T'abelle angefülırten Zahlenwerthe für die verschiedenen Wellenlïngen sind aus den betreffenden Reilhen durch Interpolation gewonnen worden und kömnen um einige Einheiten der letzten Decimale unsicher sein.

\begin{tabular}{|c|c|c|c|c|c|c|c|}
\hline Wellenlānge & Muller & Abney & Langley & WellenIāuge & Múl ler & Abney & Langley \\
\hline $760 \mu \mu$ & - & 0.954 & 0.838 & $560 \mu \mu$ & 0.819 & $0 . \$ 43$ & 0.750 \\
\hline 740 & - & 0.947 & 0.831 & 540 & 0.808 & 0.821 & 0.738 \\
\hline 720 & - & 0.940 , & $0 . \$ 24$ & 520 & 0.795 & $0.795_{30}$ & 0.724 \\
\hline 700 & - & 0.932 & 0.817 & 500 & 0.781 & 0.765 & 0.708 \\
\hline 650 & 0.551 & 0.923 & 0.809 & 480 & 0.764 & 0.729 & 0.659 \\
\hline 660 & 0.871 & 0.914 & 0.800 & 460 & 0.740 & 0.656 & 0.665 。 \\
\hline 640 & 0.861 & 0.904 & 0.792 & 440 & 0.706 & 0.637 & 0.637 \\
\hline 620 & 11.55011 & 0.592 & 0.783 & 420 & - & 0.581 & 0.6014 \\
\hline 1000 & 0.810 & u.sis & 0.772 & 400 & - & 0.522 & 0.565 \\
\hline 580 & $0.830_{11}$ & 0.562 & 0.761 & & & & \\
\hline
\end{tabular}

Die Miiller'sche und Langley'sche Reihe zeigen trotz der merklichen Unterschiede in den absolnten Werthen der Transmissionscoefficienten eine auffallende Übereinstimmung in dem Gange der Zahlen, während hei der Abney sehen Reihe nach dem brechbareren Ende des Spectrums zn die Differenzen betrïchtlich stärker anwachsen als bei den anderen Reihen. Bei Miiller und Langley sind dic Logarithmen der Transmissionscoefficienten, abgesehen ron den etwas nnsicher bestimmten Werthen an den beiden Enden des sichtbaren Spectrums, sehr nahe proportional den ungekehrten Quadraten, bei Abney dagegen ungefähr proportional den mugekehrten vierten Potenzen der Wellenlängen.

Weitere Bestimmungen dieser wichtigen Constanten sind im hohen Grade erwinscht. Soviel ist jedenfalls sicher, dass die rothen Strahlen nur etwa 10 Procent des Lichtes beim Durchgange dureh die ganze Erdatmosphäre verlieren, die hlanen und violetten dagegen to Procent, und dalss mach dem Cltrariolett zu die absorbirende Wirkung der Atmosphäre ansserordentlich rasch anwichst. Es künnen in dem Lichte der Sterne schr wohl auch strahlengattungen enthalten sein. deren Durehlïsigkeits- 
coefficienten so klein sind, dass sie bereits in den ersten Schichten der Atmosphäre gainzlich ausgelöseht werden und uberhaupt nicht bis zur Errloberfliche gelangen. Daraus wilide aber folgen, dass das Gesamntlicht eines Sternes ausserhalb der Atmosphäre viel grösser sein könnte, als man gewühnlich annimmt, und dass die auf Seite 135 zusammengestellten Transmissionscoefficienten nur obere Grenzwerthe fur die Lichtdurchlïssigkeit der Atmosphäre repriisentiren würden. Dieses Bedenken haben bereits Forbes ${ }^{1}$ ) und Croval ${ }^{2}$ geaiussert, und Ersterer hat die Wirkung der Atmosphäre mit der eines rothen Glases, verglichen, welehes bei geringer Dicke noch alle Strahlengattungen passiren liisst, dagegen bei zumehmender Dicke nur den rothen Strahlen leichten Durchgang gestattet, so dass man den Durehlaissigkeitseoefficienten fur das Gesammtlicht um so grösser finden würde, ans je dickeren Stlleken des Glases man denselben bestimmte. Langley ${ }^{3}$ ) hat diesen Einwurf noch priciser in mathematischer Form begrilndet und glaubt zn dem Schlusse berechtigt zu sein, dass alle bisherigen Bestimmungen der Gesammtenergic (Licht oder Wärme) ansserhalb der Atmosphüre betriichtlich von der Wahrheit entfernt sind, und dass der Energieverlust bei senkrechtem Strahlendurehgange anstatt der gewöhnlich angenommenen 18 Procent wahrscheinlich etwa 40 Procent betragen wird.

Da der Gegenstand flir dic Astrophotometric von nicht unerheblichem Interesse ist, so soll hier noch etwas näher darauf eingegangen werden.

Es sei $L$ die Gesammtintensitiit des Lichtes eines Sternes ausserhallb der Atmosphiare. Dieses Licht bestehe aus $n$ verseliedenen Strahlengattungen, deren Helligkeiten vor dem Eintritte in die Atmospluäre $B_{1}$, $B_{z}, B_{3} \ldots B_{n}$ sein mögen. Dann hat man:

$$
L=B_{1}+B_{2}+B_{3}+\cdots+B_{n} \text {. }
$$

Nimmt man nun an, dass die im Vorangehenden erörterten Extinctionstheorien für homogenes Licht strenge Giiltigkeit besitzen, und nennt dic Transmissionscoefficienten der ganzen A tmosphäre für die cinzelnen Strahlengattungen $c_{1}, c_{2}, c_{3} \ldots c_{n}$, so wird die Helligkeit $J_{z}$ des Sternes bei der Zenithdistanz $\approx$, wenn man die durchlaufene Luftmasse mit $\gamma$ bezeichnet, nach der Bouguer'schen Theorie ansgedriickt dureh:

$$
J_{z}=B_{1} c_{1}^{\gamma}+B_{2} e_{2}^{\gamma}+B_{3} e_{3}^{\gamma}+\cdots+B_{n} c_{n}^{\gamma} \text {. }
$$

Nun war fribher ganz allgemein gesetzt worden: $J_{z}=J_{1}{ }^{\prime \prime}$, wo $p$ der 'Transmissionscoefficient für das Gesammtlicht des sternes und $J$ die Helligkeit ausserhalb der Atmosphïre ist, wie sie durch Extrapolation

1) Phil. Trans. of the R. Soc. of London. 1842, p. 225.

2) Annales de chimie et de physique. Série 5, t. 11. p. 433 nnd t. 19, p. 167.

3 American Journal of science. 3. Ser. Yol. 24. 1. 163. 
ans den Beobachtungen ermittelt wird. Fuir zwei verschiedene Zenithdistanzen $\approx_{1}$ und $z_{2}$, denen die Luftmassen $\gamma_{1}$ und $\gamma_{2}$ entsprechen mögen, hat man daher:

$$
\begin{aligned}
& J_{g_{1}}=J p^{\gamma_{1}}=B_{1} c_{1}^{\gamma_{1}}+B_{2} c_{2}^{\gamma_{1}}+\cdots+B_{n} c_{n}^{\gamma_{1}}, \\
& J_{z_{2}}=J p^{\gamma_{2}}=B_{1} c_{1}^{\gamma_{2}}+B_{2} c_{2}^{\gamma_{2}}+\cdots+B_{n} c_{n}^{\gamma_{2}} .
\end{aligned}
$$

Erheht min die erste Gleichung zur Potenz $\gamma_{2}$, die zweite zur Potenz $\gamma_{1}$ und dividirt die beiden Gleichungen durch einander, so erhält man:

$$
J^{\gamma_{2}-\gamma_{1}}=\frac{\left(B_{1} c_{1}^{\gamma_{1}}+B_{2} c_{2}^{\gamma_{1}}+\cdots+B_{n} c_{n 1}^{\gamma_{1}}\right)^{\gamma_{2}}}{\left(B_{1} c_{1}^{\gamma_{2}}+B_{2} c_{2}^{\gamma_{2}}+\cdots+B_{n} c_{n}^{\gamma_{2}}\right)^{\gamma_{1}}} \cdot
$$

Fiir den Quotienten $\frac{L}{J}$ ergiebt sich daher der Werth:

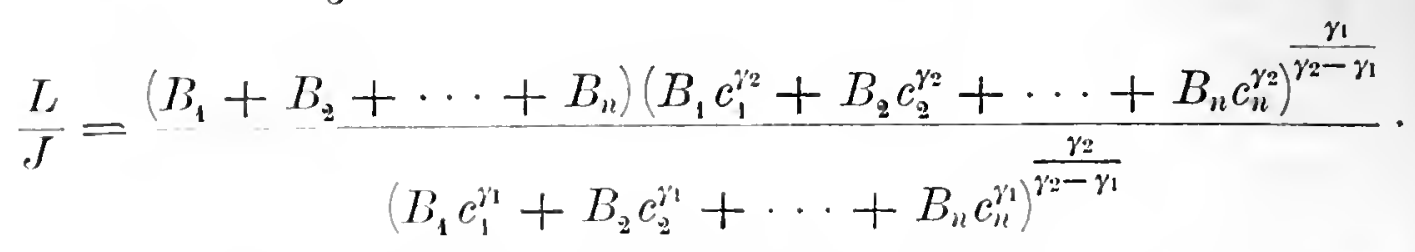

Wil wollen amnelmen, dass die Zenithdistanz $z_{2}$ grösser ist als $\approx_{1}$; dann ist alleh $\gamma_{2}>\gamma_{1}$, und wir kömnen setzen $\gamma_{2}=m \gamma_{1}$, wo $m>1$ ist. Mithin wird $\gamma_{2}-\gamma_{1}=(m-1) i_{1}$ und fermer:

$$
\begin{aligned}
& \frac{\gamma_{1}}{\gamma_{2}-\gamma_{1}}=\frac{1}{m-1}, \\
& \frac{\gamma_{2}}{\gamma_{2}-I_{1}}=\frac{m}{m-1} .
\end{aligned}
$$

Fïhrt man endlich noch der bequemeren Schreibweise wegen die Bezeichmugen ein:

$$
c_{1}^{\gamma_{1}}=b_{1}, \quad c_{2}^{\gamma_{1}}=b_{2}, \quad \ldots \quad c_{n}^{\gamma_{1}}=b_{n},
$$

so erhïlt man aus der obigen Gleichung (30) durch Substitution die nene (ileichung:

(:i) $\left(\frac{L_{1}}{J}\right)^{m-1}=\frac{\left.B_{1}+B_{2}+\cdots+B_{n}^{m-1} B_{1} b_{1}^{m}+B_{2} b_{2}^{m}+\cdots+B_{n} b_{n}^{n}\right)}{\left(D_{1} b_{1}+B_{2} b_{2}+\cdots+B_{n} b_{n}\right)^{m}}$.

Wie linke Seite der Gileichung werde mit $Z_{m}$ bezeichnet, und es sei zunärhst $m$ eine ganze Zahl. Bildet man dam entsprechend den Werth $Z_{t \prime n-1}$, so ergiebt sich:

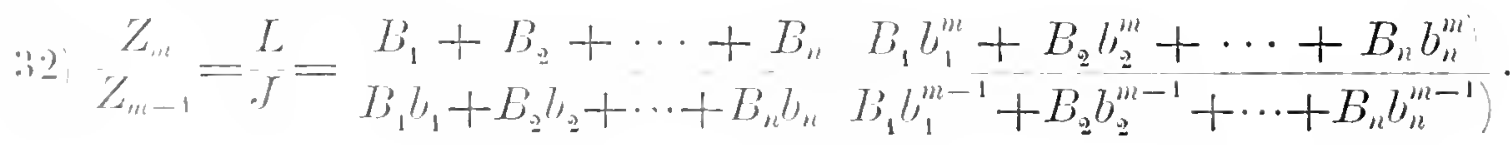

Zuihler und Nenner dieses Bruches lassen sich in der Form schreiben:

$$
\begin{aligned}
& \text { Zïhler }=B_{1}^{2} l_{1}^{m}+B_{2}^{2} l_{2}^{m}+B_{1} B_{2} l_{1}^{m}+b_{2}^{m}+\cdots \text {, } \\
& \text { Nenter }=I_{1}^{2} l_{1}^{\prime \prime}+D_{2}^{2} l_{2}^{m}+I_{1} B_{2} h_{1} b_{2}^{m-1}+b_{2} b_{1}^{m-1}+\cdots
\end{aligned}
$$




\section{Durch Subtraction erhailt man daraus:}

$$
\text { Zühler - Nenner }=B_{1} B_{2}\left(b_{1}-b_{2}\right)\left(b_{1}^{m-1}-b_{2}^{m-1}\right)+\cdots .
$$

Nun ist olne Weiteres klar, dass, wenn $b_{1}>b_{3}$ ist, damn anch $b_{1}^{m-1}>b_{2}^{m-1}$ sein muss, und elonso, wenn $b_{1}-b_{2}$ eine negative Zalul ist, auch $b_{1}^{m-1}-b_{2}^{m-1}$ negativ scin muss. Unter allon Umstiinden ist das Product der beiden Grössen positir, und da dasselbe auch fiir die weiteren Glieder der oberen Reihe gilt, so folgt, dass die Differen\% Zähler - Nenner ebenfalls eine positive Grösse ist und mithin $L>J$ wird. Damit ist also, wenigstens fir ganze $m$, erwiesen, dass die wirkliche Gesammtintensitait eines Sternes vor dem. Eintritte in die Atmosphäre stets grösser ist, als die ans Beobachtungen bei verschiedenen Zenithdistanzen nach der 'Theorie berechnete Helligkeit $J$ : und dass mithin die auf Seite 138 mitgetheilten 'Transmissionseoefficienten in der 'That, wie von Langley behauptet worden ist, nur Maximalwerthe flir diese Constante reprïsentiren kömen. Auch für beliebige Werthe von $m$ liisst sich der Beweis fulhren, dass $I>J$ ist, indem man nachweist, dass $\frac{d\left(Z_{m}\right)}{d m}$ bestindig wächst. Aus Gleichung(:33) geht noch hervor, da die Grösse $b_{1}^{m-1}-b_{a}^{m-1}$ und die entsprechenden Factoren der weiteren Glieder dem alssoluten Betrage nach um so grösser werden, je grösser $m$ ist, dass die Differenz zwischen Zähler und Nemner und demnach auch der Quotient $\frac{L}{J}$ mit wachsendem $m$ zunehmen muss. Man sollte demnach erwarten, dass die nach der Theorie berechneten Werthe von $J$ versehieden ausfillen, je nachdem man Beobachtungen mit einander combinirt, bei denen der Untersehied der Zenithdistanzen klein oder gross ist. Wenn man z. B. die Helligkeitsmessung eines Sterues im Zenith suceessive mit Messungen bei den Zenithdistanzen $60^{\circ}, 65^{\circ}, 70^{\circ}$, $75^{\circ}, 80^{\circ}$ etc. vereinigte, so miissten sich die daraus bestimmten Werthe ron $J$ bestindig kleiner ergeben oder, was dasselbe ist, die ermittelten Transmissionscoefficienten der Atmosphüre miissten anwalisen. Nun zeigt alber eine sorgfiltige Prïfung der beiden zurerliissigsten empirischen Extinctionstabellen, der Seidel'sehen sowohl wie der Potsdamer, daron keine Spur, im Gegentheil findet bis zu einer gewissen '/enithdistanz gerade das Umgekehrte statt, und man könnte schon dalsans mit einiger Wahlscheinliehkeit sehliessen, dass der Fehler, den man bei der berechnung der Helligkeit der Sterne ausserhalb der Atmosphlïire unter Anwendung der gewöhnlichen Extinctionstheorien begeht, nicht betriichtlich sein kam, jedenfalls nicht so gross, wie Langley annimmt. Seeliger") hat sich

1) Sitzungsber. der math.-phys. Classe der K. Bayer. Akad. der Wiss. Bd. 21, 1891, p. 24 i. 
neuerdings etwas eingehender mit diesem Gegenstande beschäftigt und den Versuch gemaeht, aus den Abreichungen zwisehen der Potsdamer Extinctionstabelle und den naeh der Laplace'schen Theorie berechneten Hclligkeitswerthen einen S'chluss zu ziehen anf den wahren Transmissionscoefficienten der Atmosphäre. Er findet, indem er die Langley'schen Durchlissigkiteitscoefficienten für die versehiedenfarbigen Strahlen zu Grumde legrt, dass die Helligkeit der Sterne ausserhalb der Atmosphäre noch nicht um 7 Procent grösser sein kann, als die Theorien ergeben, und dass die ahsorbirte Liehtmenge zwar mellr als 18 Proeent, wie gewïhnlich angenommen wird, aber gewiss weniger als 25 Procent betragen muss. Seeliger macht anch darauf aufmerksam, dass die physiologisehen Wirkungen der einzelnen Farben, auf die es doch bei der optisehen Photometrie fast ausschliesslich ankommt, sich anf eine verhältnissmässig schnale Zove im Gelb und Grium eoneentriren, die an Wirkung die iibrigen Partien in Spectrum so sebr ibertrifft, dass fast nur sie allein beriicksichitigt zu werden braneht; dadureh wird der fragliehe Fehler wahrseheinlieh noch mehr verringert.

Eine ganz strenge Widerlegung der Langley'sehen Bedenken ist dimit freilich noch nieht gegeben; es ist nur ihre Unwahrscheinliehkeit plansibel gemacht worden. Mit einiger Sicherheit liesse sich die wirkliche Sternhelligkeit ausserhalb der Atmosphäre nur dann ermitteln, wenn es nelainge, auf sehr hohen Bergen möglichst zahlreiche absolut zuverlässige photometrische Messungen zur Bestimmung der Extinction zu erhalten. In einer Höhe von 4000 bis 5000 Neter, wo bereits mehr als ein Drittel der gesammten Luftmasse unterhalb des Beobachters liegt, miissten nach Aler Langley'sehen Auffissung bereits Stralılungen zur Wirkung kommen, the gar nicht mehr bis zu den allemutersten Schichten der Atmosphäre grelangen; es miisste daher anch an einem solchen Punkte ans sorgfältigen Extinctionsheobachtnngen ein Transmissionscoefficient fïr die ganze Atmosphiire hervorgehen, der bereits merklich kleiner wäre, als die an tiefen stationen gefundenen. Die wenigen bisher in dieser lichtung anf hohen baren angestellten rein photometrischen Intersuchungen, sorvie die bei weitem zalhlreinheren, wem anch nicht so zurerlässigen actinometrischen Mrsumgen zeigen nichts dergleichen, und man wird daher wohl berechtigt sin. den Latingleyschen Einwendumgen keine allzn grosse praktisehe Brolentmo heizunnessen. 


\section{ABSCHNITT.}

\section{DIE PHOTOMETRISCHEN APPARATE.}





\section{Vinleitung.}

Es giebt wohl kaum einen Zweig der praktisehen Astronomie, weleher so lange und so grlindlich vernaehlässigt worden ist, wie die Lichtmessung der Gestirne. Obgleich bereits die Alten die hohe Bedeutung der Helligkeitsbestimmungen fuir die Erweiterung der mensellichen Vorstellung von der Anordmung des Weltalls erkannt hatten, existiren ans dem Alterthum doch nur Liehtschätzungen, und es ist kein Versuch bekannt geworden, Apparate zur genaneren Messung der Lichtquantitïten zu construiren. Auch in den späteren Jahrlunderten und durch das ganze Nittelalter hindurch ist anf diesem Gebiete so gut wie Nichts geschehen. Selbst die Erfindung des Fermrohres, die anf allen tibrigen Gebieten der Astronomie einen gewaltigen Umsehwung hervorgebraeht hat, ist in dieser Beziehung spurlos voritbergegangen. Noch im 18. Jahrhundert, als Bonguer und Lambert ihre grundlegenden Werke iiber die theoretisehe Photometrie rerfassten, waren die instrumentellen Hiilfsmittel, welehe diesen M:ïnnern zu Gebote standen, von der allerprimitivsteu Art. Die Photometer, deren sieh Bouguer und Latmbert bedienten, gestatteten unr die Vergleichung von ziemlich hellen Liehtquellen. Am Himmel liessen sie sich allenfalls auf Sonne und Mond anwenden, aber die Messung selbst der allerhellsten Fixsterne blieb damit unausfiihrbar. Erst im gegenwäirtigen Jahrhundert hat sich eine erfreuliche Wandlung vollzogen. Arago, der juingere Hersehel und Steinheil haben die erste Auregung zur Constrnction brauchbarer Instrumente für die Ilimmelsphotometrie gegeben, und es geblihrt diesen Mämnern das Verdienst, diesem arg vernachlliissigten und fast abgestorbeneu Zweige der Astronomie nenes Lehen eingeflösst zu haben. Seit dieser Zeit ist ein Stillstand in den Bestrebungen zur Vervollkommnung der photometrischen Apparate nicht mehr eingetreten. Erst allmählich, dann immer schneller mud allgemeiner ist d:ı Interesse für diesen Gegenstand bei den Astronomen gewachsen, nnd namentlich die letzten Jahr\%ehnte habeu uns mit einer reichen Fülle von nützlichen Instrumenten zur Liehtmessmng der Gestime leschenkt. Nicht wenig hat zu dieser Entwicklung der Umstiud beigetragen, dass die grrossartigen 
Fortschritte der Technik in Bezug auf das Beleuchtungswesen nothwendig die Einfuhrung exacter photometrischer Methoden bedingten und einen regen Erfindungseifer bei lhysikern und Technikern hervorriefen. Wenn anch die meisten der flir die Zweeke des praktischen Lebens construirten Photometer, deren Zahl bereits zu einer sehr bedeutenden angewachsen ist, nicht unmittelbar zu Messungen am Himmel verwendbar sind, so ist doch manche glickliche Idee, mancher praktische Kunstgriff auch der Himmelsphotometrie zu Gute gekommen.

Noch sind wir weit von der Erreichung des Endzieles entfernt, das uns fiir die Construction eines vollkommenen Photometers vorschwebt. Die Genanigkeit, die mit den jetzigen Hülfsmitteln erreichbar ist, bleibt verhialtnissmässig weit hinter den Ansprüchen zurïck, welche die Astronomie anf anderen Gebieten zul stellen pflegt, und ist unter allen Umstïnden nicht geniugend, um subtile Fragen, wie sie z. B. bei dem Problem der Planetenbeleuchtung, bei den Lichterscheinungen der veränderlichen Sterne u. s. w. anftreten, zu entscheiden. So lange es nicht gelingt, die Helligkeit eines Gestirnes bis auf wenige Hundertstel Grössenclassen genau zn bestimmen, fehlt es für die Lösung einer grossen Zahl von photometrischen Aufgaben an den sicheren Grundlagen.

Die meisten bisher gebräuchlichen Astrophotometer verlangen in letzter Instanz das Urtheil des menschlichen Anges; sie messen nicht die objective Helligkeit der betrachteten Lichtquelle, sondern sie erleichtern nur die Ermittlung der physiologischen Intensitiit. Es ist klar, dass auf diese Weise von vornherein allen Photometern infolge der Unvollkommenheit des Sehorgans eine Genauigkeitsgrenze gesetzt ist, welche unter keinen Umständen, anch weun der Messapparat und die demselben zu Grunde liegenden photometrischen Methoden noch so sehr verfeinert wiirden, iberschritten werden kinu. Durch lange Übung lässt sich allerdings das Ange bis zu einem gewissen Grade sehulen, und wer sich viel mit photometrisehen Beobachtungen beschäftigt lat, wird z. B. feinere Lichtunterschicde wahrzunehmen rermögen, als ein Anfinger auf diesem Gebiete. Aber die natiirlichen Mängel des Anges, die namentlich bei der Ver"leichmo rerschiedenfarbiger Lichtquellen hervortreten, stellen der Erreichung der allerhöchsten Genauigkeit fuir immer eine unüberwindliche Schranke entrgegen. Kein Auge ist im Stande, die relative Stärke zweier nerklich rou einander verschiedenen Lichteindriicke zahlenmïssig festzustellen, ebenso wenig wie es nach einem lingeren Zeitraume mit sicherheit zu constatiren vermag, ob eine Lichtruelle ihre Intensitit bis zu einem gewissen Grade bewahrt hat. Was das Auge, namentlich bei einiger Übung, mit Zurerlïssigkeit leisten kann, das ist die Beurtheilung der Gleichloit zweier nahe bei einander befindlichen gleichzeitig wahr- 
genommenen Lichteindrllcke. Dabei mltssen aber noch eine Reibe von Bedingungen erfullt sein. In erster Linie ist es erwllnseht, dass die zu vorgleichenden Gegenstïinde dieselbo seheinbare Grösse besitzen nnd in allen Theilen gleichmässig orleuchtet erseheinen. Die Vergleichung eines

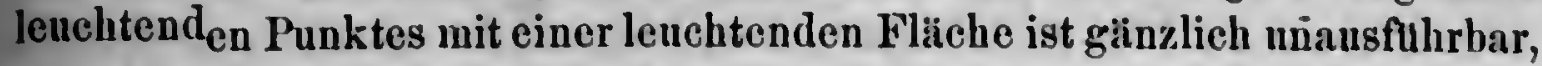
und die Beurtheilung zweier Sterne wird um so unsicherer, je mehr die Durchmesser der Diffractionsseheibehen derselben von einander verschieden sind. Zuverlässiger als Punktvergleichungen sind Fliehenvergleichungen; doeh ist es unbedingt nothwendig, dass die beiden Flïchen genan in einer geraden Linie oder, was Manehe fur wllnschenswerther halten, in irgend einer scharf begrenzten Curve aneinander stossen, sodass im Falle der vollkommenen Helligkeitsgleichheit die Grenzlinie ganz rersehwindet. Gelingt es nicht, den beiden Lichtquellen dieselbe scheinbare Grösse zu geben, so beurtheilt man in vielen Fïllen mit Vortheil ihre Intensitait nach dem Grade der Erleuchtnng, die sic auf einer weissen Fläche hervorrufen, indem man nach den Grundgesetzen der Photometrie annimmt, dass zwei Lichtquellen dieselbe Intensität haben, wenn sie auf einer weissen Fliiche, in gleichen Entfernungen und bei denselben Incidenz- und Emanationswinkeln, densclben Beleuchtungseffect hervorbringen. Durchaus erforderlich ist es ferner, dass die zu vergleichenden Lichteindriicke weder allzu intensiv noch allzu schwach sind; im ersten Falle werden die Sehnerven zu stark gereizt, und es tritt eine Abstumpfung ein, die ein richtiges Urtheil erschwert, im anderen Falle muss sich das Auge unter Umständen iibermaissig anstrengen. Endlich ist für eine sichere Beurtheilung der Gleichheit zweier Lichtquellen die gleiche Fïrbung derselben unerlässlich. Je auffallender der Farbenunterschied ist, desto schwieriger wird die Entscheidung des Auges, und desto mehr weichen die Urtheile verschiedener Beolachter von einander ab.

Aus dem Vorangehenden folgt, dass, solange das menschliche Auge bei der Lichtmessung hervorragend betheiligt ist, die Hauptaufgabe für die Construction brauchbarer Photometer sich darauf reducirt, Mittel ausfindig zu machen, um die lebendige Kraft einer Lichtquelle in messbarer Weise so weit zu verindern, bis dieselbe auf der Netzhant des Anges denselben physiologischen Eindruck hervorbringt, wie eine andere Lichtquelle. Wenn dabei ein solches Photometer noch möglichst viele der oben angeführten Bedingungen erfüllt, so wird es un so vollkommener seinem Zwecke entsprechen.

Dic zahlreichen Methoden, welche im Lanfe der Zeit in dieser Hinsicht vorgeschlagen worden sind, lassen sich in die folgenden Hanptkategorien zusammenfasseu. 
1. Anwendung der Fundamentalsitze der Photometrie, insbesondere des Gesetzes vom Quadrate der Eutfernung. Die bekanntesten und verbreitetsten Lichtmessungsapparate, wie das bereits von Lambert, später wieder von Rumford benntzte Schattenphotometer, das Ritchic'sehe Photometer und das Bunsen'sche Fettfleckphotometer beruhen auf dieser Methode. Speciell für die Astronomie sind von grosser Bedentung geworden das Herschel'sche Astrometer und das Steinheil'sche Prismenphotometer, bei denen die Gleichheit der Lichteindrücke auf der Netyhant des Auges durch Änderung der Distanzen hervorgebracht wird.

2. Veriuderung der Öffung des Fernrohrobjectirs oder des aus dem Objectiv austretenden Strahlenkegels. Diese Methode setzt rorans, dass die Intensität proportional der freien ÖffunngsHiiehe ist. Die Zahl der Photometer, bei denen man dieses Princip zur Anwendung gebracht lat, ist ungemein gross. Schon Bouguer hat sich desselben bedient, und seitdem sind bis in die neueste Zeit alle nur denkbaren Formen von Blendenöffnungen und zahlreiche meehanische Vorrichtungen zur messbaren Änderung dieser Öffumngen versucht worden, ohgleich rom theoretischen Standpunkte ans nicht unwichtige Bedenken weren diese Nethode erhoben werden können.

3. Schwichnng des Lichtes dureh absorbireude Medien. Dabei wirl rorausgesetzt, dass gleich grosse Sehichten der benutzten Substanz einen gleich grossen Procentsatz des anffallenden Lichtes auslïschen. Diese Nethode hat fast noch nrössere Verbreitung gefunden als die vorangehende, ron den primitivsten Versuchen an, wo die Schwächung durch Thereinanderlegen ron Glasplatten oder Papierscheiben oder mittelst absorbirender Flitssigkeitsschichten hervorgebracht wurde, bis zu dem relatir hohen Grade der Verrollkommunng; weleher in den nenesten formen des Leilphotometers erreiclut worden ist.

4. Zuriekwerfung des Lichtes an spiegelnden Flachen. ber Intensitaitserlust wird dabei entweder anf rein empirischem Wege mittelst irgend einer anderen photometrischen Methode bestimmt oder durch Rechmung nach den bekannten Fresnelschen Formeln ermittelt. In der Astrophotometrie sind am häntigsten spiegelnde Kugeln zur Verwendung gekmmmen.

5. Das Princip der rotirenden Scheiben. Dieselben sind mit sectorfürmigen Ansschnitten rersehen, deren Winkelüfunng sich messbar rerindern liasst. Wird eine solche Seheibe zwischen einer Lichtquelle und tem Anue in sehnelle Rotation rersetzt, so entsteht bekanntlich anf der Netzhant cin continnirlicher Lichteindruck, der um so sehwächer ist, einen je kleineren liamm diu oftenten Anssehnitte anf der ganzen Scheibe einnehmen. Besments in der technischen I'hotometrie ist dieses Prineip 
ungemein häufig zur Anwendung gekommen; doch ist es auch fur die Lichtmessung der Gestirne uutabar gemaeht worden, hauptsächlich durch Secehi, Langley und Abney.

6. Anwendung der Polarisation und Interforonz dos Lichtes. Keine Mcthode hat sich speciell fur die Himmelsphotometrie so nutzbringend gezeigt wie diese, seit $\Lambda$ rago zuerst auf sie anfmerksam genacht bat. Die vollkommensten und am meisten benutzten Messapparate, unter diesen besonders das Zölher'sche Astrophotometer und das Pickering'sche Meridianplotometer basiren auf diesem Prineip. Sie haben in erster Linie die bedentenden Fortschritte ermöglieht, welche die praktische Astrophotometrie in den letzten Jahrzehuten gemacht hat.

Dureh eine der im Vorangehenden fllehtig skizzirten Methoden ist es nun jederzeit möglieh, die Helligkeit einer Lichtquelle messbar so weit zu verändern, bis unser Auge von ihr denselben Eindruck empfängt, wie ron einer zweiten mit ihr zu vergleiehenden Liehtquelle. Bei einem Theile der fur die Messungen an Himmel bestimmten Photometèr käm auf diese Weise entweder direct das Helligkeitsverhiiltniss zweier Steme ermittelt werden, oder es kann anel, was häufig vorzuzichen ist, jedes Gestirn einzeln mit einer kïnstliehen Liehtquelle verglichen werden.

Wesentlich davon versehieden ist eine Classe von Plotometern, bei denen mit Hïlfe einer der oben aufgezïhlten Methoden die Helligkeit eines Gestirnes bis zur vollstiindigen Auslösehumg abgeschwäeht wird, so dass gar kein Lichteindruck mehr auf der Netzhaut des Auges hervorgebracht wird. Auf den ersten Blick könnte es scheinen, als ob dieses Verfahren wesentliche Vortheile böte, indem es unter der Voraussetzung, dass das Versehwinden eines Lichteindruckes ftir jedes menschliche Juge an eine bestimmte unver:inderliehe Grenze gebunden sei, rewisserm:ıssen absolute Helligkeitsmessungen gestatten wiirde. Indessen ist dies regen der Unvollkommenheit des Auges keineswegrs der Fall, und es kommt streng genommen anch bei diesem Verfahren in letzter Linie auf die Beurtheilung der Gleichheit zweier Lichteindriicke an, indem der Homent fixirt wird, wo das betraclitete Gestirn sich nicht meln von dem ungebenden Himmelsgrunde unterscheidet.

Bei der im Folgenden rersuchten Classiticirmng der photometrischen Apparate werde ich diese beiden soeben erwiihnten Arten der Beobachtung streng vou einander tremuen. Im ersten Capitel sollen diejenigen Apparate behandelt werden, welche auf dem l'rincip der Auslöschung beruhen, im zweiten Capitel diejenigen, bei denen direct die Gleichheit zweier lenchtenden Punkte oder Flächen beurtheilt wird. Die erste Classe zeichnet sich in Allgemeinen durch grössere Einfachlıeit der Construction ror der anderen ans, während sie in Bezug anf die zu erreichende 
Genauigkeit hinter ihr zurtieksteht. Bei beiden Classen von Photometern wird es sich empfehlen, noeh eine besondere Gruppirung vorzunehmen, und zwar nach den versehiedenen oben angefiihrten Hauptmethoden, welche zur messbaren Veränderung der lebendigen Kraft einer Liehtquelle benutzt werden.

Im dritten Capitel sollen dann die verschiedenen Formen der Speetralphotometer besprochen werden, bei denen die zu untersuchenden Lichtquellen vor der Vergleichung in ihre einzelnen Strablengattungen zerlegt werden, und im letzten Capitel sollen endlich noeh einige Formen von Instrumenten Erwähnung finden, bei denen das Licht eine meehanische Wirkung hervorbringt und das Urtheil des menschlichen Auges entbehrlich ist. Dabei wird namentlich auf die Anwendung der Photographie zu photometrischen Messungen hinzuweisen sein.

Obgleich es mein Bestreben gewesen ist, eine möglichst vollständige Übersicht iiber alle zu Lichtmessungen am Himmel benutzten Apparate zu geben, so wird mir doch bei der grossen Fülle derselben und bei dem bisherigen Mangel einer geordneten Zusammenstellung auf diesem Gebiete ein oder das andere Photometer entgangen sein. Manche Apparate, die sich in der Praxis bisher wenig eingebürgert haben oder fast ansschliesslich auf technischem Gebiete verwendet worden sind, sollen im Folgenden nur fliichtig beriihrt oder nur dann etwas näher besehrieben werden, wemn sie in irgend einer Beziehung besonderes Interesse bieten. Eingehende Beriieksichtigung soll in erster Linie denjenigen Photometern zu Theil werden, die mit Erfolg zu umfassenderen Beobachtungsreihen verwendet worden sind. Die Vortheile und Mängel derselben verdienen eine kritische Belenchtung, und es wird nicht ïberfliissig erscheinen, wenn hier und da praktisehe Winke zur vortheilhaftesten Handhabung dieser Apparate eingestrent werden, und wenn nebenbei anch die theoretischen Gesichtspunkte, welehe bei ihnen in Betracht kommen, wenigstens in Kiurze erörtert werden. 


\section{Capitel I. \\ Photometer, bei denen das Verschwinden ron Lichteindrücken beobachtet wird.}

Bevor wir auf die einzelnen Apparate dieser Gattung näher eingehen, sollen einige Punkte von allgemeinem Interesse herrorgehoben werden.- Alle hierher gehörigen Instrumente stellen an die Urtheilsfaligkeit des Anges ganz besonders hohe Anforderungen. Die Empfindlichkeit des Auges ist einem bestïndigen Wechsel unterworfen, und es ist gerade bei dieser Methode eine besonders lange Übung erforderlich, un zu brauchbaren Messungsresultaten zu gelangen. Wer zum ersten Male versucht, das Bild eines Sternes in einem Fernrohre zum Versehwinden zu bringen, wird sicher kein giinstiges Urtheil tiber die Methode abgeben. Jeder wird anfangs die Erfahrung machen, dass, wenn ein Stern bereits ausgelöscht scheint, häufig nur ein kurzes Schliessen und Wiederöffnen des Auges genügt, um denselben noch deutlich zu erkennen, und ganz besonders schwierig wird die Beurtheilung, wenn die Stelle des Gesichtsfeldes, wo die Auslösehung stattfinden soll, nicht durch eine besondere Einriehtung kenntlieh gemacht ist. Auf diesen Punkt sollte bei der Construction jedes auf dem Prineip des Verschwindens beruhenden Photometers in erster Linie geachtet werden. Bei der Methode der Gleichmachung zweier Lichteindrieke wird die Fixirung der richtigen Einstellnng dadurch wesentlich erleichtert, dass man nach zwei Seiten einen Aussehlag geben und das eine Object abwechselnd heller und schwächer machen kann als das andere. Bei der Auslöschungsmethode dagegen nühert man sich inmer nur der einen unteren Grenze und hat keinen sicheren Anhalt zur Beurtheilung, wie weit man eventuell diese Grenze bereits iiberschritten hat.

Bei lïngerer Übung gestaltet sich dic Sachlage allerdings etwas giinstiger. Jeder Beobachter gewöhnt sich daran, einen bestimmten minimalen Helligkeitsgrad als Verschwindnngspunkt aufzufassen, und es ist bemerkenswerth, mit welcher Genanigkeit dieser Noment (natirlich bei gleichen äusseren Umständen) immer wieder erreicht wird. Bei verschiedenen Beobachtern können selbstrerständlich grosse Unterschiede vorkommen, theils infolge grösserer oder geringerer Sehsehärfe, theils infolge der von jeder Person willkiirlich getroffenen Wahl des zu fixirenden Momentes. In Potsdam sind von Kempf und mir besondere 
Beobachtungsreihen zur Bestimmung der persönlichen Differenz an Keilphotometern angestellt worden, und es hat sich dabei mit bemerkenswerther Constanz wïhrend eines längeren Zeitraumes der ziemlich erhebliche Betrag von ungefähı einer halben Grössenclasse ergeben.

Wenn num aber auch fü jeden Beobachter ein bestimmter Grenzwerth der Auffassung existirt, so darf doch nieht übersehen werden, dass, zumal bei einer liingeren Beobachtungsreibe, dieser Grenzwerth nieht fortdanernd innegehalten wird. Bei Beginn der Messungen, wo das Auge zwar noch friseh, aber dureh die aussere Helligkeit beeinflusst ist, wird dic Auslöschung zu zeitig geschehen. Dann wächst die Emptindliehkeit des Anges und erreieht ziemlich bald den Höhepunkt, auf dem sie mit kleinen zufälligen Schwankungen bleibt, bis eine gewisse Ermiidung eintritt, infolge deren der Auslöschungspunkt ganz allmählich wieder herabsinkt. Dieser Verlauf der Empfindlichkeitseurve scheint für alle Beobachter typiseh zu sein; nur liisst sich die Zeitdauer, innerhalb weleher die Emptindlichkeit nahezu constant bleibt, nieht mit Sicherheit :mgehen. Es wird dies ganz wesentlich von der jedesmaligen Disposition des Beobachters, sowie von einer Anzahl äusserer Umstände abhängen, mid es ist klar, dass diese Unbestiindigkeit und vor Allem die iibermissige Anstrengung, die dem Ange zugemuthet wird, die Hauptschwächen der Auslöschungsmethode bilden. Es kann nicht dringend genug empfohlen werden, die einzelnen Messungsreihen nielıt allzu lange (keinesfills mehr als 30 Minuten/ auszudehnen ind ror Beginn einer nenen Reihe das Ange eine Zeit lang, womöglich im Finstern, ausruhen zu lassen, damit es die frihhere Empfindlichkeit wiedererlangen kann. Aus dem oben charakterisirten allgemeinen Verlaufe der Empfindlichkeitseurve ergeben sich noch die folgenden speciellen Regeln.

Die ersten Einstellungen jeder grösseren Beobachtungsreihe, die stets zu nichrige Anslïschngspinkte geben, sollten nicht zur Bearbeitung verwerthet werden, namentlich dam nicht, wenn das Ange vorber einer hellen Beleuchtung ansgesetzt gewesen ist. Ferner ist es unter keinen Cmstinden rathsam, weit anseinander liegende Messungen mit einander zu combiniren. Handelt es sich nm die Vergleichung zweier Objecte, so ist es am besten, die Einstellungen des einen zwisehen die des anderen einznschieben, und wenn mehrere Objecte in Betracht kommen, $\therefore$ sollte das Augenmerk stets auf eine möglichst symmetrische Anordnumg der Einstellungen gerichtet sein.

Anf das Strengste ist darant zu alchten, dass während der Messungen jedes tremde Licht ron dem Inge fern gehalten wird. Die Beobachtungen gesthehen an besten in vollkommen dunklem Raume, und es muss, wenn irgend angiangig, remieden verden, dass der Beobachter 
die Ablesungen und Aufzeichnungen selbst besorgt. Wenn man gezwungeu ist, nach jeder Auslüschung anf oine erlenchtete Seala oder einen Theilkreis oder anf ein helles Blatt Papier zu blicken, so ist eine hestandige Aceommodation des Auges nothwendig, welehe nicht nur zeitraubend ist, sondern die Beobachtungen unsicher macht. In Interesse brauchbarer Messungen muss es als eine unerlaissliehe Bedingung hingestellt werden, dass dem Beobachter entweder ein Gehulfe \%um Ablesen und Aufuotiren z.nr Seito steht, oder dass der Messapparat mit einer geeigneten Registrirrorriehtung rerselien ist.

Eine unvermeidliche Fehlerquelle bei allen anf dem Prineip der Auslösehung berulienden Photometern bildet die rerïinderliche Helligkeit des Grundes, auf welehen sich die beobachteten Himmelsobjecte projiciren. Wir könuen mit blossem Auge an 'Tage die Sterne nicht sehen, weil die Intensitiitsdifferenz zwisehen Stern und umgebendem Himmelsgrunde im Verhältniss zur Helligkeit des letzteren ausserordentlieh klein ist. Mit Hülfe des Fermrohres gelingt es wenigstens die helleren Sterne am Tage wahrzunchmen, weil durch die vergrössernde Kraft desselben das Licht des Grundes merklich abgeschwaicht und das erwaihnte Verhältniss daher vergrössert wird; aber die schwaicheren Sterne, die bei Nacht noeh mit Leichtigkeit sichtbar sind, künnen auch durch das Fernrohr nicht am Tage von dem Himmelsgrunde nuterschieden werlen. Alles dieses folgt von selbst aus dem Fechner'schen josychophysischen Grundgesetze. Ist $h$ die eigene Helligkeit eines Sternes, die er bei ganz dunklem Grunde fiir unser Ange laben wiirde, so wird, wenn die Intensität des Grundes $g$ ist, die Stelle, wo der Stern steht, fiir unser Auge die Gesammthelligkeit $g+h$ besitzen. Die entsprechende Emptindungsdifferenz dE zwischen Stern und Grund wird daher nach dem Fechnerschen Gesetze ausgedrilekt sein durch die Gleichung (siehe Seite 14):

$$
d E=c \log \frac{g+h}{g} .
$$

Je grösser $g$ ist im Verhäiltniss zu $h$, desto mehr nïhert sich der Bruch $\frac{g+h}{g}$ dem Grenzwerthe 1, und die Empfinduugsditlerenz $d E$ wird Null. d. h. der Stern unterscheidet sich nicht mehr rom Grunde. Nach den bisherigen Untersuchungen brancht das Verhiiltniss von $g$ zu $h$ gar nicht einmal sehr gross zu sein, um schon dis Terschwinden hervorzubringen. Wie bereits fribher mitgetheilt wurde, kann nuter besonders giustigen Bedingungen noch ein Helligkeitsunterschied von ungefihr $\frac{1}{10}$ emptunden werden, doch griundet sich dieser Werth fast nur auf Beohachitungen iber das Verschwinden ron ansgedehnten Lichtflichen. Bei Lichtpunkten 
scheint die Grenze noeh viel niedriger zu sein, und es braucht, wie einige Beobachter behaupten, die Helligkeit des Grundes nur ungefähr 40 Mal grösser zu sciu als die ursprüngliche Intensität des Sternes, um eine Unterscheidung zwischen Grund und Stern unmöglich zu machen. Hat man zwei Sterne von der gleichen objectiven Helligkeit $h$, die sich aber anf verschieden hellen Grund von der Intensität $g_{1}$ resp. $g_{2}$ projiciren, so werden nach dem Fechner'schen Gesetze die Empfindungsunterschiede zwischen den Sternen und dem Grunde ansgedriickt durch:

$$
d E_{1}=c \log \frac{h+g_{1}}{g_{1}}
$$

und

mithin :

$$
d E_{2}=c \log \frac{h+g_{2}}{g_{2}}
$$

$$
d E_{1}-d E_{2}=c \log \frac{1+\frac{h}{g_{1}}}{1+\frac{h}{g_{2}}} .
$$

Ist $110 n g_{1}>g_{2}$, so wird die rechte Seite negativ, d. h. $d E_{2}>d E_{1}$, und es folgt, was von vornhercin auch ganz selbstverständlich scheint, dass wenn der eine Stern auf dem Grunde $g_{1}$ gerade verschwindet, der andere anf dem schwächeren Grunde $g_{2}$ noch sichtbar ist. Bei astronomisehen Beobachtungen kommen allerdings im Allgemeinen keine sehr auffallenden Helligkeitsunterschiede des Grundes vor, und da bei der Abschwächung der Sterne die Intensität des Grundes ebenfalls vermindert wird, so erfolgt gewöhnlieh, namentlich bei den helleren Objecten, die eigentliche Auslöschung anf rollkommen dunklem Grunde. In mondlosen Nächten ist infolge dessen keine merkliche Becinflussung der Beobachtungen durch verschiedene Helligkeit des Grundes zu befürchten; dagegen dürfen auf keinen Fall Messungen in der Dämmerung oder bei Mondschein mit Messungen in dunklen Nïchten combinirt werden, und ebenso wenig ist es gestattet, bei heller Belenelitung schwache und helle sterne mit einander zu vergleichen oder Beobachtungen in unmittelbarer Nihe des Mondes mit solchen an anderen weit davon entfernten Stellen des Himmels zu vereinigen.

Durch die rorangehenden Bemerkungen ist der Bereich, innerhalb dessen die Anslijschungsmethode mit Vortheil verwendbar sein dürfte, ziemlich genau fixirt. Wie man sieht, sind die Grenzen eng genug, aber die bisherigen Erfahrungen haben gezeigt, dass bei strenger Befolgung der angedenteten Vorsichtsmassregeln sehr branchbare Resultate erhalten werden künnen. 
Von den in der Einleitung orwiahnten sechs Hauptmethoden zur messbaren Veränderung der lebendigen Kraft einer Liehtquelle sind bei der Construction der bisher bekannten Auslosschungsphotometer fast ausschliesslich die zweite und dritte zur Verwendung gekommen, wahrend die ubrigen nur gelegentlich mit zu Hulfe gezogen wurden." Wir unterscheiden daher im Folgenden nur die beiden Hauptabtheilungen: 1) Ausloschung durch Blendrorrichtungen und 2) Auslöschung durch absorbirende Medien. Ein einzig in seiner Art dastehendes Auslöschungsphotometer, das Parkhurst'selie, welehes streng genommen in keine der Hauptkategorien hineinpasst, soll im Anschlusse an die erste Abtheilung besprochen werden:

\section{Anslöschung des Lichtes durch Blendvorrichtungen.}

Wenn man eine leuchtende Fläche mit dem blossen Auge betrachtet, so entsteht auf der Netzhaut ein Bild dieser Fläche, welches sich je nach der Ausdehnung derselben uber eine grössere oder geringere Anzahl von Netzhautelementen ausbreitet. Jedes dieser Elemente empfängt eine Reizung, und man nimmt gewölnulich an, dass der Reizstärke anch dic im Nervensystem hervorgerufene Empfindungsstärke proportional ist. Dabei ist natürlich abgesehen von einer etwaigen Verschiedenheit der Empfindlichkeit einzelner Netzhautelemente oder ganzer Gruppen derselben.

Unter der scheinbaren Helligkeit einer leuchtenden Fliche versteht man die auf ein einzelnes Netzhautelement durch das optische System des Anges uibergefiihrte Lichtmenge oder, entsprechend den Definitionen im ersten Absehnitte, die auf der Netzhant hervorgebrachte Beleuchtung, mit anderen Worten die gesammte ins Ange gelangende Lichtquantitait dividirt durch die Bildfliiche auf der Netzhimut. Nun liisst sich diese Bildfläche, die wir $b$ nennen wollen, uach den Lehren der greometrischen Optik ausdriicken durch $l_{i} \frac{F^{2}}{r^{2}}$, wo $l_{i}$ ein Proportionalititsfactor ist, $F$ die Grösse der lenchtenden Fläche und $r$ die Entferunng derselben rom Auge (streng genommen vou dem vorderen Knotenpunkte des Auges) bedentet. Ferner ist die gesummte Lichtmenge $L$, welche auf die Netzhant gelangt, wenn mau die absorbirende Wirkmng der brechenden Medien des Auges ausser Aelıt liisst, identisch mit derjenigen, welche auf die vordere Öfthung des Anges, die Pupille, auffaillt, und diese lissst sich nach dem Friilıeren mit hinreichender Genanigkeit ausdriticken durch $J_{p} \frac{F}{r^{2}}$, wo $p$ die P'upillenöffnumg und $J$ die der Fläche 
innewohnende Lenchtkraft ist. Fiir die scheinbare Helligkeit $h$ der Fläche ergicbt sich daher der Werth:

$$
h=\frac{L}{b}=K J p \text {. }
$$

Daraus folgt, dass die scheinbare Helligkeit proportional der Pupillenöftnung ist und ganz unabhängig bleibt von der Entfernung der lenchtenden Fläche vom Ange. Bei unveränderter Pupillenöffnung ist also die Helligkeit einer leuchtenden Fläehe in allen Entfernungen constant, vorausgesetzt natiurlich, dass die Entfernung nicht so gross ist, dass jeder Eindruck der Flichenausdehnung rerschwindet.

Ist dies letztere der Fall und erblickt das Auge also statt einer lenchtenden Fläehe einen leuchtenden Punkt, so verhält sich die Sache allerdings wesentlich anders. Das Bild anf der Netzhant ist dann ebenfalls ein Punkt und daher klein im Vergleich zn dem minimalsten erresbaren Flichensticke der Netzhant. Es kann in diesem Falle ron ciner Beleuchtung nicht die Rede sein, und der im Ange hervorgebrachte Reiz oder die Bildhelligkeit ist der gesammten anf die Netzhant oder auf die l'upille geelangenden Lichtmenge proportional, d. h. also nicht nur rou der Öftinung der Pupille, sondern auch von der Entfernung abhïngig. Es ist schon friiher auf diesen Unterschied zwisehen Fliichenund Punkthelligkeit hingewiesen worden.

Bei den meisten photometrischen Apparaten kommt ausser dem Ange noch irgend ein dioptrisches Srstem in Betracht, und in der Astronomie speciell wird es sich un die Wirkungstreise des Gesammtsystems »Fernrohr und Ange" landeln.

Es sei df ein der Fernrohraxe nahes, zu ihr senkrecht stehendes Element einer lenchtenden Fläche. of die specifische Lenchtkraft desselben, dimn fallt anf die erste Flache des Objectirs, deren Grösse o sein möge, die Lichtmenge $Q=\frac{\text { T } d f^{\circ} 0}{r^{2}}$, wenm $r$ der Abstand der Fläche rom Objectir oder richtiger ron der crsten IIanptebene des Objectirs) ist. Nimmt maln keine Riicksicht anf das in dem Linsensysteme des Objectirs durch hetlexion and Absorption rerloren sohende Lieht und vernachläsigt zunächst auch deu Einflus der Bengung, so geht diese Lichtmenge () unrermindert anf tas rom Ohjectir entworfene Bild, dessen Flïcheninhalt $d f^{\prime}$ sein müge, iiber. Yim kamn sich nun das Bild als selbstlenchtendes onject rorstellen. welches sowohl nach vorwärts als riickwärts Licht amsstrahlt, und es witrde daher; da dieselben Liehtstrahlen auftreten, nach dem Objectir die gleiche Lichtmenge gelangen, wie ron dem lenchtenden Elencute af sellst. Nenut man also $J^{\prime}$ 
die specifische Leuchtkraft des Bildes, $r^{\prime}$ seinen Abstand vom Objectiv oder von der zweiten Hauptebene desselben, so ist auch $Q=\frac{J^{\prime} d f^{\prime} n}{r^{\prime z}}$ und folglich:

$$
\frac{J^{\prime}}{J^{-}}=\frac{d f}{r^{2}} \frac{r^{\circ}}{d f^{\prime}}
$$

Nun besteht aber nach den Sätzen der geometrisehen Optik für ein beliebiges System brechender sphärischer Flächen die Relation:

$$
\frac{d f}{r^{2}} F^{2}=\frac{d f^{\prime}}{r^{\prime z}} F^{\prime 2}
$$

wo $F$ und $F^{\prime \prime}$ die Hauptbrennweiten des Systems, erstere nach dem Objectraume, letztere nach dem Bildraume zu gereclnet, vorstellen. Da aber diese Brennweiten auch proportional sind den Brechungsindices $n$ und $n^{\prime}$ der beiden Medien, welehe den Objectraum und den Bildraum fillen, so hat man:

$$
\frac{J^{\prime}}{J}=\frac{F^{\prime \prime}}{F^{\prime 2}}=\frac{n^{\prime 2}}{n^{2}} .
$$

In den meisten Fällen sind die Indices $n$ und $n^{\prime}$ einander gleich, und es wird daher

$$
J=J^{\prime}
$$

d. h. die Lenchtkriifte in eonjugirten Punkten von Object und Bild sind einander gleich. Hat das leuchtende Object in allen Punkten dieselhe Lenchtkraft, so findet bei dem Bilde disselbe statt. In Wirklichkeit geht allerdings dureh Reflexion, Absorption ete. Licht verloren, und es wird daher $J^{\prime}$ fast immer etwas geringer sein als $J$. Eine Verstiirkung der Lenchtkraft in Bilde kamn durch ein optisches System unter keinen Umstiinden hervorgehracht werden.

Ist die lenchtende Fläche selır weit vom Objective entfernt, so liegt das Bild in rer Bremnebene desselben. Die gesamnte auf diss Objectiv auffallende Lichtmenge ist proportional der Grösse der Objectivöffumb, also gleich ko; die Bildgrösse ist proportional dem Quadrate der Bremnweite $F$ des Objectivs, also gleich' $c F^{2}$. Mithin ist die Lichtmenge, welche auf dic Einheit der Bildtiache grelangt, oder, wie man auch sagt, die objective Flaichenhelligkeit $H$ des Bremmpunktlildes ansgedriickt durch $K \frac{o}{F^{2}}$. Fiir ein zweites Objectiv mit der Öff̈nnng os und der Brennweite $r^{\prime}$, hat man die entsprechende objective Flïchenhelligkeit

$$
H_{1}=K_{H_{1}^{2}}^{o_{1}}
$$


Mithin ist:

$$
H: H_{1}=\frac{o}{F^{2}}: \frac{o_{1}}{F_{1}^{2}},
$$

oder wenn man die Durchmesser $d$ und $d_{1}$ der Objective einführt:

$$
H: H_{1}=\frac{d^{2}}{F^{2}}: \frac{d_{1}^{2}}{F_{1}^{2}} \text {. }
$$

Wemu also das Verhültniss von Objectivdurchmesser zur Brennweite in zwei Fermrölıren desselbe ist, so haben die Brennpunktsbilder in beiden gleiehe Fliiehenintensitiit.

Das rom Objectiv entworfenc Bild wird nun mit dem System $\gg$ Ocular und Ange w betrachtet, und es ist nach dem Früheren klar, dass die Helligkeit des auf der Netzhaut entstehenden Bildes oder die Beleuchtung. der Netzhaut (in letzter Linie also auch die Empfindungsstärke) proportional sein wird der Öffnung des aus dem Ocular austretenden Strahlenbiindels oder, wie man gewöhnlich sagt, der Grösse der Austrittspupille des optischen Systems ${ }^{1}$. Nennt man diese Grösse $o^{\prime}$, und ist $h$ die Helligkeit des mit dem Ferurohre gesehenen Netzhautbildes, währeud $h_{0}$ die Helligkeit des mit blossem Auge gesehenen Bildes sein möge, so hat man:

$$
h_{10}=\frac{o^{\prime}}{p} \text {. }
$$

Ist die Austrittsöffinung des optischen Systems $o^{\prime}$ gleich der Angenpupille $p$, fiillt also der aus dem System tretende Strahlencylinder gerade die Pupille aus, so wird $h=h_{0}$, d. h. das optisehe System vor dem Ange bringt in Bezug anf die Helligkeit des Netzhautbildes gar keine Änderung. hervor. Dasselbe gilt aneh noch, wemn $o^{\prime}>p$ ist; denn dann wird die Augenpupille selbst die Stelle der Austrittsöffnung einnehmen. Ist dagegen $o^{\prime}<p$, so wird anch $h<h_{0}$, das optische System bringt eine Absehwaichung des Bildes anf der Netzhaut hervor. Vernachlissigt ist dabei immer der Lichtrerlust beim Durehgange durch das optische System, welcher bewirkt, dass die vollstiindige Gleichlieit von $h$ und $h_{0}$ niemals erreieht werden kanu.

Bei jedem astronomischen Fermohre ist der Quotient $\frac{o}{o^{\prime}}$, wemn o die wirksame Objectiröffumg ist, gleich dem Quadrate der linearen Vergrösserung $r$ des Systems. Mau lat also:

$$
h_{h}=\frac{o}{h^{2}}
$$

.. Die Austrittsöfunug des optischen Systems ist nicht zu verwechseln mit der Öffnung im Angendeckel des Ocnlars. Welche bei richtig construirten Ocularen stets grösser sein sollte, als die erstere. 
Bei Abbildung ron Flïcheu dureh ein astronomisches Ferurohr verhalten sich demnach die Helligkeiten der Netzhantbilder direct wie die freien Flächen des Objectivs und ungekehrt wie die Quadrate der Vergrösserungen. Die Grösse $\sqrt{\frac{o}{p}}$ nennt man die Normalvergrösserung des Systems; bezeichnet man dieselbe mit $r_{0}$, so wird:

$$
\frac{h}{h_{0}}=\left(\frac{v_{0}}{r}\right)^{2} \text {. }
$$

Natirlich gilt diese Gleichung uur für Werthe von $v$, die grösser als $v_{0}$ sind; dẻn weun die Vergrösserung kleiner ist als die Normalvergrösserung, so muss die Austrittspupille grösser sein als die Augenpupille, und in diesem Falle ist, wie wir oben gesehen haben, die Belenchtung der Netzhaut stets gleich $h_{0}$.

Bei Betrachtung von Sternen, die sich anf der Netzhant als Lichtpunkte abbilden, wird die Helligkeit durch die gesammte Liehtmenge gemessen, welehe durch das Fernrohr dem Auge zugeftihrt wird; sie verhält sich also zu der Helligkeit des direct mit blossem Ange gesehenen Sternes wie die freie Objectivöffnung zu der Pupillenöffmung: es ist demnach:

$$
\frac{h}{h_{0}}=\frac{o}{p}
$$

Solange die Austrittsüffumng des Strahlenbïndels nicht grösser als die Augenpupille, oder mit anderen Worten, solange die Vergrössermng des Fernrohrs nieht kleiner als die Normalvergrössermug ist, geht alles anf das Objectiv fallende Licht in das Auge, und die Helligkeit des Sternes im Fernrohr in Verhältniss zur Helligkeit mit blossem Auge bleilht constant gleich $\frac{o}{p}$. Wird dagegen die Vergrössermug des Fermrohrs kleiner als die Normalvergrösserung und mithin die Austrittspupille griosser als die Angenpupille, so gelangt nur ein Theil des gesammten Lichtes in Netzhantbilde zmr Wirkung. Die Helligkeit des mit dem Fermolı gesehenen Sternes im Verlualtniss zur Helligkeit mit freiem Auge ist dam kleiner als $\frac{o}{p}$, mnd zwar ist sie, wie man leicht sieht, gleich dem Quadrate der jedesmaligen Vergrössserung.

Die Thatsache, dass bei dem System pFermoln und Ange" die Helligkeit des Netzhautbildes (sei es von einer Flaiche oder von einem Sterne), falls die Vergrösserung constant bleibt, stets der freien Objectivoffumng proportional ist, lïsst auf den ersten Blick die Ablulendungr des Objectivs oder, was dasselbe ist, des aus dem Ohjectir austretenden Strahlen- 
kegels als das einfachste und bequemste Mittel erscheinen, um die Intensität einer Liehtquelle in messbarer Weise zu verringern. Auf die Form der Blendenöffnung kommt es dabei nicht an, wenn es nur möglich ist, die Grösse der treien Flaiche genau zu bestimmen. Freilich erheben sich sofort einige gewichtige Bedenken gegen diese Methode. Auf die Mitte des Objeetivs fallen die Strahlen unter etwas anderen Winkeln auf als auf die Randpartien, und infolge dessen ist der Lichtverlust dureh Reflexion am Rande grösser als in der Mitte. Dieser Nachtheil wird dadureh wieder einigermassen anfgewogen, dass die Mittelstrahlen gewölınlich eine etwas dickere Glasschicht zu durchlaufen haben als die Randstrahlen und daher etwas mehr Licht durch Absorption einbüssen. Auch kann diesem Übelstande, wie wir später sehen werden, dadurch zum Theil abgeholfen werden, dass man das Objectiv nicht von dem Rande nach der Mitte zu abblendet, sondern fächerartige Blenden anwendet. Trotzdem wird aber eine vollkommen gesetzmässige Lichtschwiichung selten zn erzielen sein, weil kleine Fehler in der Glasmasse und vor Allem die niemals gänzlich zu beseitigende sphärische Aberration Unregelmässigkeiten in der Lichtwirkung der einzelnen Partien des Objectirs in Gefolge haben werden.

Ist schon aus diesen Griinden die Anwendung von Blenden zu photometrischeu Messungen im Princip durebaus anfeehtbar, so kommt noeh als weiteres bedenkliches Moment der Einfluss der Beugung des Lichtes au den Riinderu der Blendenöffnung hinzu. Auf die Bedeutung der Diffraction für Liehtmessungen ist bisher noeh nicht mit dem nöthigen Nachdrucke hingewiesen worden, und es diurfte daher hier am besten Gelegenheit sein, auf diesen Punkt aufmerksam zu machen und zu zeigen, dass unter Umstïnden photometrisehe Messungen mittelst Verkleinerung der Objectivöffunng infolge der Bengungswirkung zu gänzlich falschen Resultaten fülıren können.

Die Theorie der Bengungserscheinmgen, wie sie von Airy, Sehwerd, Knochenhaner, in nenerer Zeit besonders ron H. Struve und Lommel entwickelt worden ist, soll dabei als bekannt vorausgesetzt werden, und der Einfachheit wegen soll mur der Fall der Abblendung rom Rande nach der Mitte zu bei Benutzung ron kreisförmigen Blendenöffinungen etwas weiter verfolgt werden, weil diese Art der Abblendung in der Praxis wohl am hïufigsten rorkommen diurfte. Bei anders gestalteten i)ffnungen, beispielsweise dreieckigen, viereckigen n. s. w., welehe ebenfalls mitunter in der Himmelsphotometrie zur Verwendung kommen, sind die theoretischen Entwieklungen in Allgemeinen etwas complicirter. Ferner soll hier nur ron den Erscheinungen die Rede sein, welehe sich bei der Betrachtung ron Fixsternen durch das Ferurohr darbieten, während 
die schwierigeren Verhältnisse, welche bei der Abbildung vou lenchtenden Flichen auftreten, ausser Spiel gelassen werden köunen.

Wie schon Herschel bemerkt hatte, ist das mit hinreichend starker Vergrösserung in einem Fernrohr betrachtete Bild eines Fixsternes nicht ein wirklicher Punkt, sondern besteht aus einem kleinen kreisrunden Scheibchen, dessen Helligkeit von der Mitte nach dem Rande \%u abnimmt und desseu Saum gefärbt erscheint, sowie aus einigen concentrischen, abwechselnd dunklen und hellen Ringen, von denen die let\%teren ebenfalls gefirbt sind. Die Intensitiit der Ringe nimmt nach aussen zu sehr schnell ab, und die Zahl der Uberhaupt sichtbaren ist für Sterne eine sehr geringe. Es hängt dies von mehreren Umstïnden $a b$, in erster Linie naturlich von der Helligkeit des Sternes, dann von der angewandten Vergrösserung und der Helligkeit des Himmelsgrundes, auf den sich das Bild projicirt; im Allgemeinen wird man nur selten mehr als drei Ringe wahrnehmen könuen.

Ehe man diese Erseheinung richtig zu deuten wusste, nahm man an. dass die Fixsterne messbare Durchmesser besäissen, und versuchte, die Grössen derselben darans zu bestimmen. Erst Airy wies mit Sieherheit darauf hin, dass die seheibenartigen Bilder der Sterne und die sie umgebenden Ringe eine unausbleibliche Folge der Beugung des Lichtes an den Rändern der Objectivöffunng seien, und dass sich nach den Fresnelschen Untersuchungen die Lichtvertheilung iunerhalb des Beugungsbildchens mit voller Strenge theoretisch bereehnen lasse. Aus der Diffractionstheorie ergiebt sich anch die Folgerung, dass bei Verkleinerung der Objectivöffnung der Durchmesser des centralen Beugungsseheibehens grösser werden muss, und zwar umgekehrt proportional dem Durehmesser der Öftnung, eine Folgerung, die mit den Resultaten der praktisehen Messung in vollem Einklange ist.

Was die Lichtvertheilung innerhalb der in der Focalcbene des Fernrohrs entstehenden Beugungsfigur anbelangt, so folgt fïr die specifisehe Leuchtkraft $L$ irgend eines ron der optischen Axe um den Abstand $:$ entfernten Punktes aus der Lommel'sehen ') Theorie die Formel:

$$
L=C \pi^{2} r^{4}\left[\frac{2}{2} J_{1}(i)\right]^{2} \text {. }
$$

Hierin bedeutet $C$ eine Constante, $r$ den Radius der Objectivöffinung. Ferner ist gesetzt $x=\frac{2 \pi}{\lambda f} \zeta r$, wo $f$ die Brenuweite des Objectivs und $\lambda$

1) Lommel, Die Bengungserscheinnngen einer kreisrunden Öfłnung und eines kreisrunden Schirmchens theoretisch und experimentell bearbeitet (Abh. d. K. Bayer. Akad. d. Wiss. Math.-phys. Cl. Bd. 15, p. 227. 
dic Wellenlainge des zunächst als homogen angenommenen einfallenden Lichtes ist. Endlich ist $J_{1}(*)$ die bekannte Bessel'sche Function ersten Grades, nämlieh:

$J_{1}(x)=\frac{\pi}{\pi} \int_{0}^{\pi} \cos (z \cos \omega) \sin ^{2} \omega d \omega=\frac{\pi}{2}-\frac{\pi^{3}}{2^{2} \cdot 4}+\frac{\pi^{5}}{(2 \cdot 4)^{2} \cdot 6}-\frac{\pi^{i}}{(2 \cdot 4 \cdot 6)^{2} \cdot 8}+\cdots$

Durch Substitution dieses Werthes in die obige Gleichung für $L$ wird:

(2) $L=C x^{2} r^{4}\left\{1-\frac{x^{2}}{2 \cdot 4}+\frac{x^{4}}{2 \cdot 4 \cdot 4 \cdot 6}-\frac{x^{6}}{2 \cdot 4 \cdot 6 \cdot 4 \cdot 6 \cdot 8}+\cdots\right\}^{2}$.

Die nmerischen Werthe des Klammerausdruckes sind ron Lommel in einer ansfibhrlichen Tabelle für Werthe von $z$ zwischen 0 und 20 von Zehntel \%u Zehntel angegeben, und es lïsst sich daher sehr leicht in jedem Falle die Intensititsvertheilung im Bengungsbilde berechnen. Um einen bestimmten Fall zu fixiren, wollen wir ein Fernrohr von $100 \mathrm{~mm}$ Öffnung: und $1500 \mathrm{~mm}$ Focallinge annehmen und roraussetzen, dass es sich um homogenes Licht ron der mittleren Wellenliinge $0.0005 \mathrm{~mm}$ handelt; ferner wollen wil die Lenchtkraft im Mittelpunkte des Beugumgsscheibchens als Einheit annehmen. Damn ergiebt sich die Leuchtkraft in rerschiedenen Abstiuden ron der optischen txe ans der folgenden kleinen Tabelle.

Vertheilung der Leuchtkraft im Beugungsbilde eines Sternes bei einem Fernrohr von $100 \mathrm{~mm}$ öffnung und $1500 \mathrm{~mm}$ Brennweite.

\begin{tabular}{|c|c|c|c|}
\hline $\begin{array}{l}\text { Abstand } \\
\text { ron der Axe }\end{array}$ & Lenchthraft & $\begin{array}{c}\text { dhstanul } \\
\text { ron der Axe }\end{array}$ & Leuchtkraft \\
\hline $0.0600 \mathrm{~mm}$ & 1.01000 & $0.013 \mathrm{~mm}$ & 0.0160 \\
\hline 0.0111 & 10.9570 & 0.014 & 0.0105 \\
\hline 0.002 & 0.5365 & 0.015 & 11.0046 \\
\hline 0.1003 & 11.6644 & $0.011 i$ & 0.00015 \\
\hline 0.61144 & 11.4729 & 0.017 & 0.0001 \\
\hline 0.005 & 11.2949 & 0.015 & 0.0015 \\
\hline 0.0066 & 0.1542 & $0.01 !$ & 0.00133 \\
\hline 0.007 & 0.0615 & 0.020 & $0.00+1$ \\
\hline 0.0115 & 0.0141 & 0.021 & 10.0030 \\
\hline 0.1109 & 0.0001 & 0.012 .2 & 0.00021 \\
\hline 0.010 & 0.10042 & 0.023 & 0.0007 \\
\hline 0.1111 & $0.0126^{2}$ & 0.0 .4 & $0.0000 \mathrm{j}$ \\
\hline 0.012 & 0.0173 & 0.1125 & 0.011012 \\
\hline
\end{tabular}

Die drei ersten dunklen Bengmaringe halsen die Abstände 0.0091, 0.0167 und $0.0243 \mathrm{~mm}$ rom Centrum. und dis centrale Diffiractionsscheibchen 
hat denuach einen Durchmesser von $0.018 \mathrm{~mm}$ oder (rom Ohjectiv aus gesehen) von 2.5 Bogenseeunden.

Wird das Objectiv des Fernrohrs kreisformig abgeblendet, so nimmt die speeifische Lenchtkraft in der Mitte des Bildes, wie ans der obigen Formel unmittelbar liervorgeht, proportional der vierten Potenz des Halbmessers der freien Öffinung ab, gleiehzeitig vergrössert sieh aber die Dimension des Bengungsbildes proportional der Öffumung selbst, so dass also bei einer Abblendung des obigen Objectirs auf 50, 20 und $10 \mathrm{~mm}$ die Dnrehmesser der betrefienden eentralen Beugungsseheiben, in Bogensecunden ausgedrtickt, gleieh 5."0, 12.5 und 25."0 werlen.

In der Praxis komnt es weniger auf die Kenntniss der specifisehen Leuchtkraft in irgend einem Punkte des Sternbildes an, als vielmehr anf die Ermittlung der gesammten Lichtmenge, welehe von der ganzen Beugungserscheinung oder einem bestimmten Theile derselben ansgeht und sich auf einen gewissen Bezirk der Netzhant ansbreitet. Denkt man sich in dem Bengungsbilde eine ringförmige Zone mit den Radien $\zeta$ und $\zeta+d \xi$, so wird die Liehtquantitit $d Q$, welehe uber diese Zone ansgebreitet ist, dureh die Formel bestimmit sein:

$$
d Q=2 \pi \zeta d \check{L} L
$$

wenn $L$ die specifisehe Leuchtkraft im Abstande $\because$ vom Centrum bezeichnet. Die gesanmte innerhalb eines Kreises mit einem beliebigen Radius $\zeta_{1}$ eingesehlossene Lichtmenge des Beugungsbildes ist daher gegeben durch:

$$
Q=2 \pi \int_{0}^{\zeta} L \zeta d \zeta
$$

Als Einheit ist die anf die ganze Beugnugserscheinmug vertheilte Lichtmenge oder, was dasselbe ist, wenu man ron Absorption, Reflexion u.s. w. absieht, dic auf die freic Objectivöftinung auffallende Lichtquantitä zu betraehten.

Substituirt man den Werth von $\ddot{=}=\frac{\lambda f}{2 \pi r}$. und den Werth von $L$ aus Gleichung (1), so ergiebt sich:

$$
Q=2 C i^{2} f^{2} \cdot r r^{2} \int_{0}^{z_{1}} \frac{J_{1}^{2}(a)}{a} d r
$$

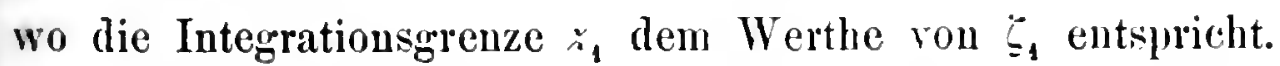


Naeh den Lommel'schen Untersuehungen über die Bessel'sehen Funetionen ist:

$$
2 \int_{0}^{\varepsilon_{1}} \frac{J_{1}^{2}(z)}{z} d z=1-J_{0}^{2}\left(z_{1}\right)-J_{1}^{2}\left(z_{1}\right) .
$$

Man hat also, wenn man noeh $C \lambda^{2} f^{2}$ durch eine einzige Constante $C_{1}$ ersetzt:

$$
Q=C_{1} \pi r^{2}\left[1-J_{0}^{2}\left(r_{1}\right)-J_{1}^{2}\left(z_{1}\right)\right] \text {. }
$$

Die numerischen Werthe der Funetionen $J_{0}$ und $J_{1}$ sind für verschiedene Werthe ron $:$ von Lommel berechnet und in Tabellen zusammengestellt worden. Daraus ergeben sich für den obigen Klammerausdruck die folgenden Zahlenwerthe:

\begin{tabular}{|c|c||c|c|}
\hline$z$ & $1-J_{0}{ }^{2}(z)-J_{1}{ }^{2}(\tilde{z})$ & $z$ & $1-J_{0}{ }^{2}(\tilde{z})-J_{1}{ }^{2}(\tilde{z})$ \\
\hline 0 & 0.000 & 7 & 0.910 \\
1 & 0.221 & 5 & 0.915 \\
2 & 0.617 & 9 & 0.932 \\
3 & 0.817 & 10 & 0.938 \\
4 & 0.535 & 11 & 0.939 \\
5 & 0.561 & 12 & 0.948 \\
6 & 0.901 & & \\
\hline
\end{tabular}

Betrachtet man nur die Gesammtliehtmenge im centralen Scheibchen, die bei Sternbeobachtungen hauptsächlich in Frage kommt, so ergiebt sieh, da in diesem Falle $a=3.5317$ zu setzen ist, für die Lichtquantität der Werth $0.8+C_{1} r^{2} x$; wenn man aber noch den ersten hellen Beugungsring hinzunimmt, so muss man für $a$ den Werth 7.0156 wählen und findet fiir die gesammte Liehtmenge den Werth $0.91 C_{1} \cdot{ }^{2} \pi$.

Es sei num das Objectir so weit abgeblendet, dass der Radius der freien Öffnung $Q$ statt $r$ ist; dam rergrössert sich der Radius des centralen Beugungsseheibehens im Verhältniss ron $r$ zn $\varrho$. Die Gesammtlichtmenge in diesem Scheibchen ist dann $=0.5+C_{1} \varrho^{2} \pi$, und es folgt also, dass die in den centralen Bengungsfiguren vereinigten Lichtquantitäten sich zu einander verhalten wie die zugehörigen freien Flïchen des Objeetirs. Wiurde das centrale Beugangsbild durch das System »Oenlar und Auge so anf die Netzhaut projicirt, dass es dort stets entweder einen kleineren Raum als ein einzelnes getrennt erregbares Element einnälmme oder wenirstens immer dieselbe Dimension besiisse, so wäre anch die Beleuchtung der Netzhant (demnach anch angenzihert die Empfindungsstiarke) der freien objectivfläche proportional, und die photometrische Methode der Abblendung wäre, was die Bengungswirkung des Fermrohrs anbetrift, durchaus einwurfstei. Dies ist aber keineswegs der Fall; 
vielmehr hängt die Grösse des Netzhautbildes wesentlich vou der Vergrüsserung des Fernrohrs ab. Ist $f^{\prime}$ die Brennweite des Oculars und $l$ : die hintere Knotenlänge des Anges, fur welche man den Werth $15 \mathrm{~mm}$ annehmen kann, so verhalten sich die Durehmesser des Brennpunktbildes und des Netzhautbildes zu einander, wie $f^{\prime \prime}$ zu $k$. Bei einem Fernrohre mit der Brenuweite $f$ und dem Objectivdnrehmesser $d$ wird der Durchmesser des centralen Brennpunktbeugungsbildes gemaiss der Formel $2 \%=\frac{\lambda f}{r \pi} \approx$, wo $\approx=3.8317$ \%u set\%en ist, ausgredrttekt durch $0.00122 \frac{f}{d}$. Der Durehmesser des Netzhautbildes wird daher gleich $0.00122 \frac{f}{d} \frac{i}{f^{\prime}}$ oder, weun man für $k$ seinen Werth einsetzt und für $\frac{f}{f^{\prime}}$ die Vergrösserung $v$ des Fernrohrs einflihrt, gleich $0.0183 \frac{v}{d} \mathrm{~mm}$.

Bei zwei rerschiedenen Fernrohren nimmt die Bengungsfigur eines Sternes nur dann den gleichen Raum auf der Netzhaut ein, wenn die angewandten Gesanmtvergrösserungen den Objectivdurchmessern proportional sind, und nur in diesem Falle verhalten sich also die Lichteindrlicke des Sternes in beiden Instrumenten genau wie die freien $\mathrm{Ob}$ jectivfliachen.

Dasselbe gilt bei der Abblendung eines und desselben Fernrohrs. Auch hier miisste fuir jede Blende die Gesammtvergrösserung entsprechend der Öff̈ung verändert werden, wenn man strenge photometrische Messungen ausführen wollte. Bleibt die Vergrösserung, wie es gewöhnlich geschieht, unverïndert, so verbreitet sich bei starker Abblendung die Beugungserscheinung iiber eine grössere Anzahl von einzeln erregbaren Netzhautelementen aus, und die im Nervensystem hervorgerufene Lichtempfindung ist infolge dessen relativ zu schwach. Man gelangt also unter Umständen zu ganz falschen Resultaten.

- Da der Durchmesser eines einzelnen Netzhautzapfens etwa 0.005 mm betriigt, so folgt noch ans dem obigen Werthe des Durchmessers des Netzhautbildes, dass das centrale Bengungsscheibchen dann ungeführ mit einem Netzhautzapfen coincidirt, wenn die Vergrösserungszahl etwa gleich dem vierten Theile des in Millimetern ansgedrïckten Objectivdurchmessers ist.

Fiir ein Fermrohr vou $100 \mathrm{~mm}$ Öff̈umg und $1500 \mathrm{~mm}$ Brennweite sind in der folgenden kleinen Tabelle die Durchmesser des centralen Beugungsscheibchens in der Brennebene sowolll als im Netzhautbilde znsammengestellt bei verschiedenen Abblendungen und verschiedenen Vergrössernugen, und zwar ausgedriickt in Millimetern. 
Dimension der centralen Beugungsfigur eines Sternes bei einem Fernrohr von $100 \mathrm{~mm}$ Öffnung und $1500 \mathrm{~mm}$ Brennweite und kreisrunder Abblendung.

\begin{tabular}{|c|c||c|c|c|c|}
\hline $\begin{array}{c}\text { Durchmesser } \\
\text { der freien } \\
\text { Offinung }\end{array}$ & $\begin{array}{c}\text { Durchmesser } \\
\text { des } \\
\text { Brennpunkt- } \\
\text { bildes }\end{array}$ & \multicolumn{4}{|c|}{ Durchmesser des Netzhantbildes } \\
\hline \hline 100 & 0.0153 & 0.0015 & 0.0037 & 0.0055 & 0.0073 \\
90 & 0.0203 & 0.0020 & 0.0041 & 0.0061 & 0.0081 \\
80 & 0.02299 & 0.0023 & 0.0046 & 0.0069 & 0.0092 \\
70 & 0.0261 & 0.0026 & 0.0052 & 0.1078 & 0.0104 \\
60 & 0.0305 & 0.0031 & 0.0061 & 0.0092 & 0.0122 \\
50 & 0.0366 & 0.0037 & 0.0073 & 0.0110 & 0.0146 \\
40 & 0.0459 & 0.0046 & 0.0092 & 0.0137 & 0.0183 \\
30 & 0.0610 & 0.0061 & 0.0122 & 0.0183 & 0.0244 \\
20 & 0.0915 & 0.0092 & 0.0183 & 0.0275 & 0.0366 \\
10 & 0.1530 & 0.0153 & 0.0366 & 0.0549 & 0.0732 \\
\hline
\end{tabular}

Wie man sieht, ist bei der schwïchsten Vergrösserung, selbst wenn das Objectiv bis anf mehr als den halben Durehmesser abgeblendet wird, das Netzhantbild noch kleiner als die Oberfläche eines einzelnen Netzhantzapfens, und der Lichteindruck auf das Ange wird also bis dahin durchans streng proportional der freien Objectivfläche bleiben. Erst wenn der Objectivdurchmesser bis auf $30 \mathrm{~mm}$ und mehr abgeblendet ist, breitet sich das Netzhantbild anf mehr als einen Netzhantzapfen aus, und die Lichtempfindung wird schwäicher, als man nach dem Verhältuisse der Objectivöffumugen erwarten sollte. Bei den stärkeren Vergrösserungen tritt dieser Fall schon bei weit geringerer Abblendung ein.

Rechnungmäissig liisst sich der Fehler, den man in jedem einzelnen Falle begeht, nicht mit Sicherheit bestimmen, schon deshalb nicht, weil die physiologische Wirkung des Auges nicht genan genug bekannt ist, insbesondere die Frage, wie sich die einzelnen Netzhantelemente hinsichtlich der Emptindlichkeit fiir Lichtreize zn einander verhalten, als keineswegs entschieden zu betrachten ist. Anch darf man nicht unberiicksichtigrt lassen, dass die Helligkeit im Bengungshilde yon der Mitte ans sehr schnell abnimmt und dass daher z. B., wemn das centrale Scheibchen sich in einem Falle iiber vier Netzhantelemente, in einem anderen nur iiber ein cinziges Element ansbreitet, die Empfindungsstärken keineswegs im Verhïltuisse $1 \mathrm{zu}+$ stehen werden. Fndlich ist nicht zu vergessen, dass die angefiihrten Zahlenwerthe mur fiir homogenes Licht von der Wellenlänge $6.0005 \mathrm{~mm}$ gelten. Fiir andere Strahlengattmugen ergeben sich etwas verschiedene Verhialtnisse, und da es sich bei den Sternbeobachtungen 
um genischtes Licht handelt, so werden die Erseheinungen noch eomplieirter; die Beugungsbilder sind mit farbigem Sanme versehen.

Fur ein weiteres Eingehen auf den angeregten Gegenstand ist hier nicht der geeignete Plat\%. Ls möge gentlgen. anf einen ḅisher nicht hinreichend beachteten Fall etwas ausfuhrlicher hingewiesen und-gezeigt z.u hahen, dass die eeutrale Abblendung bei photometrisehen Messungen infolge der Beugungserseheinungen grosse Gefahren in sich birgt, und zwar stets in dem Sinne, dass die beobaehteten Helligkeitsuntersehiede grösser sind, als die gemïss dem Verhältnisse der zugehörigen freien Objectivflichen berechneten. Die begangenen Fehler werden in Allgemeinen um so grösser sein, je erheblicher die Helligkeitsunterschiede der verglichenen Sterne sind, je weiter also das Objectiv abgeblendet werden muss; dagegen werden sich die Fehler wesentlich verkleinern, wenn man inöglichst schwache Vergrösserungen zu den Messungen benutzt.

Von den versehiedenen Photometern, bei denen die Auslösehung des Lichtes durch Ablendungsvorrichtungen bewirkt wird, sollen im Folgenden die wichtigsten angefülırt, aber nur kurz besprochen werden, weil die wenigsten von ihnen dauernde Verwendung in der Astrophotometrie gefunden haben. Am gebriuchliehsten ist die Anbringung der Blenden vor dem Objectiv, jedoch sind anch Apparate construirt worden, bei denen erst der aus dem Objeetiv austretende Strahlenkegel messbar verkleinert wird.

a. Blenden vor dem Objectiv. Die Photometer ron Köhler, Reissig, Dawes, Knobel, Thury und Lamont.

Eins der iiltesten Abblendungsphotometer ist das von Köhlery construirte. Dasselbe besteht in einer Vorrichtung, die so vor dem Fernrohrobjectiv angebracht werden kann, dass stets eine qualdratförmige öffunng frei bleibt, deren Mittelpunkt unveräinderlich mit der Mitte des Objectivs zusammenfiillt. Eine nähere Beschreibung des Mechanismus fehlt, es ist von Köhler nur angegebeu, dass sich die jedesmalige Diagonallänge des Quadrates an einer willkürlichen Scala von 0) bis 1000 ablesen liisst. Wahrscheinlich ist die Eiurichtung ähnlich einer später noch mehrfach benutzten und unter dem Namen sKatzenaugendiaphragma" bekannten, deren Erfindung allgemein s'Gravesande zugeschrieben wird, und die neuerdings wieder von Cornu und Pickering fïr photometrische Zweeke empfohlen worden ist. 
In einem fest mit dem Objectiv verbundenen Rahmen (Fig. 28) gleiten zwei Metallplatten $A$ und $B$ dicht übereinander, welche zwei gleich grosse quadratische Ausschnitte haben, deren Diagonale mit der Bewegungsrichtung parallel ist. Jede dieser Platten ist mit einer Triebstange versehen, ansserdem ist auf der unteren Platte $B$ eine feine Theilung, auf der oberen $A$ ein Indexstrich angebracht. Dureh Drehung des an dem

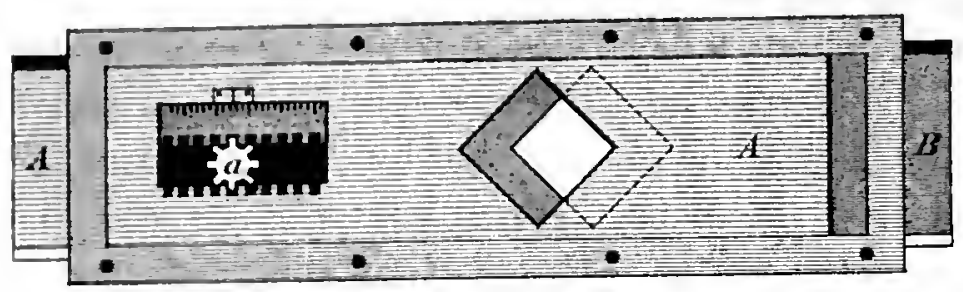

Fig. 28,

festen Theile befindlichen Triebes $a$ werden die beiden Platten im entgegengesetzten Sinne übereinander fortbewegt und zwar so, dass die Mitte der freien Öffnung, welche stets ein Quadrat ist, über der Mitte des Objectirs bleibt. Die Ablesungeu an der Seala geben direct die Lüingen der Öffnmngsdiagonalen, und die Helligkeiten zweier Sterne verhalten sich zn einander wie die Quadrate der Ablesungen, bei denen diese beiden Sterne zum Auslöschen gebracht werden. Der Apparat liesse sich sehr leicht in der Richtung vervollkommnen, dass man die Verschiebung der beiden Platten rom Ocular ans bewerkstelligte und eine Registrirvorrichtung damit in Verbindung braichte.

Ein etwas anderes Arrangement, ebenfalls mit Benutzung von quadratischen (Iffunngen, ist von Reissig') enpfollen worden. Derselbe befestigte eine Scheibe, die mit einer grossen Anzahl ron quadratischen Ausschnitten von verschiedener Grösse versehen war, in der Weise an dem Objectir eines Fermrohrs, dass bei der Drehung der Scheibe die einzelnen Öffnungen genam vor die Mitte des Objectirs gefiilurt werden komnten. Durch eine bis zum Oenlar reichende Stange wurde die Scheibe hewegt, und der jedesmalige Vortritt einer Öffumng vor die Mitte des Objectirs wurde durch das Einspringen eines kleinen Sperrkegels in einen mit der Scheibe rerbundenen Zahnkreis markirt. Bei einigermassen grossen Instrumenten lat diese Einrichtung das Unbequeme, dass die Scheibe sehr betriehtliche Dimensionen haben muss; auch ist dem zu erreichenden Genanigkeitsgrade durch die Inzahl der Öffunngen eine gewisse Grenze gesteckt.

Anstatt quadratischer Öffnungen sind am häufigsten kreisrunde in Vorschlag gebracht worden, die entweder mittelst eines dem Reissig'schen :ihnlichen Arrangements oder nit Hiilfe einer Art Sehiebervorrichtung oder durch einfaches Übereinanderlegen ror die Mitte des Objectirs gebracht

1) Berliner Astronom. Jahrbuch 1511, p. 250. 
werden konnten. Ein derartiges Verfuhreu ist \%. B. von Dawes y etwas genauer beschrieben worden, und seine Methode verdient noch deswegen eine besoudere Erwïhnung, weil er statt der Beobachtung der vollständigen Auslöschung der Sterne die Fixirung desjenigen Momentes empfiehlt, wo die Sterne gerade noch mit Muhe sichtbar sind (limit of steady visibility', und weil er alle Helligkeitsbestimmungen anf diejenige Normalibinung des Teleskops beziehen will, bei welcher die Sterne 6. Grösse diese Sichtbarkeitsgrenze erreichen.

Bei dem Knobel'schen ${ }^{2}$ Astrometer kommen dreieckige Blendenaussehnitte zur Verwendung. Diese haben nach dem Urtheile versehiedener Astronomen, unter auderen J. Hersehel's, vor anders gestalteten Öfẗnungen den Vorzug voraus, dass die eentrale Bengungsfigur sich dureh besondere Schïrfe anszeichnet, und dass auch die begleitenden Beugungserscheinungen, welche in sechs gleiehweit von einander entfernten, rom Centrum auggehenden Strahlen bestehen, verhältnissmïssig wenig störend sind.

In dem mit dem Fernrohr verbundenen Ralımen $H$ (Fig. 29) gleiten zwei Platten tibereinander. Die untere $A$ hat einen Ausschnitt in der Form eines gleichseitigen Dreiecks, die obere $B$ endet in einer scharfen zur Bewegungsrichtung senkrechten Kante. Datmit die Mitte der freien Öffunung: welehe beim Übereinandergleiten der Platten stets ein grleichseitiges Dreieck bildet, unveriindert mit dem Centrum des Objeetirs zusammenfïllt, muss die Platte $A$

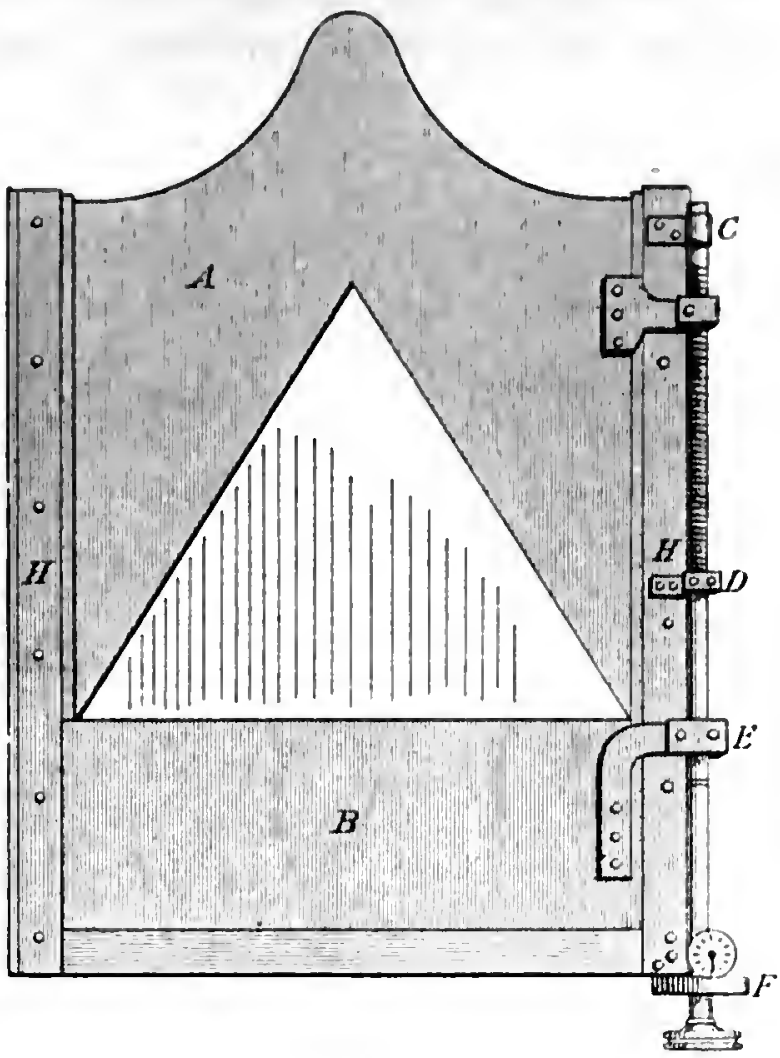

Fig. 29. sich nm eine doppelt so grosse Streeke verschieben, wie die Platte $B$, weil in gleichseitigen Dreieck der Abstand des Mittelpunktes ron den Ecken doppelt so gross ist, wie ron den Seiten. Dies wird erreicht durch die mit Links- and Rechts-Gewinde versehene Mikrometerschraube $C F$, deren oberer die Platte $A$ bewegender Theil $C D$ doppelt so grosse Steigung besitzt wie der untere $D E$. Die an dem Mikrometerkopf ange-

1) Monthly Notices. Vol. 11, p. $18 \%$.

2) Monthly Notices. Vol. 35, p. 101). 
brachte Theilung giebt ein Mass für die jedesmalige Länge der Dreiecksseite, und da der Inlaalt des Dreiecks, wenn diese Seite mit $s$ bezeichnet ist, durch $\frac{\sqrt{3}}{4} s^{2}$ ausgedriickt wird, so verhalten sich die Helligkeiten zweier \%un Versehwinden gebrachten Sterne wie die Quadrate der zugehörigen Mikrometerablesungen.

Besonders interessant ist das 'Thury'selie ${ }^{1}$ ) Photometer, welehes zwar meines Wissens niemals zu zusammenhängenden Messungsreihen am Himmel verwendet worden ist, aber schon deswegen der Vergessenheit entrissen zu werden verdient, weil bei ihm das Abblendungsprineip in der rationellsten Weise zur Anwendung gebracht worden ist.

Thu ry hat bereits in vollem Unfange den schädlichen Einfluss der Diffraction bei Helligkeitsmessungen nach der Abblendungsmethode crkannt und denselben dadurch abzuschwiichen rersucht, dass er die

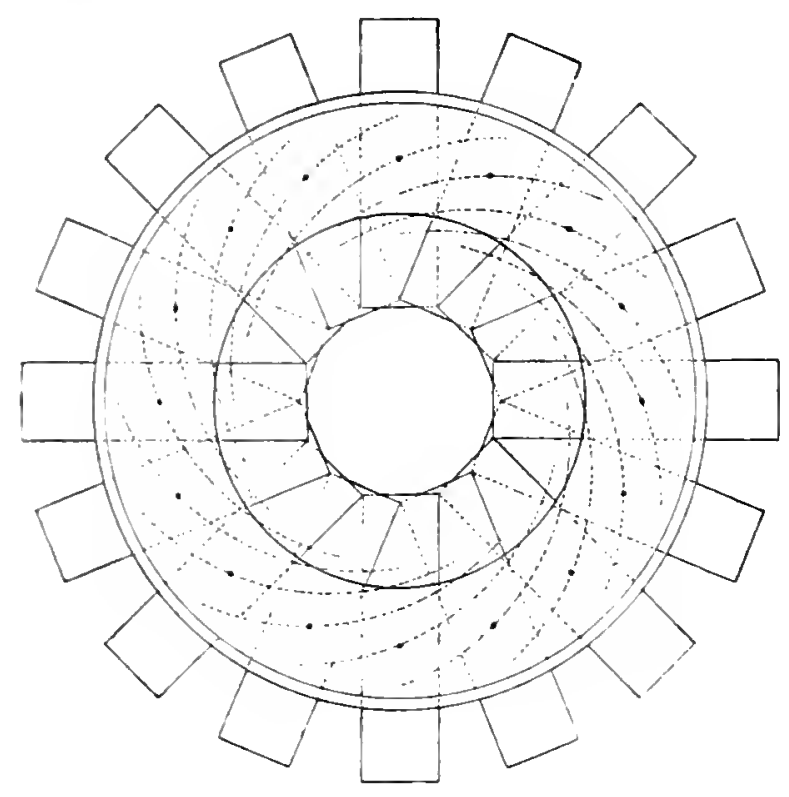

Fig. 30 .

Abblendung des Objectivs nur innerhalb mässiger Grenzen und hauptsächlich zum Zwecke der letzten feinen Auslöschung der Sterne benutzte, die Hauptschwächung aber durch Reflexe an Spiegeln hervorbrachte. Die Thury'sche Blendscheibe ror dem Objectiv (Fig. 30) besteht aus 16 iiber eineinander verschiebbaren Lamellen, welche ein gleichseitiges Polygon bilden, dessen Mittelpunkt stets die Yitte des Objectivs einnimmt. Jede einzelne Lamelle ist mit einem Stift rersehen, welcher in einen zugehörigen gekriimmten Einschnitt einer Metallscheibe eingreift. . Diese

Scheibe liasst sich drehen, und da die Einsehnitte, in denen sich die Stifte der Lamellen bewegen, die Form ron Arehimedischen Spiralen babeu, so ist die Winkelbewegung der Scheibe proportional der linearen Bewegung der Lamellen und infolge dessen anch dem freien Durchmesser des Objectivs. Die Drehnng der Scheibe kann ron dem Ocularende des Lnstrumentes ans dirigirt werden, und anf einer mit dem Bewegungsschliissel verbundenen Theilscheibe aus mattem Porzellan lïsst sich im

1 Bibliothéque miverselle et Rerue Suisse. Archives des sciences phys. et naturelles. Nourelle période. t. jl isit, p. 209. 
Finstern durch eine gan\% einfache Registrirvorrichtung der Betrag der Bewegung markiren. Das ganze Arrangement ihnelt den bei photographischen Apparaten vielfach ublichen Irisblenden.

Um bei cier Messung heller Objecte die Öfinung des Oljectivs nicht allzu sehr verkleinern zu mtissen, hat Thury dem Ocularkopfe eine besondere Einriclıtung gegeben (Fig. 31).

Ein und dasselbe Ocular kam in die Hulsen bei $a, b$ und $c$ eingeschoben werden; $m$ und $n$ sind zwei Spiegel, die unter $45^{\circ}$ gegen die Richtung der anffallenden Strahlen geneigt sind. Bei a beobachtet man die Sterne direct, bei $b$ nach eimmaliger Spiegelung an $m$ und bei $c$ nach zweimaliger Spiegelung an $m$ und $n$. Die Fassungen der Spiegrel gleiten in Schlittenfuhrungen, so dass sie je nach Bediurfuiss in den Gangr der Lichtstrahlen eingeschoben, oder aus demselben entfernt werden können. Bei grewöhnlichen Qneeksilberspiegeln mit Glas wird von dem nnter $45^{\circ}$ anffallenden Licht etwa 75 Procent zuriuckgeworfeu, und es erscheinen daher hei Benutzung solcher Spiegel die Sterne bei $b$ um ungefïhr 0.3 Grüssenclassen, bei $c$ um mehr als 0.6 Grössenclassen schwiicher als bei $a$. Ungeführ der gleiche Effect wird erreicht, wenu man anstatt der Spiegel total reflectirende Glasprismen anwendet. Versilloerte Glisspiegel reflectiren etwas mehr

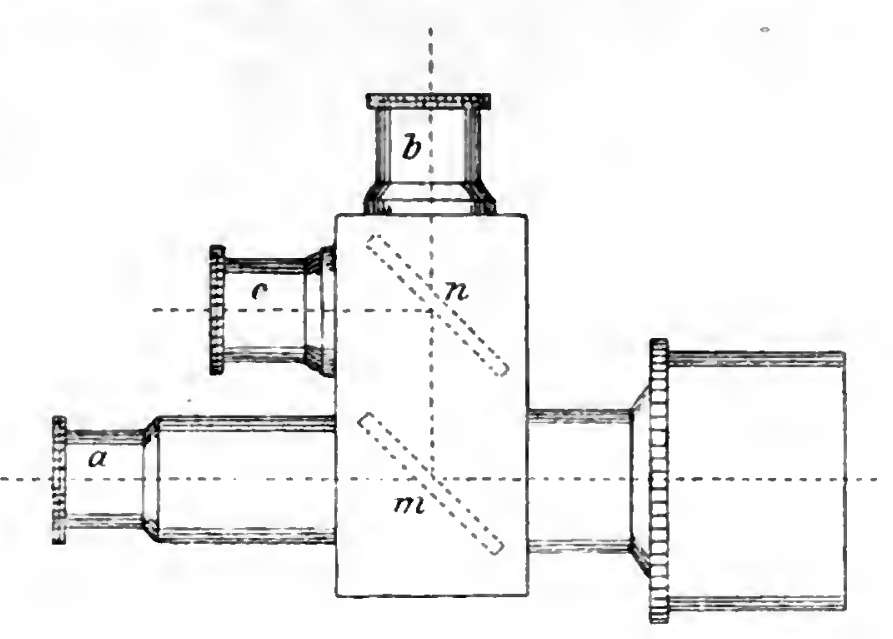

Fig. 31 .

Licht, wïhrend bei Metallspiegeln der Lichtrerlust in Allgaemeinen grö̈sser ist. Benutzt man endlich planparallele Glasplatten, so betrïigt das zuriiekgeworfene Licht mur etwa i Procent des auffallenden, und ein Stem wird daher nach eimmaliger Reflexion um 3, nach zweimaliger um 6 i rolle Grössenelassen geschwäicht. Durch Combination rerschiedener reflectirender Mittel lässt sich mit Hiilfe der 'T'hury'schen Einrichtmug innerhally gewisser Grenzen ein l,eliebiger Grad der Lichtschwichnug hervorbringen. In der Praxis ist es natiirlich, wenn man genaue photometrisehe Messungen ansfülıren will, unbedingt erforderlich, in jedem specicllen Falle die Reflexionscoefficienten der hemtzten Spiegel, P'rismen oder Glasplatten dureh besondere Untersuchungen empirisch zm bestimmen, da bei der Verschiedenheit des Verhaltens einzelner Glassorten und Metalle und bei dem Einflusse, den die Art der Politur u. s. w. besitzt, allgemeingriltige exacte Angaben iiber den Betrag des reflectirten Lichtes nicht gemacht werden 
können. Dicse umentbehrlichen Constantenbestimmungen sind ein Nachtheil der 'Thury'schen Methode, den dieselbe aber mit vielen anderen photometrischen Methoden gemeinsam hat. Beiläufig bemerkt liesse sieh dasselbe Ziel wie dureh mehrfache Spiegelung auch dureh Anwendung von verschiedenen Blendgläsern oder cines Keiles aus dunklem Glase erreiehen, die in den Gang der Lichtstrahlen zwischen Objeetiv und Oeular $a$, :un besten in der Nähe des Brennpunktes, eingeschoben werden könnten, und deren Absorptionscoefficienten durch besondere Untersuchungen im Vorans ermittelt werden miissten.

'Thury hat scinen Ocularapparat noch benutzt, um einige Untersuchungen iiber den Einfluss der Beugung auf Helligkeitsmessungen anzustellen. Er gelangt zu dem aus unseren friiheren Erörterungen unmittelbar hervorgehenden Resultate, dass, wenn in einem Fernrohre zwei verschicden helle Sterne bei den freien Objeetivflächen $o$ und $o^{\prime}$ (von denen $o$ die grössere sein möge) zum Versehwinden gebracht werden, dann dis richtige Intensitiitsverhältniss der beiden Sterme nicht dureh $\frac{o}{o^{\prime}}$ regreben ist, sondern durch $\frac{o}{o^{\prime}-x}$, wo die Correetion $x$ für jeden Werth ron $o^{\prime}$ einen anderen Betrag hat. Thury hat bei seinem Instrumente diese Correction zu ermitteln gesucht, indem er verschiedene Sterne einmal durch starke Terkleinerung des Objectirs allein und dam nach Einfiignng des einen oder der beiden Spiegel durch geringe Abblendung des Objectirs zum Verschwinden brachte. Mit Hiilfe der bekannten Reflexionsconstanten der Spiegel liessen sich daraus die Correctionen für die kleinen Öffinungen in rerhältnisse zu den grossen ableiten.

Bei den siimmtlichen im Torangehenden besprochenen Einrichtungen geschah die Abblendung des Objectirs ron dem Rande nach der Mitte zı. Da dieses Verfahren, wie ansfuilhrlich gezeigt worden ist, aus rerschiedenen Grindeu die schwerwiegendsten Nachtheile mit sich bringen kann, so ist es rithisimer, die Abblendnng so vorzunehmen, dass alle Zonen des Objectirs gleichmiissig daron betroffen werden. Ein grosser Theil der Fehlerquellen wird anf diese Weise ganz beseitigt oder wenigstens auf cin Minimum reducirt. Zur Erreichung dieses Zieles sind die rerschiedensten Vorschläge gemacht worden; am praktisehsten hat sich die Beuntzung ron sectorförmigen Ansschnitten erwiesen, welche bereits ron $B$ ongner-1) mit den folgenden Worten als die einzig riehtigen $\mathrm{Ab}$ blendungsrorrichtungen bezeichnet worden sind: „Il n'y a qu'une seule maniere légitime de diminuer l'ourerture des objectifs. Puisqu'on veut que la grandeur de la surface dn verre exprime la quantité des rayons

1. Traité d'optique, p. 36 
qui le traverse, il ne faut pas plus eouvrir les parties du eentre que celles des bords; les premières étant plus épaisses sont moins transparentes et les autres le sont davantage; mais il n'y a qu'ả les eouvrir toutes proportionnellement, et pour cela il faut se servir de diaphragmes qui aient exaetement la figure des seeteurs. \& Der Bouguer'sche Vorschlag, der lange ganz unbeachtet geblieben zu sein scheint, ist später wiederholentlich erneuert worden, unter Anderen von Lamont'), welcher die Benutzung eines rom Mittelpunkte des Objectivs ansgehenden fíeherförmig zu entfaltenden Diaphragmas empfiehlt. In Potsdam ist eine ähnliebe Blendvorrichtung in Gebranch, die zwar gewölınlieh nur zur allgemeinen $\Lambda \mathrm{b}$ sehwiiehung von Sternen bemutat wird, aber anch zu wirkliehen Helligkeitsmessungen verwendet werden könnte. Sie besteht (Fig. 32) ans drei anf einander gesteekten Metallkappen, von denen die unterste fest mit der Objectivfassung verbunden ist, wälirend die beiden anderen, einzeln oder zusammen, um die erstere gedreht werden können. An zwei Kreistheilungen liisst sich der Betrag der Drehungen ablesen. Die beiden unteren Kappen haben je vier sectorförmige Aussehnitte von $60^{\circ}$ Öffnungswinkel, die

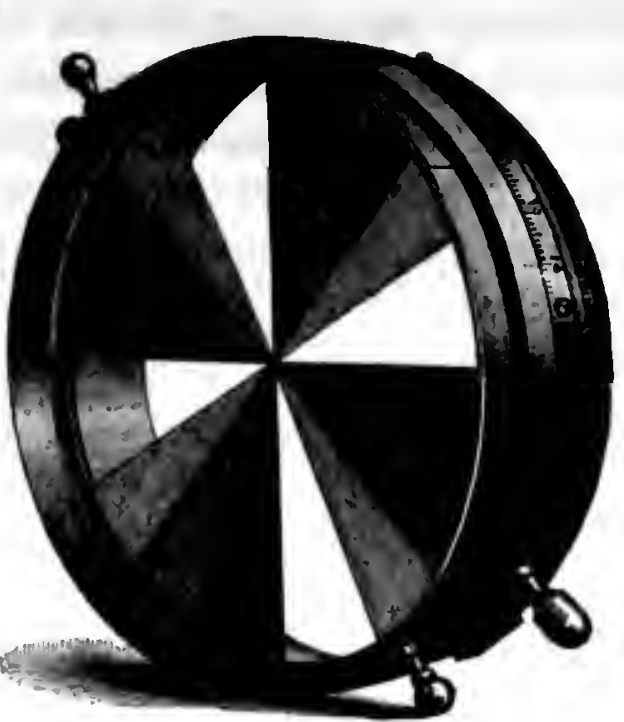

Fig. 32. dritte Kappe besitzt vier Aussehnitte mit Winkeln von $70^{\circ}$. Man kann dureh dieses Arrangement das Objectiv von $\frac{2}{3}$ bis auf $\frac{1}{9}$ der vollen Öffnung ahblenden und daher eine Lichtschwiichung von ungefähr 2 Grössenelassen hervorbringen.

b. Blenden zwischen Objectiv und Oeular. Photometer ron Hirseh, Dawes, Loewy.

Anstatt den anf das Objectiv auffallenden Strahlencylinder messbar zu verkleinern, ist mehrfach der Versuch gemacht worden, die Albblendung erst nach dem Austritte aus dem Objeetiv vorzunehmen. Eins der : iltesten auf diesem Prineip beruhenden Anslöschungsphotometer riilurt von Hirseh?) her und ist für die Sternwarte Nenchâtel ron Mer\% in München angefertigt worden. Eine Scheibe, welehe in der Mitte mit einer feinen kreisrunden Öffnung versehen ist, lïsst sich innerlalb des Fernrohrtubus vom

1) Jahresbericht der Nünchener Sternwarte für 1552 , p. 40.

2) Bulletin de la société des sciences naturelles de Neuchâtel. T.6 (1861-6)4, p.94. 
Bremupunkte nach dem Objectiv zu verschieben, und diese Verschiebung. wird an einer aussen am Rohre angebrachten Scala abgelesen. Je weiter die Blendscheibe rom Focus entfernt ist, desto mehr Licht wird abgeblendet. Ist $b$ der Durchmesser der Blendenöffnung, $d$ der Durchmesser des Objectirs und $f$ seine Brennweite, so ergiebt sich, dass, wenu die Blendscheibe um die Strecke $m$ rom Brennpunkte absteht, die Helligkeit $h$ eines Sterns ansgedriickt wird durch $h=\frac{b^{2} f^{2}}{d^{2} m^{2}}$, falls die Helligkeit ohne Blende mit 1 bezeichnet ist. Die urspriunglichen Helligkeiten zweier zum Versehwinden gebrachten Sterne verhalten sich also wie die Quadrate der zugehörigen rom Focus aus gezählten Scalenablesungen. Bei dem Hirsel'schen Apparate, welcher an einem Fermrohre von $16.2 \mathrm{~cm}$ Öffnung und $259.9 \mathrm{~cm}$ Brennweite angebracht war, hatte die Diaphragmenöffunng rinen Durchmesser ron $0.5 \mathrm{~cm}$ und liess sich innerhalb der Abstände $7.6 \mathrm{~cm}$ und $48.2 \mathrm{~cm}$ rom Brenmpunkte rerschieben. Bei der ersten Stellung wurde die Öffunng gerade von dem rom ganzen Objectiv herkommenden Strahlenkegel ansgefuilt, und die Gesammtlichtschwächung; die mit dieser Eimriehtumg zu erzielen war, betring ungefïhr vier Grössenclassen. Im Allwemeinen werden die im Innern jedes Fermrohres zur Vermeidung von scitlichen Reflexen angebrachten Scheiben einer grösseren Verschiebung. des Diaphluagnas hinderlich sein, und man wird daher, wenn man eine schr (rhebliche Liehtschwächung hervorbringen will, entweder verhältnissmässig vicl feinere diffnungen als bei dem ron Hirsch beschriebenen Apparate anwenden oder noch Blendgliser zu Hiilfe nehmen miissen.

Die Hirschische Methode hat dieselben Nachtheile wie jede Abbendmug des Objectivs. Sie bernlit ebenfalls anf der zweifelhaften Voralussetzung, dass alle Theile des Objectirs gleichmässig zur Helligkeit des Bildes heitragen, und ist dem störenden Einflusse der Beugung in nicht geringerem trade ansgesetyt. Dagegen bietet sie den Vortheil, dass die mechanische Einrichtung ansserordentlioh einfoch ist.

Eine grässere Verhreitumg hat das Verfahren der Abblendung zwischen Objectir mond Ocular niemals gefunden. Mir sind ansser dem Hirsch'schen Forschlage nur noch zwei andere bekamnt geworden, ron Dawes') und

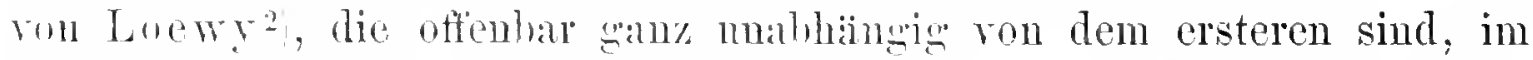
Wesutlichen aber auf dascelbe hinaskommen. Dawes hat an Stelle

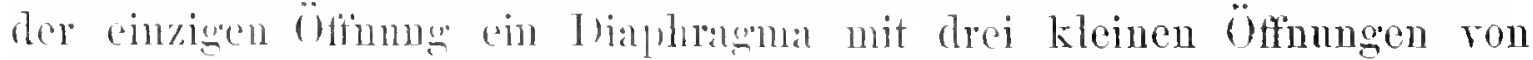

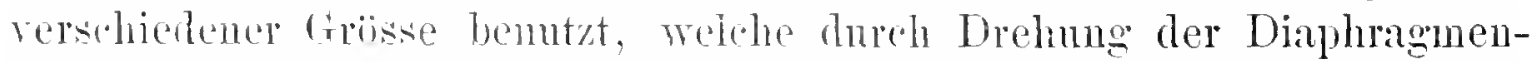
scheibe nach cinander in die Mitte des strahlenkegels gebracht werden

1 Monthly Totices. Vol. 2.5. p. 2.2n

2) Mouthly Notices. Vol. 42, p. 91 
konuteu. Loewy warnt davor, allzu kleine Öfh̉ıungen zı beuntzen oder die Verschiebung nach dem Objectiv hin sehr weit zu treiben, er will die direete Vergleiehung nur auf ein Helligkeitsintervall von j oder höehstens 6 Grössenelassen anwenden und empfiehlt fur die Beobachtung der helleren Sterne die allgemeine Abschwächung dureh Reflex von einer vor dem Oculare unter einem Winkel ron $45^{\circ}$ angebrachten Glasplatte.

\section{c. Das Parkhurst'sche Deflectionsphotometer.}

Eine ganz eigenartige Auslöschungsmethode ist in neuester Zeit ron Parkhnrst') eingefulirt und bei seinen Helligkeitsmessungen an kleinen Planeten in grösserem Umfange angewendet worden. Die Vorrichtung, welcher Parkhurst den Namen »deflecting apparatus gegeben hat, besteht im Wesentlichen ans einer sehr dunuen, etwas keilförnigen Glasplatte, welche zwischen Objeetir und Bremnpunkt eines parallaktisch montirten Fernrohres ron $22.9 \mathrm{~cm}$ Öffunng und $284.5 \mathrm{~cm}$ Brennweite, etwa $40.6 \mathrm{~cm}$ ron der Focalebene entfernt angebracht ist, und zwal so, dass die scharfe Kante derselben bis in die Mitte des Rohres hineinragt. Wird das Instrument auf irgend einen Stern gerichtet, so geht die eine Hölfte des Strahlenkegels an der Glasplatte vorbei, die andere fällt auf dieselbe und wird ein wenig abgelenkt, so dass zwei nahe bei eiuander befindliche Bilder des Sternes entstehen. Es findet also keine eigentliche Abblendung statt in dem Simne, wie es bei den bisher besprochenen Photometern der Fall war, sondern eine Zerlegung des Lichtkegels in zwei 'l'heile, und es ist klar, dass man dureh Verseliebung der Glasplatte in der Richtung senkrecht zur optischen Axe sehr leicht das directe neben der Glasplatte gesehene Bild eines Sternes zur Anslöschmm. bringen könnte. Parkhurst hat zur Erreiehung dieses Zieles einen etwas anderen Weg eingeschlagen. Er lässt bei unbeweglicher Glasplatte den zu beobachtenden Stern durch das Gesichtsfeld des Ferurohres hindurehwandern. Beim Eintritt in dasselbe geht zunichst der ganze rom Objectiv kommende Strahlenkegel an der Glasplatte vorbei, und man erblickt nur ein einziges Sternbild. Sobald aber der Mantel des Kegels die Platte erreicht hat, wird ein zweites schwaches Bild des Sternes sichtbar; waihrend das urspringliche Bild an Helligkeit abnimmnt. Man kanm anf diese Weise das vollständige Verschwinden des directen Bildes beobachten. Sterne ron versehiedener Lichtstïrke werden natiirlich an verschiedenen Stellen des Gesiehtsfeldes zum Versehwinden kommen, und die Zeit, die ron ihrem Eintritte in das Gesichtsfeld bis ' $m$ rollstindigen Auslöschung ver-

1) Annals of the Astr. Observatory of Harvard College. Vol. 1s, Nr. III. 
streicht, wirl mit Riicksieht anf die Declination der Sterne ein Mass für ihre Helligkeit gehen. Da der Eintritt in das Gesichtsfeld nieht mit der erforderlichen Genauigkeit zu bestimmen ist, so wird statt dessen der Antritt der Steme an einer dunklen Linie beobachtet, welehe auf einer in der Focalebene angebrachten Glasplatte markirt ist. Diese letztere Platte lisst sich noch verschicben und an mehreren Punkten, deren Entfernung von einander in Zeitsecunden genau bestimmt ist, festklemmen. Man kann auf diese Weise, wenn es wiinschenswerth sein sollte, die Durchgangszeit abkiir\%en. Das Ocular ist ebenfalls verschiebbar und zwar parallel zur Focalehene, um es bei der Beobachtung der Auslösehung in die vortheillafteste Position zu dem Sterne bringen zu kömnen. Die ablenkende Glasplatte kann rom Ocular ans mittelst einer einfachen Vorrichtung ganz \%uriickgeklappt werden, so dass das Gesichtsfeld nöthigen Falls vollstiundig frei wird.

Das Eigenthiimliche der Parkhurst'schen Methode besteht darin, dass die eigentliche Helligkeitsbeobachtung durch eine Zeitmessung erfolgt, ein

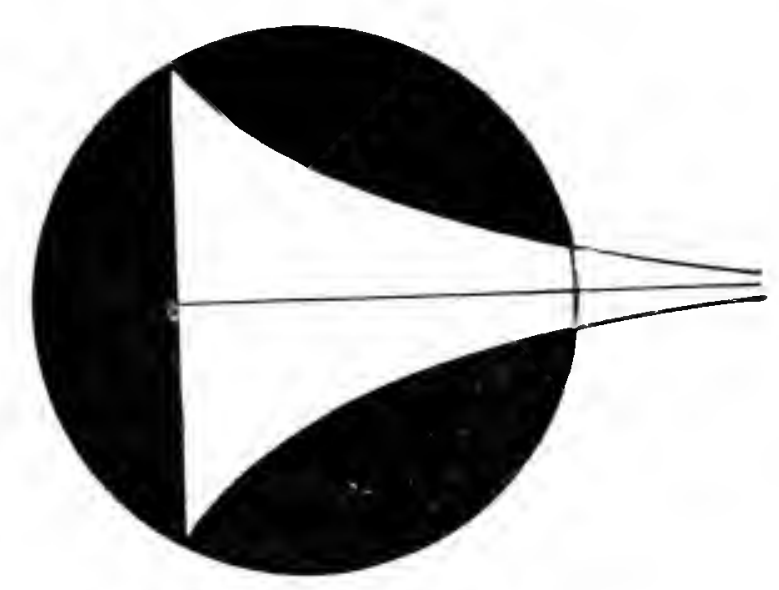

Fig. 33 . Verfahren, welches, wie wir sehen werden, anch beim Keilphotometer und zwar schou lange vor Parkhurst zur Anwendung gekommen ist. Was den Zusammenhang zwischen Durehgrangszeit und Helligkeitsabmahme beim Deflectionsphotometer anbetrifft, so liesse sich derselbe entweder dureh Berechnung des ron der ablenkenden Glasphatte ans dem Strahlenkegel ausgeschnittenen Theiles bestimmen, oder auf experimentellem Wege durch Mesinnwen an sternen rou bekannter Helligkeit oder durch irgend eine andere photometrische Vethode ermitteln. Parkhurst hat bei seinem Apparate noch eine Eimrichtung getroften, um unmittelbar aus den fiir zwei verschicdene Sterme heobachteten Durchangseiten den Helligkeitsunterwhied derselben in Crössenclassen abzuleiten. Zn diesem Zweeke hat er ror dem Objectiv sine Blendkappe angelbracht. deren eigenthimliche Construction ans Figm $3: 3$ crsichtlich ist.

Die freic öttinng der Blende wird begrenzt durch eine gerade Linie und zwei curvensticke, die srmmetrisch zu einer senkrecht auf der Geraden durch die Nitte des Objectirs gezogenen Linie liegen. Die Curven sind

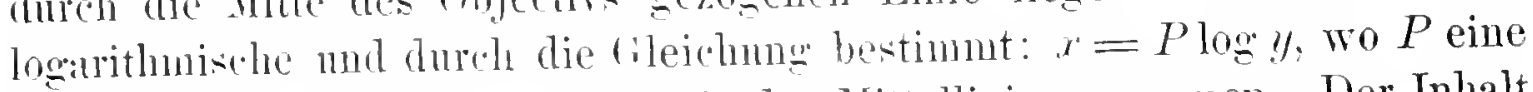
Constante ist. Wie , ixe faill wit der Vittellinie zusammen. Der Inhalt 
der Fliche, welche von irgend einer Ordinate $y$, der $x$-Axe und der asymptotisch zu derselben verlaufenden Curve gelildet wird, ist ausgedrttekt durch $P M y$, wenn $M$ der Modul der Brigg'schen Logarithmen ist. Fur zwei bestimmte Ordinaten $y_{1}$ und $y_{z}$ hat man daher die entsprechenden Flächen $P . M y$, und $P M y_{z}$. Soll nun das Verhialtniss dieser beiden Flïchen gleich sein dem Verhältuisse zweier anf ein:under folgenden Sterngrössenclassen (wofur gewöhnlich die Zahl 2.512 angenommen wird), so ist $\log y_{1}-\log y_{t}=0.4$ und demnach $x_{1}-x_{2}=0.4 P$. Parkhurst hat filr die willklirliche Constante $P$ den Werth 5 angenommen, und es ist daher bei ilm $x_{1}-x_{z}$ gleich 2 Zoll. Die Blendkappe ist so anf das Objectiv aufgesetzt, dass die Mittellinie mit der Richtung der tïglichen Bewegung der Sterne zusammenfïllt. Versehiebt sieh num, durch Bewegung des Sternes, der Strahlenkegel, dessen Quersehnitte natỉrlich liberall :ihnliche Form haben mlissen wie die freie Objectivöffunng, gegen die ablenkende Glasplatte $11 \mathrm{~m}$ ein Stllek, welches an dem Objectiv einer Strecke von 2 Zoll entspricht, so ïnlert sich die Helligkeit des Sternes um eine ganze Grössenclasse. Dic dazu erforderliehe Zeit betrïgt fur einen Äquatorstern ungefïh 40 Zeitseeunden. Will man zwei beliebige Sterne in Bezug auf ilıre Helligkeit miteinander vergleichen, so bestimmt man fur jeden die Durchgangszeit rom Antritt an die dunkle Linie bis zum vollstïndigen Auslöschen des directen Bildes. Die Differenz dieser Durchgangszeiten (in Zeitseeunden ausgedritckt), redueirt auf den Aequator und dividirt durch 40, giebt dam ummittelbar den Helligkeitsuntersehied der beiden Sterne in Grössenclassen. Da die Constante $P$ ganz willkürlich ist, und ebenso die Entfernung der ablenkenden Glasplatte von der Focalebene beliebig gewïhlt werden kam, so lïsst sich die Zeitdaner, welche zunr Hervorbringung einer Lielitabnahme von einer Grössenclasse erforderlich ist, ganz nach Gutdünken von vornherein festsetzen. Zn berticksichtigen ist noch, dass die Blendenöffinung streng genommen nach der einen Seite hin ins Unendliche sich erstrecken miisste, weil die begrenzenden logarithmischen Curven asymptotisch zur Mittellinie verlanfen. Un daher den ausserhalb des Objeetivs fallenden Theil der Öffinung in Rechnung zu ziehen, ist es nothwendig, an dieser Seite der Blendscheibe eine besondere Öftumng anzubringen, welche eine beliebige Form lıaben kann, deren Fläche aber gleich diesen ansserhalb liegenden Stiick sein muss.

Die Parkhurst'sche Objectivblende lat den Vortheil, dass die experimentelle Bestimmung der Beziehung zwischen Durchgangszeit und Helligkeitsäuderung iiberfliissig wird; dagegen diifte die exacte mechanische Herstellung der complicirten öff̈nungsform, ron der allein die zu erreichende Messungsgenanigkeit ablïngt, mit grossen Schwierigkeiten verkniipft sein. Auch sonst hat das ganze Beobachtungsverfahren mancherlei Bedenken 
gegen sich, und es ist kaum zu erwarten, dass das Parkhurst'sche Photometer weite Verbreitung finden wird.

\section{Auslïschung des Lichtes durch absorbirende Medien.}

a. Die Photometer ron Lampadius, Horner, Quetelet, Albert.

Der Gedanke, die Absorption des Lichtes in versehieden grossen Schichten eines nicht absolut durchsichtigen Mediums als Helligkeitsmass zu benutzen, ist schon verhältnissmässig früh anfgetaucht. Bouguer erwähnt in seinem 'Traité d'optique (p. 16', dass bereits im Jahre 17110 der Kapurinerpater François Marie in einer kleinen Schrift, betitelt "Nourelles découvertes sur la lumière " die Anslöschung des Lichtes dureh Übereinumderlegen melrerer Glasstiicke ron gleicher Dicke empfohlen hat. Theoretiseh liisst sich gegen dieses Prineip kanm etwas einwenden. Linter der Voranssetzung, dass die einzelnen Glasstucke nieht nur hinsiehtlich der Dicke, sondern anch hinsichtlich der Beschaffenheit des Glases rollkommen identiseh sind, kann man leieht den Lichtverhust hestimmen, der von einer beliebigen Anzahl derselben verursacht wird. Ist $J$ die Intensitait eines Lichtstrahles vor dem Eintritte in das erste Glasstiick, $J^{\prime}$ die Intensitait beim Austritte ans $n$ solchen Stiicken, so hat man nach den Erörterungen anf Seite 113 die einfache Beziehung: $J^{\prime}=J c^{n}$, wobei $c$, der sogenimnte Transmissionscoeffieient, das Verhältuiss der ron einem einzelnen Crlasstick hindureh ggelassenen Lichtmenge zu der ursprünglichen Intensitit ansdriickt. In der Praxis stellen sich diesem Verfahren und ebenso allen auderen anf dem Priucip der Absorption beruhenden Auslïschungsmethoden einige Schwierigkeiteu entgegen. Zunichst findet man nicht leicht rollkommen homogene absorbirende Medien, und noch bedenklicher ist der Lmstand, dass es Kanm eine Substanz geben dürfte, welche für strahlen von verschiedener Brechbarkeit in absolnt gleichem Masse durchlisssign wäre. Die Vergleichung versehiedenfarbiger Liehteindriicke ist daher bei jedem Absorptionsphotometer ein mehr oder weniger heikler Punkt.

Anstatt der ron Franco is In rie benutzten Glasplattensäule bediente sich Lampadius ${ }^{1}$ in . Tahre 1814 zur Bestimmmg der Helligkeit des zerstreuten Tagestichtes, sowie der Some und des Nondes, einer Röhre, in welche sn viek mit ill getrinkte Papierseheiben eingelegt wurden, bis

1 Lampadius. Beitrïge zur Atmosphïrologie. II. Phot. Beob. im Jahre 1914, p. 164. Freiberg 1,17 
jede Spur von Licht ausgelöscht war. Diese Papierscheiben ersetıte er spitter durch Hornscheiben, welche sich weit homogrener und vor Allem viel haltbarer erwiesen; ferner schlug er vor, an jeder Photometerrölire eine Theilung anzubringen und mit 100 denjenigen Punkt z.. bezeichnen, bis zu welchem die Röhre mit anfeinander gelegten Scheiben angefullt werden muss, falls gerade das Licht eines in Sanerstollyas brennenden Phosphorstilekes \%um Verschwinden gebraeht werden soll; anf diese Weise wlirde die Angalse einer beliebigen Zahl der Scala in verschiedenen derartigen Apparaten ein gan\% bestimmtes Helligkeitsmass reprïsentiren.

Eine ähnliche Einrielitung ist fast zu derselben Zeit von Horner') vorgeschlagen worden. Derselbe verwendete einen Rahmen mit 10 neben einander befindlichen, gleich grossen öfHungen, von denen die erste gan\% frei blieb, wiihrend die zweite mit einer einzelnen Lage durehsiclitigen Papieres, die dritte mit I- solchen Lagen n. s. w., die zehnte mit 9 Lagen uberzogen war. Ausserdem waren Scheiben vorhanden, die aus je 10 Lagen desselben Papieres bestanden und die in dem Photometerrohre mittelst einer Hulse festgehalten werden komnten. Bei der Beobaehtung wurden zunïehst soviel Zehmerscheiben eingesetzt, als erforderlich waren, um die Lichtquelle nahezu zum Auslösehen zu bringen, dann wurde der Rahmen so weit hineingesehoben, bis der letyte Lichteindruck versehwand, und die Nmmmer der betreffenden Rahmenöfhnung notirt. Das Verfahren ist, wie man leicht einsieht, in mancher Hinsielit bedenklich und düfte sehwerlich sichere Resultate ergelien.

Dasselbe gilt ron den zahlreichen Versuchen, Flissigkeitsschichten zur Auslöschung zu verwenden. Quetelet²) hat bereits im Jahre 18333 vorgeschlagen, in den Gamg der Lichtstrahlen ein Gefïss einzuschalten, welehes oben und unten nit parallelen Glasplatten versehlossen ist. Diese Platten können durch cine cinfache Vorriehtung einander genïhert oder von einander entfernt werden, sodass die eingesdllossene Fliissigkeitsschicht, welehe in ein seitlich angebrachtes Rohr zuriicktreten kamn, jede heliebige Lünge erhält. Dieselbe ldee ist his in die jiingste Zeit immer wieder von Neuem mit nur geringen Modifieationen anfgetancht. Am bekanntesten ist wohl das Albert'sehe Photoseop ${ }^{3}$ geworden, welches im Wesentlichen mit dem Quetelet'schen Apparate ,die grö̈ste Öhnlichkeit hat. Die Benutzung von Fliissigkeiten hat ansser vielen anderen Übelstiinden noch den Nachtheil, dass sich die selective Absorption der einzehen Farben in ganz besonders starkem Masse fiihlbar macht.

1) Bibliothèque universelle des sciences. Genère. Tome (; (1517).

2) Bibliothèque nniverselle des sciences. Genève. 'Tome 52 (1833;, p. 212.

3) Dinglers polytechnisches Journal. Bd. 100 1846, p. 20. 


\section{b. Das Keilphotometer.}

Alle im Vorangehenden erwähnten Einrichtungen und viele andere anf demselben Prineip beruhenden eignen sich wenig zu Untersuchungen am Himmel und kömmen anch nieht im Entferntesten rivalisiren mit dem hervorilgendsten Repräisentanten dieser Gattung von Instrumenten, dem Keilphotometer, welehes zweifellos iiberhaupt als das rollkommenste Auslöschungsphotometer zu bezeichnen ist. Es wird häufig auch das Pritchard'sche Keilphotometer genannt, weil Pritchard dasselbe am Eingehendsten studirt und zuerst zu umfangreiehen Messungen benutzt hat. Der Gedanke selbst ist ziemlich alt, und die Geschiehte der Entwicklung. dieses Photometers ist ein dentlieher Beweis dafür, weleh geringes Interesse stets ron Seiten der Astronomen den Helligkeitsbestimmungen der Gestirne entgegengebracht worden ist, da sonst schwerlieh eine so einfache und praktische Methode immer wieder in gainzliehe Vergessenheit gerathen wiire. Es existiren nieht weniger als fiuf rerschiedene Abhandlungen, in denen die Benutzung ron Glaskeilen zu photometrischen Zwecken als neu in Vorschlag gebracht worden ist, zum Theil bereits mit allen denjenigen Modificationen und Verbesserungen, die sieh erst seit Pritehard dinernd in der Praxis eingebirgert haben.

Der :ilteste Vorschlag scheint aus dem Jahre 1832 von dem Grafen de Inistre') herzuriihren. Derselbe beuntzte zwei Prismen ron ungefähr $11^{\circ}$ brechendem Winkel und fist 9 Zoll Lïnge, das eine ans weissem, dis andere ans blanem Glase, welehe so anfeinander gelegt waren, dass sie ein Parallelepipedum bildeten, dimit die hindurehgehenden Liehtstrahlen nieht ron ihrer urspriinglichen Richtnng abgelenkt wiirden. Das Prisma ans weissem Glase erhielt bei der Messung eine feste Stellung, wïhrend das andere mit Hiilfe einer Mikrometersehraube dagegen rersehoben werden konnte. Das de Maristresche Photometer ist ein Jahr spiter ron Quetelety in der Weise modificirt worden, dass anstatt eines weissen und eines blanen l'rismals zwei Keile ron demselben dunklen Gilse bemutat wurden: doch hat Quetelet dieses Instrument sehr bald wieder anfgegehen, weil er die Lmüghlirhkeit einsah, Glas ron solcher Färbung zon erhalten, dass die verschedenen Farben gleiehmässig dat durch absurbirt wiirden.

In den Berichten der schwedischen Akidemie beschreibt C.D.r.Sehn-

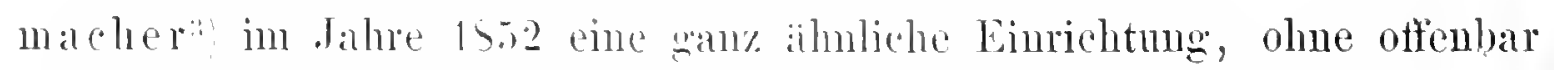
rom den friiheren Vorschligren kemutniss zu haben; er bewegt die beiden

1) Bibliothèque universelle des seiences. Genere. Tome 51 (1s32), p. 323

$\because$ Bibliotheque unirerselle des scitnces. Geneve. Tome 52 1533; p. 212.

:3 Öfrersigt at K. Vetensk. Akak. Fürh. 1与52, p. 236t. 
Keile durch eine Schraube mit Doppelgewinde gleiehmïssig gegeneinander und bringt dieselben (was als cine wesentliche Verbesserung zu bezcichnen ist) nicht vor dem Olojectiv oder Ocular, sondern in der Focalebene des Fernrohres an.

Kayser') in Danzig hat zum ersten Male die Prismen nicht getremut von einander benuty, soudern "u einem festen Doppelprisma zusammengekittet, bestehend aus einem weissen durehsichtigen und einem dunklen Keile. Sein Iustrument gleicht bis ins Kleinste unseren besten hentigen Photometern, und es ist fast unbegreiflich, dass dasselbe in der damaligen Zeit gar keinen Anklang gefunden hat. Von Kayser stammt auch zuerst der Vorsehlag, den Doppelkeil in der Brennebene eines parallaktischen Fernrohres feststehen zu lassen und zwar mit seiner Läingsansdehmung in der Richtung der täglichen Bewegung, und die Secunden zu z:ihlen von dem Antritt der Sterne an den Glasstreifen bis zu dem Momente, wo die Sterne unsichtbar werden.

Dawes ${ }^{2}$ ) hat sich ebenfalls Verdienste um das Keilphotometer erworben und insbesondere seine Anwendung ausser zu Sternbeobachtungen noch in solehen Fiillen empfohlen, wo andere photometrische Methoden schwierig $\%$ benutzen sind, beispielsweise zur Vergleichnng der Helligkeit verschiedener Partien der Mondoberfliche und zur Vergleichung der Lichtstärke der von der Somne beleuchteten Atmosphäre und der Photosphäre der Sonne selbst.

Ausser der Dawes'schen Einrichtung und einem weniger bekannten Vorschlage ron Piazzi Smytl aus dem Jahre 18+3, der nirgends veröffentlicht zu sein scheint, ist Pritchard bei der Construction seines Keilphotometers im Jahre 1881 offenbar keiner der älteren Vorschläge bekannt gewesen. Sein Apparat enthält in keiner Hinsicht etwas Nenes, im Gegentheil bedentet
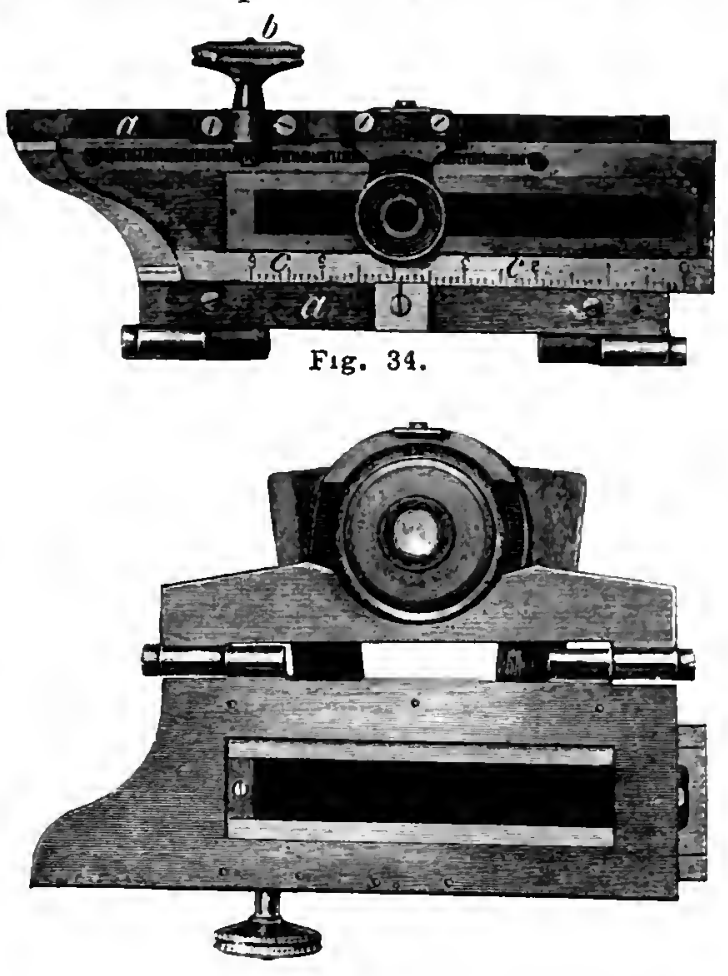

Fig. 35 . er insofern sogar einen gewissen Riickschritt, als die Verschiebung des Keiles wieder aus der Focalebene ror das Ocular verlegt worden ist. Die Figuren 34 und 35 stellen das Pritchard'sche Kcilphotometer dar. wie es

2) Monthly Notices. Vol. 25, p. 229. 
in der optischen Anstalt ron Grubb in Dublin angefertigt wird. In dem Rahmen $a$ bewegt sich mit Hilfe eines Triebes $b$ der aus weissem und nentralem Glase zusammengekittete Doppelkeil, dessen Verschiebung an der 'Theilnng' $c$ abgrelesen werden kann. Um dem Auge die nöthige Sehrichtung zu geben, ist vor dem Keil noch eine Hülse mit einer Augenifftmmg angebracht. Der ganze Rahmen $a$ lissst sich mittelst eines Scharniers herunterklappen (Fig. 35), so dass das Ocular nach Beduirfniss sofort frei ohne Keil benutzt werden kann. Diese Eimrichtung ist in mancher Hinsicht vortheilhaft, sie hat aber den grossen Nachtheil, dass der Keil viel leichter der Gefihl einer Beschädigung oder des Beschlagens durch dén Hanch des Beobachters ansgesetzt ist, als wenn er sich im Innern des Ferurohres befindet, und dass im Momente des Verschwindens der Sterne das ganze Gesichtsfeld rerdunkelt ist, was in vielen Fällen die Beobachtung erschwert. Da der Angendeckel nicht zn weit von der Ocularlinse entfernt scin darf, so ist die vorn befindliche Hülse so kurz, dass, wie Young ${ }^{\prime}$ ) bei einer Bespreehnng des Pritchard'schen Keilphotometers missbilligend bemerkt, Nase mul Stirn des Beobachters der Bewegung des Keiles muter Unstïnden hinderlich sind.

Entschiedenen Vormg vor dem Pritehard'sehen Instrumente verdient der in Potsdam eingeführte, rom Mechaniker Töpfer construirte Apparat, welcher zugleich mit einer bequemen Registrirvorrichtung versehen ist.

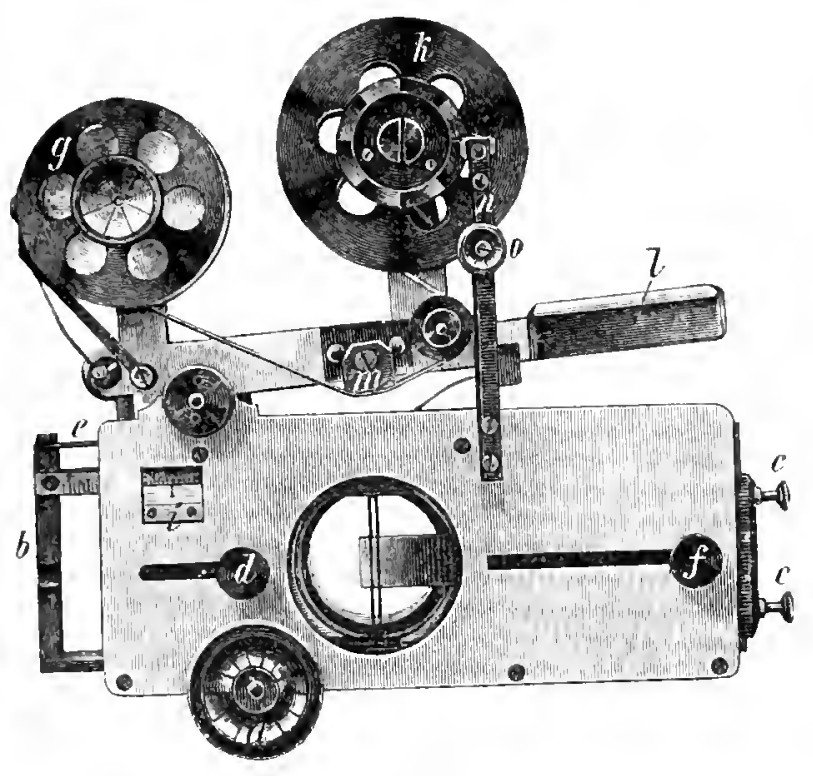

Fig. 36.

Figur 36 stellt diesen Apparat in etwar $\frac{1}{3}$ der natiirlichen Grösse (mit al)geschraubtem Ocular) dar. Er wird mittelst eines Zwischenringes an das zur Verwendung kommende Fennohr so angesetyt, dass sich der Keil mugefïhr in der Brennebene desselben befindet. In dem eigentlichen ans Aluminiumblech angefertigten Kasten des Apparates beweret sich mit Hiilfe des Triebes " ther liahnen b, in welehem der Keil mittelst der Sehrauben $c$ betestignt wird. Anf der Vorderseite dieses Ralunens ist eine Millimetertheihnng angebracht, die an dem

festen Index i abgelesen werden kamn; ausserdem befindet sich eine

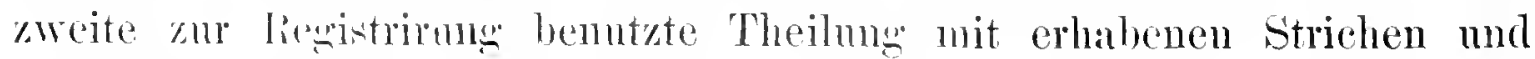

1. Investigations on light and heat published with appropriation from the Rumford Find. Ibsit. p. 3il. 
Zahlen auf der oberen Kante $e$ des Rahmens. Dureh die Mitte des Gesiehtsfeldes geht ein ans zwei schmalen Lamellen gebildeter, unmittelhar vor dem Keil sitzender Steg, welcher mit Hulfe des Knopfes'd nach Wunsch ganz aus dem Gesichtsfelde zurickgezogen werden kann. Bei der Beuntzung cines parallaktisch montirten Ferurohres wirl das Keilphotometer so angesetzt, dass dieser Steg in die Richtung der tiiglichen Bewegung zu stehen kommt und die Sterne den schmalen Streifen zwischen den Lamellen zu durchlaufen haben. Das positive Ocular, welches nicht жи stark zu wïhlen ist, wird so eingestellt, dass der Steg und die Begrenzung des Keils scharf erscheinen, und dann erst wird der ganze Apparat mittelst des Ferurohrtricbes so weit verstellt, bis auch dic Sterne scharf $\mathrm{n}, \mathrm{n}$ sehen sind. Der Umstand, dass zn beiden Seiten des Keils das Gesichtsfeld fịci bleibt, ist bei den meisten Sternbeobachtungen als ein Vortheil zu betrachten; denn erstens wird dem Auge dadurch die Mtihe erleichtert, dicjenige Stelle richtig zu fixiren, wo der Stern verschwindet, und damu giebt die Entfernung der beiden Lamellen und ebenso die Breite des Keils ein vortreffliches Mittel an die Hand, die Distanzen benachbarter Sterne in beiden Coordinaten richtig $/$ taxiren und daher bei grösseren Beobachtungsreihen Verwechslungen von Sternen zu vermeiden. Wemu es erforderlich sein sollte, kaun mittelst des Knopfes $f$ ein Schicher vorgeschoben werden, welcher das ganze Gesicbtsfeld bis auf einen schmalen Aussehnitt in der Mitte verdeckt; es kann endlich auch noch eine andere Blende mit feinen runden Öffinungen eingesetzt werden, um nach dem Dawes'schen Vorschlage einzelne Stellen der Mond- oder Somnenoberfläche, bei sehr grossen Bremupunktlildern anch rerschiedene Partien einer Planetenscheibe mit einander zn rergleichen.

Als Registrirvorrichtung, welche beim Keilphotometer durehaus unentbehrlich ist, empfichlt sich an meisten die ron E. r. Gothardi) herrührende, welche im Wesentlichen auch bei dem Potsdamer Instrument beibehalten ist. Auf das Rad $g$ ist eine Rolle schmalen Telegraphenpapieres anfgesteckt, welches sich in der ans der Figur ersichtlichen Weise auf das zweite etwas grössere Rad li aufwickelt. Durch einen Druck auf den Hebel $l$ wird dieser Papierstreifen mittelst des elastischen Kissens $m$ an die erhabene Theilnng augedriickt. Ansser dieser Theilnng presst sich noch ein an dem festen Theile des Photometers ebenfalls erhaben angebrachter Indexstrich in das Papier ein. Die Markinmo ist so deutlich, dass die Ablesung des Streifens, namentlich mittelst einer schwachen Lupe, keine Schwierigkeiten bereitet. Man kann anch noch zwischen Streifen und Theilung, wie es E. r. Gothard gethan hat, einen zweiten Streifen

1. Zeitschrift für Instrumentenkunde. Jahrg. i, p. $34 i$. 
mit Farblösuug getränkten Papieres einschieben; die Ablesung wird danu noch bequemer, indessen ist diese Complication der Einriehtung, welehe auch einige Übelstïnde mit sich führt, nicht unbedingt erforderlich. Bein Herabdriicken des Hebels $l$ fasst die starke Feder $n$ in eine Art Zahnkranz ein, weleher auf dem Rade $k$ fest aufsitzt, und beim Loslassen des Hebels wird das Rad um ein Stiick gedreht und der Papierstreifen eine kleine Strecke fortgezogen, so dass für eine nene Einstellung Raum wird. Un den Streifen nach Beendigung der Beobachtung schnell abwickeln zu können, wird die Feder $n$ mittelst der Schraube $a$ ein wenig angehoben, so dass das Rad $k$ sich frei und schnell drehen lässt.

Die Theorie des Keilphotometers ist die denkbar einfachste. Es stelle in Figur $37 A B C$ das dunkle und $A D C$ das durchsichtige Prisma dar. Die Länge des Keiles $A B$

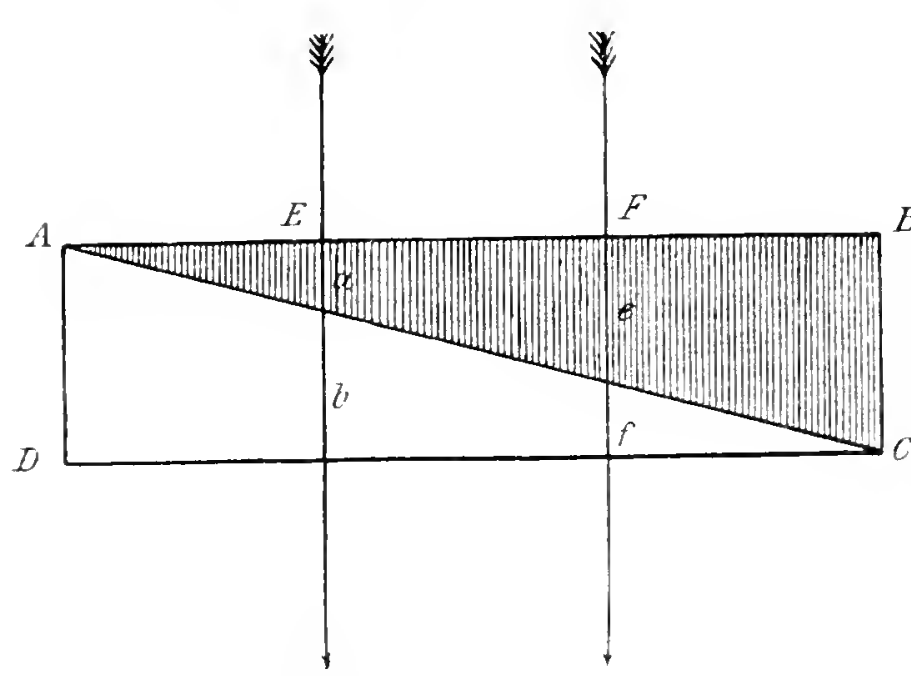

Fig. $3 \%$ sei $l$, seine Gesammtdicke $A D$ sei $d$. Die ron zwei Sternen mit den Lichtstärken $J_{1}$ und $J_{a}$ herkommenden Lichtstrahlen mögen bei $E$ und $F$ in den Keil eintreten und imnerhalb desselben die Strecken $a$ und $b$, resp. $e$ und $f$ durchlaufen. Die Liehtst:irken beim Austritt aus dem Keil seien $J_{1}^{\prime}$ und $J_{2}^{\prime}$. Nemnt man den Durchliissigkeitscoefficienten des dunklen Glases $l_{i}$, den des weissen

Glases $c$, so hat man nach dem Friberen:

$$
J_{1}^{\prime}=J_{1} l_{i}^{n} c^{b} \text {, oder: } \log J_{1}^{\prime}-\log J_{1}=n \log l_{i}+b \log c,
$$

oder eudlich, da $b=d-a$ ist:

$$
\log J_{1}^{\prime}-\log J_{1}=a \log l_{i}-\operatorname{lng} c+d \log c
$$

Eloenson ist atuch:

$$
\log J_{2}^{\prime}-\log J_{2}=\log l_{i}-\ln c+d \log c
$$

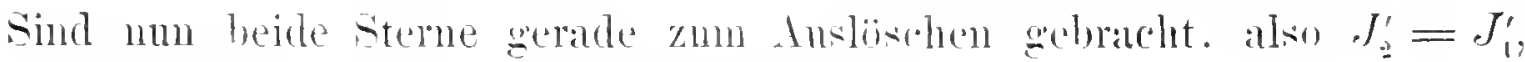
so erhälit man ane den letzton dileinhungen:

$$
\left.\ln J_{1}-\ln J_{2}=1-a\right) \ln g l_{i}-\log \theta_{2}
$$


Es ist alıer, wemn man die Streeke $E F$ mit $s$ lezeichnet, $c-a=\frac{s d}{l}$; folglich :

$$
\log e_{1}-\log J_{ \pm}={ }_{l}^{s d l}(\log k-\log c)
$$

Statt der Differenz der Helligkeitslogarithmen kaun man den Intensititsunterschied der beiden Sterne in Grössenclassen (nach der thblichen Weise dureh Division mit 0.4) einfuhren. Bezeichnet man denselben mit $g$, und ersetzt noch die verschiedenen Constanten dureh eine einzige Constante $K$, so hat man endlich:

$$
g=K s
$$

Der Grössenmuterschied zweier im Keilphotometer ausgelöschten Sterne ergiebt sieh also aus der Differenz $s$ der Scalenablesungen unmittelbar durch IIultiplieation mit einer Constante $K$, welche man die Keileonstante nennt, und die von der Besehaffenheit des dunklen Glases,' sowio von dem Winkel des Keiles abhängt. Giebt die Theilung Millimeter an, so ist $K$ die Grössenabnahme eines Sternes bei einer Verschiebung des Keiles um $1 \mathrm{~mm}$. Bestimmte Vorschriften über die Wahl dieser Constante lassen sich nicht geben. Ist der Keilwinkel sehr klein und das Glas nieht sehr dunkel, so tritt wegen der ausserordentlieh langsamen Auslöschung leicht eine Ermiidung des Auges ein; ist dagegen die Steigung. des Keiles gross, so liegt die Gefihr vor, dass ein verhältnissmäissig unbedeutendes Überschreiten des Auslöschungspunktes schon einen merklichen Fehler hervorruft. Die in England angefertigten Keile sind im Allgemeinen etwas flach und wenig stark absorbirend, sie miissen daher, um eine grössere Lichtschwächung hervorzubringen, ziemlich lang g’ewaillt werden (die beiden von Pritehard benutzten Keile waren ungefiilnr 10 resp). $16 \frac{1}{2} \mathrm{~cm} \mathrm{lang}$, die zugehörigen Keilconstanten waren $0.10 \mathrm{resp}$. 0.07 Grössenclassen), und dies bringt den Übelstand mit sich, dass die Beschaffung von so grossen Stiicken homogenen Glases schwierig ist. Die in Potsdam angervendeten Keile sind merklieh kiirzer (ungeführ nur 6 bis $8 \mathrm{~cm}$ lang), dagegen ist ihre Constante etwa doppelt so gross wie die der Pritchard'schen Photometer. Nach den in Potsdam gemachten Erfahrungen eignet sich eine Keileonstante von 0.15 bis 0.20 Grössenclassen am besten zu photometrischen Messungen. Werthe unter 0.10 und iiber 0.25 sind nach Möglichkeit $\mathrm{n}$ vermeiden.

Die genane Bestimmung der Keilconstante kam bei jedem Apparate nur auf experimentellem Wege erfolgen. l'ritchard hat dafiur awei Methoden vorgesehlagen, die Anwendung von Blendvorrichtungen und dic Benutzung ron polarisirenden Medien. Dic erstere Methode ist nach dem, 
was im Vorangeheuden ïber die Bengungswirkungen gesagt worden ist, entsehieden zu rerwerfen. Die zweite Methode ist theoretisch unanfechtbar, aber in der ron Pritchard angewandten Form nicht empfehlenswerth. Prite hard beunt zwei neben einander befindliche schmale Spalte, die eine genan bestimmte Entfermung von einander haben und setzt hinter dieselben seinen Keil. Auf die Spalte gelangt paralleles Licht, welehes nach dem Passiren des Keiles anf ein doppeltbrechendes Prisma auffält. lis cutstehen so zwei Bilder von jedem Spalt, die senkrecht zu einander polarisirt sind. Dureh ein vor dem Auge befindliehes Nieolprisma lässt sich die Gleichheit der von beiden Spalten herriihrenden Bilder herstellen und dilher die einem bestimmten Stiicke des Keiles eutsprechende Absorption ermitteln.

Gegen dieses Verfihren kanm man zweierlei einwenden. Erstens wird die Constante fiir ein viel zn kleines Stiick des Keiles bestimmt, da die Entfernung der Spalte nicht sehr gross sein darf (bei Pritchard mur $9.5 \mathrm{mmn}$; der Fehler der Messmng geht also zu stark ein. Zweitens wird dic Creichheit zweier Lichteindricke beurtheilt, der Keil also unter ganz anderen Bedingungen benutzt, als bei den Sternbeobachtungen. Spitta hat ansserdem noch darauf anfinerksam gemacht, dass bei der Pritehardshen Methode infolge der an den inneren Flïchen der Nicolprismen stattfindenden Reflexe leicht Fehler entstehen können, weun nicht ein geeignetes Diaphrigma zwischen Ange und Nicolprisma eingesetzt ist.

Anstatt des Pritchardschen Verfahrens zur Bestimmung der Keil"unstante wendet maln mit Tortheil eine der folgenden Methoden au.

1. Torschlag ron Abner. Das I'hotometer wird auf einen durch ('in geeignetes Arrangement hergestellten kinstlichen Stern gerichtet. Tor demselhen ist eine scheibe mit verstellharen sectorförmigen Ausschnitten angebracht, die in schnelle Jotation rersetzt werden kann. Man giebt nun dem kinstlichen Stern durch geeinnete Wahl der Sectoren nacheinander bestimmte Helligkeitsprade und hringt den stern jedesmal durch Verschichen des Keiles zum Terohwinden. Ant diese Weise lïsst sich die Keilconstante ans Messmengen an beliebigen stellen und iiber beliebig crosse strecken des Keiles durchans einwurstrei emitteln.

2. Forschlag ron spitta. Eine Mnalil von kleinen spiegeln, deren lietlexionsvermögen genam gleich sein muss. Wird so anfgestellt, dass ron jedem derselhen das Licht einer Flamme in oleichem Betrage auf cine sehr kleine wejse scheibe seworten wird. Diese scheibe erscheint im Photometer als winziger weisen Fleck, nud ihre Helligkeit andert sich im Verhältniss der tnzilhl de expmirten spiegel: sie wird mit Hiilfe des Keiles answeliowlit. Wie Methode ist etwat complicirter und nicht ganz 
so sicher wie die Abney'sehe, anch ist sie dadurch etwas beschrinkt, dass die Anzahl der Spiegel nicht zu gross gewïhlt werden darf. Eine sorgfiiltige Untersuchung dieser Spiegel hinsichtlich ihrer Reflexionsfihigkeit ist ein unerliissliches Erforderniss, und dadurch werden leieht Fehlerquellen herbeigeftihrt.

3. Potsdamer Methode. Am einfachsten und sichersten lïsst sich die Keileonstante mit Hulfe des Zülner'schen' Photometers bestimmen. Das Keilphotometer wird unmittelbar an Stelle des Oculars an ersteres angesetzt und der kiinstliche Stern zum Auslöschen gebracht, nachdem dessen Helligkeit durch Verstellung der Nicolprismen am Intensititskreise in messluarem Grade verïndert worden ist. Man kann das Verfahren auch in der Weise umkehren, dass man den Keil zunächst um eine ganz bestimmte Strecke versehiebt und den klnstlichen Stern durch Drehung des Intensitätskreises zum Verschwinden bringt; die eigentliche Messung geschieht dann nicht mit dem Keil, sondern mit den polarisirenden Mitteln. Das erstere Verfuluren ist entsehieden vorzuziehen.

4. Benutzung von photometrisch bestimmten Sternen. Diese Methode hat den grossen Vortheil, dass keinerlei besondere instrumentellen Einrichtungen erforderlich sind, und dass die Constantenbestimmung unter genau den gleichen iusseren Bedingungen erfolgt, wie die gewöhnlichen Beobachtungen mit dem Keilphotometer. Man sucht aus den Helligkeitscatalogen (Harvard Photometry, Uranometria nova Oxoniensis, Potsdamer Durehmusterung) Sternpaare aus, die in Bezug auf Farbe nicht allzu sehr von einander verschieden sind, dagegen betrïchtliche Intensitïtsunterschiede anfweisen, und misst dieselben mit den Keil. Um von den zufälligen Fehlern der Cataloghelligkeiten möglichst frei zu werden, thut man gut, eine sehr grosse Zahl von Sternpaareu zu benutzen.

Langley hat zur Constantenbestimmung die Anwendung des Bolometers empfohlen, und melirfach ist der Vorschlag anfgretancht, die Photographie nutzbar zu machen. Man blendet die eine Seite des Keiles bis auf zwei schmale, in einer bestimmten Entfernung von einander befindliche Spalte ab und bringt anf der anderen Seite einen Streifen photographisehen Papieres an. Lässt man dam anf die Vorderseite paralleles Licht auffallen, so giebt der Grad der Schwärzung anf dem Palpiere ein Mass fuir das Verhültniss des an den beiden betreffenden Stellen des Keiles hindurchgegangenen Lichtes. Die Langley'sche Methode beriicksichtigt nur die Wirkung des Keiles anf die Wärmestrahlen, die photographische Methode zieht nur die hrechbareren Strahlen in Betracht, die letatere ist ansserdem nur einer geringen Genanigkeit fiihig. Beide Methoden sind wenig zu empfehlen. 
Der Benutzung jedes Keilphotometers muss ausser der Constantenbestimmung, die am besten nicht nur nach einer, sondern gleichzeitig nach mehreren der oben empfohlenen Methoden geschieht, noch eine specielle Untersuchung des Keiles in Bezug auf Homogenität des Glases, Regelmïssigkeit der Gestalt und Durchlïssigkeit für verschiedene Farben vorangelıen.

Bei der Vollkommenlieit, mit welcher heutigen Tages Glas hergestellt wird, sind anffallende Mängel in der Homogenität von vornherein kaum zıl befiirchten, namentlich wenn man die Keile nicht zu lang wählt und daher anch nicht zu grosse Glasstiicke nöthig hat; dagegen bereitet das Anschleifen von absolut ebenen Flächen bei den verhältnissmässig dünnen Keilen einige Schwierigkeit, und es liegt die Gefahr vor, dass die begrenzenden Fliichen eine leichte Krïmmung besitzen, und dass infolge dessen einer Verschiebung des Keiles um gleiche Strecken nicht iiberall ein gleichmässiger Zuwachs der absorbirenden Schicht entspricht. Zur Untersuchung dieser Punkte wendet man ein älmliches Verfahren an, wie bei der Ermittlung der Theilungsfehler von Massstäben oder Kreistheilungen. Man misst ein bestimmtes Helligkeitsintervall an verschiedenen Stellen des Keiles, indem man den Endpunkt der ersten Messung zum Aufangspunkte der zweiten wählt u.s.f. iiber die ganze zum Gebranch bestimmte Länge des Keiles hiuweg. Dann nimmt man ein anderes doppelt so grosses Helligkeitsintervall, ebenso ein dreimal, riermal n. s. w. so grosses und misst anch diese ron denselben Anfangspounkten aus. Durch ein geeignetes Ausgleichnngsverfahren leitet man damn die Fehler der einzelnen Anfangspunkte her und kam anf diese Weise eine vollkommene Kalibrirung" des Keiles bewerkstelligen. Am besten eignet sich zu dieser Priifung die oben empfohlene dritte Methode mit Benutzung des Zöllner'schen Photometers; die zur Herrobringmng hestimmter Helligkeitsuntersehiede erforderlichen Einstelhngen am Intensititskreise werden dabei im Voralls berechnet.

Die Durchlïssigkeit des Keiles für verschiedene Farben hängt von der Beschaffenheit des dmuklen Glases ab. Tollkommen nentral gefärbtes Glas ist ansserst schwierig zu beschaffen. Loewy giebt an, dass er muter 50 rerschiedenen sorten nicht eine einzige grefunden habe, die seinen Anfordemugen entsumchen hïtte. In der That haben die meisten sogenamnten nentralen Giliser eine schwach wrinliche Färbung und lassen daher die rothen strahlen weniger leicht hindurds als die gelben und griinen. Man iberzent sich am einfuchsten ron der allgemeinen Absorptionswirkmng eines Glases, indem man dasselbe durch ein Spektroskop betralchtet und sieht, an welchen stellen Absorptionsstreifen anftreten. Man wird fast immer drei mehr onler weniger starke Biinder, zwei daron 
im rothen und eins im blaugrtinen Theile des Spectrums erkennen. Zur speeielleren Untersuehung bedient man sieh mit Vortheil wieder der kitnstlichen Sterne des 'Zöllıer'sehen Photometers, denen man mit Hulfe des Colorimeters die versehiedensten Farben geben kann. Man-erhält so Sterne von ähnlichen Farbenutlaneen, wie sie auch bei den wirklichen Sternen vorkommen; nur weisse oder bläuliehweisse Sterne lassen sieh nicht herstellen. Wenn man nun dasselbe Helligkeitsintervall in den versehiedenen Farben mit dem Keil misst, so kann man die Untersehiede in der Absorptionsfaihigkeit rechunngsmässig bestimmen. Die von Töpfer in Potsdan gelieferten Keile zeichnen sich in dieser Beziehung durch bemerkenswerthe Gleichförmigkeit aus.

Über den Gebrauch und den Anwendungsbereich des Keilphotometers lassen sich noch folgeude allgemeine Vorsehriften aufstellen.

Da Fehler in der Constantenbestimmung die Resultate der Messungen um so stiirker verfälschen, je grösser der beobachtete Helligkeitsuntersehied ist, so empfiehlt es sich nicht, sehr helle und sehr sehwache Sterne direet mit einander $z u$ vergleichen; jedenfalls ist es unstatthaft, den Keil ausserhalb der Strecke, für welehe speeiell die Constante bestimmt ist, zu benutzen. Bei sorgfïltig untersuchten Keilen wird man eine Differenz von 4 bis 5 Grössenclassen unbedenklich messen können.

Die Unmöglichkeit, absolut neutrales Glas zu erhalten, bedingt grosse Vorsicht bei der Vergleichung sehr versehieden gefärbter Sterne. Die Messungen rother oder röthlicher Sterne sind nach Möglichkeit ganz zu vèrmeiden.

Mit allen Auslöschungsphotometern hat das Keilphotometer die schon friber besprochenen Übelstände gemein, welche durch die Erlenchtung des Grundes und die wechselnde Empfindlichkeit des Anges herbeigefuhrt werden. Es sind demuach Beobachtungen in der Dämmerung und in hellen Mondnaichten zu unterlassen, jedenfalls diirfen unter keinen Umstiinden Sterne in der Niihe des Mondes mit anderen weit davon entfernten verbunden werden. Es ist rathsam, nur ummittelbar aufeinander folgende Differenzmessungen anszufiihren und die Beobachtungen stets vollkommen symmetrisel anzuordnen. Die ersten Messungen jedes Abends sind wegen der am Anfange der Beobachtungen besonders stark veründerlichen Empfindlichkeit des Auges am besten gar nicht zu verwerthen.

Die zuerst von Kayser empfohlene, später wieder von Pickering in Vorsehlag gebrachte Beobachtungsmethode, bei welcher die Zeitdaner des Durehganges der Sterne dureh den Keil bis zum Verschwinden als Mass benutzt wird, ist ans zwei Grijnden zu rerwerfen. Erstens ist die Genauigkeit der Auslöschungsheobachtung nicht die gleiche fiur Sterne von verschiedener Dechination, weil diese den Keil mit verschiedener 
Geschwindigkeit passiren, und zweitens erfordert diese Methode einen unverhältnissmässigen Zeitaufwand, da bei jeder einzelnen Messung der Stern inmer wieder den ganzen Weg von dem Antrittsfaden bis zum Verselıwindungspunkte durchlaufen muss. Es ist auch ein Nachtheil, dass die Auslöschung, je nach der Helligkeit der Sterne, an verschiedenen Stellen des Gesichtsfeldes stattfindet.

In Figur 38 ist die Abbildung eines nach meinen Angaben von Töpfer in Potsdam gebauten Instrumentes mit Keilphotometer beigefiigt, welches wegen seiner bequemen Handhabung sehr zu empfehlen ist. Es hat die Form cines Équatorial coudé und kann für jede Polhöhe eingestellt werden. Das Ocular ist nach dem Pole gerichtet, der Beobachter braucht also seine Stellung niemals zu verïndern. Die Declimation der Sterne wird an dem Kreise a eingestellt, welcher mit dem ror dem Objectir befindlichen drehbaren Prisma fest verbunden ist; $b$ ist der Stundenkreis und $c$ eine Vorrichtung zur Feinbewegung, um die Sterne immer in der Mitte des Gesichtsfeldes zul halten. Da das Photometer sich bei der Drehung im Stundenwinkel mitbewegt, so bleibt infolge der Spiegehung an den beiden Prismen (eins ror dem Objectir, das andere im Innern des gebrochenen Fernurns der in Keilphotometer angebrachte Steg nicht parallel der tiglichen Bewegm!ng. Das Photometer ist daher noch fiir sich im Positionswinkel drehbar, und, wie man leicht sieht, brancht man an dem Positionskreise $d$ mu die jedesmalige Declination des Sterns einzustellen, damit derselbe sich innerhalb des Steges, also senkrecht zur Richtung der Keilrerschiebung, durch das Gesichtsfeld beregt. Bei dem für das Potsdamer Observatorium construirten Apparate hat das Objectiv eine Öffinung von $5.5 \mathrm{~cm}$ und eine Brennweite ron $60 \mathrm{~cm}$. Es eignet sich in diesen Dimensimen zur Beolachtung aller Sterne bis zur achten Crösse. 



\section{Capitel II.}

\section{Photometer, bei denen die Gleichheit zweier Lichteindrücke beurtheilt wird.}

Die zweite Classe von Photometern, bei denen die zu messende Lichtquelle durch irgend welehe Mittel soweit geschwaicht wird, bis ihre Helligkeit der einer anderen Lichtquelle gleichkommt, hat vor den Auslöschungsphotometern so viele Vortheile voraus; dass ilıre grössere Verbreitung ganz selbstverständlich erscheint. Einer der Hauptvorzitge dieser Methode besteht darin, dass das Ange viel weniger angestrengt wird, als bei der Beobachtung des Versehwindens, und dass eine Änderung der Empfindliehkeit des Auges wenig oder gar keinen Einfluss auf die Messungen anstibt, weil die beiden zu vergleichenden Lichtquellen gleichmissig davon betroffen werden. Dabei ist die Sicherheit der einzelnen Einstellnng grösser als bei der Auslöschungsinethode, selion deshalb, weil die eigentliche Pointirung keine einseitige ist, vielmehr das zu messende Object abwechselnd heller und schwäeher gemacht werden kann als das Vergleichsobject. Die rerschiedene Helligkeit des Grundes kommt bei dieser Gattung von Photometern zwar auch in Betracht, der störende Einfluss derselben lässt sich aber dureh geeignete Vorrichtungen bei den meisten Apparaten so gut wie ganz unschädlich machen; es ist nämlich fast immer zu erreichen, dass sich die Helligkeiten des Grundes, anf den die zu vergleichenden Objecte, beispielsweise zwei Sterne, projicirt erscheinen, im Instrumente mit einander vermischen, so dass die Objecte auf einem gleichmäissig hellen Untergrunde sichtbar sind. Es wäre also nur die Frage zu entscheiden, ob die Genanigkeit der Vergleichnng von der grösseren oder geringeren Intensitiit dieses gemeinschaftlichen Grundes abliängt. Nach allen bisherigen Untersuchungen ist dies nicht der Fall. Die meisten Beobachter stimmen zwar darin iiberein, dass die Vergleichung fuir das Auge am Angenehmsten auszufiihren ist bei einem schwach erhellten Gesichtsfelde, dass dic Sicherheit aber keinesweg's grösser ist als bei ganz hellem oder ganz dunklem Gesichtsfelde. Die Methode hat demnach den Vortheil, dass Sterne mit einander verglichen werden können, die am Himmel selır weit ron einander entfernt sind, und dass Beobachtungen bei heller Dämmerung und in Mondscheimnäichten durchaus unbedenklich sind. Dafür treten aber auch einige Ü̉helstinde anf. Wie schon in der 
Einleitung zu dieserr Abschnitte hervorgehoben wurde, miissen die beiden Lichteindriicke gleichzeitig unmittelbar neben einander betrachtet werden, da das Ange nicht im Stande ist, den Einduck einer Helligkeit anch mur kurze Zeit festzuhalten; auch ist es unbedingt erforderlich, dass die beiden Objecte in Bezug auf ihr Aussehen und ihre scheimbare Grösse cinander vollkommen ämlich sind. Diese Bedingungen lassen sich zum 'Theil nur dureh ziemlich eomplicirte mechanische Einrichtungen erfüllen, und in dieser Beziehung stehen die meisten Gleichheitsphotometer den Auslöschmgsphotometern nach. Da die Vergleichung von Flächen im Allgemeinen sicherer ausfiihrbar ist, als diejenige von Punkten, so wäre die Benutzung soleher Apparate rorzuziehen, in denen, wie bei dem Steinheil'schen Prismenphotometer, das Licht der zu messenden Himmelskörper in eine Fliche ansgebreitet werden kann. Diese Methode ist aber wegen des bedentenden Lichtrerlustes nur auf hellere Objecte anwendbar; bei der Nessung schwächerer Sterne ist allein die Punktrergleichung möglich. (rinz besonders branchbar für die Himmelsphotometrie hat sich die Einfilhrung ron kïustlichen Vergleichsobjecten erwiesen, deren Helligkeit messban verïndert werden kamn. Man ist dabei freilich bis zu einem gewissen Grade von der Constanz der benutzten Lichtquelle abhängig, und dis Anssehen eines kimstlichen Sterns unterseheidet sich stets nicht unmerklich ron dem des wirkliehen Sterns, schon ans dem Grunde, weil diss Bild des letzteren dureh die Unruhe der Luft, durch Scintillation u.s.w. beeinthust wird; aber diese Nachtheile rerden zum grossen Theil wieder durch die grössere Einfachheit der Construction und die beqnemere Handliabung anferewogen. Ein Hauptiibelstand der Vergleichungsmethode ist der Einfluss der Farbe. Es ist schwierig, ja fast ummöglich, die Gleiehheit der Intensität zweier sehr verschieden gefübten Objecte richtig zu hentheilen, und die Angaben rerschiedener Beobachter weichen daher anch unter Umstainden sehr betrichtlich ron einander ab. So lange man keine zurerlissigen Dittel besitzt, un die Firloung einer Liehtquelle in her Weise zu indern, dass man zngleich anch angeben kann, um wie viel die lebendige Kraft derselben sich rermindert oder vergrössert hat, “" lange wird jecte Tergleichmg rersehiedenfarbiner Objecte mit einer sewissen Inoicherheit rerhunten sein.

Ans der iiberatus wrossen Zahl der zur mmittelbaren Vergleiehung zweier Lichtrindriicke bestimmten I'hotuncter sollen in Folgenden die wichtigsten besprochen und dabei spediell diejenigen berorzugt werden,

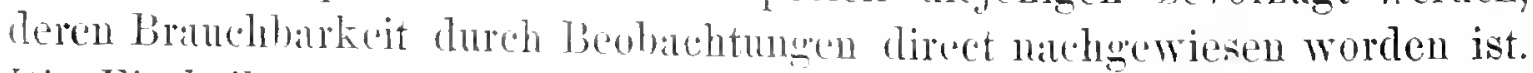
Ine Eintheihug ertolget wieder nach den in der Einleitung zn diesem Abschnitte angefihrten llanptkategurien, welche bei dieser Gattumg ron l'hotometern fiat simmolich zur Anwendung wekomuen sind. 


\section{Bentzung des photometrischen Hauptgesetzes vou Quadrate der Entfernung.}

Das erste Grundgesetz der Photometrie, nach welchem sich die lebendigen Kraifte der Ätherbewegung an zwei versehiedenen Punkten eines Lichtstrahls ungekehrt wie die Quadrate der Entfernungen dieser. Punkte von der Lichtquelle verhalten, ist maturgemäss schon sehr fruh bei der Coustruction von photometrischen Apparaten zur Anwendung gebracht worden und liefert uns anch lieute noch eins der einfachsten und sichersten Hulfsmittel zur Prufung von nenen Lichtmessungsmethoden. B o uguer und Lambert haben sich bei ihren photometrischen Untersuchungen fast ansschliesslich dieses Principes bedient, und eine ganze Reilıe der bekanntesten und weitverbreitetsten Instrumente der technisehen Photometrie beruht auf diesem Gesetze.

Da es bei den :alteren hierher gehörigen Apparaten auf eine inidirecte Vergleichung zweier Lichtquellen ankam, indem uur die von ihnen auf einer Fliche hervorgebrachten Beleuchtungen in Betracht gezogen wurden, so ist es erklärlich, dass dieselben für die Astrophotometrie, insbesondere für die Photometrie der Fixsterne, wo es sich um ausserordentlich schwache Lichteindriicke handelt, kèine wesentliche Bedeutung gewounen haben. Immerhin verdanken wir denselben die ersten branehbaren Helligkeitsmessungen an Sonne und Mond, sowic werthvolle Resultate beziglich mancher Fragen der atmosphärischen Photometrie.

Es diirfte daher nicht gerechtfertigt sein, diese älteren Instrumente ganz mit Stillschweigen zu iibergehen; es wird aber eine kurze Beschreibung derselben, ohne näheres Eingehen anf ihre Besonderheiten und ihre Handhabung, ausreichend erscheinen.

a. Die Photometer von Bouguer, Ritehie und Foucault.

Bouguer') hat zwei verschiedene Formen von Apparaten benutzt, je nachdem es sich um die Messung ron verhä̈ltnissmässig wenig ansgedehnten leuchtenden Flammen oder um die Vergleichung ron grossen Flïchen handelte. Das erste dieser Bonguer'schen Photometer (Fig. 39, Seite 196) besteht aus zwei unter einem stumpfen Winkel aneinander stossenden Brettchen, beide mit gleich grossen kreisrunden öftunngen versehen, die mit geöltem Papier bedeckt sind. Die zu vergleichenden Lichtquellen $S$ und $S^{\prime}$ sind so aufgestellt, dass ihr Licht senkrecht auf die beiden ()ffunngen auffillt, und das Auge des Beobachters befindet sich, gegen jedes fremde

1) Traité d'optique, p. 9 und 32 . 
Licht dureh geeignete Vorrichtnngen geschützt, in der den stumpfeu Winkel halbirenden Ebene. Ein drittes Brettehen ist noch in der verlängerten Halbirungsebene angebracht, um von jeder Öffnung das für sie nicht bestimmte Lieht abzuhalten. Während die eine Lichtquelle unverändert stehen bleibt, wird die andere immer in der Richtung senkreeht zur belenchteten Ehene versehoben, bis die beiden Öffnungen dem Ange gleich hell erscheinen. Die Quadrate der Entfernungen der Lichtquellen von den Öffunungen geben daun das Mass für das Helligkeitsverhältniss derselben.

Das zweite Bougner'sehe Photometer (Fig. 40) besteht aus zwei Holzröhren, die an dem einen Ende durch ein Scharnier so miteinander verbunden sind, dass sie jeden beliebigen, an einem Gradbogen einstellbaren Winkel miteinander einschliessen kömnen. Die unteren Enden dieser

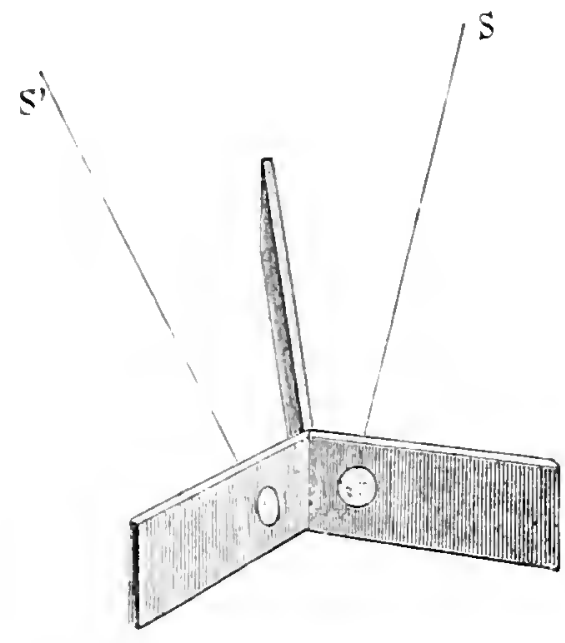

Fig. 39.

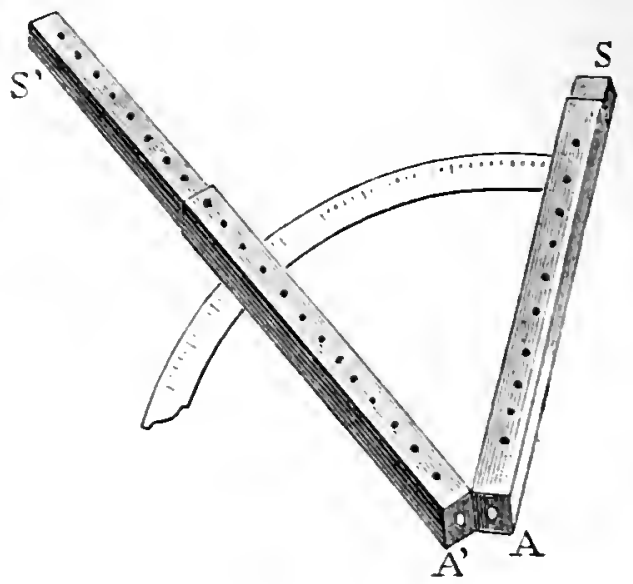

Fig. 40 .

Bühren sind mit Deckehn rerschlnssen. in welchen sich kleine, mit geöltem

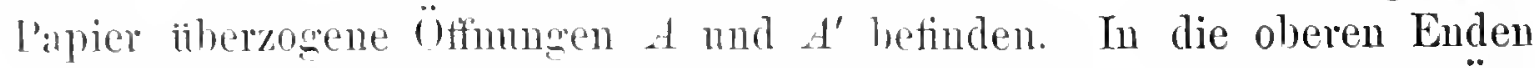
der lïhren lassen sich audere Rïhren einschieben, die mit freien öffnumen $S$ und $S^{\prime}$ rou hedentend griosserem Durchmesser als $A$ und $A^{\prime}$ versehen sind. Dic beiden Röhren werden anf die zu vergleichenden Flitchen. \%. B. auf zwei rershiedene Stellen des Himmels oder auf zwei wn der sonne helenclutete 11 inde etc.. eingestellt, und die anf die hellere Flïche werichtete hiohre wirl dam someit ansgezogen. bis die kleinen

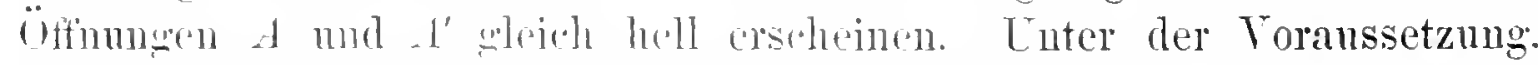
diss jerte der beiden zn verweichenden Flitchen an allen Punkten gleichmaissig hell int. dats fermer die unter cinturler gheich sind. findet man, dass die Flächenhelligkeiten sich

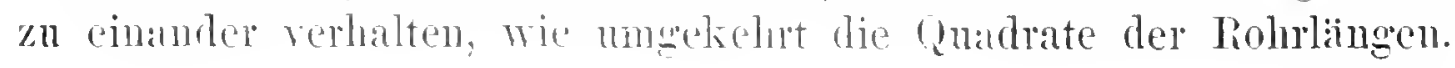


Eine Verbesserung des ersten Bougner'schen Photometers ist das viel benutzte Ritehie'sche') Photometer. Dasselbe (Fig. 41) ist ein imen geschwirzter Kasten, in dessen oberer Wand bei abc eine rechteckige Öffnung angebraeht ist, bedeekt mit geöltem P'apier. Im Innern.des Kastens sind zwei Spiegel befestigt, die bei $b$ unter einem rechten Winkel aneinander stossen. Die zu vergleichenden Lichtquellen werden vor die offenen Enden des Kastens gestellt, und der letztere wird zwischen ihnen längs eines Massstabes so lange verschoben, bis die beiden in $l$, aneinander grenzenden Theile der Öflinung gleich hell erseheinen. Man blickt auf diese Öffinung dureh eine liingere, innen geschwïrte Röhre, um

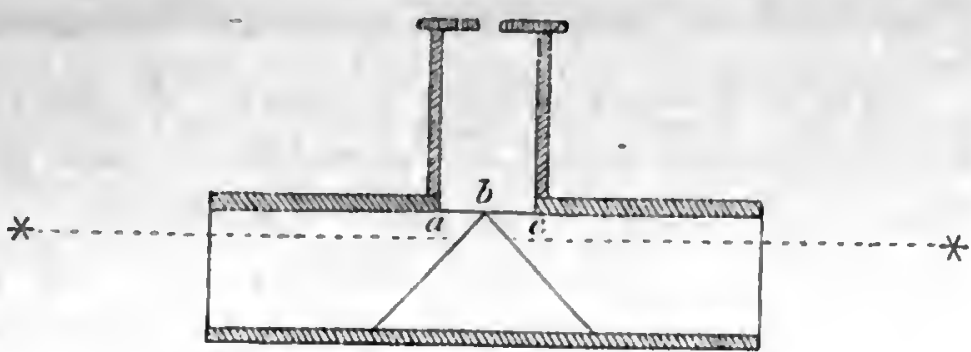

Fig. 41. fremdes Licht vom Auge fern zu lialten. Der Vortheil der Eimrichtung gegentiber dem Bongner'sehen Photometer besteht darin, dass die beiden erleuchteten Felder unmittelbar aneinander grenzen. Haben die zu vergleichenden Liehtquellen verschiedene Färbung, so empfiehlt Ritehie, die Öftumng in Photometer mit einem in kleiner Schrift bedruekten Papierstreifen zu bedeeken. Die Gleichheit der Beleuehtung wird damn als erreieht betrachtet, wenn die Sehrift ulber die ganze Läinge der Öffinung hin grleich gut gelesen werden kaun.

Fast noeh grössere Verbreitung als das Ritehie'sche Photometer lat das Foucault'sche ${ }^{2}$ ) gefunden (photomètre ì compartiment), welches ebenfalls als eine Modification des Bougner'schen Photometers zu betraehten ist. Ein innen gesehwäirter Holzkasten (Fig. 42) ist an der einen Seite offen und hat an der gegeniiberliegenden Seite eine kreisrunde Öftimung, die mit einem halbdurchsichtigen

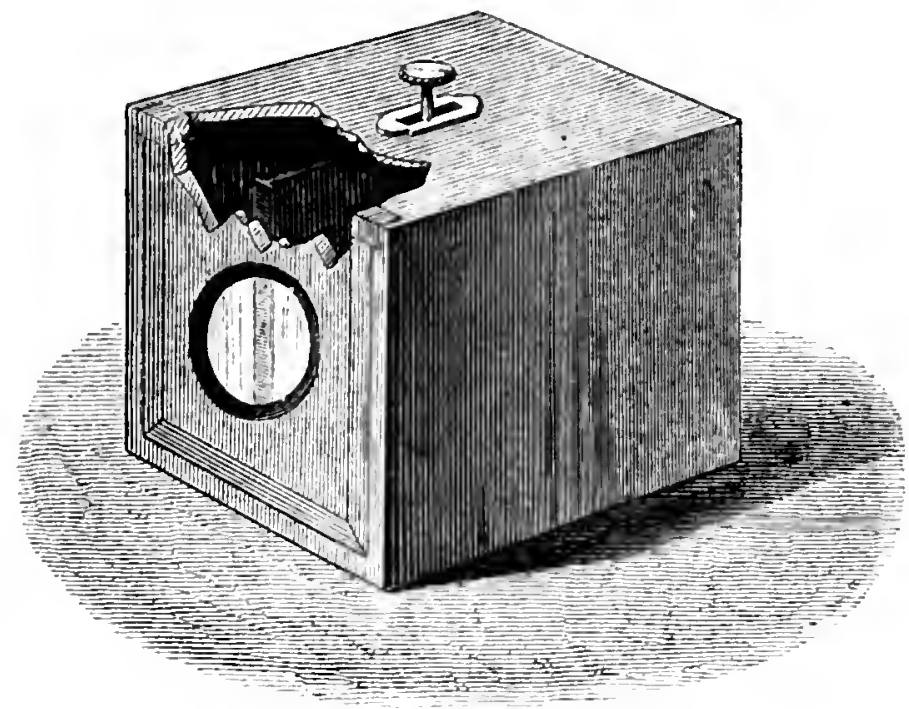

Fig. 42. Schirn bedeekt ist. Dieser Schirm wird rou zwei Glasplatten gebjildet, zwischen denen sieh eine diune gleiehförmige Stiirkemehlsehieht befindet.

1) Transactions of the R. Soc. of Edinburgh. Vol. 10.

2) Recueil des travaux scientifiques de Léon Foucault, Paris 1878, p. 100. 
Eine undurchsichtige Zwischenwand theilt den Kasten in zwei Theile und lisst sich mittels eines Knopfes vorwärts und riickwärts bewegen. Dadurch kann man erreichen, dass die von den beiden Lichtquellen beleuchteten Halbkreise in einer scharfen Linie aneinander stossen. Die Entfermungen der Lichtquellen ron dem Schirme miissen mittels Massstabes rireet remessen werden.

\section{b. Das Riumford'sche Sehattenphotometer.}

Etwas weniger genane Resultate, als die im Vorangehenden beschriebenen Aplanate liefert das gewöhnlich unter dem Namen »Rumford'sehés Schattenphotometer * bekannte Instrument 1). Dasselbe sollte eigentlich Lambertsches Photometer heissen, weil sich Lambert bei den meisten Helligkeitsmessungen einer rollkommen ähnlichen Einrichtung bedient hat. Ansser ron Lambert ist dieses Photometer anch ron anderen Beobachtern mehrfach zu Messmugen an helleren Himmelskörpern benntzt worden, und noch in der allemenesten Zeit hat Abney bei seinen Helligkeitsverglei"hungen der verschiedenen Partien des Sonnenspectrums ron diesem l'rimcipe Gebranch semacht.

Vor ciner senkrechten weissen Flïehe $A A_{1}$ (Fig. 43) ist ein rumder

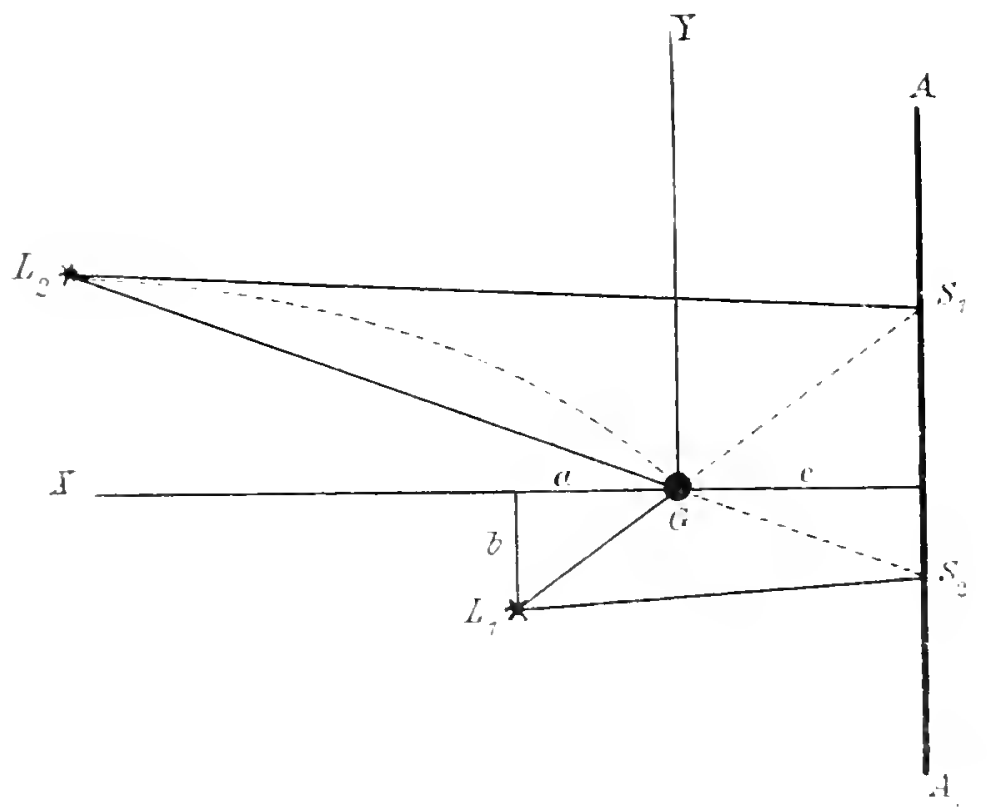
Stab $G$ senkrecht anfgerichtet, ron welchem durch die beiden zu rersleichenden Lichtquellen $L_{1}$ und $L_{2}$ die Schatten $S_{1}$ und $S_{a}$ entworfen werden. $S_{1}$ erluailt nur Licht rom $L_{2}$, dagegen $S_{2}$ num Licht rou $L_{1}$, während die übrige Flïehe ron beiden Lichtquellen zusammen belenchtet wird. $L_{1}$ bleibt in coustanter Entfermung ron dem schirme $A A_{1}$, und $L_{2}$ Fig. 43. wird num so lange rerschoben, bis die beiden

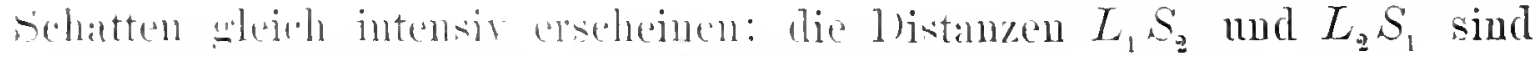

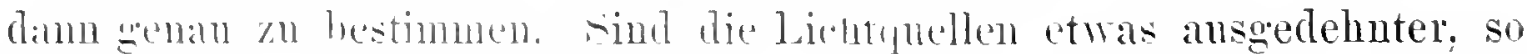

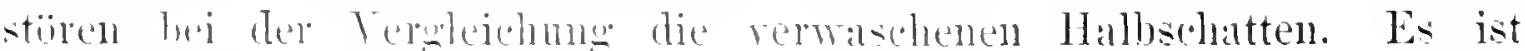

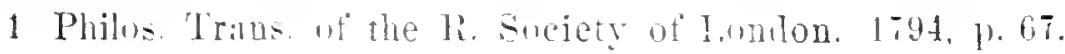


erwunseht, dass $L_{1}$ und $L_{\text {, }}$ sich in der Niihe der Normalen zum Sehirm befinden, welche dureh den Stab $G$ lindureh geht, damit einerseits die Schatten nahe bei einander liegen, andererseits die Beleuchtung von $S_{1}$ nud $S_{\xi}$ unter angenähert denselben Incidenzwinkeln erfolgt. Ist diese Bedingung nicht erfullt, und hat $L_{1}$ eine unverinderliche Position, so lisst sich selır leicht die Curve bestimmen, anf welcher $L$ a verschoben werden uuss, danit die Sehatten unter gleiehen Ineidenzwinkeln beleuchtet werden. Macht man nämlich $G$ \%m Anfangspunkte eines rechtwinkligen Coordinatensystems, dessen $x$-Axe mit der Normalen zum Sehirme zusammenfällt, nennt die Coordinaten von $L_{1}$ in Bezug auf dieses System $a$ und $b$, ferner den senkrechten Alostand des Stabes $G$ rom Selirme $c$, so findet man fur den geometrisehen Ort der Liehtquelle $L_{z}$ die Gleichung:

$$
a b x^{2}-\left(a^{2}+2 a c\right) x y+b c(c+2 a) x-a c^{2} y=0 .
$$

Dies ist die Gleiehnng einer Hyperbel, welehe dureh den Coordinatenanfang $G$ hindurch geht. Mittels der bekannten Werthe von $a, b$ und $c$

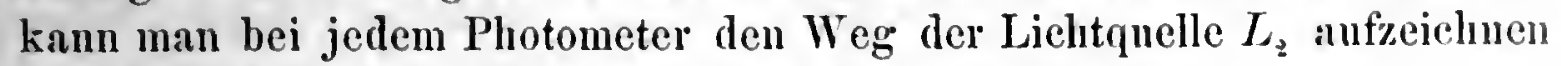
und die jedesmalige Distanz $L_{2} S_{1}$ tabellarisch berechnen.

c. Das Bunsen'sehe Fleckphotometer.

Bei diesem Instrumente, welehes in der teehnischen Photometrie eine der ersten Stellen einnimmt, werden die zu rergleiehenden Liehtıuellen zu beiden Seiten eines Papierschirmes anfgestellt, anf welehem sich eil durch Öl oder Stearin hervorgebrachter Fettfleck befindet. Derselbe erscheint bei auffallendem Lichte dunkel anf hellem Grmnde, dagegen bei durehgehendem Lichte hell anf dunklem Grunde, und wemn daher die Beleuchtung von beiden Seiten gleich stark ist, so wird der Fettfleek sich gar nicht mehr ron dem iibrigen Schirme unterscheiden lassen. Ist dieser Effeet durch Versehiebung der beiden Lichtanellen erreicht, so verhalten sich die Helligkeiten derselben zn einander, wie die Quadrate ilırer Entfermungen rom Schirme.

Die gebriuchlichste ron Riidorff $\left.{ }^{1}\right)$ empfohlene Anordnumg dieses Photometers wird dureh die sehematiselie Figur 4 (Seite 2001) erlinutert. $L_{1}$ und $L_{z}$ sind die beiden Liehtquellen, $P$ der l'hotoncterschirm mit dem Fettfleek $F$ in der Mitte, $S_{1}$ und $S_{q}$ zwei Spiegel, die einen stumpfen Winkel mit einander bilden, in dessen ILalbirungsebene der Schirm steht. Dats Auge sieht dureh eine Üftumng n in einem dunklen sichirm anf die

1, Pogg. Annalen. Jubelband, p. 234. 
Spiegel und erblickt daher die beiden Seiten des Fettfleckes. Es lässt sich leicht nachweisen, dass derselbe nie gleichzeitig auf beiden Seiten verschwinden kann, weil der nicht gefettete Theil des Schirmes mehr

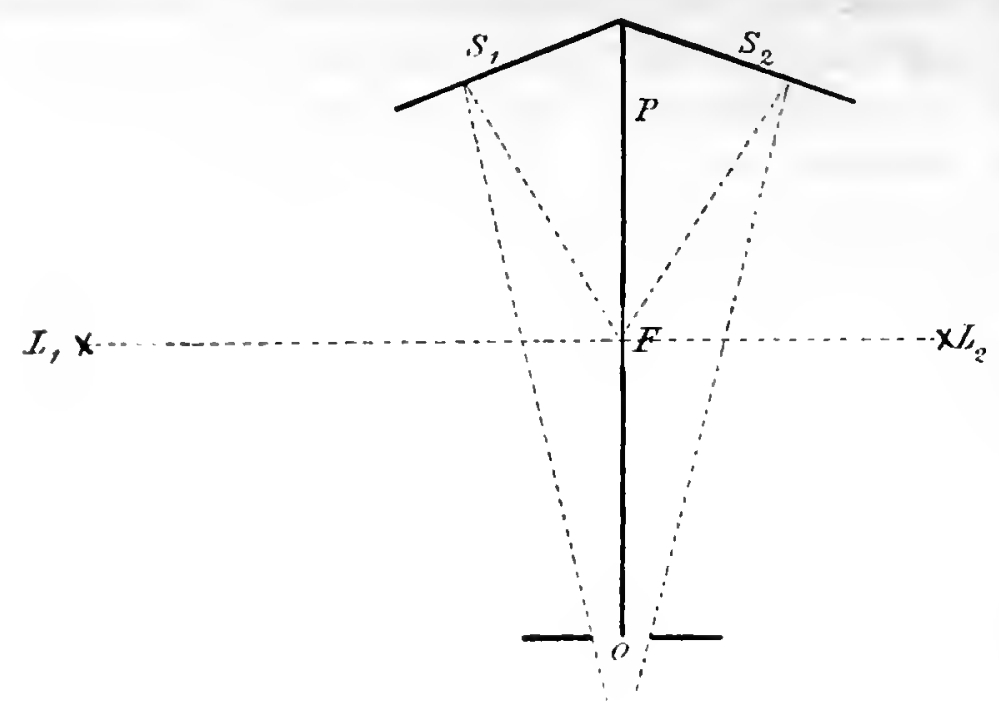

Licht absorbirt als der Fleck. Man beobachtet daher meist so, dass man erst den Fettfleck auf der rechten, dann auf der linken Seite verschwinden lïsst und das Mittel aus den gemessenen Distanzen zur Berechmung benutzt. Man kann auch eine bestimmte Hülfslichtquelle stets in unveränderter Entfermung auf der einen Seite des Papier-

Fig. 44.

sehirmes stehen lassen und die zu messcnden

Lichtquellen nach einander auf der anderen Seite in solehe Entfernungen hringen, dass der Fettfleck jedesmal anf dieser Seite nusichtbar wird.

Die Litteratur iiber das Bunsen'sche Photometer ist ausserordentlich umfingreich, nud es sind eine Menge von wichtigen Verbesserungen ron $\therefore$ Hefner-Alteneck, Kriss, Weber u. A. rorgeschlagen worden, m die Empfindlichkeit der Messungen zu steigern. Es soll hier nicht weiter anf diese Terbessermagen eingregangen werden, weil das Bunsen'sehe Photometer in der Astrophotometrie iiberlanpt nur sehr wenig Verwendung gefunden liat. In der Technik scheint es nenerdings etwas verdraingt zu werden durch die ausgezeichneten Lichtmesser ron Lummer und Brodhuny und ron Weber'), die im Princip eine gewisse Ähnliehkeit mit demselben hiben, aber eine weit grösssere Genanigkeit zu erreiehen gestatten. Aut eine nïhere Bescheibung dieser, fiur die Himmelsplotometrie ebenfalls nur in ganz heschrinktem Grade anwendbaren Instrumente muss hier rerzichtet werden.

\section{(1. Das Hersoleloche Astrometer.}

Wenn von ciner weit entfernten leuchtenden Fliche Licht anf eine Linse von sehr kurzer Brennweite antiaillt, sn entsteht in dem Foens der-

1 Zeitschrift tiil Instrumentenkunde. Jahrg. !, p. 41 und 161.

2 Wiedemann. Anmalen. Bd. 20. p. : 26. 
selben ein punktförmiges Bildehen, welches als kinstlicher Sterı benutıt werdeu kann. Betrachtet man diesen klinstlichen Stern aus verschiedenen Entferunngen mit dem blossen Ange, so ist nach dem fruher Gesagten klar, dass die auf der Netzhant hervorgebrachte Liehtempfindung umgekehrt proportional sein muss dem Quadrate der jedesmaligen Entfernung des Auges ron dem Brennpunkte der Linse. Dieses Princip hat J. Herschel') der Construction seines Photometers zu Grunde gelegt, indem er die Helligkeiten der mit blossem Auge sichtbaren Sterne mit der Helligkeit der dureh eine Linse sternartig verkleinerten Mondsclıeibe verglieh. So primitiv und mangelhaft auch der ganze Messapparat ist und so sehr er in mancher Hinsicht zu Bedenken Anlass giebt, so hat dieses Instrument

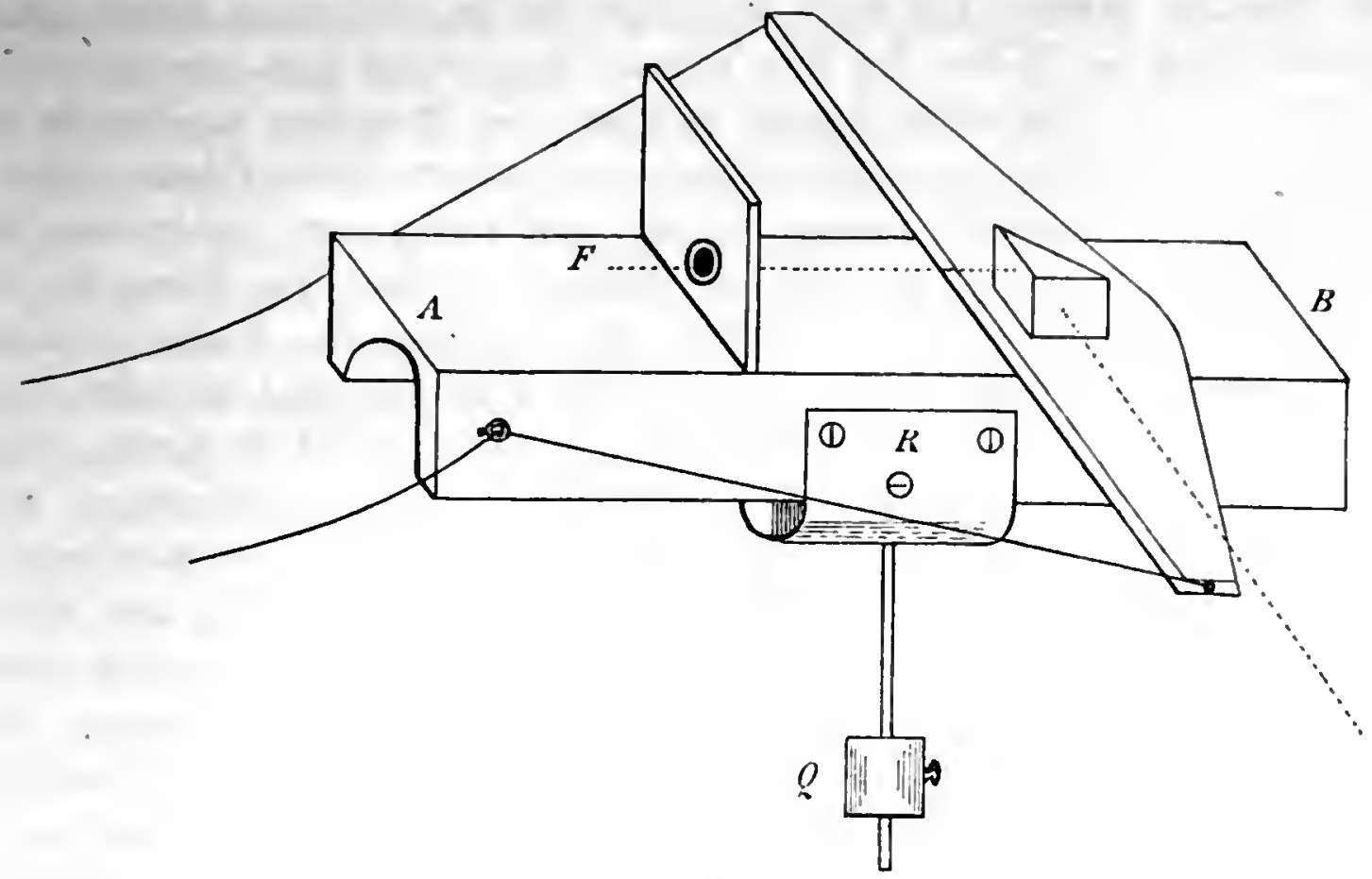

Fig. 45 .

fiir die Entwicklnngsgesehichte der Astrophotometrie doch eine gewisse Bedentung, weil mittels desselben der erste nieht auf blossen Schaitzungen beruhende Helligkeitscatalog einer Anzahl von hellen Sternen hergestellt worden ist.

Die Eimriehtung des Haupttheils dieses Photometers ist ans Figur 45 ersichtlich.

Ein Holzkasten $A B$ von ungefïhr $39 \mathrm{~cm}$ Länge kamm anf einer eylindrischen Walze von $366 \mathrm{~cm}$ Lainge hin und her geschoben werden. Durch eine federnde Vorrichtung, die an dem eisernen Bande $R$ befestigt

1) Results of astron. observ. made during 183t-1835 at the Cape of Good Hope. London 184i, p. 353 . 
ist, wird der Kasten leicht gegen die Walze gedrüekt, ohne dass die Beweglichkeit dadureh gehindert würde. Fin Gegengewieht $Q$ balancirt den Kasten aus und bewirkt, dass derselbe bei einer Drehung um die Walze in jeder Lage stehen bleiben kamn. Dic Walze selbst endet in zwei Zapfen, von denen der eine frei beweglich in einem Lager ruht, welches an einem tragharen dreifiissigen Stativ in Angenhöhe angebracht ist. An dem anderen Zapfen ist ein Seil befestigt, vermittelst dessen die Walze an einem feststehenden Balken iber eine nach allen Richtungen drehbare Rolle bis zu einer betriichtlichen Höhe hinaufgezogen werden kann. Durch diese Einrichtung und durch geeignete Aufstellung des tragbaren Stativs

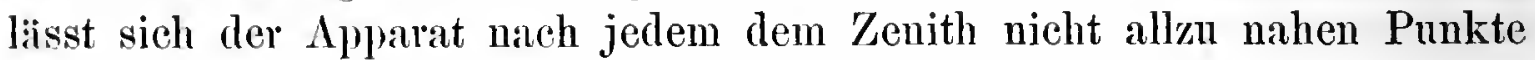
des Himmels richten, und man betrachtet die zu messenden Sterne durch Visiren liangs der Walze mit dem blossen Auge. Auf dem versehiebbaren Kisten $A B$ ist, um einen Zapfen drehbar, ein Brettchen angebracht mit einem daranf befestigten rechtwinkligen total refleetirenden Prisma. Dieses Irrettchen kam mittelst zweier Schniire ein wenig nach jeder Seite hin nm den Zapfen bewegt werden, so dass ron Stellen des Himmels, die ctwa $60^{\circ}$ his $100^{\circ}$ von dem Punkte, anf welchen die Walze gerichtet ist, abstehen, moch das Licht lïngs des Kastens hin total reflectirt wird. Eine Linse ron knmer Brennweite, die in einem anf dem Kasten senkrecelit befestigten Brette sitzt, entwirft bei $F$ ein punktartiges Bild ron dem durch das Prisma reflectirten Mondlichte. Der Beobachter erblickt den so erzengten künstlichen Stern sleichzeitig mit dem direct anvisirten Sterne und kann ilm durch Bewegen des Kopfes nach Belieben rechts. links, oben oder nuten neben den wirklichen Stern bringen. Der :nanc Kasten wird $1 m$ anf der Walze soweit dem Ange genähert oder von ihm entfernt, bis der kiinstliche und der wirkliche Stern gleich hell erscheinen, und dam wird die kntfernme des Bremununktes $F$ vom Ange möghichst senan gemessen. Hat man in derselben Weise einen zweiten Stern beobachtet, so ergieht sich das Helligkeitsverhiliniss der beiden Objecte nnmittelhar : us dem mugtkehrten Verhialtnisse der Quadrate der gemessenen Distanzen zwischen Ange mol kiinstlichem Stern. Die zu vergleichenden Gestime diirfen nicht allzn verschiedenr Abstinde vom Monde haben, weil somst die Ineidenzwinkel. nuter welken die Mondstrahlen auf die PrismenHächen anfallen, zu stark ron rinander differiren wiirden und dadureh die Constanz der Helligkeit des kïnstlichen Sterns gnefiilndet sein würde. Man wird wat thum, in Betreff dre Abstimde zwischen Mond und Sternen bei den Beobachtungen sich ctwa anf das ohen angegebene Intervall

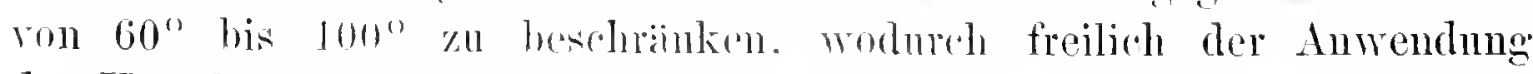
des Herachelischen Astrometers von vmuherein eine gewisse Grenze gesteckt ist. 
Solange der kfunstliche Mondstern, wie bisher voriusgesetyt ist, nur als Vergleichslichtọuelle dient, mol ledighlich Helligkeitsdifterenzen zwischen Sternen mittelst dieses Verbindungggliedes bestimmt weiden, lassen sich mit Hulfe des Herschel'sehen Photometers, so morollkommen es anch ist, ganz branchbare Messungsresultate erziclen.

Wemn der Herschel'sche Catalog, in welchem alle Helligkeiten auf einen cinzigen Stern (ce Centauri) als Einheit bezogen sind, lieute nur noch ein historisches Interesse heanspruchen kann, so liegt dies weniger an den Messungen sellst; als an der unzureichenden Bearbeitung derselben und insbesondere an der Vernachlissigung des Extinctionseinflusses. Zïllne ${ }^{\prime}$ ) hat aus den Herschel'schen Beohachtungen die Werthe fiir das Helligkeitsverhailtniss \%weier Sterne zusammengestellt, die an neun verschiedenen Abenden mit einander verglichen waren. Mit Berlleksichtigmngr der Extinetion ergiebt sich daraus fïr den wahrscheinlichen Fehler eines einzelnen Abends der Werth $\pm 0.0231 ;$ im Helligkeitslogarithmus oder \pm 0.06 Grössenclassen, ein Genauigkeitsgrad, der selbst mit den hesten modernen Photometem kaum ibertroften werden kamu.

Eine Vereinigung der an versehiedenen Abenden mit dem Astroneter angestellten Messungen, wie sie Herschel ausgefiilırt hat, ist naturlich nur damn möglich, wenn man das Geset\% kennt, nach welehem die Helligkeit des Mondes ron seiner Phase abhïngt. Hersehel hat sich zur Reduction der jedesmaligen Mondhelligkeit auf die Vollmondsintensitait der ron Euler anfgestellten Formel bedient und hat anf diese Weise für die Liehtstïrke desselben Stems an rersehiedenen Alsenden sehr erheblich ron einander abweichende Werthe erhisten. Wir suchte diese Untersohiede durch den Einfluss der verschiedenen Erlenchtung iles Himmelsarrundes bei wechselndem Abstande des Stems rom Monde zu erkliiren. bond und Zöllner haben aber nachgewiesen, dass, wem man zur lieduction der einzelnen Mondphasen auf einander anstatt der Enler'schen Fornel eine von ihnen empirisch abgeleitete Intensititscurve anwendet, die IIerseleeschen Beobachtungen desselben Stems an versehiedenen Abenden in durehaus befriedigende Übereinstimmung geblatcht werden kömuen; Boud hit anch noch direet gezeigt, dass die Eslenchtung des llimmelsgrundes auf die Messungen mit dem Herschel'sehen Astrometer nur einen geringen Einfluss ansiben kamm. Dic benutzung des Mondes zur Herorbringung des kiunstliehen Stems bleibt jedenfalls der bedenklichste Punkt dieses Photometers. II ersehel hat dies wohl sellst gefulht und daher spaiter den Vorsehlag gemaldit, anstatt des Mondes den J'laneten dupiter an benutzen, dessen Lieht, abosehen von den durch die verinderlichen Abstinde

1) Zülluer. Photometrische Untersuchungen. Leipzig 1565, p. 176. 
von Sonne und Erde bedingten Schwankungen, als himreichend constant angesehen werden darf. Mau könnte ebenso gut, wenn man sich nur auf Differenzmessungen beschränken wollte, zur Hervorbringung des kiinstlichen Sterns eine irdische Lichtquelle benutzen, die in geeigneter Weise mit dem Aplarate in Verbindung zu bringen wäre.

\section{e. Das Steinheil'sche Prismenphotometer.}

Fast genim zn derselben Zeit, in weleher Herschel sein Astrometer zu Helligkeitsmessungen am Fixsteruhimmel benutzte, trat Steinheil) mit seinem Prismenphotometer hervor. Wenu dieses Instrument anch ebenso wie das Herschel'sche hente veraltet und durch bessere verdrängt ist, so gebihht ilun doeh wegen der Eigenartigkeit seiner Construction und vor Allem wegen der ansgezeichneten Resultate, welehe Seidel mit Hülfe dieses Photometers gewommen hat, in der Gesehichte der Helligkeitsmessungen für alle Zeiten ein hervorragender Platz. Dem Herschel'schen Istrometer ist es, sowohl was die meehanische Eimrichtung als die Genanigkeit der Beobachtungen anbetrifft, weit iiberlegen.

Das von Steinheil erstrebte Endziel ist die directe Vergleichung: zweier beliebigen Sterne am Himmel, nnd das Charakteristisehe, was sein Instrument iiberhanpt von allen anderen Photometern unterseheidet, ist der zum ersten Male gemachte Versuch, die Sterne nicht im Bilde, sondern answerhall, desselben zu beobachten und die Punktvergleichung dureh die anerkanntermassen sicherere Fläehenvergleichnng zu ersetzen. Ein Naehtheil dieser Methode, welcher sofort in die Angen springt, ist der bedentende Lichtrerhust, und dieser Nachtheil ist wohl anch der haupt*iehlichste Grund, weshalb das Steinheil'sche Photometer trotz seiner grossen Vorziige keine weitere Verbreitung gefunden hat. Wollte man lasselbe fur die schwaicheren Sterne am IIimmel benutzen, so miisste man die Dimensionen so gross wïhlen, dass die Handhabung des Apparates ansserordentlich erschwert wire, und die Kosten seiner Herstellung ganz merschwinglich wiirden. Die Verwandlung der punktartigen Sterubilder in ansgedehntere Flaichen erreieht Steinheil durch Versehiebung des Ohjectivs gegen das feststehende Ocnlar. Seine Methode hat also eine gewisse P̈hnlichkeit mit dem früher (Scite 196) besprochenen zweiten Bonguersthen Plontometer, bei welchem die grösscren Öffinngen gegen dic feststehenden kleineren verschoben werden. Die Theorie des Steinheil'sehen Aplatrates ist mach den Gesetzen der geometrischen Optik ausserordentlich

1 Steinheil. Elemente der Ilelligkeitsmessungen am Sternenhimmel. Preisschritt. Denkschritten der K. Bayer. Nkad. d. Wiss Math-phys. Classe, Bd. II. Iiinchen $18 \% 6$. 
einfach. Wenn das Objectiv eines Fernrohrs sich in seiner normale॥ Stellung zum Oeulare befiudet, so wirl von einer unendlich entfernten punktformigen Liehtquelle auf der Netzhaut des Auges auch ein punktförmiges Bild entworfen. Wird aber das Objectiv dem Oculare gentiliert oder voln ihm entfernt, so fallen die Liehtstrahlen auf die vordere Fliielıe des Auges divergent oder convergent anf und verbreiten sich in beiden Fallen llber ein grösseres oder kleineres Stllek der Net\%haut, je nach der Grösse der Versehiebung des Objectirs. Aus den gewöhnliehen Formeln der Dioptrik folgt nun, wenn man Grössen von der Ordnung des Unterschiedes zwischen Tangente und Bogen vernachlissigt, dass das Flichenstluck, welches auf der Netzhaut von dem Lichtkegel ausgeschuitten wird, proportional sein muss der freien Objectivfliache einerseits und dem Quadrate der Verseliebung des Objectirs ans seiner normalen Stellung andererseits. Neunt man also die Grösse der belenchteten Fliiche der Netzhaut $F$, die benutzte freie Objectivfliche $O$ und die Verschiebung des Oljjeetivs $a$, so hat man:

$$
F=m O a^{2},
$$

wo $m$ eine Constante bedentet. Auf diese Fläehe $F$ vertheilt sich num die gesammte Lichtmenge, welche ron der freien Objectivöffinung aufgenommen wird, abgesehen natirlich von jedem durch Reflex, Absorption u. s. w. in dem ganzen Systeme herrorgebrachten Liehtrerlust. Ist $J$ die Lichtquantität, welehe ein Stern auf die Flächeneinheit des Objectirs sendet, so gelangt demnach die Quantitiit $J O$ anf die Fliiche $F$ der Netzhant, und die Flächeneinheit der Netzhaut empfängt daher die Lichtmenge $h=\frac{J}{m a^{2}} \cdot$ Es ist also die Flächenintensitait anf der Netzhant ganz nuabhängig von der Grösse der freien Öffnung. Für einen zweiten Stem. welcher auf die Fliicheneinheit des Objectivs die Lichtmenge $J^{\prime}$ sendet, wird bei einer Verschiebung $a^{\prime}$ des Objeetirs die Flichenintensitiit anf der Netzhaut $h^{\prime}=\frac{J^{\prime}}{m a^{\prime 2}}$. Beurtheilt das Auge die beiden Flächenintensitäten gleich, so ergiebt sich ummittelbar:

$$
J: J^{\prime}=a^{2}: a^{\prime 2},
$$

d. h. die Helligkeiten zweier Sterne verhalten sich wie die Quadrate der Grössen, um welche man das Objectir ans seiner nornalen Stellung rerschieben muss, damit die Fliiehenhelligkeiten auf der Netzhant gleich sind. Anf diesem Satze berulit das Steinheil'sche Photometer. Un die beiden Sternbilder gleichzeitig vor Augen zu haben, was fuir eine sichere Beurtheilung ihrer Gleichheit durchaus nothwendig ist, benutht Steinheil nicht, wie es spaiter Schwerd und De lat live gethan haben, getrennte 
Objeetive, sondern die beiden Hälften eines nnd desselben Objectivs, welches ebenso wie beim Heliometer in der Mitte durchgeschnitten ist. Die beiden Hälften sitzen in demselben Rohre und lassen sieh, jedes für sich, dem gemeinsehaftlichen Oculare messhar nähern oder von ihm ent-

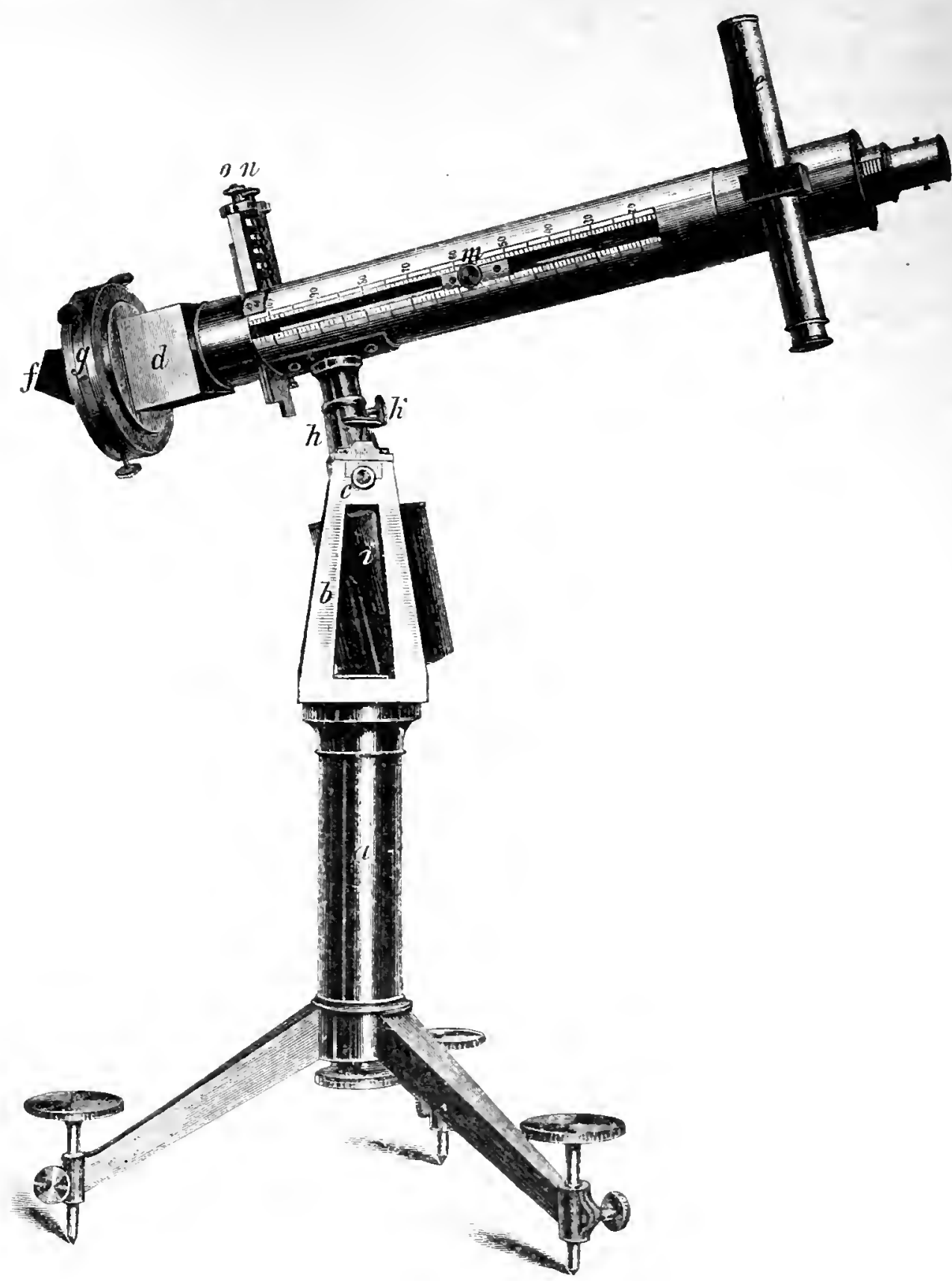

Fig. 46 .

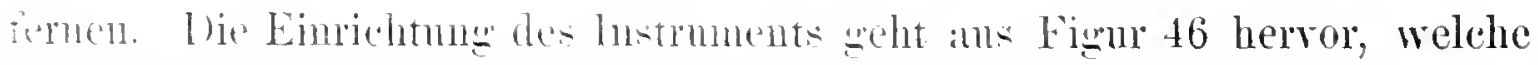
math einer photographischen Autnahme des anf der Niinchener Stemwarte hetindichen steinheilschen Orizinalphotnmeters ctwa in l. der natiorlichen

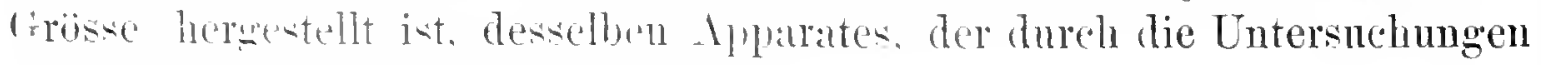

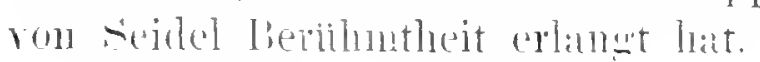


Die Säule $a$ des dreifiissigen Statirs enthïlt die verticale Drehungsaxe, mittels deren das Instrument im Azimuth beliebig bewegt werden kann. Durch den oberen 'Theil des gabelförmigen Stlickes $b$ geht die horizontale Axe c, um welche eine Bewegung in Höhe ansfuhrbar ist. Das Fermrohr hat einen wiirfelförmigen Ansatz $d$; dieser ist an der oberen Seite offen und enthïlt in Innern, in der einen Hälfte festsitzend, ein total reflectirendes rechtwinkliges Prisma, von welchem das durch die offene Seite des Wuirfels auffallende Licht auf das eine halbe Objeetiv reflectirt wird. Parallel mit der einen Seitenfliche dieses Prismas ist an dem Ocularende des Ferinrohrs ein kleiner Sucher $e$ angebracht. Wird derselbe dureh Drehung des Instrumentes in Azimuth und Höhe auf irgend einen Stern gerichtet, so erscheint derselbe anch durch Reflex an dem Prisma im Oculare des Hauptfernrohrs. Um nun anch einen zweiten Stern in das Gesichtsfeld zu bringen, ist vor dem Würel $d$ ein zweites total reflectirendes Prisma $f$ drehbar angebracht, welches durch die freie Hälfte des Wiirfels hindurch Licht auf die zweite Objectivhïlfte sendet. Die Drehnng dieses Prismas kann an dem getheilten Kreise g alogelesen werden, und man hat die Einstellung Null, wenn die siimmtlichen Seiten der beiden Prismen einander paarweise parallel sind. Das Fermrohr ist endlieb noch um die Axe ", welche das Gegengewicht $i$ trägt, drehbar und kann mittels der Schraube $k$ in jeder Lage festgeklemmt werden. Bei der Drehung um diese Axe bleibt der Sucher $e$ und das im Wiirfel festsitzende Prisma unverïnderlich auf denselben Punkt des Himmels gerichtet. Nachdem der eine Stem mit Hiilfe des Suchers in das Oeular gebracht ist, wird an dem Kreise $g$ der vorher berechnete Winkelalsstand der beiden Sterne eingestellt und dam das Fermrohr un die Axe $h$ so lange bewegt, bis der zweite Stern in Gesichtsfelde erscheint. Un dir beiden Sterne wiihrend der Daner der Messung im Gesichtsfelde zu halten, muss man um alle drei mechanischen Axen des Instruments Bewegrungen ausfiihren, was die Handhabung des Apparates betriachtlich erschwert. Dann werden die beiden Objectivhälften, von denen jede mittelst eines Knopfes $m$ in einem Schlitze längs des Rohres verschoben werden kann, so lange bewegt, bis die beiden Fliichenhelligkeiten gleich erscheinen; die Stellung der Objectiväilften wird an Scalen, die auf dem Rohre angebracht sind, abgelesen. Die Lichtflichen, in welche die Bilder der Fixsterne verwandelt werden, haben natiurlich die Form des erleuchteten Objectivtheiles und erscheinen daher ohne Abblendung als Halbkreise. Um aber die Grösse dieser Lichtscheiben beliebig reräindern zu können, was unter Umständen erwiinscht sein kam, ist noch hinter dem Würfel eine Vorrichtuug angebracht zur. Verkleinerung der Objectivhailften. Dieselbe besteht für jede Hälfte aus zwei durch die Schraluben " und o mit Links- 
und Rechts-Gewinde gleichzeitig gegeneinander verschiebbaren Metallplatten, die stets ein gleichseitiges rechtwinkliges Dreieck offen lassen. Die Hypotenuscn der beiden Dreiecke stossen genai aneinander, und wenn die Flächen gleich gross gemacht, ausserdem die beiden Lichtflïchen genan gleich hell sind, so erscheint das Gesichtsfeld als gleichmaissig helles Quadrat, in welchem die Trennungslinie der beiden Hälften gänzlich verschwunden ist. Auf die Messung der Flächenhelligkeit darf nach dem Obigen die Grösse der Objectivöffunng theoretisch keinen Einfluss haben, und anch praktisch ist es nach den Versicherungen Seidel's, ler sich am Eifrigsten mit diesem Instrumente beschäftigt hat, ohne Einfluss anf das Messungsresultat, ob die beiden Dreiccke dieselbe Grösse laben oder wesentlich von einander verschieden sind. Um die Grösse der Verschiebung der Objectivhälften genau angeben zn können, müsste man noch diejenige Ablesung der Scalen wissen, bei welcher die Brennpunkte von Objectiv und Ocular zusammenfallen. Anstatt diese $\mathrm{Ab}$ lesung durch den Versuch direct zi ermitteln, verführt man besser so, dass man immer zwei Vergleichungen nach einander ansfülırt, indem man die beiden Objectirhälften von der normalen Stellung aus einmal in der Richtmng mach dem Oculare hin, das andere Mal von ihm hinweg verschiebt und in beiden Fïllen die Helligkeitsgleichlheit herstellt. Sind die zngehörigen Ablesungen der einen Scala $m_{1}$ resp. $n_{1}$, die der zweiten $m_{a}$ resp. $n_{2}$, sind ferner $f_{1}$ und $f_{2}$ die Scalenalblesungen bei normaler Focusstellnng der beiden Objectivhälften, so ist das Helligkeitsverluältniss $P$ der vergliehenen Sterne ausgedriickt durch die Gleichungen:

$$
\begin{aligned}
& P=\left(f_{1}-m_{1}\right)^{2}, \\
& \left(f_{2}-m_{2}\right)^{2}, \\
& P=\left(n_{1}-f_{1}\right)^{2}, \\
& \left(n_{2}-f_{2}\right)^{2},
\end{aligned}
$$

ans denen munittelbar folgrt:

$$
P=\left(n_{1}-m_{1}\right)^{2} .
$$

Kleine Lnterschiede in der Reflexionsfihigkeit der Prismen, sowie in rer Absorption der heiden Objectivluälften, welehe einen sehädlichen Einthus anf die Beobachtnngen ansiiben kiinnten, lassen sieh eliminiren. wom man bei jeder Messma die Sterne abwechselnd in heiden Objectirhiilftem cinstellt. Man kamn aher anch an jedem Beobachtungsabende

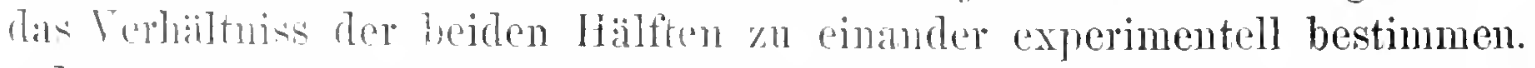

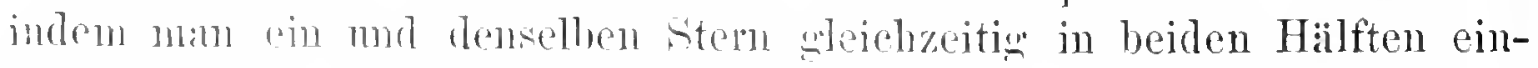
stellt mol ilm also mit sich sulbst reroleicht. Dass die verschiedene 
Helligkeit des Himmelsgrundes, auf welchen sich die Sterne projiciren, bei dem Steinheil'schen Photometer gar nieht in Betracht kommt, geht daraus hervor, dass sieh die beiden Helligkeiten, da es sich ja eigentlich un ein einziges Fernrohr handelt, zu einer mittleren Helligkeit in dem gemeinsamen Gesichtsfelde veruiselıen.

Die Genauigkeit der Messungen, welehe mit dem Steinheil'sehen Instrumente erreielit werden kann, ist sehr befriedigend. Seidel hat fur den wahrseheinlichen Fehler einer Helligkeitsvergleiehung zwischen zwei Sternen den durehsehnittlichen Werth \pm 0.024 in Logarithmus, also \pm 0.06 in Grössenclassen, gefunden und glaubt, dass unter besonders glinstigen äusseren Umständen ein noch grösserer Genauigkeitsgrad erreieht werden kann. Es ist schon oben der starke Liehtverlust als der empfindlielıste Nachtheil des Steinheil'schen Photometers bezeiehnet worden, und in der That hat Seidel bei den freilieh nur geringen Dimensionen des Apparates (das Objectir besass eine Ölfnumg rón $35 \mathrm{~mm}$ ) seine Beobachtungen kaum bis zu Sternen der fuinften Grösse ansdehnen können. Um diesem Nachtheil abzuhelfen und seine photometrisehe Methode auch auf sehwächere Sterne anwendbar zu maehen, hat Steinheil später die Construction eines Oeularphotometers') vorgesehlagen, welehes mit jedem beliebigen Refractor in Verbindung gebraelit werden kann. Dasselbe besteht in Wesentlichen aus einem um die optische Axe des Hauptfernrohrs drehbaren Rohre, in welehem ein kleines. Hülfsobjectiv mit davor sitzendem totalreflectirenden Prisma angebracht ist. Dureh dieses Hülfsobjectiv wird das Lieht eines hellen Sternes in das gemeinsame Ocular geworfen, während das Hauptfernrohr nacheinander auf die zu rergleiehenden sehwächeren Sterne geriehtet wird. Durch Verschiehung des Oculars werden diese Sterne in Lichtseheiben rerwandelt und mit dem durch Verschieben des Itiulfsoljectirs ebenfalls in eine Lichttaiche verwandelten hellen Sterne verglichen. Da dieser Apparat meines Wissens niemals zu Messungen verwerthet worden ist, so soll hier nicht näber auf denselhen eingegangen werden, ebensowenig wie ant ein zweites von Steinheil empfohlenes Ocularphotometer, bei welchem die Steme nieht als Liehtscheiben, sondern als Lichtpunkte beobachtet werden.

Dagegen verdient noch ein auf der Wiener Sternwarte befindliches Prismenphotometer Erwähnung, bei welchem eine wesentliche Vereinfachung in der llandhabung dadurch erzielt worden ist, dass dasselbe parallaktisch montirt ist. Das Hallutrohr (Fig. 47, Scite 210) ist auf einem soliden Statir in der Meridianebene nach dem Pol geriehtet und lässt sich $1 \mathrm{~m}$ seine eigene optische Axe drehen.

1) Astron. Nachr. Bd. 45, Nr. 1152. 
Das Licht der Sterne fällt nicht direct auf die vor den Objectivhälften sitzenden Prismen, sondern erst wach Reflexion von Spiegeln,

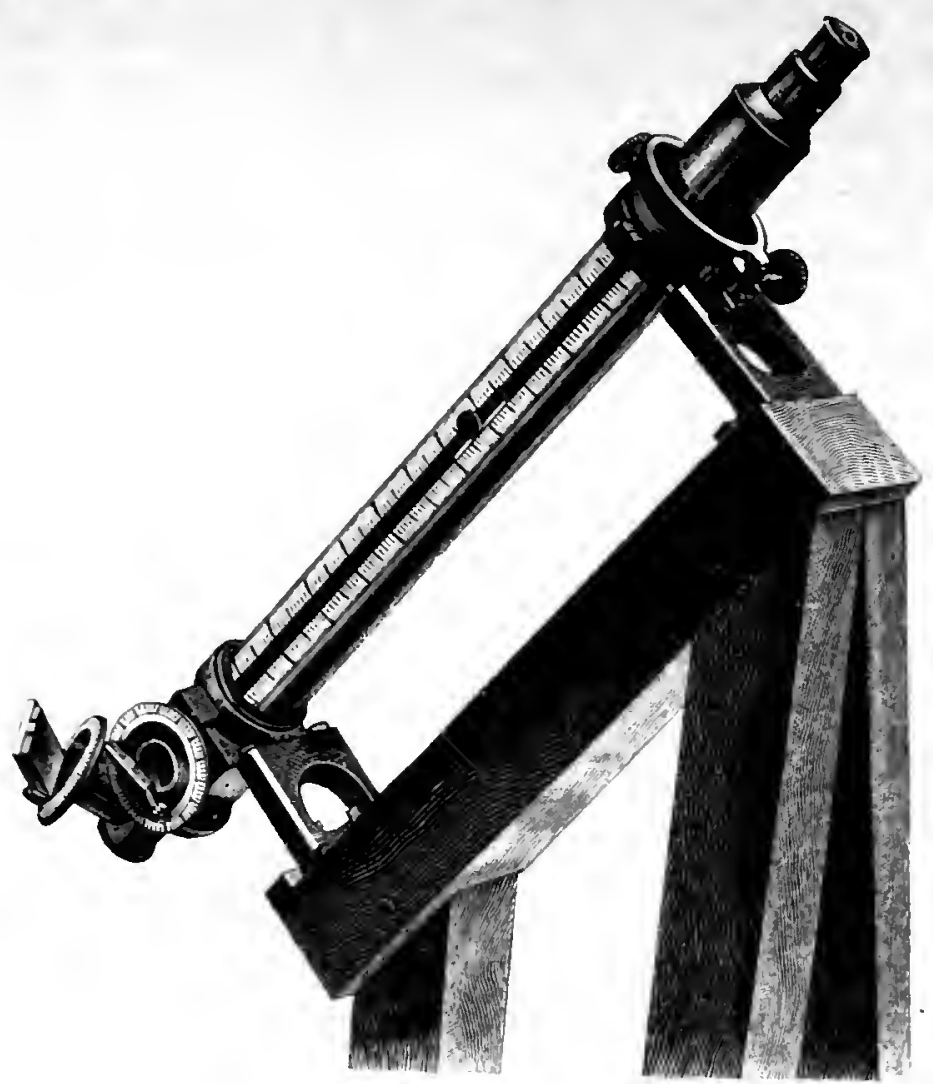

Fig. 47. die in der aus der Figur ersichtlichen Weise mit drehbaren Kreistheilungen verbunden sind. Der Vortheil der Einrichtung besteht darin, dass nicht erst vor jederBeobachtung derWinkelabstand der zu vergleichenden Sterne berechnet zu werden braueht, sondern dass unmittelbar die Stundenwinkel und Declinationen bei den Einstellungen benutzt werden, und dass ferner, wenn die beiden Sterne einmal in das Gesichtsfeld gebracht sind, sie allein dureh die Feinbewegung des Hauptrohres um seine Axe darin gehalten werden könneu. Der einzige Nachtheil des Arrangements ist der Umstand, dass der ohmehin schon grosse Lichtverlust noeh durch die Znriekwerfung an den Spiegeln gesteigert wird.

\section{‥ Anwendung ron 0hjectivblenden.}

Alles was bei den Anslöschnngsphotometern ïber die Abblendungsmethode gesagt worden ist, triffit anch bei den hier zu bespreehenden Apparaten in vollem Linfunge zu. [nsbesondere ist es die Beugungswirkung, welche sich hier vielleicht noch störender fiihlbar macht und ron vormherein nu eine beschriukte Anwendung der Methode rathsam exscheinen lisst.

\section{a. Die Methoden ron Bonguer und W. Herschel.}

Als :iltertes Instrument dieser (iattung darf wohl ein ron Boug ner') vielfarle benutytes bereichnet werden. Dasselbe besteht ans zwei Ob- 
jectiven von vollkommen gleicher Öffnung und Brennweite. Die Rühren, an deren einem Ende sich dieso Objeetire befinden, haben genau die Juinge der Brennweite und sind an anderen Ende mit Deekeln versellossen, in denen kleine kreisrunde Öffinungen von $7 \mathrm{~mm}$ bis $9 \mathrm{~mm}$ Durehmesser angebracht sind, bedeckt nnit feinem weissen Papier oder mit mattgeschliffenem Glase. Die beiden Objective werden anf die zu vergleiehenden Liehtquellen gerichtet und die Öffnung des einen dureh Sectorblenden so woit verringert, bis die kleinen in der Brennebene befindlichen Löeher fur das Ange gleich hell belenehtet erscheinen. Die Helligkeiten der beiden Lichtquellen verhalten sieh damn wie die freien Objectivöffnungen. Un etwaige kleine Untersehiede in der Beschaffenheit der beiden Objective unschädlich zu machen, kann man dieselben bei jeder Beobachtung mit einander vertausehen. Dureh eine geeignete Sehntworriehtung wird noch Sorge getragen, dass alles iussere Licht von dem Auge des Beobachters fern bleibt. Die Sicherheit der Beobachtungen mit diesem Instrumente ist ron vornherein dadureh etwas eingeschränkt, dass die belenehteten Fläehen nicht unmittelbar aneinander grenzen. Auch ist es klar, dass der Apparat nur zur Vergleichung von leuchtenden Flïchen, nicht vou Liehtpunkten verwendet werden kann. Bonguer hat damit die Helligkeit des Himmels an versehiedenen Stellen gemessen und Helligkeitsvergleichungen einzeher Partien der Sonnenscheibe angestellt.

Zur Vergleichung von Sternen hat W. Hersehel1) ein Verfahren vorgesehlagen, welehes dem Bouguer'sehen ïhnlich ist. Er benutzt zwei unmittelbar nebeneinander anfgestellte Fernrohre von gleicher Öffinung und Focallänge. Mit dem einen betrachtet er das Bild des einen der zu vergleichenden Sterne, mit dem zweiten unmittelbar darauf das des anderen and sehwiicht das hellere Bild dureh Abblenden des betreffenden Objectivs, bis ihm die Bilder in den beiden Fernrohren gleich intensiv erseheinen. Dureh Umwechseln der Instrumente lisst sich anch hier jeder durch Verschiedenheit der Objective hervorgerufene Fehler eliminiren. Wie man ubrigens sofort sieht, steht das Hersehel'sche Verfahren dem Bouguer'sehen entschieden nach, denn die Betrachtung der Bilder geschieht hier nicht gleichzeitig, sondern nacheinander, und wenn anch die Zeit, die man braueht, um von dem einen Instrument anf das andere iiberzugehen, noch so kurz ist, so vermag das Auge doch nieht die Erinnermng an den empfangenen Lichteindruck mit vollkommener Sicherheit festzuhalten. Herschel maeht bei der Besehreibung seines photometrischen Verfahrens schon selbst anf die stiorenden Einfliisse der Diffractionserscheinmugen und der Helligkeit des Himmelsgrrundes anfmerksam.

1) Philos. Trans. of the R. Soc. of London. 1517. p. 302. 
b. Dic Benutzung des Spiegelsextanten und des Heliometers als Photometer.

Von verschiedenen Seiten, unter Anderen auch ron A. v: Humboldt, ist der Gedanke angeregt worden, den Spiegelsextanten zu Helligkeitsvergleichungen am Himmel zu verwenden. Die gewöhnliche Form des Sextauten ist für diesen Zweek dahin abzuändern, dass das Fernrohr nieht fest auf die Mitte des zur Hälfte belegten, zur Hälfte unbelegten Spiegels gerichtet bleibt, sondern nach Belieben um messbare Beträge gehoben oder gesenkt werden kann. Durch den unbelegten Theil des Spiegels blickt man direct nach dem einen Sterne, wälnrend man das Bild des zweiten damit zu vergleichenden Sternes durch Reflex von dem drehbaren Spiegel des Sextauten und ron der belegten Hälfte des anderen Spiegels in das Gesichtsfeld gelangen liisst. Durch Heben oder Senken des Fermohrs wird die Helligkeitsgleichheit der Bilder hergestellt, und das Verhältniss der beiden Abschnitte des Objectirs, welche auf den belegten und unbelegten 'Theil des Spiegels gerichtet sind, giebt ein Mass für das Helligkeitsverhiltuiss der miteinander verglichenen Sterne. Natiirlich muss der durch die zweimalige Spiegelung verursachte Lichtrerlist experimentell bestimmt werden, was an Besten dadureh gesehieht, dass man das direete und das reflectirte Bild eines und desselben Sternes miteinander rergleicht.

Dic Verwendung des Sextanten zu photometrischen Messungen am Himmel unnss wegen der verhältnissmässig kleinen Dimensionen des Instrumentes und wegen der Schwierigkeit, zwei beliebige Objecte in das Gesichtsfeld des Fermrohrs zu bringen und darin wihrend der Vergleichnngen festzuhalten, anf die helleren Steme beschränkt bleiben. Anch diirtte der Tomstand, dass das Licht der reflectirt gesehenen Sterne mnter versehiedenen Incidenzwinkeln auf den ersten Spiegel anffällt, leicht zu Fehlem Anlass meben.

Handelt es sich nur un die Tergleichung nahe bei einander befindlicher Himmelsobjecte, so kamm mit Fortleil anstatt des Spiegelsextanten anch ein anderer zu Winkelmessungen am Ilimmel bestimmter Apparat, das Heliometer. benntzt werden. Bekanntlich riihrt die Bezeichnung - Helinmeter rom Bongerer her. Weleher dieses Instrument in der Form constrnirte, dass el zwei onjective mmittelbar nebeneinander in ein Rohr einsetzte und ein einziges Goular tir beide zur Anwendung brachte. Von ihm ist anch zum arsten Malie der Turschlag gemaeht worden, ein solches Tnstrument zu photometrischen Zwecken zn grebranchen, indem das eine der beiden Ohjective durch bilenden soweit rerkleinert wurde, bis die heiden sterne goledeh hell erwhiencu. In der Form, in weleher das Ileliometer hentzutane "onstruirt wird. mit einem einzigen in der 
Mitte durchschnittenen Objectiv, ist dasselbe von John s on ' i sehr angelegentlich \%u plotometrischen Beobachtungen empfohlen worlen. Johnso n fand bei der Untersuchung seines Heliometers, dass die eine Objectivhälfte ein helleres Bild gab als die andere (Helligkeitsverhältniss .100 zи 95.5), und dass bei beiden Hailften die Centralpartien verhältnissmässig durehsichtiger waren als die Randpartien. Dem ersteren Fehler liess sich bei den photometrischen Beobachtungen sehr leicht durch Vertanschen der Objectivhïlften abhelfen. Sicht man von allen Übelstïnden ab, die beim Gebranche jeder Blendvorrichtung ins Spiel kommen, und vermeidet man vor allen Dingen eine allzu starke Verkleinerung der eiuen Objectivhïlfte, so eignet sich das Heliometer ohne Zweifel sehr gut zu Helligkeitsmessungen an Doppelsternen, sowie zur Vergleichung von Veriuderlichen mit nahı dabei stehenden Stermen. Schur ${ }^{2}$ ) hat dasselbe gelegentlich einer Conjunction von Venus und Mereur auch zu Messungen der relativen Liehtstärke dieser beiden Planeten mit Erfolg verwendet.

\section{c. Das Sehwerd'sehe Photometer.}

Dieses Instrument dürfte wohl das complicirteste sein, welches jemals zu Helligkeitsmessungen am Himmel construirt worden ist, es hat daher anch trotz mancher interessanten Einrichtungen so wenig Verbreitung. gefunden, dass es heute fast ganz in Vergessenheit gerathen ist. Die Litteratur über dieses Photometer ist iiusserst spärlich. Schwerd selbst hat seine Beobachtungen mit diesem Instrumente niemals veröffentlicht: und ausser einer Beschreibung von Argelander ${ }^{3}$, der sieh sehr lebhaft für den Apparat interessirte, ist mir nur eine kleine Abhandlung von F. Berg $\mathrm{g}^{\dagger}$ ) in Wilna bekannt geworden, welcher Extinctionsbestimnungen

1) Astron. Observ. made at the Radeliffe Observatory, Oxford, in the year 1551. Vol XII, Appendix I. (Siehe ausserdem Monthly Notices. Vol. 13, p. 278.

2) Astron. Nachr. Bd. 94, Nr. 2245.

3) Sitzber. des naturhistorisehen Vereins der preuss. Rheinlande und Westphalens. Neue Folge, Jahrg. 6. 1859, p. 64, Bonn. 'Siehe aueh He is, Wochensehrift. Jahrg. 18.59 , p. 275.

4) F. Berg, Über das Schwerd'sche Photometer und die Liehtextinetion fiir den Wilnaer Horizont. Wilna 1870. - NB. Diese kleine in russischer Sprache gedruckte Schrift befindet sich in der Bibliothek der Sternwarte Pulkowa. Herr E. I, in de mann in Pulkowa hat die Guite gehabt. mir eine Übersetzung des auf das Instrument selbst beziiglichen 'Theiles dieser Abhandlung zuzusenden, der ich zum grössten Theil die obige Beschreibung entnommen habe. Herrn Lindemann verdanke ich auch die Mittheilung, dass von dem Schwerd'schen Photometer überhaupt nur vier Exemplare angefertigt worden sind, von denen zwei sich in liussland, auf den Sternwarten zu Pnlkowa und Wilna. befinden, eins im Besitze der Familie Schwerd geblieben ist, wilhrend das vierte aut der Sternwarte Bonn in einer besonderen Kuppel anfgestellt ist. Der Abbildung (Fig. 45, liegt eine photographisehe Aufnahme des letzteren Instrumentes zu Grunde. 
mit einem solchen Instrumente ansgefuihrt hat. Das Photometer (Fig. 48) besteht aus zwei Fernrohren. Das grössere ist parallaktisch aufgestellt und durch ein Uhrwerk beweglich, während das kleinere um zwei zu einander senkrechte Axen drehbar ist, von denen die eine zur optisehen Axe des grossen Ferurolirs senkreeht steht. Die Drehungen sind an zwei Kreisen ablesbar; $a a$ sind die Klemmen für den einen, $b b$ die für den inderen Kreis, $c c$ und $d d$ die entspreehenden Feinbewegungsschrauben. Ist das grosse Fermrohr auf einen Stern gerichtet, so kann man das kleinere dureh Einstellung an diesen Kreisen auf irgend eimen anderen Stern richten, weun der Abstand desselben ron dem ersteren, sowie der Positionswinkel in Bezng anf ihn bekannt sind. Zur bequemeren Einstellung des kleineren Fermrohrs dient noch ein damit fest rerbundenes Hiilfsfernröhrchen $e$.

In den beiden würfelförmigen Ansatzstiicken $f$ und $g$ sind totalreflectirende Prisinen augebracht, welche das Licht der beiden Sterne in ein gemeinschaftliches Ocular $h$ werfen. Man sieht die Sterne im Gesichtsfelde nahe bei einander, jeden aber auf den ihm zugehörigen Himmelsgrumd projicirt. Um num dem schädlichen Einflusse der verschiedenen Helligkeit des Grundes zu begegnen, werden beide Fermrohre dureh Limpenlicht erlenchtet, und diese Belenchtung laisst sich nach Belieben so moderiren, dass die beiden Hiilften des Gesichtsfeldes gleich hell erscheinen.

Die Dimensionen des Schwerd'schen Photometers sind so gewählt, dass das Verhialtniss ron Objectirdurehmesser zur Focaldistanz in beiden Fermrohren gleich ist, und zwar hat das grosse Objectir einen Durelimesser ron $5.2 \mathrm{~cm}$ und eine Brennweite ron $126 \mathrm{~cm}$, das kleine einen I) rumesser ron $2.6 \mathrm{~cm}$ und eine Brennteite ron $63 \mathrm{~cm}$. Infolge dessen erscheint ein Stern in dem kleinen Fernrohr viermal schwiicher als in dem grossen. Vor dem Objectir des ersteren ist excentrisch eine Scheibe mit rerschieden grossen kreisrunden öftinungen angebracht, die durch Irehmo der scheibe nacineinander ror die Xitte des Objectirs geführt werden kïnnen. Sind die Fermrohre auf zwei Sterne geriehtet, so wird das Lirht des helleren (im kleinen Ferurohr (ingestellten) dureh Drehung Tieser Scheibe so weit abgeschwaicht, bis die Bilder im Oeular gleiche Helligkeit besitzen. Wem die urspringliche Helligkeit des einen der heiden zu rergleiehenden sterne die des anderen

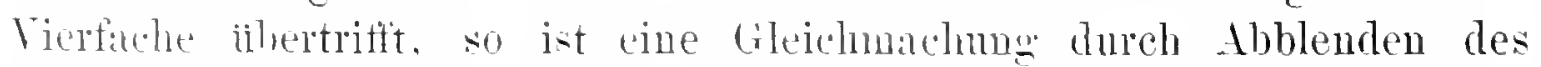
kleinem Ohjectirs nicht mögheh. und es sind daher anch fü das grosse

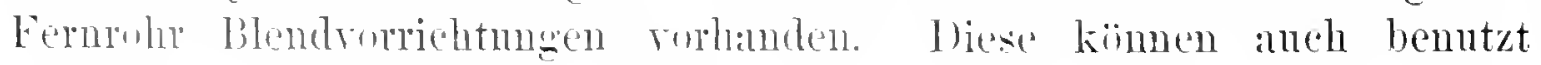
werden, un hei Einstellung beider Furnrohe anf einen und denselben stern die Giteinheit der Bilder herznstellen und anf diese Teise das 


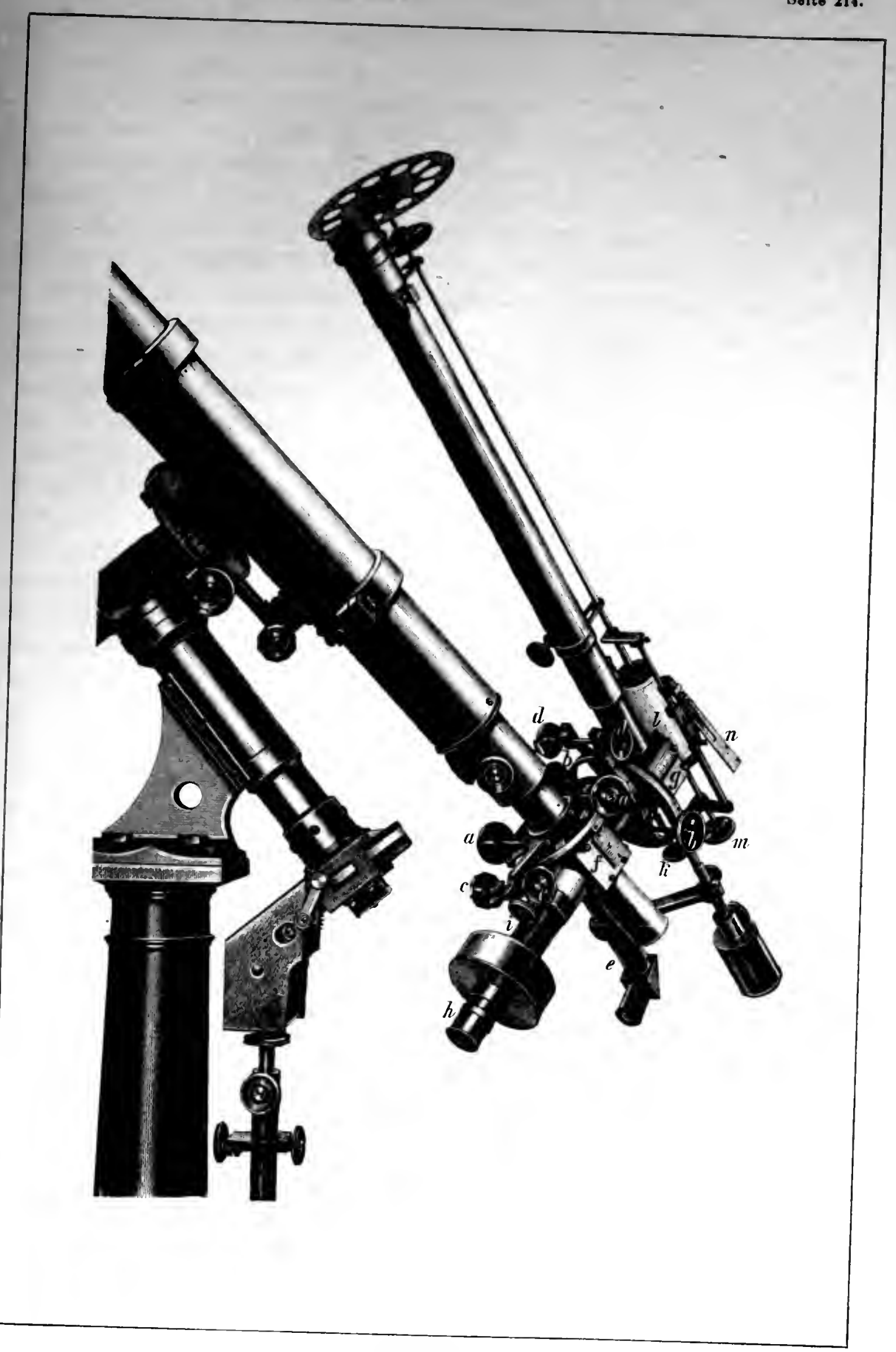



Verhältniss der beiden Objective zu einander experimentell genau zu lestimmen.

Sehwerd hat den wiehtigen Einfluss, welehen die Beugung des Liehtes boi Beuntzung von Blenden liat, richtig erkannt und infolge dessen Einrichtungen getrotien, un stets den beiden Bengungsbilderì die gleiche Grösse geben жu können. Dadurch ist allerdings jeder theoretische Einwand gegen das Prineip seiner Methode gehoben, aber zugleich auch die praktische Handhabung des Apparates wesentlieh ersehwert. Wie bereits im vorangehenden Capitel auseinandergesetyt wurde, nimmt die Beugungsfigur eines Sternes bei zwei versehiedenen Fermrolıren nur dann den gleichen Raum anf der Netzhaut des Auges ein, wenn die angewandten Gesammtrergrössernngen den Objeetivdurehmessern proportional sind, und unr in diesem Falle geben dic freien Öffunngen ein streng richtiges Mass fur das Helligkeitsverhïltniss zweier Sterne. Da bei dem Schwerdschen Plotometer fur beide Fernrohre ein gemeinschaftliches Ocular benutzt wird, so ist also Bedingung fur eine theoretisch einwurfsfreie Benutzung des Instrumentes, dass das Verhältniss von Objectivöftinung und Brennweite in beiden Fernrohren stets dasselle bleibt. Bei nicht abgeblendeten Objectiven ist diese Bedingurig durch die gewählten Dimensionen ron rornherein erfiullt, wenn aber das eine Objectiv abgeblendet wird, muss gleichzeitig auch eine Verkitrung der Brennweite desselben stattfinden. Um dies bewerkstelligen zu kümnen, hat Schwerd in beiden Fernrohren zwischen Objectiv und Brenupunkt Sammellinsen eingesetzt, die längs der optisehen Axen verschiebbar sind. Bei dem grossen Ferurohre sitzt die Sammellinse vor dem totalreflectirenden Prisma in dem langen Theile des gebrochenen Rolures, bei dem kleinen dagegen hinter dem Prisma in dem kurzen Theile. Die Schranben $i$ und $k$ dienen zur Verschiebung dieser Linsen, und der Betrag der Verschiebung liisst sich an den beiden auf den wiirfelförnigen Stiicken $f$ nud $g$ angebrachten Scalen ablesen. Sind die beiden Sammellinsen auf den Nullpuukt der Scala eingestellt, dann verhalten sich die Brennweiten der Ges:mmntsysteme »Objectir und Sammellinse zo einander, wie die nicht abgeblendeten Objectivöffinungen. Die Theilung anf der Scala steht in Berichung zu den benutaten mit Nummern versehenen Blendenöfunugen, so diass beispielsweise bei Benutzung der Blendenmummer 10 die 'iummellinse auf den 'Theilstrich 10 eingestellt werden muss, damit freie Öffinumg und Brennweite wieder das bestimmte Verlualtniss zu einander haben. Natiirlich muss anch noch das Gesammtsystem „Objectir-Sammellinse" znsammen verschiebbar sein, wemn das Ocular $h$ unverindert an seiner stelle bleiben soll.

- Die Vergleichung zweier Sterne mit dem Schwerd'schen Photometer erfordert nach dem Gesagten die folgenden Manipulationen. Der schwächere 
Stern wird mit Hülfe des Declinations- und Stundenkreises in dem grossen Fermrohre, der hellere, dessen Distanz und Positionswinkel in Bezug auf ersteren rorher berechnet sein missen, mit Hiilfe der beiden anderen Kreise in dem kleineren Fernrohre eingestellt. Durch das Uhrwerk werden beide Sterne im Gesichtsfelde fest gehalten. Dann wird das kleine Objeetiv so weit abgeblendet, bis die Bilder ungefïhr gleich hell erscheinen, die Sammellinse wird auf den Theilstrich, welcher der betreffenden Blendenoffhimng entspricht, eingestellt und das ganze System »Objectiv-Sammellinse. so weit versehoben, bis der Stern im Oculare wieder scharf erscheint. Die beiden Hälften des Gesichtsfeldes werden sodann durch Moderirung der Beleuchtung gleich hell gemacht, und die letzte feine Einstellung anf gleiche Intensität der beiden Sternbilder wird endlich durch Drehen der Blendscheibe bewirkt. Die Nummer der richtigen Blendenöfthumg griebt dann mit Hiulfe einer für jedes Instrument berechneten Tabelle unmittelbar den Helligkeitsunterschied der beiden Sterne. Bei dem von Bergi in Wilna benutzten Instrumente waren die 25 versehiedenen Blendenöffnungen, welche zu Gebote standen, so abgestuft, dass jede folgende Öffnung immer um 0.1 Grössenclassen weniger Licht hindureh liess als die rorangehende. Da $11 n$ der Helligkeitsunterschied der beiden mabgeblendeten Objective etwa 1.5 Grössenelassen betrug, so konnten mit diesem Apparate Sterne bis zu vier Grössenclassen Helligkeitsdifferenz gemessen werden. Es ist wegen des Aussehens der Bilder nicht rathsam, das Objectir mehr als bis auf etwa $\frac{1}{3}$ der Öffnung abzublenden; will man daher noch grüssere Unterschiede als vier Grössenclassen direct messen, so muss das kleine Fernrohr durch ein anderes ron noch geringeren Dimensionen ersetzt werden. Mit dem Apparate ist endlich noch eine Art Registrirrorichtung rerbunden, um die jedesmalige ror dem Objective befindliche Öffmung zu notiren. Diese Vorrichtung besteht im Wesentlichen ans einer mit Palpier iiberzogenen Trommel orler Walze l, welche anf der langen bis zur Blendscheibe reichenden Bewegungsstange fest anfgesteckt ist und mittels des Handgritfes $m$ zngleich mit der Blendseheibe geedreht wird. Durch einen Druck anf den Hebel $n$ wird auf der Walze ein Zeichen markirt mo dadurch die Stellung der Blendseheibe registrirt.

Die Handhabung des schwerd sehen l'hotometers ist, wie man aus dem Vorangehenden sieht, viel zu umstiundlich, als dass an eine Verwendung des Instrumentes zu prösteren Beohachtungsreihen zu denken wäre. Sofern das Photometer nicht im Freien Aufstelhng tindet, ist atsserdem noch eine besondere Einnichtun der Kuppel erforderlich, die beiden Fermrohre gleichzeitig nach zwei beliebigen l'nnkten des Himmels richten zu kïnnen. Das drehbatre Dach der kleinen Kuppel, in 
weleher das Bouner Instrument aufgestellt ist, besteht aus einer grossen Anzahl von Klappen, die naeh Bedurfniss einzelı geöffinet werden küuneu.

Eine grosse $̈$ Äulichkeit mit dem Sehwerd'schen Photometer besitzt ein von De la Rivol) eonstruirtes Instrument, welehes hier noch kur\% Erwilhnung finden mag, obgleich es ursprilnglich nicht zu Beobachtungen am Himmel bestimmt war. Es besteht ebenfalls aus zwei Fernrohren, die sich gleichzeitig auf zwei beliebige Punkte richten lassen. Durch ein System von Spiegeln oder totalreflectirenden Prismen werden die Lichtstrahlen in ein gemeinschaftliches Oenlar geworfen, und die gleiche Helligkeit der Bilder wird durch Abblenden der Objective erreieht. Als eine Verbesserung des Schwerd'schen Photometers kann das De la Rivesche nicht betrachtet werden, schon darum nicht, weil der schädliche Einfluss der Beugungswirkung dabei gar keine Berlicksiehtigung findet.

\section{d. Das Hornstein'sehe Zonenphotometer.}

Wie schon aus der Bezeichunng dieses Photometers hervorgeht, ist dasselbe speciell zu Zonenbeohachtungen, d. h. zur Messung von ganzen Gruppen uahe bei einander stehender, an Helligkeit nicht allzu verschiedener Sterne bestimmt. Das Prineip ist Abblenden des Objectirs und Vergleichung der Bilder mit dem Bilde eines Huilfssternes, welcher durch einen Theil des Objectivs in das Gesichtsfeld gebracht wird. Die Einriehtung greht aus der von Hornstein ${ }^{2}$ ) selbst gegebenen Abbildung (Fig. 49) hervor.

Auf die Fassung des Objectivs $O$ ist ein Ring $B B$ anfgesetzt, der sich rom Oeular ans mittelsteines Schliissels um die optische Axe des Fernrohrs drehen lässt. Mit dem lingge ist

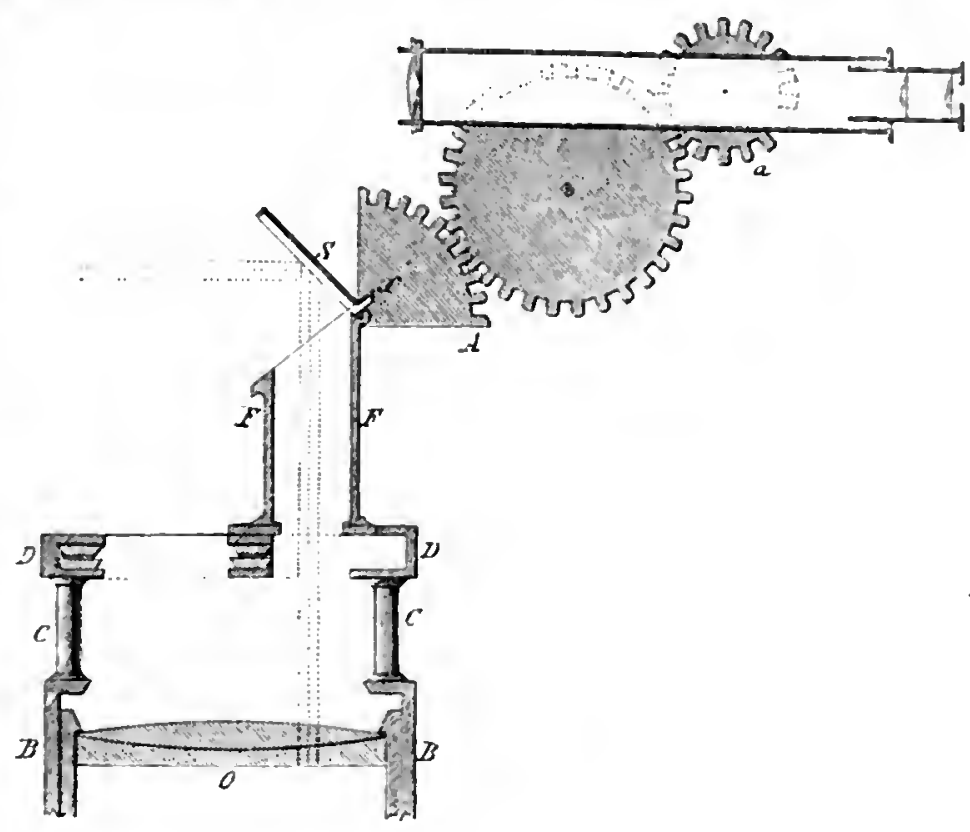

Fig. 49. durch die Sainlchen $C O$ ein Rahmen $D D$ verbunden, in welchem zwei Blendschieber zur Abblendung eines Theiles des Objectiss iibereinander verschoben werden kimmen. Anf dem liahmen sitzt ferner noch eine

1) Annales de chim. et de phys. Série 4, tome 12 1567. p. 24:3.

2 Sitzber. der K. Akxd. der Wiss zu Wien. Math.-naturw. Classe, Bd. 41, p. 2 il. 
eylindrische Röhre $F F$, an deren oberem Ende bei $x$ ein kleiner Spiegel $S$, drehbar um eine zur Zeichnungsebene senkrechte Axe, angebracht ist. Mit Hülfe dieses Spiegels, der ebenfalls vom Oéular aus dirigirt werden kann, wird nun das Bild eines Hülfssternes in das Fernrohr reflectirt, und die direct gesehenen Sterne werden durch Verschieben der Blenden so weit geschwäeht, bis sie dem Hülfssterne an Helligkeit gleich sind. Man sieht sofort, dass der Anwendungsbereich des Photometers zur Vergleichung zweier beliebigen Sterne am Himmel an gewisse Grenzen gebunden ist. Der Spiegel darf nicht so gross sein, dass er bei irgend einer Stellung etwas von dem fiir die directe Beobachtnng der Sterne bestimmten Theil des Objectivs rerdeckt, und er darf nur unter solchen Neigungswinkeln gegen die optische Axe des Fermrohrs benutzt werden, dass immer der ganze Querschnitt der cylindrischen Röhre $F F$ Licht erhält.

Um das Bild des Hiilfssternes bequem und sicher in das Hauptrolı zu bringen, wird ein kleines Hiilfsfermrohr benutzt, welches in folgender Weise mit dem Apparate verbunden ist. Mit dem Spiegel $S$ gemeinsehaftlich um Punkt $x$ drehbar ist ein gezahnter Sector $A$, dessen Drehung mit Hülfe eines gezahnten Zwischenrades anf das Rad $a$ iibertragen wird, an welchem letzteren das kleine Fermrohr befestigt ist. Da der Durehmesser ron a gerade halb so gross ist wie der von $A$, während die Breite der Zähne bei allen drei Rädern iibereinstimmt, so entspricht einer beliebigen Bewegung des Spiegels $S$ eine doppelt so grosse Bewegung des Hillfsfermrohrs. Nan probirt num an einem hellen Sterne ans, in welcher Lage das Hiilfsfermrohr mit dem Rade a zu befestigen ist, damit der Stern grleichzeitig in diesem und, durch Reflex an dem Spiegel, anch in dem Hauptfernrohre sichtbar wird. Ist diese Justirung einmal bewirkt, so wird anch jeder andere Stern, anf welchen das Hiilfsferurohr gerichtet wird, im Gesichtsfelde des grossen erscheinen. Man rerfährt bei den Beobachtungen mit dem Hornstein schen l'hotometer meistens so, dass man fiu eine ganze Reihe (Zone) ein und denselben Hiilfsstern benutzt, welcher durch Feinbewegmy des Ringes $b$ und des spiegels $S$ wihluend der Daner einer solchen Reihe bestandig in der Mitte des Gesichtsfeldes gohalten wird. Da dieser Hiilfsstem lediglich als Verbindungsgolied dient, um die Helligkeitwmterschiede der einzelnen Zonensterne gegeneinander zu ermitteln, so brancht die Helligkeit desselben gar nicht bekannt zu sein, ebensowenig wie das Intensititsverhältniss eines direct gesehenen Sternes zn seinem reflectirten libilde. Thas die Zonensterne nicht allzu weit anseinander stehen diirten. ist shom deshalb geboten, weil sonst die rom Vergleichssterne kommenden strihlen unter merklich verschiedenen Incidenzwinkeh anf den spiegel antfillen wiirden, nud die Ilelligkeit des Vergleichssternes währeud der Zone nicht constant wäre. Den Schieberm, 
mit welchen die eigentliche Lichtmessung ausgefuhrt wird, hat Hornstein Ausschnitte gegeben, welehe die Form von Hyperbeln haben; die Axen dieser Hyperbeln sind zn einander und zu der Bewegungsrichtung der Sehieber parallel. In Figur 50 ist abcde der untere, $a^{\prime} l^{\prime} c^{\prime} d^{\prime} e^{\prime}$ der obere Schieber, und $o$ ist der freie, durch die Sehieber nicht verdeckte Theil des Objectivs. In welcher Weise die Grösse der freien Fläche $o$ von der Verschiebung der Blenden abhängt, ist von Hornstein nicht angegeben. Am sichersten wilrde es wohl-sein, die Scalenwerthe empirisch durch Messungen an Sternen von anderweitig bekannter Helligkeit zu ermitteln.

Das Hornstein'sche Photometer, welches aus dem Jahre 1860 herstammt, hat tibrigens bereits im Jahre 1834 einen Vorgïnger gehabt. Infolge einer von der $\mathrm{Kgl}$. Gesellschaft der Wissenschaften in Göttingen ausgeschriebenen Preisaufgabe über Astrophotometrie waren mehrere Bewerbungsschriften eingegangen, von denen die oben citirte Steinheil'sche Abhandlung iiber das

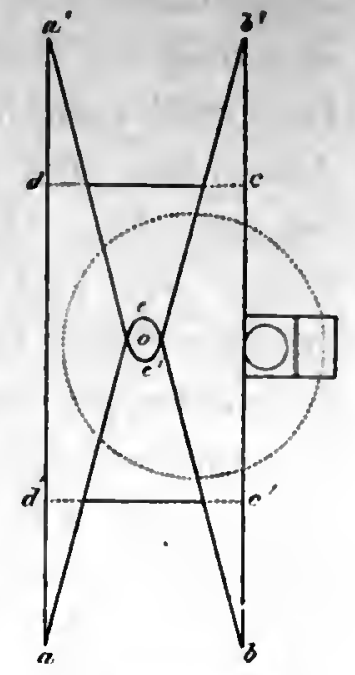

Fig. 50. Prismenphotometer mit dem Preise gekrönt wurde. Eine zweite Sclirift, deren Verfasser nicht bekannt ist, enthiilt nun die Beschreibung eines Photometers, welches fast vollkommen mit dem Hornstein'schen identisch ist. Der einzige Unterschied besteht darin, dass die eine ganze Hälfte des Objectivs (nicht bloss ein kleiner Theil desselben) ron dem Spiegel Licht erh:ilt, und dass die Abblendung nicht durch Schieber, sondem durch eine drehbare Scheibe erfolgt, welche immer eine Hïlfte des Objectivs bedeckt, die andere freiliisst.

\section{e. Die Methoden ron Searle und Cornu.}

Ganz eigenartig ist das von G. Searle $)$ empfohlene Photometer, bei welchem, ähnlich wie bei dem Parklnurst'schen Deflectionsphotometer, eine Theilnng des anf das Objectiv anffallenden Lichteylinders, also nicht eine Abblendung im eigentlichen Sinne stattfindet. Eine keilförmig geschliffene Glasplatte von ausserordentlich kleinem Winkel wird so vor dem Oljjectiv des Beobachtungsfermrohrs angebracht, dass sie iiber dasselbe hinweg. bewegt werden kann und daher einen beliebig grossen nessbaren Theil desselben bedeckt. Von jedem Sterne entstehen so zwei Bilder, deren Intensitäten dureh Verschieben der Glasplatte nach Gefallen verändert werden können. Da der Winkel des Prismas sehr klein ist, so sind die

1) Astron. Nachr. Bd. $5 \bar{i}$, Nr. 1353. 
beiden Bilder nicht sehr weit roneinander entfernt, und die prismatischen Farben des abgelenkten Bildes treten nicht wesentlich störend hervor. Hat man nun zwei Sterne von verschiedener Helligkeit, so kann man dureh Verschieben der Glasplatte das directe Bild des einen gleich dem abgelenkten des anderen machen, und das Verhältniss des bedeekten zum unl,edeckten Theile des Objectivs giebt damn unmittelbar das ursprüngliche Intensitaitsverhialtniss der beiden Sterne, vorausgesetzt, dass der durch Absorption und Reflexion an der Glasplatte hervorgebrachte Lichtverlust bekamnt ist. Derselbe kann entweder durch Vergleichung der beiden Bilder ein und desselben Sternes bestimmt oder auch dadureh zum grössten Theil eliminirt werden, dass man immer die Bilder der beiden zul messenden Sterne kreuzweise miteinander vergleieht. Grosse Helligkeitsunterschiede zu messen ist mit diesem Instrumente nicht rathsam, auch siẹt man sofort, dass die Benutzung des Photometers auf die Vergleichmng sehr nalie bei einander stehender Sterne beschränkt ist. Wie aber die von Searle mitgetheilten Beobachtungen zeigen, erweist sicli das Instrument innerhalb seines Anwendungsbereiches durehaus branchibar.

In nenerer Zeit hat $\mathrm{Cornu}^{1}$ ) noch einige Modificationen zu diesem Photometer vorgeschlagen. Un die Anwendung einer Correction wegen der Absorption in der prismatischen Glasplatte iberfliissig zu machen, emptiehlt er, anstatt cines Prismas deren zwei anzubringen, von absolnt gleichen Winkel und womöglich ans derselben Glasplatte herausgeschnitten. Dieselben stossen mit ihren scharfen Kanten gegeneinander, die Ablenkungen erfolgen daher im entgegrengesetzten Sime. Das Verhältniss der beiden bedeckten Objectirsegmente gieht dam olme jede Correction das gexnchte Intensitaitsverhältniss. Will man grössere Ablenkungen als etwil $1_{2}^{\circ}$ ' herrorbringen, so wiirden die Farben schon störend sein, mid es wäre dann rathsan, die prismatischen Glisplatten zu achromatisiren. Da die Anwendung von Objectivprismen bei Fermrohren ron grossen Dimensionen wegen der schwierigen Herstellung und der bedentenden Kosten katum möglich sein wiirte, so hat Cornu noch den Gebranch von sogemannten photometrischen Ocularen vorgesehlagen, d. h. vou gewöhnlichen terrestrischen Ocnlaren, bei denen zwischen der ersten und zweiten Linse an der stelle, wo ein reelles Bild des Fermrohrobjectirs liegt, zwei prismatische Glassticke angehracht sind, die messbar verschoben werden können und ron jeden Gestirn zwei Bilder ron variabler Helligkeit erzengen. Über praktische Versuche mit solchen Oculaphotometern ist bisher Nichts bekinnt geworden.

1 Comptes Remulus. Tome 103. 11. 1227. 


\section{Anwendung von rotirenden Scheihen. Die photometrischen Methoden ron Talbot, Secchi, Abney.}

Wenn die Augennerven durch irgend einen Lichtreiz afficirt worden sind, so dauert bekanntlich die Wirkung noch eine Zeitlang fort, nachdem die Lichtgnelle entfernt ist. Die Daner dieser Nachwirkung hïngt vou der Intensitit des Lichtes und ausserdem von dem jeweiligen Zustande des Auges ab. Sendet ein leuchtender Gegenstand intermittirend Licht auf unser Sehorgan, und folgen sich die einzelnen Inpulse in so kurzen Zwischenriumen, dass der erste Eindruck noch fortdauert, wenn der zweite eintritt, so erhalten wir die Empfindung einer vollkommen continuirlichen Belenchtung, die Helligkeit des lenchtenden Gegenstandes erseheint aber gesehwäicht und zwar in Verhältniss der Erscheinungsdauer zur Summe der Erseheinungs- und Versehwindungsdaner. Dieser Satz ist von Talbot') und Platean ${ }^{2}$ ) fast zu derselben Zeit anfgestellt und durch eine Reihe ron Versuchen mit rotirenden Scheiben bewiesen worden. Versetzt man eine weisse mit einem schwarzen Sector bemalte Scheibe in schnelle Rotation und beleuchtet dieselbe, so erscheint sie ngleichmaissig gran, und wenn man zwei soleher Seheiben, die mit verschieden grossen schwarzen Sectoren versehen sind, nahe bei einander anfstellt und die Entfernung der einen vou der Lielttgnelle so lange ver:indert, bis beide Scheiben bei der Rotation die gleiche grane Fiirbung zcigen, so findet man, dass die Quadrate der Entfernungen ron der Lichlitquelle sich umgekehrt verhalten wie die Winkelöffinnngen der beiden Sectoren, wodurch also der obige Satz bestïtigt ist. Etwals Ähnliches gilt auch, wenn es sich nicht um diffits reflectirtes, sondern um durchgehendes Licht handelt, wemn man also undurchsichtige, mit sectorförmigen Ansschnitten rersehene Scheiben ror einem leuchtenden Gegenstande rotiren liisst. Je kleiner der Aussehnitt ist, desto schwiieher erseheint der Gegenstand, und es folgt unmittelbar, dass die Helligkeit proportional ist dem Öffunngswinkel des Sectors. Entliält die Scheibe, wie es gewöhnlich der Fall ist, mehrere Ansssehnitte, so wird die IJelligkeit dureh das Verhiiltniss der Summe der Winkelöfinungen dieser Ausschnitte zum ganzen Kreisumfinge ausgedriickt, voransgesetzt, dass die Helligkeit olne die Seheibe als Einheit genommen ist. Es fragt sich, mit welcher Geschwindigkeit die Scheibe vor der Lieltequelle rotiren muss. Eine bestimmte Vorschrift dariiber liisst sich nicht geben; jedenfills ist Bedingmugn, dass

1) Philosophical Magazine. Ser. 3, Vol. 5. p. 321.

2) Pogg. Ann. Bd. 35, p. $45 \%$. 
die Lichterscheinung vollkommen continuirlich ist und jedes Flimmern oder Zittern, welches bei zu langsamer Drehung eintritt, versehwindet. Schnelleres Rotiren lat auf die Intensitiit des Lichtes gar keinen Einfluss. Nach den Versuchen von Plateau, Emsmann, Helmholtz u. A. ist eine Umdrehungsgeschwindigkeit von 24 bis $30 \mathrm{mal}$ in der Secunde unter allen Umstiinden ausreichend, um Gleichförmigkeit in der Helligkeit zu crzielen. Es kommt dabei auch wesentlich darauf an, wie die offenen und geschlossenen Abschnitte auf der rotirenden Scheibe vertheilt sind. Hat man z. B. eine Scheibe, in welcher sich nur ein einziger Ausschnitt in Grösse eines Halbkreises befindet, und daneben eine aweite Scheibe mit vier Ausschnitten von der Winkelöffnung 450, so werden diese beiden Scheiben die gleiche Lichtschwächung hervorbringen, die zweite brancht aber nicht so schnell gedreht zn werden, wie die erste.

Das Princip der rotirenden Scheiben ist schon ron Talbot zu photometrischen Zwecken empfohlen worden, und in der That ist dasselbe nicht uur in theoretischer Beziehnng durchaus einwurfsfrei, sondern auch in praktischer Beziehung so bequem anwendbar, dass es nur zu verwundern ist, dass diese photometrische Methode sich noch nicht mehr Eingang rerschafft hat.

Um nach dieser Methode die Helligkeit eines leuchtenden Gegenstandes messen zu können, muss man im Stande sein, die sectorförmigen Ausschnitte nach Belieben zu vergrössern oder

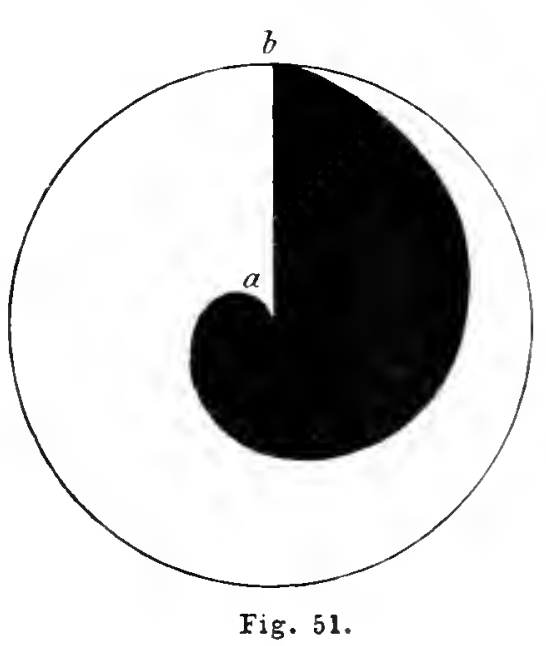
zu rerkleinern. Talbot hat zu diesem Zwecke zum ersten Male die Benutzung ron zwei Scheiben mit gleich rielen und gleich grossen Aussehnitten vorgeschlagen, welche um eine gemeinschaftliche Axe rotiren und messbar gegeneinander rerstellt werden können. Ein ailnuliches Arrangement ist schon friiher bei der Besprechung der verschiedenen Blendrorriehtungen erwïhnt worden.

Ein zweites ebenfalls rou Talbot empfohlenes Messungsmittel besteht darin, dem Ausschnitte in der Scheibe eine durch Figur 51 reprisentirte Form zn geben.

Der Ansochnitt wird hegrenzt durch den Radins $a b$ und durch eine Archimedische spirale, deren Gleichung in Polarcoordinaten bekanntlich ansgedriickt werden kann durch $r=\frac{2 \pi-r}{2 \pi}$ oder $1-r=\frac{r}{2 \pi}$, wenn der Radins der Scheibe mit 1 bezeichnet wird, und die Winkel $r$ ron 
$a b$ aus nach rechts geziahlt werden. Die Grösse $1-r$ ist der $\mathrm{Ab}$ stand rom Rande der Scheibe, und da das Verhältniss $\frac{v}{2 \pi}$ das Helligkeitsmass abgiebt, so sieht man, dass die Helligkeit eines teuchtenden Gegenstandes, wenu man ihn an versehiedenen Punkten der rotireuden Seleibe betrachtet, in demselben Verlältnisse zu- oder abnimunt, wie die Abstände dieser Punkte vom Seheibenrande. Von dieser Methode ist mehrfach Gebranch gemacht worden.

Statt der rotirenden Scheibe hat Talbot, speeiell zur Messung des Sommenliehtes, noch einen rotirenden Spiegel in Vorsehlag gebracht, weleher das Bild einer Liehtquelle im Kreise herumfuhrt. Das Auge des Beobachters wird bei jeder Undrehung des Spiegels einmal von den reflectirten Strahlen getroffen und erblickt bei gentigend seluneller Rotation ein stetiges Bild der Lielttquelle, dessen scheinbare Helligkeit sieh zur Helligkeit der Lichtquelle selbst verhält, wie dic Winkelbreite derselben zum Kreisumfange. Streng genommen ist dabei noeh der Ineidenzwinkel der auffallenden Strahlen oder der Winkel zwisehen Lichtquelle, Spiegelmittelpunkt und Auge zu berücksichtigen, da von diesem die Intensität des reflectirten Liehtes abbängt. Von der Sonne, deren seheinbarer Durchmesser ungefähr einen halben Grad beträgt, wird durch einen solchen rotirenden Spiegel eine Liehtzone hervorgebracht, deren Intensitit im centralen Streifen sich zur Intensitït der Somne selbst wie 1:720 verhiilt. Um das Sonnenlielit mehr abschwächen zu können, hat Talbot noch einen zweiten rotirenden Spiegel eingeführt, weleher zunaichst das von dem ersten kommende Licht empfängt und dasselbe dann entspreehend geschwäeht in das Ange sendet.

Fuir Helligkeitsmessungen an Sternen ist das Princip der rotirenden Seheiben zum ersten und meines Wissens bisher auch einzigen Male von Secchil) in Anwendung gebraeht worden. Derselbe verglich, allerdings nur mit blossem Auge, zwei nicht allzuweit voneinander entfernte Sterne, indem er den helleren durch eine rotirende Scheibe hindureh, den schwächeren ohne dieselbe betrachtete, und die sectorförmigen Öffnungen der Scheibe so weit rerkleinerte, bis die beiden Sterne gleich erschienen. Er bediente sich dabei, ebenso wie Talbot, theils zweier gegeneinander verstellbaren Scheiben, deren gegenseitige Stellung an einer am Rande angelorachten Theilung abgelesen werden konnte, theils einer einzelnen Seheibe mit Ausschnitten, die etwa wie in Figur 52, Seite 224, von der Mitte nach dem Rande zu immer schmäler wurden. p. 10 .

1) Atti dell' accad. Pontificia dei nuovi Lincei. Tomo 4, anno 4 (1850-1851), 
Bei dem zweiten Beobachtungsverfahren wurde diejenige Stelle auf der rotirenden Scheibe bestimmt, wo der geschwächte Stern dem direct

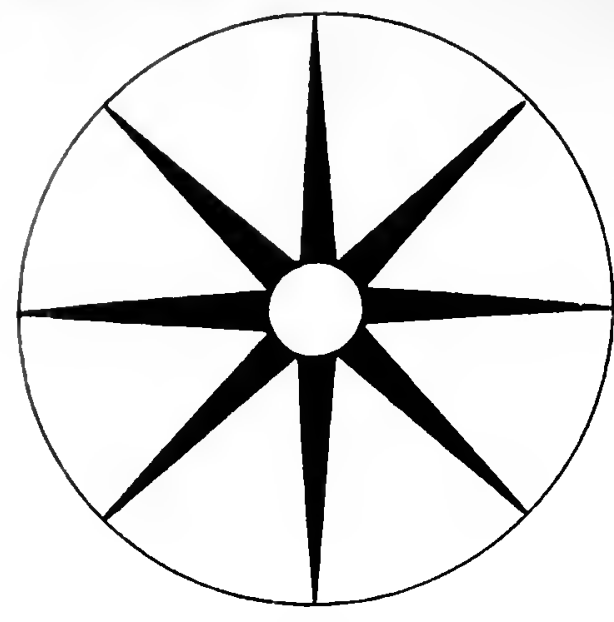

Fig. 52 . gesehenen an Intensität gleich erschicn. Aus dem gemessenen Abstande dieses Punktes vom Rande liess sich dann leicht der gesuchte Helligkeitsunterschied berechmen. Dass das erste Verfahren dem zweiten bei Weitem vorzuziehen ist, liegt anf der Hand. Da der Himmelsgrund, auf dem der hellere Stern steht, durch Verkleinerung der Ausschnitte in der Scheibe mit verdunkelt wird, so sieht man die beiden zu vergleichenden Sterne stets auf verschieden hellem Grunde, was das olmedies schon ziemlich nusichere Beobachtungsverfahren Sechi's noch weniger empfehlenswerth macht.

Es sind noch eine ganze Reibe von photometrischen Einrichtungen l,ekanut geworden, bei denen die rotirenden Scheiben in den mannigfirclisten Formen zur Verwendung kommen. Ich erwähne speciell die Vorschläge ron Guthriel), Napoli2), Hammerli3), Langley-4) und $\left.A b n e y^{5}\right\rangle$, gehe aber nicht niiher auf dieselben ein, da sie fast alle lediglich für die technische Photometrie ron Interesse sind. Nur Abney hat die Nethode anch auf die Messung der Lichtintensitit in verschiedenen Theilen des Somnenspectrums angewendet. Er und ror ihm schon Napoli hiıben insofern einen Fortschritt erreicht, als sie mechanische Eimrichtungen getroffen haben, um zwei auf derselben Axe rotirende Scheiben wïhrend der Drehme gegenein:nder um jeden beliebigen Betrag zn verschicben. Dadurch ist die Methode cigentlich erst ans einem blossen Yittel, die llelligkeit einer Lichtefnelle zn variiren, zu einem feinen Messungsverfihren umgewandelt worden.

Anch Lummer und Brodhun haben sich bei ihren photometrischen Entersuchungen eines ähnlichen Arranements zur Terstellung der Scheiben wiilnend der liotation bedient.

Diese wirlitigne Verbessermm der Methode legt den Gedanken nahe, die Secthïchen Vorschliswe wieder antinnehmen und die rotirenden Seheiben

1 The Chemical News and Jommal of phys. science. Vol. 40 (1579), p. 262.

2 Séances de la soc. France. de physique. 1sь0, p. 53.

3) Elektrotechn. Zeitschrift. Jahro. 4 148:, p. 262.

4 American Journ. of science. Ser. 3. Tol. 30 (1555. p. 210.

5) Phil. Trans of the R. Soce of Londou. 1ssh, r. 423 und 1 sss p. 547; ausserdem Proc. of the R. Soc. of London. Tol. $43, \mathrm{p} .247$. 
zur Helligkeitsmessung der Sterne nutzbar zu maehen. Versuehe in dieser Richtung konnen nicht driugend genug empfohlen werden, und es sclieint nicht allzu sehwierig, auf irgend einem Wege zum Ziele zu gelangen. Es lässt sich z. B. leicht ein compendiöser Apparat, bei welehem zwei gegeneinauder beliebig verstellbare Seheiben mit gleich grossen Ausselnitten dureh ein Uhrwerk oder irgend einen kleinen Motor in selunelle Rotation rersetzt werden, so an einem bcliebigen Refraetor aubringen, dass die Seheiben durch den rom Objectiv kommenden Lichtkegel in der Nihe des Brennpunktes lindureligehen. Wird das Fernrohr auf irgend einen Stern geriehtet, so kann man dureh Verstellen der beiden Seheiben gegeneiiiander (während der Rotation) die Helligkeit desselben so weit verändern, bis er gleieh hell erscheint mit einem kunstlichen Sterne von constanter Helligkeit, weleher dureh ein seitliehes Rohr und durch Reflex an einer unter $45^{\circ}$ gegen die optische Axe des Ferurohrs geneigten planparallelen Glasplatte (:ihmlich wie beim Zöllner'schen Photometer) in das Gesichtsfeld des Oculars gebracht wird. Auf dieselbe Weise beobachtet man einen zweiten Stern und findet so das Helligkeitsverhältniss desselben zu dem ersten. Die verschiedene Helligkeit des Himmelsgrundes hat dabei keinen schïdlichen Einfluss, weil sich stets der Untergrund des kinstliehen Stemes mit dem des wirklichen vermischt. Wir wollen annehmen, dass jede der beiden Scheiben vier Ausschnitte von $45^{\circ}$ Öffnungswinkel hat; dann wird eine rollständige Abschliessung des, Lichtes eintreten, sobald die Ausschnitte der einen Scheibe mit den undurchsichtigen Theilen der anderen coincidiren, dagegen wird die grö̈sste nutzbare Öffnung $180^{\circ}$ betragen. Man sieht iibrigens sofort, dass die Empfindlichkeit der Messungen sehr versehieden sein kann. Sind die Scheiben möglichst weit, also auf $180^{\circ}$, geöffnet, so muss man sic um $16^{\circ}$ gegeneinander verstellen, um eine Lichtsehwächung von 0.1 Grössenclassen hervorzubringen; lassen die Scheiben aber nur eine Öffumng von $t^{\circ}$ frei, so geniigt bereits eine Versehiebung von 0.4 , um denselben Effeet hervorzubringen. Je kleiner also der Öffnungswinkel ist, desto grösser nniss die Genanigkeit der Einstellung und Ablesung sein, wenn man die gleiche Genauigkeit des Resultates verbürgen will. Es wird sich daher empfehlen. nieht allzu grosse Helligkeitsdifferenzen direet zu messen. Benutzt man bei dem hier ins Auge gefassten Apparate nur Öfẗnungswinkel von 180 bis etwa $10^{\circ}$, so könute man bereits Intensitiatsunterschicde ron drei Grössenclassen messen, was für vicle Zweeke der Himmelsphotometrie ausreichend sein wiirde. 
4. Anwendung von spiegelnden Kugelı. Die photometrischen Methoden von Wollaston und Bond.

Weun von einer nicht allzu ausgedehnten Lichtquelle auf eine vollkommen spiegelnde Kugel Licht auffällt, so sieht ein Beobachter ein verkleinertes Spiegelbild der Lichtquelle, dessen Helligkeit variirt, je nachdem die Entfernung der Kugel von der Lichtquelle oder dem Beobachter zu- oder abnimmt. Diese Erscheinung ist vielfach zu Helligkeitsmessungen benutzt worden und hat sich namentlich bei der Vergleichung von sehr hellen Objecten, wie Sonne, Mond und grossen Planeten, als ein sehr werthvolles Hiilfsmittel erwiesen. Es handelt sich dabei um

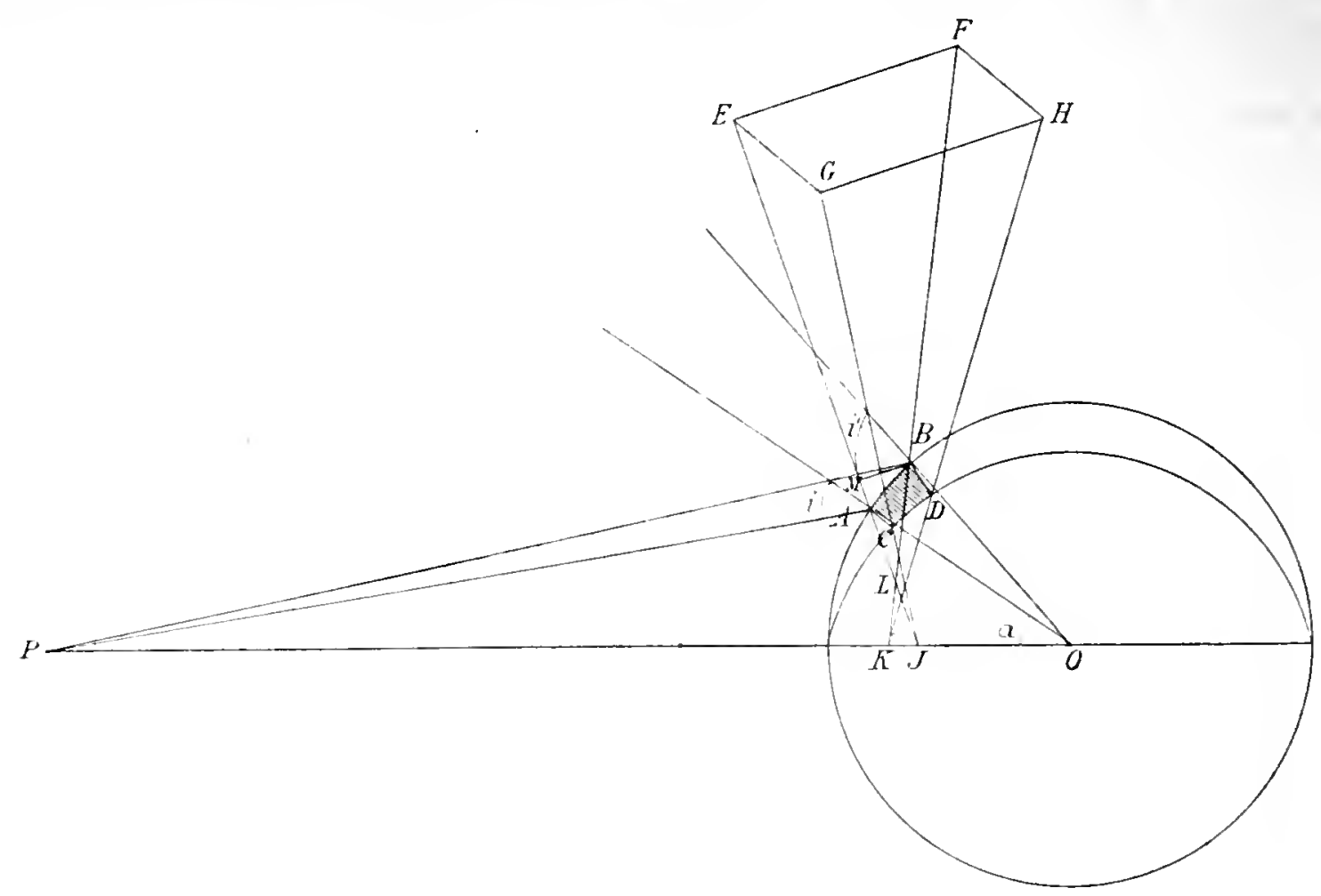

Fig. 53.

die Lüsung der folgenden Anfgabe. Das ron der spiegelnden Kugel reflectirte Licht breitet sich nach allen Richtungen in Raume aus; man soll die an irgend einer bestimmten Stelle hervorgebrachte Beleuchtung berechmen, wenn die Lenchtkraft der Lichtquelle und ihre Entfernung: ron der Kugel, ausserdem der Radius der letzteren bekannt sind. Es sei in Figur $53 P$ ein leuchtender Punkt, $A B C D$ ein kleines Element einer spiegelnden Kugel, welches ron zwei unendlich nahen Meridianen und zwei unendlich nahen Parallelkreisen hegrenzt wird.

Das Centrum der Kugel liegt in (), der Radius derselben sei $\varrho$. Ferner seien die Entfermungen $P()$ und $P A$ mit $a$ und $b$ bezeichnet, und der Winkel $\left.I^{\prime}\right) A$ mit a. Her unter dem Incidenzwinkel $i$ in $A$ anf- 
fallende Lichtstrahl $P A$ wird in der Richtung nach $A E$ reflectirt. Durch $E$ lege man eine Ebene senkreeht zur Richtung $A E$, dieselbe werde in den Punkten $F, G, H$ von den in den Punkten $B, C$ und $D$ an der Kugel zurliekgeworfenen Strahlen getroffen. Die Strahlen $A E$ und $C G$, welche von Punkten desselben Parallels herkommen, schneiden sich riltkwïrts verlingert in einem Punkte $J$, der auf der Axe $P O$ liegen muss; ebenso schneiden sich die Strahlen $B F$ und $D H$ in einem Punkte $K$ der Axe $P O$. Dagegen schneiden sich die von den Meridianpunkten $A$ und $B$ herkommenden Strahlen in dem Punkte $L$. Wir nehmen nun an, dass der leuchtende Punkt $P$ in der Entfernung 1 auf die Flaicheneinheit senkrecht die Lichtquantität $q$ sendet, dann erhält das Kugelobarflachenelement $A B C D$, dessen Inhalt mit $d f$ bezeichnet werden möge, die Lichtmenge $Q=q \frac{d f}{b^{2}} \cos i$. Nun ist aber $d f=A B \times A C$, und wenn man ron $B$ auf die Richtung $A E$ das Perpendikel $B M$ fiilt, so hat man $A B \cos i=B M$; folglich :

$$
Q=q \frac{A C \times B M}{b^{2}}
$$

Von dieser Lichtmenge wird ein bestimmter Bruchtheil, der von der Politur der Kugelfläche u. s. w. abhängt, nach dem Element EFGH reflectirt. Bezeichnet man diesen Bruchtheil dureh den Factor $l$; so erhïlt $E F G H$ die Lichtmenge $k q \frac{A C \times B M}{l^{2}}$.

Die auf die Flächeneinheit ron $E F G H$ gelangende Lichtmenge (die Beleuchtung der Flaiche), welche $\delta$ heissen möge, wird daher, wenn der Inhalt des Elementes $E F G H$ mit $d \varphi$ bezeichnet ist, gregeben durch die Gleichung:

$$
\delta=\frac{l q q}{b^{2}} \frac{A C \times B M}{d \varphi},
$$

oder, da drp ausgedriickt werden kamn dureh $E F \times E G$ :

$$
\delta=l: \frac{q}{b^{2}} \begin{aligned}
& A C \times B M G \\
& E F
\end{aligned} .
$$

Nun ist, zwar nicht streng, aber bei den vor:uszusetzenden kleinen Verhältnissen ansreichend genan:

Ferner ist streng:

$$
A C^{\prime}: E G=J A:(J A+A E) .
$$

$$
B M: E F=L M: L E,
$$

oder, wemn man das kleine Stiick $A, M$ vernachliissigt:

$$
B M: E F=L A:(L A+A E) .
$$


Durch Substitution wird daher, wenn noch die Entfernung $A E$ der auffangenden Fläche von der Kugel mit $c$ bezéichnet wird:

$$
\delta=k \frac{q}{b^{2}} \frac{J \dot{A} \times L A}{(c+J A)(c+L A)} .
$$

Es handelt sich nun noch darum, $J A$ und. $L A$ durch die Grössen $\varrho$, $i$ und $\alpha$ auszudruicken. Im Dreieck $A J O$ hat man ohne Weiteres:

$$
J A=\frac{\varrho \sin \alpha}{\sin (i+\alpha)} \cdot
$$

Etwas umstäudlicher ist die Bestimmung von $L A$. Betrachtet man $A B$ als geradlinig und bezeichnet den Incidenzwinkel bei $B$ mit $i^{\prime}$, so hat man in den beiden Dreiecken $A B P$ und $A B L$ :

$$
\begin{aligned}
& A P: A B=\sin \left(90^{\circ}-i^{\prime}\right): \sin A P B, \\
& A L: A B=\sin \left(90^{\circ}-i^{\prime}\right): \sin A L B .
\end{aligned}
$$

Mithin:

$$
A P \times \sin A P B=A L \times \sin A L B .
$$

und da der Winkel $A P B=d i-d \alpha$, Winkel $A L B=d i+d \alpha$, ferner noch $A P: \varrho=\sin a: \sin (i-\alpha)$ ist, so wird:

$$
L A=\frac{o \sin a}{\sin (i-a)} \frac{1-\frac{d a}{d i}}{1+\frac{d u}{d i}} \text {. }
$$

Es ist aber auch:

und daraus:

$$
a: \varrho=\sin i: \sin (i-a),
$$

$$
\frac{d a}{d i}=\frac{\sin a}{\sin i \cos (i-\alpha)} \text {; }
$$

daher endlich durch Substitution:

$$
L . A=\frac{\frac{1}{2} \varrho \sin \omega \cos i}{\sin u+\frac{1}{2} \sin (i-\alpha) \cos i} .
$$

Setzt man die Werthe ron $J A$ und $L A$ in die Gleichnng fiur $\delta$ ein, so erhält man eine etwas complicirte Formel, ans welcher man für jeden P'nukt der spiegelnden Kugel die Belenchtung in der Entfernung $c$ von derselben tinden kann. Die Formel rereinfacht sich aber ganz wesentlich, wenn die Entfernung des lenchtenden Punktes ron der Kugel im Verhältniss zum Kugelradius sehr gross ist. Dann kam man ohne grossen Fehler $l$, durch a ersetzen, ferner $i=$ " amnehmen. Man hat damı:

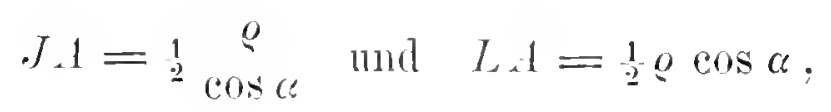


und damit:

$$
J=k \frac{q}{a^{2}} \frac{t \varrho^{2}}{\left(c+\frac{1}{2} \varrho \sec a\right)\left(c+\frac{1}{2} \varrho \cos u\right)}=l: \frac{q}{a^{2}} \frac{\varrho^{2}}{4 c^{3}+2 \varrho c(\sec \omega+\cos u)+\varrho^{2}} .
$$

Ist endlich auch die Entfernung $c$ gross im Verhältuiss zum Kugelradius Q, so ergiebt sich:

$$
\delta=k q \frac{\varrho^{2}}{1 a^{2} c^{2}}
$$

d. l. die Belenelitung ist in diesem Falle unabhängig von dem Ineidentwinkel und allein bestimmt dureh die Grösse der Kugel und die Entfernungen derselben von Lichtquelle und Auffangfliiehe. Denkt man sich an Stelle der letzteren das Auge oder das Ferurolirobjectiv, so erblickt man stets ein gleich helles Reflexbild, in welcher Richtung man anch nach der Kugel sieht. Dabei ist allerdings die Vorausset\%nng gemacht, dass der Factor $k$ für alle Incidenzwinkel derselbe bleibt, was in aller Strenge keineswegs der Fall ist.

Mau kann zu dem eben gefundeneu Resultate noch auf einem anderen viel ktirzeren Wege gelangen, wenn man einen wichtigen Sat\% aus der theoretischen Astrophotometrie (Seite 36) benutat. Wir wollen dabei noch annehmen, dass die Lichtquelle nieht ein leuchtender Punkt sei, sondern eine auf der Axe $P O$ senkrecht stehende leuchtende Scheilse mit der Leuchtkraft $q$, deren scheinbarer Radius, von der Kugel aus gesehen, $s$ sein möge. Die Lichtmenge $d l$, welche diese Scheibe auf das Element $d f$ der Kugeloberfliche sendet, ist nach dem betreffenden Satze ausgedriickt durch:

$$
d l=q d f \pi \sin ^{2} s \cos i .
$$

Es ist aber (Fig. 53) $d f=q^{2} d a \sin a d v$, wenn $d v$ der Winkel zwischen den beiden Meridianen ist, welehe das Element $d f$ einsehliessen. Mithin gelangt auf die ganze Kugel ron der leuchtenden Seheibe die Lichtquantität:

$$
l=49^{2} \pi \sin ^{2} s \int_{0}^{2 \pi} d v \int_{0}^{\frac{\pi}{2}} \cos i \sin u d u \text {. }
$$

Ist die Entfermung der Lichtquelle sehr gross im Verhältniss zu $\varrho$, so kann man $a$ durch $i$ ersetzen und erhält damn:

$$
l=q x^{2} Q^{2} \sin ^{2} s .
$$

Wir setzen wieder voraus, dass bei allen Incidenzwinkeln ein gleich grosser Bruchtheil des anffallenden Lichtes reflectirt wird; danu strahlt die Kugel die Gesammtlichtmenge lil ans, und wenn man sich concentriseh 
ım die spiegelnde Kugel eine Hohlkugel mit dem Radius $c$ gelegt denkt, so empfängt die Flächeneinheit dieser Hohlkugel die Lichtquantität:

$$
\delta=\frac{l k l}{4 \pi c^{2}}=\frac{k q \varrho^{2}}{4 c^{2}} \pi \sin ^{2} s
$$

Statt $x \sin ^{2} s$ kamu man schreiben $\frac{F}{a^{2}}$, wenn $F$ den Flïcheninhalt der leuchtenden Scheibe ausdriickt, und man hat endlich:

$$
\delta=l i q F \frac{Q^{2}}{4 a^{2} c^{2}} .
$$

Diese Formel stimmt bis auf den Factor $F$ mit der oben abgeleiteten iiberein; sie sagt aus, dass die Helligkeit des von einer spiegelnden Kugel reflectirten Bildes direct proportional ist dem Quadrate des Kugelradius und umgekehrt proportional den Quadraten der Entfermungen der Kugel von Lichtquelle und Beobachter.

Der Erste, der von diesem wichtigen Satze in der Astrophotometrie Gebranch gemacht hat, war Wollaston y. Derselbe liess das Licht der Soune von einer kleinen Thermometerkugel reflectiren und betrachtete von grosser Entfernung ans das punktartige Bildchen im Ocular eines Fermrohrs mit dem einen Ange, während er mit dem anderen Auge das von einer zweiten Thermometerkngel reflectirte Bild einer Kerzenflamme dureh eine Linse von kurzer Bremweite ansah. Dureh Verschiebung der Kerze stellte er die Gleichheit der beiden Helligkeiten her. Danu richtete er das Fermrohr direct anf einen hellen Stern und verglich denselben ebenfalls mit dem von der zweiten Kugel reflectirten Kerzenlichte. Mit Benutzung der obigen Formel liess sich anf diese Weise das Helligkeitsverhältuiss der Sonne zu dem Fixstern aus den gemessenen Distanzen und den Kugeldurehmessern berechnen. Das Wollaston'sche Verfahren hat einen bedenklichen Mangel. Da der Stern direet, das Sonnenlicht aber nach der Spiegelung betrachtet wurde, so war zur Reduction der Beobachtungen die Kenntniss des Reflexionseoeftieienten k: erforderlich. welcher sich nur schwer bestimmen lisst und ron Wollaston sehr willkiirlich gleich $\frac{1}{2}$ angenommen wurde.

Steinheil2) hat ein aihuliches Verfahren wie das Wollaston'sche zur Vergleichung vou sonne und Fixsternen in Vorschlag gebracht und hat sich zur Priifung seines Prismenphotometers eines Hiilfsapparates bedient, hei welchem das P'rincip der spiegehnden Kugeh ebenfalls zur Anwendung kim.

1) Phil. Trans. of the R. Soc. of Lundon. 1529, p. 19.

2) Elemente der Helligkeitsmessungen am Stemenhimmel, p. 33 und 4!. 
Noch empfehlenswerther ist die Methode, welehe Bond') bei Vergleichung des Mondes mit den Planeten Jupiter und Veuns, sowie bei der Messung der Liehtstiirke der versehiedenen Mondphasen eingeschlagen hat. Derselbe benutzte nur eine einzige spiegelnde Kugel, von welcher er sowohl das Licht der Himmelskörper als dasjenige der Vergleichsflamme refleetiren liess. Es kam daher uur auf die jedesmalige genane Bestimmung des Abstandes der Flamme von der Kugel an, bei weleher die mit blossen Auge ron eonstanter Entfernung aus betrachteten Reflexbilder gleich hell gesehaitzt wurden.

In ähnlicher Weise ist die Methode noch oft in der Photometrie angewendet worden. Zu spiegelnden Kugeln eignen sich sehr gut aussen versilberte oder mit Quecksilber gefullte Glaskugeln, noch vortheilhafter sind Stahlkugeln, bei denen sich leiehter die vollkommene Kugelgestalt herstellen liisst. Um ubrigens den Einfluss etwaiger Abweichungen von der regelmiissigen Gestalt, sowie anch ungleicher Reflexionsfïhigkeit an verschiedeuen Stellen der Kugel unsehïdlich zu machen, empfiehlt es sich, vor jeder einzelnen Messung die Kugel ein wenig zu drehen, damit stets möglichst viele verschiedene Partien der Kugeloberfläche zur Wirkung kommen.

\section{Benutzung der Eigenscliaften des polarisirten Liclites.}

Die merkwürdigen Eigenschaften des polarisirten Lichtes haben sehr bald, nachdem durch die epochemachenden Untersuchungen von Arago und Fresnel das Wesen der Erscheinungen richtig erkannt und die hauptsiichlichsten Gesetze auf experimentellem und theoretischem Wege festgestellt waren, den Gedanken angeregt, die Polarisation des Lichtes fiir die Photometrie nutzbar zu machen, und in der 'That hat wohl keine andere Methode die Lichtmessungen in gleicher Weise grefürdert. Nicht ganz mit Unrecht nennt man daher bisweilen Arago, der die ersten wichtigen Schritte auf dieseni Gebiete gethan hat, den Begrinder der modernen praktischen Photometrie. Auch die Himmelsphotometrie verdankt dieser Methode ihre besten instrumentellen Hiilfsmittel.

Da man es bei den meisten photometrischen Aufgaben, sowohl in der Technik als am Himmel, mit natiirlichem oder nur partiell polarisirtem Lichte zu thmu hat, so kommt es in erster Linie daranf an, Mittel zu besitzen, um aus solchem Lichte vollkommen polarisirtes herzustellen. Es giebt eine Menge Wege, welche zu diesem Ziele führen. Für die Photometrie haben sich hauptsichlich zwei als branchbar erwiesen: erstens

1) Memoirs of the Amer. Acad. of science. New series, Vol. S, p. 221. 
die Reflexion an der Oberfliche isotroper Medien und aweitens die Doppelbrechung in Krystallen.

Bekanntlich hat Malus durch Zufall 'die Entdeckung gemacht, dass das von der Oberfläche des Wassers oder einer Glasplatte reflectirte Licht die Eigenschaften des natuirlichen Lichtes verloren hat und je nach der Grösse des Incidenzwinkels melı oder weniger polarisirt ist. Alle (lurchsichtigen festen und flüssigen Substanzen besitzen diese Eigenschaft, und fur jede existirt ein ganz bestimmter Incidenzwinkel, bei welchem die Polarisation des reflectirten Strahles vollständig wird. Man nenut diesen Incidenzwinkel den Polarisationswinkel der Substanz. Für Glas betrïgt derselbe etwa $56^{\circ}-60^{\circ}$, für Wasser etwa $53^{\circ}$. Brewster ${ }^{1}$ ) hat das nach ihm genannte Gesetz anfgestellt, welches aussagt, dass bei jeder durchsichtigen Substanz das zuriickgeworfene Licht dann vollständig polarisirt ist, wenn der reflectirte Strahl auf dem gebrochenen senkrecht steht. Ist $p$ der Polarisationswinkel einer Substanz, $n$ der Brechungsexponent derselben und $r$ der Brechungswinkel, so ist $\sin p=n \sin r$, und da nach dem Brewster'schen Gesetze $p+r=90^{\circ}$ sein soll, so hat man $n=\operatorname{tang} p$, wodurch also für jeden durchsichtigen Körper der Polarisationswinkel bestimmt ist. Die Kenntuiss dieses Winkels giebt, wie man sieht, ein vortreffliches Mittel, gewöhnliches Licht in vollständig polarisirtes zu verwandeln, doch ist dabei zu beachten, dass für jede Farbengattung ein besonderer Polarisationswinkel existirt, und dass infolge dessen bei Benutzung von zusammengesetztem Lichte streng genommen niemals eine rollkommene Polarisation stattfinden kann. Je stärker hrechend die spiegelnde Substanz ist, desto grösser ist natiirlich die Quantitiit des unpolarisirt bleibenden lichtes, doch wird in der Praxis der störende Einfluss meistens vou geringer Bedentung sein, wenn man den Spiegel auf denjenigen Polarisationswinkel einstellt, welcher der intensirsten Strahlengattung entspricht.

Fällt das Licht muter einem anderen als dem Polarisationswinkel auf, so findet nur cine partielle Polarisation statt, und das reflectirte Licht ist ans natiirlichem und polarisirtem gemischt; wenn man aber dieses zuriickgeworfene Licht noch mehrmals und zwar unter beliebigen Winkeln reflectiren lïsst, so wird dasselbe endlich, wie schon Brewster hervorgehoben hat, beinahe vollstïndig polarisirt. Man kann also anch mehrfache Reflexion unter beliebigen Winkeln zur Herstellung von vollkommen polarisirten Licht benutzen, hat aber natiirlich mit dem Übelstande zu kimpfen, dass eine anserorlentliche Lichtschwaichung eintritt.

Was noeh die in durchsichtigen Medien gebroehenen Strahlen an-

1 Phil. Trans, of the R. Soc. of London. 1815, p. 125. 
betriftt, so sind dieselben niemals yollstiindig polarisirt, sie enthalten naturliches und polarisirtes Licht, und zwar ist die Polarisationseliene des letzteren senkrecht \%ur Polarisationsebene der reflectirten Strahlen. A rago hat das wichtige Gesetz aufgestellt, dass, weun uatirliches Licht uuf ein durchsichtiges Medium anffallt, der reflectirte und der gebrochene Strahl gleiche Quantititen polarisirten Lichtes enthalten. Da nun bei nicht allzu grossen Incidenzwinkeln das reflectirte Licht schwächer ist als das durchgehende, so folgt, dass das letatere unr eine particlle Polarisation aufweisen kann. Wemn man aber eine grössere Anzahl von durchsichtigen plauparallelen Platten tibereinander legt, so wird bei dem Durchgange durch jede folgende immer ein neuer Bruchtheil des Lichtes polarisirt, und schliesslich ist fast alles durchgehende Lieht polarisirt. Die sogenamnte Glasplattensïule dient also ebenfalls als Polarisator und ist als solcher z. B. bei dem Wild'schen Photometer verwendet worden. Neumann') hat speciell die Theorie dieser Glassaiule sehr ausfuhrlich behandelt.

Die bei weitem gebrïnchlichsten Polarisatoren, speciell in der Himmelsphotometrie, sind die doppeltbrechenden einaxigen Krystalle, und zwar benutzt man fast ansschliesslich entweder das Rochon'sche und Wollaston'sche Prisma, bei welehen beide Strahlen, sowohl der ordentliehe als der ausserordentliche, zur Wirksamkeit gelangen, oder die verschiedenen Formen des sogenanuten Nieol'schen Prismas, bei welchem der ordentliehe Strahl durch Totalreflexion fortgeschafft wird. Einige Bemerkungen iiber diese wichtigen Hülfsmittel der Photometrie mögen hier am Platze sein ${ }^{2}$ ).

Das Rochon'sche ${ }^{3}$ ) Prisma, das iilteste von allen Polarisationsprismen, wird gewöhnlieh aus Bergkrystall (seltener aus Kalkspath) angefertigt und besteht aus zwei vollkommen gleichen rechtwinkligen Prismen, welche mit ihren Hypotenusenfliehen an einander gekittet sind. Die Hauptaxe des Krystalles steht in dem einen Prisma auf der Eintrittsfläche senkrecht, in dem anderen liegt sie parallel der breehenden Kante. Ein Strahl natiirlichen Lichtes, welcher senkrecht auf die Vorderfliche des ersten Prismas auffiilt, geht daher ohne Ablenkung und Zerlegung dureh dasselbe hindurch und wird erst bei dem Eintritte in das zweite Prisma in zwei Strahlen zerspalten, die nach dem Verlassen des Doppelprismas

1) Neumann, Vorlesungen iiber theoretische Optik, heransg. von E. Dorn. Leipzig, 1885 , p. $14 \bar{i}$.

2) Ausfüllrliche Angaben ïber die verschiedenen Polarisationsprismen findet man in den beiden folgenden Abhandlungen: W. Grosse, Die gebrïuchlicheu Polarisationsprismen mit besonderer Berücksichtigung ihrer Anwendung in Photometern. Clausthal, 1886, und: K. Feussner, Über die Prismen zur Polarisation des Lichtes Zeitschr. für Instrumentenkunde, Jahrg. 4, 1584, p. 41.

3) Recueil de mémoires sur la mécanique et snl la physique. Brest, 1873. Siehe anch Gilberts Annalen. Bd. 40, p. 141. 
vollkommen polarisirt sind, und zwar in Ebenen, die anfeinander senkreeht stcheu. Der ordentliehe Strahl behält nach dem Austritte aus dem Doppelprisma die Richtung des auffallenden Lichtes bei, erleidet aber meistens dureh die Kittsehicht eine kleine seitliehe Versetzung. Nur wenn die Kittsubstanz genau denselben Brcehungsexponenten wie der ordentliche Strahl hat, findet gar keine solche Versehiebung statt. Der ausserordcutliehe Strahl tritt abgelenkt von der urspriinglichen Richtung ans dem Doppelprisma aus in einer Ebene, die auf der brechenden Kante senkrecht steht. Die Grösse der Ablenkung hängt von dem breehenden Winkel der Prismen und von dem Untersehiede der Brechungsexponenten des ordentlichen und ansserordentlichen Strahles ab. Ist bei Verwendung von Quarz der Prismenwinkel ungefähr $60^{\circ}$, so beträgt die Ablenkung fast eimen ganzen Grad. Wenn das Doppelprisma aus Kalkspath angefertigt ist, so wird entspreehend der grösseren Differenz zwischen den Brechnng:sexponenten des ordentlichen und ausserordentliehen Strahles anch eine grössere Ablenkung der austretenden Strahlen erzielt werden, was unter Unständen von Wichtigkeit sein kann. Das ordentliche Bild beim Rochon'sehen Prisma ist farblos, wälırend das ansserordentliehe gefürbt erseheint. Dies macht sieh als empfindlieher Naehtheil bemerklieh, wenn man das ordentliche Bild einer Lichtquelle mit dem ausserordentlichen einer anderen direet vergleichen will.

Eine Modification des Rochon'schen Prismas, bei weleher eine besonders weite Tremnung der beiden anstretenden Strahlen erreieht ist, rialirt ron Wollaston') her. Bei dieser Form ist das erste Prisma so hergestellt, dass die Hauptaxe des Krystalls (Quarz oder Kalkspath) parallel zur Eintrittsfliche und senkrecht zur brechenden Kante liegt. Das zweite Prisma ist genan so gearbeitet, wie bei dem Rochou'sehen Polarisator. Die beiden anstretenden Strahlen sind von der urspriinglichen Richtung um gleiche Betrïige nach entgegengesetzten Seiten abgelenkt, und die Gesammttrennung ist doppelt so gross, wie bei dem Rochonschen Prisma. Ordentliches und ausserordentliches Bild erscheinen in gleicher Weise gefürbt.

Häntig wird in photometrischen Apparaten anch von dem sogenannten achromatisirten Kalkspathprisma Gelmanch gemacht. Bei diesem besteht die eine Hailfte aus Kallispath, die andere aus Crownglas, dessen Brechungsenetticient nahe mit dem des ansserordentlichen Strahles im Kalkspath iibereinstinment. Als Kittungsmittel ist Canadabalsam verwendet. Die ansserordentlichen Strahlen grehen olme Ablenkung und fast ohne jede seitliche Versehiebung hindurch und sind bei geeigneter Wahl

Phil. Trans. of the R. Soc. of London. 1920. part. I, 1. 126. 
des brechenden Winkels fast vollkommen achromatisirt; die ordentlichen Strahlen erfalıren eine ziemlich starke Totalablenkung und zwar, wenn die Kalkspathhälfte dem auffallenden Lichte zngekehrt ist, eine etwas grössere, als wenn das Licht znerst die Glashälfte passirt.

Von der grössten Bedeutung für die Photometrie sind das Nicol'sche Prisma und die versehiedenen Modificationen desselben, die im Laufe der Zeit eingefuhrt worden sind. Diese Prismen sind aus zwei Kalkspathstueken zusammengesetıt, die so aus dem Krystall herausgeschnitten und mit geeigneten Kittsubstanzen wieder vereinigt sind, dass der ordentliche Strahl dureh Totalreflexion ganz beseitigt wird, und nur der ausserordentliche unabgelenkt hindurchgeht. Von den verschiedenen Formen sind die gebrïnehlichsten: das ursprllngliche Nicolprisma mit schrigen Endflïchen, das Nicolprisma mit geraden Endflichen, das HartnackPrazmowski'sche, das Foueault'sche und das Glan-Thompson'sche Prisma. Sie unterscheiden sich roneinander durch die Art des Sehnittes und durch die Schicht zwischen den beiden Hälften. Bei den drei ersten Formen wird zum Kitten Canadabalsam oder Copaivabalsam oder Leinöl benutzt, bei den beiden letzten Formen ist die Kittschicht ganz weggelassen und durch eine dunne Luftschicht ersetzt. Jede dieser Formen hat ihre Vorzlige und Naehtheile, und man wird je nach dem Zwecke, den man erreichen will, von einer oder der anderen Gebrauch machen. Ein Hanptubelstand fast aller Nicolprismen ist die nicht zu vermeidende geringe seitliche Abweichung. Dadurch wird bewirkt, dass in photometrischen Apparaten, wo Nicolprismen und Liusen combinirt werden, die Bilder etwas seitlich von der optischen Axe liegen, und da meistens Drehungen der Prismen erforderlich sind, so findet infolge dessen eine Rotation des Bildes um die Axe, ein sogenanntes Schlendern, statt. Bei photometrischen Beobachtungen ist dieses Schleudern sehr störend; an Besten ist dem Fehler bei Nicolprismen mit geraden Eudfliichen abgeholfen, und in dieser Beziehung eignen sich dieselben in erster Linie zur Verwendung in Photometern. Sehr gefährlich sind die Vebenreflexe, welche an den Seitenflaichen der Prismen und an der //wischenschicht auftreten und nieht uur die Reinheit der Bilder erheblich beeintrïchtigen, sondern auch zur Entstchung ron elliptisch polarisirtem Lichte und zur directen Verfiilschung der Messungsresultate beitragen können. Durch sorgfältige Schwärzung der Seitenfliichen und vor Allem durch passende Anwendung von Diaphragmen, welehe nur den Hauptlichtkegel frei hindurchgehen lassen, kann dieser Fehler wesentlich abgeschwäeht werden, und es sollte bei der Construction ron Photometern niemals verabsäumt werden, die Prismen in Bezug auf diesen Punkt einer genanen Prüfung za unterwerfen. Das Foucault'sche und Glansehe Prisma stehen in 
Bezug auf Reinheit der Bilder den anderen nach, weil die Reflexionen innerhalb der Luftschicht eine nicht unbeträchtliche Trübung. hervorbringen. Bei manchen photometrisehen Aufgaben kommt es auf ein möglichst urosses Gesichtsfeld an, und in dieser Beziehung verdienen die älteren Formen des Nicolprismas, und das Hartnack-Prazmowski'sche den Vorzug vor den anderen. Was endlich noch die Lichtstärke anbetrifft, so ist von vornherein zu bedenken, dass infolge der Trenmung in ordentlichen und ausserordentlichen Strahl bei keiner der erwähnten Formen mehr als die Hälfte des einfallenden Lichtes zur Ausnutzung kommen kilnn, und dass durch die Absorption und Reflexion im Prisma selbst noeh ein weiterer Lichtverlust eintritt. Das eigentliche Nicolprisma ist das lichtstärkste ron allen, es lässt etwa 40 bis 45 Procent des auffallenden Lichtes lindurch. Dann folgt das Prazmowski'sche und erst hinter diesem das Foucanlt'sche und das Glan'sche Prisma. In der Astrophotometrie, wo es fast immer auf die iusserste Ausnutzung des vorhandenen Lichtes ankommt, rerwendet man daher mit Vorliebe das Nicolprisma und zwar ans den oben schon erwïhnten Griinden dasjenige mit senkrechten Endflichen.

Wir haben im Vorangehenden die verschiedenen in photometrischen Apparaten iiblichen Hiilfsmittel zur Hervorhringung ron vollständig polarisirtem Lichte besprochen. Um nun aus den Eigenschaften dieses so erhaltenen Lichtes anf die ursprüngliche Intensität schliessen zn könuen, muss in jedem Photometer noch ein sogenannter Analysator zur Verwendmg kommen, welcher es ermöglicht, die Beschaffenheit des polarisirten Lichtes zu untersuchen. Nan benutyt hierzu fast ausschliesslich eine der erwïhnten Formen des Nieol'sehen Prismas. Die theoretische Berechmmg der Lichtstirken stiitzt sich dann auf das wichtige Malus'sche Gesetz oder, wie es gewöhnlich genannt wird, das Cosinusquadratgesetz. Dieses Gesetz sagt aus, dass, wenn ein geradlinig polarisirter Lichtstrahl anf einen doppeltbrechenden Krystall anff̈̈llt, die Lichtstärke des austretenden ordentlichen Strahles proportional dem Quadrate des Cosinus, die des ansserordentlichen proportional dem Qnadrate des Sinus desjenigen Winkels ist, welchen die Polarisationsebene des anffallenden Lichtes mit dem Hauptsehnitte des Krystalls bildet. Hat man als Polarisator ein Rochon'sches oder Wollaston sches Prisma rerweudet, so theilt sich der auffallende Strahl, dessen Intensitiit $J$ sein möge, in zwei gleichstarke Strahlen, ron denen der ordentliche in der Ehene des Hauptschnittes, der ausserordentliche in der Ebene senkrecht zum Hauptschnitte polarisirt ist. Bezeichnet man die Helligkeiten derselben mit $\theta$ und $E$, so hat man, wenn noch $m$ einen Sehwïchnngsfactor bein Durchgange Aureh die Substanz ausdriickt:

$$
0=\frac{1}{3} m . J \text { und ehenso } E=\frac{1}{2} m J \text {. }
$$


Fallen diese beiden Strahleu auf ein Nicolprisma als Analysator, so liefert jeder nur einen einzigen anstretenden Strahl. Die Intensitäten derselben mögen $O^{\circ}$ und $E^{\prime}$ heissen. Bildet dann der Hauptsclunitt des Nicols mit dem Hauptschnitte des Polarisators den Winkel $q$, so laat-man nach dem Malus selien Gesetze:

$$
\begin{aligned}
& O^{\prime}=\frac{1}{2} m^{2} J \sin ^{2} \uparrow, \\
& E^{\prime}=\frac{1}{2} m^{2} J \cos ^{2} r .
\end{aligned}
$$

Wir denken uns nun zwei Liehtquellen mit den ursprüinglichen Intensitäten $J_{1}$ und $J_{2}$, welehe ihr Licht nebeneinander anf den Polarisator werfen. Dann treten ans dem analysirenden Nicol im Ganzen vier Lichthundel heraus mit den Intensitäten:

$$
\begin{array}{ll}
O_{1}^{\prime}=\frac{1}{2} m^{2} J_{1} \sin ^{2} \varphi, & O_{2}^{\prime}=\frac{1}{2} m^{2} J_{2} \sin ^{2} \varphi, \\
E_{1}^{\prime}=\frac{1}{2} m^{2} J_{1} \cos ^{2} \varphi, & E_{2}^{\prime}=\frac{1}{2} m^{2} J_{2} \cos ^{2} \varphi .
\end{array}
$$

Man findet stets eine Stellung des Nicols, bei weleher die Werthe $O_{i}^{\prime}$ und $E_{\text {q }}^{\prime}$ einander greich sind. Wird der dieser Stellung entsprechende Werth ron $\varphi$ mit $\alpha$ bezeichnet, so folgt:

$$
\frac{J_{2}}{J_{1}}=\operatorname{tang}^{2} \alpha \text {. }
$$

Ebenso giebt es eine zweite Stellung des Nicols, hei welcher dic Werthe $E_{\mathrm{i}}^{\prime}$ und $O_{a}^{\prime}$ einander gleich werden. Heisst der entsprechende Winkel $a^{\prime}$, so wird:

$$
\frac{J_{1}}{J_{z}}=\operatorname{tang}^{2} \alpha^{\prime}
$$

und es folgt daher numittelbar: $\alpha^{\prime}=90^{\prime \prime}-\alpha$.

Wenu man als Polarisator austatt des Rochon'schen oder Wollastonschen Prismas ein Nicolprisma benutzt, so liefert eine Liehtquelle nit der urspriłnglichen Intensität $J$ nur einen einzigen aus dem analysirenden Nicol anstretenden Strahl, dessen Intensität gegeben ist dureh:

$$
E=\frac{1}{2} m^{2} J \cos ^{2} r \text {. }
$$

Wiihlt man diese Liehtquelle als Vergleiehsohjeet fïr eine andere mit der Intensitiit $J_{1}$, die mau ohme polarisirende Medien direct neben derselben erblickt, so kann man $E^{\prime}$ durch Drehung des analysirenden Nicols so weit verindern, bis es gleich $J_{1}$ wird. Man hat daum, wenn der entsprechende Winkel zwischen den Hauptschnitten der beiden Nieols mit $\omega_{\text {, }}$ bezeichnet wird:

$$
J_{1}=\frac{1}{2} m^{2} J \cos ^{2} \omega_{1} .
$$

Für cine dritte Lichtquelle mit der Intensitiit $J_{2}$ wird ebenso bei einem 
grewissen Winkel $\omega_{g}$ die Gleichheit mit dem Vergleichslichte hergestellt werden köuneu. Man erhält:

Ind mithin:

$$
J_{2}=\frac{1}{2} m^{2} J \cos ^{2} \alpha_{2}
$$

$$
\frac{J_{1}}{J_{2}}=\frac{\cos ^{2} \alpha_{1}}{\cos ^{2} \omega_{2}}
$$

Wenn endlich noch als Polarisator eine unter dem Polarisationswinkel gegen die auffallenden Strahlen geneigte reflectirende Glasplatte dient, so lat der einzige aus dem analysirenden Nicol anstretende Strahl die Intensitiit:

$$
E^{\prime}=m J \sin ^{2} \uparrow .
$$

Hier bedentet of den Winkel, den die Einfallsebene des Lichtes mit dem H:uptschnitte des Nicols bildet. Beuntzt man die Lichtquelle $J$ wieder als Vergleichslicht für zwei andere Lichtquellen mit den Intensitäten $J_{1}$ und $J_{2}$ und stellt nacheininder durch Drehung des analysirenden Nicols die Gleichheit der Helligkeiten dar, so ergiebt sich:

$$
\text { 3) } \quad \frac{J_{1}}{J_{2}}=\frac{\sin ^{2} c_{1}}{\sin ^{2} \iota_{0}} \text {, }
$$

wo $c_{1}$ und $a_{2}$ die entsprechenden Werthe des Winkels $\varphi$ sind.

Jede der drei im Vorangehenden angedenteten Methoden hat in der Photometrie Yerwendung gefunden, die erste z. B. bei den Pickering'schen Photometern und dem Glan-Togel'schen Spectralphotometer, die zweite bei dem Zöllner"schen Astrophotometer, und die dritte bei dem ersten Vild schen Photometer.

Was die Richtigkeit des zu Grunde liegenden Cosinusquadratgesetzes anbelangt, so ist dieselbe durch zahlreiche Beobachtungen innerhalb der bei Lichtmessungen zn rerbiirgenden Genanigkeit nachgewiesen. In der That stimmen die meisten Beobachter darin iiberein, dass der Helligkeitsmuterschied zwischen den beiden durch ein doppeltbrechendes Prisma erzeugten Bildern nicht nehr als etwa $\frac{1}{50}$ oder $\frac{1}{3}$ der Intensität betragen kamn. Die ganz strengen Ausdriicke für die Intensitäten $O$ und $E$ des ordentlichen und ansserordentlichen strahles, in welche ein linear polarisirter Lichtstrahl von der Intensitiit $J$ beim Durchgange durch einen doppeltbrechenden Krystall zerlegt wird, sind ron Wild $\left.{ }^{1}\right)$ anf Grund der ron Xeumann gegebenen Theorien anfgestellt worden; sie lanten:

$$
\begin{aligned}
& 0=\frac{16 a^{2}}{\left(1+a^{2}\right.} J \cos ^{2} u, \\
& E=\frac{16\left[a^{2}-\left(a^{2}-a^{2} \sin ^{2}\right)\right]}{1+\sqrt{\left.\left.a^{2}-a^{2}-c^{2}\right) \sin ^{2}\right)^{4}}} J \sin ^{2} u .
\end{aligned}
$$

1 Poggend. Annalen. Bd. 11s. 1. 1:1:3. 
Hierin ist w der Winkel zwisehen der Polarisationsebene des einfallenden Lichtes und dem Hauptschnitte des Krystalls, ferner bedeuten $a$ und $c$ die reciproken Brechungsindices des ordentlichen und ansserordentlichen Strahles und $v$ den Winkel zwischen optischer Axe und Einfallsloth. Fur $y^{\prime}=0$ und fit $a^{2}-c^{2}=0$ gehen die Formeln in das einfache Malussche Gesetz ulber; dasselbe wirl also um so besser erfullt sein, je geringer die Doppelbrechung des benutzten Polarisationsprismas ist. Der Bergkrystall rerdient in dieser Beziehung - den Vorzug vor dem Kalkspath. Man sicht noch, dass die Formeln (2) und (3) auch mit Benutzung der strengen Wild'schen Ausdrlicke ganz einwurfsfrei sind, da stets nur Werthe von der Form $O$ oder $E$ miteinander combinirt sind. Nur bei Formel (1) whrde die Abweichung rom einfachen Malus'schen Gesetze in Betracht kommen; doch darf man dieselbe bei allen Aufgaben der Himmelsphotometrie unbedenklich ausser Acht lassen.

Bei den drei oben'erwïlnten Methoden kommt es auf die Beurtheilung der Gleichheit zweier Lichteindricke an. Arago hat noch ein anderes Polarisationsprineip zu photometrisehen Messungen vorgeschlagen, welches von Babinet und namentlich von Wild mit Erfolg angewendet worden ist. Nach diesem Principe verhalten sieh gleiche Quantitäten senkrecht zu einander polarisirten Liehtes bei ihrer Misehung wie natiorliches Licht. Nun giebt aber das bekannte Polariskop ein vortreffliches Mittel, auch die geringsten Mengen von polarisirtem Lichte nachzuweisen, da in einem solchen Apparate bei vollständig oder particll polarisirtem Lichte Interferenzfiguren auftreten, während solehe bei natiirlichem Lichte nicht rorhanden sind. Kann man also von zwei Lichtquellen Strahlenbiischel zum Zusammenfallen bringen, die senkreeht zu einander polarisirt sind, und deren Intensität durch Drehung eines Polarisators nach Belieben um messbare Quantitäten ge:indert werden kanu, so braucht man diese Drehung. nur so weit anszufuiluren, bis in einem Polariskope dic Interferenzfiguren verschwinden. Die gemischten Quantititen sind dann nach Obigem goleich, nnd die Drehung des Polarisators erlaubt die Berechnung des urspriinglichen Lichtrerhältnisses. An Stelle der Gleichheitsbeurtheilung tritt also bei dieser Methode die Beobachtung des Auftretens oder Verschwindens ron Interferenzerscheinungen, welche bei einiger Übung ansserordentlich fein ist.

In der folgenden Besprechung der wichtigsten Polarisationsphotometer sind in erster Linie diejenigen berorzugt worden, welche bei lieobachtungen am Himmel ausgedehnte Verwendung gefunden haben; rou den iibrigen sind nur solche lervorgehoben, die fuir die ganze Entwicklung dieser Classe von Instrumenten bedeutungsvoll sind, oder die durch 
besonders eigenthïmlielıe Einrichtungen Interesse verdienen. Eine Gruppinung der einzelnen Apparate nach einem bestimmten Gesichtspunkte, etwa mach den versehiedenen im Vorangehenden erwähnten Methoden, ist nicht durehgefiihrt worden; es ist vielmelr bei der Zusammenstellung lediglich die ehronologische Reihenfolge massgebend gewesen.

\section{a. Die Photometer von Arago, Bernard, Babinet.}

Von den zahlreichen Apparaten, welche Arago zur Lichtmessung vorgesehlagen hat, wird gewöhnlich einer mit dem speciellen Namen des Arago'schen Photometers bezeiehnet; bei welchem die Helligkeitsinderungen des ron einer planparallelen Glasplatte unter verschiedenen Winkeln refleetirten und dureligelassenen Lichtes zur Verwendung kommen. Dieses Instrument ist von $\operatorname{Arago}^{1}$ ) erst verhältuissmässig spät (im Jahre 1S50) beschrieben worden, während seine ersten Vorschläge zur Verwendung der Polarisationserseheinnngen in der Photometrie bereits aus den dreissiger Jalıren herriihren. Der Apparat (Fig. 54) besteht aus einem kreuz-

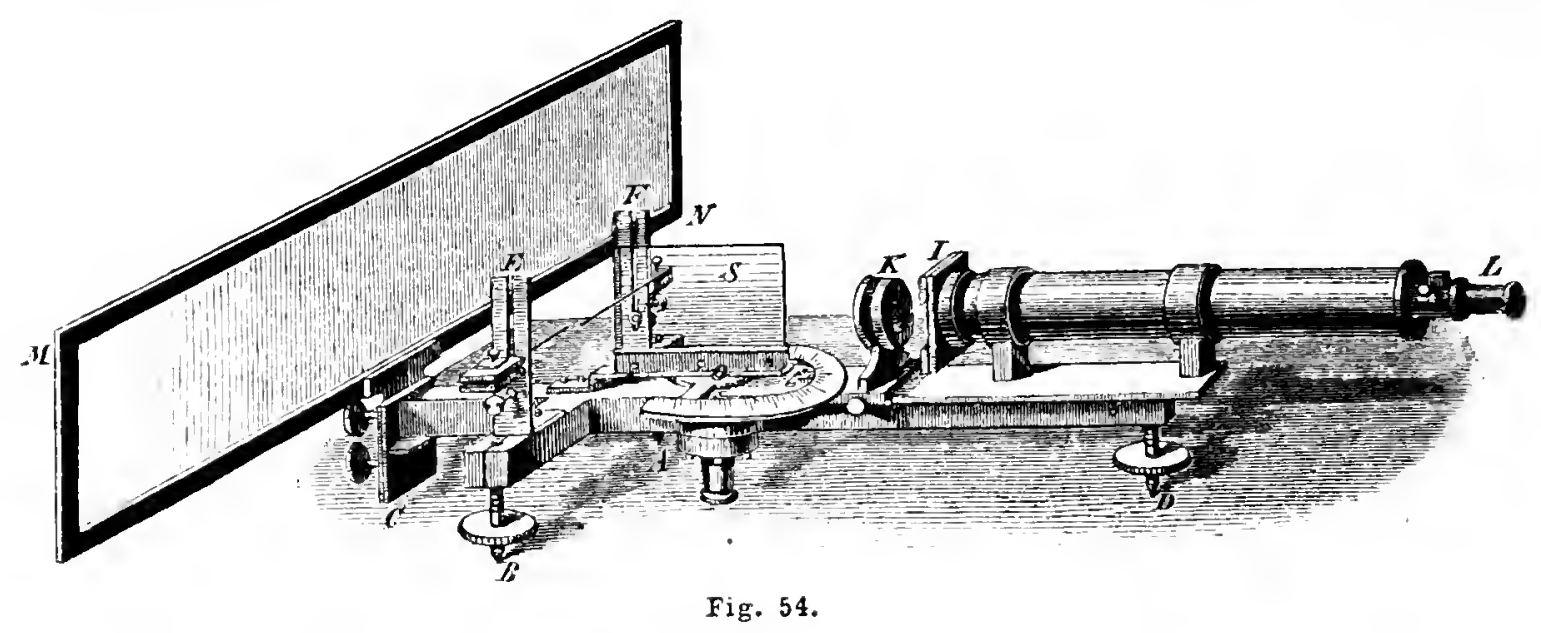

f̈̈rmigen Lntergestell, welches mittels der drei Fussschrauben $A, B, D$ horizontal gestellt werden kann. Bei $C$ ist ein transparenter senkrecht stehender l'apierschirm ML angebracht, welcher von den zu untersuchenden Lichtquellen belenchtet wird. Senkrecht zum Horizont und zur Ebene dieses Schirmes steht die plauparallele Glasplatte $S$. Zu beiden Seiten derselben zwischen ilı mond dem Schime betinden sich die Träger $E$ und $F$, welche zwei hurizontale Stiibchen oder Nadeln enthalten, die in jeder Hïle festogeklemmt werden kijnnen. Anf dem Statir befestigt ist fermer ein getheilter Kreis, dessen Mittelpunkt () genan nuter der Glasplatte liegt. Um einem durch () wehenden Zalufen liisst sich ein das Roln $I I$, tragencier horizontaler Arm frei drehen, so dass dieses Rohr muter jedem

1) Aratso. Simmtliche Werke. Deutsche Ausgabe von Hankel. Bd. 10, p. 156. 
beliebigen Winkel ron beiden Seiten her auf die Glasplatte gerichtet werden kann. Der Betrag der Drehung wird mit Hulfe eines gleichyeitig mit dem Arme beweglichen Nonius abgelesen. Das Rohr enthält keine Linsen, die Beobachtungen werden mit blossem Auge ausgefuhrt, und an Stelle des Objectivs befindet sich ein schmaler verticaler Spalt, welcher das Gesiehtsfeld beseluriukt.

Ist der Sehirm $M I N$ gan\% gleichmïssig dureh eine Liehtquelle von linten erleuchtet, und sicht man durch das Rohr auf die Glasplatte, so erblickt man gleichreitig einen 'Theil des Schirmes dureh die Platte hindurch und einen anderen Theil gespiegelt. An der Stelle, wo das gespiegelte ${ }^{-}$Bild der einen horizontalen Nadel erscheint, sieht man nur das durchgelassene Licht des Schirmes, und an der Stelle, wo die andere Nadel im durchgehenden Lichte sichtbar ist, sieht man bloss das gespiegelte Lieht des Sehirmes. Man kann das Rohr so weit gegen die Glasplatte drehen, bis die beiden schwarzen Streifen, welche man nebeneinander anf dem gleichmässig hellen Untergrunde erblickt, gleich intensiv erseheinen; dann weiss man, dass bei dieser Stellung das gespiegelte und durchgelassene Licht gleich sind. Um nun empirisch feststellen zu können, wie sich die Quantitäten des reflectirten und durchgehenden Lichtes bei jedem beliebigen anderen Winkel zu einander verhalten (theoretisch liesse sich dies nach den Formeln von Fresnel und Neumann berechnen), benutate Arago doppeltbrechende Krystallplatten, die in einer Hiilse $K$ und am Ende des Rohres bei $I$ angebracht werden konnten, and bestimmte zunïchst dureh Versuche diejenigen Stellungen des Beohachtungsrohres, bei denen das reflectirte Licht das Vicrfache, Doppelte, Ilallbfache, Viertelfache des durehgehenden betrug. Durch Interpolation ergab sich dann eine Tabelle, aus der für jeden beliebigen Winkel das betreflende Helligkeitsverhältuiss entnommen werden kounte. Mit einem derartig anf empirischen Wege kalibrirten Photometer liess sich mun das Helligkeitsverhältniss zweier beliebigen Lichtquellen ermitteh, wemn dieselben so aufgestellt waren, dass die erste nu die eine Hiilfte des transparenten Schirmes, die zweite nur die andere Hailfte belenchtete.

Weiter auf dic Theorie dieses Instrumentes und die Vorsichtsmassregeln, welche bei seiner Anwendung erwinscht sind, einzugehen, diirfte schon aus dem Grunde iiberflïssigr erscheinen, weil dasselbe ausser von Arago (und auch ron diesem nur zur Priifung der Polarisationsgesetze) niemals wieder benutzt worden ist und in der Himmelsphotometrie jedenfalls nur in ganz beschriuktem Grade zur Verwendumg kommen könnte.

Für die Lichtmessungen der Gestime hat Arago cine ganze Reihe anderer Eimrichtungen emp,fohlen, die fast alle spaiter fiur die Construction von Photometern massgebend gewesen sind, von ihm selbst aber nur in 
ganz wenigen Fällen zu wirklichen Beobachtungen am Himmel benutzt worden sind. Er hat zuerst auf die Wichtigkeit der Doppelbrechung im Kalkspath und Bergkrystall für die Photometrie hingewiesen und unter Anderem das Rochon'sche Prismenfermrohr (ein gewöhnliches astronomisches Fernrohr, bei welchem durch ein in der Nähe des Brennpunktes in den Strahlengang eingesetztes Rochon'sches Doppelprisma zwei Bilder eines Objectes hervorgerufen werden, deren Helligkeiten durch ein vor das Ocular gesetztes Nicolprisma beliebig verändert werden können) zur Vergleichumg der centralen Partien der Sonnenscheibe mit den Randtheilen und zur Vergleichung des aschfarbenen Mondlichtes mit dem übrigen

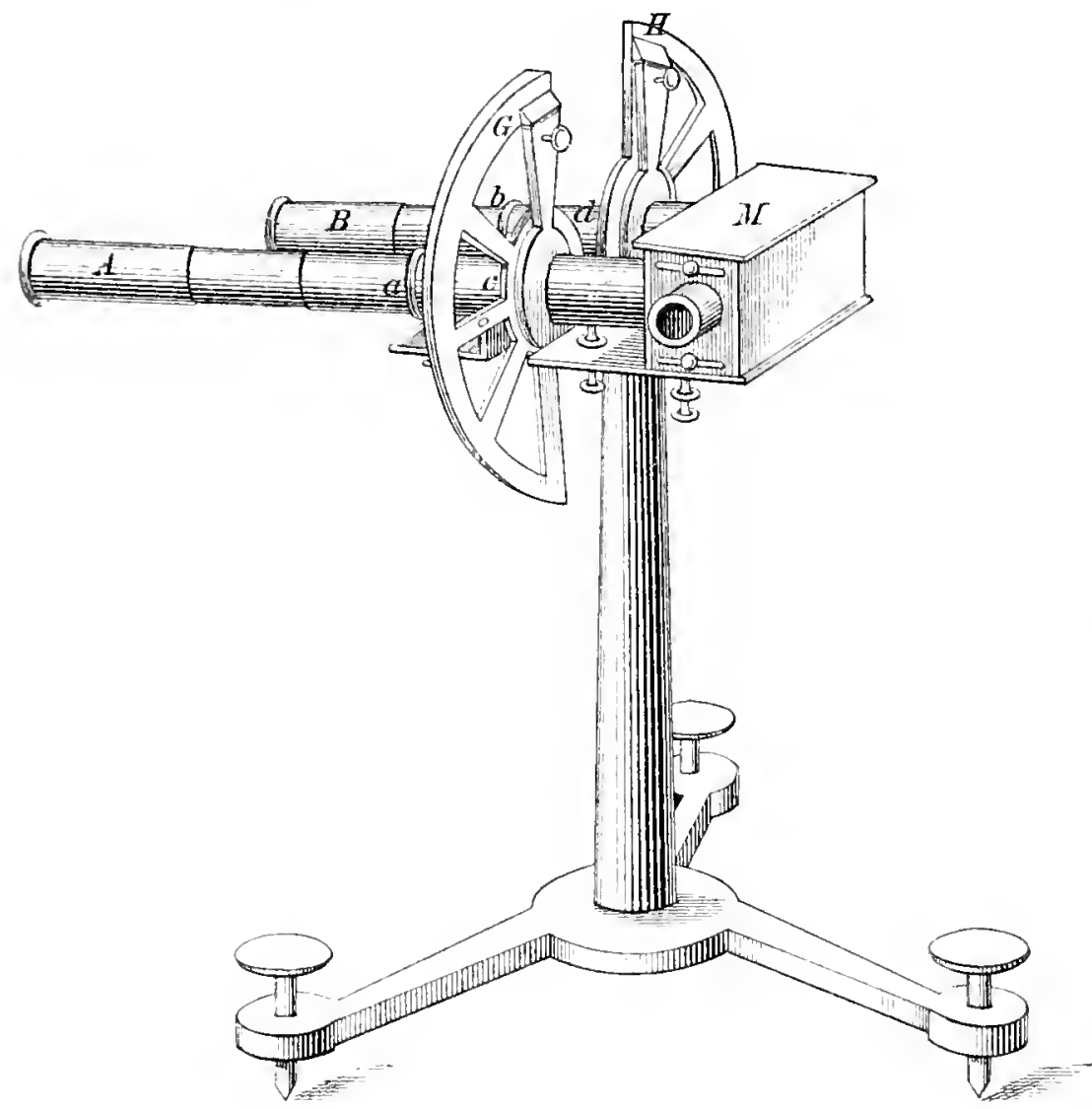

Fig. 55 . Mondlichte empfohlen. Von ihm rührt auch der Vorschlag her, das Licht der Sterne mit einem ganz ähnlichen Apparate in der Weise zu messen, dass man bei jedem Stern diejenige Stellung des Nicols bestimmt, bei welcher das eine Bild verschwindet.

Die Arago'schen Vorschläge sind ohne Zweifel fuir die Photometer von B e rnard! und Babi$\left.\mathrm{net}^{2}\right)$ vorbildlich gewesen. Diese Instrumente sind zwar in erster Linie zu technischen Zwecken bestimmt worden; ihre Verwendung bei gewissen Anfgaben der Himmelsphotometrie scheint aber keineswegs ausgeschlossen. Berinard hat sein Photometer insbesondere zu Absorptionsuntersuchungen benutat. Die Eimrichtung desselben ist aus Figur 55 ersichtlich.

Die beiden Rühren $A$ und $B$, welche an dem einen Ende durch Diaphragmen verschlossen sind, werden anf die zu vergleichenden Lichtquellen oder beleuchteten Flïchen gerichtet. Im Innern dieser Röhren

1: Ammales de chimie et de physique. Série :3, tome 35, p. 355.

2 Compter Rendus. Tome 37, p. 774. 
sind bei $a$ und $b$ \%wei Nicolprismen fest eingesetzt, zwei andere Nieolprismen sind bei $c$ und $d$ beweglich angebracht, die Drehungen können an zwei getheilten Halbkreisen $G$ und $H$ abgelesen werden. In dem Kasten $M$ sitzen zwei total reflectirende Prismen, welehe das von den kleinen Diaphragmenöfhinungen herkommende Lielit nebeneinander in ein gemeinschaftliches Ocular werfen. Dureh Drehung eines der beiden beweglichen Prismen oder anch beider liisst sich Intensitiitsgleichlheit herstellen, und dureh Vertauschen der beiden Liehtquellen lassen sieh die etwaigen Untersehiede der beiden optischen Systeme eliminiren. Das Cosinusquadratgesetz ermöglieht dann dic Bereehnung des Intensitiitsverhailtnisses. Wenn man die Diaphragmen an den Enden der Röhren durch Fernrohrobjective ron gleicher Grösse ersetzte und vor denselben drehbar totalreflectirende Prismen anbriichte, so liesse sich das Beruard'sehe Instrument, falls noch die Fernrohre in die Richtung Ost-West gestellt wiurden, in aihnlicher Weise, wie wir es sp:iter bei dem Piekering'sehen Instrumente sehen werden, als Meridianphotometer am Himmel benutzen. Im Prineipe ganz ähnlich dem Bernard'schen Photometer sind die ron Beer') nnd Beequerel2) construirten, auf die hier nieht näher eingegangen werden soll.

Wesentlich anders ist das Babinet'sehe, welches in erster Linie zur Vergleichung der Helligkeit von Gasflammen bestimmt war. Dasselbe (Fig. 56) bestelit aus einer Röhre $A B$, in welche seitlich unter einem Winkel von etwa $60^{\circ}$ eine zweite Röhre $C D$ einmlindet. Beide Röhren sind durch mattgesehliffene Glasplatten oder durch Diaphragmen

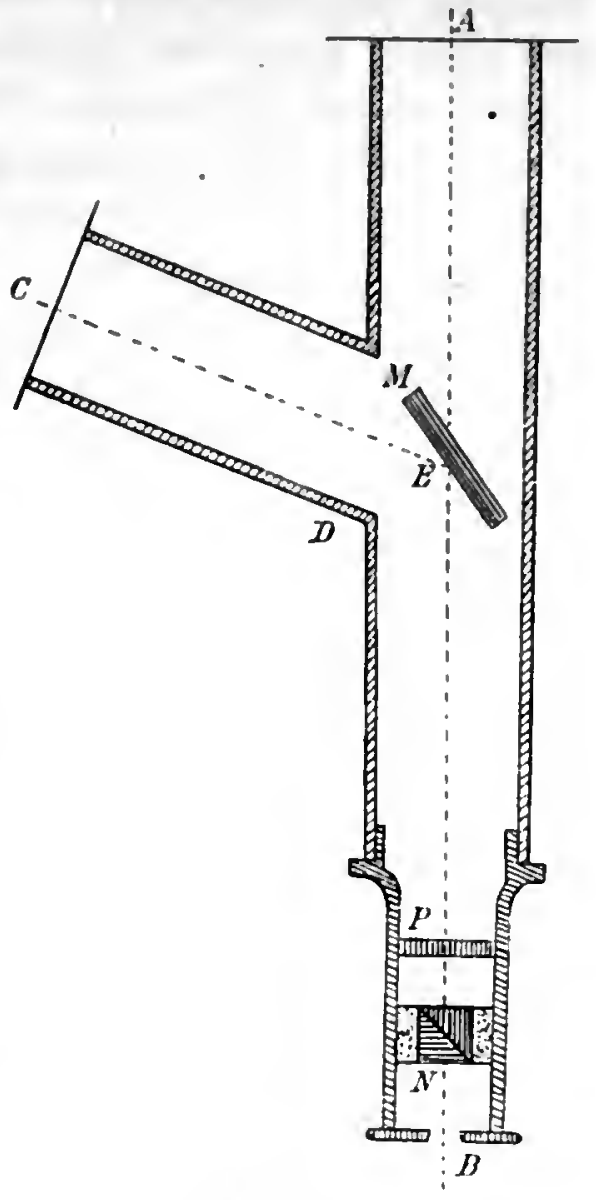

Fig. 56. mit messbar veriinderliehen Öffnungen verschlossen. Bei $E$ ist im Innern der Röhre $A B$ ein Glasplattensatz $M$ eingesetzt, welcher den Winkel der beiden Röhren halbirt. Die zu vergleichenden Lichtquellen befinden sich vor den Öffnungen $A$ und $C$. Das bei $C$ eindringende Lichtbindel wird nahe unter dem Polarisationswinkel ron der Glassiule reflectirt und ist daher beinahe vollstindig in der Einfallsebene polarisirt; dagegen besteht das durch die Glassïnle hindurehgegangene, ron $A$ herkommende Licht-

1) Pogg. Annalen. Ba. 56, p. 78.

2) Annales de chimie et de physique. Série 3, tome 62, p. 14. 
blindel zum Theil ans natiirlichem Lichte, zum Theil aus solchem, welches in einer zur Einfallsebene senkrechten Ebene polarisirt ist. Sind die beiden Lichtquellen gleich intensiv, so enthalten nach dem früher erwähnten Satze von Arago das reflectirte und das durchgelassene Lichtbuindel gleiche Quantitäten von entgegengesetzt polarisirtem Lichte, und das aus beiden zusammengesetzte Doppellichtbiindel $E B$ verhält sich ganz wie natirliches Licht. Sind aber die Lichtquellen ungleich intensiv, so bleibt dieses Doppellichtbündel partiell polarisirt. In das Rohrende $B$ ist ein Soleil'sches Polariskop eingeschoben, bestehend ans dem analysirenden Nicol $N$ und einer davor befindlichen Doppelquarzplatte $P$, die aus zwei ancinander gekitteten Hälften zusammengesetzt ist, von denen die eine rechtsdrehend, die andere linksdrehend ist. Wenn auf ein solches Polariskop partiell oder vollstaindig polarisirtes Licht auffällt, so erscheinen die beiden Hälften des Bildes versehieden gefürbt, dagegen sind sic gleich gefiurbt, falls das auffallende Licht natiurliches ist. Bei Benutzung des Babinet'schen Photometers stellt man entweder durch Verïnderung der Distanzen der Lichtquellen von der Eintrittsfl̈̈che oder durch Variirnng der Diaphragmenöffumugen die gleiche Färbung der beiden Hälften im Polariskol, her und kann daraus das Helligkeitsverhältniss der beiden Lichtquellen ermittehn.

\section{b. Die Züllner'schen Photometer.}

Von den beiden Instrumenten, welche Zöllner in die Photometrie eingefiilurt lat, stanmt das eine aus dem Jahre $1955^{\circ}$ ), das zweite, das bekimute Astrophotometer, ist zuerst in einer im Jahre 1861 ersehienenen Abhandlnng'2) beschrichen worden, die urspringlich als Bewerbungssehrift fiir eine ron der Ikademie der Wissenschaften in Wien ansgeschriebene Preisanforbe eingereicht war. Beide Instrumente sind dann nach wesentlichen Modificationen und Verbesserungen ansfiihrlich in Zöllners »Photometrischen Tntersuchumgen behandelt worden mit Beriicksichtignng derjenigen Formen, die nachler im Grossen und Ganzen massgebend geblieben sind.

Dals erste Instrument war ansser zu technischen Zweeken nur fiir die Beobarlitumg der allerhellsten Ilimmedkörper bestimmt und ist ron Zöllner selbst zu seinen Lichtnessmgen ron Simne und Mond verwendet worden. Es kommt dabei anf die Tergleichmng der Helligkeit zweicr ummittelbar aneinander senzenden Flädhen an, und es wird die durch Reflex herrorgerufene Polanisation rerrerthet.

1) Pogg. Annalen. Bd. 100, p. 351

2 Zïllner, Grundziige einer allgemeinen Photometrie des Himmels. Berlin, 1861. 
In einem Stativ $C$ (Fig. 57) ist der Haupttheil des Apparates un die horizontale Axe $A B$ drehbar und kann in jeder Zenithdistanz. festgekleinmt werden. Wine Petrolenmlampe $F^{\prime}$ ist anf einem starken Arme befestigt und dreht sich in Azimuth \%ugleich mit dem gam\%en Apparate um eine verticale Axe. Das Licht der Flamme a fillt durch ein Diaphragma $r$ auf die Convexlinse $b$, tritt aus dieser parallel aus, gelangt auf den kleinen Silberspiegel $c$, ron diesem anf den Polarisationsspiegel $f$ ans seliwarzem Glase, dessen Normale mit der Axe $D E$ den Polarisationswinkel fir Glas einschliesst, und tritt endlich durch die Convexlinse $g$ und das Nicolprisma $h$ in das Auge bei $o$. Der Spiegel $f$ stelit so, dass die scharfe Kante das kreisfürmige Gesichtsfeld halbirt, und wenn die Linse $g$ auf diese Kante eingestellt ist, so erblickt man die eine Hälfte des Feldes durch das in der Ebene der Zeichnung polarisirte Lieht der Flatumme a belenelitet. An dem Ende des Hauptrohres ist der ebenfalls aus sehwarzem Glase gefertigte Polarisationsspiegrel ' $d$ augeloracht, und zwar so, dass

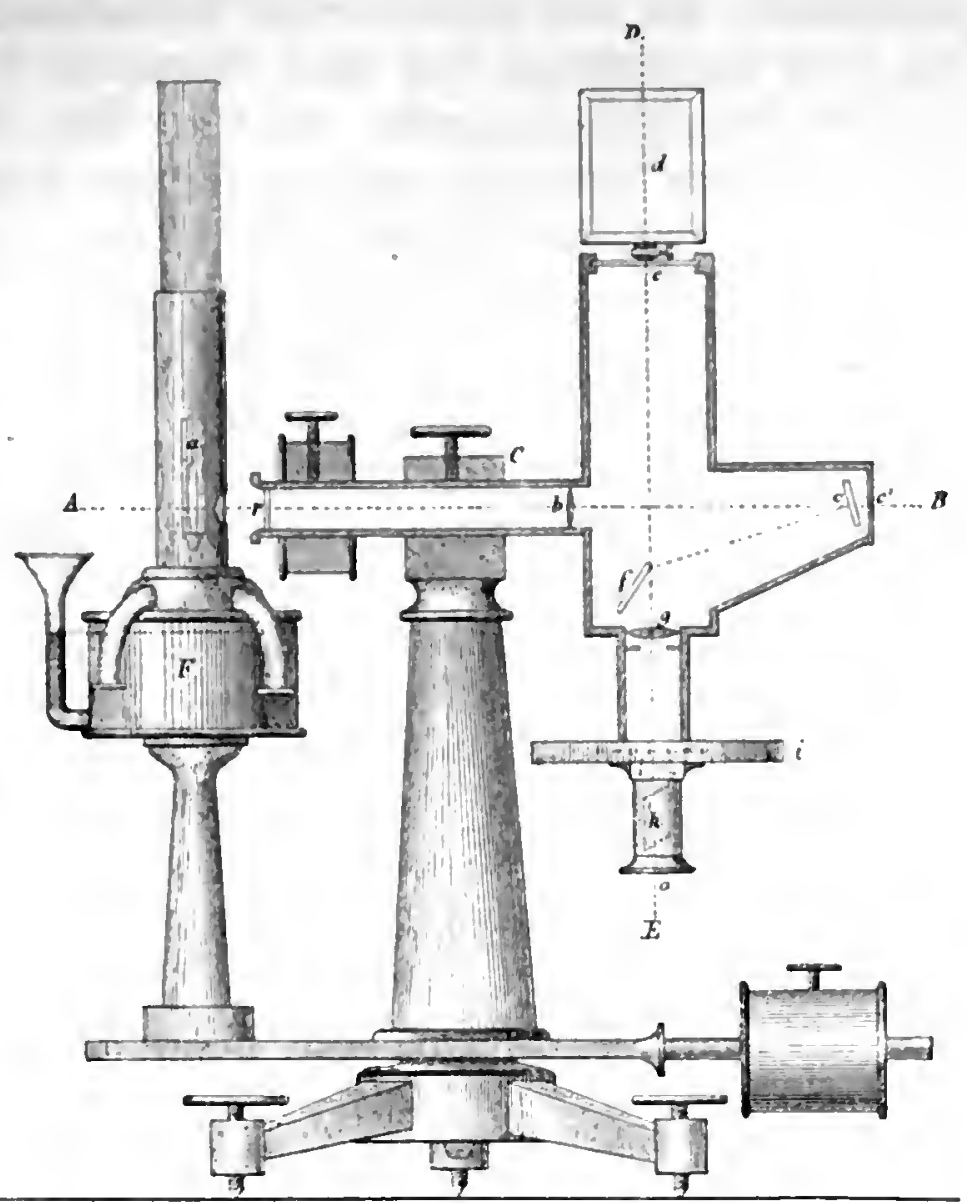

Fig. 57 . seine Ebene senkrecht liegrt zu einer dureh $D E$ normal zur Zeichnungsfliiche stehenden Ebene, und dass ausserdem die Normale zu diesem Spiegel mit der Axe DE den Polarisationswinkel bildet. Das ron d reflectirte Licht einer Lichitruelle greht bei $f$ rorbei und beleuchtet die zweite llälfte des Gesichtsfeldes. Das Lieht ist senkreelit zur Ehene der \%eichnung polarisirt und kamn daher dureh Drehung des Nicols h dem Liehte der Tergleiehsflamme grleich gemacht werden. Die Beriihrung der beiden Ilälften des Gesichtsfeldes ist so vollkommen, dass bei eintretender Gleiehheit der Intensitiit das Gesichtsfeld als eine einzige leuehtende Scheibe erscheint. Das Quadrat 
der Tangente des Drehungswinkels des Nicols, welcher an dem Kreise $i$ abgelesen wird, giebt dann das Verhältniss der Helligkeit der beobachteten Lichtquelle zum Vergleichslichte. Úm noch die bei den meisten Beobachtungen störende röthliehgelbe Färbung der von der Lampe erleuchteten Hälfte zu beseitigen, wird in den Blecheylinder der Lampe ein Stiick blanen Kobaltglases eingesetzt, welehes der Flamme einen gelblichweissen Farbenton giebt. Handelt es sich um die Messung des Sonnenlichtes, so wird vor dem Spiegel $d$ noch eine mattgeschliffene Glasplatte :mgebracht, um eine gleichförmige Erlenchtung zu erzielen; auch können bei $e$ zur Schwächung des allzu intensiven Sonnenlichtes Blendgläser in den Strahlengang eingesetzt werden. Die Genanigkeit der Messungen ist bei diesem Apparate ansserordentlich gross. Nach Zöllner beträgt der walnscheinliche Fehler einer einzelnen Vergleichung zweier Licht(quellen nur etwa 2-3 Procent des Helligkeitsrerhültnisses. Sehr sorgfilltig ist darauf zu achten, dass die zu untersuchenden Lichtquellen kein polarisirtes Licht enthalten, da sonst die Resultate erheblich verfälscht werden kïmnten.

In dieser Bezichung ist jede Gefahr ansgeschlossen bei dem zweiten Züllner'schen Photometer, welches ausschliesslich zn Himmelsbeobachtungen bestimmt ist, und bei welchem die polarisirenden Medien mur zur Verandermg der Helligkeit der Verghleichsflamme benutzt werden. Die Form, welche Zöllner nach manchen Ändermgen diesem Instrumente gegeben lat, ist durch Figur os illustrirt.

Die Fermrohraxe $A B$ wird durch Bewegonng in Höhe und dureh Drehung des ganzen Apparates im Azimith anf das zu messende Himmelsobject geriehtet, und das in der Brennebene bei $b$ entstehende Sternbild wird mit zwei in derselben Ebene durch die Petroleumlampe $F$ entworfenen kiinstlichen Sternen gg rerglichen. Zur Erzengung der kiunstlichen Sterne dienen die rerschiedenen in der seitlichen Axe CD angebrachten Medien. Bei o' ist eine feine Öfinung (in den neneren Instrumenten befindet sich an dieser Stelle eine drehbare Scheibe mit verschieden grossen öftumgen, mm den kiunstlichen Sternen beliebige Grösse geben zu lï̈nnen); durch diese Öfỉunng fällt das Licht der Lampe anf die Biconcarlinse m, welche die Bestimmung hat, das Bild der Öffnung zu rerkleinern. Das Licht passirt damm das Nicolprisma $k$, die senkrecht zur Ixe geschliffene Berokrytallplatte $l$, fermer die beiden Nicolprismen $i$ und $h$ und wird dam durch die sammellinse $f$ auf die planparallele Glasplatte $e e^{\prime}$ geworfeu und endlich zu zwei punktförmigon Bilderu durch Reflex an der rorderen und hinteren Glasfläche, gg vereinigt. Die Bilder werden mit dem schwarh rerorisseruden Oculare o betrachtet. I'm sleichzeitig die wirklichen und die kinstlichen Sterne scharf einstellen 
zu küunen, war bei deu älteren Formen des Züllner'sehen Photometers die Convexlinse $f$ versehiebbar; diese Verschiebung gestattete die Pointirung anf die kilustliehen Sterne, naehdem der wirkliche Stern mittelst des Oculares o eingestellt war. Bei den neneren Formen des Apparates wird meistens das Objectiv $O$ durch 'Trieb verstellbar eingerichtet und die Convexlinse $f$ bleibt muverindert. Das letzte Nicolprisma $h$ sitzt in dem seitlichen Rohre fest, und da das anstretende polarisirte Licht von

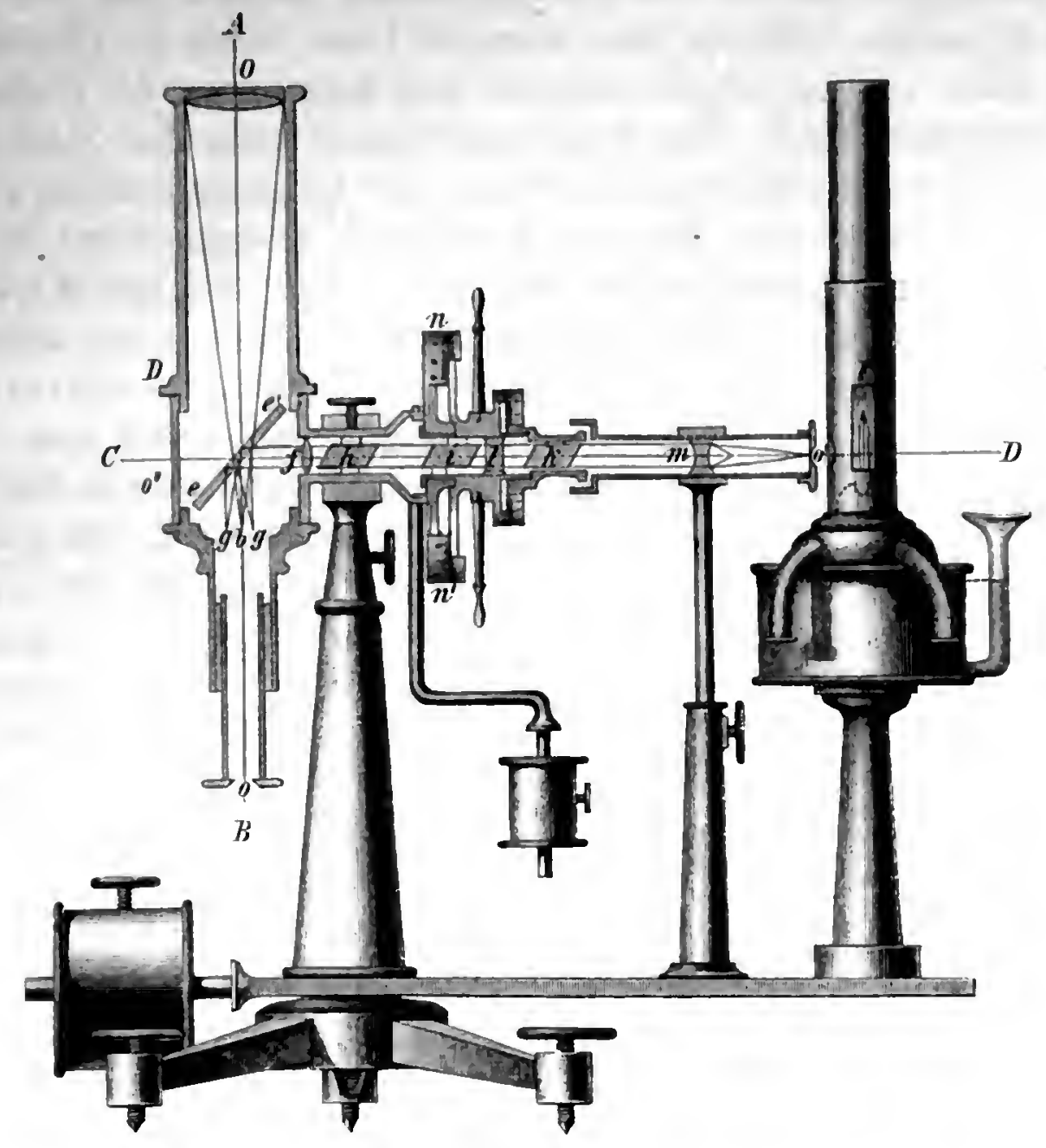

Fig. 58.

der Glasplatte zuriickgeworfen wird, so ist zur Erzichumg rles grösssten Lichteffectes erforderlich, dass der Ilauptschnitt dieses Prismas in der durch die Figur reprïisentirten Schnittebene liegt, eine Vorschrift, die nicht immer geniigend beachtet wird. Die beiden Nicolprismen $i$ und $k$ mit der zwischen ihnen befindlichen Bergkrystallplatte sind gegen das feste Prisma $h$ drehbar, und die Drehnug kanm an zwei Nonien $n$ und $n^{\prime}$ abgelesen werden. Der mitgehende getheilte Kreis, der Intensitiitskreis, wird an besten in jedem der rier Quadranten von $\left(0^{\circ}\right.$ bis $90^{\circ}$ 
getheilt, und die Stellung von $i$ wird so regulirt, dass bei der Ablesung 0" gar kein Licht anf die Glasplatte fällt; dann wird bei irgend einer anderen Ablesung die Helligkeit des künstlichen Sternes nach dem Malusschen Gesetze proportional dem Quadrate des Sinus des abgelesenen Winkels. In der Figur ist die Biconeavlinse $m$ auf einer besonderen Siiule montirt und bleibt sowohl bei Bewegung des ganzen oberen Instrumenttheiles mit dem Fernrohre $A B$, als auch bei der Drehung des Intensititskreises fest ror der Lampenöffinung stehen. Bei den meisten neneren Apparaten bleibt die Sänle weg; die Linse, sowie die Diaphragmenscheibe sitzen in dem seitliehen Rohre und nehmen an der Drehung des Intensitiitskreises 'Theil. Die Bergkrystallplatte $l$ und das Nicolprisma $k$ habeu den Zweek, den kiinstlichen Sternen eine beliebige Färbung zu geben. Dals l'risma $h_{i}$ ist nämlich fiir sich (gewöhnlich zusammen mit $m$ nnd $o^{\prime}$ ) gegen die anderen polarisirenden Medien drehbar, und der Winkel, den der Hamjtsehnitt ron $l_{i}$ nit demjenigen ron $i$ bildet, kann mittelst der Indices $c$ mol $c^{\prime}$ an einem getheilten Kreise, dem Colorimeterkreise, algelesen werden. Ist dieser Winkel bekannt, und nimmt man eine bestimmte Dieke der Bergkrystallplatte an (man wählt gewöhnlich $5 \mathrm{~mm}$ ), so ist die Farbe des Sternes unzweidentig charakterisirt. Die ganze Einrichtung dient in erster Linie dazu, die Farbe der kiinstlichen Sterne möglichst der der wirklichen Sterne gleich zu machen, sie kann aber anch zu directen Farbenmessungen der Gestirne benutzt werden. Will man auf den letzteren Zweck von rornherein rerzichten, so wäre es einficher, l und li ganz fortzulassen und dafiur ein geeignetes blanes Glas in den Gang der ron der Lampe kommenden Strahlen einzuschalten, welches den kiinstlichen Sternen eine mittlere Sternfarbe giebt. Die mechaniche Ausfühmng des Apparates wiirde dadurch erheblich vereinfacht werdeu.

Es ist iblugens merkwiirdig. dass ansser Zö̈llner Niemand ernstlich rersucht hat, das colsimeter zu umfangreicheren Farbenmessungen am Himmel zu rerwenden. Die hervorgebrachten Farben sind zwar eigent-

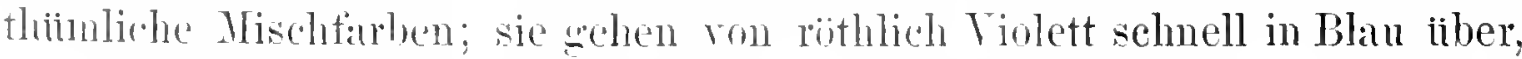
damn etwats laugsaner durch grimliche Niancen nach Hellgelb und dann rehr allmihlich dureh die rerschiedenen Stufen des Gelb nach Orange nnd Purpuroth. Die Farbe der am Himmel am meisten verbreiteten weisclichen nud gelblichweisen steme lisst sich iiberhanpt nicht rollständig herstellen; dagegen finden sich fiir alle gelblichen, gelben und röthlichen steme cutspreclende Farben ann colorimeter, und es wiirde zweitelhs eine hïchst rerdienstliche und lohnende Arbeit sein, an solehen Sternen. zu denen anch die meisten Verindlerlichen gelö̈ren, ansgedehnte Farbenmesungen mit dem Zöllnersehen Colorimeter amzustellen. Das 


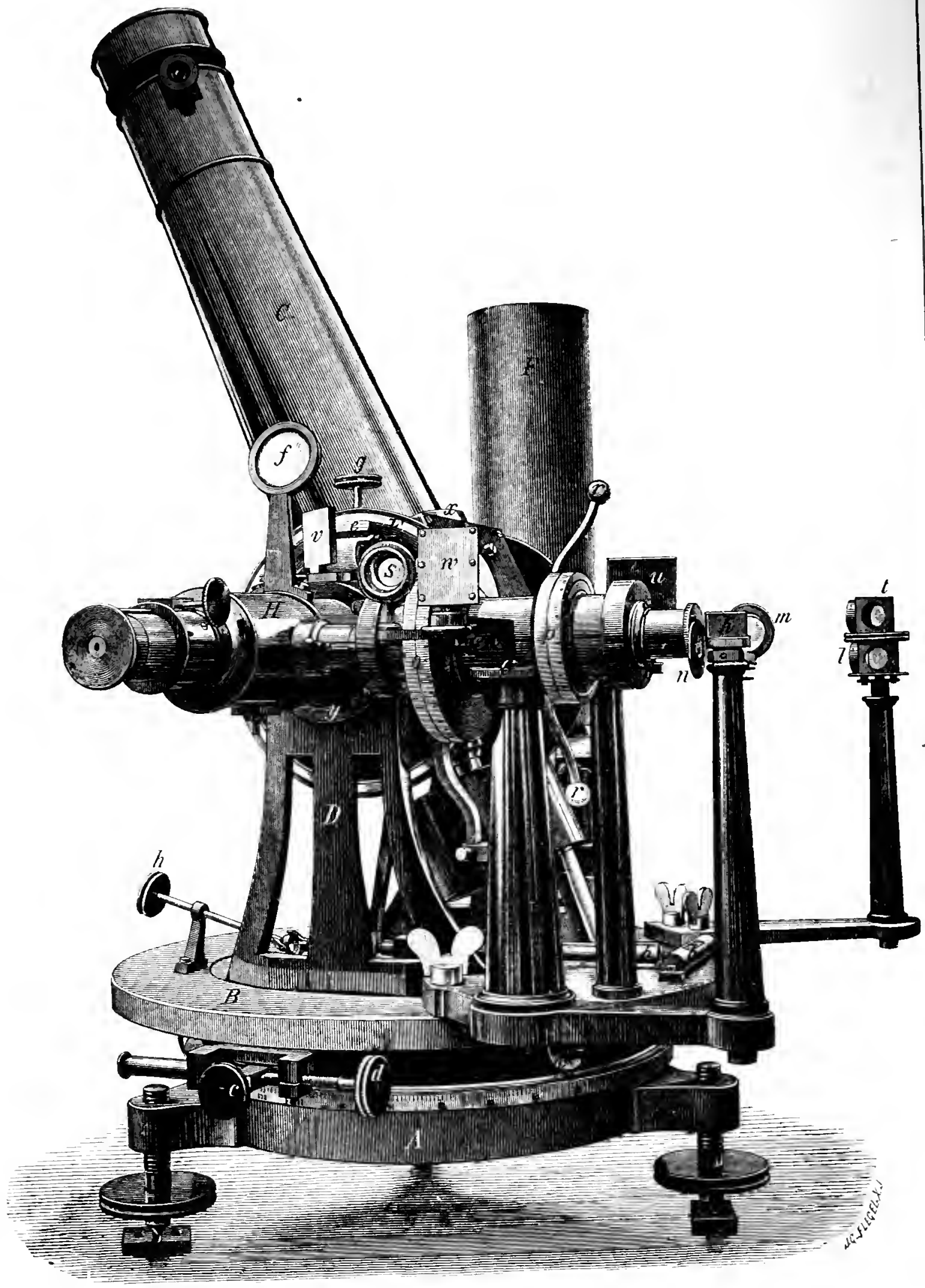

Fig. 59. 
Colorimeter kamn noch zu einer interessanten Untersuchung llber die Beurtheilung der Gleichheit versehieden gefïloter Sterne benutzt werden. Man riehtet das Ferurohr auf irgend einen Stern am Himmel, giebt den kllustlichen Sternen durch Einstellen auf bestimmte Striehe des Colorimeterkreises verschiedene. Farben und stellt die Glejehheit am Intensititskreise her. Dureh Vergleiehung der von verschiedenen Beobachtern auf diese Weise erlaltenen sogenamten Intensititstabellen ist es möglich, ein Urtheil uber die Farbenanffussung derselben zu gewimnen. Wie eine von mir gegebene Zusammenstellung') zeigt, kommen, mit Ausualime bei den allerextremsten Farben, keine sehr merklichen Auffassungsunterschiede vor.

Zur Vervollstïndigung der Beschreibung der alten Form des Zölnerschen Photometers ist noeh zu bemerken, dass der obere Theil des Fernrohrs bei $D$ abgeschraulit und naeh Bedarf dureh Objeetive von längerer oder klirzerer Brennweite ersetzt werden kann. Ferner muss vor das Ocular o bei den meisten Beobachtmugen, um die unbequeme Lage des Kopfes zu vermeiden, ein total reflectirendes Prisma gesetzt werden, was leider die Sieherheit der Messungen ein wenig beeinträehtigt.

Figur 59 stellt ein anf der Potsdamer Sternwarte befindliches Zöllnersehes Photometer dar, bei welehem der znletzt erwïhnte Übelstand rermieden ist. Dasselbe hat die Form eines Passageninstrumentes; der Beobachter blickt daher stets in horizontaler Richtung in das Ferurohr. Die Lampe befindet sich nicht unmittelbar vor der Diaphragmenöfhnung, sondern steht dem Oeular gegeniiber anf dem festen im Azimuth drehbaren Untergestelle $B$. Das Licht gelangt durch Reflex an den total reflectirenden Prismen $i$ und $k$ in den seitlichen Theil $G$ des Photometers, weleher die drei Nicolprismen mit der Bergkrystallplatte enthiilt. Zwei Linsen $l$ und $m$, welehe in den Gang der Lichtstrahlen eingefitgt sind, sammeln das Licht und entwerfen auf der Diaphragmenscheibe " ein scharfes rundes Lichtbildchen. Was die Handhalmmg dieses $A_{p}$ prarates noch mehr erleiehtert, ist der Umstand, dass alle Kreise von der Photometerlampe sellsst mit Hiilfe des total reflectirenden P'rismas t und der Spiegel $u, v$ und $w$ belenchtet werden, so dass dir Einstellungen und Ablesmngen olne Beobachtungslampe ansgefuhrt werden können. Das grosse Objectiv von 67 mun öfinme.. nul 700 mm Brennweite laisst sich mit zwei kleineren (36.5) mm (Offinmug mnd 350 mm Bremweite, resp. $21.5 \mathrm{~mm}$ Öfinm tanschen, die an den in der Figur mit $x$ und ! bezeichneten Stellen eingesetzt werden kömen. Auf diese Weise ist es möglich, mit deu

1) Publ. des Astrophys. Obs. zil Potsdam. Bd. 3. p. 24.5. 
Apparate Sterne von der siebenten Grösse bis zn den allerhellsten zu beobaehten und auch die grossen Planeten in den Messungsbereieh zu ziehen, da diese mit dem kleinsten Objective durchaus punktförmig erscheinen ').

Un aueh die schwäehsten Sterne beobachten zu können, hatte bereits Zölluer eine Einriehtumg getroffen, die gestattete, sein Photometer mit jedem beliebigen Refractor in Verbindung zu bringen. Eine dafür sehr geeignete handliche Form des Apparates, welehe in Potsdam benutzt wird, ist in Figu 60 dargestellt.

In dem Ringe $A$, weleher an Stelle des Oculars an den Refraetor angeschranbt wird, dreht sich das Photometer frei, so dass die Axe $C D$ bei

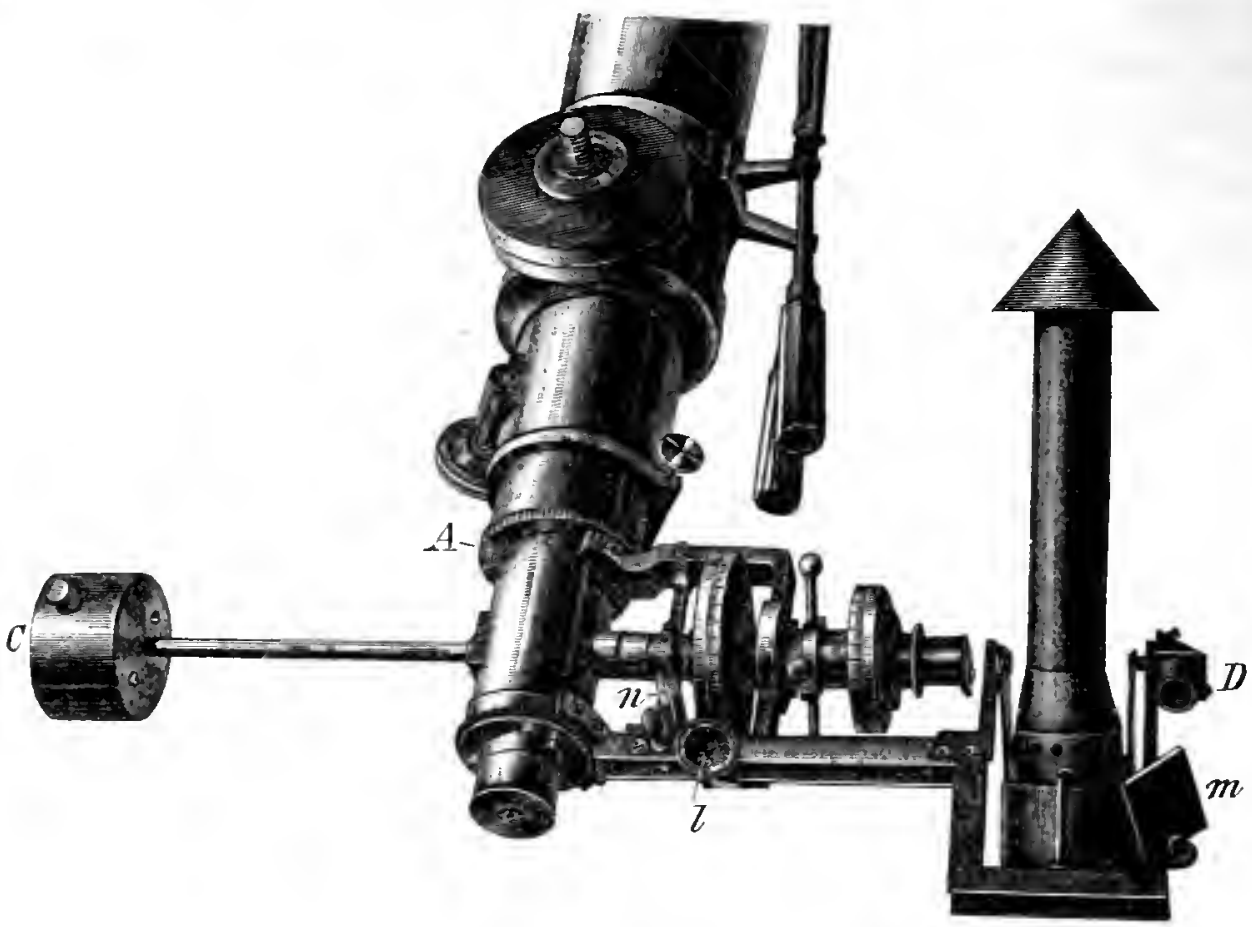

Fig. 60 .

jeder Stellumg des Ferurohrs horizontal bleiben und die bewegliche Lampe genan rertical hängen kam. Anch hier ist es so eingerichtet, dass das Licht der Flamme zur Bcleuchtung des Intensitaitskreises beuntzt wird, indem dasselbe mittelst des Prismas $D$ und der Spiegel $m$ und $n$ anf die Theilung geworfen wird; die Ablesung geschieht mit Hiilfe der schwach vergrösseruden Lupe .

Beachitenswerth ist ein Torshlag. den Ceraski²) gemacht hat, um ohue Vertanschen der Objective sonvohl schwache als anch sehr helle Sterue beobachten zn kömnen. ('eraski bringt in der Axe $C D$ (Figur 5s)

1 Eine ausfibliche Beschreibung des Aprarates findet sich in den Pnbl. des Astrophys. Obs. zu Poisdam. Bd. S, p. 17

2 Anuales de lobs de Lloscon. Sirrie 2. Vol. I. lirr. 2. p. 13. - Siehe anch Astr. Nachr. Bd. 120. Nr. 2670. 
an dem der Lampe entgegengesetaten Ende ungefalur bei $o^{\prime \prime}$ ein zweites Ocular an, mit welehem der kllustliche Stern dịreet gesehen werden kaun, wihlirend die wirklichen Sterne von der Glasplatte $e e^{\prime}$ in 'das Ocular reflectirt werden. Da der Liehtverlust bei der Reflexion unter. $45^{\circ}$ etwa 3 bis 4 Grüssenclassen betriggt, so kaum man mit Hulfe des doppelten Oculars ein sehr betriichtliches Helligkeitsintervall durehmessen.

Über den Gebrauch des Zölhner'sehen Photometers und die mit demselben жu erreichende Genauigkeit mögen noch einige Bemerkungen Plat\% finden. Gewisse Vorurtheile haben dem Instrumente vicht diejenige Verbreitung versehafft, welche es ohne Zweifel verdient. Man macht ihm hauptsiichlich die Benutzung des kunstlichen Vergleiehslichtes zum Vorwurfe. Zöllner hatte sich anfangs bemitht, den kilnstliehen Sternen dureh Construction einer besonderen Gaslampe constante Helligkeit zu geben, um so zu versehiedenen Zeiten und an versehiedenen Orten angestellte Messungen direet miteinander vergleichbar zu maehen. Die Einriehtung erwies sich jedoeh als viel $/$ eomplicirt, und Züllner fuhrte daher eine einfache Petroleumlampe ein, deren Flamme auf eine durch ein Diopter bestimmte Höhe eingestellt wurde. Anch heute bedient man sich noch anssehliesslich dieses Hülfsmittels. Man kam freilich nicht erwarten, dass auf diese Weise die kiinstliehen Sterne lange Zeit hindureh constante Helligkeit besitzen. Dies wird, alggesehen von anderen Unständen, dadureh unmöglich gemacht, dass sich an der die Cylinderöffiuung abschliessenden Glasplatte Russtheilehen und an den Öfhinngen der Diaphragmenseheibe Staubpartikelchen ansetzen, welche allniihlich die Helligkeit verringeru. Züllner hat zwar bei seinen Vergleichungen von Sonuenund Mondlicht viele Wochen hindureh die unverinderte Intensitiit der Lampe zu constatiren vermocht und infolge dessen die Messungen unbedenklich anf die Lampenlıelligkeit als Eimheit bezogen. Indessen diurfte dieses Beispiel doch nicht nachahmenswerth sein, und man sollte es sich zur strengeu Regel machen, die kinstlichen Sterne stets nur als Verbindungsglied zu benutzen und lediglich Differenzmessungen am Himmel anzustellen. Alle Beobachter, die sich eingehend mit dem Zöllner'schen Photometer beschiaftigt haben, stimmen darin iiberein, dass an ein und demselben Beobaehtungsabende die Lampe stundenlang vollkommen gleichmässig brennt, namentlich wenn mau die nöthigen Vorsichtsmassregeln nicht ausser Acht liisst, nïmlich erstens Flachbremer (nicht Rundbrenner) benutzt, zweitens fiir sorgfailtige Reinhaltmig des Dochtes sorgt und endlieh nur das beste Petroleum verwendet. Ceraskiy) lat ausführliche Untersuchungen iiber diesen Gegenstand angestellt, insbesondere den

1 Annales de l'obs. de Moscou. Série 2, Vol. I, livr. 2, p. 1:. 
wirksamsten Theil der Flamme bestimmt und Einrichtungen zur genauen Einstellung anf denselben empfohlen; er findet, dass bei gehöriger Vorsicht die Flamme 10 Stuiden lang constant bleibt. Nach meinen eigenen Erfahrmugen halte ich es, auch wenn man nicht besondere Vorsicht anwendet, für durchaus unbedenklich, sich ein bis zwei Stunden lang auf die Constanz der Lampe zu verlassen. Längere Zeit wird bei zweckmässiger Anordumng der Beobachtungen kaum erforderlich sein. Empfehlenswerth ist es, vor Beginn der Beobachtungen die Lampe erst einige Zeit (vielleicht 10 bis 15 Min.) brennen zu lassen, weil sich die Helligkeit bald nach dem Anzïnden gewöhulich etwas ändert. Eine grosse Gefahr ist das durch Wind und Luftzug hervorgebrachte Flackern der Flamme, welehes namentlich das Beobachten im Freien wesentlich erschwert. Man kamm sich zwar dureh zweckmässige Construction der Blechcylinder, wie es bei den Potsdamer Photometern geschehen ist, theilweise dagegen schiitzen, es wiirde aber eiue wesentliche Verbesserung des Apparates erzielt werden kömnen, wenu es geelänge, austatt der Petroleumlampe das elektrische Licht nutzbar zu machen. Bei der gegenwiirtig erreichten grossen Verrollkommunng der elektrischen Beleuchtungscinrichtungen und nach den Erfuhrungen, die z. B. in juingster Zeit in Bezug auf die constante Helligkeit der Gliihlampen in der technisehen Reichsanstalt in Charlottenburg gemacht worden sind ${ }^{1}$ ), erscheint die Sache keinesweg's atussichtslos, und es kimn nicht dringend geemng zu Versuchen in dieser Richtung anfgefordert werden.

Ein zweiter Einwnrf gegen die Benutznug der kïnstliehen Sterne beim Zölhner'schen Photometer, der viel sehwieriger als der Vorwurf nicht geniigend gleichmïssiger Lichtintensitit zuriiekznweisen ist, bezieht sich anf das nicht rollkommen gleichartige Anssehen der wirklichen und der kinstlichen Steme. Hier liegt wirklich cin Mangel ror. Denn die Bilder der kimstlichen Steme sind kleine schart begrenzte runde Scheibchen rou etwas mattem Anssehen, die sich roin den strahlenfömigen Sternbildem anf den ersten Blick nuterscheiden, besonders anffallend dann, wemu die letzteren durch starke Luftumbule in wallende Bewegung versetzt werden. Ls gehört eine ziemlich lange Übung dazn, bevor das Ange sich an das rerschiedene Anssehen wewöhnt und das Gefuhl der Unsicherheit verhoreu hat, und die Gefahr ist uiemals ganz ansgeschlossen, dass bei directer Terghleichung sohr heller und seh" schwacher Sterne Anffassmugsehler ins spiel kommen. Ton der grössten Wichtigkeit ist daher die Wahl der I)iaphragmenöthmug: die sich stets nach der speciellen Anfgabe, die man in Ange hat, riviten sullte. Man wird am Besten

1) Zeitschr. f. Instrumentenkunde, Jalur@. 10 1690, 1. 119. 
eine solehe Öflinung benutzen, dass die Bildgrösse der künstlichen Sterne etwa in der Mitte liegt zwischen den Bildgrössen der hellsten und der schwäichsten Sterne, die man beobachten will. Je grösser die zu messende Helligkeitsdifferen\% ist, desto mehr wird sich die Versehiedenheit des Aussehens geltend machen, und es ist bedauerlich, dass der dadurch herbeigefuhrte Fehler die Resultate stets in einem bestimmten Sinne beeinflusst. Man misst die schwachen Sterne verhältnissmïssig zu hell und dio hellen verhültnissmässig zu schwach, und die Folge davon ist, dass man im Allgemeinen ein bestimmtes Helligkeitsintervall mit dem Zöllnersehen Photometer zu klein findet. Um diesem Mangel naeh Möglichkeit abzuhelfen, ist es streng zu vermeiden, grosse Helligkeitsdifferenzen direet zn messen. In dieser Beziehung ist etwa ein Helligkeitsintervall von drei Grössenclassen als Grenze anzusehen, und es sollte als Regel gelten, wenn es irgend angeht, nur Ablesungen zwischen $10^{\circ}$ und $40^{\circ}$ am Intensitätskreise zu benutzen. Wenn es erforderlich ist, grössere Unterschiede zu messen, so ist es entsehieden rathsam, das Intervall zu theilen und verschiedene Objective und Diaphragmenöftunngen, eventuell auch Blendglïser, zu verwenden und zur Übertragung Sterne von mittlerer Helligkeit zu benutzen. Bei einiger Übung lernt man sehr bald die gecignetsten Vorsichtsmassregeln kennen, um den gefährliehen Einfluss des versehiedenartigen Aussehens, wenu nicht ganz zu beseitigen, so doch auf ein Minimum zu besehränken.

Infolge der Reflexion von der Vorder- und Riickflïche der Glasplatte sieht man, wie schon erwïhnt, im Zöllner'sehen Photometer zwei kinstliehe Sterne, deren Distanz von der Dicke der Glasplatte ablängt, und von denen der eine etwas schwächer als der andere ist. Da sie nicht gleichzeitig seharf erscheinen, so benutzt man zur Vergleichung gewöhnlieh nur den helleren und betrachtet den anderen nur nebenbei zur Controle. Die neisten Beobachter bringen den wirkliehen Stern in eine bestimmte Stellung zn dem kiunstlichen, und zwar möglichst nahe an denselben heran. Es ist aber vielleicht besser, die beiden Bilder in verschiedenen Positionswinkeln zu vergleichen, damit nicht stets dieselben Stellen auf der Netzhaut von ihnen eingenommen werden. Bei der photometrischen Durchmusterung in Potsdam wird so beobachtet, dass der wirkliehe Stern der Reihe nach links, oben, rechts und unten neben den kinstliehen gebracht und gleichzeitig: anch mit den vier Quadranten des Intensitiitskreises abgeweehselt wird. Letzteres Verfahren ist deswegen erwünscht, weil auf diese Weise der Indexfehler des Intensitätskreises und der Excentricitätsfehler der Nicolprismen eliminirt wird.

Was die Sicherheit der Messungen mit dem Zölher'schen Photometer anbetrifft, so ist zunächst klar, da die Helligkeit sich proportional 
dem Quadrate des Sinus des Drehungswinkels der Nicolprismen ändert, dass der wahrscheinliclic Fehler einer einzelnen Einstellung, in Winkelwerth ansgedriickt, bei kleinen Ablesungen des Intensitätskreises viel geringer sein muss als bei grossen. Wenn man aber den wahrscheinlichen Fehler in Helligkeitslogarithmen oder in Grössenclassen ausdriickt, so zeigt sich, dass die Genauigkeit der Einstellung bei den meisten Beobachtern fast iiber die ganze Ausdehnung des Intensitätskreises von etwa $5^{\circ}$ bis $50^{\circ}$ nahezu dieselbe ist; nur Lindemann $\left.{ }^{1}\right)$ kommt zu dem Resultate, dass bei grösseren Einstellungen am Intensitätskreise, also im Allgemeinen bei Beobachtung hellerer Sterne, die Messungen am sichersten sind. Als wahrscheinlichen Fehler einer einzelnen Einstellung am Photometer findet man im Durchschnitt bei einer grossen Zahl von geübten Beobachtern \pm 0.092 Grössenclassen und mithin fiur einen Mittelwerth aus vier Einstellungen \pm 0.046 Grössenclassen. Berechnet man aber für eine an verschiedenen Abenden gemessene Helligkeitsdifferenz zweier Sterne den wahrscheinlichen Fehler eines Abends, so findet man, allerdings nur in besonders grinstigen Fällen, wenn z. B. die Sterne nahe bei cinander stehen und in Farbe nicht wesentlich verschieden sind, den Werth \pm 0.06 Grössenclassen oder etwa 6 Procent des Helligkeitsverhiiltnisses. Diese Genanigkeitsgrenze ist bisher mit keinem anderen Sternphotometer iiberschritten worden und wird wohl anch schwerlich bei Messungen am Himmel iibertroffen werden kömmen, weil die von Tag zu Tage, ja vou Stunde zu Stunde schwankenden Durehsichtigkeitsverhältnisse der Atmosphäre und die Unsicherheit der Extinctionscorrectionen nuiberwindliehe Hindernisse in den Weg stellen, die um so stärker einwirken, je weiter die zu vergleichenden Sterne am Himmel roneinander entfernt sind.

\section{c. Die Wild'schen Photometer.}

Man riihnnt den ron Wild zu Helligkeitsmessungen construirten Apparaten allgemein eine Emptindlichkeit nach, wie sie bei keinem anderen Photometer erreieht worden ist, und es diirfte schon aus diesem Grunde gereehtfertigt erscheinen, diesclben hier zu crwilhnen, obgleich sie bisher in der Astrophotometrie nicht benutzt worden sind und auch kiunftig. höchstens zи Messungen der allerhellsten Himmelsobjecte Verwendung finden kïmnten. Eine kure Besihreibung möge hier genïgen. In Betreff der ansfilhrichen, etwas complicirten Theorie muss anf die Abhaud-

1) Observations de Poulkova. Supplément iI, p. 11 . 
lungen von Wild') und die neucen Arbeiten von Moller ${ }^{2}$ ) verwiesen werden, weleher auch einige $\Lambda$ bänderungen an den Apparaten vorgeschlagen hat.

Von den beiden versehiedenen Formen des Wild'schen Photometers beruht dic erste (Fig. 61) auf einer Idee von Neumann's) und ahnelt im Principe dem oben bescliriebenen Babinet'scheu Photometer.

Die zu vergleichenden Lichtquellen senden ihr Licht durch die kurzen Röhren $A$ und $B$. Die ron $A$ kommenden Strahlen fallen unter dem Polarisationswinkel auf eine in den Rahmen $C$ befindliche, senkrecht stehende planparallele Glasplatte, werden von dort anf eine in Rahmen $D$ sitzende mit $C$ parallele Glasplattensäule reflectirt und von dort in die Beobachtungsrölire $E$ zurilckgeworfen. Diese Strahlen sind vollständig in der Horizontalebene polarisirt. Die aus $B$ kommenden Strahlen gehen zunächst durch einen im Rahnen $F$ befestigten Glassatz, dann durch die Glasplattensänle in $D$ und treten zugleich mit den reHectirten in $E$ ein. Ein Theil des durchgehenden Lichtes bleibt unpolarisirt, ein Theil ist in der Verticalebene polarisirt, und die Intensitiit des letzteren :indert sich mit dem

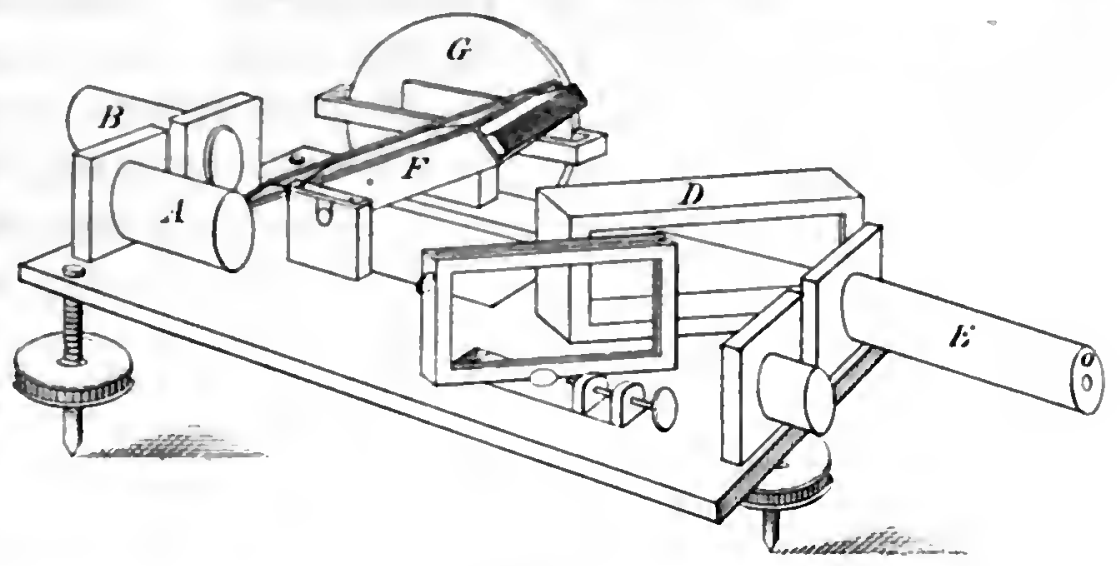

Fig. 61.

Winkel, den der Glassatz in $F$ mit der Horizontalebene bildet und der an einer Kreistheilung auf der Scheibe $G$ abgelesen werden kann. In der Beobachtungsröhre $E$ ist ein Polariskop, bestehend ans einer senkrecht zur Axe geschliffenen Kalkspathplatte und einem 'Turmalin, angebracht. Wenn nun die Quantitïten entgegengesetzt polarisirten Lichtes in den beiden sich vermischenden Strahlenbiindeln gleich sind, so erblickt das Auge in o Nichts ron der sonst sichtbaren Interferenzerscheinung. Man kann aber durch Drehung des Rahmens $F$ stets erreichen, dass die Interferenzfarben verschwinden, und da mit Hiilfe der Nenmann'sehen Formeln für jede Stellung ron $F$ die Menge des in $E$ eintretenden, in der Verticalebene polarisirten Lichtes in Theilen der urspriinglichen ron $B$

1) Pogg. Annalen. Bd. 99. p. 235 und Bd.115, p. 193. - Ausserdem Bull. de l'Acad. Imp. des sciences de St. Pétersb. Vol. 28, p. 392.

2) Wiedem. Annalen. Bd. 24. p. 266 und p. 446.

3) Ne umann. Vorles. iiber theor. Optik: herausg. von Dorn. Leipzig, 1885, p. 152. 
herkommenden Lichtmenge ansgedriiekt werden kann, ebenso ein für alle Male die Menge des von $A$ kommenden in der Horizontalebene polarisirten Lichtes, so liisst sich das ursprüngliche Helligkeitsverhältniss der beiden Lichtquellen berechnen.

Dic Complieirtheit der zur Berechnung erforderlichen Formeln und der Umstand, dass wegen des erheblichen Lichtverlustes bei der zweimaligen Reflexion nur die Vergleichung von verhältnissmässig intensiven Lichtquellen möglich war, veranlasste Wild zur Construction seines zweiten Photometers, bei welchem die Doppelbrechung zur Benutzung kommt. Die Anordnung des Apparates geht aus der sehematischen Zeichnung (Fig. 62) hervor.

$A_{1}$ und $A_{2}$ sind zwei total reflectirende Prismen, auf welche das Licht der zu vergleichenden Lichtquellen anffällt. Die aus der Nähe der

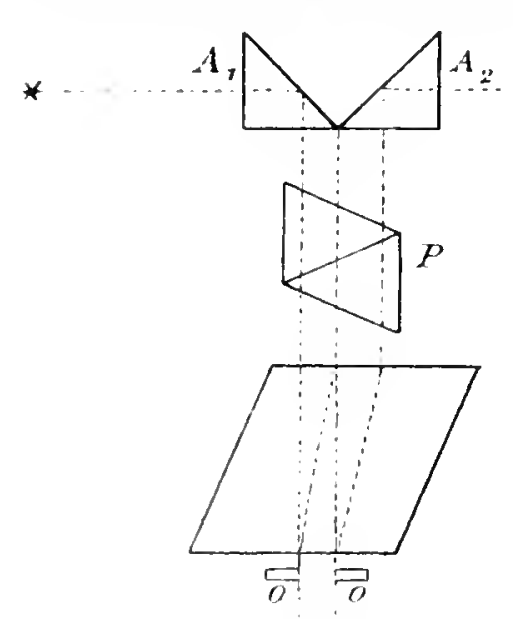
Trennungslinie der Prismen herkommenden beiden Strahlenbüdel werden zunächst dureh das Nicolprisma $P$ polarisirt und fallen dann senkrecht auf die vordere Begrenzungstläche eines Kalkspathrhomboëders. An der Austrittsfläche dieses Rhomboëders ist ein Diaphragma $O$ angebracht von solchen Dimensionen, dass vom Prisma $A_{2}$ nur die ordentlich gebrochenen, rom Prisma $A_{1}$ nur die ausserordentlich gebrochenen Strahlen austreten können. Das vereinigte Strahlenbündel

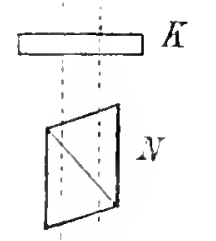
greht dann dureh ein Savart'sches Polariskop, bestehend ans einer Krystallplatte $K$ und einem analysirenden Nicol $N$. Die Interferenzstreifen verschwinden, wemn das aus $O$ anstretende vereinigte Fig. 62. strahlenbiindel gleich grosse Mengen senkrecht zu einander polarisirten Lichtes euthailt. Nach dem Mahus sehen Gesetze ist dies aber der Fall, wenn man fiir die mropringlichen lichtintensituiten $J_{1}$ and $J_{0}$ die Relation hat:

$$
\frac{J_{1}}{J_{2}}=C \tan g^{2} \rho,
$$

wo of Rer Winkel ist, den der Inaptschnitt ron $P$ mit dem Hauptschnitte dus Kalkspathomboüders bildet. Die Grösse $C$, welche naeh der Nenmann'selen Theorie heredhet werden kamn und bei strenger Giiltigkeit des Malns shen Gesctzes gleich 1 sein sollte, laist sich experimentell durch Vergleichnug zweier oleich intensiven Lichtquellen bestimmen. 
Moller hat den Apparat in der Weise abgeilndert, dass er das Polariskop fortgelassen und den Gang der Strahlen so eingerichtet hat, dass die beiden aus dem Kalkspathrhomboëder austretenden Lichtbindel nicht zusammenfallen, sondern in oiner scharfen Treunungslinio nneinander grenzen. Anstatt des Polariskops wird ein kleines Ferurohr benutzt, welches in der Brennebeno mit einem Diaphragma versehen ist, um das Gesichtsfeld bis auf dio beiden erleuehteten Felder abzublenden. Dio Gleiehheit der Helligkeit dieser Felder wird dann dureh Drehung des Prismas $\boldsymbol{P}$ hergestellt, und das Quadrat der 'Tangente des Drehungswinkels giebt das gesuchte Intensitätsverhältniss.

d. Das Chacornac'sche Sternphotometer.

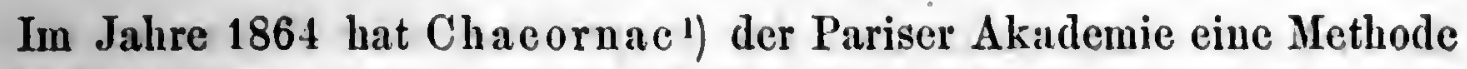
zur Helligkeitsvergleichung zweier Sterne vorgesehlagen, die, wenn auch umständlich und nicht sehr genau, doch von einigem Interesse ist. Ein parallaktisch montirtes Fernrohr wird auf den einen der zu vergleichenden Sterne gerichtet, und der zweite Stern wird, ahnlich wie bei dem Hornstein'schen Zonenphotometer, mit Hillfe eines Spiegels in das Gesichtsfeld gebracht, der in einer Ringfassung um den Objectivrand drehbar ist und ausserdem beliebig gegen die Ebene des Objectivs geneigt werden kann. Die eine Hälfte desselben erlält Licht von dem reflectirten, die andere von dem direet eingestellten Stern. Durch die Dimensionen des Spiegels ist der Anwendungsbereich des Apparates etwas eingeschriinkt. Bei dem Chacornae'schen Arrangement konnten nur Sterne miteinander verglichen werden, deren Winkeldistanz am Himmel zwischen $20^{\circ}$ und $160^{\circ}$ betrug. Das Licht beider Sterne geht durch ein in der Niihe des Oculars befindliches doppeltbrechendes Prisma und wird mit Hiilfe eines zwischen Oenlar und Auge drehbar augebrachten Nicols analysirt. Bildet der Hauptsehnitt des Nieols mit dem Hauptsehnitt des doppeltbrechenden Prismas den Winkel $q$, und ist $J_{1}$ die Intensitiit des direct gesehenen Sternes, so werden die beiden Bilder desselben nach dem Malus'schen Gesetze die Helligkeiteu haben:

$$
\left\{\begin{array}{l}
J_{1}^{\prime}=\frac{1}{2} \% J_{1} \sin ^{2} \varphi, \\
J_{1}^{\prime \prime}=\frac{1}{2} \% J_{1} \cos ^{2} \varphi,
\end{array}\right.
$$

wo * den Sehwächmugscoefficienten beim Durchgange des Lichtes durch beide polarisirenden Prismen repriisentirt.

Für den reflectirten Stern ist die Berechnung der Helligkeiten etwas schwieriger, weil bei der Reflexion ein Theil des Lichtes polarisirt wird.

1) Comptes Rendus. Tome 58, p. 657. 
Die urspriungliehe Liehtstïrke des zweiten Sternes sei $J_{q}$. Ein gewisser Procentsat\% Lieht gelıt von vornherein bei der Zuriiekwerfung verloren. Bezeichnen wir den Reflexionseoefficienten des Spiegels mit $k$, so gelangt auf das doppeltbrechende Prisma von dem zweiten Stern die Lichtmenge k $J_{\sharp}$. Dieselbe setzt sich aus zwei Theilen zusammen, einer Quantität in der Einfallsebene polarisirten Liehtes, die wir $k L_{2}$ nennen wollen, und einer Quantitiit natiirlichen Lichtes, die demnach gleich $k\left(J_{2}-L_{z}\right)$ ist. Es bilde zuniehst die Einfallsebene mit dem Hauptschnitte des doppeltbreehenden Prismas den Winkel $\omega$; dann erhält man für die beiden im Gesichtsfelde siehtbaren Bilder des reflectirten Sternes die Intensitäten:

$$
\begin{aligned}
& J_{2}^{\prime}=k_{i}\left[\frac{1}{2}\left(J_{2}-L_{2}\right)+L_{2} \cos ^{2} \omega\right] \sin ^{2} \varphi, \\
& J_{2}^{\prime \prime}=k_{i}\left[\frac{1}{2}\left(J_{2}-L_{2}\right)+L_{2} \sin ^{2} \omega\right] \cos ^{2} \varphi .
\end{aligned}
$$

Zur Vereinfachung kann man das doppeltbreehende Prisma so drehen, dass sein Hauptschnitt mit der Einfallsebene zusammenfällt. Dann ist $\omega=0$, ind man hat, wenn der Winkel zwischen den Hauptschnitten des doppeltbrechenden Prismas nud des Nicols wieder $\varphi$ heisst, für die beiden Bilder die Intensitäten:

$$
\left\{\begin{array}{l}
J_{2}^{\prime}=\frac{1}{2} \% l_{i}\left(J_{2}+L_{2}\right) \sin ^{2} \varphi, \\
J_{2}^{\prime \prime}=\frac{1}{2} \% l_{i}\left(J_{2}-L_{2}\right) \cos ^{2} \varphi .
\end{array}\right.
$$

Durch Drehung des analysirenden Nicols lässt sich, wenm man die zu vergleichenden Bilder der beiden Sterme nahe aneinander gebracht hat, die Gleichheit ron $J_{1}^{\prime}$ und $J_{2}^{\prime \prime}$ herstellen. Ist der betreffende Drehungswinkel $f_{1}$, so hat man:

$$
J_{1} \sin ^{2} r_{1}=I_{i}\left(J_{2}-L_{2}\right) \cos ^{2} \varphi_{1} .
$$

Ebeuso kaun man die beiden Bilder des reflectirten Sternes durch Drehung: des Nicols gleich machen. Heisst der hierbei abgelesene Drehungswinkel $u_{1}$, so ergiebt sich:

$$
\left(J_{2}+L_{2}\right) \sin ^{2} \alpha_{1}=\left(J_{2}-L_{2}\right) \cos ^{2} \alpha_{1} .
$$

Ans den beiden letzten Formeln erhïlt man durch Elimination von $L_{a}$ die Gleichmo:

$$
\frac{J_{1}}{J_{3}}=27_{i} \sin ^{2} c_{1} \cot ^{2} r_{1} .
$$

Hierans wiirde man umnittelbar das gesnchte Helligkeitsverhältniss der beiden Sterue erhalten, wenn der Reflexionscoefficient des Spiegels bekamnt wiire. Ist dies nicht der Fall, so kann man $k$ dadurch eliminiren, dass man zwei weitere Beobalchtungen ansfiihrt, indem man die Sterne miteinander rertauscht, d. h. den vorher refleetirt gesehenen Stern direct betrachtet und den anderen rom spiegel reflectiren lässt. Man erhält 
dann statt der obigen Formeln (1) und (2) vier andere, die sich von den ersteren nur dadureh unterseheiden, dass die unteren Indices 1 und 2 miteinander vertanseht sind. Selbstrerstiindlich ist dabei die Voraussetzung, dass auch hier wieder der Hauptschnitt des doppeltbrechenden Prismas иu der Einfallsebene des reflectirten Sternes parallel gestellt ist. Macht man dann wieder dureh Drehung des Nicols das erste Bild des direct gesehenen Sternes und das zweite des reflectirt gesehenen einander gleich, ebenso die beiden Bilder des reflectirt gesehenen Sternes uutereinander gleich, so ergiebt sich, wenn die betreffenden Drehungswinkel $\varphi_{2}$ und $\alpha_{2}$ heissen, entspreehend der Formel (3) die Gleichung:

$$
\frac{J_{q}}{J_{1}}=2 k: \sin ^{2} \alpha_{2} \cot ^{2} r_{2} \text {. }
$$

Aus (3) und (4) folgt dann endlich:

$$
\frac{J_{1}}{J_{2}}=\frac{\sin \alpha_{1}}{\sin \alpha_{2}} \cot \tau_{1} \operatorname{tang} \tau_{2}
$$

Wie man sieht, ist das ganze Beobachtungsverfahren ausserst umständlieh. Denn es sind nicht nur vier getrennte Messungen zur Bestimmung der Winkel $\varphi_{1}, \alpha_{1}, \tau_{2}, \alpha_{2}$ erforderlich, sondern es muss anch noch zweimal der Hauptschnitt des doppeltbrechenden Prismas zu der Einfallsebene des rom Spiegel refleetirten Sternes parallel gestellt werden. Übrigens ist die Endformel nur dann streng riehtig, wenn der Reflexionscoeffieient des Spiegels fiir die beiden in Betracht kommenden Einfallswinkel als gleich angenommen werden darf, was keineswegs immer statthaft ist. Die ganze Methode ist schon ans diesem Grunde wenig zu empfehlen. Chacornac scheint sie auch selbst nicht in grösserem Umfange angewendet zu haben, wenigstens sind von ilum ausser einer Helligkeitsvergleichung ron Sirius und Aretur keine weiteren Bestimmungen bekannt geworden.

\section{e. Die Pickering'schen Photometer.}

Das Verdienst, welches sich Pickering um die Himmelsphotometrie durch seine alle Gebiete derselben umfassenden Arbeiten erworben hat, wird noch dadurch erhöht, dass er eine Anzahl voll Apparaten construirt hat, die zwar im Princip nichts wesentlich Neues enthalten, bei denen aber ältere Vorschlïge in so zweekentsprechender Weise verwerthet sind, dass sie genug des Lehrreichen und Nachahmenswerthen bieten. Je nach der Aufgabe, deren Lösung Pickering im Ange hatte, unterscheiden sich die verschiedenen Formen dieser Instrumente voneinander. Der eine Typns umfasst alle diejenigen Apparate, welche speciell zur Messung 
nahe bei einander stehender Himmelsobjecte, insbesondere der Doppelsterne, bestinımt waren; ein zweiter Typus repräsentirt alle Instrumente, nit denen die schwiichsten Objecte am Himmel, vornehmlich die Planetentrabanten, gemessen werden sollten. Den hervorragendsten Platz unter allen aber nimmt das Meridianphotometer ein, mit welchem Pickering seine grossen Helligkeitseataloge, die umfangreichsten, die wir bisher besitzen, hergestellt liat. Mit Recht wird dieses Instrument in gleicher Linie mit dem Pritelıard'schen Keilphotometer und dem Zöllner'schen Photometer zu den besten modernen Huilfsmitteln der Astrophotometrie gerechnet.

Als Hauptrertreter des ersten Typus ist das in Figur 63 abgebildete Instrument zu erwähnen. Dasselbe gleicht vollkommen dem unter dem Namen Roehon'sches Fernrohr bekannten Mikrometer, welches zu Messungen kleiner Distanzen am Himmel vielfach Verwendung findet.

Die Röhre $E$ wird an dem Oeularende eines parallaktiseh montirten Ferurohrs eingesehoben. In derselben kaun ein Roehon'sches Bergkrystall-

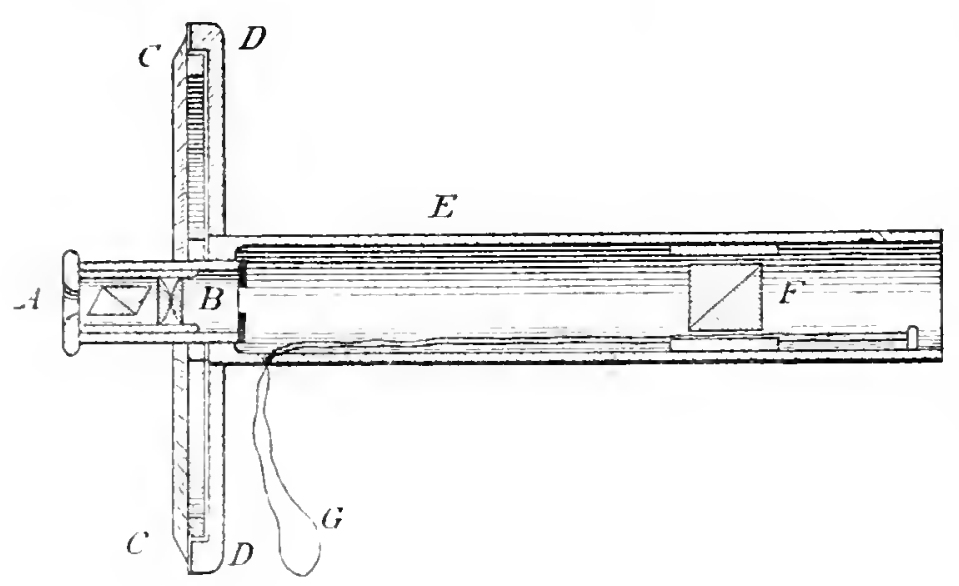

Fig. 63. prisma $F$ mittelst eines Sehnurlaufes $G$ dem Objectiv des Fernolurs genähert oder von demselben entfernt werden. Tor dem Ocular $B$, dessen Gesichtsfeld durch ein geeignetes Diaphragma etwas abgeblendet ist, befindet sieh eim Nicolprisma $A$, welches zugleich mit dem getheilten Kreise $C$ drehbar ist; die jedemalige Stellung des Kreises wird an den Indices $D$ abgelesen. Ton zwei nahe stehenden Objecten werden durch das Rochon'sche Prisma je zwei Bilder hervorwebracht, deren Distanzen durch Nähern oder Entfernen des Prismas imnerhall, gewisser Grenzen nach Belieben verändert werden kömnen. Man bringt durch Drehung des Nicols das ordentliehe Bild des einen und dis ansserordentliche Bild des anderen Objects anf gleiche Intensitit. Ist of die Ablesung, bei welcher das eine Bild ganz rershwindet, dagesen of die Ablesung, bei welcher die beiden Bilder einander gleich sind, so erhizlt man das gesnchte Helligkeitsverhältniss $\frac{J_{1}}{J_{z}}$ der beiden rerolichenen Objecte ans der Gleichmg:

$$
\frac{J_{1}}{J_{2}}=\operatorname{ting} g^{2}\left(p-f_{0}\right)
$$


Die Gleichheit der Bilder kann in allen rier Quadranten des Kreises $C$ hergestellt werden; dadureh eliminirt man einerseits einen etwaigen Fehler in der Stellung der Axe des Nicols, andererseits " naelit man die Bestimmung der Nullinge $p_{0}$ uberflissig. Fur den speciellen Zweek, zu welehen der Apparat bestimint ist, erweist er sieh ausserordentlich werthvoll. Ein Nachtheil ist nur der erhebliche Liehtverlust, der dureh die Treunnng in zwei Bilder bedingt wird, und ferner die Beselurinkung auf die Messung rerhailtnissmässig sehr kleiner Distanzen. In Verbindung mit einem Fernrolır ron $35 \mathrm{~cm}$ Öfrumig und $653 \mathrm{~cm}$ Brennweite liessen sich nur Objecte messen, die weniger als 64" voneinander entfernt waren, und mit - Anwendung eines Fernrohrs von $12.7 \mathrm{~cm}$ Öftunng und $231 \mathrm{~cm}$ Brenuweite betrug die grösste verwendbare Distanz etwa 190". Um dem letzteren Übelstande abzuhelfen, hat Piekering ${ }^{1}$ ) in, allernenester Zeit eine Modifieation dieses Photometers vorgesehlagen. Lr befestigt das doppeltbrechende Prisma in der Nible des Brennpunktes. und bringt zwisehen Foeus und Objeetiv nebeneinander zwei achromatisclıe Prismen von kleinem Winkel in, die sich lïngs des Rolıres im Innern mittelst Triebwerkes lin und her bewegen lassen. Die Winkel des doppeltbrechenden Prismas und der achromatisehen Prismen sind so gewïlit, dass bei der Verbindung dieses Photometers mit dem ersten der oben genannten Refractoren noch die Bilder von zwei Sternen, die $35^{\prime}$ auseinander stehen, zusammengebracht werden künnen; dabei haben die achromatischen Prismen den grösstmöglichen Abstand (10 em) ron der Focalcbene. Sind die Prismen aber in nmmittelbarer Nïhe der Bremebene, so fallen die Bilder von zwei Sternen zusammen, dio uur eine Distanz von 3' haben. Man kann auf diese Weise durch Bewegung der Prismen Stermpare mit Distanzen zwischen $3^{\prime}$ und $35^{\prime}$ photometrisch messen.

Der Nachtheil des Lichtverlustes durch die Trennung in zwei Bilder ist ron Pickering bei der zweiten Classe ron Photometern vermieden worden, welche speciell zur Beobachtung der lichtschwiichsten Objecte an Himmel bestimmt sind. Figur 64 (Seite 262) stellt eins dieser Instrnmente dar. Der schwache Stern wird direct in dem grossen Fermrohre durch das Ocular $A$ in der einen Hälfte des Gesichtsfeldes betrachtet, wiihrend das Bild eines hellen zur Vergleichung dienenden Sternes dureh das Prisma $B$ in das seitliehe Hülfsfernrohr mit dem Objectir $D$ reflectirt und nach dem Austritt ans demselben dureh das Prisma $F$ in die andere Hïlfte des Gesichtsfeldes gebracht wird. Das Prisma $B$ ist $\mathrm{um}$ die Axe des Hiilfsfernrohrs drehbar, und das ganze Photometer kamn endlich noch um die

1) The Astrophysical Journal. Vol. 2, p. 89. 
Axe des Hauptinstrumentes gedreht werden, so dass es möglich ist, jeden beliebigen Stern zur Vergleichung zu benutzen.

In dem seitlichen Rohre sitzen die beiden Nieolprismen $C$ und $E$; das letztere ist zusammen mit dem Kreise $G$ drehbar, und der Betrag

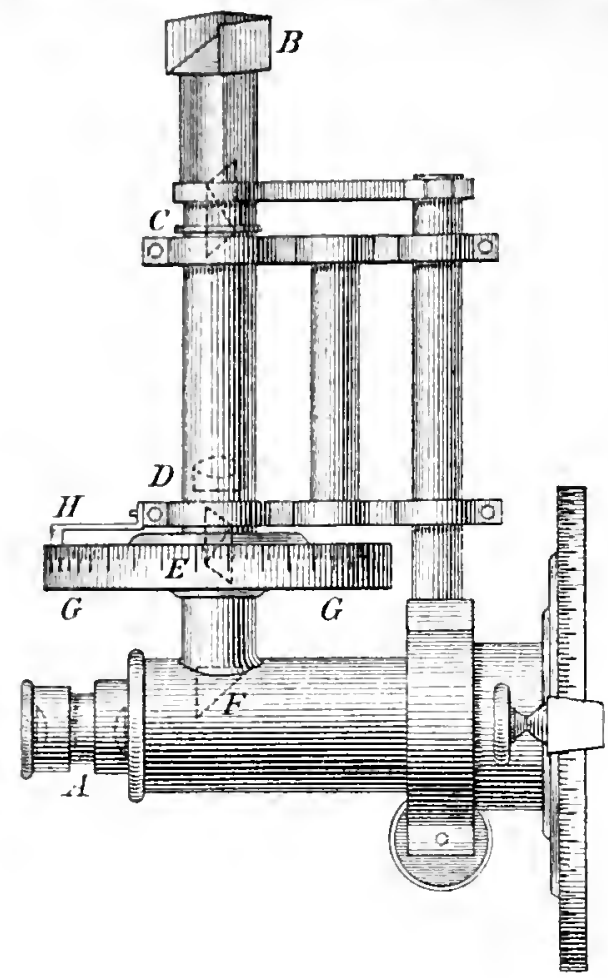

Fig. 64. der Drehnng wird an dem Index $H$ abgelesen. Durch Bewegung von $E$ wird der Vergleichstern so weit abgeschwächt, bis er dcm direct gesehenen an Helligkeit gleichkommt. Die Eimrichtung hat drei bedenkliche Übelstände. Erstens erscheinen die beiden verglichenen Sterne auf verschiedenem Himmelsgrunde, zweitens wird das Bild des Vergleichsternes durch die Nicolprismen merklich verschlechtert, so dass sein Aussehen von dem des direct geseheneu etwas verschieden ist, und drittens ist die Constante des Photometers, d. h. das Verhältniss eines im Hiilfsfermrohr bei parallel gestellten Nicols gesehenen Sternes zu seinem Bilde im Hauptfermrohre, durch besondere Versuche zu ermitteln. Der erste Übelstand kann, wie schon Pickering selbst bemerkt hat, dadurch beseitigt werden, dass das Prisma $F$, ebenso wie beim Zölhner'schen Photometer, durch eine planparallele Glasplatte ersetzt wird, dem zweiten Nachtheil hat Pickering später dadureh zu begegnen rersucht, dass er an Stelle der Nicolprismen eine Abblendungseinrichtung ror dem Objectir $D$ angewandt hat. Dadurch ist aber keine Terbesserung erzielt, rielmehr kömnen durch die bekannten Mängel der Abblendungsmethode nuter Umstinden merkliche Fehler verursalcht werden. Der dritte Ühelstand ist iiberhaupt nicht zn beseitigen, und da die Bestimmmg der Constante des Photometers, für welche Pickering verschiedene Methoden in Torschlag gebracht hat'), ziemlich schwierig ist, so steht die hier besprochene Form ron Photometern an Genanigkeit hinter dem ersten Tylus zuriick.

Wir kommen mu zu dem wiclitigsten der Pickering'schen Apparate, dem Meridianphotoneter, welches dazn bestimnt ist, die Sterne beim Durchgange durch den Meridian zu messen. Pickeriug hat zwei solcher Instrumente construirt; Figur 6.5 stellt das grössere derselben dar.

1. Annals of the Astr. Obs. of Harvard College. Vol. 11, part II, p. 195. 


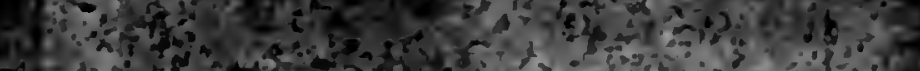

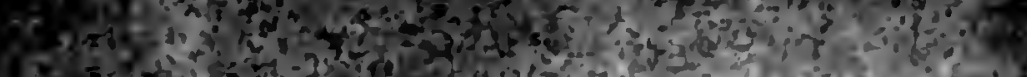
35.

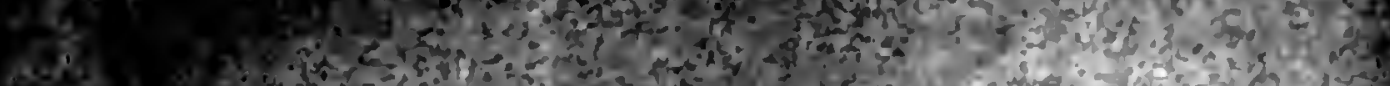

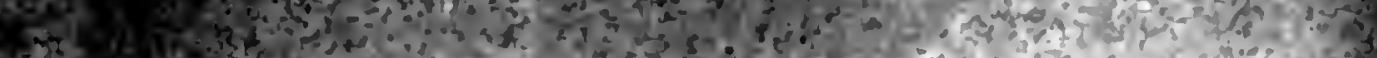

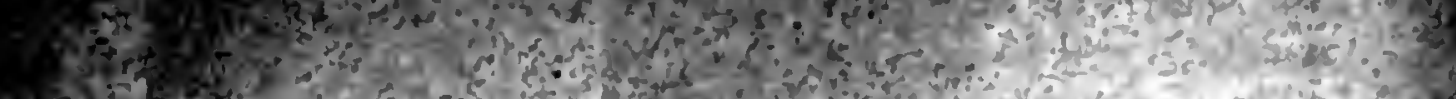

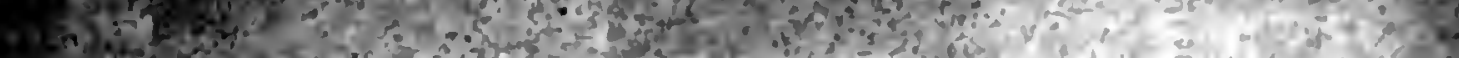

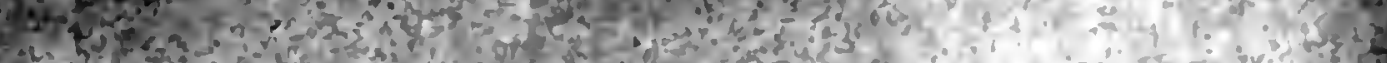

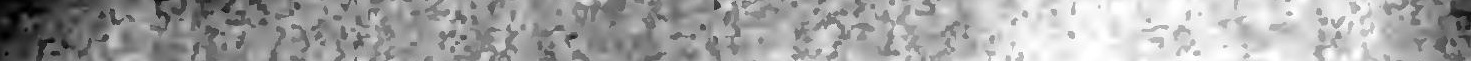

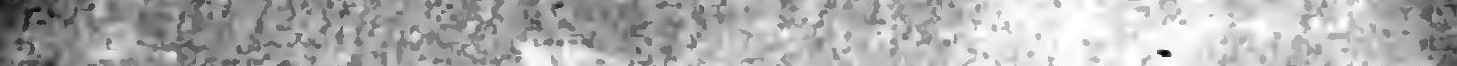

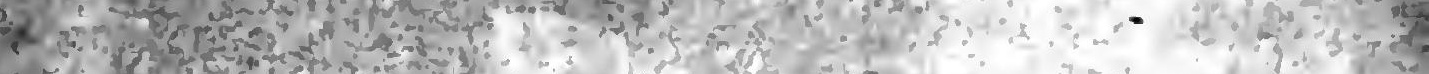

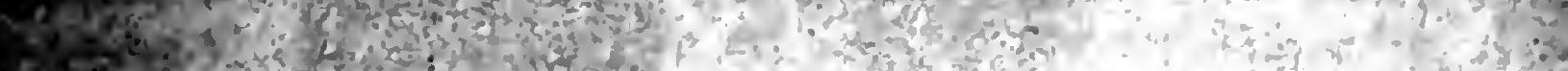

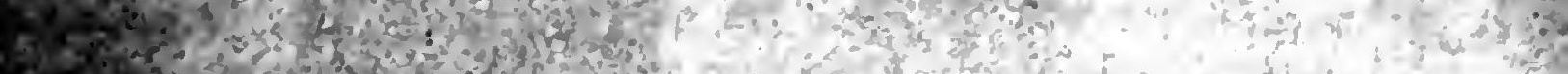

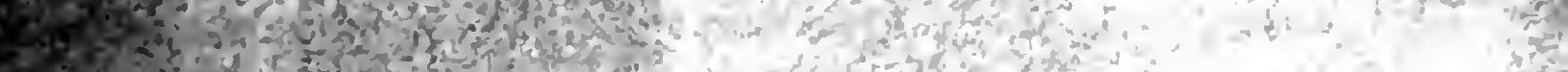

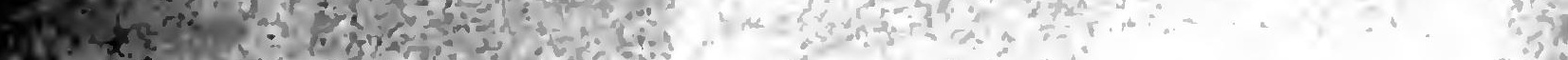
Q

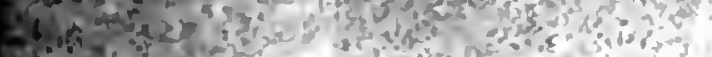

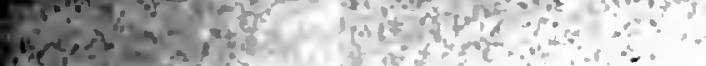

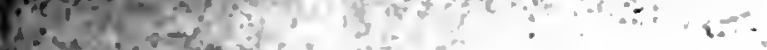

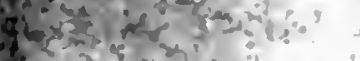

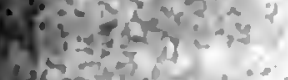

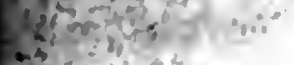

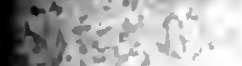




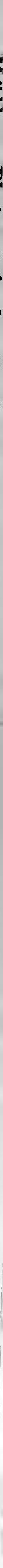


Ein Holzkasten ist in der Richtung Ost-West anf Pfeilern fest montirt. Au dem östlichen Ende des Kastens sind zwei Rohren $A$ und $B$ angesetzt, welehe zwei gleich grosse Objective von $10.5 \mathrm{~cm}$ Öfinung enthalten. Vor diesen Oljectiven sitzen, unter $45^{\circ}$ gegen dieselben greneigt, die versilberten Glasspiegel $C$ und $D$, welehe mit ihren Fassungen vermittelst der langen Triebstangen $E$ und $F$ um die optischen Axen der Objective gedreht werden können. An zwei Kreistheilungen lassen sich bei richtiger Justirung des Apparates unmittelbar die Declinationen der Sterne einstellen. Das slidliche Objectiv (im Rohre A) hat eine etwas lingere Brenuweite $(166 \mathrm{~cm})$ als das nördliche $(145 \mathrm{~cm})$; es ragt nach Osten zu um 21 en luber das nördliche hinaus, und für den Spiegel $C$ ist daher die ganze Meridianebene frei. Vermittelst der Schraube $S$ kann man die Neigung des Spiegels $C$ gegen das Objectiv innerhalb kleiner Grenzen variiren, um während der Beobachtung den eingestellten Stern an derselben Stelle im Gesichtsfelde zn halten. Die Scliraube $S$ wird durch einen uber mehrere Rollen geführten Schnurlanf vom Ocular aus inittelst des Griffes $G$ bewegt, und an einer auf der Längsseite des Kastens angrebrachten Seala $H$ kann die jedesmalige Stellung des Spiegels (die Collimation desselben) abgelesen werden. Eine iilnliche Bewegungseinrichtung fuir den Spiegel $D$ befindet sich auf der entgegengesetzten Seite des Apparates. Dieser zweite Spiegel ist dazu bestimmıt, das Bild eines Polsternes (Pickering hat $\lambda$ Ursae minoris grewïllt) in das Gesiehtsfeld zu bringen. Mit diesem constanten Vergleichsobject werden die in $C$ eingestellten Sterne beim Passiren des Meridians vergliehen. In dem Ocularansatze an dem westlichen Ende des Instrumentes sitzt in der Naihe der Brennebene ein doppeltbrechendes achromatisirtes Kalkspathprisma. Zwischen Oeular und Auge befindet sich endlich noch ein drehbares Nicolprisma, dessen Stellung gegen das feste doppeltbrechende Prisma an einem getheilten Kreise alggelesen wird. Die Winkel der beiden Theile des doppeltbrechenden Prismas, von denen der eine ans Glas, der andere aus Kalkspath besteht, sind so gewiihlt, dass das ordentliche Bild eines mit $C$ betrachteten Sternes genau coincidirt mit den ansserordentlichen Bilde eines mit Hialfe von $D$ gesehenen Sternes. Dadurch, dass das doppeltbrechende Prisma in der Niihe des Focus steht, wird der Vortheil erreicht, dass die Bilder sehr wenig gefärlot erscheinen, und dass ausserdem ordentliches und ausserordentliches Bild desselben Objeetivs möglichst weit voneinamder getrennt sind. Die beiden nieht zur Beobachtung benutzten Bilder sind durch den Augendeckel des Oculars rom Gesichtsfelde ausgeschlossen. Ist $J$ die Helligkeit des Meridianstemes, $J_{0}$ diejenige des Polsternes, ist ferner op der am Kreise alogelesene Winkel, wenn die in Betracht kommenden Bilder der beiden Sterne grleich hell 
erscheinen, und endlich $\varphi_{0}$ dic Ablesung am Kreise, wenn das Bild des Polsternes verschwindet, so hat man:

$$
\frac{J}{J_{0}}=\operatorname{tang}^{2}\left(\varphi-\varphi_{0}\right)
$$

Es verstelt sich von selbst, dass die Einstellungen in sämmtlichen Quadranten des Intensitïtskreises ausgeführt werden müssen, wenn man den Indexfehler desselben, sowie eine etwaige schiefe Stellung der Axe des Nicols eliminiren will; auch empfiehlt es sich, wie bei den Beobachtungen mit dem Zöllner'sehen Photometer, die Vergleichungen bei verschiedenen Stellumgen der beiden Bilder zu einander anzustellen, um von Auffassungsfehlem möglichst frei zu sein. Nicht ganz unbedenklich ist die Benutzung der Spiegel vor den Objectiven, weil bei der Reflexion eine partielle Polarisation des. Liehtes stattfindet, und der Winkel, den die Einfallsebene mit dem Hanptsehnitte des doppeltbrechenden Prismas bildet, je mach der Meridianhöhe des Gestirns verschieden ist. In dieser Hinsicht ist bei dem kleineren, von Piekering zuerst construirten Meridianplrotometer ${ }^{1}$ ), dessen Objective nur Öffnungen ron $4 \mathrm{~cm}$ und Brennweiten ron $80 \mathrm{em}$ besitzen, und bei wclehem $\alpha$ Urs. min. an Stelle von i. Crs. min. als Vergleichstern dient, jedes Bedenken ausgeschlossen, weil statt der Spiegel total reflectirende Prismen zur Anwendung gekommen sind. Bei grö̈sseren Dimensionen können freilieh solche Prismen wegen der Sehwierigkeit und Kostspieligkeit ihrer Herstellung nicht in Frage kommen.

Da bei dem Neridianphotometer die beiden zn vergleichenden Sterne dureh verschiedene Objective abgebildet werden, so ist bei jedem Instrumente die Bestimmung einer Constante erforderlich, welche das Helligkeitsverhiiltniss der beiden Ohjective zu einander angiebt. Diese Constante wird sehr einfach dadurch ermittelt, dass ein und derselbe Stern in heiden Objectiren eingestellt und mit sich selbst verglichen wird, und da diese Constante durch äussere Einfliisse, wie Staub ete, sich von Tağ zn Tag verändern kann, so emptiehlt es sich, nach dem Vorgange Pickering's, dieselbe am Anfange und am Ende jeder grösseren Beobachtumgsreile zu bestimmen.

Ans der rorangehenden Beschreibung des Pickering'sehen Meridianphotometers geht liervor, dass der Tlauptrorzug desselben ror vielen anderen Photometern darin zu selen ist, diss direet zwei Sterne am Himmel miteinander zur Vergleichung kommen, deren Bilder, mit fast gleichen Objectiren nud mit demselben Ocular betrachtet, ein absolut

1) Annals of the Astr. Obs. of Harrard College. Vol. 14, part I, p. 1. 
gleiches Aussehen haben, abgesehen natilrlieh von den Untersehieden der Fürbung. Diesem sehr hoch $z$ schlitzenden Vortheile stehen freilich einige Mingel gegenliber. Durch die feste Aufstellung des Apparates wird zuniichst der Anwendungsbereieh desselben wesentlich beschrïnkt. Man kann nicht jederzeit beliebige Sterne am Himmel miteinander vergleichen, und die mehrmalige Beobachtung eines Sternes an ein und demselben Abend ist nnmöglich. Der Umstand, dass ein Polstern als constantes Mittelglied benutat wird, bringt den Nachtheil mit sich, dass die beiden verglichenen Objecte unter Umstiinden weit am Himmel roneinander entfernt sind, und dass infolge dessen Verschiedenheiten in der Durchsichtigkeit der Atmosphäre schïdlichen Einfluss auf die Messungen haben können. Au Beobachtungsorten in niedrigen Breiten ist von der Benutzung des Photometers ganz abzurathen, weil infolge der geringen Höhe der Polsterne tiber dem Horizonte die Extinction eine allzu bedenkliche Rolle spielt. Ferner ist es ein empfindlicher Übelstand, dass dureh die Anwendung des doppeltbrechenden Prismas eine starke Lichtverminderung herbeigefulirt wird, und dass das Instrument daher, weun es für die Beobachtung schwilcherer Sterue dienen soll, verh:̈iltnissmäissig grosse Dimensionen haben muss. Die Theilung in ordentliches und ausserordentliches Bild reducirt schon die ursprlingliche Lichtmenge anf die Hiilfte, und da noch eine weitere Verminderung bei der Gleichmachung mit dem Vergleichsterne stattfinden muss, auch etwas Licht durch Absorption und Reflexion verloren geht, so wird man nur solche Sterne in den Bereich der Messungen ziehen düfen, die mindestens $1 \frac{1}{2}$ bis 2 Grössenclassen heller sind als die schwïchsten, welche man mit dem betreffenden Objective ohne polarisirende Medien gerade noch wahruehmen kann. Die Benutzung des Intensitiitskreises bei sehr kleinen Winkeln ist ebenso wenig rathsam als beim Zöllner'schen Photometer, weil schon granz geringfüigige Ablesungsfehler einen grossen linfluss anstiben. Macht man bei einer Einstellnng ron $4^{\circ}$ einen Ablesefehler von 0.1, so ïndert dies schon die berechnete Helligkeit, da das Quadrat der Tangente in Frage kommt, um 0.05 Grössenclassen, ein Betrag, der als unzulässig zu bezeichnen ist. Will man daher solche oder noch kleinere Winkel benutzen, so miisste dic 'Theilung genauer als bis auf 0 ? 1 abzulesen sein, und anch die Bewegung des Kreises muisste feiner bewirkt werden kïnnen, als es bei der Drehung mit der Hand möglich ist. Grössere Helligkeitsunterschiede als etwa vier Grössenclassen direct zn messen scheint daher bein Pickeringschen Meridianphotometer kaum statthaft. Pickering ist mit dem kleineren seiner beiden Instrumente nicht viel ïber die 6. Grössenclasse, mit dem grösseren nicht weit tiber dic 9. Grössenclasse linausgegangen, und es fragt sich, ob er nicht damit bereits die zulaissige Grenze iiberschritten 
hat. Wollte man gar noch ganz schwache Sterne in den Messungsbereich des Meridianphotometers ziehen, so müssten die Dimensionen noch erheblich gesteigert werden, und damit wïrden die Kosten der Herstellung unverhältnissmïssig wachsen. In dieser Hinsicht steht das Meridianphotometer jedem anderen Photometer, welches sich mit einem beliebigen Refractor in Verbindung bringen lïsst, entschieden wach.

\section{Capitel III.}

\section{Die Spectralphotometer.}

I. Die Methoden von Framliofer, Vierordt, Draper, Crova, Abney zur Bestimmmng der Helligkeitsvertheilung im Sommenspectrum.

Wemn man das ron rersehiedenen Lichtquellen ansgesandte Licht mit Hiilfe eines Prismas in die einzelnen Strahlengattungen zerlegt und auf irgend eine Weise die Gleichheit der Intensitiit in einem bestimmten Farbenbezirke bei simmtliehen Speetren herstellt, so sieht man, dass an anderen Stellen diese Gleiehheit nieht mehr besteht. Bei einzelnen Liehtquellen iiberwiegen die weniger brechbaren, bei anderen die brechbareren Strahlen. Die Lichtrertheilung im Spectrmm ist durchans charakteristisch fiir jede Lichtquelle; sie wird im Allgemeinen bedingt dureh die Temperatur und die damit im Zusammenhang stehende Färbung derselben. Je höher die Temperatur einer Lichtquelle und je weisser infolge dessen gewöhnlich anch ihre Färbung ist, desto reicher ist ihr Speetrum an blanen und rioletten Strahlen. Lngekehrt macht sieh eine niedrigere Temperatur dirch das stiirkere Hervortreten der rothen Strahlengattungen bemerkbar. Es geht hieraus herror. wie wichtig die Kenntniss der Lichteurve des Spectrums für die Beurtheihnu einer Liehtrnelle ist, es ist aher anch nmmittelbar klar, dass die Bestimmung dieser Curve, da es sirll un die Tergleichnu" rerschiedener Farben handelt, ans physiolonischen Grimden grose schwierigkeit bereitet.

Der Erste, welcher den Versuch gemacht hat, rersehiedene Partien des sumenspectrums in Bezug anf Helligkeit miteinander zu 
vergleichen, war Frannhofer 1 . Er bediente sich dabei der folgenden Methode.

Vor dem Objectiv $A$ eines Fernrohrs (Fig. 66) wurde ein Prisma $P$ anfgestellt und das entstehende Sonnenspectrum durch das Qcular $B$ hetrachtet. In Innern des Rohrs ist ein kleiner, unter einem Winkel rou $45^{\circ}$ gegen die optisehe Axe geneigter Metallspiegel $s$ angebracht, auf dessen scharfe, bis in die Mitte des Rohrs reichende Kante das Ocular $B$ eingestellt wird. In der rom Spiegel nieht rerdeekten-Hälfte des Gesichtsfeldes, welehes durch ein Diaphragma beschrïnkt ist, erblickt man ein Stlick des prismatischen Spectrums. Vom Spiegel $s$ wird das Licht einer kleinen Lampe $L$ reflectirt, aus deren Flamme dureh die Blende $b$ ein kleiner Theil lieransgeblendet ist, und die in einem seitlichen,

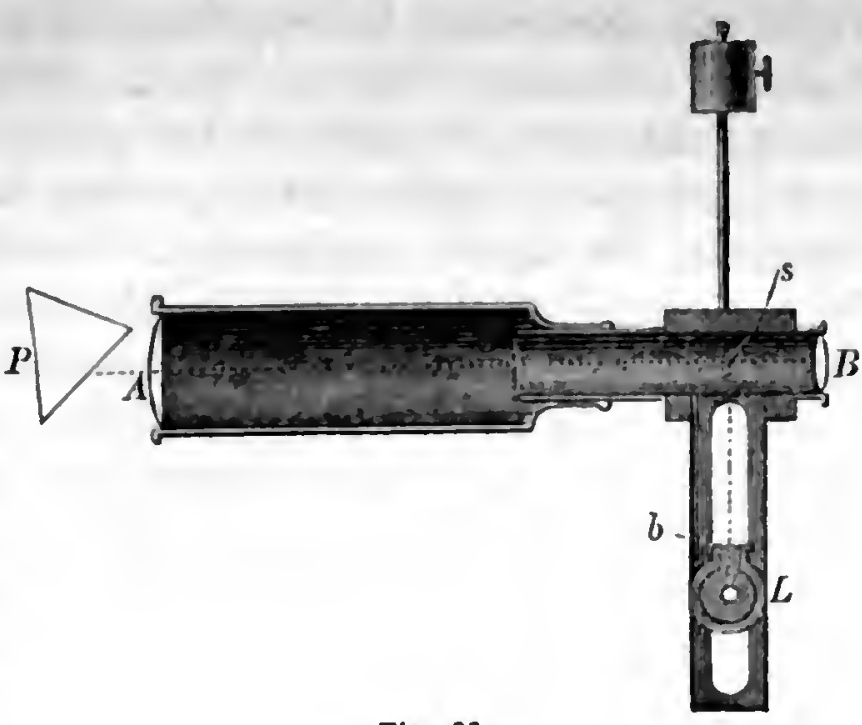

Fig. 66. oben und unten durehbrochenen Rohre messhar verschoben werden kann. Die Intensität der von diesern Vergleichslichte ausgehenden Beleuchtung indert sieh umgekehrt proportional dem Quadrate des Abstandes der Lampe vom Spiegel. Man verschiebt nun die Lampe jedesmal so weit, bis die von ilır beleuchtete Hälfte des Gesichtsfeldes mul der in der anderen Hïlfte sichtbare Theil des Spectrums grleich hell erscheinen. Einer sehr grossen Genauigkeit ist diese Beobachtungsmethode, welche Franuhofer auf acht versehiedene Bezirke des Sounenspectrums angewandt lat, nicht fühig, weil die Vergleichung verschieden gefiirbter Flichen ungemein sehwierig ist. Dic cinzelnen Messungsreihen von Fraunh ofer zeigen daher anch grosse Abweichungen untercinander, und noch stïrkere Unterschiede wiirden zwischen verschiedenen Beobachtern zu erwarten sein.

Dic Frannhofer'schen Versnche zur Vergleichung verschiedener Spectralbezirke sind erst cin halbes Jahrhundert spiiter von Vierordt?) wieder anfgenommen worden, und zwar nach einer griinzlich anderen Methode.

1) Denkschriften der K. Bayer. Akad. der Wiss. Math.-phys. Classe, Bd. 5 (181i), p. 193. - Siehe auch Gilberts Annalen, Bd.56, p. 26.1.

2) Vierordt, Die Anwendung des Spectralapparates zur Messung und Vergleichung der Stairke des farbigen Lichtes. Tüibingen, 18i1. - Siehe auch Pogg. Annalen, Bd.13i, p. 200. 
In dem Scalenrohre eines gewöhnlichen Spectralapparates wird an Stelle der Scala ein horizontaler Spalt angebracht, welcher durch eine Petroleumlampe beleuchtet wird. Durch ein vorgesetztes blaues Glas kann die Farbe der Flamme in Weiss verwandelt werden. Das Bild des Spaltes grelangt durch Reflex an der letzten Prismenfläche in das Beobachtungsferurohr und projicirt sich als weisser Streifen auf das Spectrum. Die Höhe des Spaltes ist so bemessen, dass das Spectrum ober- und unterlalb des Streifens sichtbar bleibt. Das Licht der Lampe kann dann durch vorgesetzte Blendglïser von verschiedener Absorptionsfähigkeit so weit geschwiicht werden, bis der weisse Streifen in den einzelnen Farbenbezirken, dic durch gecignete Ocularschieber herausgeblendet werden können, verschwindet. Sind die Absorptionscoeffieienten der Blendgläser durch besondere Untersuchungen bekannt, so lässt sich aus den Quantitiiten des zugemischten weissen Lichtes das gesuchte Helligkeitsverhältniss der betreffenden Spectralbezirke berechnen. Um nicht allzu viele verschiedene Blendgliiser nöthig zu haben und nm die Messungen noch mehr zu verfeineru, hat Vierordt den Vorschlag gemacht, eine Abschwächung der Vergleiehsflamme imnerhalb kleiner Grenzen durch Verengung des Hiilfsspiltes zn erreichen. Die Vierordt'sche Methode leidet ebenso wie die Fraunhofer'sehe an dem Mangel, dass die Empfindlichkeit des Auges sieh fuir die verschiedenen Farben indert und auch mit der absoluten Intensitait des Spectrums variirt. Fast in noch stäkerem Grade trifft dies eine von W. Draper $\left.{ }^{1}\right)$ empfohlene Methode, weleher das Spectrum auf eine von weissem Licht belenchtete Fliiche projicirt nud nntersucht, bei welchen Intensitiiten des weissen Lichtes die einzchen Spectralbezirke nicht mehr von der erlenchteten Fliiche unterschieden werden können. Zu erwähnen ist noch, dass Vierordt der Erste gewesen ist, welcher sein Verfahren anch für die Untersnchung der Sternspectren in Vorschlag gebracht hat ${ }^{2}$ ).

Ein wesentlich anderer Weg ist ron Crova und Lagarde ${ }^{3}$ eingesehlagen worden. Diese bringen ror den Spalt cines gewöhnlichen Spectrilapparates eine Glasplatte, anf welcher eine Anzahl feiner Striche eingeritzt sind. Letztere projiciren sich als schwarze Querstreifen anf das Spectrum, und indem das Licht desselben durch polarisirende Medien weindert wird, lissst es sich erreichen, dass die Striche in den einzehnen Farbenregionen verschwinden. Die Sehschïrfe des menschlichen Auges ist also hierbei der entscheidende Factor. und es unterliegt keinem Zweifel, dass die Methode einer etwas grösseren Genanigkeit fühig ist, als die rorher besprochenen.

1) Philos. Mag. Ser. 5, Tol. 8 (157!', p. 75.

2 Astr. Nachr. Iid. is, No. 1563.

3 Comptes Rendus. 'T. 93 (1881, p. 959. 
Eines ähnlichen Verfahrens haben sieh anch Macé de Lépinay and Nicati') bedient.

Besondere Beachtung gebuhrt endlich noch der Methode, welche in nenerer Zeit ron $\mathrm{A} b$ ney und Festing ${ }^{2}$ ) bei ihren furbenphotometrischen Untersuchnngen angewendet worden ist. Die ron einem Heliostat kommenden Sonnenstrahlen $R R$ (Fig. 67) werden dureh die Linse $L_{1}$ zu einem Bilde auf dem Spalt $S_{1}$ des Collimators rereinigt. Nach dem Austritt aus dem Collimatorobjectiv $L$ gehen die Strahlen dureh dic. Prisunen $P_{1}$ und $P_{2}$, fallen anf dic Linse $L_{3}$ und bilden auf dem schrigg zu ihrer Richtang stehienden Sehirme $D$ ein reines Sonnenspectrum. Der Sehirm $D$ enthält eine spaltfürmige Öftinung $S_{a}$ und lïsst sieh parallel mit sich selbst verschieben, so dass. $S_{2}$ anf jede beliebige Stelle des Spectrums eingestellt werden kann. Man erhält dann mit Hulfe der Liuse $L_{4}$ auf einem weissen Sehirme ein monochromatischesBild $F$ von der Austrittsöffinung des zwreiten Prismas; die Farbe desselben kann dureh Versehieben von $D$ beliebig geändert werden. Als Vergleichslicht kommt keine Lampe zur Verwendung, sondern es werden dazu die von der ersten Prismen-

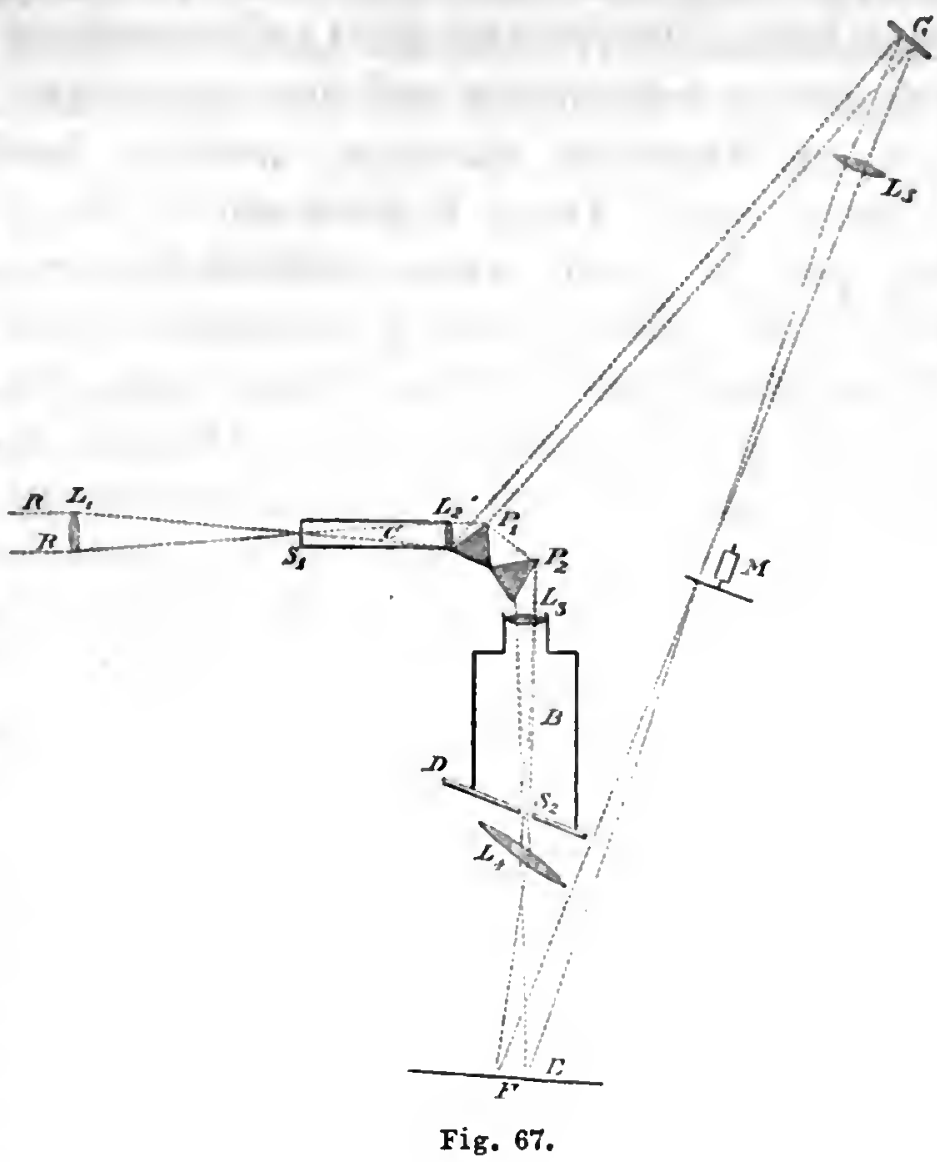

fliiche reflectirten Sommenstrahlen beuntzt. Dieselben fallen anf einen versilberten Glasspiegel $G$, werden dureh die Linse $L_{s}$ gresammelt und bilden bei $F$ ein weisses Bild der freien Prismenfliche. Vor dem anffangenden Schirme bei $F$ steht ein diunner senkrechter Stab, und es kommt, genau so wie beim Rumford schen Photometer, auf die Beurtheilung der von diesem Stabe herrorgebrachten Schatten an. In den Gaug der von $G$ kommenden Strahlen ist bei $M I$ noch ein kleiner elektrischer Motor

1) Annales de chimie et de physique. Série 5, t. $24(1551)$, p. 289 und t. 30 (1883), p. 145 .

2) Phil. Trans. of the R. Soe. of London. 1886, part II, p. 455. 
cingefugt, welcher eine mit sectorförmigen Ausschnitten versehene Scheibe in schnelle Rotation versetzt. Durch Vergrösserung oder Verkleinerung der Ausschnitte lïsst sich die Gleichheit der Schatten auf dem Schirme herstellen. Die Methode hat den Vortheil, dass Spectrum und Vergleichslicht vou derselben Lichtquelle herstammen, und dass infolge dessen kleine Schwankungen des Sonnenlichtes während der Messungen die Resultate nicht schädlich beeinflussen können.

Die Lichtenrve des Sonnenspectrums, die man mit Hülfe einer der im Vorangehenden beschriebenen Methoden findet, hängt ganz und gar von der Dispersion des benutzten Spectralapparates ab. Um allgemein vergleichbare Werthe für die Lichtvertheilung zu erhalten, muss man die gefundenen Intensitäten auf das sogenannte Normalspectrum reduciren, d. h. anf dasjenige Speetrum, welches durch Diffractionsgitter hervorgebracht wird. Diese Reductionen bestimmt man auf folgende Weise. Hat man in irgend einem prismatischen Spectrum für einen gewissen Bezirk, der zwischen den Wellenlängen $\lambda$ und $\lambda+d \lambda$ enthalten ist, und dessen lineare Ausdehnung $d x$ sein mögee, die mittlere Flächenhelligkeit $i$ nach einer der obigen Methoden gefunden, so ist die Gesammtlichtmenge des betreffenden Spectralstreifens proportional dem Werthe $i d x$. In dem Normalspectrum wïrde der entsprechende Bezirk eine lineare Ausdehnung haben, die direct dem Wellenlängenintervall $d \lambda$ proportional ist. Nennt man daher die Flächenintensität an dieser Stelle des Normalspectrums $J$, so hat man:

$$
J d \lambda=C i d x
$$

wo $C$ eine Constante bedentet. Darans ergiebt sich:

$$
J=C i \frac{d x}{d \lambda} \text {. }
$$

Rechnet man die Abstande $x$ im prismatischen Spectrum ron irgend einem Anfangspunkte aus, so liisst sich nach den Dispersionsgesetzen $x$ angenähert ausdriicken dureh die Formel:

$$
x=a+\frac{b}{\lambda^{2}}+\frac{c}{i^{2}},
$$

wo $a, b, c$ Constanten sind, die von der Beschaffenheit des Prismas u. s. w. abhängen. Man hat also:

und folglich:

$$
d x=-2\left(\begin{array}{l}
b \\
\lambda^{3}
\end{array}+\frac{2 c}{\lambda^{5}}\right) d \lambda,
$$

$$
\frac{J}{i}=K\left(\frac{b}{i^{3}}+\frac{2 c}{i^{5}}\right),
$$

wo eine nene Constante $K$ statt $-2 C$ eingefiihrt ist. 
Bezicht man alle Intensititen anf eine bestimmte Stelle des Spectrums, so ergeben sich die Werthe von $J$ aus den entsprechenden beobachteten Werthen von $i$ durch Multiplication mit dem Ausdrucke $\frac{b}{i^{3}}+\frac{2 c}{\lambda_{0}^{5}}$, der fur jeden Spectralapparat zu ermitteln ist. Man kann die gesuchten Reductionen austatt durch Reehnung auch durch einfaches graphisehes Verfahren bestimmen.

Das Maximun der Liehtintensitiit liegt im Normalspectrum etwa in der Mitte zwischen den Spectrallinien $D$ und $E$, also ungefithr bei der Wellenlänge $558 \mu \mu$, dagegen in prismatischen Spectrum ungefihr in der Gegend von $D$.

Aus der beobachteten Lichteurve eines Spectrums ergiebt sich, noch die gesammte Intensitiit $L$ der untersuchten Lichtquelle mittelst der Formel:

$$
L=k \cdot \int_{\lambda_{1}}^{\lambda_{2}} J d \lambda
$$

wo $k$ eine Constante bedentet, und $\lambda_{1}$ und $\lambda_{2}$ diejenigen Wellenlïngen an den beiden Enden des Spectrums sind, wo jede Lichtwirkung aufhört. Hat man den Zusammenhang zwischen $J$ und $\lambda$ an hinreichend vielen Stellen durch Messungen ermittelt, so kann man $L$ durch mechanische Quadratur bestimmen.

Alle Versuche, die verschiedenen Partien eines und desselben Speetrums in Bezug anf ihre Helligkeit miteinander zu vergleichen, haben hauptsächlich wegen der Sehwierigkeiten, die sich in physiologischer Hinsicht entgegenstellen, nur wenig befriedigende Resultate ergeben. Weit fruchtbarer hat sieh der Gedanke erwiesen, die Speetra zweier Lichtquellen nebeneinander zu bringen und die verschiedenen Partien des einen mit den gleichgefïbten Partien des anderen zu vergleichen. Dureh dieses Verfahren erhält man nicht nur sehr zuverliissige Werthe für das Helligkeitsverhältniss der in beiden Lichtquellen enthaltenen Strahlengattungen, woraus sieh damn auch leicht das Verhiiltniss ihrer Gesammtintensitiiten finden liisst, sondern man gewimnt gleichzeitig auch eine ungefïlure Vorstellung von dem Temperaturverhäiltniss derselben. Wem nämlich die Spectra zweier Liehtquellen in den mittleren Partien grleiehe Intensitit haben, dageren an dem breehbaren Ende starke Intensitiitsunterschiede zeigen, so weiss man, dass diejenige Lichtquelle, deren Helligkeit im Blan iiberwiegrt, die höhere 'Temperatur besityt, und wenn man Flammen ron bekannter Temperatur zur Vergleichung benntzt, so kann man anf rein optischem Wege eine Art Temperaturbestimmung ausführen. Das Spectrum einer Petroleumflamme sieht neben dem Sonnenspectrum, wenn die Gleichheit in den griinen Theilen hergestellt ist, in 
den blanen und violetten Partien vollkommen dunkel aus, und das Speetrum des elektrischen Lichtes tibertrifft dasjenige einer Gasflamme durch einen Überschuss an brechbaren Strahlen.

Die Bedeutung der spectralphotometrischen Methode für die Technik, bei der die Frage nach der Ausuutzung einer Leuchtkraft im Vordergrunde des Interesses steht, liegt auf der Hand; sie ist fiur dieselbe von unschätzbarem Werthe geworden. Aber auch furr die Himmelsphotometrie ist diese Methode zweifellos von der allergrössten Wichtigkeit. Die Vergleichung verschiedener Sternspectren giebt einen Begriff von den Temperaturverhältnissen der betreffenden Himmelsobjecte und erlaubt in Verbindung mit spectralanalytischen Forschungen einen Sehluss auf das Entwicklungsstadium, in welchem sich dieselben befinden. Bei sehr verschieden gefärbten Sternen, wo die directe photometrische Vergleichung mit Schwierigkeiten verknüpft ist, darf man von spectralphotometrischen Messungen bessere Resultate erwarten. Von allerhöchstem Interesse sind solehe Untersuchungen in Bezng anf den Lichtwechsel der veränderlichen Sterne. Leider ist eine erfolgreiche Anwendung der Methode auf alle Probleme der Himmelsphotometrie wegen der verhältnissmässig geringen Lichtstïrke der meisten Gestirne nur mit Benutzung der mächtigsten Instrumente möglich.

Die Zahl der bisher speciell zn spectralphotometrischen Beobachtungen construirten Apparate, der sogenannten Spectrophotometer oder spectralphotometer, ist bereits ansserordentlich gross. Fast alle in den vorangehenden Capiteln erörterten Verfahren kommen dabei zur Anwendung, am häufigsten dic Polarisationsmethode. Im Folgenden sollen nur die wichtigsten derselben einer etwas eingehenderen Besprechung unterworfen werden.

\section{Das Govi'sche Spectralphotometer.}

Das erste Spectralphotometer riilnt ron Gori') her, welcher sich hereits im Jahre 1550 mit dem Plane zu diesem Instrumente beschäftigt latte, aber erst im Jahre 1860 eine Nittheilmug dariiber an die Pariser Akademie gelangen liess. Sein Apparat hat grosse Ähnlichkeit mit dem Ritche'sehen Photometer. Ein länglicher viereckiger Holzkasten hat an den beiden Enden zwei rollkommen gleiche verticale Spalte, auf welche das Licht der zu untersuchenden Lichtquellen fällt. Im Innern des Kastens, mugefihlu in der Mitte, sind zwei total reflectirende Prismen so angebracht, dass sie das ron den spalten herkommende Licht anf eine 
in der oberen Seite des Kastens befindliche Öffnung werfen. Vor dieser Öffnnng sitzt eine achromatisehe Linse, welehe das aus beiden Prismen austretende Licht parallel macht. Die Strahlen fallen damn auf ein grosses Flintglasprisma, dessen Kante der Längsrichtung des Kastens parallel ist, und welehes auf das Minimum der Ablenkung fur mittlere Strahlen eingestellt ist; die beiden entstehenden Spectra werden auf einer matten Glasscheibe aufgefangen. Dureh eine versehiebbare Platto mit schmalem Aussehnitt kann ein kleines Stllek ans ihnen heransgeblendet werden. Die gleiche Helligkeit der beiden Spectralstreifen wird dann durch Versehiebung der einen oder beider Liehtquellen hergestellt; - stwaige Unterschiede in den Spectralspalten und den reflectirenden Prismen können dadureh unschädlich gemaeht werden, dass der ganze Apparat um $150^{\circ}$ gedreht wird oder, was dasselbe ist, die Liehtquellen miteinander vertauscht werden.

Es ist von melhreren Seiten, besonders von Vierordt1), versucht worden, Govi die Prioritait der Erfindung des Spectralphotometers streitig zu machen. Jedenfalls mit Unrecht. Deun wenn sieh Govi anch nicht der ganzen Bedeutung und vollen Anwendungsfähigkeit seines Apparates, den er selbst > photomètre analyseur * nennt, bewusst gewesen ist, so entspricht derselbe, mit geringen Modificationen, so vollkommen allen Anforderungen, dic man heute an ein Spectralphotometer stellt, dass er unbedingt als Vorbild flir diese Classe ron Instrumenten ancrkannt werden muss, wenn auch bei den späteren Apparaten dieser Gattung eine wesentlich andere Form und ein anderes Beobachtungsverfahren gewählt worden ist.

\section{Das Vierorlt'sche Spectralphotometer ${ }^{2)}$.}

Dasselbe ist ein gewöhlulicher Spectralapparat, dessen Spalt durch einen besonders eonstruirten Doppelspalt ersetzt ist. Die eine Schncide desselben ist fest, wiihrend die andere in zwei Hïlften getheilt ist, von denen jede fiir sich mittelst einer feinen Mikrometerschraube hin und her bewegt werden kann. Die beiden Spalthïlften entwerfen im Beobachtungsfernrohre von einer Liehtquelle zwei scharf aneinander grenzende Spectra, welehe gleieh liehtstark sind, sobald die Spalthiilften gleichweit geöffnet sind. Um einen beliebig grossen Spectralbezirk benutzen zu kömnen und nicht von den angrenzenden Theilen beeinflusst zu werden, kann man in der Bremebene des Oculars dureh zwei gegeneinander

1) Wiedem. Annalen. Bd. 3, p. 375.

2) Pogg. Annalen. Bd. 140, p. 172.

Múller, Photometrie der Gestirne. 
verschiebbare Metallplatten einen Ocularspalt von willkürlicher Breite herstellen. Gewölınlieh wird der Apparat direct auf die zu untersuchende Lichtquelle gerichtet, während die andere Spalthälfte durch ein davor gesetytes total reflectirendes Prisma Licht von einer Vergleichsflamme (einer Petrolcumlampe ete.) erhält. Die Gleichheit der Helligkeit in den beiden Speetren wird durch Veränderung der Spaltbreiten bewirkt. Erweitert man den Spalt, so wächst die Liehtstärke des Spectrums proportional der Breite dessclben. Denn man kann sich den Spalt in lanter nebeneinander befindliche Spalte getheilt denken, ron denen jeder für sich ein Speetrum entwirft, und da die einzelnen Speetra sich übereinander lagern, so nimmt die Helligkeit direct proportional der Spaltbreite zu. Je breiter der Spalt ist, desto mehr Elementarspectra legen sich übereinander, desto unreiner werden aber anch die Farben in dem entstehenden Gesammtspeetrum, weil die einzelnen Farben sich nieht genau decken. Man darf also, um diesen Naehtheil zu vermeiden, den Spalt nicht über eine gewisse Grenze hinaus iffnen. Andererseits ist es aber auch nicht rathsam, den Spalt allzu sehr zu verengen. Denn in diesem Falle können Unrollkommenheiten der Spaltbaeken, anhaftende Staubpartikelehen u. s. w. sehr leieht die Proportionalität zwisehen Öffunng und Intensität stören. Unter allen Umstïnden wird man demnaeh mit dem Vierordt'sehen Doppelspalt nicht sehr grosse Intensitïtsuntersehiede direct messen diurfen. Vierordt hat diesen Mangel sehon selbst erkannt und daher vorgeschlagen, die stirkere der zu vergleiehenden Lichtquellen dureh rorgesetzte Blendglïser zu schwächen; da es aber sehwierig, wenn nieht ganz ummöglich ist, Blendgliiser zu erhalten, welehe alle Farben ganz gleiehmässig absorbiren, so bleibt es bei Anwendung solcher Gläser eine sehr listige aber unumgängliche Forderung, die Absorptionseoeffieienten derselben fiir möglichst riele rerschiedenen Farben zu bestimmen. Der Tierordt'sche Doppelspalt hat in nenerer Zeit noch eine wesentliehe Verhesserung erfahren. Urspringlich war die eine Spaltsehneide fest. Wenn daher die beiden Hälften verschieden weit geöffnet werden mussten, dann fielen ihre Mitten nicht zusammen, und die Folge davon war, dass die beiden entstehenden Spectra ein wenig gegeneinander versehoben walren, und nicht rollkommen gleiche Farbenbezirke rergliehen werden konnten. Diesem Übehstande ist ron Kriiss alogeholfen worden, welcher die beiden spralthïlften on eingerichtet hat, dass bei jeder beide Baeken sich srmmetrisch bewogen, so dass die Mitten der heiden Hälften stets zusimmenfallen.

Vierordt hat seinen Apparat hauptsiichlich zu Untersuehungen der

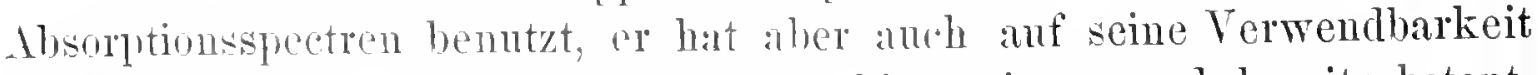
fiir Messungen an den Ilimmelskibruern hingewiesen und bereits betont, 
dass die Kenntniss der Helligkeiten der Einzolfarben in den Sternspectren ebenso wichtig, wenn nicht wichtiger sei, als die der Gesammthelligkeiten. Meines Wissens sind Versuche in dieser Riehtung niemals angestellt worden, aher ohne 'Zweifel eignet sich gerade das Kierordt'sche Spectralphotometer sehr gut zu Beobachtungen an Sternspectren, schon deshalb, weil bei ihn dio erste Bedingung, die volle Ausnutzung des vorhandenen Lichtes, viel besser erfillt ist, als bei den meisten anderen Spectralphotometern. Als ein Vorzug der Vierordt'schen Methode ist die grosse Gonanigkeit anzusehen, die sieh mittelst derselben erreichen liasst, sowie der Unstand, dass diese Genanigkeit flir alle Grade der Intensitii, bei welehen die Vergleiehungen ausgefuhrt werden, dieselbe bleibt, was \%. B. bei den auf dem Polarisationsprineipe beruhenden Spectralphotometern uieht der Fall ist. Ans einer grösseren Reihe von Messungen mit einem Vierordt'schen Apparate habe ich als wahrscheinliehen Fehler einer Helligkeitsvergleichung in den grunen Theilen des Spectruns 0.61 Procent, in den blanen Theilen 0.75 Procent des gemessenen Intensitiitsrerhältnisses gefunden.

\section{Das Glan-Vogel'sche Spectralphotometer.}

Dasjenige Spectralphotometer, welehes am meisten verbreitet ist und bisher allein von allen ausgedehntere Verwendung in der Astrophotometrie gefunden hat, ist das unter dem Namen des Glan-Vogel'schen bekannte. Das Prineip und die allgemeine Einrichtung rihrt von Glan') her, wihrend Vogel2) dem Apparate diejenige Form gegeben hat, in weleher er hente gewöhnlich benutzt wird, und die sich am hesten zu Untersuchungen am Himmel bewihhrt hat; auch sind von Vogel die eingehendsten Studien an diesem Instrumente angestellt worden. Der folgenden Beschreibung. ist dasjenige Photometer zu Grunde gelegt, welehes fuir das Potsdamer Observatorinm von Schmidt und Hïnseh in Berlin angefertigt worden ist. Der Apparat (Fig. 65, Seite 276) kann entweder auf ein festes Holzstativ aufgelegt werden oder er wird mittelst des Rohrendes $O$ in den Ocularstutzen eines grösseren Refractors so weit eingeschoben, dass der Spalt in die Brennebene fällt.

Der im Innern des liohres liegende Spalt, welcher dureh die Sehraube $s$ symmetrisch zur Mitte geöfhnet oder geschlossen werden kann, wird durch

1) Wiedem. Annalen. Bd. 1, p. 351.

2) Monatsber. der K. Preuss. Akad. der Wiss. 15it, p. 104. - Eine ausführliche Kritik des Glan'schen Photometers findet sich in einem Aufsatze von Ketteler und Pulfrich in Wiedem. Annalen, Bd. 15, p. 337. 
einen etwa $2 \mathrm{~mm}$ breiten Metallsteg in zwei Hälften getheilt. Die eine Hälfte empfäingt direet von $O$ her das Licht der zu untersuchenden Lichtquelle, während die andere Hälfte durch eine zur Vergleichung dienende Petroleumlampe $l$ beleuehtet wird. Diese Lampe, durch ein Gegengewicht $G$ ansbalaneirt, ist in den Gabeln $g g$ beweglich, ausserdem noch un eine andere Axe drehbar, so dass sie bei allen Lagen des Apparates eine senkrechte Stellung behalten kann; eine Wasserwage $w$ dient zur Controle der riehtigen Lage. Das Licht der Lampe fällt zunäehst auf das total reflectirende Prisma $p$ und gelangt von diesem auf ein zweites unmittelbar vor dem Spalt sitzendes Prisma (in der Figur

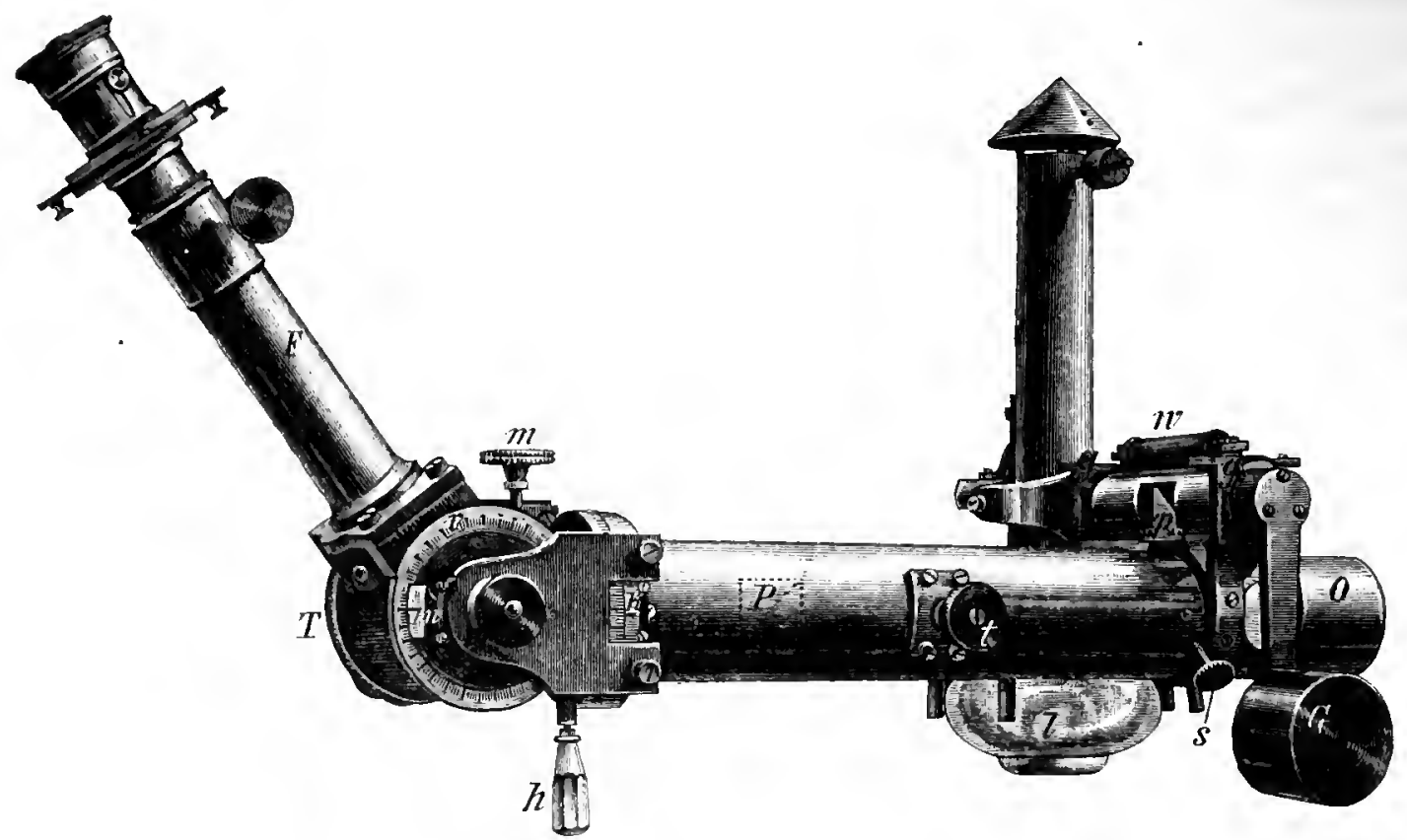

Fig. 68.

nicht sichtbar), welches sich durch eine einfache Vorrichtung nach Belieben ror die eine oder andere Hiilfte des spaltes oder auch ganz bei Seite schieben liisst. Das Vergleichslicht kaun anf diese Weise sowohl durch die eine als durch die andere spalthialfte in das Photometer geleitet werden oder anch ganz daron answeschlossen bleiben. Für die Jnstirmg des Aplarates ist dies ron Vortheil. Die von den beiden Spalthälften kommenden Lichtstralnlen werden durch eine Collimatorlinse, welche mit Ifilfe des Triebes t bewegt werden kamn, parallel gemaeht und gelangen dimn anf ein doppelthrechendes Bergkrystallprisma $P$, desseu Ifauptselnitt der Spaltrichtmog parallel ist. Das Ende des Hauptrohres nimmt ein Ninolprisma als Analysator ein, welches mittelst des Handgriffes he gedreht werden kamm; mit demselben fest verbunden ist eine Kreistheilung li, die an zwei einander gegentiberliegenden Nonien 
abgelesen wird. Anf dem runden tellerartigen Stllek $T$ ist ein stark zerstreuendes Flintglasprisma befestigt und zwar in der Weise, dass seine breehende Kante der Spaltrichtung parallel ist, und dass es fur Strahlen von mittlerer Brechbarkeit im Minimum der Ablenkung steht. Das Beobachtungsfernrolır $F$ ist wie bei den Vierordt'schen Apparaten mit Schieberrorrichtungen in der Brennebene versehen, welehe gestatten, eine rechteckige Öfrnung von belicbiger Breite und Höhe herzustellen. D Durch die Wirkung des doppeltbreehenden Prismas $P$ und des Flintglasprismas entstehen im Beobachtungsfernrohre vier Spectra, zwei von jeder Spalthiilfte, von denen je zwei senkrecht zu einander polarisirtes Licht enthalten. Die Breite des vor der Mitte des Spaltes sitzenden Steges ist so bemessen, dass zwei Speetra unmittelbar aneinander grenzen, während die beiden anderen dureh die Sehieberplatten verdeckt werden. Mittelst der anderen Schieber lïsst sich noch ein beliebig schmaler Streifen ans den Spectren heransblenden, und durch Drehung des analysirenden Nicols kann die Helligkeit beider gleich gemaeht werden. Das Intensitäitsrerhältniss der untersuchten Liehtquelle zu der Petroleumlampe an der betrefienden Stelle des Spectrums wird dann bei richtiger Justirung der einzelnen Theile durch das Quadrat der Tangente des an der Kreistheilung $k$ abgelesenen Winkels gegeben. Natiirlieh ist es rathsam, behufs Elimination des Index- und Exeentrieitätsfehlers, wie bei jedem Polarisationsphotometer, die Einstellungen in allen vier Quadranten zu machen. Das Fernrohr $F$ liisst sieh noch mittelst der Sehraube $m$ um eine dureh die Mitte des Flintglasprismas, parallel zn seiner breehenden Kante gehende Axe bewegen, und diese Drehung kann mit Hiilfe des Nonins $n$ an der Kreistheilmug $v$ abgelesen werden. Man ist so im Stande, jeden beliebigen Theil des Spectrums in die Mitte des Gesichtsfeldes zu bringen. Um die Wellenlïnge der untersuchten Stelle aus den Ablesungen an $v$ angenähert angeben zu können, muss für jerlen Apparat auf graphisehem Wege eine Tabelle hergeleitet werden, welche den Zusammenhang zwischen Wellenlainge und Einstellung am Kreise angiebt. Zu diesem Zwecke wird am Besten das Sonnenspectrum benutzt. Man bringt die bekanntesten Fraunhofer'sehen Linien der Reihe nach in die Mitte des selumalen Ocularspaltes, notirt die entsprechenden Ablesungen am Gradbogen $v$ und leitet daraus graphisch eine Einstellungstabelle ab. Da der Ocularspalt offer mit oder ohne Absieht veräindert wird, so muss man sich vor jeder Beobachtungsreihe iberzeugen, ob die Tabelle noch Giiltigkeit hat; man stellt zn diesem Zweeke den Kreis $v$ auf diejenige Ablesung, welehe nach der Tabelle einer bestimmten Speetrallinie, z. B. der $D$-Linie, entsprieht, und verïindert eventuell die Stellung. der Ocularschieber, bis dic Linie genau in der Mitte des Spaltes erseheint. 
Es empfichlt sieh im Allgemeinen nicht, diesen Ocularspalt zu breit zu wihlen, weil dann, namentlich in den ziemlich dieht zusanmengedrängten weniger brechbaren Theilen des Spectrums, ein viel zu grosser Wellenlïngenbezirk mit einem Male ibersehen wird; andererseits darf aber der Ocularspalt anch nieht zu eng gemacht werden, weil dann die Sicherheit der Beobachtungen leidet. Grossen Vortheil würde die Verwendung von Reflexgittern anstatt der Dispersionsprismen bieten, denn in diesem Falle wiirde man an allen Stellen des Spectrums ein gleich grosses Wellenlïngenintervall iibersehen, und die Einstellungen an dem Gradbogen $v$. wïren nmmittelbar den WellenIängen proportional. Die directe Benutzung des Normalspectrums hat freilieh aneh den Nachtheil, dass das Spectrum noch lichtschwäelier ist, auch wird man meistens nur das Spectrum erster oder lï̈chstens zweiter Ordnung benutzen diurfen, weil sonst Übereinanderlagerungen störend wären.

Ein grosser Vorzug des Glan-Vogel'schen Spectralphotometers ror dem Vierordt'schen besteht darin, dass viel grössere Helligkeitsuntersehiede direct ohne Zuhiilfenahme von Blendg]ïsern gemessen werden können, und dass die Spectralfarben im Allgemeinen viel reiner sind, weil der spectralspalt nicht weiter geöfnet zn werden braueht, als gerade nöthig. ist, damit die störenden Framuhofer'schen Linien versehwinden. Ein Nachtheil ist dagegen der Lichtverhst, weleher durch die Trennung in zwei Lichthindel bedingt wird; derselbe erschwert insbesondere die Ausfiihrung der Hessungen in den brechbareren Theilen des Speetrums. Was dic Genanigkeit anbetrifft, welehe bei den Messungen zu erreichen ist, so scheint im Grossen und Ganzen der Vierordt'sche Apparat iberlegen zu sein. Wiihrend meine Messungen nit diesem für den wahrscheinlichen Fehler ciner Beobachtung Werthe zwischen 0.61 und 0.75 Procent crgabeu, erhielt irli ans Messungen an dem Glan-Vogel'schen Instrumente Werthe awischen 1.2 and 2.5 I'rocent. Die meisten Beobachter finden eine verhälnissmaissign grössere Genanigkeit in den mittleren Theilen des spectrums, als im iussersten loth und Violett; anch von der absoluten Intensitiat, hei welcher die Gleichheit in boiden Spectren stattfindet, seheint der Genauigkeitsgrad ahlïngig, und zwar in der Weise, dass die Sicherheit bei nittleren Helligkeitsgraden an wrissten ist, bei sehr heller Belenchtung am kleinsten. Bei Ablesungen des Intensitätskreises $l_{i}$ in der Xïhe von $11^{\circ}$ oder $(y)^{\circ}$ wïre es dbenso wie bei dem Pickering'schen Meridianphotometer erwinscht, das Nienlyrisma feiner als aus freier Hand drehen zu kimmen und den Kreis selbst genaner als bis auf Zehntel Grade abzulesen, weil das liesultat schou durch sehr geringe Fehler in dieser Bezielung merklid, beeinflusst wird.

Ein Hamptmangel, der dem Glan-Vogel'schen Instrumente zur Last 
gelegt wird, bezicht sich darauf, dass die beiden zu vergleichenden Spectra nicht der. ganzen Länge nach in einer scharfen Linie aneinander stossen. Es ruhrt dies von der Wirkung des doppeltbrechenden Prismas her, welches fur violettes Licht das ordentliche und ansserordentliche Bild etwas weiter auseinander bringt, als fur rothes Licht. Da dies nun fur die Bilder beider Spalthalften gilt, so milssen die beiden aneinander grenzenden, entgegengesetzt polarisirten Speetra, falls sie sich an einer bestimmten Stelle, \%. B. in Grlin genau berilhren, in den violetten Partien etwas Ubereinander liegen, dagegen im Roth durch einen dunklen Zwischenraum getrennt sein. Um diesen Übelstand, weleher die Sicherheit der Messungén beeintrïiehtigt, „u beséitigen, kamn mau nach Glans Vorgange das Collimatorohjectiv mit Hulfe des Triebes $t$ dem Spalt nähern oder von ihm entfernen. Eine derattige Verschiebung bringt eine Änderung in tem Gange der Strahlen hervor und bewirkt, dass ordentliches und ausserordentliches Bild derselben Spalthïlfte weiter auseinanderfallen oder nïher zusammenriicken. Auf diese Weise lassen sich die beiden Spectra in jedem beliebigen Farbenbezirke zum Contact bringen; freilich ist dabei auch jedesmal eine entspreehende Verstellung des Fernrohroculares erforderlich, danit die Linie, in weleher sich die Spectra bertihren, seharf erscheint. Croval) hat ein einfaches Mittel vorgesehlagen, um die listige und nicht ganz unbedenkliche Verschiebung von Collimatorobjectiv und Fernrohrocular zu vermeiden. Dasselbe hesteht darin, dass man als Trenumngssteg einen Metallstreifen benutzt, dessen Riinder nicht prarallel sind, sondern einen kleinen Winkel miteinander bilden, und der vermittelst einer Schranbe in der Richtung senkrecht zur Spaltlïnge verschoben werden kamn. Dadurch ist ein Steg von variabler Breite hergestellt, und die genane Berilurmug der Speetren liisst sich an jeder beliebigen Stelle erreichen.

Zn erwähnen ist noch, dass durch innere Reflexe an den verschiedenen Linsen und Prismenfliichen sehr leicht diffuses Licht erzenght wird, welches sich wie cin diumer Nebel iiber das ganze Spectrum rerbreitet und die Messungen erschwert. Es wird dies namentlich dann fiilulbar, wemn die untersnchte Liehtquelle im Vergleich zur Petrolemmlanpe sehr intensir ist; denn in diesen Falle wird die nicht ans dem Nicol anstretende ordentliche Componente in Innern desselben melırfache Reflexion erleiden und einen Lichtsehimmer auch auf das Spectrum der auderen Spalthïlfte werfen. Han sieht daher häufig, anch wenn das Licht der Petrolenmlampe ganz abgeblendet wird, diejenige Stelle im Gesichtsfelde, wo das Spectrum derselben linfällt, nicht rollkommen dunkel. Am Auf-

1) Annales de chimie et de physique. Série 5, t. 19, p. 495. 
fallendsten und Störendsten treten diese Nebenliehtwirkungen in den brechbareren Partien des Spectrums hervor. Bis zn einem gewissen Grade lïsst sich dureh sorgfältige Schwärzung der inneren Theile des Apparates Abhtilfe schaffen.

\section{Das Crova'sche Spectralphotometer.}

In mancher Hinsieht verdient das ron Croral) construirte Spectralphotometer den Vor\%ug vor dem Glan-Vogel'schen. Bei demselben könneu entweder einfache Prismen oder Prismensaitze à vision directe zur Verwendung kommen; die letzteren haben den Nachtheil, dass die Absorption im Blau und Violett sehr bedeutend ist, und dass ansserdem die Kittung der einzelnen Theile Verinderungen unterworfen ist und die Bilder daher

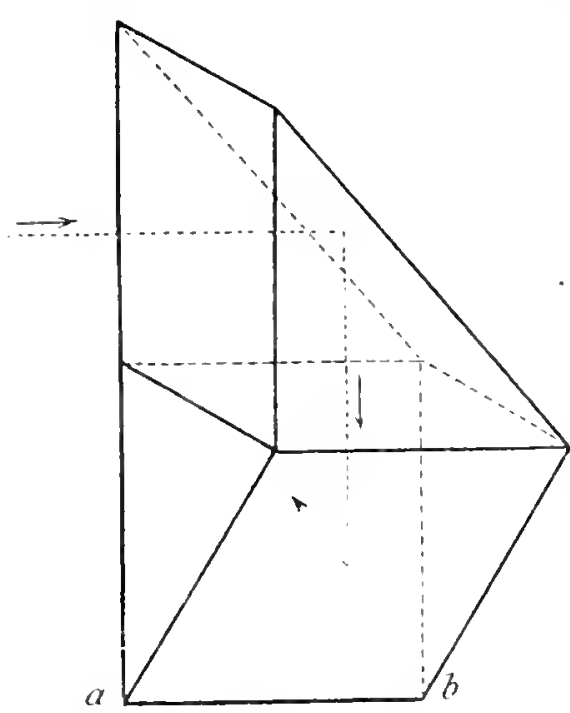

Fig. 69 .

mit der Zeit leicht triibe werden. Crova benutzte einen Satz ron fünf Prismen, von denen das mittelste ohne Ablenkung ron den Strahlen mittlerer Breehbarkeit durchlanfen wurde, wälnend die anderen parweise srmmetriseh dazu standen. Die Stellung des Beobachtungsfermrohrs lässt sich, wie bei den meisten Spectroskopen a rision directe, an einem Gradbogen ablesen, und mit Hiilfe einer Tabelle kann man jeden belicbigen Theil des Spectrums in die Mitte des Gesichtsfeldes einstellen. Das Ocular ist mit der uiblichen Sehiebereinrichtung rersehen. Vor der einen Hälfte des gewöhnlichen Spectroskopspaltes sitzt cin Prisma mit doppelter tutaler Reflexion, welches die ans Fig. 69 ersichtliche Gestalt hat.

Dasselbe besteht ans zwei rechtwinkligen Prismen, ron denen das eine so ror der Spaltplatte befestigt ist, dass die seharfe Kante $a b$ stukrecht zur Spaltrichtung ist. Das zweite Prisma sitzt anf dem ersten, und zwar in der Weise, dass die eine Kathetenfliche nach unten, die indere nach der seite gekelurt ist. Eine seitlich anfgestellte, oder :ihnlich wie beim Glan-Togel sehen Phntometer beweglich aufgehäng'te Teroleichstampe sendet dimn ihr Licht anf dem in der Figur dureh Pfeile angedenteten Wene in die eine spalthiilfte, wïhreud die andere ron der zu untersuchenden Lichtquelle belenchtet wird. Das Arrangement hat den

1) Annales le elimie et de physique. Série 5, t. 29, p. 556. 
Vortheil, dass die beiden Speetra der ganzen Länge nach in einer feinen Linie zusammenstossen, was selten vollkommen zu erreichen ist, weun man nur ein gewöhnliches totalreflectirendes Prisina anwendet. In einem kurzen seitlichen Rohre, dureh welehes die Vergleichsflamme-ihr Licht sendet, befindet sich ein Nicol, dessen Drehung an einem 'Theilkreise abgelesen wird; dasselbe dient als Polarisator. Ein zweites festes Nicolprisma ist ebenfalls in dem seitlichen Rohre munittelbar hinter dem ersten, also vor dem total reflectirenden Prisma angebracht. Ist $J$ dic Intensitit der direet beobachteten Liehtquelle, $J^{\prime}$ diejenige der Vergleichsflamme, und ist $\alpha$ der an bewegliehen Nicol abgelesene Winkel fur den Fall, dass an irgend einer Stelle die Gleichheit der Spectren hergestellt ist, so findet man das Helligkeitsverhältniss der beiden Lichtquellen fuir die betreffende Spectralfarbe ausgedritekt durch die Formel:

$$
\frac{J}{J^{\prime}}=\frac{1}{2} k \sin ^{2} \alpha,
$$

wo $k$ eine Coustante ist, die von der Absorption des Lichtes in den Prismen abhïngt. Dabei ist die Stellung des festen Nicols so regulirt, dass am Kreise der Winkel 0 abgelesen wird, wenn gar kein Licht durch die beiden Nicols hindurch gelangt. Wie man sofort sieht, hat das Crova'sche Spectralphotometer den grossen Vorzug, dass die zu untersuchende Lichtquelle nicht, wie beim Glan'schen, dureh Doppelbreehung gesehwächt wird, und dass anch ein etwaiges Vorhandensein von polarisirtem Lichte keinen Fehler in die Messung bringt. Das Crora'sche Instrument eignet sich daher mehr zur Untersuchung schwächerer Lichtquellen, und es empfiehlt sich, dasselbe für die Sternspectren in Anwendung zn bringen. Da um die seitliche Veroleichsflamme geschwicht werden kaun, so muss dieselbe stets heller und zwar mindestens zweimal so hell sein, als die zu untersuchende Lichtquelle. Wenn dies nicht der Fall ist, so muss man die direet gesehene Lichtquelle durch Blendgliiser oder irgend ein anderes Verfahren absehwiichen. Ls ist dies ein Nachtheil des Crovatschen Apparates.

Eine ganze Anzahl ron Speetralphotometern, bei denen ebenfalls die Polarisation des Lichtes zur Verwendung kommt, sind dem Crova'schen :ihnlich. Unter ihnen sind besonders herrorzuheben die Photoneter von Gouy $\left.{ }^{1}\right)$ und Glazebrook ${ }^{2}$, welche sich dadmeh rou den bisher erwähnten unterseheiden, lass für die beiden zu vergleichenden Lichtquellen zwei besondere Collimatoren benutzt werden, was fuir manche Untersnchungen ron Vortheil ist.

1) Annales de chimie ct de physique. Série 5, t. 18, p. 1.

2) Proc. of the Cambridge Philus. Soc. 'T. 4. p. 304. 


\section{b. Das Interferenz-Spectralphotometer von Trannin.}

Kurze Erwiilnumg verdient noch eine Classe von Spectralphotometern, bei denen das Verschwinden von Interferenzstreifen beobachtet wird. Das bekimnteste dieser Instrumente, welches noch älter als die Photometer ron Glan und Crova ist, rüht von Trannin') her. Die beiden zu vergleichenden Lichtquellen werfen ihr Lieht von entgegengesetzten Seiten anf zwei total reflectirende Prismen, welche die obere nud die nutere Spalthälfte eines Spectralapparates bedecken. Beim Austritt ans dem Collimator werden die Strahlen durch irgend einen Polarisator (Nicol'sehes oder Foncault'sches Prisma), dessen Hauptschnitt der Spaltrichtung parallel ist, polarisirt, passiren damn eine senkrecht zur Axe geschnittene Quarzplitte ron etwa $1 \mathrm{~cm}$. Dieke, deren Hauptschnitt einen Winkel ron $45^{\circ}$ mit dem des Polarisators bildet, gehen ferner durch ein Rochon'sches oder Wollaston'sches Prisma, dessen Hauptsehnitt wieder parallel dem des lolarisators ist, und werden damn erst durch die Prismen des Spectralalplarates in die einzelnen Farben zerlegt. Im Beobachtungsfernrohre crblickt man vier Spectra, fon denen je zwei entgegengesetzt zu einander polanisirt sind, und ron denen die beiden mittleren (den beiden Licht'pnellen zugehörig. zum Theil iibereinander liegen. Durch die Quarzplatte werden in allen vier Spectren Interferenzstreifen hervorgebracht, und zwar wechseln in den senkrecht zn cinander polarisirten Spectren die dunklen nnd hellen Streifen miteinander al). In dem Theile, wo die Spectra iihereinander liegen, und der allein beobachtet wird, versehwinden die streifen rollstandig, sobald das Licht beider Lichtquellen in dem betretfenden Spectralbezirke greich ist. Han stellt diese Gleichheit dadurch her. dass man eine der beiden Lichtrnellen oder anch beide rersehiebt; das Ferhähtniss der Ruadriste der Distanzen ron dem Spalt giebt damn das enestchte Helligkeitsverhältuiss. Dibei ist Raicksicht zu nehmen anf

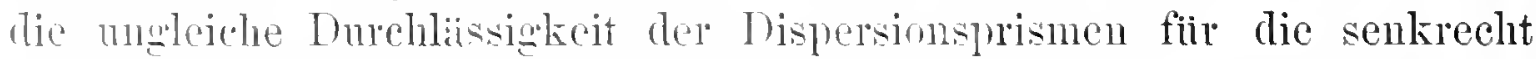
20 cinander polarisirten strahlensyteme. was dadurch emeicht werden Kann, dass man während der Beobachtungen das doppelthrechende Prisma nun Ist" lleht. lons Terfalnen wird noch exacter, wenn man stets eine Aritte constante Lichtynelle, die unveriunderlich mit dem Apparate in Terhindung wetracht werden kann. als Teroleichsolject benutzt, and diese

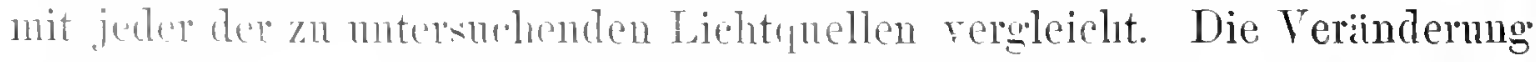

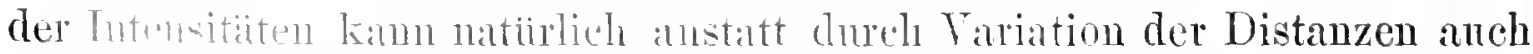

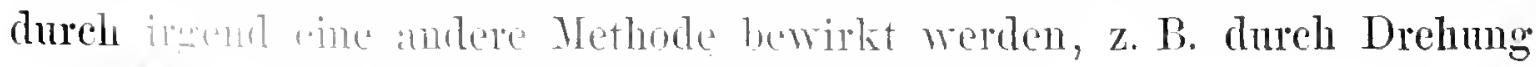

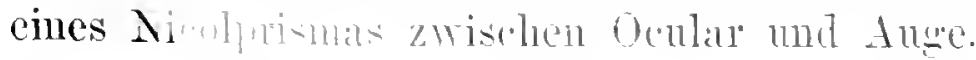

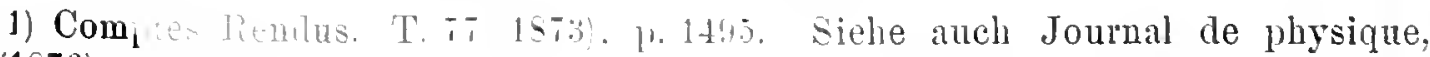
T. $5(1576)$ : p. 
Auf ganz ahnlichen Principien wie das 'Irannin'sche Photometer bernhen die Apparate ron Gouy ${ }^{1}$ ), Krech ${ }^{2}$ ) und Violley). Auch Wild') hat sein zweites Photometer in ein Spectralphotometer umgewandelt. Sämmtliche Instrumente gestatten eine grosse Genauigkeit der Nessungen, leiden aber au dem gemeinsamen Übelstande, dass ein betrichtlieher Theil des Lichtes alssorbirt wird, und dass sie daher nur zur Messung selir intensiver Strahlungen geeignet sind. Filr die Sternphotometrie dirften sie kaum rerwendbar sein.

\section{Spectralphotometer mit Alssorptionskeil.}

Zn Untersuchungen uber die Helligkeitsvertheilung im Speetrum der Sonne und des Mondes ist in neuester Zeit flir das Potsdamer Observatorium nach meinen Angaben von Töpfer ein Apparat eonstruirt worden, bei welchem das Auslöschungsprincip zur Anwendung kommt. Der :iusseren Form nach ist das lnstrument (Fig. 70, Seite 281) vollkommen ähnlich dem grossen Potsdamer Keilphotometer (Seite 192); es ist parallaktisch inontirt, und das Beobachtungsfernrohr $F$ ist nach dem Pol gerichtet.

Der Collimator $C$ ist seitlich in den Würfel $w$ eingeschraubt, und der Spalt wirl dureh die Schraube $s$ symmetriseh zur Yitte verbreitert oder verengt. Vor dem Spalte sitzt in dem Rohre $R$ ein total reflectirendes rechtwinkliges Prisma. Das Rohr ist $\mathrm{mm}$ die Axe des Collimators drehbar, und an der Kreistheihng $k$ liisst sich die Declination des betrachteten Himmelsobjectes einstellen. Eine kleine Linse $l$ an Ende des Rohres entwirft auf der Seheibe $m$ ein punktförmiges bild der Sonne oder des Mondes, und die Justirung der einzelnen Theile ist so angeordnet, dass das Bildchen sich auf die Mitte dieser Scheibe projicirt, wenn das Licht genau auf die Mitte des Spaltes fällt. Wiihrend der Beobachtung hat ein Gehiilfe durch langsames Drehen an der Feinbewegung $f$ des Stundenkreises datiir zu sorgen, dass das Sonnenbildchen bestiindig anf der Scheibeumitte bleibt. In Innern des Wuirfels $w$ ist ein Rowland'sches Diffractionsgitter angeloraclit, dessen Striche der Spaltrichtung parallel sind. Dieses Gitter kimm mittelst des Knopfes $n$ um

1) Comptes Rendus. T. 83 (1876), p. 269 und Annales de chimie et de physique, Série 5, t. 18, p. 15.

2) Krech, Photometrische Untersuehungen. (Wissenseh. Beilage zum Programm des Luisenstädtischen Gymnasiums zu Berlin, 1853.)

3) Annales de chimie et de physique. Súrie 6. t. 3, p. 391.

4) Bull. de l'acad. Imp. des sciences de St. Pétersbourg. T. 28, p. 392. - Siehe auch Wiedem. Annalen, Bd. 20, p. 452. 
grösscre Betrïge, mittelst der Feinbewegung $i$ um minimale Strecken gedreht werden, und die Drehung wird an dem Gradbogen $v$ mit Hülfe von Nonius und Lupe abgelesen. Anf diese Weise wird jeder beliebige Theil des Spectrums in die Mitte des Gesichtsfeldes gebracht. An dem Ocularende des Beobachtung:sferurohrs ist das auf Seite 184 beschriebene Keilphotometer angesetzt; an Stelle des dort erwähnten Steges wird durch den Knopf $r$ ein Schieber unmittelbar vor den Keil eingeführt, welcher das ganze Gesichtsfeld bis auf eine schmale spaltförmige Öffnung, dic genan parallel den Spectrallinien zu stellen ist, abblendet.

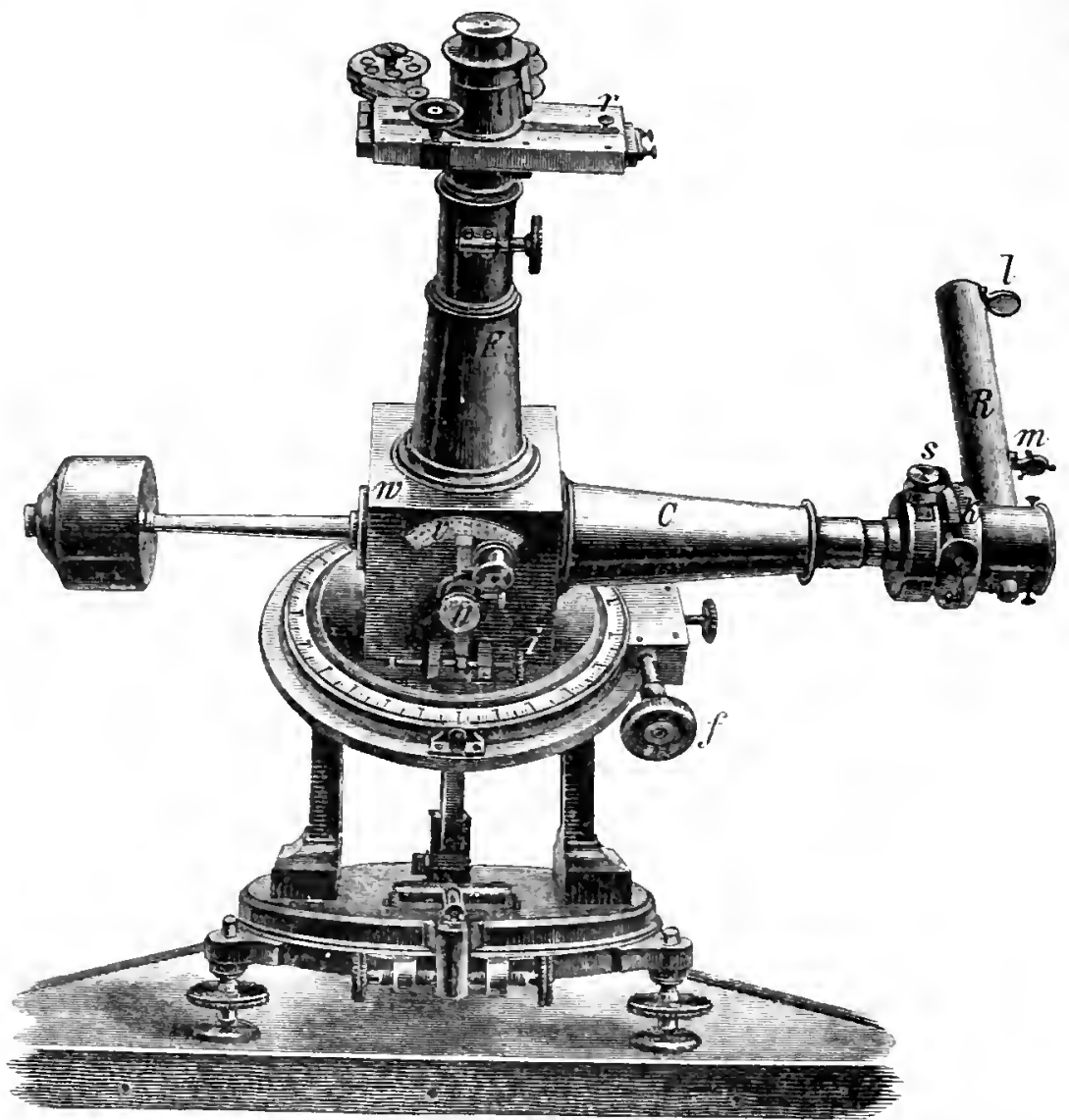

Fig. 70.

Man bringt nun durch Drehung des Gitters die einzelnen Spectralbezirke in diesen Ocularspalt und löscht dieselben mit Hülfe des Keiles aus. Es versteht sich ganz ron selbst, dass der Beobachter durch Bedecken mit einem dunklen Tuche rur jedem inssereu Lichte geschuitzt sein muss. Die friiher erwiihnten Übelstinde des keilphotometers machen sich natiirlich im vollen Grade fiuhlbal, mol wan hesonders bedenklich ist hier der Umstand, dass die dunklen Glissorten, ans denen die Keile gefertigt werden, selten alle Farben gleithmissig absorbiren. Eine sorgfältige Untersuchung der benutzten Keile in dieser Beziehmng ist durchaus er- 
forderlieh, und es ist rathsam, fur alle diejeuigen Spectralgegenden, in denen man Messungen vornelumen will, die Keileonstante besonders zn bestimmen.

In Grossen und Ganzen bietet die Auslöschungsmethode in der Spectralphotometrie manehe Vortheile und durfte sich vielleicht, mit entsprechenden Modificationen, auch zur Beobachtung der Sternspeetren eignen.

\section{Capitel IV.}

\section{Einiges ubber Lichtmessungsverfahren, bei denen nicht das Urtheil des Auges zur Anwendung kommt.}

In den vorangehenden Capiteln sind nur solche lichtmessenden Apparate besprochen worden, bei denen es in letzter Instanz auf dis Urtheil des Auges ankommt, sei es, um das Verschwinden eines Lichteindruckes auf der Netzhant zu constatiren, sei es, un die Gleichheit zweier verschiedenen Liehteindrieke zu beurtheilen. Wenn wir die Definitionen Lichtstärke, Helligkeit u. s. w. anssehliesslieh auf diejenige Wirkung der Ätherbewegung bezichen, welche in unserem Auge eine Gesichtsempfindung hervorruft, so ist damit das Gebiet der Photometrie streng abgegrenzt, und unsere messenden Hiilfsmittel sind mit den bisher anfgezählten nahezu ersehöpft. Gleichzeitig ist damit aueh die Genauigkeitsgrenze, die bei photometrischen Messungen iiberhaupt erreichbar ist, festgelegt; sie hängt ganz und gar ron der Empfindlichkeit der Netzhant ab, und da wir wissen, dass diese von Person zu Person versehieden ist, und selbst bei demselben Beobachter im Lanfe der Zeit merklichen Schwanknngen unterworfen sein kann, so sind wir gezwungen, nus mit einem Grade der Sicherheit zu begnügen, den wir durch keine Verfeinerung unserer instrumentellen Hiilfsmittel erhöhen können. Der berechtigte Wunseh, diese Grenze, wenn irgend möglich, noch zu iiberschreiten, hat immer wieder den Gedanken angeregt, bei den Helligkeitsmessungen das unvollkommene Sehorgan ganz entbehrlich zu machen und 
die physiologische Wirkung durch irgend eine andere weniger subjective zu ersetzen. Insbesondere hat man versucht, die von jeder Lichtquelle ausgehende Wärmewirkung und die von ihr hervorgerufenen chemischen Processe als Mass der Helligkeit zu verwerthen. Die Berechtigung zu diesen Versuchen bedarf keiner Vertheidigung, und da jede Veränderung der Liehtstïrke (in dem gewöhnlichen Sinne) auch von einer Veränderung der Wiirme und der ehemischen Wirkung begleitet ist, so kann man mit gewissem Fug und Recht Apparate, welche zur Messung dieser Wirkungen dieneñ, auch Photometer nennen; uur darf man nicht vergessen, dass photographische, optische und thermische Photometrie, wenn man sie-so nennen will, wesentlich voneinander verschieden sind, und dass ein directer Zusammenhang zwischen ihnen, wenn iiberhaupt, jedenfalls nur mit grossen Schwierigkeiten ermittelt werden kann. Man nimmt zwar nicht mehr an, wie es friiher häufig gesehah, dass allein die am Wenigsten breehbaren Strahlen eine Wärmewirkung, die brechbarsten lediglich eine chemische Wirkung ausiiben, während die mittleren die Gesichtsempfindung hervorrufen; man weiss jetzt, dass innerhalb des ganzen sichtbaren Spectrums sowohl eine erwärmende als eine chemische Wirkung der Strahlen rorhanden ist, und dass nur das Maximum derselben sich verschiebt, je nach der Beschaffenheit derjenigen Körper, welche dem Lichte ausgesetat sind. Das Maximum der Warmewirkung im Spectrum bei Terwendung von gewöhnlichen Glasprismen liegt im Roth, dagegen bei Benutzung eines Wasserprismas im Gelb; ebenso kanu das Maximum der photographischen Wirkung aus dem Violett nach jedem anderen Theile des siehtbaren Spectrums riicken, je nach den Substanzen, mit denen man die empfindlichen Platten imprägnirt. Die Frage, ob es möglich ist, aus der Stärke der einen Wirkung unmittelbar auf die der anderen zu schliessen, und ob man daher berechtigt ist, die rerschiedenen Wirkungen wach Gefallen durch einander zu ersetzen, ist im Princip durchans zu rerneinen. Eine I'roportionalitait findet unter gewissen Bedingungen allerding statt. Iiandelt es sich z. B. um zwei Lichtquellen, welehe nur Strahlen ron ein und derselben bestimmten Wellenlänge aussenden, so wird man finden, dass, wenn die eine doppelt so hell erscheint als die andere, damn anch die thermische und chemische Wirkung der ersten doppelt so stark ist als diejenige der zweiten. Etwas Älnnliches wird sich ergehen, wenl es sich nicht um homogenes Lieht handelt, sondern m zusammengesetztes Licht von solcher Beschaffenheit, dass die einzelnen Strahlengattmugen hei heiden Lichtquellen in gleichem Verhïlnisse rorkommen. Ilat man \%. B. zrrei Sterne ron genau gleichem Spectraltypus, so wird man fur ihr Energiererluiltniss dieselben Werthe erwarten können, sei es, dass man sie mit Iliilfe der Thermosäule oder 
eines unserer optischen Photometer oder auf photographisehem Wege miteinander vergleicht. Handelt es sieh jedoch um Licht voll gan\% versehiedener Zusammensetzung, so hört jede Proportionalitit zwisehen den drei fraglichen Wirkungen auf, und man kaun nicht unmittelbar von der einen auf die andere sehliessen. Bestimmt man 7. B. die Extinetionseurve in der lirdatmosphatire durch thermische, optische und photographische Messungen, so ist der Verlauf derselben in allen drei Fiillen ein absolut anderer, weil die selective Absorption der Atmosphäre die Znsammensetzung des Speetrums beständig verïndert. Aus dem Gesagten dttrfte bereits zur Gentige hervorgehen, dass Apparate, welehe speeiell zur Messung der thermisehen und eliemisehen Wirkung des Lichtes bestimmt sind, nur in sehr beschrïnktem Masse die physiologischen Photometer zu ergïnzen oder gar zu ersetzen vermögen. Es wïre daher in einem Lehrbuche der Astrophotometrie, welehes in erster Linie die physiologisehe Wirkung des Lichtes behandeln will, olne Zweifel erlaubt, von diesen Apparaten ganz abzusehen und in Betreff derselben auf die ausfuhrlichen Sehriften iber Aetinometrie und Photographie zu verweisen. Vielleicht wird aber doeh ein kurzer Überblick uber die wichtigsten Hulfsmittel und Messungsverfahren anf diesen Gebicten nicht unerwilnseht sein. Insbesondere dirfte ein Hinweis auf die Anwendung der Photographie vollanf berechtigt erscheinen, sehon ans dem Grunde, weil hente die photographisehen Helligkeiten der Fixsterne neben den optisehen bereits Bedeutung erlangt haben. Ieh möelte aber ansdrlicklich herrorheben, dass dieses Capitel weder anf Vollstiindigkeit noch auf besondere Griundlichkeit Anspruch macht.

1. Apparate zur Messung der thermischen Wirkungen des Lichtes.

a. Die wiehtigsten Actinometer.

In seiner "Photometria * (S $\$ S 6$ ) erwïhnt Lambert die Versuche, die er zur Bestimmung der Extinction des Sonnenlichtes in der Erdatmosphäre angestellt hat. Diese Versuche sind nicht mit eigentlichen Photometern ausgefuhrt worden. viehnehr hat sich Lambert dabei der thermisehen Methode bedient, indem er ein gewïhnliches Thermometer den directen Sonnenstrahlen anssetzte, dasselbe mit einem zweiten, im Sehatten liegenden Thermometer verolich und die Differenz der Ablesungen ale Mass fuir die Stairke der Somnenstralilung betrachtete. Dass Lambert diese Messungen ganz gleichbedentend mit irgend welchen anderen Lichtmessungen auffasste, geht ans seinen Worten unzweidentig hervor; man 
wird sich aber wohl mit Ruicksieht auf- die grundverschiedenen Verfahren kaum wundern diirfen, dass die Lambert'schen Extinetionsresultate sehr erheblich von allen anderen, auf rein photometrischem Wege abgeleiteten abweichen.

Nach Lamberts Vorgange ist noch häufig die Differenz der Angaben \%weier Thermometer zu Strahlungsmessungen benutzt worden, und um die Empfindlichkeit zu erhöhen, hat man Thermometer mit geschwärzten oder versilberten Kugeln benutzt. Von den älteren Apparaten dieser Art sind besonders das Heliothermometer von de Saussure 1), das Differentialthermometer von Leslie ${ }^{2}$ ), dem der Erfinder selbst den Namen Photometer beigelegt hat, das Rumford'sehe ${ }^{3}$ ) Thermoskop und das Ritchie'sche ${ }^{4}$ ) Photometer, welches eine Modification des Leslie'schen Instrumentes ist, zu erwähnen. Die drei letzten Apparate sind Luftthermometer mit zwei gleieh grossen Glaskugeln, die durch eine gebogene Röhre miteinander verbunden sind; in der Röhre ist irgend eine Fliussigkeit enthalten. Wird die eine Kugel von einer Liehtquelle bestrahlt, wïhrend die andere durch einen Sehirm vor Bestrahlung gesehuitzt ist, so bewegt sich die Fliissigkeit, sobald die Luft in den beiden Gefässen sich ungleieh ausdehnt. Die eine Kugel ist gewöhnlieh gesehwärzt. Die Genauigkeit der Differentialthermometer wird nieht unwesentlieh beeinträichtigt durch den Umstand, dass die Strahlung erst das Glas zu passiren hat, ehe sie anf die Luft einwirken kaun, und ferner noeh mehr dadureh, dass sieh in der benutzteu Fliissigkeit Dämpfe entwiekeln, deren Expansion die Bewegung der Fliissigkeit mit beeinflusst und daher die Angaben des Instrumentes verfälscht.

Bei den meisteu der vorher erwähnten Apparate kommt es darauf an, zu constatiren, wann der Überschnss der Temperatur der bestrahlten iiber die unbestrahlte Thermometerkngel den Höhejunkt erreieht hat. Man nemt diese Methode die statische. Haiufig wird an Stelle derselben die dynamisehe Methode angewandt, welehe darin besteht, die Verschiebnng der Flissigkeit in den Thermometern wïhrend eines bestimnten Zeitintervalles, abwechselnd bei Bestrahlung nud Niehtbestrahlung, zu messen. Das erste Instrument dieser Art ist das Aetinometer von J. Hersehelis), welehes aus einem sehr emptindlichen Thermometer mit grosser Kugel und einer dunkel getirbten Flissigkeit besteht. Man wendet dasselbe in der Weise an, dass man es eine Minute lang der

1) De Sanssure, Toyage dans les Alpes. 13d. 2, p. 294.

2) Leslie, Inquiry into the nature and propagation of heat. London, 1804.

3) Phil. Trams. of the R. Soc. of Lombon. 1604, p. 77.

4) Phil. Trans. of the R. Soc. of Loudon. 1825, p. 141.

5) The Edinbureh Journal of science. Vol. 3 (1525, p. 107. 
Strahlung aussetzt und das Auwachsen der 'Temperatur wïhrend dieser Zeit beobachtet, daun das Instrument eine Minute lang vor den directen Strahlen durch einen Schirm sehtitat und das weitere Anwachsen oder Abnehmen der Temperatur wïhrend dieser Zeit beobachtet. Ein Anwachsen im Schatten deutet dauauf hin, dass das Steigen während der Bestrahlung nicht dem Einflusse derselben allein \%muschreiben war, sondern \%um Theil von indireeter Wärmezufulır (\%. B. durch die umgebenden Gegenstainde oder die Theile des Apparates u. s. w.) herrilhrt. Um den reinen Einfluss der Strahlung und somit ein Mass fur ihre Intensitait zu erhalten, hat man also in diesem Falle die Zumahme in Sehatten von der Zunahme wärend der Bestrahlung \% subtrahiren. Im anderen Falle, wenn eine Alunahme im Sehatten eintritt, muss der Betrag derselben zu dem Anwachsen wïhrend der Bestrahlung hinzuaddirt werden.

Noch branchbarer als das Herschel'sche Actinometer hat sich. das Pouillet'sche Pyrheliometer') erwiesen, welehes in Wesentlichen aus einem flachen, cylindrisehen, mit Wasser gefüllten Gefaisse hesteht, dessen vordere geschwiirzte Fläche die Sonnenstrahlen senkrecht auffingt. In das Gefiiss taucht ein Thermometer ein. Da die Quantitait des Wassers bekannt ist, so kann man aus dem Steigen des Thermometers wiahrend eines bestimmten Zeitraums (nach Pouillet is Min.) die Strahlungsmenge bestimnen, welche die gesehwillyte Oberfliehe in diesel Zeit von der Somne empfängt. Um den durch Ausstrahlung in die Umgebnug bewirkten Verlust zu beriicksichtigen, beobachtet man während weiterer 5 Minuten das Sinken des Thermometers im Schatten und addirt diesen Betrag zu der vorher erhaltenen strahlumgsmenge hinzu. Als Masseinheit fiir die Strahhung betrachtet man diejenige Wirmemenge, welche erforderlich ist, um $1 \mathrm{~kg} \mathrm{~W}^{\mathrm{a}}$ asser $u m 1^{\circ}(\mathrm{C}$.) zu erhöhen.

Aus nenerer Zeit stammen eine grosse Inzahl von Actinometern, bei denen zum Theil die im Vorangehenden erwähnten Methoden mit wichtigen Verbesserungen zur Anwendung kommen, zum Theil noch empfindlichere Hiilfsmittel, insbesondere die Thermosiinle in Verbindung mit dem Galvanometer, benutzt werden. Die wichtigsten dieser Instrumente, anf die hier nicht nïher eingegangen werden kann, sind die Actinometer von Secehiy, Violley), Croval) und Eriesson $\left.{ }^{5}\right)$.

1) Comptes Rendus. Tome i, p. 24.

2) Bollettino dell'osserv. del Collegio Romano. 1863, p. 19.

3) Comptes Rendus. 'Tome is, p. 1425 ; t. 82, p. 729 und 896.

4) Crova, Mesures de lintensité calorifique des radiations solaires et de leur absorption par l'atmosplière terrestre Mém. de l'acad. des sciences et lettres de Montpellier). Paris 1876.

5) Nature. Vol. 4, p. 204 n. 449: Vol. 5, p. 28i, 344, 505; Vol. 12, p. 517. 


\section{b. Das Langley'sche Bolometer.}

Die vorhergenannten Apparate zur Messung der Lichtstrahlung werden an Empfindlichkeit wesentlich durch das von Langley') erfundene Bolometer ibertroffen, welches die allergeringsten Strahlungsänderungen anzeigt und daher vielleicht auch fuir die lichtschwaehen Himmelsobjecte nutzbar zu werden verspricht. Das Bolometer bermbt auf der bekaunten 'Thatsache, dass sich der elektrische Widerstand in dünnen Metallstreifen unter dem Einflusse einer Bestrahlung ändert. Es besteht aus einer Wheatstone'schen Bricke, in deren beide Zweige je ein System von diunen Eisen- oder Platinstreifen eingeschaltet ist. Das eine dieser Systeme wird der zu untersuchenden Strahlung ausgesetzt. Hierdurch vermehrt sich der elektrische Leitungswiderstand im entsprechenden Stromzweige, und das Gleichgewicht in der Wheatstone'sehen Bricke wird gestört. Das in diese Briicke eingesehaltete Galranometer, welches sich bei gleicher Temperatur der beiden Streifensysteme in Rulhe befindet, wird mun bei Tersehiedenheit des Leitungswiderstandes einen Anssehlag. zeigen, und die Grösse dieses Anssehlages ist ein Mass für die Intensitiit der ansgeibten Strahlungswirkung. Langley hat mit diesem empfindlichen Instrumente seine epochemachenden Untersnehungen iiber die Vertheilung der Energie im Diffractionsspectrum der Some ausgefuilırt, und es ist ihm gelungen, die Strahhungswirkungen nicht mur in allen Theilen des sichtbaren Spectrums, sondern anch bis weit iber die Grenzen desselben hinaus zu bestimmen. Das kleine Instrument, dessen Behandlung allerdings wegen verschiedener Fehlerquellen die alleräusserste Vorsicht erfordert, ist in juingster Zeit noch in mancher Hinsicht verfeinert worden *).

\section{(C) Das Boys sche Radiomikrometer.}

Tom Boys3 ist zur Messmng sehr schwacher Strahlungen ein Instrument construirt worden, bei welchem ein empfindliches Thermoelement zur Terwendung kommt. Dieses Element besteht im Wesentliehen ans einem Rahmen, gebildet ron sehr schmalen Lamellen ans Antimon und Irismuth, die mit ihren nnteren Enden an ein dïnnes Kupferscheibchen gelöthet sind, wïhrend die oberen Enden durch einen gekrimmten Kupferdraht miteinander verbunden sind. Wer Rahnen hängt an einem diinnen

1 Proce of the Amer. Acad. of alts mul sciences. Tol. $16(1550-81)$, p. 342.

2, Witulem. Anualen. Bd. 4ti, 1, 204.

3) Proc at the R. Soc. of Lonrion. Vol. 42, 1). 1s!\%. Ansserdem Phil. Trans. of the R. Soc. of Londm. 15>9 A. p. 159. 
Qnarzfuden zwischen den Polen eines kriftigen Magneten. Wird die Lïtlstelle vou einer Strahlung getrofien, so tritt der eryeugte Thermostrom mit dem magnetischen Felde in Weehselwirkung, und der Rahmen erhallt daher eine Ablenkung aus der Ruhelage, welehe der Strahlungsintensitiit proportional ist. Eine einfache Spiegelablesung mit Scala giebt den Betrag der Drehung an. Das Instrument lat neben hoher Empfindliehkeit noeh den Vortheil, dass es von den störenden Eiuwirkungen der äusseren Temperatur und des insseren Magnetismus unabhängig ist. Boys hat dasselbe zu Versuchen Fixsternen ansgehende Strahlungsintensitait benut\%t') und ist dabei im Gegensatze zu anderen Beobachtern zu dem Resultate gekommen, dass sich eine Sternenstrahlung nicht mit Sicherheit nachweisen liisst.

\section{d. Das Crookes'sche Radiometer und das Zöllner'sche Scalenphotometer.}

Ein gewisses Aufsehen hat das von Crookes ${ }^{2}$ ) erfundene Radiometer erregt, bei welchem die von einer Lichtquelle ausgesandte Strahlung direet in Bewegung umgesetzt wird. Ein vierarmiges Kreuz ans Aluminiumdraht ruht mittelst eines Glashitehens auf einer feinen Spitze. An jedem Arme ist ein Blättchen aus Glimmer befestigt, welches anf der einen Seite mit Russ tiberzogen ist; die schwarzen Seiten aller vier Bhättehen sind nach derselben Drehungsrichtung hingewandt. Die ganze Vorrichtung hefindet sich innerhalb einer Glaskugel, in weleher die Luft ansserordentlich verdiinnt worden ist. Unter der Einwirkung einer Lichtstrahlung beginnt das Kreuz zu rotiren und zwar stets in solchem Sinne, dass die nicht berussten Fliiehen vorangehen. Die Bewegun! ist 1 m so schueller, je stärker die Strahlung ist, und dureh die verschiedenartigsten Versuche ist naehgewiesen, dass die Rotationsgeschwindigkeit direet der Strahlungsintensität proportional ist. Z Zölner $\left.{ }^{3}\right)$ hat nach dem Crookes schen Princip) ein sehr eompendiöses Instrument construirt, dem er den Namen Scalenphotometer gegeben hat, und bei dem das liadjometerkrewz wie bei einer Drehwage an einem Coconfaden anfgehängt ist. Massgebend für die Sehnelligkeit der Drehung ist der Grad der Verdiinnung der in dem Glasrecipienten enthaltenen Luft; im Allgemeinen steigert sich die Empfindlichkeit mit zunehmender Verdiinnung, jedoch nur bis zu einer

1) Proc. of the R. Soc. of I,ondon. Vol. 47, p. 4911.

2) Phil. Trans. of the R. Soc. of London. 1873, p. 277.

3) Zöllner, Das Scalen-Photometer, ein neues Instrument zur mechanischen Messung des Lichtes etc. Leipzig, 1679. 
gewissen Grenze. Ferner wird die Bewegliehkeit um so grösser, je ungleichartiger die beiden Seiten der Radiometerflügel in Betreff ihres Strahlungsvermögens sind. Lampenruss und Versilberung sind die wirksamsten Contraste; bei gleieher Beschaffenheit der Seiten findet überhaupt keine Drehung statt.

Lässt man nur Strahlen von bestimmter Brechbarkeit auf das Radiometer einwirken, so findet man, dass die Bewegungsgeschwindigkeit um so grösser wird, je weniger brechbar die Strahlen sind, ein Beweis, dass dic thermischen Wirkungen den hauptsäeblichsten Einfluss auf die Vorg:iinge ansiiben. Meistens nimmt man zur Erklärung der Erscheinungen die kinctische Gastheorie zur Hülfe, nach welcher die einzelnen Moleküle eines Gases in rascher Bewegung nach allen Richtungen begriffen sind und daher theils gegeneinander, theils gegen die umgebenden Gefiisswïnde und andere Hindernisse anprallen und zurückgestossen werden. Aı den lïher erwärmten geschwärzten Flächen werden die Moleküle hiiufiger zuriickgeworfen als an den nicht geschwärzten, und dadureh wird die lieaction auf die beweglichen Blättchen bestimmt. Obgleieh diese Hypothese nicht alle Einzelheiten der Erscheinung erkliirt, so verdient sie doch vor vielen anderen weit complicirteren Hypothesen den Vor\%llg.

\section{Apparate zur Messung der chemischen Wirkungen des Lichtes.}

a. Das chemische Photometer ron Bunsen und Roscoe.

Yon der schon friher bekannten Fähigkeit des Lichtes, chemische Veränderungen lervorzubringen, ist mehrtach zu Strahlungsmessungen Gebranch gemacht worden. Nachdem im Jahre 1543 Draper einen Apparat construirt hatte, bei welchem die Stralhlungsintensität aus der Wirkung. des Lichtes anf ein Gemenge ron Chlor und Wasserstoff bestimmt wurde, ist diese Methode ron Bunsen und Roseoel) weiter ausgebildet und rervollkommnet worden. Das von ihmen construirte chemische Photometer ist eine thermometerartige Vorrichtung, bestehend ans eimem kleinen zum Auffangen der Strahhng bestimmten Gefïsse, einem diumen mit Scala verschenen Tiohre und einem grïsseren Behïiltnisse am anderen Ende. Dieses Behältniss und ein Theil des kleinen Gefäisses sind mit Wasser gefüillt. Durch das Ganze wird mittelst eines besonderen Verfabrens ein Gemenge

1) Pogg. Ammalen. Bd. 96, p. 373; Bd. 100, 1). 43 u. 481; Bd. 101, p. 235; Bd. 108, 1. $193 ;$ Bd. $11 \pi$, p. 529 . 
von gleichen Volumtheilon Chlor und Wasserstoff so lange lindurclsgeleitet, bis die Fltssigkeiten vollstïndig gesittigt sind und der llbrige Raum damit erfullt ist. Wird nun das Insolationggefiss allein dem Lichte exponirt, so verbindet sich unter der Einwirkung desselben der Wasserstoff mit dem Chlor zu Salzsĭure, und da diese von dem Wasser im Gefasse absorbirt wird, so bewirkt die Volumverminderung des Gasgemenges ein Vorrtleken der Fllissigkeit aus dem grösseren Behälter in das Scalenrohr. Aus der Grösse der Verschicbung liisst sich die Stürke der Lichtwirkung bestimmen, und zwar folgt aus zahlreichen Versuchen, dass die eliemische Wirkung (d. h. die Volumverinderung des Gases) direct der Intensität der Strahlung proportional ist. Dabei sind freilieh eine Menge von Nebenumstiinden zu beachten, insbesondere ist daranf Rlicksicht zu nehmen, dass der Beginn des Processes eine gewisse Zeit verlangt, dic von der Lieltstärke ablïingt, und dass ebenso wieder von dem Begimu der chemischen Wirkung his zur Erreichung des Maximums eine Zeit vergeht, deren Daner mit der Intensität der Strahlung variirt. Auf diese und andere damit zusammenhängeude Fragen kann hier nicht nüher eingegangen werden.

\section{b. Das Selenphotometer.}

Das krystallinische Selen besitzt die merkwürdige Eigensehaft, unter Einwirkung des Lichtes die Elektrieitiit besser zu leiten als im Dunklen. Dieses Verhalten des Selens ist merst von Sale1), damn besonders eingehend ron Siemens') studirt worden, und es sind mehrfach Versuche gemacht worden, dasselbe zur Construction von Photometern zu verwerthen. Ein vollstiundiger Erfolg ist bisher nicht zu er\%ielen gewesen, hauptsäichlich wegen der Unbeständigkeit der Erseheinungen. Eine lïngere Dauer der Beliehtung hat nämlich eine Abnahme der Wirkung, eine »Ermïdung « der Sulstanz zur Folge, und ein ähnlicher Effect wird durch allzu intensive Liehtstrahlungen hervorgebracht, ferner wird durch die geringste Verunreinigmo des Selens mit anderen Metallen die Lichtempfindlichkeit vermindert. Nach den Untersnchungen von Siemens nimmt die Leitungsfähigkeit nicht proportional der Liehtstiirke selbst zu, sonderm nahezu proportional der Quadratwurzel aus derselben. Die Wirkung geht hauptsaichlich ron den sichtbaren Strahlen des Spectruns aus; sie beginnt mit den Violett, steigt von da ziemlieh gleichmaissig bis zum Roth, ist noch in Ultraroth zil constatiren, rersehwiudet alber dariber hinaus.

1) Proc. of the R. Soc. of London. Vol. 21, 1. 283.

2) Monatsb. der K. Preuss. Akad. der Wiss. 1875, 1. 280; 18i6. p. 95; 187 , p. 299. 
Siemens erklärt die Erscheinungen dadurch, dass die Lichtstrahlen direet eine Molekularveränderung des Selens hervorbringen, indem sie das krystallinisehe Selen zu metallischem, viel besser leitendem reduciren; nach Aufhören der Beleuchtung bildet sich die metallische Selenoberfliche wieder in krystallinisches Selen zurück.

Siemens lat noch anf eine andere eigenthiimliche Eigenschaft des Selens in Verhalten gegen die Lichtstrahlen aufmerksam gemacht'1). Er hat nämlieh gefunden, dass manche Selenplatten bei der Beleuchtung nicht besser leitend werden, dagegen selbst elektromotoriseh wirken, als ob die Energie des Lielites unmittelbar in elektrische Energie nmgewandelt wiirde. Die auffallende Erscheinung ist in nenerer Zeit von Minchin ${ }^{2}$ ) weiter verfolgt worden. Derselbe hat photoelektrische Elemente, ähnlich den galvanischen Elementen, construirt, bestehend aus Selen, Aluminium und einer Fliissigkeit. Im Dunklen zeigen dieselben keinerlei Wirkungen, dagegen entwickeln sie bei Belichtung des Selens eine elektromotorische Krilft, die mittelst eines empfindlichen Elektrometers gemessen werden kamm. Die einwirkende Lichtquantitit ist direct dem Quadrate der beobahleten elcktromotorischen Kraft proportional. Minchin hat in allerjiin.ster Zeit ${ }^{3}$ ) solche photoclektrischen Elemente in einem grossen Teleskop an Stelle des Oculares angebracht und rersucht, mittelst derselben die ron den Sternen ansgehende Energie zu bestimmen. Bei der Versleichung gleich gefärbter Sterne zeigen scine Resultate eine rollkommene İbereinstimmung mit den Ergebuissen der photometrischen Messungen. Eine weitere Ansbildung der im hohen Grade beachtenswerthen photoelektrischen Iethode kömnte für die ganze Plotometrie des Fixsternhimmels bedentungsoll werden.

\section{c. Die Photographie als photometrisches Hiilfsmittel.}

Bald natchdem durch die epochemachende Entdeckumg Dagueres im Jahre 1439) die Jögliehkeit gegeben war, das auf einer jodirten silberplatte unter Einwirkung des Lichtes arzengte latente Bild durch Behandlumg mit Quecksiberdänpen hervornuten und danernd auf der Platte zu erhalten, tanchte der Gedanke aut, das neue Terfihren auch zu quantitativen Lichtmessungen nutzbar zu machen. Arago ist wohl der Erste gewesen, weleher anf die Bedentung der Photographie für Lichtmessungen lingewiesen hat, und anf scinen Einfluss sind anch die ersten girindlichen

1) Sitzungsber. der K. Preuss. Akiul. der Wiss. 1S55, 1. 14i.

2) Philos. Mag. Ser. 5, Yol. 31, p. 207

3) Nature. Vol. 49, p. 270: Vol. 22, 1. 241;. - Ausserdem Proc. of the R. Soc. of London. Tol. 5s, p. 142. 
Untersuchungen anf diesen Gebiete zurlickzufulıren, die im Jahre 1844 von Fizean und Foucault') angestellt worden sind. Diese gingen von dem durchaus plansibel erselieinenden Grundsatze ans, dasss die von einer Lichtquelle anf einer emptindlichen Platte geleistete Arbeit, die sieh in der Zersetzung der Schicht bemerkbar macht, direct proportional sein milsse der ursprilngglichen Intensitait der Lichtquelle und femer der Zeitdaner der Belichtung. Hat man also versehiedene Lichtquellen, welehe mit den Intensitiiten $i_{1}, i_{2}, i_{3} \ldots$ anf eine Platte einwirken, und bei den Expositionszeiten $t_{1}, t_{3}, t_{3} \ldots$ dieselbe Arbeit anf der empfindlichen Sehicht ansulien, so ist naelı dem objigen Grundsatze:

$$
i_{1} t_{1}=i_{2} t_{1}=i_{3} t_{3}=\ldots ; \quad \text { allgemein }: i t=\text { const. }
$$

Man findet also für \%wei zu untersuchende Liehtruellen das Intensitätsverhältniss $\frac{i_{1}}{i_{2}}$, nachdem die Expositionszeiten $t_{1}$ und $t_{2}$ bestimmt sind, bei denen von ihnen der gleiche Etlect anf der Platte hervorgebracht wird, ans der Gleiehnng:

$$
\frac{i_{1}}{i_{0}}=\frac{t_{3}}{t_{1}}
$$

Um ein fir alle Male einen bestimmten Grad der Veränderung in den benutzten Jodsilberplatten als Fixpunkt fiir die Liehtvergleichungen zu normiren, wihlten Fizeau und Foueault denjenigen Moment, wo die empfindliche Schicht die Quecksilberdämpfe gerade zu verdiehten begiunt, wo also das photographische Bild zn entstehen anfingt. Ihre Lichtmessungen gesehahen in der Weise, dass sie das in einer Camera entworfene Bild einer Liehtquelle nacheinander anf verschiedene Stellen derselben Platte einwirken liessen, wobei sie jedesmal die Expositionszeit um kleine Betrïge verinderten. Elsenso verfuhren sie nit einer zweiten Liehtquelle, und indem sie nun in beiden lieihen von Aufnahmen denjenigen Punkt bestimmten, wo bei der Entwieklung das Bild grerade zu entstehen begann, erhielten sie aus dem Verhïltnisse der zugehïrigen Expositionszeiten nach der obigen Gleichung das Intensitiitsverhiiltniss der beiden Liehtquellen. Die Untersuchungen von Fizean und Foueanlt erstreckten sich anf das Licht der Somne, anf dals elektrische Kohlenlicht und auf das Kalklicht und lieferten unter Anderem das interessinte Resultat, dass fiir diese Lichtquellen die chemischen Inteusititen den optischen gleich seien. Bei den Mängeln, die den Dagnerreotypieplatten amhaften, sind die Liehtmessungen von Fizean und Foucault nur als erste Versuche in dieser Richtung zu betrachten, die anf grosse Genanig-

1) Comptes Rendus. Tome 18, p. 746 11. Sio. 
keit keinen Anspruch machen können. Es ist übrigens von grossem Interesse, dass die beiden französischen Gelehrten in der citirten Abhandlung bereits durch besondere Versuche nachgewiesen habeu, dass das von ihnen benutzte Gesetz von dem reciproken Verhältniss der Expositionszeiten und Intensitäten zweier Lichtquellen bei gleicher chemischen Wirkung nur innerhalb beschrïnkter Grenzen Gültigkeit hat, und zwar dass Abweichungen von diesem Gesetze schon bemerkbar werden, wenn das Verhältniss der Intensitiiten oder Expositionszeiten das Zehnfache iibersteigt.

Anstatt der jodirten Silberplatten versuchte man unmittelbar nach der Entdeckung der Dagnerreotypic auch das billiger herzustellende Chlorsilberpapier zu Messungen der chemischen Lichtstärke zu benutzen. Es sind Untersuchungen von Jordan, Hunt, Herschel und Anderen in dieser Richtung bekaunt, die aber zu keinen befricdigenden Resultaten fiihrten, weil es nicht gelang, ein photographisches Papier von vollkommen gleich bleibender Empfindlichkeit herzustellen, sowie eine gesetzmässige Abhängigkeit der Schwäizung des Papiers ron der Intensität und Expositionsdaner aufzufinden. Erst Bunsen und Roscoe') haben die Methode so weit verrollkommnet, dass sie zu exacten Messumgen branchbar geworden ist. Durch zahlreiche sehr sorgfältige Versuche haben sie zunächst den Satz bewiesen, sdass imnerhalb sehr weiter Grenzen (Änderung der Lntensitiit un das 25fache) gleichen Producten ans Lichtintensität und Belichtungsdaner gleiche Schwäiznngen anf Chlorsilberpapier von gleicher Sensibilitiit entsprechen«. Die Einschriinkungen, welchen dieses Gesetz nach den Untersuchnngen von Fizean und Foucault bei den Daguerreotypieplatten unterworfen ist, und welche nach den nenesten Untersuchungen anch fiir die photographischen Trockenplatten giiltig sind, kommen also bei den lichtempfindlichen mit Chlorsiber geträinkten Papieren in Wegfall. Beşonderen Fleiss haben Bunsen und Roscoe anf die Herstellung eines unverianderlichen photographischen Normalpapieres verwandt, und ihre Versuche zeigen, dass es in der That möglich ist, ein solehes Papier von hinlänglich constant bleibender Lichtemptindlichkeit zu bereiten. Um ferner bei der Beurtheihurg. des Schwiirzungsorades einen bestimmten unveränderlichen Farbenton als Ansgangspunkt henutzen zu kömnen, der jederzeit anch leicht wieder hergestellt werden kamn, bereiteten sie eine Mischung ron 1000 Theilen Zinkoxyd und I Theil reinen Lampenruss, die auf Papier anfgetragen wich als cine Normalsehwärze vou gleicher und unverinderlicher IBeschatfenheit erwies. Mls willkürliehe Masseinheit für alle ihre photometrischen bestimmungen lenten Bunsen und Roscoe

1) Phil. Trans. of the R. Soc. of London. 186i3, 1. 139. - Pogg. Anmalen. Bd. 117, 1. 529. 
diejenige Lichtintensitiit \% Grunde, welche in einer Secunde anf dem Normalpapier die Normalsehwirze hervorbringt.

Bunsen und Roseoe haben ihre photometrische Methode, die einer betraichtlichen Genauigkeit filhig ist, hauptsaichlieh zu Messụngen der chemischen Intensituit des Tageslichtes an versehiedenen Beobachtungsorten und zur Aufsuchung der Berichung zwischen Sonnenböhe und Tageslicht angewandt, ausserdem hat Roseoe') nach dieser Methode Bestimunungen der chemisehen Helligkeit an versehiedenen Stellen der Sonnenoberfläche ausgefilhrt. Dass das Verfahren in der Astronomie keine weitere Verbreitung gefunden hat, liegt wohl, abgesehen von der Umstïndlichkeit, die mit der Herstellung des photographisehen Normalpapieres verbunden ist, besonders daran, dass bei sehr sehwachen Liehteindricken die Empfindlichkeit nicht ansreichend ist, um genaue Bestimmungen zu ermöglichen. Fur die technische Photographie sind dagegen die Untersuchungen von Bunsen und Roseoe von der grössten Bedeutung gewesen, weil ihr Verfalıren vorbildlich geworden ist flir dic Construction einer ganzen Reihe von sogenannten chemischen Photometern und Sensitometern.

Wïhrend Fizeau und Foucault, ebenso Bunsen und Roscoe ron den Himmelskörpern nur die Sonue in den Bereich ihrer Untersuchungen zogen, wandten Bond ${ }^{2}$ ) und nach ihm Warren de la Rue ${ }^{3}$ ) bereits in den fünfiger Jahren die Photographie anch zn quantitativen Bestimmungen am Monde und an den helleren Planeten an. Sie machten anfings anf Dagnerreotypic-, damn auf Collodiumplatten die ersten gelungenen Aufuahmen von diesen Himmelskörpem und schlossen ans den Expositionszeiten, die erforderlich waren, nm fertige Negative zu erlangen, auf die ehemischen Intensitätsverhailtnisse dieser Gestirne. So erhielt Bond die ersten, wemn aneh nicht sehr zuverliissigen Werthe fiir das photographische Helligkeitsverhäitniss vou Some zu Mond und ron Mond zu Venus, Jupiter und Saturn und fand unter Anderem in Übereinstimmung mit Warren de la Rue das bemerkenswerthe liesultat, diss Jupiter ron einer bestimmten Menge anftallenden Sonnenlichtes einen viel grösseren Theil der ehemischen Strahlen reflectirt als der Mond. Ferner ergaben sich aus diesen Photographien interessante Anfischliisse iiber die Vertheilung der Helligkeit auf den sichtharen Scheiben dieser Himmelskijrper; so zeigte sich Jupiter im Centroun der scheibe betriichtlich heller als an Rande, während beim Mond eine Zunahme der Ilelligkeit ron der. Mitte nach dem Rande hin angedentet war. Es ist merkwiirdig, dass die Bond'schen

1) Proc. of the R. Soc of London. Vol. 12. p. 1848.

2) Memoirs of the American Acad. New Series. Tol. S, p. 221.

3) Monthly Notices. Vol. 18. 1. 54. 
Versuche in dieser Richtung später nicht weiter verfolgt und zu genaucren Messungen der Helligkeitsvertheilung auf Planetenscheiben ausgebildet worden sind; gerade auf diesem Gebiete könnte die Photographie ein sehr werthvolles Hülfsmittel für die Photometrie werden.

Der Gedanke, auch die Helligkeiten der Fixsterne anf photographischem Wege zu ermitteln, ist ebenfalls von Bond ${ }^{1}$ ) zuerst angeregt worden. Er fand, dass die Durchmesser der in der Focalebene eines Fernrohrs anf ciner photographischen Platte erzeugten Sternscheibchen je nach der Expositionszeit verschieden gross waren, und folgerte daraus, dass Durehmesserbestimmungen verschiedener Sterne auf derselben Platte cin Mass fïr ihre relativen Helligkeiten liefern miissten. Die wichtigsten Resultate, zu denen Bond durch die Discussion einer grossen Zahl vou Stemaufnahmen gefiihrt wurde, gipfeln in den folgenden Sätzen: 1) * Das erste Bild eines Stermes entsteht anf der photographischen Platte ganz plötzlieh, und dieser Moment kann mit grosser Genanigkeit festgestellt werden." 2) "Der Flicheninhalt eines Sternscheibchens nimmt direct proportional der Expositionszeit zu. " 3) »Für jeden Stern ,und jede Platte silt die empirische Gleichnng: $P t+Q=y^{2}$, wo y der zur Expositionszeit $t$ gehörige Durchmesser des photographischen Scheibchens ist und Q eine Constante bedeutet. Wendet man diese Gleichung auf sterne ron verschiedener Helligkeit an, so könuen die daraus abgeleiteten Werthe ron $P$ ein Mass fïr die photographische Intensität derselben albobeli. *

Bond hat durch seine wichtigen Untersuchungen zweifellos den Grund zu einer exacten photographischen Photometrie der Fixsterne gelegt, und es ist fast unbegreiflich, dass seine Vorschlige bis in die Nenzeit gänzlich unbeachtet geblieben sind, ja so rollstindig in Vergessenheit gerathen komuten, dass die meisten Ergebnisse, zu denen er bereits in deu Jahren 15.57 und IS5̆ gelangt war, gamz von Nenem hergeleitet werden mussten.

Der Erste, der nach Bond dis Problem der photographischen Fixsternhelligkeiten, und zwar anf einem ganz anderen Wege, in Angriff nahm, war Janssen\%). Er hatte dabei dals specielle Ziel im Ange, das Inteusitaitsverhialtniss der some zu deu helleren Fixstermen zu ermitteln, und construirte zu diesem Zwecke ein Instrument, welehes er photographisches l'hotometer namte, und welches dazu hestimmt war, die Beziehnngen festzulegen zwischen der Intensität der Strahlung und dem Dichtigkeitsorade des silberniederschlages, deu die-

1) Astron. Nachr. Bd. 4i, Nr. 1105; Bd. 4h, Nr. 1129: Bd. 4!, Nr. 115-1159.

2. Comptes Rendus. Tome !1:, 1. 521 . 
selbe auf der Platte hervorbringt. Dieses Photometer besteht in Wesentlichen aus einer Cassette, welehe die empfindliehe Platte enthilt. Vor derselben wird mit Hulfe eines Uhrwerks, oder wenn eine sehr grosse Schnelligkeit erwtinseht ist, mit Hulfe ron starken Federn ein.undurchsichtiger mit einer Öfhung versehener Schieber mit constanter Geschwindigkeit vorbeigeflutıt. Hat die Öfthung die Form eines Rechtecks, so wird die Platte Hberall die gleiche Sehwarzung annehmen; ist die Öffnung aber ein Dreieck, welches parallel zu der einen Seite tiber die Platte hinweghewegt wird, so erseheint dieselbe an demjenigen Ende, welehes dieser Seite des Dreiceks entspricht, dunkler als an dem entgegengesetzten Ende, wo die Spitze des Dreiecks vorbeigeht, und da die Bewegung gleichförmig ist, so wird man unter Berlteksichtigung der Dimensionen des Dreiecks fitr jede Stelle der Platte den Zusammenhang wwischen Schwirzung und Intensitiit findeu. Janssen hat mit diesem Apparate das bereits von Fizeau und Foucault naclgewiesene Resultat bestaitigt, wonach die Zunahme der Schwiirung nicht streng proportional bleibt der Zunahme der Lichtintensität, sobald dieselbe sehr betrïchtlich ist, und er hat daher, un diesen Fehler zu corrigiren, den Seiten der Schieberöffnung eine Currenform gegeben, welehe dic gefundenen Abweichungen beriicksichtigt. Mit einem solehen corrigirten Instrumente hat Janssen zahlreiche Anfuahmen der Sonne gemacht und auf diese Weise eine Reihe von Sonnensealen erhalten. Zur Vergleichung der Fixsterne mit der Soune empfiehlt er, die ersteren in einiger Eutfernung von der Focalebene aufzunehmen, die so erhaltenen Scheibchen mit den Sonnensealen zu vergleichen und auf denselben den Punkt der grleichen Schwäizung aufzusuchen. Aus den zugehörigen Zeiten laisst sich dimn das photographisehe Intensititsverhiälniss der betreftenden Himmelskijr|er ermitteln; wenn noeh gebiihrende Riicksieht anf den Abstand der photographisehen Platte von der Brennebene bei den Fixsternanfnalmen genommen wird. Eine praktische Anwendung dieser Methode in grösserem Unfange seheint weder ron Janssen noch von Anderen versneht worden au sein; anch ist sehr aweifelhaft, ob diesellse eine hinreichende (ienanigkeit gestattet.

Die Frage nach den photographischen Fixsternhelligkeiten trat erst. danernd in den Vordergrund des Interesses bei Gelegrenheit der Vrorbereitungen und der ersten Arbeiten fiir die internationale Himmelskarte, and ist anch hente noeh lange nicht als abgesehlossen zu betrachten. Gan\% allgemein wurde bald die Methode der Durchmesserbestimmun" als die bei Weitem beste anerkamut. Die Vorbedingmugen fiir die Anwendung" dieser Methode sind hentzutage viel güinstiger als zu der Zeit, wo Bond sie zuerst in Vorsehlag brachte. Durch die Construction besonderer 
plotographischer Objective, welche speciell für die chemisch wirksamen Strahlen achromatisirt sind, gelingt es, den Sterubildchen auf der Platte vollkommen scharf begrenzte regelmässige Form zu geben. In dieser Bexiehung diurfte der Ausbildung der photographischen Photometrie kaum noch ein erustes Hinderniss im Wege stehen, zumal wenn man gewisse Vorsichtsmassregeln nieht ausser Acht lässt und namentlich nicht in zu grossen Abständen von der optischen Axe auf der Platte Messungen ausfuihrt. Dass die Sternscheibchen am Rande der Platte etwas anders aussehen, als in der Mitte, beruht, abgesehen ron nurermeidlichen kleinen Mängeln des Objectivs, schon darauf, dass die exaete Abbildung der Sterne auf einer Kugelfläche erfolgt, während die Platte eben ist. Man hat zwar, um diesem Übelstande zu begegnen, die Benutzung von gekriimmten Platten vorgeschlagen; indess diurfte dies in der Praxis ans rerschiedenen Griinden schwer ausfiihrbar sein. Gewöhnlich haben die Bilder am Rande der Platte eine elliptische, in der Richtung nach dem Centrum der Platte zn verlängerte Form. Es empfiehlt sich daher, wenn man die Randbilder nicht ganz von den Helligkeitsbestimmungen ansschliessen will, die Durchmesser sowohl in der radialen als in der dazu senkreehten Richtung zu messen und die Mittelwerthe aus beiden Bestimmungen zu benutzen. Bei gruten photographischen Objectiven ist iibrigens der Unterschied in der Form der Sternscheibchen bis zu nicht unbetrichtlicher Entfermung ron der Nitte der Platte kamm merklich. Im Allgemeinen erhält man ans Messungen am Rande der Platte die Helligkeiten der Sterne etwas zu gross.

Toranssetzung ist natiirlich bei allen photographisch-photometrischen Bestimmungen, dass die stembilder richtig ansexponirt sind. Ist die Expositionszeit rerhältnissmaissig sehr gross, so werden die hellsten Sterne iiberexponirt, und die Begrenzmg der Scheibchen wird dann durch rerschiedene Ursachen, insbesondere durch Reflexe ron der Riickseite der Platte, unschart und rerwaschen. Dagegen wirl bei sehr schwachen unterexponirten Sternen infolge der kleinen Miingel des Objectirs, ferner infolge der nieht in aller Strenge erreichbaren exarten Focussirung und anderer Ursachen der erste Bildeindruck auf der Platte rerhailtnissmissig einen zu grossen Durchmesser haben. jedoch nicht rollstindig schwar\% erscheinen. Es ist daher nicht gerathen, die Helligkeiten der schwiichsten sowohl als der hellsten Steme auf einer l'latte ans den Durchmesserbestimmungen an ermitteln, und man wird sich an Besten auf ein gewisses Helligkeitsinterrall, ïber dessen Grenzen bei jeden Ohjective die Erfalmung entsolheiden muss. heschrinken.

Während Bond bei seinen Helliwkeitsmessungen im hohen Grade von den Wängehn der damaligen photographischen Verfahren, insbesondere 
auch von den Unvollkommenheiten der Dagnerreotypieplatten und der Collodiumplatten abhïngig war, ist hente in dieser Beziehung bei den ansserordentlichen Fortschritten der photographisehen Technik so gut wie keine Gefalr mehr zu befürehten. Die trockenen Bromsillor-Gelatineplatten werden in den verschiedenen renommirten Fabriken, sowohl was Gleichmässigkeit der empfindlichen Schicht als deren Feinheit anbetrifit, in solcher Glite hergestellt, dass inan sogar Aufuahmen auf verschiedenen Platten derselben Emulsion nubedenklich "n vergleichenden Helligkeitsbestimmungen benutzen kaun, wenn man nicht gerade die höchsten Anforderungen an Genauigkeit stellt. Selbstverstaindlieh muss die Entwickelung - soleher Aufualunen unter genau gleichen Bedingungen erfolgen, und die Aufnahmen durfen zeitlich nicht allzu weit auseinander liegen, weil erfahrungsmiissig. die Empfindlichkeit der Platten bei längerer Aufbewalurung sieh allmällich etwas verändert. Da man in nenerer Zeit photographische Platten hergestellt hat, die nicbt nur für die violetten und ultravioletten, sondern anch für andere Strahlen des Spectrmms empfindlich sind, so liegt der Gedauke nahe, solche Platten zur photographisehen Photometrie zu verwenden. Wem es gelïnge, einen Sensibilisator zn finden, der die Platten gerade nur fïr die im Auge wirksamen Strahlen empfinglich macht, so wiirde die Platte munittelbar das menschliche Auge vertreten, und die photographischen Helligkeiten wären direct mit den optisehen vergleichbar. Vorläufig ist dieses Ziel noch nicht erreicht, und es bereitet insbesondere Schwierigkeit, orthochromatische Platten von vollkommen grleichartiger Beschatfenheit herzustellen, aber es unterliegt wohl keinem Zweifel, dass die Überwindung dieser Sehwierigkeit nur eine Frage der Zeit ist.

Bedenklicher für die gauze photographische Photometrie ist ein Übelstand, gegen den sich roranssichtlich ibberhaupt keine vollstiindige Ahhiilfe treffen laisst. Es ist dies der enorme Einfluss der Luftumruhe auf die Durchmesser der photographisehen Sternscheibchen. Bei unruhiger Luft werden die Bilder grö̈sser als bei ruhiger Luft, und demuach werden in ersten Falle die Helligkeiten zu gross gefunden. Nilch Untersuchungen von Scheiner kann der Fehler his \% einer halben Grössenclasse und dariiber anwachsen. Es folgt daraus, dass, wenn es sich mm Erreichung der höehsten Genanigkeit handelt, die Helligkeitsvergleichungen nur anf Messungen an ein und derselben Aufnahme beschriakt bleiben sollten, wo die Durchmesser aller Sterne im grleichen Sime durch die Luftumme beeinflusst worden sind. Die besten Resultate wird man daher bei der Ausmessung rou dicht gedräingten Sterngruplen, sowie bei der Vergleichung von Verinderlichen mit wahestehenden Vergleichsternen erwarten können. Am Günstigsten liegen in dieser Beziehumğ die Bedingungen an 
hochgelegenen Beobachtungsstationen, wo im Allgemeinen die Ruhe der Inft gleichmässiger zu sein pflegt.

Auch in Betreff der Extinction des Lichtes in der Erdatmosphäre ist bei den photographischen Helligkeitsbestimmungen viel grössere Vorsicht geboten als bei den photometrischen Messungen. Die chemisch wirksamen Strahlen werden durch die Atmosphäre am Stärksten absorbirt, und es ist daher zur Reduction der photographischen Helligkeiten eine besondere Extinctionstabelle erforderlich. Da aber die Bestimmung einer solchen 'labclle namentlich für die grösseren Zenithdistanzen mit grosseu Schwierigkeiten verbunden ist (bisher existiren über diesen Gesenstand nur zwei keineswegs ansreichende Untersuchungen von Schäberle1) und Pickering $\left.{ }^{2}\right)$ ), so ist es dringend gerathen, sich bei der photographischen Photometrie mur auf Beobachtungen in kleinen Zenithdistanzen zu beschränken.

Um aus den Durchmesserbestimmungen der photographischen Sternscheibchen die Helligkeiten abzuleiten, ist es nöthig, eine gewisse Masseinheit fiir dieselben zu Grunde zu legen. Man könnte eine solehe natïrlich ganz willkiirlieh wählen, doch dräingt sich von selbst der Gedanke anf, die photographische Helligkeitsscala in möglichste Übereinstimmung mit der iiblichen optischen Sterngrössenseala zu bringen und demnach die Intensititsdifferenz zweier Sterne, deren Helligkeiten sich zu einander wie $1: 2.512$ verhalten, als eine Grössenclasse zu definiren. Es handelt sieh dann darum, die Beziehungen zwischen optischen Sterngrössen und den photographisehen Durchmessern zu ermittelı. Über diesen Gegenstand sind im letzten Jahrzehnt eine ganze Reihe ron Untersuchungen, ins-

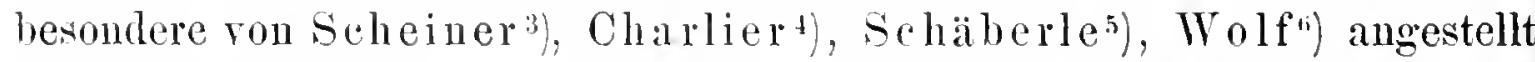
worden, anch in dem »Bulletin dn comité international pour l'exécution photographinque de la carte du ciel " finden sich zahlreiche wichtige Notizen von Seiten verschiedener Astronomen. Am Griindliehsten und Erschöpfendsten ist die Frage bisher ron Charlier mud Scheiner behandelt worden. Ersterer tindet fiur die Relation zwischen dem Durehmesser $D$ und der sterngrisse m den dusarnok:

$$
m=a-b \log D,
$$

Worin a und $b$ Grojsen sind, die fiir jede Anfnalnne bestimmte Werthe haben. Die Grösse a hïngt answer rou der Empfindlichkeit der Platte und dem Inftzustimde hauptsächlich von der Expositionszeit $t a b$,

1 Schaberle, Terrestrial atmospheric absorption of the photographic rays of lipht. Contributions from the Lick Ohservatory. No. 3. Sacramento 1893.

2 Anuals of the Astr. Ohs of Harvall College. Vol. 19, part II, p. 247.

3 Astr. Nachr. Bd. 121, Nr. 2s-4: 134. 121. Nr. 2969: Bd. 12s, Nr. 3054.

4 Publ. der Astr. Gesellschatt, Nr. XIX.

5) Publ. of the Astr. Soc of the Pacific. Vol. I. p. 51.

6) Astr. Nachr. Bd. 126. Nr. 3006. 
dagegen kaun / fur ein bestimmtes Instrument und fur eine bestimmte Plattensorte innerhalb gewisser Greuzeu als constant betrachtet werden. Vergleicht man zunächst uur Sterne auf derselben Aufnalıne miteinander, so ist anch $a$ als coinstant anzusehen, und man erhïlt fur die Grössendifterenz жweier Sterne die Gleichnng:

$$
m_{1}-m_{2}=b\left(\log D_{2}-\log D_{1}\right) \text {. }
$$

Scheiner hat gefunden, dass, wenn man nieht allzu grosse Helligkeitsdifferenzen in Betracht zieht, die Zunahme der Sterngrössen direct der Zunahme der Durchnesser proportional gesetzt werlen kann, so dass also die noch einfachere Relation besteht:

$$
m_{1}-m_{2}=l_{i}\left(D_{2}-D_{1}\right) \text {. }
$$

Beide Formeln, ebenso wie alle anderen bisher aufgestellten, sind nur als Interpolationsformeln anfanfassen und haben keine plyysikalische Bedentung. Mit Hulfe derselben kamn man anf jeder Aufnahme filr alle ansexponirten Sterne die photographische Grösse ermitteh, nachdem ans einer Anzahl von Sternen derselben Aufiahne, deren optisehe Grössen genau bekannt sind, die Constanten der Formel abgeleitet worden sind. Diese Anhaltsterue sind am Besten aus ein und derselben Spectralelasse und zwar der ersten (weisse Sterne), welche an Meisten an Himmel verbreitet ist, auszuwählen. Fill diese Spectralclasse wird so ein engrer Anschluss der photographischen und optisehen Grössen erreicht, während natiorlich fib Steme anderer Speetralelassen sehr erhebliche Untersehiede zwischen den beiden Systemen bestehen kümen.

Etwas weniger zurerliissige photographisehe Grössen lassen sich in Ermanglung einer genligenden Anzahl von photometrisch gut bestinmten Anhaltsternen auf folgende Weise erlangen. Man henutat hei jeder Himmelsatufuahne nur einen gewissen Theil ler l'latte, \%. B. die Hailfte, und nimmt jedesmal unmittelbar vor-oder naehher anf dem anderen Theile der Platte mit Beibehaltung der betrefienden Expositionszeit eine hestimmte Region des Himmels anf, welehe eine Menge sorgfuiltig photometriseh gemessener Sterue aller möglichen Grössen enthiilt; diese Sterne dienen dam \%ur Berechmmog der Constanten fiir die Platte. Dal die beiden Aufnahmeu nicht absolut gleich\%eitign gemacht sind, so wirken bei diesem Verfahren kleine Schwankungen in der luftheschaffenheit störend ein; auch ist man nicht immer von der Verpflichtnng befreit, die Extinction des Lichtes in der Atmosphäire in Rechmmog zu ziehen. Immerhin gestattet anch dieses Verfaluren bei einiger Vorsicht in der IValul der Beobachtumgstage eine recht befriedigende Genanigkeit, zumal wem mam sich nicht nur anf eine cinzige Gruppe ron Anhaltsternen beschriukt, sondern mehrere an versehiedenen Stellen des llimmels answählt mo in jedem gegehenen Fille die giinstigste benutzt. 
Ein drittes Verfahren, welches jedoch nur beschränkte Genauigkeit giebt, besteht darin, dic photographischen Sternscheibehen mit einer festen Seala zu vergleichen. Eine solche Scala kann man sich dadurch verschaffen, dass man von einem Sterne bekannter Helligkeit, etwa dem Polarstern, auf derselben Platte nebeneinander eine Anzahl von Aufnahmen bei gleicher Expositionszeit macht, indem man jedes Mal durch irgend eine Methode, sei es durch Abblendnng des Objectivs, sei es durch die einwurfsfreiere Verwendung von rotirenden Scheiben, die Helligkeit um einen bestimmten Betrag, etwa eine ganze Grössenclasse, verändert. Man erhiilt dam eine Reihe von Seheibchen, welche Helligkeitsdifferenzen vou je einer Grössenclasse entsprechen, und wenn man diese feste Seala mit den zu untersuchenden Aufnahmen vergleicht, so kann man für jeden Stern derselben die Stellung innerhalb dieser Scala angeben und dureh Schiitzung die Helligkeit bis anf Zehntel Grössenclassen ermitteln. Abgesehen daron, dass hier in letzter Linie alles auf eine Beurtheilung des Auges ankommt, wirken Veränderungen in der Luftbeschaffenheit, Extinction n. s. w. ebenso störend wie bei der zweiten Methode, und ausserdem kommt noch die verschiedene Empfindlichkeit der Platten in Betracht. Die Methode kamn daher nur als ein Näherungsverfahren angesehen werden. Pickering $\left.{ }^{1}\right)$ hat sich einer :ihnlichen Methode bei seinen zahlreichen photographiseh-photometrischen Lntersuchmoen bedient; er hat aber in den meisten Fiallen nicht die Durehmesser der Sterme in Betracht gezogen, sondern die Spuren, welche dieselben auf der Platte einzeichnen, wemn man wïlnend der Exposition das Lhrwerk des Fermrohrs entweder ganz ausser Thätigkeit setzt oder demselben eine geringere Geschwindigkeit giebt, als der täglichen Bewegun der Sterne entspricht. Das Anssehen dieser Striche wirl ebenfills dureh Schätzung mit einer festen Scala rerglichen, die durch Aufuahnnen der Spuren eines oder mehrerer bekannter Sterne bei messbar rerïnderter Helligkeit derselhen erhalten ist. Ua der Schwiirzmosorad der Striche wegen der rerschieden grossen linearen bewegung der Steme ron der Declination derselben abhängt, so ist bei dieser Nethode noch eine besondere Correction erforderlich, und die Pickering'schen photographisehen Sternhelligkeiten kömnen schon ans diesem Grunde kamm einen hijheren Werth beamsuruchen, als er z. B. blossen Itelligkeitsschiitzunֻen zukommt.

1) Memoirs of the American Acad. Vol. 11. p. 17\%. Ausserdem Anuals of the Astr. Obs. of Harrard College. Vol. 1s. 1. 119; Vol. 26. part. I; Vol. 32, part. I. 


\section{ABSCHNIT'T.}

\section{RESULTATE}

\section{DER PHOTONETRISCHEN BE0BACHTUNGEN}

\section{AII HIIIVEL.}





\section{Capitel I.}

\section{Die Sonne.}

Der Centralkörper unseres Planetensystems, ron welehem alle Glieder lesselben Licht und Wiirme empfangen, nimmt bekanntlich als Stern unter den ibrigen Stemen keineswegs einen hervorragenden Platz ein. Wie gewaltig auch die Lichtfülle ist, die bei der verhältnissmässig geringen Entfernung der Erde von der Soune unser Auge trifft', so wiirde doeh ron einem der näehsten Fixsterne aus betrachtet die Somne nur als ein Lichtpunkt von bescheidenem Glanze erscheinen. Wem es gelingt, das Helligkeitsverhältniss der Sonne zu anderen Himmelskörpern mit grosser Genauigkeit za bestimmen, so wird uns dadureh nicht nur Aufsehluss gegeben, welehe Stellung dem Sonnensystem unter den ithrigen Welten znkommt, sondern wir gewinnen auch ein einigermassen sicheres Fundament zu weiteren Speenlationen iber die Dimensionen der anderen Weltkörper, sowie uiber die ganze Anorduung des Weltalls. Aber aneh aus anderen Grïnden ist eine genaue Kenntniss der Intensitiit des Somnenlichtes erwiuscht. Wir haben bereits im ersten Abschnitte bei Berechnumgr des ron einem Planeten reflectirten Sonnenlichtes gesehen, dass eine sichere Bestimmung der Albedo des Planeten nur dann möglich ist, wenn das Helligkeitsverhältniss desselben zur Sonne bekannt ist; wir kïnnen also nur auf diesem Wege Aufsehliisse iber die physische Beschaffenheit unserer Naehbarplaneten zu erlangen hoffen. Anch die immer wieder auftanchende Frage, ob die Somne ein verinderlieher Stem ist, lässt sich nur durch die sorgfiltigsten photometrischen Messungen beantworten. Bei den gewaltigen Veränderungen, die sich beständig vor unseren Augen auf der Sonnenoberfliche abspielen, ist die Amnahme durehaus bereehtigt, dass auch Lieht und W:ïme Sehwankungen unterworfen sind, deren Kemntniss schon im Hinblick anf den bedeutenden Einfluss, den diese 
Factoren auf alles organische Leben an der Erdoberfläche ausilben, von allerhöchstem Interesse sein muss. Leider ist die Erforschung der Lichtverh:̈̈ltnisse des Sonnenkörpers bisher so mangelhaft geblieben, dass auch nicht im Entferntesten daran zu denken ist, auf Grund derselben Fragen wie die eben angefiihrten zu entscheiden. Mit einem gewissen Widerstreben haben sich offenbar die Astronomen aller Zeiten an dieses Problem gewagt, und das bisher vorhandene Beobachtungsmaterial, welches in den folgenden Paragraphen in mögliehster Vollständigkeit zusammengestellt und kritisch besprochen werden soll; giebt zunächst nur einen keineswegs zuverlissigen Werth fiir eine der wichtigsten Constanten der Astrophotometrie, weleher dringend der Verbesserung bedarf. Der Grund, weshalb das Studium der Somnenintensität bisher so sehr vernachlässigt worden ist, liegt wohl hauptsäehlich darin, dass eine directe Vergleichung der Sonne sowohl mit irdischen Lichtquellen als auch mit anderen Himmelskörpern wegen der enormen Helligkeitsunterschiede schwer ansfuhrbar ist. Um mit unseren bisherigen instrumentellen Hiilfsmitteln derartige Intensit:itsdifferenzen zu bestimmen, muss das Somnenlieht zuvor in messbarem Grade erheblich abgesehwïcht werden, und gerade die dazu erforderlichen Zwischenoperationen sind es, welche die Genauigkeit der Resultate betrïchtlich verringern. Nur dureh zahlreiche, immer wieder abgeänderte Versuche und durch Verbessermng der photometrischen Methoden lässt sich in Zukunft ein Fortsehritt auf diesem Gebiete erwarten. Jedenfalls bleibt hier fiir den Astrophysiker noch ein reiches und lohnendes Feld der Thätigkeit offen.

\section{Das Licht der Somne verglichen mit anderen Lichtquellen.}

a. Sonne und kinstliehes Lieht.

Der erste Versuch, das Licht der Sonne mit dem einer Kerze zu rergleichen, ist meines Wissens ron Bouguerl) im Jahre 1725 gemacht worden. Bonguer liess das Sonnenlicht durch eine kleine Öffnung, welche mit einer Concarlinse ron 2.25 $\mathrm{mm}$ Durchmesser rersehlossen war, in ein duukles Zimmer fallen und ting dasselbe in einer Entfermung von etwa 150 em anf einem weissen Schirme anf. Da das Licht sich anf dem schirme iiber einen Kreis ron nugefüh $24.3 \mathrm{~cm}$ Durchmesser ausbreitete, so ergalb sich die Dichtigkeit der Belenchtung anf dem Schirme 11664 mal geringer, als die Dichtigkcit der Beleuchtung beim Anftallen anf die Linse. Eine Wallhskerze in der Entfermung von $43.3 \mathrm{~cm}$ belenchtete den Schirm

1 Traité doptique, p. 55. 
etwa ebenso hell, wie die Sonne dureh die Linse. Un aber eine 11664 mal stilrkere Beleuchtung hervornubringen, mtlsste die Kerze dem Sehirme bis auf eine Distanı ron $4.01 \mathrm{~mm}$ nalie gebracht werden. Es folgt also daraus leicht, dass in der Entfernung von 1 Meter rund 62000 Kerzen aufgestellt werden mussten, um den gleichen Beleuchtungseffeet wie die Sonne zu crzielen.

Bei diesem Versuche hatte die Some eine Höhe von $31^{\circ}$ ther dem Horizonte. Berlleksichtigt man die Extinction in der Erdatmosphäre, so findet man, dass die Sonne, im Zenith gedacht, eine Fliche senkrecht ebenso stark belenchten wilrde, wie rund 75600 Kerzen in der Entfermung von 1 Meter. Da bei dieser Berechnung anf die Absorption des Sonnenlichtes in der Linse gar keine Rucksicht genommen ist, und da ferner die Vergleichnng wegen der Versehiedenlieit der Farben von Sonnen- und Kerzenlicht nothgedrungen sehr schwierig sein musste, so kann das abgeleitete Resultat nur als ein erster Näherungswerth betraehtet werden.

Nicht viel besser steht es mit dem Resultate. zu welchem Wollaston') im Jahre 1799 gelangt ist. Derselbe bediente sich der Rumford'schen Selıttenmethode, indem er das Sonnenlieht durch eine kleine Öfthung (olme Linse) in ein dunkles Zimmer eindringen liess, in gewisser Entfernung von der Öfnung einen dinnen eylindrischen Stab und ummittellbar dahinter eine weisse Fliche anfstellte und eine Kerzenflamme so lange verschob, bis die beiden anf dem Schirme entstehenden Schatten gleich intensiv ersehienen. Ist $e$ die Entferuung der Kerze rom Auffungschirme in Metern, und ist $d^{\prime}$ die Lichtrumuntitit, welche anf die Fliichencinheit in der Entfernung von 1 Meter senkrecht anffällt, so erlä̈lt der sommenschatten von der Kerze die Beleuchitungr $\frac{d^{\prime}}{e^{\underline{a}}}$. Ist ferner d die Lichitquantität, welche von der Sonne anf die Flächeneinheit senkrerlit anffällt, so wird infolge der kleinen Öffumug anf die Flächeneinleeit des Schirmes nur ein Theil gelangen kïnnen, der sich zu $d$ selhst verhailt wie der rom Schirme ans dureh die kleine Öffinmg hindureh sichthatre Theil der Sonne zu der ganzen scheinbaren Somnenscheilse. IDieses Verhailtniss ist aber gleich dem Verhailtnisse der Quidrate der Tamyenten der seheinbaren Hallmesser von Öfmum (rom Schirme ans gesehen) und Sonne. Ist also $D$ der wirkliche Durchmesser der kleinen ölfinung in Metern, $E$ die Entfernung derselben vom Schirme, und $S$ endlich der scheinbare Sonnenradins, so erhält der Kerzenschatten anf dem Sichirme von der Sonne die Belenchtung $\frac{d \times L^{2}}{4 E^{2} \tan r^{2} S^{\prime}}$.

1 Phil. Trans. of the R. Soc. of London. 1529, p. 19. 
Da nun die Kerze bei den Versuchen so weit versehoben wird, bis die Belenchtungen der beiden Schatten einander gleich erscheinen, so lat man:

und mithin:

$$
\frac{d^{\prime}}{e^{2}}=\frac{d \times D^{2}}{4 E^{2} \operatorname{tang}^{2} S^{\prime}}
$$

$$
\frac{d}{d^{\prime}}=\frac{4 E^{2} \operatorname{tang}^{2} S}{e^{2} D^{2}}
$$

Aus den zwölf einzelnen Messungen, welche Wollaston angestellt hat, erruieht sich im Nittel $\frac{d}{d^{\prime}}=59881$, d. h. die Sonne belenehtet die Flächéncinheit ebenso stark wie 59881 Kerzen in der Entfernung ron 1 Meter.

Was die Sicherheit der Messungen und die angewandte Methode betriff't, so wiirde der Wollaston'sehe Werth entschieden den Vorzug vor dem Bonguer'schen verdienen; leider verliert derselbe aber dadureh an Bedeutung, dass die Sommenhöhen, bei denen die Beobachtnngen angestellt wurden, nicht angegeben sind, und daher der Einfluss der Extinction nicht bestimmt werden kamn. Nimmt man an, dass die Messungen stets gregen Mittag ausgefiuhrt wurden, so wiurde die durchschnittliche Sonnenhïhe, da die Beobachtungen Anfang. Juni stattfanden, etwa $60^{\circ}$ betragen haben, und es wiirde daraus folgen, dass die Beleuchtung durch die Sonne im Zenith der Beleuchtung durch $61+46$ Kerzen in der Entfernung von 1 Meter gleichkommt. Dieser Werth ist als Minimalwerth anzusehen; er wiurde sich, falls die Beobachtumgen in grösseren Entfernungen vom Meridian angestellt wären, noch melu dem Bouguer sehen nähern.

Aus nenerer Zeit sind norh zwei weitere Bestimmungen des Helligkeitsrerhältnisses ron Somnen- und Kerzenlicht bekannt geworden, und zwal ron Thomson't und Exner"2). Ersterer hat seine Beobachtungen ebenso wie Wollaston mach der Rumford'schen Schattenmethode angestellt. Ans den mitgetheilten Zahlen ergielst sich, mit Beriicksichtigung der Extinction in der Atmosphitie, für die Liehtwirkung der Somne im Zenith der Werth 36104 Meterkerzen, der betrïchtlich kleiner als die Werthe von Bonguer und Wollastou ist. Da die Beobachtungen an cincm Wintertage bei einer Somnenhöhe von nur $g^{\circ}$ ausgefiihrt worden sind, so ist wegen der Lnsicherheit der Extinctionsrednction eine stärkere threichung leicht exklälich. Man wird daher dem Thomson'schen Werthe kein sehr wroses Gewicht geben diurfen.

Nature Vol. 2i, p. 275.

2 sitzmush, ler K. Akad. der Wiss. zu Wien. Math-naturw. Classe. Bd. 94 $1956, p, 545$. 
Exner hat sich bei seinen Messungen eines Ritehie'schen Photometers bedient, in welehem die Spiegel durch ein rechtwinkliges Prisma aus Gyps ersetat waren, so dass nur diffuse Reflexion stattfinden konute. Das Somnenlieht wurde, ehe es anf das Prisma fiel, durch rotirende Scheiben mit verstellbaren sectorförnnigen Aussehnitten in messbaren Grade geschwilcht, und dic letzte feine Vergleiehnng mit dem Liehte der benutzten Normalkerze wurde dureh die Drehung des Prismas un cine zu der Kante desselben parallele Axe ausgefuhrt, wodureh der Ineidenzwinkel der Somnenstrahlen verïndert wurde. Die Abhïngigkeit der Intensitiit von dem Ineidenzwinkel wurde empiriseh durch besondere Versuche bestimmt. Die Messungen selbst gesehahen bei Einsehaltung von furbigen Medien (Gliiser und Flussigkeiten) zwischen Prisma und Auge in drei verschiedenen Farben, Roth, Grllu und Blan. Zieht man mu die dureh das grỉne Glas hindurchgelassenen Strahlengattungen, welche etwa das Spectralgebiet zwischen $D$ und $F$ umfassen, in Betracht, so folgt im Nittel aus alleu einzelnen Bestimmumgen mit Berlieksichtigung der Extinction für die von der Sonne in Zenith ausgehende Beleuchtung der Werth 166150 Meterkerıen.

Mit Rucksieht darauf, dass die Intensität einer Kerzeuflamme kein absolut eonstantes Helligkeitsmass!) ist, und dass \%. B. schwerlich vollkommene Gleichheit zwischen der von Bouguer und der von Exner benutzten Kerze vorausgesetzt werden kann, wird man von vonherein starke Differenzen zwischen verschiedenen Bestimmungen erwarten können, wenn auch nicht so grosse, wie sie in den angefihhrten Zahlen zu 'Tage treten. An meisten Vertranen verdient der Exner'sche Werth, der sich aber mur anf die griinen Strahlen bezieht. Man wird jedenfalls anf Grund der bisherigen Untersuchungen nicht mehr sagen diurfen, als dass die Sonne in mittlerer Entfernung (im Zenith) eine ebenso starke Beleuehtung hervorbringt, wie etwa 50000) Normalkerzen in der Entfernung von 1 Meter. Diese Zahl wiirde noch nun ungefihr 20 Procent zu vergrössern, also durch b(0000) zn ersetzen sein, wemn inan die Gesammtabsorption unserer Erdatmosphïire in Rechunng briugen und die Lichtwirkung der Some ansserhalb der Atmosphitire betrachten wollte.

1) Unter Normalkerze versteht man gewöhnlich die englische Wallrathkerze, welche eine Flammenhühe von $44.5 \mathrm{~mm}$ hat und in der Stunde $7.77 \mathrm{~g}$ verbraucht. Ausser dieser Kerze wird als Lichteinheit hïufig noch die tranzüsische Stearinkerze und die dentsche Vereinskerze benutzt, deren Lichtstiirken sich zu derjenigen der englischen Normalkerzo nach Bestimmungen von Violle wie 1.15 resp. $1.13 \mathrm{zu} 1$ verhalten. Die von Violle vorgeschlagene L'latinlichteinheit entspricht in ihrer Wirkung etwa 18.5 englischen Normalkerzen, und die besonders in Frankreich gebrïnchliche Carcellampe kommt etwa 9 solcher Kerzen gleich. - Nïheres iiber diese und andere irdische Normallichtquellen findet man in dem linche von H. Krïss $\gg$ Die elektrotechnische Plotometrie. Wien, P'est, Leipzig, $1886^{\circ}$. 
Denkt man sich endich Kerzen in einer Entfernung von der Erde, die gleich der Sonnenentfernung ist, anfgestellt, so würde eine.Anzahl von etwa $134 \times 10^{25}$ erforderlich scin, un die gesammte Lichtwirkung der Sonne zu ersetzen.

Wir haben bisher nur voll der Beleuchtung gesprochen, welche das gesammte ron der Sonne ausgestrahlte Licht hervorbringt. Wesentlich rerschieden davon ist die mittlere scheinbare Helligkeit der Somnenoberflaiehe. Wollen wir diese im Verhältnisse zu anderen Lichtquellen ausdriicken, so müssen wir die scheinbaren Grössen der ausstrahlenden Flächen beriicksichtigen. Die Fläche einer Kerzenflamme betriigt etwa $3 \square \mathrm{cm}$; sic erscheint daher, als Kreis gedacht, in der Entferumig von $1 \mathrm{~m}$ unter cinem Winkel von $1^{\circ} 7^{\prime} 11^{\prime \prime}$. Mit Zugrundelegung. des obigen Werthes von 50000 Kerzen für die Sonnenbeleuchtung findet man daher, dass die scheinbare Helligkeit der Sonnenoberfliche ungeführ 220420 mal so stark ist, wie dic scheinbare Helligkeit einer englischen Normalkerze.

Vereinzelt sind Versuche gemacht worden, das Sounenlicht auch mit intensiveren irdisclen Lichtrqnellen als dem Kerzenlichte zu rergleichen. So haben Fizeau und Foucault anf photographischem Wege festgestellt, diss die mittlere scheimbare Helligkeit der Somnenoberflïche $146 \mathrm{mal}$ so hell ist wie das Drummond'sehe Kalklicht und ungefähr 3 mal so hell wie der elektrische Flammenbogen. Ferner hat Langley das von geschmolzenem Eisen ansgestrahlte Licht mit der Sonne verglichen und gefunden, dass die scheinbare Helligkeit desselben etra 5300 mal geringer ist als die der Sonne.

\section{b. soune und Vollmond.}

Lnter allen llimmelsiorpern kommt der Mond an Helligkeit der simne am nächsten, und es ist daher begreiflich, dass man der Bestimnumg des Intensitiitsrerhibituisses dieser beiden Gestirne besonderes Interesse gewidmet hat. Da eine gleichzeitige Beobachtmg derselben nicht möglich ist, so ist man leider gezwungen, als Mittelglied bei ihrer Vergleichumg kimstliches Licht zn henutzen, und dadureh wird die Sicherheit der fiesultate niclit unwesentlich beeintriielitigt. Dazu kommt, dass die Hollingeit des Mondes mit der P'lanse variirt, und dass, wenn man die Boobarlitungen nicht zu der genanen Zeit des Vollmondes anstellen kamm, Reductionen erforderlich sind, die ein weiteres Element der Unsicherheit bilden. Es ist daher nicht zu rerwundern, dass die bisherigen Angaben sehr erhebliche sinwankmugen zeigen, und dass der Endwerth, den man 
ans den besten Bestimmungen ableiten kann, mindestens noch un sechs Proceut unsicher ist.

Bis vor wenigen Jahrzehnten waren nur zwei Werthe fur das Helligkeitsverhältniss ron Sonne und Vollmond bekannt, die sich anch in allen astronomischen Lehrbilchen finden, obgleich sie miteinander gänzlich unvereinbar sind. Bouguer') giebt die Zahl 300000 und Wollaston ${ }^{2}$ ) den Werth 801072 an. Bouguer hat sowohl Somne als Mond nach seiner im Vorangehenden erwiihnten Methode mit dem Lichte einer Kerze verglichen, und da er nur Vollmondnächte (im Ganzen vier) benutzt, ausserdem beide Gestirne stets nahe in gleiehen Höhen Hber dem Horizonte gemessen hat, so wfirde- man ron vomherein geneigt sein, dem ron ihm angegebenen Mittelwerthe ein gewisses Vertrauen \%u schenken, wenn er nicht selbst die Genaugkeit desselben durch die Bemerkung in ZWeifel gestellt hätte: - les grandes diffieultés qu'il y a à déterminer un semblable rapport, font que je n'ose pas le regarder eomme exacte. Offenbar ist sein Eudwerth betrichtlich su klein.

Was den Wollaston'schen Werth hetrifft, so ist derselbe entschieden zu verwerfen, obgleich die Messungen selbst (Vergleichung von Some und Mond mit Kerzenlicht nach der Runiford'schen Schattemmethode) den Bouguer'sehen Messungen tiberlegen sind. Dic Verwerfung ist aus dem Grunde geboten, weil es zweifelhaft bleibt, ob die Extinetion bei den Wollaston'schen Beobachtungen berịeksichtigt worden ist. Die Höhen der beiden Gestime und die Beobachtungszeiten sind voil Wollaston nieht angegeben; da aber der Mond an den beiden Beohachtungstagen eine sehr grosse siidliche Declination besass, so dass er nicht hölıer als etwa $11^{\circ}$ ibler dem Horizonte von London gestanden hahen kamm, so ist die Wollaston'sche Zahl nur dam zu aceptiren, wemn anch die Somnenmessungen bei demselben tiefen Stande gemacht sind. Dariber findet sieh keine Angabe, die Voransset\%ung ist alher deshalb kaum zullissig, weil sich dan ein iibermäissig grosser 1 Trerth für das Itelligkeitsverhältniss von Sonnen- und Ker\%enlicht ergeben wiirde. Welchen Einfluss alber die Vemachlässigmug der Extinetion anf das Endresultat laaben kamn, geht daraus hervor, dass man unter der Annahne, Somne mid Mond wären beide in der Nïhe des Meridians beobalchtet worden, statt 801072 den Werth 372450 finden wiirde, also eine Zathl, die der Bongner'sehen nahe kïme. Die Unklarheit, welche iiber diesen wichtigen Punkt herrseht, bedingt jedenfalls ein winzliches Anssehliessen des Wollaston'sehen Werthes.

1) 'Iraité d'optique, p. si.

2 Phil. Trans. of the R. Soc. of London. 16.29, p. 27. 
Die zuverliissigsten Bestimmungen des Helligkeitsverhältnisses von Soune und Vollmond verdanken wir den Untersuchungen von Bond ${ }^{1}$ ) aus dem Jahre 1860 und von Zöllner ${ }^{2}$ ) aus dem Jahre 1864. Ersterer hat bei seinen Beobachtıngen versilberte Glaskngeln benutzt. Eine solche Kugel wurde den Sonnenstrahlen ausgesetzt, das durch Reflex entstehende Bildchen ron einer zweiten kleineren Kugel aufgefangen und zugleich mit dem Bilde einer künstlichen Lichtquelle betrachtet, welehes ebenfalls von dieser zweiten Kugcl entworfen wurde. Durch Verstellen der Kugeln gegeneinander und dureh Verschieben des Vergleichslichtes liess sich die gleiche Helligkeit der Bilder herstellen, und aus den Dimensionen der Kugeln, sowie aus den gemessenen Entfernungen ergab sich nach den bekaunten Formeln (siehe Seite 229) das Intensitätsverhältniss von Sonne und Vergleichslicht. In derselben Weise geschahen die Messungen am Monde. Als Vergleichslicht diente das Lieht von bengalischen Flanmen (Bengola lights). Nit Beriicksiehtigung aller Reductionsgrössen findet Bond für das Verhiiltniss von Sonne zu Vollmond, beide Himmelskörper in mittleren Entfermungen yon der Erde gedacht, den Werth 470980. Troty der geringen Zahl vou Messungen, auf denen dieser Werth beruht, erscheint er durehaus rertranenswiirdig. Bedenklich duirfte höchstens die Anwendung des ron Boud gewählten Vergleichslichtes sein, uiber dessen Constanz jegliche näheren Angaben fehlen.

Zöllner hat bei seinen Vergleichungen ron Sonne und Mond die beiden rou ihm construirten Photometer bemutzt; seine Resultate beruhen daher anf zwei ganz verschiedenen Beobachtungsmethoden, da bei dem ersten Züllner'schen Photometer Flächen, bei dem zweiten Punkte miteinander rerglichen werden. Als Zwischenglied diente bei beiden Methoden die mit dem Photometer rerbundene Petroleumlampe. Das Somnenlicht wurde durch eine Combination von Blendglïsern, deren Absorptionscoefficienten genau bestimmt waren, abgeschwächt. Bei dem zweiten Photometer kam an Stelle des gewölnnlichen Objeetivs eine besondere Linsencombination zur Terwendung, welche punktartige Bilder ron Some und Mond lieferte. Die Mondbeobachtungen wurden endlich nit Hiilfe der ron Züllner abgeleiteten Phasencurve (siehe nächstes Capitel) auf Tollmondhelligkeit reducirt. Gegen das Zölluer'sche Vertahren ist der Einwurf zu erheben, dass die Petrolenmlampe nicht während lingerer Zeitrïume als genigend ronstantes Vergleichslicht betrachtet werden kann. Bei geeigneten Vorsichtmassregeln wird zwar eine gleich-

1 Lemoils of the American Acail. New series. Tol.s, p. 257.

2 Zïlluer. Photometrische Untersuchungen etc. Leipzig. 1865, p. 73-11\%. 
missige Helligkeit innerhalb vieler Stunden erzielt werden kömnen, aber die Annahme, dass die Helligkeit whihrend mehrerer Monate, wie es bei den Zölhner'sehen Beohachtungen gefordert wurde, unveriindert bleibt, ist nach den Erfahrungen Aller, die sich mit photometrischen Messungen beschäftigt haben, durchans unzuliissig. Wenn die 'Zöllner'sehen Messungen trotzlem ganz ausgezeichnet miteinander ubereinstimmen, so . kann dies nur einem glitekliehen Zufalle zugeschrieben werden. Bei einer Wiederholuıg der Zölher'sehen Versuche, die sehr wilnsehenswerth ist, wird man gut thun, nur solehe Beobachtungen von Sonne und Mond zn eombiniren, die einige Stunden auseinander liegen, so dass merkliche Änderungen der Lampenhelligkeit nicht zu befureliten sind.

Die Werthe, welehe Zöllner aus seinen sämmtliehen Messungen flir den Quotienten $\frac{\text { Somne (in mittl. Entf.) }}{\text { Vollmond (in mittl. Entf.) }}$ abgeleitet hat, sind 618000 nach der einen und 619600 nach der anderen Methode.

Trotz der vortrefflichen Übereinstimmung dieser beiden Werthe ist den Zölhner'sehen Zahlen doch sehwerlich grössere Bedentung einzurïumen als dem Bond'schen Werthe, schon deshalb nicht, weil der absolute Betrag bei Zöllner ganz und gar von den angenommenen Absorptionseoefficienten der benutzten Blendgliiser abhïngt, deren sichere Bestimmung mit Schwierigkeiten verbunden ist. Bis bessere Bestimmmngen vorhanden sind, diirfte es sich empfehlen, das Mittel ans den beiden Zöllner'schen Werthen und dem Bond'schen zu benutzen und daher abgerundet zu setzen:

$$
\frac{\text { Sonne }}{\text { Vollmond }}=569500 \text {. }
$$

Der wahrseheinliche Fehler dieses Werthes diirfte schwerlich unter 6 Procent betragen.

Da die beiden Himmelskörper nahezn dieselbe scheinbare Grösse besitzen, so giebt die obige Zalhl atuch gleichzeitig das Verhiiltniss ilırer mittleren scheinbaren Helligkeiten an.

Will man den Intensitiitsmuterschied zwischen Somne und Mond, wie es jetzt in der Astronomie allgemein tiblich ist, in Sterngrössenclassen ansdrucken, so folgt atus dem obigen Werthe, dass die Sonne um rund 14.4 Grössenclassen heller ist als der Mond. Es ist dies mugefïhr derselbe Betrag, um welchen der Planet Mars seine Trabanten an Lichtstärke iibertriffit. 


\section{c. Sonne und Fixsterne.}

Die bisherigen Versuche, das Helligkeitsverhältniss der Sonne zu einem Fixsterne direct zu bestimmen, sind sehr spärlich und haben zu stark voneinander abweichenden Resultaten geführt, was bei der Schwierigkeit derartiger Vergleichungen kaum in Verwunderung setzen kann. Die erste Bestimmung rüht, soweit bekannt ist, von Hugghens') ler, welcher eine Helligkeitsvergleichnng zwischen Sonne und Sirius in seinem "Kosmotheoros « ansführlich beschreibt. Bei der Ungenaugkeit seiner Beobachtungsmethode, die darin bestand, dass er das Sonnenbild durch winzige Öffnungen so weit verkleinerte, bis es ihm der Erinnerung nach ebenso hell zu sein schien, wie der Sirins bei Nacht, kann das Huyghens'sche Resultat, welches die Helligkeit der Sonne 765 Millionen mal grösser giebt als die Helligkeit des Sirins, nur ein historisches Interesse beansprnchen. Der Werth ist zireifellos viel zu klein.

Ausser der Huyghens'schen Bestimmung sind nur noch zwei Versuche zur directen Vergleichung ron Somnen- und Fixsternlicht bekannt geworden, und zwar ron Wollaston2) ans (lem Jahre 1827 und von Zöllner:3) ans dem Jahre 1S64. Wollaston hat die Some ebenfalls mit Sirius verglichen und zwar vermittelst einer Kerze als Zwischenglied. Er beobachtete das ron einer kleinen Thermometerkugel reflectirte Sonnenbild durch ein 'Teleskop und rerglich es mit dem durch eine Linse ron kurzer Brennweite betrichteten Bilde einer Kerze, welches ron einer :nderen Kugel reflectirt wurde. Die gleiche Helligkeit der Bilder wurde durch Veränderung der Entfermung der Kerze hergestellt. In ähulicher Theise wurde der im Teleskop eingestellte sirius mit dem reflectirten Kerzenbildchen verglichen. Mit Bericksichtigung der Distanzen der Kerze und der Durchmesser der Kugeln erhich Wollaston ans sieben rerschiedenen Tersnchen fiur das Helligkeitsverhältniss rou Somne zu Sirius im Mittel den Werth 1085092. Dabei int der Liehtrerlust nicht beriicksichtigt, den die Somne bei der IReftexion ron der Thermometerkugel erfillren hat. Indem Wollastun ganz willkiirlich dafuir ungefähr 50 Procent :anuilum, leitete a den Endwerth ab:

$$
\frac{\text { Somne }}{\text { Sirius }}=20000 \text { Millionen. }
$$

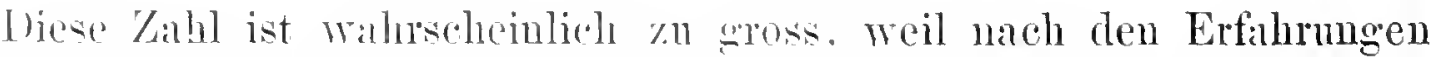

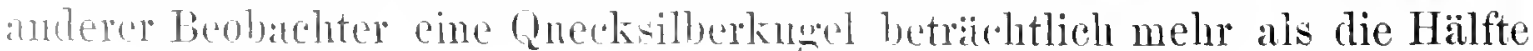

Christini Hugenii opera raria. Helansw. ron G. J. sGravesande. Lugduni Batarorm. 1724. Tomus tertins, 17. 717

? Phil. Trans ut the R. Soc of Lundom. 1529. 1) 19.

Zïlluer. Hhotmetrische Lutersuchungen etc., 1). 120-125. 
des auffallenden Liehtes zurltekwirft. Der Werth $15000($ Millionen wilrde vermuthlich der Wahrheit näher kommen. Leider wird dureh die Unsicherheit dieses Reductionselementes die Bedeutung der im Übrigen durehaus vertranenswurdigen Wollaston'schen Bestimmung etwas beeintrichtigt.

Zöllner hat das Intensitaitsverhältniss der Sonne z.u a Aurigae vermittelst seines Astrophotometers hestimmt, indem er durch eine. Linsencombination ein punktartiges Bild der Sonne herstellte und dasselbe mit dem kunstlichen lhotometerstern verglich, während er mit dem.gewöhnlichen Objectiv des Instrumentes die Helligkeit von a Aurigae in Verhältuiss zum kunstlichen Stern ermittelte. Dureh besondere Messungen musste däs Verhältniss der bei der Sonne angewandten Linseneombination zn dem gewöhnlichen Objectiv bestimnt werden, und zur Abschwiichung des Sonnenlichtes waren ansserdem Blendgliiser erforderlich. Zöllner findet:

$$
\frac{\text { Sonne }}{a \text { Aurigae }}=55760 \text { Millionen; }
$$

er schreibt dieseru Werthe, nach der inneren Übereinstimmung der einzclnen Messungen, einen wahrscheinlichen Fehler von 5 Procent zu. Die Unsicherheit des absoluten Betrages ist aber jedenfalls ans denselben Grinden, wie bei dem Helligkeitsverhältnisse von Sonne und Vollmond, viel grösser.

Interessint ist noch eine Vergleichung des Zölher'schen Werthes mit derjenigen Zahl, zu der man auf indireetem Wege gelangt, wenn man das Helligkeitsverhïltniss von Somne zu Vollmond mit dem anderweition bestimmten Helligkeitsverhältniss von Mond zu Fixstemen (siehe nächstes Capitel) combinirt. Es ergiebt sich dann:

$$
\frac{\text { Sonne }}{a \text { Aurigae }}=37165 \text { Millionen, }
$$

also ein Werth, der von dem Zöllner'sehen um mehr als 30 Procent des letzteren verschieden ist.

Will man die Helligkeit der Somne in Sterngrössen ansdricken (wobei man natirlich anf negative Grössen kommt), so ergiebt sich, da ce Aurigae nach den nenesten Messungen die Grösse 0.27 besitat, dass die Some ein Stern ist von der Grïsse -26.60 (nach Zöllner) oder - 26.16 (nach der indireeten Methode).

Unter der Voranssetzung endlich, dass c Aurigae eine jihrliche Parallaxe von 0.11 hat, folgt noch aus den obigen Bestimmungen, dass mis die Some in derselben Entfernung wie "Aurigae als ein Stern von der Grösse 6.5 erscheinen wiirde. Demmach miisste, da sich natch den spectral- 
analytischen Untersuchungen die beiden Gestirne höehstwahrscheinlich in demselben Entwicklungsstadium, also auch in gleichem Glühzustande befinden, die Somne ein viel kleinerer Weltkörper sein als a Aurigae.

\section{Die Vertheilnng der Helligkeit auf der Sonnenscheibe.}

Es ist in den vorangehenden Abschnitten schon mehrfach darauf hingewiesen worden, dass die Somnenscheibe uns nicht als eine gleichmiissig leuchtende Fläche erscheint, sondern dass die Helligkeit am Rande merklich geringer ist als in der Mitte. Diese heut allgemein anerkannte Thatsache ist friiher ein vielumstrittener Punkt gewesen. Galilei und später Huyghens hielten die Some an allen Punkten für gleieh hell. Der Jesuitenpater Scheiner scheint der Erste gewesen zu sein, der dieser Ansicht entgegengetreten ist, ohne dass jedoch sein Widersprueh die verdiente Beachtung gefunden hat. Zwei um die Photometrie so hoch verdiente Mainner wie Bouguer und Lambert wichen noch um die Mitte des rorigen Jahrhunderts in dieser Frage, wie schon fribher betont wurde, durchans roneinander ab. Während Lambert in seiner »Photometria (S 73) ausdriicklich sagt, dass wohl Niemand lengnen wird, dass das Ange die Oberfliche der Sonne iiberall gleich hell erblickt, hat Bouguer ${ }^{1}$ ) uicht nur die entgegengesetzte Meinung rertreten, sondern er hat aueh die ersten Versuche zur quantitativen Bestimmung der Helligkeitsabnahme ron der Mitte der Sonnenscheibe uach dem Rande hin angestellt. Scin Resultat gipfelt darin, dass die Lichtintensität im Centrum sich zur Intensitait einer um $\frac{3}{4}$ des Radius rom Centrum entfernten Stelle wie ts zu 35 rerlialt. Es ist bemerkenswerth, dass diese Angabe des ansgezeichneten franzüsischen Physikers, dessen Beobachtungsergebnisse anf fast allen Gebieten der Photonetrie auch heute noch die höchste Beachtung verdienen, schr sut mit den besten neneren Bestimmungen hiumonirt. Das Bonguer'sche Resultat ist spiiter noch mehrfach angezweifelt worden, unter Anderen sogalr ron einer Autoritiit wie Arago, der zwar eine Helligkeitsabnalme nach dem Rande lin nicht gänzlich in Abrede stellte, aber anf Grund seiner Yersuche zu dem Schlusse kam, dass der Lnterschied zwischen der Intensitit am Rande und der in der Mitte nicht mehr als etwa $\frac{1}{4}$ betragen kijnnte. Mam kam diese mit zurerlässigen Messulgen durchaus unverembire Zahlenangabe wohl kaum anders erKliren als durch die Unzulinglichkeit der ron Arago benutzten Hülfsmittel, insbesndere durch die Kleinheit der Somnenbilder, mit denen er 
operirt hat. Durch eine Auyahl von wichtigen Untersuchungen ist seitdem die Vertheilung der Helligkeit auf der Sonnenseheibe so sorgfiltig atudirt worden, dass die gewonnenen Resultate bereits als werthvolle Grundlage zu weiteren Betrachtungen llher die Ausdehnung und Besohaffeuheit der Sonnenatmosphäre, wolche diese Intensitiitsverschiedenheiten bedingt, dienen küunen.

Fine der ersten neneren Messumgsreihen, die wenig bekannt ${ }^{-} \eta n$ sein seheint, rlihrt ron Chacorna $\mathrm{y}^{\mathrm{J}}$-her. Derselbe blendete in einem grösseren Ferurohre aus dem Bremumuktsbilde der Sonne mittelst zweier in einem undurehsichtigen Schirme augehrachten kleinen öflnungen \%wei Partien heraus, eine in der Mitte, die andere in bestimnter Entfernung rom Rande, brachte dam durch ein doppeltbrechendes Prisma das ordentliche Bild des einen Lichtscheibehens neben das ausserordentliche des anderen und stellte endlich die gleiche Helligkeit dieser beiden Bilder mit Hnlfe eines drehbaren Nicolprismas her. Die Resultate, zu denen er gelangte, sind die folgenden, wenn der Radius der Somnenscheibe mit 1 und die Intensitiat in der Mitte mit 100 bezeichnet wird.

$\begin{array}{cc}\text { Abstand ron } & \text { Intensitiit } \\ \text { der Sonnenmitte } & 100 \\ 0.000 & 100 \\ 0.292 & 92 \\ 0.523 & \end{array}$

Fur Punkte in der Nïhe des Randes fand Chacornac die Intensität höchstens halb so gross wie diejenige des Centrums. Er hat ausserdem auf die versehiedene Firbung von Nitte und Rand anfmerksam gemacht, welehe solche Messungen wesentlich erschwert, und schon damals auf die Wichtigkeit spectrophotometriseher Beobachtungen hingewiesen, die erst viele Jahre spuiter von Vogel zur praktischen Ausfiilumg gebracht worden sind.

Eine sehr umfangreiche Untersuchung iiber die fragliche llelligkeitsvertheilung ist im Jahre 1859 von Liais²) angestellt worden, deren Ergebnisse jedoch wegen der Unzuverlassigkeit der angewandten Methode kein sehr grosses Vertranen verdienen. Liais brachte in der Focalebene seines Ferurohrs einen beweglichen Schirm an, mittelst dessen er einen beliebigr grossen Theil der Somnenscheibe verdecken kounte. Das abgeblendete Bild wurde durch Ausziehen des Oculars in vergrössertem Massstabe anf einen weissen Schirm projicirt, der durch die direct auf ihn fallenden Somnenstrahlen gleichmissig beleuchtet war.

1) Comptes Rendus. 'I. 49, p. $\$ 06$.

2) Mémoires de la société des sciences de Cherbourg. Vol. 12 (1866), p. 27i. 
Durch die bewegliche Blende wurden dann nacheinander verschiedene Partien der Sonnenscheibe herausgeblendet, und in jedem Falle wurde das Ocular so weit verschoben, bis das projicirte Bild auf dem Schirme nicht mehr vom Untergrunde unterschieden werden konnte. Die Grösse der Verschiebung des Oculars gab ein Mass für das Intensitätsverhältniss der untersuchten Stellen. Es unterliegt keinem Zweifel, dass diese Verschwindungsmethode gerade bei der grossen Lichtfïlle der Sonne wenig geeignet ist. Die Liais'schen Zahlen ergeben die Intensitätsabnahme von der Mitte nach dem Raude hin offenbar zu gering.

Zuverlässiger sind die Resultate, welche Pickering und Strangey im Jahre 1874 nach einem etwas ähnlichen Verfahren erhalten haben. Sie projicirten in einem dunklen Raume vermittelst eines kleinen Fernrohrs ein Sonneubild von etwa $40 \mathrm{~cm}$ Durchmesser anf einen Schirm, in welchem eine Öffnung ron $1.9 \mathrm{~cm}$ Durchmesser angebracht war. Das durch diese Öffnung hindurchgehende Licht traf auf ein Bunsen'sches Photometer und wurde mit dem Lichte einer Normalkerze verglichen. Anf diese Weise kounte das Helligkeitsverhältniss beliebiger Stellen der Sonneuscheibe mit ziemlicher Sicherheit ermittelt werten. Ein Nachtheil dieser Methode liegt in der Terwendung des Kerzenlichtes als Mittelglied, weil etwaige Schwankungen des Luftzustandes während einer Messungsreihe die Ergebnisse verfälschen können, was bei der Liais'schen Methode nicht zu befiirchten ist. Ans den ron Pickering und Strange angestellten Messungen lïsst sich die folgende Tabelle ableiten:

\begin{tabular}{c|c|c|c}
\hline $\begin{array}{c}\text { Abstand } \\
\text { ron der } \\
\text { Mitte }\end{array}$ & Intensităt & $\begin{array}{c}\text { Abstand } \\
\text { ron der } \\
\text { Mitte }\end{array}$ & Intensitāt \\
\hline 0.00 & 100.0 & 0.70 & 82.3 \\
0.10 & 99.2 & 0.75 & 78.5 \\
0.211 & 97.6 & 0.80 & 74.5 \\
0.30 & 45.7 & 0.5 .5 & 69.2 \\
0.40 & 93.5 & 0.90 & 63.2 \\
0.50 & 91.3 & 0.95 & 55.4 \\
0.60 & 57.4 & 1.00 & 37.4
\end{tabular}

Bei Weitem die ausfiihrlichsten und zuverlissigsten Beobachtungen iiber die Helligkeitsabnahme nach dem Rande hin sind ron H. C. Vogel²) im Tahre 1sit angestellt worden. Der hohe Werth dieser Messungen liegt hanptsiichlich darin, das sie sich nicht, wie die friiheren, auf das

1) Proc of the American Acad. of arts and sciences. New Series, Vol. II, p. 42S.

2 Monatsber. d. K. Prenss. Akad. d. Wiss. 15ii, p. 104. 
Gesammtlicht der Sonue, sondern auf die cinzeluen Strahlengattungen bezichen und das wichtige Resultat ergeben, dass die Intensitatsabnalime von der Sonneumitte nach dem Rande hin fur die violetten Strahlen beträchtlich grösser ist als fitr die rothen. Vogel hat sich des Spectralphotometers bei seinen Beobachtungen bedient und dadureh, dass er das Spectrum dor einzelnen Partien der Sonnenoberfliche stets mit dem Spectrum des Gesammtlichtes verglich, den bei den Piekering'sehen Verfuliren anftretenden Übelstand rermieden. Die Vogel'sehen Werthe sind in der folgenden Tabelle enthalten, wobei durchgaingig die Helligkeit in der Sonnenmitte mit 100 bezeichnet ist.

\begin{tabular}{|c|c|c|c|c|c|c|}
\hline \multirow{2}{*}{$\begin{array}{c}\text { Abstand } \\
\text { ron der } \\
\text { Sonnenmilto }\end{array}$} & \multicolumn{6}{|c|}{ Intensitat for die Strablou von der Wellonlango } \\
\hline & $105-112 \mu$ & $1.10-116 \mu$ & $167-173 \mu \mu$ & $510-515 \mu$ & $573-565 \mu \mu$ & $635-666 \mu 11$ \\
\hline 0.00 & 100.0 & 100.0 & 100.0 & 100.0 & 100.0 & 100.0 \\
\hline 0.10 & 99.6 & 99.7 & 99.7 & . 99.7 & 99.5 & 99.9 \\
\hline 0.20 & 99.5 & 98.7 & 95.8 & 99.7 & 99.2 & 99.5 \\
\hline 0.30 & 96.3 & 96.5 & 97.2 & 96.9 & 95.2 & 98.4 \\
\hline 0.40 & 93.4 . & 94.1 & 94.7 & $9 \cdot 1.3$ & 96.7 & 95.0 \\
\hline 0.50 & 88.7 & 90.2 & 91.3 & 90.7 & 94.5) & 96.7 \\
\hline 0.60 & 82.4 & 84.9 & 87.0 & 56.2 & 90.9 & 94.5 \\
\hline 0.70 & 74.4 & 77.8 & 80.8 & 80.0 & S.1.5 & 91.0 \\
\hline 0.75 & 69.4 & 73.0 & 76.7 & 75.9 & 80.1 & 88.1 \\
\hline 0.80 & $63 . \overline{7}$ & 67.0 & 71.7 & 70.9 & 74.6 & 54.3 \\
\hline 0.85 & 56.7 & 59.6 & 65.5 & 6.1 .7 & 67.7 & 79.0 \\
\hline 0.90 & 47.7 & 50.2 & 57.6 & 56.6 & 59.0 & 71.0 \\
\hline 0.95 & 34.7 & 35.0 & 45.6 & 4.1 .0 & 46.0 & 59.0 \\
\hline 1.00 & 13.0 & 14.0 & 16.0 & 16.0 & 25.1 & 30.0 \\
\hline
\end{tabular}

Diese Tabelle zeigt den erheblichen Untersehied in dem Verhalten der rothen und violetten Strahlen. Es geht daraus hervor, dats die Färbung der Sonne am Rande eine andere scin muss als in der Mitte, eine Thatsache, die ausser ron Chacornac anch von Secelii, Langrley und Anderen betont worden ist, und die mit dirzu beitriignt, dic directe Vergleichung von Mitte- und Randpartien zu ersehweren.

Die Pickering'sehen Angaben stimmen mit den Voucl'schen Irerthen fiur dic gelben und rothen Strahlen zienlich befrjedizend iherein; nur die Zahlen fïr die ainssersten Randtheile weichen nerklich ab, was ather wohl darauf zurickzufilhren ist, dass bei lem Pickering sollen Vorfahren uberhaupt nicht der eigentliche Rand, somdern stets ein messharer Theil der Seheibe bei der Beobachtnng benutyt wird, und diher im Allgemeinen zu grosse Angaben erwartet werden miissen. In dieser beziehung ist die spectralphotometrische Methode, bei der jedesmal mur der wingige anf den 
Spalt fallende Theil des Sonnenbildes beriicksichtigt wird, allen anderen Methoden ülierlegen, und es kann nicht dringend genug empfohlen werden, sich bei weiteren Untersuchungen ïber den Gegenstand derselben ausschliesslich zu bedienen.

Eine allerdings nur kurze Messungsseihe aus dem Jahre 1882 von Guy und Thollon') mit einem Spectralphotometer von $G \mathbf{u} y$ bestätigt in befriedigender Weise die Vogel'schen Resultate. Da Vogel seine Messungen zur Zeit des Sonnenfleckenminimums angestellt hat, so dürfte es vou Interesse sein, dieselben mit ebensolcher Genauigkeit zur Zeit des Maximums zu wiederholen, wo möglicher Weise die absorbirende Wirkung der Somnenatmosphäre etwas ander's sein kamn. Anch ist es empfehlenswerth, derartige Untersuchungen an der Sonnenscheibe nicht auf eine bestimmte Richtung, z. B. anf diejenige rom Centrum nach den Polen hin, zu beschränken, sondern dieselben iiber möglichst viele verschiedene Positionswinkel anszudehnen, $r m$ Fragen nach etwaigen Unterschieden zwischen nördlicher und südlicher Hemisphärre der Sonne u. s. w. mit Sicherheit zn entscheiden. Alles, was in dieser Beziehung bekannt gerorden ist, geht iiber die Berleutung blosser Muthmassungen nicht hinaus und darf daher unbedenklich iibergangen werden.

Dagegen ist es der Vollstindigkeit wegen erforderlich, wenigstens kurz anf die Tersuche hinzureisen, die gemacht worden sind, um iber die Vertheilung der Energie anf der Somnenscheibe auf anderem als rein optischem Wege, und awar durch das Stndium der chemischen und thermischen Wirkmugen des Sonnenlichtes Anfschluss zu erhalten. In ersterer Hinsicht liegen bisher zwei Messungreihen ror, eine ron Roscoe ${ }^{2}$ aus dem Jahre IS63 und eine ron H. C. Togel'3) ans dem Jahre 1S72, beide mach der bekaunten Bunsen-Roscoe'schen Methode ansgefiihrt. Roscoe hat die Messungen ansser in der Mitte der Somnenscheibe nur noch in zwei Entfermungen rom Centrum. allerdings in rerschicdenen Positionswinkeln, angestellt, wihloend Togel die chemische Intensitit in sehr vershiedenen Abtinden rom Centrum bestimmt hat. Wie ans der folgenden

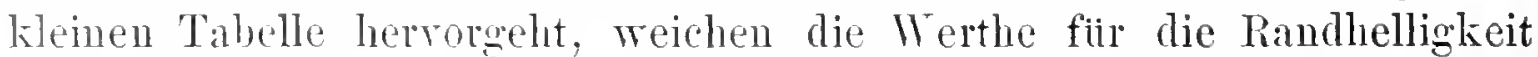
hei Roscoe und Togel nicht unerheblich roneinander ab; dagegen stimmt die Togel'sche Reihe recht sut mit den Resultaten der spectralphotometriahen Lntersuchungen fiur dic violetten und dunkelblanen Strahlen iiberein.

1 Comptes Rendus. T. 95, p. $\$ 34$.

2 Ploc. of the R. Soc. of London. Tol. 12 1863. p. 645 und Pogg. Annalen. Fid. 1211. p. 331 .

Jer. iiher die Terhandl. d. K. Sïchs. Ges. d. Wiss. Bd. 24 1872), p. 135 und Pogg. Anualen. Ind 14. p. 161. 


\begin{tabular}{c|c|c}
\hline $\begin{array}{c}\text { Abstand } \\
\text { rou der } \\
\text { Mttto }\end{array}$ & \multicolumn{2}{|c}{$\begin{array}{c}\text { Cheviscle Iotenoitst } \\
\text { nach }\end{array}$} \\
\hline \hline 0.00 & 100.0 & 100.0 \\
0.20 & - & 95.7 \\
0.40 & - & 91.2 \\
0.60 & - & 82.9 \\
0.80 & - & 59.6 \\
0.85 & 50.9 & 50.3 \\
0.90 & - & 39.5 \\
0.95 & - & 27.1 \\
1.00 & 29.7 & 13.5 \\
\end{tabular}

Was den Untersehied der Wärmewirkung zwisehen einzelnen Stellen der Sonnenscheibe betriflt, so ist das bisher gesammelte Beobachtungsmaterial ziemlich umfangreich. Es seien hier nur die Bestimmungen

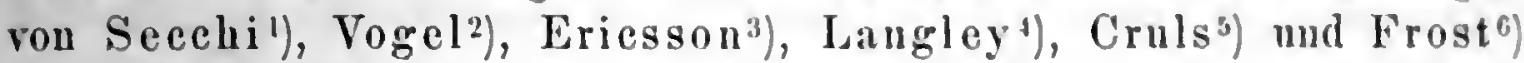
namlaft gemacht. Aus den zuverliissigsten dieser Beobachtungsreihen sind in der folgenden Tabelle für verschiedene Stellen der Sonnenoberflïehe einige Intensitätsangaben zusammengestellt.

\begin{tabular}{|c|c|c|c|c|}
\hline \multirow{2}{*}{$\begin{array}{c}\text { Abstand } \\
\text { ron der } \\
\text { Sonnenmitte }\end{array}$} & \multicolumn{4}{|c|}{ Wărmeintensităt der Sonnenschoibe nach: } \\
\hline & $\begin{array}{c}\text { Secchi u. } \\
\text { Vogel }\end{array}$ & Jangley & Frost & Mittal \\
\hline 0.00 & 100 & 100.0 & 100.11 & 1001 \\
\hline 0.20 & 99 & 99.5 & 99.4 & 99 \\
\hline 0.41$)$ & 9 & 96.4 & 96.3 & $: 4$ \\
\hline 0.60 & $9 \cdot 4$ & 92.2 & $5: 1,4$ & 92 \\
\hline 0.71 & $\varsigma 9$ & 88.4 & 54.6 & $-i$ \\
\hline 0.50 & \$2 & 82.5 & 77.9 & $\$ 1$ \\
\hline 0.90 & 69 & $i 2.6$ & (is.1) & (11) \\
\hline 0.96 & $(5 i)$ & 61.9 & $5 \pi: 2$ & 59 \\
\hline 0.95 & 47 & 50.1 & 511.0 & 19 \\
\hline 1.00 & 40 & - & 39 & $(10)$ \\
\hline
\end{tabular}

1) Mem. dell' Osserv. del Collegio Romano. 15.il, Apl. :3 und Astron. Nachr. Bd. 34, Nr. Su6; Bd. 35, Nr. s:33 und Mem. della Soeicti degl. Spettroc. It:1l. Vol. 4 $(1875)$, p. 121.

2) Monatsber. d. K. Preuss. Akad. d. Wiss. 197, 1. 135.

3) Nature. Vol. 12, p. 517 ; Vol. 13, p. 114 und 221.

4) Am. Journal of seience. Ser. 3, Vol. 114(16:5. p. 15:1. Ansserdem Comptes Rendus. t. 80 , p. $7+6$ und $\$ 19$; t. 51 , p. 4336 . - Nl3. Die Langley'schen Resultate sind nirgends ansfïhrlich publicirt; es finden sich iiberall nur kurze Ausziige und Notizen.

5) Comptes Rendus. T. S5, p. 570 .

6) Astron. Nachr. Bd. 1319. Nr. 3105-3106. 
Die ausgezeichnete Ubereinstimmung der drei Messungsreihen lässt die Mittelwerthe aus ihnen.sehr vertrauenswerth erscheinen, und die Vergleichung dieser Zahlen mit den spectralphotometrischen Messungen Vogels zeigt, dass die thermischen Bestimmungen sehr gut mit den Resultaten für die äussersten sichtbaren rothen Strahlen des Spectrums harmoniren.

Wie bereits mehrfach betont worden ist, rührt die Abnahme der Energie von der Mitte der Sonne nach dem Rande hin von der Absorption einer die Sonne umgebenden Atmosphäre her, und es ist vou Interesse zu wissen, um wieviel uns die Sonne heller resp. wärmer erscheinen wïrde, wenn diese Atmosphäre nicht vorhanden wäre. Laplace hat bereits auf Grund der oben erwähnten Bougner'schen Beobachtungen diese Frage zu beantworten versucht und ist mit Zugrundelegung seiner bekannten Extinctionstheorie zu dem Resultate gelangt, dass die Sonnenatmosphäre nicht weniger als $\frac{11}{12}$ des gesammten Lichtes absorbirt. Dieser Werth ist aber unrichtig, weil Laplace fiur die Berechnung der von einer selbstleuchtenden Kugel ausgehenden Lichtstrahlung das Euler'sche Gesetz angenommen hat, wonach eine solche Kugel ohne Atmosphäre am Rande heller erscheinen muisste als in der Mitte, während nach den neneren Forschungen für selbstleuchtende Körper aussehliesslich das Lambert'sche Emanationsgesetz zu Grunde gelegt werden muss. Pickering und Vogel laben bei der Anwendung der Laplace'schen Extinctionstleorie auf ihre Sonnenbeobachtungen diesen Fehler vermieden und finden daher fiur die Absorption der Sonnenatmosphäre erheblich kleinere Werthe als Laplace. Nach Ersterem wiirde die bcobachtete Helligkeitsabnahme hervorgebracht werden können durch eine homogene Atmosphäre von derselben Höhe wie der Somnenradius und ron solchem Absorptionsrermögen, dass bei senkrechter Ausstrahlung etwa 26 Procent des Lichtes hindurchgelassen wiirden; das Gesammtlicht der Sonne wiirde mach Pickering, wem gar keine Itmosphäire vorhanden wäre, $4.6+$ mal stairker sein als in Wirklichkeit.

Togel findet aus seinen Beobachtungen für die Transmissionswefficienten der Sonnenatmosphirie Werthe, die ron $0.79 \mathrm{im}$ Roth bis 1). Is im Vinlett abnehmen, und macht darauf aufmerksam, »dass die Extinction in tnhetracht der enormen Dimensionen der Chromosphäre ansserordentlich gering ist". Nach ihm wiirde das Gesammtlicht der Sonne olne Atmonliaive fuir violettes Licht $3.01 \mathrm{mal}$, für rothes Licht $1.49 \mathrm{mal}$ heller erscheinen als bei Anwesenheit der Atmosphäre. 
Die Vogel'sehen Beobaehtungen gestatten noch, wie Seeliger') in jungster Zeit bei einer Neubearbeitung derselben gezeigt hat, einige interessante Ausblicke auf die Beschaffenheit der Sommenatmosphlibe, die hier noch eine kurze Erwiihnung verdienen. Ist $J_{0}$ die Helligkeit im Centrum der Somnenscheibe, $J$ diejenige an irgend einer beliebigen Stelle, ist ferner $z$ der Winkel, den der von dieser Stelle ansgegangene und in das Ange gelangende Lichtstrahl mit dem verlangerten Sonnenradius bildet, und bedeutet endlich (Refr.) die Refraction, welehe dieser Lichtstrahl in der Sonnenatmosphïre erleidet, so gielıt die Auwendung der Laplace'schen Extinctionstheorio (Seite 122) die folgende Gleichung:

$$
\log J=-K \frac{\text { (Refr.) }}{\sin z} .
$$

Unter der Voraussetzung, dass die Refraction auf der Some ebenso wie auf der Erde ansgedrilekt werden kann dureh a tang $z$, wo der Vereinfachung wegen zuniichst a als const:unt fiir alle Werthe von $z$ angenommen werden soll, ergielst sich:

$$
\log J=-K^{\prime} \sec \approx,
$$

wo $K^{\prime}$ statt $\hbar \alpha$ gesetzt ist.

Ferner ist fuir $z=0$ :

$$
\log J_{0}=-K^{\prime}
$$

Mithin wird:

$$
\log _{0} \frac{J}{J_{0}}=-K^{\prime \prime}(\text { sec }:-1) \text {. }
$$

Es sei in Figur 71 (Seite :326) $C$ der Mittelpunkt der Somme. $P$ ein Punkt der Oberflache. Der wahre Sonnenradius sei $a$, und die Entfermum Sonne-Erde möge mit $\angle$ bezeichnet werden. Die gekrlimmte Linie I'F ist die Refractionsenrve; der Winkel $\sigma$ giebt cin Mass fur den scheinharen Abstand des in Betracht gezogenen Punktes rom Centrum der Scheibe. Nimmt man an, dass die Somenatmosphine concentrisch zeschichtet ist. so gilt ftir irgend einen Punkt $P^{\prime}$ der Refractions'urre die bekinnte Gleichung:

$$
r u \sin i=\text { Const. }
$$

wobei $r$ die Entfermung des Punktes $P^{\prime}$ vom Somnemnittelpunkt, "t der Brechungsexponent der Sommenatmosphiare in Punkte $P^{\prime}$ und $i$ der Winkel ist, den der Radins $C P^{\prime}$ mit der liefractionscurve einsehliesst.

1) Sitzber. d. matb.-phys. Classe d. K. Bayer. Akad. d. Wiss. Bd. 21, p. 264. 
Fiir die beiden Punkte $P$ und $E$ der Refractionscnrve gelten die entsprechenden Gleichungen:

$$
\begin{aligned}
& a \mu_{0} \sin \approx=\text { Coust. } \\
& \Delta \sin \sigma=\text { Const. }
\end{aligned}
$$

wo noch $\mu_{0}$ der Brechungsexponent an der Sonnenoberfläche ist. Mau hat also:

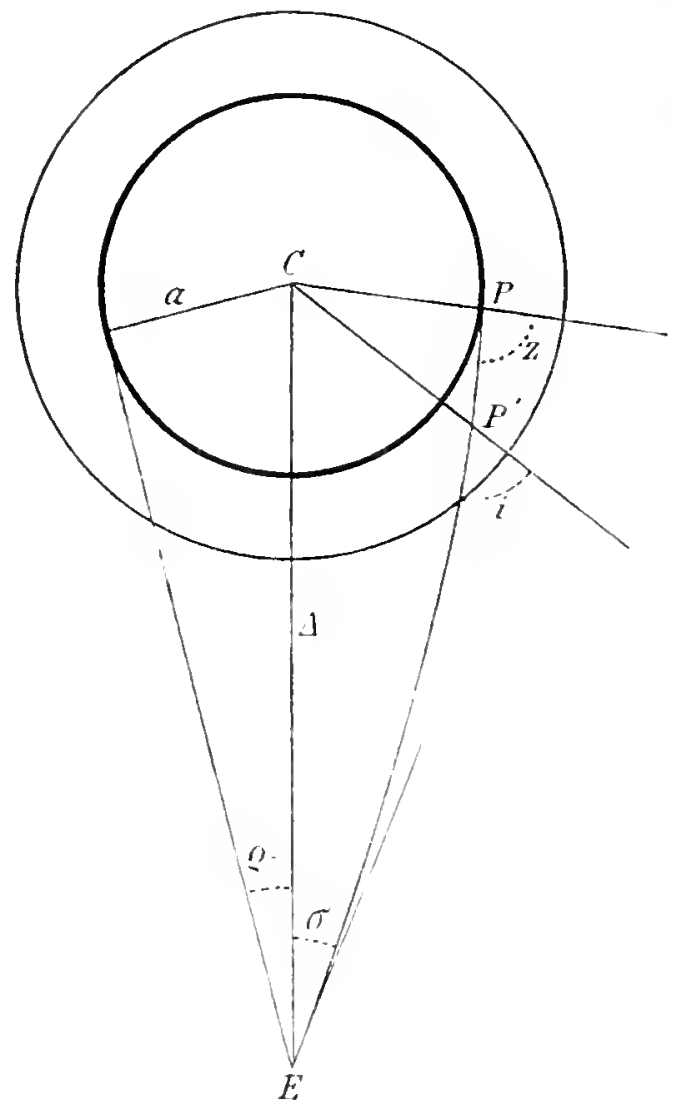

Fig. 71 .

$$
a \mu_{0} \sin \approx=\Delta \sin \sigma .
$$

Nun ist aber $\frac{a}{d}=\sin \varrho_{0}$, wenn $\varrho_{0}$ den Winkel bedentet, nuter welchem der Somuenradins ohne Vorhandensein einer Somnenatmosphäre rou der Erde aus erscheinen wiirde. Daher:

$$
\sin r=\frac{\sin \sigma}{\mu_{0} \sin \varrho_{0}} .
$$

Fuir $x=90^{\circ}$ beriihrt die Refractionscurve die Somnenoberflaiche, $\sigma$ geht daun in den scheinbaren Somnenradius (Sonne + Atmosphäire) iiber, der mit $Q$ bezeichnet werden soll, und man hat:

$$
\sin \varrho=\|_{0} \sin \underline{Q}_{0} \text {. }
$$

In Gleichung (2) darf man noch mit geniigender Nïherung den Quotient $\frac{\sin \sigma}{\sin g_{0}}$ durch den scheinbareu Abstand des in Betracht gezogenen Punktes der Sonnenscheibe ron der Mitte derselben (ansgedrickt in Theilen des scheinbareu Radins)

ersetzen. Nennt man diesen Abstand d, so wird:

$$
\sin \because=\frac{d}{"_{0}} \text {. }
$$

Fuir jeden anf der Somnenseheibe gemessenen Abstand $d$ kann man hieraus, wem $\mu_{0}$ bekannt ist, den Winkel s bestimmen und dann mit Hiilfe rou Cteichung (1) das Helligkeitsrerhialtniss irgend eines Punktes der Scheibe zul Jitte derselben berechnen.

Die Gleichung (1) kann watirlich nur als eine erste Näherung betrachtet werden, weil die Refraction auf der Sonne schwerlich dureh " tang: anit constantem a) ausgedrickt werden darf. $Z n$ einem etwas 
genauereu Resultate wilrde man gelangen, weun man die Refraction ausgedruckt hiitte dureh \& tang $z+\beta$ tang $\xi^{3} z$. Die Formel (1) wilrdo dann libergehen in:

$$
\log \frac{J}{J_{0}}=-K^{n}\left\{\sec x-1+\frac{\beta}{\alpha} \frac{\sin ^{2} x}{\cos ^{3} x}\right\} \text {. }
$$

Seeliger hat die Gleichmugen (1) und (1) anf die Vogel'schen speetralphotometrischen Beobachtungen und zwar auf die Helligkeitslogarithmen, nicht anf die Helligkeiten selbst, angewendet und ge\%eigt, dass, wenu " zunächst durchweg gleich 1 gesetzt wird, die Messungen in Roth und Gelb durchans gentigend, dagegen die Messungen in den anderen Farben nur mangelhaft dargestellt werden, dass jedoch eine beinahe vollkommene Darstellung der Beobachtungen in allen Farben erhalten werden kann, wenn man $\mu_{0}$ flir verschiedene Wellenlingen andere Werthe amnehmen lïsst. Hierans wilirde der Schluss zu zichen sein, dass die Sonnenatmosphäre eine merkliche Dispersion besitzen mlusse. Filr die Transmissionseoefficienter ergeben sich nach der Seeliger'schen Bearbeitung die folgenden Werthe:

\begin{tabular}{c|c}
\hline Wellenlinge & $\begin{array}{c}\text { Transmissions- } \\
\text { coeflicient }\end{array}$ \\
\hline $662 ! 1 !$ & 0.77 \\
579 & 0.66 \\
513 & 0.633 \\
470 & 0.174 \\
443 & 0.57 \\
$40 !$ & 0.54
\end{tabular}

Die Sommeuatmosphäre absorbirt also dantell immerhall des muter-

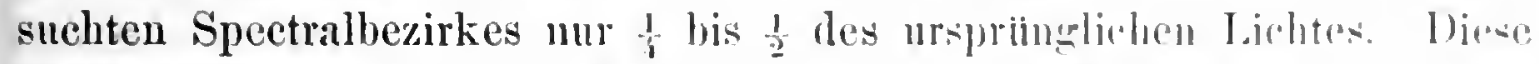
relativ geringe Absorptionsfahigheit kamm nur erkliat worklen, wann die

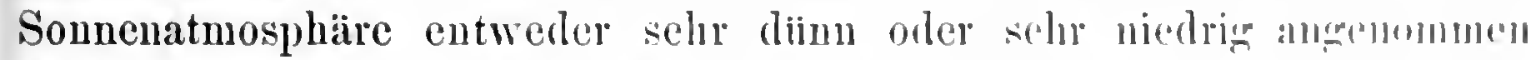
wird. Die erstere Ammalme ist nicht sehr wahreheinlide, woil hei niner diunen Atmosphäre keine starke Dispersion stattfinden kiinnte, wit site

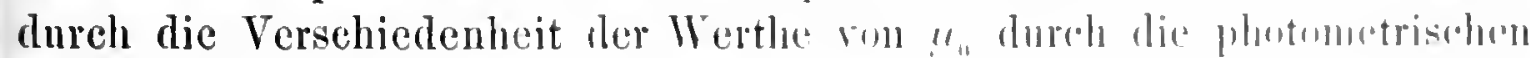
Beobachtungen angedeutet ist. Nan wirl also anf einc rolatir niedrige Sonnenatmosphäre schliessen miisis'll.

Aus der Versehictenheit der Werthe von " "wiirde naln nowh, mit

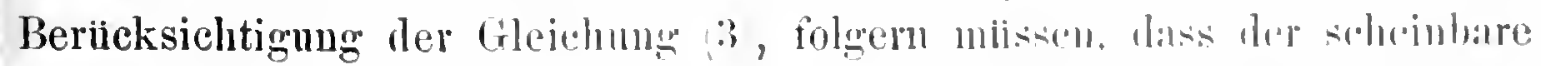

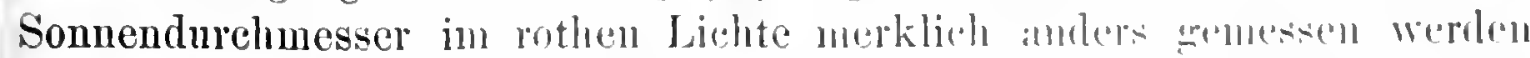

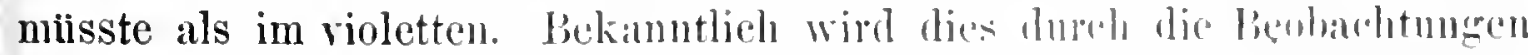

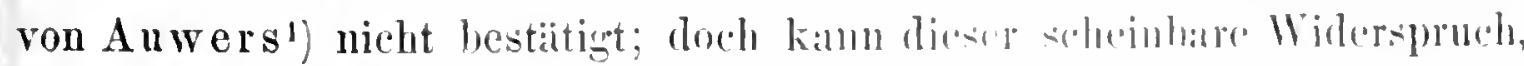

1) Astron. Nachr. Bd. 123, Nr. 2935. 
wie Seeliger nachgewiesen hat, dadurch erklärt werden, dass bei einer niedrigen und dichten Atmosphäre am Sonnenrande totale Reflexionen anftreten müssen, welche sehr wohl eine Vergrösserung der scheinbaren Durchmesser verhindern können.

Zum Schlusse dieses Paragraphen soll noch kurz anf die Versuche hingewiesen werden, die gemacht worden sind, um die Strahlungsintensitait der Sonuenflecke im Verhältniss zu den benachbarten Theilen der Sonnenoberfläche zu bestimmen, obgleich diese Versuche bishẹr nur selur ungeniigende, einander widersprechende Resultate ergeben haben. Dass die Somnenflecke keineswegs so wenig Licht aussenden, als man geneigt ist, nach dem blossen Augenscheim aus der Contrastwirkung anzunehmen, ist laingst bekannt gewesen, und schon Schwabe in Dessau hatte bei Gelegcnheit eines Voriiberganges des Mercur vor der Sonnenscheibe auf den beträchtlichen Unterschied der Intensität zwischen Planet und Somnenfleck anfmerksam gemacht. Aber die Frage, um wie viel die Photosphäre den Kern und die Penumbra eines Fleckes an Helligkeit iibertrifft, ist noch nicht als endgiiltig beantwortet zu betrachten. W. Herschel ${ }^{1}$ ) schätzte die Intensität der Penumbra gleich 47, die des Kernes gleich 0.7, wem die mittlere Fliichenintensität der Sounenscheibe gleich 100 angenommen wurde. Chacornac ${ }^{2}$ constatirte, dass die Penumbra eincs etwa $1 m$ ein Drittel des Radius von der Mitte entfernten Sonnenfleckes heller war als ein gleich grosses Stiick der Scheibe in numittelbarer Niihe des Randes; es wiirde daraus folgen, dass die Intensitait der Penumbra höchstens halb so gross sein kamn als die Intensität im Centrum (der Scheibe. Nach Liaisis) ist die Helligkeit der Fleckenkerne etwa 10 mal geringer, als die der umgebenden Photosphäre, und aus seinen Beobachtungen folgt ferner, dass die Intensitit der Sonnenfackeln in einer Entfermung ron 1' rom Rande gleich der des Centrums der Sonnenscheibe ist. Die spectralphotometrischen Messungen ron Guy und Tho $110 \mathrm{n}^{4}$ ) endlich ergaben für das Helligkeitsrerhältniss eines Kernes zu benachbarten Stellen der Somnenscheibe (fiir Strahlen von der Wellenlänge 680 !ul in THbereinstimmung mit Liais den Werth 0.1 .

Alle diese Angaben, ron denen allerdings keine ein besonderes Vertrineu beansunuchen kam, weichen sehr stark ron den Resultaten ab, die in Betreff der Wïrmewirkmug der Somnenflecke gewounen worden

1) Phil. Trans. of the R. Soc. of London. 1501, p. 35 t.

2 Comptes liendus. T. 49 , p. Soti.

3. Mém. de la soc. des sciences de Cherbourg. Tol. 12, p. 27.

4. Comptes Rendus. T. 95, p. 94. 
sind. Nachdem zuerst Henry und Alexandery in Princeton festgestellt hatten, dass die thermische Wirkung eines Sonnenfleckes geringer ist, als die seiner Ungebung, sind derartige Bestimmungen inehrfich gemacht worden, unter Anderen von Laugley ${ }^{2}$ ) und in neucster Zeit von Frost3). Aus den Langley'schen Messungen folgt fur die W'irmeausstrahlung eines Sonnenfleckenkerns der Werth is, fur diejenige der Peumbra der Wertl! S0, wem die Strahlung der unmittelbstren Nachbarschaft des Fleckes mit 100 bezcichnet ist. Frost findet den Unterschied zwischen Fleck und Photosphäre noch kleiner als Langley; seine Beobachtungen zeigen ferner, dass, wenn ein Fleek sich auf der Mitte der Scheibe befindet, der Wärmennterschied zwisehen ilın und der unmittelbaren Umgebung grösser ist, als wenn er sich in der Nüic des Randes befindet, was darauf hindenten witrde, dass die Flecke eine geringere Absorption in der Sonnenatmosphäre erfaliren als die Photosphiire. Dieses Resultat, welehes fur die Beurtheilung der physischen Beschaffenheit der Sonne von hohem Interesse sein witrde, bedarf freilich erst noch einer definitiven Bestiitigung. Es sind hieriber ansgedehnte systematische Beobaehtungsreihen sowohl zur \%eit des Sonnenfleckenmaximuns als des Minimums nieht nur mit der Thermosïule, soudern auch mit dem Photometer im hohen Grade erwinscht.

\section{Die Helligkeit der Sonnencoroni.}

Alles was wir bisher iiber die Helligkeit der äussersten Lmhlllum der Sonne, der Corona, wissen, berult anf den spirlichen lienhaldhtumps-

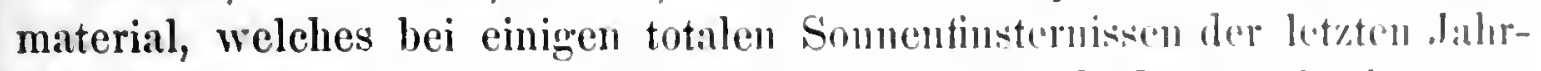

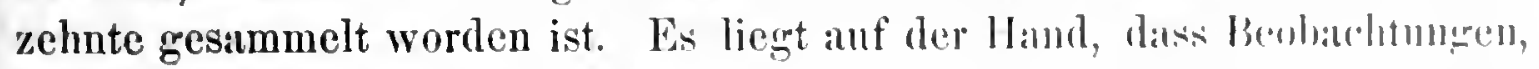
welche innerhalb der wenigen Minnten ciner solchen Erscheinm,g, meistens:

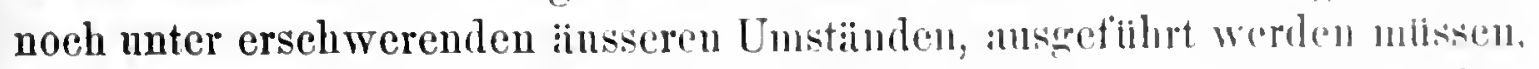
von vornherein nieht das hiehste Vertranen heanspruchen kimnem, nnd es darf daher auch kaum verwundern, dass die bistrerigen Eropelmisse betriichtliche Abweichungen mntereinander zeigen. Trutslem wird man die grossen Unterschiede in den liesultaten nicht allein der Inusirherheit

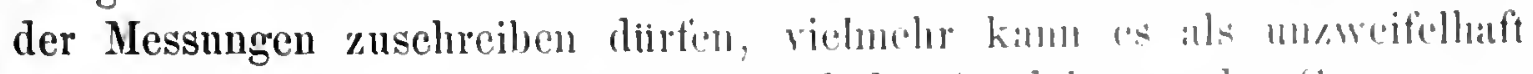
gelten, dass, ebenso wie die Form und dir Aushlehmun der Cormal ron

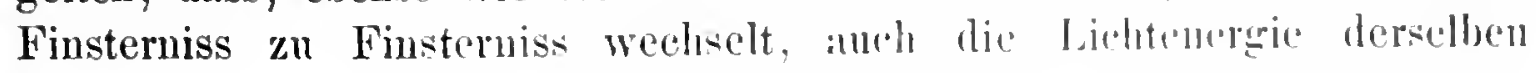

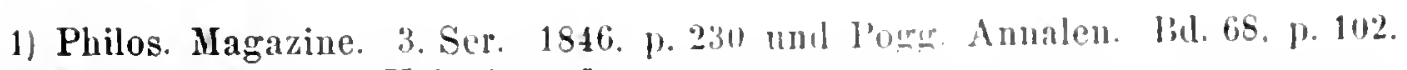

2 Monthly Notices. Yol. 37, p. 5.

3 Astr. Nachr. Bd. 130. Nr. 3110.5-3106. 
starken Schwankungen unterworfen ist. Ob diese Schwankungen in engem Zusammenhange stehen mit den beobachteten Vorgängen auf der Sonnenoberfliiche und daher auch denselben periodischen Verlauf nehmen wie diese, dariiber können erst länger fortgesetzte sorgfältige Beobachtungen Aufschluss geben. Soviel scheint schon jetzt festzusteheu, dass zur Zeit des Maximums der Sonnenthïtigkeit auch die Lichtentwicklung der Corona besonders lebhaft ist.

Die bisherigen Angaben iiber die Helligkeit der Corona beruben nur zum 'Theil auf directen photometrischen Messungen, zum grössten Theile sind sie aus photographischen Aufnahmen abgeleitet. Bei der ersteren Methode muss man sich hauptsächlich auf die Bestimmung der gesammten von der Corona ausgestrahlten Lichtmenge, der von ilı hervorgebrachten Beleuchtung, beschrïnken, da eingehende Untersuchungen iiber die Flächenhelligkeit der Corona an möglichst vielen Punkten derselben während der kurzen Daner einer totalen Somnenfinsterniss schwer durchfiihrbar sind. Die Entscheidnng iiber diese bei Weitem interessantere Frage muss daher nothgedrungen in erster Linie der photographischen Methode iiberlassen werden.

Bei den directen photometrischen Messungen hat man sich bis jetzt ausschliesslich des Bunsen'schen Photometers bedient, und in der That eignet sich dieses oder ein im Princip) ilhm ähnliches (etwa das Ritchieselie oder das in nenerer Zeit viel in Anwendungebrachte Weber'sche) vortreftlich zu solchen Bestimnungeu. Ein im Inneren sorgtältig gesehwärztes Rohr wird so anf die rerdunkelte Somne gerichtet, dass das Licht der Corona scnkrecht auf einen im Rohre angebrachten, mit einem Fettfleck rersehenen Papierschirm fïllt, welcher ron der anderen Seite her dureh eine rerschiebbare kiunstiche Lichtquelle beleuchtet wird. Die Läinge des Rohrs mos dibei so bemessen sein, dass möglichst wenig Licht ron den an die Corona angrenzenden Partien des Himmelsgrundes mit zur Wirkung gelangt, dass aber anch audererseits nichts ron dem ('oronalichte abgeschnitten wird. Der wunde Punkt bei diesen und allen :ihnlichen Helliskeitsbestimmungen bleibt immer die Benutzung einer irdischen Vergleichslichtquelle. So lange wir noch keine einwurfstreie Lichteinheit besitzen, welche willnend beliebig langer Zeiträume als absolut murerindert gelten kimn. so linge hahen wir mit einer Fehlerquelle zu kimpren, welche die Veroleichng der bei rerschiedenen sonnenfinsteruissen erhaltenen liesultate erhehlich nusicher macht.

Uie folgende: kleine Tabelle sieht eine Übersicht iiber die Resultate ter bisherigen photometrischen Bestimmungen der Leuchtiraft der Corona. bei den heiden Finsternissen ron 1571 und 1578 sind Normalkerzen zur Verwleinhme benntyt worlen, wihlend 1546 eine Glihlampe und Iss9 
ein Careelbrenner zur Verwendung kamen. In der Zusammenstellung ist Alles in Meterkerien ungewandelt und ansserlem noeh der Einfluss der Extinetion in der Erdatmosphiäre berlleksiclitigt worden. Die Zahlen gehen daher an, wie viel Normalkerzen in der lintfermung ron I Meter die gleiche Beleuchtung herrorbringen, wie die gesammte Sonneneorona, let\%tere in Zenith gedaeht. In der letzten Colmme ist noch die Lenchtkraft der Corona in Finheiten der Leuehtkratt des mittleren Vollmondes angegehen, welehe gleich 0.234 Meterkerzen zu setzen ist. (Sielie niichstes Capitel.)

\begin{tabular}{|c|c|c|c|c|}
\hline \multirow[b]{2}{*}{ Datuen } & \multirow[b]{2}{*}{ Beobachler } & \multirow{2}{*}{$\begin{array}{l}\text { Zenitl- } \\
\text { dlatanz der } \\
\text { Sonne }\end{array}$} & \multicolumn{2}{|c|}{ Zenithbelligkeit der Corona in } \\
\hline & & & Selerkerzen & $\begin{array}{l}\text { Elobeiten } \\
\text { det Yollmond- } \\
\text { bolligkeit }\end{array}$ \\
\hline 18io December 22! & W. 0. lioss & $62^{\circ}$ & 3.75 & 24.6 \\
\hline 1878 Juli 292 ) & J. C. Smith & 46 & $0.1 i 4$ & 2.7 \\
\hline 1556 August $29^{3}$ ) & A. Dollglas & 71 & 0.34 & 1.4 \\
\hline 1859 Januar 14) & A. 0 . Leuschner & 66 & 0.12 & 18.5 \\
\hline
\end{tabular}

Die beträchtlichen Unterschiede zwisehen den einzelnen Resnltaten lassen sich kaum dureh die Unsicherheit der Messnugen allein erklitren; sie sind zum grossen Theile wahrscheinlich darauf zuriackufluhren, dass die Beziehungen der benutzten Vergleichslichtyneilen zu einamer nicht genau genug bekannt sind, theils deuten sie ant wirkliche Helligkeits:indernngen der Corona hin. Mit einiger Sieherheit wird man daher ans den bisherigen Messungen nur folgern dürfen, dass die Belenthtum dureh die Corona im Durehsehnitt stairker ist als dureh den Volluombl.

Will man den Versuch machen, die Intensitiitsuertheilumge innorhallb

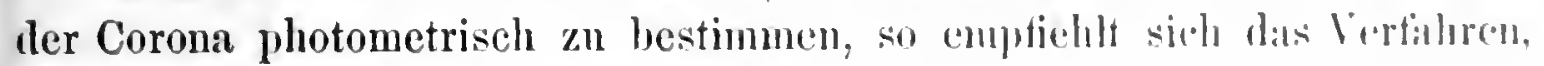

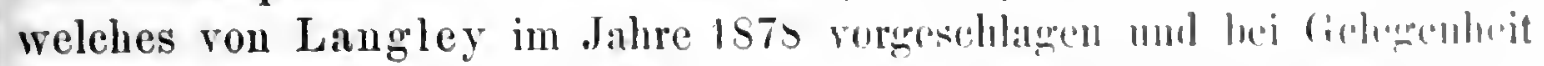

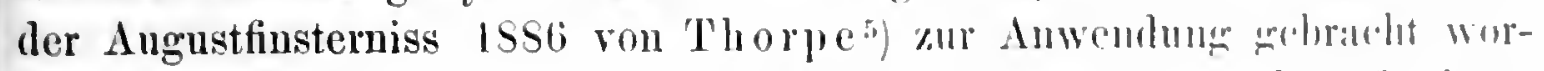
den ist. Dureh eine Linse ron linger Brennwoite wird anf cinm Sehirme in einem geschwärzten Photometerkasten ein Premummktshilal

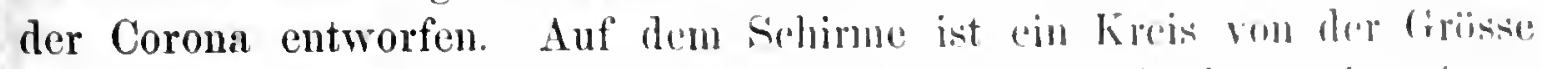

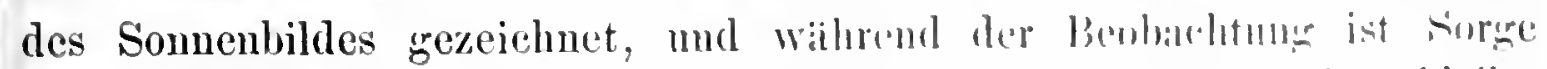

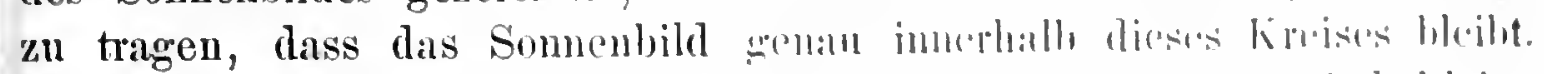

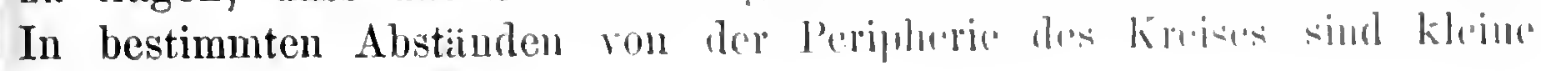

1) U. S. Coast Survey Repurt. 1870. p. 173.

2) Washington Observations. 1876, Appendix III. 1. 34-1;.

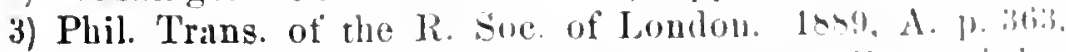

4) Reports on the observations of the total celipses of the san of Jan. 1. 1559,

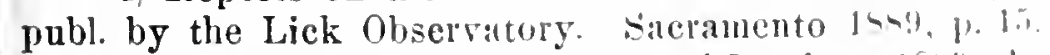

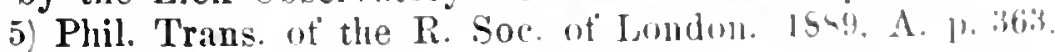


Öffnungen in dem Schirme angebracht, die mit geöltem Papier überdeckt sind. Auf diese Öffnungen fallen nun verschiedene Partien des Coronabildes, und man kann nach der Bunsen'schen Methode in verhältnissmässig kurzer Zeit für eine ganze Anzahl von Punkten die Flächenintensität ermitteln. Die Thorpe'schen Messungen, die allerdings unter sehr ungünstigen atmosphärischen Verhältnissen ausgeführt worden sind, ergaben, dass die Helligkeit der Corona in einem Abstande von etwa 1.5 Sonnenradien vom Centrum der Sonne ungefähr dreimal so hell war, wie in einem Abstande von 3.5 Sonnenradien. Eine Vergleichung mit der mittleren Flächenhelligkeit des Vollmondes fülurte zu dem Resultate, dass die Intensität der Corona in einem Abstande von 1.5 Sonnenradien bei der Augustfinsterniss 1886 nngefähı $15 \mathrm{mal}$ geringer war als die mittlere Flächenintensitiit des Vollmondes.

Weit besser als derartige photometrische Messungen eignen sich, wie bereits bemerkt ist, zu Untersuchungen iiber die Helligkeitsvertheilung innerhalb der Corona die photographischen Aufnahmen derselben, wenn sie uns anch nur Anfschluss iiber die chemische Wirkung des Coronalichtes geben. Ein von Abuey empfohlenes Verfahren, welches ebenfalls bei der Augustfinsterniss 1586, sowie bei den Finsternissen im Januar und December 1859 angewandt wurde, ist das folgende. Anf den zur Anfuahme der Corona bestimmten Platten werden ror der Finsterniss kleine quadratische Stiicke in der Nïhe des Randes dem Lichte einer Vergleiehsflamme ansgesetzt (während der iibrige Theil der Platte rerdeckt bleibt), und zwar jedes derselbeu währeud einer versehieden langen Zeitdamer; z. B. 1 Sec., 2 Sec., 4 Sec. u. s. w. Die exponirten Stellen werden mit dunklen Papierstreifen iiberdeckt, mud erst nachdem die Anfnahme der Corona anf derselben Platte erfolgt ist, zugleich mit dieser entwickelt. Die Dichtigkeit des Silberniederschlages an beliebig vielen Stelleu der Corona wird dann mit den rerschiedenen Quadraten am Rande der Platte verglichen, und fiir jeden Punkt das an Intensitiit gleiche Quadrat aufgesucht. Nimmt man num an, dass die Flächenhelligkeit dieser Quadrate direct proportional ist der Expositionsdaner, so lassen sich leicht die Curren gleicher Intensitit anf der Corona bestimmen.

Das Verfahren ist in der soeben besehriebenen Form nicht nachahmenswerth, weil nach den nenesten Untersuehungen die photographische Intensitait keineswegs proportional der Belichtungsdaner vorausgesetzt werden darf. Man wird daher besser so rerfahren, dass man sïmmtliche Hiilfsquadrate dem Vergleichslichte so lange exponirt, wie es fuir die Coronaanfuahmen beabsiehtigt ist, und die Helligkeitsabstufungen in der Weise herrorbringt, dass man die Intensitait des Vergleiehslichtes mittelst eines vorgeschobenen Keiles oder noch besser mittelst der rotirenden 
Quadrate seines Abstandes rom Sonnenrande, und dass ferner die Abhängigkeit der Intensität rom Positionswinkel des betreffenden Punktes ausgedruickt werden kann durch die einfache Relation $a+b \cos v$, wo $a$ und $b$ Constanten sind. Man wiirde also zu setzen haben:

$$
f(v, s)=\frac{a+b \cos v}{s^{2}}
$$

und unter dieser Annahme liesse sich das obige Integral leicht berechnen.

Der von Harkness voransgesetzte einfache Zusammenhang zwischen den Grössen $h, v, s$ ist bei keiner späteren Sonnenfinsterniss bestätigt gefunden worden. Vielmehr scheint die Vertheilung der Helligkeit innerhalb der Corona ziemlich ungleichmässig zu sein, und die Hoffnung, einen einfachen Ausdruck für die Funetion $f(v, s)$ zu finden, ist äusserst gering. Um trotzdem ans den photographischen Aufnahmen einen angenäherten Werth für das Gesammtlicht der Corona abzuleiten, haben Holden und Barnard ein zwar etwas primitives, aber ganz zweckmässiges und ausreichendes Verfahren eingesehlagen. Sie zeichneten auf starkes Cartonpapier um einen die Somnenseheibe repriisentirenden Kreis in entsprechenden Dimensionen die aus den photographisehen Aufnahmen der Corona bestimmten Linien gleicher Helligkeit. Den Flächeninhalt der einzelnen auf diese Teise entstandenen Zonen der Corona ermittelten sie dann dadurch, dass sie dieselben heraussebnitten, ihr Gewicht genau bestimmten und dasselbe mit dem Gewichte des der Somnenscheibe entsprechenden Kreises rerghlichen. Fiir alle Punkte einer Zone wurde als Flïehenintensitiit der fiir die begrenzende Curve festgestellte Werth angenommen. Durch Multiplication mit dem betreffenden Fliicheninhalte ergab sich daun sofort das Cresammtlicht der cinzelnen Zonen, und die Summe aller dieser Wertle lieferte endlich den gewiinschten Endwerth für das Gesammtlicht der Corona in der bei den Messungen zu Grunde gelegten Lichteinheit (Meterkerze, Carcellampe u. s. w.).

Die wiehtigsten Ergebnisse der bisherigen photographischen Helligkeitshestimmungen der Corma nach. der ron Holden I) gegebenen Zusammenstellung sind in der folgenden kleinen Tabelle enthalten, wo Alles in der oben definirten Lichteinheit der Careellampe ansgedriickt ist. Die Extinction des Lichtes in der Erdatmosphäre ist dabei nicht beriieksiehtigt, doch hat dies auf die Tergleichbarkeit der Resultate keinen merkliehen Einfluss, weil bei den drei in Betracht kommenden Finsternissen die somnenhöhen sehr wenig rerschieden waren.

1) Reports on the observ. of the total eclipse of the Sun, Dec. 21-22, 1859. Publ. by the Lick Observ. Sacramento. 1591, p. 14. 


\begin{tabular}{|c|c|c|c|c|}
\hline Datum & Aulorilal & $\begin{array}{l}\text { Ylhebeahellig. } \\
\text { Leil der folen. } \\
\text { sivolen Blellen } \\
\text { der Corona }\end{array}$ & 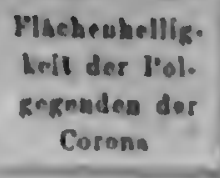 & $\begin{array}{c}\text { Gemagellicht } \\
\text { der } \\
\text { Corous }\end{array}$ \\
\hline IS86 Angust 28-29 & WV. II. Pickering') & 0.031 & - & $3: 0$ \\
\hline 1859 Januar 1 & Holden u. Barnard & 0.079 & 0.033 & 60.5 \\
\hline 1S5! Docember $21-22$ & Holden и. Barnard & 0.029 & 0.016 & 26.2 \\
\hline
\end{tabular}

Nach den Bestimmungen von W. H. Pickering, die allerdings noch weiterer Bestatigung bedurfen, ergieht sich noch flir die mittlere photographisehe Flächenhelligkeit des Vollmondes, ansgedruckt in derselben Einheit wie die voranstehenden Zahlen, der Werth 1.6it unl ferner fur die Flichenhelligkeit des Himmelsgrundes in einer Entfernung von $1^{\circ}$ von der Sonne (ausser der Zeit einer Finsterniss) der Werth 41. Die Richtigkeit dieser Zahlen voransgesetyt whrde also folgen, dass eine kleine Stelle des Vollmondes im Mittel mindestens eine 20 mal so starke photographische Wirkung-auslibt, als eine goleich grosse Stelle aus den hellsten Partien der Corona. Ferner wilrde sich ergeben, dass, selbst weun man nur den höehsten der obigen 'Tabellenwerthe bericksichtigte, die Fliachenhelligkeit der liehtstiirksten Stellen der Corona nur den 500sten Theil von der Helligkeit der nïchsten Ungehung der Somne betriigt, und dass daher die Bemilhungen, directe Anfuahnen der Corona anch amsser der Zeiten einer totalen Sonnenfinsterniss $\%$ erhalten, von vmulerein so gut wie gänzlich aussichtslos sind.

\section{Capitel II.}

Der Mond.

Wenn dem Trabanten der Erde wetremut rou den ibluigen Ciliedern

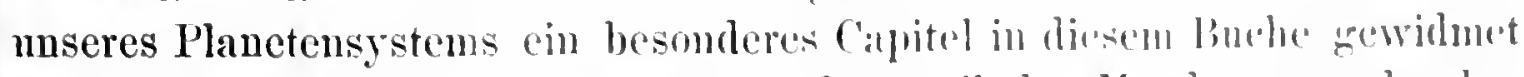
wird, so gesehieht dies ans dem cimule. weil der Homd wrenen der be-

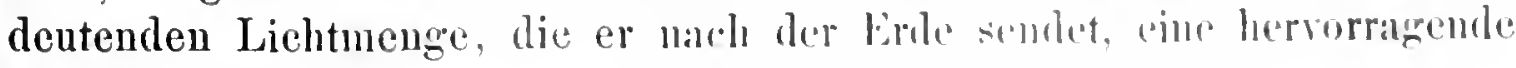

1) Annals of the Astr. Obs. of Harvard Collece. Fol. 15. p. 105. 
Rolle in der Astrophotometrie spielt. Er bildet, wie wir bereits gesehen haben, ein wichtiges, ja fast unentbehrliches Mittelglied bei den Vergleichungen zwischen dem Lichte der Sonne und dem der anderen Gestirne. Da er kein eigenes licht ausstrahlt, sondern nur das empfangene Sonnenlicht zuriickwirft, und da er ferner bei allen möglichen Beleuchtungsphasen, von der fast vollkommen verdunkelten Scheibe bis zum Vollmond, beobachtet werden kann, so bietet er, wie kein anderer Himmelskörper, die Möglichkeit, die Gesetze, welche für die Zurückwerfung des Lichtes zerstreut reflectirender Körper anfgestellt worden sind, zu prüfen. Die verhältnissmässig grosse Ausdehuung der scheinbaren Mondoberfläche gestattet ferner ein eingehendes Studium der Lichtvertheilung anf einer beleuchteten Kugel, und es ist wohl nur der Unvollkommenheit unserer photometrischen Hülfsmittel zuzuschreiben, dass die bisherigen Ergebnisse auf diesem Gebiete noch liickenhaft geblieben sind. Zweifellos werden anf diesem Wege, wenn nur ibberhaupt erst ein regeres Interesse für photometrische Untersuchungen bei den Astronomen erwacht sein wird, manche wichtigen Aufschliisse iiber die physische Beschaffenheit unseres Trabanten zu gewinnen sein.

\section{Das Licht des Mondes verglichen mit anderen Lichtquellen.}

\section{a. Mond und kiinstliches Licht.}

Die bisherigen Untersnchungen in dieser Richtung beziehen sich fast ausschliesslich auf Kerzenlicht, und es gilt daher von ihnen dasselbe, was bei der Besprechung der Vergleichungen von Some und Kerzenlicht gesagt worden ist. Insbesondere kann das Licht einer Kerze nicht zu allen Zeiten als ein constantes Helligkeitsmass angesehen werden, und ferner ist eine Vereinigung der an verschiedenen Orten und zn verschiedenen Zeiten erhaltenen Resultate anch nur unter der weiteren Voraussetzung zuliissig, dass sieh alle Angaben anf eine und dieselbe Höhe des Mondes beziehen, und dass die Mondbeobachtungen anf gleiche Phase redneirt sind.

Die bekanntesten Versuche riihren ron Bouguer1), Lambert2), Wollaston ${ }^{3}$, Plummert) und Thomson'i) her; alle sind nahe zur Zeit des Tollmondes angestellt. Bouguer, Wollaston und Thomson haben

1) Traité l'optique, p. 56 .

2) Lambert, Fhotometria. Deutsche Ausgabe von Anding, Heft 3, § 1075.

3) Phil. 'Trans of the R. Soc. of London. 18:9, p. 27.

4 Monthly Notices. Tol. 36, p. 354.

5 Nature. Tol. $2 i$, p. 277. 
dem Horizont gestanden hat; die Reductionen sind in diesem Falle unter der Annahme berechnet, dass der Mond im Meridian beobachtet worden ist.

\begin{tabular}{l|c}
\hline Beobachter & $\begin{array}{c}\text { Anzahl } \\
\text { der Kerzen } \\
\text { in Entfernung } \\
\text { von 1 Meter }\end{array}$ \\
\hline Bouguer & 0.2959 \\
Lambert & 0.2491 \\
Wollaston & 0.1650 \\
Plummer & 0.2282 \\
Thomson & 0.2336
\end{tabular}

Bildet man aus diesen Zahlen, die verhältnissmässig starke Unterschiede aufweisen, das Mittel, so ergiebt sich, dass •die Beleuchtung einer Fläche durch den Vollmoud im Zenith äquivalent ist der Beleuchtung durch 0.234 Kerzen in der Entfernung ron 1 Meter oder, was dasselbe ist, durch 1 Kerze in der Entfernung rou 2.07 Meter.

Daraus wiirde noch unter Beriicksichtigung der scheinbaren Grössen von Vollmond und Kerzenflamme folgen, dass die mittlere scheinbare Helligkeit des Mondes ungefüihr 1.09 mal grösser ist als diejenige einer Kerzenflamme.

b. Mond verglichen mit Planeten und Fixsternen.

Weit zuverlïssiger als das Intensitïtsverhiiltniss des Mondes zu kiinstlichem Lichte ist dasjenige zn den grossen Planeten und den hellsten Fixstemen bekannt. Bereits Ste inheil') hatte eine Vergleichung zwischen Vollmond und Aretur rersucht, indem er das Bild des Sternes in einem Fermrolne durch Ausziehen des Oculars in eine Scheibe rerwandelte, den Mond aber bei normaler Ocnlarstellung in demselben Fermrohre betrachtete mnd das Objeetir soweit abblendete, his die Helligkeit der Steruscheibe der des Mondes rergleichbar wurde. Als Verbindung'sglied diente kiinstliches Licht. Seidel²) hat diese Bcobaclitungen neu bearbeitet und macht darauf anfmerksam, dass bei der Steinheil'schen Methode zugleich mit dem Lichte des Sternes auch das rom Himmelsgrunde reflectirte Iondlicht in das Ange gelangt; indem er dafuir eine ziemlich willkiurliche

1) Steinheil. Elemente der Helligkeitsmessungen am Fixsternhimmel. München, 1936, p. 31 .

2 Abhand. 1. K. Bayer. Akad. der Wiss. II. Classe, Bd. 6, p. 629. 
Correction in Rechnung brachte, fand er fir den Quotienten $\frac{\text { Vollmond }}{\text { Aretur }}$ in runder Zahl den Werth 20000, der aber wegen der grossen Unsicherheit der Reduction kein grosses Vertranen heanspruchen katun.

J. Herschel') hat aus den photometrischen Nessungen, die er im Jahre 1836 mit seinem Astrometer am Cap der guten Hoffunug ausgefuhrt hat, fur das Helligkeitsverhialtniss von Vollmond \%u Centuuri im Mittel ans 11 Vergleichungen die Zahlı 27405 abgeleitet. Diese Zahl ist aber, wie Bond und Zöllner nachgewiesen haben, zu klein, weil sich Herschel zur Reduction der bei verschiedenen Mondphasen angestellten Beobaehtungen der Euler'sehen Belenchtungsformel hedient hat. Bond findet, indem er die Hersehel'schen Messungen mittelst der von ihm altgeleiteten empirischen Lichteurve anf den Vollmond redneirt, statt des obigen Werthes die Zahl 41400. Geht man noch von a Centauri auf einen hellen Stern am nördlichen Himmel, z. B. $\alpha$ Aurigac ${ }^{2)}$, uber, so folgt aus den Hersehel'schien Beobachtungen:

$$
\frac{\text { Mittl. Vollmond }}{\text { a Aurigate }}=64170 \text {. }
$$

Bond ${ }^{3}$ ) hat im Jahre 1860 den Mond mit den Planeten Jupiter und Venus verglichen unter Benutzung der von versillherten Giasknugeln reflectirten Bilder. Er findet:

$$
\begin{aligned}
& \frac{\text { Nittl. Vollumond }}{\text { Jupiter in mittl. Opl. }}=6431 \text {, } \\
& \text { Nittl. Vollmond } \\
& \text { Venus in mittl. Entf. beim l'hasconwinkel bs:5 }=1315 .
\end{aligned}
$$

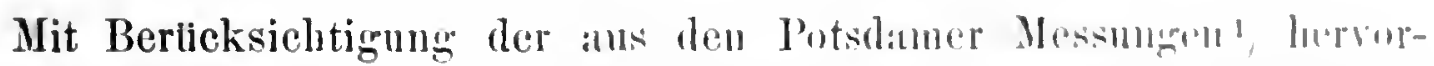
gehenden Helligkeiten der beiden Planeten ergehen sich fllr dias luten-

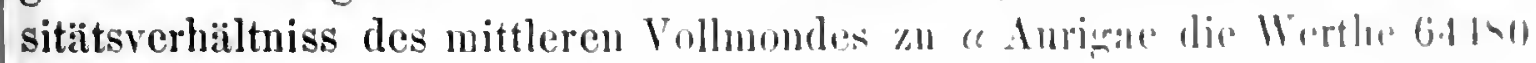

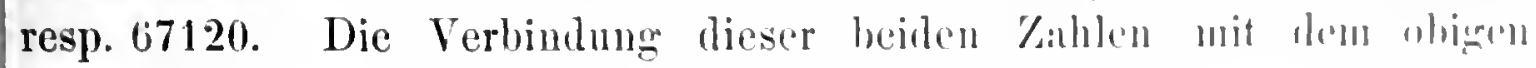

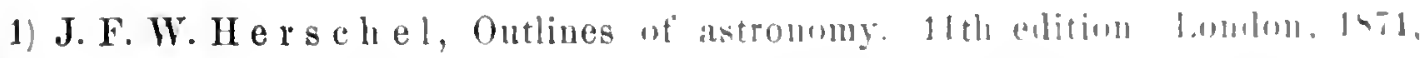
p. 595 .

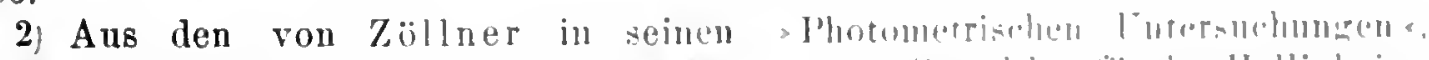
p. $171 \mathrm{ff}$. neu redueirten Herschel'schen Messungen an ('all fulet fiir dis Helligkits-

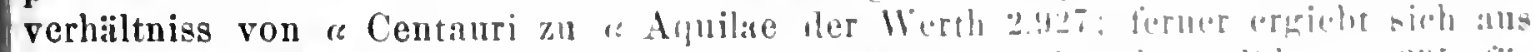

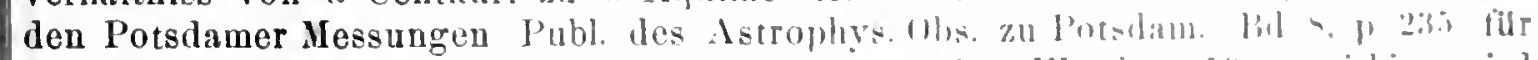
das Verhailtniss ron "Aquilate za "Anrigae Her Wurth 11.jul, mithin wirs $\frac{\text { « Centauri }}{\text { "A urigae }}=1.550$.

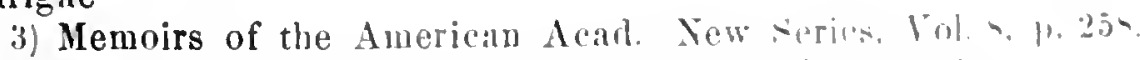

4) Publ. d. Astrophys. Obs. zu l'otsdatm. lid. ?. H. iliti. 
Herschel'schen Werthe liefert endlich den als zuverlässig zu betrachtenden Endwerth:

$$
\frac{\text { Mittl. Vollmond }}{\text { a Aurigae }}=65260
$$

dessen Unsicherheit auf höchstens 1 Procent zu schätzen ist.

Neuere Bestimmungen fiir diese wichtige Constante liegen nicht vor. Eine von Plummer') im Jahre 1876 nach der Rumford'schen Schattenmetlode ansgeführte photometrische Vergleichung zwischen Vollmond und Venus ist wegen der Schwierigkeit, mit welcher der schwache und undeutliche von der Veuus entworfene Schatten beobachtet werden konnte, als durehaus inzureichend zu bezeichnen; sie giebt fuir das gesuchte Verhältniss einen entschieden zu kleinen Werth.

Aus dem obigen Mittelwerthe folgt noch, dass der Vollmond in mittlerer Entfernung an Helligkeit einem Sterne von der Grösse - 11.i7 gleichkommt. Wäre aber der Mond ron der Somme ebenso weit entfernt, wie etwa der Planet Jupiter, so wuirde er uns als ein Stern von der Grösse 7.9 erseheinen.

\section{Die Lichtstärke der Mondphasen.}

Im theoretischen Theile sind Formeln abgeleitet worden zur Bereehmung der ron den Phasen eines beleneliteten Himmelskïrpers nach der Erde refleetirten Liehtmengen. Diese Formeln basiren auf drei verschiedenen Belenthtungsgesetzen, und es ist daher ron Interesse, an der Hand ron beobachteten Helligkeitswerthen zn prifen, welches dieser drei Ciesetze den Torzug rerdient. Gerade der Mond eignet sich am Besten zu einer solchen Lntersuchung, weil er infolge seiner Stellung zur Erde in allen Phasen beobachtet rerden kann. Merkwürdiger Weise ist aber das rorhandene Material so iiberans spialibeh und so wenig iibereinstimmend, dass eine definitice Entscheidun" zur Zeit noch nicht möglich ist. Wir besitzen bisher nur drei einigermassen Vertranen verdienende Tessungreihen iber die Helligkeit der Mondphasen, ron Herschel²), Bond: und Zijllnerit; aber nur die Bondsche Reilhe erstreckt sich

1 Monthly Notices. Vol. 36. p. 351.

2 II erschel. Results of Astr. Obs. made during 1534-1838 at the Cape of Good Hope. London. 15t7, p. 353.

3 Nemuils of the American Acad. New Series. Vol. 8. p. 250.

4 Zïllner. Photometrische Untersuchungen ete. Leipzig, 1865, p. 102. 
III. Resultate der photometrischen Beobachtungen am Himmel.

\begin{tabular}{|c|c|c|c|c|c|c|}
\hline \multirow{2}{*}{$\begin{array}{l}\text { Phasen- } \\
\text { winkel }\end{array}$} & \multicolumn{3}{|c|}{ Beobachtete Helligkeitslogarithmen } & \multicolumn{3}{|c|}{ Berechnete Helligkeitslogarithmen } \\
\hline & J. Herschel & Bond & Zöllner & Lambert & $\begin{array}{l}\text { Lommel- } \\
\text { Seeliger }\end{array}$ & Euler \\
\hline $0^{\circ}$ & 0.000 & 0.000 & 0.000 & 0.000 & 0.000 & 0.000 \\
\hline 10 & 9.903 & 9.965 & 9.928 & 9.994 & 9.989 & 9.997 \\
\hline 20 & 9.805 & 9.917 & 9.844 & 9.975 & 9.966 & $9.98 \overline{1}$ \\
\hline 30 & 9.705 & 9.855 & 9.748 & 9.945 & 9.934 & 9.970 \\
\hline 40 & 9.602 & 9.778 & 9.639 & 9.903 & 9.894 & 9.946 \\
\hline 50 & 9.494 & 9.686 & 9.516 & 9.850 & 9.847 & 9.915 \\
\hline 60 & 9.378 & 9.578 & 9.377 & 9.785 & 9.792 & 9.875 \\
\hline 70 & 9.250 & 9.454 & 9.220 & 9.706 & 9.730 & 9.827 \\
\hline 80 & 9.105 & 9.313 & - & 9.613 & 9.658 & 9.769 \\
\hline 90 & - & 9.155 & - & 9.503 & 9.576 & 9.699 \\
\hline 100 & - & 8.979 & - & 9.373 & 9.482 & 9.616 \\
\hline 110 & - & 8.783 & - & 9.220 & 9.373 & 9.517 \\
\hline 120 & - & 8.564 & - & 9.037 & 9.246 & 9.395 \\
\hline 130 & - & $8.31 \mathrm{~s}$ & - & 8.815 & 9.092 & 9.252 \\
\hline 140 & - & 8.038 & - & 8.536 & 8.903 & 9.065 \\
\hline 150 & - & 7.698 & - & 8.171 & 8.656 & 8.826 \\
\hline
\end{tabular}

Aus dieser Znsammenstellnng geht zunächst hervor, dass die bisherigen Beobachtungen iiber die Lichtstiirke der Moudphasen keineswegs geniigend nutereinander iibereinstimmen, und dass daher weitere sorgfriltige Messungen dringend erwiunscht sind. Die Herschel'schen und Zöllner'schen Zahlen für das Intervall rom Vollmond bis in die Nähe der Quadraturen harmoniren allenfalls noch leidlich unter sich, sie differiren aber von den Bond'schen Werthen nm Beträge (bis 0.6 Grössenclassen), die bei photometrischen Beobachtungeu durchans muzulaissig sind. Es wiirde daher nicht gerechtfertigt sein, anf Grund des rorliegenden Materials schon jetzt weitergehende Schliisse anf die physische Beschaffenheit des Mondes zichen zu wollen, doch diurfte aus der Vergleichung der beobachteten und berechneten Helligkeiten mit grosser Bestimmtheit hervorgehen, dass keine der bisher anfgestellten Belenchtungstheorien die wirklich stattfindenden Helligkeitsïnderungen darzustellen vermag. Die beobachtete Intensititsabuahme ist grö̈sser, als die theoretisch berechnete. Wïhrend nach der Euler'schen Theorie die Helligkeit des Mondes im ersten oder letzten Viertel gleich $\frac{1}{2}$, nach der Lambert'schen nugefähr weich $\frac{1}{3}$ der Vollmondshelligkeit sein sollte, ist dieselbe nach der Bondschen Lichtenre nur etwa gleich $\frac{1}{7}$.

Da alle Belenchtungstheorien einen gewissen idealen Zustand des difins reflectirenden Himmelskurpers roranssetzen, ror Allem eine gleichmässige Oberfliclıe, so ist es von vornherein klar, dass gerade beim Monde, von dem wir wissen, dass er ein Kürper olme merkliche Atmosphäre und 
dass dieselben ungefähr mit den Werthen für Quarz und Thonmergel iibereinstimmen. Natïrlich kann eine solche Gegenüberstellung nur ein ganz nebensächliches Interesse haben. Denn die angefülurten Albedowerthe beziehen sich ja nur auf die gesammte von der Mondoberfläche "zuriickgestralılte Lichtmenge; sie geben also nur einen ungefähren Begriff von der mittleren Reflexionsfahigkeit des Mondes, nicht aber von der verschiedenen Wirkungsweise einzelner Theile der Oberfläche. Schon der blosse Anblick der Mondscheibe zeigt ganz beträchtliche Unterschiede in der Helligkeit einzelner Partien, und da zweifellos damit eine stoffliche Verschiedenheit dieser Stellen im Zusammenhange sein wird, so ist es fiir die Erkcuntniss der physischen Beschaffenheit des Mondes von viel grösserem Interesse, die Reflexionsfähigkeit an möglichst vielen Punkten der Scheibe und bei den mannigfachsten Beleuchtungsverhältnissen zu studiren, als nur das Gesammtlicht in Betracht zu ziehen. Das Brennpunktsbild des Mondes ist bereits in mittelstarken Fernrohren gross genug, um eine photometrische Untersuchung bestimmter einzelner Partien zu ermöglichen; aber die wenigen Versuche, die bisher in dieser Richtung. gemacht worden sind, haben wegen der grossen Schwierigkeiten, die sich bei Anwendung der gebräuchlichen Photometer den Messungen entgegenstelleu, noch zu keineu verwerthbaren Ergebnissen gefiihrt. Ausser einigen mehr allgemeinen Angal)en iiber das Helligkeitsrerhältniss verschiedener Stellen der Mondoberfliche ron Bongner, Arago und Bond sind systematisch durchgeführte Beobachtungsreihen uiber die Helligkeitsvertheihung anf der Mondscheibe mur ron Pickering bekannt geworden, und anch diese Messmen sind offenbar noch viel zu unsicher, um weitere Schliisse daranf zn grinden. Nach Bonguer'y) ist die dunkle Stelle im Grimaldi funf- bis sechsmal lichtschwiicher, als die Mitte des Mare humornm. Aragoz) fand, dass sieh im Mittel die Intensität des Mondrandes zu der Intensität der srossen Flecke anf der Scheibe verhält wie 2.7 z 1 . Eine sehr shinzende Stelle des Raudes ïbertraf nach seinen Messungen einen dunklen Fleck mo das $15 \frac{1}{2}$ fache, nnd ein isolirter, nicht weit ron der schattenwrenze selegener glïnzender Punkt soll wach ilm soggar 109 mal heller wewesen sein, als die allgemeine Oberfläche

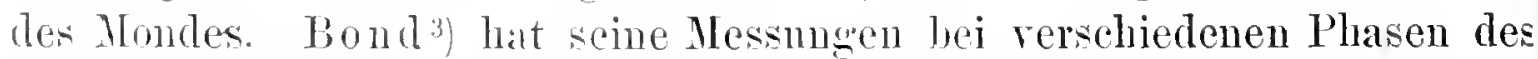
Moudes angestellt. In der Nïhe des ersten Viertels $\left(a=81^{\circ}\right)$ fand er, wem die Intensitit der hellsten P'artien anf der Nondscheibe mit 100 bezeidnet ist, die folgenden Werthe:

1) Traité dintique. p. 122.

2 Alagus Werke. Wentsche Ansgabe ron Hankel, Bd. 10, p. 239.

3 Memoirs of the American Acad. New Series. Tol. S, p. 267-276. 
Hellste Stellen auf dem Monde.

Mare crisium (Mitte der slldl. Hiilfte desselben) $=60.8$

Mare tranquillitatis (dunkelster 'Theil) . . . . = $=7.9$

Stelle mahe der Mitte des Mondes, etwa 1 Min. voin der Schattengrenze entferit . . . . . . $\}=9.3$

Fliehenstlick, etwa $\frac{1}{2}$ Min. von der Schattengrenze entfernt..

line zweite Beobachtnngsreihe rou Bond, einige Tage spater angestellt $\left(\iota=39^{\circ}\right)$, gab fllr das Mare erisium die Helligkeit 47.5.

Die photometrischen Messungen Pickerings') erstrecken sich anf 60 verschiedene Stellen der Mondoberfliche. Sie sind mit einem Photometer ansgefuhrt, welches dem anf Scite 262 beschriebenen ahmlich ist. Die einzelnen Objecte wurden dabei direet rerglichen mit einem g:m\% kleinen Mondbilde, welehes dureh ein Hulfsfernrohr in das Gesichtsfeld des Hauptteleskopes reflectirt wurde. Die Pickering'sehen Zahlen heruhen auf mehrfach wiederholten Messungen, die fast saimmtlieh in der Nihhe des Vollmondes ansgeftilırt sind. In der folgenden Zusammenstellung sind statt der Pickering'schen Grössenclassenangaben die Intensititen selbst angefuhrt, und zwar ist die Intensitiit des hellsten Objeetes der Mondscheibe, des inneren Walls und der Centralspitze vou Aristarch, mit 100 bezeichnet.

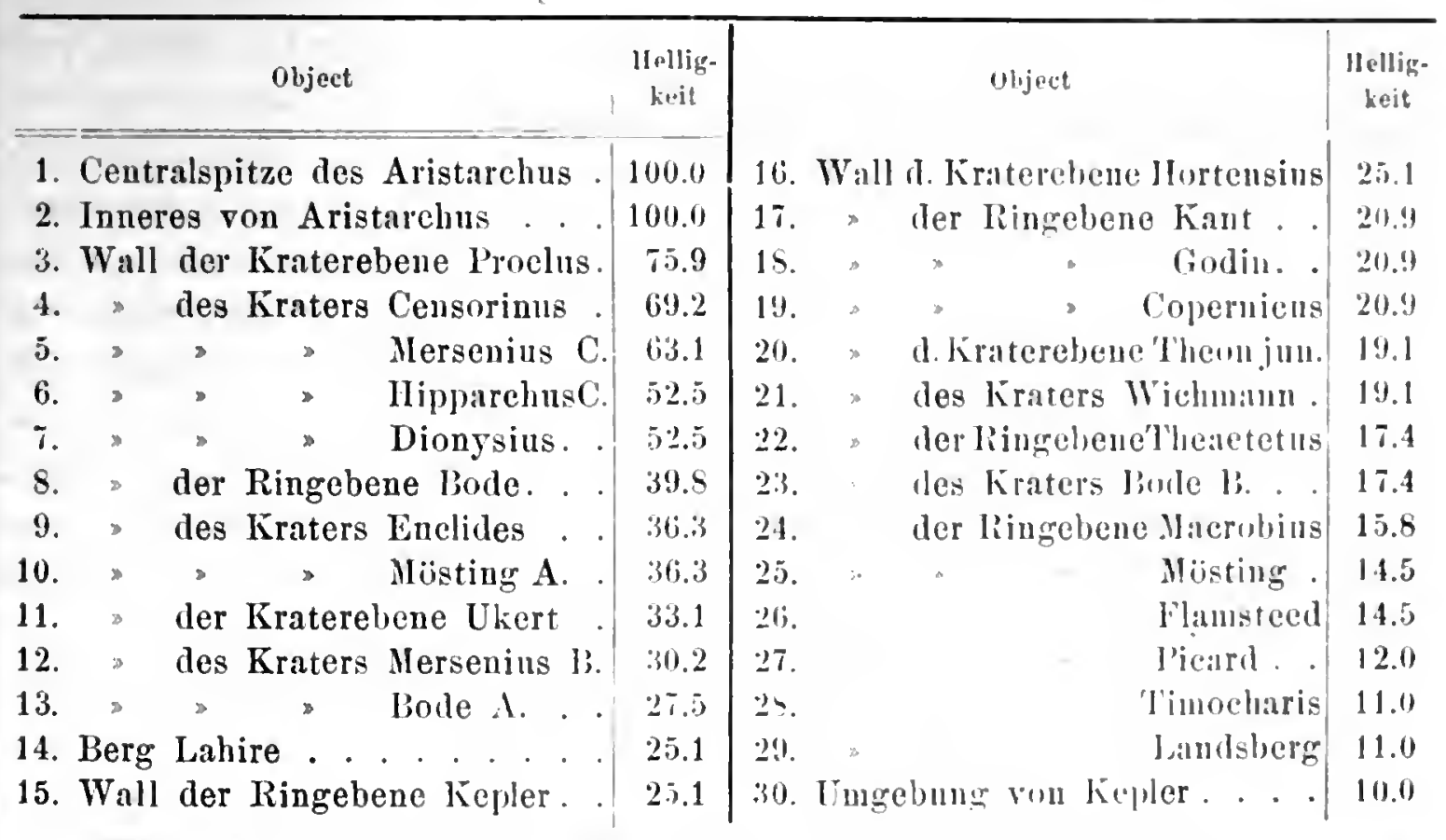

1) Die Pickering'schen Beobachtungeu sind in them selenumraphical Journale für 1882 verüffentlicht, welehes mir leider nicht zugingling gewesen ist. Ich ver. danke die obigen Angaben einer brieflichen llitheilun von Herrn F. C. Pickering. welcher mir eine Copie seines Manuscriptes wititist \%ur Verfibung gestellt hat. 


\begin{tabular}{|c|c|c|c|}
\hline object & $\begin{array}{c}\text { Hellig- } \\
\text { keit }\end{array}$ & Object & $\begin{array}{c}\text { Hellig- } \\
\text { keit }\end{array}$ \\
\hline 31. Wall der Wallebene Langrenus & 8.3 & 46. Inneres von Mercator & 1.9 \\
\hline 32. Inneres von Guericke. .... & 8.3 & 47. Wallebene Endymion & 1.7 \\
\hline 33. Sinus Medii . . . . . . . & 8.3 & 48. Inneres von Pitatus. & 1.6 \\
\hline 34. Umgebung ron Archimedes. & 8.3 & 49. $\gg$, Hippalus . & 1.6 \\
\hline $35 . \quad, \quad$ Aristillus . . & 7.6 & $50 . \gg$, Taruntius .... & 1.6 \\
\hline 36. Inneres von Ptolemäus . & 6.9 & 51. Flïche der Ringebene Fourier a & 1.1 \\
\hline 37.,$\Rightarrow$ Manilius . . . & 6.3 & 52. Inneres von Flamsteed. . . . & 1.1 \\
\hline 38. Fliiche der Ringebene Hansen. & 5.5 & 53. Inneres d.Wallebene Jul. Caesar & 1.0 \\
\hline 39. Wall der Ringebene Arago . . & 4.8 & 54. Inneres von Vitruvius . . . & 1.0 \\
\hline 40. Flïche von Mersenius. . . & 4.4 & 55. $\gg$ d. Wallebene Grimaldi & 0.9 \\
\hline 41. Wall des Kraters Bessel. & 4.0 & der Ringebene Crüger & 0.9 \\
\hline 42. Inneres von Theophilus. . . & 2.3 & von Zupus . . . . . & 0.8 \\
\hline 43. Innere Flïche von Archimedes & 2.3 & $»$ Le Monnier . . & 0.8 \\
\hline 44. Inneres von Azout . . . . . & 2.1 & $»$ Billy . . . . & 0.8 \\
\hline 45. $\gg \quad \gg$ Marius. . . & 2.1 & $\gg$ Boscovich & 0.6 \\
\hline
\end{tabular}

Aus dieser Zusammenstellung geht hervor, dass die dunkelsten Stellen der nahezu roll boleuchteten Mondscheibe um das 160- bis 170 fache an Intensitiit ron den allerhellsten Punkten iibertroffen werden. Pickering hat scine Jessungen mit den Helligkeitsschätzungen verglichen, die ron zahlreichen Mondbeobachtern nach einer allgemein gebrä̈chlichen Scala ausgefiihrt worden sind. Dicse Scala ist zuerst ron Schröter in seinen "Selenotopographischen Fragmenten in Torschlag gebracht, später ron Beer und Miidler etwas abgeändert worden und dient in dieser letzteren Form jetat allgemein als Richtschnur. Danach sind 10 rerschiedene Helligkeitsgrade festgesetzt, ron denen die Grade 1-3 als dunkelgran bis gran, $4-5$ als lichtgram, $6-7$ als weiss nnd $8-10$ als glänzend weiss zu bezeichnen sind. Die schatten der Mondberge wiirden in dieser Scala den Helligkeitsgrad 1) haben, der Grad I wird den dunkelsten Schatten im Innern ron Ring- nud Villebenen zugesehrieben. $2^{\circ}$ nnd $3^{\circ}$ ist die gewöhuliche Flächenhelligkeit der Neere, die Ränder der meisten Ringwrebirge mnd Wallehenen sind $t^{\circ}$ bis $7^{\circ}$, und die drei höchsten Lichtgrade kommen latuptsidlilich bei Kratern und Ringgebirgen vor. Nach den Lnteruchungen Pickering ist das photometrisch bestimmte Helligkeitsverhältniss. welches je zwri anfeinamder tolgenden Lichtgraden entspricht, als constant zu betrachten und zwal ungetäh gleich 1.7.1, oder mit anderen Worten: zwei anfeinmder folsende Lichtorade unterscheiden sich um $0.2 t$ im lhelliwkeitsluginithmus oder 140.6 in sterngrössenclassen.

Alle Helligkeitswhiatzmngen anf dem Monde und ebenso die photometrischen Vessungen werden durch die Terschiedenheit der Fiirbung der einzelnen Ghjecte, die sehom auf den ersten Blick deutlich erkembar 
ist, ersehwert. Wahrend das Mare serenitatis eine grunliche Farbung besitzt, zeigt das Mare imbrinm einen bräunlicligelben Farbenton, und der Palus somuii hat einen goldbriunliehen, fast robthlichen Schimmer. Filr die Kenntniss der physisehen Besehaffenheit des Moudes ist es zweifellos von der grössten Wichtigkeit, die relative Albedo der eimalnen Objecte fur verschiedene Strahlengattungen, nicht bloss fur das masammengesetzte Licht, zu bestimmen. Es empfichlt sich daher, wenn es auf die genaueste Erforsehung der Helligkeitsvertheilung anf der Mondscheibe ankommt, spectralphotometrisehe Messungen anzustellen. lün dahin gehender Vorschlag ist bereits im Jahre 1577 von Petruscheffsky l) gemacht worlen, und vor langerer Zeit haben Vogel und ich eine Reihe ron Beobachtnngen mit einen Glan-Vogel'sehen Speetralphotometer begonnen, die aber noch nicht zum Abschlusse gebracht ist. Solehe Untersuchungen, bei verschiedenen Beleuchtungen des Mondes ausirefthrt und verglichen mit entsprechenden Messungen an irdischen Substanzen, können an lihesten da\%n dienen, unsere Vorstellungen von der stofflichen Zus:ummensetzung der Mondoberfliche zu erweitern. Auch die mehrfach aufgestellte Vermuthung, dass die Helligkeit einzelner Punkte der Mondseheibe veriuderlich ist, was nur möglieh wïre, wenn dis Reftexionsvermögen der betreflenden Regrionen Schwankungen unterworfen wïre, liisst sich erst anf Grmnd langjähriger photometrisehen Messmugen mit sicherheit entscheiden.

\section{Capitel III.}

\section{Die Planeten und ihre Trabanten.}

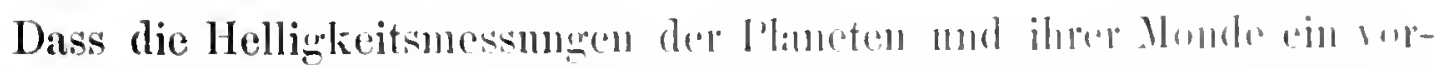

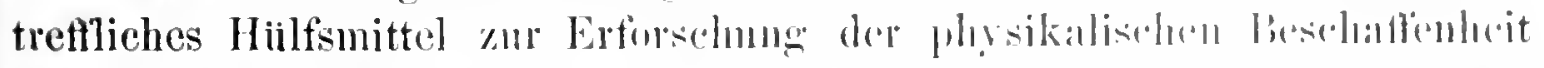
dieser Himmelskörper lieforn, unterliegrt wohl keinem \%weifel; mur muss

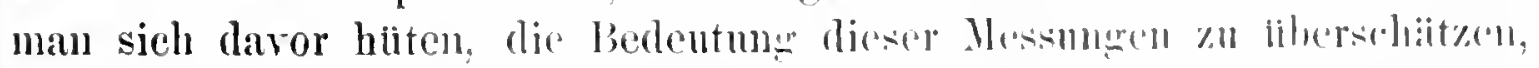

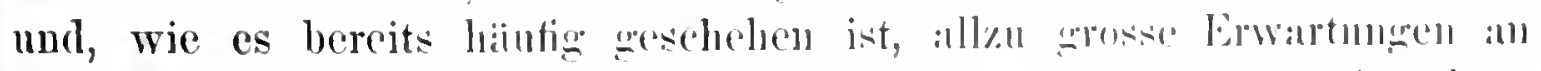

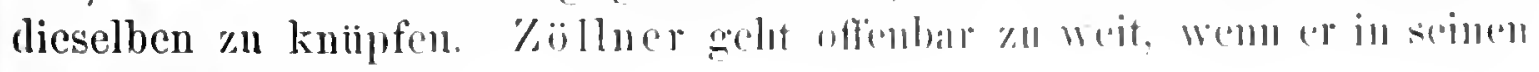

1 Astr. Nachr. IBd. 91. Nr. 2173. 
"Photometrischen Untersuehungen " die Ansieht ausspricht "dass die Anwesenheit einer Atmosphäre oder partiell spiegelnder Substanzen in den Helligkeitsänderungen der Phasen eines Planeten ihren bestimmten und gesetzmässigen Ausdruek finden wird, so dass man aus der besonderen Beschaffenheit dieser Änderungen mit grösserer Sieherheit die physikalisehe Eigenthümlichkeit der Planetenoberfläehen wird ermitteln können, als dies bisher auf dem Wege directer Beobachtung möglieh gewesen ist .

Wie sehwierig es ist, aus den blossen Messungen des Gesammtliehtes eines Planeten auf seine wirkliche Oberflächenbeschaffenheit zu schliessen, lässt sich am Besten beurtheilen, wemn man sich vorstellt, welehen Anblick unsere Erde einem Beobachter anf einem der anderen Planeten darbieten wiirde. Die grossen Wassermengen, welche wie mächtige Spiegel wirken, die Schnee- und Eismassen, welche zum Theil immerwährend, zum Theil nur in bestimmten Zeitepochen grosse Strecken der Erde bedecken, die gewaltigen Flächen bebauten und unbebauten Landes, die hohen Gebirgsziige mit den Schatten, die sie bei verschiedener Beleuchtung werfen, endlich die Atmosphäre mit den beständig wechselnden Wolkengebilden - alle diese Factoren, zu denen noch die Rotation des Erdballes hinzukommt, wiirden sich in den Helligkeitserscheinungen zu einer Gesammotwirkung rereinigen, in welcher sich anf keinen Fall der Einfluss der einzehnen Ursachen erkemen liesse. Etwas auders, aber nicht riel besser verhält es sich mit denjenigen Planeten, welche, abweichend von der Erde, mit einer so dichten Atmosphüre nmgeben sind, dass die Sonmenstrahlen zum grossen Theil ron derselben reflectirt werden und kanm bis zu der eigentlichen festen Oberfläche gelangen; lier ist natiirlich gar keine Anssicht vorhanden, aus den Lichtmessungen etwas Näheres iiber die physikalische Beschatfenheit derselben zu erfahren.

Wenn es geliinge, die Helligkeit eines Planeten an jedem beliebigen Punkte seiner Scheihe mit derselben sicherheit zu bestimmen, wie sein Gesammtlicht, dann wiirle sich vielleicht eher Anfsehluss iber manche der in Betracht kommenden Fragen finden lassen. Solange dies aber nicht gelungen ist, muss man jede optinistische Anffassmo bei Seite lassen und sich damit hernigen. Analogien zwischen den einzelneu Gliedern des Sonnensystems anfunsuchen. Es ist wohl kaum zu bestreiten, dass Himmelskörper, welche nuter denselben BelenchtungsverhäItnissen dieselben Helligkeitserscheinnngen zeigen, hinsichtlich ihrer physischen Beschatfenheit eine gewisse Terwandtschaft miteinander haben miissen. die sich nicht nothwendig Jis in die genaneste stoffliche Übereinstimmung zn erstrecken braucht, die aber doch gerade für diese Körper dhakteristisch ist. Fs liewt anch nahe, festmustellen, nach welchen Gesichtspmeten eine Classiticirung der Plancten zn erfolgen hä̈te. Es wird 
sich dabei lanptsätehlich um den grösseren oder geringeren (irad der Dichtigkeit handeln, welehen die Planetenatmosphinren besitzen. Bei Körpern mit sehr diehter Atmosphäre wird man voransichtlich das hibehste Reflexionsvermögen finden, ansserdem wird man die Helligkeitserscheinungen ihrer Phasen am Leichtesten durch eine rationelle Theorie darstellen können. Bei denjenigen Planeten, welehe, wie unsere Erde, von einer wenig dichten, bestïndigen Verinderungen unterworfenen Atmosphilire ungeben sind, wird die l'hasenlichteurve wahrscheinlich gan\% unregelmăssig verlaufen und der Theorie wenig oder gar nicht zuginglich sein. Diejenigen Himmelskörper endlich, die, wie unser Mond, so gut wie gatr keine Atmosphïre haben, werden an wenigsten Licht reflectiren, mod es scheint bei ihnen die Möglichkeit nicht ausgeschlossen, aus der Vergleichung mit dem Reflexionsrermögen irdiseher Substanzen einen Rllckschluss anf ihre Beschaffenheit zu ziehen, wenigstens in dem Simne, dass hesonders anffallende Erseheinungen, wie sie z. B. eine ganz nit Sehnee und lis bedeckte Oberfliche darbieten whirde, richtig gedeutet werden könnten. Anch wiire es denkbar, dass bei einer anssergewöhnlichen Vertheihugr von hellen und dunklen Partien anf der Ohertliehe eines solchen Planeten seine Rotation durch sorgfiltige photometrische Beobachitungen mit einiger Zuverlïssigkeit bestimmt werden kïmte.

Hiermit ist das Gebiet näher fixirt, imnerhalb dessen in lBe\%ng anf die physische Beschaffenheit der Planeten und 'Trahanten von der Photometrie Erfolge zu hoften sind. Es giebt aber noch einc Inzahl rou

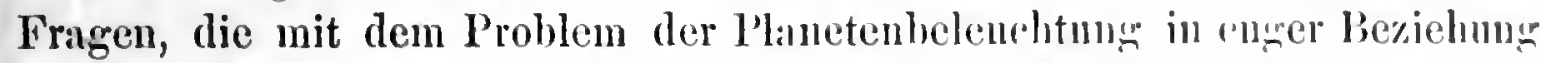
stehen. Dazu gehürt vor Allem die Frage nach der Verimlerlichkeit drer Sonnenstrahlung. Es ist bereits im Friilheren erörtort worden, mit welehen Sehwierigkeiten die Lichtmessungen der Somme verhmolen sind, nurl wie

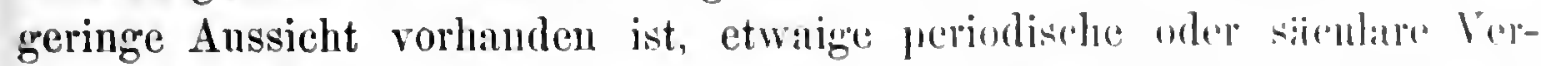

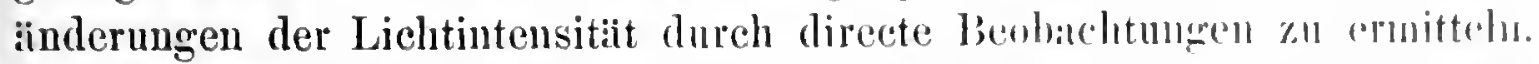

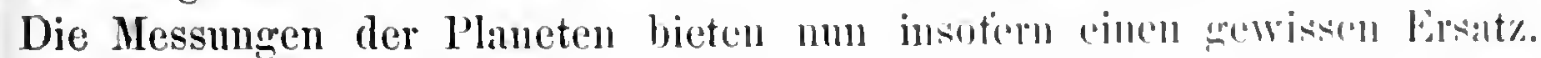

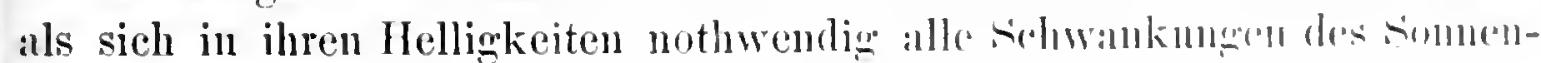
lichtes wiederspiegehn missen, und da die Lutensititshestimnnmenen der

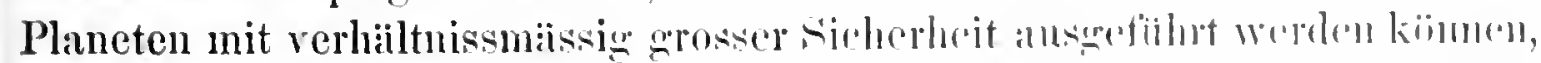

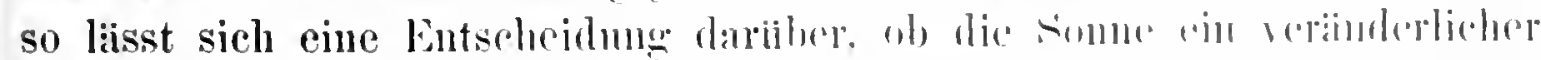

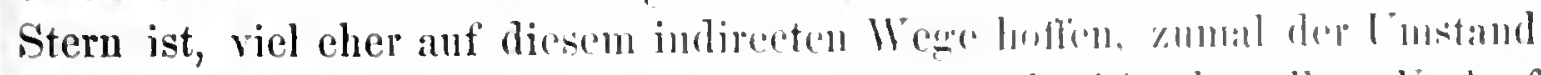

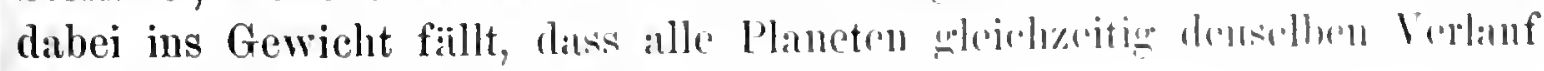
der Erscheinung zeigen minssen.

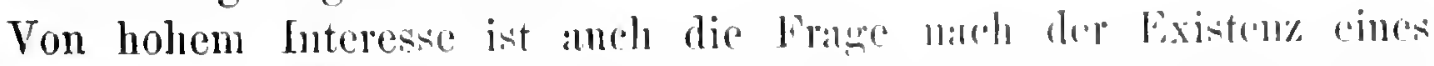

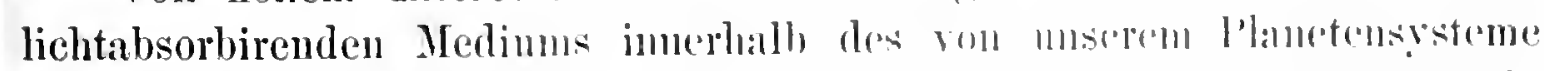
eingenommenen Weltrammes. Dass die Photometrie dere l'laneten cin

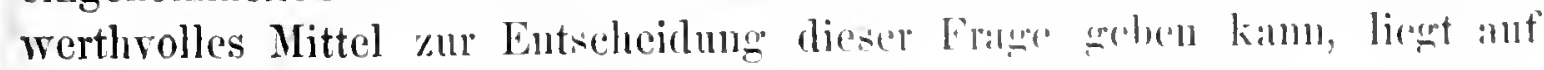


der Hand. Eine einfache Betrachtung auf Grund der im Capitel über die Extinction des Lichtes in der Erdatmosphäre gefundenen Resultate lehrt, dass ein gleichmässig den Planetenraum erfüllendes Medium, wenn seine Dichtigkeit auch 30 Millionen mal geringer wäre als diejenige der untersten atmosphärischen Schichten, doch in den Lichtquantitäten, die ein Planet einmal in seiner grössten Erdnähe, das andere Mal in seiner grössten Erdferne uns zusenden würde, einen Helligkeitsunterschied von 0.2 bis 0.3 Grössenclassen hervorbringen könnte, ein Betrag, der durch sorgfältige photometrisehe Messungen noch mit vollkommener Sicherheit festzustellen ist.

Es verdient hier endlich noch anf die hohe Bedeutung hingewiesen zu werden, welche die Lichtmessung der kleinen Planeten und der Satelliten fuir die Bestimmung der Dimensionen dieser Himmelskörper hat. Gegenwärtig bietet noch bei der iiberwiegenden Mehrzahl derselben die Photometrie das einzige Mittel, Werthe für ilıre Durchmesser zu erhalten. Wenn diese Angaben auch verhältnissmässig unsicher sind, weil sie auf uncontrolirbaren Ammahmen über die Reflexionsfähigkeit der betreffenden Himmelskörper beruhen, so werden sie doch voraussichtlich nicht allzu weit von der Wahrheit entfernt sein und uns eine ausreichende Vorstellung von den Grössenverhältnissen im Somnensysteme geben.

Dals grosse Verdienst, die ersten werthvollen Messungen über die Helligkeiten der Planeten ausgefiihrt zu haben, gebiihrt Seidel und Züllner, und obgleich ihre Resultate in mancher Beziehung der Verbesserung bediirfen, so bilden sie doch einen unschätzbaren Ausgangspunkt fiir alle Untersuchnngen anf diesem Gebiete. Leider hat ihr Beispiel nur wenig Nachahmer gefunden, und erst in nenerer Zeit ist durch die Satellitenbeobachtungen Pickerings und durch die langjährigen Planetenmessungeu in Potsdam ein ausreichendes Naterial geliefert worden, um anf dem ron seidel und Zijllner gesehaffenen Fundamente weiter bauen zu könnelı.

\section{Mercul.}

Die Helligkeitsheobachtungen des Mereur sind wegen der grossen Somnentihe dieses I'aneten mit ansserordentlichen Schwierigkeiten verbunden. In mittleren Breiten wird or nur zu gewissen Zeiten und bei besonders guten Luftrerhialtnissen fuir das blosse Auge sichtbar, und es scheint durehaus nicht unglanblich, dass es Copernicus trotz vieler Miihe nie gegliickt sein soll, diesen I'laneten zn Gesicht zu bekommen. Riceioli nemt in der Torrede zu seinem "Almagest " den Mereur ein sidus 
dolosun, und v. Zach') erahhlt, dass ihm der Planet, bei grosser Digression von der Sonne, ungeachtet aller angewandten Vorsicht, doch öfter melirere Tage nacheinander unsichtbar geblieben sei. Da Messungen seiner Lichtstiirke an hellen Tage, in mumittelbarer Nithe der Sonne, nieht ansnihrhar sind, und da der Planet in unseren Breiten höchstens $1 \frac{1}{2}$ Stunden vor der Sonne aufgeht oder naeh ilır mitergeht, so beschrïnkt sich die Müglichkeit der Beobaehtungen anf kurze Zeitriume in der Morgen- und Aienddiimmerung, wobei er natillich dem Horizonte so nahe steht, dass seine Siehtharkeit dureh die Extinetion in der Atmosphaire noeh wesentlich heeintriichtigt wird. Nur an Beobachtungsorten, die sieh eines besonders reinen unä dunstfreien Hori\%ontes erfrenen, wird es gelingen, den Planeten wiblend längerer Zeitrimne hintereinander $\%$ beobachten. Es ist daher anch nicht zu verwundern, dass das bisher gesammelte Beobachtungsmaterial sehr spärlich geblieben ist. Im Allgemeinen sind die Epochen der grössten östliehen und westlichen Elongation des Mereur von der Some fllr seine Anffindung am ginstigsten, und unter besonders guten luftrerhältnissen ist es möglieh, den Planeten etwa S-10 Tage zn beiden Seiten von diesen Epochen zu verfolgen. In Jahre 1876 konnte Denningr2) in Bristol den Mereur in der Zeit rom 5. bis 2S. Mai an jedem Abend, wo der Himmel genitgend klar war, mit blossem Auge erkemnen, am letyten Tage allerdings nur mit grosser Miilue; nach seiner Ansicht ist der l'lanet :un Vortheilhaftesten fuir das blosse Auge sichtbar wenige Tagne vor der grössten östlichen oder wenige Tagre nach der griossten westlichen Elongation. Freilich spielt dabei die Entfermung des Nerenr von der Somne cine wichtige Rolle, da es bei der grossen Excentricitait der Mcrourbalnu wesent-

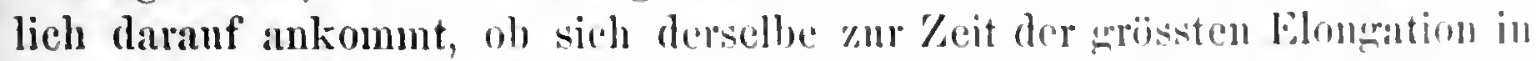
der Niihe seines Perihels oder $A$ phels betindet. In Pontedinn ist es in den Jahren 1878 bis 1859 selten gelmogen, den Planeten ibler cimen laingeren

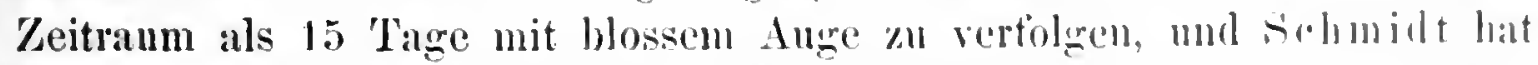

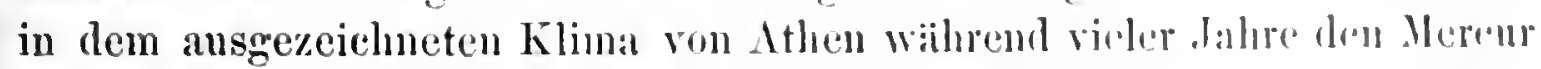

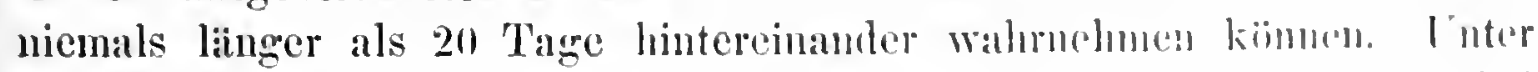

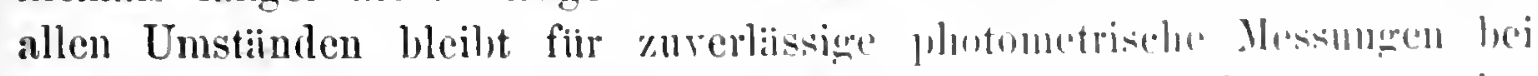

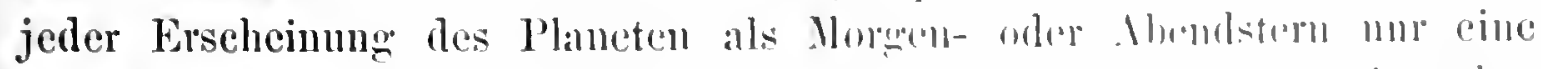

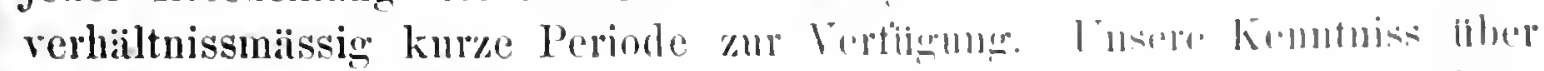

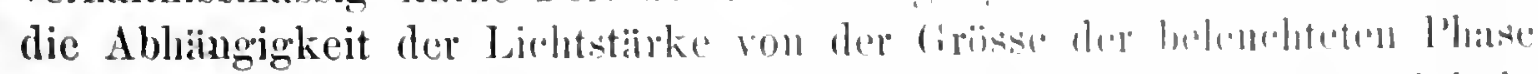

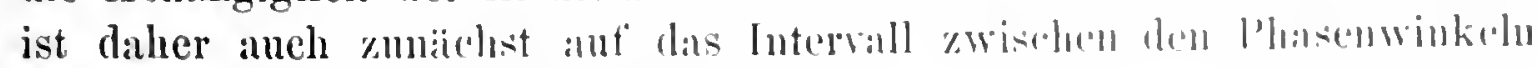
$50^{\circ}$ und $120^{\circ}$ beschrimkt weblieben.

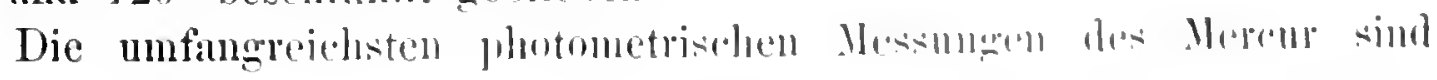

1) Bodes astron. Jahrbuch fiir 1794, 1. 14h.

2) Monthly Notices. Vul. 36, p. 345. 
bisher in Potsdam ') ansgefiilıt worden; ausser diesen sind nur drei vereinzelte Bestimmungen von $\mathrm{Zölln} \mathrm{er}^{2}$ ) und zwei von $\mathrm{Vogel}{ }^{3}$ ) bekannt geworden, welche gut mit den Potsdamer Resultaten iibereinstimmen. Eine grössere, in Stufenschätzungen des Planeten bestehende Beobaehtungsreihe von Schmidt ${ }^{4}$, welche sieh in den hinterlassenen Papieren dieses Astronomen befindet, ist vor einigen Jahren von mir bearbeitet worden und giebt eine erwiinsehte Ergänzung fiir die Potsdamer Liehteurve.

Der Umstand, dass die photometrischen Beobachtungen des Mereur fast ausschliesslich bei sehr grosseu Zenithdistanzen ausgeführt werdeu müssen, beeinträchtigt dieselben in nicht unerheblichem Grade. Die Genatuigkeit der Resultate bleibt daher, namentlich infolge der Sehwankungen, welehe die Absorption der Erdatmosphäre in der Nähe des Horizontes erfahrnngsgemäss erleidet, ein wenig hinter der bei den photometrisehen Beobachtungen der übrigen Planeten erreiehteu Genanigkeit zuriiek.

Die grösste in Potsdam beobachtete Helligkeit des Mereur, von dem Einflusse der Extinction befreit und in Grössenclassen ausgedrüekt, ist - 1.2, dagegen die kleinste 1.1. Da in dem ersten Falle der Planet seinem Perihel selır nahe, in dem zweiten nieht weit ron seinem Aphel entfernt war, nud da es nur ganz ansnahmsweise möglich sein wird, ilun noch in grösserer Nïhe der oberen oder unteren Conjunetion zu messen, so kamn man die angefiihrten Helligkeitswerthe ungeführ als die änssersten Grenzen bezeichnen, innerhalb deren die beobachtete Lichtstïrke sehwanken kann. Nercur erreicht also im Maximum etwa die Helligkeit des Sirins und sinkt im Minimum bis zur Helligkeit des Aldebaran hinab; er wiirde demnach, wenn er hoch am Himmel beobachtet werden könute, eine glainzende Erscheinmog darbieten.

Wegen der starken Excentricitit der Meremrbahn kaun, wie schon oben erwälnt, die Helligkeit bei derselben Elongation sehr verschieden sein, und zwalr zeight die Rechnung, dass der Unterschied bis zu einer vollen Grössenclasse, also bis zum 2.5 fachen, anwachsen kann. Darans erklïrt sich an Besten dic oft bemerkte Thatsache, dass der Planet in mamchen Erscheiunngen leichter anfzutinden ist als in anderen. Im Allgemeinen lehren die photometrischen Nessungen, dass die Lichtstärke des Mreren am thenthimnel wïhrend seiner ganzen Sichtbarkeitsdaner beständig abninmmt, dagegen am Norenhimmel bestïndig anwächst, dass er also als Abendstern am hellsten ist, wenn er nus zun ersten Male sichtbar wird, mud als Morenstern, wemn wir ihn zum letzten Male

1) Publ. des Astroiliys. Ohs. zu l'otsdam. Ld. S, p. 305.

2) Poggend. Annalen. Jubelband. p. ti2.t.

3/ Bothkamper Benbachtmeneu. Thett II. p. 133.

4 Publ. des Astomliys. (1)s. zu lotsdam. Bd. 8, p. 372. 
erblicken; nur der Umstaud, dass er bei diesen Stellungen schon жu sehr im Bereiche der Sonnenstrahlen ist, verhindert die augenfillige Constatirung dieser Thatsache. Von einem grossten Glanze des Mereur in dem Sinne, wie wir es bei der Venus sehen werden, dass nămlich seine Helligkeit wïhrend ein und derselben Erscheinung erst anwilehst und daun wieder abninmt oder umgekehrt, kaun demnach keine Rede sein.

Zur Ableitung der Curve, welehe die Abhängigkeit der Lichtstïrke des Mereur allein von der Grösse der belenehteten Phase darstellt, mllssen die beobachteten Helligkeitswerthe zuvor von dem Einflusse der verschiedenen Distanzen befreit werden. Man reducirt sie gewöhnlich auf die mittlere Entfermung 0.35710 des Planeten von der Somne und anf seine mittlere Entfernung 1 von der Erde. Die simmtlichen in dieser Weise bearbeiteten Potsdamer Messungen haben sich innerhalb des Phasenintervalles von $\alpha=50^{\circ}$ bis $\alpha=120^{\circ}$ durch die Formel darstellen lassen:

$$
h=-0.901+0.02838\left(\alpha-50^{\circ}\right)+0.0001023\left(\alpha-50^{\circ}\right)^{z},
$$

worin $h$ die jedesmalige mittlere Lichtstiirke beim Phasenwinkel a, in Grössenclassen ausgedriekt, bedeutet, und -0.901 die mittlere Grösse beim Phasenwinkel $50^{\circ}$ bezeichnet.

Fast ebenso gut entsprieht den Beobachtungen anch eine gerade Linie, welche gegeben ist dureh die Gleichung:

$$
h=-1.041+0.03679\left(c e-50^{\circ}\right) .
$$

Die Helligkeitsschaitzungen von Sehmidt, welche mahezu dasselhe Phasenintervall wie die Potsdamer Messungen umfassen, an Genauigkeit allerdings wesentlich hinter jenen zuriickstehen, sind ebenfalls durch eine gerade Linie darstellbar, deren Gleiehung $h=-0.969+10.035 .45\left(6-510^{\prime \prime}\right)$ hinreichend mit der obigen Formel (II) iblereinstimmt.

Sowohl ans den Athener als ans den Potsdamer Werthen geht hervor, dass zwischen den Morgen- und Abendbeobachtungen keine systematischen Untersehiede vorhanden sind, und ferner erwieht sich, dass in dem frim\%en Zeitranme ron 1861 bis 1S98, den diese Beobachtmugen umfassen, die mittlere Helligkeit des Mereur keine nachweisbaren sohwankungen queeigt hat. Für die Helligkeit bei voller Belenchtm" $\left.u=\|^{\prime \prime}\right)$ wiirde sich, wenn der Formel (I) noch ausserhatl, des Intervalles rou ${ }^{2}=51^{\circ}$ bis $x=120^{\circ}$ Guiltigkeit zukime, der Werth - 2.06 areben. Ganzallgenein ässt sich für einen beliebigen Zeitpunkt, fïr welchen die butfermungen

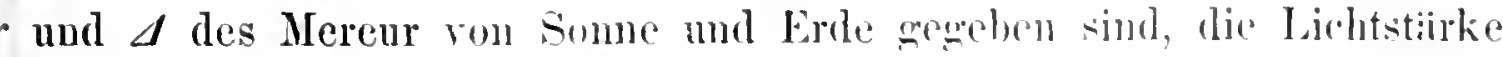
lesselben in Grössenclassen ans der Formel volansheredhen:

$i=\frac{1}{0 . t} \log \frac{r^{2} \Delta^{2}}{(0.38710)^{2}}-0.901+0.028 .3 \mathrm{~S}(u-5)^{\prime \prime}+0.100111023\left(\varepsilon-50^{\circ}\right)^{2}$. 
Von Interesse ist die Vergleichung der aus der. empirisehen Liehtcurve (I) herrorgehenden Helligkeiten des Mercur mit den Werthen, die sieh aus den versehiedenen Beleuehtungstheorien ergeben, sowie ferner mit den entsprechenden Helligkeiten-des Mondes. Eine Übersicht giebt die folgende kleine Tabelle, in weleher von $10 \mathrm{zu} 10 \mathrm{Grad}$ Phasenwinkel zwischen $\alpha=50^{\circ}$ und $\alpha=120^{\circ}$ dic betreffenden Lichtstärken (auf mittlere Entfernungen reducirt) zusammengestellt sind, wobei Alles in Grössenclassen ausgedruickt ist, und die Helligkeit bei $a=50^{\circ}$ ïberall dem aus Formel (I) hervorgehenden Werthe -0.90 gleichgesetzt ist. Für den Mònd sind die Bond'schen Zahlen (Seite 342) zu. Grunde gelegt.

\begin{tabular}{r|r|r|r|r|r}
\hline & $\begin{array}{c}\text { Beob. } \\
\text { Helligk. des } \\
\text { Mercur }\end{array}$ & \multicolumn{2}{|c|}{ Helligkeit nach der Theorie von } & \multicolumn{1}{c}{$\begin{array}{c}\text { Beob. } \\
\text { Helligk. des } \\
\text { Mondes }\end{array}$} \\
\hline $50^{\circ}$ & -0.90 & -0.90 & -0.90 & -0.90 & -0.90 \\
60 & -0.61 & -0.73 & -0.76 & -0.80 & -0.63 \\
70 & -0.29 & -0.54 & -0.60 & -0.68 & -0.32 \\
80 & 0.04 & -0.30 & -0.42 & -0.53 & 0.03 \\
90 & 0.40 & -0.03 & -0.22 & -0.36 & 0.43 \\
100 & 0.77 & 0.29 & 0.01 & -0.15 & 0.57 \\
110 & 1.17 & 0.68 & 0.29 & 0.10 & 1.36 \\
120 & 1.59 & 1.13 & 0.61 & 0.40 & 1.90
\end{tabular}

Von den Theorien stellt keine die Beobaehtungen geniigend dar; am Näichsten der empirisehen Curve kommt noeh die Lambert'sehe Formel, obgleich anch bei dieser die Abweiehungen bis zn einer halben Grössenclasse gehın. Dagegen zeigt sich eine bemerkenswerthe Übereinstimmung zwischen den Lichtemren des Mereur und des Mondes. Sehon Zöllner hatte anf rrund seiner vereinzelten Beobachtungen den Satz ausgesproehen, diss der Mereur ein Körper ist, dessen Oberflïehenbeschaffenheit mit derjenigen des Inondes selur nahe iibereinstimmt, der also aneh, wie der Nomd, wahrscheinlich keine wirkliche Atmosphäre besitzt«. Da der rou Zobllner versuchte Beweis bei dem unzureiehenden Naterial nur indireet und wenig iberzengend sein konnte, so hat man diesem Satze niemals besondere Bedeutung beigemessen; erst dureh die umfangreiehen neneren Nessmgen ist er iiber den Werth ciner blossen Hypothese hinansgeriekt worden. In der That wird man es fiir in lohen Grade wahrseheinlieh halten diuffen, dass zwei Hinmelskörper, welche in Bezug anf die Zuriiekwerfung des sumenliehtes ein on iihnliches Verhalten zeigen, anch hinsichtlich ihrer Oherflïchenbeschaftenheit nicht wesentlieh voneinander rerschieden sein kimnen. 
Dass der Mereur, ebenso wie der Mond, ein Kïrper ist, der von dem auffallenden Sonuenlichte nur einen zicmlich geringen Betrag zurthekstrahlt, geht ans seiner kleinen Albedo hervor. Wirl die inittlere Helligkeit der Sonne in Grössenelassen nach Z,811ner (siehe Seite 317) gleich - 26.60 gesetzt, ferner fur die Lichtstiirke des Mereur bei mittlerer Eutferumng und voller Belenchtung nach der Formel (I) der Werth - 2.06 augenommen, so ergeben sich ans den Gleichungen (14) (Seite 65) die folgenden Albedowerthe des Mereur:

$$
\begin{aligned}
& A_{1}=0.140 \quad \text { (Lambert'sche Definition), } \\
& A_{3}=0.187 \quad \text { (Seeliger'sehe Definition). }
\end{aligned}
$$

So unsicher diese Zahlen anch sind, so beweisen sie doch, dass die mittlere Reflexionsfähigkeit des Mereur jedenfalls nur gering sein kamn; sie entsprieht etwa der Albedo von Thonmergel. In Vergleich zu allen anderen Planeten erseheint Mereur als ein relativ dunkler Körper, und man wird daraus schliessen durfen, dass das Sonnenlicht in der Hanptsache von den festen Theilen des Planeten zurldekgeworfen wirl, und dass die ihn umgebende Atmosphïre nur sehr dumn sein kamn.

Diese Schllisse werden zum Theil auch durch die topographischen Beobaehtungen des Mercur bestitigt. Die von verschiedenen Beohachtern, unter Anderen von Vogel') gemachte Wahruehmung, dass die Grösse der gemessenen Phase meistens kleiner als die herechnete ist, liisst sich bei einer mit Erhebungen bedeckten und ron einer sehr dimnen Atmosphiare umgebenen Oberfliche unschwer durch Schattenwurf erklitren. Anch die neueren Untersuchungen von Sehiaparelli2) und Anderen, durch welche das Vorhandensein von bestimmten Gebilden anf der Mereurscheibe nathgewiesen ist, deuten anf eine bis zum gewissen Grade durchsichtigne Itmosplüre hin. Freilich folgt gleielzeitig ans der unhestimmten Begrenzm!r der Fleeke und ans ihrer verinderlichen Intensitit, diss in der ungebenden Hiille zeitweilig Condensationen stattfinden missen, die fiir ms einen :ihnlichen Anblick hervorbringen, wie ihn etwil die Ertatmosphibre fiir einen auf dem Mereur befindliehen beobachter hedingen wiirde.

\section{Venus.}

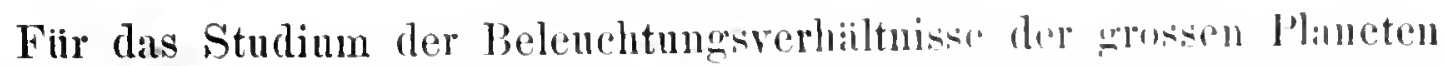
ist keiner besser geeignet als die Venus, weil dieselhe in dem grriissten Theile ihrer Bahn pluotometrisch beobachtet werden kilnm. Freilich wind

1) Bothkamper Beobachtungen. Heft II. 1) 127 und 13.4.

2) Astr. Nachr. Bd. 123, Nr. 2944. 
die Messungen, insbesondere in der Nähe der Conjunctionen, durch den tiefen Stand des Planeten in der Morgen- oder Abenddämmerung etwas erschwert, und dazu kommt, dass der ỉberaus grosse Glanz des Gestirnes, welcher meist die Benutzung von Blendgläsern erforderlich macht, die Gənanigkeit der Bestimınungen ein wenig beeinträchtigt.

Zusammenhängende Beobachtungsreihen sind zuerst von Seidel in den Jahren 1852 bis 1857 angestellt worden; später haben Bond, Zöllner, Plummer und Pickering den Helligkeitserscheinungen des Planeten Aufmerksamkeit gewidmet; die umfassendsten Beobachtungen sind aber in den Jahren 1877 bis 1890 in Potsdam ausgefübrt worden, und durch diese ist die Lichtcurve der Venus für das Phasenintervall von $\alpha=22.5$ bis $a=157^{\circ} .5$ mit relativ grosser Genauigkeit festgelegt. Über diese Grenzen hinaus ist bisher nur eine einzige Helligkeitsbestimmung der Venus bekannt geworden, und zwar von Bremiker bei Gelegenheit der totalen Sonnenfinsterniss am 18. Juli 1860, wo die Venus einen Phasenwinkel von 172.2 besass; doch bedarf diese Bestimmung, welche nur in einer fliichtigen Schätzung bestand, sehr der Bestätigung und verdient nicht die Bedentung, die ihr mehrfach zugeschrieben worden ist.

Einen Überblick über die Helligkeitserscheinungen der Venus während der Dauer ihrer Sichtbarkeit giebt die folgende Tabelle, welche aus den Potsdamer photometrischen Messungen abgeleitet ist. Argmment derselben

\begin{tabular}{|c|c|c|c|c|}
\hline $\begin{array}{c}\text { Inzahl } \\
\text { ror oder } \\
\text { obereu } \\
\text { Conjunction }\end{array}$ & $\begin{array}{l}\text { der Tage } \\
\text { nach der } \\
\mid \begin{array}{c}\text { unteren } \\
\text { Conjunction }\end{array}\end{array}$ & $\begin{array}{c}\text { Elongations- } \\
\text { winkel }\end{array}$ & $\begin{array}{l}\text { Phasen- } \\
\text { winkel }\end{array}$ & $\begin{array}{c}\text { Beobachtete } \\
\text { Helligkeit }\end{array}$ \\
\hline 60 & 232 & 15.4 & $21: 6$ & -3.25 \\
\hline 50 & 212 & 20.4 & $2 S .9$ & -3.29 \\
\hline 100 & 192 & 25.3 & 36.3 & -3.34 \\
\hline 120 & 172 & 30.1 & 43.9 & -3.40 \\
\hline 140 & 152 & 34.6 & 51.7 & -3.48 \\
\hline 160 & 132 & 35.7 & 59.9 & -3.57 \\
\hline 180 & 112 & 42.3 & 68.6 & $-3.6 i$ \\
\hline 200 & 92 & 45.1 & 78.2 & -3.50 \\
\hline 210 & $S 2$ & 45.9 & $83 . \tilde{3}$ & -3.57 \\
\hline 220 & 72 & 46.3 & 89.3 & -3.95 \\
\hline 230 & 62 & 46.0 & 95.5 & -4.04 \\
\hline 240 & 52 & 44.5 & 103.2 & -4.14 \\
\hline 250 & 42 & 42.1 & 112.0 & -4.26 \\
\hline 2604 & 32 & 37.4 & 122.9 & -4.28 \\
\hline 270 & 22 & $29 . i$ & 136.7 & -4.11 \\
\hline 250 & 12 & 18.2 & 154.4 & -3.75 \\
\hline
\end{tabular}


ist die Anzahl der Tage vor oder nach der oberen und unteren Conjunction; daneben ist der Elongationswinkel des Planeten ron der Somne, der Phasenwinkel und die rom Einflusse der Extinetion befreite Lichtstlirke (in Grüssenelassen) angegelıen. '/u bemerken ist, dass bei Anfatellung der Tafel die Balunen ron Venus und Erde als kreisförnig vorausgesetzt sind. Da die Excentrieititen in beiden Fillen unbedentend sind, 80 weiehen die thatsichliehen Verhiiltnisse nicht erhehlich ron dem mittleren Verlaufe ab.

Aus dieser Tabelle und der Figur 73 geht hervor, dass imnerhalh des betrachteten Zeitraumes von 220 Tagen die Ilelligkeit der Venus nur

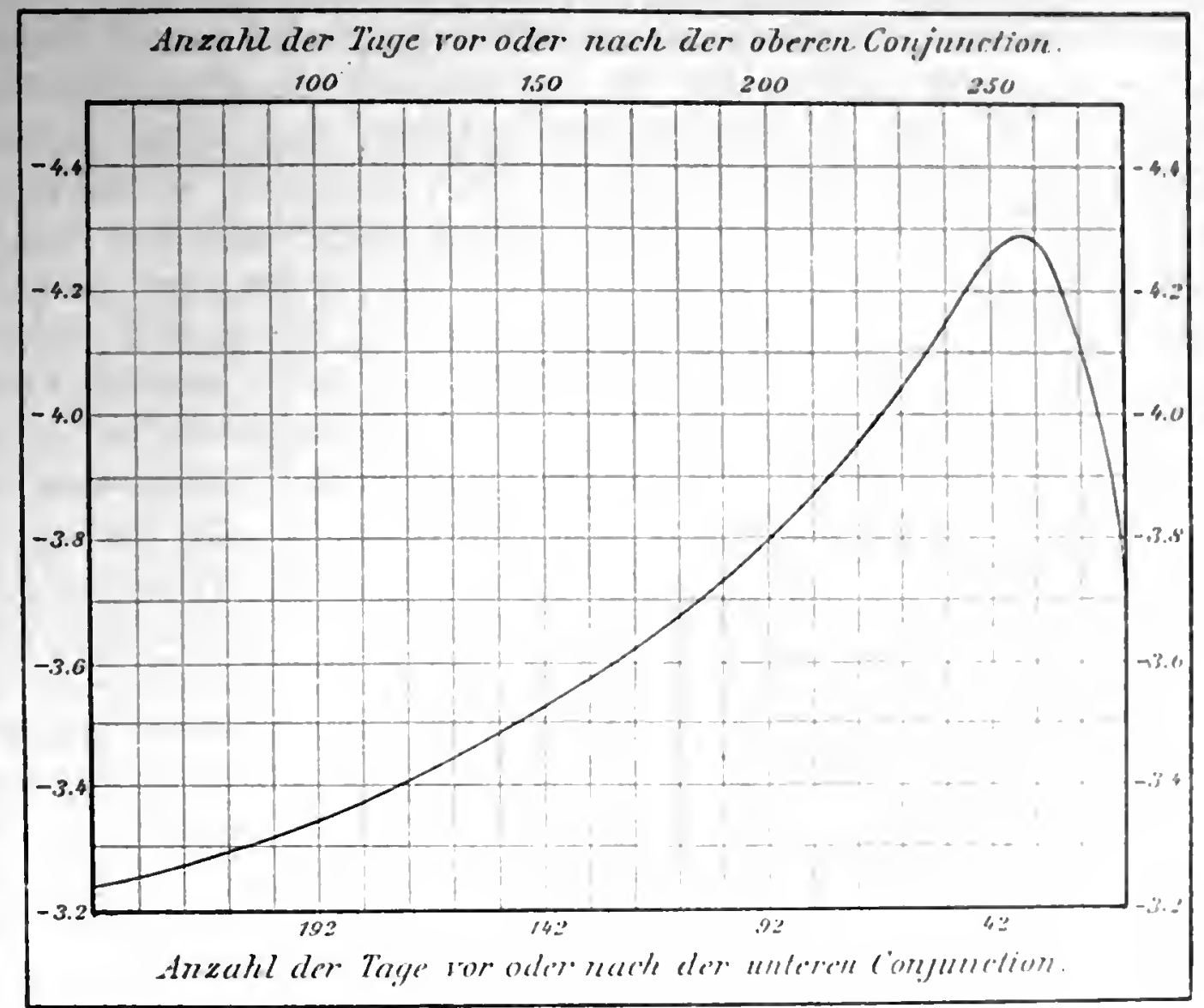

Fig. 73.

um den verhältnissmässig kleinen Betrag ron etwats mohr als einer Grössenclasse schwankt. Die Ïnderumgen, welche die Lichtstïrke infolge der wechselnden Entfermungen des Planeten von der Erik cröthrt, werden also zum grössten Theile durch die l'hasenwirkmunen wirder ansgeglichen.

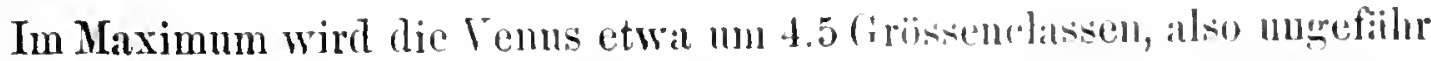
60 mal heller als Aretur. In der Nille der oberen Conjunction scheint die Helligkeit nahezn constant zu bleiben; erst in gribserer Entfermmg ron 
derselben beginnt sie allmählich ziemlich gleichmässig anzuwachsen bis etwa 35 oder 36 Tage vor der unteren Conjunction, von welehem Zeitpunkte an sie dann sehr sehnell abnimmt. Dieser letztere Theil der Lichteurve ist noeh etwas unsicher bestimmt, weil die Beobachtungen in unmittelbarer Nähe der unteren Conjunetion ziemlieh spärlich und verhältnissmässig am ungenauesten sind. Nach dem blossen Augensehein werden die Lichtschwankungen der Venus gewöhnlieh viel grösser geschätzt, als ans der obigen Tabelle hervorgeht; es ruihrt dies wohl daher, dass die Beobaelitungen, insbesondere am Anfang und am Ende der Siehtbarkeitsdauer, hïufig bei schr tiefem Stande des Planeten angestellt werden miissen, und der starke Einfluss der Extinetion dabei nieht genügend berücksiehtigt wird.

Es ist bekannt, dass die Venus am hellen Tage, selbst um die Mittagszeit, für das blosse Auge sichtbar ist. In friiheren Zeiten hat man diese Erscheinung fuir eine anssergewöhnliche gehalten, und es ist ans den rorigen Jahrhunderten eine Reihe von Fällen y) iiberliefert, wo die Sichtbarkeit der Venus am Tage grosses Anfsehen erregt hat. Man braueht deswegen keineswegs anzunehmen, dass zu diesen Zeiten eine besondere Liehtentwieklung auf dem Planeten stattgefunden habe; vielmehr erklärt sieh das grosse Aufsehen in den meisten Fällen dadureh, dass die Venus fuir einen Cometen gehalten und ron dem abergläubischen Volke als die Vorbedeutung drohenden Ungliickes angesehen wurde. Bei Anwendung der nöthigen Vorsichtsmassregeln, insbesondere wenn man das direete Sonnenlicht abhält, bereitet es keine Sehwierigkeiten, die Venus wïhrend ihrer ganzen Siehtbarkeitsdaner zn jeder Tageszeit mit blossem Ange anfzufinden. natiirlich nur bei besonders guinstigen Luftrerhältnissen. Im Jahre 1590 sind ron Cameron in Tarmonth (Nenschottland) und ron Bruguiere in Marseille umfassende Beobaehtungen iiber diesen Gegenstand angestellt worden $\%$. Ersterer hat die Tenus $26 \frac{1}{2}$ Tage nach der oberen Conjunction bei einer Elongation ron $6 \frac{1}{2}^{\circ}$ zum ersten Male mit blossem Ange sehen kümnen, Letzterer hat sie noeh bis ti Tage vor der unteren Conjunction rerfolgt: danach ist also der Planet 259 Tage lang: mit blossen Ange sichthar gewesen. Diese Zeitdaner wiirde sich sogar now un 14 Tage vergrössern, wenn man der Angabe Baldwins Glauben schenken will. wekher in Denver Colorado in Sommer 1880 die Venus 12 Tage nach der oberen Conjunction um Mittag ohne Fermrohr gesehen zu lahen behauptet.

1 Eine Zusammenstelhng solcher Fälle findet sich in der Abhandlung: Wurm. Über den grössten Glanz der Tents. sammt Tafeln für diese periodische Erscheinung. Allg. geograph. Ephemeriden. herausg. von v. Zach. Bd. ‥ p. 30.5.

- Tature. Tol. 45 p. 623. Obserfatury. Tol. 3, p. 573. 
Was die Ablängigkeit der Lichtstärke der Venus lediglich von der Grösse der erlenchiteten Plase betrifit, so hat sich aus den Potsdamer Vessungen, nachden dieselhen anf die mittlere Entfernung 0,72333 des Planeten von der Sonne und auf die Entfernung 1 von der Erile reducirt waren, ergeben, dass die Helligkeit (in Grössenclassen) dureh die eınpirische Formel dargestellt werden kann:

$$
h=-4.707+0.01322 a+0.0000004247 a^{3} \text {. }
$$

Dass die nach dieser Formel berechneten Werthe mit keiner der bekannten Belenchtungstheorien vollständig harmoniren, \%eigt die folgende kleine Tabelle, in weleher durchweg die Lichtstiirke fur $a=20^{\circ}$ dem aus der Formel hervorgehenden Werthe -4.44 Grössenclassen gleich gesetzt worden ist.

\begin{tabular}{|c|c|c|c|c|}
\hline $\begin{array}{l}\text { Phaseu- } \\
\text { Wiukel }\end{array}$ & $\begin{array}{l}\text { Empiriselie } \\
\text { Lichtcurve }\end{array}$ & $\begin{array}{c}\text { Lambert'sches } \\
\text { Gesetz }\end{array}$ & $\begin{array}{l}\text { Loivinel- } \\
\text { Secliger schers } \\
\text { Geset. }\end{array}$ & $\begin{array}{l}\text { Eulerselien } \\
\text { Gesclz }\end{array}$ \\
\hline $20^{\circ}$ & -4.14 & -1.14 & -4.44 & -1.41 \\
\hline 30 & -4.30 & -1.37 & -1.36 & -1.39 \\
\hline 40 & -4.15 & -4.26 & -4.26 & -4.33 \\
\hline 50 & -3.49 & -4.13 & -1.1 .1 & -4.26 \\
\hline 60 & -3.92 & -3.96 & -1.00 & -4.11 \\
\hline 70 & -3.63 & -3.77 & -3.5 .1 & -1.11 .4 \\
\hline So & -3.43 & -3.53 & -3.60 & — \\
\hline 90 & -3.21 & $-3.21 i$ & -3.46 & -3.72 \\
\hline 100 & -2.96 & -2.94 & -3.23 & -3.31 \\
\hline 110 & -2.69 & -2.55 & -2.95 & -3.26 \\
\hline 120 & -2.39 & -2.10 & -2.813 & - 2.9ti \\
\hline 130 & -2.06 & -1.51 & -2.25 & -2.60 \\
\hline 140 & -1.69 & -0.5 .4 & -1.75 & -2.11 \\
\hline 150 & -1.29 & 0.07 & -1.16 & $-1 . .8$ \\
\hline 160 & -0.85 & 1.37 & -11.25 & -11.187 \\
\hline
\end{tabular}

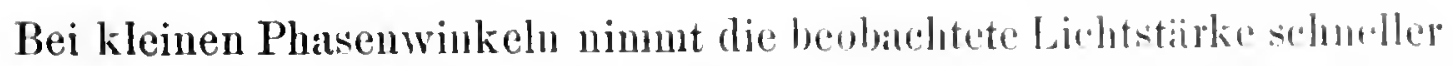
ab, als es die Theorien verlangen, wihlend in der Xïhe der unteren Com-

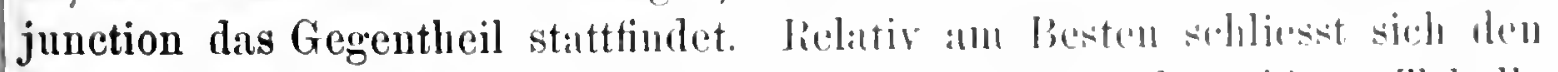

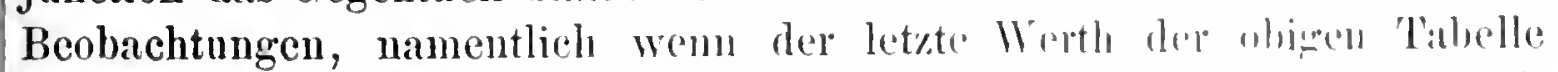

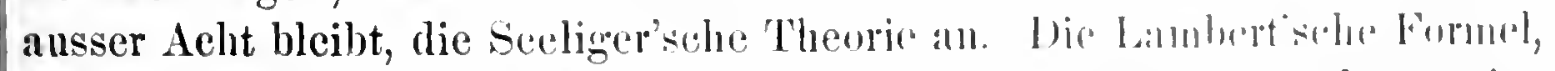

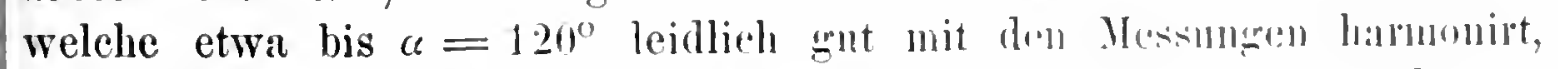
zeigt dariber hinaus so starke Abweirhungen, liss sio rntsohieden zu

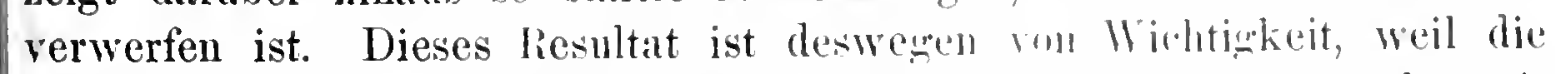
photometrischen Vemusheobachtumgen seidels mul Zibllners, die nit einer einzigen Ausnahme bei Phasenwinkehn miter 120" angestellt wurken. 
stets als Beweis dafür angeführt worden sind, dass das Lambert'sche Emanationsgesetz auch auf die Phasen einer Planetenkugel anwendbar sei.

Die für die Venus ans den photometrisehen Messungen ermittelte Phasencurve weieht gänzlieh von derjenigen des Mondes und des Mercur ab. Während sich die Liehtstärke des Mercur von $\alpha=50^{\circ}$ bis $\alpha=120^{\circ} \mathrm{um}$ 2.5 Grössenclassen indert, findet bei der Venus iunerhalb des gleiehen Intervalles nur eine Änderung ron 1.6 Grössenelassen statt. Dieses gänzlieh verschiedene Verhalten der beiden Planeten dentet auf bemerkenswerthe Unterschiede in der physischen Beschaffenheit ihrer Oberflïehen oder ihrer atmosphärisehen Umhüllungen hin. Dass die Venus eine ausserordentlieh diehte Atmosphäre besitzen muss, geht einerseits daraus hervor, dass es bisher noeh nieht gelungen ist, deutliche Gebilde von längerer Daner auf der Seheibe zn erkennen, andererseits ans der starken Refraction, welehe sich ans der Verlängerung der Hörnerspitzen ergiebt. Naeh den Untersuchungen ron Neisou'), weleher die Beobachtungen von Midler und Lyman zu Grunde gelegt hat, beträgt die Horizontalrefraction auf der Venus ungefiihr 5 $54^{\prime} .7$; darans wiirde folgen, dass die Diehtigkeit der Atmosphäre an der Oberfläehe des Planeten fast doppelt so gross ist, wie die der Erdatmosphäre. Wahrseheinlieh ist sie noeh beträichtlich grö̈sser anzunehmen, wie man anch aus der anffallenden $A b-$ nahme des Liehtes nach der Beleuchtungsgrenze hin schliessen könute, und es ist sehr wohl denkbar, dass der grösste Theil des Sonneulichtes unmittelbar von den diehteu Wolkengebilden der Venusatmosphäre refleetirt wird und gar nicht zn der eigentliehen Oberfliche des Planeten gelangt. Im Einklange damit steht das aussergewöhnlich grosse Reflexionsvermögen der Venus, welches kaum durch die Zurïckstrahlung von einer festen, etwa nuserer Erde ähnlichen Oberfläche zu erklären wäre. Wird die Helligkeit der Tenus bei mittlereu Entfernungen ron Sonne und Erde und bei voller Beleurlitung naeh der obigen empirisehen Formel zn - 4.707 angenommen, so ergeben sich ans den Gileichnngen (14) (Seite 65) die folgenden Werthe fiur die Albedo der Tenus:

$$
\begin{aligned}
& A_{1}=0.758 \quad \text { (Lambert'sehe Definition), } \\
& A_{\beth}=1.010 \quad \text { (Seeliger'sche Definition). }
\end{aligned}
$$

Der letzte Werth wiirde mit der Annahme, dass das ron dem Planeten zu nus gelangende Licht nur diffus reflectirtes Somnenlicht ist, gänzlich unverembar sein; es ist aber nicht zu vergessen, dass die abgeleiteten Zahlenwerthe wegen der grossen L'nsicherheit, die dem zu Grunde gelegten Werthe der sonnenhelligkeit anhaftet, keineswegs als sehr zurer- 
lässig anzusehen siud. Jedenfalls ist so viel klar, dass die Albedo der Venus sehr gross sein muss. Dieser Umstand hat mehrfach z.l der Vermuthung Veranlassumg gegeben, dass die Venusoberfliche spiegelnde Bigenschaften besitzt. Besonders lebhaft ist fur diese \%nerst von Brett anfgestellte Behauptung (hristie') eingetreten, welcher in den Jahren 1876 und 1578 mit einem Polarisationsocular Beobachtungen ansgefuhrt hut, nach denen sich eine bestimmte Stelle des erleuchteten Theiles der Scheibe etwa 7 inal heller ergab, als die Randpartien. Wurde die Helligkeit der Venusscheibe allmälilich abgeschwiicht, so blieb bei den verschiedenste॥ Beleuchtungsverhailtnissen des Planeten \%ulet»t immer ein undentlich begrenzter Lichtfleck mit einem kleinen intensiven Punkte in der Mitte Ilbrig, und zwar an einer Stelle, wo nach der Vorausberedunugr bei einer vollstïndig spiegelnden Kugeloberfliiche ein Reflexbild der Sonne entstehen musste. Das verschwommene Aussehen des Lichtfleckes erklarte Christie dureh die Zerstrenung' der Sonnenstrahlen in der Venusatmosphïre. Von versehiedenen Seiten, nnter Anderen ron Noble, Neison und /enger, ist gegen die Christie'schen Erklärungsversuehe Einspluch erhoben worden, und die ganze Erscheinung bedarf der Bestïtigung dureh weiter ansgedehnte Untersuchungen.

Ein anderes, ebenfills noch nicht vollstindig anfigekliirtes Phïnomen, welehes schon im vergangenen Jahrhundert die Aufmerksankeit der Astronomen auf sich lenkte, ist das sogenamute aschfarbene Jicht der Venus, welches besonders zu der Zeit, wo der erlenchtete Theil als schmale Sichel erscheint, ähnlich wie beim Monde sichtbar ist. Die erste grenaue Beschreibung dieser Erseheinmog rilirt von den Berliner Astronomen ('h. Kirch her, weleher in den Jahren 1721 und 1726 dentlich die dunkl. Seite der Venus erkamnte. Nach ihm wurde dassello l'hïmmen von Derham, Harding, Schröter, Groithnisen wahrentommen, in nenerem Zeit dann unter Anderen ron Engelmann, Noble, Browning, Safarik, Winneeke und Webb bestitigt. Alle Beobalchter stimmen in der Besehreibung ihrer Wahrnehmungen so nahe iiherein, dass an der lieilitiit

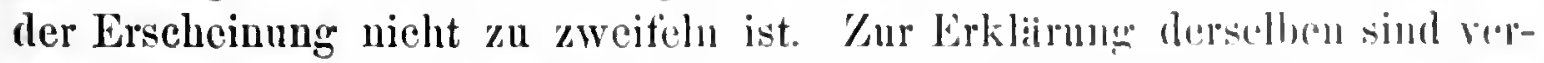
schiedene Hypothesen anfgestellt worden. Einign haben sio tiir wine hlosso Contrastwirkung gehalten. Harding, später Hershel mul Ollwers

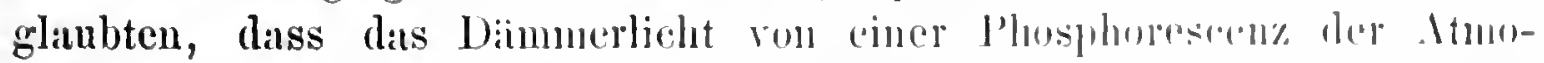
sphäre oder des festen Kemes des Planeten herrilhe. nud Harling fimd

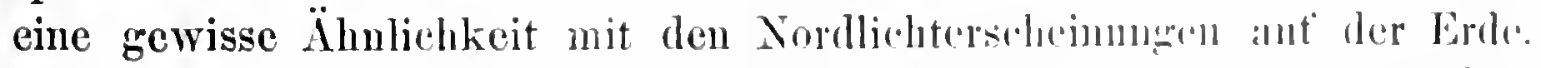
Diese letatere Analogie ist anch von anderen Beobisehtern behauptet worlen.

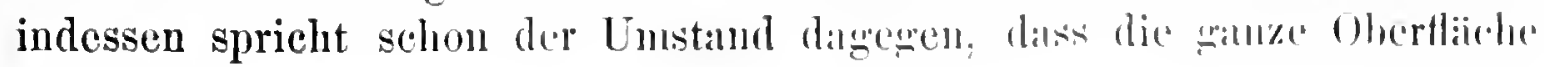

1) Monthly Notices. Yol. 37, p. 90 und Vul. 35, 11. 104. 
des Planeten der Sehauplatz solcher Lichtentwicklungen sein müsste, und dass ferner dieselben bisher nur in der Nähe der unteren Conjunetion bemerkt worden sind.

Die ebenfalls hier und da vertretene Ansicht, dass das Dämmerlicht der Venus von einem Monde derselben hervorgebracht sein könnte, wird dadurch so gut wie ganz ansgeschiossen, dass es bis heute trotz vieler Bemülıungen nieht gelungen ist, einen Venussatelliten aufzufinden. Aus photometrischen Versuchen, welche Pickering ${ }^{1}$ ) mit künstlichen Venusbegleitern angestellt hat, geht hervor, dass ein Venusmond, selbst wenn er nicht grösser als die Marstrabanten wäre, schwerlich der Aufmerksamkeit der Astronomen hätte entgehen können; es liegt aber auf der Hand, dass ein so kleiner Körper nicht im Stande sein wïrde, die dunkle Seite der Venus mit dem beobachteten Dämmerlichte zu erlenchten.

Am meisten Verbreitung hat die Ansicht gefunden, dass das secundäre Licht der Venus, :̈hnlich dem aschfarbenen Mondlichte, ron dem von der Erde zuriickgeworfencn Sonnenlichte herriihrt. Aber anch diese Hypothese ist nicht gauz eimwurfsfei, weil die uach den bekannten Belenchtungstheorien berechnete Helligkeit durchaus nicht geniigend sein wiirde, um die beobachtete Erscheinung rollstïndig zn erklären.

Die beobachtete Phasenlichtcurve der Venus zeigt, wie bereits erwähnt, ein Maximum der Helligkeit zwischen der muteren Conjunction und der grössten (östlichen oder westliehen) Elongation des Plameten. Die Voransberechnung des Zeitpunktes dieses grössten Glanzes ist stets ein beliebtes Problem gewesen, und die Litteratur iiber diesen Gegenstand ist sehr unfangreich. Da man bis in die neneste Zeit ans Mangel an ansreichendem Beobachtungsmaterial lediglich anf theoretische Betrachtungen angewiesen war, so mussten die Resultate je nach den Annahmen ïber die Phasenbelenchtumgsgesetze rerschieden sein; es ist daher anch nieht zu verwundern, dass dic Augaben füir die Epochen des grössten Glanzes in den rerschiedenen astronomischen Ephemeriden häutig um mehrere Tage roneinander differiren. Zum ersten Male ist das Problem bereits im Jahre 1716 ron Haller ${ }^{2}$ ) behandelt worden, welcher zu seiner Untersuchung dureh die damals allgemeines Anfsehen erregende Sichtbarkeit der Yemn am hellen Tage reranlasst wurde. Mit Zugrundelegung des spiiter nach Enler zenamnten Belenchtnngsgesetzes ergab sich die folgende einfache mathematische Lïsmg der Lufgabe. Nimmt man die Lichtstärke der Venus in mittlerer oberer Conjunction, wo die Distanzen SomneVenus und Erde-Venus die Werthe $r_{0}$ und $r_{0}+1$ haben mögen, als

1) Annals of the Astr. Obs. of Harvard College. Vol. 11, part II, p. 294.

2 Phil. Trans. of the R. Soc. of Lomilon. 1716. 1. 466. 
Finheit an, so wird die Lichtstïrke $\boldsymbol{h}$ des Planeten zu irgend einer anderen Zeit, wo die hetreffenden Entfernumgen $r$ und $\Delta$ und der zongehörige Phasenwinkel " heissen mögen, nach der liuler'schen Formel ansgedrnckt durch:

$$
h=\frac{r_{0}^{2}\left(r_{0}+1\right)^{2}}{r^{2} d^{2}} \cos ^{2} \frac{1}{2} \text {. }
$$

Unter der vorlänfigen Anualume, dass die Bahnen der Venus und der Erde kreisfürmig sind, bleiben in dieser Gleichung nur die Grössen " nud $/$ variabel, und die Bedingung des grössten Glanzes reducirt sich daher darauf, dass der Bruch $\frac{\cos \frac{u}{2}}{\Delta}$ ein Maximun wird. Es folgt also die Bedingungsgleiehung:

$$
\frac{d \Delta}{d c}=-\frac{1}{2} \Delta \text { tang } \frac{\alpha}{2} .
$$

Nun gilt in dem Dreieck Sonne-Venus-Vrde die Gleichung:

$$
R^{2}=r^{2}+d^{2}-2 r d \cos \approx,
$$

wenn $R$ die Entfernung Erde-Sonne bedentet. Durch Differentiation der letzten Gleichnng wird:

$$
\frac{d A}{d c}=-\frac{r d \sin \omega}{d-r \cos \alpha} .
$$

Ferner hat man noch, wenn der Elongationswinkel der Venus, d. h. der Winkel an der Erde im Dreieck Somne-Venns lirle, mit e lrezeichnet wird, die Beziehmngen:

$$
\left\{\begin{aligned}
r \sin \omega & =R \sin \rho \\
d-r \cos \alpha & =R \cos e .
\end{aligned}\right.
$$

Dnreh Substitution in (3) wirl also:

$$
d A=-I \text { timge, }
$$

und ans (1) und (5) ergiebt sich dimm die einfarhe Italley sille Formel:

$$
2 \text { tang }=\operatorname{tang}: 4 .
$$

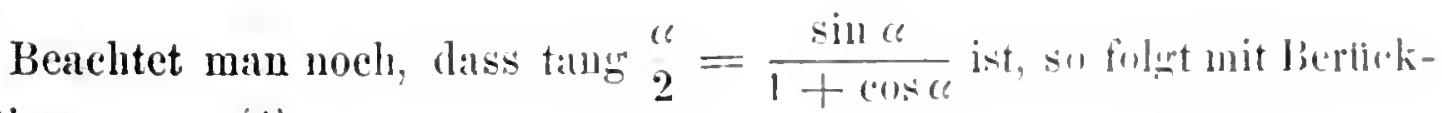
sichtigung von (4):

$$
2 \operatorname{tang} e=\frac{r^{2} \sin e}{1+\sqrt{1-\frac{r^{2}}{l^{2}}}} \cdot
$$


Daraus ergiebt sich leicht:

$$
\cos ^{2} e+\frac{4}{3} \frac{r}{R} \cos e=\frac{4}{3},
$$

und wenu man endlich den Hülfswinkel $x$ einführt mittelst der Substitution:

$$
\operatorname{tang} x=\frac{R}{r} \sqrt{3}
$$

so erhält man zur Bestimmung desjenigen Elongationswinkels, bei welchem der grösste Glanz der Venus eintritt, die einfache Gleichung:

$$
\cos e=\sqrt{\frac{4}{3}} \operatorname{tang} \frac{x}{2} .
$$

Zu ähnlichen Resultaten wie Halley sind auf etwas verschiedenen Wegen später auch Euler ${ }^{1}$, Lalande ${ }^{2}$ ), Boscorich ${ }^{3}$ ) und Delambre ${ }^{4}$ ) gelangt. Im Nautical Almanac werden noch hente die Epochen des grössten Glanzes der Vemus wach den Halley'schen Formeln angegeben.

Aus den Gleichungen (7) und (8) erhält man $e=39^{\circ} 43^{\prime}$, nod der zugehörige Phasenwinkel wird $117^{\circ} 56^{\prime}$. Das grösste Licht tritt danach also ungefähr 36 Tage ror und nach der unteren Conjunction ein.

Wollte man auf die Excentricitäten der Tenus- und Erdbahn Riicksicht nehmen, so wäre eine strenge Lösung der Aufgabe nicht möglich; indessen ist der Fehler, welcher bei der Annahme ron Kreisbahnen begangen wird, nur umbedentend. Nach einer Untersuchung ron Kiess) schwanken die Werthe des Phasenwinkels fuir den grössten Glanz, je nachdem man die kleinsten, mittleren und grössten Entferunngen der beiden Planeten ron der Somne zu Grunde legt und dieselben auf alle möglichen Weisen miteinander combinirt, zwischen den Grenzen $116^{\circ} 46^{\prime}$ und 119 $3^{\prime}$; und dem entspricht in der Zeitangabe des grössten Lichtes ein Spielraum ron ungefïlır 6 Tagen.

Welches Interesse der Frage nach dem grössten Glanze der Venus friiher entgegengebracht worden ist, geht daraus herror, dass sogar ein kleiner Apparat construirt worden ist, an welchem direct der Phasenwinkel, bei welchem die Erscheinung eintritt, abgemessen werden kann. Da die sinnreiche, ron J. A. Herschel b) angegebene Einrichtung wenig

1) Hist. et Mémoires de lacad. R. des sciences et belles lettres de Berlin. 17in, p. 280.

2. Lalande, Astronomie. :3. édition. tome I, p. 475.

3 Boscorich, Opera pertinentia ad opticam et astronomiam. Tomus 4, p. 358.

4. Delambre, Astronomie théorique et pratique. Tome II, p. 513.

5) Hist. et Mémoires de lacad. R. des sciences et belles lettres de Berlin. 1750,7 p. 215.

6) The Qutarterly Journal of pure and applied mathematics. Vol. $t$ (S61), p. 232. 
bekannt sein durfte, so möge eine kurze Besehreibung derselben hier Platz finden; sie beruht auf der unmittelbar aus den obigen Formeln (1) und (3) hervorgehenden Bedingungsgleiehung fur den grössten Glan\%:

(9)

$$
\Delta=2 r+3 r \cos a \text {. }
$$

An einer Schiene $A B$ (Fig 74 ) ist um ein Scharnier bei $V$ eine Stange $V C$ drehbar, an weleher in der Mitte bei $S$ eine \%weite Stauge $S E$ befestigt ist, ebenfalls un ein Seharnier frei beweglich, nud \%war so, dass bei der Drehung des Armes $V C$ das freie Ende $E$ stets liings der-Sehiene $A B$ hingleitet. Die Länge $V C$ $=V B$ entspricht der doppelten Entfernung Sonne - Venus, und die Länge $S E$ der Entfernung Soune - Erde. Die Dimensionen sind daher so gewählt, dass wenn z. B. SE eine Lünge ron $10 \mathrm{~cm}$ hat, die Stange $V C$ 14.4 bis $14.5 \mathrm{~cm}$ lang ist. Die Punkte $B$ und $E$ sind durch eine lose Schumr verbunden, von deren Mitte ein Loth $P$ herabhängt;

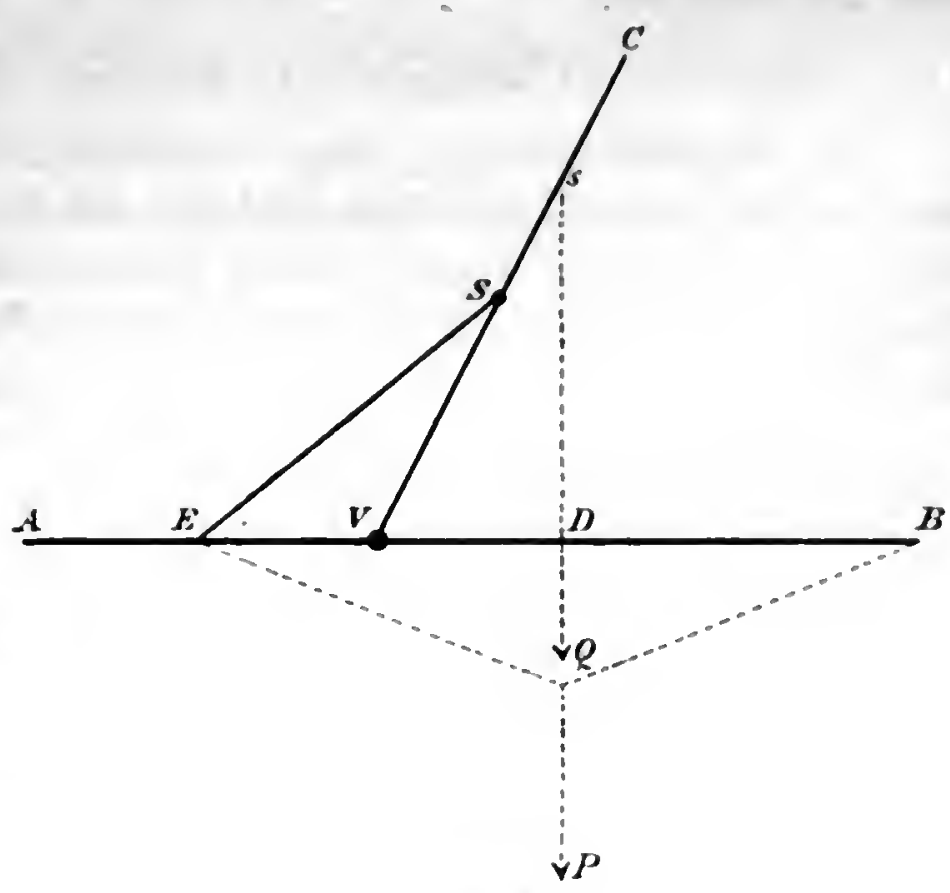

Fig. 74.

ein zweites Loth $Q$ ist im Punkte $s$, der Mitte zwischen $S$ mnd $C$, :m der Stange VC befestigt. Wird num diese Stange so weit fedroht, bis die beiden Lothe in eine Linie fallen, so gielst der Winkel CIF den Phasenwinkel an, bei welehem der grrösste Glan\% der Venus stattfindet. Denn bezcichnet man im Dreicek $S V E$ die Seiten $S V$ und $E V$ rusp. mit $r$ und $\Delta$, den Winkel $S V E$ mit a, so ist:

$$
\Delta+V D=V B-V D=2 \cdot-V D .
$$

Ferner hat man:

$$
V D=V s \cos \omega=-\frac{3}{2} r \cos u,
$$

mithin :

$$
d=2 r+3 r \cos \kappa,
$$

entsprechend der obigen Bedingungsurgleichung (9). Ler Y'erfertiger dieses kleinen Instrumentes hat für den Winkel STE durch Messung den Werth $117^{\circ}$ gefunden, in naher Übereinstimmung mit dem ans der Rechmung hervorgegangenen Werthe. 
Wird statt des Euler'schen Beleuchtungsgesetzes das Lambert'sche zu Grunde gelegt, so ergiebt sich die Lichtstärke der Venus, falls die Helligkeit in mittlerer oberer Conjunction wieder als Einheit gewählt ist, aus der Formel:

$$
h=\frac{r_{0}^{2}\left(r_{0}+1\right)^{2}}{r^{2} \Delta^{2}} \frac{\sin \alpha+(\pi-\alpha) \cdot \cos \alpha}{\pi} .
$$

Die Bedingung für das grösste Licht wird danach (unter Voraussetzung ron Kreisbahnen):

$$
(\pi-\alpha) \sqrt{1-r_{0}^{2} \sin ^{2} \alpha}=2 r_{0}[\sin \alpha+(\pi-\alpha) \cos \alpha] .
$$

Aus dieser Gleichung, welche nur durch successive Näherungsrechnungen lösbar ist, folgt für deu Wiukel $\propto$ der Werth $103^{\circ} 46^{\prime} .5$; die Epochen des grössten Glanzes liegen danach etwa 51 Tage ron der unteren Conjunction entfernt, weichen also um 15 Tage ron den Epochen der Halley'schen Formel ab.

Nach der Lommel-Seeliger'schen Theorie endlich, welche sich den Helligkeitsbeobachtungen verhältnissmässig am Besten anschliesst, ergiebt sich die Lichtstärke der Venus aus der Gleichung:

$$
h=\frac{r_{0}^{\varphi}\left(r_{0}+1\right)^{2}}{r^{2} J^{2}}\left[1-\sin \frac{a}{2} \operatorname{tang} \frac{a}{2} \log \cot \frac{\alpha}{4}\right],
$$

und daraus folgt als Bedingung für den grössten Glanz die Formel:

$\left(2 \operatorname{tang} e+\frac{1}{2} \operatorname{tang} \frac{\alpha}{2}\right)\left(1-\sin \frac{\alpha}{2} \operatorname{tang} \frac{\alpha}{2} \log \cot \frac{\alpha}{4}\right)-\sin \frac{\alpha}{2} \log \cot \frac{\alpha}{4}=0$.

Dieser Gleichung geniigt der Werth $\iota^{\prime}=116^{\circ} 0^{\prime}$; die Epochen der Maximalhelligkeit liegen etwa 38 Tage ror und nach der unteren Conjunction, nähern sich also wieder der Halley'schen Bestimmung.

In Betreff der fraglichen Erscheimung ist iibrigens noch zu bemerken, dass, wie die photometrischen Messungen ergeben haben, der ganze Betrag, um den sich die beobachtete Lichtstiirke der Veuns innerhalb eines Zeitratumes vou to Tagen (gerechnet von dem Zeitpunkte an, wo die Venus noel 60 Tage ron der unteren Conjunction entfernt ist, bis zu dem Momente, wo diese Entfernung mur 20 Tage betriigt) andert, nur etwa 0.25 Grössenclissen ansmacht, ein Werth, der bei einem so schwierig zu beobachtenden Ohjecte, wie die Venus, un durch sehr znverlissige Messungen verbuirgt werden kann. Es folgt hieraus, dass in Wirklichkeit der grösste Glauz der Tenus lieine sehr anffallende Erscheinung ist und durchaus nicht die Bealehtung rerdient, die ilm bisher immer beigelegt wordeu ist.

Lur liestimmung der definitiven Epochen empfiehlt es sich jedenfalls, statt eines der theoretischen Ansdriicke, ron denen keiner die thatsïch- 
lichen Lichterscheinungen der Venus ansreichend darstellt, die empiriselie Formel zu benutzen. Naeh dieser erhält man die Helligkeit der Venus zu irgend einer Zeit, in Grössenelassen ausgedruckt, durch die Gleichung:

$$
h=-4.707+0.01322 a+0.0000004247 \alpha^{3}+\frac{1}{0.4} \log \frac{r^{2} d^{3}}{r_{0}^{2}} .
$$

Unter der Annahme von Kreisbahnen lautet damn die Bedingungsgleichung für den grössten Glan\%:

$$
0=0.01322+0.0000012741 a^{2}-5 \operatorname{Mod} \cdot \frac{\pi}{180} \frac{r \sin a}{\sqrt{1-r^{2} \sin ^{2} a}},
$$

und darans ergiebt sieh $\iota=115^{\circ} 37^{\prime}$.

Innerhalb weleher Grenzen die Ejpochen des grössten Glanzes und die Maximalhelligkeiten selbst sehwanken können, wenn die Exeentricitiiten der Venus- und Erdbahn in Betracht gezogen werden, \%eigt die folgende Zusammenstellung, in weleher die betrefienden Werthe nebst

\begin{tabular}{|c|c|c|c|c|}
\hline $\begin{array}{l}\text { Stellung } \\
\text { der Erde }\end{array}$ & $\begin{array}{l}\text { Stellung } \\
\text { der Venus }\end{array}$ & $\begin{array}{l}\text { Phasen- } \\
\text { wiakel }\end{array}$ & $\left|\begin{array}{c}\text { Anzahl der 'Tage } \\
\text { ror oder nach } \\
\text { der unleren } \\
\text { Conjunction }\end{array}\right|$ & $\begin{array}{l}\text { Gròsste } \\
\text { Ilelligkeit }\end{array}$ \\
\hline 1) Perihel. . & Apliel & $120^{\circ} \mathrm{s}^{\prime}$ & 32.5 & -1.55 \\
\hline 2) Perihel. & Mittl. Entt. & 11943 & 33.4 & -1.411 \\
\hline 3) Perihel. . & Perihel & 11916 & 34.3 & -1.35 \\
\hline 4) Mittl. Entf. & Apliel & $119+$ & $34 . \dot{7}$ & -4.30 \\
\hline 5) Mittl. Entf. & Mittl. Entt. & $1193 i$ & $35.1 \%$ & -4.25 \\
\hline 6) Mittl. Entf. & P'erihel & 11510 & 36.5 & $-1.2 i$ \\
\hline 7) Aphel . . & Apleel & $1175 !$ & 37.0 & -4.19 \\
\hline 8) Aphel & Mittl. Enti. & 11731 & 37.9 & -1.15 \\
\hline (1) Aphel. . & Periliel & 1174 & 35.5 & $-1.11 \mathrm{i}$ \\
\hline
\end{tabular}
den жugehörigen Phasenwinkeln fïr neun verschiedene Fïlle angegeben sind.

Mit Hiilfe dieser Tabelle liasst sich der Zeitpmnkt des grö̈sten lichtes in jedem Falle angenïhert vorherbestimmen. Ist eine wenanerre Ingalbe erwtinscht, so verfiilırt man am Besten so, dass maln natele finmel (I0)

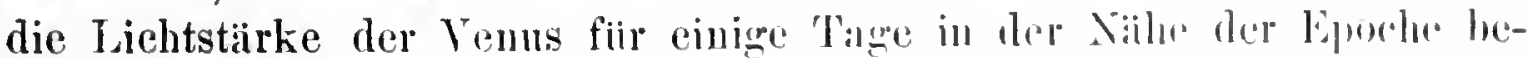

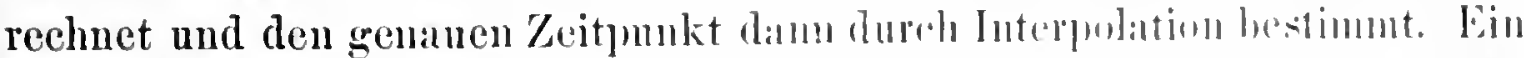

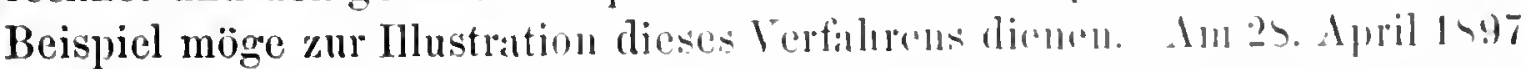
befindet sich Venus in der muteren Conjunction; dey yroste Cilan\% ist

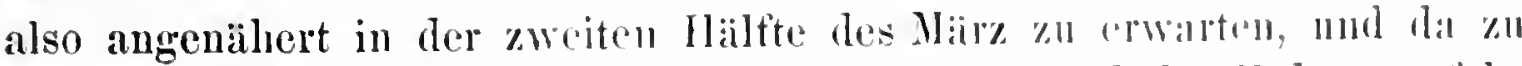

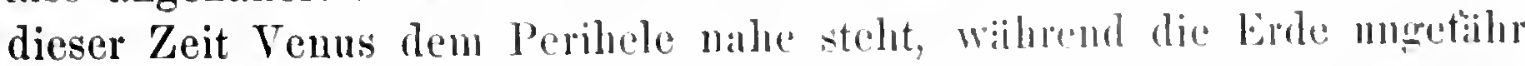
ihre mittlere Entfernumg hesitzt, so faillt uach der uhigen Zusammenstedlumer

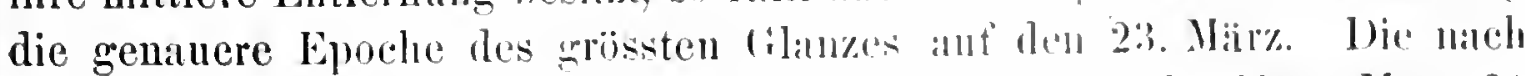

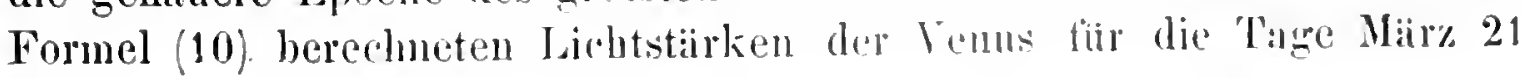


bis März 25 sind nun, mit Zugrundelegung der Werthe $r$ und $\Delta$ aus dem Nautical Almanac:

1897 März 21, $0^{\dot{u}}$ M. Z. Greenwich $h=-4.2840$ Grössenclassen

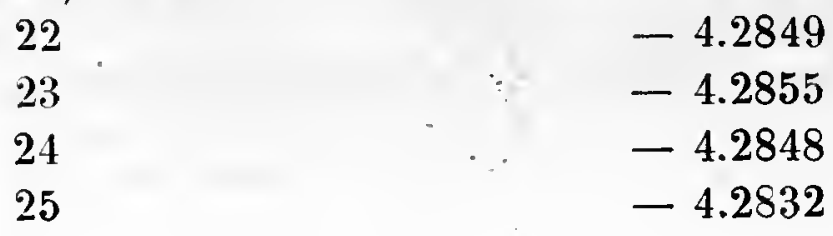

Darans ergiebt sich als Zeitpunkt des grössten Lichtes: $1897 \mathrm{März} \mathrm{22,}$ $21^{\text {h }}$ mittl. Zeit Greenwich.

Im Berliner astronomisehen Jahrbuche für 1897 sind zum ersten Male die Epochen des grössten Glanzes der Venus nach diesem Verfahren berechnet worden, nachdem in den früheren Jahrgängen bis 1867 die Lambert'sche Theorie, und von 1868 bis 1896 eine von Bremiker ${ }^{1}$ ) aufgestellte Formel zu Grunde gelegt worden war.

Nicht ohne Interesse ist noch eine Zusammenstellung der Ergebnisse aller bisher an der Venus angestellten photometrisehen Messungen, nachdem dieselben in einheitlicher Weise auf eine bestimmte Stellung des Planeten redncirt worden sind. Wird dazu die mittlere obere Conjunction gewählt, so ergeben sieh mit Benutzung der aus den Potsdamer Messungen abgeleiteten Lichteurve die folgenden Jahresmittel:

\begin{tabular}{|c|c|c|c|c|c|c|c|}
\hline Jihr & Beobachter & $\begin{array}{c}\text { Anzahl } \\
\text { der } \\
\text { Beob. }\end{array}$ & Helligkeit & Jahr & Beobachter & $\begin{array}{c}\text { Anzahl } \\
\text { der } \\
\text { Beob. }\end{array}$ & Helligkeit \\
\hline 1852 & Seidel & 23 & -3.63 & $1980 / 81$ & Miiller & 38 & -3.48 \\
\hline 1857 & 2 & 10 & -3.49 & 1884 & " & 13 & -3.39 \\
\hline 1865 & Zijllner & 8 & -3.56 & $1885 / 86$ & $\Rightarrow$ & 8 & -3.56 \\
\hline $1877 / 75$ & Miiller & 4 & -3.49 & 1887 & 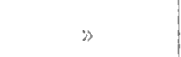 & 18 & -3.61 \\
\hline $187 \mathrm{~s}$ & $\gg$ & 10 & -3.52 & $1888 / 89$ & $\gg$ & 33 & -3.65 \\
\hline $187 !$ & 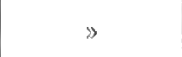 & $2 !$ & -3.50 & 1890 & $»$ & 5 & -3.47 \\
\hline
\end{tabular}

In der Potsdamer Reihe seheint ein Minimum der Helligkeit fiir das Jahlr 1854 mol ein Maximum fiur die erste Hailfte des Jahres 1889 angedentet zu sein; indessen sind die Untersehiede so unbedeutend, dass sie allenfalls noch dureh die Unsieherheit der Messungen erklärt werden k̈̈nnen. Aus der Vergleiehung des Potsdamer Gesammtmittelwerthes - 3.53 mit den Resultaten von Seidel und Zïllner geht mit Sieherheit hervor, dass die mittlere Helligkeit der Venus, also voraussiehtlich auch ihre Albedo, in dem Zeitraume von 1852 bis 1590 keine merklichen Änderungen erfihren hat.

1 Ionatsber. Mer K. Prenss. Akad. der Wiss. Jahrg. 1560, p. 707. 


\section{Mars.}

Während die beiden inneren Planeten in sehr rerschiedeuen Phasen sichtbar sind, ändert sich bein Mars die Grösse des beleuchteten Theiles der Scheibe nur wenig, dn der grösste Phasenwinkel, welcher Iherhaupt vorkommen kann, noch nicht $50^{\circ}$ ubersteigt. Die Helligkeitsiunderungen, welche ron der Phase herruhren, sind infolge dessen bei diesem Plancten viel unbedeutender als bei Mereur und Veuns; dagegen sind die lielitsehwankungen, welche dureh die stark verinderlichen Entfernungen des Mars von der Erde hervorgebracht werden, so erheblich wie bei keinem anderen Planeten. Die grösste Helligkeit tritt zur Zeit der Opposition ein; doch variirt auch diese Lichtstitike wegen der betriichtlichen lixeentrieitit der Marsbahn selır merklieh. In glinstigsten Falle, wemn Mars zur Zeit der Opposition den Perihel nahe, und gleichreitig die Lrde im Aphel ist, erreicht der Planet ungefähr die Grösse - 2.8; er ist dann heller als Jupiter und niehst Veuns das glinzendste Gestirn an Himmel. Befindet er sich dagegen zur Opposition im Aphel, während die lirde zugleich den kleinsten Abstand ron der Somne hat, so wird die Helligkeit nur -1.0 ; er gleicht dam an Glanz etwa dem Sirius. You der Opposition an' bis zu dem Zeitpunkte zwisehen Quadratur und Conjunction, wo der Planet wegen allzu grosser Niilie der Sonme nicht mehr am Morgenoder Abendhimmel beobaehtet werden kann, nimmt seine Helligkeit heständig ab, so dass er zuletzt nur noch a Leonis oder " (ieminorum gleichkommt, also etwa die Grösse 1.6 besitzt.

Die gesammten messbaren Lichtschwankungen des Mars kïnnen narls dem Gesagten den Betrag von heillahe 4.5 Grijssenclisisen arreirlens, d. h. die Maximalhelligkeit iibertrifft nuter Umstinden mehr als tio mal die: kleinste Lichtstäirke. Diese starken IJelligkeitsuntersehiede arsolhwall die Genanigkeit der photometrischen Bestimmungen ein wenim, und dizu kommt noeh, dass die röthliehe Farbe des Planeten stïrourl wirkt umb die Gefahr von Auffassungsdifferenzen bei verschiodenen Berilindutern mit sich fuihrt.

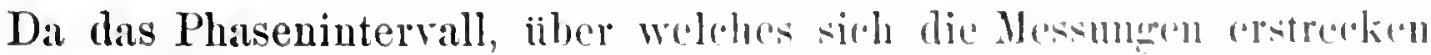

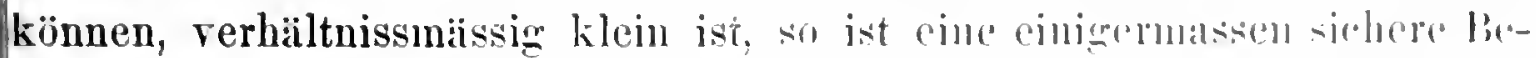

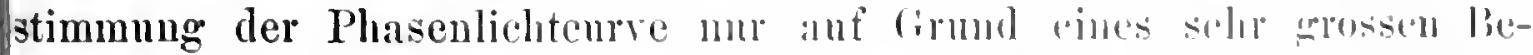
obachtungsmateriales möglicl. Seidel, rom denn ilie erste zus:ammenhaingende Messungsreihe des Mars herrihnt, hat diaher sedu licellt dalran

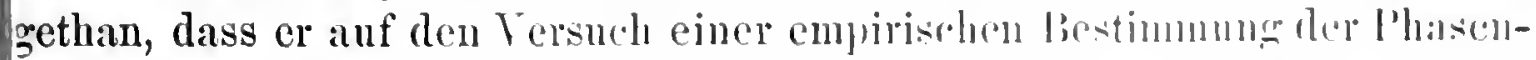
ourve verzichtet und seine wenigen Beohadhtumeren, fon denen nur droi

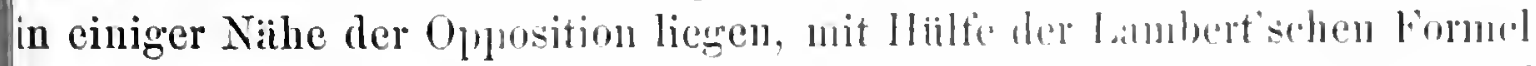
berechnet hat. Weniger vorsichtig ist Zijllun verfihren, weleher anf 
Grund eines noch geringeren Beobachtungsmaterials als das Seidel'sche zu dem Schlusse berechtigt zu sein glaubte, dass die Lambert'sche Theorie auf die Marsphasen keine Anwendung finden könne, dass vielmehr die Lichteurve des Mars eine gewisse Ähnlichkeit mit der des Mondes besitze, in der Nähe der.Opposition sogar noch steiler als diese verlaufe, und dass infolge dessen auf der Marsoberfläche, ähulich wie auf dem Monde, selir starke Erhebungen anzunehmen seien.

Sehr beachtenswerth sind zwei Beobachtungsreihen, welche von Kononowitsch') in Odessa bei den Marsoppositionen 1875 und 1881/82 ausgefuihrt worden sind und welche bisher nur deshalb wenig bekanñt geblieben sind, weil die betreffende Abhandlung in russischer Sprache erschienen ist. Kononowitsch hat ausser seinen eigenen 35 Messungen auch die Seidel'schen und Zöllner'schen Beobachtungen einer Neubearbeitung unterworfen und aus allen 69 Bestimmungen eine empirische Formel abgeleitet, nach welcher die Reductionen anf volle Beleuchtung (in Grössenclassen) dureh den Ausdruek $\frac{1}{0.4} \log \{1-\sqrt{0.00616} \alpha\}$ gegeben werden. Die hierdurch charakterisirte Liehtcurve zeigt zwar auch ein etwas stïrkeres Anwachsen der Helligkeit in der Nähe der Opposition, verläuft aber im Ganzen viel weniger steil als die Zöllner'sehe.

Die umfangreichsten Messungen der Marshelligkeit sind in den Jahreu $1 S 77$ bis 1599 in Potsdam angestellt worden. Eine graphische Darstellung der sämmtlichen 176, auf mittlere Opposition (Entfernung Mars-Sonne $=1.52369$ und Entfermung Erde-Sonne = 1) reducirten Helligkeitswerthe zeigt, dass die Grössenänderungen sehr nahe den entsprechenden Phasenändernngen proportional sind, und dass sich die jedesmalige Lichtstärke des Planeten am Besten aus der einfachen Formel

$$
\lambda_{1}=-1.787+0.01486 a
$$

berechnen lässt, worin - 1.757 die Grösse des Mars in mittlerer Opposition ansdriickt. Bei deu einzelnen Oppositionen seheinen nicht ummerkliche Unterschiede rorhanden zu sein; mamentlich die Beobachtungsreihe ans dem Jahre 1879 dentet im Vergleich zu den anderen anf einen steileren Verlauf der Phasencurve und auf ein etwas stärkeres Anwachsen der Helligkeit bei den kleinen Phaseuwinkehn hin. Ob diese Untersehiede nur ron grösseren zufalligen Messungsfehtern herriihren oder auf wirkliche Inderungen im Reflexionsvermögen des Mars zuriickzuführen siud, laisst sich nicht mit Sicherheit entseheiden. An und fur sich diurfte die letatere Annahme nichts Befremdliches haben; denn die topographisehen

Kononowitsch. Photometrische Untersuchungen der Planeten Mars, Jupiter und saturn. Denkschriften der K. Neuruss. Universitiit. Bd. 37, 1883. 
Beobachtungen des Mars deuten auf erhebliche Ïnderungen der Oteerflachengebilde und wahrseheinlich auch der atmósphărisehen Zustinde hin, und man könute sich woohl vorstellen, dass dadurch zu gewissen 'Zeiten eine besonders intensive Zurllekstrahlumg des Sonnenlichtes beglunstigt wilrde. Schon die Annahme, dass die Rotation des Planeten in den photometrischen Messungen zum Ausdruck kommen könnte, insofern uns bald dunklere, bald hellere Partien der Oberfliche zugekehrt worden, ist nieht ganz von der Hand zu weisen. Sehunidt in Athen glanbte mehr als ein Mal sieher bemerkt zu halıen, dass die Anwesenheit von grossen dunklen Flecken auf der Marsseheihe eine wirkliehe Vermindering des Lieltes herbeifulhte. Freilieh steht diese Walıruehmung bisher nur vereinzelt da, und es ist klar, dass es zahlureicher, besonders zu diesem Zweeke angestellter Messingen bedarf, wenn eine sichere Entscheidnng Utber diese Frage getroffen werden soll.

Zur Vergleiehnng der fuir den Mars gefundenen Phaseneurve mit denjenigen des Mondes und der Venus dient die folgende Tabelle, in weleher von $4 \mathrm{zn} 4 \mathrm{Grad}$ Phasenwinkel die Reduetionen auf volle Belenehtmig angegebon sind. Die letzte Columne enthialt noch die entsprechenden aus der Lambert'schen Theorie herrorgehenden Werthe.

\begin{tabular}{|c|c|c|c|c|c|}
\hline \multirow{3}{*}{$\begin{array}{l}\text { Phasen- } \\
\text { Winkel } \\
\end{array}$} & \multicolumn{5}{|c|}{ Reductionen auf rolle Beleuchtung } \\
\hline & $\mathrm{M}$ & • & Yond & Venus & Lambert'sche" \\
\hline & Kononowitsch & Múller & (a)eh Zölluer) & (nach Molles) & Theorin \\
\hline 0 & 0.00 & 0.00 & 0.00 & 0.100 & 0.110 \\
\hline 4 & 0.12 & 0.06 & 0.07 & 0.06 & 11.00 \\
\hline 8 & 0.24 & 0.12 & 0.14 & 10.11 & 0.01 \\
\hline 12 & 0.34 & $0.1 \mathrm{~s}$ & 0.22 & 0.17 & $0.0 \%$ \\
\hline 16 & 0.42 & 0.24 & 0.30 & 11.22 & 0.0 .1 \\
\hline 20 & 0.48 & 0.30 & 0.39 & 0.27 & 0.01 \\
\hline 24 & 0.54 & 0.36 & 0.48 & 0.33 & $(1.119$ \\
\hline 28 & 0.60 & 0.42 & 0.58 & 0.35 & 0.12 \\
\hline 32 & 0.66 & 0.45 & 0.69 & 0.44 & $0.11 i$ \\
\hline 36 & 0.71 & 0.53 & 0.79 & 0.50 & 11.21 \\
\hline 40 & 0.75 & 0.59 & 0.90 & $0.51 ;$ & 11.21 \\
\hline 44 & 0.50 & 0.65 & $1.02^{\circ}$ & 0.612 & 0.29 \\
\hline 48 & 0.55 & 0.71 & 1.15 & 11.69 & $(1, .35)$ \\
\hline
\end{tabular}

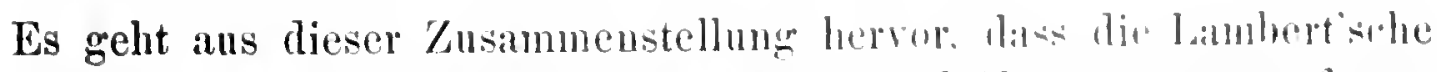
Theorie beim Mars ehenso wie bei Mercur und Venus versut; fermer ergiebt sich, dass die Marscurve imerhall, des hetrachteten l'halsenintervalles weniger steil verlaiuft als die Mondemrer, mul dass liaher dire Zölner'sche Anuahme ciner Verwandtsohate \%wishen dirsen heiden Himmelskörpern zuribkzuweisen ist. Ditgergen tritt zwisthen den l'lat-

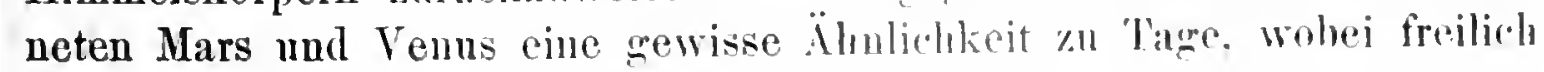


nicht übersehen werden darf, dass die Werthe für die Venus zum Theil nicht direct aus den Beobachtungen hergeleitet, sondern nur durch Extrapolation gewonnen sind.

Ausser den angeführten Untersuchungen über die Marshelligkeit sind noch einige kürzere Messungsreihen von Copeland') und Pickering ${ }^{2}$ ), sowie eine grosse nur auf Schätzungen beruhende Reihe von Schmidt ${ }^{3}$ ) bekannt geworden, die zwar sämmtlich keine weiteren Beiträge zur Ergänzung der Phasencurve liefern, weil sie entweder ein zu kurzes Phasenintervall umfassen oder nicht sicher genug sind, die aber doch für die betreffenden Beobachtungsepochen ganz brauchbare Mittelwerthe der Lichtstärke geben. Erwähnenswerth ist anch noch die älteste bisher bekannte Helligkeitsbestimmung des Mars von Olbers ${ }^{4}$ ), welcher am 23. Februar 1801 den Planeten fast gleich hell mit a Tauri und ein wenig schwächer als a Orionis schätzte. Da die Gestirne nahe dieselbe Farbe besitzen, auch die Zenithdistanzen von Mars und a Tauri zur Zeit der Beobachtung nicht erheblich voneinander verschicden waren, so hat diese Schätzung fast den Werth einer guten Messung.

Die folgende Zusammenstellung giebt einen Überblick über die Resultate aller bisherigen Helligkeitsbeobachtungen des Mars, nachdem dieselben in einheitlicher Weise mit Hiilfe der Potsdamer empirischen Phasenformel und mit Zugrundelegung der in Potsdam bestimmten Helligkeitswerthe der Vergleichsterne auf mittlere Opposition reducirt und für die verschiedenen Beobachtungsepochen zu Mittelwertlien rereinigt worden sind.

\begin{tabular}{|c|c|c|c|}
\hline $\begin{array}{l}\text { Beobacht.- } \\
\text { Epoche }\end{array}$ & Beobackter & $\begin{array}{c}\text { Zahl } \\
\text { der } \\
\text { Beobacht. }\end{array}$ & $\begin{array}{l}\text { Mittl. Oppos.- } \\
\text { Helligkeit }\end{array}$ \\
\hline 1801 & Olbers & 1 & -1.65 \\
\hline $184.5-1558$ & Seidel & 19 & -1.55 \\
\hline $1845-1880$ & Sehmidt & - & -1.65 \\
\hline $1864-1865$ & Züllner & 15 & -1.87 \\
\hline 1875 & Kononowitsch & 20 & -1.72 \\
\hline 1850 & Copeland & $s$ & -1.92 \\
\hline $15>0-1582$ & Pickering & $1 ! 1$ & -1.655 \\
\hline $18 \$ 1-1882$ & Kononowitsch & 15 & -2.03 \\
\hline $157-1890$ & Miiller & 176 & -1.79 \\
\hline
\end{tabular}

Monthly Notices. Vol. 40, p. 390.

2 Ammals of the Astr. Obs. of Harvard College. Vol. 14, part II, p. 410.

3 Astr. Nachr. Bd. 97, Nr. 2310.

1 v. Zach's monatliche Correspondenz. Bd. s, p. 293.

Der von Pickering mitgetheilte Werth ist - 1.29: da aber die Phasencorrectionen ron Pickering nach der Enler'schen Formel angebracht sind, so war eine Ändernng erforderlich, um den Werth auf die Potsdamer Formel zu beziehen. 
Dic hier zu Tage tretenden Unterschiede sind grösser, thls man nach der Gonanigkeit der einzelnen Resultate erwarten sollte. Man braucht deswegen aber noch nicht an wirkliche Helligkeitsinderungen des Mars zu denken, da boi der stark röthlichen Farbe des Planeten Auffassuugaunterschiede von :ihnlichem Betrage durehaus nichts Befremdliches halven wurden, zumal wenn man berlleksichtigt, dass der kleinste Werth von allen, der Seidel'sehe, init Benntzung des Steinheil'sehen Prismenphotometers gefunden ist, wo das Licht anf eine Fliche ausgebreitet wird, und die Beurtheilung der Gleichheit verschiedenfarbiger Eindrllcke besonders schwierig ist.

Die Albedo des Mars ist näclıst der des Mondes und des Mereur die kleinste unter allen Himmelskörpern. Mit Benutzung des Werthes - 1.787 fur die mittlere Oppositionshelligkeit des Mars und des Zöllner'sehen Werthes -26.60 für die mittlere Helligkeit der Some ergeben sich ats den Formeln (14) (Seite 65) die folgenden Albedowerthe:

$$
\begin{aligned}
& A_{1}=0.220 \text { (Lambert'sche Definition) } \\
& A_{q}=0.293 \text { (Seeliger'sche Definition). }
\end{aligned}
$$

Das verhältnissmässig geringe Reflexionsvermögen dirfte wohl hauptsächlich darin begrtindet sein, dass die Sonnenstrahlen die nicht sehr dichte Atmosphäre des Planeten leicht durchdringen und erst ron der festen Oberfläche zurtickgeworfen werden. Filr diese Annilhme spricht auch die röthliehe Farbe des Planeten. Denn da die Marsatmosphäre nach den spectroskopisehen Beobachtumgen wahrscheinlich eine granz :ilunliche Zusammensetzung hat wie die Erdatmosphïre, so wird sie rorminsweise die blanen Strahlen absorbiren, und das reflectirte Sommonlicht, welches die Atmosphärensẹicht hweimal passirt hat, wirl ror\%ussweise gelbe und rothe Strahlen enthalten. Linsere Erde, die nisch allen bisherigen Forschmngen viel $̈$ hhnliehkeit mit dem Mars besitzt, wible. von diesem aus betrachtet, wahrseheinlich eine aihnliche farthe und ein gleiches Reflexionsrermögen zeigen.

\section{Die Marstribanten.}

Die Satelliten des Mars, welche bei der suimstipen Oppusition des Jahres 1877 vou Hall in Washington entdeckt wurlen, gehören kleinsten Körpern unseres Somnensystems. Die licstimmung ihrer Helligkeit ist wegen der Nähe der blendenden Narsscheihe nit grossen Schwierigkeiten verbunden: doch kïnnen braurhbare fiesultate erhalten werden, 
wenn die Trabanten mit ganz nahen Fixsternen vergliehen werden; deren Helligkeiten später, sobald der Planet sich etwas weiter von ihnen fortbewegt hat, mit Sicherheit bestimmt werden können. Hall hat in den ersten Tagen nach der Entdeckung die Helligkeiten der Trabanten etwa 12. bis 13. Grösse 'geschätzt; an einigen Tagen' schien die Lichtstärke des einen Mondes in der Nähe der Elongation sogar noeh ein wenig beträchitlieher zu sein als 12. Grösse. Im Allgemeinen giebt er dem inneren Satelliten Phobos eine etwas grössere Helligkeit als dem äusseren Deimos, und nur ein Mal schätzt 'er beide 'gleich hell. Holden dagegen hat den Intensitätsuntersehied der beiden Trabanten zu fast zwei Grössenelassen taxirt und für die Oppositionshelligkeiten derselben die Werthe 11.5 (Phobos) und 13.5 (Deimos) angegeben. Anch von anderen Beobachtern liegen Helligkeitsschätzungen, namentlich des äusseren Mondes, vor, z. B. rou Watson, Wagner, Trourelot, Erck, welche alle dem Deimos etwa die Grösse 12 bis 13 zusehreiben. Die Erck'schen Schätzungen ${ }^{1}$ ) verdienen deswegen besonders hervorgehoben zu werden, weil es diesem Beobaehter gelungen ist, Deimos noch mit einem Refraetor von $19 \mathrm{~cm}$ Oeffumng zu sehen, und weil er zuerst den Versueh gemaeht hat, aus Vergleiehungen mit den Planeten Mars und Vesta einen angenäherten Werth fuir den Durelmesser des Trabanten abzuleiten; er giebt als wahrseheiulichen Werth dafuir 13.6 engl. Meilen oder 21.9 Kilom. an, ein Betrag, der allerdings beträehtlieh zu grross sein diurfte.

Zurerlissigere Werthe fuir die Dimensionen dieser kleinen Himmelskörper gehen aus den photometrisehen Messungen Pickering ${ }^{2}$ ) herror, welcher bei den Oppositioneu 1877, 1 S79 und IS81, S2 mit Hiilfe eines der rom ilım eonstruirten Photometer die Trabanten mit dem steruartig verkleinerten Bilde des Mars vergliehen hat. Fuir die Helligkeitsdifferenzen zwischen Planet in mittlerer Opposition) und Satellit ergeben sich ans allen Bestimmungen die Werthe 14.47 (Phobos) und $14.5:$ (Deimos), d. h. ler Planet ist 614000 mal resp. 649000 mal heller als die Satelliten; er ibertrifft sie also mugefähr ebenso sehr an Helligkeit, wie die Somne den Volhnond. Wird die Lichtstärke des Mars in mittlerer Opposition zu - 1.79 angenommen, so erhält man für die Oplositionsgrössen der Trabanten die Zahlen 12.65 (Phobos) und 12.74 Leimos.

Ans den gremessenen Helligkeitidifferenzen folgen mu unter der Annahme, dass die Reflexionsfähigkeit der satelliten die gleiche ist wie die

1 Astromonical Register. Tol. 16. p. 201.

2 Annats of the Astr. Obs. of Harvart College. Vol. 11, p. 226 und 311. Ansserden Astr. Nachr. Bd. 102. Nr. 2437. 
des Mars, nach den Formeln (16) (Seite 66) die folgenden Werthe fur die Durchmesser:

$\begin{array}{lccc}\text { Entfernung I } & \begin{array}{c}\text { Vom Mars } \\ \text { aus gesehen }\end{array} & \text { In Kilom. } \\ \text { Phobos } & 0.0119 & 190^{\prime \prime} & 8.6 \\ \text { Deimos } & 0.0116 & 74 & 8.4\end{array}$

Aus den Beobachtungen des Jahres 1879 glaulte Pickering auf Veränderungen in Liehte des uusseren Trabanteu schliesseu \% durfeu, und zwar in dem Sinue, dass derselbe anf der Westseite des Planeten stets lieller erschien als auf der Ostseite. Da ein ähnliches Verhalten bei einem der Saturntrabanten mit. Sicherheit nachgewiesen ist, so ware dieso Beobachtung an und fur sieh durehaus nicht unwahrseheinlich. Aber mit Rlleksicht anf die Geringfugigkeit des wahrgenommenen Helligkeitsuntersehiedes und besonders weil die Beobachtungen der anderen Oppositionen nichts Ḧhnliehẹs zeigen, ist dieses Resultat \%unïchst noch mit Vorsielit aufzunehmen.

\section{Die kleinen Planeten.}

Bei dem ansserordentlich regen Interesse, mit welchen die \%wischen Mars und Jupiter befindliehen Asteroiden stets von den Astronomen beobachtet worden sind, bleibt es auffallend, dass die Helligkeitsrerhältuisse dieser kleinen Himmelskörper bis in die allerneueste Koit so gut wie gä̈nzlich vernachliassigt worden sind. Schon hald nidch lintederkmg der ersten kleinen Planeten an Anfange dieses Jahrhunderts bihon fians und Olbers auf die Wichtigkeit guter Ilelligkeitshestimmungen lor lstoroiden hingewiesen, und spaiter, als bereits eine wrissere lumblhl derselben bekannt war, ist namentlich Argelanderl) sehr cifiring fur ihre photometrisehen Beobachtungen eingetreten. Die folgenden Ausfihlumuren

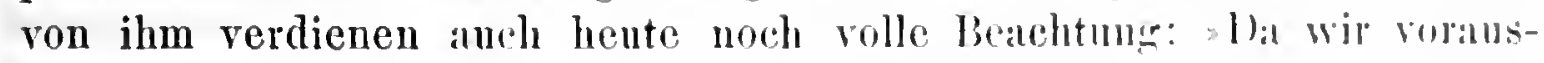
setzen miissen, dass alle die kleinen Plancten wwis.hen Mars mul Jupiter

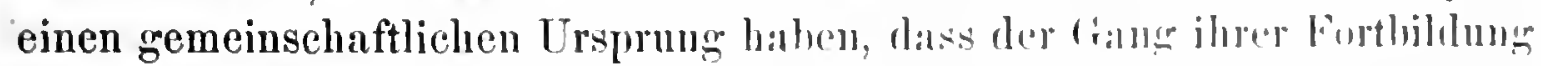

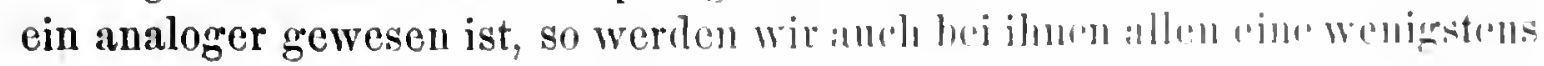

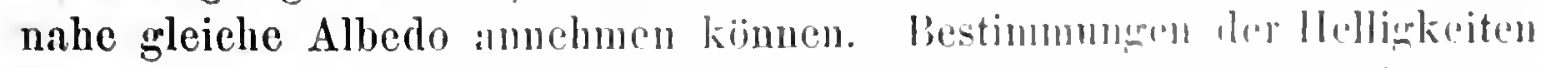
der einzelnen werden uns daher auch ihre relativen firiven mit zilun-

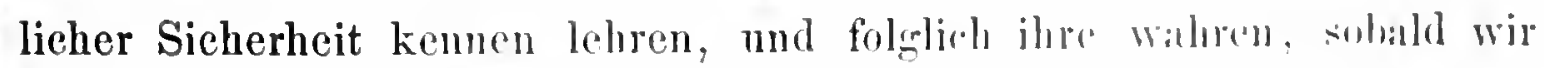
nur von einem von ihnen den scheinbaren IMrdunesser zu messen in Stande sind."

1) Astr. Nachr. Bal. 42, Nr. 996. 
Trotz der warmen Fürsprache von Seiten einer solchen Antorität, und trotzdem später noch mehrfach, besonders lebhaft von Hornstein ${ }^{\mathbf{1}}$, auf die Wichtigkeit des Gegenstandes aufmerksam gemacht worden ist, hat sich niemals ein regeres Interesse für die Helligkeitsverhältnisse der kleinen Planeten kundgegeben. Man hat sich auf gelegentliche Grössenschätzungen derselben beschränkt, und es existiren bis in die Neuzeit nur ganz vereinzelte zusammenhängendere Beobachtungsreihen. Die Werthe für die mittleren Oppositionshelligkeiten, welche im Berliner astronomischen Jahrbuche mitgetheilt werden, sind Mittelwerthe aus den vercinzelten Schätzungen verschiedener Beobachter und können schon wegen der Ungleichartigkeit des Materials anf keine grosse Genanigkeit Anspruch machen.

$\mathrm{Ab}$ und $\mathrm{zu}$ ist die Vermuthung ausgesprochen worden, dass Lichtänderungen bei einzelnen Asteroiden stattgefunden haben, aber die Angaben, welche beispielsweise schon Olbers und Schröter über Helligkeitsschwankungen bei den Planeten Ceres, Pallas, Juno und Vesta, später Ferguson in Betreff der Clio und Goldschmidt in Betreff der Pales gemacht haben, sind so unbestimmt, dass an eine weitere Verwerthumg derselben gar nicht zu denken ist. Nur in einigen grösseren Reihen von Helligkeitsschätzungen, insbesondere ron Tietjen an Melete und Niobe, ron C. F. Peters an Frigga, in nenerer Zeit ron Harrington an Vesta und ron Pickering an Ceres, lassen sich ganz regelmässige Lichtainderungen erkennen, an deren Realität trotz der Unsicherheit der Bestimmungen nicht zu zweifeln ist, die aber keinenfalls, wie z. B. Harrington bei der Vesta annimmt, durch Axendrehung der Planeten zu erkliiren sind, sondern, wie die neueren Untersuchungen gezeigt haben, offenbar rom Phaseneinflusse herriihren. Es könnte befremdlich erscheinen, dass man nicht schon friiher auf diese Deutung gekommen ist, aber es darf nicht rergessen werden, dass, solange das Euler'sche oder Lambertsche Phasengesetz als giiltig angesehen wurde, ein merklicher EinHuss der Phase bei den Asteroiden ron vornherein ausgeschlossen scheinen musste. Denn da der Phasenwinkel bei diesen Himmelskörpern im Maximum nur etwa $30^{\circ}$ betragen kann, so waren mit Beriicksichtigung dieser Phasengesetze hüchstens Lichtinderungen ron 0.11S oder 0.14 Grössenchassen zu erwarten, also Quantititen, die durch Helligkeitsschätzungen iiberhaupt nicht mehr mit Sicherheit zu bestimmen sind. Erst nachdem durch genane photometrische Messungen der grossen Planeten nachgewiesen war, dass die bekannten Plissentheorien unzureichend sind, und 1. 261

1) Sitzungsber. der K. Akad. der Wiss. in Wien. Math.-Naturw. Classe, Bd. 41. 
dass die beobaehteten Liehtinderungen viel grösser sind, als von vornherein vorauszusetzen war, liess sieh ein analoger Schluss anch anf die kleinen Planeten ziehen. Die in den Jahren IS81-1856 von mir an einer Anzahl derselben ausgefuhrten Beobachtungsreilien') habei \%uerst auf den Phaseneinfluss aufmerksan gemacht, und seitdem auch die Messungen von Parkhurst²) zu ganz :ihnlichen Resultaten gefulirt haben, durfte es wohl kaum einem Zweifel unterliegen, dass alle bisher an den Asteroiden wahrgenommenen Helligkeitsïnderungen in erster Linic anf die wechselnden Belenehtungsverhiiltnisse \%urlick\%fulıren sind. Erst wenn es sich zeigen sollte, dass in gewissen Fällen diese Frklärung versagt, whirde es statthaft sein, an wirkliche Änderungen der Oberflichenbeschaffenheit oder an Rotationswirkungen u. dergl. zu denken. Bisher lient hierzu jedenfalls kein zwingender Grund vor.

Aus den Potsdamer Beobachtungen und ebenso ans den Messungen von Parkhurst ergiebit sieh, dass bei den meisten der bisher untersuchten Asteroiden die Änderungen der auf mittlere Opposition reducirten Helligkeitswerthe (in Grössenclassen) den entspreehenden Phasenänderungen proportional sind. Nur bei einigen seheint die Lichteurve von der geraden Linie abzuweichen und in der Nähe der Opposition etwas steiler zu verlaufen als bei grösseren Phasenwinkeln; jedoch ist \%u einer sicheren Entscheidung dieser angedeuteten Verschiedenheit noch ein grösseres Beobachtungsmaterial erforderlich, und es wird daher der kinfachheit wegen zunachst gestattet sein, bei allen Asteroiden die Lichtcurven als grerade Linien vorauszusetzen.

Die folgende Zusammenstelhng enthïlt die Resultate aller hisherigen Untersuchungen aber die Helligkeitsschwankungen der kleinen Planeten. wobei ausser den Potsdamer und Parkhurst'schen Messungen noch cinigge grössere Schätzungsreihen beriicksjehtigt worden sind, die ein gewisses Vertranen verdienen. Anfgenommen sind in die Tabelle nur solche l'laneten, bei denen die Beobiehtmugen ein grösseres Phasemwinkelintervall als $8^{\circ}$ umfassen. In der Tabelle ist ausser der Zahl der Beohachtungen und dem benutaten Phasenintervall noch die mittlere Oplnsitionswriosse und die aus den Beobachtmngen al)geleitete llelligknitsindermir fur je $1^{\circ}$ Phasenwinkel angegeben.

1) Astr. Nachr. Bd. 114, Nr. 2724 und 2725. - Publ. des Astrophys. Obs. zu Potsdam. Bd. 8, p. 355.

2. Annals of the Astr. Obs. of Harvard Cullege. Vibl. 15, p. 2! und Vol. 29. p. 65. - Siehe auch Astr. Journal. Vol. 9, p. 127. 


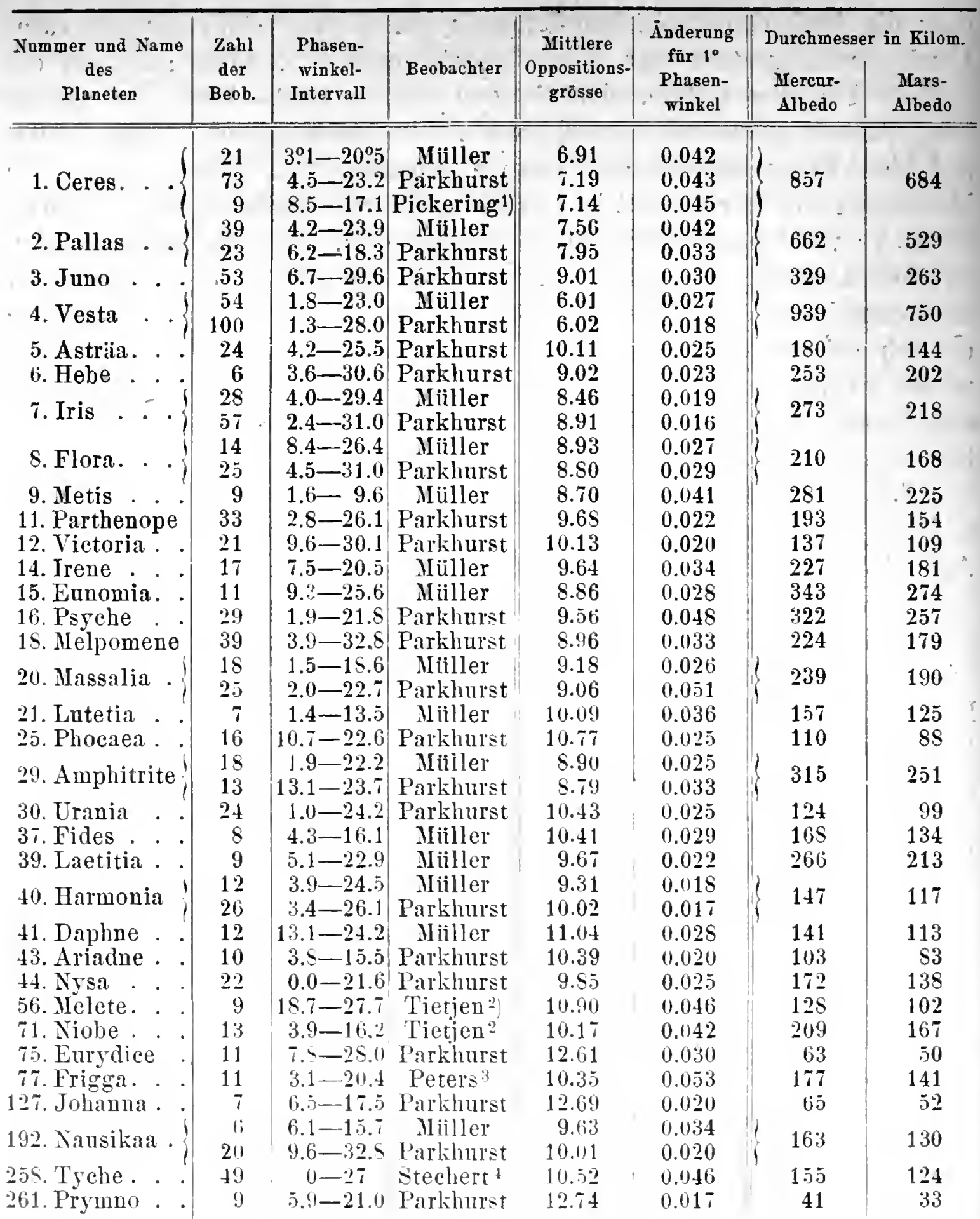

Tach dieser Zusammenstelhng schwanken die Phasencoefficienten wrischen den Werthen 0.016 , Iris und 0.053 Frigga; einem Phasenintervall ron $20^{\circ}$ entspricht also hei dem letzteren Planeten eine Grössen-

1 Annals of the Astr. Obs. "It Ilarrard College. Vol. 24: 1. 265.

- Astr. Nachr. Bd. 135 , Nr. 3227.

3 Astr. Nachr. Bd. 114. Nr. 2724.

t) Mitheilungen der Hamburor Sternwate. Nr. 2. p. $31 \mathrm{ff}$. 


\begin{tabular}{c|c|c}
\hline Ceres & Pallas & Vesta \\
\hline \hline 0.170 & 0.258 & 0.810 \\
0.227 & 0.344 & $(1.0810)$
\end{tabular}

Danach wiirde Ceres das Licht etwas stärker reflectiren als Mercur, Pallas etwas stärker als Mars, und das Reflexionsvermögen der Vesta wïrde noch cin wenig dasjenige der Venus iibertreffen. Es ist nicht sehr wahrscheinlich, dass die kleinen Planeten, bei denen man in Bezug anf die physische Beschaffenheit eine gewisse Verwandtschaft voraussetzen darf, so starke Albedounterschiede haben sollten, wie aus den Barnard'schen Zahlen hervorgeht. Namentlich scheint der letzte Werth, der eine nahezu spiegelnde Oberfläche oder eigenes Licht bei dem Planeten Vesta bedingen wiirde, kaum mit nnseren Anschaunngen iiber die Entstehung und die Beschaffenheit der kleinen Himmelskörper vereinbar. Es geht daraus hervor, dass auch die nenesten directen Durchmesserbestimmungen noch Manches unaufgeklärt lassen und erst noch durch weitere Untersuchungen nach wesentlich verfeinerten Messungsmethoden bestïtigt werden miissen. Gegenwärtig wird man sicher noch zu besseren Resultaten gelangen, wenn man unter Annahme einer gleichen Albedo für alle Asteroiden aus den photometrischen Bestimmungen ihre relativen Dimensionen ableitet, als wenn man umgekehrt ihr Reflexionsrermögen aus den durch Schätzungen gewonnenen oder mit dem Fadenmikrometer erhaltenen Durchmessern zu bestimmen rersucht. Schon Stampfer 1 ) hat auf die Verweudung der photometrischen Beohachtungen der kleinen Planeten zu Durchmesserbestimmungen hingewiesen, und Argelander ${ }^{2}$ ) hat anf Grund der Stampfer'schen Formeln im Jahre 1855 für 26 derselben Durchmesser mitgetheilt. Diese Werthe sind aber offenbar beträchtlich zu klein, weil die Albedo dabei gleich derjenigen der grossen Planeten Saturn, Uranus und Neptun vorausgesetzt war. Spaiter sind noch ron Bruhns 3 ) für die ersten 39 und ron Stone ${ }^{4}$ fiir die ersten 71 Asteroiden Durchmesser berechnet worden; aber anch diese Werthe sind zweifellos zu klein, schon ans dem Grunde, weil die Oppositionshelligkeiten der Planeten wegen Nichtberiicksichtigung der Phasencorrection meistens zu gering angesetzt waren. Nachdem die photometrischen Messungen an einer grösseren Anzahl der Asteroiden gezeigt haben, dass die Beträge der ron der Phasenänderuuğ ablängigen Lichtrariation nicht wesentlich verschieden sind ron

1) Sitzungsber. der K. Akad. der Wiss. zu Wien. Bd. т, p. 756.

2 Astr. Nachr. Bd. 41, Nr. 982.

Bruhns, De planetis minoribus inter Martem et Jovem circa solem versautibus. Berolini, 1565, p. 15.

4 Monthly Notices. Vol. 27, 1. 302. 
den bei den Planeten Mereur und Mars constatirten, wird man zu besseren Durchmesserwerthen gelangen, weun man die Albedo der kleinen Planeten gleich der von Mereur oder Mars annimunt. Berechnet man die Durchmesser fur beide Hypotheson, so erhält man zwei Grenzwerthe, zwischen deuen voranssiehtlich die walıren Werthe liegen werden. In der obigen Zusammenstellung siud fur die bisher mit einiger Sicherheit photometrisch beobachteten kleinen Plancten in den letzten Columnen dic berechneten Durehmesser in Kilometern angegeben. Damach hat der grösste dieser Asteroiden, Vesta, im Mittel einen Durchmesser ron S.55 Kilom. und Uhertrifit an Grösse den kleinsten, Prymuo, um melır als das zwanzigfache. Der fur Vesta berechnete Werth ist mehr als doppelt so gross, wie der aus den Barnard'schen Messungen hervorgehende.

Naturlich werden die ans den photometrischen Beobachtungen abgeleiteten Durchmesserwerthe um so mehr Vertrauen verdienen, je sorgfaltiger die Oppositionshelligkeiten bestimmt sind. Es wäre daher im höchsten Grade zu wïnscheu, dass in Zukunft den Lichterscheinungen dieser Himmelskürper eine regere Theilnalıme entgegrengebracht wlirde, als bisher. Es liegt hier noch ein weites Feld der 'Thitigkeit offen. Wenn die zahlreichen Beobachter der kleinen Planeten die geringe Mthe nicht schenten, mit jeder Positionsbestimmung eine Helligkeitshestimmung zu verbinden, und zwar nach dem Argelander'schen Vorschlage durch Stufenvergleichungen mit passend gewiilıten Fixsternen, deren Lichtstiirken dann auf photometrischem Wege gen:u ermittelt werden kïnnten, so wïrde sehr bald ein unfangreiches Material gesammelt sein, welehes nnsere Kenntniss von diesen kleinen Weltk̈̈pern wesentich hereichern und vielleicht $\%$ manchen interessunten Folgernumen hinsiehtlich ihror physischen Beschaffenheit führen wiirde.

\section{Inpiter.}

Die Helligkeitsïnderungen, welche beim Jupiter dureh die werhsehden Entfernungen von Sonne und Erde hervorgehnatht werden, sind int Vergleich zu den der Somne näheren I'laneten mbindrutemd. In der

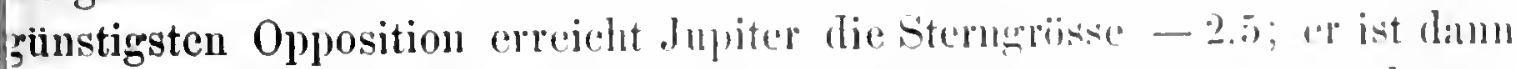
ast genau so hell wie Mars in semer minstiosten yplusition und etwil sine Grössenclasse schwaicher als Venus in ihrer durchsehnittlichen Lichtstäke. Wie Arago in seiner populairen Astrmonnic angieht, haben rerschiedene Beobachter, nuter Anderen bereits Cialilei, die Wahrmohmmm ;emacht, dass Jupiter bei dieser Stellung hinter undurchsightizen Kiirpern

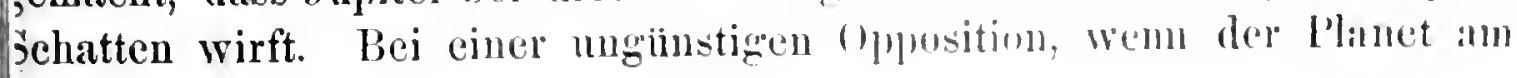


weitesten rou der Sonne entfernt und gleichzeitig die Erde ihr am naichsten ist, wird die Helligkeit etwa gleich - 2.0. In der Nähe der Conjunction endlich, zu der Zeit, wo Jupiter fuir die Beobachtungen unzug̈änglich-wird, sinkt seine Lichtstärke ungefähr bis zur Grösse- - 1.5 hinab, er ist dann nur wenig heller als Sirius. Maximum und Minimum der iiberhaupt beim Jupiter messbaren Helligkeiten verhalten sich etwa wie $2.5 \mathrm{zu} 1$.

Der Phasenwinkel schwankt nur 'zwischen den Grenzen $0^{\circ}$ und $12^{\circ}$; die davou herrührenden Helligkeitsänderungen können also unter allen Umständen nur geringfiigig sein. Schwankungen, wie sie unter Zugrundelegung der rerschiedenen Phasentheorien fuir das Intervall von $0^{\circ}$ bis $12^{\circ}$ zu erwarten wären (im Maximum etwa 0.04 Grössenclassen), lassen sich iiberhaupt nicht durch photometrische Messungen nachweisen. Verhielte sich Jupiter genau so wie Mars, so wuirde die gesammte Helligkeitsänderung wegen Phase 0.17 Grössenclassen betragen, eine Quantitiit, die durch sehr zahlreiche sorgfältige Messungen vielleicht eben noch zu bestimmen wäre. Wenn dagegen Jupiter das Sonnenlicht in derselben Weise reflectirte, wie die kleinen Planeten oder wie Mercur und der Mond, so könnte die durch die Phasen berrirkte Lichtänderung bis zu 0.3 oder 0.4 Grössenclassen anwachsen und würde dann schon durch einigermassen zuverlässige photometrische Beobachtungen mit Sicherheit ermittelt werden kümnen. Alle bisher ausgeführten Lichtmessungen des Jupiter zeigen num keinerlei Einwirkung der Phase. Weder in der Seidel'schen Reihe aus den Jahren 1845-1557, noch in den Beobachtungen ron Zöllner und Kononowitsch aus den Zeiträumen 1862-1864 und 1875-1892 ist ein Anwachsen der Lichtstärke in der Nïhe der Opposition zu erkemnen, und noch deutlicher tritt dies aus den Potsdamer Messungen herror, welche sich iber einen ganzen Cmlauf des Planeten um die Somne erstrecken. Soviel folgt mit sicherheit ans diesem umfangreichen Material, dass Jupiter in seinem photometrischen Verhalten durchans rerschieden ist rom Ionde, ron den Planeten Mercur und Mars und den Asteroiden, und dass wir daher bei ihm eine wesentlich andere phrsische Besehaffenheit als bei diesen Himmelskörpern roranssetzen diurfen. Nach Allem, was wir durch die topographischen Beohachtungen des Jupiter wissen, ist derselbe mit einer ausserordentlich dichten Atmosphäre nmgeben, und es ist daher sehr wahrseheinlich, dass die sumenstrilhlen zum grijssten Theile ron den Wolkengebilden dieser Atmorshïre zurickgeworfen werden und nur in relativ geringer Menge rou den festen Theilen des eigentlichen .Jpitersphïroides. Diese Ausicht wird anch bestitigh durch das ansergewiohnlich grosse Reflexionsvermögen des Inpiter, welches sanz besonders deutlich in seiner photo- 
graphischen Wirkung zu'Tage tritt. Warren de la kue l) hat auf diesen Punkt hingewiesen, und von Bond ${ }^{2}$ ) existiren ausfuhrliche Untersuchungen daruber. Letzterer findet, dass Jupiter ron den ehemischen Strahlen des Sonnenlichtes etwa $14 \mathrm{mal}$ melir reflectirt als der Mond, falls aber nur die hellen Stellen des Planeten und die Centralregionen des Mondes in Betracht gezogen werden, sogar 27 mal mehr. Neuere Versuche von L,ohse zeigen eine ähnliche Überlegenheit der photographischen Wirkung des Jupiter uber diejeuige des Mars.

Auch die optisehe Albedo des Jupiter ergiebt sich ans den bisherigen photometrischen Beobachtumgen sehr gross. Mit Zugrumdelegung des aus den Potsdamer Messungen hervorgehenden Werthes - 2.233 fitr die mittlere Oppositionshelligkeit des Planeten erhïlt man die folgenden Albedowerthe:

$$
\begin{aligned}
& A_{i}=0.616 \text { (Lambert'sche Definition), } \\
& A_{i}=0.821 \text { (Seeliger'sehe Definition). }
\end{aligned}
$$

Das Reflexionsvermögen des Jupiter ist hiernach zwar etwas geringer als dasjenige der Veuus, aber im Vergleieh \%u irdischen Sulsstanzen doeh noch so betriehtlich, dass wiederholt die Vermuthung ausgesprochen worden ist, dass Jupiter uns nicht uur reflectirtes Licht \%usendet, sondern auch eigenes Licht ausstrallit.

Was die Abstufungen der Helligkeit anf der Jupiterscheihe anbetrift; so lehrt sehon eine fliichtige Betrachtmug, dass die liandpartien im Allgemeineu schwächer sind als die centralen lieurionen, eine Erseheinnngr: die dureh die Annahme einer sehr dieliten Atmosphiare unschwer zor erklären ist. Der llelligkeitsuntersehied ist sehr hedentend, win solun daraus hervorgeht, dass dic Trablanten des Jupiter, die hein Voriibergange vor der Mitte der Scheibe als dunkle lelecke assheinen, hein linoder Austritt sieh nur wenig ron dem l'ntergrunde abheben. winderholentlich sogar als helle Flecke ant dunklem Grunde zesehen worden

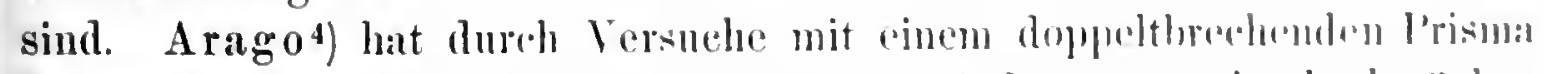

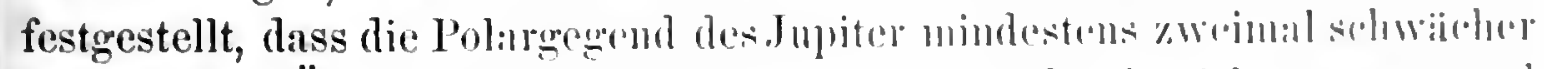

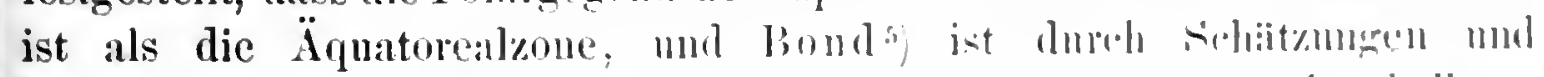

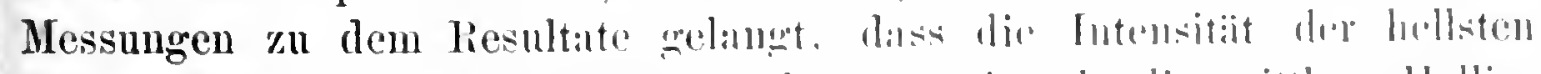

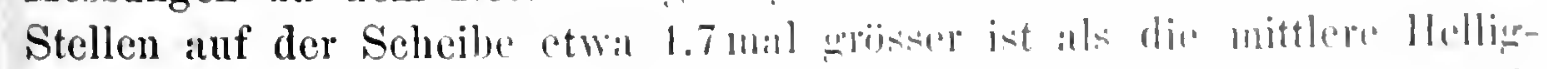

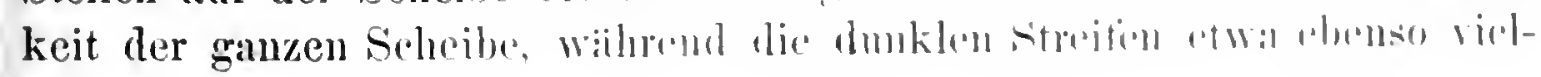

1) Monthly Notices. Vol. 1ৎ, p. 55.

2) Memoirs of the American Acul. New Series. Vul. 4, p. 201

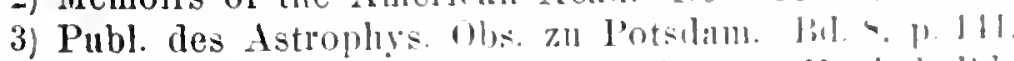

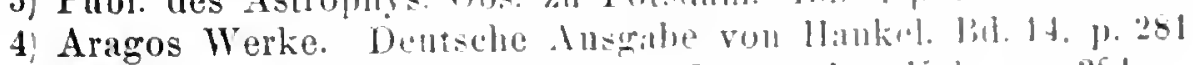

5) Memoirs of the American Acal. New series. Vul. -, p. 25. 
mal schwächer sind. Nach Browning ${ }^{1}$ ) ist eine sehmale Zone nördlich vom Äquator bei Weitem die hellste Partie anf der ganzen Planetenseheibe. Zenger ${ }^{2}$ ) hat zur Ermittlung von relativen Helligkeitsunterschieden auf der Planetenseheibe die Bestimmung der Zeitpunkte empfohlen, zu welchen in der Morgen- und Abenddämmerung die verschiedenen Details verseliwinden oder zum Vorschein kommen.

Weitere Untersuehungen in dieser Richtung sind im hohen Grade erwiinseht, und insbesondere verdient die Frage nach etwaigen Veränderungen der relativen Intensitäten eingehende Beruicksichtigung. Dass zeitweilig gewaltige Revolutionen auf der Jupiteroberfläche vor sich gehen, die sieh uns durch Farben- und Helligkeitsänderungen einzelner Partien, sowie durch das Auftreten und Verschwinden heller und dunkler. Fleeke (z. B. des bekannten rothen Fleeks in den letzten Jahrzehnten) bemerkbar machen, ist eine allgemein constatirte Thatsaehe. Huggins und Ranyard haben darauf hingewiesen, dass die Epochen lebhafter Verinderungen anf der Jupiterseheibe mit den Epoehen der Sonnenfleckenmaxima im Zusammenhange zu stehen scheinen, und Letzterer ${ }^{3}$ ) hat eine Anzahl von Fällen angefiihrt, die zu Gunsten dieser Vermuthung sprechen, so die Beobachtungen von Cassini (1692), von Hersehel (1778-1780), ron Lassell und Dawes(1845) und von Huggins, Lassell und Airy(1858 bis 1860). Weitere Beispiele zur Unterstittung der Ranyard'schen Annahme sind von Lohset) reröffentlicht worden, weleher eine ausfiihrliche Zusammenstellıng der Litteratur über diesen Gegenstand gegeben hat. Naturgemäss dräing't sich die Frage auf, ob ähnliehe Veränderungen von periodischem Charakter etwa anch in den Messungen der Gesammthelligkeit des Jupiter zu Tage treten. Eine Zusammenfassung aller bisher bekannten zuverlissigen Beobachtungsreihen liefert die folgenden auf mittlere Opposition reducirten Mittelwerthe.

\begin{tabular}{|c|c|c|c|c|}
\hline Eporhe & Beubachter & $\begin{array}{l}\text { Zillil } \\
\text { der } \\
\text { Mess. }\end{array}$ & $\begin{array}{l}\text { Wittlere } \\
\text { Oppos.- } \\
\text { Helligkeit }\end{array}$ & Bemerkungeu \\
\hline $1845-1846$ & Seidel & 5 & -2.05 & 1StS Sommenfleckenmaximum \\
\hline 1852 & $\gg$ & 12 & -2.04 & 1860 Maximum \\
\hline $1862-1864$ & Zöllner & i & $-2.3 \ddot{3}$ & 1871 Jaximum \\
\hline 1975 & Kononowitsch & $S$ & -2.21 & \\
\hline 1882 & $\gg$ & 8 & -2.06 & \\
\hline 1578 & Miiiller & 26 & -2.11 & \\
\hline
\end{tabular}

1 Monthly Notices. Vol. 31, p. 33.

2) Honthly Notices. Vol. 35, p. 65.

Nonthly Notices. Vol. 31, p. 34.

4. Bothkamper Beobachtungen. Heft 2. p.92. 


\begin{tabular}{|c|c|c|c|c|}
\hline Epoche & Beobachler & $\begin{array}{l}\text { Zabl } \\
\text { der } \\
\text { Mese }\end{array}$ & $\begin{array}{l}\text { Mritlese } \\
\text { Oppos.. } \\
\text { llelligkelt }\end{array}$ & Remerl whrew \\
\hline $1879-1880$ & Malier & 53 & -2.23 & 1579 Minimuแ \\
\hline $1880-1881$ & , & 27 & -2.26 & \\
\hline $1881-1882$ & , & 15 & -2.33 & \\
\hline 1853 & , & 5 & -2.30 & 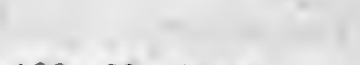 \\
\hline $1883-18 S 4$ & - & 12 & -2.35 & 1554 Maximum \\
\hline 1885 & , & 1i) & -2.31 & \\
\hline 1886 & , & 20 & -2.28 & \\
\hline 1887 & , & 7 & -2.25 & \\
\hline 1889 & , & if & -2.16 & 1889 Miniıum \\
\hline 1890 & > & 21 & -2.14 & \\
\hline
\end{tabular}

In der Potsdamer Reihe, welehe wegen der grösseren Zalıl der Beobachtungen die sichersten Werthe enthält, tritt ein dentlicher Gang auf, und zwar in dem Sinne, dass die Helligkeit des Planeten in dem Zeitraume von $1878-1884$ bestiindig zunimunt und ron da an wieder bestindig kleiner wird. Da die Anfangs- und Endepochen nahe mit Somnenfleckenminimis, das Jahr 1854 mit einem Sonnenfleckenmaximun zusannenfallt, so könnte man in den photometrischen Messungen eine Best:itigung dafür finden, dass die grösste Lichtentwieklung auf dem Jupiter mit der grössten Thätigkeit auf der Sonne im Zusammenhange steht. Indessen sind die Potsdamer Beobachtungen allein noch keineswegs ansreichend, um die Frage mit Sicherheit $" u$ entscheiden.

\section{Die Jupitersatelliten.}

Die Helligkeitsverhältnisse der Jupitertrahanten siml von joher Gegenstand des Iehhaftesten Interesses hei den Astrononen focwesen. Schon Cassini in der zweiten Ilialfte des 17. Jahrhunderts mud Il a raldi am Anfange des 1S. haben auf die ejgenthiumliehen Erscheimungen anf-

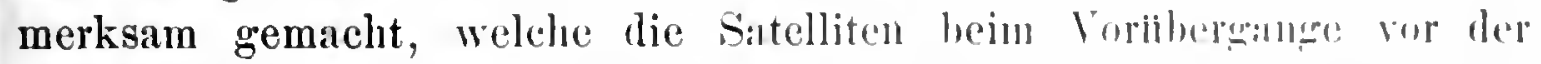

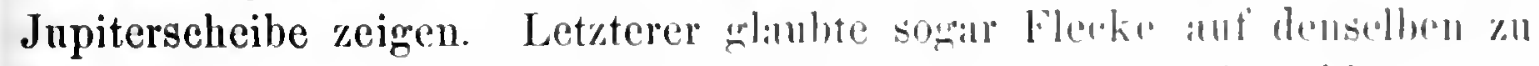

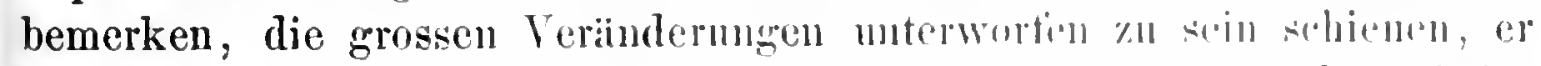

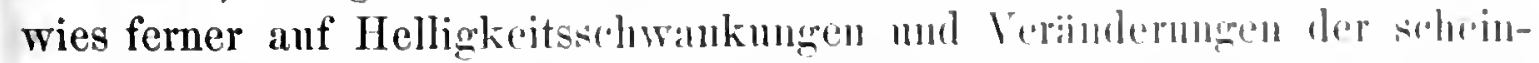
baren Grössen hin mol schloss daraus anf Rotation drerselhen. Die ersten

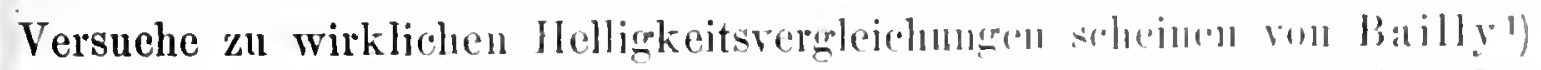

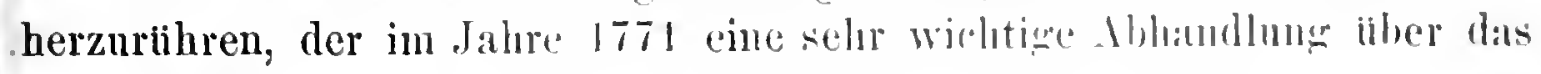

1) Mémoires de l'acad. I. des sciences de Paris. Anmie 17i1, p. 550. 
Problem der Verfinsterung der Jupitertrabanten veröffentlicht und darin auch Resultate aus Beobachtungen der Lichtstärke mit Benutzung von Diaphragmen vor dem Objective des Fernrohrs mitgetheilt hat. Nach ihm ist die Reihenfolge der Satelliten in Bezug auf ihre Helligkeit die folgende: $3,4,2,1$ (die beiden letzten gleich hell), oder in Zahlen ausgedrückt, wenn die Lichtstïrke des dritten Trabanten gleich 1 gesetzt ist:

Trabant $3=1.00, \quad$ Trabant $4=0.30, \quad$ Trabant 1 und $2=0.24$.

Bei Weitem ansführlichere Angaben verdanken wir W. Herschel ${ }^{1}$ ). Aus seinen Schätzungen ergiebt sich das Resultat, dass die Jupitermonde veränderliche Helligkeit haben. Nach ilım ist die Reihenfolge der Lichtstiirken: 3, 1, 2, 4. Der erste Mond erscheint nach Herschels Angaben in seinem grössten Glanze, wenu er sich zwischen Conjunction und grösster östlicher Digression befindet. Dasselbe gilt vom zweiten Trabanten, bei welchem aber die Lichtschwankungen innerhalb engerer Grenzen als bei jenem bleiben. Am wenigsten veränderlich ist Trabant 3, welcher das Maximnm der Lichtstärke in den grössten Elongationen erreicht, und abweichend von allen andereu verhält sich der vierte Trabant, bei welchem der grösste Glanz kurz ror und nach der Opposition eintritt. Herschel

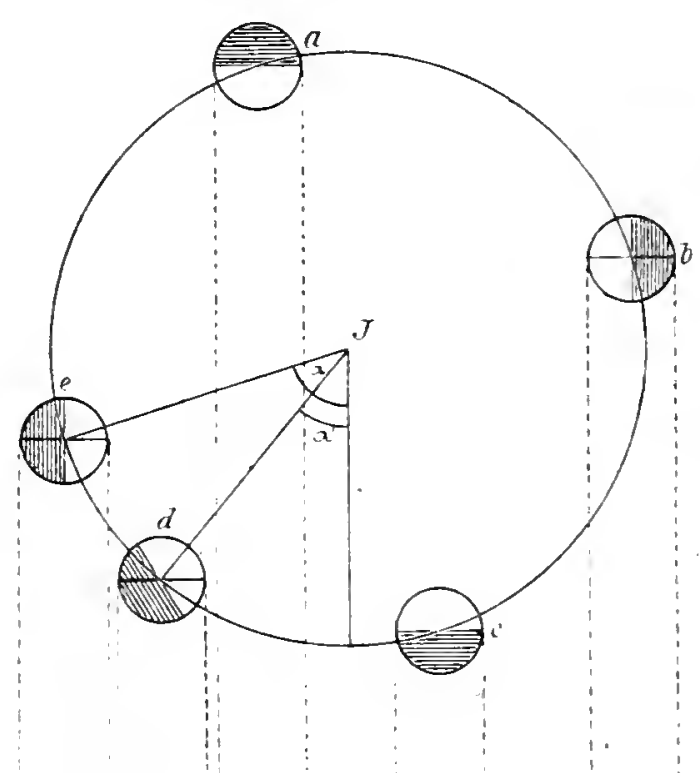

Erde

Fig. 75. setzt die Helligkeitsvariationen als durchans periodisch rorans und nimmt zu ihrer Erklärung an, dass die Trabanten mit Flecken bedeckt sind und sich in derselben Zeit um den Jupiter bewegen, in welcher sie eine Umdrehung um ihre eigene Axe vollenden. Dass eine solche Annahme in der That einen periodischen Lichtwechsel nngezwungen erklären kann, ist ans der nebenstehenden Figur 75 leicht crsichtlich, in welcher $J$ das Jupitercentrum vorstellt, und $a, b, c, d, e$ rerschiedene Stellnugen des Trabanten in seiner Bahn markiren. Nimmt man der Einfachheit wegen au, dass eine ganze Hemisphäre des Trabanten hell, die andere relatir dunkel ist, so wird derselloe. da er nach der Herschel'schen Voraussetzung dem Planeten stets dieselhe Seite zukehren muss, rou der Erde ans gesehen in a am lıellsten, in e am schwächsten, in b und e ron mittlerer Lichtstärke 
Mädler') im Jahre 1836, deren Beobachtungen in Bezug auf die Veräiderlichkeit von Trabant 3 ebenfalls zur Bestätigung der Ergebuisse von Herschel und Schröter angeführt werden.

Die Untersuchungen von Secehi, Lassell, Dawes, Noble und vielen Anderen beziehen sich mehr auf die merkwiirdigen Erscheinungen, welche die Satelliten beim Voriibergange vor dem Jupiter darbieten, als auf die absoluten oder relativen Lichtstärken derselben. Ueber diesen letzteren Punkt sind erst wieder im Jahre 1870 eingehendere Untersuchungen von Engelmann ${ }^{2}$ ) angestellt worden; dieselben verdienen um so mehr Beachtung, als es die ersten sind, welche auf zuverlässigen photometrischen Messungen basiren. Engelmann hat mit Hülfe eines Zöllner'schen Photometers die Satelliten unter einander und mit benachbarten Fixsternen rerglichen, daun mit Zugrundelegung des Lambert'schen Beleuchtungsgesetzes aus jeder einzelnen Beobachtung die Albedo der Trabanten berechnet und schliesslich untersucht, ob dieselbe irgend welchen Änderungen, speciell periodischen, unterworfen ist. Die Resultate seiner Beobachtungen gipfehn in den folgenden Sätzen: "Fïr die beiden inneren und kleinsten Trabanten, besonders den zweiten, ergiebt sich eine grössere, rasche und unregelmaissige, fuir die beiden äusseren und grössten, insbesondere für den vierten Trabanten, eine kleinere und mehr periodische Änderung der Albedo; bei den ersten scheint Fleckenbildung in raschem Wechsel nud während der ganzen Rerolutions- und Rotationsdaner stattzufinden, bei den letzten nur zu gewissen Zeiten und namentlich bei der unteren Conjumetion ausser den Flecken ron nahe unveränderlicher Gestalt noch schmelle Verdunklung der Oberflächen rorzukommen. Von den Principien der Kant-Laplaceschen Kosmogonie ausgehend, wie sie Zöllner benutzt hat, um an der Hand der dureh die Astrophysik gelieferten Thatsachen die rerschiedenen Entwicklungsstufen der Himmelskörper physikalisç zu begriiuden und darzulescen, diufte demuach angenommen werden, dass die beiden änsseren Trabanten, rornehmlich der vierte, in weiter fortgeschrittenem Entwicklmngsustande als die inneren sich befinden. Mit Riicksicht auf die geringe Albedo kïnnte seibstrerständlich an eigene Lichtentwicklung nicht gedacht werden; anch Erlebungen scheinen, wenigstens bei dem 1., 3. und 4. Trabanten, nicht rorhanden zu sein, da bei den schon ziemlith rerschiedenen Phasenwinkeln sieh sonst der Einfluss der Mangelhattigkeit des zu Grunde liegenden Lambert'schen Princips geltend machen wiirde. Die Rotationsdaner ist beim t. Trabanten sicher gleich

i lieep und Iiadler. Beiträge zur physischen Kenutniss der himmlischen Firine im sonnensrsteme. Weimar. 1541. p. 101

Engelmain. C̈ber die Helligkteitsrerhälnisse der Jupiterstrabanten. 
der Umlaufszeit um Jupiter $\left(16^{\mathrm{d}} 8^{\mathrm{h}} 5^{\mathrm{m}}\right.$ synodisch); bei den ulorigen ist dasselbe zwar wahrseheinlicher als eine andere Annahne, allein die Beobachtungen liegen in noch zn geringer Zahl und zum Theil \%weidentiger Form vor, als dass die Frage dureh sie schon entsehieden werden kïnnte.e

Die Engelmann'schen Resultate weichen zum Theil gan\% erheblich von denen Hersehels ab und scheinen uur fur den 4. Trabanten mit einiger Sicherheit einen periodischen Lichtweehsel zu beweisen, wihhrend sie fur die anderen Trabanten nur unregelmiissige Schwankungen andeuten.

Von sonstigen Helligkeitsbeobachtungen der.Jupitersatelliten sind noch die ausgedehnten Schaitunngsreihen von $\Lambda$ uwers, Flammarion, Zenger und Dennett, vor Al!em aber die photometrischen Messungen Piekerings und Spittas hervoralieben.

Die A uwers'schen ${ }^{1}$ ) Schät»ungen bestiitigen in den wesentlichsten Punkten die Engelmam'schen Resultate.

Flammarion ${ }^{2}$ findet bei allen vier Satelliten Lichtrariationen, die geringsten bei Trabant 3, die stïrksten bei Trabant 4. Nach ihm haben aber die Helligkeitsschwankungen keinen Zusammenhang mit der Rotation; sie rthien nieht vou permanenten Oberflächengebilden, sondem von wolkenartigen Prodneten der Atmosphären her, die sehr schnellen gewaltigen Veräinderungen unterworfen sind.

Zenger ${ }^{3}$ ) hat die relativen Helligkeiten der Trabanten durch Beobachtung der Zeitpunkte ermittelt, zn welehen dieselben in der Morgendiimmerung verschwinden. Beim 2. und 4. Trabanten glanht er periodische Lichtänderungen wahrgenommen zu haben, deren Daner mit den Undrehungszeiten um den Jupiter iibereinstimmt.

Nach Dennetts $\left.{ }^{4}\right)$ Angaben sind saimmtliche Satelliten veriinderlich; sie gruppiren sich in Bezug anf die absolute llelligkeit in der lieilsenfolge 3, 1, 2, 4, in Bezug auf den Betrag der Lichtvariationen in der Reihenfolge 4, 2, 1, 3. Satellit 1 soll an hellsten sein in dem unteren westlichen Quadranten, am wenigsten verïnderlich in dem oheren westlichen, am stärksten variabel in dem unteren ijstlichen Qnadranten. Trabant 2 ist nach Dennett heller im Osten als in Westen und in allen Theilen der Balm veränderlich. Trabant 3 ist am hellsten im oberen ostlichen und am schwichsten im unteren östlichen Quadranten, zur Zeit scines Maximums

1 Die Auwers'schen Beobachtungen, welche in Schätzungen nach der Argeander'schen Stufenmethode bestehen, sind in der oben eitirten Abhaullung von Ingelmann (Seite 69) publicirt und verarbeitet.

2) Comptes Rendus. T. is, p. 1295; T. 79, p. 1491; T. ᄂ1, 1. 145.

3) Monthly Notices. Vol. 38, p. 65.

4) Astr. Register. Vol. 17, p. 49. 
:indert sich die Helligkeit am stärksten.' Satellit 4 endlieh, welcher nach Dennett ebenso wie nach Flammarion bis unter die 10. Grösse sinken soll, ist am hellsten im oberen westlichen Quadranten.

Sehr auffallend ist, dass die photometrischen Messungen Pickering ' $^{1}$ aus den Jahren 1877 und 1878 bei keinem der Trabanten, auch nicht beim 4., gesetzmässige Helligkeitsänderungen erkennen lassen. Pickering hat die Satelliten mit Hülfe eines der von ihm construirten Polarisationsphotometer theils unter einander, theils mit einem punktartig verkleinerten Bilde des Jupiter verglichen, und wenn die Messungen auch wegen der Sehwierigkeit der Beobachtung stärkere Abweichungen aufweisen, als soust bei photometrischen Untersuchungen vorzukommen pflegen, so zeigt sich in den Abweichungen doch keine Spur von systematischer Vertheilung, so dass sie unbedingt nur als zufällige anzusehen sind.

Die Pickering'schen Angaben werden fast vollkommen durch Messungen vou Spitta ${ }^{2}$ ) bestaitigt, weleher mittelst eines Keilphotometers die Trabanten mit dem Jupiter selbst verglichen hat. Besondere Untersuchungen iiber etwaige periodische Lichtinderungen der Trabanten sind ron ihm nicht amgestellt worden; dagegen hat er noch eine Reihe ron interessanten Versuchen an kleinen Scheibehen von verschiedener Reflexionsfähigkeit, welehe vor einer weissen Kngel beobachtet wurden, ausgefiihrt, um zu zeigen, dass die merkwiurdigen Erscheinnngen der Trabanten beim Vorübergange vor dem P'laneten kiinstlich hervorgebracht werden können und nur auf Contrastwirkung zuriickzufiihen sind. W'emn der Albedounterschied zwischen kiunstlichem Planet und kiinstlichem Satellit einen bestimmten Werth hat, dam erscheint der letztere am Rimde der Planetenscheibe hell, verschwindet damn allmiallich, wemn er iiber die Scheibe hinweg bewegt wird, und wird in der Mitte als dunkler Fleck sichtlsar, genan in derselben Weise, wie es wiederholt am Himmel beobachtet worden ist. Man brancht also zur Erklairmo dieser Phänomene nicht besondere plịsische Vorgänge auf den Satellitenoberfliichen oder in deren Atmosphiairen anzmuehmen, wie es ron friblheren Beobachtern geschehen ist.

Ein Überblick iiber alle bisherignen L'ntersuehungen iiber die Lichtverhailtnisse der Jupitertrabanten zeigt, dass trotz der zahlreiehen Bemiilnngen eine volle Klarheit noch nicht gewonnen ist. Die Mehrzahl der Beobachter stimmt zwar dariu iibcrein; dass der 4. Trabant einem perindischen Lichtwechsel unterliegt; aber in Betreff der Epochen und der Amplitude der Helligkeitsschwanknngen gehen die Angaben weit auseinander: cine Antwitiit wie l'ickerings stellt sogar die Lichtändermng 
uberhaupt in Abrede. Noch zweifelhafter sind die Resultate hinsichtlich der anderen drei 'Trabanten. Weitere sorgfiltịge Beobachtungen derselbeu sind daher mehr als je erwunscht. Freilich gehören diese zu den schwierigsten Aufgaben der Photometric, weil die Nihe des Hauptplaneten störend wirkt, und die Erleuchtung des Untergrundes, zumal bei den Schlitzungen, eine wichtige, sehwer controlirbare Rolle spielt. Die Frage, ob der Plaseneintluss in den Helligkeitswerthen der Satelliten \%u erkenmen ist, harrt noch giinzlich der Lösung und kamu erst damu mit Erfolg behandelt werden, wenu sicher entsehieden ist, ob und nach welehen Gesetzen periodische Lichtschwankungen vor sich gehen.

Die folgende Tabelle enthïlt eine Zusammenstellung der wichtigsten Angaben uber die mittleren Liehtstïrken der vier grossen Jupitersatelliten, und zwar nuter der. Übersehrift $A$ in Einheiten der Lichtstiarke vou Trabant 3, unter der Überschrift $B$ in Sterngrössen und reducirt auf mittlere Opposition.

\begin{tabular}{|c|c|c|c|c|c|c|c|c|c|}
\hline \multirow[t]{2}{*}{ Jahr } & \multirow[t]{2}{*}{ Beobachter } & \multicolumn{2}{|c|}{ Satellit 1} & \multicolumn{2}{|c|}{ Satellit 2} & \multicolumn{2}{|c|}{ Satellit : } & \multicolumn{2}{|c|}{ Salellit 4} \\
\hline & & $A$ & $B$ & $A$ & B & $d$ & b & $A$ & 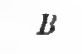 \\
\hline 1771 & Bailly & $0: 24$ & & 0.24 & & 1.00 & & 0.30 & \\
\hline $1802-1806$ & Flaugergnes & 0.62 & & 0.57 & & 1.00 & & 0.5 .1 & \\
\hline $1858-1860$ & Auwers & 0.60 & 6.43 & 0.52 & 6.59 & 1.00 & 5.87 & 0.44 & 6.76 \\
\hline 1870 & Engelmann & 0.83 & $5.5:$ & 0.70 & 5.70 & 1.00 & 5.32 & 11.11 & 6.28 \\
\hline $1874-1875$ & Flammarion & 0.44 & 6.4 & 0.36 & 6.6 & 1.00 & 5.5 & 0.21 & 7.2 \\
\hline 1877 & Zenger & 0.96 & & 0.97 & & 1.00 & & 0.92 & \\
\hline $1877-1878$ & Pickering & 0.71 & 5.90 & 0.63 & 6.04 & 1.00 & 5.53 & 0.35 & 6.66 \\
\hline 1857 & Spitta & 0.80 & $5.8: 1$ & 0.62 & 6.17 & 1.00 & 5.65 & 0.46 & 6.50 \\
\hline
\end{tabular}

Da die Durehmesser der Jupitersatelliten einigermassen sicher bustimmt sind, so lassen sich aus den photometrischen liesultaten angenäherte Werthe für die Albedo derselben ableiten. Mit Benntzum dè neuesten Durchmesserbestimmmugen von Barmardl) und der Oppositionshelligkeiten, wie sie sieh im Mittel ans den Beobachtmugen won I'ickering und Spitta ergeben, erhält man die folgenden Albedowerthe:

\begin{tabular}{|c|c|c|c|}
\hline & & $\begin{array}{c}\text { Lambert'sche } \\
\text { Albedo }\end{array}$ & $\begin{array}{c}\text { Seeliger'sche } \\
\text { Albedo }\end{array}$ \\
\hline 'lrabant & 1 & 0.412 & 10.5 .50 \\
\hline 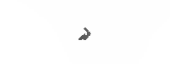 & 2 & 0.459 & 0.652 \\
\hline , & 3 & 0.259 & 0.346 \\
\hline , & 4 & 0.118 & 0.157 \\
\hline
\end{tabular}

1 Monthly Notices. Vol. 5.5, p. $3 \$ 2$. 
Dis Reflexionsvermögen des 4. Trabanten ist danach noch geringer als das des Planeten Mereui und nahe gleich dem unseres Mondes, während die Albedo der iibrigen 'Trabanten zwischen der' Mars- und Jupiteralbedo liegt.

Über die Lichtstärke des im Vorangehenden noch nicht erwähnten 5. Jupitersatelliten, welcher erst im September 1892 auf der Lick-Sternwarte ron Barmard entdeckt wurde, sind sichere Angaben bisher nicht bekannt geworden. Er gilt für ein noch schwierigeres Object als die beiden inneren Uranusmonde und ist nur mit den lichtstärksten Instrumenten sichtbar, und zwar nicht als kleines Scheibchen, sondern auch unter den güustigsten Verhältnissen nur als Lichtpunkt, dessen Helligkeit mit Riicksicht auf die blendende Nähe des Jupiter etwa einem Sterne 13. Grösse gleich geschätzt werden kann.

Wie bereits im ersten Abschnitte herrorgehoben worden ist, bieten die photometrischen Beobachtungen der Jupitersatelliten zur Zeit ihrer Verfinsterung ein rortreffliches Mittel dar, um bestimmte Momente dieses Phïnomens, also etwa den Anfang oder die Mitte oder das Ende der Verfinsternug. mit ansserordentlich grosser Sicherheit zu berechnen und dadurch das Problem der geographischen Längenbestimmung wesentlich zu fürdern. Die bisherige Methode bei den Beobachtungen der Trabantenrerfinsterungen bestand in der Feststellung desjenigen M[omentes, wo der Trabant beim Eintritte in den Schattenkegel eben unsichtbar wurde oder beim Anstritte ans demselben gerade anflenchtete. Da bei einer solchen Beobachtung die Grözse des benutzten Fermrohrs, die Helligkeit des Grundes, die Luftbeschaffenheit, die Empfindlichkeit des Auges und andere Lmstände mitspielen, so liisst sich eine grosse Genauigkeit auf diesem Tege nicht erreichen: man kamn die Lnsicherheit der Bestimmungen auf 5 bis 10 secunden schiitzen.

Der Gedanke, anstatt den Verschwindmosmoment zu bestimmen, die Helligkeit des Trabanten wïhrend der ganzen Dauer der Verfinsterung zu messen und dadurch die Lichtcurre abzuleiten, muss als ein sehr clicklicher bezeichnet werden. Er ist zuerst ron Pickering $\left.{ }^{1}\right)$ ansgesprochen worden, weleher anch die ersten Messungen in dieser Richtung angestellt hat: dagegen gebiihrt Corn n ${ }^{2}$. welcher mabhingig ron Pickering die photometrischen Beohachtungen der Trabantenverfinsterungen empfohlen hat. das Verdienst, bestimmtere Torschlige gemacht und insbesondere darauf

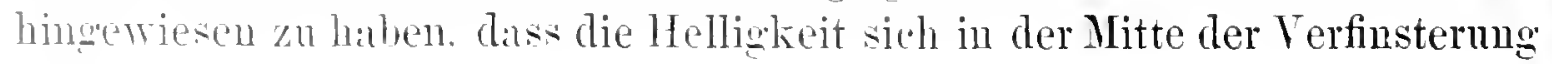

1 Ammal reprort of the director of the Harvard College Observ. for the year $187 \mathrm{s.}$

2 Comptes Rendus. 'T. 96. p. 1609 und 1515. 
an sehnellsten und zwar der 'Leit proportional andert, und dass daher die einzelnen Beobachtungen an Besten auf denjenigen Moment \%u reduciren sind, wo der Satellit die Hailfe der Helligkeit erlangt lat.

Die theoretische Seite des Problems ist im ersten Absehnitte behandelt worden; in Bezug auf die praktische Seite ist vielleicht noch zu betonen, dass es sich empfiehlt, auch unmittelbar vor oder nach der Verfinsterung die Helligkeit des Trabanten sehr sorgfiiltig «u bestimmen und ansserdem, wenn irgend möglich, noch einen anderen etwa sichtbaren Trabanten zu beobachten. Ferner ist es rathsam, zu diesen Beobachtungen ein Photometer zu wihlen, welehes in mögliehst kurzer Zeit die meisten Einstellungen zu machen gestattet, und endlieh durfte gerade bei diesen Messungen die Anwendung einer Registrirvorriehtung besonders erwilnseht sein.

Bisher sind ausser einer einzigen Beobachtungsreihe von Obrecht'), welche mehr zur Illustrirung des ganzen Verfahrens dienen sollte, keine zusammenhängenden Messungen maeh der Cornu'sehen Methode verötlentlicht worden. Man wird daher mit um so grösserem Interesse der Verarbeitung des umfangreiehen Materials entgegensehen diirfen, welehes nach den Pickering'selien Jahresberieliten auf der Sternwarte des Harvard College seit dem Jahre 1878 ununterlorochen gesimmelt worden ist.

\section{Saturn.}

Die eigenthümliche Beschaffenheit des Saturusystems macht sieh anch in den Helligkeitserscheinmugen desselben bemerkbar und stellt sowohl der theoretischen als der praktisehen Photometric wiolitige Aufuablien. Das von den Ringen reflectirte Somnenlicht bildet einen wesentlichen Bruchtheil des Gesammtlichtes, und da die Ringe wiilıend eines vollen Umlaufes des Planeten um die Somne der Erde zweimal die selnmale, kaum sichtbare Kante zukehren, andererseits zweimal weit greïhnet erseheinen, so zeigen im Zusammenhange danit die photonetrischen Beobachtungen des Planeten einen periodischen Lichitwechs(d, bei welchen die Zeit zwisehen Maximum und Minimum der Ilelligkeit ungefalur 7 . Julre 4 Monate beträgt. Waire von der Existenz der linge Niclıts bekamut, so wirden die periodischen Liehtschwankmogen allein \%u der Annahme zwingen, dass entweder die Refiexionsfähigkeit des l'laneten einer periodischen Verinderung unterliegen misse, was sehr unwalurshenenlich wire, oder dass die Gestalt des Planeten merklich ron der Kugelgestalt ahweichen miisse.

1) Comptes Rendus. 'T. 97, p. 112s 
Wie aus den photometrischen Messungen hervorgeht, beträgt der Lichtzuwachs, den Saturn durch seine Ringe, wenn sie am weitesten geöffnet sind, erhält, etwa cine volle Grössenclasse. Bei weit geöffnetem Ringe und in einer besonders günstigen Opposition (Erde im Aphel und Saturn in Perihel) erreicht die Lichtstärke des Systems den grösstmöglichen Wertl; Saturn erscheint dann ungefähr um 0.4 Grössenclassen heller als Arctur. In der Nïhe der Coujunction ist die Helligkeit im Allgemeinen am geringsten. Als unterste Grenze wird man (bei verschwundenem Ringe) die Sterngrösse 1.5, also etwa die Lichtstärke von a Leonis, annehmen diirfen. Danach beträgt also - die grösste Helligkeitsdifferenz, die bei photometrischen Beobachtungen des Saturu iberhaupt rorkommen kamn, fast zwei ganze Grössenclassen, entspricht also etwa dem Helligkeitsverhältuiss 6 zn 1.

Der Phasenwinkel kam bei Saturn im Maximum nur den Werth 6.3 crreichen, und es wäre daher a priori ein Einfluss der Phase auf die Helligkeit des Planeten noch viel weniger zu erwarten als beim Jupiter. Auffallender Weise ist aber durch langjährige Beobachtungen in Potsdam ein solcher Einfluss im höehsten Grade wahrscheinlich gemacht worden. Die bei zwölf rerschiedenen Erscheinungen des Planeten ausgefiihrten Messungen zeigen dentlich eine Zumahme der Helligkeit vor der Opposition und eine Abnahme nach derselben, und zwar von solchem Betrage, dass die Lichtstärke des Systems 60 Tage vor oder nach der Opposition etwa 79 Procent der Oppositionshelligkeit ansmacht. Wenn diese Differenz allch so gering ist, dass sie nicht erheblich die mrermeidliche Unsicherheit der photometrischen Messungen uibersteigt, so scheint sie doch durch das iibereinstimmende Terhalten in verschiedenen Erscheinnngen des Planeten ziemlich sicher rerbiirgt zu sein. Wird dic Helligkeitsänderung der Einfachheit wegen proportional der Phasenwinkelinderung angenommen, so folgt ans den Potsdamer Lntersulnmgen für jeden Grad Plasenwinkel eine Zn- odel" Abnahme der Liehtitiolke von 0.0436 Grössenclassen. Bemerkenswerth ist, diss die im ersten Abchnitte belandelte Seeliger"sche Theorie des saturnringes ebenfalls cine Helligkeitsainderung des Systems im Znsammenhange mit der Phase fordert.

Was die Abhïnigkeit der Lichtstairke des ganzen Saturnsystems ron der Erhebung der Frde iiber der Ringebene ambelangt, so hat sich aus den simmtlichen Potsdamer Beolachtnngen, nachdem dieselben wegen des soeben erwähnten Phaseneinfluses corrigirt und anf mittlere Opposition reducirt waren, die empirische Formel croben:

$$
l=0.577-2.5965 \sin l+1.2526 \sin ^{2} l,
$$

wn 0.57 die mittlere Oppositionshelligkeit bei rershwundenem Ringe ansdritkt. nnd / der Elevationswinkel der Erde iiber der Ringebene ist. 
Ausser den Potsdamer Messungen eiguen sich zu Untersuchungen uber den Einfluss der Ringöfrnung anf die Helligkeit des gan\%en Saturnsystems nur noch die zahlreichen Helligkeitsschaitzungen vou Schmidt, welche in den hinterlassenen Papieren desselben anfgefunden und von mir bearbeitet wordeu sind $)$. Dieselben haben deshalb noeh ein besonderes Interesse, weil sie fast die gan\%c Zeit umfassen, in weleher die Nordseite des Ringes der Erde zugekelirt war, während sich die Potsdamer Messungen nur auf das ron der Sulseite reflectirte Somenlieht bezichen. In Grossen und Ganzen besteht eine befriedigende Übereinstimmung \%wischen deu beiden Reihen.

Die älteren Saturnbeobachtungen von Seidel und Zöllner, sowic die neueren von Kononowitseh und Pickering sind weder yahlreich genug, noch uber einen ansreichend grossen Zeitraum vertheilt, un die Abhängigkeit der Helligkeitsvariationen von der Ïnderung des Eilevationswinkels erkennen zu lassen.

Mehrfach ist der Versueh gemacht worden, die Helligkeitsiinderungen des Saturnsystems auf theoretischem Wege abzuleiten. Die ron Albert²) und Seidel'3) zu diesem Zwecke aufgestellten Formeln sind so liherans umständlicb und nubequem, dass ihre praktisehe Verwendung von vornherein fast gainzlich ansgeschlossen scheint. Ausserdem hat Zöllner hinsichtlieh der Seidel'schen Theorie darauf hingewiesen, dass sich nath derselben aus den Seidel'schen Messungen, Welche simmotlich bei weit geöffinetem Ringe ansgefiilnt sind, für die Ilelligkeit hei verschwundencm Ringe ein Werth ergiebt, weleher den durch directe lieuhiolitungern hestimmten um $3+$ Procent iibertrift.

Zöllner ${ }^{4}$ ) hat selbst eine Formel tour Realution von silturuluobachtungen angegeben, welche auf der einfichon . Imbihme hermht, dats

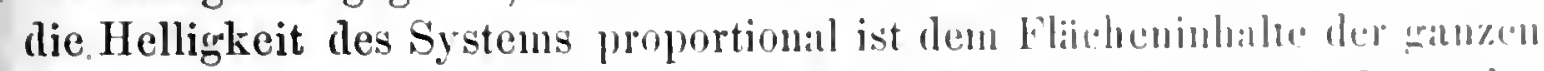
scheinbaren Figur. Ist $J^{\prime}$ die Lichtmenge bei ciner belichignen Lalper llos Ringes, $J$ diejenige bei rerschwundenem Ringe, wirl ferner dir schlin-

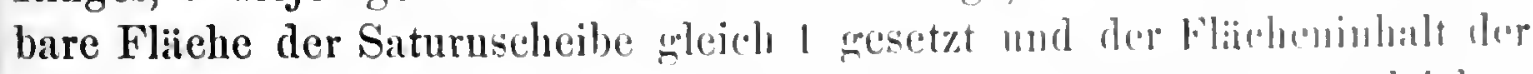

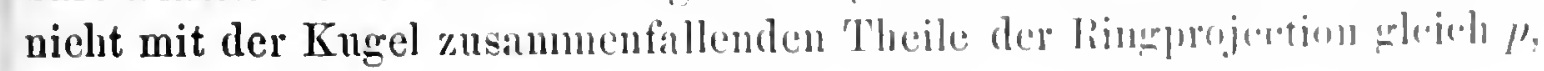
so ist nach Zöllner:

$$
J^{\prime}=J 1+I^{\prime}
$$

1) Publ. des Astrophys. Obs. zu Potslim. Bil. 6, p. 37:2.

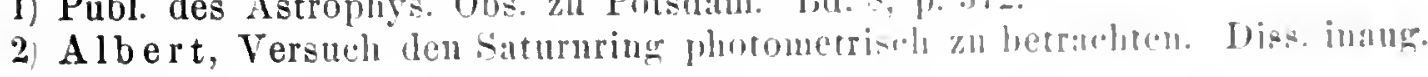
Miinchen, 1832.

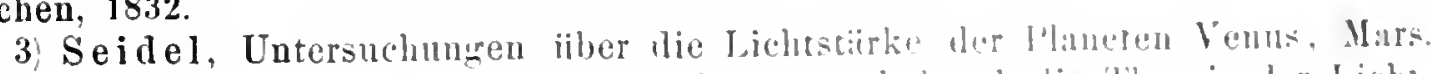

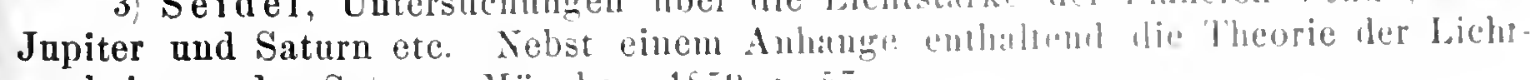
erscheinung des Saturn. Wiinchen. 165\% 1) is.

4 Zöllner, Photometrisehe Untersuchugen ute Leipig, 1-6i. p. 1 tu. 
Da Zöllner den Ring als eine feste zusammenhängende Masse betrachtet, so ist klar, dass die Annahme einer Unabhängigkeit der Beleuchtung von Incidenz- und Emanationswinkel; wie sie der Formel zu Grunde liegt, nicht zulässig ist. Man wird daher diese Formel, welche zufällig die Beobachtungen sehr gut darstellt, nur als eine Interpolationsformel ansehen diurfen.

Von wirklicher Bedeutung ist nur die im ersten Abschnitte besprochene Saturntheorie von Seeliger, welche sich auf die moderne Anschaung iiber die plysische Constitution des Ringes stützt und eine leichte praktische Anwendung gestattet. Nach dieser Theorie ergiebt sich die jedesmalige Helligkeit des Saturnsystems aus der Gleichung (Seite 100):

$$
Q_{B}=m x+n y,
$$

wo $m$ und $n$ Grössen sind, die mit Hülfe der Elevationswinkel und der Phase aus Tafehn zn entnehmen sind, wo ferner $y$ die auf verschwundenen Ring reducirte Liehtmenge bedentet, endlich $x=$ Const. $\ngtr y$ ist. Seeliger hat seinen theoretischen Untersuchungen sowohl das Lambert'sche als das Lommel-Seeliger'sche Beleuchtungsgesetz zu Grunde gelegt und kommt in beiden Fällen zu der gleichen Endformel, in welcher nur die Constanten andere Werthe haben. Es ist sehwerlich zu erwarten, dass es durch Beobachtungen des Saturn jemals gelingen wird, zn Gunsten des einen oder anderen Gesetzes zu entscheiden. Unter Benutzung des Lambert'schen Gesetzes und mit Zugruudelegrung der Potsdamer photometrisehen Messungen ergiebt sieh nach der Seeliger'schen 'Theorie die folgende Formel, ans welcher die Helligkeit des Saturn in Grössenclassen zu entnehmen ist:

$$
h=-\frac{1}{0.4} \log [0.1656 m+0.4163 n] .
$$

Diese Formel sehliesst sich den vorhandenen Beobachtungen so gat an, dass man, wie bereits Seeliger hervorgehoben hat, zu dem Schlusse berechtigt ist, dass die Maxwell'sche Saturnringhypothese in den photometrischen Messungen eine nene und nicht muwichtige Stiitze gaefunden hat.

Die folgende Zusammenstellung enthält die Reductionen auf verschwundenen Ring (in Grüssenchassen), wie sie sich auf empirischem Wege ans meinen und Sehmidts Beobachtnngen ergeben, ferner wie sie aus der 'Zölhner'schen und Seeliger'schen Formel hervorgehen. Argument ist rer Elevationswinkel der Erde iiber der Ringebene. 
Satura.

\begin{tabular}{|c|c|c|c|c|}
\hline \multirow{2}{*}{$\begin{array}{c}\text { Elevations- } \\
\text { winkel } \\
\text { der } \\
\text { Erde } \\
\end{array}$} & \multicolumn{4}{|c|}{ Ireduction auf rersebwundenen flog } \\
\hline & $\begin{array}{c}\text { Mallers } \\
\text { Messungeu }\end{array}$ & $\begin{array}{c}\text { Sctrmidts } \\
\text { Schstzungen }\end{array}$ & $\begin{array}{l}\text { Zollners } \\
\text { Formal }\end{array}$ & $\begin{array}{l}\text { Seollisero } \\
\text { Thoorio }\end{array}$ \\
\hline $0^{\circ}$ & 0.00 & 0.00 & 0.00 & 0.00 \\
\hline 2 & 0.09 & 0.117 & 0.05 & 0.09 \\
\hline 4 & 0.17 & 0.15 & 0.16 & 0.15 \\
\hline 6 & 0.26 & 0.22 & 0.23 & 0.27 \\
\hline 8 & 0.34 & 0.30 & 0.30 & 0.35 \\
\hline 10 & 0.41 & 0.37 & 0.36 & $0.4 i$ \\
\hline 12 & 0.49 & 0.44 & 0.43 & 0.50 \\
\hline 14 & 0.55 & 0.52 & 0.49 & 0.35 \\
\hline 16 & 0.62 & 0.59 & 0.35 & 0.65 \\
\hline 18 & 0.65 & 0.67 & 0.61 & 0.73 \\
\hline 20 & 0.74 & 0.74 & 0.67 & 0.50 \\
\hline 22 & 0.80 & 0.81 & 0.74 & 0.55 \\
\hline 24 & 0.85 & 0.89 & 0.80 & 0.96 \\
\hline 26 & 0.90 & 0.96 & 0.87 & 1.0 .1 \\
\hline $28^{\circ}$ & 0.94 & 1.04 & 0.94 & 1.11 \\
\hline
\end{tabular}

Aus den bisher bekannten Helligkeitsbeobachtungen des Saturn ergeben sich, wenn dieselben in einheitlicher Weise auf verschwundenen Ring, anf Phase 0 und auf mittlere Opposition redueirt werden, fur die versehiedenen Zeitepochen die folgenden Mittelwertlıe.

\begin{tabular}{|c|c|c|c|c|}
\hline $\begin{array}{r}\text { Jabr } \\
\end{array}$ & Beobachter & $\begin{array}{l}\text { Zahl } \\
\text { der } \\
\text { beob. }\end{array}$ & $\begin{array}{c}\text { Helligkeit } \\
\text { in miltl. Opy. } \\
\text { bei verschwund. } \\
\text { lling }\end{array}$ & Bemerkungen \\
\hline 1717 & Kireh & 1 & 1.15 & Schiitznng \\
\hline 1803 & Olbers? & 1 & $0.5 \mathrm{~S}$ & s \\
\hline $1852-1858$ & Seidel & s & 1.04 & Photom. Messungen \\
\hline $1858-1880$ & Sehmidt & 493 & 1.04 & Schïitungen \\
\hline $1862-1865$ & Zöllner & 14 & 0.95 & Photom. Messungen \\
\hline $1875-1882$ & Kononowitsch & 16 & 0.52 & , $\quad$. \\
\hline $1877-1891$ & Miüler & 252 & 0.55 & , \\
\hline
\end{tabular}

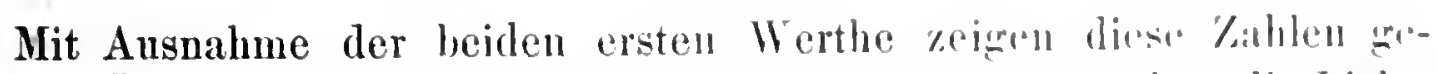
niggende Üebereinstimmung und rechefertignen dens Sulluss, dists die Licht-

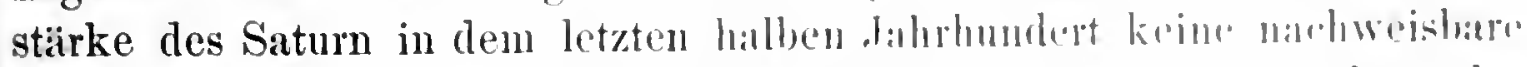

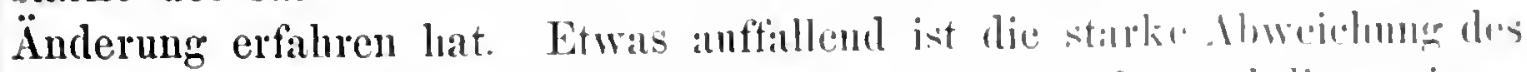
Olbers'sehen Werthes; dnel wiirde es gewant sein, anf firmul dirser rinen.

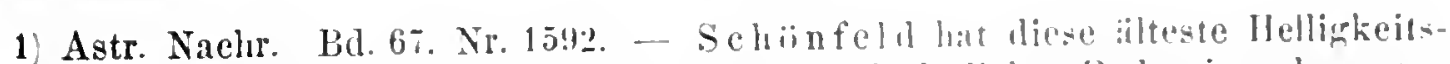
beobachtung des Saturn anfgefunden und mit allen erforlorlidicu Rolucrionselementen veröffentlicht.

2) v. Zachs monatliche Correspondenz. bid. ?. 1.30ti. 
noch dazu etwas unbestimmten Helligkeitsangabe auf eine besonders grosse Lichtstärke des Saturn am Anfange des 19. Jahrhunderts sehliessen zu wollen.

Die Reflexionsfähigkeit des Saturn ist grösser als die der anderen Planeten, ausgenommen allein die Venus. Unter Annahme des Werthes 0.88 für die Liehtstärke in mittlerer Opposition bei verschwundenem Ringe ergeben sich die Albedowerthe:

$$
\begin{aligned}
& A_{1}=0.721 \text { (Lambert'sche Definition), } \\
& A_{2}=0.961 \text { (Seeliger'sche Definition). }
\end{aligned}
$$

Aus dem hohen Reflexionsvermögen kann man mit einiger Wahrscheinlichkeit folgern, dass Saturn voraussichtlich ebenso wie die Planeten Jupiter und Venus mit einer sehr dichten Atmosphäre umgeben ist. Dafuir sprieht ja anch der Umstand, dass keine deutlichen Gebilde auf seiner Oberfläche zu erkennen sind.

Genauere Messungen über die Vertheilung der Intensität auf der Saturnscheibe sind nicht bekamnt. Allgemein gilt die Äquatorealzone als die hellste. Nach Seechi soll dies von dem dureh den Ring auf die Kugel reflectirten Lichte herriihren; doch ist dieser Annahme von verschiedenen Seiten, namentlich von Dawes, widersprochen worden.

Anch iiber das Helligkeitsverhältniss von Ring und Kugel existiren keine sicheren Angaben. Im Allgemeinen nimmt man an, dass der Ring ein intensiveres Licht besitzt als der Planet, und diese Ansicht wird insbesondere durch die photographischen Anfnahmen bestärkt. Über die Intensitaitsvertheilung auf dem Ringsysteme sind zwar Messungen nicht vorhanden, doch gilt es nach den Beobachtmgen von W. Struve ${ }^{1}$ ) als sicher, dass der änssere Ring bedeutend weniger Glanz hat als der innere, und dass der letztere nach dem Planeten zu weniger seharf begrenzt ist und ein matteres Aussehen hat. Etwas ansführlichere Angaben daruber sind in nenerer Zeit von Trourelot²) gemacht worden. Nach ihm befindet sich die allerhellste Stelle auf dem inneren Ringe an der Haupttheihnng, dann folgt in Bezng anf Intensitait eine daran grenzende Zone nach dem I'laneten ru, daun die an die Haupttheilung angrenzende Zone des änsseren Ringes; die allerschwïchste Region des ganzen Systems endlich ist die dem Plameten zunächst befindliche.

1) Astr. Nachr. Bd. 5, Nr. 97.

2 American Journal of science. 3 Ser. Vol. 11, p. 447. 


\section{Die Saturusatelliten.}

Von den aeht Monden des Saturn bietet der an Weitesten rom Planeten entferute Japetus in photometrischer Beyichung das herrorragendste Interesse, weil bei ihm ein regelmissiger periodischer Lichtweehsel in Zusammenhange mit seiner Rotations\%eit ziemlich sicher lachgewiesen ist. Schon der iltere Cassin i hatte im Jahre 1673, \%wei dahre nach Entdeckung dieses Trabanten, die Beobachtung gemacht, dass derselbe in der Nibe der grössten östlichen Digression wilırend eines ganzen Monats unaichtbar blieb, dagegen in der grössten westlichen Digression gut zu sehen war. Ïhnliche Liehtunregehnissigkeiten sind spiater anch von dem jungeren Cassini wahrgenommen worden und ebenso ein Jahrlundert spaiter ron Bernard in Paris, welcher fand; dass dapetus ron der östlichen Digression bis zu seiner unteren Conjunction nur mit Muhe sichtbar ist, dass er dagegen seinen grössten Glan\% vor der westliehen Digression erreieht und noch gut siehtluar ist in der Niihe der oberen Conjunction. W. Herschely hat diese Lichtschwankungen noch etwas genaner verfolgt. Nach ihm ist Japetus : 1 h hellsten, wenn er sich in dem Bogen seiner Bahn zwischen $65^{\circ}$ und 129 (gez:ihlt von dem Durchgange dureh die untere Conjunction) befindet; es kommt daun an Lichtstiirke etwa dem Trabanten Jitan gleich. An schwichsten erscheint er nach dem Passiren der Opposition bis wieder zul unteren Conjunction: er ist damn weniger hell als Rhea mul iabertrifit kamm Dione nul thetrs. Der Untersehied zwischen Maximal- und Minimalhelligkeit des dalprotus betrïgt nach Hersehel fast drei volle lirissendlassen.

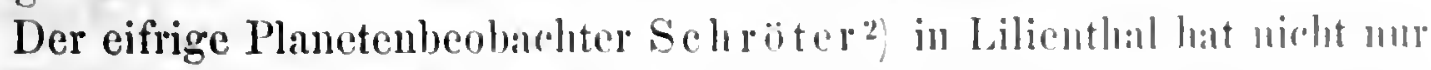
die Herschel'sehen Beobachtungen in Brour anf dipletus in der IJanpt-

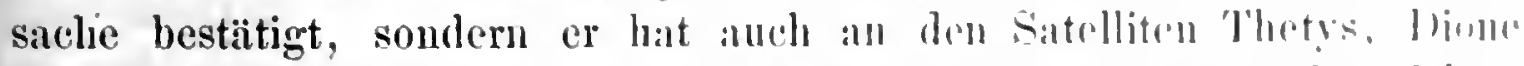

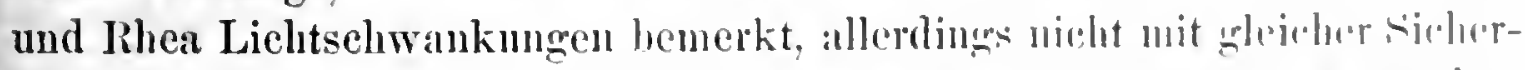

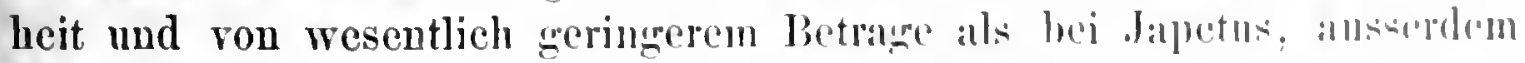
mit dem Untersehiede, diss die grijste Linhtstiirke niwht in dir westliche, sondern in die östliche Ijigresision trift"t.

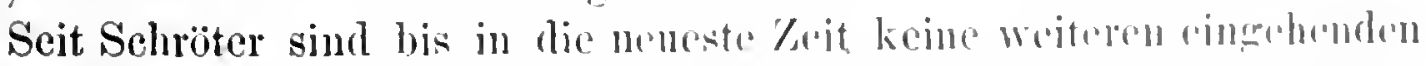

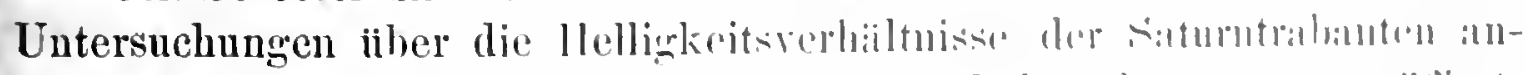

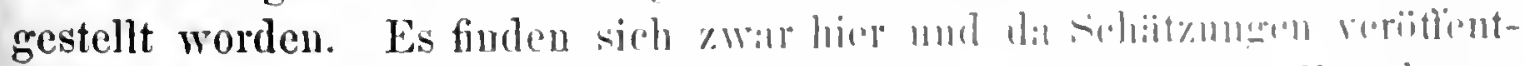

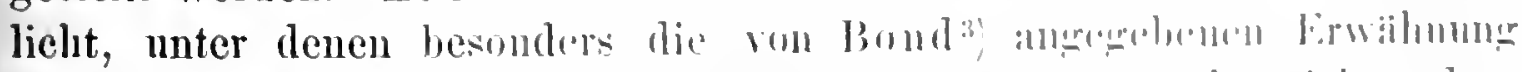

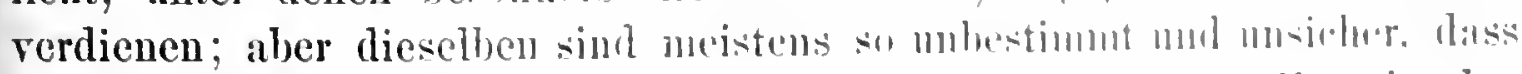

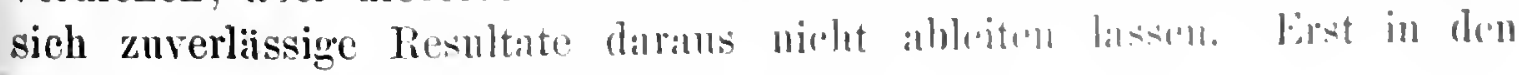

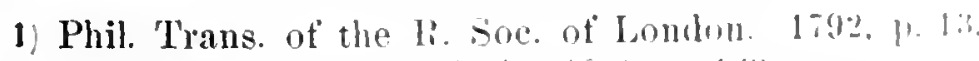

2. Berliner astr. Jahrbuch tiir 1500 . p. J6!?.

3) Annals of the Astr. Ms. of Harvard College. Vol. 2. part I 
Jahren 1877-1879 hat Pickerin ${ }^{\circ}{ }^{1}$ ) sorgfältige photometrische Messungen an allen acht Satelliten ausgefiihrt, indem er sie mit dem sternartig verkleinerten Bilde des Saturn verglich. Die Pickering'schen Resultate sind in der folgendeu Zusammenstellung mitgetheilt, und zwar erstens die aus den Messungen abgeleiteten Grössenunterschiede zwischen Trabanten und Planet ohne Ring, wobei die Reductionen auf versehwundenen Ring der Zöllner'schen Formel entnommen sind, feruer die mittleren Oppositionsgrössen der Trabanten, berechnet unter der Annahme von 0.88 für die Oppositionsgrösse von Saturn ohne Ring, und endlich die ans den photometrischen Werthen berechneten Durchmesser der Trabanten, wenn die Reflexionsfähigkeit derselben gleich der des Saturn angenommen ist.

\begin{tabular}{|c|c|c|c|c|}
\hline $\begin{array}{l}\text { Nummer und } \\
\text { Name des } \\
\text { Trabanten }\end{array}$ & $\begin{array}{l}\text { Grössendiff. } \\
\text { gegen } \\
\text { Saturn ohne } \\
\text { Ring }\end{array}$ & $\begin{array}{c}\text { Mittlere } \\
\text { Oppositions- } \\
\text { grösse }\end{array}$ & $\begin{array}{l}\text { Scheinbarer } \\
\text { Durchmesser in } \\
\text { mittl. Entf. } \\
\text { Trabant-Sonne }\end{array}$ & $\begin{array}{c}\text { Dorchmesser } \\
\text { in } \\
\text { Kilom. }\end{array}$ \\
\hline 1. Mimas. & 11.91 & 12.79 & $0.06 \mathrm{~S}$ & 470 \\
\hline 2. Enceladus & 11.40 & $12.2 S$ & 0.086 & 594 \\
\hline 3. Thetys. & 10.46 & 11.34 & 0.132 & 916 \\
\hline 4. Dione. & 10.57 & 11.45 & 0.126 & 871 \\
\hline ๖. Rhea & 9.88 & 10.76 & 0.173 & 1197 \\
\hline 6. Titan. & 8.50 & $9.3 \mathrm{~S}$ & $0.32 \pi$ & 2259 \\
\hline 7. Hyperion & 12.81 & 13.69 & 0.045 & 310 \\
\hline S. Japetus. & 10.80 & $11.6 \mathrm{~s}$ & 0.113 & 783 \\
\hline
\end{tabular}

Bei den Satelliten Nr. 1-i hat Pickering keinerlei Lichtschwankungen ron periodischem oder unregelmässigem Charakter constatiren können, dagegen ist durch seine Messungen der periodische Lichtwechsel beim letzten Trabanten mit rollkommener Sicherheit nachgewiesen und die Form der Lichteure recht genau bestimmt worden. Maximum und Minimum der Helligkeit treten, wie schon die friiheren Beobachter gefunden hatten, wahe zu den Zeiten der srössten westlichen resp. östlichen Elongation ein, dagegen folgt aus den photometrischen Messungen fiur den Cresammtbetrag der Lichtschwankung ein viel kleinerer Werth als aus den Schätzungen Herschels; Pickering findet zwischen Maximum und Minimun nur eine Grössendifferenz von 1.36. Zur Erklärung des Lichtweehsels nimmt Pickering ebenso wie Cassini, Herschel, Schröter und Andere an, dass der Satellit in derselben Zeit um seine Axe rotirt, in welcher er einen Unulauf um den Saturn rollendet, und dass er auf verschiedenen Seiten das Somnenlicht sehr ungleich reflectirt; er hält es ferner nicht tiin ansgeschlossen, dass die Gestalt des Trabanten merklich ron der Kugelgestalt abweicht und daher bei der Rotation als rerschieden grosse

Anuils of the Astr. Obs. of Ifarrard College. Vol. 11, part II, p. 24 . 
Scheibo erseheint. Zur Darstellung der beohachteten lichtenscheinumgen des Japetus ist von Pickering eine Interpolationsfornel volu der Gentalt: $h=a+b \sin v+c \cos v+d \sin 2 v+e \cos 2 v$ versucht worlen, wo $v$ die Linge des Trabanten in seiner Bahn, von der Opposition an greshlhlt, bedeutet. Ist dio mittlere Liehtstiirke des Japetus mit 100 bezeiclunet, so folgt aus den Pickering'schen simmntlichen Messungen, weun die Husserst klein sich ergebenden Coefficienten von $\cos v$ und sin $2 v$ vernnehlissigt werden, die Gleichung:

$$
l=100-50 \sin v+10 \cos 2 v,
$$

welehe sich den Beobaehtungen sehr gut anschliesst.

Fur verseliedene Stellungen des Japetus in seiner Balın ergeben sich ans dieser Formel die folgenden Helligkeiten, ansgedrllekt in Procenten der mittleren Lichtstïrke des Trabinten, und ausserden noch in Grössenclassen.

\begin{tabular}{|c|c|c|}
\hline I.ångo & $\begin{array}{l}\text { Lichtstarke } \\
\text { in l'roce dur } \\
\text { mittl. Liclitst. }\end{array}$ & $\begin{array}{l}\text { von Japetus } \\
\text { in mittleren } \\
\text { Ojpros.-Gröson }\end{array}$ \\
\hline $0^{\circ}$ & 110 & 11.55 \\
\hline 30 & 80 & 11.92 \\
\hline 60 & 52 & 12.39 \\
\hline 90 & 10 & 12.67 \\
\hline 120 & 52 & $12.34)$ \\
\hline 150 & S11 & 11.92 \\
\hline $1 S 0$ & 110 & 11.55 \\
\hline 210 & 130 & 11.10 \\
\hline 240 & 138 & 11.333 \\
\hline 270 & 140 & 11.31 \\
\hline 300 & $1: 18$ & $11.38 \%$ \\
\hline $3: 30$ & 130 & 11.40 \\
\hline
\end{tabular}

10. Uranus.

Alles, was wir uber die Lichtstiirke lles l'lineten I'ranms wiston, he-

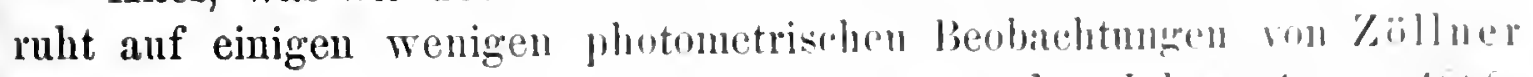

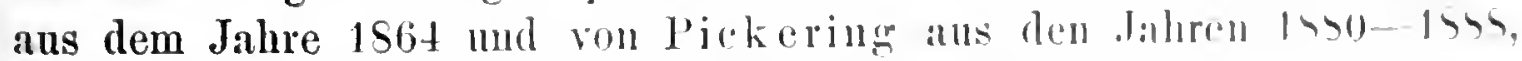
ausserdem auf einer umfingreicheren Messumgereihe, die in len dahren 1878-1888 ron mir in Potsdan auszefilhrt worden ist. Ans frliherer \%eit sind nur ganz vereinzelte, nicht sehr zuverliisige Hellighkeitsschiitzmugen anfzufinden. 
Die Oppositionshelligkeit des Uranus kann wegen der Excentrieitäten der Erd- und Uranusbahn um 0.4 Grössenclassen variiren und erreicht im Maximum, wie es im Jahre $1884^{\circ}$ der Fall war, die Grösse 5.5 bis 5.6. Die kleinste Helligkeit zeigt der Plánet in der Nähe der Conjunetion; er sinkt dann unter Umständen bis zur Grösse 6.3 hinab. Es geht daraus hervor, dass der Planet zu allen Zeiten, falls er nicht zu tief am Horizonte steht, mit blossen Augen aufgefunden werden kann.

Eine Einwirkung der Phase anf die Lichtstärke des Uranus ist bei dem äusserst kleinen Werthe $(3.1)$, den der Phasenwinkel im Maximum erreicht, von vornherein so gut wie ausgeschlossen. Zöllner hat zwar ans seinen Messungen eine derartige Einwirkung vermuthet, und in der That zeigen diese Beobachtungen ein Anwaehsen der Helligkeit nach der Opposition zu; indessen ist die Zahl der Messungen viel zu klein, und die Abweiehungen zwischen den einzelnen Werthen bleiben durchaus innerhalb der Beobachtungsungenanigkeit, sodass eine sichere Entscheidung nieht zul treffen ist. Ans den viel zahlreicheren Potsdamer Messungen lässt sich jedenfalls keine Spur eines Phaseneinflusses nachweisen, und man hat daher zunächst anch keine Veranlassung, beim Uranus ein gänzlieh abnormes Verhalten im Vergleich zu deu iibrigen Planeten anzunehmen.

Fuir die mittlere Oppositionshelligkeit des Uranus liefern die bisherigen photometrischen Bestimmungen die folgenden Mittelwerthe:

\begin{tabular}{c|c|c|c}
\hline Jahr & Beobachter & $\begin{array}{c}\text { Zahl } \\
\text { der } \\
\text { Beob. }\end{array}$ & $\begin{array}{c}\text { Nittlere } \\
\text { Oppositions- } \\
\text { helligkeit }\end{array}$ \\
\hline 1564 & Züllner & 4 & 5.73 \\
$1550-1$-1sss & Pickering & 6 & 5.66 \\
$1575-1585$ & Nuiller & 93 & 5.56
\end{tabular}

Zu der Potsdamer Reihe ist noeh zu bemerken, dass, weun die Beohachtmugen zn einzehnen Jahresmitteln zusammengefasst werden, diese nuter einander grössere Abweichungen zeigen, als man nach der Sicherheit der einzelnen Messungen erwarten sollte, und dass sich insofern eine gewisse Gesetzmissigkeit zu erkennen giebt, als die Helligkeit von 1s7s an bestiindig znzunehmen scheint bis zn einem Maximnm Anfang der stler tahre, und dam wieder zu einem Minimum gegen das Ende des Jahrzehnts herabsinkt. Ob diese Helligkeitsänderungen als reell anzusehen sind, bleibt noch fraglich; immerhin ist bemerkenswerth, dass ein :alnliches Terhalten in noch stiirkerem Grade beim Jnpiter nachgewiesen ist und anch bei Mars und Safurn schwach angedentet zu sein scheint, so dass man anf die Termuthung kommen könnte, dass eine gemeinsame 
Ursache, etwa ein Lichtweehsel der Sonne, zu Grunde lage. Bei den Pickering'schen Uranusbeobachtungen ist es elienfalls aufiallend, dass die zwei Messungen ans dem Jalıre 1851 eine besonders grosse Helligkeit ergeben, dagegen die Messung ans dem Jahre 1859 eine sehr geringe.

In Bezug anf das Reflexionsvernbgen :hnelt Uranus am meisten dem Jupiter. Ans den Potsdamer Helligkeitsangaben resultiren die Albedowerthe:

$$
\begin{aligned}
& A_{1}=0.604 \text { (Lambert'sche Definition), } \\
& A_{2}=0.505 \text { (Seeliger'sche Definition). }
\end{aligned}
$$

Wahrscheinlich ist Urauns, ebenso wie Jupiter, mit einer dichten und ansgedehnten Atmosphaire mugeben, was anch ans den Beohachtungen seines Spectrums folgt, in welchem eine Anzahl von kraftigen Absorptionsstreifen $z \mathbf{u}$ erkennen ist.

Seeliger') hat noeh auf einen Punkt hingewiesen, der beim Ura!us Beachtung verdient. Bekanntlich ist die Frage noeh nicht entschieden, ob die Rotationsaxe des Planeten mahe in rer Ekliptik liegt, und ferner ob derselbe eine merkliche Abplattung besityt. Wire dis Enstere der Fall, und erreichte ansserdem die Abplattung deu von einigen Beobachtern angegebenen Werth 0.1, so misste nach Seeligers Berechumug die Helligkeit des Uranus je nach der Stelhng in seiner Bahn um etwa 0.17 Grössenclassen versehieden sein können, ein Betrạ́, der durch sehr sorgrfailtige Messungen noch schr wohl eonstatirt werlen könnte. Maximum und Minimum der Liehtstirke wiirlen um $\frac{1}{4}$ der Lmlanfszit des Lraun, also etwa um 21 Jahre, anseinander liegen missipll. Die bisheriggen

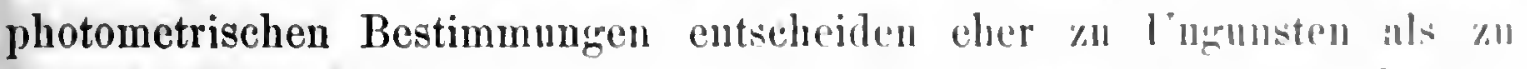
Gunsten dieser Hypothese; doeh ist dies nicht massonolume, weil date lice-

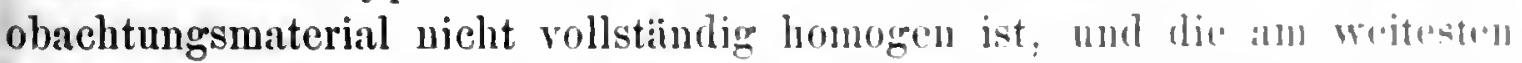

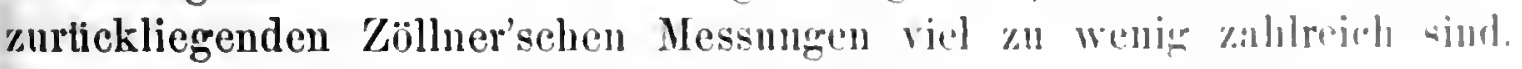
Die Frage verdient jedenfalls noch weiter verfolgt zil werden.

\section{Die Lranussatelliten.}

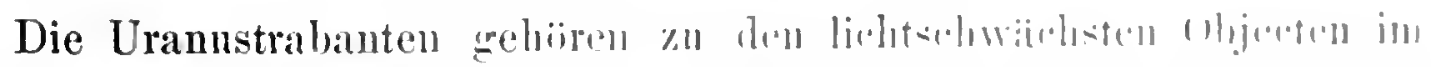

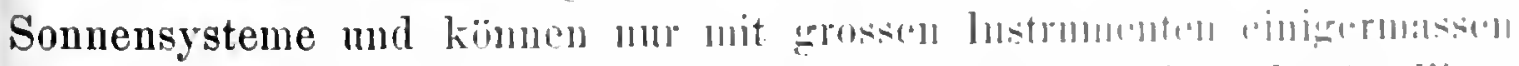

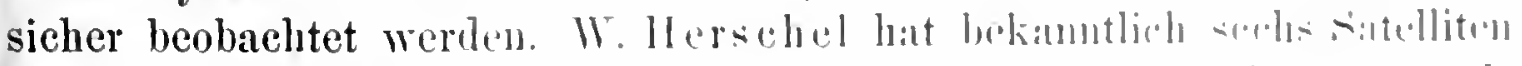

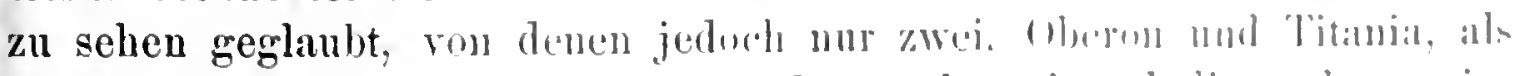

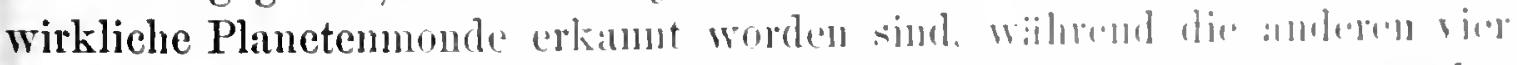

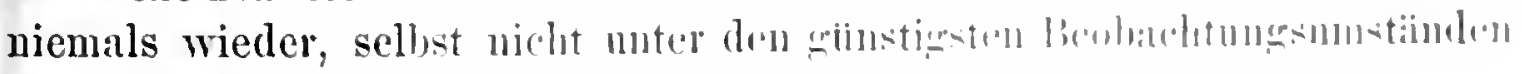

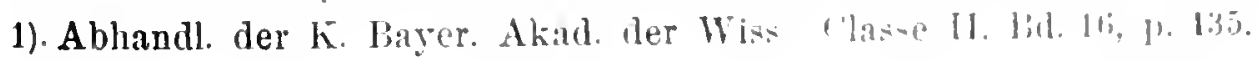


und mit den mächtigsten Instrumenten, aufgefunden werden konnten. Dagegen lat Lassell anf Malta im Jahre 1851 zwei weitere Trabanten des Uranus entdeckt, die dem Planeten noch näher stehen als Titania und Oberon, und die von J. Herschel die Namen Ariel und Umbriel erhielten. Nach Lassells Ansicht können sich die vermeintlichen Satellitenbeobachtungen von W. Herschel nur anf Fixsterne bezogen haben, und es gilt ihm als ganz unwahrscheinlich, dass es mehr als vier Uranussatelliten geben sollte. Dieselbe Meinung ist später anch von Nercomb ausgesprochen worden.

Ueber die Helligkeiten der vier Trabanten existiren nnr ganz spärliche Angaben. Lassell schätzt die äusseren Monde etwa doppelt so hell als die inneren. Newcomb taxirt Ariel etwas heller als Umbriel und letzteren ungefähr halb so hell wie Titania. Aus Schätzungen von Hall und Holden') folgt, dass Titania und Oberon nahe gleich hell sind, vielleicht der erstere sogar ein wenig heller als der letztere, ferner dass Titania gerriss doppelt so lichtstark ist wie Ariel, und Umbriel ein wenig schwächer als dieser.

Die einzigen photometrischen Messungen, welche bekannt geworden sind, ribuen ron Pickering ${ }^{2}$ ) her, welcher die beiden äusseren Trabanten mit Uranns rerglichen und im Mittel ans allen Beobachtungen die folgenden Grössendifferenzen zwischen Satellit und Planet gefunden hat:

\section{fiir Titania 8.79 und fiir Oberon 8.95.}

Darans ergeben sich, wenn die mittlere Oppositionsgrösse ron Uranus zn 5.86 angenommen wird, für die mittleren Oppositionsgrösseu der Trabanten die Werthe 14.65 (Titania) and 14.81 (Oberon). Fiir die Durchmesser dieser kleinen Himmelskörper resultiren endlich unter Voraussetzung. gleicher Albedo ron Planet und Trabant die Werthe $942 \mathrm{~km}$ (Titania) und $875 \mathrm{kin}$ (Oberon).

Bei Ariel hat Newcomb Helligkeitsinderungen rermuthet, weil er diesen Trabanten trotz der giinstigsten Luftrerhältuisse wiederholt nicht mesehen hat, wïhrend Lmbriel bei weniger rortheilhafter Stellung gut sichtbar blieb. Nach seinen Angaben wiirde die grösste Helligkeit ungeführ beim Positionswinkel $0^{\circ}$, dagegen ein Minimum bei $180^{\circ}$ zu erwarten sein. Auch leei Titania sollen naeh H. C. Toggel Helligkeitsschwankungen rorkommen. Doch sind naturgemaiss bei den iberaus schwierigen sichtharkeitsrerhïltnissen der Trabanten alle derartigen Vermuthungen mit einer gerrissen Torsicht anfzunehmen.

1 American Journal of science. 3. Ser. Yol. 15. p. 195.

2 Ammals of the Astr. Obs. of Harrard College. Vol. 11, part II. p. $2-1$. 


\section{Neptun.}

Von den Hauptplaneten ist Neptun der einzige, welcher niemals nir das blosse Auge sichtbar ist. Er hat die Helligkeit eines Stenes 7. bis 5. Grüsse, und die Lichtïnderungen, welche durch die weehselnden Eutfernungen des Planeten von Sonne und Erde hervorgebracht werden, sind so geringfugig, dass sie dureh blosse Schitzungen kaum erkenubar sind und nur durch sorgfiltige photometrische Messungen nachgewiesen werden können; sie betragen im Maxinum während eines ganzen Umlaufes des Neptun um die Soune nur 0.23 Grössenclassen.

Der Phasenwinkel bleibt beim Neptun stets kleiner als $2^{\circ}$, und es ist daher eine Einwirkung der Phase anf die Lichtstirke noch viel weniger denkbar als beim Uranus.

Aus den bisher bekaunt gewordenen photometrischen Bestimmungen, welche sich auf einige Messungen von Zöllner und Pickering, sowie auf eine grössere Messungsreihe in Potsdam beschrïnken, ergehen sieh die folgenden mittleren Oppositionsurö̈ssen des Neptun:

\begin{tabular}{|c|c|c|c|}
\hline Jahr & Beobachter & $\begin{array}{l}\text { Zoabl } \\
\text { der } \\
\text { Beob. }\end{array}$ & $\begin{array}{l}\text { Millere } \\
\text { Oppositions. } \\
\text { helligkoit }\end{array}$ \\
\hline 1864 & Zijllner & 4 & 9.16 \\
\hline $1882-1855$ & Pickering & 25 & 7.71 \\
\hline $1578-1557$ & Miiller & 135 & i.titi \\
\hline
\end{tabular}

Der erste Werth weicht rou den beiden anderen viel stïrker alh. als naeh der Genanigkeit der photometrischen Bestimmungen zn erwarten ist, nnd man wird daher, falls man njeht bei den Ziillner schen Beobichtungen eine Verweehslnng oder irgend ein anderes Versehen annehmen will, zu dem Schlusse gredrängt, dass die ILelligkeit des Neptun scit 1Stil sehr beträchtlich angewachsen ist. Imnerhalb, des Zeitranms, welchen die Potsdamer Messungen umfassen, lassen sich Ilelligkeifinderumben nicht mit Sicherheit erkennen, es ist höehstens anzufuluren, dass dir ein\%elnen

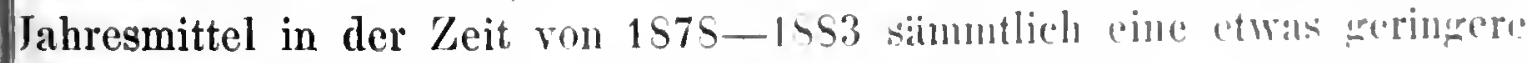
Lichtstairke ergeben, als in der Zeit ron 1SS1-1S47.

Nach Helligkeitssehätzungen bon Maxwell Hally soll Xeptun : In Ende des Jahres 1853 Lichtinderungen his zum Betring ron beinahe biner ganzen Grössenclasse gezeigt habcu, welche sich durch Annahmo siner nahezu achtstindigen Rotationsdaner des Planeten erkliirun liesicun.

1) Monthly Notices. Vol.44, p. 257. 
Es ist von mir nachgewiesen worden 1), dass die Hall'șchen Angaben weder mit den Potsdamer Messungen noch mit denjenigen Pickerings in Einklang zu bringen sind, und es ist ausserdem durch eigens zu diesem Zwecke in Potsdam ausgeführte Beobachtungen in den Jahren 1884 und 1885 dargethan, dass jedenfalls zu diesen Zeiten keine Helligkeitsschwankungen von kurzer Periode vorgekommen sind. Entweder beruhen also die Hall'schen Werthe auf irrigen Schätzungen, oder es müsste die g:ïzlich unwahrscheinliche Voraussetzung gemacht werden, dass nur während einer ganz kurzen Zeit periodische Lichtänderungen beim Neptun stattgefunden haben.

Für die Albedo des Neptun giebt Zöllner die Zahl 0.465 an; doch ist dieser Werth offenbar zu klein, entsprechend der verhältnissimässig geringen Lichtstärke, welche Zöllner für den Neptun gefunden hat. Aus den Potsdamer Beobachtungen ergeben sich die folgenden Albedowerthe:

$$
\begin{aligned}
& A_{1}=0.521 \text { (Lambert'sche Definition), } \\
& A_{2}=0.694 \text { (Seeliger'sche Definition). }
\end{aligned}
$$

Danach reflectirt Neptun das Somnenlicht beinahe ebenso stark wie Uranus, und es ist daher sehr wahrscheinlich, dass die Atmosphären der beiden Planeten eine gewisse Ähnlichkeit miteinander haben. Dafür spricht anch der Umstand, dass im Spectrum des Neptun dieselben Absorptionsstreifen beobachtet worden sind, wie in demjenigen des Uranus.

\section{Der Neptunsatellit.}

Bisher ist nur ein einziger Trabant des äussersten Planeten bekannt, welcher im Jahre 1847 ron Lassell entdeckt wurde. Mehrfach ist die Vermuthung ausgesprochen worden. dass noch ein zweiter Trabant rorhanden ist; doch hat sich dies nicht bestätigt, und Lassell ist der festen Überzengung, dass, wenn wirklich ein zweiter Satellit existirt, dieser im Verhültniss zum ersten mindestens so lichtschwach sein muss, wie die Satmrnsatelliten Dione und Rhea im Verhältniss zn Titan.

Die Helligkeit des Neptuntrabanten wird allgemein 13. bis 14. Grösse reschïtzt; er ist ein ziemlieh schwieriges Object, aber jedenfalls bedeutend leiehter zu sehen als die beiden imneren Urannssatelliten. Pickering ist es gelungen, denselben an 7 Tagen im Jahre 1578 photometrisch zu messen; er findet im Nittel fiir den Helligkeitsunterschied zwischen Teptun und seinem Trabanten den Werth 5.93 Grössenclassen, und daraus

1. Publ des Astrophys. Obs. zu Potsdam. Bd. s, p. 351. 
folgt fur die mittlero Oppositiousgrosse des Satelliten, wenn diejenige des Neptun zu 7.66 angenommen wird, der Werth 13.59. Der Durehunesuer des Trabanten ist demzufolge, wenn man demselhen gleiche All;edo wie dem Planeteu zuerkennt, gleich $3630 \mathrm{~km}$; er wllrde also fust dem ersten Satelliten des Jupiter an Grösse gleichkommen.

\section{Capitel IV.}

\section{Die Cometen und Nebelflecke.}

So fruchtbringend sich die Anwendung der Spectralanalyse schon seit der kurzen Zeit ihres Bestehens fur die Erkenntuiss der Natur der Cometen erwiesen hat, so wenig hat die Photometrie trot\% ilhres hohen Alters zur Aufklärnng der einfachsten und wichtigsten Fragen hinsichtlich der physischen Beschaffenheit dieser merkwirdigen Himmelskirper boigetragen. Die vorhandene Litteratur iber die Cometenhelligkeiten ist mgemein dürtig. Nur von einer versehwindend kleinen An\%all aller bisher erschienenen Cometen besitzen wir zusammenhingende Benbahtuntreseihen. und diese wenigen, zumeist anf blossen sohitmmen horuhenden An-

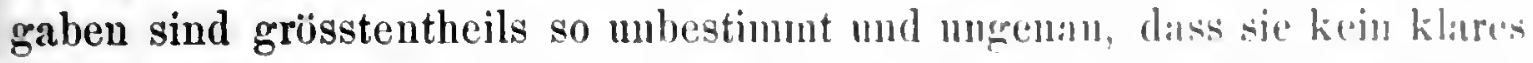

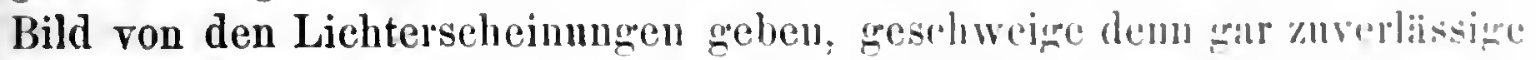
Schlisse anf die Natur der Cometen gestatten. Von wirkfichull photu-

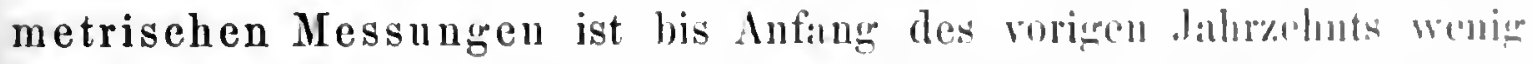
oder nichts bekannt geworden. Die Versurhe, dir seithlen ron ver-

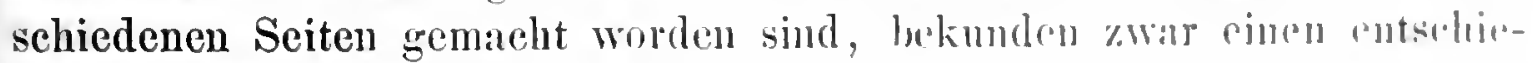

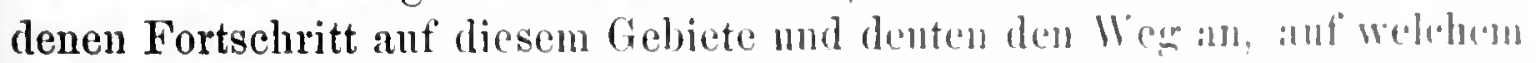

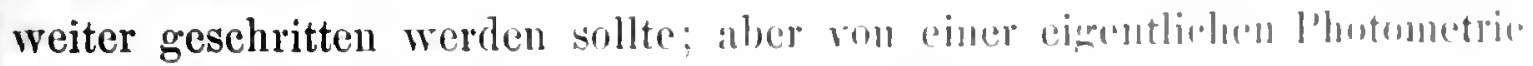
der Cometen kann hente noch katum die lioh sein.

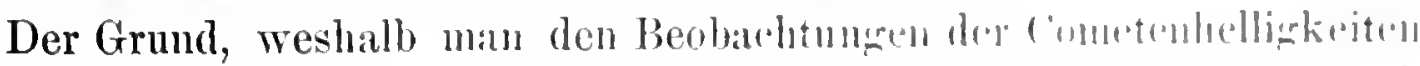

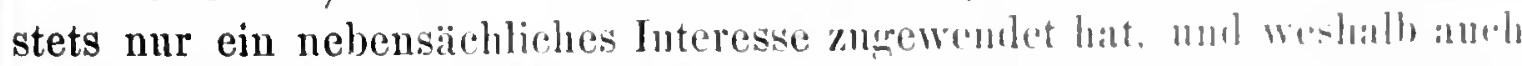

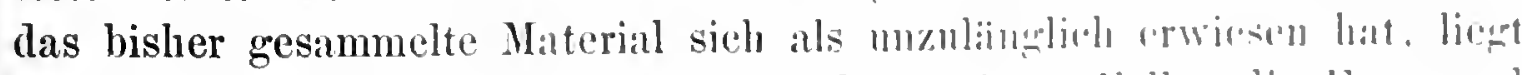

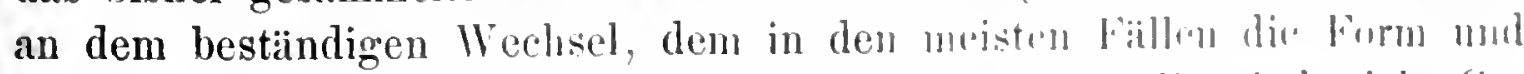
das ganze Ausselien eines Cometen unterworfen int. los sind vich rin-

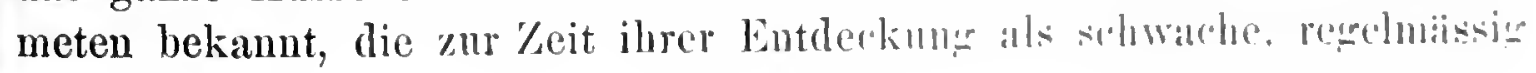


gestaltete Nebelflecke mit mehr oder weniger starker centraler Verdichtung erschienen, die sich aber in der Nähe des Perihels zu einer glänzenden Erscheinung mit blendend hellem fixsternartigen Kern, mit einer ausgedehnten ebenfalls hellleuchtenden Umhüllung und einem mächtigen Schweif entwickelten. Bei vielen Cometen, namentlich bei den der Sonne nahe kommenden, traten hierzu noch zeitweilig fächerfờrmige Ausstrahlungen aus dem Kern, die das Anssehen des Cometen vollständig verïnderten. Die Unmöglichkeit, solche gïnzlich heterogenen Erscheinungen miteinander zu vergleichen, hat zweifellos die Astronomen von einer systematischen Beobachtung des Lichtwechsels der Cometen abgeschreckt; sie ist auch Schuld daran, dass von den wenigen vorliegenden Beobachtungsreihen manche als gänzlich werthlos zu bezeichnen sind, und es darf fraglich erscheinen, ob es iiberhaupt jemals gelingen wird, für so extreme Lichterscheinmngen ein gemeinschaftliches einwurfsfreies Mass zu finden.

Das Hauptinteresse hat sich von jeher anf die viel umstrittene Frage concentrirt, ob die Cometen eigenes Licht besitzen oder uns, wie die iibrigen Glieder des Somnensystems, nur reflectirtes Licht zusenden. Während Newton und Olbers die Cometen für dunkle Weltkörper hielten, nahmen Herschel und Schröter dieselben als selbstlenchtend an, und beide Ansichten sind noch bis in die neneste Zeit mit lebhaften Gründen gegen einander vertheidigt worden. Als Hauptargument gegen die erstere wurde friiher liäufig geltend gemacht, dass die Cometen bei ihren stark wechselnden Stellungen zu Sonne und Erde keine Lichtphasen wie die Planeten zeigen. Es ist zwar mehrfach das Vorhandensein ron Phasenerscheinnngen behauptet worden, so z. B. von De la Hire am Cometen des Jahres 1682, ron Cassini am Cometen ron 1744 und besonders ron Cacciatore am Cometen ron 1819; aber alle diese Wahrnchmungen sind keineswegs als verbiirgt anzusehen und lassen sich dureh unregelmässige Gestalt der betreffenden Cometenkerne migezwnngen erklïren. Man kam es in der That heute als erwiesen betrachten, dass die Cometen keine Spur von Lichtphasen zeigen. Dies ist aber durchaus kein directer Beweis gegen die Annahme ron reflectirtem Sonnenlicht. Denn es ist schon längst durch zahlireiche Beobachtungen, insbesondere bei Bedeckungen ron Fixsternen durch Cometen, festgestellt, dass die Cometenmaterie nicht nur im Schweif, smdern anch im Kern und der umgebenden Hiille ausserordentlich dimm scin muss, so dass ron einer eigentlich festen Masse ähnlich wie z. B. hei den Planeten nicht die Rede sein kamn. Sind aber die Cometen, wie es als sicher anzunehmen ist, Aggregate ron zahllosen getremnten Partikelchen, die in Kern an dichtesten zusammengedrängt sind, so ist es nicht zu verwumlern, dass wir an ihnen, selbst wemn sie nur Sonnenlicht reflectirten, keine regehiasigen Plasenerscheinungen bemerken. 
Man hat ferner eine Entscheidung Ulher den Ursprung des Conetenlichtes ans den Helligkeitssehatzungen bei verschiedenen Entferumngen von Sonne und Erde zu gewimnen gesucht. Wenu ein Comet laliglich eigenes Licht ausstrahlte (und \%war von unverinderlicher lenelitkraft), so mtisste nach den Grundsät\%en der Photometrie die beobachtete Gesammtintensitit stets dem Werthe $\frac{1}{J^{2}}$ proportional sein, wo $I$ die lintfenumb Comet-Erde bedentet; die beobachtete Flächenintensitit musste in diesem Falle bei allen Entfernungen die gleiehe sein. Wäre dagegen der Comet nur dureh reflectirtes Sonnenlicht sichthar, so mtlsste (rorausgesetzt dass die Dimensionen desselben unverindert bliebeu) die beobachtete Gesammtintensitiit ebenso wie bei den Plancten proportional der Grösse $\frac{1}{r^{2} d^{2}}$ sein, wo $r$ die Entfernung des Cometen ron der Somne ist; dic beobachtete Flïehenintensitiit wiire dann proportional der Grösse $\frac{1}{r^{3}}$.

Es liegt nun der Gedanke nahe, ans den Helligkeitsheobachtungen. je nachdem sich dieser oder jener Ausdruck denselben an Besten :usehliesst, zu Gunsten der einen oder anderen Hypothese zu entseheiden. Indessen ist die Sache in Wirklichkeit keineswegs so eiufuch. Dem abgesehen davon, dass, wenigstens bei den meisten bisherigen Conetenbeobachtungen, eine gewisse Unklarheit dartiher herrscht, ol, die Angraben sich auf die Gesanntintensitait oder die Fliichenlulligkejt beziehen, so wird anch häufig dureh die Gestaltsändermugen der Cometern. besonders aber dureh plïtzliche Lichtansbriiche, fler rexpluminsige Verlant der Lichteurve so wesentlich modificirt, dass von vornherein jedr Hollinm:-

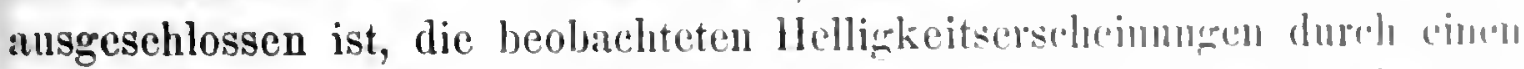
der obigen einfachen Ansdricke darzustellen. Die 'Tlustsallhe, diss an einigen Cometen eigenthiimliche Lichtentwicklumpen in Furm von Ans-

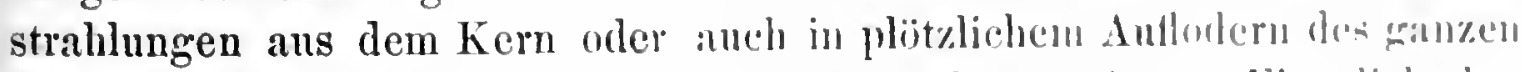

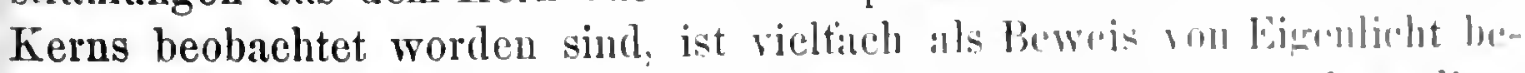

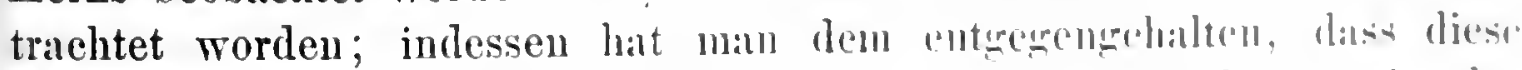

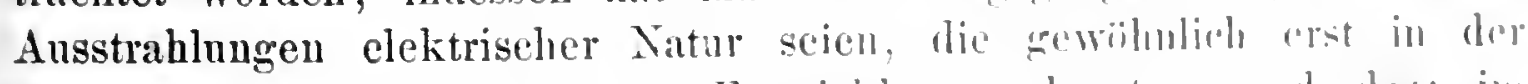
Sonnennähe des Cometen zur Entwicklmug trelangtoll, und diss im Übrigen sehr wohl das Cometenlirht in der Hanptsild he onler ansinghlies:lich reflectirtes Sommenlicht sein kimnte.

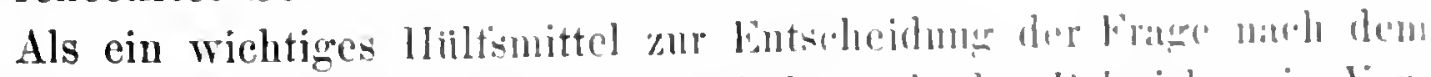
Ursprunge des Cometenlichtes ist vielfach aluch lats l'ulariaknp in Von-

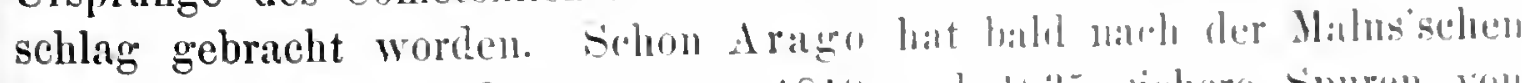

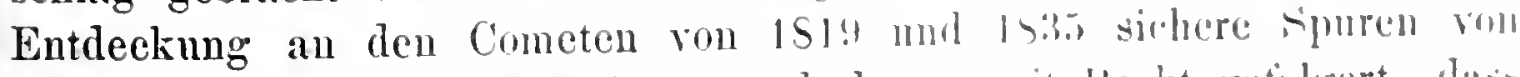
polarisirtem Lichte nachgewicsen und dalralls mit lienht gefolgert, das 
diese Cometen nicht ausschliesslich eigenes Licht nach der Erde senden könnten. Später sind auch an vielen anderen Cometen Polarisationserscheinungen naehgewiesen worden, und nur bei einigen wenigen Cometen hat sich keine Spur von Polarisation gezeigt. Da aber der Grad der Polarisation sehr wesentlich von der Grösse des Einfallswinkels abhängt, so kann es vorkommen, dass bei gewissen Elongationen des Cometen fast gar keine Polarisation wahrzunehmen ist. Im Allgemeinen darf man es dureh die bisherigen Untersuchungen in dieser Richtung für erwiesen annehmen, dass in der That wenigstens ein Theil des Cometenlichtes von Reflexion herrührt, wenn man aueh über den Betrag desselben schon ans dem Grunde nichts Sieheres angeben kann, weil die Polarisation keine vollständige ist.

Vollkommene Klarheit über die Beschaffenheit des Cometenlichtes hat uns erst das Speetroskop gebraeht. Bei den meisten der bis jetzt untersuchten Cometen ist ein mehr oder weniger intensives continuirliches Speetrum und ausserdem eine Anzahl von hellen Bändern eonstatirt worden, die nach dem rothem Ende hin scharf begrenzt, nach dem violetten hin verwasehen sind, und deren Position und Aussehen vollkommen mit den Bändern in den Spectren der Kohlenstoffverbindungen übereinstimmen. Bei einigen Cometen, z. B. vom Jahre 1881 und 1882, sind bei der Annäherung derselben an die Sonne ausser diesen Bändern noch andere helle Linien, insbesondere die Natriumlinien, bemerkt worden. Das Vorhandensein von hellen Linien und der Umstand, dass dieselben, wenigstens die Kohlenwasserstofflinien, in allen Theilen des Cometen und bei allen Entfernungen desselben sichtbar sind, beweisen nun ohne Weiteres, dass gliihende Gase vorhanden sind. Mag der Gliihznstand durch eine gewaltige Erhitzung des ganzen Körpers hervorgernfen sein, oder, wie es die Meteoritenhypothese verlangt, die Folge von Zusammenstïssen zwischen den festen Partikelchen sein, die in der gasförmigen Hiille zerstreut sind, oder mögen endlieh, was das Wahrselıeinliehste ist, elektrisehe Entladungen im Spiele sein, so viel steht jedenfalls fest, dass durch die speetroskopischen Beobachtungen das Selbstlenehten der Cometen iiber jeden Zweifel gestellt ist. Auch das continuirliche Spectrum künnte an und fïr sieh von Eigenlicht der Cometen herriihren; da jedoch auf den photographischen Speetralanfnahmen einiger Cometen dentlieh Fraunhofer sehe Linien gesehen worden sind, so ist die Annahme gereehtfertigt, dass das continuirliche Spectrum der Cometen wenigstens zum Theil dem zuriickgeworfenen Somnenlichte den Ursprung rerdankt. Dem Spectroskop wird es roraussichtlich aneh in Zukunft vorbehalten bleiben, in erster Linie iiber Alles, was hinsiehtlich der hier besproehenen Frage noch unanfgeklint ist, Auskunft zu geben, und in dieser Beziehung werden die 
Helligkeitsbeobachtungen der Cometen stets an Bedeutung hinter den spectroskopischen Untersuchungen zurlickstehen. Trotzdem sollten die ersteren keineswegs so wie bisher vernachlassigt werden. Kin genanes Stndium der Sichtbarkeitsverhiltnisse, namentlich bei den periodiseh wiederkehrenden Cometen, ist fur das Wiederaffinden derselben von grossem Werthe, und die mehrfach ausgesprochene Vermuthung, dass periodische Liehtsehwankungen bei den meisten Cometen vorkommen, kann unr durch sorgfaltige Helligkeitsbestinmungen entschieden werden. Soll aber auf diesem Gehiete etwas Erspriessliches erreicht werden, so muss \%nnlichst die gegenwärtig herrsehende Unklarheit beseitigt und eine Verständigung darllber angestrebt werden, was unter Helligkeit eines Cometen \%u verstehen ist.

In den Cometenephemeriden wird von jeher als Helligkeit eine Grüsse angefuhrt, die dem Ausdrucke $\frac{1}{r^{2} A^{2}}$ proportional ist; meistens wird dabei als Einheit der Helligkeiten der Werth angenommen, den dieser Ansdruck zur Zeit der Entdeckung des Cometen besass. Voraussetzung hei dieser Bezcichnungsweise ist nach dem fribler Gesagten, dass der Comet nur reflectirtes Licht aussendet, und dass seine Reflexionsfithigkeit während der D:mer seiner Erseheinung unveründert bleibt. Da diese Vorausset\%ung nach dem, was das Speetroskop uns lehrt, keineswegs rollkommen zutreffend ist, und sicher wenigstens ein Bruchtheil des Cometenlichtes von Selhstlenehten herruhrt. so ist klar, dass die bisher gebrïuchliche Voransberechnung der Helligkeiten nngenan sein muss. Aber selbst wem das zurilekgeworfene Licht bei einem Cometen ganz und gar iiberwiegen sollte, so withde anch diun der wbige Ansdruck nur gelten, wem es sich mu dats Gesimmtlipht, nicht un dic Flächenhelligkeit an einer bestimmten stelle des ('onoton handelte: im

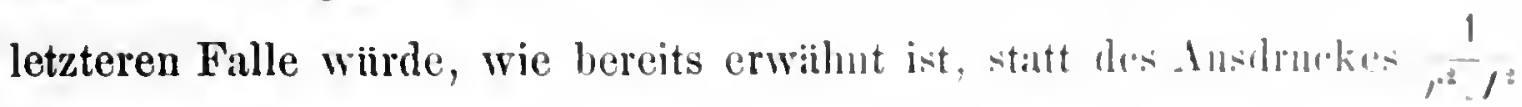
der Werth $\frac{1}{r^{2}}$ für die Voransberechumug zn benutzen sein. lis ist in fler jüngsten Zeit mehrfach ïber die Berechtigung des cinen oder andreren diesere Ausdrücke gestritten worden; wie mir scheint, durdhans "lute zwingenden Grund. Nach den ersten Grundsitzen der l'hotometrie halnoll heide Ans-

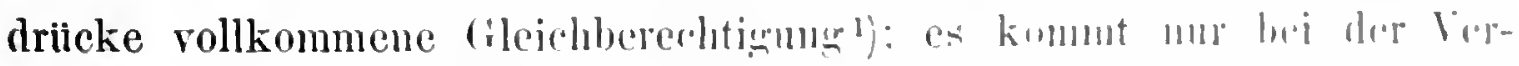

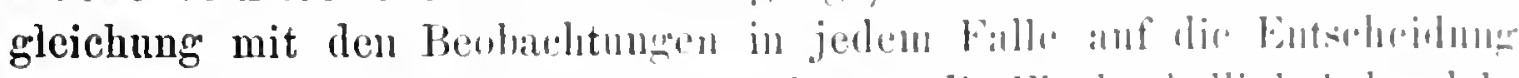

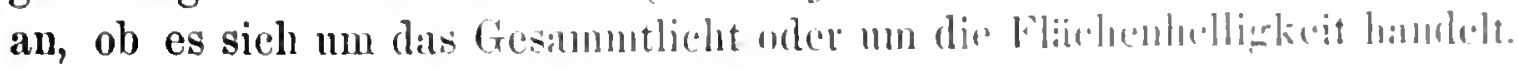

1) Es wiire sehı erwiinseht, wie auch schon mehrfich won anderer seite hervor.

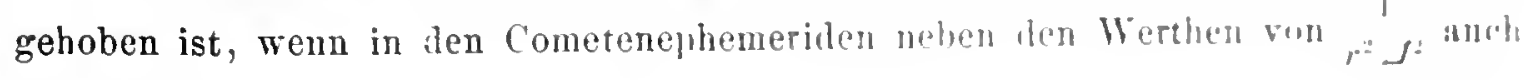
die Werthe $\frac{1}{r^{2}}$ angegeben wiirlen. 
Wie sind nun bisher gewöhnlich die Helligkeitsbeobachtungen der Cometen angestellt worden? Meistens ist die Lichtstärke in Sterngrössen angegeben, aber es hängt wesentlich von dem benutzten Hülfsmittel ab, worauf diese Angabe zu beziehen ist. Wird ein Comet so hell, dass er mit blossen Angen sichtbar ist, so erscheint er gewöhnlich, vom Schweif abgesehen, wie ein nebelartiger Stern, dessen Vergleichung mit benachbarten Sternen zwar äusserst schwierig, aber bei einiger Übung doch ausführbar ist. Der Comet wird dabei durchaus als Stern anfgefasst, und die Grössenschätzung, so nnsicher sie auch ist, kamn als guiltig für das Gesammtlicht betrachtet werden.

Bei Anwendung von Fernrohren kommt es einerseits auf die Beschaffenheif des Cometen selbst an, dann aber auch auf die instrumentellen Hülfsmittel, insbesondere auf die angewandte Vergrösserung. Hat dër Comet ron Anfang an einen deutlichen sternartigen Kern, so wird man diesen Kern mit lichtstarken Fermrohren und mittlerer Vergrösserung fast ebenso gut wie jeden beliebigen anderen Fixstern in Bezug auf seine Grösse schätzen oder mit anderen Sternen vergleichen können. Diese Schätzungen des Kerns allein sind gewöhnlich die zurerlässigsten and geben die brauchbarsten Lichtcurren; man wird bei ihnen, da es sich um Punktyergleichungen handelt, am Ehesten den Ausdruck $\frac{1}{r^{\circ} \Delta^{2}}$ zur Anwendung bringen können. In kleineren Ferurohren und bei Benutzung recht schwacher Vergrösserung wird häufig der Kern des Cometen zusammen mit der umgebenden Hiille, also der ganze Kopf, wie ein punktartiges Nebelobject erscheinen, welches sich zur Noth mit benachbarten Fixsternen, namentlich wemn man dieselben etwas ansserhalb des Focus betrachtet, rergleichen lässt.

Hat der Comet gar keinen sternartigen Kern, sondern erscheint im Fermrohr als matter, höchstens nach der Mitte zu etwas verdichteter Nebelfleck ron merklicher Ausdehumg. so sind Grössenschätzungen so gut wie unansführbar. Die einzig mögliche Art der Helligkeitsbestimmungen sind damn Messungen der Flächenintensität mittelst irgend eines dazu sceeigneten Instrumentes, etwa des Steinheil'schen Prismenphotometers oder noch besser des Keilphotometers.

Rechnet man zu den bisher anfgezühlten Schwierigkeiten, die sich einer einheitlichen Beobachtungsweise der Cometenhelligkeiten entgegenstellen, noch den Linstand, dass bei den meisten Cometen Veränderungen in der Form und dem ganzen Aussehen eintreten, so ist klar, dass exacte Resultate ans den Helligkeitsbeobachtungen niemals erwartet werden dïrfen, und dass sich auch kaum bindende Vorschriften für die Anstellung der Beobuchtungen geben lassen. Soriel geht jedenfalls aus dem Gesagten hervor. dass unter allen Lmstiinden nur Helligkeitsangaben mit 
einander vereinbar sind, die von demselhen Beohachter mit demselhen Instrumente und der gleichen Vergrossermg gewomnen sind.

Welche Unterschiede bei den Grössenschätzungen eines Cometen je nach den benutzten Hulfsmittelu herrortreten künnen, möge im Folgende॥ an dem Boispiele des Cometen 157.4 III (Coggia) gezeigt werden, dessen Helligkeit von Sehmidt ") in Athen auf dreifache Art untersucht wordeu ist. Die beiden ersten Beobachtungsreihen sind am liefraetor angestellt, und zwar die eine mit Benutyng eines stark vergrössernden Oculars, die andere mit eimem seliwachen Ocular; beide beziehen sich anf die Helligkeit des Kerns allein. Die dritte Reihe enthilt die Schitrungen mit unbewaffuetem Ange und bezieht sich auf die Helligkeit des ganzen Cometenkopfes. In der folgenden Zusammenstellung iler Schmilt'schen Grössenangaben sind nur diejenigen Beobachtungstage heransgegriffen worden, an denen alle drei Schat\%ungsmethoden \%ugleich angewandt wurden. Da der Comet in Juli einen riemlich tiefen Stand hatte, so wird der Einfluss der Extinction nicht gan\% unbedeutend gewesen sein; es ist aber anzunehmen, dass ein so gellbter Beobachter wie Schmidt bereits bei den Schätzungen selbst auf die Extinetion Rlleksicht genommen hat ${ }^{2}$. Zur Vergleichung mit den Beobachtungen sind in der Zusammenstellung noch die berechneten Helligkeiten angefthrt und \%war sowoh! mit Benutzung des Ausdrueks $\frac{1}{r^{2} d^{2}}$ als $\frac{1}{r^{2}}$. Die Logarithmen dieser Werthe sind dureh Division mit 0.4 in der Whlichen Weise in (irüsses.classen umgewandelt, und zu den so crhiltenen Kablulen ist dam eince Constante hinzugefugt worden, $1 m$ die berechnote llelligkeit filr do." ersten Beobachtungstag (Juni 1) in genane Übereinstimumug mit de. heobachteten Grüsse in der ersten keihe zu bringen.

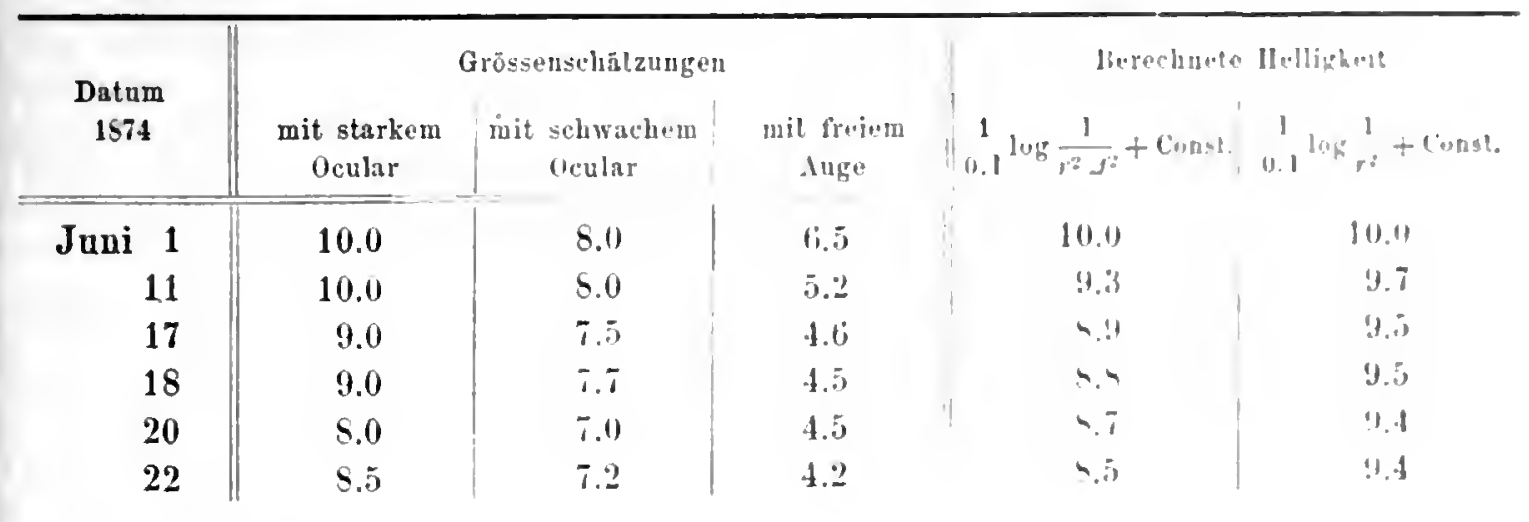

1) Astr. Nachr. Bd. ५i, Nr. $206 i$

2) Bei Gelegenheit der Nittheilung seiner Helligkeifswehitzungen an dem

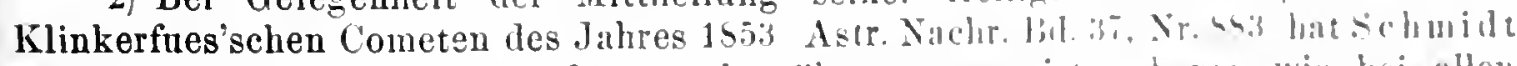
ansdrücklich hervorgehoben. dass er bemiht gewesen int. ebrenso wie bei allew seinen Beobachtungen der veriaderlichen Sterne. lie Extinction des Lichtes in der Atmosphäre zu berieksichtigen. 


\begin{tabular}{|c|c|c|c|c|c|}
\hline \multirow{2}{*}{$\begin{array}{l}\text { Datum } \\
\text { 1574 }\end{array}$} & \multicolumn{3}{|c|}{ Grössenschătzungen } & \multicolumn{2}{|c|}{ Berechnete Helligkeit } \\
\hline & $\begin{array}{l}\text { mit starkem } \\
\text { Ocular }\end{array}$ & $\begin{array}{l}\text { mit schwachem } \\
\text { Ocnlar }\end{array}$ & $\begin{array}{l}\text { - mit freiem } \\
\text { Auge }\end{array}$ & $\frac{1}{11.4} \log \frac{1}{r^{2} \Delta^{2}}+$ Const. & $\frac{1}{0.4} \log \frac{1}{r^{2}}+$ Const. \\
\hline Jnni 24 & 8.0 & 6.8 & 4.0 & 8.4 & 9.3 \\
\hline - 27 & 9.0 & 7.0 & 4.0 & 8.1 & 9.3 \\
\hline 30 & 8.5 & 7.2 & 3.5 & 7.8 & 9.2 \\
\hline Juli 2 & 7.5 & 6.7 & 3.2 & 7.7 & 9.2 \\
\hline 4 & 7.5 & 6.0 & 3.0 & 7.5 & 9.2 \\
\hline 6 & $\pi .5$ & 6.7 & 2.9 & 7.3 & 9.2 \\
\hline 8 & 7.0 & 6.0 & 2.5 & $\tau .1$ & 9.1 \\
\hline 10 . & 7.5 & 5.5 & 1.9 & 6.9 & 9.1 \\
\hline 12 & 7.0 & 5.0 & 1.5 & 6.7 & 9.2 \\
\hline 13 & 6.5 & 4.7 & 1.5 & 6.6 & 9.2 \\
\hline
\end{tabular}

Dieses Beispiel ist in mehrfacher Beziehung lehrreich. Erstens sieht man, wie stark die verschiedenen Helligkeitsangaben roneinander abweichen. Für das blosse Auge erschien am letzten Tage der Cometenkopf als Stern 1. bis 2. Grösse, während im Fermrohre der Kern allein als Stern 6. bis 7. Grösse taxirt wurde; die Schätzungen des Kerns mit verschiedenen Vergrösserungen differiren um durchschnittlich 1.5 Grössenclassen roneinander. Man sieht hierans, dass Grössenangaben für einen Cometen ohne nähere Bezeichnung, anf welchen Theil sich dieselben beziehen, und ohne genane Mittheilung der angewandten Instrumente und Vergrösserungen vollständig werthlos sind. Ferner ergiebt sich aus dem obigen Beispiele, dass die Form der Lichtcurre für die einzelnen .Reihen wesentlich rerschieden ist. Während die beiden ersten für den Kern allein giiltigen Reihen noch in leidlicher Übereinstimmung immerhalb des betrachteten Zeitraumes eine Helligkeitszunahme ron ungefähr 3.5 Grössen ergeben, folgt ans der dritten Reihe ein Anwachsen von 5 Grössen, offenbar weil bei den Schätzungen mit blossem Auge die Lichthüllen um den Kern, die bei der Amiiherung des Cometen an die Somne fast immer grösser und intensicer werden, wesentlich zur Verstïrkung des Eindrucks beitragen. Die Vergleichung: mit den berechneten Intensitaiten zeigt endlich. dass der Ansdruck $\frac{1}{r^{2}}$, welcher für den grössten Theil des betrachteten Zeitintervalles constant bleibt, anch nicht im Entferntesten die Beobachtumgen darstellt. Dagegen schliesst sich der Ansdruck $\frac{1}{r^{2} d^{2}}$ den ersten Reihen im Grossen und Ganzen leidlich an, wemn anch einige Abweichungen zwischen Beobachtung und Rechmmg rorkommen, die kaum duroh blosse zufallige schätzungsfehler erklärt werden können. Jedenfalls wiirde in diesem Falle, da es sich mu nm die Schätzmg des Kermes, also eines nihezu pmiktartigen Objectes handelt. der Sehluss gerechtfertigt 
erseheinen, dass das Licht des Cometenkernes wilhrend der Zeit ron Juni I bis Juli 13 in der Hauptsache refleetirtes Sonnenlicht gewesen ist.

Die Zahl der Cometen, an denen ebenso ausnihrliche und sichere Helligkeitsbeobachtungen wie die ehen besprochenen angestelt worlen sind, ist ausserordentlieh gering. Das meiste Material ist Schmidt zu verdanken, weleher ausser ron dem Cometen $157.4 \mathrm{III}$ noeh von den Cometen 1850 I (Petersen), 1853 III (Klinkerfues), 1554 II (de Mcnciaux), 1862 II (Schmidt) zusammenhängende Sehaitzungsreilien veröftentlicht hat. Weiter verdienen noch Erwihnung die von Pasehen') an den beiden Kernen des Biela'schen Cometen im Jahre 1S46 ausgenhrten Vergleichungen, die besonders deswegen bemerkenswerth sind, weil dabei die Steinheil'sche Methode der Vergleiehung ausser dem Bilde durch Ausziehen des Oeulars benutzt wurde; ferner die Schütmungen der Cometen 1581 III ('Tebbutt) und 1S81 IV (Schaeberle) ron Schwabs), und endlich die in Potsdam4) mit dem Züllner'sehen Photoneter angestellten Messungen an den Cometen $1882 \mathrm{I}$ (Wells), 185.1 I (Pons-Brooks), 1856 I (Fatury') 1586 II (Barnard). Die meisten dieser Beobachtungsreihen bezichen sich auf den Kern allein oder wenigstens auf den Kern unit seiner unmittelharen Umgebung; sie werden daher im Allgemeinen besser durch den dusiluck $\frac{1}{r \cdot \Delta^{2}}$ als durch $\frac{1}{r^{2}}$ dargestellt, obgleich auch der erstere, wie xu erwarten ist, sich keineswegs als ausreichend erweist. Es kommen nicht nur un-

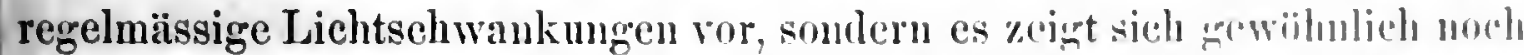
in der Nähe des Perihels eine besonders starke lichtounalmure, die hiiutin von Ausströmnngen ans dem Kerm, wahrscheinlich clektrisshen l'réprung-

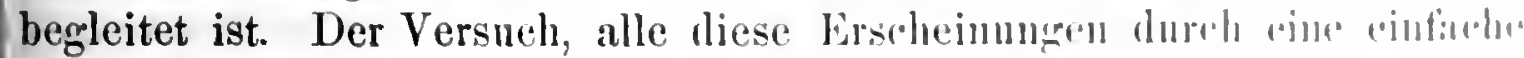

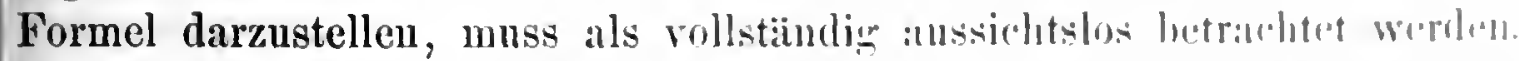

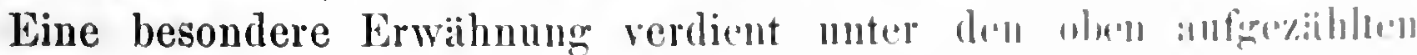

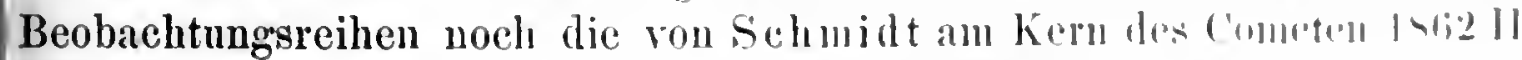

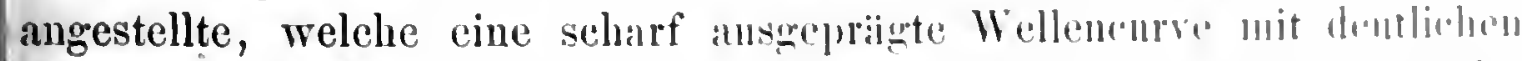

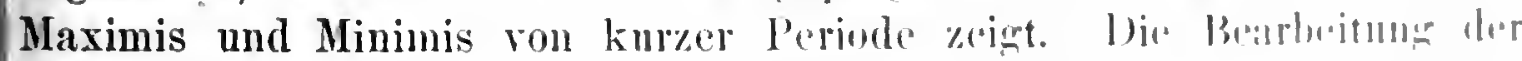

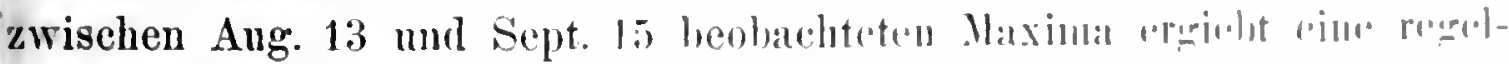

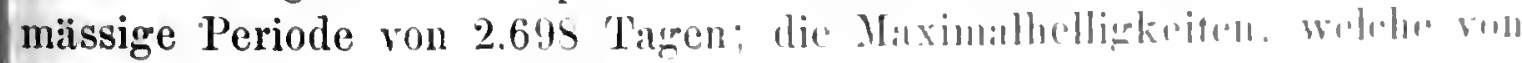

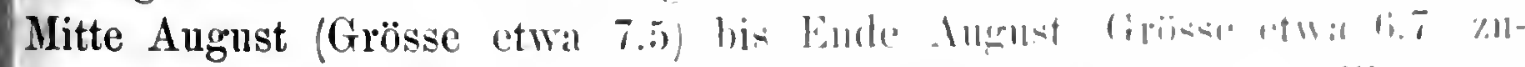

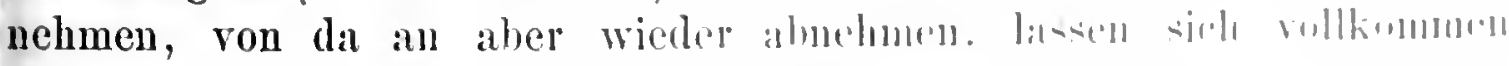

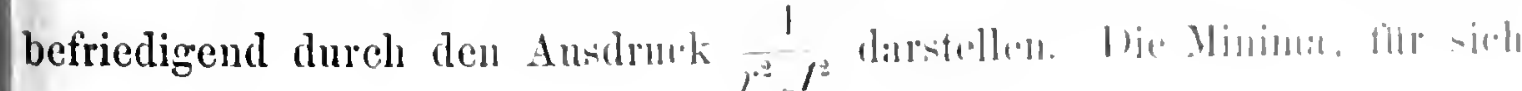

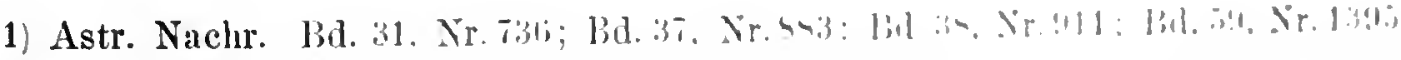

2 Astr. Nachr. Bd. 24. Nr. 5ti2.

3) Astr. Nachr. Pd. 101. Nr. 2412.

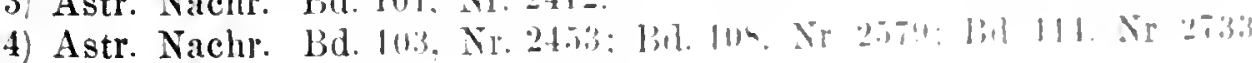


behandelt, ergeben fast genan dieselbe Periode (2.707 Tage); dagegen fiigen sich die Minimalhelligkeiten durchaus nicht dem einfachen Ausdrucke $\frac{1}{r^{2} \Delta^{2}}$. Die Helligkeitsdifferenz zwischen einem Maximum und dem darauf folgenden Minimum betrug Mitte August etwa $1 \frac{1}{4}$ Grösse, stieg dann bis Ende August (bis kurz nach dem Perihel) auf etwa vier Grössen und sank endlich bis Mitte September wieder auf etwa zwei Grössen zurück. Die Pulsationen des Cometenlichtes sind also in der Nähe des Perihels am lebhaftesten gewesen. Interessant ist, dass diese Erscheinungen in deutlichen Zusammenhange standen mit den drehenden Bewegungen, welche an den Ausströmungen aus dem Kerne bemerkt wurden. Die scheinbaren Neigungswinkel des Strömungsfächer's gegen die Schweifaxe erreichten in einer Periode ron 2 bis 3 Tagen ihre Maxima und Minima, und zwar coincidirten die Maximazeiten mit den Zeiten der grössten Kernlelligkeit und ebenso die Minima mit den Zeiten der geringsten Helligkeit. Die iiberaus interessanten Beobachtungen Schmidts, an deren Realitiit nicht zu zweifeln ist, zeigen, von welcher Bedentung sorgfältige Helligkeitsschätzungen für die Erforschung der physischen Beschaffenheit der Cometen erentuell sein können.

Periodische Helligkeitsändermugen, allerdings ron langer Dauer, sind auch bei einigen anderen Cometen rermuthet worden, insbesondere bei dem Encke'schen Cometen. Berberich ${ }^{1}$ hat sich im Jahre 1888 der verdienstlichen Arbeit unterzogen, Alles, was ïber die Helligkeit dieses Cometen bei den 24 Erscheinungen desselben zwischen 1786 und 1885 bekannt geworden ist, zusammenstellen. Es sind dies allerdings meistens nur ganz rohe Angaben; nur vereinzelt finden sich wirkliehe Schätzungen der Lichtstärke, und anch diese sind nur mit Vorsicht zu rerwerthen, weil bei dem nebelartigen Aussehen dieses Cometen, an dem fast niemals ein wirklicher Kern wahrgenommen ist, die Entscheidung schwierig ist, ob die Beobachtung sich anf das Gesammtlicht oder die Flächenintensituit bezieht. Berberich glanbte ans dem Naterial den Schluss ziehen zn können, dass die Lichtstärke des Enckeschen Cometen in den einzelnen Erscheinungen nicht unmerklich rerschieden gewesen ist. Dabei schien ein Znsammenhang mit der Sonnenthätigkeit in der Weise angedentet, dass die hellen Erseheinnngen mit den Zeiten der Maxima, die schwachen mit den Zciten der Minima der Somnenthätigkeit zusammenfallen; selbst die Lnregelmässigkeiten in der 11 jührigen Somnenperiode sollen sich nach Berberichs Ueinung in der Cometenlielligkeit abspiegeln. Wemn anch das ungenitgende Material sichere Schliisse in dieser Beziehnng nicht 
gestattet, so ist doch bei der un\%weifelhaften Linwirkung der some nuf die Lichterscheinungen der Cometen die Berberich'sche Vermuthung nicht ohne Weiteres von der Hand \%u weisen.

Zum Sehlnsse mögen noeh einige Bemerkungen Platz finden ther die bisherigen Versuche, bei den Helligkeitsbestimmungen der Cometen die Sehätzungsmethode durch photometrisehe Messungen zu ersetzen. Dass wirkliche Messungen vor den blossen Sehit»ungen den Vorıug verdienen, bedarf wohl kaum der Erörtermug; unr durfte es sich fragen, welche Methode gerade hier am Vortheilhaftesten zu verwenden ist. Bestimute. Vorsehriften lassen sich naturlich von vornherein nieht geben, weil es in jedem einzelnen Falle auf das Aussehen des betrefienden Cometen ankommen wird; indessen unterliegt es keinem 'Lweifel, dass das Keilphotometer sich wie kein anderes Instrument \%u Untersuchungen des Cometenlichtes eignet, nieht nur bei solehen Cometen, die einen scharfen fixsternartigen Kern haben, sondern aueh bei solehen, die nur eine nebelartige centrale Verdiehtung aufweisen. Empfehlenswerth ist anch das Verfahren, welehes von mir bei den Helligkeitsmessungen einiger Cometen bereits mit Erfolg angewendet worden ist. An einem Zölner'schen Photometer wird an Stelle der Diaphragmenseheibe fitr die kunstliehen Sterne ein Blendglas ron dunklem blauen Glase in geeigneter liassun? vor der Lampenöftinung angebracht. In dieses Blendglas, welches firr das Lampenlieht fast vollstiindig undurehsichtig scin muss, wird eine kleine Kngelschale eingeschliffen, sodass dasselbe in der Vlitte mur noch eine Dieke von etwa $0.3 \mathrm{~mm}$ behiilt und stark durdhsichtig wird, wihrend

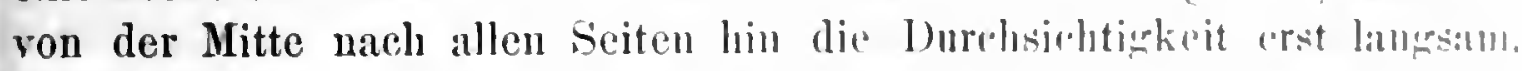

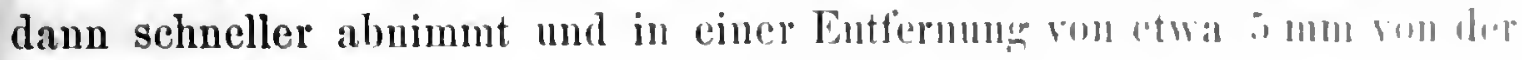

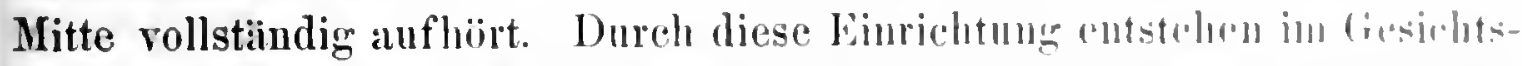
felde des Photometers anstatt der kinstlichen Sterne zwei rumb Xichelbildehen, die am Rande gant verwaschen sind und, je nallo der form

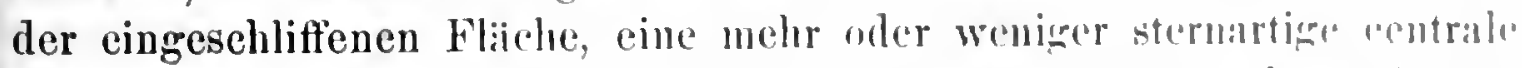

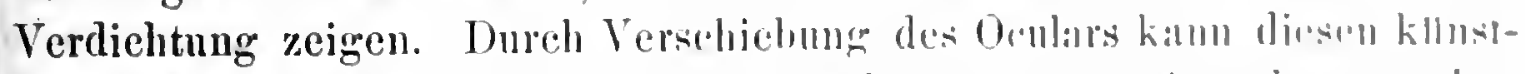

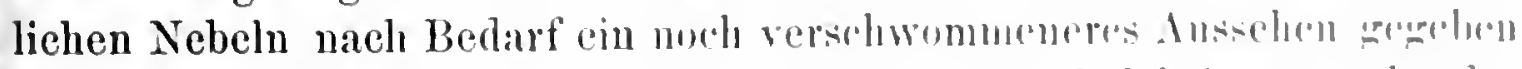

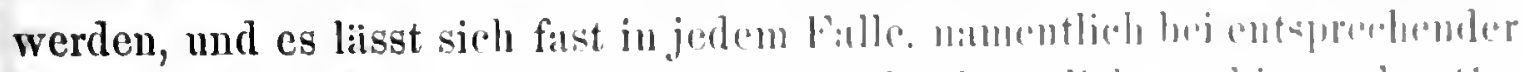

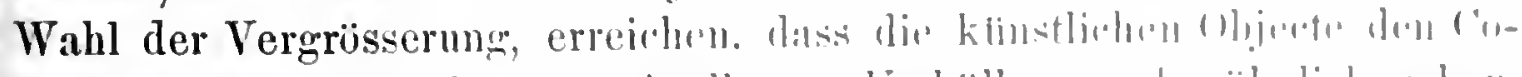

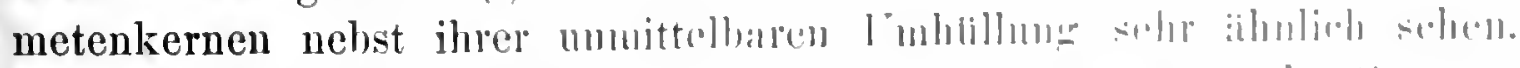

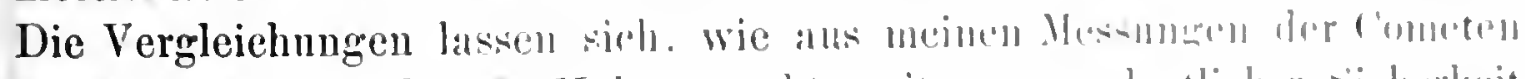

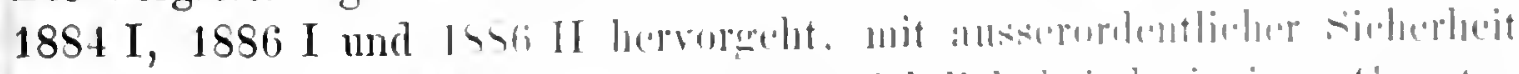

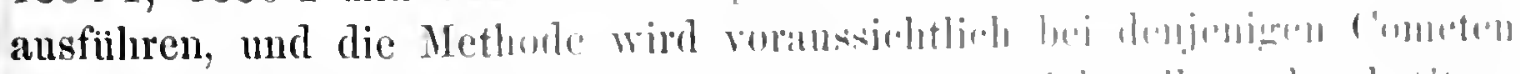

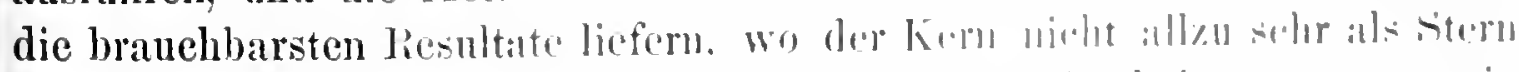

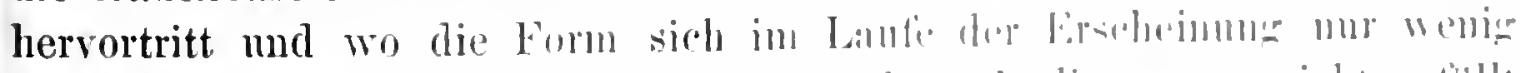

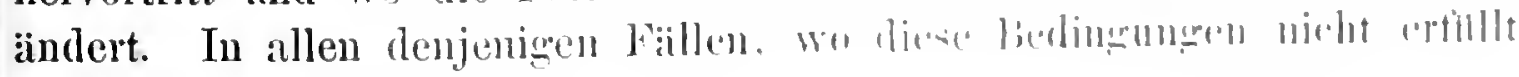
Múller, Photonetrie der Gestirne. 
sind, besonders aber dann, wenn Lichtaússtrahlungen aus dem Kern und ganz plötzliche Änderungen des ganzen Aussehens eintreten, versagt natürlich dieses photometrische Verfahren genau ebenso wie iiberhaupt jeder Versuch, solche g:ïnzlich heterogenen Lichterscheinungen miteinander vergleichen zu wollen.

Noch weniger als auf dem Gebiete der Cometenphotometrie ist bisher auf demjenigen der Nebelphotometrie geleistet worden, obgleich bei dieser Classe von Himmelskörpern die Sachlage insofern etwas giinstiger ist, als reflectirtes Licht dabei gar nicht in Frage kommt, und im Allgemeinen Form und Aussehen dieser Objecte keinen oder nur ganz geringfügigen Änderungen unterworfen sind. Alles was wir über die Helligkeiten dieser Weltkörper wissen, beschränkt sich im Wesentlichen auf die kurzen beschreibenden Notizen, die ron den Beobachtern in den verschiedenen Nebelcatalogen mitgetheilt worden sind. Als Richtschnur hat dabei bis hentigen Tages die von Hersehel in seinem Generalcataloge eingeführte Bezeichnungsweise gedlient, nach welcher die folgenden zehn hauptsächlichsten Helligkeitsstufen unterschieden werden (man benutzt auch hente noch gewöhulich die Herschel'schen Abkiirzungen):

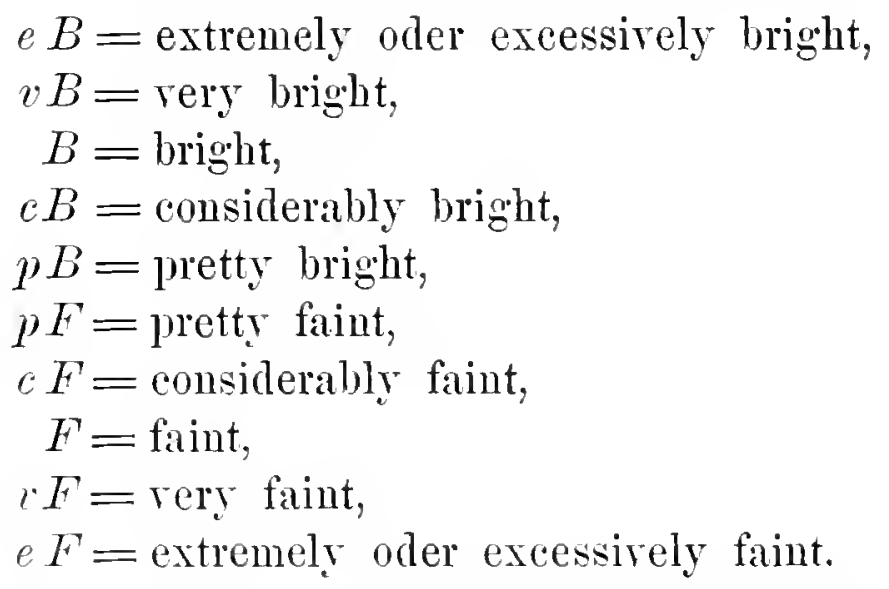

Diese durchaus willkiurlich gewïhlte Scala macht keineswegs den Anspruch darauf, ganz grleichmässige Helligkeitsabstufungen anzugeben; sie soll nur allgemein eine "Reihung" der Nebel nach ihrer Helligkeit ermöglichen. Aber anch dieses Ziel ist schon schwierig genug zu erreichen in liticksicht auf die enormen Unterschiede in dem Aussehen der einzelnen Tehelclissen. Ein sehr answedehnter Nebel ron unregelmässiger Gestalt und glechmaissiger Helligkeitsrertheihng wird z. B. ebenso gut mit $v B$ bezeichnet, wie ein kleiner runder Nebel mit einer starken fixsternartigen Verdichtun in der Nitte, obgleich streng genommen beide Objecte durehans nicht miteinander rergleichbar sind. Abgesehen ron dieser fast uniberwindlichen schwierigkeit hat eine derartige "Reihung" streng ge- 
nommen nur Bedentung fur die Schätaungen eines und desselben Beobachters mit Benutzung desselben Instrumentes und der gleichen Vergrösserungr. Es ist bekannt, wie sehr die Sichtbarkeit und Helligkeitsbeurtheilung namentlich ausgedehuter sehwacher Nebelflichen von der Grösse des Objectivs und insbesondere von der angewandten Vergrösserung ablängt. Berilcksichtigt man fener noch, dass Ungleichmassigkeiten in der Lufturehsichtigkeit und die Helligkeit des Grundes bei den Nebelbeobachtungen eine besonders wichtige Rolle spiclen, und dass meh die versehiedene Sehschärfe der Beobachter bei diesen sehwierigen Ohjecten in Betracht kommt, so liegt es auf der Hand, dass Helligkeitsschitumngen rersehiedener Beobachter nicht ohne Weiteres uiteinander vereinbar sind. Man wird sich daher anch nicht wundern diurfen, diss \%. B. ein und derselbe Nebel in zwei verschiedenen Catalogen mit $B$ und $F$ bezeichnet ist, und dass häufig noch grössere Differenzen vorkoumen. Verhältnissmaissig am Besten stimmen die Helligkeitsangabeu fir diejenigen Nebel untereinander iberein, die eine starke sternartige Concentration in der Mitte zeigen. Man hat bei diesen Nebeln hisweilen auch rersucht, die Helligkeit des Kerns mit benachbarten Fixsternen zu vergleichen und direct in Grössenclassen auszndricken.

An Vorsehligen, die Lichtstiirke der Nehelflecke mit Hulfe ron photometrisehen Vorriehtungen zn messen, hat es nieht gefehlt; doeh sind bisher niemals grössere Beobachtungsreihen angestellt worden. Am meisten Beachtung verdienen in dieser Bezichmng die Vorshlibige von Huggins'), Piekering2) und Holden:3). Ersterer emptiohlt die Bunutzung eines Instrumentes, in welehem zwei bereits fon Dal wes ang-

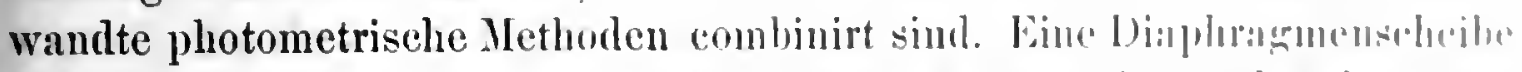
mit einem kleinen Loeh in der Nitte kitmm mittelst einer sidhruln messbar vom Brennpunkte nach dem Objective him bewent werden; ansonerlem

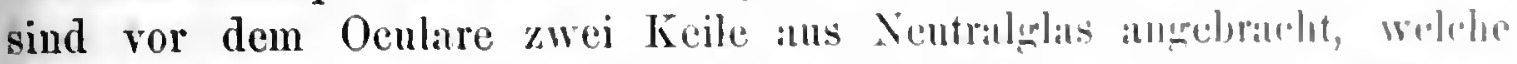

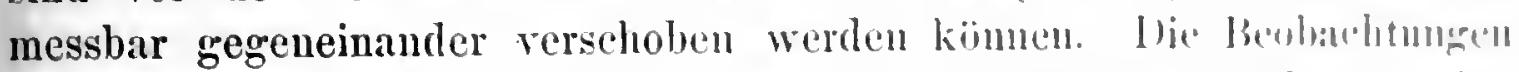

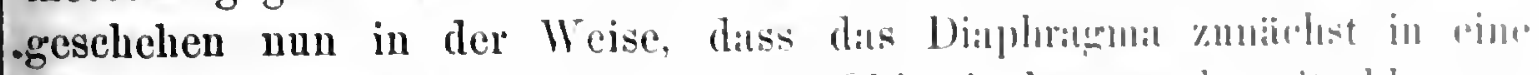

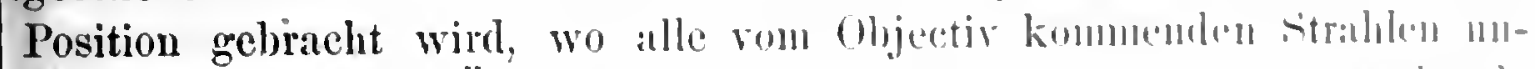

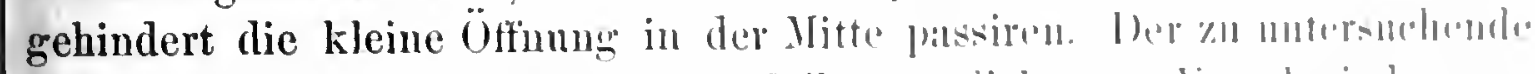

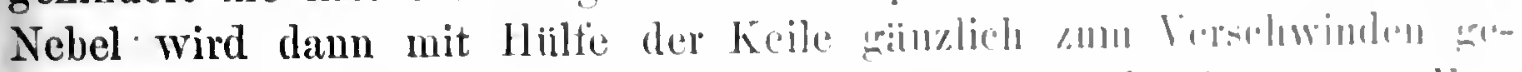

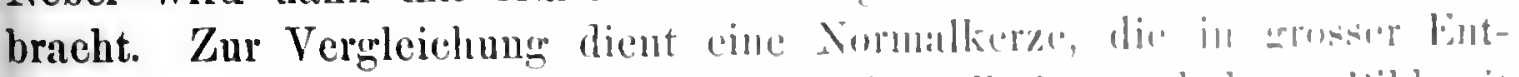

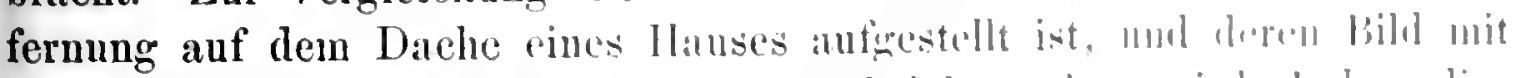

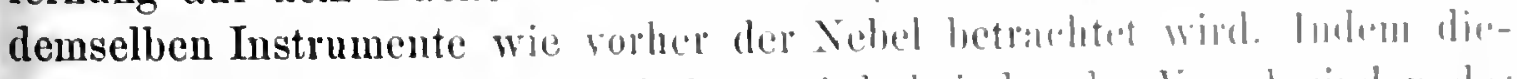

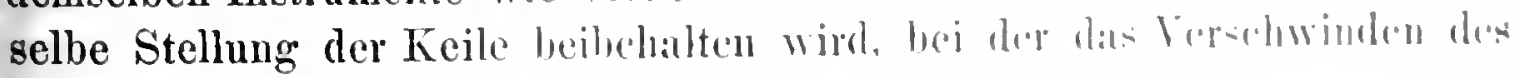

1) Plil. Trans. of the R. Soc. of Iondon. 1stili, p. :int.

2) American Jonrnal of science. 3. Ser. Vol. 11 1 $>1,-4,11.42$

3) Nature. Vol. 21, p. 346 . 
Nebels beobachtet war, kann unn durch Bewegung des, Diaphragmas auch das Kerzenbild ausgelöscht werden; die Grösse dieser Verschiebung: giebt danu nach den bekannten Principien der Photometrie ein Mass fuir das Verhältuiss der Flächenhelligkeiten ron Nebel und Kerzenbild. Huggins hat auf diese Weise den Nebel G.C. Nr. $4628=\mathrm{h} 2098$, den Ringnebel in der Leier und den Dumbbell-Nebel mit der Kerzenflamme verglichen, und seine Messungen ergeben, wenn die Helligkeit des ersteren Nebels mit 1 bezeichnet wird, die Helligkeitswerthe:

$$
\begin{aligned}
& \text { G. C. Nr. } 4628 \text { Helligkeit }=1 \\
& \text { Ringuebel } \\
& \text { Dumbbell-Nebel } \\
& \begin{aligned}
\therefore \quad & =\frac{1}{4} \\
& =\frac{1}{13} .
\end{aligned}
\end{aligned}
$$

Natiirlich beziehen sich diese Angaben nur anf die hellsten Partien der betreffenden Nebel.

Pickering hat zur Beobachtung der kleinen regelmässig gestalteten Nebel, insbesondere der sogenannten planetarischen Nebel, das folgende Verfalıren in Vorschlag gebracht. Durch ein seitlich an dem Hauptfernrohre angebrachtes Hiilfsfermrohr wird, ihnlich wie bei einem der friiher beschriebenen Pickering'schen Steruphotometer (Seite 262), das Bild eines hellen Sternes in das Gesichtsfeld neben den zn untersuchenden Nebel gebracht, und durch Verschiebung des Hiilfsfernrohrobjectirs (sowohl nach dem Brennpunkte hin als ron demselben hinweg') wird die Flächenhelligkeit des in eine rerwaschene Scheibe rerwandelten Stemes der des Nebels gleichgemacht. Die Grösse der Verschiebung giebt ein Mass fuir das Intensitätsverhältniss der beiden Objecte. Benutzt man denselben Stern (mit Vortheil liesse sich z. B. bei grösseren Beobachtungsreihen der Polarstern rerwenden zur Vergleichnng mit rerschiedenen Nebeln, so erhïlt man ans den Versehiebungen am Hiilfsermrohre das Verhältniss der Flächenhelligkeiten derselben. Man kann daun entweder alle Helligkeiten auf einen bestimmten Tebel beziehen, oder man kann als Einheit der Flächenhelligkeiten, wie Pickering rorschlägt, die Intensitait wïhlen, welche das Veroleichsteruscheibehen annimmt. wenn es anf einen Raum. rou 1 Bogenminute im Durchmesser ausgebreitet ist. Ans dem Verhältnisse der Flichenhelligkeiten versehiedener Tebel lisst sich endlich noch, mit Beriicksichtigung ihrer Dimensionen, das Terhältuiss der ron ihnen ansgesandten Gesammtlichtmengen berechnen. Pickering hat nach dieser Methode den plametarisclen Nebel B.D. $+41^{\circ} 4004$ mit a Croni rerglichen und gefunden, dass der Nebel uns 590 mal weniger Licht zusendet als dieser Stern. und dass daher seine Gesammtintensitit der cines Sternes S.6 ter Grösse gleichkommt.

'Zur Beolychtung' grösserer, umegelmaissig' gestalteter Nebelflecke hat l'ickering noch eine andere Mlethode empfohleu. hei welcher das Princip 
des Bunsen'schen Photometers benutzt wird, und bei welcher es sich um die Messung der Flichenhelligkeit an verschiedenen Stellen des Nelrels haudelt. In der Praxis seheiut diese Methode, die kaum einer grossen Genanigkeit fihig sein durfte, niemals in Anwendung gekommen zu sein.

Wesentlich rerschieden davon ist eine Methode, die Holden zur Bestimmung der Flaichenintensitiit versehiedener P'strien des Orionnehels angewandt lıat. Zwischen den beiden den Auge zunichst befundlichen Linsen eines terrestrischen Oculars, mit welehem der Nehel betrachtet wird, befindet sich im Brenupunkte der vordersten Liuse ein kleiner Silberspiegel. Dieser erhilit rou einem seitlich an Rohre hefestigten Schirme, der dureh eine bewegliche Lampe belenchtet wird, diffus reflectirtes Lieht und erseheint, durch die vorderste Linse betrachtet, in Gesichtsfelde als ein kieiner heller Fleck, der auf jede Stelle des Nebels projicirt werden kann. Dureh Verschiebung der Lampe lisst sich die Beleuchtung des Schirmes so moderiren, dass der helle Fleck mit jeder helichigen Partie des Nebels gleich hell gemacht werden kaum. Der Betrag der Verschiebung liefert das Mass filr das Intensitiitsverhiltniss der vergliehenen Theile des Nebels. So ungenau diese Methode anch sein mag, so giebt sie doch eine ungefuilure Vorstellung von der Helligkeitsvertheilung innerhalb des Orioninebels. Holden glauht aus seinen Messungen sogar anf Helligkeitsvariationen einzehner lartien solliessen zu durfen; doch bedarf diese Vermuthung noch weiterer Bestaitizung. Instatt dor Iblden-

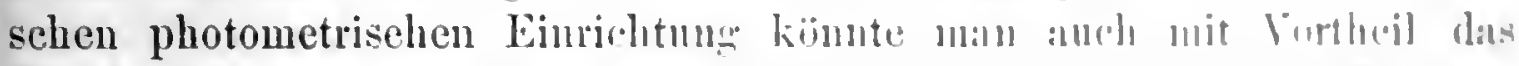

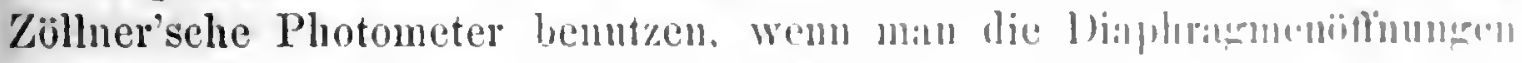

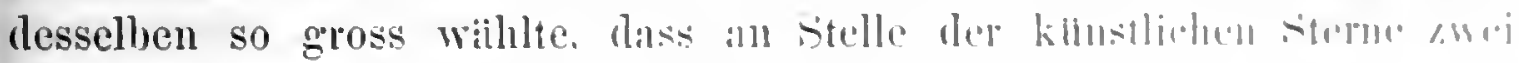

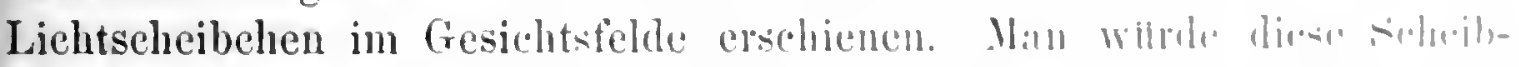

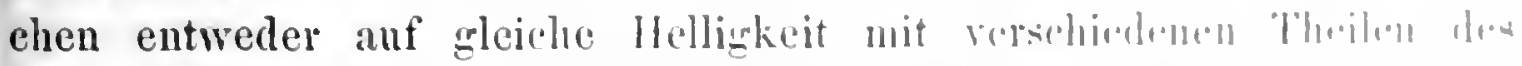

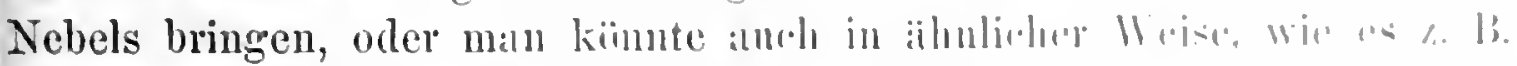

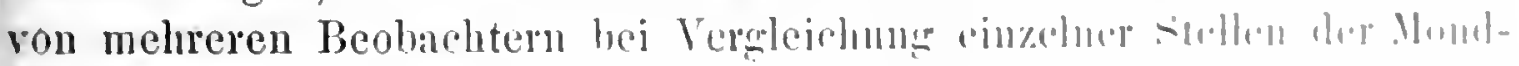

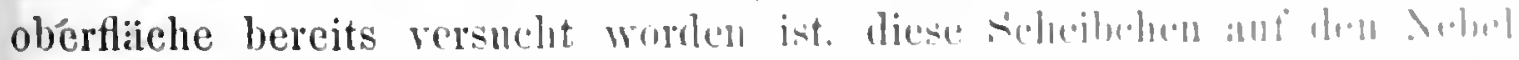

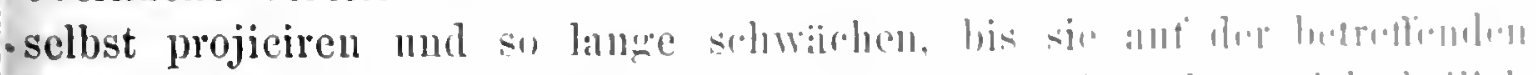

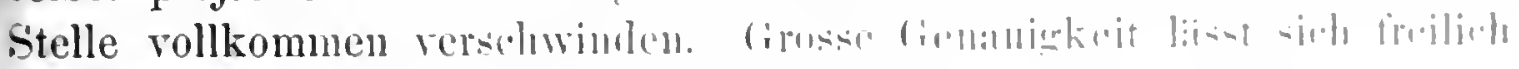
bei keinem dieser Verfulhern urwathll.

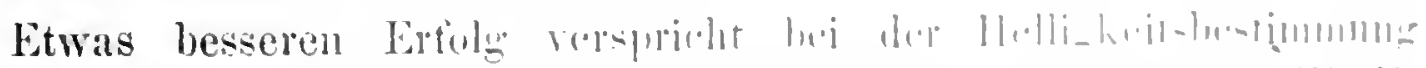

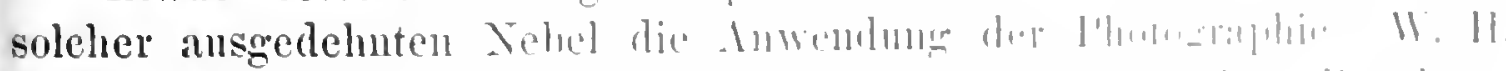

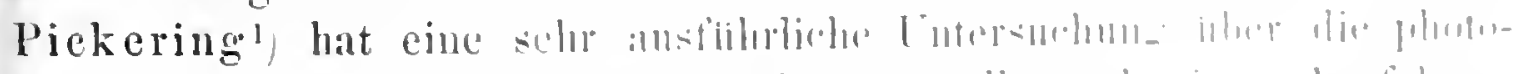

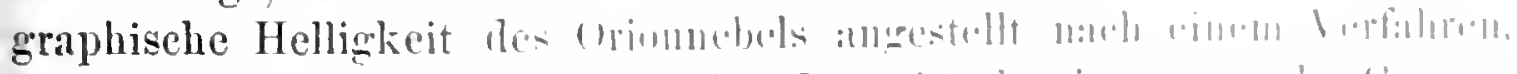

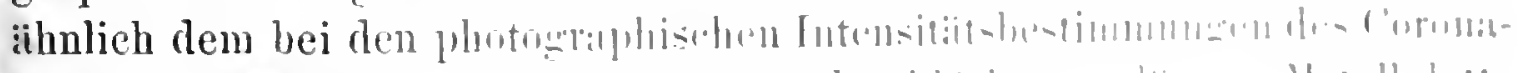

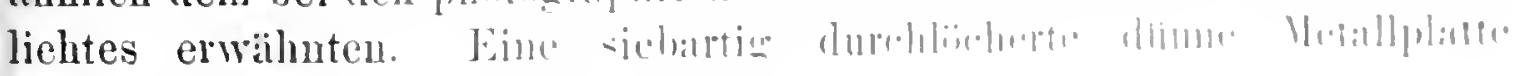


wird in die Brennebene des Fernrohrs unmittelbar vor die dort angebraehte photographisehe Platte gesetzt, anf welcher das Bild des Nebels aufgenommen werden soll. Diéses photographisehe Bild besteht dann aus einer Anzahl ron verschieden intensiven Punkten, von denen jeder einer anderen Stelle des Nebels, die sieh dureh Vergleichumg mit einer zweiten ohne die Metallplatte gemaehten Aufnahme leieht bestimmen lïsst, entsprieht. Als Vergleichslicht dient eine Normallampe (Carcellampe), aus dereu Flamme ein bestimmter Theil herausgeblendet ist. Auf den Rand derselben photographischen Platte, auf welcher der Nebel aufgenommen wird, werden nun von dieser Lampenöffnung mit vorgesetzter durchlöeherter Metallseheibe eine Anzahl rou Aufnahmen gemacht, wobei jedesmal die Intensitiit der Lampe durch Änderung der Expositionszeit oder, da dieses Verfahren zu Fehlern Anlass giebt, dureh irgend eine photometrische Eimrichtung (Nicolprismen, rotirende Scheiben u. s. w.) um einen messbaren Betrag veriudert werden kaun. Die Vergleichung der von verschiedenen Stellen des Nebels herrihrenden Punkte auf der Platte mit den von der Vergleichslampe erzengten Punkteu ermöglicht danu die Bestimmung des Helligkeitsverhältuisses der betrachteten Nebelpartien und die Feststellung der Linien rou gleicher Helligkeit anf dem Nebel. Das ganze Verfahren ist der Beachtung werth und kann ohne Zweifel noch in mancher Hinsicht rerrollkommuet werden.

Vou allen im Vorangehenden erwähnten Vorschlïgen zu photometrischen Nebelbeobachtungen scheint der Huggins'sche am empfehlenswerthesten zu sein; namentlich bei den ansgedehnten Nebelfleeken diirfte die Anwendung des Keilphotometers die besteu Resultate versprechen. Bei den planetarischen und ganz regelmässig gestalteten kleinen Nẻeln wäre vielleicht noch besser das Zöllner'sche Photometer zu beuntzen, mit der bereits bei den Cometenbeobachtnugen erwähnten Modification, dass an Stelle der kiinstlichen Sterue kimstliche Nebelflecke rerwendet werden. Dieselben kümnen gewissen Classen rou Nebeln am Himmel so vollkommen iilulich gemacht werden, dass die photometrische Bestimmung solcher Objecte mit grosser Genauigkeit ansfubrbar ist.

Der Hauptwerth exacter Helligkeitsmessungen au Nebelflecken liegt offenbar darin, dass sie die Möglichkeit gew:ihren, etwaige Lichtrerïuderungen zu constatiren. Gerade bei diesen noch in einem frïhen Eutwicklungsstadimm befindlichen Himmelskörpern duirften solche Änderungen von vornherein durchans walnscheinlich sein. Ton Seiten rerschiedener seiibten Beobachter sind anch in der That bereits Lichtschwankungen bei einigen Nebeln rermuthet worden, allerdings lediglich auf Grund rou Helligkeitssehätzmen oder gar nu von Notizen iber die Dentlichkeit des sichtbarseins. Bei dem schwer controlirbaren Einfluss, den einerseits 
die atmosphärischen Zustinde, andererseits die angewandten optischen Hulfsmittel, nanentlich die Vergrösserung, anf die Siehtharkeit der Nehelflecke ausuben, mllssen solehe blossen Sehiitzungsangaben mit der aller¿ussersten Vorsieht aufgenommen werden, und es unterliegt keinem Zweifel, dass bei den meisten der fitr variabel erklärten Nebel die beobacliteten Helligkeitsunterschiede auf solche Einflusse zurllek»ufuhren sind.

Mit einiger Sieherheit kann man bisher eigentlich nur von drei Nebeh die Veränderlichkeit behaupten; und aueh bei diesen ist es gegenwärtig noch unmöglich, den Betrag der Helligkeitsschwankungen zahlenmässig au»ugeben und irgend eine Gesetzmässigkeit des Lichtwechsels aufzufinden. Der eclatanteste Fall ist der berilhmte Hind'sehe Nebel (Nr. 1555 in Dreyers New General Catalogue of nebulaee; Position fur 1560,0: $a=4^{\mathrm{b}} 13^{\mathrm{m}} 45^{\mathrm{s}}$ und $\left.\delta=+19^{\circ} 11 \cdot 2\right)$. Dieser Nebel wurle im Jahre 1552 von Hind als ein ziemlich schwaches Objeet mit einem Durehmesser von nieht mehr als $30^{\prime \prime}$ entdeckt. In den folgenden Jahren wurde er von verschiedenen Beobachtern, zum Theil mit missigen Instrumenten, leicht gesehen, und d'Arrest bezeichnete ihn 1855 und 1556 sogar als sehr hell. Dagegen konnte ihn Sehönfeld im Jahre 1861 mit dem achtfiissigen Refractor der Mamnheimer Sternwarte nicht auffinden, und auch d'Arrest gelang es in diesem und dem folgenden Jahre nicht, im Kopenhagener Refractor eine Spur ron dem Nebel walırzmelumen. Mit dem Lassell'schen Teleskop auf Malta und dem Pulkowaer Refrictor wurde das Object noch in den folgenden .Jahren mit Miihe erkalnut, aber im Jahre 1568 war es anch fuir das Pulkowaer Instrument grinzlich unsichtbar. Später scheint man dem Nebel keine weitere Beachtung geschenkt zu haben, und erst in der allerueuesten Zeit jst rou l'uruluan' und Barnard $\left.{ }^{2}\right)$ von Neuem anf denselben antmerksiun gemachlit worlen. Mit dem 36-Zöller der Lick-Stemwarte konuten diese beiden Beohanhter in Jahre 1890 und später im Februar 1895 den Nebel dentlich walunchmen, und Barnard glanbt sogar behaupten zu kïnnen, dass or h!la ctwals beller gewesen ist als 1590 . Im September 159. hat batruall noch mehrere Male nach dem Nebel gresucht, ohne datss es ihm grelmugen ist.

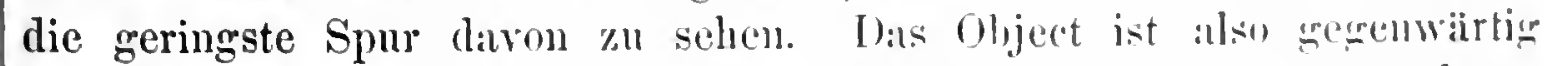
auch für das mïehtige Liekfermrohr msichthar; und es moterliegt latuer keinem Zweifel, dass seine Liehtstairke sich rerindert hat. In munittelbarer Nïhe des Hind'schen Nebels (etwa 2* folpend und 226" niirdlicher steht der rerinderliche Stern 'T' Tauri, welcher von den beiden gen:mutcu Beobachtern in der nenesten Zeit ebenfalls als Vebelstern erkamut worlen

1) Monthly Notices. Tol. 51, p.94.

2) Monthly Notices. Vol. 55, p. 442; Vol. 56. p. liti. 
ist. Im Jahre 1890, als der Stern sich im Minimum der Lichtstärke befand, bildete er den dentlichen Kern eines sehr kleinen, ziemlich hellen, länglich geformten Nebels; dagegen war im Februar 1895, wo der Stern selbst viel heller war, nur eine ganz schwache Andeutung von diffuser Nebelmasse um denselben zu erkennen.

Der. zweite Nebel, bei welchem zweifellos ebenfalls eine merkliche Helligkeitsänderung vor sich gegangen ist, steht merkwürdiger Weise ganz in der Nähe des Hind'schen Nebels. Es ist dies Nr. 1554 des Dreyer-

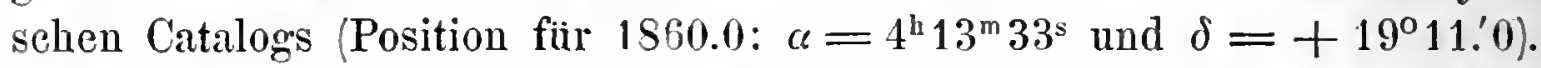
Er wurde $1 \$ 68$ rou 0 . Strure aufgefunden und nachher mehrere Male ron d'Arrést beobachtet, welcher sicher zu sein glaubte, dass friiher an dieser Stelle kein Nebel rorhanden gewesen war. D'Arrest bezeichnete ihn als ziemlich klein und fast rund mit excentrischem Kern und schätzte ihn schwächer als den Hind'schen Nebel, etwa der Herschel'schen Classe II angehörig. Im Jahre 157 (November 8) konnte Tempel in Arcetri den Strure schen Nebel noch deutlich erkenuen, dageg*en sah er ihn am 12. December desselben Jahres uicht mehr, und nur zwei schwache Sternchen waren an der Stelle des Nebels sichtbar, ron denen der eine anch bereits im Norember bemerkt worden war. Im Jahre 1890 haben Burnham und Barnard mit dem grossen Lickfermrobr den Nebel nicht mehr aufgefunden, und anch 1595 war er unsichtbar; ron den beiden Tempel'schen Sternchen liess sich nu der eine constatiren. Es unterliegt keinem Zweifel, dass der Strure sche Tebel ebenso wie der Hind'sche gregenwärtig gäinzlich unsichtbar geworden ist. Die ganze Umgebung dieser beiden merkwiurdigen Objecte rerdient andanernd die sorgtailtigste Beachtung ron Seiten der Astronomen.

Der dritte als repbiirgt zu betrachtende Fall eines reränderlichen Tebels stitzt sich zwar nur anf das Zengniss eines einzigen Beobachters, aber die Angahen sind so klar, dass an der Realitit kanm zu zweifeln ist. Im Jalne 1sss entleckte Barmard'1 mit dem 12-Züller der Licksternwarte einen kleinen ziemlich lıellen Nebel, der in keinem Cataloge zu finden war, und den el etwa einem sterne 9. bis 10. Grösse gleich shaitzte. Seine Position wurde genau liestimnt und ergab sich für

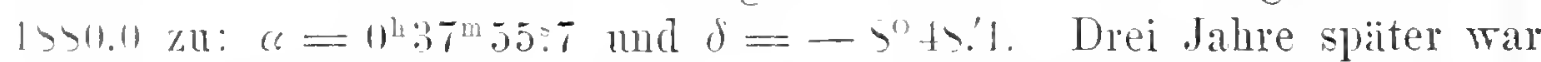
dieser Xehel mit demselben Instrumente nur ämserst schwierig anfanfinden mul ist anch lis hentigen Tages ein ganz sehwaches object geblieben. tuser den drei angefuhrten Tebehn sind noch viele der Teränderlishkit rerdichtigt worden, ron denen hier zum schlusse noch zwei nanhaft emacht werden sollen, weil bei ihnen die Yöglichkeit rou 
periodisehen Lichtsehwankungen vorhanden ist. Es sind dies die beiden Tebel Nr. 955 und Nr. 3666 in Dreyers Nebeleatalog oder h 229 und

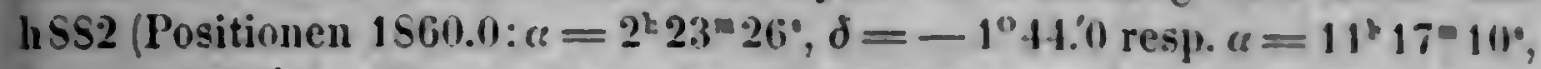
$\delta=+12^{\circ} 6^{\prime} 6$. Winnecke' hat die wichtigsten Beobachtungsangaben uber diese beiden Objecte zusanmengestellt, und es gelit darans Folgendes hervor. Der erstere Nebel ist rou den beiden Hersehel 1755 resp. 1827 als $p B$ be\%eichnet worden, dagegen ist er 1556 ron d'Arrest selir schwach genannt und 1561 ron Schünfeld und ebenso spater 1565 von Vogel vergeblich gesucht worden; 1S6S wurde er rou Scliönfeld wieder deutlich gesehen und $157 \mathrm{~T}$ ron Winnecke sogar als secht helle bezeiehnet; im Jahre 1SSt war er noeh ein leidlich helles Object. Der andere Nebel, den W. Hersehel vB nanute, ist ron J. Hersehel $1 \$ 30$ und 1 S31 wiederholt als $F$ oder sogar $e F$ anfgefthit; Winnecke rechnete ihn 1 SiS - ISi9 wieder zur zweiten Hersehel'schen Classe, während Dreyer ihn 1557 nur mit der grüssten Schwierigkeit wahrnehmen konnte.

Dic periodische Variabilitait scheint hiernach bei beiden Objecten ziemlich sicher zu sein, und es ist zu hoffen, dass dureh exate photometrische Messungen die Dauer der Periode und der Betrag der Lichtinderung festgestellt'werden kaun.

\author{
Capitel $\mathrm{r}$. \\ Die Fixsterne.
}

1. Die Helligheitserzeichnime ler Fixitelde.

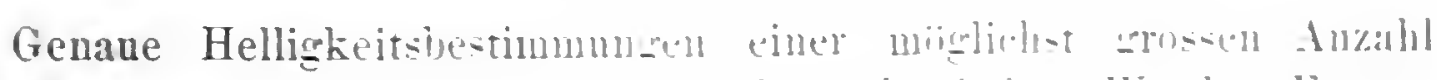

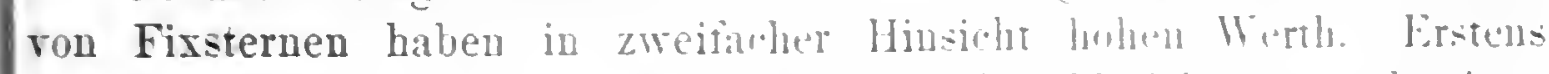

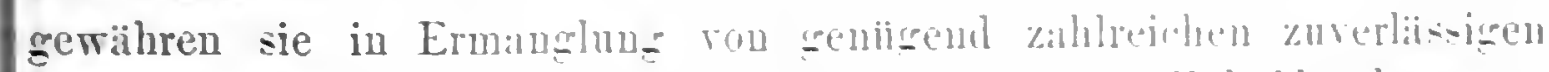

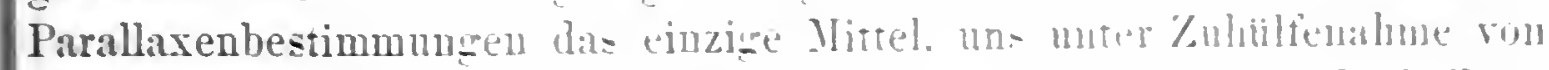

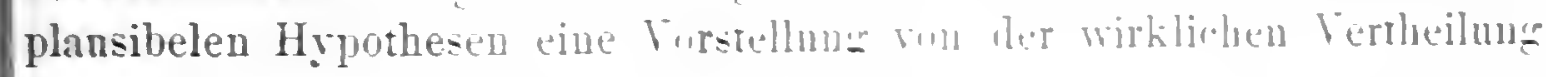

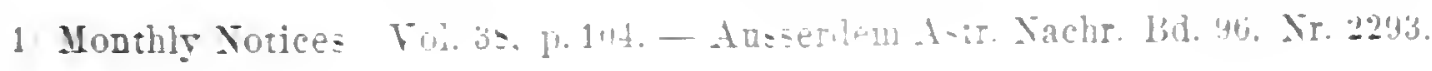


der Sterne im Raume und von der Anordnung des Weltalls zn bilden; und zweitens geben sie uns Aufschluss iiber Veränderungen, welche in der physischen Beschaffenheit der Gestirue vor sich gehen. In letzterer Beziehung handelt es sich nicht uur um die Auffindung aller mit dem speciellen Namen „Veränderliche" bezeichneten Objecte, bei denen Lichtschwankungen von grösserem oder geringerem Betrage schon in verhältuissmässig sehr kurzen Zeitrïumen beobachtet werden können, sondern von viel grösserer Tragweite ist die Entscheidung der Frage, ob im Laufe der Jahrhunderte eine gleichmaissige Zunahme oder Abnahme des Lichtes bei sämmtlicheu Fixsternen oder wenigstens in bestimmten Regionen des Himmelsraumes und bei gewissen Classen von Sternen, beispielsweise bei denjenigen vou gleicher Färbung, stattfindet. Da ein Stillstand in dem Entwicklungsprocesse eines Weltkörpers nudenkbar ist, so wird man ohne Weiteres zn der Annahme berechtigt sein, dass auf jedem Fixsterne Veränderungen vor sich gehen, die sich, wenn auch möglicherweise erst nach vielen Jahrtansenden, durch ein Anwachsen oder eine Verminderung: der Lenchtkraft offenbaren miissen.

Der Photometrie ist seit wenigen Jahrzehnten ein mächtiger Bundesgenosse entstanden in der Spectralanalyse, welche sich nicht mit der Untersuchung der Quantität, sondern der Qualität des von den Fixsternen zu uns gelangenden Lichtes beschäftigt und uns gelehrt hat, dass die Gestirue sich in ganz versehiedenen Entwicklungsstadien hinsichtlich ihrer physischen Beschatfenheit befinden. Noch ist dieser bliihende Zweig der Astrophysik viel zu jung, um auch nur den kleinsten Theil aller dabei auftretenden Fragen zu entscheiden. In absehbarer Zeit ist gar nicht daran zu denken, dass das Spectroskop sicheren Aufschluss dariiber geben könnte, ob bei allen Fixsternen ein gleicher Entwicklungsgang vorausgesetzt werden darf, und zwar in dem Simne, dass alle Spectra der ersten Classe. allmïhlich in solche der zweiten und diese wieder in solche der dritten Classe übergehen, dass also eine allmähliche Abkiihlung und infolge dessen anch Lichtabnahme aller Fixsterne eintritt, oder ob entsprechend den Lockyer'schen Hypothesen ebenso oft Übergïnge ans niederen in höhere Spectralclassen wie nugekehrt vorkommen, und ob demnach Znnahme der Temperatur bei einem Theile der Fixsterne ebenso walhseheinlich ist wie Abkiihlung bei den iilurigen. Anch darf nicht vergessen werden, dass bei dem gegenwärtigen Stande der instrumentellen Hiilfsmittel vorlia fig uur an den helleren Fixsternen siehere spectralanalytische T'ntersuchungen möglich sind. Bei dem nnermesslichen Heere der schwächeren sterue werden wir voraussichtlich uoch anf lange Zeit hinaus, wenn wir nach Verändermugen in ihrer physischen Beschaffenheit fragen, ledighich auf photometrische Messungen angewiesen sein. Es goht daraus herror, dass es Pflicht 
jedes Zeitalters sein sollte, ein muglichst getreues Bild von den Helligkeitsverhältnissen an Fixsternhimmel zu entwerfon und damit den kommenden Gesehlechtern das Material »u weiteren erfolgreiehen Forsehungen »u lieferu. Im hohen Grade befremdlieh bleibt es, dass diese Erkenntniss so wenig beherzigt worden ist, nud dass die Entwieklung der Fixsternplotometrie unendlich weit hinter der anderer Zweige der $\Lambda$ stronomie \%urllekgebliehen ist.

Der älteste Positionseatalog von Fixsternen, den wir im Almagest des Ptolomans besitzen, ist ungleich anch das erste Helligkeitsverzeichniss. Aber während ron der Zeit des Ptolemäus an in Bezug auf die Positionsbestimmnngen der Sterne ein stetiger Fortsclıritt \%n erkennen ist, bis zu dem relativ hohen Grade der Vollkommenheit, der heute erreieht ist, kann in Bezug auf die Helligkeitsbestimmungen der Fixsterne bis fast in die Mitte des gegenwirtigen Jahrhunderts nur ein rollkommener Stillstand eonstatirt werden. Die im .Jalıre 1843 erschienene Uranometria nova von Argelander gielst die Intensitit der Sterne nach blossen Sehätzungen in fast denselben unvollkommenen Unteribtheilungen an, welehe bereits die Beobachter des Almagest eingefuhrt hatten. Der Fortsehritt von den Zeiten des Ptolemäins bis \%u Argelander ist ausserordentlieh nubedentend. Die Sicherheit der Helligkeitsangaben zu beiden Epoehen ist so gering, dass es ummöglich sein wilrde, säculare Helligkeitsänderungen von nicht allzu grossem Betrage zu entdecken. Innerhalb dieses langen Zeitrammes kann von einer eigentlichen Fixsternphotometrie nielit die Rede sein; eine solche datirt erst ron I. Hersehel, Seidel und Zöllner, welche zuerst photometrische Apparate anf den Fixsternhimmel angewendet und wirkliche llelligkeitseataloge anfigestellt haben. Warum das Beispiel dieser Männer bis in die Nenzeit noch nicht diejenige Nachahmung gefunden hat, die der Wichtigkeit des (ierentustandes entspricht, und warum atueh heute noch ein wrosser Theil der Astronomen der Photometrie der Fixsterne gnleichgiiltig geventibersteht, ist sehwer zu sagen und $1 \mathrm{~m}$ so unbegreiflicher, als gerade dic l'flege dieses Zweiges rerhältnissmässig beseheidenc instrumentelle Iliilfsuittel erfordert, wie sie auch der kleinsten stemwirte \% Gebute steden wiirden. Vielleicht liegt der Grund zum Theil dirrin, diss viele Astronmen den blossen Helligkeitssehitzungen einen uibertrichenen Werth beilenen, datgegen den verschiedenen photometrischen Instrunenten cin gewisses Miss-

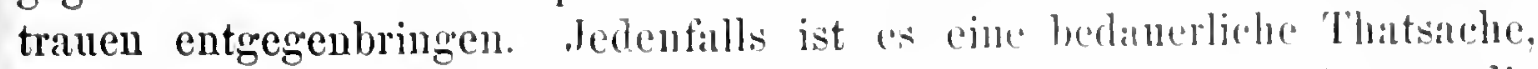
dass, während hentzutage fuir mehrere llunderttansende von sternen die allergenauesten Positionen bekannt sind, wir kilnm für den dreissigsten Theil derselben photometrisch bestimmte Hellipkeitsin wallen besit\%en, die noch dazu fast alle den Beniihungen ciner vinzigen stemwarte, der des Harrard College in Cimbridge (Amerikil), zu verdanken sind. Gerate 
auf einem so ausgedehnten Gebiete lassen sich nur durch ein planmässiges Zusammenwirken grosse Fortschritte erzielen.

Im Folgenden soll eine kritisehe Übersicht über die wiehtigsten Helligkeitsverzeichnisse von Fixsternen, die wir besitzen, gegeben werden, und zwar in erster Linie iiber diejenigen, welche auf blossen Schätzungen beruhen, zweitens ibber diejenigen, welehe aus photometrisehen Messungen hergeleitet sind; endlich sollen die Beziehungen erörtert werden, welche zwischen diesen beiden Classen von Helligkeitscatalogen existiren.

\section{a. Helligkeitsverzeichnisse, welche auf Grössenschätzungen berulien.}

Das älteste Helligkeitsverzeichniss, welehes bis auf unsere Zeiten gekommen ist, findet sich, wie schon erwälnt, in der $\mu \varepsilon \gamma \alpha^{\prime} \lambda \eta \sigma \dot{v} \nu \tau \alpha \xi \iota s$ des Ptolemäus. Die Epoche, welehe Ptolemäus seinem Cataloge zuschreibt, ist etwa das Jahr 138 n. Chr.; es steht aber fest, dass die Beobachtungen, auf welehen der Catalog beruht, nicht von Ptolemäius selbst herriihren, sondern ans einer viel friiheren Zeit stammen. Die Meisten schreiben sie dem Hipparch (etwa 150 v. Chr.), einige sogar einem noch friheren Astronomen, dem Eudoxus (etwa 366 v. Chr.), zu. Fiur die Beurtheilung der Genanigkeit der Positionen ist diese Streitfrage von der allergrössten Wiehtigkeit, wiihrend sie in Betreff der Helligkeitsangaben ron geringerem Belange ist. In dieser Bezielıng geniigt die Angabe, dass der Ptolemäus'sche Catalog ein Bild von den Helligkeitsverhältnissen der helleren Sterne ungeführ zu Beginn der cluristlichen Zeitreehmung giebt. Hentzutage ist man wohl kaum noch im Kweifel, dass der Hauptwerth dieses Cataloges grerade in der Eintheilung der Sterme nach ihren Intensititen besteht, und es ist in hohem Grade bemerkenswerth, dass die ron Ptolem ï us eingefiihrte Classificirung mit geringen Modificationen bis jetzt beibehalten worden ist. Ptole mä us hat zherst das Wort úgevos (Grösse) für die Bezeiehmung. her Sternhelligkeiten henutzt und hat fiil die mit blossem Auge sichtbaren steme, die bei ihm allein in Betracht kommen kounten, ganz willkiirlieh sechs Hauptabtheilungen oder Grössenchissen (er hätte natiirlich ebenso g̈ut anch deren acht oder zehn wählen künnen) festgesetzt, indem er die allerhellsten Objecte an Himmel Sterne erster Grösse, die schwäehsten Steme sechster Groisse nannte und die dazwischen liegenden so einzntheilen suchte, dass der Holligkeitsunterschied zwischen zweiter und dritter trönse ungefihn ehenso gross wurde, wie der zwischen dritter und vierter Griose 1. s. w. Eine Gefahr, die bei einem derartigen Eintheilungsversuche, iiberhangt hei allen blossen Helligkeitsschitzungen, sofort auftritt, liegt darin, dass die Zahl der schwaicheren Sterne am Himmel betrïehtlich 
grösser ist als die der helleren. Infolge dessen wirl das Ange von voruherein versucht sein, hei den weniger hiufig vertretenen cine verhliltuissmässig grössere Zahl zu ciner Hauptelasse zusanmen\%ufussen, als hei den schwächeren, und es kamn daher sehr leieht kommen, dass die eingefthrte Helligkeitsscala eine ungleichmissige wird. Wir kommen hierauf spaiter noch ausfillirlicher \%urllek.

In den iilteren Handschriften des Almagest finden wir bei einer Anzahl von Sternen zu der Zahl, welehe die Grösse angiebt, noch die Bnch-

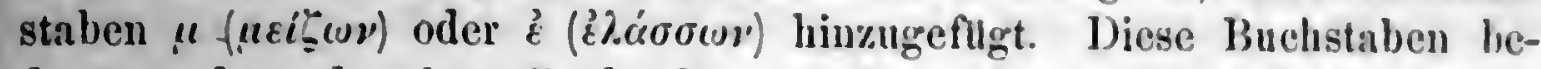
deuten, dass der betreffende Stern heller oder sehwiicher ist als die angegebene volle Grössenclasse. Die Beobachter des Alunagest haben also das Unzulïngliche einer. Eintheilung in nur sechs verschiedene Hauptclassen bereits gefilhtt und versucht, Unterabtheilungen einzufuliren, oftenbar mit der Absicht, dass auch diese Unteralıtheilungen einem gan regelmässigen Helligkeitsverlaufe entspreehen sollten. Dass erst durch diese Zwischenstufen der Helligkeitscatalog des Ptolemä us seinen vollen Werth erhält, liegt anf der Hand, und es ist \%u bedanern, dass in den beiden am meisten verbreiteten Ausgaben des Ptolemäus'schen Sternverzeichnisses, sowohl in der Halmas als in der Bailys, die Bezeichnung der Zwisehenstufen fortgelassen ist. Die verschiedenen Handschriften des Almagest zeigen leider in Betreff der himzugefügten " und ż betrïchtliche Unterschiede; häufig stehen die Buchstaben zwischen zwei aufeinander folgenden Zeilen, so dass nicht zu entscheiden ist, zu welcher derselhen sie gehören, bei manehen Sternen sind sie ganz fortgrelassen, an anderen Stellen sind - sie wahrscheinlich miteinander vertanscht n. s. w. l'eiree') hat eine sehr dankenswerthe Vergleichmon zwischen alcht der hekanntesten Hand-

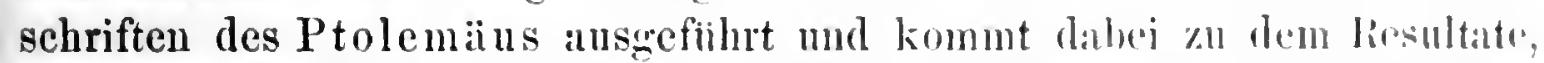
dass die aus dem 9. Jahrhundert stammende Hands(hrift Nir. 23:3!) in der Sammlung der Bibliotlieque nationale zu Paris, welche anch der Halma sishen Ausgabe zu Grunde gelegen lat, bei weiten die zuverliissigste ist mol mur

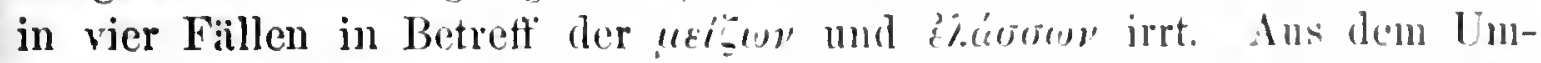
stande, dass nach der Peirce'sehen Identificirun ron den siinntlichen

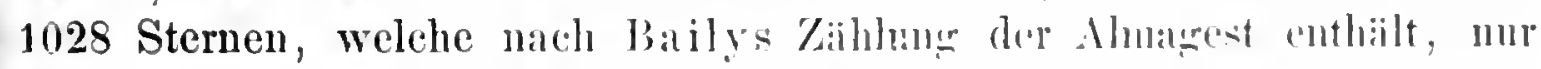
154 den neun bei Ptolemains rorkommenden Zwischenstufon anrehören,

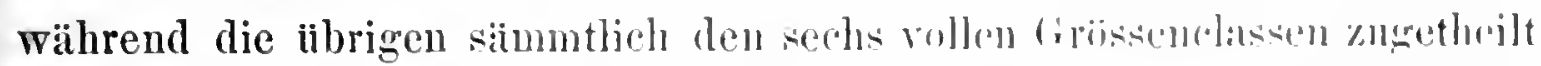

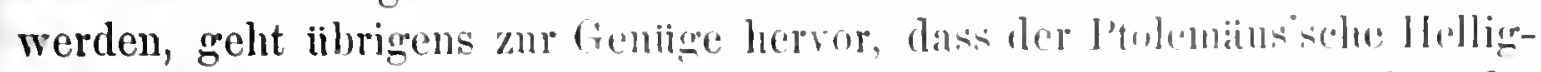
keitscatalog kein vollkommen homogenes Material cuthialt. Xinr firl einen Bruchtheil der Sterne sind engere lutensitittsintervalle eingefiuht worden; bei dem Gros der Steme schreiten dir l unterschiede von Griissen-

1) Annals of the Astr. Obs. of Harvard College Vol. 4. p. 39. 
classe zu Grössenclasse vorwärts. Die häufig wiederkehrende Angabe, dass der Almagest die Helligkeiten der Sterne in Drittelgrössenclassen enthalte, ist also in dieser allgemeinen Form nicht richtig. Bei einem grossen Theile der Ptolemüus'schen Sterne ist überdies infolge ungenauer Ortsangabe die Identificirung schwierig. Pickering ${ }^{1}$ ) hat bei einer Vergleichung der Sterngrössen des Almagest mit den photometrischen Messungen auf dem Harvard Observatorium naeh Ausschluss aller "nicht mit absoluter Sicherheit zu identificirenden Objecte nur 757 von den Sternen des Ptolemäus benutzt, darunter 111, bei denen Zwischenstufen angegeben sind. Aus der Piekering'schen Vergleichung geht hervor, dass die einzelnen Hélligkeitsabtheilungen keineswegs gleichmässig sind, und dass, wie von yornherein zu erwarten stand, bei den helleren Sternen eine Grössenclasse ein viel grösseres Helligkeitsintervall umfasst, als bei den schwäeheren Objecten. Ferner zeigt sich, dass zwischen je zwei benachbarten Unterabtheilungen nur ganz geringe Intensitätsunterschiede vorhanden sind, und dass also die Bezeichnungen $3 \vec{\varepsilon}$ und $4 \mu$, ebenso $4 \vec{\varepsilon}$ und $5 \mu$ u. s. w. fast dasselbe besagen; es kann also schon aus diesem Grunde nieht von einer Eintheilung in Drittelgrössen bei Ptolemäus die Rede sein. Für den wahrseheinlichen Fehler einer Helligkeitsangabe des Almagest wird man waeh Pickering die Zahl \pm 0.3 Grössenclassen annehmen diirfen.

Ein Zeitraum von ungefähr 800 Jahren liegt zwischen dem Erseheinen des Almagest und dem Zeitalter des persischen Astronomen Abd-al-Rahman Al-Sîfi, der von 903-986 lebte und uns in seinem Werke »Besehreibung der Gestirne" einen Helligkeitseatalog hinterlassen hat. Dieses Werk, friiher nicht genug beachtet, ist erst dureh die vortreffliche Übersetzung Sehjellerups ein Gemeingut der Astronomen geworden. Sûfi hat zwar seinem Werke den Catalog des Ptolemïus zu Grunde gelegt, sich aber keineswegs vollständig von demselben beeinHussen lassen. Er hat nicht nur die Örter des Almagest einer Prüfung unterworfen, sondern vor Allem die Helligkeiten der Sterne revidirt, und man kann wohl mit Sicherheit amnehmen, dass er uns ein Bild des Fixsternhimmels iiberliefert lat, wie es sich den Blieken seiner Zeitgenossen zeigte. Es scheint sogar fast, als wäre die Ermittlung der Sterngrössen der Hauptzweck seiner Arbeit gewesen. Der Catalog enthält 1145 Objecte, es sind also zn den Sternen des Almagest noch einige, meist schwaichere, hinzugenommen worden. Die Grössenseala des Ptolemïns ist unveriindert beibehalten, und es trifft daher im Grossen und Ganzen dasselbe zu, was oben bemerkt wurde. Die Zwisehenstufen sind

1) Annals of the Astr. Obs. of Harvard College. Vol. 14, part II, p. 329. 
uicht planmissig eingefuhrt, sondern offenbar mur gelegentlich benutzt worden, und der Unterschied zwischen je \%wei benachliarten Unterabtheilungen ist verschwindend klein; es kann ulso auch bei Sinfi nicht von einer Trenuung in Drittelgrössen, höchstens ron einer solehen in halbe Grïssen gesprochen werden. Dagegen ist die Identifieirung der Sterne sicherer als bei Ptolemius, und anch die Genungkeit der Schïtzungen scheint eine etwas grössere \%u sein. Nach Pickerings Untersuchungen ist der wahrscheinliehe Fehler einer Suff'schen Helligkeitsangabe gleich \pm 0.24 Grössenclassen.

Von Snfis Zeit bis zum Ende des 1S. Jahrhmolerts ist kein wesentlicher Fortschritt in den Helligkeitsschätzungen der Fixsterne zu verzeiehnen. Wir besitzen zwar aus diesem langen Zeitraume eine Anzahl rou Sterneatalogen, in denen anch Grössen angegeben sind; jedoch beruhen die let»teren entweder nur auf :ilteren Schät\%ungen oder sind viel zu ungenau, um Vertranen zu verdienen. Der viel gerihmte Catalog von Ulıgh Begh für die Epoehe 1437, der besonders durch die Hyde'sche Ausgabe ans dem Jahre 1665 in der astronomisehen Welt bekannt geworden ist, giebt offenbar nur die Sûti'schen Grïssen unverindert wieder. Von Tyeho Brahe sind uns zwei Sterneataloge liberliefert, der eine in seiner Schrift "De nova stella u. s. w.e, enthaltend 77 Sternc, bei denen die Helligkeiten in ganzen Grüssen und l'tolemäis'schen 'Zwischenstufen angegeben sind, der andere in keplers sabulac Rudolphinae. mit 1005 Sternen, die aber bloss in ganzen Grössen ansigedrlickt sind. Nur das erste dieser Helligkeitsverzeichnisse kinn cinen zewissen Werth beanspruchen.

Offenbar beeinflusst dureh Tycho Bralue sind in iluren Itellipkeits-

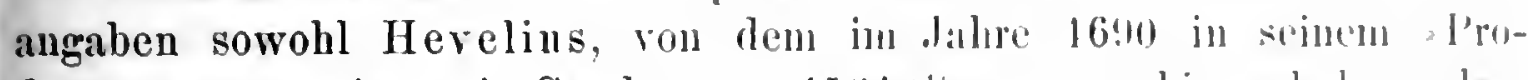
dromus astronomiae e ein Catalog ron 1.364 sternen ersolicu, als besonders

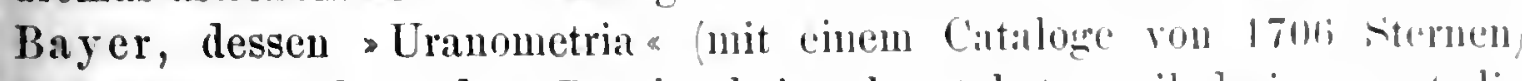
deswegen eine besondere Beriihmtheit erlangt lat. Weil darin zunerst dir

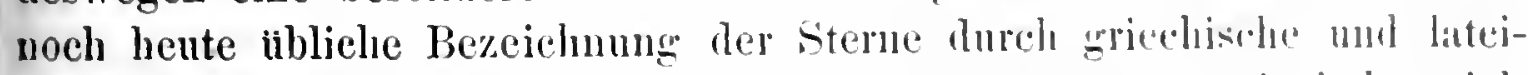

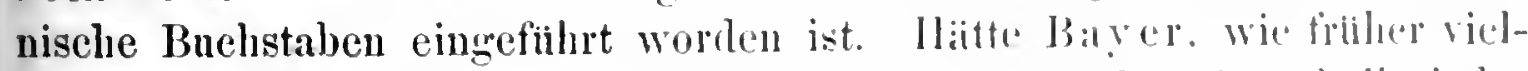

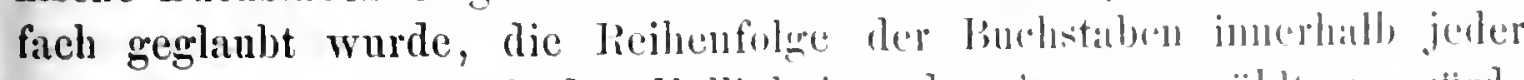

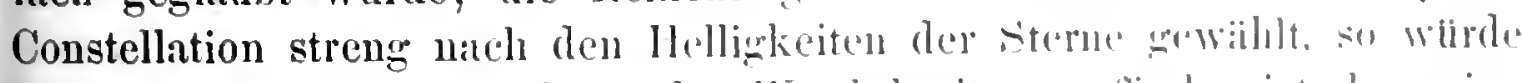

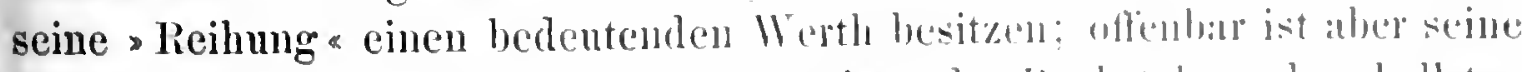

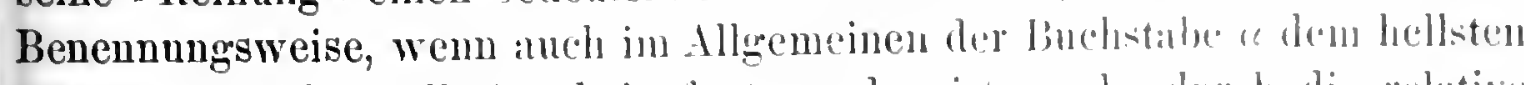
Sterne jeder Constellation beigefiigt worlen ist. methr dureh dic relative

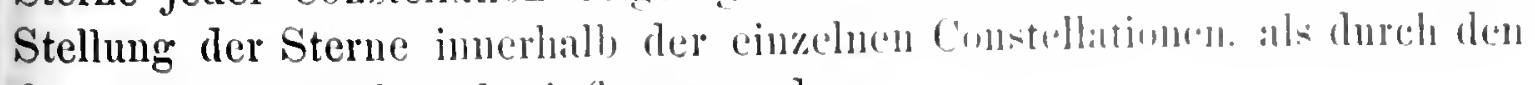
Grad ihrer Helligkeit beeinflusst worden.

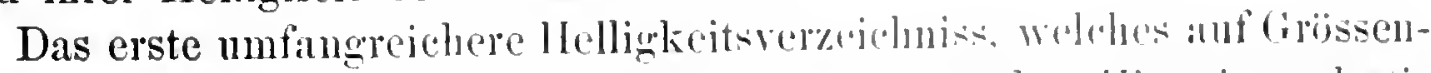
schätzungen am Fermrohre heruht. ist in Flamstedes ollistoria coelestis 
Britannica a aus dem Jahre 1725 enthalten. Nach der Baily'schen Rerision dieses Werkes beträgt die Zahl der Flamsteed'schen. Sterne 2913. Es scheint, als ob die Benutzung. des Teleskops den Helligkeitsschätzungen anfangs nicht sehr förderlich gewesen sei. Jedenfalls stehen die Flamsteedschen Helligkeitsangaben an Genanigkeit denen seiner Vorgänger, die mit blossem Ange beobachtet haben, wesentlich nach.

Einen wichtigen Abschnitt in der Geschichte der Intensitätsschätzungen der Fixsterne bezeichnen die Beobachtungen W. Herschels. Nur dem Umstande, dass diese Untersuchungen unrollständig und in einer Form reröffentlicht sind, die erst eine weitere Bearbeitung behufs Herstellung eines wirklichen Helligkeitscataloges erforderlich macht, ist es zuzuschreiben, dass die Herschel'sche Arbeit bis hente noch nicht diejenige Beachtung gefunden hat, die ihr zweifellos gebiihrt. Herschels Absicht war es geweseu, die relaticen Helligkeiteu aller in Flamsteeds Catalog euthaltenen Sterne zu bestimmen. Dieser Plan ist auch durchgefïhrt worden, aber mur ein Theil des ganzen Werkes ist im Druck erschienen ${ }^{1}$. Das iibrige Mannscript hat sich, wie ans einer Mittheilumg Pickering s $^{2}$ ) herrorgeht, in den hinterlassenen Papieren Herschels, rollstïndig' zum Druck fertig gestellt, rorgefunden. nnd es wäre mit Frende zu begriissen, wem anch dieser Theil der Arbeit der wissenschaftlichen Welt zngänglich gemacht wiurde. Herschel hatte erkannt. dass die bis dahin uibliche Eintheilung der Fixsterne in ganze. allenfalls in halbe oder drittel Grössenclassen fiur genanere Untersnchnngen keineswegs ansreichend sei; das ron ihm eingeschlagene Verfahren ging daher daranf hiuans, jeden einzelnen Stern direct mit einem anderen oder anch mit mehreren benachbarten zu rergleichen, die sich so wenig wie möglich an Helligkeit ron ilım unterschieden, nud anf diese Teise eine Gruppirung der Steme in minimalen Intensitätsstnfen herzustellen. Seine Methode ist rorbildlich gerresen für die später von Aroelander eingefiulnte Stufenschätzungsmethode, die mit so grossem Erfolge hei den Beohachtnngen der veränderlichen Sterne zur Anwendung kommt: sie unterscheidet sich ron ihr eigentlich mur durch die schreibweise. Herschel hat die drei Zeichen

- und die rerschiedenen Combinationen dieser Zeichen bemutzt. 1 m rerschicden grosse Intensitiitsunterschiede zwischen zwei Sternen zn kienuzeichnen. Ein Punkt zwischen den Xmmmern zweier Sterne soll andriteken, das dieselben entweder guz gleieh hell erscheinen, oder dass hïchstens der voranstehende ein wenig iberwiegt. Das Comma sagt ans,

Phil. Trans. of the R. Soc. of Lomion. 1796. p-166 und 452; 1797, p. 293; 1799.11 .121$.

2 Annals of the Astr. Obs. of Harrari College. Tol. 14, part II. p. 345. 
dass der zuerst genannte Stern entwehieden, wem anch unr in geringeu Betrage, heller isi als der «weite. Der Strich endlich bedentet einen merklichen Unterschied. Noch stiirkere Differen\%en werden gelegentlich durch die Zeichen $(-$,$) und (--)$ insgedrllckt, und neue Zwischenstufen finden sich bisweilen noch durch nudere Zusummenstellumgen der Zeichen markirt. Die einfuehen Symbole kommen an Hilufigsten vor. Pickering hat den Helligkeitswerth der eimzelnen Herschel'schen Stufen aus der Vergleichung mit den photometrischen Nessungen des Harvard College abgeleitet und dabei nicht bloss die vier publieirten Herschel'sehen Sternverzeielnnisse, sondern anch die beiden nur im Manuscript vorhandenen benutzt. Unter allen mit Sieherheit zu identificirenden Sternen kommt das Symbol (.) 385 mal, das Symbol (,) 868 mal und das Symbol (-) 505 mal vor. Im Mittel ergiebt sich, dass diese drei Zeichen Helligkeitsuntersehieden von $0.06,0.23$ und 0.35 oder rund rou 1,2 und 4 Zehutel Grössenelassen entsprechen. Etwas weniger sichere Werthe ergehen sich fulr die eomplicirteren, seltener rorkommenden Hersehel'schen Be\%eichnungen. Bemerkenswerth fir die Zuverlissigkeit der Herschel'schen Schätzungen ist, dass der Werth der einzelnen Symbole innerhalb des ganzen Helligkeitsgebietes, welches die Beobachtungen umfassen, nahezu constant ist.

Die Kenutniss der durch die einzelnen Zeichen ansgedrllekten Ilellixkeitsuntersehiede kann nun dazu dienen, aus den Herschel'schen Beobachtungen einen Intensititscatalog alozuleiten. Win deratigere Versuch ist bereits von Peirce') gemacht worden mit Zugrumlelegnm ron etwats anderen Werthen für die einzelnen Symbole als den oben angugebenen; da jedoch dabei nur die im Druck ersehienenen Herschel'schen Perobarlitungen benutet und ausserdem als Fundament die fuir diesen Zwerk keineswegs ausreichenden Grössen der Bonner Durchmustermugr herangezongen worden sind, so kann diese Bearbeitung nicht als definitive he\%eichnet werden. Dasselbe grilt rou der Pickering schen Bearbeitung, bei der zwar sämmtliche Herschel'schen Beobachtungen mit wirklichen photonmetrischen Messungen rerglichen wurden, die aber in b̈hrigen viel \%u summarisch ansgeführt und in viel zn weuig iibersichticher l'mm mit-

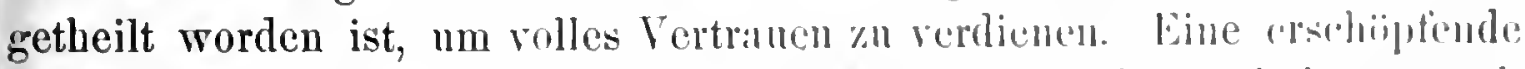
Behandlung des ganzen Herseleclschen Matterials hliche anch helute nowh

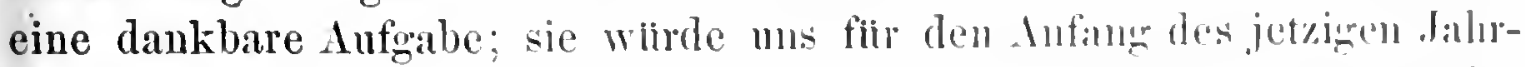
hunderts einen Helligkeitscatilog bon mehr als $200 n 0$ sternen liefim, der allen anderen aus dieser und noch ans suiteren lipulhen stimmenden bedeutend überlegen witre und beinalie mit den mondernen photometrischen

1) Annals of the Astr. Obs. of Harvard College. Vol. 9, 1\% 51\%. 
Catalogen concurriren könnte. Welchen Fortschritt die Herschel'schen Bestimmungen gegenüber den älteren bezeichnen, geht daraus hervor, dass der wahrscheinliche Fehler einer Helligkeitsangabe nach Pickering $n m r^{\circ} \pm 0.15$ Grössenclassen beträgit.

Nicht ganz so werthvoll wie die Beobachtungen des älteren Herschel sind die etwa 40 Jahre später von seinem Sohne am Cap der guten Hoffuung ausgeführten Intensitätsschätzungen, deren Bedeutung hauptsaichlich dariu liegt, dass es die ersten zuverlässigen Angaben über den Glanz der helleren Steme am siidlichen Himmel sind. J. Herschel') hat ebenfalls die Methode der directen Schätzung in Grössenclassen aufgegeben und ein Verfahren angewendet, welches ähnlich wie das seines Taters in einer Aneinanderreihung der Fixsterne bestand, jedoch mit dem wesentlichen Unterschiede, dass nicht bloss einzelne nahe bei einander stehende Sterne rerglichen wurden, sondern dass in ein und derselben Nacht eine grosse Anzahl von Sternen (bis 80) in der Reihenfolge ihrer scheinbaren Helligkeit anfnotirt wurden, die nun eine Stufenleiter ron nicht rollkommen gleichen, aber im Allgemeinen minimalen Intensitätsunterschieden bildeten. Um diese Stufenfolgen in den sämmtlichen 46 Beobachtungsreihen in die iibliche Grössenscala umzuwandeln, verglich J. Herschel dieselben mit den im Cataloge der Royal Astr. Society rom Jahre 1827 angegebenen Sterngrössen mnd leitete durch ein graphisches Ausgleichnngsrerfahren schliesslich einen Catalog ron ungefähr 300 Sternen ron der ersten bis zur fünften Grössenclasse ab, der lange Zeit als das genaneste Helligkeitsrerzeichniss angesehen wurde. Der Werth dieses Cataloges ist jedoch dadurch ein wenig beeintrïchtigt, dass bei den Schätzungen auf die Extinction des Lichtes in der Erdatmosphäre keine Rïicksicht genommen ist. Bei den einzelnen Serien kommen Zenithdistanzen bis zu $60^{\circ}$ und $70^{\circ}$ vor, und es wiurden daher an die Beobachtungen unter Umständen Correctionen ron 3 bis 5 Zehntel Grössenclassen anzubringen sein. Die urspringlich beobachteten Reihungen der Fixsterne wirrden also eine wesentliche Umgestaltung erfahren. Dazu kommt noeh, dass bei den Schïtzungen anch Monduäichte nicht vermieden sind, und dass infolge dessen die rerschieden helle Erlenchtung des Himmelsgrundes die Stufenfolge nicht nmmerklich beeinflusst haben kamn. Bei einer crentuellen Nenbearbeitung der J. Herschel'schen »Sequenzen", die sich ant' genane photometrische Messungen stiitzen miisste, wäle auf diese beiden wichtigen Punkte Riicksicht zn nehmen.

1. J. Hersche1. Results of Astr. Obs. made during 1834-3s at the Cape of Good Hope. London 1847. Chapter III, p. 304. 
Das Beispiel der beiden Herschel hat trot\% der grossen Vorallge der von ihnen eingefuhrten Sehitzungsmethoden wegen der Umsthindlichkeit der Bearbeitung keine Nachahmung gefunden, und die spulteren Helligkeitsverzeiehnisse, wenn sie auch eine betrïchtlich grössere Anzahl von Sternen enthalten, können in Be\%ug auf Genanigkeit nicht als ein Fortsehritt betrachtet werden. Dies gilt zuniehst von der Argelandersehen s Uranometria Nova e, die im Jahre 1S43 veroffentlieht wurde und die Grüssen ron allen im mittleren Europa mit blossen Augen sichtbaren Sternen enthalten sollte. Argelander kelırte dabei wieder \%n der sehon ron Ptolemäns und Sûfi benutzten Eintheilung in 6 Grössenclassen mit je zwei Unterabtheilungen zurthek. Flir die Bezeichnung der Zwisehenstufen wihlte er eine wohl zuerst ron Flansteed angewandte Schireibweise, indem er dureh $3.4^{\mathrm{m}}$ einen Stern kennzeichnete, der etwas sehwäeher als dritter Grösse ist, dagegen dureh $4.3^{\text {m }}$ einen solehen; der etwas heller als vierter Grösse ist. Um eine Verwechslung dieser Sehreibweise mit der Bezeichnung von Zeluntelgrössen zı vermeiden, wurde' später hei den Zwischenstufen der Punkt nicht unten zwischen die beiden Zahlen, sondern obenhin gesetat, von einigen Astronomen wurde auch die Benut\%ung von mehreren Punkten oder eines Striches vorgeschlagen, also entweder $(3 \cdot 4)$ oder $(3 \ldots 4)$ oder $(3-4)$ u.s.w. geschrieben. Wem auch die Genanigkeit der Sehätzungen der Uranometrie nicht unbetriiehtlich grösser ist als in den alten Catalogen, so tritt doeh auch hier der Übelstand zu Tage, dass die Unterabtheilungen verhältnissmässig zu spärlich vertreten sind und dass sie keineswegs genan Drittelgrössen entsprechen. Xach Pickering̨, der von den 3256 Objecten der Uranometria Nova 3158 mit seinen photonmetrischen Messungen verglichen hat, sind die ersten Zwischenstufen $2 \cdot \%$, $3 \cdot 4,4 \cdot 5,5 \cdot 6 \mathrm{im}$ Durehschnitt gleichbedentend mit 2.25, 3.29, 4.25, 5.29, d:1gegen die zweite Stufe $3 \cdot 2,4 \cdot 3,5 \cdot 4,6 \cdot 5$ gleichbedeutend mit 2.52, 3.5.2, 4.52, 5.52. Das Material der Uranometria Nova ist also durchauls nicht homogen, und die Argelander'sche Scala ist cine ungrleichfoirmige. Wie fast bei allen anf Schatzungen hasirten Helligkeitserzeichnissen ergicht sich anch hier, dass bei den helleren Stemen eine Grïssenclatse ein weiteres Intensitätsintervall umfasst, als bei den schwiahsten. I)er wahrscheinliche Fehler einer einzelnen Grössenangabe diufte anf rund 0.2 Grössenclassen zu taxiren sein.

Eng an die Uranometria Nova sehliesst sich der inn Jahne 1572 erschienene Atlas coelestis norns von IIeis an. Vervelle entlailt cine Revision der Argelander'schen Grössen, die schwrrlich als fran\% mathhängig zu betracluten ist. Was jedenfalls meln Worth hat, ist die Fortführung des Argelander"schen Werkes bis zn den Sternen der Crijse 6.T, soweit sie von Heis noch mit blossem Ange gesehen werden komnten. Die 
Zahl der Objecte betrïgt im Ganzen 5421. In Betreff der Scala gilt dasselbe wie für die.Uranometria Nova; anch die Genauigkeit der Beobachtungen diurfte ungefähr dieselbe sein.

Als eine Fortsetzung der Arbeiten von Argelander und Heis ist der *Atlas des südlichen gestirnten Himmels * von Behrmann (erschienen 1874) anzusehen, welcher die Schätzungen der mit blossem Auge sichtbaren Sterne zwischen 20 Grad südlicher Declination und dem Siidpol in der Argelander'schen Scala enthält. Die Genauigkeit dieser Beobachtungen scheint eine verhältnissmässig geringe zu sein, wie sowohl aus der von Gould als von Pickering angestellten Vergleichung hervorgeht. Nach Letzterem ist der w. F. einer Behrmann'schen Helligkeitsangabe grösser als 0.2 Grössenclassen.

Nicht besser steht es um die Angaben der von Houzeau im Jahre 1878 herausgegebenen »Uranométrie générale . Dieselbe enthält die Helligkeitsschätzungen von allen sowohl am nördlichen als südlichen. Himmel mit blossem Auge sichtbaren Sternen, im Ganzen von 5719 Objecten, wèlche Houzeau während eines Aufenthalts in den Tropen angestellt hatte. 'Dadurch dass diese Schätzungen direct nur in halben Grössenclassen statt in Drittehn ansgefiihrt sind, ist schon von voruherein der zu erreichenden Genanigkeit eine Grenze gesetzt, und das an und für sich verdienstliche Unternehmen bezeichnet in der Geschichte der Helligkeitsbestimmungen im Vergleich zu seinen Vorgängern jedenfalls keinen bemerkenswerthen Fortschritt.

Bei Weitem das hervorragendste Helligkeitsverzeichniss, nicht nur unter den bisher angefiihrten, sondern iiberhanpt unter allen, die anf Grössensehätzungen beruhen, ist die »Uranometria Argentina « von Gould (1879), deren Bedentung bisher noch nicht geeniigend gewürdigt zu sein scheint. Was dieses Werk weit iiber andere derartige emporhebt, ist der Umstand, dass sämmtliehe Schätzungen direct in Zehntelgrössen ausgefiihrt sind, und zwar nicht nach einer bloss im Gedïchtniss beruhenden Scala, soudern im Anschluss an einen Guirtel ron Hanptstemen, deren Grössen durch die sorgfältigsten Vergleichungen als Fundament der ganzen Arbeit vorher festgelegt wurden. Es ist dies das einzig richtige Verfahren bei einer derartigen Catalogisirung der Sterne. Denn dadnrch: dass die willkürlich gewialte Intensitaitsscala immer wieder von Nenem, zn Rathe gezogen wird, ist eine vollkommene Gleichmiissigkeit der Schätzungen von roruherein gesichert; die Methode kommt dadurch, ähnlich wie die Herschel'sche, anf die Benrtheilung von minimalen Helligkeitsunterschieden himans, und es darf mit Recht behauptet werden, dass die Genanigkeit der Resultate beinalue diejenige von Messungen mit photometrischen Apparaten erreicht. Als ein besonderer Vorzng der Gould- 
schen Uranometrie ist ferner auzfnliren, dass die Schatznngen stets von mehreren Beobachtern angestellt sind. Das Werk unfasst den ganzen audlichen Himmel nud den Gurtel zwisehen Ïquator und + 100 Deelinution und erstreckt sich Ilber alle mit blossem Auge sichtharen Sterue bis znr Grüsse 7.0 linab, geht also noch etwas llber die von Heis gesteckte Grenze hinans. Der Catalog entlılt 7756 Objecte, welehe heller als 7.1 gesehatat sind, wobei eine Anzalıl von Veränderliehen und Nebelflecken mitgercehnet ist; ansserdem ist noch eine nicht unbetrichtliche Zahl (981) von sehwiicheren Objecten hinzugefligt, bei deren Einreihumg das Opernglas lind eventuell das Teleskop "u Hulfe genommen wurde. Die Seala ist so gewiihlt; dass sie sich eng an die der Argelander'schen Uranometria Nora anlelunt. $\mathrm{Zn}$ diesen Zweek sind die Anlialtsteme in dem Gurtel zwisehen $+5^{\circ}$ und $+15^{\circ}$ Declination ansgesucht, welcher in Bonn und Cordoba nalue dieselben Zenithdistanten erreicht, und die Grössen derselben sind so festgelegt, dass der Durchselınittswerth jeder Hanptabtheilung mit dem entsprechenden Werthe bei Argetander Ubereinstimmt. Vou den so heransgegriffenen 1800 Sternen wurden dicjenigen 722 als eigentliche standards a beibehalten, bei denen die Schïtzungen von vier Beobachtern vollkommen miteinander harmonirten. Diese 722 Hauptsterne bilden ein ganz besonders wertholles Material, da die zufälligen Schätzungsfehler jedenfalls ansserordentlich treringfiggig sein werden; sie sind das eigentliche Gerilst, anf welchem die Uranometria Argentina aufgebaut ist. Zur Erleichterung sind an lie Hauptserie der Vergleichstene noch einige Nebenserien in sullichen Declinationen angesehlossen worden, $m$ in allen Theileu des Jimmels Anhalt-

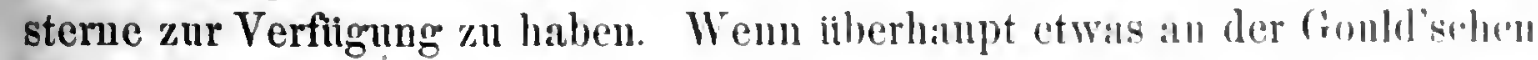
Uranometrie zn tadeln wäre, so kïmnte sich dies hïchstens daranf beziehen, dass, wie bei den meisten der anfgezihlten Lamometrien, die Sterne nach Stembildern und nicht in der Reihenfolge der liectassensiomen zusammengestellt worden sind, wodureh die Ühersichthichkeit und das Aufsuchen der einzelnen Objecte etwals erschwert ist.

Wir kommen nun zm Besprechmur derjenign Ilelligkeitserzeichnisse, deren Angaben auf Sehitzungen am Fermohr beruhen. Fast ille Positionseataloge, mögen sie aus planmaissign allgestollten Zumenhenhachtungen hergeleitet sein oder mehr einen grelegentlichen ('halrakter tragen, enthalten Helligkeitsschätzungen von griisserem wher wringerem Werthe. Bei allen ist die Eintheilung in Grïssenelassen beibehalten worden; doch findet zwischen den versehicdenen Beobachtern hinsidhtlieh des I mfanges der einzelnen Classen keineswegs dieselbe Ühereinstimmung stutt. wie bei den belleren Stermen. Es hat dies whl hamptsibllidh darin soinen Grund, dass eine unterste Grössenclasse für die teleskopischen Sterne nicht ron 
vornherein festzulegen ist, und dass die ganze Eintheilung mehr in eimer Extrapolation als in einer Interpolation besteht. Am meisten voneinander abweichend sind die von W.Struve und J. Herschel eingeführten Scalen. Nach der ersteren sind die schwächsten mit einem Fernrohre von $24 \mathrm{~cm}$ Öffnung gerade noch sichtbaren Sterne als Sterne 12. bis 13. Grösse zu bezeichnen, während dieselben nach Herschel bereits zur 20. Grössenclasse gerechnet werden. Der verschiedene Gang der beiden Scalen ist aus der folgenden kleinen Zusammenstellung zu erseben:

\begin{tabular}{|c|c|c|c|}
\hline Herschel & Struve & Herschel & Struve \\
\hline 7. Grösse & 6.3 & 13. Grösse & 10.6 \\
\hline 8. » & 7.2 & 14. & 10.8 \\
\hline 9. & 8.1 & 15. & 11.0 \\
\hline 10. & 8.8 & 16. & 11.2 \\
\hline 11. & 9.6 & $\vdots$ & $\vdots$ \\
\hline 12. & 10.2 & 20. & 12.0 \\
\hline
\end{tabular}

Die ron Argelander in der grossen Bomner Durchmusterung gewählte Scala stimmt sehr nahe mit der Strure'schen überein, und da dieselbe, wie später bei der Vergleichung mit den photometrischen Messungen gezeigt werden soll, angenähert einem gleichmïssigen Stufengange der Helligkeiten entspricht, so rerdient zweifellos die Strure sche Scala den Vorzug vor der Herschel'schen. Sie ist anch jetzt fast allgemein adoptirt worden, und die Herschel'sche Scala findet nur noch rereinzelte Anhänger in England.

Das umfangreichste und werthrollste Terzeichniss von teleskopischen Grössenschätzungen ist die Bomner Durchmusterung ron Argelander, Schönfeld und Krïger, welche die Helligkeiten aller Sterne bis zur Grösse 9.5 vom Nordpol bis zur Declination - $2^{\circ}$ angiebt, und die Fortsetzung derselben, die Siidliche Durchmusterung ron Schönfeld fiur den Giurtel ron $-2^{\circ}$ bis $-23^{\circ}$ Declination. Daran schliesst sich die nach demselben Plane auf dem Observatorium in Cordoba ron Thome unternommene Durchmusterung des iibrigen siidlichen Himmels, rou welcher der erste Theil $\left(-22^{\circ}\right.$ bis $-32^{\circ}$ Dechination) bereits rerüflentlicht ist. Nach Fertigstellung dicses Werkes werden wir von mehr als einer Million Stemen Helligkeitsschätzungen besitzen. Da in rielen Jahrzehnten, rielleicht in Jahrhunderten, schwerlich Anssicht rorhanden ist, dass diese Schïtzungen dureh genane photometrische Messungen ersetzt werden, so liegt die hohe Bedentung derselben klar zu Tage. Sie werden noch fur lange Zeit das Fundament fuir alle Speculationen sein, die an die Helligkeiten der Sterne geknipht werden können, und es ist daher 
von der grössten Wichtigkeit, Uber den Genauigkeitsgrad dieser Grössenschitzungen vollkommen unterrichtet \%u sein. Fs seheint dies $11 \mathrm{~m}$ so nothwendiger, als gar nicht selten in astronomisehen Kreisen die Neigung vorhanden ist, die Bedeutung der Helligkeitsangahen der Bonner Durchmusterung zn llbersehätzen und wohl gar hesondere photometrische Messungen fur uberfllssig zu halten. Dem gegentber ist zu betonen, dass der Hauptzweek jenes gewaltigen Unternehmens die Bestinimung der Positionen der Sterne war, nicht die ihrer Grössen, und dass man daher von vornherein den Helligkeitsangaben keine höhere Bedentung beimessen sollte, als ihnen der Natur aer Sache nach zukommen kann, und als ihnen die Beohachter selbst von Anfäng an zugesehrieben haben. Bei der Schnelligkeit, mit weleher besonders in sternreichen Gegenden die Sterne anfeinander folgen, konnten selbstverständlich die Helligkeitssehätznngen nehen den Positionsbestimmungen nieht mit derjenigen kuhe und Sicherheit gemacht werden, die unter anderen Verhältnissen eher möglich gewesen wïre, und es ist wohl nur der grossen Übung und der ausserordentliehen Gewissenhaftigkeit der Beobaehter "zuzusehreiben, dass die Endresultate einen verhiiltnissmässig so hohen Genaujgkeitsgrad besit\%en. Entsprechend den Hauptzweeke der Arbeit ist ferner anf die Answahl der Beobachtungsnäichte nicht so ïngstlich geachtet worden, wie es bei photometriselsen Messungen erwhinscht ist. Es sind daher Beobachitungen ron solehen Tagen mitgenommen, wo der Himmel mit leichtem Dunstsehleier bedeckt war, und helle Mondnäichte, die für blosse Grössensehäit\%nngen ebenfalls sehr gefährlich sein können, sind natiirlich nicht vermieden worden. Datı kommt der Einfluss der Extinction, der besonders bei den siddichen Sternen schwer ins Gewicht faillt, und der trot\% der Bemiihungen der Beohalliter, ihre Sehätzungsseala naeh dem durch die Zenithdistan\% hedingten Anssehen der Steme zu modifieiren, keineswegs als vollkommen heseitignt angesehen werden kann.

Die Grössen in der Durchmnsterung sind in \%ehuteln angerrehen; es darf aber nicht unbeachtet bleiben, dass bei einem erhehlichen P'rocentsatze aller Werthe diese Zehntel un Rechenresultat sind (ent-stunden durch die Vereinigung der zweimaligen Beobachtungren zu Mitteln). Die eigentliehen Schïtzungen sind zum Theil nur in hallben ofler viertel Grissen gemacht. Vollkommene Klarheit iiber die Schätzmusweise der Bomer Durchmusterung giebt ein Brief ron schoijnfeld an l'eirer, den Letzterer in seinen "Photometric Researehes " it)gedruckt hat. Lamadr sind drei verschiedene Theile zu unterseheiden. In der ursten Periode, welche etwa 20 Procent aller Beobachtumgen mufisst, ist cine in wleichen Inter-

1) Annals of the Astr. Obs. of Harvard College. Vol.9. 1). 27. 
vallen von einer halben Grössenclasse fortschreitende Scala zu Grunde gelegt, also: $1^{\mathrm{m}}, 1 \cdot 2^{\mathrm{m}}, 2^{\mathrm{m}}, 2 \cdot 3^{\mathrm{m}}, 3^{\mathrm{m}}$ u. s. w. Bei der Mittelbildung von ziwei Beobachtungen, die um ein Intervall voneinander. verschieden waren, wurde im Allgemeinen das Zehntel so abgerundet, dass der Stern schwächer angenommen wurde als das genane Mittel; es kommen also aus dieser Periode die Zehutel $0,3,5$ und 8 fast ausschliesslich vor. In der zweiten Periode, welche beinahe 50 Procent aller Beobachtungen enthält, haben die Beobachter noch Zwischenstufen zwischen je zwei aufeinander folgende halbe Grössenclassen eingefügt, jedoch nur besouders anffällige Helligkeitsunterschiede hervorgehoben. Es sind daher nicht genau Viertelgrössen, in denen die Schiitzungen ansgefiihrt sind. Die Hinzufiigung des Zeichens » $s$ * (schwach) zur Grösse 7 z. B. sollte ausdriicken, dass der Stern merklich schwächer war als 7. Grösse, aber doch näher der Grösse 7 als der Grösse $7 \cdot 8$; ebenso sollte die Bezeichumng $7 g t(g t=g u t)$ einem Sterne zwischen $6 \cdot 7^{\mathrm{m}}$ und $7^{\mathrm{m}}$, aber näher an $7^{\mathrm{m}}$, angehören. Es sind also in der zweiten Periode fünf Stufen zwischen zwei aufeinander folgenden Grössenclassen zu unterscheiden, und es entspricht z. B.

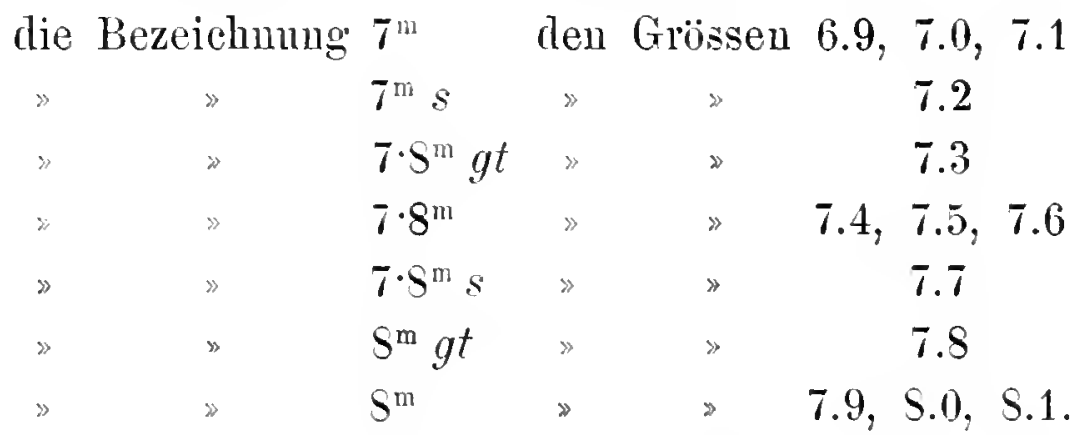

In der dritten Periode endlich (mit etwa 30 Procent aller Beobachtungen) sind die Schätzungen direct in Zehnteln ansgefiihrt worden; doch sind anch hier die Zehntel 1, 4, 6, 9, besonders aber 1 und 6 viel seltener benutzt worden als die übrigen. Dieser letzte Theil erstreckt sich fast mu auf Deelinationen iber $50^{\circ}$, also anf Gegenden des Himmels, die im Durchschnitt weniger reiche Zonen enthalteu. wo also die Schätzungen mit etwas mehr Musse gemacht werden komnten.

Man sieht. dass das Material der Bomer Durehmusterung ziemlich ungleichfürmig ist, und dass eine regelmässige Eintheilung in ZehntelGrössenclassen nicht stattfindet. Die Zehutel 1 und 6 kommen riel zu selten ror. daun folgen in der Hänfigkeit die Zelntel 4 und 9, dann 2 und 7.9 und 5 und endlich in gauz gleichmässiger Vertheilnng die Zehntel 1$)$ und 5. Zu beachten ist moch, dass dem urspriuglichen Plane gennäss die B. D. die Sterne des nürdlichen Himmels bis zur Grösse 9.0 nahezu rollstinding enthiilt. dass aber die letzten Unterabtheilungen 9.3, 
9.4, 9.5 insofern aus der Seala heransfallen, als sie eine grosse Anzahl von viel schwicheren Sternen, bis zur Grösse 10 und dariber hinaus, einschliessen. Bei der Schönfeld'schen Sudlichen Durehnusterung, die in Allgemeinen ein gleichmissigeres Material enthilt, ist die Vollstïndigkeit bis zur Grösse 9.2 oder 9.3 erstrebt worken, und die letzten Unterabtheilungen unfassen nicht ein so grosses Helligkeitsintervall, wie bei der nördlichen Durehmusterung.

Über die Genanigkeit der Grössenaugahen der Bonner Durchmusterung hat Argelander in der Einleitung \%u diesen Werke eine Untersuchung angestellt, die allerdings nur eine mngefihre Vorstellung geben kann, weil sie anf der Vergleichung mit den Bessel'schen und Lalandeschen Grössenschïtzungen in den Meridianzonen berulıt, die selbst offenbar viel unsicherer sind, als die Bonner Schätzungen. Nach Argelanders Rechnung ist der wahrscheinliche Fehler einer Grössenangabe der B. D. inı Durchschnitt etwa $= \pm 0.16$; er stellt sich fur die schwitcheren Sterne, wic von vornherein zu erwarten ist, wegen der grösseren Menge dieser Sterne und der dadureh erleichterten Vergleichung betrïchtlich kleiner heraus, als fiir die helleren. Fur alle Sterne bis etwas ther die sechste Grösse hinaus, hat Piekering ans der Vergleichung mit seinen photometrischen. Messungen den wahrscheinliehen Fehler der Durchmuisterungsgrössen zu \pm 0.18 bestimmt. Noch etwas grösser, ungefihihr zu \pm 0.2 , ergielot sich dieser Werth ans der Vergleichung aller Sterue bis zur Grösse 7.5 innerhall, des Gultels zwischen $+0^{\circ}$ mul $+20^{\prime \prime}$ Deelination mit den Potsdamer photometrischen Messungen").

Ftir die Südliche Durchmusterung haben Schönfeld² und ścheiner $\left.{ }^{3}\right)$ zur Beurtheilung des Genanigkeitsgratles eine :ilmliche Lntersuchung wie Argelander für den nürdliehen 'Theil angestellt, indem sie die Bomner Grössen mit denen anderer Cataluge verglichen haben. Ninch ilıen ergiebt sich der wahrseheinliche Fehler im Durchschnitt zu etwal \pm 11.2 Grössen, und es zeigt sieh anch hier, dass die schwiieheren sterne sicherer bestimmt sind, als die helleren. Sehönfeld hat nuch fiir cine grosse Anzahl ron zweimal beobachteten Sternen ans den Abweichungen von einander den w. F. berechnet. Das liesultat wird anf diesen Were etwas günstiger. Der w. F. einer Cataloggrässe schwankt damn zwischen \pm 0.06 bei den Sternen 9.5 Grösse und \pm 0.211 bri den Sternen

5. Grösse.

1) Publ. des Astrophys. Obs. zu Potsdam. lid. 9. 1) d4.\%

2) Astr. Beob. aut' der Sternw. Bonn. Bd. - 1. 34 it.

3) Astron. Nachr. Bd. 116, Nr. 2766. 
Ein vollkommen richtiges Bild von dem Genauigkeitsgrade der Bonuer Schätzungen wird man erst erhalten können, wenn sorgfältige photometrische Messungen von einer bedeutend grösseren Anzahl von Sternen vorliegen werden. Soviel kann man jedoch schon aus den bisherigen Untersuchungen schliessen, dass die Genauigkeit der einzelmen Werthe durchschnittlich nicht grösser ist als 0.2 , und dass die Zahl der Sterne, bei denen Fehler von mehr als einer ganzen Grössenclasse vorkommen, gar nicht gering ist. Weitere Fragen, ob und in welchem Grade die Sicherheit der Schätzungen in verschiedenen Zonen des Himmels wechselt, und ob dieselbe insbesondere von der Sterndichtigkeit u. s. w. abhängt, harren noch der Entscheidung. Als eine sehr wichtige Ergänzung der Bonner Durchmusterung ist das munmehr bald vollendete Zonemunternehmen der Astronomischen Gesellschaft zu betrachten, welches anch eine Rerision der Sterngrössen enthält. Wenu anch diese nenen Schätzungen der Natur der Sache nach im Allgemeinèn nicht sicherer sein werden als die Bonner, so ist doch zu hoffen, dass bei dieser Gelegenheit besonders anffallende Schätzungsfehler der Entdeckung nicht entgehen werden.

Die folgende Znsammenstellung gielot einen vergleichenden Überblick uiber die Schätzungsscalen der wichtigsten im Vorangehenden besprochenen Cataloge, zn denen noch die häufig benutzten Helligkeitsschätzungeu der Lalande'schen und Bessel'schen Meridianzonen, sowie der Strnve'schen Doppelsternbeobachtungen hinzugenommen sind. Als Vergleichsmassstab fuir alle Cataloge dient die Bonner Durchmusternng. Fuir die in den Überschriften der einzelnen Columnen angegebenen ganzen und halben Grössen der verschiedenen Sterncataloge sind in der Tafel die entsprechenden Grössen der B. D. anfgefülurt. So bedenten also beispielsweise die Zahlen der letzten Columne, dass ein Stern $9^{\mathrm{m}}$ in den Catalogen ron Lalande, Bessel, Strure und Schönfeld (Südl. Durchm.) durchschmittlich an Helligkeit gleich ist einem Stern in der B. D. ron der Grösse S.5, 8.5, 9.3 und 9.1. Die Tergleichung begimnt erst bei der Grösse 3.0, weil die helleren Sterne zn wenig zahlreich und meistens auch nicht sicher genug bestimmt sind. Wem in einem Cataloge zwei Unterabtheilungen zwischen je zwei anfeinander folgenden rollen Grössenclassen cingefülırt sind, so ist das Mittel ans diesen Abtheilnngen für die halben Grössen angesetzt worden. Zur Ableitnng der Tafel sind die hereits ron Argelander, Schönfeld, Gould und Pickering ansgefiilnten Vergleichnngen benntzt worden. Auf alleränsserste Genanigkeit machen die mitgetheilten Zahlen keinen Anspruch. 


\begin{tabular}{|c|c|c|c|c|c|c|c|c|c|c|c|c|c|}
\hline Calalog & 3.0 & 3.5 & 4.0 & 4.5 & 3.0 & 3.5 & 6.0 & 6.3 & $\therefore .0$ & 8.8 & 5.0 & 6.5 & 0.0 \\
\hline Ptolomäus & 3.1 & 3.6 & 4.4 & 4.7 & 5.0 & 5.3 & 5.5 & & & & & & \\
\hline Sûfi & 3.0 & 3.5 & 4.1 & 4.6 & 4.9 & 3.1 & 5.4 & 3.9 & & & & & \\
\hline Argel. Uranom. & 3.0 & 3.4 & 4.0 & 4.5 & 5.0 & 3.4 & 6.0 & & & & & & \\
\hline Hois & 3.0 & 3.4 & 4.0 & 4.5 & 5.0 & 5.3 & 6.0 & $(6.5)$ & & & & $f$ & \\
\hline Houzean & 2.5 & 3.3 & 3.9 & 4.4 & 4.9 & 5.4 & 5.9 & 6.3 & 6.6 & & & & \\
\hline Uran. Argent. & 2.9 & 3.4 & 4.0 & 4.4 & 4.9 & 5.4 & 6.0 & 6.5 & 7.0 & & & & \\
\hline Lalande & 2.9 & 3.3 & 3.9 & 4.5 & 4.9 & 5.5 & 6.2 & 6.8 & 7.2 & 7.6 & 7.9 & $\$ .3$ & 8.5 \\
\hline Bessel & 3.1 & 3.4 & 3.7 & 4.2 & 4.7 & 5.2 & 5.7 & 6.2 & 6.8 & 7.4 & 7.9 & 8.4 & 8.5 \\
\hline Struvo & 3.2 & 3.7 & 4.4 & 4.8 & 5.2 & 5.7 & 6.2 & 6.6 & 7.2 & 7.7 & 8.3 & 8.8 & 9.3 \\
\hline Schünfeld (S.D.) & & & & & 4.9 & 5.4 & 6.0 & 6.6 & $i .2$ & 7.6 & 8.1 & $\$ .5$ & 9.1 \\
\hline
\end{tabular}

Trot\% einiger nieht unbetriehtlichen Differenzen \%wischen einzehen Reihen, insbesondere \%wischen Struye und Bessel und zwischen Iatlande und Bessel, sind im Allgemeinen die Schiatznngsscalen nicht so sehr voneinander versehieden, wie man vielleicht erwarten mbichte. Die Scala der B. D. entspricht, wie man sieht, durehweg sehr nahe dem Mittel der sämmtlichen oben angefilurten Cataloge.

b. Helligkeitsverzeichnisse, welche ans photometrischen Messungen hergeleitet sind.

Es ist sehon mehrere Male daranf hingewiesen worden, datss J. Hersehel zuerst den Versuch gemacht hat, einen Ilellinkeits"atalog unter Anwendung von instrumentellen Itiilfsmitteln herzustellen. Hit dem rom ihm erfundenen Astrometer hat er die Helligkeit bon 69 meist sid llichen Sternen dureh Vergleichung mit einem verkleinerten Mondlibldreln hestimmt; die Resultate sind in einem (atalosel) zusammengefitsit, in welehem die Intensitäten in Einheiten der Hellinkkeit ron c ('entinn'i ansgedruekt sind. Wie schon friher bemerkt wurle, hist diesor l'atalong deshalb kaum ein anderes als ein historisches Interesse, weil anf dic KXtinetion des Liehtes in der Erdatmosphäire ran keine liticksicht fornommen ist, und vor Allem, weil bei der Reduction der Mondphatsen anfoinander die mnrichtige Enler'sche Formel benutyt worden ist.

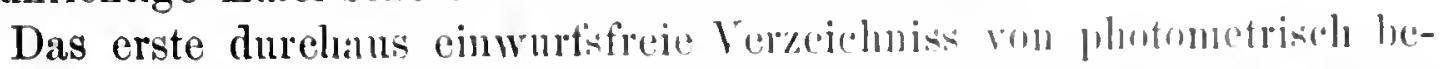
stimmten Sternen verdanken wir Seidel\%. Dissolle cuthialt zwar nur 208 hellere Fixsterne, die Messmugen zeichuen sich alher dureh soldehe Genaugkeit aus, dass dieser Catalog als wrundlenewd fir die moderne

1) J. Herschel. Results of Astr. Obs. male during 1-34-1 - as at the Cape of Good Hope. London 1847 , p. 367 .

2) Abhandl. der K. liayer. Akad. del Wiss. I1. Classe. lid. !. p. t2l. 
Astrophotometrie zu bezeichnen ist. Die Beobachtungen sind mit Benutzung des Steinheil'schen Prismenphotometers in den Jahren 1852-1860 ausgeführt, und es ist wohl lediglich dem Umstande, dass bei diesem Photometer infolge der Vergleichung ausserhalb des Focus ein bedeutender Lichtverlust stattfindet, zuzusehreiben, dass Seidel seine Messungen nicht weiter als bis zu Sternen der fünften Grösse ausgedehnt hat. 'Die Sterne sind parweise miteinander verglichen, einzelne Paare mehrfach, die meisten nur einmal beobachtet worden. Aus dem so erhaltenen regellosen Netz von Kreuz- und Querverbindungen sind dann unter strenger Beriieksichtigung der Extiuetion dureh ein etwas complieirtes Näherungsverfahren die Endwerthe des Cataloges gefunden worden. Seidel hat die Intensitäten aller Sterne auf diejenige von $\alpha$ Lyrae als Einheit bezogen und durchweg die Logarithmen der Helligkeitsverhältnisse, nicht dic Zahlen selbst bei seiner Verarbeitung benutzt.

Fast gleiehzeitig mit der Seidel'sehen Arbeit ersehienen die »Grundziige einer allgemeinen Photometrie des Himmels s von Zöllner, welche in erster Linie der Besehreibung und Untersuchung des von Zöllner erfundenen Astrophotometers gewidmet sind. In diesem Werke finden sieh auch photometrisehe Messungen von mehr als 200 Sternen, die ZölIner wohl mehr in der Absieht, die Branehbarkeit seines Apparates darzuthun, ausgefiihrt hatte, als um einen zusammenhängenden Helligkeitscatalog herzustellen. Die Beobaehtungen sind daher ohne Plan angestellt. Jede Reihe enthält eine Anzahl von Sternen, deren Helligkeiten auf einen beliebigen Stern in derselben bezogen sind. Eine Vereinigung der versehiedenen Reihen ist mur dann möglich, wenn einzelne Sterne in mehreren derselben vorkommen und so sämmtliche Sterne auf die Helligkeit einer einzigen Gruppe redneirt werden künnen. Der Versueh zu einer derartigen Verarbeitung der Zölner'schen Beobaehtungen ist vor einigen Jalren ron Dorsti) g'emacht worden, aber da eine gerwisse Willkiur bei der Vereinigung der Gruppen unvermeidlich ist, so hat der abgeleitete Catalog nicht denjenigen Werth, weleher der Genanigkeit der Messungen entspricht.

Das ZöIher'sehe Astrophotometer ist in grösserem Umfange zur Catalogisinung von Steruhelligkeiten zuerst von Peiree ${ }^{2}$ ) und Wolff ${ }^{3}$ ) benutzt worden. Von Ersterem besitzen wir einen Catalog von 495 Sternen, hamptsaichlieh solehen der Argelander'sehen Uranometrie, in dem Giurtel zwischen $+40^{\circ}$ und $+50^{\circ}$ Declination. Diese Zone war von Peirce

1) Astr. Nachr. Bd. 118, Nr. 2522-23.

2) Annals of the Astr. Obs. of Harvard College. Vol. 9.

3) Wolft, Photometrische Beobachtungen an Fixsternen. Leipzig, 1877 und Berlin, 1>44. Zwei Abhandlungen. 
ausgewahlt worlen, um zu allen Zeiten in jeder beliebigen Zenithdistauz Anhaltsterne zu hahen, mit denen die Helligkeiten anderer Sterne bei $\mathbf{\Lambda b}$ leitnng eines unfassenderen Cataloges verglichen werden könuten. Peirce giebt den wahrscheinlichen Fehler seiner endgrtltigen Helligkeitswerthe zu \pm 0.09 Grössenelassen an, also ein wesentlieher lortschritt gegenther dem Genauigkeitsgrade der blossen Helligkeitsschlit»ungen.

Wolff hat zwei Helligkeitseataloge veröflentlicht mit zusammen uber 1100 Sternen, von denen nahezu die Hälfte in beiden Verzeichnissen vorkommt. Der Plan war, sämmtliche Sterne der Argelander'sehen Uranometrie bis etwa zur Grösse 5.6 dureh\%ubeobachten; es sind aber anch noch schwiichere Sterne mit hinzngenommen worden. Die Intensituten sind-wie bei Seidel in Logarithmen angegeben, aber nicht bezogen anf einen einzelnen Stern, sondern anf die Helligkeit des kilnstlichen Sternes im Photometer. Da nun eine gleichförnige Helligkeit der Flamme \%war innerhalb kurzer Zeitrïume, aber keinesfalls von 'lag zu 'Tag vorauggesetzt werden darf, so muss das Intensitïtsverhililtuiss der Lampe bei verschiedenen Bcobachtungsreihen dureh Vermittlung von Sternen, welche gemeinsehaftlich in denselben vorkommen, bestimmt werden, nm schliesslich dureh wiederholte Übergänge alle Angahben auf die Lampenhelligkeit einer einzigen Beobachtungsreihe als Einhcit beziehen zı können. Dieses Verfahren, welches in ahnlicher Weise anch bei der oben erwälunten Beartbeitung der Zöllner'schen Messungen, sowie bei der Reduction der Peirce'schen Beobachtungen Anwendung gefunden hat, ist so unstiudlich und wenig Vertranen erweekend, dass es in keiner Beziehung Nachahmumg verdient. Betreff's der Wolff'schen l'eobachtungen ist noch daranf hinzuweisen, dass dieselben zwar eine ganz vortreffliche innere Ühereinstimmmur zeigen, dass sie aber, wic die Vergleichung mit anderen photonetrischen Catalogen, speciell mit Seidel, Pickering und Pritchard lehrt, mit nicht nnerheblichen systematischen Fehlern behaftet sind. Die leclleren Sterne sind von Wolff oftenbar verhiiltnissmissig zu schwarh, und dic schwächsten Sterne zu hell gemessen worden. Solche principielle Unterschiede zwischen verschiedenen Catalognen sind an und fiur sich infolge der individuellen Auffassumg jedes Beobatchters nicht befremdlidh, doch ist der Betrag der Abweichung hei Wolff so betriichtlich, dass er hesondere Beachtung verdient. Es liegr nale, den fimul dieser ligigenthimlichkeit in der Benutzung des 'Zïlner'schen Photonncters zu suchen, bei welchem das versehiedene Anssehen der kimstlichen mud wilklichen Sterne eine gewisse Gefahr hinsiehtlich der abweichenden A uffis:mug verschiedener Beobachter in sich birgt. Es ist bereits bei der ans fithrlichen besehreilunug dieses Instrumentes auf diese Gefilnr hingewiesen und nilher erörtert worden, dureh welehe Vorsichtsmassregehn dieselbe zu hereitigen ist. Oflenbar hat 
Wolff an solche Vorsichtsmassregeln nicht gedacht; insbesondere hat er verabsäumt, allzu kleine Ablesungen des Intensitätskreises durch Wahl geeigneter Diaphragmen für die. künstlichen Sterne zu vermeiden, und dadurch ist bei ihm der Auffassungsfehler so bedenklich gross geworden. Die Angaben der Wolff'schen Cataloge sind jedenfalls nicht ohne Anbringung von Scalencorrectionen, wie sie z. B. von Pickering abgeleitet worden sind, zu benutzen. Dass fast alle anderen Beobachter mit dem Zöllner'schen Photometer bemerkenswerthe Auffassungsfehler vermieden haben, beweisen ausser dem oben erwähnten Cataloge von Peirce noch die vortrefflichen Arbeiten von Lindemann, unter denen besonders die Vergleichungen der Plejadènsterne, die Revision der Bonner Durchmusterungsgrössen und die Ausmessung des Sternhaufeus $h$ Persei hervorzuheben sind, ferner die Untersuchungen Ceraskis, der sich besonders mit Helligkeitsmessungen von Circumpolarsternen beschäftigt hat, und endlich die Potsdamer Arbeiten.

Alle im Vorangehenden erwähnten photometrischen Cataloge können wegen der rerhältnissmässig beschränkten Anzahl der darin enthaltenen Sterne nur als Vorlänfer betrachtet werden zu den umfangreichen Verzeichnissen, welche in den letzten Jahren von den Observatorien zu Cambridge (Mass.), Oxford und Potsdam veröffentlicht worden sind und als Ansgangspunkte einer neuen Aera in der Fixsternphotometrie betrachtet werden können. Pickering gebihht das grosse Verdienst, zuerst eine planmässige Durchmusterung des Fixsteruhimmels begonnen und diejenige Bezeichnnngsweise für die Helligkeiten der Sterne eingeführt zu haben, welche nummehr hoffentlich definitiv in der Astronomie Geltung behalten wird. In seinem unter dem Namen »Harvard Photometry " bekannten Cataloge sind die Helligkeiten von 4260 Sternen, abgeleitet aus Messungen mit dem Meridianphotometer, enthalten, mit einer Genanigkeit, die zwar noch nieht das Änsserste reprïisentirt, was bei Anwendung ron instrumentellen Hiilfsmitteln erreicht werden kamn, die aber doch alle aus blossen Schätzungen erhaltenen Resnltate weit iiberflïgelt. Die Harvard Photometry umfasst alle Sterne bis zur 6. Grösse und noch eine grosse Anzahl schwïcherer zwischen dem Nordpol und etwa $30^{\circ}$ siidlicher Declination. Die Intensititen sind, entsprechend dem Principe des benutzten Instrnmentes, beim Neridiandurchgunge der Sterne durch Vergleich mit dem Polarstern abgeleitet. So einfach dieses Verfahren anch ist, und so sehr dadurch namentlich die Reduction der Beobachtungen erleichtert wird, so diirfte doch, wemu es sich nm Erreichung der höchsten Genanigkeit handelt, die Methode nieht unmmschränkt zu empfehlen sein nud zwar hauptsïchlich wegen des sehwer zu bestimmenden Einflusses der Extinetion. Es kommt ia, wie sehon im ersten Abschnitte betont wurde, nicht nur anf 
die yerschiedene Höhe der beiden zu vergleichenden Sterne, sondern auch anf iliren Azimuthunterselied an, und es tritt nicht selten der Fall cin, dass in seheinbar ganz. klaren Nibhten die Durehsichtigkeit in verschiedenen Regionen des Himmels wesentlich anders ist, wobei namentlich locale Einflisse eine grosse Rolle spielen können. Bedingung fir die allervollkommensten photometrischen Messungen ist die unmittelbare Nibe der mit einander zu vergleichenden Objecte, und gerade diese Bedlingung ist bei dem Pickering'sehen Verfahren durchaus ausser Aeht gelassen. Was die in der Harvard Photometry gewihlte Masseinheit fur die Helligkeitsangaben betrifft, so lat Piekering einen wichtigen und entscheidenden Sehritt gethan, indem er statt der voin Seidel und Wolff henutzten Helligkeitslogarithmen den Begriff der photoinetrischen Sterngrösse festgesetzt hat. An und fir sich ist die Bezeichnnng in Helligheitslogarithmen durehans rationell und einwurfsfrei; da aber die Astronomen seit den Zeiten des Ptolemäus gewöhnt sind, die Intensitïten in Grössenelassen auszudricken, und da wir ferner noch lange Zeit für den griossten Theil der Steme auf die Grössensehait\%ungen der B. D. und anderer Cataloge angewiesen sein werden, so wibrle die binfuhrung einer gan\% nenen Scala auf Widerspruch stossen. Es ist daher durchans zu billigen, dass Pickering die Bezcichung Sterngrösse beibehalten hat. Nach seinem Vorschlage versteht man unter einer photometrischen Sterngrösse den Intensititsuntersehied zweier Sterne, für welche der Logarithmus ihres Helligkeitsverhialtnisses gleieh 0.4 ist; man hat also die Logaritlunen der instrumentell gemessenen Helligkeitsverhiiltnisse mit 0.4 \% dividiren, wn die Differenzen der betreffenden Objecte in photometrisehen rrössen z.ll erhalten. Wir

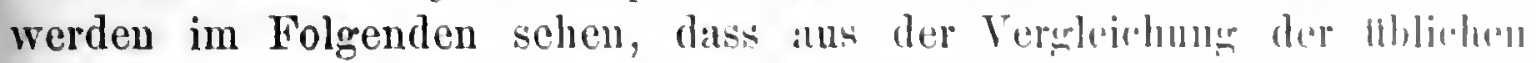
Grössensehätzungen mit photonetrisehen Messungen für den Luratrithums

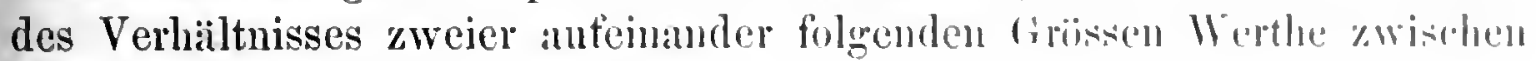
0.3 und 0.4 resultiren.

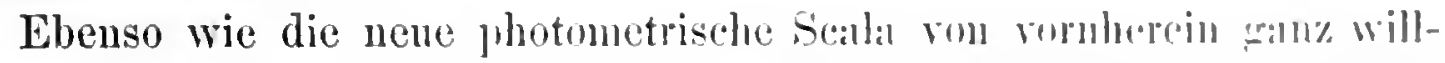
kiurlich gewählt werden konnte, so liesse sich anch der Xullpunkt diuses Systems ganz beliebig festset\%en. Man kiinnto \%. P. dem allerhullsten Fixsterne (dem Sirins) die photrmetristhe (iriisse 0.0 heilexpen und wille anf diese Weise negative Sterngrösen remeiden. Interessint ist ancll

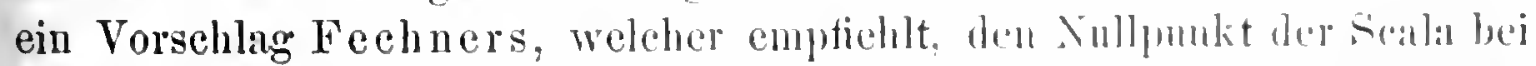

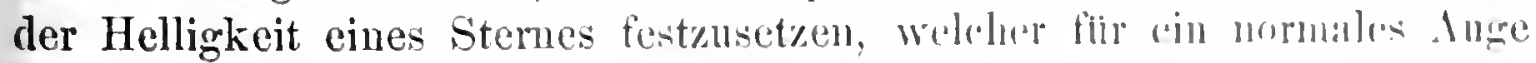

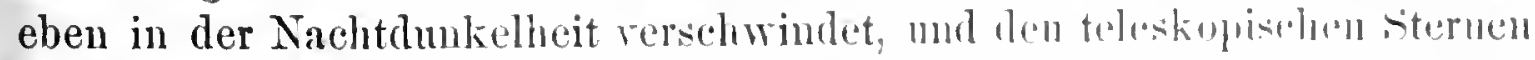
negative, den mit blossen Ange sichtbaren unitive Griistenwerthe hoizn-

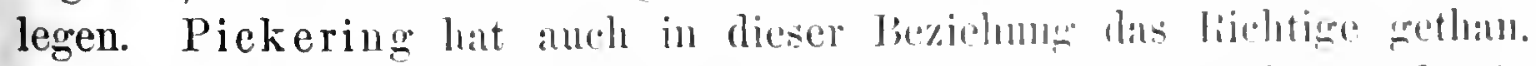

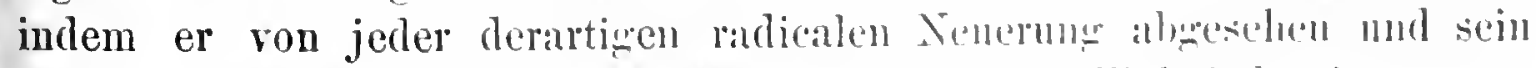
System so gewaihlt latt, dass das Mittel ans reinen llelligkeitshestimmungen 
von 100 Circumpolarsternen der 2. bis 6. Grösse mit dem entsprechenden Mittel aus den Werthen der B: D. zusammenfiel. Sein System, in welchem der Polarstern die Grösse 2.15 besitzt, trifft also etwa bei der 4 . bis 5. Grösse geuau mit dem Schätzungssystem der B. D. zusammen. Zur Reduction der Grössen des einen Cataloges auf die des anderen sind nur verhältnissmässig geringe Beträge erforderlich. Wie bei allen genanten photometrischen Catalogen, ist auch in der Harvard Photometry der Einfluss der Extinction in Rechnung gebracht worden und zwar nach der Laplace'schen Theorie; die sämmtlichen Helligkeitswerthe gelten für das Zenith von Cambridge. Jeder Stern der Harvard Photometry ist an mindestens drei rerschiedenen Abenden beobachtet worden, manche auch noch viel öfter. Der wahrscheinliche Fehler eines Catalogwerthes ergiebt sich im Durchschnitte zu etwa \pm 0.075 Grössenclassen, derjenige einier einzelnen Beobachtung zu etwa \pm 0.15 Grössenclassen. $\mathrm{Zu}$ bedanern ist es, dass bei einer nicht unerheblichen Zahl ron Sternen zwischen den an verschiedenen Abenden gemessenen Helligkeiten ganz aussergewöhnlich starke Abweichungen (bis zu einer ganzen Grössenclasse und dariber) auftreten. Solche Differenzen können natiirlich nicht durch blosse Messungsfehler erklärt werden; sie sind zweifellos entweder anf unrorsichtige Wahl der Beobachtungsuächte oder, was wahrscheinlicher ist, auf Verwechslungen mit nahe stehenden Sternen zuriickzufuihren. Bei der auffallend grosseu Schnelligkeit, mit welcher die Cambridger photometrischen Messungen angestellt worden sind, kann ein häufiges Vorkommen solcher Verwechslungen gar nicht in Verwundermng setzen. Es ist dies ein Vorwurf, der dem rerdienstlichen Werke nicht erspart werden kann, und der leider vielfach das Vertrauen auf seine Zurerlässigkeit etwas beeinträchtigt hat. Pickering hat iibrigens in den letzten Jahren eine Neubeobachtung sämmtlicher Sterne der Harrard Photometry nnternommen, die gegenwärtig dem Abschlusse nahe ist und jedenfills zur Aufklärung mancher Z

Ausser der Harvard Photometry ist noch ein zweiter weit umfangreicherer Helligkeitscatalog ron Pickering veröffentlicht worden, welcher gewöhnlich nuter dem Namen »Photometric Rerision of the Durchmustemug" bekannt ist. Dieser umfasst kein zusammenhängendes Gebiet am Himmel, sondern enthïlt in kleinen, meist uur $20^{\prime}$ breiten Streifen, deren Mitten in Declination um je $5^{\circ}$ roneinander entfernt sind, die sämmtlichen Sterne der beiden Bomner Durchmusterungen bis zur Grösse 9.0 und anserdem eine beträchtliche Zahl ron schrächeren Sternen, im Ganzen nahe an J7000 Objecte. Der Zweck dieser Arbeit war, den Scalenwerth der Brmmer Durchmusterumg fü Sterne aller Helligkeiten in rerschiedenen Regrionen des Himmels photometrisch zn bestimmen und ausserdem die 
Mittel zu geben, die Grössenschätzungen in den einzelnen Abschuitten des voll der Astronomisehen Gesellschaft herausgegebenen Sterneataloges untereinander vergleichbar zu machen. Neben der Erreichung dieses Hauptzweckes liegt der Werth dieses Cataloges darin, dass wir unumelır fur eine sehr grosse Anzahl ron sehwiicheren Sternen Ibberall an Himmel photometrische Grössen besit\%en, die bei den Beobachfungen rou verhinderlichen Sternen, kleinen Planeten n. s. w. als Anhaltspunkte dienen und bei Grössenschäitzungen zur Controle der gewihlteu Scula benut»t werdeu können. Die meisten Sterne sind nur \%weimal beobachtet, sodass im Allgemeinen die Sicherheit der Endwerthe vielleicht etwas geringer ist, als in der Harvard Photometry; man wird aber anch hier den wahrscheinlichen Fehler einer Cataloghelligkeit nicht grösser als \pm 0.1 Grössenclassen annehmen dilrfen. Im Übrigen gilt das oben liber die Cambrilger plintometrischen Messungen Gesagte anch fir dieses Werk; die \%alıl der auffallend starken Abweichungen zwischen Beobachtungen dessellen Sternes ist grösser, als es bei Benutzung von instrumentellen Ilulfsmitteln und bei vorsiehtigster Auswahl der Beobatehtungsibende der Fall sein sollte.

Eine werthvolle Ergïnzung der beiden genannten sternveracichnisse bildet ein dritter, ganz kiirzlich ron Piekeringl) veröftentlichter Helligkeitseatalog von 7922 slidlichen Stemen. Die Beobichtungen zn diesem Cataloge sind gelegentlich der von der Harvard-Sternwarte uach Sildamerika entsendeten Expedition an verschiedenen Stationen durch Bailey ausgefithrt und später von Piekering in ähnlicher Weje wie lic lienbaldhtungen am nördlichen Himmel bearbeitet worklen. Anch bei diesen Messungen kam das Meridiamphotometer in Gelurach, und als Polstern

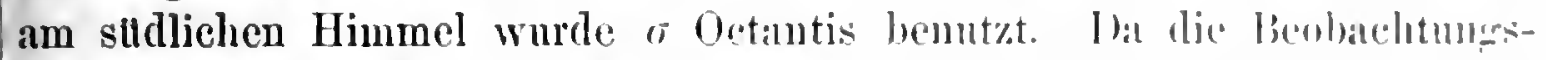
stationen sämmtlieh in verhailtnissmiissigr niedrigen lireite'n lityen, sn killu wegen des tiefen Standes des Polsternes die Anwendum des Moridianphotometers bei diesem Unternehmen nicht als cine grlinkliche Wall he-

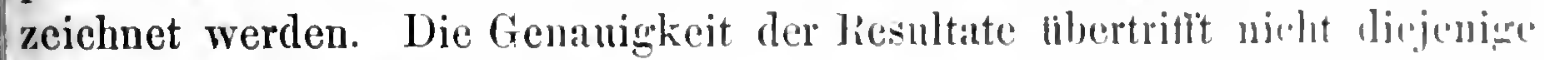
der Cambridger Messungen.

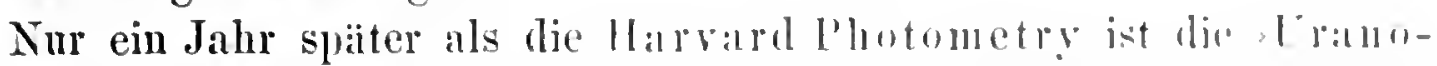

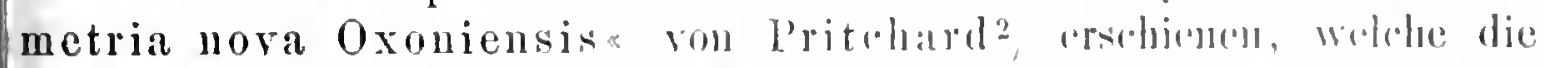

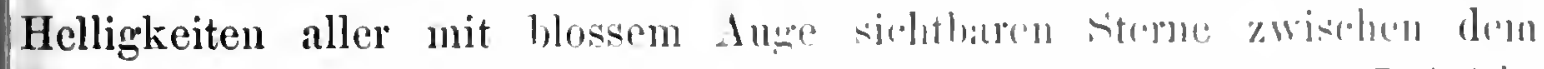

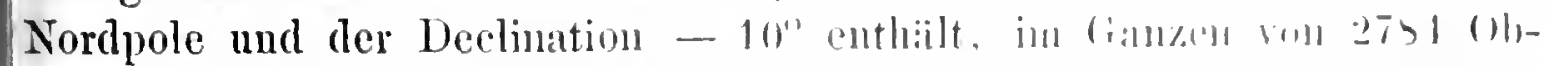

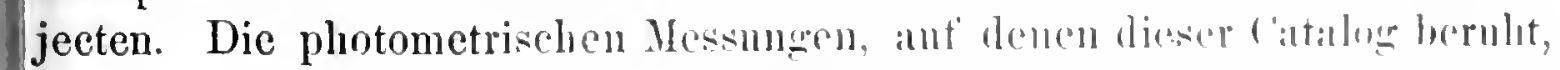

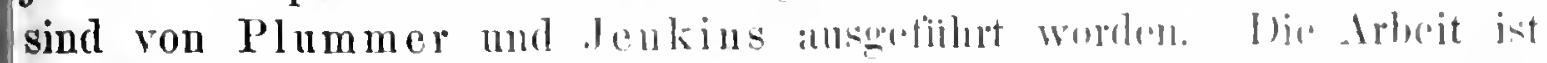

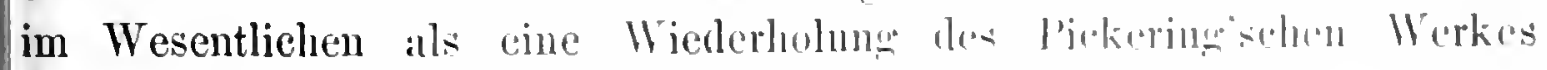

1) Annals of the Astr. Obs. of Harvard College Villo 31.

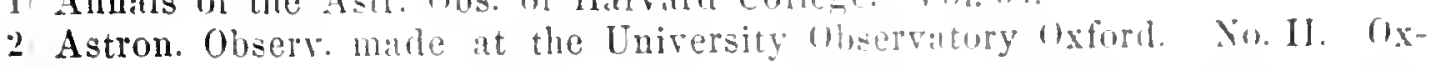
ford, 1885 . 
anzusehen, sie hat aber neben demselben deshalb eine durchaus selbständige Bedeutung, weil die Beobachtungen nach einem ganz anderen photometrischen Princip, und zwair mit Hülfe des Keilphotometers ausgeführt sind. Pritchard hat dieselbe Grössenscala wie Pickering angewendet und ebenso wie dieser sämmtliche Sterne mit dem Polarstern verglichen. Da er fuir letzteren die Zenithhelligkeit 2.05 (statt 2.15) zu Grunde gelegt hat, so ist zwischen den beiden Catalogen von vornherein eine constante Differenz,von $0.1 \mathrm{zu}$ erwarten. Um negative Sterngrössen zu vermeiden, hat Pritchard die Helligkeiten aller Sterne, welche die Grösse 1.0 iiberschreiten, dureh Vorsetzen des Zeichens + ausgedrückt, so dass also z. B. seine Schreibweise +1.95 für den Sirius dasselbe bedentet, was sonst allgemein durch -0.95 bezeichnet wird. Diese Neuerung ist entschieden nicht empfehlenswerth, und es ist kein Grund einzusehen, warum die Continuität der photometrischen Grössenscala unterbrochen werden sollte; die negativen Grössen, die nur bei sehr wenigen Fixsternen und bei den hellsten Planeten vorkommen, können unmöglich zu Missverständnissen Anlass geben. Die Genanigkeit der Oxforder Cataloghelligkeiten ist im Durehschnitt nicht grösser als die der Cambridger Verzeichnisse, schou deshalb, weil bei Weitem der grösste Theil der Sterne (iiber 90 Procent aller) nur an einem Abend beobachtet worden ist; man wird den wahrscheinlichen Fehler einer Catalogangabe jedenfalls nicht kleiner als 0.1 Grössenelassen annehmen dürfen.

Die genamesten Helligkeitswerthe, welche wir gegenwärtig besitzen, diurften in der Potsdamer 》Photometrischen Durchmusterung ${ }^{1}$ ) enthalten sein, von weleher bis jetzt erst ein Theil (ron $0^{\circ}$ bis $+20^{\circ}$ Declination) ersehienen ist. Das Werk, welehes in der Fortsetzung bcgriffen ist, soll am uördlichen Himmel alle Sterne der Bonner Durehmusterung bis zur Grösse 7.5 umfassen, und wird demnach, wenn es rollendet ist, einen Catalng ron mehr als 14000 Objecten bilden.

Die bisherigen Nessmgen sind ron Kempf und mir mit Benutzung ron Zöllner schen Photometern ansgefiihrt, und jeder Stern an zwei Abenden, alle diejenigen, bei denen die Bestimmungen der beiden Beobachter um mehr als 11.3 Grössenclassen roneinander abwichen, an vier Abenden gemessen worden. Das Beobaehtmosserfahren ist resentlich ron dem Piekerings sehen und dem Pritehard'sehen versehieden, und serade der Anwendung dieses Tertahrens diirfte wohl in erster Linie die befriedigende Genanigkeit der licsultate zuzuschreiben sein. Statt cines einzigen Vergleichsternes ist ein System rou 144 Hauptsternen answewählt worden, welche bei drei rersehiedenen Declinationen $\left(10^{\circ}, 30^{\circ}\right.$ 
and $60^{\circ}$ ) je in durehschnittlichen Interrallen von 30 \%eitminnten anfeinander folgen. Diese Hauptsterne, welehe das eigentlielıe Fundament des ganzen Cataloges bilden sollen, sind durch mannigfache Kreuz. rerbindungen aneiuauder angeschlossen und so oft leobachtet worden, dass die Ungenanigkeit der abgeleiteten Fndwerthe schwerlich mehr als wenige Hundertstel Grössenelassen betragen kann. Unter Benutzung der Pickering'sehen photometrischen Grössenseala ist das Potsdamer System so festgelegt worden, dass der mittlere Helligkeitswerth der 1.14 Fumbmentalsterno mit dem entsprechenden Mittelwerthe der Bonner Durchmusterungsgrössen ubereinstimmt. Die beiden Systeme fallen ungefthr bei der Grösse 6.0 miteinander zusammen.

An die Hauptsterne sind dann die Catalogsterne in der Weise angesehlossen, dass dieselben in Zonen ron ungefihr je 12 \%usammengefasst und mit zwei in ihrer Nihe befindliehen Fundameutalsternen verglichen wurden, welehe am Anfange, in der Mitte und am Ende der Zonen gemessen wurden. Dieses Verfahren, welehes bei derartigen Catalogunternehmungen das empfehlenswertheste sein diurte, hat den grossen Vortheil, dass die Extinetionseorrectionen stets mbedentend sind, und dass vor Allem Durchsichtigkeitsunterschiede an rerschiedenen Stellen des Hinmels und partielle Triilnngen nicht so schïillieh wirken, wie bei dem Pickering'sehen und Pritchard'sehen Messungssverfahren.

Es ist hier nieht der Platz, niher auf das Detail der Potsdamer Durelimusterung einzugehen; es genijgt hier noch heroryuleben, dass in dem publicirten ersten Theile der wahrscheinliche Fehler einer einzelnen

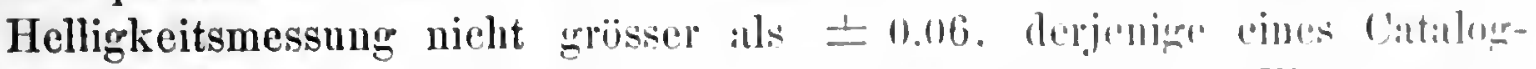

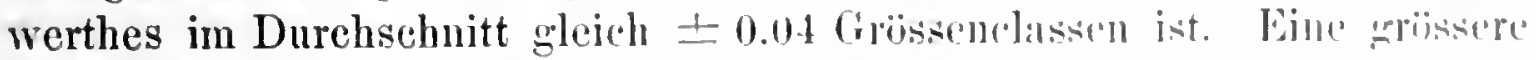
Genaugkeit ist bei photometrisehen Messungen hisher molh nibht arreicht worden.

Der vollendete Potsdamer Catalog wird simmentliche sterme des nïrdlichen Himmels, welehe in der Jlarrat l'hotumetry mul in tee Uranometria nova Oxoniensis rorlanden sind, whenfalls colthiltem. und die Vereinigung aller drei Cataloge wird uns fiir dats Pimle des

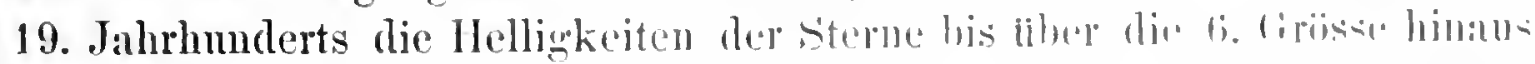

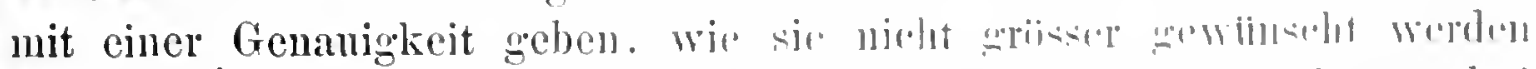

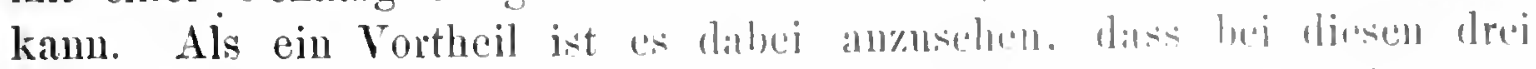

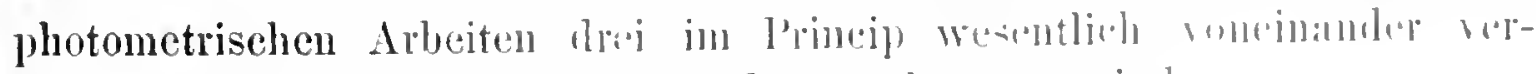

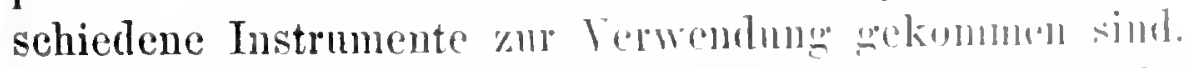

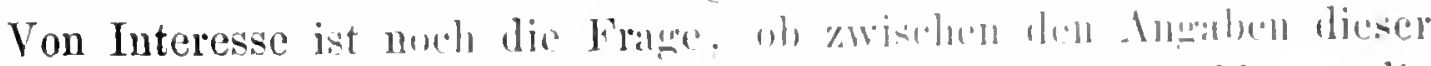

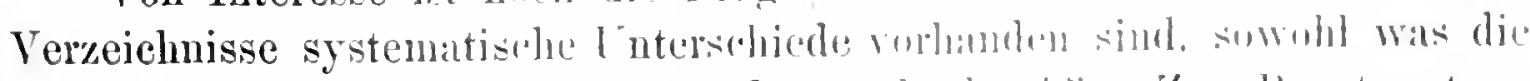

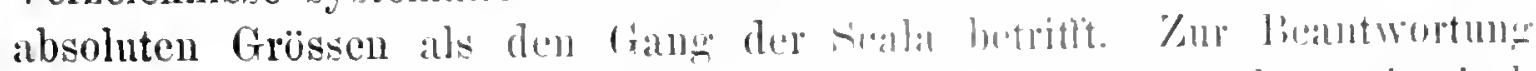

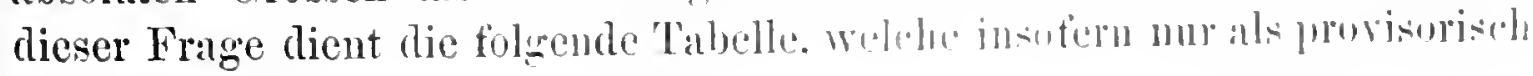


anzusehen ist, weil sich die Vergleichnng lediglich auf die in Potsdam vollendete Zone von $0^{\circ}$ bis $20^{\circ}$ Declination bezieht und daher die Anzahl der gemeinschaftlichen Sterne verhältnissmässig gering ist. Argument der Tabelle sind die Grössenangaben der in den Überschriften genannten Cataloge, zu denen noch die-Bonner Durchmusterung hinzugenommen worden ist. Fiir jeden Catalog sind unter der Überschrift "Diff. « die Reductionen anf. Potsdam angefiilurt, $d$. h. die Werthe, welche zu den Grössenangaben des betreffenden Verzeichnisses hinzugefügt werden müssen, um die entsprechenden Grössen der Potsdamer Durchmusterung zu fiuden. Das positive Vorzeichen bedeutet also, dass die zugehörige Sternclasse in Potsdam schwïcher gemessen ist, als in dem betreffenden Cataloge. Die Sterne heller als 3. Grösse sind wegen ihrer kleinen Anzahl zu einer Gruppe zusammengefasst worden.

\begin{tabular}{|c|c|c|c|c|c|c|c|c|}
\hline \multirow{2}{*}{ Helligkeit } & \multicolumn{2}{|c|}{ Harvard Photometry } & \multicolumn{2}{|c|}{ Photom. Revision } & \multicolumn{2}{|c|}{ Uranom. Oxoniensis } & \multicolumn{2}{|c|}{ Bonner Durchmust. } \\
\hline & Dift. & Anzahl & Diff. & Anzahl & Diff. & Anzahl & Diff. & Anzahl \\
\hline Heller als 3.0 & +0.28 & 16 & - & - & +0.34 & 17 & +0.55 & 9 \\
\hline $3.00-3.49$ & +0.16 & 11 & - & - & +0.22 & 12 & +0.42 & 21 \\
\hline $3.50-3.99$ & +0.16 & 41 & +0.13 & 3 & +0.24 & 39 & +0.36 & 26 \\
\hline $4.00-4.49$ & +0.16 & 54 & +0.04 & \pm & $+0.1 t$ & 47 & +0.19 & 42 \\
\hline $4.50-4.99$ & +0.13 & 75 & $+0.0 \mathrm{~s}$ & 4 & $+0.0 \mathrm{~s}$ & 55 & +0.08 & 45 \\
\hline $5.00-5.49$ & +0.20 & 162 & +0.27 & 14 & +0.12 & $15 \tau$ & +0.04 & $10 \mathrm{~S}$ \\
\hline $5.50-5.99$ & +0.19 & 253 & +0.21 & 72 & +0.11 & 195 & -0.06 & 167 \\
\hline $6.00-6.49$ & +0.13 & 150 & +0.16 & 145 & +0.11 & 149 & +0.01 & 327 \\
\hline $6.50-6.93$ & +0.13 & 29 & +0.13 & 262 & +0.15 & 21 & +0.06 & 658 \\
\hline $7.00-7.49$ & - & - & +0.10 & 205 & - & - & +0.02 & 1252 \\
\hline 7.50 u. darunter & - & - & $+0.0 t$ & 90 & - & 一 & -0.03 & $6 \varsigma 0$ \\
\hline Znsammen: & +0.17 & 791 & +0.13 & 799 & +0.13 & 691 & +0.02 & 3335 \\
\hline
\end{tabular}

Die Differenzen der Harrard Photometry können, mit Ausuahme der ersten nicht sehr sicher bestimmten, als constant betrachtet werden, wor:uls also folgt, dass die Grössensealen beider Systeme denselben Gang haben. Die constante Differenz von +0.17 ist theilweise dadurch zu erkliiren, dass das Potsdamer Srstem etwa bei der 6. Grüsse, das Pickering sche dagegen etwa bei der 5 . an die Bonner Durchmustermg angeshlossen ist.

Die Veroleichung mit der Photometric Revision liefert ebenfalls nithezu constante Differenzen; der geringe Gang, der fiur die sehwächeren Stemo angedentet zu sein scheint, kamn noch nicht als rerbiirgt angesehen werten. Ilie beiden Pickering'schen srsteme stimmen jedenfalls genigend miteinander ibserein, und es wird in thlgemeinen anseichend sein, für den Fall dass man eine Cambridger Helligkeitsangabe anf das Potsdamer 
System reduciren will, an dieselbe die Correetion + 0.15) (den Mittelwerth aus den constanten Differenzen der beiden Cambridger Cataloge) anzubringen.

Die Uranometria Oxoniensis giebt die Differenzen fur die Sterne bis zur Grösse 4.0 etwas grösser als fir die Sterne von 4.0 bis 7.0 ; innerhalb jeder dieser beiden Gruppen durfen aber die Unterschiede mit Rucksicht auf die den Zahlen noch anhaftende Unsicherheit als constaut gelten. Will man daher bei der Rednetion einer Oxforder Helligkeits. angabe anf das Potsdamer System nicht die mittlere Differenz ans allen Sternen +0.13 als Correction benntyen, so kann man filr Sterne bis \%ur Grösse 4.0 die Correction +0.26 , fur die Ubrigen +0.11 anwenden.

Was endlich die Vergleichung mit der Bonner Durehmusterung anbelangt, so spricht sich fur die. Sterne bis \%ur 6. Grösse ein dentlicher Gang in den Differenzen aus, während fur die schwïcheren Sterne die Unterschiede als constant zn betrachten sind. Die Grössenschät»ungssealat stimmt also keineswegs fur das ganze in Betracht kommende Helligkeitsintervall mit der photometrischen Grössenscalal Uberein. (Siche (len folgenden Paragraphen.)

Es ist im Frihteren sehon hier und da betont worden, dass die Farbe eines Sternes einen wiehtigen Einfluss anf die Helligkeitssehät/ungen und Messungen ausibben muss, da es bekannt ist, dass die Angen der einzehen Beobachter fur die versehiedenen Farben ungleich empfindlich sind. Dis verhältnissmässig sichere Material, welches die besprochenen photometrischen Cataloge enthalten, hat die Möglichkeit geboten, die hierhei vorkommenden Unterschiede zahlenmässig fest\%ustellen, whlei sich die in der folgenden Tabelle zusammengefassten liesultate erselien hahen. Die Sterne sind in Bezug anf die Farbe in die fünf l'nterahtheihnugn: Woiss, Gelblich-Weiss, Weissheh-Gelb, Gelb, Rïthlieh-(iell, his linth :ugeondnet worden, und die Differenzen der versehiedenen Cataloge sind wiedler gregen den Potsdamer und zwar im Sime: Potsdam-Caltalongebildet, wobei noch die beiden Cambridger Cataloge, welche sich oflenhalr pentu fnleich verhalten, zusammengezogen wurden.

\begin{tabular}{|c|c|c|c|}
\hline Farlee & $\begin{array}{l}\text { l'otadata - } \\
\text { l'ivkering }\end{array}$ & $\begin{array}{l}\text { Pefedinn - } \\
\quad \text { "expers }\end{array}$ & 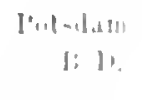 \\
\hline Weiss. . . & $+0.2 ! 1$ & +11.21 & $f-10.11 !$ \\
\hline Gelblich-Weiss. & +0.23 & +11.211 & +1113 \\
\hline Weisslich-Gelb. . & +0.177 & $+11.116 j$ & $-11,112$ \\
\hline Gelb ...... & $-0.0: 3$ & -11.013 & $-0.9 ! 4$ \\
\hline Rüthlieh-Gelb bis Rotlı & -11.13 & $-11.1 \%$ & $-0.11 i$ \\
\hline
\end{tabular}


Es folgt hieraus, dass im Potsdamer Cataloge die Helligkeitsdifferenz zwischen einem weissen und röthlichen Stern kleiner ist als in den anderen Catalogen, und zwar im Vergleich zu Cambridge und Oxford um rund 0.4, im Vergleich zur B. D. um 0.25. Zwischen weissen und gelbliel-weissen Sternen ist der Abstand nur gering; von da an aber wachsen die Differenzen von einer Farbenabtheilung zur anderen um nahe gleiche Beträge. Die B. D. steht ungefähr in der Mitte zwischen dem Potsdamer Cataloge und den Catalogen von Piekering und Pritchard. Diese systematischen Unterschiede werden ihren Grund zum Theil in der Eigenthiimlichkeit der verschiedenen angewandten Instrumente haben, wie es ja z. B. von vornherein klar ist, dass beim Keilphotometer eine leichte Färbung des Glaskeiles derartige Abweichungen hervorbringen kann; aber zweifellos muss der grösste Theil der Unterschiede in der ungleichen Empfindlichkeit der Augen gesucht werden. Jedenfalls sind die oben constatirten Beträge im Verhältniss zu der Sicherheit moderner photometrischen Messungen so beträchtlich, dass bei der Vergleichung von Catalogen unbedingt anf die Farben Riicksicht zu nehmen ist. Man wird gut thun, bei der Reduction der Cambridger und Oxforder Helligkeitswerthe anf das Potsdamer System, wenn es sich um die grösste Genanigkeit handelt, anstatt der constanten Correctionen +0.15 und +0.13 das obige Taifelchen zu benutzen. Eine weitere Verfolgung des wichtigen Gegenstandes, der bisher wenig oder gar nieht beachtet worden ist, wïre im hohen Grade erwiinscht, und es sollte bei keiner photometrisehen Catalogabeit, iiberhaupt bei keiner genanen Helligkeitsmessung rerabsäumt werden, sorgfïltige Farbenschäitzungen auszufiihren. An eine selbstandige, anf unanfechtbaren Prineipien begriundete instrumentelle Colorimetrie der Fixsterne ist ja leider gegenwartig noch nicht zn denken.

e. Beziehungen zwisehen den Grössensehätzungen und den photometrischen Messungen.

Seit man in der Astronomic begonnen hat, die Helligkeitsverhältnisse der Gestirne unter Anwendung ron instrumentellen Hiilfsmittehn zu bestimmen, ist anch immer ron Nenem die Frage anfgestellt worden, welche Beziehung zwischen den gemessenen Lichtrerhältnissen und den bis dahin iiblichen, auf einer ganz willkiirliehen Schaitzungsscala beruhenden Grössenangaben besteht. Diese Frage, wehehe durch die bisherigen Untersnchungen keineswegs als endgiiltig gelijst zu betrachten ist, hat gegentwärtig, soweit es die helleren Steme bis etwa zur 7 . Grösse hinab betrift', allerding nur ein rorwiegend theoretisches Interesse. Denn da wir für die meisten dieser Sterne in den oben besprochenen photometrischen 
Bezichnngen zwischen den Grissenschitzuggen und den photous. Messungen. (Һ.,

Catalogen jetzt genane Helligkeitswerthe besityen, so wird es unr in gan\% seltenen Fïllen erforderlich sein, anf die frllheren Grüssenschilt»ungen zurllckzukommen. Dagegen ist die Frage bei den schwlicheren Sternen, bei denen wir voranssichtlich noch lange Zeit lediglich auf die Schatzungsangaben angewiesen sein werden, von emincut praktischer Bedeutung, weil wir die Mittel zu haben whinschen, diese Angahen eventuell unf dus fur die lielleren Sterne eingefthrte photonetrisehe Grüssensystem ж॥ reduciren.

Die theoretisehe Seite der Frage ist bereits in ersten Abschnitte diesis Buches bei Besprechung des Fechuer'sehen psychophysischen Grunderesetzes berilhrt worden. Nich diesem Gesetze mltsste das Helligkeitsverhïltniss zweier anfeinander folgenden Grössenelassen constant sein. Werden also die Intensititen von Sternen, deren Schiitzungsgrössen $1,2,3 \ldots n$ sind, mit $h_{1}, h_{2}, h_{3} \ldots h_{n}$ bereichnet, und bedeutet o eine Constante, so mlisste sein:

$$
\frac{h_{1}}{h_{2}}=\frac{h_{3}}{h_{3}}=\cdots=\frac{h_{n-1}}{h_{n}}=\varphi .
$$

Hieraus ergiebt sich, wie friher gezeigt wurle, gan\% allgemein fur zwei Sterne von den Grüssen $m$ und $n$ dic Gleichnng:

$$
\log \frac{h_{m \prime \prime}}{l_{n}}=(n-m) \log ? .
$$

Diese Gleichung gestattet, ans den gemessenen Holligkeitsverhailtnisse

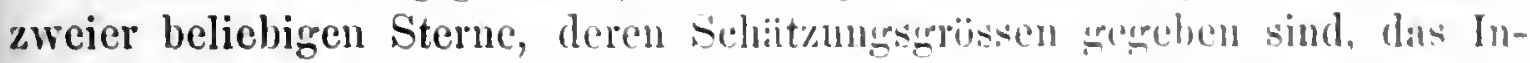
tensitaitsverhälniss o zweier aufeinander folgenden dirissemelassen zu berechnen und zu entseheiden, ob dieser Wrerth in der That flir alle Hellin-

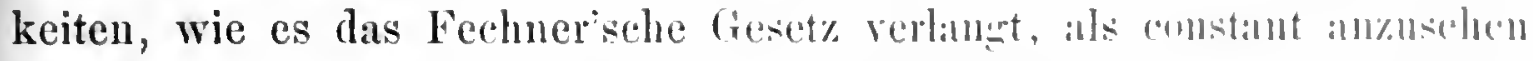
ist. Bei den meisten bisherigen Lntersuchungen hirriber simd din Grissensehätzungen ans Argelanders tranometrie oder diejenigen de linmer

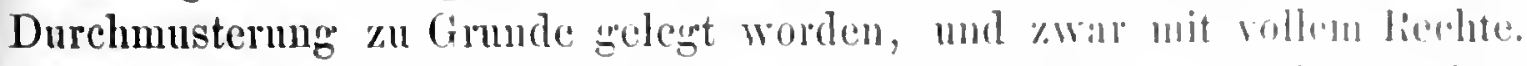

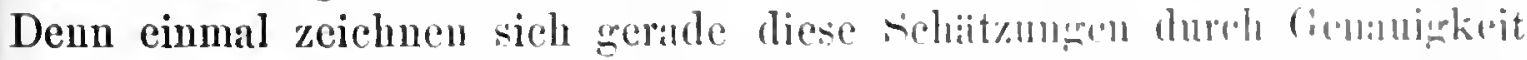

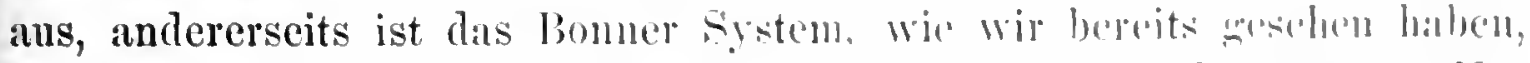

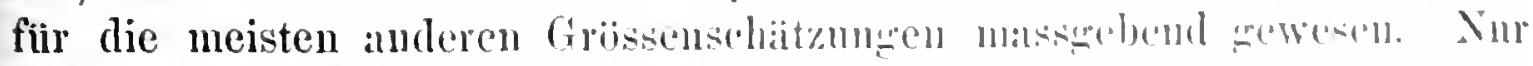

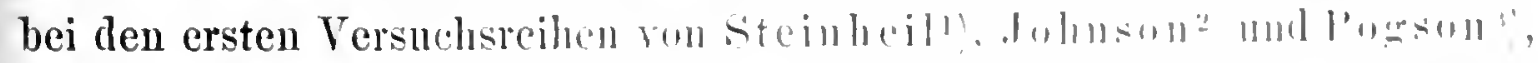

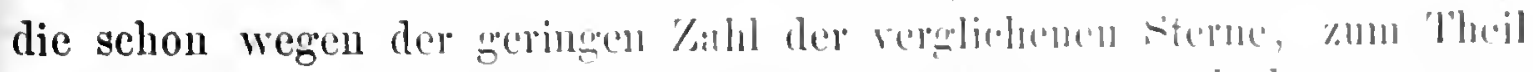

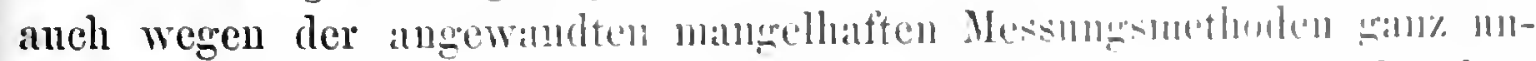
sicher sind und daher lediglich historisches Intererio hesityen, siml andere

1) Steinheil, Elemente der Ilelligkeitsmessungen an strnenhimuel. Minchen. 1836, p. 21.

2) Astron. Obserw. at the Radelifte Observatury. 11xford. Vul. 12. Apwendix.

3) Astron. Observ. at the Radeliffe Observatury. (Ixiord. Vul. 15. P. 29li. 
Grössenschätzungen zu Grunde gelegt worden. Steinheil hat nur die Messungen von 30 Sternen von der 1 . bis 6 . Grösse verwendet und bemerkt selbst ausdriicklich, dass die Werthe weder genan genng bestimmt noch zahlreich genug sind, um ein zuverlässiges Resultat zu liefern. Johnson und Pogson haben sich des keineswegs einwurfsfreien Prineips der Objectivabblendung bedient. Ersterer hat nach dieser Methode am Heliometer die Helligkeitsverhältnisse ron wahe stehenden Sternen (im Ganzen nur ron 39 Pararen) bestimmt und die Resultate mit eigenen Grössenschätzungen verglichen; die Endwerthe können schon deshalb nicht sehr zuverlässig sein, weil bei der nahen Stellung der verglichenen Objecte störende Beeinflussung zn erwarten ist, ansserdem auch bei einigen Paaren die gemessene Helligkeitsdifferenz viel zu klein ist. Pogson hat das Verschwinden von Sternen bei Verkleinerung der Objectivöffnung beobachtet und diese Helligkeitsmessungen mit den Schätzungen ron Bessel, Argelander, Groombridge, Lalande und Piazzi verglichen; die Zahl der beobachteten Sterne, anch die Grössen derselben sind nicht angegeben.

Die Endwerthe, welche sich ans diesen drei ältesten, zweifellos unsicheren Beobachtungsreihen ergeben, sind:

\begin{tabular}{ll|l}
\hline Beobachter & $\because$ & $\log !$ \\
\hline Steinheil & 2.53 & 0.452 \\
Johnson & 2.65 & 0.423 \\
Pogson & 2.40 & 0.350
\end{tabular}

Von Pogson ist zuerst der Vorsehlag ausgegangen, der Bequemlichkeit der Berechnung wegen für das Helligkeitsrerhältniss zweier aufeinander folgenden Grössenclassen dic Zahl 2.512, deren Logarithmus 0.400 ist, zu benutzen.

Die folgcnde Zusanmenstellung giebt eine Übersicht iiber die Werthe ron $\log \varrho$, welche ans den zuverlaissigsten neueren Messungsreihen durch Tergleichung mit den Schätzungen der Argelander schen Uranometrie oder der B. D. abgeleitet worden sind. Eine Lnterscheidung zwischen den beiden Bomner Schätzungssystcmen schcint bei der nahen Übereinstimmung derselben iiberfliissig. Dic meisten der im Torangehenden besprochenen photometrischen Cataloge sind bei dieser Zusammenstellung verwerthet worden, ansserdem sind noch die eigens zu diesem Zwecke angestellten Messungsreihen ron liosén ${ }^{1}$ ) und Lindemaninz) linzugezogen, die deshalb besonders wichtig sind. weil es bisher die einzigen sind, die für die schwïcheren Sterue einigermassen sichere Werthe ron $\log _{0}$ liefern. Die

1 Linll. de lacial. Imp. de St.-Pétersb. Tome 14. 1sio. p. 95.

2 sulplément II anx Observations de Ponlkova. St.-Pétersb. 1ss9. 
Zahlen der Tabelle sind, soweit sie nicht von den Beobachtern selbst angegeben sind, einer Abhandlung von Chandler') (iber das lichtrerhïltniss aufeinander folgender Grössenclassen entuommen. Filr die Sterne der ersten Grössenclasse ist die Berechunng you $\log \varrho$ gailuzlich illusorisch, da dieselbe alle Sterne ron Sirius bis a Leonis und a Geminorum umfasst, also Objecte, die in der photometrischen Seala un mehr als 2.5 Grössenclassen auseiuander liegen. Die Zusammenstellung beginnt dalier enst fir die zweite Grössenclasse, und auch fur diese sind die Werthe von log $\varrho$ wegen der geringen Zahl der verwendbaren Sterne ausserordentlich usicher.

\begin{tabular}{|c|c|c|c|c|c|c|c|c|c|}
\hline $\begin{array}{c}\text { llouner } \\
\text { Schstzungen } \\
\end{array}$ & Zơllner & Seidel & P'eirce & Wolf & $\begin{array}{l}\text { llarvard } \\
\text { I'hotom. }\end{array}$ & $\begin{array}{l}\text { Uranom. } \\
\text { oxoninn. }\end{array}$ & $\begin{array}{l}\text { Potmel. } \\
\text { Durchim. }\end{array}$ & Ilose'n & $\begin{array}{l}\text { Lindre } \\
\operatorname{san} a t\end{array}$ \\
\hline 2-3 Grösso & 0.406 & 0.487 & - & 0.365 & 0.396 & 0.424 & 0.329 & - & - \\
\hline $3-1$ & $0.2 \$ 3$ & 0.362 & 0.391 & 0.325 & 0.365 & 0.365 & 0.329 & - & $\operatorname{lng} 9$ \\
\hline $4-5$ & 0.315 & 0.342 & 0.310 & 0.230 & 0.325 & 0.36 .1 & 0.329 & 一 & \\
\hline $5-6$ & 0.209 & - & 0.437 & 0.178 & 0.352 & 0.377 & 0.329 & 0.355 & 0.303 \\
\hline li-i & - & - & - & - & - & - & 0.100 & $0.35 S$ & 0.394 \\
\hline $7-5$ & - & - & - & - & - & - & $(0.100\}$ & 0.36 .3 & 0.3912 \\
\hline$s-9$ & - & - & - & - & - & - & - & $0.37 !$ & $0.43 i$ \\
\hline A us allen Sternen $2{ }^{m}-6^{m}$ & 0.341 & - & 0.371 & 0.305 & 0.356 & 0.355 & 0.329 & - & $0.2 \mathrm{SO}$ \\
\hline$>, \quad, \quad 6-9$ & - & - & - & - & - & - & $(0.400)$ & 0.350 & 0.102 \\
\hline
\end{tabular}

Betrachtet man in dieser Zusammenstelhung \%uniichst mur die Sterne bis zur 6. Grösse, welche in den meisten Reihen rorkommen, so sieht man, dass sehr beträichtliche Unterschiede \%wischen den einzelnen lorobachten vorhanden sind. Ein systematischer Gamg in den Werthen von

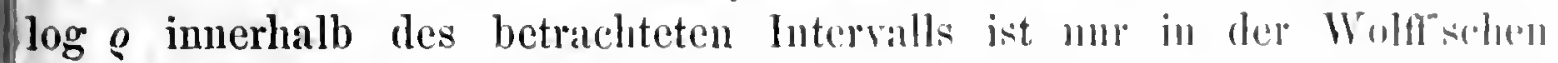
Reihe deutlich ansgesprochen, und nach dem, wats in Friberen wher die Wolffschen Cataloge benerkt worlen ist, miterlicut es keinen \%weifel, dass dieser Gang in der Eigenthiinlichkeit der Woltf"schen Messungen, nicht in den Grössensehait»ungen seine Erklairmng findet. Dis anderen Reihen zeigen niehts Deratiges; sie greben im Allgemoinen zwal fur die Sterne 2. bis 3. Grösse etwas höhere Werthe. dagergen liisst sinh fur die

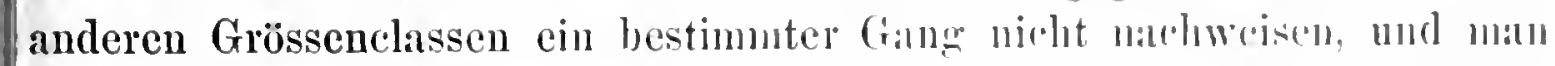

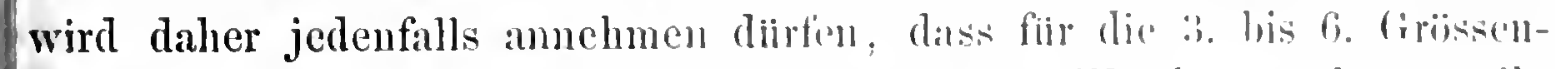
classe der Bonner Ver\%eichnisse ein constanter Wirth rom log a grilt. Werden dic in der vorletyten Horizontatreihe der ohipen liahelle ange-

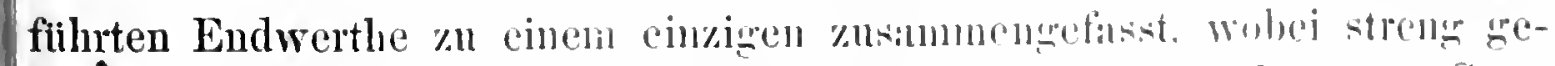
nommen die auf einem grijsseren Material heruhenden Cintalone won Cambridge, Oxford und Potsdam stiirkeres Gewieht als die anderen crhalten

1) Astr. Nachr. Bd. 115. Nr. 274ti. 
sollten, so ergiebt sich für den Logarithmus des Helligkeitsverhältnisses zweier aufeinander folgenden Grössenelassen innerhalb des Intervalles von der 2. bis 6. Grösse mit geniigender Annäherung der Werth:

$$
\log \varrho=0.340 \text {. }
$$

Fiir die Seidel'sche Reihe ist kein Endwerth abgeleitet, weil die ZahI der Sterne unter der 4: Grösse in dieser Reihe viel zu gering ist.

Was die sehwächeren Sterne anbelangt, so stimmen die versehiedenen Werthe von $\log \varrho$ weit besser untereinander, als bei den helleren Steinen; indessen ist das bisherige Material nicht ausreichend, um mit Sicherheit $z \mathfrak{u}$ entscheiden, ob $\log _{Q} \varrho$ für das ganze Intervall von der 6 . bis 9 . Grösse als constant zu betrachten ist. Macht man zunïchst diese Annahme, so folgt. aus der obigen Tabelle als plausibelster Werth fuir die teleskopischen Sterne:

$$
\log \varrho=0.394 \text {. }
$$

Wenn auch die abgeleiteten Endwerthe noch ziemlich unsicher sind, so steht doch soviel fest, dass für die schwächeren Sterne ein anderer und zwar unter allen Umständen ein grösserer Werth von log $\varrho$ gilt, als fuir die helleren. Bemerkenswerth ist, dass diese Änderung ganz plötzlich einzutreten seheint, und zwar bei der Grösse 6.0, also bei derjenigen Helligkeit, bis zu welcher die Grössenschätzmngen mit blossem Auge angestellt werden.

Es gelit ferner ans den bisherigen Untersuchungen hervor, dass die znerst von Pogson vorgeschlagene und jetzt allgemein acceptirte Zahl 0.400 sehr wahe mit dem fuir die teleskopischen Sterne giiltigen Werthe von $\log _{0} Q$ iibereinstimmt. Lässt man also die photometrische Grössenclasse 6.0, wie es z. B. bei der Potsdamer Durchmusterung geschehen ist, mit der Bonner Durchmusterung angenïhert zusammenfallen, so sind auch für alle schwächeren Sterne die Grössenangaben der letzteren und ebenso die der meisten anderen anf Sehïtzungen beruhenden Cataloge direct mit dem photometrischen Grössen vergleichloar. Fiir die hellereu Steme bringt freilich die Benutzung des Werthes 0.4n merkliche Abweichungen zwischen Schützmngsungaben und photometrischen Grössen hervor; doch ist dies praktisch nicht von Bedentung; weil man von den ersteren schwerlich in Zukmuft Gebrauch machen wird, seitden ron allen Stermen bis zur 6. Grösse sorgfüiltige Helligkeitsmessungen vorliegen.

\section{Die verïnderlichen Sterne.}

1)asjenige Gebiet der Fixsternphotometrie, welches etwa seit der Mitte des gregenwairtigen Jahrhunderts relativ am Meisten gepflegt worden ist, 
falls $b$ der hellere ist. Eine sofort auf den ersten Anblick hervortretendr Verschiedenheit gilt fiir drei Stufen und eine noch auffallendere Differenz für vier Stufen; die Bezeichnnngen dafür sind $a 3 b$ resp. $b 3 a$ unc $a 4 b$ resp. $b 4 a$. Noch grössere Unterschiede zu schätzen, wie es z. B Schmidt und Andere gethan haben, ist nicht rathsam, weil daun dic Sicherheit der Beurtheilung abnimmt; schon die Benutzung der vierter Stufe ist nach Möglichkeit zu vermeiden. Dagegen hat Argelande? bei den niedrigeren Stufen die Methode noch verfeinert, indem er halbr Stufen einführte, wenn ihm der Unterschied zu gross erschien, um ihr zu der einen, zu klein, um ihn zu der nächst grösseren zu rechneu Ausserdem empfiehlt er unter Umständen bei Beobachtung der Veränder lichen noch Vergleichnngen mit dem Mittel zweier anderen Sterne, ror denen der eine heller, der andere schwächer ist als der Veränderliche Die Schreibweise ist dann so gewählt, dass die beiden Sterne neben einander in Klammern gesetzt werden und die Anzahl der Stufen hinzug.efiigt wird, um die der Unterschied zwischen dem Veränderlichen une dem einen Sterne denjenigen zwischen dem Veränderlichen und dem anderen iibertrifft. So bedentet z. B. die Bezeichnung $v 2(a b)$, dass de Veränderliche $r^{\circ}$ an Helligkeit zwischen $a$ und $b$ liegt und zwar den helleren Stern $a$ um 2 Stufen näher als dem schwächeren $b$, dass er also um eine Stufe heller ist als die Mitte zwischen $a$ und $b$.

Pickering $\left.{ }^{1}\right)$ hat noch eine Modification dieser letzteren Schätzungs. weise rorgeschlagen, indem er empfiehlt, das Interrall zwischen den Variablen and dem helleren Vergleichsterne mit dem Interrall zwischer den beiden Vergleichsternen, welches gleich $10 \mathrm{zu}$ setzen ist, zu rergleichen. So wiirde die Schreibweise $a 3 b$ bedenten, dass der Helligkeitsunterschied zwischen Stern a und dem Veränderlichen 3 Zehntel des Helligkeitsunterschiedes zwischen $a$ und $b$ beträgt. Eine wesentliche Verfeinerung der Methode wird dadurch nicht erreicht.

Die Argclander'schen Definitionen sind so klar und nuzweideutig; dass sich Jeder leicht mit der. Methode rertrant machen kann. Es ist anch bemerkenswerth, wie gut die Stufenwerthe rerschiedener Beobachtes nntereinander iibereinstimmen. Bei geringer Übung wird man zwar im Allgemeinen geneigt scin, die Intervalle etwas zu gross zu wählen; abes man sewöhnt sich sehr bald an cine engere scala, die man dann auch meist nnverändert beibehält. Bei der Mehrzahl der Beobachter schwankt der Werth einer Stufe zwischen 0.115 und 0.10 Grüsscnclassen.

Der wichtigste Punkt bei der Anwendung der Argelander'schen Stufensorhitzungsmethode auf die Beobachtung der Veräinderlichen ist die Aus- 
wahl geeigneter Vergleichsterne. Da die Schittungen um so sicherer ausfallen, je seluneller man mit dem Auge oder mit dem Fernuhohre ron einem Objecte zum anderen Ubergehen kann, so ist vor allen Dingen erwthnseht, dass die Vergleichsterne dem Verinderliehen nahe stehen; ferner missen sie so gewililt werden, dass der hellste von ihnen den Veränderliehen in seinem Lichtmaximum noch thertrifit, wahrend das Lieht des schwächsten noeh unter der Minimalhelligkeit des Veränderlichen bleibt. Zwischen diesen beiden Grenzen sind mögliehst viele Zwischengrlieder einznschalten, an Besten so, dass die einzehen Vergleichsterne in Interrallen von 4 bis 5 Stufen aufeinander folgen. Das strenge Innehalten dieser Vorschrift bedingt natthlich bei Verinderliehen, die einem sehr starken Lichtweehsel unterworfen sind, eine grosse Zahl von Vergleichsternen, die in unmittelbarer Niihe des Variablen in der gewlinseliten Stufenfolge nur selten zu finden sind. Von Wichtigkeit wiire es, wemn alle Beobachter der Verïnderlichen sich derselben Vergleiehsterne bedienten, und aus diesem Grunde waire fur alle bekannten Verinderliehen die Herstelhng von Kirtehen erwllnseht, anf denen die geeigneten Vergleichsterne bezeichnet wären. Eine derartige, im hohen Grade verdienstliche Arbeit ist in nenester Zeit auf dem Observatorium des Georgetown College in Washington und auf der Sternwarte in Bamberg in Angriff genommen worden.

Ist die wichtige Vorarbeit der Auswahl ron passenden Vergleichsternen erledigt, so gilt bei jeder Beohachtung eines Verïiderlichen die Regel, denselben mit so vielen ron diesen Vergleichstemen zu verbinden, als mit Vermeidung von allzu grossen Stufenuntersehieden irgend möglich ist, jedenfalls aber mindestens zwei zu benutzen, von lenen einer lieller. der andere sehwiicher als der Verïnterliche sein muss.

Bei den Schätzungen der Stufenuntersehiede ist es rathsium, erst das eine Object eine kume Zeit lang (bei genaner Einstellung in die Mitte des Gesichtsfeldes) zu fixiren, his sich der Helligkeitsejudruck dem Gediichtnisse fest eingepright hat, dimn das zweite Object in golejeher Weise zu betrachten, und so abwechsehd mehrere Male ron einem Sterne auf den anderen tiberzugelıen, bis man ein festes Lrtheil gewonnen halt. Mit blossem Auge oder mit dem Opernglase liist sich in Allgemeinen diesere Übergang sehneller bewerkstelligecn, als wenn ein Teleskop bentyt wird, und ans diesem Grunde sind die Vergleichungen der teleskopischen Veranderlichen eher etwas schwieriger ansanfiihren, als dic der helleren Variablen.

Eine gleiehzeitige Betrachtung der beiden zn vergleichenden objecte (falls sie nahe genug bei einander stehen ist weniger emptehlenswerth als die successive Beobachtung, weil die beiden sternbilder anf rer- 
sehiedene Stellen der Netzhaut fallen, und daher eine geringe Veränderung der Kopflage des Beobachters unter Umständen bereits merklich Unterschiede in der Helligkeitsbeurtheilung hervorbringt. Will man dif simultane Beobaehtungsweise doch beibehalten, so thut man gut, sich eines sogenannten Reversionsoculares $\mathrm{zu}$ bedienen, vermittelst dessen man die Bilder zweier Sterne in beliebige Positionswinkel zu einander bringen kann. Man schätzt damn den Stufenunterschied zweier zu vergleichenden Sterne einmal mit dem gewöhnlichen Ocular, und ein zweites Mal unmittelbar darauf mit Benutzung des Reversionsoculares, nachdem man mit Hiilfe desselben die gegenseitige Stellung der Sterne vertauseht, also dén rechtsstehenden nach links, oder den obenstehenden nach unten gebracht hat. Der Mittelwerth ans den beiden Beobachtungen is dann von jedem Auffassungsfehler frei.

Was die Berechnung der Beobachtungen nach der Stufensehätzungsmethode betrifft, so wird nach Argelanders Vorgange die Lichtstärke ausgedriickt in Stufenwerthen über einem willkiurlichen, für jeden Veranderlichen verschiedenen Nullpunkt. Es ist daher das erste Erforderniss, die Stufenuntersehiede der einzelnen Vergleichsterne eines Veränderlichen gegen diesen Nullpunkt, für den man gewöhulich die Liehtstärke des schwächsten Vergleichsternes amnimmt, auf das Genaueste zu bestimmen. Zu diesem Zwecke benutzt man sämmtliche Beobachtungen des Veränderlichen, bei denen er gleichzeitig mit einem helleren und einem schwächeren Vergleichsterne verbunden ist, und leitet ans diesen die Stufendifferenzen fiur die einzelnen Paare ab. Hat man z. B. den Veränderliehen $v$ mit den Vergleichsternen $a$ und $b$ rerglichen und gefunden $a 2 v$ und $v 3 b$, so erhiilt man lieraus unmittelbar $a 5 b$. Entsprechende Gleichnngen ergeben sich für die anderen Sternpare, und ans der Combination aller (entweder streng nach der Methode der kleinsten Quadrate oder durch irgend ein Nïherungsverfilnen) findet man die Stufenmnterschiede sämmtlicher Vermleichsterne gegen den sehwïhsten unter ihnen, dem gewölnnlich der Helligkeitswerth 0 beigelegt wird.

Da jeder Beobachter sich seine Stufenscala selbst bildet, so ist ron rornherein klar, dass die Resultate verschiedener Beobachter nicht ohne Weiteres mitein:uder vergleichbar sind. Dies ist nur danu möglieh, wemn fiu jeden der Stufenwerth in ein und demselben unverïnderlichen Masse, an Besten in Sterugrössenclassen, bekannt ist, und die Helligkeiten der Tergleichsterne dưch sorgtiiltige photometrische Messingen ein für alle Malle festerelegt worden sind.

'̌n Beurtheilung der Sicherheit, welche bei einiger Übung nach der Stufenschitzungsmethode zu erreichen ist, dient eine Angabe von Argelander, whach der wahrscheinliche Fehler einer Helligkeitsrergleichung 
unter gunstigen Umstiuden etwa 0.5 bis 0.6 Stufen, oder da eine Stufe angefaihr 0.1 Grössenclassen entspricht, etwa 0.05 Grössenclassen betrilgt. Zu ahnlichem Resultate sind auch andere Beobachter gelangt, und es geht daraus hervor, dass die Genauigkeit der Stufenschäitzungen, sofern es sich nur um die innere Übereinstimmung melırfach wiederholter Vergleichungen desselben Sternpaares handelt, kaum hinter den besten photometrischen Messungen zurlicksteht.

Es ist im Vorangehenden etwas ausfuhrlich bei der Stnfenschattaungrsmethode verweilt worden, weil fast das gesammte Beobachtungsmaterial, welches wir gegenwärtig ibber die Veränderliehen besitzen, anf dieser Methode beruht, und weil dieselbe auch heute noch in Ermanglung von geeigneten instrumentellen Hiilfsmitteln, namentlich fur Liebhaber der Astronomie, durehaus zu empfehlen ist. Nur muss dringend davor gewarnt werden, die Bedeutung dieser Methode, wie es häufig sogar von Seiten der Fachastronomen geschieht, zu iiberschätzen. Ls kamn nicht oft genug betont werden, dass jede Messung mit einem erprobten Photometer einer Beobachtung nach dem Argelander'sehen Verfahren vorzuzichen ist. Wo die instrumentellen Hiilfsmittel rorhanden sind, sollte diese Methode gänzlich aufgegeben werden, deren einzige unbestrittene Überlegenheit in einer kleinen Zeitersparniss bestehen diurfte. Unter allen Umstainden miisste dafuir gesorgt werden, dass die bedenklichsten Punkte der Argeliunder'sehen Methode nach Möglichkeit unsehädlich gemacht würden. Dahin gehört in erster Linie die Bestimmmng der Lichtstïrken der Vergleichsterne. Dieselben alus den Vergleichungen mit dem Veränderlichen selbst herzuleiten, wie oben auseinandergesetat wurle, ist im hohen Grade bedenklich, weil sich die einmal gesehiitzten Untersehiede zwischen tlen einzehen Paaren dem Gedäiehtuisse sehr bald so fest einpräigen, dass die späteren Vergleichungen sehr leicht dadurch beeinflusst werden, gainz abgesehen daron, dass die Resultate versehiedener Beobachter nicht direct miteinander verolcichbar sind.

Fiir cine erspriessliche Weiterentwiekelung der Photometrie der Veränderlichen wïre es von hïchsten Werthe, wenn die Helligkeiten der Vergleichsterne cin für alle Male durch sorgfältige photometrische Messungen festgelegt wïrden. Es wïre auf diese Weise ein Fundument gesthatten, welehes dazu beitragen wiirde, das IVillkiirliehe der Argelander'schen Methode zu beseitigen nud die cinzelnen Beobatchter in den stand zu setzen, jederzeit ihren Stufenwerth zu controliren. l'iekering hat anf diesen wichtigen Umstand schon wiederholt lingewieven und ist selbst mit gutem Beispiele vorangegangen, indem er für eine betriichtliche Zahl von Verinderlichen die Helligkeiten der Vergleichsterne photometrisch bestimmt hat. 
Bei Weitem der wundeste Punkt der Argelander'schen Methode ist jedenfalls die Voreingenommenheit, die sich beim besten Willen und ungeachtet aller Vorsicht nicht vermeiden lässt, und die je nach der Aufgabe, welche man im Auge hat, und nach der Natur der einzelnen Variablen mehr oder weniger schädlich wirken kann. Bekanntlich sind es zwei Hauptanfgaben, die sich bei dem Problem der veränderlichen Sterne ganz von selbst darbieten, erstens die Ermittlung der Zeitpunkte, zu denen der Veränderliche das Maximum oder das Minimum der Lichtstärke erreicht, also die Bestimmung der Periodendauer, und zweitens die Feststellung der Form der Lichteurve, insbesondere Untersuchungen darüber, ob dieselbe durchweg continuirlich und symmetrisch verlänft oder ob sich Unregelmässigkeiten (Nebenmaxima und Nebenminima u. s. w.) zeigen.

Ist die Periode lang und die gesammte Lichtänderung beträchtlich, so dass die Beobachtungen einerseits nur in grösseren Zeitintervallen zu erfolgen brauchen, andererseits immer neue Vergleichsterne zur Verwendung: kommen, so werden die Schïtzungen weniger dureh Voreingenommenheit beeinflusst sein. In solchen Fällen sind infolge dessen auch die Beobachtungen nach der Stufenschätzungsmethode unbedenklich zu empfehlen. Die einzige Gefahr liegt dann darin, dass sich im Laufe der langen Zeit anch bei dem einzelnen Beobachter der Stnfenwerth verändem könnte; diese Gefahr verliert aber an Bedentung, wenn nach der obigen Vorschrift die Vergleichsterne photometriseh bestimmt sind, und der Stufenwerth daher beständig gepriift werden kann.

Ist die Periode dagegen kurz und der ganze Liehtwechsel nur unbedeutend, so liegt die grosse Gefalır vor, dass jede folgende Beobachtung. durch die vorangehende noch im Gedichtniss haftende beeinflusst wird, und dass sich eine ganz bestimmte Vorstellung von dem Verlanfe der Lichtcurre bildet, die nnwillkiurlich immer wieder anf das Urtheil einwirkt. Am Gefährlichsten ist dies bei denjenigen Veräiderlichen, bei denen sich der ganze Lichtwechsel innerhalb weuiger Stunden abspielt. Nach wiederholten Beobachtungen eines solchen Veränderlichen weiss z. B. der Beobachter, anch wemn er sich absichtlich vorher nicht den genanen Zeitpunkt des Minimums ans den Ephemeriden gemerkt hat, selır wohl aus seinen Schäitzungen, wann ungefähr die geringste Lichtstïrke erreicht ist, und das Bewnsstsein, dass nun wieder eine Zunahme der Helligkeit erfolgen muss, wirkt in hohen Grade störend anf die weiteren Schätzungen. Alle Eigenthiimlichkeiten in der Form der Lichtcurven, die bei einigen dieser kurz verinderlichen Steme ron versehiedenen Beobachtern bemerkt worden sind, miissen mit insserster Vorsicht anfgenommen werden, und wenn irmendwo, so sind gerade bei dieser Classe ron Verinderlichen unbeeinflisste exacte photometrische Messungen durchans mentbehrlich. 
Dic Zahl der gegenwatrtig als sicher verilnderlich erkannten Sterne ist verhältnissmaissig noch gering; sio betrïgt kaum 400. Die meisten derselben sind erst in nenerer Zeit entdeckt worden, und nur sehr wenige waren bereits in fruheren Jahrhunderten bekannt. Das alteste Verzeichniss von veräniderlichen Sternen ruhrt von Pigott') ans dem Jahre 1786 her und enthiilt nur 12 Sterne, deren Verïnderlichkeit damals unzweifelhaft festgestellt war, ausserden noeh 38 Objecte, bei denen clie Verïnderlichkeit weiterer Bestiitigung bedurfte. Ein Catalog von Pogson ${ }^{2}$ ) aus dem Jahre 1856 umfasst erst 53 sicher bestimmte verïnderliche Sterne, und das von $\mathrm{Chambers}^{3}$ ) im Jahre 1865 veröftentlichte Verzeiehniss enthïlt 113 bekannte nnd 15 zweifelhafte Objecte. Die grössten Verdienste um die Catalogisirung der Verïnderliehen haben sich Schönfeld $\left.{ }^{4}\right)$, Gore ${ }^{3}$ ) und Chandler6) erworben. Von Ersterem besitzen wir zwei Verzeichuisse, eins ans dem Jahre 1566 mit 119, das zweite ans dem Jahre 1874 mit 143 Veränderlichen. Gore ziihlt in seinem ersten Cataloge (18S4) bereits 191, in seinem dritten (1SS8) 243 Variable auf, während er in den zweiten (1885) eine Zusammenstellung 'aller der Verïnderlichkeit verdiichtigen Sterne, im Ganzen 736, giebt. Die zuverliissigsten neuesten Daten enthalten die drei Cataloge von Chandler (1588, 1593, 1596). In dem letzten derselben sind für nahezu 400 einigermassen sicher bekannte Verïnderliche die aus den besten Bestimmungen abgeleiteten Elemente des Lichtweehsels mitgetheilt; er giebt uns in Verbindung mit den hinzugefigten Noten einen Gesammtiiberblick iber den Stand unserer Kemntuiss von den veränderlichen Sternen fiur den Anfang des lahres 1896.

In der Vierteljahrssehrift der Astronomischen Gesellschaft werden alljährlieh die Epoehen der Maxima und Minima der wichtigsten Verïnderlichen für das folgende Jahr vorausberechnet.

Die Benennung der Veränderlichen geschieht nach den von Schönfeld und Winneeke j) gemaehten Vorschliigen in der Weise, dass dieselben nach den Sternbildern und ausserdem mit den Buehstaben des grossen lateinischen Alphabetes bezeichnet werden, jedoch erst von $R$ an, un Verwechslungen mit den von Bay er eingefiilurten Benennungen zu vermeiden. Ausgenommen

1) Plil. Trans. of the R. Soc. of London. 17\$6, p. 189.

2) Astr. Obs. made at the Radcliffe Observatory, Oxford. Vol. 15. 1. 281.

3) Monthly Notices. Vol. 25, p. 205.

4) Schönfeld, Catalog ron veriinderlichen Sternen und Zweiter Catalog von veränderlichen Sternen. Jahresbericht des Mannheimer Vereins für Naturkunde, 1866 und 1s74.)

5) Proc. of the R. Irish Acad. II. Ser., Vol. 4, No. 2 und No. 3; III. Ser., Vol. 1, No. 1.

6) Astronomical Journal. Vol. 8, p. \1; Vol. 13, 1. 69; Vol. 15, p. 51; Vol. 16, p. 145 .

i) Vierteljahrsschrift der Astron. Gesellschaft. Jahrg. 3. p. 66. 
sind dabei einige der sogenannten Novae und eine kleine Zahl von Sternen (wie o Ceti, $\eta$ Aquilae, $\delta$ Cephei u. s. w.), für welche sich die früheren Bezeichnungen bereits fest eingebürgert hatten. Da die Zahl der zur Verfügung stehenden Buchstaben in einzelnen Sternbildern sehr bald erschöpft war, so musste die Schönfeld'sche Nomenclatur erweitert werden, und es fand daher ein Vorschlag ron Hartwig $\left.{ }^{1}\right)$ allgemeine Anerkennung, nach welchem in solchen Fällen zwei Buchstaben anzuwenden sind, so dass der 10. Verïnderliche in einem Sternbilde die Bezeichnnng $R R$, der 11. die Bezeichnung $R S$; u. s. w., dann weiter der 19. die Bezeichnung $S S$ n. s. w. erhïlt. Im Sternbilde des Schwans ist die erste dieser nenen Combinationen bereits verbraucht, und der nächste Veränderliche in diesem Sternbilde bekommt die Bezeichnung SS Cygni. Bemerkenswerth ist noch die Art der Numerirung, welche Chandler in seinen Catalogen eingefiuhrt hat. Um bei der roraussichtlich sehr schnell zunehmenden Zahl der Veräinderlichen und der infolge dessen auch sehr häufig erforderlich werdenden Anfertigung von nenen Verzeichnissen die jedesmalige Angabe des betreffenden Cataloges und der laufenden Nummer desselben iiberfliissig zu machen. wählt Chandler als Nummer des Veränderlichen den 11. Theil seiner Reetascension für das Aequinoctium 1900, ausgedrückt in Zeitsecunden. So hat z. B. der Stern $U$ Ophiuchi, dessen Rectaseeusion für 1900 gleich $17^{\text {h }} 11^{\text {m } 2} 7^{\text {s }}$ oder gleich 61587 Secunden ist, die Nummer 6189. Es kann natiirlich rorkommen, dass nach diesem Prineipe zwei oder sogall mehrere Sterne dieselbe Nummer erhalten miissten, und dies ist ein bedenklicher Nachtheil der im Grossen und Ganzen empfehlenswerthen Bezeichnungsmethode; es muss in solchen Füllen, wie es anch Chandler bereits mehrfach gethan hat, ron der strengen Vorschrift abgewichen und die richtige Nummer um eine oder mehrere Einheiten geändert werden.

In Bezug anf die Lainge der Periode und den Intensitaitsunterschied zwischen Maximum und Minimum herrscht bei den Veranderlichen die grösste Mannigfaltigkeit. Es sind sterne bekamnt. bei denen die ganze Verinderung nur eine halbe Grössenclasse betriigt, während die Lichtstairke anderer um 6 bis s Grössenclassen zn- und abnimmt; ferner griebt es sterue. hei denen die Lichtschwankungen in wenigen Stmuden ror sich gehen, dagegen andere. hei denen die Periode beinahe zwei Jahre unfinst. Zweifellos gieht es eine zrosse Zahl ron Yerïnderlichen. bei henen regehmissige sehwinkungen in noch riel lïngeren Perioden epfolgen; aber da zuverlissige Helligkeitsbeobachtungen ans friherer Zeit nicht whliegen, on ist es bisher nicht münglich gewesen. solche Fälle mit

Vierteljahrsschrift der Astr. Gesellschaft. Jahrg. 16 1ss1 . p. 256. 
Sicherheit zu constatiren. Ebenso sind bisher wegen der unzureichenden Genanigkeit der Beobnelıtungen alle diejenigen zahlreieheu Verinderlichen unentdeckt geblieben, bei denen die gesammte Liehtvariation weniger als etwa 0.5 Grössenclassen beträgt. Solche Objecte werden auch in Znkunft nur unter besouders gllustigen Umstinden und mit Hulfe eines ausserordentlich grossen Beobachtungsmaterials aufaufinden sein.

Die Periodendaner der Variablen von langsamer Lielitänderung wird in den Catalogen meistens nur auf ganze oder höehstens Zehntel Tage angegeben, und die Unsicherheit in der Bestimunung der Zeitpunkte des grössten oder kleinsten Lichtes betriigt bei ihnen oft melirere Tage. Dagergen wird bei den schnell Verinderlichen, insbesondere hei denjenigen vom sogenannten Algoltypus, in. den Catalogen die Periodenlinge bis anf Bruchtheile der Zeitseennde angegeben, und die Epoehen lassen sich ans den Beobachtungen bis nahe auf die Minnte sicher ableiten. Bei diesen letzteren Sternen ist es daher von Wichtigkeit, auf den Unstaud Riteksicht zu nehmen, dass die Erde infolge ihrer Bewegung um die Sonne dem Stern bald naiher kommt, bald sich von ihm entfernt, und dass daher auch die Liehterseheinungen verfrtiht oder verspätet beobachtet werden. Man redneirt bei diesen Sternen die Beobachtungen gewöhnlich auf den Sonnenmittelpunkt, d. h. man bereehnet die Zeiten, zu denen die betreffende Erseheinung, z. B. das Minimum des Lichtes, von der Sonne aus wahrgenommen sein wiirde. In den Ephemeriden der variablen Sterne werden für diese Objeete meist die heliocentrischen Zeiten der Minima mitgetheilt. Die Reduetion auf den Somnenmittelpunkt, die sogenamnte Lichtgleiehung, berechnet sich, wie leicht ersichtlich ist, für jeden Stern aus der Formel:

$$
\text { Helioe. Zeit }- \text { Geoc. Zeit }=-497.5 R \cos \beta \cos (\odot-i),
$$

worin $\beta^{\beta}$ und $\lambda$ Breite und Länge des betreftenden Veränderlichen, bezogen auf die Ekliptik, bedenten, $\odot$ die Länge der Sonne, $R$ der Radiusvector der Erdbahn und 497:S die Liehtzeit ist, d. h. die Zeit, welche das Licht braucht, um die halbe grosse Axe der Erdbahn zu durchlaufen. Für Sterne, welche dem Pol der Ekliptik nabe stehen, wird diese lieduction versehwindend klein, für Sterne in der Ebene der Ekliptik erreicht sie die extremsten Werthe -5.3 und +5.3 Minuten. mol zwar zn denjenigen Jahreszeiten, wo somnen- mol Sternlänge einander greich resp. um $150^{\circ}$ voneinander versehieden sind.

Die Entdeckung der hisher bekannten verinderlichen sterne ist fast ohne Ausnahme dem blosen Zufatl zu verdanken. Ein planmäissiges Snchen mach solchen Objecten ist anch gainzlich anssichtslos, solange nieht irgend eine Gesetzmiissigkeit in Betreff ilurer Vertheilnug am Himmel 
oder in Bezug auf ihre Eigenschaften ermittelt ist. Wesentlich erleichtert und begünstigt ist das Auffinden von Veränderlichen seit dem Erscheinen der verschiedenen Uranometrien und insbesondere der Bonner Durchmusterungen; und eine ganz neue Epoche für die Entdeckung von Verinderlichen wird zweifellos durch die neuen photometrischen Cataloge, zum nicht geringen Theile auch durch die Anwendung der photographischen Yethoden eingeleitet werden. Freilich wächst durch die Vermehrung der Zahl auch die Arbeit, die zur weiteren Verfolgung und zur sicheren Festlegung der Elemente ihres Lichtwechsels erforderlich ist; es wäre daher dringend erwiinscht, dass sich die Astronomen mehr als bisher diesem interessanten Zweige zuwendeten und nicht die Hauptsorge dafuir den Liebhabern der Astronomie überliessen. Eine strenge Regel sollte es sein, um Verwirrung in den Benennungen zu vermeiden, nur solche Objecte in die Verzeichnisse aufzunehmen, bei denen die Veränderlichkeit durch mehrere Beobachter ansser jeden Zweifel gestellt ist, ausserdem die Daner der Periode und die Hauptepoche möglichst genau bekannt sind. Selbst der vortreffliche Chandler'sche Catalog enthält noch eine ganz beträchtliche Anzahl von keineswegs sicher bestimmten Veränderlichen, die besser in die Liste der noch zu bestätigenden Objecte gehörten. Für die Sterme mit sehr starkem Lichtwechsel fehlen häufig zuverlässige Angaben über das kleinste Lieht, weil die Instrumente, mit denen gewöhnlich die Veränderlichen beobachtet werden, eine Verfolgung dieser Objecte bis zum Minimum nicht gestatten. Es wäre mit Freude zu begriissen, wenn diese Liicken an Sternwarten, die im Besitz sehr lichtstarker Fernrohre sind, ergänzt wiirden.

Alle Versuche, die bisher gemacht worden sind, um ein Gesetz fuir die Vertheilung der Veränderlichen am Himmel festzustellen, sind als. gescheitert zu bezeichnen; sie werden auch in Zukunft noch so lange rerfriiht sein, bis nicht die Zahl der bekannten Ver:̈inderlichen erheblich stärker angewachsen ist. Zwar glaubte Espin ${ }^{1}$ ) bereits im Jahre $18 \$ 1$ anssprechen zu diirfen, dass die variablen Sterne eine deutlich markirte Zone repriisentirten mit einer Neigung fon $15^{\circ}$ oder $20^{\circ}$ gegen den Äquator, eine Zone, welche siidlich vom Äquator in enger Beziehmng zur Vilchstrasse stehen und sich dort, ebenso wie diese, in zwei Streifen tremnen sollte. Dieses Resultat hat sich aber später an einem grösseren Material nicht bestaitigt. (Chandler'2) findet im Jahre 1889, dass die Teränderlichen von kurzer Periode, mit Ausualume derer vom Algoltypus, zum grijsten Theile nahe in der Ebene der Milchstrasse liegen, dass aber

Ohservatory. Vol. 4, p. 250.

2) Astronomical Journal. Vol. 9, p. 1. 
die Variablen von langer Periode in Bezichnug zur Milehstrasse genau so liegen, wie es bei einer ganz zuftilligen. Vertheilung derselben von vornherein zu erwarten ist. Weitere Untersuchungen uber diesen: Gegenstand mltssen der Zukunft ulıerlassen bleiben. Dagegen verdienen schon jetzt zwei allgemein charakteristische Eigenschaften hervorgehoben $z u$ werden, die einigermassen verbilirgt erseheinen. Erstens findet, weun man die Daner der Perioden ins Auge fasst, keine Gleiehförmigkeit in der Anzahl der Veriinderlichen statt; es geht dies aus der folgenden kleinen Zusammenstellung hervor, welche die Zahl der zn bestimmten Periodenlängen gehörigen Sterne angiebt. Es sind zu dieser 'Tabelle inur diejenigen Sterne des letzten Chandler'sehen Cataloges benutzt worden, fur welehe die Perioden sicher genug bekannt sind.

\begin{tabular}{|c|c|c|c|c|}
\hline \multicolumn{4}{|c|}{ Periodeulänge } & $\begin{array}{l}\text { Anzahl der } \\
\text { Verănderlicben }\end{array}$ \\
\hline \multicolumn{4}{|c|}{ Kiirzer als 20 Tage } & 35 \\
\hline Zwischen & 20 & und 50 & Tagen & 6 \\
\hline 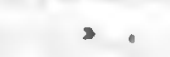 & 50 & . 100 &, & 7 \\
\hline$>$ & 100 & $=150$ & s & $s$ \\
\hline , & 150 & $=200$ & . & 14 \\
\hline , & 200 &, 250 & . & $2 i$ \\
\hline , & 250 & .300 & , & 33 \\
\hline . & 300 & $=350$ & . & $3 i$ \\
\hline$>$ & 350 & $=400$ & s & $2 ! 9$ \\
\hline , & 400 & $=450$ & , & 14 \\
\hline , & 450 & $=500$ & , & 5 \\
\hline Gri & isser & als 500 & 'I'age & 3 \\
\hline
\end{tabular}

Abgesehen ron der ersten Gruppe, welehe die simmtlichen Sterne des ganz eigenartigen Algoltypus umfasst, bemerkt man ein dentlielıes Häufigkeitsmaximum bei Perioden zwischen 3001 und 350 Tagen. Es ist dies um so bemerkenswerther, weil gerade das Auftinden ron Veriunderlichen mit Perioden von nahe einem Jahre dadureh erschwert ist, dass diese Sterne zu denjenigen Zeiten, wo sie am Bequemsten zu beobachten sind, sich immer wieder nahe in demselben Stadium der Helligkeit befinden.

Ein zweiter, fast noch auffallenderer Zusanmenhang findet zwischen der Länge der Periode und der Fürbung der Verïnderlichen statt. Während die Variablen von kurzer Periode, vor Allem die Algolsterne, fist ausschliesslich weiss sind, haben diejenigen mit langer Periode vorwiegend gelbe und röthliche Farben, und man kann als Regel anfstellen, dass, je röther ein Veränderlicher ist, desto lïnger grewöhnlich auch seine Periode sich ergiebt. Es geht dies sehr deutlich aus der folgenden kleinen 
Tabelle hervor. In dieser sind zur Bezeichnung der Farben die Zahlen der Chandler'schen Scala benutzt; in. welcher 0 dem weissen Lichte und. 10 dem tiefsten röthlichen Farbentone, wie er z. B. bei den Sternen $V$ Cygni und $R$ Leporis vorkommt, entspricht. Anch bei dieser $\mathrm{Zu}-$ sammenstellung sind die Angaben des letzten Chandler'schen Cataloges zu Grunde gelegt.

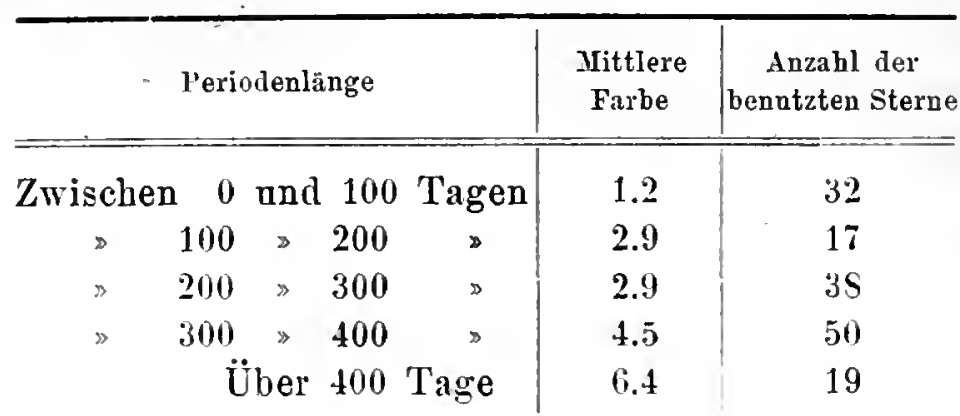

Der Zusammenhang tritt so klar zu Tage, dass an der Realität kaum zu zweifeln ist, trotzdem die bisherigen Farbeuschätzungen keine sehr grosse Sicherheit besitzen. Behufs weiterer Verfolgung dieser interessanten Beziehung sollten es sich alle Beobachter der reränderlichen Sterne zur strengen Regel machen, mit jeder Helligkeitsbestimmung anch eine sorgfältige Farbensehätzung zu rerbinden, wo möglich nach einer einheitlichen Seala. Auf diese Weise liesse sich allein anch die Frage entscheiden, ob mit dem Lichtwechsel eines Sternes gleichzeitig auch eine Farbenändermug eintritt. Fast noch wichtiger wäre eine sorgofältige Untersnehung der Speetren der Verïnderliehen, und zwar sowohl hinsichtlieh des allgemeinen Charakters, als auch in Bezug auf die Helligkeit in rersehiedenen Farben. Leider treten der Ausfiihrung dieser Untersnchungen in grösseren Massstabe gegenwärtig noch bedentende Schwierigkeiten entgegen, weil die Lichtschwäche der meisten Veränderlichen die Anwendung der mächtigsten optisehen Hiilfsinittel erfordert.

Von verschiedenen Seiten sind noch Versuche gemacht worden, den Gesammthetrag der Lichtinderung rom Maximum zum Minimum, und ferner das Verhältniss der Zeitdaner der Lichtabnahme zu der der Lichtzunahme bei den einzelnen Veränderlichen mit der Länge der Periode in Zusammenhang zn hringen. Aber alle diese Tersuche schweben ans Mangel an geniigendem Iaterial rollstaindig in der Luft und brauchen an dieser stelle nicht niaher besprochen zu werden.

Eine strenge Classifieirung der Teränderlichen ron irgend einem einfachen Gesichtspunkte aus ist bei der Mamnigfaltigkeit der Erscheinungen und ter rerhältnissmissig geringen Zahl der Objeete gegenwärtig noch nicht möglich. Ich werde mich diaher bei der folgenden Bespreehumg der 
wichtigsten Frgebnisse der bisherigen Forsehungen an die von Pickering ${ }^{\text {) }}$ vorgeschlagene Eintheilung in funf Hauptclassen halten, obgleich diesellen keineswegs scharf gegeneinander abgegrenzt sind, und anch nicht alle heobachteten Erscheimungen darin untergeordnet werden kümuen. Die Pickering'schen Classen sind die folgenden.

I. Die temporären oder neuen Steme, welehe plötrlich erscheinen und im Verlaufe von relativ kurzer Zeit wieder his fast \%ur Unsichtbarkeit hinabsinken. Der berilhunteste Vertreter dieser Classe ist der Tyehonisehe Steñ vom Jahre 1572.

II. Die Verïnderlichen von langer Periode, welehe in Zeitrïunen von einem halben Jahre bis zu zwei Jahıren nnd darther einen mehr oder weniger regelmässigen Lichtwechsel rom Maximun zum Minimum und wieder zum Maximum zurick vollenden, wobei die gesammten Lichtiinderungen meist selı betrïchtlich sind. Als interessantestes Beispiel wird gewöhnlich der Stern Mira Ceti angefuhrt, und der ganze Typus lieisst daher anch allgemein der Mira-Typus.

III. Die umregelmässig verïnderlichen Sterne, bei denen die Lichtsehwankungen gan\% regellos verlaufen und bei denen weder eine Periodenliinge noch die Maximal- und Minimallehligkeiten mit Sicherheit angegeben werden können. Die Sterne « Cassiopejae und « Orionis gehören zn diesem Typus, der anch bisweilen der Orion-Typus genannt wird.

IV. Die Verinderlichen von kurer Periode, hei denen der Lichtwechsel mit grosser Regelmässigkeit im Laufe von wenigen Tagen vor sich geht. Meistens treten neben dem Hauptmaximum und Ilauptminimum noch Nebenepochen anf. Als Beispiel gelten die Sterne $\delta$ Cephei und $\beta$ Lyrae; nach letzteren heisst diese Clisse anch der Lyra-Typus.

V. Der Algol-Typus, umfassend alle diejenigen Sterne, die wihrend längerer Zeit constante Helligkeit besitzen, aber nach ganz regrelnässigen Intervallen im Verlaufe ron wenigen Stunden einen betriichtlichen Theil ihres Lichtes verlieren und ebenso schuell wiedergewimnen. Der 'Typus ist nach dem ältesten und bekamntesten Vertreter, dem Stern p I'sersei oder Algol, benaunt.

Die wichtigsten Hypothesen, welche zur Erkliirumg der verschiedenen Phänomene des Lichtwechsels der Ver:̈nderlichen antgrestellt worden sind, sollen im Folgenden bei Besprechung" der einzelnen Typen kur\% berihurt werden.

1) Proc. of the Amer. Acad. New Series. Vol. 8 1s51, p. 17 und 257. 


\section{a. Die temporären oder neuen Sterue.}

Das Charakteristische dieser Classe von Sternen besteht darin, dass sie an Stellen des Himmels, wo vor ihrer Entdeckung keine oder nur ganz sehwache Objecte zu erkènnen waren, plötzlich in hellem Lichte aufflammen, verhältnissmässig kurze Zeit in dieser Helligkeit verharren und daцn zuerst langsam und meist mit geringen Sehwankungen, zuletzt mit grosser Schnelligkeit ari Liehtstärke abnehmen, bis sie entweder gänzlich dem Blicke entschwinden oder als ganz schwaehe Sternehen sichtbar bleiben. Friiher war man der Ausicht, dass man es bei diesen Sternen mit neuen Weltbildungen zu thun hätte, und daher stammt die auch heute noch iibliche Bezeichnung "Novae* für dieselben. Es unterliegt wohl aber kaum einem Zweifel, dass diese Sterne keine Nenbildungen sind, sondern dass durch irgend welche Katastrophen ein plötzliches Aufleuchten und Wiederverselıwinden von sehr lichtschwachen Objeeten hervorgebraeht wird, die bei Anwendung der allergrössten Fermrohre sowohl vor dem Auftanchen als naeh dem Erlöschen erkannt werden könnten. Man wird daher die „Novae" als Verïnderliehe ansehen dïrfen, welche nur einem eimmaligen aussergewöhnlieh starken Liehtweehsel unterworfen sind. Die Vermuthung, dass es Veränderliehe mit Perioden vou vielen Jahrzehnten oder Jahrhunderten seien, entbehrt zunäehst der Begriundung. Die Angaben eines Zeitgenossen Tyeho Brahes, nach denen ungefähr in der Gegend der Tyehonisehen Nova rom Jahre 1572 bereits in den Jahren 945 und 1264 nene Sterne bemerkt worden seieu, so dass also anf eine etwa 314 jährige Periode gesehlossen werden könnte $)^{\prime}$, sind nieht als erwiesen zu betrachten.

Lin besonderes Merkmal aller nenen Sterne ist die iiberaus grosse Schnelligkeit, mit welcher das Anwaehsen bis zum grössten Liehte erfolgt. Bei einem derselben, $T$ Coronae, ist eine Lichtzunahme ron mehr als drei Grössenclassen innerhalb $2 \frac{1}{2}$ Stunden nachgewiesen, und ans den zurerlïssigen Angaben bei den in den letzten Jahrzehnten entdeekten nenen Sternen geht hervor, dass der ganze Aufleuchtungsprocess stets innerhalb weniger Tage ror sich gegangen ist.

Man reehnet heute gewöhnlich 11 Sterne zu der Classe der neuen sterne, von denen aber zwei als zweifelhaft zu bezeichnen sind. Piekering. hat in den letzten Jahren noch drei weitere, anf photographisehem Wege cutdeckte, hinzugefiigt; doeh scheint es fraglich, ob diese nieht besser zu den Variablen mit langer Periode zn zählen sind. Auch friiher sind mehrfiuch Objecte als Novale bezeichnet worden. die damn spaiter als regel-

Ton verschiedenen Seiten ist im Zusammenhange daunit die Vermuthung anfgestellt worden, dass anch der biblische Stern ron Bethlehem als eine frihere Erscheinng der 'Tychonischen Nora anznsehen wire. 
mässig verinderliche Sterne erkaunt wurden, wie z. B. vor einer Reile von Jahren der bei $\varkappa_{1}$ Orionis entdeckte Variable $U$ Orionis. In der folgenden 'Tabelle ist eine Zusamnenstellung der 11 nenen Sterne mit den Oerteru filr 1900 in der Reihenfolge ihrer Entdeckung gegeben. Etwas aufallend ist die grosse Zeitlucke zwischen dem vierten und finften Stern.

\begin{tabular}{|c|c|c|c|c|c|c|c|c|}
\hline \multirow{2}{*}{ Same } & \multicolumn{3}{|c|}{ A. R. 1800} & \multirow{2}{*}{ Decl. 1900} & \multicolumn{2}{|c|}{ Nelligkeit } & \multirow{2}{*}{$\begin{array}{c}\text { Jaht } \\
\text { der } \\
\text { Fat- } \\
\text { deekung }\end{array}$} & \multirow{2}{*}{$\begin{array}{c}\text { Name } \\
\text { des } \\
\text { Entderkers }\end{array}$} \\
\hline & & 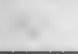 & & & Jax. & Slin. & & \\
\hline$B$ Cassiopejae . & $0^{\text {h }}$ & $19^{\mathrm{n}}$ & $15^{\circ}$ & $+63^{\circ} 35.5$ & $>1$ & $?$ & 1572 & Tycho Brahe \\
\hline P Cygni. . .... & 20 & 14 & 6 & +37.43 .3 & 3.5 & $<6$ & 1600 & Janson \\
\hline Nova Serpentarii & 17 & 24 & 35 & -2123.7 & $>1$ & $?$ & 1604 & Brunowski \\
\hline 11 Vulpeculae . & 19 & 43 & 25 & $+27 \quad 4.2$ & 3 & $?$ & 1670 & Anthelm \\
\hline Nova Ophiuchi & 16 & 53 & 54 & -1244.4 & 5.5 & 12.5 & 1845 & Hind \\
\hline T Scorpii . . & 16 & 11 & 5 & -2243.6 & 7.0 & $<12$ & 1560 & Auwers \\
\hline T Coronae & 15 & 55 & 19 & +2612.2 & 2.0 & 9.5 & 1866 & Birmingham \\
\hline Q Cygni. . . & 21 & 37 & 47 & +4223.1 & 3 & 14.8 & 1876 & Schmidt \\
\hline S Andromedac. & 0 & 37 & 15 & $+40 \quad 43.2$ & $i$ & $?$ & $188 j$ & Hartwig \\
\hline$T$ Aurigae. . & 5 & 25 & 34 & +3022.2 & 4.5 & $<15$ & $1 S 92$ & Anderson \\
\hline$R$ Normae. & 15 & 22 & 11 & $\begin{array}{ll}-50 & 13.9\end{array}$ & $i$ & 13 & 1593 & Harvard Coll. Obs \\
\hline
\end{tabular}

Die Angaben nber den Verlanf der Lichterscheinungen sind bei den älteren von diesen Sternèn sehr liiekenhaft und unsieher und entbehren schon deswegen der Vollstïndigkeit, weil sie nicht iber die Sichtbarkeitsgrenze für das blosse Auge hinaus verfolgt werden konnten. Die grösste Liehtstïrke von allen scheint der Tychonische Stern erreicht $\%$ haben. Nach Tychos Angaben soll er Ende November 1572 der Venus an Helligkeit grleich gewesen sein, und die Lichtabnahme bis zum Verschwinden für das unbewaffinete Auge erfolgte innerhall, eines Zeitraums rou 1 Jahr und 4 Monaten. Bereits im Februar und Mail\% 1573 war er bis zur Helligkeit eines Sternes erster Grösse heralggesunken, im Juli und August erreichte er die dritte, im October und November die vierte Grösse und im Februar 1574 erschien er als Stern 5-6. Grösse. Ob seine Lichtstairke in den Zwischenzeiten contimuirlich abgenommen hat oder geringen Fluetuationen unterworfen gewesen ist, steht nicht fest, dagegen seheint mit der Helligkeitsverminderung ein auffillender Farbenwechsel verhunden gewesen zu sein. Nach Tychos Versichermg Wal die Noval im Maximum weiss, wurde damn gelblich und röthlich, etwal ron der Farbe des Mars, zeigte aber rom Mai 1573 an bis zum Verschwinden wieder eine entsehieden weissliche Firbung. Die Frage, ob an der Stelle der Tychonischen Nora sich jetat ein sehwaches Object findet, ist wegen der verhältnissmäissig unsicheren Positionsaugahen der damaligen Zeit nicht sieher zn entscheiden; nach Untersuchungen ron d Arrest steht etwa $49^{\prime \prime}$ siddich 
von dem Tycho'schen Orte ein Sternchen $10 \frac{1}{2}$ Grösse, welches möglicher Weise mit der Nova identisch ist.

Den zweiten Stern der obigen Liste, $P$ Cygni, wollen manche Astronomen ganz aus der Reihe der nenen Sterne streichen. Soweit die diurftigen Angaben erkennen lassen, wurde der Stern, der im Jahre 1600 . von dem Geographen Janson zuerst gesehen war, zwei Jahre später von Kepler als Stern dritter Grösse beobachtet. 1621 war er für das blosse Ange nnsichtbar, erreichte aber 1655 nach Cassinis Angaben wieder die dritte Grösse, verschwand abermals, wurde 1665 von Hevel schwiicher als dritter, Grösse wiedlergefunden und sank bis zum Jahre 1677 bis zur fiunften Grösse hinab, in welcher er seitdem bis heute unver:indert geblieben ist. Wenn der Stern ror 1600 schon die jetzige Helligkeit besessen hat, was zwar nicht erwiesen, aber sehr wohl möglich ist, so wäre. er allerding:s nicht zu den neuen Stemen im gewöhnlichen Sinne zu rechmen. Anch das mehrmalige Aufleuchten nahe in derselben. Helligkeit. widerspricht der obigen Definition der nenen Sterne.

Über die Nova Serpentarii, die von einem Schiiler Keplers am 10. October 1604 entdeckt wurde, hat $\mathrm{Kepler}^{1}$ ) selbst eine klèine Schrift. veröffentlicht, ans der hervorgeht, dass dieser Stern in seinem Lichtwechsel grosse $̈$ Ähnlichkeit mit der Tychonischen Nova gehabt hat. Die sichtbarkeitsdaner für das blosse Ange betrug nahezu 1 Jahr und 5 Monate. Im Maximmm erreichte der Stern nicht die grösste Lichtstärke der Tychonischen Nova, iibertraf aber an Glanz noch den Planeten Jupiter. Im Januar 1605 war er sehon wieder etwas schwächer als Arctur, Ende März glich er einem Sterne dritter, im October einem Sterne sechster Grösse, und im März 1606 wurde er für das unbewatfnete Ange unsichtbar. Über aluffallende Farbenänderungen finden sich in der Kepler'schen Schrift keine Yittheilungen; der Stern wird als weiss bezeichnet. An der Stelle dieses Sternes ist heute anch mit Hiilfe der stirksten Fernrohre kein Object zu tinden, welehes sicher mit ihm identificirt werden könnte.

Anch die Authelm sche Nora rom . Jahre 1670 (am 20. Juni entdeckt). ist, ähnlich wie PCrgni, insofern nicht ganz streng zn den nenen Sternen zn rechnen, als bei ihr ein mehrmaliges Auflenchten constatirt worden ist. Bei der Entdeckung besass er die dritte Grösse, nalım sehr bald an Helligkeit ab und war schou nach zwei Monaten verschwunden. Im Miirz 1671 tanchte er ron Nellem auf und wurde ron Dom. Cassini als Stern vierter Grösse mit kleinen schwankungen beobachtet. Im Febrnar 1672 war er nicht sichthar, disegen lenchtete er noch einmal in Mir" 1672 als Stern sechster Grösse ant, um dann für immer zu rer-

1) Kepler. De stella nora in pede Serpentarii. Pragae, 1606. 
schwindeu. In der Nühe des nicht ganz sicher bestimmiten Ortes findet sich ein Sternchen elfter Grösse, welehes von Hind fur verinderlich gehalten wurde und vielleicht mit der Nova identisch ist.

Weit znverlissigere Angaben als uber die alteren Nora besitzen wir uber die in gegenwirtigen Jalırhundert entdeckten. Bemerkenswertlı ist, dass keine ron diesen im Maximum aneh nur entfernt die Lichtstïrke der Tychonisehen oder Kepler'schen Nova erreicht hat, und dass bei den meisten der Lichtwechsel sich in verhältnissmässig viel kltrzeren Zeitrainmen abgespielt hat.

Hinds Nova, die als Stern 6. Grösse am 27. April 1545 entleckt wurde, muss ziemlich schmell aufgetancht scin, denn mach Hinds Versicherung war noch am 3. April kein Object heller als $9 \frac{1}{2}$ Grösse an dem Orte sichtbar gewesen. In Jahre 1850 war der stark röthlich gefiribte Stern sehon wieder unter die 10. Grösse gesunken, und seit 1876 ist er ohne merkliche Änderungen als Stern $12 \frac{1}{2}$ Grösse sichtbar gewesen.

Bei Weitem die kurzeste Sichtharkeitsdamer ist bei der $A$ uwers'sehen Nova $T$ Seorpii nachgewiesen, die am 21. Mai 1860 in dem kugelförmigen Sternhaufen Messier 80 an einer Stelle, wo noch am 19. Mai nichts Auffallendes bemerkt werden kounte, als Stern 7 . Grösse anftanchte, aber so schnell wieder an Licbtstïrke abnahm, dass sie schon nach Verlauf eines Monats nieht mehr von dem Nebellichte zu unterseheiden war. Auch spaiter ist an der bezeichneten Stelle keine Spunr ron einem Stemehen wiedergefunden worden.

Das schnellste Auflenchten ist bisher bei $T$ ' Coronite constatirt worden. weleher bereits vor der Katastrophe als Stern 9.5 Grösse bekanut war und anch nach derselben eonstant unter 9. Grösse geblieben ist. Derelbe wurde am 12. Mai 1866 um 12 Uhr Pariser Keit von Birmingham als Stern 2. Grösse (etwa so hell wie a Coronac) erlolickt und noch in derselben Nacht auch von anderen Beobachtem fast ehenso hell geschaity. Da nach der bestimmten Angabe von Schmidt in Athen an der betreftenden Stelle

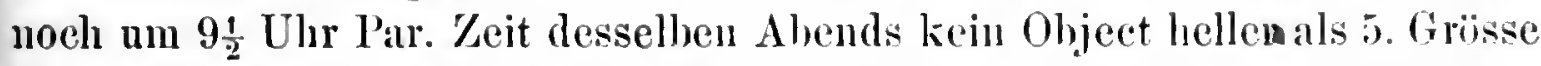
sichtbar gewesen ist, so muss das Auflenchten innerhall, weniger Stunden erfolgt sein. Schon am 13. Mat war die lichtstärke geringer weworden, nach 9 Tagen wurde der Stern fiir das blosse Auge unsichtbatr, und nach Verlauf eines Monats war bereits die 9. Grösse erreicht. Der Stern ist dann nach zuverlissigen Angaben noch einigre Male heller geworden, olne sich jedoch jemals wieder iiber die 7.5 Grösse zu erheben. In den Jilnren 1894 und 1895 ist seine llelligkeit mrerindert 14.5 (irüsse gewesen. $T$ Coronae ist die erste Nora, ron welcher spectroskopische Beobaclitungen vorliegen. Nach den Lintersuchungen von 11 uggoins bestand das Spectrum aus dnnklen und hellen Linien, ron denen zwei nit den Wasserstofflinien 
$C$ und $F$ identificirt werden konnten. Damit war der Beweis erbracht, dass das Auflodern des Sternes mit dem Auftreten von glïhenden Gasmassen im Zusammenhange stand. Heute zeigt das Spectrum von $T$ Coronae nichts Auffallendes.

Ein zweites Beispiel von aussergewöhnlich schnellem Anwachsen der Helligkeit bietet die am 24 . November 1876 von Schmidt entdeckte Nova $Q$ Cygni. Dieselbe tauchte als Stern 3 . Grösse an einer Stelle auf, wo in der Bonner Durchmusterung kein Object verzeichnet ist, und wo noch an 20. November von Schmidt bestimmt kein Stern heller als 5. Grösse gesehen war. Seine Sichtbarkeitsdauer für das blosse Auge betrug 21 Tage, und die Helligkeitsabnahme erfolgte ohne bemerkenswerthe Fluctuationen zuerst schneller, dann etwas langsamer. Anfang 1877 war er 8. Grösse, 1878 sank er unter die 10. Grösse, und gegenwärtig ist er nur noch mit starken Instrumenten sichtbar.

Die zuverlïssigsten Kenntnisse über den Verlanf der Lichtänderungen besitzen wir von den nenen Sternen $S$ Andromedae und $T$ Aurigae. Während die Helligkeitsangaben bei den friiheren Novis nur auf Schätzungen beruhten, sind diese beiden anch mehrfach photometrisch gemessen worden, und das vorhandene Material ist namentlich bei $T$ Aurigae ziemlich umfangreich. Beziuglich des ersteren im Andromedanebel am 30. August 1885 aufgetauchten Objectes, dessen Entdeckung gewöhnlich dem Freiherru ron Spiessen zugeschrieben wird, der aber schon friiher von Hartwig wahrgenommen worden ist, lïsst sich nicht mit Sicherheit der Zeitpunkt des Anfleuchtens constatiren; nur soviel diurfte feststehen, dass etwa Mitte August das Aussehen des Nebels noch nichts besonders Auffallendes gezeigt hat. Seine Anfangshelligkeit wird 7 . Grösse geschüitzt, doch duirfte diese Angabe, sowie die iibrigen Helligkeitssch:atzungen wegen des störenden Einflusses des umgebenden Nebels wenig Vertranen verdienen. Meine photometrischen Messungen ${ }^{1}$ ) geben für September 2 die Helligkeit $\mathbf{7 . 9 5}$, fiir Ende September 9.50; Nitte October war die Lichtstärke schon unter die 10. Grösse gesunken. Die aus den Potslamer Messungen abgeleitete Lichteurve (Fig. 76) zeigt um die Nitte September einen Stillstand in der continuirlichen Lichtabnahme, der anch durch die Beobachtungen ron Hartwig bestiitigt wird. Der Ort der Nova faillt nicht genan mit der Mitte des Andromedanebels und auch nicht mit dem Orte eines Sternchens 11. Grösse zusammen, das schon "friiher bekannt war. Nach dem rollstindigen Verschwinden soll die Nova, wie r. Köresligethy versichert, noch eimmal (Ende September 1856) aufgetaucht sein; doch ist diese Behauptung nicht durch andere Beobachter bestiitigt worden, und es hat 
moglieher Weise eine Verwechslung mit dẹm oben erwilhnten Sternchen 11. Grosse stattgefunden.

Von der Nova $T$ Aurigae liegen bei Weitem die zahlreicheten und sichersten Helligkeitsbestimmungen vor. Dieselben siud von Lindeman n') gesammelt und in sehr grlindlieher Weise bearbeitet worden. Der Stern wurle von Anderson am 24. Januar 1892 zuerst wahrgenommen, jedoch erst am 31. Januar als Nova erkannt. Er besass damals dio Helligkeit 5. Grosse, nalm in den ersten Tagen des Februar noch etwas an Licht

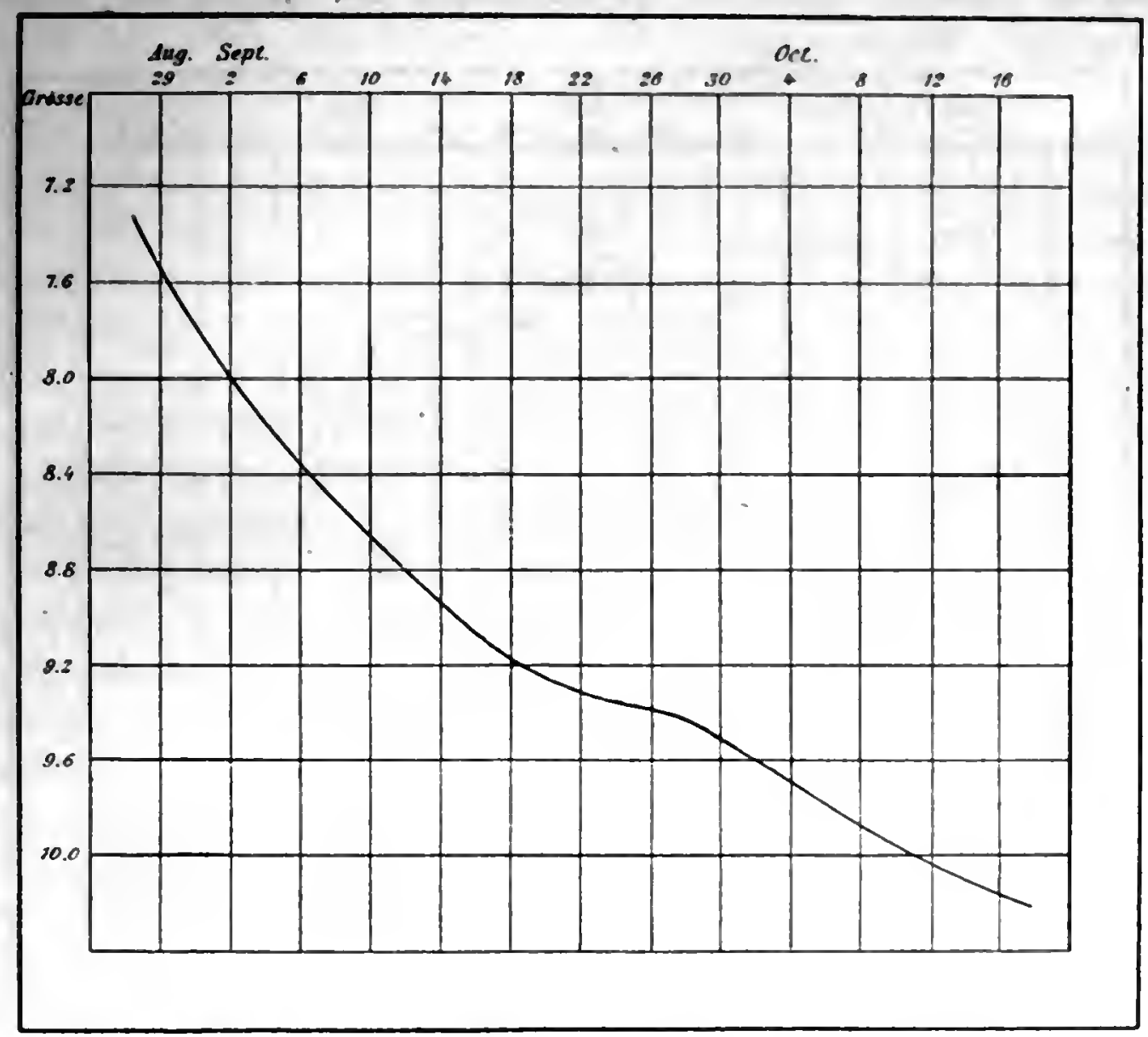

Fig. 76. Lichteurve des neuen Sterns im Anclromedanebel, iss5.

zu, sank dann im Lanfe des Februar ganz langsam und mit deutlich ansgeprägten Nebenmaximis bis zur 6. Grösse herab und nalnm erst im Monat März mit grosser Sehnelligkeit continuirlich bis fast zur vollstindigen Unsiehtbarkeit ab. Höchst eharakteristisch für diese Nova ist, dass sie verhältnissmässig lange Zeit nahezn die Maximalhelligkeit behalten hat. Wie aus einer Reihe von photographischen Anfnahmen auf der sternwarte des Harvard College in der Zeit von 1891 December 10 bis 1592 Januar 20 hervorgeht, ist die Nova bereits vom 10. December an als Stern 5. Grösse

1) Bull. de l'acad. Imp. des sciences de St.-Pétersb. Nouv. Sér. III (35), p. 50 i. 
sichtbar gewesen; sie hat sich also fast drei Monate lang nur wenig an Helligkeit verändert. Auf photographischen Aufuahmen zwischen October 21 und December 1 findet sich die Nova nicht; ihr Aufleuchten fallt mithin in die Zeit zwischen December 1, und December 10. Die von Lindemann, mit Hinzuziehnng der Cambridger Aufnahmen abgeleitete Lichtcurve von $T$ Aurigae ist in Fig. 77 dargestellt. Ende !August 1892 ist ein nochmaliges Auflodern dieses. Sternes constatirt worden, wobei er jedoch nicht heller als 9 . bis 10 . Grösse wurde. In dieser Lichtstärke scheint er daun mit geringen Schwankungen bis fast in die Mitte des

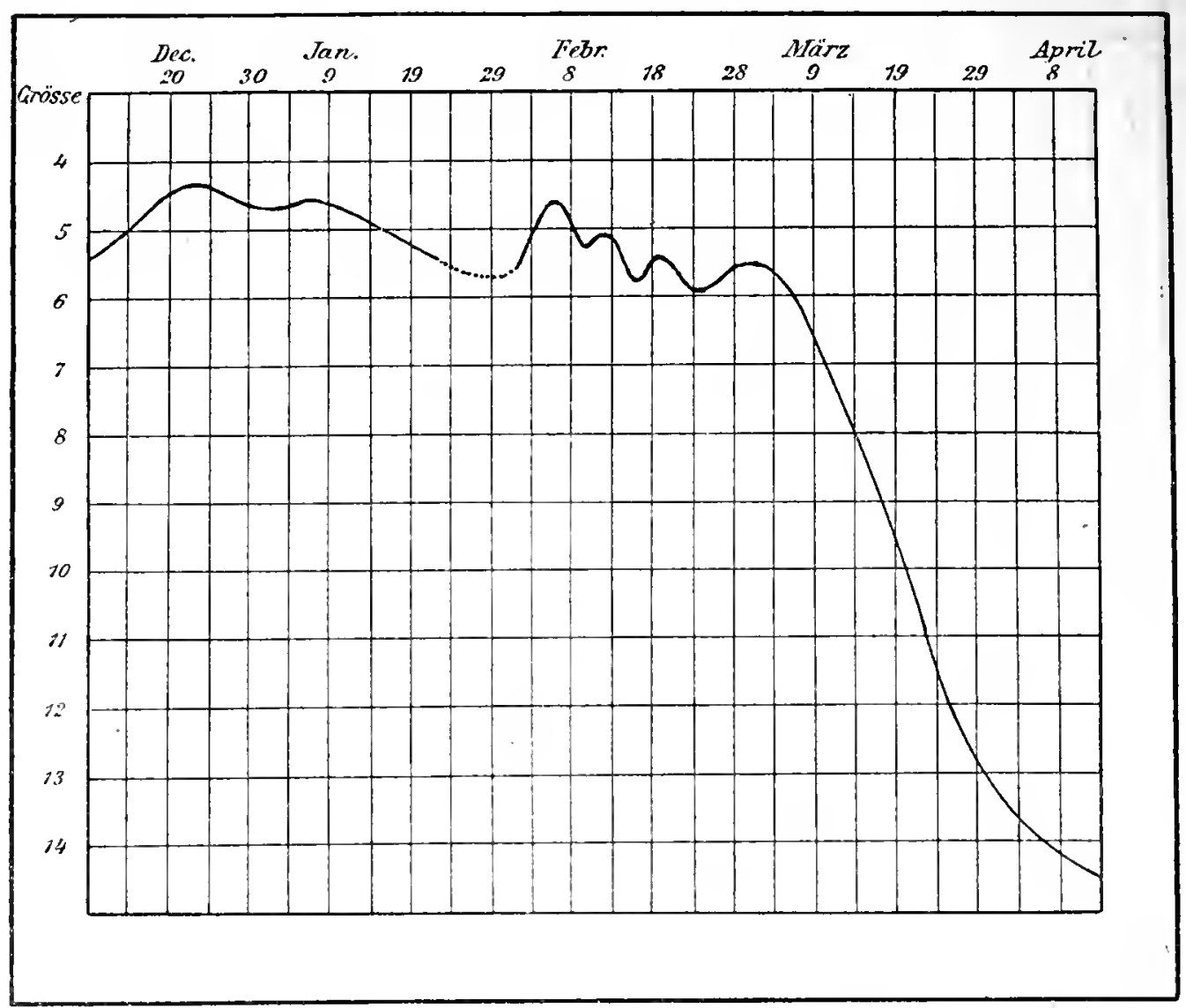

Fig. 77. Lichteure der Nova T Aurigae, 15:2.

Jahres 1594 geblieben zu sein; erst ron da an ist er allmählich wieder schwaicher geworden, ohne jedoch bis hente rollkommen unsichtbar zu werden.

Der letzte Stern in der obigen Liste, $R$ Normac, ist mit Hiilfe der Photographie entdeckt worken. Anf einer an 10. Juli 1893 in AreInipa ron Bailer gemachten Sternspectralanfuahme, welche auf dem Hatrard College Observatorinum ron Mrs. Fleming untersucht wurde, find sich ein spectrum, welches rollkommen identisch zu sein schien nit dem Spectrum der Nora Anrigae. Line zweite Spectralaufwahme rom 
21. Juni 1993 zeigte keine Spur ron dem Sterne, und da er anch auf zuhlreichen photographisehen Karten aus der Zeit von 1589 Juni 6 bis 1893 Mai 27 fehlte, so ergab sich als sieher, dass der Stern zwischeu dem 21. Juni und dem 10. Juli 1893 an einer Stelle anfigeleuchtet war, wo vorher entweder gar kein oder nur ein schwaches Object gestanden hatte. Dic Helligkeit des Sternes wurde nach der Aufuahme rom 10. Juli nngefihr 7. bis 8. Grösse geschät\%t. Directe Beobachtungen dieser Nova sind nur von Campbell bekamnt geworden; demnach war die Lichtstïrke Mitte Februar 1894 etwa 9.5 Grüsse, sie sank damn langsam weiter, und an 23. Mai 1895 war bereits die 13. Grösse erreicht. Seitdem sind keine weiteren Beobachtungen reröflentlicht worden.

Zur Erkliirung der Natur der sogrenannten neuen Sterne laben die Helligkeitsbeobachtıugen derselben verhältnissmässig wenig beigetragen; sie haben uur festgestellt, dass bei diesen Sternen der Vorgang des Auflenchtens von vollstiindiger Unsichtbarkeit oder wenigstens von sehr geringer Lichtstiirke an bis "n enormer Helligkeit zum Theil in gan\% kurzer Zeit erfolgt, und dass dann sehr bald ein ganz allmähliches Almehmen! der Lenchtkraft eintritt, zuweilen noch durch ernente kurze Anschwellungen des Lichtes unterbrochen, bis der urspriingliche Zustand wieder erreicht ist. Darans geht \%weifellos hervor, dass die betrefienden Himmelskörper durch ein plötzlich eintretendes Ereigniss vorübergehend in einen ungemein hohen Gltihzustand versetzt worden sind; aber einen näheren Aufschluss tiber das Wesen dieser Katastrophe erhalten wir ans den heobachteten Lichterscheinungen nicht. In dieser Beziehung hil)en die speetroskopischen Untersuchungen, obgleich sie erst bei den zuletzt erschienenen nenen Stemen zur Auwendung kommen kounten, bereits riel mehr Anhaltspunkte geteliefert als alle photometrischen Beobachtungen. Die verschiedenen 11ypothesen, die zur Erklairung der Erseheinmgen der neucn Sterne anfgestellt worden sind, miissen daher in erster Linie anf Grund der spectroskopischen Ergebnisse gepriift werden, und eine kritische Besprechmng dieser Hypothesen grehört in ein Lehrbuch iiber die spectral:unalyse der Gestime 1). Im Folgenden soll nur der Vollst:indigkeit wegen ein kur\%er Überblick iber die wiehtigsten Lrklïlnuggsversuche gegeben werden ohne näheres Eingehen anf dieselben.

Sehon vor Anwendung der Spectralanalyse hat Zijllner2y eine Ilypothese anfgestellt, welche die beobahteten Erscheinumgen in der IIantsache zu erklïren vermay. Nath ihm sind die nenen sterne Weltkörper, bei deneu sich dureh allmähliche Abkiihlnng eine feste Schlatckendecke

1) Siehe Scheiners Spectralanalyse der Gestirne. Leipzig, 1590. p. 300-305.

2. Zöllner, Photometrisehe Untersuchungen etc. Leipzig, 1 1 655, 1. 247. 
gebildet hat, die plötzlich aus irgend einer Ursache gewaltsam durchbrochen wird, so dass die im Innern eingesehlossene Gluthmasse sich iiber die ganze Oberfläche oder einen Theil derselben verbreiten und dadureh Licht und Wärme hervorbringen kann. Dabei werden auch noch die bereits an der erstarrten Oberfläche vorhandenen chemischen Verbindungen zersetzt, und diese Zersetzung ist gleichfalls mit einer starken Lichtentwieklnng verbunden. Je gewaltiger die herausgetretenen Gluthmassen sind, desto länger wird der Verbrennungsprocess dauern, und desto langsamer wird auch die allmähliche Abkühlung vor sich gehen. Über die Ursache des plötzlichen Ausbruches macht Zöllner keine bestimmten Annahmen, er deutet nur gelegentlich an, dass- der Zustand der Erstarrung lediglich durch änssere Einflüsse, wie z. B. durch den Zusammenstoss mit einem anderen Weltkörper oder durch den Einsturz eines Méteors wieder aufgehoben werden kaun.

Eine gewisse Verwandtsehaft mit der Zöllner'schen Hypothese hat die ron Lohse') anfgestellte. Nach dieser ist die Abkiihlung des Sternes nicht bis zur Bildung einer festen Schale, sondern nur bis zur Bildung einer liehtabsorbirenden Atmosphäre vorgeschritten, welche aber nieht immer so dieht zu sein brancht, dass der Stern gänzlich unsichtbar wird, sondern sehr wohl noch, wie es in einigen Fällen constatirt ist, als schwaches Object vor und nach der Katastrophe rorhanden sein kann. Die Katastrophe selbst besteht in der plötzlichen Bildung von ehemisehen Verbindungen, die bei ganz bestimmten Graden der Abkïhlung eintreten und infolge der enormen Wärmeentwicklıng ein Auflodern des Sternes bedingen. Da in rerschiedenen Stadien der Erkaltung bei ein und demselben Sterne alle mögliehen Terbindungen erfolgen können, so findet nach dieser Hypothese ein mehrmaliges eruentes Aufflackern eine ungezwungene Erklärung.

Anf wesentlich anderen Grundsaitzen beruht die ron Wilsing ${ }^{2}$ ) erweiterte Klinkerfues'sche ${ }^{3}$ Fluthbyothese, nach weleher die nenen Sterne als schwaeh leuehtende Himmelskörper mit diehter Atmosphäre anfzufassen sind, die von cinem relatir dunkleren Begleiter in sehr excentrischer Balm umkreist werden. Beim Durchgange durch das Periastron findet eine so starke Deformation der Atmosphäire des Hạptsternes statt, dass ein Theil der Oberfliehe ganz freigelegt und infolge dessen cine bedeutende Helligkeitsznnahme bewirkt wird. Ist die Annäherung gamz besonders gross, so kömen dadurch, dass nicht nur in der Atmoshliare, sondern aneh in den gliiheuden Gasmassen im Innern des

Honatsber der K. Preuss. Akad. d. Wiss. 147i, p. 826.

Astron. Nachr. Bd. 124, Nr. 2960.

Nachr. von der K. Ges. d. Wiss. zu Güttingen. Jahrg. 1865. 
Hauptsternes Flnthwellen entstehen, Eruptionen hervorgeloracht werden, die noch wesentlich zum plützlichen Anwachsen der Lenchtkraft beitragen und die auch das Auftreten der hellen Linien in Spectrum am Finfaclasten erklïren. Nach dem Durchgange durch dis Periastron bedeckt sich mit zunehmender Entfernung des Begleiters die Oberfliche des Hanptsternes wieder allmählich mit der Atmospläre, und infolge dessen verringert sich die Leuchtkraft. Die Annahme eines Doppelsternsystemes willde natirlich ein periodisch wiederkehrendes Auffammen verlangen, und da ein solches bei keinem der bisher bekannten nenen Sterne beobachtet worden ist, so mtlsste man, wenigstens bei den ailteren derselben, sehr grosse Umlanfszeiten voraussetzen, die aber sehr wohl möglich siud.

Manche Astronomen haben die Erseheinungen der nenen Sterne anstatt durch Vorgainge in einem zusammengehörigen Systeme durch zulfaillige Annäherung zweier gauı getrennten Weltkörper zu erklïren gesucht, oder anch, wie Vogel'), durch das Zusammentreffen eines Himmelskörpers mit einem dem nuseren ähulichen Sonnensysteme, dessen Centralstern dureh allmähliche Abklihlung seine Leuchtkraft verloren hat. Sehr beachtenswerth ist anch die von Secliger ${ }^{2}$ ) anfgestellte Hypothese, welche den Eintritt eines Weltkörpers in ein wolkenartiges Gebilde von sehr dium verstrenter Materic annimmt. Ähnlich wie bei dem Eindringen cines Meteors in dic oberen Schichten der Erdatmosphïire tritt eine plïtzliche Erhitzung der Oberfliche ein, die so lange bestehen bleiben muss, als der Stern innerhalb der kosmisehen Wolke sich bewegt, vielleieht mit geringem Auf- und Absehwanken der Helligkeit, je nachdem die Materie dieliter oder dtinner vertheilt ist. Nach dem Anstritte des Stemes ans der Wolke beginnt die Helligkeit sofort ziemlich schnell abzunelımen.

Zn erwiihnen ist endlich noch die Meteoritenhypothese Lockyers, welcher die Erseheinungen der neuen Sterne dureh die Collision rom Metcoritenschwärmen zn erklïiren versuclit.

\section{b. Die Verinderlichen vou langer Periode.}

Das Unzulaingliche der Pickering sehen Eintheihnug der Verïnderlichen tritt am Dentlichsten bei der zweiten Classe heror; zu welcher hei Weitem der grösste Theil aller Variablen zu rechnen ist. Eine scharfe Abgrenzung gegeniiber der dritten und vierten Girupe sowohl in Bezug anf die Länge der Periode als anf die Art und Weise des Lichtweehsels ist nicht imnezuhalten, und durch Sterne wie , Arons scheint ein Übergang zu der Gruppe der nenen Sterne angedentet zu sein. Man rechnet

1) Mathem. Abhandl. der Kgl. Preuss. Akad. der Wiss. 1693, 1. 1.

2) Astr. Nachr. Bd. 130, Nr. 3116. 
zu dieser Classe gewöhnlich alle Veränderlichen, bei denen die Lichtvariationen in bestimmter Gesetzmässigkeit in Perioden von etwa drei Monaten bis zu zwei Jahren und darüber vor sich gehen. Der bekannteste Vertreter dieser Classe ist der Stern o Ceti, von Hevel wegen -seines merkwurdigen Lichtwechsels »der Wunderbare (Mira) «enanut. Er wurde von Fabricius im Jahre 1596 als Stern 2. Grösse entdeckt und galt, weil er nach einigen Monaten für das blosse Auge verschwand und auch in den nächsten Jahren nicht weiter beobachtet wurde, längere Zeit für eine Nova: Erst von Holwarda wurde im Jahre 1638 seine Eigensehaft als Variabler endgiiltig festgestellt. Durch zahlreiche Beobachtungen seit dieser Zeit ist constatirt, dass der Stern im Minimum bis zur 9. Grösse und darunter herabsinkt, und dass seine Maximalhelligkeit zwischen 2. und 5. Grösse schwanken kann. Das hellste Maximum scheint im November 1779 stattgefunden zu haben. Die Periode beträgt etwa 331.6 Tage, doch ist diese Dauer veränderlich und zwar, wie die Bcobachtungen zeigen, selbst wieder periodisch veränderlich, Argelander hat eine Formel mit mehreren Sinusgliedern aufgestelit, welche die Berechnung der Periodenlänge für jede beliebige Zeit ermöglichen soll, sich aber keineswegs stets als vollkommen ausreichend erwiesen hat. Die Zunahme des Lichtes rom Minimum bis zum Maximum erfolgt bei o Ceti viel schneller als die Abnahme bis zum nächsten Minimum, welche etwa eine doppelt so lange Zeit in Ansprnch nimmt. Es ist dies eine Eigenthümlichkeit, welche ebenso wie die Veränderlichkeit der Periodendauer fuir die meisten der zu dieser Classe gerechneten Variablen charakteristisch ist. Erst bei einer verhältnissmäissig geringen Zahl derselben sind die Erscheinungen des Liehtwechsels so gründlich studirt worden, dass es möglicb gewesen ist, Formeln zur Berechnnng der verïnderlichen Periodendaner anfzustellen und die Gestalt der mittleren Liehteurven mit einiger Zuverlässigkeit zu ermitteln. Verhältnissmäissig gut bekannt sind die am Friihesten entdeckten Glieder der Gruppe, darunter besonders \% Cygui, $l$ Aquarii, $S$ Serpentis, $R$ Pegasi, $R$ Cancri, $R$ Ursae majoris u. s. w. Eine der kiirzesten P'erioden in dieser Gruppe besitzt der Stern $U$ Geminorum, welcher in weniger als 20 Tagen vou der 13. bis zur 9. Grösse anwiachst und dann in 60 bis 70 Tagen wieder zum Minimum herabsinkt. Ob der bereits oben erwïhnte Stern $\eta$ Argus zur zweiten Classe zu rechnen ist, kann noch als zweifelhaft gelten, da eine wirkliche Gesetzmässigkeit im Lichtwechsel nicht nachzuweisen ist; jedenfalls nimmt er eine extreme Stellung imnerhalb der Gruppe ein. Halley zïhlte diesen Stern im Jahre 1677 zur 4. Grösse, 1657 und später 1751 wurde er 2. Grösse geschiätyt, 1\$11-1815 besass er wieder die 4. Grösse, und 1827 wurde er von Burehell heller als a Virginis und a Aquilae geschiitzt. Nach 
J. Herschel erreichte er in Jahre 1837 fast die Lichtstiłke von Sirius und verblieb in dieser Helligkeit mit geringen Schwankungen bis Mitte 1843. Seitdem hat er bestüudig abgenommen und ist seit 1865-constant 7. bis S. Grüsse geblieben. Interessant. ist, dass der Stern sich immitten eines Nebelfleckes befindet, weleher ebenfalls der Veriunlerlichkeit verdiehtig ist. Nach Loomis soll $\eta$ Argus eine Periode von ungefihhr 7) Jahren besitzen, doch fehlen zunïehst noch sichere Anlıaltspunkte zur Bestiitigung dieser Behauptung:

Bei allen Sternen der zweiten Classe ist der Helligkeitsunterschied zwisehen Maximum und Minimum selır bedeutend; er betrigt fast immer mehrere Grössenclassen, und es giebt auch ansser Mira Ceti und ๆ Argus eine ganze Anzahl Sterne, deren Maximallichtstïrke un melır als das 500 fache die Minimalhelligkeit ibertriftt. Bemerkenswerth ist, dass diese Veründerlichen fast siimmtlich eine gelbe oder röthliche Fïrbung besitzen, was darauf hindeuten wilrde, dass wir es bei ihnen mit Himmelskörpern zu thun haben, deren Abkuhlung bereits so weit vorgeschritten ist, dass der Zustand des Rothgluhens cingetreten ist. Dafur sprechen auch die speetroskopischen Beobachtungen, naeh denen die Sterne dieser Gruppe meistens zum III. Spectraltypus gehören. Bei einigen derselben, wie z. B. bei o Ceti, sind zur Zeit des Maximums anch helle Linien in. Spectrum beobachtet worden. Die Speetralanalyse, die sich bisher noch wenig mit diesen Sternen besehiftigt hat, wird hier noch manche interessante Anfschlusse geben kömnen. Leider bereitet die geringe Lichtstïrke im Minimum einer andanernden spectroskopischen Verfolgung dieser Verïnderlichen grosse Sehwierigkeit.

Zur Erklärung der Liehterseheinnngen dieser Variablen, bei dentu es sich nicht mm eine eimmal eintretende gewaltize Katastrophe, sondern un periodisch wiederkehrende Vorgäinge handelt, wird mit Vorliebe die Zöllner'sehe Schlackentheorie herangezogen. Dieselbe setzt voraus, dass die Abkiihlung bei diesen Himmelskïrpern nicht gleichnässig anf der ganzen Oberflaiehe erfolgt, sondern dass sich mehr oder weniger grosse dunkle Flecke bilden, und dass die Erseheinungen des Lichtweehsels von der Rotation des Himmelskörpers herriihren. So einfach und plansibel die Hypothese auf den ersten Blick erscheint, so stijsst sie doch anf manche Schwierigkeiten. Um das im Vergleich zur Abuahme schnellere Anwachsen der Helligkeit zu erkliiren, muss eine besondere Configuration der Schlackenfelder bei allen hierher gehörigen Variablen angenommen werden, und die Verïnderlichkeit in der Daner der Periode kimn nur durch eine Verschiebung der Schlackenfelder erkliurt werden. Die letzteren dürfen also nicht als feste unveränderliche Gebilde, sondern eher wie fliissige oder wolkenartige Condensationsproducte aufgefusst werden; 
aber auch dann ist die Erklärung der mehrfach beobachteten Erscheinung, dass die Veränderlichkeit der Periodendauer selbst wieder einen periodischen Charakter hat, eine missliche Sache. Gyldén $\left.{ }^{1}\right)$ hat, um diese Schwierigkeit zu beseitigen, die Zöllner'sche Hypothese erweitert, indem er nicht bloss den besondercn Fall ins Auge fasst, wo die Rotationsaxe des Himmelskörpers mit der Hauptträgheitsaxe unveränderlich .zusammenfällt, sondern indem er ganz allgemein voranssetzt, dass die Rotationsaxe ihre Lage zu den Massentheilchen des Körpers beständig verändert. Dadurch würde eine eigenthiimliche Drehung desselben entstehen, die auch eine periodische Veränderung der Umdrehungszeit erklären könnte. “Eine strenge mathematische Behandlung des interessanten Problems ist von Gyldén durchgeführt worden. Die Zöllner-Gyldén'sche Hypothese muss anch mit der Thatsache des enormen Intensitätsunterschiedes zwischen Maximum und Minimum, sowie ferner mit der grossen Dauer der Periode rechnen; sie muss also eine sehr weit vorgeschrittene eigenthiimlich vertheilte Schlackenbildung und eine langsame (im Vergleich zur Sonnenrotation sogar sehr langsame) Umdrehungszeit voraussetzen.

In vieler Beziehung sympathischer erscheint daher eine Hypothese, die zwar ebenfalls das Vorhandensein ron Abkiihlungsproducten an der Oberfläche oder in der Photosphäre des Gestirns annimmt, jedoch von der Rotation ganz absieht und nur voraussetzt, dass diese Abkühlungsproducte, ähnlich wie wir es an der Somne beobachteu, in periodischen Zeiträumen sich anflösen und von Nenem wieder bilden. Die Veränderlichkeit der Periode hat nach dieser Annahme nichts Befremdliches an sich, da das Gleiche von der Somnenfleckenperiode bekannt ist. Anch die Ungleichmässigkeit in der Daner der Licht-Zunahme und -Abnahme findet eine Analogie bei den Erseheinungen der Somnenflecke. Dagegen besteht ein grosser Unterschied darin, dass die Fleckenbildung. a uf den Veränderlichen in viel grösserem Umfange stattfinden und sich in viel kiuzeren Zeitrïmmen wiederholen muss, als anf der Sonne. Bei letzterer ist bekanntlich nicht mit Sicherheit ein Anwachsen und Abnehmen der Lichtstärke im Zusammenlange mit der periodischen Fleckenbildung nachzureisen. Wie gewaltig müssen also die Unwälzungen anf den anderen Gestirnen sein, um Lichtïndermgen von 6 oder 7 Grössen"lassen hervorzubringen! Hier liegt der schwache Punkt dieser Hypothese, ganz abgesehen davon, dass man sich ron der Ursache des periodischen Entstehens und Vergehens so grosser Abkiihlungsprodncte nu schwer eine klare Vorstellung machen kann.

1) Gyldén, Versuch einer mathematischen Theorie zur Erkliirung des Lichtwechsels der verinderlichen Sterne. Acta societatis scientiarum Fennicae, Vol. XI, $18 \rightarrow 0$ 
Selır viele Auhäuger hat in Bezug auf die Veränderlichen vom MiraTypus anch die bereits bei den neuen Sternen erwilhute Wilsing-Klinkerfues'sehe Fluth-Hypothese. Wenn der Trabant, weleher in der selir hoch und selır dieht vorauszusetzenden Atmosphäre des Hauptsternes eine Fluthwelle herrorbringt, zur Zeit seines Durehganges durch das Periastron, wo die Flnthwirkung (insbesondere bei selır excentrischen Bahnen) an allerstiirksten ist, gerade auf der ron der Erde abgewandten Seite des Hauptsternes steht, so wird die grösste Aufhellung eintreten, weil die absorbireude Hulle zum grössten Theile ron der uns zugekehrten Seite hinweggezogen ist und die eigentliche Photosphäre zu Tage tritt. Freilich muss die absorbirende Kraft der Atmosphäre im Vergleich zur Sonipenatmosphüre ganz ansserordentlich gross angenommen werden, nm die Lichtzunahme bei Stemen wie o Ceti, $\chi$ Cygni u. s. w. plausibel zu machen. Im Übrigen lassen sich die beobachteten Erscheinungen durch die FluthHypothese leidlich gut erklïren. Zur Begrindung der Verinderlienkeit der Periodendauer mitssen Störungen zı Hülfe genommen werden, die von weiteren Trabanten des Systems ausgelıen. Da diese ebenfalls grössere oder geringere Fhuthwellen je nach der Lage ihrer Bahuen und nach ihren Dimensionen bewirken können, so liisst sich die Versehiedenheit der Lichtstiirke in verschiedenen Maximis und die Ungleichlheit der Perioden ungezwnugen deuten.

Weniger annehmbar als diese Hypothese erscheint die ebenfalls bereits kurz erwïhnte Lockyer'sehe Collisionstheoric. Lockyer stellt sich die Verïnderlichen vom Mira-Typus nicht als einzelne compacte Weltkörper vor, sondem als ziemlich dichte Meteoritenschwïme, jeden begleitet von einem zweiten kleineren Meteoritensehwarn, der sich in einer excentrischen Bahn um den Hauptschwarm bewegt. Wenn die Periastrondistanz sehr klein ist, so treffen im Periastron die äusseren 'Theile der beiden Sehwärme direct aufeinander; zwischen den einzelnen Partikelchen finden zahllose Zusammenstösse statt, und dadurch wird eine starke Lichtentwieklung hervorgebracht. Abgesehen davon, dass die ganze Vorstellungsweise etwas erkiinstelt ist, bereitet die Erklärung nancher beobachteten Thatsachen, insbesondere der grossen Unregrelmaissigkeit der Perioden, erhebliche Schwierigkeiten. Es ischeint nicht, als ob diese Hypothese bisher weitere Vertheidiger gefunden hïtte.

c. Die unregelmässig Verinderlichen.

Alle diejenigen Steme, bei denen zwar Lichtinderungen mit Sicherheit constatirt sind, die aber keinerlei Gesetzmiissigkeit erkennen lassen, 
werden zu der dritten Pickering'schen Gruppe der Veränderlichen gerechnet. Thre Überwachung und Verfolgung ist eine der schwierigsten und undankbarsten Aufgaben fuir: den Astronomen, und es ist daher leicht erklïrlich, dass das Interesse für die meisten dieser Veränderlichen sehr bald erlahmt und unsere Kenntniss von ihnen durchaus lückenhaft geblieben ist. Dázu kommt, dass die gesammte Lichtänderung bei sehr vielen dieser Variablen äusserst geringfügig ist, bei einigen nur wenige Zehntel Grössen umfasst, sodass die allergenauesten Messungen und Schätzungen dazu gehören, um überhaupt die Veränderlichkeit zu erkemnen. Da die meisten hierher gehörigen Sterne röthlich gefärbt sind, so ist ihre Beobachtung von vornherein erschwert, und es giebt zweifellos manche unter ihnen, bei denen die vermeintliche Variabilität lediglich auf physiologische Einfliisse zurückzufiihren ist. Man sollte gerade bei dieser Gruppe mit der Behanptung der Veränderlichkeit sehr vorsichtig. sein und nur solche Sterne in die Cataloge aufnehmen, bei denen die Gesammtänderung der Helligkeit mindestens 0.5 Grössen beträgt und von mindestens zwei Beobachtern vollkommen übereinstimmend constatirt worden ist. Es ist durehaus gerechtfertigt, dass $\mathrm{Chandler}$ ans der Liste der Veränderlichen einen Stern wie $\delta$ Orionis entfernt hat, der frither allgemein als variabel galt und fiir den sogar eine Periode von 16 Tagen angenommen wurde, der aber nach dem ibereinstimmenden Urtheile verschiedener Beobachter sehon längst keine Helligkeitsainderungen gezeigt hat, die iiber die erlaubten Unsicherheitsgrenzen der Schätzungen hinausgingen. Vielleicht wäre es empfehlenswerth, noch manche anderen Sterne, bei denen ein ähnliches Verhalten beobachtet ist, ans der Liste der als sicher veränderlich bezeichneten in diejenige der nur verdächtigen zu iibertragen.

Die bekanntesten Vertreter der dritten Pickering'schen Classe sind die hellen Sterne a Herenlis, a Cassiopejae und "Orionis, nach welehem letzteren die Gruppe häufig benamnt wird. Da gerade bei diesen hellen Sternen die Anwendung der Schiitzungsmethode durch den Nangel an gneniigend zahlreichen, nahe gleich hellen und nahe gleich gefïrbten Vers.leichsternen sehr erschwert ist, so sollten dieselben nur mit Hiilfe yon Photometern verfolgt werden.

Die Lichtstairke von “ Orionis sehwankt etwa zwischen den Grüssen 0.7 und 1.3. Bisweilen findet ein sanz regehmissiges Abnehmen und Auwahsen der Helligkeit statt, sodass sich die Zeit des Minimums mit zicmlicher Genamigkeit ableiten laisst; in anderen Jahren sind aber die Lirhtiinderungen während längerer Zeit ganz unmerklich. Nach Argelander witre eine Periode ron etwa 196 Tagen anzunehmen, doch ist diese bahl durch nencre Beobachtungen durchans fraglich geworden. 
Fast ebenso rergeblich wie bei a Orionis sind die Versuche gewesen, bei den anderen Stemen der Gruppe hestimmte Periodendanern unehauweisen, und es ist daher anch kaum möglich, die ganz unregehnassigen Lichterseheinungen dureh eine einheitliche Theorie zu erkliiren. Die Flnth-Hypothese-dinfte kanm anwendbar sein, weil man nur durch die Annahme von mehreren Satelliten und vou eomplicirten Störmugen unter denselben zn einer gekthnstelten Deutung der Vorgäuge gelangen künte. Anch die Fleckenhypothese in einer der in vorigen Paragraphen erwiluten Formen durfte fur sich allein nieht ausreichend sein, und man wird daher in Ermanglung von etwas Besserem zur Combination von mehreren Hypothesen die Zuflueht nehmen mussen. So kömnte mau sieh vorstellen. dass in der Photosphäre alnnliche Rerolutionen wie anf der Soine, aber in noch unregelmïssigeren Zeitrïumen vor sich gehen, und dass gleichzeitig eine Rotation des Sterues stattfindet. Manche haben anch noch eine neue Hypothese hinaugefilgt, indem sie eine von der Kugel oder dem Rotationsellipsoid abweichende Gestalt des Sternes vorausset\%en, sodass bei einer Axendrehung desselben verschieden grosse Theile der Oberfliehe flir uns sichtbar werden könneu. Alle diese und ähnliche Combinationen haben jedoch eine befriedigende Deutungr der Erseheinungen nicht z.ll geben vermocht. Auch die speetroskopischen Beobachtungen dieser Classe von Verïnderlichen haben bisher keine bemerkenswerthen Anhaltspmikte geliefert.

d. Die regelmassig Verinderlichen von kurzer leriode. Der Lyra-Typus.

Die vierte Classe der Verïnderlichen hat ihren Sinnen ron dem interessantesten Vertreter derselben, dem Sterne $\beta$ Lyrale. If:m rechmet zu ilır alle diejenigen Variablen, bei denen die Periodendaner zwischen wenigen Tagen und etwa 2 his 3 Monaten selswankt, und bei denen ein continuirlieher Lichtwechsel stattfindet. Eine strenge lbgrenzmg gegen die anderen Typen, namentlich gregen die aweite l'ickering'sche

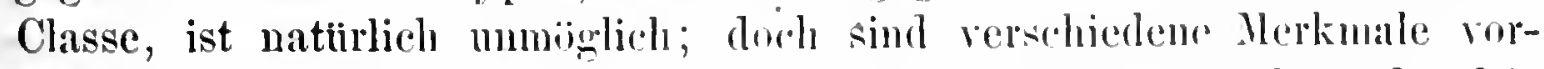
handen, die gerale für diese Verinderlichlen dharakteristisch sind. Die

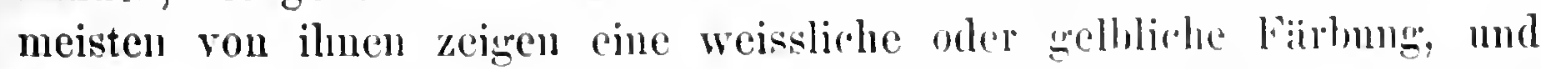
ihre Spectra gehören dem ersten oder zweiten Siertraltypus an; es sind also wahrscheinlich Weltkörper, die sich noch nicht in so vorgeschrittenem Entwieklungsstadium befinden, wie die Verinderlidhen ron Mirat'Tyms. Die gesammten Helligkeitsinderumgen sind verläiltuis:milissign mbedcutend, und es scheint beathenswerth, dass bei ciner wossen Zahl dieser Variablen 
der Intensitätsunterschied zwischen Maximum und Minimum nahezn den gleichen Betrag von etwa einer Grössenclasse hat. Ein weiteres Merkmal ist, dass bei den meisten hierher gehörigen Veränderlichen neben dein Hauptmaximum und Hauptminimum mehr oder weniger deutlich ausgeprägte Nebenmaxima und Nebenminima auftreten. Die regelmässigste Lichtcurve von allen zeigt wohl $\beta$ Lyrae, dessen Veränderlichkeit schon 1784 von Goodricke entdeckt wurde, dessen Periode aber anfangs zu klein angenommen wurde, weil man den Unterschied zwischen Haupt- und Nebenminimum nicht richtig zu erkennen vermochte. Erst durch die ausführlichen Untersuchungen ron Argelande ${ }^{1}$ ), Oudemans ${ }^{2}$ ) und Schönfeld ${ }^{3}$ ) ist der Lichtwechsel mit grosser Genauigkeit bestimmt worden. Nach

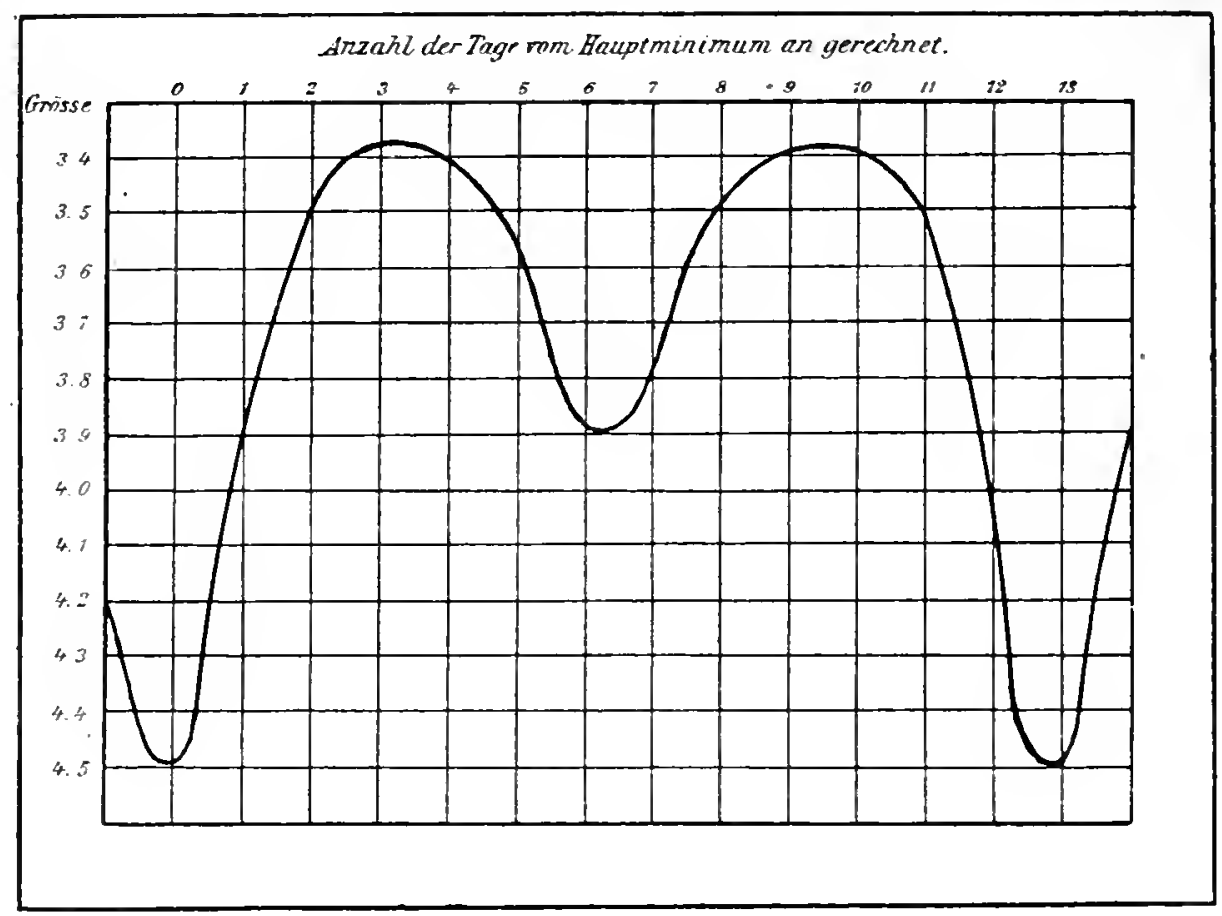

Fig. 78. Lichtemre ron 3 Lyrae.

Letzterem hat $\beta$ Lyrae im Hauptminimum die Grösse 4.5. Die Lichtstiirke steigt ron da innerhalb $3^{\mathrm{d}} 3^{\mathrm{h}} 3$ bis zum ersten Maximum (Grösse 3.4), sinkt dann in weiteren $3^{\text {d }} 5^{\text {h. }}$ zum Nebenminimum (Grösse 3.9) hinab, erhebt sich wieder in $3^{4} 2^{\mathrm{h}} 9 \mathrm{zmm}$ Maximum 3.4 und erreicht endlich nach $3^{\text {th }} 9^{\text {h }}$ ron Nenem das Haiptminimum. Der ganze Vorgang spielt sich in $12^{\mathrm{d}} 2$ l: $^{\mathrm{h}} \mathrm{S}$ ab, und die Lichteurve (Fig. 78) zeigt zwei gleich grosse Erhebungen mit einer dazwischen liegenden Einbuchtung.

1. Argelander. De stella 3 Lylae variabili disquisitio. Bonnae, 184 und be stella $\beta$ Lyrae rariabili commentatio altera. Bonnae, 1559.

$?$ Oudemans. Zweijahrige Beobachtungen der meisten jetzt bekannten verinderlichen Sterne. Amsterdam. 1550.

Astr. Naclur. Bd. 75. Nr. 177\%. 
Die regelüissig Veriinderlichen von kuraer Periode. Der Lyra.'Typus. 459

Nenere Untersuchungen vou Schur'), welehe sich auf die von ihm angestellten Helligkeitsschätzungen in den Jahren 1577-1855 grinden, bestatigen im Grossen und Gauzen die Form der Argelander'sehen und Sehönfeld'sehen Lichtenrve. Dagegren deuten die Untersuchungen Lindemanns'), bei denen Beobachtungen von Plassmann ans den Jahren 1888-1893 \%u Grunde gelegrt sind, auf eine nicht unwesentliche Anderung der Lichteurve hin, insofern sich fur die Zwischenzeiten zwischen den Hauptmomenten anstatt der oben angegebenen Kalilen die folgenden Werthe ergeben:

$\begin{array}{llr}\text { Min. I bis Max. I } & 3^{\mathrm{d}} & 12 \text { 孔0 } \\ \text { Max. I * Min. II } & 3 & 3.6 \\ \text { Min. II * Max. II } & 3 & .1 .2 \\ \text { Max. II * Min. I } & 3 & 5.0\end{array}$

Eine weitere Bestätigung dieser Änderungen der Lichteurre, womöglich durch photometrische Messungen, bleibt abzuwarten.

Zur Berechnung der Epochen der Hauptminima von $\beta$ Lyrae kamn man die von Selur anfgestellte Formel benutzen:

1855 Jall. 6, $15^{\mathrm{b}} 28^{\mathrm{m}}$.0 (Mittl. Z. Bomm) $+12^{\mathrm{d}} 21^{\mathrm{h}} 47^{\mathrm{m}} 23^{\mathrm{s}} .72 \mathrm{E}$

$$
+0.315938 E^{2}-0.00001211 E^{3} \text { : }
$$

wo $E$ die Anzahl der Perioden ist, die seit dem angenommenen llauptminimum im Jahre 1855 verflossen sind. Die Gesammtlinge der Periode ist kleinen Schwankungen muterworfen, die selbst wieder einen periodisehen Charakter tragen; ansserdem ist noch eine siiculare Zunahme derselhen angedentet.

Ebenso lange bekannt wie $\beta$ Lyrae sind zwei andere Sterne dessellen 'Yypus, $\eta$ Aquilae und $\delta$ Cephei, beide gleielffalls in Jahre 175.1 entdeckt, ersterer von Pigott, letzterer von Goodricke. Ilure Lichtcurven sind nicht so symmetrisch wie diejenige von $\beta$ Lyrae. Bei $\gamma$ Aquilate ist das zweite Maximum nur sehwach ausgeprïgt, und bei $\delta$ Cephei ist es sogar fast ganz verwischt. Die Lainge der Periode betrïgt bei dem ersteren Stern $7^{\mathrm{d}} 4^{\mathrm{b}} 13^{\mathrm{m}} 59.3$ und ist oftenbar etwas veriuderlich. Nach dem Minimum (Grösse 4.7) steigt die Helligkeit innerhall, $2^{4}\left(6^{\text {he }}\right.$ zum Han!tmaximum (Grösse 3.5) an ; nach Verlanf roul $1^{\text {d }} 15^{\text {h }}$ ist dats Nebenminimum mit der Helligkeit 4.1 erreicht, dam erhelst sich die Lichtstärke noch einmal in etwa $13^{\text {h }}$ \%u einem zweiten Maximum 3.S. $11 m$

1 Astr. Nachr. Bd. 137, Nr. 32S2.

2. Ball. de l'acad. Imp. des seiences de St.-Pétersb. Nonv. Sér. IV XXXVI, 1893, p. 251. 
dann endlich wieder in $2^{\mathrm{d}} 18^{\mathrm{h}}$ zum Hauptminimum herabzusiuken. (Fig. 79.)

Bei $\delta$ Cephei ist die Periode noch kïrzer; sie betriigt nach den Bestimmungen von Argelander $5^{\mathrm{d}} 8^{\mathrm{b}} 47^{\mathrm{m}} 39.974$ und diirfte, wie die neueren Beobachtungen ron Schur zeigen, jetzt wohl noch eine Secunde kleiner anzunehmen sein. Die Grenzwerthe der Helligkeit sind $4.9 \mathrm{im}$ Hauptminimum und 3.7 im Hanptmaximum; zwischen diesen beiden Epochen liegt ein Zeitraum von etwa $1^{\mathrm{d}} 14^{\mathrm{h}}$. Nach den Beobachtungen von Argelander und $\mathrm{Heis}$ findet etwa $20^{\mathrm{h}}$ nach dem Hauptmaximum ein Stillstand in der Liçhtabnahme statt, entsprechend dem Nebenminimum und Nebenmaximum bei $\beta$ Lyrae und $\eta$ Aquilae. Doch ist diese Einbiegung nicht

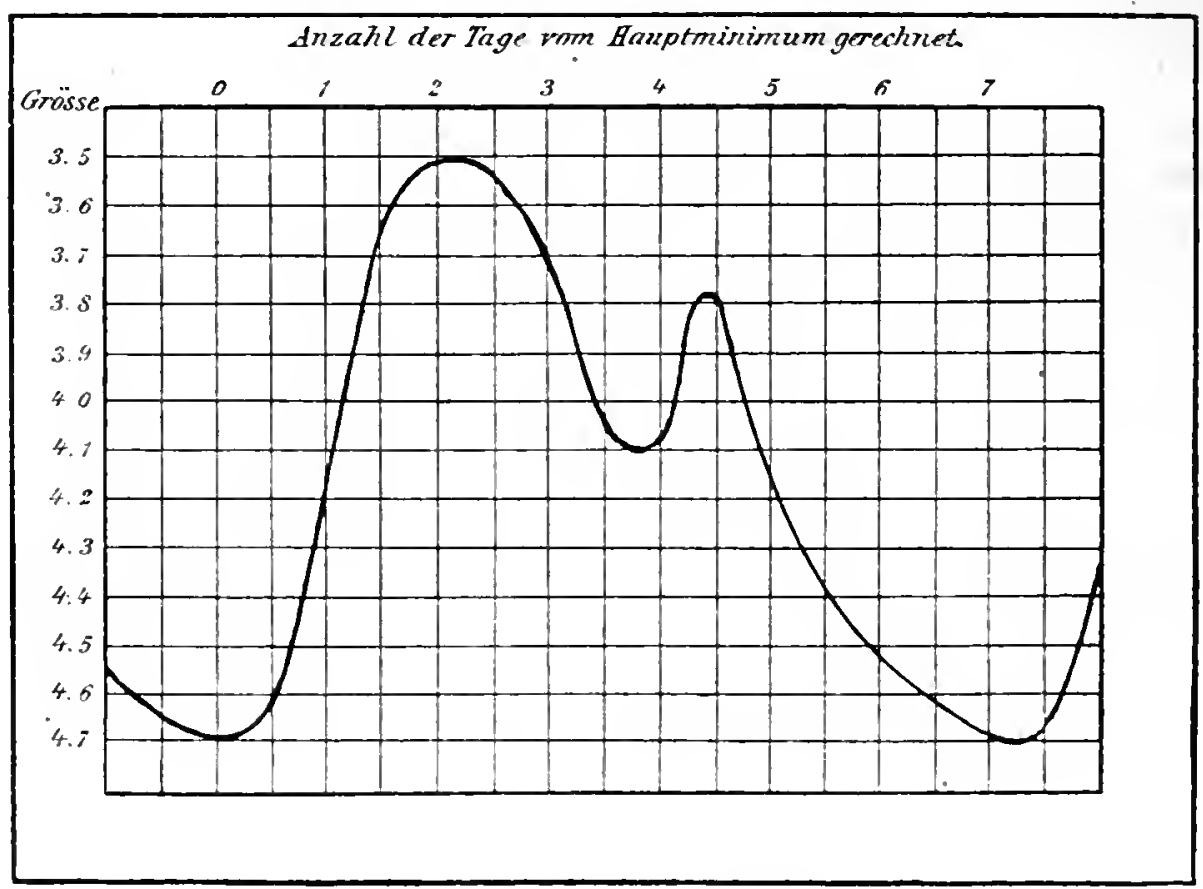

Fig. 79. Lichteurve von ، Aluilae.

als sicher rerbiirgt zu betrachten; die Beobachtungen ron 0 udemans erzeben den Stillstand in der Lichtabnahme erst etwa 3 Tage nach dem Hauptmaximum, und die Sehur'sche Lichtemve (Fig. 80) lisst nur wenig davon erkennen.

Die übrigen Steme, welche man mit einigem Reehte zu der vierten l'irkering'schen Classe zihhlen kam, sind bei Weitem noeh nicht so strindlich in Bezug anf den Verlauf der Lichtaindermoen untersucht, wie die drei eben besprochenen. Einige scheinen ein ganz ähnliches Verhalten wie r Aquilae zu haben, und man spricht daher bisweilen ron einem besonderen , Aquilae-Typus. Bei anderen ist die Einbiegung der Curve nach dem Naximm nu nusicher angedentet; man rechnet sie zu rinem $\delta$ Cephei-Tylus. Bei allen zeigt die Periodendaner kleine sienlare 
Unregelmissigkeiten. Die bekanntesten Vertreter sind $T$ Monocerotis, $\zeta$ Geminorum, $S$ Sagittae und $T$ Vulpeculae. -

Was die Erkliirungsyersuche fir die Lichterseheinungen bei den Sternen der vierten Gruppe anbelangt, so ist olme Weiteres ersiclitlich, dass die Annalıme von periodisch erfolgenden Fleckenbildungen, ailnlich wie bei ler Sonne, kaum mit einem so kurzen nnd so unregelmaissig verlaufenden Lichtwechsel réreinbar sein ditrfte. Auch die Züllner'sehe Hypothese stosst anf manche Schwierigkeiten. Um eine so symmetrische Lichteurre, wie bei o Lyrae, zu erklären, milsste man annehmen, dass die Abkithlungsproducte anf zwei gerade entgegengesetzten Sciten des Sternes angehiuft

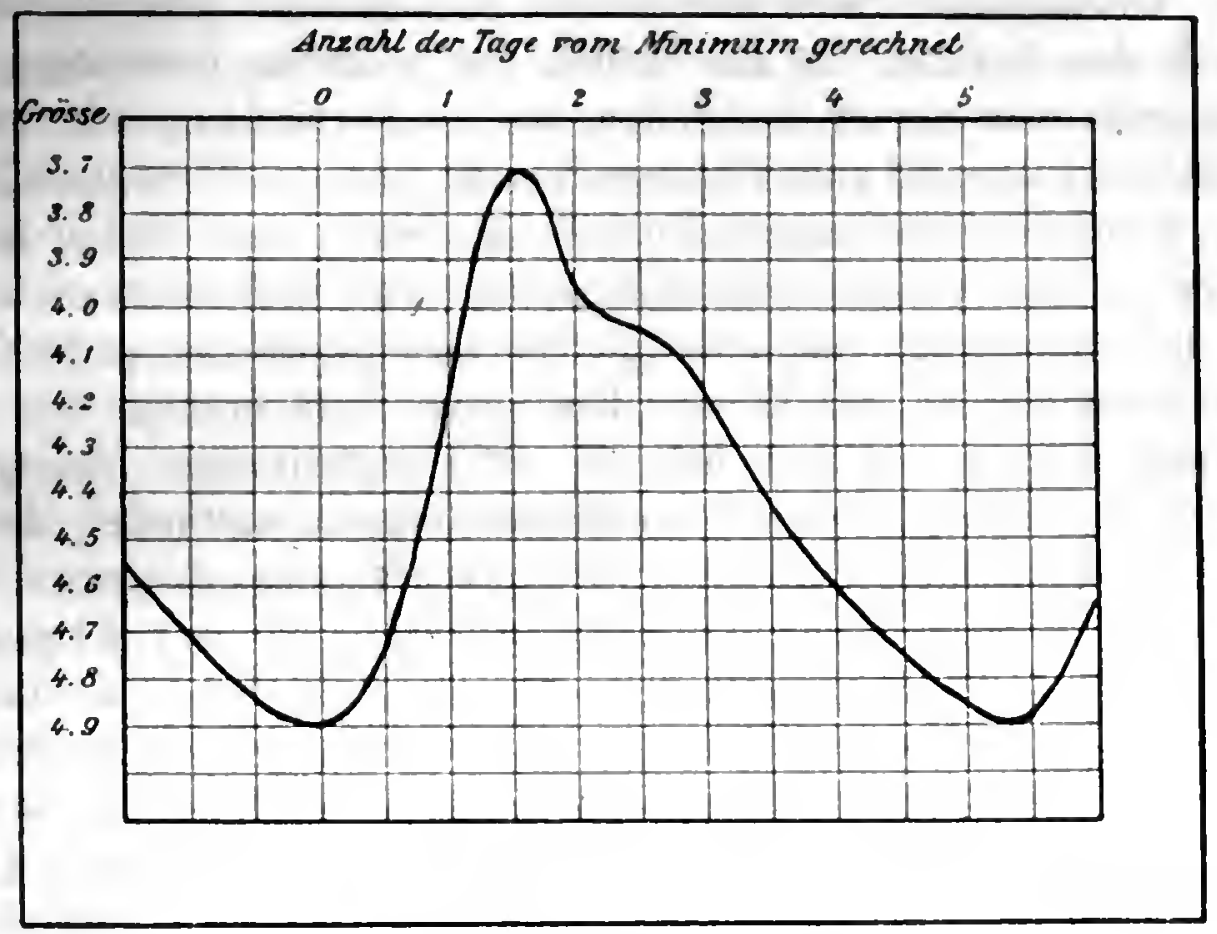

Fig. B0. Lichteurve ron de ciphei.

seien, und zwar auf der einen Seite in betrïichtlich wrösserer Menge als auf der anderen, wïhrend die dazwischen befindliehen helleren Oherfliichentheile zwei ebenfalls gerade gegeniiberstehende Maxima hesitzen muissten. Selu wahrscheinlich ist eine solehe Vertheilung rom dunklen und hellen Partien nicht, und noch unwahrschemlicher ist es, disis bei der ziemlich sehnellen Rotation des Sternes keine merkliche Versohiehung dor

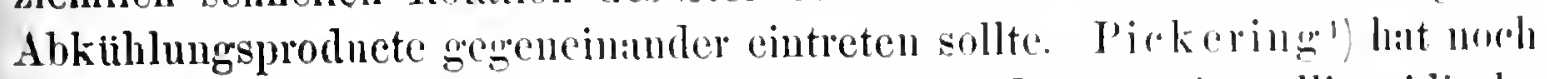
cine zweite Hypothese zu Hiilfe genommen, inden er einc cllipsuidische Gestalt des mit unregehnaissigen Schlackenfeldem bedeckten Körpers voralussetzte und die kiilzeste dxe sieh als Rotationsixe daldite. Dher wem es ihm anch gelungen ist, unter diesen Voramssetzmugen empirische Formeln

1) Proc. of the Amer. Acal. New series. Vol. VIII. p. 2.57. 
abzuleiten, welche die beobachteten Lichterscheinungen bei $\beta$ Lyrae, $\eta$ Aquilae, $\delta$ Cephei und $\zeta$ Geminorum befriedigend darstellen, so wird man sich schwerlich dazu verstehen, die physikalische Bedeutung dieser Interpolationsformeln in vollem Umfange anzuerkennen.

Viel näher liegt bei den 'Veränderliehen vom Lyra-Typus der Gedanke an eine bisher noeh nicht berïhrte Hypothese, welche den Lichtweehsel durch die gegenseitige Verdeekung zweier (oder mehrerer) um einen gemeinsamen Sehwerpuukt sieh bewegenden Himmelskörper erklären will. Denkt man sieh die Erde gerade in der Bahnebene eines solchen Systems und nimmt zunäehst zwei gleich grosse und gleieh helle Sterne an, die sich in einer kreisförmigen Bahu umeinander bewegen, so wird die Gesammthelligkeit des Systems zu den Zeiten der genauen Bedeckung gerade halb so gross sein als zu den Zeiten, wo die Verbindungslinie der beiden Componenten senkrecht zum Visionsradius ist und das Licht derselben sich addirt. Während einer ganzen Umdrehung des Systems finden in diesem Falle zwei gleieh grosse Helligkeitsmaxima und Helligkeitsminima statt. Haben die Componenten nicht die gleiche Leuehtkraft, so modificiren siek die Erseheinungen in der Weise, dass beim Voriibergange des weniger lenchtenden Körpers vor dem helleren ein Hauptminimum, dagegen nach einer weiteren halben Rotation ein Nebenminimum stattfindet; die beiden Helligkeitsmaxima bleiben gleich. Die Liehteurve eines derartigen Systems wïrde vollkommen mit der beobachteten Liehteurve von $\beta$ Lyrae übereinstimmen. Ist die Erde nicht genau in der Bahnebene, so dass keine eentrale Bedeekung eintreten kann, so werden die Helligkeitsunterschiede zwischen Maximum und Minimum in Allgemeinen geringer sein, und weitere Modifieationen der Liehterseheinungen werden in dem Falle bedingt werden, wenn die Bahn der beiden Himmelskörper eine elliptisehe ist, deren grosse Axe irgendwie gregen den Visionsradius geriehtet ist. Nimmt man endlich noch statt eines doppelten ein dreifaches oder mehrfaches System an, wo wiederholte Bedeckungen stattfinden und ausserdem leicht Störungen der Bewegung rorkommen kïmnen, so werden Ungleichmässigkeiten der Liehteurve und der Periodendaner die unvermeidliehe Folge sein.

Man sieht also, dass fast alle photometrischen Erseheinungen, die wir an den Stcrnen der vierten Piekering'sehen Classe beobachten, so leieht und ungezwungen dureh die V'erdeckungshypothese erklärt werden kömnen, dass man ihr unbedenklich ror allen anderen den Vorzug geben wird, falls sie nicht mit anderen als photometrischen Beobachtungsthatsichen im Widerspruche ist. Hierbei ist in erster Linie an die Ergebnisse der spectralanalytischen Forschung zu denken, welche ein so mächtiger Bundesgenosse der Photometrie geworden ist. Einige der wiehtigsten Resultate, 
hinweg, im anderen dagegen nach der Erde hin. Im Gegensatze hierzu findet er ans den Messungen der dunklen Linien während der ganzen Periode des Lichtwechsels die Verschiebungen nach einer und derselben Seite des Spectrums hin (nach Violett). Das Verhalten der dunklen Linien wiirde also auf eine stetige Bewegung des Systems nach der Erde hin schliessen lassen, während das Verhalten der hellen Linien damit im Widerspruche steht. Es ist hier nicht der Ort, näher auf diese Ergebnisse einzugehen, es geniigt hervorzuheben, dass dieselben zwar auf Berregungen innerhalb eines zweifachen oder mehrfachen Sternsystems mit Sicherheit hinweisen, dass es aber zunächst noch nicht gelingen will, die spectroskopischen und photometrischen Erscheinungen durch eine einheitliche Annahme iiber die Constitution und die Berregung eines solches Systems zu erklären.

Ansser $\beta$ Lyrae ist noch ein anderer Veränderlicher der vierten Pickering'schen Gruppe, $\delta$ Cephei, in neuerer Zeit von Belopolsky 1) spectroskopisch untersucht worden. Das Spectrum dieses Sternes unterscheidet sieh wesentlich ron demjenigen ron $\beta$ Lyrae, indem es keine hellen Linien anfweist, sondern fast rollkommen dem Sonnenspectrum ähnlich ist. Bemerkenswerthe Verïnderungen im Anssehen des Spectrums sind in Zusammenhange mit dem Lichtweehsel nicht zu constatiren, nur scheint in Allgemeinen die Intensitit des continuirlichen Spectrums zur Zeit des Maximums grösser zn sein als zu den iibrigen Zeiten. Die Ausmessungen der Spectrogramme zeigen Verschiebungen der dunklen Linien gegen die Linien des irdischen Vergleichspeetrums, diese Verschiebungen sind aber nicht während der ganzen Liehtperiode constant. Man hat es hier zweifellos mit einem Doppelsternsysteme zu thun, dessen Componeten ausser einer gemeinschaftlichen Translation im Raume Bewegungen un den Schwerpunkt des Systems ansfiuhren. Lnter der Annahme, dass die Visionsrichtung nahezu in die Bahnebene des Srstems fällt und dass die Umdrehungszeit gleich der Liehtperiode ist, hat Belopolsky aus den semessenen Geschwindigkeiten im Tisionsradius die Bahn des hypothetisehen Doppelsternsystems nach den ron Lehmann-Filhés²) entrickelten Formeln berechnet und gefunden, dass die Excentricitiat nicht unbetrïchtlich ist, und dass der Periastrondurehgang etwa einen Tag nach dem Helligkeitsminimum stattfindet. Diese Resultate sind zunächst als provisorische anzusehen und bediurten noch der Bestätigung durch weitere spectroskopische Beobachtungen; Vielleicht lissen sich dann die Veränderungen im spectrum in noch besseren Einklang mit den photometrischen

Bull. le lAcad. Imp. des sciences de St.-Pétersb. V. Sér. t. 2 1594, p. 267.

$\because$ Astr. Nachr. Bd. 13t, Nr. 324.2. 
Erscheinungen bringen. Auch an den ubrigen Verïnderliehen der vierten Grnppe sind speetroskopiselie Untersuchungen dringend erwilnselst. Die bisherigen 'Versuche sind an der geringen Liehtstiirke der meisten von ihnen geseheitert, und man weiss daher von ihmen nicht viel mehr, als dass ihre Spectra, dom allgemeinen Anblicke nach, eher mit dem Spectrum von $\delta$ Cephei, als mit demjenigen von $\beta$ Lyrae ubereinzustimmen scheinen.

\section{e. Die Vexinderlichen rom Algol-Typus.}

Verhailtnissmïssig am Schärfsten abgegrenzt gegen die Ubrigen Gruppen der Veränderlichen ist die letzte Pickering'sehe Classe, zn welcher diejenigen Stene gezihlt werden, bei denen uur imerhalb kurzer periodisch wiederkehrender Zeitriiume Lichtinderungen vor sich gehen, wiihrend sie sonst constante Helligkeit besitzen. Diese Classe hat ihren Namen nach dem Sterne \& Persei oder Algol, dessen Lichtweehsel unter allen Veränderlichen bisher wohl am Sorgfältigsten und kifrigsten studirt worden ist. Seine Variabilitiit wirde schon 1667 oder 1669 von Montanari benerkt, aber erst Goodricke stellte 1782 die besondere Art der Lichtanderungen fest, die daun durch zahlreiche andere Beobachter, insbesondere durch Sehönfeld, Pickering, Scheincr und Chandler anf das Genameste ermittelt wurden. Der Stern hat gewöhnlich die Grijsse 2.3 ; dann beginnt er plötzlich abzunchmen und erreicht in $4^{\mathrm{h}} 37^{\mathrm{m}} .5$ die Grösse 3.5, wiichst dam wieder in etwa derselben Zeit bis zur Anfangshelligkeit 2.3 und bleibt in derselben wïhrend $2^{\text {' }} 11^{\mathrm{b}} 33^{\mathrm{m}}$, worauf $\mathrm{ron}$ Nenem ganz in der gleichen Weise die Lichtiuderungen beginnen. Die, Daner der Periode, d. h. die Zeit zwisehen zwei anfeinander folgenden Minimis, ist in den letzten Jahrzehnten wiederholt sehr sicher bestimnt worden, und es hat sich herausgestellt, dass dieselbe siicularen Schwankungen unterworfen ist. Nach den Angaben Chandlers1), welcher in nenerer Zeit fast das gesammte Beobachtungsmaterial iiber Algol bearbeitet hat, betrug die Periode zil Goodriekes Keiten etwa $2^{4} 20^{\mathrm{h}} 48^{\mathrm{m}}$;5:0. Sie wuchs mit kleinen unregelmäissigen Schwankungen bis $z u 2^{\mathrm{d}} 200^{\mathrm{L}} 48^{\mathrm{m}} 59.2$ im Jahre 1830, simk dimn inn Jahre 1855 bis $22^{\mathrm{d}} 20^{\mathrm{h}} 45^{\mathrm{m}} 52.8$ herah und erreichte, nach einem nochmaligen geringen Anwachsen, im . Jalure 1 s77 den Werth $2^{\mathrm{d}} 20^{\mathrm{h}} 4 \mathrm{~S}^{\mathrm{m}} 51 \mathrm{~s}$, , weleler sich lange \%eit constant grehalten hat. gegenwärtig aber wieder zuzmehmen scheint. Zur Berechmung der Minimaepochen hat Chandler ans seinen Untersuchungen die foligende Formel abgeleitet:

1) Astr. Journ. Vol. T, Nr. 165-16i. 
1888 Jan. 3, $7^{\mathrm{h}} 21^{\mathrm{m}} 29 \mathrm{~s} 23$ (Mittl. Z: Greenw.) $+2^{\mathrm{d}} 20^{\mathrm{h}} 48^{\mathrm{m}} 55.425 E$

$+173^{\mathrm{m}} 3 \sin \left(\frac{1}{50} E+202^{\circ} 30^{\prime}\right)+18^{\mathrm{m}} 0 \sin \left(\frac{3}{40} E+203^{\circ} 15^{\prime}\right)$

$+3^{\mathrm{m}} 5 \sin \left(\frac{1}{6} E+90^{\circ} 20^{\prime}\right)$

worin $E$ die Anzahl der Perioden bedeutet, welche seit der gewählten Anfangsepoche verflossen sind.

Die Lichtciurve Algols (Fig. 81) hat eine sehr einfache und regelmässige Form. Manche Beobachter haben zwar Abweichungen von der regelmässigen Form der Lichtcurve sowohl beim absteigenden als aufsteigenden Zweige derselben vermuthet, andere wollen auch während der Zeit des vollen Lichtes kleine Schwankungen der Helligkeit bemerkt haben; doch sind alle diese Angaben keineswegs als verbürgt zu betrachten, weil sie

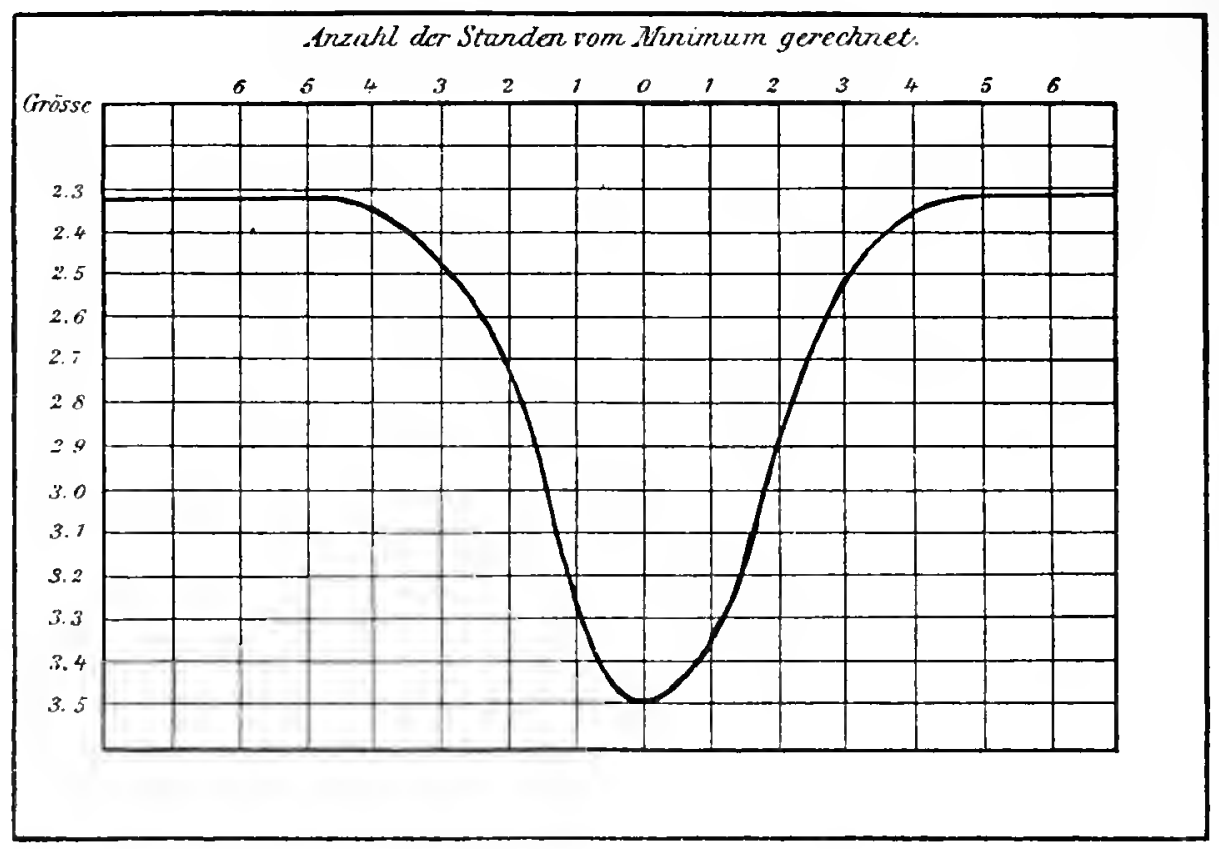

Fig. 81. Lichteurve ron $p$ Persei.

fast ansschliesslich anf Beobachtnngen nach der Stufenschätzungsmethode beruhen, bei der gerade in diesen Fïllen die Gefahr der Voreingenommenheit sehr gross ist. Nur sorgfiltige photometrische Messungen, an denen es bisher leider fehlt, können uns vollkommene Gewissheit über etwaige Einbiegungen der Lichteurve verschaffen.

Die ubrigen Sterne der Algolgruppe zeigen im Allgemeinen einen ihnlichen Charakter des Lichtweehsels wie Algol; nur sind bei den meisten rom ihnen Ungleichmaissigkeiten mit Sicherheit constatirt, und insbesondere stellt es fest, dass hei einigen die Lielitabnahme in kiirzerer Zeit elfolgt als die Lichtzunahme. Man kennt gegenwärtig erst 14 Sterne, die mit einiser Bestimmtheit zu dieser Classe von Verinderlichen zn rechmen sind. Näclist $\beta$ Persei sind am Lïngsten bekannt $\lambda$ Tanri und $S$ Caneri, 
die im Jahre 1848 entdeckt wurden, und von denen der erstere die grüssten, noch nieht zur Genllge erforseliten Unregelmässigkeiten anfweist. Die Periodendaner ist bei den 14 Sternen ansserordentlich versehieden;

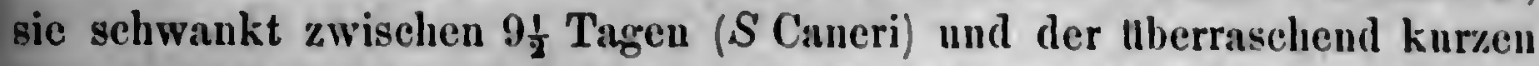
Zeit ron 7.8 Stunden (S Antliae). Das Verhibltniss des Zeitraumes, innerhalb desseu die Liehtvariationen vor sich gehen, zur ganzen Periodendamer ist ebenfalls bei den einzelnen Steruen selır versehieden; es schwankt ungefilhr zwischen $\frac{1}{2}$ und $\frac{1}{T}$. Zweifellos existiren am Himmel noch viele Verinderliche vom Algoltypus; doch ist ilıre Auffindung nugemein schwierig und nur durch glileklichen Zufall möglich.

Besonderes Interesse verdienen zwei Vertreter dieser Gruppe, Y Cygni und $Z$ Hereulis, weil sie eine doppelte Periode besitzen. Geht man näimlich von einer bestimmten Minimumepoche aus, so ergiebt sich das Zeitintervall zwischeu einer geraden und der folgenden ungeraden Epoehe merklich verschieden von dem Intervalle wwisehen einer ungeraden und der niichstfolgenden geraden Epoche, während die Hauptperiode, d. h. die Zwischenzeit zwisehen zwei benachbarten geraden oder zwei benachbarten ungeraden Minimis nahezu constant ist. Nach den Untersuehungen von Dunér') sind bei $Y$ Cygni die beiden Theilperioden gleich $1^{\mathrm{d}} 10^{\mathrm{b}} 11^{\mathrm{nt}} 10^{\mathrm{s}}$ und gleich $1^{\mathrm{d}} 13^{\mathrm{b}} 43^{\mathrm{m}} 43^{\mathrm{s}}$, also die Hauptperiode gleich $2^{\mathrm{d}} 23^{\mathrm{h}} 54^{\mathrm{m}} 53^{\mathrm{g}}$.

Bei $Z$ Herculis sind die Bestimmungen noch etwas unsicher. Die Theilperioden ergeben sich fiir 1895 ungefïhr $z 1^{\mathrm{d}} 22^{\mathrm{h}} 49^{\mathrm{m}}$ und $\% 2^{\mathrm{ll}} 0^{\mathrm{h}} 59^{\mathrm{m}}$, die Hauptperiode etwa $\% u \quad 3^{\mathrm{d}} 23^{\mathrm{h}} 48^{\mathrm{m}} 20^{\mathrm{s}}$. Bei diesem Sterne ist anch ein nicht unmerklieher Helligkeitsunterschied zwisehen den greraden und ungeraden Minimis eonstatirt.

Ansser den beiden eben erwihnten Sternen zeigt noch ein anderer Vertreter der Algolgruppe, $U$ Cephei, gewissermassen eine doppelte Lichteurve, indem die Minimalhelligkeit der geraden Epoehen stets un einige Zehntel Grössenclassen von derjenigen der ungreraden Epochen verschieden ist. Der Charakter der doppelten Periode ist aber nicht so schilrf ausgeprägt wie bei $Y$ Cygni ind $Z$ Herculis, da die Epochen der Nebenminima fast genan in die Mitte der Hauptepochen fallen.

In der folgenden Tabelle sind noch die wichtigsten Daten für die jetzt einigermassen sicher bekannten Algolsterne zusammengestellt; sie sind geordnet nach der Linge der Periode. Ausser der Position fiir 1900 ist die Maximal- und Minimalhelligkeit, die Periodenlainge, die Daner der Lichtänderungen und der Nane des Entdeckers mit Hinzufiigung des

1) Öfversigt af K. Svenska Vetensk.-Akad. Fürhand!. 1\$92, p. 325. - Ausserdem Astrophys. Journ. Vol. I, p. 255. 
Jahres der Entdeckung angegeben. Bei $Y$ Cygni und $Z$ Herculis ist nur die Länge der Hauptperiode verzeichnet.

\begin{tabular}{|c|c|c|c|c|c|c|c|c|c|}
\hline \multirow{2}{*}{ Name } & \multicolumn{4}{|c|}{ Position für 1900} & \multicolumn{2}{|c|}{$\begin{array}{l}\text { Helligkeit } \\
\text { im }\end{array}$} & \multirow{2}{*}{$\begin{array}{l}\text { Länge } \\
\text { der } \\
\text { Periode }\end{array}$} & \multirow{2}{*}{$\begin{array}{c}\text { Dauer } \\
\text { der } \\
\text { Licht- } \\
\text { ânde- } \\
\text { rang }\end{array}$} & \multirow{2}{*}{ Entdecker } \\
\hline & & A. $\mathrm{F}$ & & Decl. & $\operatorname{Max}$. & Min. & & & \\
\hline S Caneri. . & & 38 & n14s & $\left|+19^{\circ} 23.6\right|$ & 8.2 & 9.8 & $9^{\mathrm{d}} 11^{\mathrm{b}} 37^{\mathrm{m}} 45^{\mathrm{s}}$ & $21 \div 5$ & Hind, 1848 \\
\hline S Velortmm. & & 29 & 27 & $|-44 \quad 45.9|$ & 7.8 & 9.3 & 5222421 & 15 & Woods, 1894 \\
\hline W Delphini . & 20 & 33 & 7 & +1755.9 & 9.3 & 12.0 & $\begin{array}{llll}4 & 19 & 21 & 11\end{array}$ & 14 & Miss Wells, 1895 \\
\hline Z Herculis. . & 17 & 53 & 36 & +158.8 & 7.1 & 8.0 & $\begin{array}{llll}3 & 23 & 49 & 30\end{array}$ & 5 & $\begin{array}{l}\text { Hartwig, Chand } \\
\text { ler, 1894 }\end{array}$ \\
\hline >. Tanri . . & & $\check{55}$ & & +1212.5 & 3.4 & 4.2 & $\begin{array}{llll}3 & 22 & 52 & 12\end{array}$ & 10 & Baxendell, 1848 \\
\hline$U$ Coronae. & 15 & 14 & 7 & +320.8 & 7.5 & 8.9 & $\begin{array}{llll}3 & 10 & 51 & 12.4\end{array}$ & 10 & Winnecke, 1869 \\
\hline I Cygni. . & 20 & 48 & 4 & +3417.0 & 7.1 & 7.9 & $22354 \quad 43$ & 8 & Chandler, 1886 \\
\hline$\beta$ Persei & & 1 & 40 & +4034.2 & 2.3 & 3.5 & $\begin{array}{llll}2 & 20 & 48 & 55.425\end{array}$ & 9 & Montanari, 1669 \\
\hline$U$ Cephei . . & 0 & 53 & 23 & +8120.2 & 7.1 & 9.2 & $\begin{array}{llll}2 & 11 & 49 & 38.25\end{array}$ & 10 & Ceraski, 1880 \\
\hline$R S$ Sagittarii & 18 & 10 & 59 & $\left|\begin{array}{ll}-34 & 8.5\end{array}\right|$ & 6.4 & 7.5 & $\begin{array}{lll}9 & 59 & 24\end{array}$ & 13 & $\begin{array}{l}\text { Gould, } 1874 ; \text { Ro } \\
\text { berts, } 1895\end{array}$ \\
\hline$\delta$ Librae. & 14 & 55 & 38 & $-\begin{array}{ll}- & 7.3\end{array}$ & 5.0 & 6.2 & 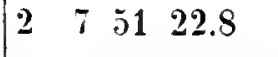 & 12 & Schmidt, 1859 \\
\hline$R$ Canis majoris & 7 & 14 & 56 & $-16 \quad 12.4$ & 5.9 & 6.7 & $\begin{array}{llll}1 & 3 & 15 & 46.0\end{array}$ & 5 & Sawyer, 1Ss7 \\
\hline$U$ Ophinchi . & 17 & 11 & 27 & +119.3 & 6.0 & 6.7 & $\begin{array}{llll}0 & 20 & 7 & 42.56\end{array}$ & 5 & $\begin{array}{l}\text { Gould, 1871; } \\
\text { Sawyer, is81 }\end{array}$ \\
\hline$S$ Antliae & 9 & 27 & 56 & $|-28 \quad 11.2|$ & 6.7 & 7.3 & 74645.0 & 3.5 & Paul, 18ss \\
\hline
\end{tabular}

Sämmtliche Sterne haben eine weisse oder gelblichweisse Farbe, und ihre Spectra, soweit sie bekannt sind, gehören dem ersten Spectraltypus an.

Man hat friiher versucht, die Lichterscheinumgen der Algolsterne durch Axendrehung dieser Himmelskörper bei ungleichartiger Oberflïchenbeschaffenheit zu erklïren, und noch im Jahre 1891 hat Bruns') nachgewiesen, dass unter dieser Ammahme bei passender Wahl der Bestimmungsstiicke anf theoretischem Wege eine Lichteurse abgeleitet werden kann, die sich dem bei den Algolsternen beobachteten Helligkeitsverlanfe durchans befricdigend anschliesst. Indessen ist diese Theorie, sowie alle anderen Erklärungsversuche, gegenwärtig vollstiindig rerdrängt durch die Verdeckungshyothese. Piekering 2 hatte bereits im Jahre 1 s\$0 eine Dentung der Lichtrerhailtnisse bei Algol durch die Annahme rou zwei Sternen versucht, von denen der grïssere hellere durch einen weniger lenchtenden Begleiter, der sich in einer Kreisbahn nu den ersteren bewegt, zeitweilig" hedeckt wird. Ans der Periodendaner, dem Helligkeitsmuterschiede zwischen Maximmm nud Minimum und den beobachteten Momenten von Anfang: Mitte und Ende des eigentlichen Lichtwechsels hatte Pickering sogar

1) Monatsber. A. K. Preuss. Akad. d. Wiss. Berlin. 1s51, p. 4 .

2 Proc of the Amer. Acad of arts and sciences. New series. Vol. s, p. 1. 
eine provisorische Berechnung der Bahu dieses hypothetischen Doppelsternsystems unternommen. Eine weseutliche Stutze hat diese Hypothese seitdem iurch die spectroskopischen Beobachtungen Vogels') erhalten. Aus diesen geht hervor, dass die Linien im Algolspectrum gegen die Linien eines irdischen Vergleichspectrums zeitweilig nach der einen und reitweilig nach der anderen Seite versehoben sind; es folgt also nach dem DopplerFizean'schen Principe, dass der Stern, weleher das Spectrum giebt, sich bald von der Erde hinweg, bald auf dieselbe zu bewegt. Durch sorgfiltige Ausmessung der Verschiebungen hat sich ergeben, dass etwa 1 'hug und 10 Stunden lang vor einem Helligkeitsminimun eine Bewegung von uns hinweg, dagegen in dem gleichen Zeitranme nach dem Minimun eine Bewegung auf uns $\%$ stattfindet. Dadurch ist aber die Existen\% \%weier um einen gemeinschaftlichen Schwerpmukt rotirenden Körper, von denen der eine relativ dunkel ist, so gut wie \%weifellos festgestellt. Die Vereinigung der photometrischen und spectroskopischen Ergebnisse gestattet unn eine viel genauere Bestimmung der Bahn dieses Systems, als die Verwerthung der Helligkeitsmessungen allein. Die photometrischen Beobachtungen lieferu die Umlaufszcit und die ungeführen Dimensionen der heiden Körper (letztere aus der Zwischenzeit zwischen den Punkten der lichteurve, wo die Krimmung merklich zu werden anfïngt); die spectroskopischen Beobachtungen geben die im Visionsradius gelegene Gesch windigkeitscomponente. Unter Voraussetzung einer kreisförmigen Bahn, deren Ebene nur sehr weuig gergen die Gesichtslinie geneigt ist, so latss die Bedecknng nahezu central ist, ferner unter der Annahme grleicher Dichtigkeit beider Körper hat Vogel die folgenden Werthe berechnet:

Durchmesser des hellen Hauptsternes

$=1707000 \mathrm{~K} l m$.

Durchmesser des relativ dumklen Abstand der Mittelpunkte

Bahngeschwindigkeit des Hauptsterues

Bahngeschwindigkeit des Begleiters

Masse des Hauptstermes

Masse des Begleiters

$$
\begin{aligned}
& =13360000 \text { \% } \\
& =5194000 \text {, } \\
& =42 \% 1 \text { in der } \\
& =\quad 55 \text {. I Secunde. } \\
& =\text {; vionnen111sse }
\end{aligned}
$$$$
=\frac{1}{1}
$$

Von den übrigen Algolsternen liegen bisher wegren der uperingen Liehtstiirke der meisten keine zurerlissigen spectroskopischen lieobiehtungen ror, aus denen mit derselhen Sicherheit wie bei Algol die Bahnbewerungen und die Beselatfenheit des Systems berechnet werden kïmten. Indessen dräugt sich bei dem analogen Verlaufe der Lichterscheinungen der Gedanke an Bedeckungen so selbstrerstindlich auf. dass man hentzutage

1) Publ. des Astrophys. Obs. zu Potsdam. Bd. T, Theil I, p. 111. 
kaum einer anderen Hypothese Berechtigung zuerkennen wird. Alle $\mathrm{Ab}$ weichungen von der regelmässigen Algolcurve lassen sich ohne Weiteres durch plansible Annahmen über die relativen Helligkeiten der Componenten, iiber die Lage der Bahnebene zum Visionsradius, über die Excentricität der Bahu n. s. w. erklären, und auch Erscheinungen, wie sie z. B. die beiden Sterne $Y$ Cygni und $Z$ Herculis bieten, finden ungezwungene Erklärung. Nach Dunér besteht das System $Y$ Cygni aus zwei Sternen von gleicher Grösse und gleicher Helligkeit, die sich in $2^{\mathrm{d}} 23^{\mathrm{h}} 54^{\mathrm{m}} 43^{\mathrm{s}} .26$ um einander in einer elliptischen Bahn bewegen, deren Ebene durch die Sonne geht, und deren Apsidenlinie einen gewissen Winkel mit dem Visionsradius bildet. Noch genauere Angaben macht Dunér über $Z$ Herculis. Von den beiden gleich grossen Componenten dieses Systems ist die eine doppelt so hell als die andere; die Ebene der elliptischen Bahn geht ebenfalls durch die Sonne, die Excentricität ist 0.2475 , und die Apsidenlinie bildet einen Winkel von $4^{\circ}$ mit dem Visionsradius. Die Umdrehungszeit ist $3^{\mathrm{d}} 23^{\mathrm{h}} 48^{\mathrm{m}} 30^{\mathrm{s}}$.

Das Hypothetische, welches diesen Untersuchungen gegenwärtig noch anhaftet, wird zweifellos bald rerschwinden, sobald es gelungen ist, mit den mächtigsteu optischen Hiilfsmitteln der Neuzeit auch die schwächeren Algolsterne in den Bereich exacter spectrographischer Messungen zu ziehen.

\section{Die spectralphotometrischen Beobachtungen der Fixsterne.}

Bei der Besprechung der gebränchlichsten Formen der Spectralphotometer ist bereits auf die Wichtigkeit der Anwendung dieser'Art von Instrumenten für die Photometrie der Himmelskörper hingewiesen worden. Dadurch dass anstatt des Gesammtlichtes die einzelnen Strahlengattungen miteinander verglichen werden, ist es möglich, einen Überblick iiber die verschiedene Znsammensetzung des Lichtes der Gestirme und damit bis zu einem gewissen Grade auch iiber die verschiedenen Entwicklungsstadien derselben zu gewinnen. Die grossen Schwierigkeiten, die bei der directen Vergleichung verschiedenfarbiger Sterne aus physiologischen Griinden anftreten, kommen bei der spectralphotometrischen Methode gar niclit in Betracht. Die Helligkeit eines Gestirnes, bezogen auf eine bestimmte Lichtquelle als Einheit, wird bei dieser Methode nicht durch eine einzige Zahl, sondern durch eine Reihe ron Zablen, giiltig fuir die verschiedenen mutersuchten Stellen des Spectrums, ansgedriickt. Je mehr einzelne Speetralhezirke rerolichen werden, desto klarer wird das Bild, welches wir von den Lichtrerhältuissen der Sterne erhalten, nnd wemn der Zusammen- 
hang zwisehen Intensität und Wellenlïnge fir eine genligende Anzahl von Punkten ermittelt ist, so lissst sich, wie hereits im Capitel ther die'Spectralphotometer angegeben ist, rechungsmissig die gesammte imnerhalb) der sielitbaren Grenzen des Speetrums enthaltene Liehtquantitait bestimmen. Es ist auf diese Weise möglich, die Ergehnisse der directen Messungen der Gesammthelligkeit zu eontroliren und zu ergäinzen.

So deutlich aber aneh die Vortheile der spectralphotometrischen Methode vor Augen liegen, so ist doch die Auwendung derselben auf die Fixsterne in der Praxis mit sehr. grossen Schwierigkeiten verbunden. Die Spectra derselben erscheinen bei Anwendung eines Refractors und eines damit verbundenen Spectralphotometers als schmale Linien, dic an sich schou, ansser bei den allerhellsten Sternen, wenig liehtstark sind, aber noch viel schwächer werden, wenn man zum Zweeke der Verbreiterung derselben eine Cylinderlinse benutz. Will man daher schwichere Sterne in den Bereich der Untersuchung ziehen, so sind ziemlich bedentende instrumentelle Hiilfsmittel erforderlich. Lin Übelstand ist ferner, dass das verbreiterte Fixsternspectrum infolge der chromatischen Abweichung des Fernrohrobjectirs nicht in seiner ganzen Ausdehmung von parallelen geraden Linien begrenzt ist. In den einzelnen Spectralbezirken ist also das Lieht auf einen mehr oder weniger breiten Streifen zusammengedräugt, wïhrend das Spectrum der kilnstlichen Liehtquelle, welche man bei den meisten Spectralphotometern zm Vergleichung benut\%t, als gleichmaissigg breiter Streifen erscheint. Da es sich nun mu Flichenhelligkeiten landelt, so ist es zur Erlangungr vergleichbarer Resultate erforderlieh, die versehierlene Breite des Stemspectrums in Rechunng zn ziehen. Dieser Üluelstand würde wegfallen, wenn mam anstatt eines Refrautors ein Spiegelteleskoj, henutate, bei welchem alle Strahlen in einem Brennpmute rereinigrt werd(n.

Es ist ferner zu bedenken, dass die nit verschiedenen Apparaten gefundenen Resultate nicht ohne Weiteres untereinander vergleichlyar sintl. Je nach den bemutaten Prismen indert sich die Dispersion im spectrmu, und damit andert sich anch die Fliichenhelligkeit fü einen hestimmten Wellenlängenbezirk. Es ist also erwinscht, die unemessenen Lntensitäten in der Weise, wie es fribler Seite 270, eribtert worlen ist, anf dias Normalspectrum zu redneiren. Die Benutzmng ron l)illiactionsuttern, bei denen diese Rednction iiherflissin sein wiirle. empfiehlt sich wegen der geringeren Lichtstiirke nicht.

Zu diesen Schwierigkeiten komnt noch himzn. diss bei nielit gan\% regehmässig functionirendem Lhrwerke das Sternlyild wihrend der baner der Messung nicht leicht auf dem Spalte zn halten ist; selhst wenn dies aber dureh Benutzung eines Jeiffermrohrs erevelit werden kilm, so bewirkt doch die muermeidliche Luftumbule. dass das stemspectrmu rom 
zahlreichen hin- und herschwankenden dunklen Längsstrichen durchzogen erscheint und ein wesentlich anderes Aussehen hat, als das ruhige Vergleichslichtspectrum. Sehr störend sind endlich noch, namentlich bei den Steruen vom dritten Spectraltypus, die Absorptionslinien im Spectrum. Um diese zu beseitigen, muss der Spalt verhältnissmässig weit geöffnet werden, und dies hat wieder den Nachtheil im Gefolge, dass die Farben unrein werden und die Vergleichung dadurch erschwert wird.

In vollem Umfange ist die Methode bisher nur von H. C. Vogel') angewandt worden, aber auch nur an einer geringen Zahl der allerhellsten Fixsterne. Zur Benutzung kam dabei ein Glan-Vogel'sches Spectralphotometer, wclches, mit dem grossen Refractor des Potsdamer Observatoriums verbunden wurde. Als Vergleichslicht diente eine am Apparate aufgehängte Petroleumlampe. Die Verbreiterung der Sternspectren geschalı nicht mit Hülfe einer Cylinderlinse, sondern einfach dadurch, dass der Spalt des Spectroskops etwas ansserhall des Focus des Fernrohrobjectivs gestellt wurde. Die Messungen wurden an sieben verschiedenen Stellen des Spectrums ausgefiihrt; sie sind aber nicht auf das Normalspectrun reducirt, sondern nur wegen der verschiedenen Breite des Spectrums corrigirt. Die in der folgenden Tabelle mitgetheilten Resultate sind daher nur untereinander vergleichbar, Ausser den sechs untersuchten Stermen, die absichtlich ans verschiedenen Spectraltypen ausgewählt sind, ist in der Zusammonstellung noch die Sonne angefiihrt. Die Zahlen geben das Helligkeitsverhältniss des Petroleumspectrums zu den Spectren der einzelnen Himmelskörper fiir die verschiedenen Wellenlïngen an, wobei durchgängig der Werth fuir die Wellenlïnge $555 \mu \mu$ gleich 100 gesetzt worden ist.

\begin{tabular}{|c|c|c|c|c|c|c|c|}
\hline \multirow{2}{*}{ Wellenlänge } & \multicolumn{7}{|c|}{ Helligkeitsverhăltniss des Vergleichslichtes zum Stern } \\
\hline & a Can. maj. & a Lyrae & a Aurigie & "Buotis & $\alpha$ 'Timri & a Oxionis & Sonne \\
\hline $633 \mu \mu$ & 285 & 270 & 232 & 200 & 218 & 202 & 232 \\
\hline 600 & 200 & 191 & 173 & 153 & 159 & 153 & 175 \\
\hline 555 & 100 & 100 & 100 & 100 & 100 & 100 & 100 \\
\hline 517 & 49 & 50 & 46 & 71 & 70 & 61 & 52 \\
\hline 496 & 24 & 27 & 20 & 57 & 53 & 47 & 27 \\
\hline 464 & 14 & 16 & 14 & 50 & 48 & 39 & 15 \\
\hline 444 & 11 & 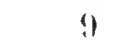 & 12 & 46 & 11 & 32 & 11 \\
\hline
\end{tabular}

Wenn diese Zahlen anch kanm eine grössere Genanigkeit als höchstens 5 I'rocent besitzen werden, so geht ans der Znsammenstellung doch deutlich hervor, wieriel mal heller die weissen Sterne in den brechbareren 
Theilen des Spectruns sind als die gelbliehen und rothen. Ferner ergiebt sich, dass die Intensitiitsvertheilung im Somenspectrum fust vollständig mit derjenigen im Spectrum von a Aurigae Ibereinstimut; mau wird also schliessen durfen, dass die Sterne von diesen Typus sich in ihnlielıem Glihzustande befinden wie die Sonne, wilirend die Temperatur der weissen Sterne weit Ilber, die T'mperatur der rothen Sterne weit unter der Temperatur der Sonue gelegen ist.

Bei dem grossen Interesse, welches der Gegenstand im Hinblick auf die Entwicklungsgeschiehte der Fixsterne hat, wäre eine Fortfihrung der Vogel'schen Untersuchungen in grösserem Massstabe durchaus erwlinselit. Bisher scheint die Schwierigkeit der Beobachtungen von weiteren Versuchen abgeschreckt zu haben.

In gewissem Zusammenhange damit stelit eine ganz eigenartige Photometrie der Fixsteme, welehe Pickeringl) in seinem sDraper Cataloge in Anwendnng gebracht hat, und welehe sieh ebenfalls anf die Speetra der Sterne gründet. Obgleieh diese anf photographischem Wege ausgetuhrten Helligkeitsbestimmungen insofern keine vollstiindigen spectralphotometrisehen Angaben liefern, weil die Vergleichungen nur an einer einzigen Stelle des Speetrums gemaeht sind, und obgleich ausserdem Vieles gegen die Methode und die Art der Bearbeitung einzuwenden ist, so verdient das Unternehmen doch hier erwähnt \%u werden, schon deshalb, weil es sich anf mehr als 10000 Sterne erstreckt und in Verbindung mit anderen photometrischen Bestimmungen in Zukmuft noch ron grossem Nutzen sein kann. Der Halut\%weck der Pickering'schen Arbeit war eine Classificinung der Spectren der Steme his etwat zur sichenten Grösse vom Nordpol bis zur Declination - 25". Die sipectra wurden mittelst eines Objectivprismas ron $20 \mathrm{~cm}$ Öffiung und 13" hrechendem Winkel erhalten, welehes vor einem parallaktisch montirten Voigtlinderschen Objective ron $20 \mathrm{~cm}$ Durchmesser und 115 cm Focallinge so angebracht war, dass die brechende Kante parallel der tiiglichen Bewegung stand. Dadureh dass dem Uhrwerk eine etwas andere Geschwindigkeit als nach Sternzeit ertheilt wurde, bewegte sich dis Spectrum langsim tiber die photographische Platte und erschien daher ein wenig verbreitert. Bei einer durchsehnittlichen Expositionszeit vol is Minuten fìr Äquatorsterne hatten die spectral anf der Platte cine Jäinge von 1 cm und eine Breite von etwa $1 \mathrm{~mm}$. Zur Bestimmung" der Helligkeiten der Spectren bediente sich Piekering eines photographischen Streifens, welcher durch kiinstliches Lielit hervorgehracht wall und an dem rinen bude ganz dunkel, an den anderen Ende vollkmumen durchsichtig crschien,

1) Annals of the Astr. Obs. of Harvard College. Vol. 2ti, part. I and Vol. 27. 
ähnlich wie ein keilförmig geschliffenes Stück dunklen Glases. Dieser Vergleichsstreifen wurde durch ein Verfahren erhalten, welches mit dem friiher beschriebenen Janssen'schen (Seite 299) eine gewisse Ähnliehkeit hat. Die Helligkeitsabstufung des Streifens liess sich durch Reehuung ermitteln, und eine daran angebrachte Scala gab fiir jeden Punkt desselben die Intensitiit unmittelbar in Sterngrössenelassen an, bezogen auf einen beliebigen Ausgangspunkt. Dieser photographische Massstab wurde nun neben die aufgenommenen Sternspectra, aus denen durch ein Diaphragma stets ein bestimmter Theil herausgeblendet war, gelegt und dann diejenige Stelle des Streifeus aufgesucht, wo die Dichtigkeit des Silberniedersehlages die gleiche zu sein sehien, wie anf dem herausgeblendeten Bezirke des Sternspectrums. Die Ablesungen an der Seala gaben danu fuir zwei verschiedene Sterne ummittelbar den Helligkeitsunterschied in Grössenclassen, griltig für die untersuchte Stelle des Spectrums. Sämmtliche Messungen wurden nur au einem einzigen Punkte in der Nähe der Wellenlïnge 432 "u ausgefiuhrt; es stiude aber natürlich Nichts in Wege, die Vergleichungen auf beliebig viele Stellen des Spectrums auszudehnen.

Die Einheit, anf welche die so erhaltenen Spectralhelligkeiten bezogen werden, kaun willkiirlieh gewiihlt werden; Pickering hat aber, um die Angaben mit den optischen Helligkeiten der Sterne in einen gewissen Znsammenhang zu bringen, das folgende Verfahren eingeschlagen. Auf jeder Platte, welche gewöhnlich eine beträchtliche Anzahl ron Speetren enthielt, wurden diejenigen Sterne aufgesucht, deren Spectrum dem ersten Typus angehörte, und fur welche in der Harvard Photometry die optische Helligkeit angegeben ist. Mit Zugrundelegung dieser Helligkeitswerthe wurde nun ans den photometrischen Spectralmessungen der betreffenden Sterne fiur jede Platte eine Constante berechnet, und mit Hïlfe dieser Constante wurden dann die endgiiltigen Helligkeiten für sïmmtliche Sterne des Draper Cataloges abgeleitet. Das System schliesst sich natiulich mu für die Sterne rom ersten Tylus an das Srstem der Harrard Photometry an; fïr alle anderen Sterne sind die Grössen des Draper Cataloges nicht direet mit den optischen Grössen vergleichbar. Zwei Sterne, die in diesem Cataloge als gleich hell bezeichnet sind, können, wemn sie rerschiedenen Typen angeliören, in der Harvard Photometry nmm mehr als zwei Grössen roneinander verschieden sein.

so interescant in mancher Hinsicht das Pickering sche Verfahreu ist, anf welches hier nicht nïher eingeginngen werden kann, so wird doch schwerlich eine Photometrie, die sich mur auf Vergleichnngen in einer cinzigen Strahlengattung grindet, allgemeinen Anklang finden. Erst wenn der Tersuch pemacht wiirde. die spectralphotometrischen Messungen ïher mighlichst viche stellen des Spertrums ausudehnen und daraus das 
Gesanmmtlicht der Sterne "u bereehnen, wltrde der Weg \%u einer durchaus rationellen und einwurfsfreien l'hotometrie der Fixsterne angebahnt sein. Die Hindernisse, die sich jeder photographischen Lichtmessung entgregenstellen, treten auch bei der Piekering'sehen Methode in rollem Umfange auf. Die Genanigkeit der Resultate steht entschieden hinter der bei optischen Messungen erreichbaren zurtlek. Insbesondere ist die Bestimmung der photographischen Vergleichsscala ein heikler Punkt, abgesehen vou allen anderen Schwierigkeiten, die dureh die :iusseren Unstände, namentlich dureh die Unrnhe der Luft, die Absorption in der Atmosphäre, die versehiedene Empfindlichkeit der benutaten Platten u. s. w. herbeigefuhrt werden.

\section{Die photographischen Helligkeiten der Fixsterne.}

Nachdem in letzten Capitel des zweiten Abschnittes die photogratphischen Methoden zur Bestimmung des Gesammitlichtes der Gestirne bereits so ansfuhtlich, als es in diesem Buche winschenswerth schien, besprochen worden sind, brancht hier nur noeh kur\% anf die wichtigsten Ergebnisse dieser Methoden an Fixsternhimmel hingewiesen zu werden. Die grossen Hoffinngen; die man im rorigen Jalurzehnt atuf die lintwicklung der photographischen Photometrie gesetzt hatte, sind freilich nicht in Erflilhng gegangen, und es ist gregenwärtig sogar ein Stillstand in den Bemühungen auf diesen Gebiete eingetreten. Aber der Hanptvortheil, der bei der photographisehen Methode darin liegt, dass man in verhältnissmässig sehr ku\%er Zeit vou einer betrichtlichen Anzahl von Sternen Aufnahmen erhält, die zur Bestinmmug der llelligkeit in aller Bequemlichkeit ansgemessen werden kömen, ist so in die Angen fillend, dass es verfehlt sein wiirde, von weiteren Versuchen alyzustehen, selhst wenn es nicht gelingen sollte, alle im Frihheren erwïhnten schwierimkeiten zu iberwinden und die Genanigkeit so weit zu treiben, als man anfangs erwartet hatte. Dass die photographisele Photometrie der Fixsteme sich jemals der optiselh-physiologischen so weit iiluerlewen zeigen sollte, dass man auf die letatere ginzlich vergichten kiomte, ist durchans unwahrseheinlich; dagegen werlen die photographischen llelligkeitshestimmungen immer eine sehr willkommene lirainzung der bisherigen physiologisehen Resultate bilden; fuir yewisse specielle dufgaben wird man unbedenklich schon hente eine answedehntere Anwendung der Photographie empfehlen kïmnen.

Nan hat sich bisher davor gehiitet, eine besondere Intensitiitsscala fuir die photographisehen Helligkeiten einzufiihren. Lis ist viehnehr der Begrifl" 
der Sterngrösse zunächst streng festgehalten und ein möglichst enger Anschluss an die optische Grössenscala erstrebt worden. Solange die photographische Photometrie noch nicht auf geniigend sicherem Fundamente ruht, mag eine derartige Verbindung das Beste sein; aber, da nun doch einmal wegen der verschiedenartigen Zusammensetzung des Fixsternlichtes eine directe Vergleichung der photographischen und optischen Helligkeiten für alle Sterne unmöglich ist, so wird man in Zukunft schwerlich auf einen allzu engen Anschluss Gewicht legen und eventuell nicht davor zuriickschrecken, die photographisehen Grössen vollkommen unabhängig zu máchen. Gegenwärtig benutzt man gewöhnlich die weissen, dem ersten Spectraltypus angehörigen Sterne als Verbindung:sglieder und bestimmt aus ihnen, wie bereits friiher anseinandergesetzt ist, die Constanten der Formeln, welche zur Ableitung der photographischen Grössen dienen. Damit ist allerdings erreicht, dass wegen des Überwiegens des ersten Spectraltypus für den grössten Theil der Sterne am Himmel die photographischen Helligkeitsangaben mit den gebrïuchlichen Cataloghelligkeiten direct vergleichbar sind; aber für die Sterne der anderen Spectraltypen, die weniger reich an photographisch wirksamen Strahlen sind, geben die photographischen Bestimmungen verhältnissmässig zu kleine Helligkeiten. Man kann im Durchschnitt annehmen, dass ein Stern rom III. Typus photographisch um etwa 2.5 Grössenclassen schwächer gemessen wird als optisch. Die blosse photographische Helligkeitsangabe fuir irgend einen Stern giebt uns also nur damn eine ungefähre Vorstellung von der physiologischen Helligkeit desselben, wemn gleichzeitig anch sein Spectraltypus bekannt ist.

Die bisherigen Arbeiten anf rem Gebiete der photographischen Fixsternphotometrie sind zum grössten Theile nur als Vorversuche zu betrachten; sie waren in erster Linie dazn bestimmt, die verschiedenen Arten der Ausmessung zu erproben und iiber die wichtigsten principiellen Fragen Klarheit zn schaffen. Von zusammenhängenden Messungsreihen, die einen ungefïhren Überblick iiber die zu erreichende Genanigkeit geben könnten, sind eigentlich nur die Ansmessung der Plejadengruppe ron Charlier') nnd die von Pickering? reröffentlichten photographischen Helligkeitsrerzeichnisse hervorzuheben.

Charlier hat sich bei seiner Irbeit der Methode der Durchmesserbestimmmg der photographischen sternscheibchen bedient, die zweifellos ror allen anderen Methoden den Vorzug verdient. Das ron ihm bentzte chemisch achromatisirte Objectir ron $\$ 1 \mathrm{~mm}$ Öfr̈mug und $100 \mathrm{~cm}$ Brennweite war an dem Stockholmer Refractor befestigt, nud die Aufnahmen

1. Publ. der Astron. Gesellschaft, Nr. 19.

"- Aunals of the Astr. Obs. of Harvard College. Vol. 1s, No. VII, p. 119. 
nmfussten einen Raum von etwa 20 Quadratgraden an Himmel. Den Untersnchungen z.n Grunde gelegt wurlen vier Platten, die bei Expositionszeiten zwischen 13 Minuten und 3 Stunden anfgenommen waren. Auf jeder derselben wurden die Durchmesser von 52 der Bessel'sehen Plejadensterne sorgfiltig ausgemessen, und mit Zugrundelegung der von Lindemann flir diese Sterne photometriseh bestimmten Helligkeiten wurden die Constanten der Formel $m=a-b \log D$ berechnet, wo $m$ die photographisehe Grösse und $D$ den gemessenen Durehmesser des Sternseheibchens reprisentirt. Zur Ableitung des Helligkeitscataloges der Plejadengruppe wurde nur die am Laingsten exponirte Platte benutzt. Anf dieser wurden im Ganzen 364 Sternseheibchen ausgemessen und daraus die Intensititen mittelst der betreffeuden Formel bestimmt, ausserdem wurden noch von 168 sehwiicheren Sternen der Platte die photographisehen Helligkeiten angenähert durch Sehäitzungen des Schwärzmngsgrades erhalten. Die Vergleichung der vier Charlier'sehen Plejadenanfuahmen gestattet ein Urtheil uber die bei dieser Methode der Helligkeitsbestimmung zu erreichende Genauigkeit. Dasselbe ist ausserordentlich guinstig. Grössere Abweichungen \%wischen den vier Bestimmungen eines Sternes als 0.4 Grössenclassen kommen nieht vor, und der wahrseheinliche Fehler einer einzelnen photographischen Helligkeit ergiebt sich im Durchsehnitte zu \pm 0.10 Grössenclassen; die Genanigkeit bleibt also nir sehr wenig hinter den besten photometrisehen Messungen zurlick. Die Ausmessung der Plejadengruppe ist freilich insofern eine der günstigsten Aufgaben fiur die photographische Photometrie, als die sämmtlichen $z u$ vergleichenden Steme anf derselben Platte enthalten sind, welehe auch gleichzeitig geniigend zablreiche zur Constantenbestimmung geeignete Anhaltsterne anfweist. Dalum werden vou romherein die bedenkliehsten Schwierigkeiten, die bei der photographischen Methode anftreten, beseitigt; insbesondere kommt die rerschiedene Empfindlichkeit versehiedener Platten gar nicht und der Einfluss der Extinction nur versehwindend wenig in Betracht; die iiberans grefiihrliche Einwirknng der Unruhe der Luft trifłt alle zu vergleichenden Stemhildehen in demselben Grade. Endlich ist eine Vergleichung der photographischen Resultate mit den optiseh photometrischen gerade hei der Plejarlengmppe ausserordentlich giinstig, weil fast alle Stemr dieser fruple den ersten Spectraltypus angehören. Alles in Allem hetrachlet wird man den Charlier'sehen Helligkeitscatalog der Plejaden unbedenklich fiir cinen äusserst werthrollen Beitrag zur Fixstemphotometrie erkliiren dirfen. Auf ahnliche Aufgaben, insliesondere auf die Ansmessung noch dichterer Sterngruppen, die den optisehen Helligkeitshestimmungen grosse Sehwierigkeiten bereiten, sollte die photographische l'hotometric klinftig in erster Linie ihr Angenmerk richten. 
Bei Weitem weniger günstig lautet das Urtheil über die Pickeringschen photographischen Helligkeitsverzeichnisse. Von diesen bezieht sich das eine ebenfalls auf die Plejadengruppe, und die ron Charlier angestellte Vergleichung zwischen seineu und den Pickering'schen Resultaten lïsst einen systematischen Gang erkennen, der zweifellos dem Pickeringschen Verfahren zur Last zu legen ist. Das zweite Verzeichniss umfasst alle Sterne (1009 an der Zahl) heller als fünfzehnter Grösse, die nicht weiter als $1^{\circ}$ vom Nordpol abstehen. Ein dritter Catalog endlich enthält 1131 Sterne zwischen der dritten und neunten Grösse innerhalb des schmalen Gürtels ron $-2^{\circ}$ bis $+2^{\circ}$ Declination. Die benutzten 'Aufuahmen sind zum Theil mit beweǵtem, zum grössten Theil mit ruhendem Fermrohr gemacht worden; die Helligkeiten sind also in der iiberwiegenden Zahl durch Vergleichung der von den Sternen auf der photographischen Platte beschriebenen Striche (trails) abgeleitet worden. Steht diese Methode, wie friiher betont worden ist, an und für sich schon hinter der Methode der Durchmesserbestimmung der Sternscheibchen zuriick, so ist auch in mancher anderen Hinsicht das ganze Verfahren Pickerings keineswegs nachahmenswerth. Er bediente sich wieder, wie bei den spectralphotometrischen Messungen des Draper Catalogs, einer photographisehen Scala, die er in der Weise herstelite, dass er ron einem bestimmten Sterne eine Reihe ron gleich langen Aufnahmen unter Anwendung ron rerschiedenen Blenden ror dem Objectir machte. Die Blendenüffnungen waren so abgemessen, dass das Verhältniss je zweier anfeinander folgenden freien Objectirflaichen gleich 2.5 war. Unter der Annahme, dass das Abblendungsprincip durchaus einwurfsfrei ist, wiirde der Unterschied in dem Aussehen zweier aufeinander folgenden Striche der Seala einem Helligkeitsunterschiede ron einer Grössenclasse entsprechen. Pickering hat die ron dem gewälhlten Vergleichsterne beschriebenen Striche ans der photographischen Platte herausgeschnitten und benutzt dieselben als feste Scalen zur Teroleichung mit allen anderen Strichanfuahmen, wobei noeh Zehntel der einzelnen Intervalle geschiitzt werden. Abgesehen ron der verhiltnissmissig grossen Unsieherheit dieser Schiitzungen ist die Abblendungsmethode ans rersehiedenen Griinden, insbesondere wegen des schidlichen Einflusses der Bengung bei sebr kleinen Öfrnungen, entschieden zu rerwerfen. Nicht viel besser ist das Verfahren, welches Pickerins bei der Terwerthung der mit bewegtem Fermrohr gemachten Sternscheibchenanfuahmen angewendet hat. Anch hier ist eine feste Scala beuntzt worden, bestehend ans einer Reihe ron Bildern eines bestimmten Sternes ant derselben Platte, die bei verschiedenen Exponsitionszeiten erhalten waren. Die Expositionszeiten waren so gewählt, dass der Lnterschied zwischen je zwei aufeinander folgenden Jildr'n der Seala wieder einer Intensitiitsindermg rou einer ganzen 
Grössenclasse entsprechen sollte. Zehntel des Intervalles wurden dureh Schätzung erhalten. Da der Zusammenhang zwischen der Dichtigkeit des Silberniedersehlages und der Expositionszeit keineswegs durch ein einfaches Geset\% ausgedrickt werden kann, sondern empirisch ermittelt werden muss, so ist die Herstellung der festen Vergleichsseala eine der schwierigsten Anfgaben bei diesem Verfahren. Um von den Fehlern der Seala einigermassen frei $\mathbf{z}$ werden, hat Pickering auf jeder Platte noch eine Gruppe von Polsternen aufgenommen, deren Helligkeiten mit dem Meridianphotometer nnd einem Keilphotometer bestimmt waren, und die zur Frmittlung von Rednetionsgrössen fur jede Platte dienen sollten. Danit ist zugleich anch der Anschlnss der photographischen Helligkeiten an das System der Harvard Photometry, den Pickering bei allen seinen photographischen Helligkeitsverzeichnissen streng festhält, erreicht.

Die Hauptsehwierigkeiten, die sich einer allgemeinen photographischen Fixsternphotometric entgegenstellen, sind in den eben besprochenen Vorarbeiten nicht beseitigt, vielmehr erst in ihrem vollen Unfange erkannt worden, und es bleibt der Zukunft tiberlassen, inwieweit es gelingen wird, dieser Schwierigkeiten Herr zu werden und die bisherigen unvollkommenen Methoden durch einwurfsfieiere zu ersetzen.

Ein specielles Gebiet der Fixsternphotometrie, wo auch schon jetzt die Photographie mit Aussicht auf ginstigen Erfolg angewendet werden könnte, umfasst die rerïnderlichen Sterne, speeiell diejenigen von kurzer Periode. Es ist leicht möglich, auf ein und derselben l'latte eine grosse Anzahl von Aufnahmen eines Verïnderlichen, alle von gleicher Expositionsdauer, nahe nebeneinander zn machen. Der Vortheil, den dieses Verfahren namentlich für die Sterne vom Algoltypus gewïhren kamn, wo es auf die Festlegung mögliehst vieler Punkte der Liehteurve ankommt, liegt auf der Hand. Zur Ableitung der Helligkeiten sollte dabei ausschliesslich die Methode der Bilddurehmesserbestimmung benutzt werden, und ferner sollte es Regel sein, nur Differenzmessungen gegen einen benachbarten Stern, von denen wohl stets einer auf der Platte vorhanden sein wird, zu verwerthen. Es wird keine Selwwierigkeiten bereiten, die zugehörigen Sternbildehen auf der Platte zu unterscheiden. Die Differenzmessung macht den Einfluss der Extinetion fast granz unschaidlieh, und anch die wechsehnde Luftunrulıe, einer der greführlichsten Feinde der photographischen Photometrie, kommt dabei nicht in Betracht. Handelt es sich nur um die Festlegrung der Epoehen der Minima oder Maxima und um die Bestimmung der blossen Form der Lichteurve, so geben die gemessenen Unterschiede zwischen den Bilddurchmessern des Veräinderlichen und des benutzten Vergleichsternes, gan\% gleichyiiltig, in welcher Einheit sie ausgedriickt sind, alles erforderliche Material. Bei der 
Anwendung dieser Methode auf die Algolsterne wird es am Ehesten möglich sein, kleine Einbiegungen -und Unregelmässigkeiten der Lichtcurven, die bei optisch photometrischen Bestimmungen der Aufmerksamkeit leichter entgehen können, zu entdecken; auch sind alle Täuschungen, die durch etwaige Voreingenommenheit des Beobachters veranlasst sein könnten, von vornherein ausgeschlossen. Schon hente kanu man mit Sicherheit behaupten, dass anf diesem beschränkten Gebiete die photographische Photometrie der optischen zum Mindesten ebenbiirtig ist.

Handelt es sich nicht nur um die Form der Lichtcurve, sondern soll der Betrag der Helligkeitsschwankung in einem bestimmten Masse angegeben werden, so ist eine Constantenbestimmung für jede Platte erforderlich, und es treten danu sofort alle im Friiheren erwähnten Schwierigkeiten auf. Am Besten wird man in diesem Falle noch zum Ziele kommen, wenn mehrere dem Veränderlichen nahe stehenden Sterne von verschiedener Helligkeit mit auf der Platte enthalten sind. Eine sorgfältige Bestimmung der photometrischen Grössen derselben ermöglicht dann den Anschluss der photographischen Helligkeitsscala an das iibliche optische System.

Bisher sind nur an Algol photographische Helligkeitsbestimmungen versucht worden, und zwar von Charlier') und Townley ${ }^{2}$. Die Urtheile beider Beobachter weichen wesentlich voneinander ab. Waihrend Charlier der Meinung ist, dass unter giinstigen atmosphärischen Bedingungen durch die photographische Methode weit bessere Resultate erreicht werden können, als durch die optische Methode, erkennt Townley keineswegs die Überlegenheit der Photographie an und hebt die Schwierigkeiten, die sich dabei entgegenstellen, nachdriicklich hervor. Freilich ist das von Letzterem eingeschlagene Verfahren, bei welchem der Einfluss der atmosphäirischen Extinction sowie der Unruhe der Luft sich in rollem Grade geltend machen, durchans zu verwerfen. Das unginstige Urtheil sollte daher aneh anf keinen Fall von weiteren Bemiihnngen, die Photographie für die Helligkeitsbestimmnngen der Verïnderlichen nutzbar zu machen, abschrecken.

1) Bihang till K. Svenska Vetensk.-Akad. Handlingar. Bd. 18, Afd. I, No. 3.

2) Publ. of the Astr. Soc of the Pacific. Vol. 6, 1594, p. 199. 


\section{ANHANG.}

\section{Tafel der nach den Theorien von Lambert, Lommel-Seeliger und Ruler berechneten, vom Phasenwinkel abhångigen Reductionen auf volle Beleuchtung eines Planeten.}

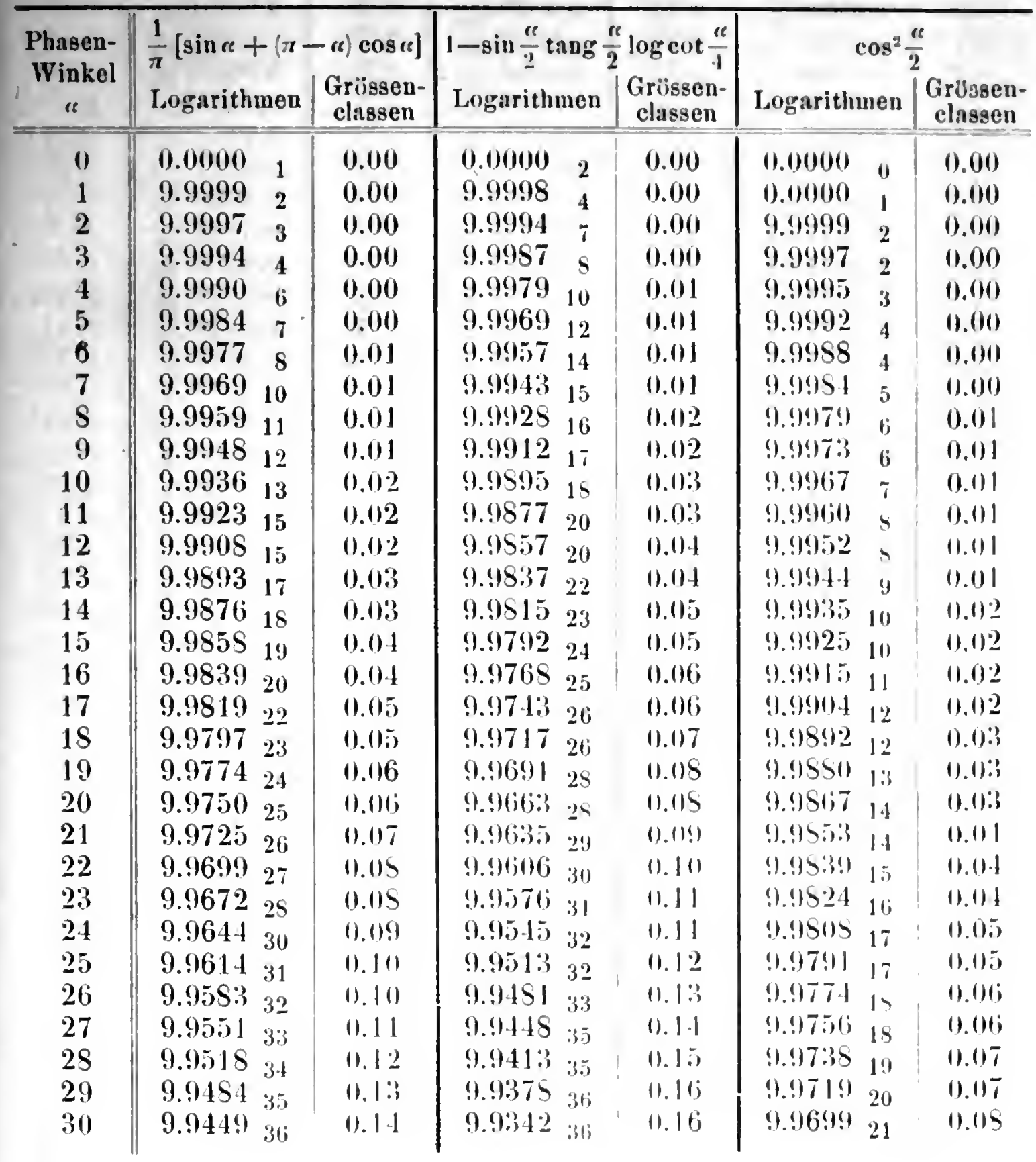




\begin{tabular}{|c|c|c|c|c|c|c|}
\hline $\begin{array}{c}\text { Phasen- } \\
\text { Winkel } \\
a\end{array}$ & $\begin{array}{l}\frac{1}{\pi}[\sin \alpha+(\pi- \\
\text { Logarithmen }\end{array}$ & $\begin{array}{c}-(c) \cos (c] \\
\text { Grüssen- } \\
\text { classen }\end{array}$ & $\begin{array}{l}1-\sin \frac{\alpha}{2} \text { tang } \\
\text { Logarithmen }\end{array}$ & $\begin{array}{c}\log \cot \frac{\alpha}{4} \\
\text { Grössen- } \\
\text { classen }\end{array}$ & $\begin{array}{r}\cos ^{2} \\
\text { Logarithmen }\end{array}$ & $\begin{array}{l}\frac{\ell}{2} \\
\begin{array}{c}\text { Grössen- } \\
\text { classen }\end{array}\end{array}$ \\
\hline 31 & $9.9413 \quad 38$ & 0.15 & $9.9306 \quad 38$ & 0.17 & 9.967821 & 0.08 \\
\hline 32 & $9.9375 \quad 39$ & 0.16 & $9.9268 \quad 38$ & 0.18 & $9.9657_{22}$ & 0.09 \\
\hline 33 & $9.9336 \quad 40$ & 0.17 & $9.9230 \quad 39$ & 0.19 & $9.9635 \quad 22$ & 0.09 \\
\hline 34 & $9.9296 \quad 41$ & 0.18 & 9.919140 & 0.20 & 9.961324 & 0.10 \\
\hline 35 & $9.9255 \quad 42$ & 0.19 & 9.915140 & 0.21 & 9.958925 & 0.10 \\
\hline 36 & 9.9213 .43 & 0.20 & $9.9111_{41}^{4}$ & 0.22 & 9.956425 & 0.11 \\
\hline 37 & $9.9170^{45}$ & 0.21 & $\begin{array}{l}9.9070 \quad 42 \\
\end{array}$ & 0.23 & $\begin{array}{l}9.9539 \quad 26 \\
26\end{array}$ & 0.12 \\
\hline 38 & $9.9125 \quad 45$ & 0.22 & $9.9028 \quad 42$ & 0.24 & 9.951326 & 0.12 \\
\hline 39 & $9.9080 \quad 47$ & 0.23 & $9.8986 \quad 43$ & 0.25 & $9.9487_{27}$ & 0.13 \\
\hline 40 & 9.903345 & 0.24 & $9.8943 \quad 44$ & 0.26 & $9.9460 \quad 28$ & 0.13 \\
\hline 41 & $9.5985 \quad 49$ & 0.25 & $9.8899 \quad 45$ & 0.28 & $9.9432 \quad 29$ & 0.14 \\
\hline 42 & 9.893650 & 0.27 & 9.885445 & 0.29 & $9.9403 \quad 30$ & 0.15 \\
\hline 43 & $9.8886 \quad 52$ & 0.28 & $9.8809 \quad 46$ & 0.30 & 9.937330 & 0.16 \\
\hline 44 & $9 . \$ 834 \quad 52$ & 0.29 & 9.576347 & 0.31 & $9.9343 \quad 31$ & 0.16 \\
\hline 45 & $9 . \$ 782 \quad 54$ & 0.30 & 9.871648 & 0.32 & $9.9312 \quad 32$ & 0.17 \\
\hline 46 & $9 . \$ 72855$ & 0.32 & 9.8668 4s & 0.33 & $\begin{array}{l}9.9280 \\
32\end{array}$ & $0.1 \mathrm{~s}$ \\
\hline 47 & $9.5673 \quad 56$ & 0.33 & $9 . \$ 620 \quad 49$ & 0.34 & $9.9248 \quad 33$ & $0: 19$ \\
\hline 18 & $9 . \$ 61757$ & 0.35 & 9.557150 & 0.36 & $9.9215 \quad 34$ & 0.20 \\
\hline 49 & $9.55 \% 60 \quad 59$ & 0.36 & 9.852151 & 0.37 & $9.91 \$ 1 \quad 35$ & 0.20 \\
\hline 50 & 9.550160 & 0.37 & $9.8+7051$ & 0.38 & $9.9146 \quad 36$ & 0.21 \\
\hline 51 & $9 . \$ 44161$ & 0.39 & $9.5419 \quad 52$ & 0.40 & $9.9110 \quad 37$ & 0.22 \\
\hline 52 & $9 . \$ 380 \quad 62$ & 0.40 & $9 . \$ 367 \quad 53$ & 0.41 & $9.9073 \quad 37$ & 0.23 \\
\hline 53 & 9.831864 & 0.42 & 9.531454 & 0.42 & 9.9036 38 & 0.24 \\
\hline 54 & $9 . \$ 25465$ & 0.44 & $9.5260 \quad 54$ & 0.43 & $9.8998 \quad 39$ & 0.25 \\
\hline 55 & 9.518966 & 0.45 & 9.820655 & 0.45 & $9.5959 \quad 40$ & 0.26 \\
\hline 56 & $9 . \$ 12367$ & 0.47 & $9 . \$ 151 \quad 56$ & 0.46 & $9 . \$ 919 \quad 41$ & 0.27 \\
\hline 57 & $9.8056 \quad 69$ & 0.49 & $9.5095 \quad 57$ & 0.48 & $9.557 S_{41}$ & $0.2 S$ \\
\hline $5 S$ & $9.7957 \quad 70$ & 0.50 & 9.503557 & 0.49 & $9.8837 \quad 43$ & 0.29 \\
\hline 59 & $9.7917 \quad 71$ & 0.52 & 9.7981 55 & 0.50 & $9 . \$ 794 \quad 43$ & $0.3^{0}$ \\
\hline 60 & $9.78+6 \quad 72$ & 0.54 & $9.7923 \quad 59$ & 0.52 & 9.575145 & 0.31 \\
\hline 61 & 9.777474 & 11.56 & $9.786 \pm 60$ & 0.53 & $9.5706 \quad 45$ & 0.32 \\
\hline 62 & 9.770075 & 0.57 & 9.750461 & 0.55 & $9.8661 \quad 46$ & 0.33 \\
\hline 6.3 & $9.7625 \div$ & 11.59 & $9.77+361$ & 0.56 & $9 . \$ 615 \quad 4$ & 0.35 \\
\hline 61 & $9.75+8$ is & 11.61 & 9.7652 & $0.5 \mathrm{~S}$ & $9 . \$ 565 \pm 8$ & 0.36 \\
\hline 6.5 & $9.7470 \quad 79$ & 0.63 & 9.761963 & 0.611 & $9.8520 \quad 49$ & 0.37 \\
\hline $64 ;$ & 9.739181 & 11.65 & 9.755664 & 0.61 & $9.5+7150$ & 0.35 \\
\hline 67 & $9.7311 \mathrm{~s} 2$ & 0.67 & 9.749265 & 0.63 & 9.542150 & 0.39 \\
\hline $6 \mathrm{~S}$ & $0.722 S \$ 4$ & 11.69 & $9.7427 \quad 66$ & $0.6 t$ & $9 . \$ 37152$ & 0.41 \\
\hline 69 & $0.7144 \$ 5$ & 11.71 & 9.736166 & 0.66 & 9.531952 & 0.42 \\
\hline 70 & $9.7059 \quad 56$ & 11.74 & $9.7295 \quad 68$ & (1). $6 \mathrm{~S}$ & $9 . \$ 267_{54}$ & 0.43 \\
\hline 71 & 1.6973 ss & 0.76 & 9.722765 & 0.69 & 9.521354 & 0.45 \\
\hline 72 & 9.1 is5. 59 & 11.75 & $9.7159 \quad 69$ & 0.71 & $9 . \$ 159 \quad 56$ & 0.46 \\
\hline $7:$ & 9.679691 & 0.50 & 9.71191170 & 0.73 & $9 . \$ 10356$ & 0.47 \\
\hline $7 t$ & $9.67(1592$ & 0.52 & $9.70211-11$ & $0.7 t$ & 9.504757 & 0.49 \\
\hline 75 & 9.661391 & 11.55 & $9.69+972$ & 11.76 & $9.7990 \quad 59$ & 0.511 \\
\hline
\end{tabular}




\begin{tabular}{|c|c|c|c|c|c|c|c|c|c|}
\hline $\begin{array}{c}\text { Phasen- } \\
\text { Winkel } \\
\text { " }\end{array}$ & $\begin{array}{l}\frac{1}{\pi}[\sin r+ \\
\text { Logarithn }\end{array}$ & $\begin{array}{l}+\{x- \\
\text { men }\end{array}$ & $\begin{array}{l}-(8) \cos r \text { ] } \\
\text { Grisssen- } \\
\text { clnssen }\end{array}$ & $\begin{array}{l}1-\sin \frac{\pi}{2} t \\
\text { Logarith }\end{array}$ & & $\begin{array}{l}\log \cot \frac{1 !}{1} \\
\text { Grüssen- } \\
\text { clnssen }\end{array}$ & I.ogarithn & $\begin{array}{l}\cos ^{2} \frac{18}{2} \\
\text { ment }\end{array}$ & $\begin{array}{l}\text { Griissen- } \\
\text { clnssen }\end{array}$ \\
\hline 76 & 9.6519 & 96 & 0.87 & 9.6877 & 73 & 0.78 & 9.7931 & 60 & 0.52 \\
\hline 77 & 9.6423 & 97 & 0.89 & $9.65(1)$ & 74 & 0.50 & 9.7871 & 61 & 0.53 \\
\hline 79 & 9.6326 & !9 & 0.92 & 9.6730 & 75 & 0.82 & 9.7510 & 62 & 0.65 \\
\hline 79 & 9.6227 , & 100 & 0.94 & 9.6655 & 76 & 0.84 & 9.77 .15 & 63 & $11 . \overline{6} 6$ \\
\hline S1) & 9.6127 & 102 & 0.97 & 9.6579 & it & 0.56 & 9.7655 & 64 & $0 . \overline{5}$ \\
\hline$\$ 1$ & 9.6025 & 104 & 0.99 & 9.6502 & is & 0.87 & 9.7621 & $6 i t$ & 0.59 \\
\hline$\$ 2$ & 9.5921 & 105 & 1.02 & 9.6424 & in & 0.59 & $9.755 \%$ & 66 & $0.6 i 1$ \\
\hline S.3 & $9.5 S 16$ & 107 & 1.05 & 9.6345 & $s_{0}$ & 0.91 & 9.7159 & 65 & 0.63 \\
\hline S4 & 9.5709 & $10 !$ & 1.07 & 9.6265 & 81 & 0.93 & 9.7 .121 & (6) & 0.64 \\
\hline 55 & 9.5600 & 110 & 1.10 & 9.6154 & 83 & 0.95 & 9.7352 & 70 & 0.66 \\
\hline S6 & 9.5490 & 113 & 1.13 & 9.6101 & S3 & 0.97 & 9.7252 & I & $0.6 \mathrm{~S}$ \\
\hline 87 & |9.5377 & 114 & 1.16 & 9.6018 & 85 & 1.00 & 9.7211 & 72 & 0.70 \\
\hline SS & $9 . j 263$ & 116 & $1.1 \mathrm{~S}$ & 9.5933 & $\mathrm{~s} 6$ & 1.02 & 9.7139 & 74 & 0.72 \\
\hline S9 & 9.5147 & 118 & 1.21 & $9.5 \$ 47$ & 87 & 1.04 & 9.7065 & 76 & 0.73 \\
\hline 90 & 9.5029 & 121 & 1.24 & 9.5760 & 88 & 1.06 & 9.6959 & 76 & 0.75 \\
\hline 91 & 9.4905 & 121 & 1.27 & 9.5672 & \$9 & 1.08 & 9.6913 & $\pi s$ & 0.77 \\
\hline 92 & 9.4787 & 124 & 1.30 & 9.5583 & 90 & 1.10 & 9.6535 & 79 & 11.79 \\
\hline 93 & 9.4663 & 126 & 1.33 & 9.5493 & 92 & 1.13 & 9.6756 & $\$ 1$ & 11.51 \\
\hline 94 & 9.4537 & 128 & 1.37 & 9.5401 & 93 & 1.15 & $9.6(575)$ & 82 & 0.83 \\
\hline 95 & 9.4409 & 131 & 1.40 & 9.5309 & 95 & 1.17 & 9.6593 & $8: 3$ & 0.55 \\
\hline 96 & 9.4278 & 132 & 1.43 & 9.5213 & 96 & 1.20 & 9.6510 & 55 & 0.57 \\
\hline 97 & 9.4146 & 135 & 1.46 & 9.5117 & 97 & 1.22 & 9.6 .125 & 56 & 11.59 \\
\hline 95 & 9.4011 & 137 & 1.50 & 9.5020 & !s & 1.25 & 9.633339 & ss & 0.92 \\
\hline 99 & 9.3874 & 139 & 1.53 & 9.4922 & 100 & 1.27 & $9.6 i 251$ & 90 & 0.94 \\
\hline 100 & 9.3735 & 142 & 1.57 & 9.4822 & 102 & 1.29 & $9.616 i 1$ & $! 1$ & 10.916 \\
\hline 101 & 9.3593 & 144 & 1.60 & 9.4720 & 103 & 1.32 & $9.1 \mathrm{i} 0711$ & 93 & 0.15 \\
\hline 102 & 9.3449 & 146 & 1.64 & 9.4617 & 105 & 1.35 & ד & 14 & 1.111 \\
\hline 103 & 9.3303 & 149 & 1.67 & 9.4512 & 106 & 1.37 & 4.5553 & 96 & 1.113 \\
\hline 104 & 9.3154 & 152 & 1.71 & 9.4406 & 106 & 1.40 & 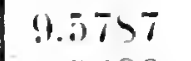 & is & 1.115 \\
\hline 105 & 9.30112 & 154 & 1.75 & 9.42 .95 & 109 & $1.4: 3$ & !.:̈tis? & $100)$ & 1.115 \\
\hline 106 & 9.2848 & 157 & 1.79 & 9.4159 & 111 & 1.45 & $(1.5 \% 49$ & 102 & 1.10 \\
\hline 107 & 9.2691 & 159 & 1.53 & 9.4075 & 113 & $1.4 \mathrm{~S}$ & 9.5187 & $10: 3$ & 1.13 \\
\hline 108 & 9.2532 & 163 & 1.57 & 9.3965 & 115 & $\mid .51$ & $9.534+1$ & 106 & 1.16 \\
\hline 109 & 9.2369 & 165 & 1.91 & 9.3550 & 116 & $1 . \overline{1} 1$ & 9.5275 & 107 & 1.18 \\
\hline 110 & 9.2204 & 169 & $1.9 \%$ & $9.37: 3.1$ & 119 & 1.57 & 9.5171 & $10 !$ & 1.21 \\
\hline 111 & 9.2035 & 171 & 1.99 & 9.3615 & 120 & 1.60 & 0.501192 & 111 & 1.23 \\
\hline 112 & 9.1864 & $1 \% 4$ & 2.113 & 9.8495 & 123 & $1.6: 1$ & $9.4 ! 101$ & 11.1 & $1.26 i$ \\
\hline 113 & 9.1690 & $17 s$ & 2.05 & 9.3372 & 124 & 1.66 & $(0.44: 37$ & 115 & $1.2 !$ \\
\hline 114 & 9.1512 & $1 \leqslant 1$ & 2.12 & 9.3245 & 127 & 1.69 & 0.4722 & 115 & 1.32 \\
\hline 115 & 9.1331 & 154 & 2.17 & 9.3121 & 125 & 1.72 & $9.16(1) .1$ & 120 & 1.35 \\
\hline 116 & 9.1147 & 165 & 2.21 & 9.29913 & 131 & 1.75 & 0.4191 & 122 & 1.35 \\
\hline 117 & 9.0959 & 191 & 2.26 & 9.2562 & 133 & $1.7 s$ & 0.43612 & 125 & 1.41 \\
\hline $11 \mathrm{~s}$ & 9.0765 & 195 & 2.31 & 9.2729 & 135 & 1.92 & 9.1237 & 125 & 1.44 \\
\hline 119 & 9.0573 & 199 & $2.36 j$ & 9.2594 & 138 & 1.5 & $9 .+109$ & 130 & 1.47 \\
\hline 120 & $9.037+$ & 203 & $2 .+11$ & $9.2+56$ & 141 & 1.49 & 9.3979 & $1: 32$ & 1.51 \\
\hline
\end{tabular}




\begin{tabular}{|c|c|c|c|c|c|c|}
\hline $\begin{array}{c}\text { Phasen- } \\
\text { Winkel } \\
\alpha\end{array}$ & $\begin{array}{l}\frac{1}{\pi}[\sin r+(\pi) \\
\text { Logarithmen }\end{array}$ & $\begin{array}{c}\text { - c) } \cos (t] \\
\text { Grössen- } \\
\text { classen } \\
\end{array}$ & $\begin{array}{l}1-\sin \frac{\alpha}{2} \text { tang } \\
\text { Logarithmen }\end{array}$ & $\begin{array}{l}\log \cot \frac{\ell}{4} \\
\begin{array}{l}\text { Grössen- } \\
\text { classen }\end{array}\end{array}$ & Logarithmen & $\begin{array}{l}\frac{2}{\text { Grössen- }} \\
\text { classen }\end{array}$ \\
\hline 121 & 9.0171207 & 2.46 & $9.2315 \quad 143$ & 1.92 & $9.3847 \quad 136$ & 1.54 \\
\hline 122 & $8.9964_{211}$ & 2.51 & $9.2172 \quad 146$ & 1.96 & $\begin{array}{l}9.3711 \quad 138 \\
0\end{array}$ & 1.57 \\
\hline 123 & $8.9753 \quad 215$ & 2.56 & $\begin{array}{ll}9.2026 & 149\end{array}$ & 1.99 & $9.3573 \quad 141$ & 1.61 \\
\hline 124 & $8.9538 \quad 219$ & 2.62 & $9.1577 \quad 152$ & 2.03 & $9.3432 \quad 144$ & 1.64 \\
\hline 125 & $8.9319 \quad 224$ & 2.67 & $9.1725 \quad 154$ & 2.07 & $9.3288 \quad 147$ & 1.68 \\
\hline 126 & $8.9095 \quad 229$ & 2.73 & $\begin{array}{llll}9.1571 & 157\end{array}$ & 2.11 & $9.3141 \quad 150$ & 1.71 \\
\hline 127 & $8.8 \$ 66 \quad 234$ & 2.78 & $9.1414 \quad 160$ & 2.15 & $9.2991 \quad 154$ & 1.75 \\
\hline 128 & 8.8632239 & 2.84 & $9.1254 \quad 164$ & 2.19 & $9.2837 \quad 157$ & 1.79 \\
\hline 129 & $8.8393 \quad 244$ & 2.90 & $\begin{array}{lll}9.1090 \quad 167\end{array}$ & 2.23 & $9.2680 \quad 161$ & 1.83 \\
\hline 130 & $\$ .8149250$ & 2.96 & $\begin{array}{l}9.0923 \quad 171 \\
\end{array}$ & 2.27 & $9.2519 \quad 164$ & 1.87 \\
\hline 131 & S.7\$99 256 & 3.03 & 9.0752174 & 2.31 & $\begin{array}{lll}9.2355 & 169\end{array}$ & 1.91 \\
\hline 132 & $\$ .7643261$ & 3.09 & $9.0578 \quad 179$ & 2.36 & $9.2186 \quad 172$ & 1.95 \\
\hline 133 & $8.7382 \quad 268$ & 3.15 & $\begin{array}{l}9.0399 \quad 183 \\
\end{array}$ & 2.40 & $9.2014 \quad 176$ & 2.00 \\
\hline 134 & $5.711+274$ & 3.22 & $9.0216 \quad 157$ & 2.45 & $9.1838 \quad 181$ & 2.04 \\
\hline 135 & 5.6540281 & 3.29 & $9.0029 \quad 191$ & 2.49 & $9.1657 \quad 185$ & 2.09 \\
\hline 136 & 8.6559288 & 3.36 & $8.983 S_{196}$ & 2.54 & $9.1472 \quad 190$ & 2.13 \\
\hline $13 \bar{T}$ & S.6271 295 & 3.43 & $5.96+2200$ & 2.59 & $9.1252 \quad 195$ & 2.18 \\
\hline $13 \mathrm{~S}$ & $\begin{array}{l}5.5976 \quad 303 \\
\end{array}$ & 3.51 & $\$ .9+42205$ & 2.64 & $9.10 S 7200$ & 2.23 \\
\hline 139 & $5.5673 \quad 311$ & $3.5 \mathrm{~S}$ & 5.9237211 & 2.69 & $9.0 S S 7206$ & 2.28 \\
\hline 140 & $5.5362 \quad 319$ & 3.66 & $\$ .9026 \quad 216$ & 2.74 & $9.0681 \quad 211$ & 2.33 \\
\hline 141 & $8.5043 \quad 328$ & 3.74 & $8.5510 \quad 222$ & 2.511 & $9.0470 \quad 217$ & 2.35 \\
\hline 142 & $8.4715 \quad 338$ & 3.52 & S.85SS 229 & 2.55 & $9.1) 253223$ & 2.44 \\
\hline 143 & $8.4377 \quad 347$ & 3.91 & S.\$359 235 & 2.91 & $9.0030 \quad 230$ & 2.49 \\
\hline 144 & $5.4030 \quad 358$ & 3.99 & $8.512+2+2$ & 2.97 & $5.9800 \quad 237$ & $2.5 \mathrm{3}$ \\
\hline 145 & $5.3672 \quad 368$ & $4.0 \mathrm{~s}$ & S.7SS2 250 & 3.03 & $8.9563 \quad 244$ & 2.61 \\
\hline 146 & $8.3304 \quad 380$ & 4.17 & 5.7632 .257 & 3.09 & $\$ .9319 \quad 252$ & 2.67 \\
\hline 147 & $8.292+392$ & 4.27 & $5.7375 \quad 265$ & 3.16 & 5.9067260 & 2.7 .3 \\
\hline $14 \mathrm{~S}$ & 8.2532406 & 4.37 & 5.7110272 & 3.22 & $5.5507 \quad 269$ & 2.50 \\
\hline 149 & $5.2126+19$ & 4.47 & $5.6 \$ 35 \cdot 280$ & 3.29 & $\$ . \$ 53 S_{278}$ & 2.57 \\
\hline 150 & 5.1707433 & 4.57 & S.655S 291 & 3.36 & 5.82611258 & 2.94 \\
\hline 151 & $5.127+450$ & 4.65 & 5.6267302 & 3.43 & 5.7972 .298 & 3.01 \\
\hline 1.12 & $5.1152+t 66$ & 4.79 & 0.5965314 & 3.51 & 8.7674310 & $3.0 \mathrm{~S}$ \\
\hline 153 & 5.11355485 & +.91 & 5.5651326 & 3.59 & $8.736+322$ & 3.16 \\
\hline 154 & 7.9873504 & 5.113 & $5.5325 \quad 335$ & 3.67 & $5.70+2 \quad 335$ & 3.24 \\
\hline $15 \%$ & 7.99369526 & 5.16 & 5.4957352 & 3.75 & S.67117 349 & 3.32 \\
\hline 156 & $7.58+3545$ & 5.29 & $5.4635 \quad 368$ & 3.54 & S.635S 365 & 3.41 \\
\hline 1.7 & $7.5295 \quad 573$ & 5.43 & $5.4267 \quad 355$ & 3.98 & $8.5993 \quad 381$ & 3.50 \\
\hline $1 \% S$ & 7.7722600 & 5.55 & -.3552402 & 4.03 & 5.5612399 & 3.60 \\
\hline $15 !$ & $7.7122 \quad 630$ & 5.72 & $5.3+1511+21$ & 4.13 & 8.5213420 & 3.70 \\
\hline 160 & $7.6+92 \quad 664$ & 5.54 & 5.30 .59444 & 4.24 & 5.4793441 & 3.511 \\
\hline 161 & $7.5 \supset 25699$ & 6.114 & 3.261 .1468 & 4.35 & $\$ .4352465$ & 3.91 \\
\hline 162 & 7.5129740 & 6.22 & 5.2147494 & 4.46 & $S^{5.3857_{493}}$ & 4.03 \\
\hline 163 & T.t.is!) 756 & 6.40 & -16.33525 & 4.59 & $5.339+523$ & 4.15 \\
\hline 164 & 7.36113 S36 & 6.60 & 5.1125560 & 4.72 & 5.2971557 & 4.25 \\
\hline 16,5 & 7.2767 & 6.51 & 5.0 .565 & 4.56 & 5.2314 & 4.42 \\
\hline
\end{tabular}


lla. Mittlere Extinctionstabellen für Potsdam (Meereshōhe $100 \mathrm{~m}$ ) und
für den Gipfel des Săntis (Meereshőhe $2500 \mathrm{~m}$ ) von Grad zu Grad. in Helligkeitslogarithmen und Grössenclassen.

\begin{tabular}{|c|c|c|c|c|c|c|c|c|c|}
\hline \multirow{2}{*}{$\begin{array}{l}\text { Wahre } \\
\text { Zenith- } \\
\text { distanz }\end{array}$} & \multicolumn{2}{|c|}{ Potsdam } & \multicolumn{2}{|c|}{ Siintis } & \multirow{2}{*}{$\begin{array}{l}\text { Walse } \\
\text { Zenith- } \\
\text { distanz }\end{array}$} & \multicolumn{2}{|c|}{ Potsclam } & \multicolumn{2}{|c|}{ Sintis } \\
\hline & Logarith & (irỏssen & Logarith. & Cirỏssen & & Ioogarilh. & Girôssen & loogrith. & Grüasen \\
\hline $11^{\circ}$ & 0.0006 & 0.110 & 0.0010 & 0.00 & $50^{\circ}$ & 0.0152 & & $0.0 .311)$ & 0.015 \\
\hline 12 & 0.0008 & 0.00 & 0.0012 & 0. & i) & & & 0.0325 & 0.115 \\
\hline 13 & $0.1) 010$ & 0.010 & $0.001+1$ & 0.110 & 52 & $0.1) \div 49$ & 0.14 & 0.0345 & (1.6)!) \\
\hline 14 & 0.0013 & 0.100 & 0.0017 & 0.00 & 53 & 0.0556 & (1.15) & 0.0369 & $0.0 !)$ \\
\hline 15 & 0.0016 & 0.00 & 0.0019 & 0.00 & 54 & $0.1)(625$ & 0.16 & $0.0: 3 ! 91$ & 0.10 \\
\hline 16 & 0.0019 & 0.110 & 0.0022 & 0.01 & 55 & 0.0667 & & .0415 & 0.10 \\
\hline 17 & 0.0023 & 0.01 & 0.0025 & 0.01 & 56 & 0.0711 & & & 0.11 \\
\hline 18 & 01.00127 & 0.01 & $0.0(129$ & 0.01 & 57 & 0.0758 & 0.19 & 66 & 0.12 \\
\hline 19 & 0.0032 & 0.01 & 0.0032 & 0.01 & is & $0.1150 \mathrm{~S}$ & 0.20 & 0.04 & 0.12 \\
\hline 20 & 0.0037 & 0.01 & 0.01036 & 0.01 & 5 & & 0. & & $0.1: 3$ \\
\hline 21 & 0.0042 & 0.101 & 0.0040 & 0.01 & 60 & 0.0920 & 0.23 & (0.0556 & 0.11 \\
\hline 22 & 0.0045 & 0.01 & 0.0044 & 0.01 & 61 & $0.09 \$ 2$ & $0.2 \vdots$ & 0.0 & 15 \\
\hline 23 & 0.0054 & 0.01 & 0.0048 & i). 101 & 6 & 0.1 & & 627 & 0.16 \\
\hline 24 & 0.0061 & 0.02 & 0.0053 & 1 & & & & 0.11667 & 0.17 \\
\hline 25 & 11.0068 & 0.012 & 0.0058 & 0.01 & & & $0 .:$ & 710 & $0.1 \mathrm{~s}$ \\
\hline 26 & 0.00176 & 0.112 & 0.0063 & 0.02 & $6:$ & 11.1276 & 0.32 & $1) .07 .57$ & 1).1!) \\
\hline 27 & 0.0054 & 0.02 & $0.1) 068$ & 0.112 & & 0.1361 .4 & 0.31 & 0.11305 & (1.21) \\
\hline 29 & 0.0093 & 0.112 & 0.01074 & 0.02 & 6 & & $11 .:$ & & 11.22 \\
\hline 29 & 0.0102 & 0.113 & 0.0050 & 0.02 & 6 & $0.15(j .1$ & $0 . .3$ & 1122 & $11.2: 3$ \\
\hline 30 & 0.11112 & 0.03 & 10.0086 & $0.1) 2$ & i) & 0.1676 & 0.4 & 957 & 0.25 \\
\hline 31 & 0.0 & 0.03 & 1).0093 & 0.02 & 70 & 0.1794 & 0.15 & 59 & $11.26 ;$ \\
\hline 32 & 0.0 & & 10.0100 & & 71 & 0.19131 & 0.45 & 0.1 & $(1.2)$ \\
\hline 33 & 0.0144 & 0.04 & 0.0107 & 0.03 & 72 & 0.2 & 0.72 & 11.1225 & 0.31 \\
\hline 34 & 0.0156 & 0.04 & 0.0115 & 0.113 & 73 & $11.22: 12$ & $0 . .16$ & 0.1327 & 0.333 \\
\hline 35 & 0.0169 & $(1) .1) 4$ & 0.0123 & 0.03 & 74 & $0.240 \pi$ & 0.60 & 0.1 .135 & $10.31 ;$ \\
\hline 36 & 0.0182 & 0.05 & 0.0132 & 0.013 & 7.1 & (1).2.5!11; & 11. & $0.15(3.3$ & 0.39 \\
\hline 37 & 0.0196 & & 0.0141 & 0.04 & & 0.2507 & & $(1.1710)$ & $11.4: 3$ \\
\hline 38 & 0.0211 & 0.115 & 0.0150 & 0.04 & 77 & $11 .: 3010$ & $0.71 ;$ & $0.1>6 i$ & 10.47 \\
\hline 39 & 0.0227 & $0.1) 6$ & 0.016010 & $11.11-1$ & T5 & 0.32015 & $0 .>2$ & 11.2011 .77 & $11 . \vdots 1$ \\
\hline 40 & 0.0244 & 0.06 & $0.017(1)$ & 0.114 & $7 !$ & $(1.33 .5 .5$ & $0.911)$ & 11.2277 & 11.57 \\
\hline 41 & 0.0262 & $0.1) 7$ & 0.0151 & 0.115 & 50 & $0 .: 3(10)$ & 0.91 & 1).2.5:36 & $11.63: 3$ \\
\hline 42 & $0.02 S 1$ & 0.117 & 0.11192 & 0.0 .5 & 31 & $0.127 !$ & 1.07 & $0.2 \bigcirc 45$ & 11.71 \\
\hline 43 & 0.0301 & 0.115 & 0.020 .4 & 0.05 & $\supset 2$ & 0.4715 & 1.15 & 11.33221 & 0.51 \\
\hline 44 & 0.0823 & 0.118 & 0.11217 & $0.0 \%$ & $5: 3$ & $(1 . .52(i)$ & $1 . \because 2$ & 1).:3(is) & 0.92 \\
\hline 45 & $0.03+6$ & 11.119 & $0.012: 31$ & $0.1)(i$ & +1 & (1...)!(.)!) & 1.19 & 1). 1277 & 1.07 \\
\hline 46 & 0.0370 & 0.09 & 0.0245 & 0.06 & 5.7 & $0.1 ; 5 ! 12$ & 1.72 & $0 . .311: 14$ & 1.26 \\
\hline 47 & 0.0396 & 0.10 & $0.021 ; 0$ & $0.06 j$ & $56 i$ & $11 . \succ \mid 1 ; 1$ & 2.01 & $0.603 \%$ & 1.51 \\
\hline 48 & $0.0+23$ & 11.11 & 0.10276 & 0.117 & 57 & $11.91912 ! 1$ & 2.45 & 11.74115 & 1.55 \\
\hline 49 & $0.04 \div 2$ & 11.11 & 0.0293 & 0.117 & is & $1.2+11)$. & $: 1.111$ & $0.91 \%$ is & 2.31 \\
\hline
\end{tabular}




\section{IIb. Mittlere Extinotionstabelle für Potsdam zwischen $50^{\circ}$ und $88^{\circ}$ Zenithdistanz von Zehntel zu Zehntel Grad in Helligkeitslogarithmen.}

NB. Einheiten der vierten Decimale.

\begin{tabular}{|c|c|c|c|c|c|c|c|c|c|c|}
\hline $\begin{array}{l}\text { Wahre } \\
\text { Zenith- } \\
\text { distanz }\end{array}$ & 0.0 & 0.1 & 0.2 & 0.3 & 0.4 & $0 . \overline{5}$ & 0.6 & 0.7 & 0.8 & 0.9 \\
\hline $50^{\circ}$ & 482 & $4 S 5$ & 488 & 491 & 495 & 499 & 501 & 504 & 507 & 511 \\
\hline 51 & 514 & 517 & 521 & 524 & 528 & 531 & 535 & 538 & 542 & 545 \\
\hline 52 & 549 & 553 & 556 & 560 & 564 & 567 & 571 & 575 & 578 & 582 \\
\hline 53 & 586 & 590 & 594 & 597 & 601 & 605 & 609 & 613 & 617 & 621 \\
\hline 54 & 625 & 629 & 633 & 637 & $6+2$ & 646 & 650 & 654 & 658 & 663 \\
\hline 55 & 667 & 671 & 676 & 680 & $6 S 4$ & 659 & 693 & 698 & 702 & 706 \\
\hline 56 & 711 & 716 & 720 & 725 & 729 & 734 & 739 & 744 & 748 & 753 \\
\hline 57 & $75 \mathrm{~S}$ & 763 & 768 & 773 & 778 & $7 S 3$ & 788 & 793 & 798 & 803 \\
\hline 58 & 808 & 813 & $\$ 18$ & $\$ 24$ & $\$ 29$ & 835 & 840 & 845 & 851 & 856 \\
\hline 59 & $\$ 62$ & $\$ 68$ & 573 & 579 & $\$ 55$ & 891 & 896 & 902 & 908 & 914 \\
\hline 60 & 920 & 926 & 932 & 938 & 944 & 951 & 957 & 963 & & 976 \\
\hline 61 & 982 & 988 & 995 & 1002 & 1008 & 1015 & 1021 & 1028 & & 1041 \\
\hline 62 & 1045 & 1055 & 1062 & 1069 & 1076 & 1083 & 1090 & 1097 & 1104 & 1111 \\
\hline $6: 3$ & 1118 & 1125 & 1133 & 1140 & $11+8$ & 1155 & 11 & 1171 & & 1186 \\
\hline 64 & 1194 & 1202 & 1210 & 1218 & 1226 & 1234 & $12+2$ & 1251 & 1259 & 1267 \\
\hline 65 & 1276 & 1285 & 1293 & 1302 & 1310 & 1319 & & 37 & & 1355 \\
\hline 66 & 1364 & 1373 & 1383 & 1392 & 1401 & 1411 & 1421 & 1430 & 1440 & 1450 \\
\hline 67 & $1+60$ & 1470 & 1480 & $1+90$ & 1501 & 1511 & 21 & 1532 & 43 & 1553 \\
\hline 68 & 1564 & 1575 & 1586 & 1597 & 1608 & 1619 & 1630 & 1642 & 1653 & 1664 \\
\hline 69 & 1676 & 1688 & 1700 & 1712 & 1724 & 1736 & $17+8$ & 1760 & 1773 & 1785 \\
\hline 70 & 1798 & & & $18: 37$ & & & & & & 1917 \\
\hline 71 & 1931 & 1945 & 1959 & 1973 & 1987 & 2002 & 2016 & 2031 & & 2060 \\
\hline 72 & 2075 & 2090 & 2106 & 2121 & 2137 & 2152 & 21 & 21 & 00 & 2216 \\
\hline 73 & 2232 & 2249 & 2265 & 2282 & 2299 & 2316 & 2334 & 2352 & 2369 & 2387 \\
\hline 74 & 2405 & 2423 & $24+2$ & 2460 & 2479 & 2498 & 2517 & 2537 & 56 & 2576 \\
\hline 75 & 2596 & 2616 & 2637 & 2657 & 2678 & 2699 & 2720 & 2742 & 2763 & 2785 \\
\hline 76 & 2507 & 2529 & 2552 & 2875 & $2 \$ 99$ & 2921 & 2944 & 2968 & 2992 & 3016 \\
\hline 77 & $30+0$ & 3065 & 3090 & 3115 & 3140 & $3166^{\circ}$ & 3192 & $321 \mathrm{~s}$ & 3244 & 3271 \\
\hline 78 & 3298 & 3325 & 3353 & 3351 & 3409 & & & & & 3555 \\
\hline 79 & 3585 & 3616 & 3647 & 3675 & 3710 & $37+2$ & 3775 & 3808 & & 3874 \\
\hline 80 & & & & & & & & & & 4239 \\
\hline$S 1$ & 4279 & 4319 & 4360 & 4402 & 4444 & 4488 & & 4577 & 4623 & 4670 \\
\hline 82 & $471 \mathrm{~s}$ & 4767 & 1517 & 4868 & 4920 & +1973 & 5028 & 5084 & 41 & 5200 \\
\hline $8: ;$ & 5260 & 5322 & 5385 & 5450 & 517 & 5586 & 5656 & 5725 & 5803 & $5 \$ 50$ \\
\hline$-\frac{1}{1}$ & 5959 & 6040 & 6124 & 6211 & (i299 & 13391 & & $65 \$ 2$ & $66 \$ 2$ & 6785 \\
\hline 5.5 & 6592 & 7002 & 7115 & 7232 & 7353 & 7477 & 7606 & 7739 & $7 \$ 76$ & S01S \\
\hline$S 1 i$ & 5164 & 831.5 & 5471 & 5632 & 5799 & $\$ 971$ & 9150 & 9335 & 9526 & 9724 \\
\hline 87 & 9929 & 10141 & 111360 & 111556 & 10821 & 11063 & 11314 & 11573 & $11 S 42$ & 12120 \\
\hline
\end{tabular}




\section{Litteraturverzeichniss.}

Obgleich die wichtigsten Arbeiten auf dem Gebiete der Astrophotometrie bereits bei den einzelnen Capiteln des Buches angemerkt sind, dilrfte doch vielleicht Vielen, die sich nither mit diesem Zweige der Astrophysik beschäftigen wollen, eine besondere nach bestimmten Gesichtspunkten georduete Znsammenstellung der einsehligigen Litteratur nicht nnwillkommen sein. Fine absolnte Vollstindigkeit ist dabei nicht angestrebt worden; der leitende Gedanke war, in erster Linie alle diejenigen Sehriften anzufithren, deren Kemutniss für ein näheres Studium der Astrophotometrie entweder unung:inglich nothwendig oder weuigstens in irgend einer Beziehung lehrreich nnd förderlich erscheint, daggegen von vornherein Alles anszusehliessen, was gïmzlich werthlos ist oder lö̈chstens nur ein nebensïchliches Interesse bieten kamn. Ans diesem Grunde sind \%. B. ohne Weiteres blosse Ankündigmugen oder gelegentliche kurze Notizen iiber Gegenstände der Astrophotometrie, namentlich wemn sie in schwer zugänglichen Zeitschriften zo finden sind, ganz unberickssiclitigt gehliehen. Es sollte denjenigen, die sich mit der Litteratur des Faches vertraut machen wollen, die Miilhe erspart werden, eine gante Anzahl vou Schriften, deren Titel in irgend einem Zusammenhange mit der Astrophotometrie zu stehen scheinen, deren Inhlalt aber liäutig wenig oder gar nichts d:unit zu thun hat, vergeblich zn liathe zo ziehen, eine Niihe, der ich mich selhst bei den Vorarbeiten zin diesem Buche nicht entziehen konnte.

Bei Weitem die meisten der in der Übersicht :ufgezilhlten Werke sind ron mir selhst durchgesehen worden, sodass, sowohl wats ihren In-

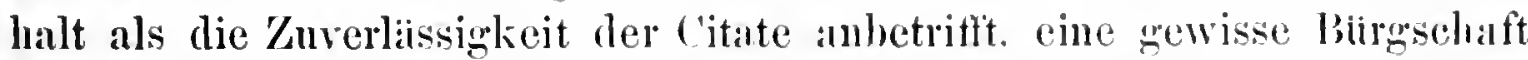
ibhernommen werden kann; nur bei einer verlialtnissmiissig kleinen Anzahl von Abhandlungen, die mir nicht zngäinglich gewesen sind, halbe ich mich auf das Zengniss anderer Quellen verlassen miissen. Wenn eine Abhlaullung in melreren Zeitsehriften oder in Übersetznngen mo Anszitigen erschienen ist, so ist die Originalyublication entweder allein oder wenigstens an erster Stelle angefülnt. Blosse Referate iil)er astrophotometrische Arbeiten sind 
nur ausnahmsweise beriicksichtigt worden. Bei denjenigen Artikeln, die vom Verfasser nicht mit einem eigenen Titel versehen sind, ist eine kurze Inhaltsangabe (in eckigen Klámmern) zu dem Namen des Autors hinzugefügt.

Die geewählten Abkiirzungen der Citate werden durchweg ohne weitere Erlänterungen verstïndlich sein; es verdient höchstens noch hervorgehoben zul werden, dass die fettgedruckte Zahl sich stets auf den Band oder Jahrgang, die daneben stehenden Zahlen auf die Seiten beziehen. Die in Klammern dabei gesetzte Jahreszahl nennt fast immer dasjenige Jahr, in welchẹm der betreffende Band erschienen ist.

Der gesammte Stoff ist im Folgenden in acht Abschnitte eingetheilt worden, wobei im Allgemeinen die in diesem Buche gewählte Disposition massgebend gewesen ist. Noch mehr Unterabtheilungen zn wählen schien bei der nicht allzu grossen Zahl der vorhandenen Titel kanm erforderlich zu sein. Innerhalb jedes Abschnittes sind die Schriften in der alphabetischen Reihenfolge der Namen der Verfasser geordnet.

\section{Theoretisches und Allgemeines.}

Albert, L. A. Versuch, den Saturnring photometrisch zu betrachten. Diss. inaug. Niinchen, 1832.

Anding, E. Photometrische Untersuchungen iiber die Verfinsterungen der Jupiterstrabanten. Preisschr. der Univers. München. München 1589.

_- Die Seeliger'sche Theorie des Saturnringes und der Beleuchtung der grossen Planeten iiberlaupt. Astr. Nachr. 121, Nr. 2851 (1589).

- Über die Lichtvertheilung auf einer unvollständig beleuchteten Planetenscheibe. Astr. Nachr. 129, Nr. 3095 (1892).

Arago, Fr. Sieben Abhandlungen iiber Photometrie. Aragos Werke; deutsche Ausg. von Hankel. Bd. $10(1859)$.

Beer, A. Vier photometrische Probleme. Pogg. Ann. 88, 114 (1853).

Grundriss des photometrischen Calciiles. Braunschweig, 1854.

Bouguer, P. Essai d'optique sur la gradation de la lumière. Paris, 1729.

- Traité d'optique sur la gradation de la lumière. - Onvrage postbume', publié par l'abbé de Lacaille. Paris, 1760.

v. Bezold, W. Einige analoge Sitze der Photometrie und Anziehungslehre. Pogg. Ann. 141, 91 1870.

Bruns, H. Bemerkungen iiber den Lichtwechsel der Sterne vom Algoltypus. Honatsber. d. Berliner Akad. 1581. 4 s.

Carstaedt. Über die Abnilhme der Lichtstärke mit dem Quadrate der Entfernnng. Pogg. Ann. 150, 551 (1873.

Charlier. C. V. L. Astrophotometrische Studien. Bil. Srensk. Vet.-Akad. Handl. 14. Afd. 1. Nr. ? (15ss).

Chwolson, O. Photometrische Untersuchungen iiber die innere Diffusion des Lichtes. Bull. Acad. St.-Pétersh. 31. 2131557.

(trundziige einer mathematischen Theorie der inneren Diffusion des Lichtes. Bull. Acad. St.-Pétersb. Nouv. Sér. 1 33, 221 1590. 
Eulor, L. Réflexions sur les divers degtés de lumiére du soleil et des autres corps célestes. Hist. et Mém. de l'Acad. R. de Berlin 1550, 250.

Fechner, G. Th. Uber ein psychophysisches Grundgeset\% und dessen Bezichung zur Schätzung der Sterngrüssen. Abhandl. d. K. Siichs. Ges. d. Wiss. \$, 455 (1859). - Siehe auch Ber. Uuber die Verhandl. d. Siichs. Ges. 11, $58(18.9) ; 16,1$ (1864).

Guther, S. Studien zur theoretischen I'hotometrie. Diss. inaug. Firlangen, 1872.

Gyldén, H. Versuch einer mathematischen Theorie zur Firklïrung des Liehtwechsels der veründerlichen Storne. Acta Soc. scient. Fennicae 11, 3 (1850).

Karsten, W. J. G. Lehrbegriff der gesammten Mathematik. Achter Theil: Die Photometrie. Greifswald, 1777.

Kr liss, H. Dio Grandlagen der Plotometrie. Abb. d. Naturw. Vereins in Hamburg (7) 9,28 (1552).

Dio clektro-technische Photometrie. Wien, Pest, Leipzig, 1886.

Lambert, J. H. Photometria sive de mensura et gradibus luminis, colorum et umbrae. Augustae Vindelicorum, 1760. (Deutsch herausgegeben von K. Anding. Ostwald's Klassiker der exacten Wissenschaften, Nr. 31-33. Leipzig, 1892.)

v. Langsdorff, K. C. Grundlehren der Photometrie oder der optischen Wissenschaften. Abtheilung I u. II. Erlangen, 1803 u. 1805.

Lommel, E. Über Flnoreseenz. Abschnitt 1: Ưber die Grundsätze der Photometric. Wieder. Ann. 10, 449 (1550).

- Die Photometrie der diffusen Zurïckwerfung. Sitzungsber. der Munchener Akad. II. Cl. 17, 95 (1887).

Mascart, E. Traité d'optique. Chapitre XVI: Photométrie. Tome III, p. 145-271. Mcisel, Fr. Über die Bestrahlung einer Kugel durch eine Kugel. Zeitschr. f. Math. u. Phys. 27, 66 (1852!

Messerschmitt, J. B. Über diffuse Reflexion. Diss. inaug. Leipzig, 1888.

Obrecht, A. Étude sur les éelipses des satellites de Jupiter. Annales de l'Obs. de Paris. Mémoires, tome 15 (1885).

Plana, J. Note sur la manière de ealeuler le déeroissement d'intensité que la photosphère du soleil subit en traversant, l'atmosphire qui l'entoure. Astr. Nachr. 34, Nr. 813 (1852).

Recknagel, G. Lamberts Photometrie und ibre Beziehung zum gegenwirtigen Standpunkt der Wissenschaft. Von der philos. Facultit in Miinehen gekrönte Preisschrift als Dissertation. Miinchen, 1861.

Rheinauer, J. Grundzüge der Photometrie. Halle, 1562.

Searle, A. 'The phases of the Moon. Proc. Amer. Acad. New Ser. 11, $310 / 1584$. Seeliger, H. Zur Photometrie des Saturnringes. Astr. Nachr. 109, Nr. 2612 (1884). Bemerkungen zu Zijllners "Photometrischen Untersuchungen d. Astr. Ges. 21, 216 (1586).

Zur Theorie der Beleuclitung der grossen Planeten, insbesondere des Saturn. Abhandl. der Miinchener Akad. II. Cl. 16. 4051585.

Zur Photometrie zerstreut reflectirender Substanzen. Sitzungsher. der Minchener Akad. II. Cl. 18, 201 (1S5s).

- Theorie der Beleuchtung staubförmiger kosmischer Massen. insbesondere des Saturnringes. Abhandl. der Miinchener Akad. II. Cl. 1s, 1 1893.

- Über den Schatten eines Planeten. Sitzungsher. der Miinchener Akad. II. Cl. 24, 423 (1894.

Wellmann, V. Zur Photometrie der Jupiters-T'Tabanten. Berlin. 1857. 
Wesely, J. Analytische und geometrische Auflösung einiger photometrischer Problewe und ein neues Photometer. Zeitschr. f. Math. u. Phys. 16, 324 (18i1). Wislicenus, W. F. Abriss der Astrophotometrie und Astrospectroskopie. Breslau, 1896. Sonderdruck aus dem Handwörterbuch der Astronomie, herausgeg. von W. Valentiner.

Zöllner, Fr. Photometrische Untersuchungen. Pogg. Ann. 100, 381, 474, 651 (1857); 109, 244 (1860).

Grundzüge einer allgemeinen Photometrie des Himmels. Berlin, 1861.

- Photometrische Untersuchungen mit besonderer Rücksicht auf die physische Beschaffenheit der Himmelskörper. Leipzig, 1865.

- Einige Sätze aus der theoretischen Photometrie. Pogg. Ann. 128, 46 (1866).

- Resultate photometrischer Beobachtungen an Himmelskörpern. Pogg. Ann. 128, 260 (1866); Astr. Nachr. 66, Nr. $1575\langle 1866$ \%

\section{Photometrische Apparate und Methoden.}

Abney, W. Note on the scailing of Dr. Spitta's wedge by means of photography. Monthly Not. 50, 515 (1890).

On the estimation of star magnitudes by extinction with the wedge. Monthly

Not. 52, 426 (1692).

Graduating wedges. Monthly Not. 54, 368 (1894).

Abney, W. und Festing, E. R. Colour photometry. Phil. Trans. 177, 423 $(1556) ;$ 179, 54 i (1885); 183, 531 '1592.

Arago, Fr. Über das Gesetz des Cosinusquadrats fïr die Intensität des polarisirten Liclits, welches von doppeltbrechenden Krystallen durchgelassen wird. Pogg. Ann. 35, $444(1535)$.

Argelander, Fr. [Über das Schwerd'sche Photometer.] Sitzungsber. des naturhist. Vereins der Preuss. Rheinlande und Westphalens. Jahrg. 16, 64 (1559).

Babinet, J. Note descriptive du photomètre industriel. Compt. Rend. 37, 7i4 (1853).

Becquerel, E. Recherches sur divers effets lumineux qui résultent de l'action de la lumière sur les corps. Ann. Chim. et Phys. $3,62,5$; $1 \mathrm{~s} 61$,

v. Berg, F. Über das Schwerd'sche Photometer und die Lichtextinction für deu Wilnaer Horizont. Aus den s. Sipisslic der Petersburger Akademie, 1S73. - (In russischer Sprache.

Bernard, F. Thése sur labsorption de la lumière par les milieux non cristallisés. (Darin entbalten Beschreibung und Abbildung eines Photometers.) Ann. Chim. et Phys. $\langle 3,35.355,15522$.

- Note sur lia description et l'emploi d'un nouveau photomètre. Compt. Rend. 36,$7251553 ;$.

Bohn. C. Photometrische Lntersuchungen. Pogg. Anu. Ergänzungsband 6, 356 1573

Bruhns, C. Über ein nenes Photometer. Vortrag auf der Astronomenversammlung in Leiden, 15i5.] Vierteljahlrsschr. der Astr. Ges. 10, 23.5 1575\%.

Carl. Ph. Zülner's Astrophotometer. Carl's Repert. 1, 15 i 15666.

Ceraski. W. Über Helligkeitsbestimmng sehr heller Sterne mit dem Zülner'schen Photometer. Astr. Nachr. 107, Nr. 2561 1554.

_ Über das Züllner'sche Photometer. Astr. Nitchr. 110, Nr. 2621; 112. Nr. 2658 $1 \times 5$. 
Ceraski, W. Nouvelle construction de l'astrophotométre de Zïllner et le collimateur photométriquo. Annal. de l'obs. de Moscon. Sér. 2, Vol. I, livr. 2, p. 13 (1556,. - Sur lo photomètre do Zollner a deux oculnires. Astr. Nachr. 120, Nr. 25io (1859).

Petit appareil à l'usage de ceux qui étudient les magnitudes des étoiles. Annul. de l'obs. de Moscon. Sér. 2, Vol. II, 173 (1890).

Chacornac, J. Sur un moyen de comparer avec précision l'éclat de deux étoiles. Compt. Rend. 68, 65i (1564).

Christie, W. H. M. On the colonr and brightness of stars as measured with * new plotometer. Monthly Not. 34, 111 (1874).

Cornu, A. Études photoinétriques. Séances de la Soc. Franç. de physique, 1851, 50. Sur quelques dispositifs permettant de réaliser, saus polariser la lumière, des photométros biréfringents. Compt. Rond. 103, 122i (1556).

Crookes, W. On the measurement of the luminous intensity of light. Proe. $R$. Soc. London 17, 166, $358\langle 1569\rangle$.

Crova, A. Étude des radiations émises par les corps incandescents. Mesure optique des hautes températures. Ann. Chim. et Phys. (5) 19, 4i2 (1850).

Étude des aberrations des prismes et de leur influence sur les observations spectroscopiques. Ann. Chim. et Phys. (5) 22, 513 (158I).

- Étude sur les spectrophotomítres. Compt. Rend. 92, 36 〈1881).

- Comparaison photométrique des sonrces lımineuses de teintes différentes. Compt. Rend. 93, 512 (1851).

- Description d'ın spectrophotomètre. Ann. Chim. et Phys. (5) 29, 556 (1853).

Crova, A. et Lagarde, H. Détermination du pouvoir éclairant des radiations simples. Compt. Rend. 93, 959 (18S1).

Czapski, S. Einrichtung der Spalten an Polarisationsphotometern, un auch ohne Achromatisirung der Kalkspathprismen vollstïndige Achromasie der Grenzlinie zu erhalten. Zeitschr. f. Instrum. 12, 161 (1892).

Dawes, W. R. Description of an aperture-diminishing eye-piece and of a photometer of neutral-tint glass. Monthly Not $\mathbf{2 5}, 229 / 1565$.

Dove, H. [Beschreibung eines Photometers.] Monatsber. d. Berliner Akad. 1861, 493. Espin, T. E. Observations of $U$ Monocerotis and Lalande 14551, with a new photometer. Monthly Not. 43, 431 (1583).

Finck, E. W. .J. V. Alberts neuer Lichtmessapparat, beschriehen und mitgetheilt von E. W. Finck, Mechaniker. Dinglers polytechn. Journal 100, 20, 1846

Foucanlt, L. [Photometre a compartiment.] Recueil d. trav. scient. de L. Foucault. Paris 1878, 100.

Fuchs, Fr. Über (in neues Interferenzphotometer. Wiedem. Ann. 11. 465 (1540. Vorschläge zur Construction einiger optischer Vorrichtungen. 11. Sprectrophotometer. Zeitschr. f. Instrum. 1. 349 (15s1.

Glan, P. Über ein newes Photometer. Wiedem. Anu. 1. $351(14 i j\rangle$.

Glazebrook, R. T. On a spectrophotometer. Proc. Cambridge Plit. Soc. 4. 3114 (1853).

v. Gothard, E. Keilphotometer mit Typendruck-Apparat. Zeitschr. f. Instrm. $\mathbf{7}$ $34 \mathrm{i}(155 \mathrm{i})$.

Gouy. Recherches photométriques sur les flammes colorées. Ann. Chim. et Phys. (5) $18,5(15 ; 9$.

Govi, G. Note sur un photometre analysenr. Compt. Rend. 50, 156 (1560. 
Grosse, W. Über Polarisationsprismen mit besonderer Berücksichtigung ihrer Anwendung in Photometern. Clausthal, Grosse'sche Buchb. (1887).

Über eine neue Forın von Photometern. Zeitschr. f. Instrum. 7, 129 (1887); 8,95 (1888).

Guthrie, F. On a new photometer. Chem. News 40, 262 (1879).

$\mathrm{H} a \mathrm{mmerl}$, H. Über eine Methode zur Messung der Intensität sehr heller Lichtquellen. Elektrot. Zeitschrift 4, 262 (1883).

Heis, E. [Über das Schwerd'sche Photometer.] Heis' Wochenschrift. Nene Folge $2,275(1859)$.

Herschel, J. Account of some attempts to compare the intensities of light of the stars one with another by the intervention of the Moon, by the aid of an astrometer adapted to that purpose. Restrlts of astr. obs. made during $1534-38$ at the Cape of Good Hope. London, 1847; p. 353.

Herschel, W. On the power of penetrating into space by telescopes; with a comparative determination of the extent of that power in natural vision and in telescopes of various sizes and constructions. Phil. Trans. 90, $49\langle\mathbf{1 8 0 0}\rangle$.

Astronomical observations and experiments tending to investigate the local arrangement of the celestial bodies in space, and to determine the extent and condition of the milky way. Pbil. Trans. 107, $302\langle 1817\rangle$.

Hirsch, A. Description d'un photomètre. Bnll. de la Soc. des sciences naturelles de Neuchâtel 6, 94 (1861-64).

Horner, J. K. Description d'ın photomètre. Bibl. univers. de Genève 6, 162 (181i).

Hornstein, C. Über Helligkeitsmessungen bei kleinen Fixsternen. II. Beschreibung des Zonen-Photometers. Sitzungsber. d. Wiener Akad. II. Cl. 41, 263 (1860).

Hiifner, G. Über quantitative Spectralanalyse und ein neues Spectrophotometer. Journal für prakt. Chemie, Neue Folge 16, 290 (187i).

Janssen, J. Sur la photométrie photographique et son application à l'étude des pouvoirs rayonnants comparés du soleil et des étoiles. Compt. Rend. 92, 821 (1881).

Johnson, M. J. Remarks on the application of the heliometer to the photometry of the stars. Radcliffe Observations 12, Appendix I; Monthly Not. 13, 2is (1853).

Kayser, E. Ein Photometer zur Bestimmung der relativen Helligkeiten der Sterne. Astr. Nachr. 57, Nr. 1346 1562.

Ketteler, E.. und Pulfrich, C. Photometrische Untersuchnngen. [Theil I. Über das Glan'sche Photometer.] Wiedem. Ann. 15, 337 (1882).

Klein, H. J. Über eine einfache Abänderung des Steinheil'schen Prismenphotometers zur Messung lichtschwacher Sterne. Heis' Wochenschrift. Neue Folge 5, $319,331,355(1862$

Knobel. E. B. On a new astrometer. Nonthly Not. 35, $100\langle 18 \overline{i 5}\rangle$. On the application of the method of limiting apertures to the photometry of waked-eye stars. Monthly Not. 35. 3S1 1875.

Köhler, J. G. Ü̈ber ein nenes Photometer nach dem Princip der Abblendung.] Bodes astr. Jahrb. für 1792. 233.

Krech. G. Photometrische Untersuchungen. Wissensch. Beilage zum Programm. des Luisenstädtischen Gymn. in Berlin. Ostern 1853. Berlin, 1583.

Kritiss. H. Eine nene Form des Bunsen'schen Photometers. Abhandl. d. naturwiss. Vereins in Hamburg 8. 55 ' $18 \$ 4$.

Das Mischungs-Photometer nach Dr. Wr. Grosse. Zeitscbr. f. Instrum. S, $3+1656$ 
Lagrunge, K. ot Stroobant, P. Une nouvello inćthodo astrophotométrique. Bull. de l'send. IR. de Belgique 1892, 811.

Lampadins, W. A. Beiträge ziIr Atmospliünlogic. Stllck II, Photometrisehe Beobachtungen im Jahre 1814. Freiberg, 1817. - NB. Ein nusfilirlicher Auszng, fast wörtlich, findet sich in sSehweiggers Journnl filr Chemie und Physike, 11, 361.

Langley, S. P. Note on the transmission of light by wire gauze sereeus. American Journal (3) 30, 210 (1855).

Langley, S. P., Young, C. A. and Piekering, E. C. Pritchard's wedge photo. meter. Investigations on light and heat published with appropriation from the Rumford fund, 1896, p. 301.

Lehunann; A. Über Photometrie mittelst rotirender Scheiben. Naturforscher 20, 285 (188i).

Lohmann, E. W. Über ein Photometer. Wiedem. Aun. 49, 672 (1893).

Loewj, M. Remarques sur la méthode proposéo par M. le professeur Pritchard pour la mesure de l'éclat des astres. Monthly Not. 42, 91 (1552).

Lummer, 0. und Brodhun, E. Photometrische Untersuchungen. Zeitschr. f. Instrum. 9, 41, 461 (1859); 10, 119 (1890); 12, 41, 132 (1892); 16, 299 (1896).

II acé de Lépinay, J. et Nicati, W. Recherches sur la comparaison photozétrique des diverses parties d'un même spectre. Aun. Chim. et Phys. 5; $_{;}$24, 259 (1581); 30, 145 (1583).

de Maistre, Xaver. Description d'un photométre destiné a comparer la splendeur des étoiles. Bibl. univers. de Genéve 51, 323 (1832). - Siehe auch das Referat in Pogg. Ann. 29, 186.

Masson, A. Études de photométrie électrique. Ann. Chim. et Physs. (3, 14, 129 (1545).

Minchin, G. M. Electromotive force from the light of the stars. Nature 49, 2iu (1894).

- The electrical measurement of starlight. Nature 52, 246 1895\%.

Möller, W. Photometrische Untersuchungen. Elektrot. Zeitschr. 5, 370, 405 (1854.

— Über das Wild'sche Photometer. Wiedem. Ann. \$4, $446 / 1585$

Iüller, G. Photometrische Untersuchungen. Erster Absehnitt. Publ. d. Astrophys.

Obs. Potsdain 3, 236 (1583).

Nagand. [Ein neues Photometer.] Naturforscher 3, 350 1870.

Napoli, D. Un nouveau photometre. Séances de lia Soc. Franc. de physique. $1880,53$.

Neumann, F. E. Photometrisches Verfahren, die Intensitiat der ordentlichen und ausserordentlichen Strahlen sowie die des reflectirten Lichtes zu bestimmen etc. Pogg. Ann. 40, 497 (1837).

Parkhurst, H. M. [The deflecting photometer.] Ann. llarvard Coll. Obs. 18. 29. -

Siehe auch das Referat in Viertejjahrsschr. d. Astr. Ges. 23, 297 '1590.

Pernter, J. M. Die Methoden der Messung der chemischen lntensitiit des Lielites. Zeitschr. d. Österr. Ges. f. Meteor. 14, 254 1879 .

Pickering, E. C. A nebula photometer. Americau Journ. (:3 11. 452 1876.

- [Neue Formen von Photometern.] Ann. Harv. Coll. Observ. 11, 1 1s79; 14. 1 (1884); 23, 1 (1890).

- The wedge photometer. Proc. Amer. Acad. New Ser. 9. 231 1882.

A new form of stellar photometer. Astrophys. Journ. 2, $\$ 9$ (1595.

Plateau, A. F. J. Sur un principe de photométrie. Bull. Acad. de Bruxelles 2. 52 1835'. 
Pritchard, C. On a simple and practicable method of measuring the relative apparent brightnesses or magnitudes of the stars with considerable accuracy. Monthly Not. 42, 1 (1882 .

Notes on Mr. Loewy's remarks relative to the wedge-extinction method of stellar photometry. : Monthly Not. 42, 223 (1882).

On certain deviations from the law of apertures in relation to stellar photometry; and on the applicability of a glass wedge to the determination of the magnitudes of coloured stars. Monthly Not. 43, 1, 100 (1883).

__ On Dr. Wilsing's experimental examination of the wedge photometer and on the degree of accuracy attainable by means of that instrument. Monthly Not. 46, 2 (1886).

On the verification of the constants employed in the Uranometria nova Oxoniensis. Monthly Not. 50, 512 (1590).

Quetelet, A. Sur un photomètre proposé par M. de Maistre pour mesurer la splendeur des étoiles. Bibl. univ. de Genève 52, 212 (1833). - Siehe Refer. Pogg. Ann. 29, 187.

Reissig. [Beschreibung einer photometrischen Einrichtung.] Bodes astr. Jahrb. für 1811, 250.

Ritchie, W. On a new photometer with its application to determine the relative intensities of artificial light. Phil. Trans. 115, $141(1825)$.

- On a new photometer, founded on the principles of Bouguer. Trans. R. Soc. Edinburgh 10 II, 443 (1826).

de la Rive, A. Note sur un photomètre destiné ì mesurer la transparence de l'ail. Ann. Chim. et Phys. (4 12, 243 '1867,.

Rood, 0. N. Photometrische Untersuchungen. I. Theil. Über ein einfaches Photometer zur Bestimmung der vou Metallfiachen bei verschiedenen Einfallswinkeln reflectirten Lichtmengen. Repert. d. Phys. ?. 204 (15i1).

Roscoe, H. E. Einfaches Instrument zı meteorologischen Lichtmessungen in allgemein vergleichbarem Masse. Pogg. Ann. 124, 353 (1865).

Rosén, P. G. Studien und Messungen an einem Züllner'schen Astro-Photometer. Bull. Acald. St.-Pétersb. 14, 95 (1870.

Riidorff. Fr. Über das Bunsen'sche Photometer. Pogg. Ann. Jubelbd., 234 (1874). Rumford, B. Th. An acconnt of a method of measuring the comparative intensities of the light emitted by luminous bodies. Phil. Tirans. 84, $67\langle 1794\rangle$.

Sabine, E. A wedge and diaphragm photometer. Nature 27, 201 1883).

schafhïll. Abbildung und Beschreibung des Universal-Vibrations-Photometers.

Abh. der Miinchner Akad. II. Cl. 7, 465, 1855.

v. Schumacher. C. D. Instrument till bestammande af stjernornas relativa klarhet och ljusstyrka. öfvers. K. Vetensk. Akad. Förhandl. 9, 236 (1852.

Searle, G. [Vorschlag zu einem nemen Photometer.] Astr. Nachr. 57, Nr. 1353 1862, secehi. A. Sopra un nuovo fotometro destinato specialmente a misurare l'intensiti relativa della luce delle stelle. Atti dell' accad. Pontif. dei nuori Lincei 4, 10 $1850-51$.

Silliman, B. and Porter. H. Fotice of a photometer and of some experiments therewith apon the comparative power of sereral artificial means of illumination. American Journ. $2 \mathbf{2 3}, 315$ (155i)

Simon. H. Z̈ber ein nenes photographisches Photometrirverfahren und seine Anwendung anf die Photometrie des ultravioletten Spectralgebietes. Wiedem. Ann. 59. $91 \quad 1596$ 
Simonoff, L. Sur un plotométre optique. Compt. Rend. 97, 1055 (1853).

Spiith, J. L. Photometrische Untersuchung liber die Deutlichkeit, mit welcher wir entfernto Gegenstände vermittelst dioptrischer Fernröhro beobnchton kUnnen ete. Leipzig, 1789.

Spitta, E. J. A compound wedge photometer. Proc. R. Soc. London $47,15(1890)$

Some experiments relating to the method of olitining the coefficient of absorption of the wedge photometer. Monthly Not. 50, $319(1890)$.

Some experiments relating to the photometric comparison of points of light with objects of sensible area. Monthly Not. 51, 32 (1891).

A note on some photometric experiments connected with the application of the law of limiting apertures to small object glasses. Monthly Not. 52, 45 (1892). On the scaling of a wedge. Observatory 17, 176 (1894).

Steinheil, C. A. Eleınente der Helligkeits-Messungen am Sternenhinmel. Denkschr. der Mïnchner Akad. II. Cl. 2 (1837).

[Verbesserte Form seines Prismenphotometers.] Münchner gelehrte Anzeigen 15, 9. Beitrige zur Photometrie des Himmels. Astr. Nachr. 48, Nr. 1152 (185S).

Talbot, H. F. Experiments on light. § 2: On photometry. Pliil. Mag. (3) 5, 321 (1834).

Thury, M. Description d'un photomètre astronomique et considérations sur la photomútric. Bibl. nniv. et Revue Suisse. Arch. des sciences phys. et nat. Nouv. Période 51, 209 (1874).

'Trannin, H. Mesures photométriques dans les différentes régions du spectre. Journ. de phys. 5, 297 (1876).

Varley, H. Ein neues. Photoneter mit directer Ablesung. British Association meeting at Leeds, Reports. 1890, 759.

Vierordt, C. Beschreibung einer photometrischen Methode zur Messung und Vergleichung der Stärke des farbigen Lichtes. Pogg. Ann. 13i, 2001869.

Die Messung der Lichtabsorption durchsichtiger Medien nittelst des Spectralapparates. Pogg. Ann. 140, 172 1870\%

- Die Anwendung des Speetralapparates zur Messung und Vergleichung der Stärke des farbigen Lichtes. T'ibingen. 1871.

- Die Messung der Lichtstärke der Steruspeetren. Repert. d. I'hys. 7, 392 (187).

Die Anwendung des Spectralapparates zur Photometrie der Absorptionsspectren und zur quantitativen chemischen Analyse. T'iibingen, 1873.

- Zur quantitativen Spectralanalyse. Wiedem. Ann. 3, $357 / 1876$.

Voller, A. Über die Anwendung von Dispersionslinsen bei photometrischen Messungen. Abh. d. naturw. Vereins in Hamburg (7 \&, to 1882 .

Weber, L. Mittheilung iiber einen photometrischen Apparat. Wiedem. Ann. $\mathbf{2 0}$ 326 (1883).

Zar Theorie des Bunsen'schen Photometers. Wierlem. Ann. 31, 676 1887,

Eine neue Montirung des Milehglasplattenphotometers. Zeitschr. f. Instrum. 11, $6: 1891$.

Wild, H. Über ein newes Photometer md Polarimeter nebst cinigen damit angestellten Beobachtungen. Pogg. Ann. 99. 2351656.

- Photometrische Untersuchungen. Pogg. Ann. 118. 193 1863:.

Über die Umwandlung meines Photometers in uin Spectro-Photometer. Bull. Acad. St.-Pétersu. 28, 3921853 ; Wiedem. Anu. 20, 452 $185 \%$.

- Polarisations-Photometer fiir technische Zwecke und Lntersuchung von WenhanGaslampen mit demselben. Bull. Acall. St.-Pétersh. 82. 193 (1565. 
Wilsing, J. Versuche mit dem Wedge-Photometer. Astr. Nachr. 112, Nr. 2680 und Nr. 2681 (1885).

Wilson, W. E. A new photographic photometer for determining star magnitudes. Monthly Not. 52, 153 (1892).

Wolf, M. C. Photometrische Untersuchungen. Reper. d. Physik 8, 22i (1872).

Wollaston, W. H. On a method of comparing the light of the Sun with that of the fixed stars. Phil. Trans. 119, 19 (1S29); Pogs. Ann. 16, 328 (1829).

Zenker;, W. Das nelue Spectrophotometer ron Crova verglichen mit dem ron Glan, nebst einem Vorschlag zur weiteren Verbesserung beider Apparate. Zeitschr. f. Instrum. 4, $83(1884)$.

[Photometer zur Vergleichung zweier Sterne.] Göttingische gelehrte Anzeigøn 1835, Stiick 34 n. 35.

\section{Sonne und Mond.}

A bney, W. The photographic values of moonlight and starlight compared with the light of a standard candle. Proc. R. Soc. London 59, 314 (1s96).

Abney, W. and Thorpe, T. E. On the determination of the photometric intensity of the coronal light during the Solar eclipse of Ang. 28-29, 1886. Phil. Trans. 180, $363 / 1589$.

- On the determination of the photometric intensity of the coronal light during the Solar eclipse of 16th April, 1593. Proc. R. Soc. London 60, 15 (1896).

d'Arrest, H. Über die ungleiche Vertheilung der Wärme auf der Sonnenoberfläche. Astr. Nachr. 37. Nr. 879 1554.

Boeddicker, O. Lunar radiant heat, measured at Birr Castle Observatory, during the total eclipse of Jan. 2s, 1ss8. 'l'rans. R. Dublin Soc. 2 Ser. 4, 4\$1 (1s\$8-92).

Bond. G. P. On the results of photometric experiments upon the light of the Noon and of the planet Jupiter, made at the observatory of Harvard College. Mem. Amer. Acad. New Ser. 8, 221 1Sb1.

- Comparison of the light of the Snn and Moon. Mem. Amer. Acad. New Ser. $\mathbf{S}, 28 i(1861)$.

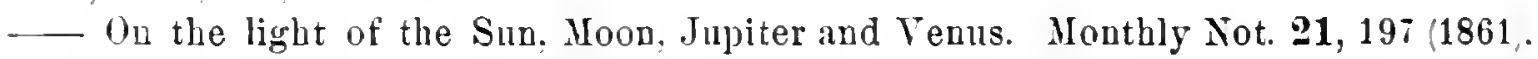

Bunsen, R. und Roscoe, H. E. Photochemische Untersuchungen. Fiinfte Abhandlung: Die Sonne. Pogg. Ann. 108. 193 (1859).

Chacornac, J. Intensité lumineuse du centre du soleil comparée á celle des bords.] Lettre de Chacornac a Lererrier. Compt. Rend. 49. S06 1559. - Siehe auch Monthly Not. 20.92.

C'lark. A. The sun and stars photometrically compared. American Journal 2 36,761963 .

Crova. A. Sur la photométrie solaire. Compt. Rend. 95, 1271 1S52: 96, 1241583. Cruls. L. und La Caille. J. O. Sur la distribution de la chaleur a la surface du soleil. Compt. Rend. S\&, 570 1 s79.

Ericsson. J. The difference of thermal energy transwitted to the earth by radiation from different parts of the solar surface. Natme 12.517 185;: 13. 114. 224 18:6'.

Exner. Fr. Zm Photometrie der Sonne. Sitzungsber. d. Wiener Atad. II. Classe. 9411.3451586

Faye, H. A. E. Sur l'atmosphère dn soleil. Compt. Rend. 49. 6961559. 
Frost, E. B. Observations on the therual absorption in the solar atmosphere. Astr. Nachir. 130, Nr. 3105-3106 (1S92).

Gore, J. E. [The Sun's stellar magnitude.] Koowledge, Juni 1895; Nature b\&, $135(1895)$.

Guy und 'Thollon. Mesures spoctrophotométriques en divers points dı disque solaire. Compt. Rend. 95, 834 (1852).

Harkness, W. Note on the brightness of tho corona. Aus: Reports on the total Solar eclipses of July 29, 1878 and January 11, 1580. Wash. Observ. 1876, $\mathrm{pp}$. III, 386.

v. Hepperger, J. Über die Helligkeit des verfinsterten Mondes unil die scheinbare Vergrüssernng des .Erdschattens. Sitzungsbor. dor Wiener Akad. II. Classe. 10411 ^, 189 (1895).

Holden, E. S. Reports on the Observations of the total eclipse of the Sun, Dec. 21-22, 1859, and of the total eclipse of the Moon, July 22, 15s5. Coutributions from the Lick Observatory, No. 2. Sacramonto, 1891. - Siehe speciell den Abschnitt: Photographic photometry of tho Corona, p. 1.

Langley, S. P. Sur la temperature relative des diverses régions du soleil. Compt. Rend. 80, 746, 819 (1875).

Étudo des radiations superficielles du soleil. Compt. Rend. 81, 136 (1575).

The solar atinosphere, an introduction to an account of researches made at the Allegheny observatory. American Journ. (3) 10, 459 (1575).

On the temperaturo of the Sun. Proe. Amer. Acad. New Ser. 6, 10ti $(1579)$.

Sur la distribution de l'énergie dans le spectre solaire normal. Compt. Rend. 92, 701 (1881); 93, 140 (1851).

The temperature of the Moon. Mem. National Acad. of sciences 411, 105 (1859).

Langley, S. P., Very, F. W. und Keeler, J. E. On the temperature of the surface of the Moon. Mem. National Acad. of sciences 3 I, 13 (155i).

Liais, E. Sur l'intensité relative de la lumière dans les divers points da diśnue du soleil. Mém. Soc. d. sc. de Cherbourg 12, 277 1566; - Ref. dariiber Fortschr. d. Phy8. 23, $266(1 S 67)$.

Petruscheffsky, Th. Einige Worte iber eine spectrophotometrische Untersuchung der Mondoberflache. Astr. Nachr. 91, Nr. 2173 (18is.

Pickering, E. C. und Strange, D. P. Light absorbed by the atmosphere of the Sun. Proe. Amer. Acad. New Ser. 2, 428 (1S74-75).

Pickering, W. H. 'Total eclipse of the Sun, Aug. 29, 1,56. Siche den Abschnitt: Brightness of the corona. Ann. Ilarv. Coll. Obs. 18, $100(1556-1590 \%$

Provenzali, P. F. S. Sulla misura dell' intensitil della luce solare. Atti dell' accul. Pont. dei nuovi Lincei 25, 32, 311 (1572); $26,245(1573)$.

Roscoe, H. E. On the measurement of the chemical brightness of various portions of the Sun's disk. Proc. R. Soc. London 12, wh 14ti3: - Dentsch in Pogg. Ann. 120, 331 (1863).

Rosse, L. P. On the radiation of heat from the Mloon, the law of its absorption by our atmosphere and its variation in amount with her phase. Phil. Trans. 163, 587 (1873).

Secchi, A. Sull' intensiti del calore nelle virie parti del disco solare. Memor. dell' Osserv. del Coll. Roman. 1851, App. 3 und App. ¿. - Astr. Nachr. 34. Nr. 806 (1852); 35, Nr. $\$ 33$ (1553.

Sur l'intensité lumineuse des diverses parties du disque solaire. Compt. Rend. 49, $931(1959) ; 62,1060(1>66)$. 
Secchi, A. Recenti ricerche intorno alla distribuzione del calore sul disco solare. Mem. della Soc. d. Spettroc. Ital. 4, 121 (187j).

Seidel, L. Über die Helligkeit der Sonne, verglichen mit Sternen, und über die Licht reflectirende Kraft der Planeten und des Mondes. Abh. der Münchener Akad. II. Cl., 6, 623 (1S52).

'Thomson, W. Approximative photometric measurements of Sun, Moon, cloudy sky and electric and other artificial lights. Nature 27, 277 (1883).

Very, F. W. Photometry of a Lunar eclipse. Astrophys. Journ. 2, 293 (1895).

Violle, J. Mémoire sur la température moyenne de la surface du soleil. Ann. Chim. et Phys. (5) 10, 289 (1877).

Vogel, H. C. Über die Absorption der chemisch wirksamen Strahlen in der Atmosphäre der Sonne. Verhandl. der Sächs. Ges. d. Wiss. II. Cl., 24, 135 (1572); Pogg. Ann. 148, 161.

- Spectralphotometrische Untersuchungen, insbesondere zur Bestimmung der Absorption der die Sonne umgebenden Gashiille. Monatsber. der Berliner Akad. 1877, 104.

Weber, L. Photometrische Beobachtungen während der Sonnenfinsterniss 1587, Aug. 1S-19. Astr. Nachr. 118, Nr. 2810 (1SSS).

\section{Planeten und Satelliten.}

Albert, L. A. Über die Berechnung des grössten Glanzes der Venus. Gruithnisen's naturw.-astr. Jahrbuch 5, 101 1S43-44.

Alexander, Stephen. [Mittheilungen über das Aussehen und die Helligkeit der Japitertrabanten bei ihren Voriibergängen vor der Jupiterscheibe.] Astr. Nachr. 83, Nr. 1986; 84, Nr. $20121 \varsigma$ 14.

Arago, Fr. Untersuchung des Lichtes des Jupiter und seiner Monde. Arago's Werke, deutsche Ausg. 10, 241 (1843).

Arcimis, A. T. On the visibility of the unilluminated portion of the disk of Venus. Monthly Not. 37, 259 167i).

Argelander. Fr. Über die Lelligkeiten der kleinen Planeten. Astr. Nachr. 41, Nr. 9521855.

- Vorschlag zu Beobachtnngen iiber die Helligkeiten der kleinen Planeten. Astr. Nachr. 42, Nr. $996,1556$.

Bailly, J. S. Némoire sur les inégalités de la lumière des satellites de Jupiter, sur la mesure de leurs diamétres et sur un moyen, anssi simple que commode, de rendre les olsservations comparables. en remédiant à la différence des rues et des lunettes. Mém. de l'acad. R. des sciences de Paris 1771, 550.

Baldwin, H. L. Visibility of Venus in the daytine. Observatory $\mathbf{3}, 573 \quad(1890)$. Beer. W. und Mädler. J.H. Beiträge zur physischen Kenntniss der himmlischen Körper im Sonnensysteme. Weimar 1S41, p. 101.

Bond, G. P. On the light of the Sum. Moon, Jupiter and Venus. Monthly Not. 21, 197 1\$61!.

Bremiker. C. Über die grüsste Helligkeit der Venus.] Monatsber. d. Berliner Akad. 1560, 706.

Burtou. C. E. Note on the appenrence presented by the fourth satellite of Jupiter in transit in the years 1si-1si3. Nonthly Not. 33. 472 1873).

Cassini. Dom. Réflexions sur les observations des satellites de Saturne et de son annean. Mém. de lacad. des sciences de Paris 1705, 14. 
Christie, W. H. M. Note on the gradation of light on the disk of Venus. Monthly Not. 87, 90 (1SiT).

Note on specular reflexion from Venus. Monthly Not. 38, 105 (16is).

Cornu, A. Sur la possibilité d'uccroitre dans une grunde proportion la précision des observations des éclipses des satellites de Jupiter. Compt. Rend. O6, 1609 (1883).

- Sur les inéthodes photonétriques d'observation des satellites de Jupiter. Astr. Nachr. 114, Nr. $272 i$ (1886).

Cornu, A. et Obrecht, A. Études expérimentales relatives á l'observation photométrique dos éclipses des sutellites de Jupiter. Compt. Rend. 96, 1815 (1583).

Dawes, W, R. On the appearance of Jupiter's satellites while transiting the lisk of the planet. Mfontlily Not. 20, $245\{1860\}$.

Dennett, F.C. Jupiter's satellites. Astr. Register 17, 45 (1579).

Donning, W. F. Note on the visibility of Jupiter. Monthly Not. 38, 179 (1873).

- Naked-eye observations of Jupiter's satellites. Monthly Not. 84, 309 (1574).

Visibility of Mereury and of Venus in sunshine. Monthly Not, 36, 345 (1876).

Jupiter's third satellite in transit, April 11, 1586. Monthly Not. 46, 394 (1856).

Draper, H. On a photograph of Jupiter's spectrum showing evidence of intriusic light from that planet. Monthly Not. 40, 433 (1580).

Engelmann, R. Über die Helligkeitsverhailtnisse der Jupiterstrabanten. Habilitationsschrift. Leipzig, 1871.

Erck, W. Satellite of Mars. [Helligkeit von Deimos.] Astr. Register 16, 20 (1879). Ferguson, J. Resuits of observations for determining the relative brightness of the asteroids made with the Washington equatoreal. Astr. Nachr. 34, Nr. 802 (1852).

Flammarion, C. Phénomènes observés sur les satellites de Jupiter. Compt. Rend. 78, 1295 (1574):

- Sur les changements d'éclat des satellites de Jupiter. Compt. Rend. 79, 1490 (1574).

Observation des satellites de Jupiter pendant les oppositions de $15 i \cdot 4$ et 18 is. Détermination de leurs différences d'aspect et de leurs variations d'éelat. Compt. Rend. 81, 145 (15i5).

— Variations d'éclat du quatrième satellite de Jupiter. Déductions relativess it sa constitution physique et it son monvement de rotation. Compt. Rend. 81. $233(1875)$.

v. Glasenapp, S. Observations des satellites de Jupiter. Bull. Acad. St.-Pétersb. $18,90\langle 1873)$.

Grunert. J. A. Venus im grössten Glanze. Grunert's Archiv der Math. u. Phys. 20, $288(1853)$.

Hall, A. Observations and orbits of the satellites of Mars. Washington, 1675.

Hall, Maxwell. Variation in the light of Neptune. from Nov, 29) to Dece 14. 14a3. Monthly Not. 44, 25i 1854).

Halley, E. An account of the late remarkable appearance of the planet Venns, seen this summer for many days together in the daytime. Phil. 'Trans. $29.466 \mathbf{1 7 1}$ ).

Harding, K. L. Beobachtungen der Nachtseite der Venuskunel. Bodes astr. Jahrb. fiir 1809. 167 .

Harrington, M. W. A brief study of Vestal. American Journ. 3 26.461 15533. Heis, E. Die Venus in ihrem grüssten Glanze. Unterhalt. im Gehiete der Astron., Geogr. u. Meteor. 11. 951557. 
Herschel, A.S. Wheu is Venus briglitest? Quarterly Journ. of pure and applied mathematics 4, 232 (1861).

Herschel, W. On the ring of Saturn and the rotation of the fifth satellite upon its axis. Phil. Trans. 82, 1 (1792). - Theilweise übersetzt in Bodes astr. Jahrb. füir $1796,88$.

Observations of the changeable brightness of the satellites of Jupiter and of the variation in their apparent magnitudes; with a deterwination of the time of their rotatory motions on their axes. Phil. Trans. 87, part II, 332 (1797).

Hold en, E. S. On the inner satellites of Uranus. Monthly Not. 35, 16 (18i5).

- Note on the brightness and the stellar magnitude of the third Saturnian satellite 'Tethys. American Journ. (3) 17, 49 (1879).

Huggins, W. On the periodical changes in the belts and surface of Jupiter. Monthly Not. 22, 294 (1862).

Kies, J. Sur le plus grand éclat de Vénus, en supposant son orbite et celle de la Terre elliptique. Hist. et Mém. de l'acad. de Berlin, 1750, 218.

Klein, H. J. Über das secundäre Licht der Venus. Kleins Wochenschrift, Neue Folge 10, 329 (1867).

- Über dic Helligkeitsverhältnisse der Jupitersmonde. Astr. Nachr. 71, Nir. 1684 (1865).

Kononowitsch, A. K. Photometrische Untersuchungen der Planeten Mars, Jupiter und Saturn. Memoiren der K. Neurussischen Univers. 37. Odessa, 1883. (In russ. Sprache.

_ Über die Albedo des Planeten Mars. Astrou. Nachr. 109. Nr. 2604 (18S4).

Lalande, J. Sur le diamètre et la lumière du quatriẻme satellite de Jupiter. Mém. de l'acad. $R$. des sciences de Paris 178S, 209.

Lambert, H. Vom Glanze del Veuts. Berl. astr. Jahrb. fuir 1780, Theil 2, 59.

Lassell, W. Physical observations of Jupiter's satellites. Monthly Not. 20, 5 i 1560 ;

Leslie, J. Remarks on the light of the Moon and of the planets. Edinburgh Philos. Journ. 11, 393 1524. - Deutsch in Schweiggers Journ. f. Chemie u. Phys. 43, 185 1825.

Lindsay. J. B. On the relatire star magnitude of Mars in February and March 1850. MLonthly Not. 40, 3פ0 1550 .

Marth, A. Note referring to observations and estimations of the brightness of Mars. which ought to be made in February and March 1sso. Monthly Not. 40, $1 j 9\langle 1550$.

- Note on the computation of the brightness of the planets with some ephemerides for the observations of the brightuess of Mercury. Montlily Not. 54, 388 (1594.

Miiler. G. Helligkeitsmessungen des Planeten Neptun. Astr. Nachr. 109. Nr. 2600 $185 t$.

Resultate aus Helligkeitsmessungen des Planeten Saturn. Astr. Nachr. 110, $\mathrm{Nr} .2631$ 1555.

- Beobachtungen über den Einfluss der Phase auf die Lichtstärke kleiner Planeten. Astr. Narhr. 114, Nr. 2724-2725 1556.

- Über den grössten Glanz der Venus. Astr. Nachr. 132. Nr. 3162 1593;.

- Helligkeitsbestimmungen der grossen Planeten und einiger Asteroiden. Publ.

d. Astroplycs Obs. Potsdam \$. 193 1593.

Über die Lichtstäke des Planeten Mercur. Astr. Nachr. 133. Nr. 3171 (1593. 
Mlller, G. Helligkeitsänderungeu der l'luneten (2) Meleto und (i) Niobe. Astr. Nachr. 135, Nr. 3227 (1894:-

Nasmyth, J. Relative brightuess of Venus and Morcury. Observatory 2, 225 (1579\%. Neison, E. On the ntmosplere of Venus. Monthly Not. 36, 3471576.

On the position of the point of maximum brightness on Venus. Monthly Not. 87, 59 (1577).

Noble, W. On the appearance of Jupiter's thirul sutellite on the disk of the plnnet. Monthly Not. 20, 24i (1560).

Observations of Venus. Monthly Not. 36, 350 1576 .

Obroeht, A. Observation photométrique d'une éclipse dı premier satellite lo Jupiter. Compt. Rend. 97, 1128 (1553).

Olbers, W. Mars und Aldebaran. v. Zatehs monatl. Corresp. 8, 293 (1803).

Parkhurst, H. M. Photometric observations of asteroids. Anmals Harr. Coll. Obs. 15, $29(1890)$; 29, 65 (1593); Astron. Journ. 9, Nr. 20 S $(1590$ ).

Poters, C. H. F. Über die Helligkeit der Frigga (7). Astr. Nachr. 97, Nr. 2314 $1550 \%$.

- Zur Geschichte photometrischer Beobachtungen der Jupiterstrabanten - Verfinsturungen. Astr. Nachr. 114, Nr. 2721 (1566).

Pickering, E. C. Conjunction of planets. Annals IIarr. Coll. Obs. 11, 9S (1S79). - [Photometrische Messungen der Satelliten von Mars, Jupiter, Saturn, Uranus und Neptun.] Anuals Harv. Coll. Observ. 1111, 226-276, 311 (1579).

Photometric observations of planets and of Jupiter's satellite III, made at the Harvard College Observatory. Astr. Nachr. 102. Nr. 2434 1882;.

- Photometric observations of the satellites of Mars, made at the Harvard College Observatory 1881-\$2. Astr. Nachr. 102, Nr. 2437 (1882.

- Photometric observations of Neptune at the Harvard College Observatory. Observatory 7, 134 (1881).

Photometric observations of Ceres (1), Pallas (2) and Vesta (1) at the llarvard College Obscrvatory. Observatory 8, 238 (1555.

Plummer, J. Photometric experiments upon the light of Venus. Nonthly iot. 36, 351 (1S76).

Pogson, N. Magnitude constants for fiftly-seven of the minor plinets. Monthly Not. 21, 33 (1861).

Ranyard, A. C. On periodical changes in the plysical condition of Jupiter. Monthly Not. 31, 34, 224 (157).

Rheinauer, J. Die Erleuchtung des Planeten Venus durch die Erde. Freiburg i, 13 . 1859. Beigabe zum Programm des Gymnasiums in Offenburg, 155!?.

Roberts, G. W. Observation of transit of Jupiter's fourth satellite. Monthly Not. $33,412(1873)$.

Rodgers, J. Observations of the brightness of the satellites of Lrams. Anerican Jonrn. (3) 15, 195 (15is.

Rogerson, G. R. On the visibility of Oberon and Titania. Honthly Not. 36, 331 (1876,

Safarik, A. Über die Sichtbarkeit der dunkelen Halbkugel der Venns. Sitzungsher. der K. Bölım. Aliad. 1573, Juli.

Sehmidt, J. F. J. Helligkeit des Planeten Mars. Astr. Nachr. 97. Nr. 2310 , 15s0, Schön feld, E. [Mittheilung der Kirch'sehen Beobachtungen des aschgramen Lichtes der Venus.] Astr. Nachr. 67, Nr. 1556 (1566.

- - Über eine iiltere Ilelligkeitsbestimmung des Pianeten Saturn. Astr. Nachr. 67, Nr. $1592 / 1866$. 
Schröter, J. H. Fragmente zur genaueren Kenntniss der Jupiterstrabanten, ihrer Naturanlage, wahren Grössenverhältnisse, Rotationsperioden und Atmosphären.

Beitrïge zu den neuesten astron. Entdecknngen, Bd. 2. Göttingen, 179s.

- Beobachtung der Nachtseite der Venuskugel. Bodes astr. Jahrb. für 1809, 164.

Seechi, A. Ricerche sopra il pianeta Giove. Mem.' dell' Osserv. Coll. Romano $1852-55,114$.

Seeliger, H. Zur Reduction von photometrischen Messungen des Saturn. Astr. Nachr. 110, Nr. 2639 (1885).

Seidel, L. Untersuchungen über die Lichtstärke der Planeten Venus, Mars, Jupiter and Saturn, verglichen mit Sternen, und iiber die relative Weisse ihrer Oberflichen. Monumenta saecnlaria der Münchener Akad. II. Classe (1859;.

Spätb,'J. L. Photometrische Untersuchung 'über die Beobachtungen der Verfinsterungen der Jupitersmonde. Bodes astr. Jahrb. für 1795, 153.

Spitta, E. J. The fourth satellite of Jupiter during superior conjunetion on the night of April 5, 1\$s6. Monthly Not. 46, 451 (15\$6).

- On the appearances presented by the satellites of Jupiter during transit, with a photometric estimation of their relative albedos, and of the amount of light reflected from the different portions of an unpolished sphere. Monthly Not. 48, 32 (18SS.

Stampfer, S. Über die kleinen Planeten zwischen Uars und Jupiter. Sitzungsber. der Wiener Akad. II. Cl. 7. 756 1551).

Stone, E. J. Approximate relative dinensions of seventy-one of the asteroids. Monthly Not. 2i, $302,186 \bar{i}$.

Tebbutt, J. Observations of Jupiter's third satellite. Monthly Not. 34, i3 (1Sit); 3S. 73 18is!.

Tietjen, F. [Grössensch:itzungen der Planeten Welete und Niobe.] Astr. Nachr.

57. Nr. 1359 (1862).

Vogel. H: C. Über die Sichtbarkeit der Uranusmonde in Fernröhren mittlerer Grösse. Astr. Nachr. S7, Nr. $2069\langle 1576$.

Webb, T. W. Dark side of Venus. Astr. Register 16. 76 1579\%.

Winnecke. A. Wotiz betreffend die Sichtbarkeit des unbeleuchteten Theiles der Venusscheibe um Mittag.) Astr. Nachr. 7S, Nr. 1863 (1872.

Beobachtungen wiibrend der Conjunction von Mercur und Venus am 30. Sept. $157 \pi$, angestellt auf der provisorischen Lniversitiitssternwarte zu Strassburg. Astr. Nachr. 94. Nr. 2245 1979.

Wittstein, 'T. Das grüsste Licht der Yenus. Heis' Wochenschrift 6, 243 (1S63), Wurm, J. F. Über den grüssten Glanz des Nereurs. Berl. astr. Jahrb. für $\mathbf{1 7 9 7}$, 137,145 .

Über den grüssten Glanz der Tenus sammt Tafeln für diese periodische Erscheinung. v. Zachs allgem. geogr. Ephem. 2. 30.5179.

- Allgemeine Tafeln. um die grössten Digressionen der Venus. ihre oberen und unteren Conjunctionen, auch die Zeiten ihres grössten Glanzes. für alle Jahrbunderte zu berechnen. Bodes astr. Jahrb. für 150: 153.

Zenger. C. Y. Absorption of the light of Yenus by dark violet glass plates. Ionthly Not. 37. 460 15i5).

_- On a new astrophotometrical method. Helligkeitsbest. der Jupiterscheibe und der Tupitertrabanten. Nonthly Not. 38. 65 1sis

- On the risibility of the dark side of Venus. Ifonthly Not. 43, 331 1953. 
Zülner. F. Photometrisehe Uutersucbungen liber die plyssische Beschaffenheit dea Planeten Mercur. Pogg. Anu. Jubelbanil, 6i2. (15ii).

\section{Cometen und Nebelfecke.}

d'Arrest, H. Vorliufigo Mittheilungen, betreffend eine auf der Kopenhagener Stern. warte begounene Revision des Himmels in Bezug nuf die Nubelflecken. Astr. Naclır. 5\%, Nr. 1366 (1862).

Auffindung oines zweiten variablen Nebelflecks in Stier. Astr. Nuchr. bs, Nir. 1378 (IS62).

Auffindung eines dritton variablen Nebelfecks. Astr. Nachr. 58, Nr. 1339 1562. Über den Nebel bei Merope und oinen zweiten Nebel in den Plejaden. Astr. Nachr. 59, Nr. 1393 (1863).

Uiber einen angeblich von Maskelyne beobachteten, gegenwirtig unsiclitharen Nebelfleek. Astr. Nachr. 60, Nr. 1440 (1863).

- Strnve's Beobachtung eines neuen Nebelflecks nahe bei Hind's variablem Nebel in 'Inurus. Astr. Nachr. 71, Nr. 1659 (1S6S).

A 11 wers, A. [Bemerkungen iber drei der Verïnderlichleit verdichtige Nebel.] Astr. Nachr. 58, Nr. 1391 (1562).

Backhouse, 'T. W. The relative brightness of comets. Observatory 16, is (1593) Barnard, E. E. 'I'wo probable variable nebulae. Astr. Nachr. 130, Nr. 3097 (1592). - On the variable nebulae of Hind G.C. 1555) and Struve G.C. 1554) in Tuurus and on the nebulous condition of the variable star ' $T$ 'auri. Monthly Not. 55, 442 (1595).

- Invisibility of Hind's variable nebula (G. C. 1555). Monthly Not. 66. 66; (1596). Berberich, A. Die Helligkeit des Eneke'sehen Cometen. Astr. Nachr. 11!, Nr, 2sist; $\because-37$ (1558).

Bessel, F. W. Beobaehtungen iber die physische Beschatfenheit des llalley'schen liometen und dadureh veranlasste Bumerkungen. Astr. Nachr. 13. Nr. 30(1-31)? (1536).

Bruhns, C. Bemerkungen iiber die Erscheinung des Cometen V. 14j4. Astr. Ninchur. 51, Nr. 1205 (1S59.

Burnham, S. W. Note on Hind's varible nebula in 'lanlus. Montluly Not. מI, 14 (1591).

Chacornae, J. On the missing nebula in Coma Berenices. Monthly Not. 2a, $2 \pi(1562)$

Chandler, S. C. On the outburst in the light of the Comet l'ons-13rouks. Sint. 21-2:3. Astr. Nachr. 107. Nr. 2553 lint.

Deichuiller, Fr. Über die Vormsberechnung der Comptun-llolligkeiten. Astr. Nacbr. 131, Nr. $3123 \quad 15 ! 13$

Zur Photometrie der Cometen. Astr. Nitchr. 131. Ni. 313:1 1-41\%.

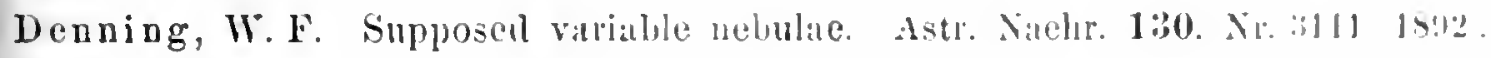

Dreyer, J. L. E. On some nebulac litherto suspecten of variability or propere motion. Monthly Not. 47. 112 1657.

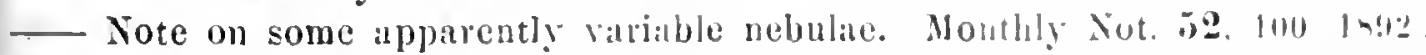

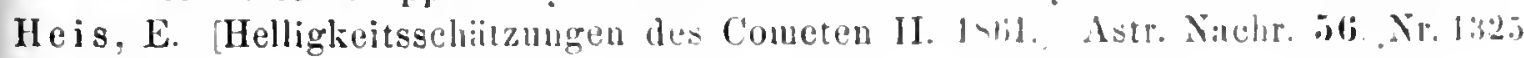
(1562).

Hersehel, J. On the disappearence of al Hebula in Comal Berenices. Mlonthly Not. 22. 2451562 . 
Hind, J. R. Note on the variable nebula in Taurus. Monthly Not. 24, 65 (1864). Holden, E.S. Monograph of the central parts of the nebula of Orion. Part II: Reduction of photometric observations made at Washington. Wash. Obs. 1878, App. I, 191.

Holetschek, J. Helligkeitsschätzungen der Cometen $18 \varepsilon 6$ I (Fabry) und 1886 II (Barnard). Astr. Nachr. 115, Nr. 2739 (1886).

Über die Beobachtung und Berechnung von Cometen-Helligkeiten. Astr. Nachr. 131, Nr. 3135 (1893).

Über die Berechning von Cometenhelligkeiten, insbesondere fïr periodische Cometen. Astr. Nachr. 135, Nr. 3237 (1894).

— Untersuchungen iiber die Grösse und Helligkeit der Cometen und ihrer Schweife. I. Die Cometen bis zun Jahre 1760. Denkschr. del Wiener Akad. II. Cl. 63, $317\langle\mathbf{1 8 9 6}$.

Huggins, W. Further observations on the spectra of some of the nebulae with a mode of determining the brightness of these bodies. Phil. Trans. 156, 381 (1866).

Knobe1, E. B. Note on the comparative brightness of the nebula of Orion. Monthly Not. 41, 312 (1881).

I iiller, G. Photometrische Beobachtungen des Cometen 1882 Wells. Astr. Nachr. 103, Nr. 2453 (1882).

___ Über einen zweiten merkwiirdigen Lichtausbruch an dem Cometen Pons-Brooks. Astr. Nachr. 107, Nr. $256 \mathrm{~S}$ (1SS4).

- Photometrische Beobachtungen des Cometen Pons-Brooks. Astr. Nachr. 108, Nr. 2579 (1S54).

- Über die Helligkeit der Cometen 18s6, Fabry und Barnard. Astr. Nachr. 114, Nr. $2733(1 £ 86)$.

Olbers, W. Einige Bemerkungen über das Licht der Cometen. Berl. astr. Jahrb. für $\mathbf{1 8 1 9}, 190$.

Paschen, F. [Helligkeitsvergleichungen der beiden Biela'schen Cometen, 1846.] Astr. Nachr. 24, Nr. 562 (1546).

Pickering, E. C. Light of Webb's planetary nebula, D. M. $+41^{\circ}, 4004$. Nature 21, $346(1880$.

Roberts, J. Photographic evidence of variability in the nucleus of the great nebula in Audromedi. Nonthly Not. 51, 116,1691 .

Saw yer, E. F. The apparent brightness of comet b, 1593. Astr. Journ. 13, Nr. 30 j 1894).

S chmidt, J. F. J. [Helligkeitsschïtzungen des Petersen'schen Cometen, 1S50.] Astr. Nachr. :1, Nr. $736 \quad 15.51$.

____ Über den von Klinkerfues entdeckten Cometen, 1553. Astr. Nachr. 37, Nr. 853 1654 ).

_ Bemerkungen iiber den Cometen im April 1554. Astr. Nachr. 38, Nr. 911 (1854). [Helligkeitsschätzungen des Brorsen'schen Cometen, 1S57.] Astr. Nachr. 46, $\mathrm{Nr} .1090 \quad 1857$.

- Über veründerliche Nebelgestirne. Astr. Nachr. 57, Nr. $1360 / 1 \$ 62$. Über die Sichtharkeit des Nelels in den Plejaden.] Astr. Nachr. 58. Nr. 1391 18 riv.

- [Helligkeit des Cometen II, 1662.] Astr. Nachr. 59, Nr. 1395 1663. Beobachtungen iiber den grossen Cometen im Jahre 1574. Astr. Nachr. 87. Nr. 20671676. 
Schðnfold, E. Uber len Nobelfeck $+30^{\circ}, 548$ des Bonner Sternverzeichnisses, mit einigen Bemerkungen ilber die Nobelbeobachtungen in der Bonuer Durchmusterung ilberhaupt. Astr. Nachr. 58, Nr. 1391 (1862).

Schultz, H. [Bemerkungon Ilbor oinen wahrscheinlich verïnderlichen Nebelfeck.] Astr. Nachr. 65, Nr. 1556 (1865).

Schwab, Fr. Beobachtungen Hber dle Helligkeit und den Schweif der Coneten 1881, III und IV. Astr. Nachr. 101, Nr. 2412 (1582).

Stone, 0 . Herschel's estimates of l,rightuess of nebulas expressed in magnitudes. Astr. Jourd. 13, Nr. 294 (1894).

Struve, 0. On the missing nobula in Taurus. Monthly Not. 22, 242 (1562).

Winnecke, A. On tho evidence of periodic variability of the nebula H. II, 2 is. Monthly Not. 38, 104 (15is).

[Bemerkungen ubor zwei cler Veriinderlichkeit verdichtige Nobel.] Astr. Nachr. 59, Nr. 1397 (1863).

Uber die periodische Verïnderlichkeit in der Helligkeit des Nebelflecks h $\$ \$ 2$, nebst einigen Bemerkungen llber andero Nebolflecko. Astr. Nachr. 96, Nr. 2293 (1580).

Wolff, Th. Photometrische Beobaclitungen des Cometen Pons-Brooks. Astr. Nuchr. 10S, Nr. 2583 (15S4).

\section{Fixsterno.}

NB. In Betreff der iberaus umfangreichen Litteratur iiber die Beobachtungen de⿰ einzelnen veränderlichen Sterne ist auf die von $\mathbf{K}$ nobel gegebene Übersicht (Monthly Not. 36, 372 [1876]), ferner anf die Generalrogister der Astronomischen Nachrichten und die Einzelregister des Astronomical Journal zu verweisen.

Abney, W. On crrors that may arise in estimating star magniturles by photography. Monthly Not. 54, 65 (1894).

Argelander, Fr. Neue Uranometric. Darstellung der im nittleren Europa nit blossen Augen sichtbaren Sterne nach ihren wahren, unmittelbar vom Himmel entnommenen Grössen. Sternverzeichniss und Atlas. Berlin, 1513.

- Aufforderung an Freunde der Astronomie zur Anstellung von eb nso interessanten und nuitzlichen, als leicht auszufiihrenden Beobachtungen iiher mehrere wichtige Zweige der Himmelsknnde. Schumachers Jahrbuch fiir 1S44, 122.

De stella $\beta$ Lyrac variabili disquisitio. Bonnae, 1 . 1.

De stella $\beta$ Lyrae variabili commentatio altera. Bonnac, $15 \% 9$.

Beobachtungen und Rechnungen iiber verinderliche Sterne. Astron. Beob. auf der Sternw. Bonn. Bd. 7, 315 (1 569 ).

Auwers, A. Bemerkungen iiber die sogenannten nenen Sterne und Beobachtungen der Nova Scorpii von 1860. Astr. Naclır. 114, Nr. 2715 1sh(i).

Ceraski, W. Photometrisehe Beobachtungen. Annales de l'Ohis. de Moscour. Qu,

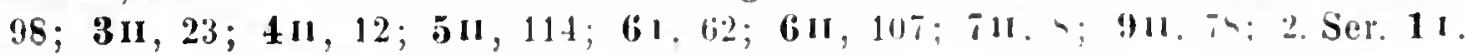
71; 2. Ser. 111,$83 ; 2$ Ser. 31 , $70(1876-1593$.

Über die Berechnung der Beobachtungen von verïinderlichen Sternou. Astl. Nachr. 99, Nr. 2371 (1\$81.

Über die Bercehnung des Liehtverhailtnisses für sterne von aul cinamler fol. genden Grössenclassen. Annales de l'Obs. de Moseou 10 11. 1.5.) 1501.

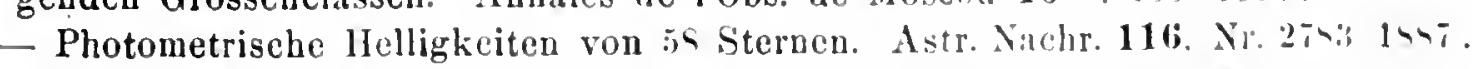
Observations photométriques de l'étoile nouvelle alparue dans la constellation du cocher. Aunales de lObs. de Moseon 2. Ser. :11, 107 1 16:33. 
Chambers, G. F. A catalogne of rariable stars. Astr. Nachr. 63, Nr. 1496 (1965; Nonthly Not. 95, 205 1565:

Chandler, S. C. On the light-ratio unit of stellar magnitudes. Astr. Nachr. 115, Nr. $2746,1 \leq s 6$.

- Inrestigation of the light rariations of $U$ Ophiachi. Astr. Jonrn. 7. Nr. 161 u. 1621555 .

On the period of Algol. Astr. Journ. 7, Nr. 165-167 (15ss).

Catalogue of variable stars. Astr. Jouru. S. Mr. 179-150 1599.

On the obserration of the fainter minima of the telescopic Variables. Astr. Journ. S, Nr. $1 \$ 3$ 1 $15 \$ 9$.

- On some remarkable anomalies in the period of 1 Crgni. Astr. Journ. $\mathbf{3}$, Tr. 155 . 1559 .

On the colors of the rariable stars. Astr. Journ. S. Nr. 156 1ss9;.

Contributions to the knomledge of the inequalities in the periods of the rariable siars. Asir. Journ. S. Mr. 159 11. 190 1ss9:9. Nr. 205 1590:10. Nr. 229 1591; 11. Ir. 242. 255.2501292.

- On the general relations of variable star phenomeua. Astr. Journ. 9, Nr. 193 1590.

- On the light rariations of $t^{2}$ Cephei. Astr. Journ. 9. Mr. 199 '1590.

- Sppplemeut to irst eüition of the caialogue of variable stars. Astr. Journ. 9, I.- 216 1500.

- Un the observations of rariable stars with the meridian-photometer of the

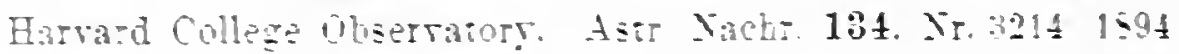

- On the Hartara phorometric obserations. Astr. Yachr. 136. Mr. 3246 '1594.

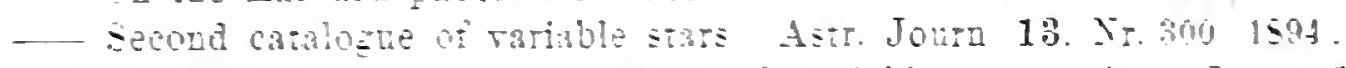

- Supplemen: to second catalogne of rariable siars. Asir. Jouru. 14. Mr. 319 105

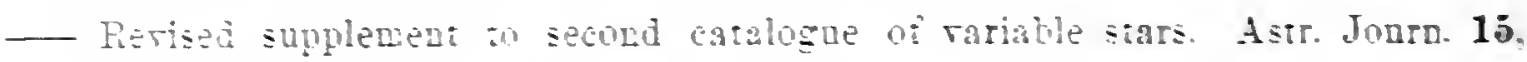
X. $4: 1998$

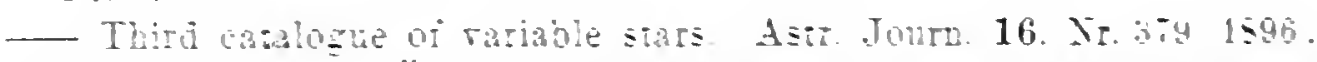

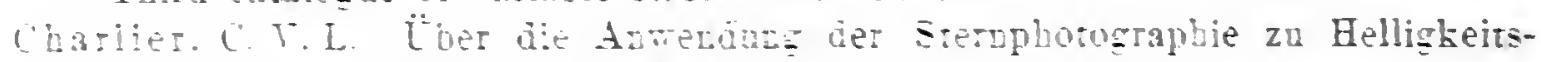

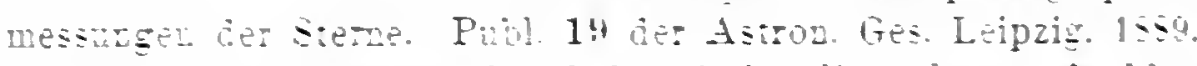

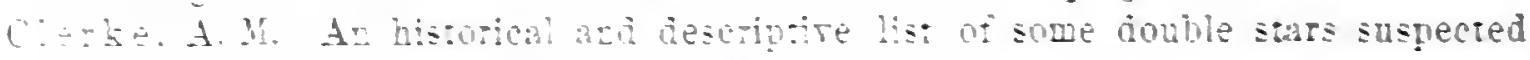

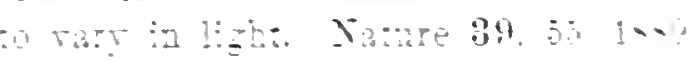

If

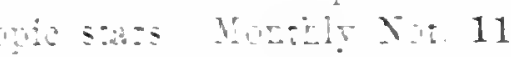

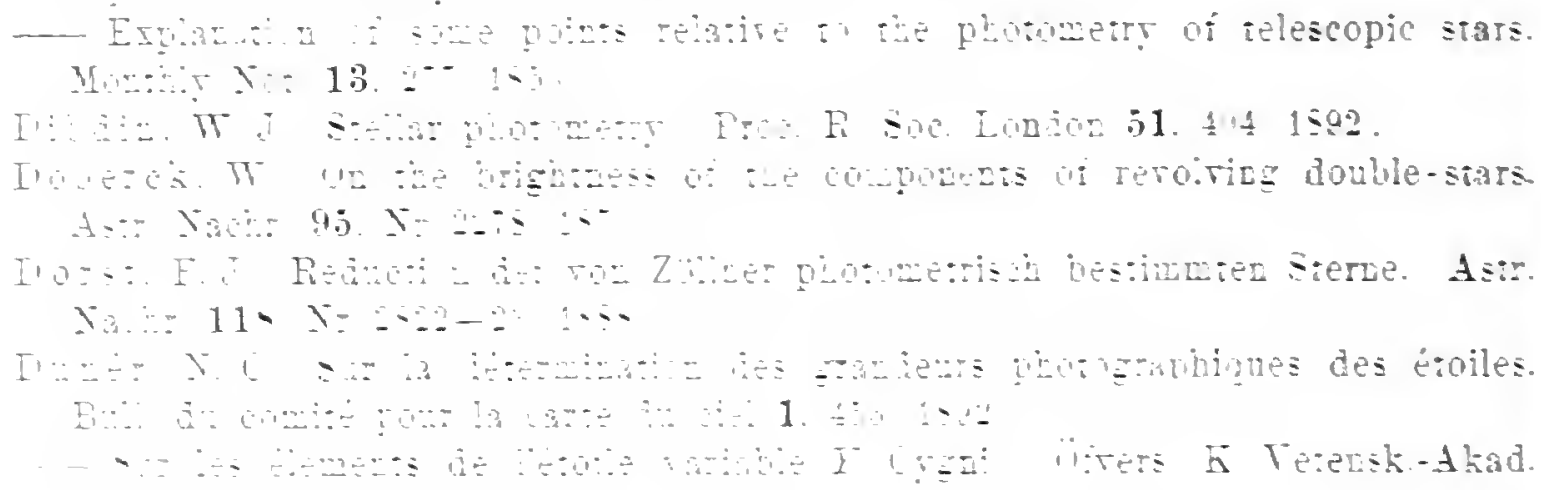

$1 \div \div 2 \div \div$ 
Espid. T. E. The distribution of the rariable stars. Observatory \&, 230 19s1; 5,7 1652:

Gore. J.E A catalogue of known rariable stars. With notes and olservations. Proc Irish Acad. $\$$ \$, 149 1ss4.

A catalogue of suspected rariable stars. With notes and observatiocs. Proc. Irisb Acad. (2 4, 26: 1554.

A revised catalogue of rariable stars, with wotes and obserrations Proc. Irish Acad. (3! 1, 9; (15s:?

Gould, B. A. Urancmetria Argentina. Brightuess and position of every fixed stat, down to the sereoth magaitude. within one bundred degrees of the south pole. With an àtlas. Buenos Aires, $15 i 9$.

Ueis, E. De magnitadine relatira numeroque acenrato stellarnm quae solis oculis conspiciantur fixaram. Colonise, 1552.

Seuer Himmels-Atlas. Darstellung der im wittleren Europa mit blossen Augen sichtbaren Sterne nach ibren wabren, unmittelbar rom Himmel entnommenen Grüssen. Sterarerzejchniss und Atlas. Küln, 15:2.

Herschel, J. Astrometry, or the numerical expression of the apparent magnitndes of the stars. Results of astr. obs. made daring the years 1 SH-1\$35 at the Cape of Good Hope. Loudon, 1S47. chapter 111, 304.

Herschel, W. On the wethod of observing the changes that happen to the fired stars; with some remarks on the stability of the light of our Sun. To which is added a catalogue of comparatire brightness, for sscertaining the permanency of the lustre of stars. Phil. Trans. \$6, 166 (1796.

On the periodical star a Herculis, with remarks tending to establish the rotatory motion of the stars on their ares. To which is added a second catalogue of the comparatire brightness of the stars. Phil. Trans. $\$ 6,1521796$.

A third catalogue of the comparatire brightness of the stars etc. Phil. Trans. S7. 2931797.

A fourth catalogue of the comparative brightness of the stars. Phil. Trans. \$9. $121 / 799$.

Holden. E. S. Note on a relation between the colors and magnitudes of the components of binary stars. Amer. Journ. 3 19. thit $1: 0$

- Sur la détermination des gradeurs stellaires a loaide de la photographie. Bull. du comité pour la carte da ciel 1. 2011 1592

Honzeau. J.C. Cranowétrie génerale arec une étude sur la jistriburion des étoiles risibles a l'oil nu. Annales de lons de Brustlles. Sour. Śr. 1 lsi

Jäger. G. Über die Beziehung zwischen Helligkei cad Eigenteregung der Fixsterne. Sizungsber. der Wiener Akad 11. Cl. 10311a. 145 1-94.

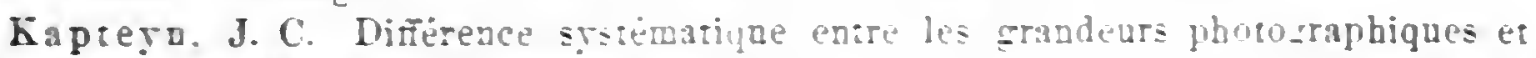
visuelles dass les difiérentes région du ciel. Bali a comiti pour la carte du ciel :. 131 159\%

Klinkerfues. E. F. II. Zbe deg I,ichtwechsel der Verinderlicher. Nachrichten der K. Ges. d. Wis: Gütim_ten $196 \%$. 1

Knobel. E. On Al-sint's star maguirudes. Monthly Nut 45. 417 ls.5.

r. Küresligethy. R. Beirage zur Erkeznidis der linur variabler Sterne. Astr. Nachr. 10S. Nr. $25 \leq 5$ 1s:4.

Lindemann. E. Ïeber Holligkeirsbestimmangen ron Fixseraen mis dem Züllaerschen Photoneter und durch stafenschäzungey. Bnll. Acai. Si-Pétersb. $\mathbf{Q} 0$. $3: 815 \% 3$. 
Lindemann, E. Zur Beurtheilung der Veränderlichkeit rother Sterne. Mém. Acad. St.-Pétersb. (7) 30, Nr. 4 (1882).

Über den Lichtwechsel des Sterns $V$ Cygni. Bull. Acad. St.-Pétersb. 29, 302 (1884).

Helligkeitsmessungen der Bessel'schen Plejadensterne. Mém. Acad. St.-Pétersb.

(7) 32, Nr. 6 (1884).

Die Grössenclassen der Bonner Durchmusterung. Astr. Nachr. 118, Nr. 2816 (1888). Photometrische Bestịmmung der Grössenclassen der Bonner Durchmusterung. Supplément II aux Observations de Poulkova. St.-Pétersb. 1889.

Ü̉ber eine von Prof. Ceraski angedentete persönliche Gleichung bei Helligkeitsvergleichungen dér Sterne. Bull. Acad. St.-Pétersb. Nouv. Sér. II (34), $77(1892)$.

__ Die Liehtcurve des neuen Sterns von 1892 ( $T$ Aurigae). Bull. Acad. St.-Pétersb. Nouv. Sér. III (35), 507 (1894).

Helligkeitsmessungen im Sternhaufen $\hbar$ Persei. Bnll. Acad. St.-Pétersb. Sér. 5, Q, 55 (1895).

Lockyer, N. On the canses which produce the phenomena of new stars. Phil. Trans. 182, $397(1891)$.

- On the variable stars of the $\delta$ Cephei class. Proc. R. Soc. London 59, 101 (1896).

Lo omis, F. C. Periodic stars. Inang.-Dissert. Göttingen, 1869.

II i iler, H. Über das Helligkeitsverhültniss der Doppelsternpaare. Astr. Nachr. 16, Nr. $364(1839)$.

Miiller, G. [Helligkeitsmessungen des neuen Sterns ím Andromeda-Nebel.] Astr. Nachr. 113, Nr. $2690\langle 1586\rangle$.

II üller, G. und Kempf, P. Photometrische Durchmusterung des nördlichen Himmels, enthaltend alle Sterne der B. D. bis zur Grösse 7.5. Theil I. Zone $0^{\circ}$ bis $+20^{n}$ Declination. Publ. Astrophys. Obs. Potsdam 9 1894.

Oudemans, J.A.C. Zweijährige Beobachtungen der meisten jetzt bekannten verinderlichen Sterne. Abhandl. d. mathem.-phys. Classe der K. Niederl. Akad. d. Wiss. 1856.

Über die Änderung der Helligkeit der Fixsterne zufolge der eigenen Bewegung in der Richtung der Gesichtslinie. Astr. Nachr. 137, Nr. 3275 1895).

Parkhurst, II. Ml. Photometric observaltions of the new star in Auriga. Astr. Journ. 11, Nr. $262: 1592$.

Observations of variable stars. Annals Harv. Coll. Observ. 99, s9 (1893).

Peirce, C.S. Photometric researches. Wade in the years 1872-i5. Annals Harv. Coll. Ous. 9 ( $15 ; 8$.

Peters, C. H. F. Über Ulugh Beg's Sterugrössen. Astr. Nachr. 99, Nr. 236i (1851). Pickering, E. C. [Photometrische Hessungen von Doppelsteruen.] Annals Harv. Coll. Observ. 11, 105, 277 (1579).

Dimensions of the fixed stars, with especial reference to binaries and variables of the Algol type. Proc. Amer. Acald. New Ser. S, 1 (1581).

Variable stars of short period. Yroc. Amer. Acad. New Ser. 8, 257 15\$1).

-.. Photometric measurements of the variable stars ${ }^{3}$ Persei and D. M. 81 ${ }^{\circ}, 25$, made at the Harvard College Observatory. Proc. Amer. Acad. New Ser. 8, 3701881 .

Observations with the IIeridian photometer during the years 1879-1ss2. Hallid Photometry.] Annals Harv. Coll. Observ. 14 1584-1885. 
Pickering, E. C. Magnitudes of circumpolar stars determined nt the obtervatories of Moscow and of Harvaril College. Astr. Nachr. 117, Nr. 2793 (185i).

Magnitudes of stars employed in various Nautical Almanacs. Annuls Hars. Coll. Observ. 18, 1 (1590).

Discussion of the Uranometrin Oxoniensis. Annals Harv. Coll. Observ. 18, $15\langle 1590\rangle$.

A photographic determination of the brightness of the stars. Anmals Harr. Coll. Observ. 18, 119 (15!0).

— Index to observations of variable stars. Annuls Harv. Coll. Observ. 18, 215 (1590;.

- Results of observations with the Meridinn photometer during the years 1552

-88. [Photometric revision of the Durchmusterung.] Aumals IIars.

Coll. Observ. 24 (1590).

The pholometric catalogues of the Harvard College Observutory. Astr. Ninelir. 135, Nr. 3229 (1894).

Comparison of the photometric magnitudes of the stars. Astr. Naclir. 137, Nr. 3269 (1595); Astrophys. Jouru. 1, 15. (1595). - Bemerkungen zu diesem Aufsatz von 'Turnor (Astr. Naclar. 137, Nr. 3274) unl ron Muller u. Kempf (Astr. Niclir. 137, Nr. 3279 (1895) )

Pigott, E. Observations and remarks on those stars which the astronomers of tho last century suspected to bo changeable. Phil. 'Trans. 76, 189 (1is6).

Plassmann, J. Die verinderlichen Sterne. Darstellung der wichtigsten Beobachtungs-Ergebnisse und Erkliirungs-Versuche. Köln, 1858.

Plumner, J. On the collective light and distribution of the fixed stars. Monthly Not. 37,436 (1577).

Pogson, N. Catalogue of 53 known variable stars, with notes. Astron. Obs. Radeliffe Observ. Oxford 15, 281 (155ii).

Pritchard, C. Photometrie determination of the relative brightness of the brighter stars north of the equator. Mem. R. Astr. Soe. London 47, 35:3 (1583.

- Uranometria nova Oxoniensis. A photometric determination of the magnitudes of all stars visible to the naked eyo from the pole to ten degrees south of the equator. Oxford, 155.5 .

Note on the comparison of the photometric magnitudes of the same stars observed at Harvard College and at the University Observatory, Oxforl. Monthy" Not. 45, 33 (1S55.

On some points of difference between the Harvard and Oxford stellar photometry. Monthly Not. 45, 111 1885.

Photometric Observations of the Nova Andromedac. Monthly Not. 4ti. is isst.

- Further experience regarding the magnitudes af stals as ohtained by photography in the Oxford University Observatory. Monthly Not. 51, 13:1 15:11.

Roberts, A.W. Certain considerations concerning the aceuracy of eye-estimates of magnitude by the method of serpenees. Astrophys. Iourn. 4. 194 159ti.

- Notes on a method of determining the value of the light-ratio. Astrophys. Journ. 4, 265 (896.

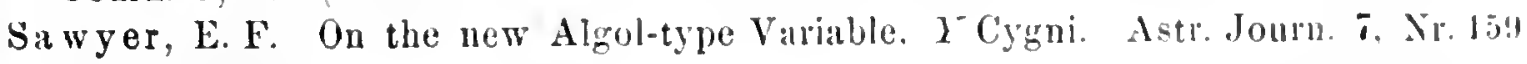
u. 161 (15S8).

Catalogue of the magnitudes of southern stius firom $11^{\prime \prime}$ to - $30^{n}$ declination, to the magnitude 7.0 inelusive. Mem. Amer. Acall. 12. 1 1593.

Sehaeberle, J. M. On the photographic brightness of the lixed stars. I'ubl. Astr. soc. of the Pacific 1. 5l 159!!. 
Scheiner, J. Untersuchungen über den Lichtwechsel Algols nach den Mannheimer Beobachtungen von Prof. Schönfeld in den Jahren 1869 bis 1875. Diss. intug. Bonn, 1882.

- Vergleichung der Grössenangaben der Südlichen Durchmusterung mit.denen anderer Cataloge. Astr. Nachr. 116, Nr. 2766 (1887).

Über die Bestimmung der Sterngrössen aus photographischen Aufnahmen. Astr. Nachr. 121, Nr. $2984(1899$; 124, Nr. 2969 (1890).

— Photographisch-photometrische Untersuchungen. Astr. Nachr. 128, Nr. 3054 (1S91).

Application de la photographie a la détermination des grandeurs stellaires. Bull. du Comité intern. pour l'exécution photogr. de la carte du ciel 1, 22i (1592). Schjellerup, C. Eine Uranometrie aus dem zehnten Jahrhundert. Astr. Nachr. 74, Nr. 1759 (1869).

- Description des étoiles fixes composée an milien du dixième siècle de notre ère par l'astronome Persan Abd-Al-Rahman Al-Sûfi. St.-Pétersbourg, 18 i4. Schünfeld, E. Beobachtungen von veränderlichen Sternen. Sitzungsber. der Wiener Akad. II. Cl. 42, 146 (1560).

Catalog von veränderlichen Sternen mit Einschluss der nenen Sterne. 32. Jahresbericht des Mannheimer Vereius für Naturkunde für $1 \$ 66$.

Zweiter Catalog von ver:̈̈nderlichen Sternen. Mit Noten. 40. Jahresber. des Mannheimer Vereins fiil Naturkunde fiir 1874.

Schönfeld, E. und Winnecke, A. Verzeichniss von veränderlichen Sternen zur Feststellnng ilırer Nomenclatur. Vierteljahrsschr. d. Astr. Ges. 3, 66 (1868).

Seeliger, H. Über den senen Stern im Andromeda-Nebel. Astr. Nachr. 113, $\mathrm{Nr} .2710$ 1856\%.

Seidel, L. Untersuchungen über die gegenseitigen Helligkeiten der Fixsterne erster Grösse und ïber die Extinction des Lichtes in der Atmosphäre. Abhandl. der Miinchener Akad. II. Cl. 6, $541(1550-1552$.

Resultate photometrischer Messungen an zweihundert nud acht der vorziiglichsten Fixsterne. Abhandl. der Muinchener Alad. II. Cl. 9, 421 (1863).

Thome, J. II. Cordoba Durchnusterung. Brightness and position of every fixed star down to the tentl magnitude comprised in the belt of the heavens between 22 and 32 degrees of sonth declination. Results of the Nittional Argentine Observatory. Yol. 16. Buenos Aires, $14,2$.

Togel, H. C. Resultate spectralphotometrischer Untersuchnngen. Monatsber. der Berliner Akad. 1850, sll.

Westphal, J.H. Über die periodisch verïnderlichen Sterne. Neneste Schriftend. naturf. Ges. zu Danzig. Bd. I. Ileft 2 (1 20 . - Ausserdem Zeitschr. fiir Astr. von Lindenan 11 . Bohnenlerger 4, 195, 316: 6, 252.

Über die verhïltniss:nässige Helligkeit der Sterne. Neneste Schriften d. naturf. Ges. zu Danzig. 18ะ0,61.

Wilsing. J. Untersuchngen iiber den Lichtwechsel ron $U$ Cephei. Astr. Nachr. $109, \mathrm{Nr} .2 .996 \mathrm{i}, 158 \mathrm{~s}$

Üher den Lichtwechsel Algols und iiber die Klinkerfues'sche Erklïrnng des rerinderliehen Lichtes bei Sternen der III. Spectralclasse. Astr. Nachr. 124, N1: 2960 15:10.

Wolt', M. Photographisehe Messung der Sternhelligkeiten im Sternhaufen G. C. 4410. Astr. Nachr. 126, Nr. 3019) (1 491.

Wolff J.Th. Photometrische Beobachtungen an Fixsternen. Leipzig. 1Sit. 
Wolff, J. Th. Photometrische Beolsachtungen an Fixsternen aus den Juhren 1576 bis 1553. Berlin, 1554.

Photometrische Arbeiten Uber dio Sterne der Bonner Durchmusternng. Vierteljahrsschrift der Astr. Ges. 28, 36it; 1857$\}$.

\section{Extinction des Lichtes in der Erdatmosphäre.}

A bnoy, W. On the atmospheric transwission of visual and photographically active light. Montbly Not. 17,260 (1557).

- Transmission of suilight through the earth's atmosphere. Plit. Truns. 178, 251 (1587); 184, I (1593).

Abney, W. and Festing, E. R. The influence of water in the ntmospliere on the solar spectrum and solar temperature. Proc. R. Soc. Iondon 35, 325 (185\%.

Angstrüın, K. Beitrïgo zur Kenntniss der Absorption der Wiirmestrahlen dureh die verschiedenen Bestandtheilo der Atmosphïre. Bilı. Svenskin Vet.-Acall. Hnndl. 15, Nr. $9\langle 1590\rangle$ - Siehe auch Wiedem. Ann. 39, $267\langle 1590\rangle$.

- Beobachtungen über die Strahlung der Sonne. Bil. Svenska Vet.-Acad. Handl. 15, Nr. 10. - Siehe anch Wiedem. Ann. 89, $294(1590 ;$.

Cornu, A. Sur la limite ultra-violette du spectre solaire. Compt. Rend. 88, 1101 (15i9).

Sur l'ubsorption par l'atmosphére des radiations ultra-violettes. Compt. Rend. 88, $1255(1879)$.

Observation de la limite ultra-violette du spectre solaire a diverses altitudes. Compt. Rend. 89, S0S ! 1579 \%

- Sur la loi de répartition suivant l'altitude le la substance absorbant dans l'atmosphère les rudiations solaires ultra-violettes. Coinpt. Rend. 90, 940 (1580. - Sur l'observation comparative des raies telluriques et métilliques, comme moyen d'évaluer les pouvoirs absorbants de l'atmosphère. Compt. Rend. 95, 801 1552.

Crova, A. Mesure de l'intensité calorifique des radiations solaires, et de leur absorption par l'atmosphère terrestre. Ann. Chim. et Phys. 5 11, 433 (1sii; 19 . $16 i$ (isso).

- Sur la transmissibilité de la radiation solaire par l'atmosphère terrestre. Compt. Rend. 104, $1475(1 \$ \varsigma i)$.

Elster, J. und Geitel, H. Beobachtungen des atmosphairischen Potentialgefailles und der ultravioletten Sonnenstrahlung. Theil 4: Über die Absorption des ultravioletten Sonnenlichtes in der Erdatmosphäre. Sitzungsher. d. Wienel Akad. II. Classe $101 \mathrm{IIa}$; 335 ; 1592 .

Forbes, J. D. On the transparericy of the atmosphere and the law of extinction of the solar rays in passing through it. Phil. Trans. 132. 2251442.

Frülich, 0 . Über das Gesetz der Alssorption der Sounenwiirme in der Atmusphiire. Meteor. Zeitschr. 5, 342 (1856.

Hausdorff, F. Über die Ahsorption des Lishtes in der Atmosphäre. Sitzungsber. Saichs. Ges. d. Wiss. 1895. 401.

v. Hepperger, J. Über den Einfluss der selectiven Absorption auf die Extinction des Lichtes in der Atmosphire. Sitzungsher. d. Wiener Akad. II. Cl. 105 IIa, 173 '1896.

Hill, S. A. On the constituent of the atmosphere which absorbs radiant heat. Proc. R. Soc. London 33, 2161552 . 
Hodgkinson, G. C. Actinometrical observations among the alps, with the description of a new actinometer. Proc. R. Soc. London 15, 321 (1867).

Langley, S. P. Olsservations on Mount Etna. American Journ. (3) 20, 33 (1850). Sunlight and skylight at high altitudes. American Journal (3) 24, 393; Nature 26, $586(1882)$.

The selective absorption of solar energy. American Journal (3) 25, 169 (1883). On the amount of the atmospheric absorption. American Journal (3) 28, 163 (1884).

- Researches on solar heat and its absorption by the earth's atmosphere. A report of the Mount Whitney expedition. Prof. papers of the Signal Service Nr. $15\langle 1884$.

Laplace, P.S. Traité de mécanique céleste. Tome IV, Chap. III: De l'extinction de la lnmière des astres dans l'atmosphère terrestre, et de l'atmosphère du soleil.

Maurer, J. Die Extinction des Fixsternlichtes in der Atmosphäre in ihrer Beziehung zur astronomischen Refraction. Diss. inaug. Zürich, 1882.

_- Über die atmosphärische Absorption ron strahlender Würme niedriger Temperatur und die Grösse der Sternenstrahlung. Vierteljahrsschr. naturf. Ges. Ziirich 34; Repert. d. Phys. 25, $642(1889$.

Michalke, C. Untersuchungen iiber die Extinction des Sonnenlichtes in der Atmosphäre. Astr. Nachr. 113, Nr. 2691 (1886).

Miiller, G. Untersuchungen iiber die Helligkeitsïnderungen in verschiedenen Theilen des Sonnenspectrums bei abnehmender Höhe der Sonne iiber dem Horizont. Astr. Nachr. 103. Nr. 2464 (1882).

- Photometrische Untersuchungen. Zweiter Abschnitt: Untersuchungen iiber die Extinction des Lichtes in der Atmosphäre. Publ. Astrophys. Observ. Potsdam 3, Nr. 12, 227 (1583).

- Photometrische und spectroskopische Beobachtungen, angestellt auf dem Gipfel des Süntis. Publ. Astrophys. Observ. Potsdam 8, Nr. 27, 1 (1891).

Pouillet, C. S. MI. Némoire sur la chaleur solaire, sur les pouvoirs rayonnants et absorbants de l'air atmosphérique et sur la température de l'espace. Compt. Rend. 7, 24 (183) .

Roscoe, H. E. and Baxendell, J. Note on the relative chemical intensities of direct suulight and diffuse daslight at different altitudes of the sun. Proc. R. Soc. London 15, 20 (186̣7.

Schaeberle, J. M. Terrestrial atmospheric absorption of the plotographic rays of light. Contrib. Lick Observatory, Nr. 3. Sacramento, 1 s93.

Schlagintweit, H. Bemerkungen über die Durchsichtigkeit der Atmosphäre und die Farbe des Himmels in grösseren Hühen der Alpen. Astr. Nachr. 31, Nr. it2 1851 .

Secchi, A. Considerazioni sulla vera maniera di valutare il raggiamento solare e ricerche sulla forza assorbente dell' atmosfera terrestre. Mem. dell'Osserr. dell'universiti Greg. del Collegio Rom. 1S51. App. II.

Seeliger, II. Über die Extiuction des Lichtes in der Atmosphüre. Sitzungsber. d. Miinchner Akad. II. Cl. 21, 24i 1591.

Seidel. L. Untersuchungen iiber die gegenseitigen Helligkeiten der Firsterne erster Grüsse und iiber die Extinction des Lichtes in der Atmosphäre. Abhandl. der Nliinchener Akad. 1I. Cl. 6, 511 15.50-1552. 
Trépiod, Ch. Sur la photométrio des étoiles et la transparenco do l'air. Compt. Rend. 82, $55 i$ (18i6).

Violle, J. [Absorption atmosphérique. Role de la rapeur d'eau.] Ann. Chim. et Phys. (j) 17, 433 (1879).

\section{Verschiodenes.}

Abney, W. On the photometry of the inagnetoelectric light. Proc. R. Soc. Iondon 27, 157 (18TS).

Abney, W. and Festing, E. R. On photometry of the glow lamp. I'roc. R. Soc. London 43, 247 (1858).

Adam8, W. G. The action of light on Selenium. Phil. Trans. 16i, 313 (157i).

Brennand, W. Photometric Observations of the sun and sky. Proc. R. Soc. I.on. don 49, 255 (1591).

Brosinsky, A. Über die Vergrüsserung des Erdschattens bei Mondfinsternissen. Diss. inaug. Berlin, 1559.

Bunsen, R. und Roscoe, II. E. Photo-chemical researches. Phil. Trans. 14;, 355, 381, 601 (155i); 149, 879 (1559); 153, 139 (1563). - Dentsch in I'ogg. Ann. 96,$373 ; 100,43,451 ; 101,235 ; 108,193 ; 117,529$.

Christie, W. I. M. On the relation between diameter of image, duration of exposure and brightness of objects in photographs of stars taken at the Royal observatory, Greenwich. Monthly Not. 52, 125 (1592).

Clausius, R. Über die Lichtzerstreumng in der Atmosphïire. Journ. f. reine u. angewandte Matliem. 34, 122 (1547).

Über die Intensitït des durch die Atmosphïre reflectirten Sounenlichtes. Journ. f. reine u. angewandte Nathem. 36, 195 (1845:

Conroy, J. Some observations on the amount of light reflected and transmitted by certain kinds of glass. Phil. 'Trans. 1S0, 245 (1589.

Crosby, W. 0. Light of the sky. Proc. Amer. Acad. New Ser. 21555.

Crova, A. Sur l'analyse de la lumiere diffusée par le ciel. Compt. Rend. 10 s. 493 (1899); Ann. Chim. et Pliys. (6) 20,4S0 (1>90, 25, 534 15:12\%.

Dobrowolsky, W. Die Empfindlichkeit des Auges gegen Lnterschiede der Lichtintensität verschiedener Spectralfarben. Monatsber. der Berliner Akad. 1872. 119.

Dove, H. Über den Einfluss der Helligkeit einer weissen Belcuchtung anf die relative Intensitiit versehiedener Farben. Monatsber. der Berliner Akad. 1852. 69.

Fizeau, H. L. et Foucault, L. Recherehes sur l'intensité de la luniere émise par le clarbon dans l'expérienee de Davy. Compt. Rent. 1S, 746 u. S60 1s.4.

Glan, P. Über die Intensitiit des rom Glase reflectirten Lichtes. Monatsber. der Berliner Akad. 1874, 511.

Gouy. Sar la mesure de l'intensití des raies d'absorption et les raies obseures du spectre solaire. Compt. Rend. S9, 10331579.

Hankel, H. Messungen iber die Absorption dur chemischen Strahlung des Sonncnlichtes. Ablundl. K. Siichs. Ges. d. Wiss. 11. Cl. 6. j3 1 blit.

Hartmann. J. Die Vergrösserung des Erdschittens bei Mondfinsternissen. |Ahhandl. K. Säcbs. Ges. d. Wiss. 11. Cl. 17. $363(1591)$.

- Die Beobachtung der IIondfinsternisse. Abhandl. K. Sichs. Ges. d. Miss. II. Cl. 23. $369(1596)$.

Künig, A. Über den Ilelligkeitswertlı der spectralfalben hei versehiedener absuluter Intensitiat. Beitriige zur Psychologie und Plysiologie der Sinnesorgane. Festsehrift fuir H. v. Helmholtz. Hamburg 15:1, 309. 
Kononowitseh, A. Bestimmung der Albedo des weissen Cartons unabhängig von der Lambcrt'schen Berechnung. Sehriften der math. Abth. d. Neuruss. Naturf. Ges. Bd. 2 (1879) Odessa. (In russischer Sprache.) - Referat in den Fortschr. d. Phys. 1879, 430 .

Über die Reflexion des Lichtes verschiedener Wellenlänge von der Oberfläche des Gypses. Sehriften d. K. Neuruss. Universitait. Bd. 29 (1880). (In rusisseher Sprache.)

Krïss, H. Über den Lichtverlust in sogenannten durehsichtigen Körpern. Abhandl. d. naturwiss. Vereins in Hamburg 11, Heft 1 (1890).

Lagarde, H. Mesure de l'intensité photométrique des raies spectrales de l'hydrogène. Compt. Rend. 95, 1350 (1882).

Langley, S. P. Energy and vision. Amer. Journ. (3) 36, 359 (1888).

v. Littrow, K. Zühlung der nördliehen Sterne im Bonner Verzeichniśs nach Grössen. witzungsber. der Wiener Akad. II. Cl. 59, Abth. II, 569 (1869); 61, Abth. II, $263(1870)$.

Olbers, W. Über die Durchsichtigkeit des Weltraums. Berl. Astron. Jahrb. für 1826, 110.

Piekering. W. H. Photometric researehes. Proc. of the Amer. Acad. New Series 7, $236(1850)$.

Preeee, W. H. On a new standard of illumination and the measurement of light. Proc. R. Soc. London 36, 270 (18\$4).

Pritehard, C. A photometric comparison of the light transmitted by certain. refracting and reflecting telescopes of equal aperture. Monthly Not. 45, 29 (1885).

Rayleigh, J. W. On the intensity of light reflected from certain surfaces at nearly perpendicular ineidence. Proc. R. Soc. London 41. 275 (1887).

Rood, O. N. Photometrische Untersuehungen. Zweiter Theil. Über die Lichtmenge, die durch polirte Crownglasplatten bei senkrechter Incidenz durchgelassen wird. Repert. d. Phys. 7, 213 (1871).

Roscoe, H. E. On a method of meteorological registration of the chemical action of total daylight. Phil. Trans. 155, 605 (1865); Pogg. Ann, 124, 353 (1865.

- On the chemical intensity of total daylight at Kew and Para, 1865-1867. Phil. Trans. 157. 555 (1867); Pogg. Ann. 132. $404(1867$.

Roscoe, H. E. and Thorpe, T. E. On the relations between the Suns altitude and the chemical intensity of total daylight in a clondless sky. Phil. Trins. 160, 309 (1570); Pogg. Ann. Ergiinzungsbd. 5, 177 (1871).

Sehumann. O. Über die Farbe und Helligkeit des elektrischen Glühlichtes. Elektr. Zeitschr. 5, 220 (1584).

śe eliğer. H. Über die Vertheilung der Sterne auf der nördliehen Halbkugel nach der Bonner Durchmusterung. Sitzungsber. der Miinehener Akad. II. Cl. 14, 5211884 .

___ Über die Vertheilıng der Sterne auf der siillichen Halbkugel nach Schünfelds Durchmusterung. Sitzungsber. der Miunchener Akad. II. Cl. 16, 220 1886\%.

- Die seheinbare Vergrösserung des Erdschattens bei Mondfinsternissen. Abh. der Mianchener Akad. II. Cl. 19. 345 1891;.

Stampfer, S. Üher den suheinbaren Durchmesser der Fixsterne. Denkschriften der Wiener Akıd. II. Cl. 5. $91 / 1952$.

stelling. E. Photuchemische Beobachtungen der Intensitï des gesammten Tageslichts in St. Petersburg. Repert. f. Meteor. 6. Nr. $6(1879)$ 
Stokes, G. On the inteusity of the light reflected from or transmitted trungil a pile of plates. Proc. R. Soc. Londen 11, 545 (1862).

Stonc, E. J. On apparent brightness as an indication of distance in stellar mases. Monthly Not. 87, 232 (1877).

Strehl, K. Ubber die Helligkoitsmessung der Gestirne. Klein's Wochenschr. 1891, 103. Tumlirz, 0. Das mechanischo Äquivalent des Lichtes. Sitzungaber. d. Wiener Akad. II. Cl. 98 I a, 526, 1121 (1889).

Vierordt, C. Die Phocomotrie der Franhofer'schen Linien. Whedem. Ann. 13, 339 (1851).

Violle, J. Sur l'étalon absolu de luwière. Ann. Clim. et Plıys. (6) 3, 373 (1654) Wober, L. Intensititsı́essungen des difusen 'Tageslichtes. Wiedern. Ann. 26, 374 (15S5).

Wild, H. Über die Lichtabsorption der Luft. Pogg. Ann. 134, 56s (1665); 135, 99 (1868).

Phorometrisclıe Bestimmung des difusen Himmelsliclıtes. Bull. Acıu. St.-Iétersb. 21, 312 (1876); 23, 290 (1577).

Wilsing, J. Über die Lichtabsorption astronouscher Objective und uber piotographische Photonetrie. Astr. Nichr. 142, Nr. 3400 (1897! 


\section{Namen- und Sachregister.}

Abblendung des Objectivs, Einwirkang der Bengung des Lichtes dabei 162-169. Abblendungsphotometer, bei denen die Auslöschung des Lichtes beobachtet wird 169-177; bei denen die Gleichheit zweier Lichteindrücke beobachtet wird 210-220.

Ablenkungsphotometer, von Parkhurst 17 -180 .

Abney, W. Transmissionscoefficient der Erdatmosphäre 138; Durchläissigkeit der Atmosphiire fiir verschiedene Wellenliingen 140; Nethode zur Bestimmung der Constante beim Keilphotometer 185: photographische Helligkeitsbestimmung der Sonnencorona 332 .

Abney, W. und Festing, E. R. Methode zur Bestimmung der Helligkeitsvertheilung im Sonnenspectrum 269.

Absorption, der Sonnenatmosphiire 324328.

Absorption, selective, der Erdatmospliare $139-144$.

Absorptionsphotometer 180-192.

Absorptionstheorie bei diffus reflectirenden Fläclien, von Lommel 44-52.

Actinometer, ron J. Herschel $2 s s$.

Airy, G. B. Beugungserscheinungen an Fenrohren 163.

Albedo, Begriffsbestimmung nach Lambert und Seeliuer 52-55: irllischer Substanzen 52; Formeln zur Berechnung derselben fiir einen Himmelskörper 64-65: des Mondes 343 ; des Mercur 355; der Venus 360: des Mars 373; der kleinen Planeten Ceres, Pallas, Vesta 3̈S0; des Jupiter : 393 ; der Jupitertrabanten 391: des Saturn 398; des Uranus 403: des Neptun 406.

Albert. L. A. Photoskop 191.

Algol, Lichtwechsel 495-496.

Algoltypus der Veriinderlichen $495-500$

Al-sifi. Hellinkeitskatalog 430.

Andersun. Th. D. Nora $T$ Aurigae ti.
Anding, E. Über selbstleuehtende Flïchen mit Mittelpunkt 35-37; iiber die Lichtvertheilung auf einer Planetenscheibe 68; Verfinsterung der Jupitertrabanten 102 .

Andromedanebel, Neuer Stern in denselben 476 .

Anthelm. Nova 11 Vulpeculae 474.

$\eta$ Aquilae, Lichtcurve 489.

Arago, Fr. Empfindungsgrenze 13: Polarisationsphotometer 240; Vertheilung der Helligkeit auf der Sonnenscheibe 318; Intensitïtsvertheilung anf der Mondoberfliche 344; Helligkeitsvertheilung auf dem Jupiter 3\$3; Polarisation des Cometenlichtes 409 .

Argelander, F. W. A. Photometrie kleiner Planeten 375; Uranometria Nova 435; Bonner Durchmusterung 438-142; Stufenschiitzungsmethode 459; Lichtwechsel von 3 Lyrae 489; Lichtwechsel von $\delta$ Cephei 490.

$\eta$ Argus, Lichtwechsel 482.

Ariel, Lichtstïke 404.

d'Arrest. H. Veränderliche Nebel 423 $-424$

Asch farbenes Mondliclıt, Theorie desselben $82-85$.

Asteroiden, Helligkeitsbestimmungen und Phasenlichtcurven 375-381.

Astrometer. von Knobel 171; von J. Herschel 200-204.

Astrophotometer. Züllner'sches 246-249; Potsdamer Form desselben 249; in Vel" bindung mit Refractoren 250; Abinderungen rou Ceraski 250; allgemeine iorscliriften iiber den Gebrauch desselben 251-253; Genanigkeit der Messuugen mit demselben 254 .

Atmosphäre der Erde. Extinction in derselben 110-138: selective Absorption derselben $139-144$.

Atmosphäre der Sonne. Absorptionswirkming 324-325. 
Ausgestrahlte Lichtmenge, Definltion des Begriffs 26.

Ausgleichung photometrischer Beobachtungen nach dem Fechner'schen Gosetz $17-18$.

Anslobchungspliotoneter, allgemeino Vorseliriften filr die Beobachtungen init denselben 153-15T, Ansloschungsphotometer mit Abblendung 169-179; Auslüschungsphotometer mit absorbirenden Medien 180-192.

A uwers, A. Helligkeitsschiitzungen der Jupitertrabanten $3 \$ 9$; Nova $T$ Scorpii 475.

Babinet, J. Polarisationsphotometer 243.

Bailoy, S. J. Helligkeitscatalog slidlicher Sterne 44!.

Bailly, J. S. Helligkeitsschätzungen der Japitertrabanten 386.

B aldwiu, H. L. Sichtbarkeit der Venus am Tage 358.

Barnurd, E. Durehgang des Iapetus durch den Schatten des Saturnsystems 101; Helligkeit der Sonnencoron: 334; Durchmesser der kleinen Planeten Ceres, Pallas, Vesta 379; verïnderliche Nebel $423-424$.

Bayer, J. Uranometrie 431.

Beor, A. Über das Lambert'sche Emanationsgesetz 30 .

Beer, W. und Mỉdler, J. H. Ilelligkeitsscala für den Mond 346; Helligkeit der Jupitertrabanten 385 .

Behrmann, C. Atlas des siidlichen Hinmels 436.

Beleuchtang, von Fliichen durch lelichtende Punkte 19-25; von Flaichen durch leuchtende Fliichen $25-38$.

Beleuchtung der Netzliaut 157-162.

Beleuchtungsgesetz, von Lambert 29-33. $39-40$; von Lommel-Seeliger $44-52$; von Euler 57.

Beleuchtungsmeridiane auf beleuchteten Flaichen 23.

Belenchtungsparallele auf beleuchteten Fliichen 23.

Beleuchtungstheorie, der Planeten 56-67; der Planetentrabanten $79-52$; eines Systems kleiner Körper $56-94$ : des Saturnringes 94-101.

Belopolsky, A. Spectroskopische Beobachtungen von \& Lyrac und $\delta$ Cephei $493-494$.

Berberich, A. Helligkeit des Enckeschen Cometen $\$ 16$.

r. Berg. F. Das Schwerd'sche Photometer 213.

Bernard, F. Polarisationsplotometer 242; Lichtiindermngen des Japetus 399.
Bessel, F.W. DioHelligkeitsschlitzungen dessulben vergllehen mit der Bonner Dirchmusterung 4.13.

Beuging des Lichtes an den Rlindern des Objectivs, Einfluss derselben auf Jichtmessungen 162-169.

Beugungstigur eines Sternes, Vertheilung ler Intensitiit innerhalb derselben 16.; Dimensionen der centrulen Bougungsfigur bei kreisformiger $\Delta$ bblendung lis.

Birmingham, J. Nova 'T' Coronae 4is.

Blenden, Verwendung derselben in der Photometrie $169-175$.

Blendkappe, beim Parkhurst'schen Photometer 178.

Blendscheibe, von 'Thury 1i2; beim Horastein'schen Zonenphotometer 219.

Blendvorrichtung, sectorfürnige 175.

Bolometer, von Iangley 291.

Bond, G. P. Verwendung spiegelnder Kugeln zn photometrischen Messungen 231; Anwendung der Photographie zu IIelligkeitsmessungen 297-29S; Helligkeitsverbialtniss von Sonue und Vollmond 314; Helligkeitsverhailtuiss zwischen Mond und len Planeten Jupiter und Veuss 33:; I,ichtstiirke der Mondpliasen 341-342; Lichtverthoilung auf der Mondscheibe 344; photographischo und optisehe Albedo von Jupiter 383.

Bonner Durchmusterung 43S-442; Vergleichung derselben mit der Potsdamel photometrisehen Durclunusterung 452.

Bouguer, P. limpfinlungsgrenze 13; Retlexionstheorie 41 ; Extinctionstheorie 116-122; Weglingen in der Erdatnosphitre und \%enitlureductionen 135 ; Transmissionscoefficient der Frdatmosphire 135; Sectorblenden 174; photometrische Apparate 195-196.211; Vervendung des Heliometer's zn photometrischen Mlessungen 212; Sonne und Kerzenlicht 30s; Sonne und Vollmond 313; Vertheilung der Helligkeit :uff der Sonnenscheibe 318 ; Mond und Kerzenlicht 33S; llelligkeitsverhiiltniss verschiedener Stellen der Mondoberfliche 31.4.

boys. C. V. Radiomikrometer 2911.

Bremiker. C. Helliekeit der Venus 356.

Brennpanktshilder in Fernroliren. Hellinkeit derselluen 158-160.

bruguicir. II. Sichthatkeit ler Venus am 'lage 35 .

Bruhns, ('. Durchmesser kleiner Planeten alms Helligkeitsschiitznngen $3 \backsim 0$.

bruns, II. Therorie dep Verinderlichen vom Aliroltyjus 496.

Buch holz. II. Japetusverfinstorung durch das batumersystem 101.

Bunses sclies lileckphotometer 199-200.

Bunsen. R. und Roscoe. H. L. Chemi- 
sches Photometer 292; photographische Lichtmessungen 296.

Burnham, S. W. Veränderliche Nebel $423-424$.

Cacciatore, N. Phasenerseleinungen der Cometen 408.

Calueron. Sichtbarkeit der Venus am Tage 358.

Campbell, W. Nova $R$ Normae 479 .

Cassini, D. Lichtsehwankungen des Jalpetus 399; Phasenerscheinungen am Cometen von 1744 408; Nova P Cygni und Nova Vulpeculae 474.

d' Cephei, Lichtenrve 490.

Ceraski, W. Einrichtungen am Zöllnersehen Photometer 250; Helligkeiten von Circumpolarsternen 446.

Chacornac, J. Sternphotometer 257259; Vertheilung der Helligkeit auf der Sonnenscheibe 319; Intensitït der Sonnenflecke 325 .

Chambers, G. F. Catalog verïnderlicher Sterne 465.

Chand ler. S. C. Lichtverh:iltniss zweier aufeinander folgenden Grössenclassen 457; Cataloge ron veränderlichen Sternen 465; Bezeichnung der Veriinderlichen 466; Tertheilung der Ver:inderlichen am Himmel 465; Algol 495.

C'harlier. C. Y. L. Beziehnng zwischen optisehen Sterngrössen und photographischen Durehmesseru 302; photographische Helligkeit der Plejadensterne 506: photographische Helligkeit Algols 510 .

Chemisehes Photometer, ron Bunsen und Roscoe 292.

Chemische Wirkung rerschiedener Partien der Sonnenscheibe 322.

Christie. W.H.M. Spiegelung der Tenusoberfliche 361 .

Colorimeter. am Züllner'schen Astrophotometer 245 .

Cumeten, Phasenerscheinungen und Eigenlicht 40s; polarisirtes Licht 409 ; speetroskopische Beobachtungen $\$ 10$ : Gesilmmtintensitit und Fliichenintensitiit 411: Beobachtungsmethoden 412; Eluelonisse 413-416: photometrische Messungen 417 .

Comet 1562 II Schmidt periodisehe Helligkeitsschwankungen 415.

comet 18 it III Coggia), Helligkeitsschitzungen ron sehmidt 413.

Cimet 1si2 I Wells, photometrische Messungen 415.

Comet 19541 Pons-brooks. photometrische Messmyen 415 .

Comet 1ssb I Fabry und 1ss6 II Baruard. photomerrische Mlessmgen $\$ 15$.
Copeland, R. Helligkeit des Mars 372.

Cornu, A. Verfinsterung der Jupitersatelliten 102,109, 392; photometrische Methoden 220.

Corona der Sonne, Helligkeitsbestimmungen derselben $329-335$.

Cosinusquadratgesetz, von Malus 236.

Crookes, W. Radiometer 291.

Crova, A. Verbesserung am Glan-Vogelschen Spectralphotometer 279; neues Spectralphotometer $280-281$.

Crova, A. und Lagarde. Methode zur Bestimmung der Helligkeitsvertheilung im Sonnenspectrum 268.

Curven gleicher Helligkeit, auf beleuchteten Fliichen 23-25; auf einer Planetenscheibe $70-73$.

Dimmerlieht, des Planeten Venus 361.

D a wes, W. R. Abblendungsphotometer 171; Verwendung von Diaphragmen zwisehen Objectiv und Ocular zu photometrischen Messungen 176; Keilphotometer 183.

Deflectionsphotometer, von Parkhurst 177 -180 .

Deimos, Lichtstïrke 374; Durelımesser 375.

De la Rue, Warren. Anwendung der Photographie zu Helligkeitsmessungen 297.

Dennett, F. C. Lichtrariationen der Jupitertrabanten 359.

Denning, W.F. Sichtbarkeit des Mereur mit blossem Auge 351.

Dichtigkeit der Belenchtung 26-27.

Dichtigkeit des Lichtes, Definition des Begriffes derselben 20.

Differentialthermometer, ron Leslie $28 \mathrm{~s}$.

Diffraction, Einfluss derselben auf Lichtmessungen 162-169.

Dione, Liehtstärke und Durchmesser 400.

Dorst, F. J. Bearbeitung der Zöllnersehen photom. Fixsternmessungen 444.

Dove, W. Helligkeitsverhältuiss rerschiedener Farben 11.

Draper, W. Methode zur Bestimmung der Helligkeitsvertheilung im Sonnenspectrum 26 s.

Dumbbell-Nebel. Helligkeit 420 .

D unér. $\mathbf{X}$. C. Lichtweehsel ron I Cygni und $Z$ Herenlis 497, 500.

Durehliissigkeitscoeffieient der Erdatmosphïre: Zusammenstellung der verschierlenen Bestimmungen 138; für verschiedene Welleuläugen 140.

Durchlässigkeitscocficienten der Sonnenatmosphïre fiir Strahlen rerschiedenel Wellenliuge $32 \%$.

Durhuesserbestimmung. photometrische, Formeln dafiir 66; der Marstrabanten 
375 ; der kleinen Planeten 375; derSaturnastelliten 400; der Uranustrabanten 40.; des Neptuntrabanteu 407 .

Durchmusterung, Bonner 435; Slldliche von Schünfelil 411; Potsdumer photometrische 450.

Kigoulicht, des Auges 14.

Eigenlicht, ler Cometen 410.

Ellipse, spluarische, die von ciner selbstleilchtenden ansgesand to lichtwenge 37.

Emanationsgesetz, Lambert'sches 29-33, 39.

Einanationswinkel 26.

Eulp findungsgrenze, bei Beurtheilung von Iutensitïtsunterschieden 13.

Enceladus, Helligkeit und Durehmesser 400.

Eucke'scher Couet, Lichtstiirke desselben 416.

Engelmann, R. Plotometrische Messungen der Jupitertrabanten 385.

Entfernungsgesetz in der Photomotrio 6 -i; die auf deinselben beruhenden Photometer $19 j-210$.

Erck, W. Ilelligkeitssehiitzungen der Marstrabanten 374.

Erdatmosphiire, Extinction des Lichtes in derselben 110-138; selective Absorption dorselben 139-144.

Erhebungen auf den Planetenoberflichen, Einfluss derselben anf dis scheinbare Helligkeit $74-i s$.

Espin, T. E. Vertheilnng der Veriinderlichen an Himmel 465 .

Euler, L. Abhïngigkeit der Helligkeit rom Emanationswiokel 25; Belenchtungsgesetz 57.

Exner, F. Vergleichung von Sonuenund Kerzenlicht 311.

Extinetion des Lichtes, in der Erdatmosphäre 110-144; in der Sonnenatmosphïre $324-32 \pi$.

Extinctionstabelle, fiir Minchen 131; fiir Potsdan 132, 515-516; fiir den Sintis 134,515 ; theoretische 135 .

Extinctionstbeorie, von Lambert 112-116; ion Bouguer 116-122; von Laplace 122-128; von Naurer 125-131.

Farben der Fixsterne, Einfluss auf Ilclligkeitsmessungen 453 .

Farbenphotometer, von A bney und Festing 269.

Fechner, G. T. Psychophysisches Grundgesetz 12-1s.

Fernrohr, Helligkeit der Brenupunktsbilder $15 \mathrm{~s}$.

Fizeau, H.L. und Foucalult, L. Photographische Lichtmessungen 295.
Fla mu arion, C. Liehtrariationen der Jupitersatelliten 359.

Fla ms teod, J.' Sterncutalog 132.

Fla ugergues, H. Helligkeltsscliatzungen iler Jupitertrabanten $35 \%$.

Fleckphotometer, von Bunsen 199-200.

Fluthliypothese, zur Erkliirung des Lichtweelisels der neueu und variubleu Sterno $480,485$.

Forbus, J. D. Selective Absorption der Erdatmosphiire 141.

Foueault'aches Photometer 197.

Fourier, J. Whirmeemanationsgesetz 30.

I'raunh ofer, J. Bestiminung der Liclitvertheilung in Sonnenspectrum 267.

Frost, E. B. Wainnewirkung verschiedener 'Theile der Sonnenscheibe 323; Strahlungsintensitiit der Sonnenflecke 329.

Glan, I. Spectralphotometer 275-250.

Glazobrook, R. 'I'. Spectralphotometer 251.

Goodricke, W. Veriinderliche Sterue $455,459$.

Go r e, J.E. Cattaloge veränderlicher Sterne 465.

v. Gothard, E. Registrirvorrichtıng beïı Keilphotoweter 185.

Gould, B. A. Uranometria Argentinu $436-437$.

Guuy. Spectralphotometer 281, 283.

Gouy und 'Thollon. Spectralphotometrische Untersuchnngen an der Sonue 322,325 .

Govi, G. Spectralphotometer 272-273.

Grüssenclassen de' Fixsterne, nach Schïtungen +25 ; nach photometrischen Nessungen 417; I.jchtverhiiltniss zweier aufeinauder foigenden $455-455$.

Gyldén, II. Veritmlerliche Sterne 4s4.

II a II, A. Helligheitsschätzungender Marstrabanten 374; der Uranustrabanten 404.

Hall, Maxell. Lichtiuderungen des Neptun 405.

I alley, E. Grüsster Glanz der V'enus 362. II a rding. K. I. Diimmerlicht d. Venus 341.

Il arkuess. W. Intensitiitsvertheilung in der Somencorona 334.

II arrington, M. W. Hulligkeitsinderungen der Vesta 376.

Hartwig. E. Benennung der Veriuderlielien 4titi; Nova is Andromedae 476.

Harvard Photometry, von Pickering 446: Verglei(hung derselben mit der Potsdamer photometrischen Durchmusterung 452.

Heis, E Atlas coelestis norns 435. 
Heliocentrische Zeiten der Epochen bei verïnderlichen Sternen 467 .

Heliometer, Verwendung desselben in der Photometrie 212.

Heliothermometer, von de Saussure $28 S$.

Helligkeit eines leuchtenden Elements, wirkliche, 27; scheinbare 29.

Helligkeit des Himmelsgrundes, Einfluss derselben auf die Beobachting des Verschwindens der Sterne 155 .

Helligkeitscataloge aus Schätzungen, ron Ptolemäus 428 ; von Sîfi 430 ; von Ulugh Begh, Tycho Brahe, Hevel, Bayer, Flamsteed 431 ; von W. Herschel 432; von J. Herschel 434; vou Argelander, Heis 435; von Behrmann, Houzean, Gould 436; von Argelander, Schönfeld, Krüger (Bonner Durchmusterung) 435--442; Vergleichung der verschiedenen Cataloge 443 .

Helligkeitscataloge aus photometrischen ILessungen, von J. Herschel, Seidel 4433 : von Zöllner, Peirce 4t4; von Wolff 445 ; von Pickering 446 ; von Pritchard 449: von Miiller und Kempf 450 .

Helligkeitslogarithmen, als Mass in der Astronomie 15.

Helligkeitsvertbeilung im Sonnenspectrum. Methoden zur Bestimmung derselben. von Friunhofer 267; von Vierordt 265: von W. Draper, Croval und Lagarde 26s: ron Abuey und Festing 269.

v. Hepperger, J. Helligkeit des verfinsterteu Hondes 101

Herschel, A. Apparat zur Bestimmung des grössten Glanzes der Venus 365.

Herschel, J. Astrometer 200-204; Actinometer 2S5; Helligkeitsverhältuiss zwischen IIond nud \& Centauri 339: Lichtstïrke der Mondpliasen 342; Helligkeitscaltalog siidlicher Sterne ans Sequenzen 434: Helligkeitscatalog aus photometrischen Messingen 443 .

Herschel. W. Sein photometrisches Verfilhren 211: Intensitit der Sonneuflecke 329: Helligkeitsschïtzmingen der Jupitertrabanten 356: Lichtschwankungen des Japetus 399: Schïtzungsscalal fiir Yebelflecke 415: Fixsternhelligkeiten 432.

Hevelius, J. Sterncatalog 431.

Ilimmelsg rund. Einfluss der Helligkeit desselben inf die Messungen mit den Anslüschunesphotometern 155.

Hind. J. R. Tora Ophiuchi tis.

II ind's rerïnderlicher Yebel 423.

$\mathrm{H}$ irsch, A. Yerwendung ron Bleudscheiben zwischen Objectir und Oenlar Z11 plotometrischen Vlessungen 175.

Holden. E.S. Coronallelligkeit 33t: Helligkeit der M:Irstralbanten 37t: Schiitzmugen der Urannstrabanten 404; Hellinkeit des Orionnebels t?2!
Horner, J. K. Auslüschungsphotometer 181.

Hornstein'sches Zonenphotometer 217 -219 .

Houzeau, J. C. Uranométrie Générale 436.

$\mathrm{H} \mathbf{n g}$ gins, W. Helligkeitsbestimmungen von Nebelflecken 419 .

H nyghens, Chr. Helligkeitsvergleichung vou Sonne und Sirins 316.

Hyperion, Helligkeit und Durchmesser 400.

Janson. Nora $P$ Cygni 474.

Janssen, P.J.C. Photographisches Photometer 298.

Japetus, Lichtvaliationen desselben 401.

Jenkins, B. G. Uranometria nova 0xoniensis 449 .

Incidenzwinkel, Gesetz vom Cosinus desselben 20.

Intensität des Lichtes, allgemeine Begriffsdefinition 5; physiologiscle Intensität 9.

Intensitätskreis, beim Zöllner'schen Astrophotometer $24 \overline{7}$.

Intensitïtsrertheilung, im Beugungsbilde eines Sternes 164-165.

Interferenz-Spectralphotometer von Trannin 252 .

Johnsou, S.J. Verwendung des Heliometers als Photometer 213; Lichtrerhältuiss zweier aufeinander folgenden Grössenclissen 456 .

Isophoten. Curven gleicher Helligkeit 23 $-25$.

Jupiter. Albedo 353 ; Helligkeitsvertheilung anf der Oberfläche 353; Zusammenstellung der von verschiedenen Beobachtern gefundenen Helligkeitswerthe $3 \varsigma 4$.

Jupitersatelliten, Theorie der Verfinsterung derselben 101-109; Helligkeitsbeobachtungen derselben $355-390$; Albedo derselben 391 .

Kalkspathprisma, achromatisirtes 234.

Katzenaugendiaphragma 169.

Kayser, E. Keilphotometer 1 \&3.

Keilphotometer. ron de Mastre, Quetelet, Schumacher 152; ron Kayser: Dawes, Pritchard 15.3; das Potsdamer Keilphotometer 194: Theorie des Keilphotometers 156: Bestimmung der Keilconstante 1ss: allgemeine Vorschriften iiber den Gebrauch desselben 191; parallaktisch anfgestelltes in Potsdam 19?.

Keilspectrilphotometer, Potsdamer $2 \$ 3$.

Kempf. P. Extinction in der Erdatmosph:ire 136.134; persönliche Unterschiede beim Keilphotometer 153; Potsdamer photometrische Durchmusterung 450 .

Kepler. J. Nova Serpentarii 4 it. 
Kies, J. Über den grossten Glanz ler Venus 364.

Kleine Planeten, Resul tate photometrischer Messungen 375.

Klinkerfues, W. Nene Sterne und Verinderliche $450,455$.

Knobel, E. B. Astrometer 171.

Köhlor, I. G. Abblendungsphotometer 169.

Kunig, A. Helligkeitswerth der Spectralfarben 11.

$\therefore$ Küvesligethy, R. Novi $S$ Andromedae 476 .

Kononow: tseh, A. K. Phaseălichteurve des Mar's 371; Helligkeit des Jupiter 384; Helligkeit des Saturn 397.

Kr tiger, A. Bonner Durchmusterung 438.

Kugeln, spiegelnde, Auwendung derselben zu photometrisehen Mlessungen 226-231.

Lalande, J. Helligkeitsschiltzungen der Fixsterne 443.

Lam bert, J. H. Emanationsgesetz 2933, 39-40; Begriff der Albedo 52; Extinctionstlieorie 112-116; Mond- und Kerzenlicht $33 i$.

Lam ont, J. F"ieherfürnige Abblendung eines Objectivs bei Lichtinessungen 175 .

Lampadius, W. A. Auslöschungsphotometer 180.

Langley, S. P. 'Transmissionseoefficient der Erdatmosphäire 138; Durehliissigkeit der Atmosphïre fiir Lieht von versehiedener Wellenlïinge 140; theoretisehe Untersuchungen iiber den Energieverlust in der Erdatmosphäre 141; Methode zur Bestimmung der Coustante heim Keilphutometer 189; Bolometer 290; Vertheilung der Wiirme auf der Sonnenseheibe 323 ; Strahlungsenergie der Sonnenfleeke 329.

La place, P. S. Abhängigkeit der Licht ausstrahlung vom Emanitionswinkel 28 Extinetionstheorie 122-128; Weglingen in der Erdatmosphaire und Zenithreduetionen 135 .

Lassell, W. Helligkeitsscliatzungen der Uranustrabanten 404.

Leslie, J. Differentialthermometer 258.

Leuchtende Flïchen mit Mittelpunkt, Theorie derselben 35-38.

Leuehtkraft eines leuclitenlen Elementes. Definition 2 fi.

Li a is, E. Ifelligkeitsvertheilung anf der Sonuenscheibe :319; Intensitiit der Sounenflecke $32 \subseteq$.

Licht, allgemeine Eigeuschuften nach der Undulationstheorie :-4; Begrill der Intensität 5 .

Lichtither, Bewegungsgleiclungen der 'Theilehen nach der Undulationstheorie 4 .
Lichtemptindliches P'upier, Anweutung dessellien in der Photonetrie 296.

Líchtgleichung, f. veriinderlielie Sterne 46i.

Lichtverthcilung, auf einer I'lanerenscheibe 67-7i; innerhalb der Bengungsfigur eines Sternes 163-105; in Sonnenspectrum $266-271$.

Lindomann, E. Genauigkeit der Messungen mit den Zöllner'sehen Pliotometer 254; photometrisehe Fixsternuessungen 446; Lichtverhaltniss zweier aufeinander folgenden Girüssenclassen 457; Nova 7' Aurigae 477; $\beta$ Lyrne 459.

Loekyer, N. Meteoritenhypothese 451, 485.

Loewy, M. Verwendung von Blendscheiben zwischen Objectiv und Ocular 17.

Lohise, 0. Photographische Wirkung des Jupiter 383 ; neue Sterno 480.

Lommel, E. Beweis des Lambert'scheu Emanationsgesetzes fúr selbstlenchtende Fliichen 31-33; Belenchtungsgesetz filir zerstreut reflectirende Substanzen d.1; Liehtvertheilung innerhalb dor Beugungsfigur eines Sternes 163-165.

3 Lyrie, Lichteurve 458.

Lyra-Typus der verïnderlichen Sterne 45i -495 .

de Maistre, X. Keilphotometer 15\%.

Mars, Phasenlichteurve 370; Zusammenstellung der von verschiedenen Beobachtern gefundenen llelligkeitswerthe 372; Albedo 373.

Narstrabinten, Ilelligkeit derselhen 373.

Il assoll, A. Empfindungsgrenze thes Anges 13.

Il anrer, J. Extinctionstheorie 124-1:13: Wegliingen in der Erdatmosphïre und Zenithreductionen 135.

Mereur, Sichtbarkeit mit blossem Auge 351; Helligkeitsmessungen 352; 1'hasenlichteurve 353; Albedo 355.

Meridianphotometer, von Pickering 262266 .

Messerschmitt, J. B. Zerstrent reftectirende Substanzen 411. 51.

Mimas, Lichtstitlke und Durehmesser 400. II inehin, (t. II. I'hotoelektrisehe Elemente $2 !$.

Mlira Ceti, Jichtwechsel 45:.

Mirn-Typus der verinderlichen sterne tol $-48 \%$

Mittelpunktsfliichen, selhstleuchtende. Theorie derselben 35 -35.

Möller. Wr. Experimentelle Bestitigung des Einanatịnnggesetzes fuir gliihente Kürper 33; Änderningen an Wild'schen Photometer $25 \%$. 
Mond, Intensitätsverhältniss desselben zur Sonne 312-315; Vergleichang mit Kerzenlicht 336-338; verglichen mit Planeten und Fixsternen 338-340; Albedo 343.

Mondlicht, aschfarbenes, theoretische Berechnung desselben 82-85.

Mondoberffïche, Vertheilung der Helligkeit auf derselben $344-347$.

Mondphasen, Lichtstärkc derselben 340343.

Nebelflecke, Schätzungsscala von Herschel 415; photometrische Methoden 419-421; photographische Helligkeitsbestimmungen 422 ; Veränderlichkeit 423-425.

Neptun, Oppositionshelligkeit 405; Albedo 406.

Neptuntrabant, Helligkeitsmessungen ron Pickering 406.

Netzhaut, die auf derselben hervorgebrachte Beleuchtnng 157-161.

Teue Sterne, Beobachtungen und Lichtcurven 473-178; Erklärungsversuche $479-481$.

Neutrales Glas, Verwendung desselben in Keilphotometern 190.

Newcomb, S. Helligkeitsschätzungen der Uranustrabanten 404.

Nicol'sche Prismen, iiber die Verwendung derselben in der Photometrie 235.

Tormalspectrum der Sonne, Helligkeitsrertheilung in demselben 270 .

Nova $S$ Andromedae 476.

Nora $T$ Aurigae 4i.

Nora $B$ Cassiopejae, Tychonischer Stern 473.

Nova $T$ Coronae 475.

Nova $P$ Cygni 47 i.

Nova $Q$ Cygni 476.

Nova $R$ Normae 4 is.

Nora Ophiuchi 475.

Nora T Scorpii 475.

Nova Serpentarii 47.

Nova 11 Tulpeculae 474.

\section{Oberon. Lichtstïrle und Durchmesser} 4 t).

Uijectivoffnung. Abhängigkeit der Lichtstälke ron derselben $159-165$.

Obrecht. A. Terfinsterung der Jupitersatelliten 102.

Ucularphotumeter. ron Steinheil 209; ron Cornu 220.

Olbers. W. Helligkeit des Mars 372: Helligkeitsschätzung des Saturn $39 \%$

() Mionis. Lichtäinderung 456 .

(1) ionnebel. Helligkeitsuntersuchungen 421.

() n l emans. J.A.C. Teriinderliche Sterne 450.490.
Parkhuirst, H. M. Deflectionsphotometer 177; Helligkeitsmessungen kleiner Planeten $377-378$.

Pasch en, F. Helligkeitsschätzungen des Biela'schen Cometen 415.

Peirce, C. S. UỦber das Ptolemäus'sche Helligkeitsverzeichniss d. Fixsterne 429; Bearbeitung der Helligkeitsschätzungen von W. Herschel 433; Helligkeitscatalog von Fixsternen 444; Lichtverhältniss zweier aufeinander folgenden Grössenclassen 457 .

Peters, C.F. Helligkeitsschätzungen der Frigga 378.

Phasenbelenchtung eines Himmelskörpers, 'Theorie derselben 55-64.

Phasenhelligkeit, des Mercur 353; der Venus 359 ; des Mars 371 ; der kleinen Planeten 3\%.

Phobos, Lichtstitire 374; Durchmesser 375.

Photoelektrische Elemente, von Minchin 294 .

Photographie, Anrendung derselben in der Photometrie 294-304.

Photographische Helligkeiten der Fixsterne $505-510$.

Photographisches Photometer, von Janssen 298.

Photometrische Durchmusterung, Potsdamer $450-451$.

Photometrische Oculare, ron Cornu 220.

Photometric Rerision of the Durchmusterung, von Pickering 448; Vergleichung clerselben mit der Potsdamer Durchmusterung 452.

Pickering, E. C. Photometrische Apparate 259-262; Neridianphotometer 262 - 266; photographisch-photometrische Methoden 304; Helligkeitsvertheilung auf der Sonnenscheibe 320; Helligkeit verschiedener Stellen der Mondoberfïche 345-346; Helligkeit des Mars 372; Lichtstärke der Marstrabanten 374; die Jupitersatelliten 391; die Satulnsatelliten 400; Helligkeit des Uranus 102; die Uranustrabanten 404; Lichtstärke des Neptun 405; der Neptunsatellit 406; photometrische Methode fiir Nebelflecke 420; Genanigkeit der Ptolemäus'schen Fixsternhelligkeiten 430: iber Sîfis Helligkeitsangaben 431: Bearbeitnng der Helligkeitscataloge von W. Herschel 433: Helligkeitsstufen der Argelander'schen Uranometria 435: Genauigkeit der Grössen der Bonner Durchmusterung 441: Harvard Photometry 446: Photometric Rerision 445; Schätzungsmethode bei verïinderlichen Sternen 460 : Classificirung der Veränderlichen 4il: Algol 498: Draper Catalog 503; photogralphische Helligkeitscataloge jos. 
Pickering, W. II. Photographische Helligkeit der sonnencorona 335; Helligkeit des Orionnebels 421.

Pigot t, E. Cutalog verïnderlielier Sterne 465; $y$ Aquilae 459.

Planctentrabianten, 'Tlieorie der Beleuchtung dersolben 7 ? -82.

Planetoiden, Resultate photometrischer Messungen 378.

Plassmann, J. Beobachtungen ron Lyrae 459.

Platean. A. F. J. Rotirende Scheilen in ller Photometrio 221.

Plejaden, photographisehe Helligkeit derselben 506.

Plummer, J. Mond verglichen mit Kerzenlicht 335; photometrische Vergleichung zwischen Vollmond und Venus 340; Uranometria nova Oxoniensis 449.

Pogson, N. Lichtierhältniss zweier anfeinander folgenden Grössenclassen 456; Catalog verïnderlicher Sterue 465.

Polarisation des Lichtes, Anwendung derselben in der Photometrie 231-266.

Polarisationsphotometer, von Arago 240; von Bernard 242; von Babinet 243; von Zöllner 244-254; von Wild 254-25ij; von Chacornac 25i-259; von Pickering 259-266.

Polarisationsprismen, die wichtigsten in der Photometrie benutzten 233-236.

Potsdam, Extinctionstabelle 132-136, 515 -516; photometrische Durclimusterung 450.

Pouillet, C. S. M. Pyrheliometer 259.

Prismenphotometer, von Steinheil, 'Theorie desselben 205; Beschreibung des anf der Niunchener Sternwarte befindlichen 207; parallaktische Aufstellung desselben in Vien 210.

P'ritchard, C. Transmissionsenefficient der Erdatmosphiire 138; Keilphotometer 183-184; Bestimmnng der Keilconstante 187; Uranometria nova Oxoniensis 449 -454 .

I'sychophysisches Gesetz. von F'echner 13 -15 .

P'tolemäus. Helljgkeitscatalog 424-430.

Purkinje, J. E. Physiologische Intensitiit 10 .

Pyrheliometer, von Ponillet 269 .

Quetelet, A. Anslüschnuspluotumeter mit absorbirender Fliissigkejt 151: Anslöschungsphotonneter mit keil 152.

Rildiometer, von Crookes 291.

Radiomikrometer, von boys $2 ! 0$.

Reflexionstheoric, von Bouguer $41-44$.
Registrirrorrichtung, beim Kellphotometer $185-156$.

Reissig. Abbloudungahotometer 1:0.

Rhea, Ilelligkeit und Durchmesser 100.

Rheiuauer, J. Das Lamiert'sche Fimauntionsgesetr 30.

Ringnebel in der I.eler, Helligkeit 120.

Ritchie, W. Optischey Plotometer 107; thermisches I'hotometer 255.

de la Rive, $\Lambda$. I'hotouseter 218.

Rochon'sches l'risma 233.

Roscoe, H. F. Chemisches Photoncter 292; photographische Photometrie 296; chomische Intensitiit verschiodener Partien der Souneuscheibe 323.

Rosén, P. G. Ilchtverhiiltniss z.weier aufeinander folgenden Grissenclassen 457.

Rotationsellipsoid, Belenchtungsformeln fiir dasselbe nach Seeliger 6i.

Rotirende Scheiben, itber die Verwendung derselben in der Photometrio 221-22\%.

Rotirencle Spiegel, ilber die Vorwendung dorselben zur Messung des Sonneulichtes 223.

Rildorff, I'. Anordnung des Bunsenschen l'hotometers 199.

Ruinford, B.'T'l. Schattenphotometer 195 -199; 'Thermoskop 2ss.

Siotis, Extinctionstabelle 134,515

Saturn, Phaseneinfluss anf die llelligkeit 394; Abhingigkeit der lichtstirke von der Lange des linges 394; Darstellung der Beobatitungren durch die l'orumeh ron \%üllner und ilie Suchligur'sche 'Ther. rie 397; \%usammenstellung der von verschiedenen bioblichtern erlalten Wertbe 397 ; Albedo $3: 16$; Ilelligkeiss.

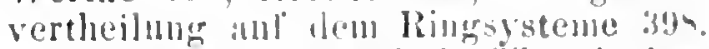
saturnong, die Seeliger'sche 'Therie desselben :H-101: 'Jibelle \%ur Iiernetion photometrischer Messungen les Satmprn ant versehwundenen Ring 390.

saturnsatelliten, photometrische Messmren rou lickuring fun.

desinussure, 11. B. Melinflurmometer2an.

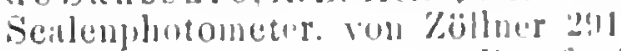

schattenphotometer. rom Rumford 1!n$19 ! \%$

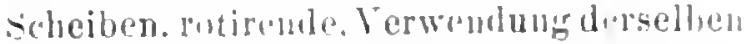
in der $\Lambda$ strophutometrie 221 - 225.

scheinbare Helligkoit. oiner lenchtenden Fliiche $2-35:$ ainer helenchteten l'lanetenscheibe $6:-7:$

selheiner. I. Buikhng zwischen optischen Stemeriossm nud photographi-

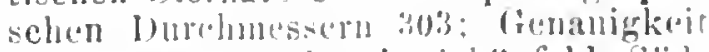
her Gröscuangiblen in sehönfelds Sid licher Durehmustermun 141 ; Algol 49\%. 
Schlackenhypothese, von Zöllner, zür Erklärung des Lichtwechsels veränderlicher Sterne 483.

Schmidt, J. F. J. Helligkeitsbeobachtungen des Mercur 352; Helligkeitsschaitzungen des Mars 372; Helligkeitsschätzungen des Saturn 39i; Beobachtungen der Cometen 1850 I, 1853 III, 1854 II, 1862II, 1874 III 415; periodische Lichtschwankungen des Cometen 1862 II 415-416; neue Sterne 475-476.

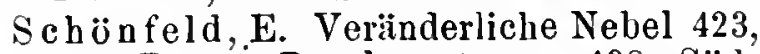
425; Bonner Durchmustermng 438; Südliche Durchmusterung 441; Cataloge veräinderlicher Sterne 465; Lich twechsel von $\beta$ Lyrae 48s; Lichtwechsel von Algol 495.

Schrüter, J.H. Helligkeitsscala für den Nond 346; Helligkeitssehätzungen der Jupitertrabanten 387 ; Lichtvariationen der Saturntrabanten 399.

v. Schumacher, C. D. Koilphotometer 182.

Schur, W. Anwendung des Heliometers zII photometrischen Messungen 213; $\beta$ Lyrae $489 ; \delta$ Cephei 490.

$\mathrm{S}: \mathrm{hwab}, \mathrm{F}$. Helligkeitsschätzungen der Cometen 1881 III und 1 SSI IV 415.

Schwerd'sches Photumeter 213-217.

Searle, A. Über die Züllner'sche Theorie der Ilondphasen 77.

Searle, G. Photometer 219.

Secchí, A. Über die Verwendung der rotirenden Scheiben in der Sternphotometrie 223; Wärmevertheilıng auf der Sonnenscheibe 323 .

Sectorblenden, vor dem Objectiv eines Fernrohrs 175 .

Seeliger, II. Ausgleichung photometrischer Beobachtungen 18; zerstreut reflectirende Substanzen 40, 51 ; die Bongner'sche Reflexionstheorie 4-44: Begriff der Albedo 53-55; Beleuchtungsformeln fiir ein Rotationsellipsoid $b i$ : iiber die Zölnner'sche Theorie der Mondphisen i7; Belenchtung eines Systems kleiner Körper $56-94$; Belenclitungstheorie des Saturnsystems 94-101; Verfinsterung der Jupitersatelliten 102109; Extinction des Lichtes in der Erdatmosplaïre 144; Absorption der Sonnenatmosphäre 325-32s: Darstellung der Saturnbeobachtungen durch die Theorie 397; Einfluss der Abplattmug anf die llelligkeit des Planeten Uranus 403 nelle sterne $4 \$ 1$.

Seidel. L. Rechmngsverfahren bei photometrischen Messungen 18; Extinctionstahelle fiir Niiuchen 1:31; Transmissionscoefficient der Erdatmospliäre 1:38:
Helligkeit der Venus 36s; Helligkeit des Mars 372 ; 'Helligkeit des Jupiter 384 ; photometrische Messungen des Saturn 397; Helligkeitscatalog -443; Lichtverhältniss $\mathrm{z}$ weier aufeinander folgenden Grössenclassen 457 .

Selective Absorption, der Erdatmosphäre 139-144.

Selenphotometer, von Siemens 293.

Sequenzen, Schätzungsmethode von J. Herschel 434 .

Sichtbarkeit der Sterne am Tage 12.

Siemens, W. Einwirkung des Lichtes auf Selen 293-294.

Sonne, verglichen mit Kerzenlicht 308312 ; verglichen mit Vollmond 312-315; verglichen mit Fixsternen 316-318; Vertheilung der Helligkeit anf der Sonnenscheibe 318-323.

Sonnenatmosphäre, Absorptionswirkung derselben 324-328.

Sonnencorona, Helligkeit derselben 329 335.

Sonnenflecke, Helligkeit derselben 328329.

Sonnenspectrum, Vertheilung der Helligkeit in demselben 266-271.

Spectralphotometer, von Govi 2i2; von Vierordt 273; von Glan-Vogel 275; von Crova 280; vou Gouly und vou Glazebrook 2S1; von Trannin 282; mit Absorptionskeil 283.

SpectralphotometrischeBeobach tungen der Fixsterne 500-505.

Spiegelnde Kugeln, ïber die Verwendung derselben in der Photometrie 226-231.

Spiegelsextant, seine Verwendung als Photometer 212.

Spitta, E. J. Bestimmung der Constante beim Keilphotometer 18s; Lichtstärke der Jupitertrabanten 390.

Stampfer. S. Transmissionscoefficient der Erdatmosph:ire $13 \mathrm{~s}$.

Stechert, C. Helligkeitsschïtzmgen des Planeten Tyche $3 i s$.

Steinheil, C. A. Empfindungsgrenze des Auges 13: Prismenphotometer 204205 ; Ocularphotometer 209 ; Verwendung von spiegelnden Kugeln zu photometrischen Messungen 230; Helligkeitsverhältniss ron Ifond und Aretur 33s; Lichtverhïltniss zweier anfeinander folgenden Grössenclassen 456 .

Strange, D. P. Helligkeitsvertheilung anf der Somnenscheibe 320.

Struve, 0 . Ver:inderlicher Nebel 424

Struve, W. Schätzungsscala für Fixsterne 43 s.

Stufenschützungsmethode, von Argelander $459-464$. 
Tulbot, W. H. F. Anwendnng rotirender Scheiben in der Photometrie 222.

Tow pel, E. W.I. Struves veriinderlicher Nebel 424.

Theruoskop, vou Rumford 288.

Thetye, Lichtstiirke und Durchinesser 400.

Thome, J. M. Cordoba-Di:rchinusterung 438.

Thomson, W. Vergleichung von Sonnenund Korzenlicht 310 ; Vergleichung von Mond- und kerzenlicht 338.

'Thorpe, 'T. E. Helligkeitsbestinmung der Sonnencorona 331.

Thury, M. Photometer mit Blendscheibe 172.

Tietjen, F. Helligkeitsschiitzungen der kleinen Planeten Meleto und Niobe $3 i 8$.

Titan, Helligkeit und Durchmesser 400.

Titania, Lichtstïrke und Durchmesser 404:

'Townley, S. D. Photographische llelligkeit von Algol 510.

Trannin, H. Interferenz-Spectralphotouneter 282.

I'ransmissionscoefficient der Erdatmosphïre, Zusammenstellung der verschicdenen Bestimmungen 135; fiir verschicdene Wellenlängen 140.

Transmissionscoelficienten der Sonnenntmosphäre $324,327$.

Trépied, C. Transmissionscoefficient der Erdatinosphiire 138.

Tronvelot, L. Ilelligkeitsvertheilung auf den Suturnringen 398.

Tycho Bralie, Sterncataloge 431; neuer Stern in der Cassiopeja 473.

Ulugh Begh. Helligkeitseatalog 431 .

Umbriel, Lichtstïrke 404.

Undulationstheorie des Lichtes 3.

Uranometria Argentina, von Gould 436.

Uranometria Nova, von Argelander 435.

Uranometria novit Oxoniensis 449; Vergleichung mit der Potsdamer photometrischen Durchmnsterung 452.

Uranus, Oppositionshelligkeit 402; Albedo 403; Einfluss der Alpplattung ulf die Helligkeit 403 .

Uranustrabanten. Helligkeitsschiitzungen nnd photometrische Messungen 4114.

Venus, Helligkeitstabelle 356 ; Sichtbarkeit am Tuge mit blossem Ange 35s: Phasenlichtcurve 359; Albedo 360; spiegelnde Eigenschaft 361; Dimmerlicht 361: grösster Glanz 362-365: Zusanmenstellung der von verschiedenen Beobachtern gefundenen Helligkeitswerthe 365 .

Veriinderliche Nebel $423-425$

Veriinderliche Sterne, Schaitzungsmethode von Argelander 459: Zahl derselben und
Catalogo 465; Benenuung 46ib; lieliocentrische Zoiten der Fpochen 467 ; Verthellung an Himmel 465; Cruppiruog nach der Poriodenlïnge 169 ; Znsammenhang zwischen Farbe und Periodenlikng 170; Classificirung nach l'jekering til.

Verfinaterung der Jupitersntelliteu, Theorie derselben $101-109$.

Vergrösserung cines Fernolira. Iinfluss derselben anf die Bildhelligkvit 161.

Yerschwindung 3 - P'hotometer 153-192.

Vierordt, C. Methode z.ur Bestiumung der lichtvertheilung in Sonnenspectrum 265; Spectralphotoweter 273-275.

Vogel, H. C. Spectralphotoneter 2i5; Ifelligkeitsvertheilung atf der Sonnen. scheibe 320; Vertheilung der chenischen Intensitiit auf der Sonnenscheibe 322; Warmevertheilung auf iler Sonnenscheibe 323; 'Transmissionscoefticienten der Sonnenatinosphitire 324; Mercur 352; newe Sterne 481; spectroskopische Be:obachtungen von \& Lyrae 493: Algolsystem 499 ; spectralphotometrische Mlegsungen au Fixsternen 502.

Wiirmeintensitiit verschiedencr 'I'heile der Sonnenscheibe 323.

IVeglïngen in der Fidatmosphiire, Tabelle derselben nuch den Theorien von Bonguer, Laplace und Maurer 135.

Wellmanu, V. Verfinsterung der Jupitertrabanten 102.

IVild, ll. Polarisationsphotomoter 254 -257 .

Wilsing. J. Nene sterne und Verindlerliche $480,455$.

Winnecke, 1 . Periodisch verinderliche Neleel 425

Wolff, 'Th. 'I'ransmissionseuefficient der Frolatmosphiire 1:15; photometrische Castilloge 445: Lichtverhältniss zweier auteinander folgenden Grössenclassen $455^{\circ}$

Wollaston, F. Verwerdung von spiegelnden kingeln zu photometrischen Messungen 230: Polarisationsprisma 234: Sonne mud Kerzenliclıt 309 : Sonne und Vollmond :313; Somne mul sirins 316: Ilond und Kerzenlicht 335

Young. C. A. Ïluer lats keilphotometer $1,1$.

Zenger, C. V. Ilelligkeit der Jupitertribanten 35 !.

Zerstrent reflectirende Substanzen 3y-52.

Ziillner. Fr. Lamberts Linanationgesetz 30; Alleelowerthe irdischer substanzen 52 ; Einfluss der Erhebungen ant lie 
Lichtstärke eines Himmelskörpers 7578; Genauigkeit der photometrischen Messungen von J. Herschel 203; Polarisationsphotometer 244-254; Scalenphotometer 291; Sonne und Vollmond 314; Sonne und ic Aurigae 317; Lichtstärke der Mondphasen 342; Mercur 352; Venus 368 ; Mars 372 ; Jupiter 384 ;
Formel zal Reduction der Saturnbeobachtungen 395 ; . Lichtstärke des Saturnsystems 397; Uranus 402; Neptun 405; Helligkeitscatalog von Fixsternen 444; Lichtverhältniss zweier aufeinander folgenden Grössenclassen 45i; neue Sterne 479; Schlackenhypothese 483.

Zonenphotometer, von Hornstein 217 .

\section{Berichtigungen.}

pag. 8 Zeile 2 von unten lies: $\cos \vartheta$ statt: $\vartheta$.

-22 $>2$ > das Complement des Winkels $i$ statt: der Winkel $i$. -40>1,D, Messerschmitt.

$\Rightarrow 72>10$ oben $\Rightarrow 1-2 b \cos a+b^{2}$ statt: $1+2 b \cos a+b^{2}$.

- \$5 In der Formel für $\frac{h_{1}}{h_{1}^{\prime}}$ lies im Nenner: $\cos \omega^{\prime}$ statt: $\cos \omega$.

- 160 Zeile 6 von oben lies: dasselbe statt: desselbe.

$>365>6$, unten $V s \cos (\pi-a)$ statt: $V s \cos \alpha$.

-38S .2 » oben " Trabant 4 statt: Trabant 3.

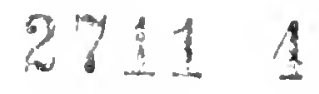




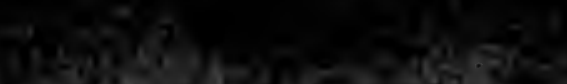

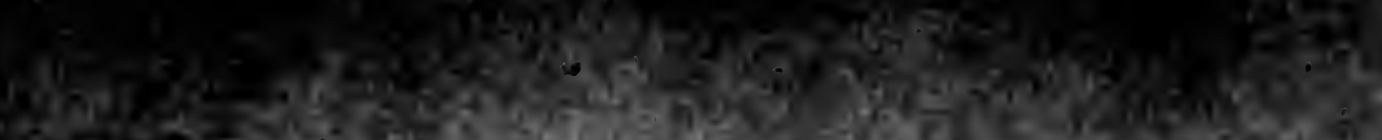

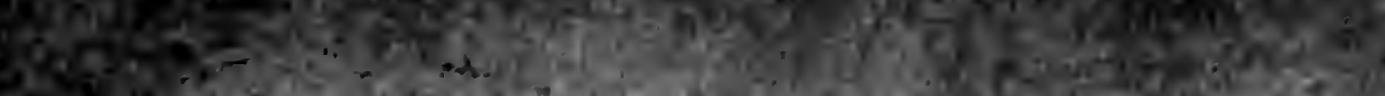

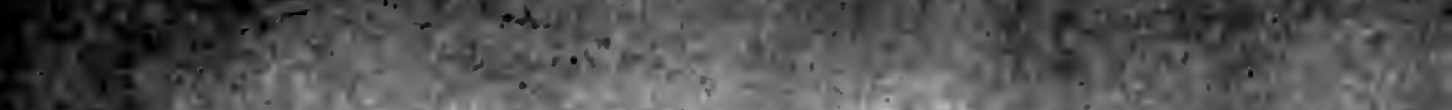

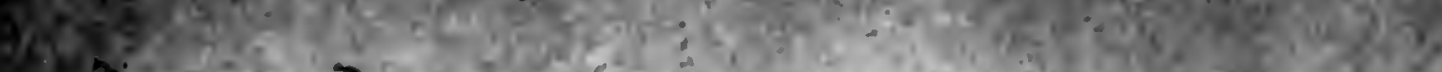
i.

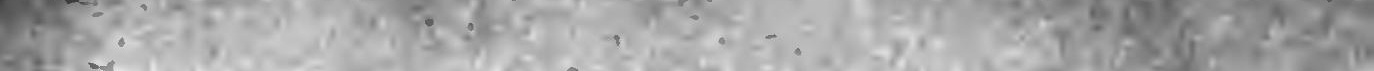

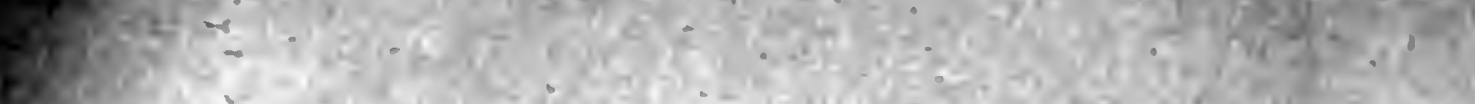
(2)

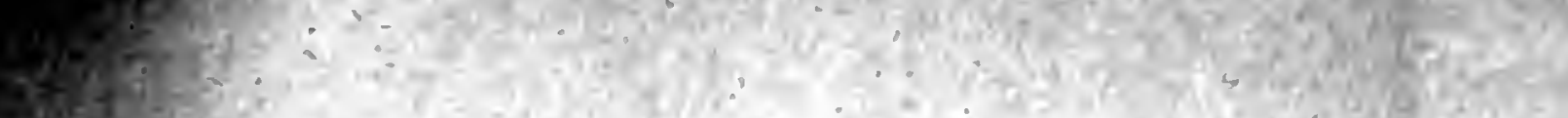

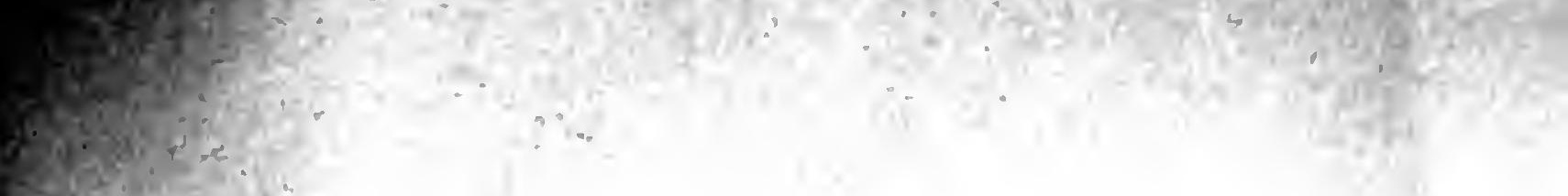





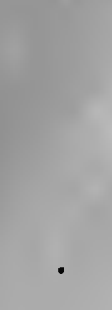

8

$x^{2}+x^{2}$

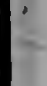

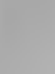





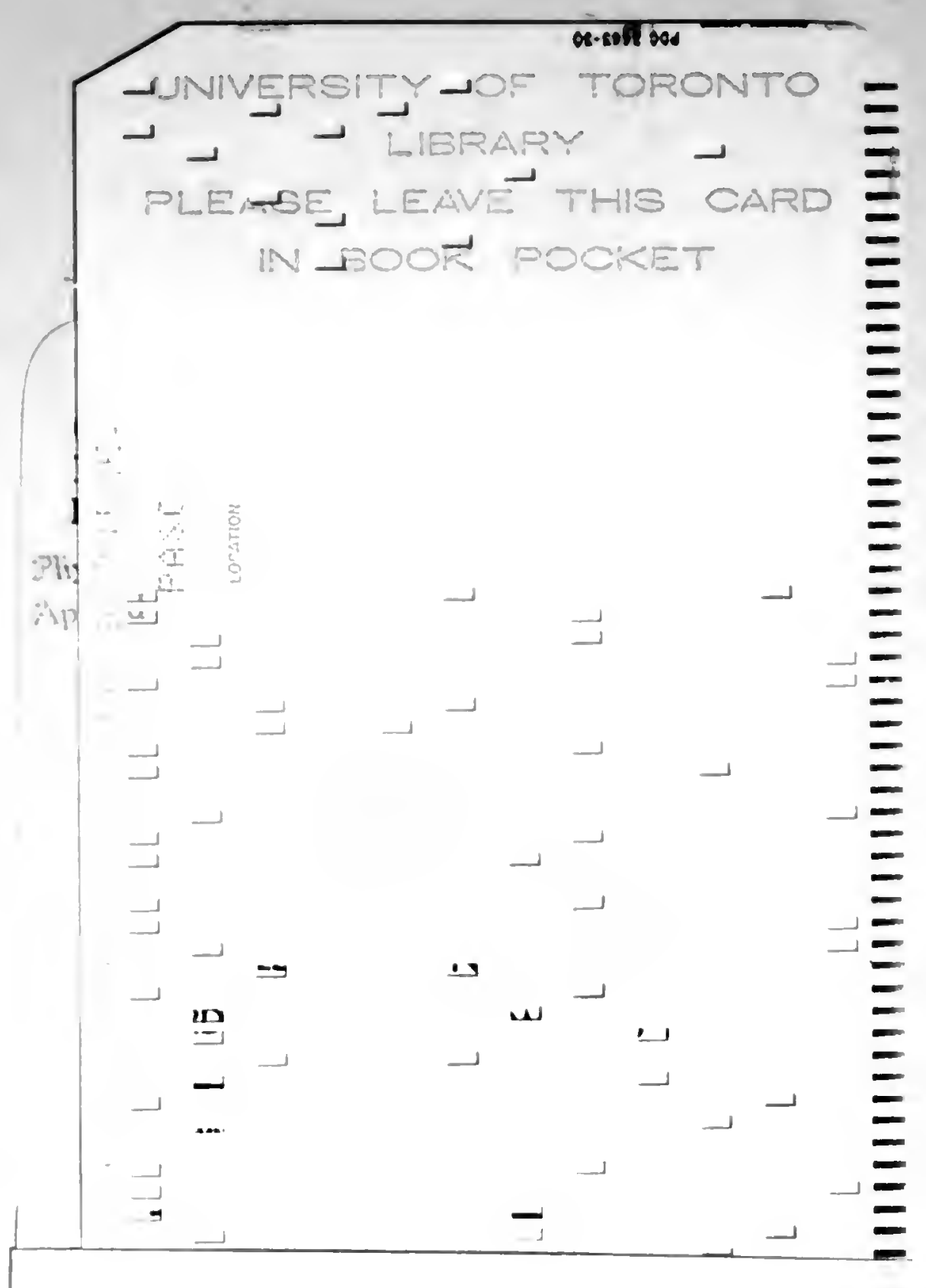




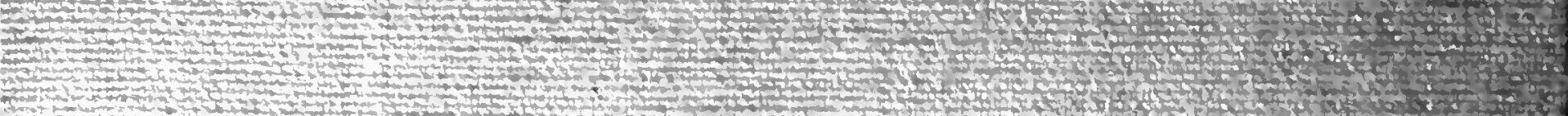

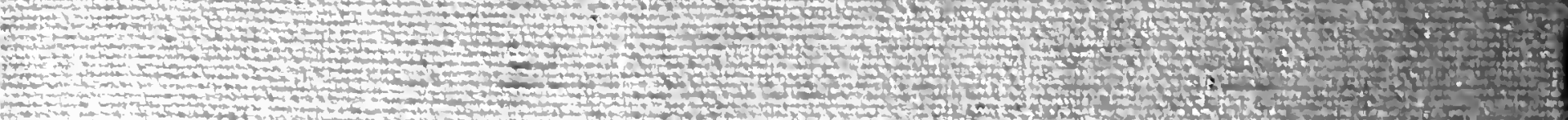

